\title{
ETHNOPHARMACOLOGY IN CENTRAL AND EASTERN EUROPE IN THE CONTEXT OF GLOBAL RESEARCH DEVELOPMENTS
}

EDITED BY: Judit Hohmann, Anna Karolina Kiss, Judith Maria Rollinger and Atanas G. Atanasov

PUBLISHED IN: Frontiers in Pharmacology

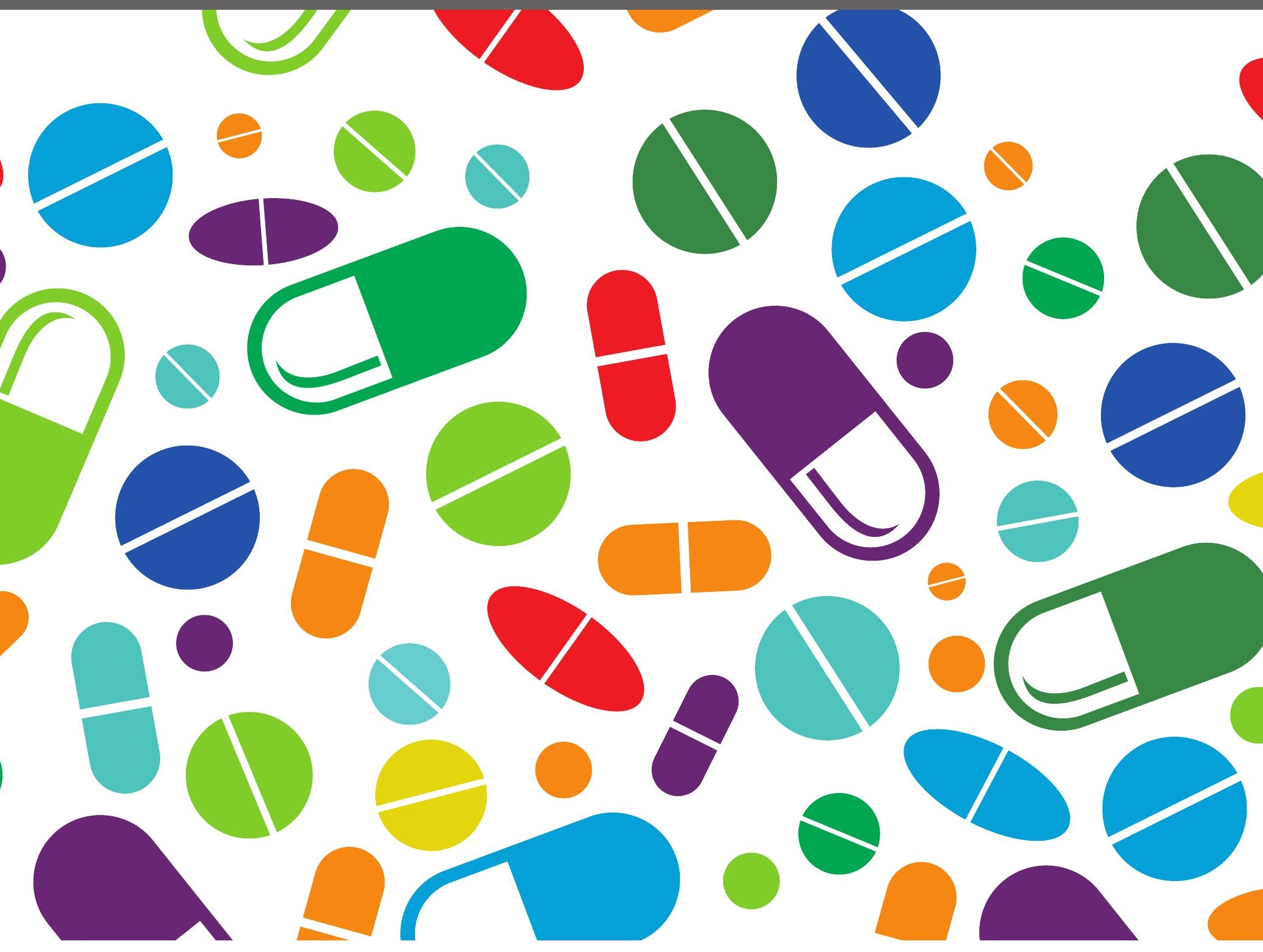




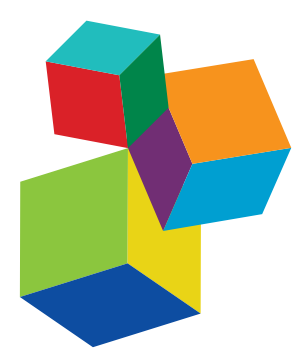

Frontiers Copyright Statement

C Copyright 2007-2019 Frontiers Media SA. All rights reserved.

All content included on this site, such as text, graphics, logos, button

icons, images, video/audio clips, downloads, data compilations and

software, is the property of or is

licensed to Frontiers Media SA

("Frontiers") or its licensees and/or subcontractors. The copyright in the text of individual articles is the property of their respective authors, subject to a license granted to Frontiers.

The compilation of articles constituting this e-book, wherever published, as well as the compilation of all other content on this site, is the exclusive property of Frontiers. For the

conditions for downloading and copying of e-books from Frontiers website, please see the Terms for Website Use. If purchasing Frontiers e-books from other websites or sources, the conditions of the website concerned apply.

Images and graphics not forming part of user-contributed materials may not be downloaded or copied without permission

Individual articles may be downloaded and reproduced in accordance with the principles of the CC-BY

licence subject to any copyright or other notices. They may not be re-sold as an e-book.

As author or other contributor you grant a CC-BY licence to others to reproduce your articles, including any graphics and third-party materials supplied by you, in accordance with the Conditions for Website Use and subject to any copyright notices which you include in connection with your articles and materials.

All copyright, and all rights therein, are protected by national and international copyright laws.

The above represents a summary only.

For the full conditions see the Conditions for Authors and the Conditions for Website Use.

ISSN 1664-8714

ISBN 978-2-88945-890-5

DOI $10.3389 / 978-2-88945-890-5$

\section{About Frontiers}

Frontiers is more than just an open-access publisher of scholarly articles: it is a pioneering approach to the world of academia, radically improving the way scholarly research is managed. The grand vision of Frontiers is a world where all people have an equal opportunity to seek, share and generate knowledge. Frontiers provides immediate and permanent online open access to all its publications, but this alone is not enough to realize our grand goals.

\section{Frontiers Journal Series}

The Frontiers Journal Series is a multi-tier and interdisciplinary set of open-access, online journals, promising a paradigm shift from the current review, selection and dissemination processes in academic publishing. All Frontiers journals are driven by researchers for researchers; therefore, they constitute a service to the scholarly community. At the same time, the Frontiers Journal Series operates on a revolutionary invention, the tiered publishing system, initially addressing specific communities of scholars, and gradually climbing up to broader public understanding, thus serving the interests of the lay society, too.

\section{Dedication to Quality}

Each Frontiers article is a landmark of the highest quality, thanks to genuinely collaborative interactions between authors and review editors, who include some of the world's best academicians. Research must be certified by peers before entering a stream of knowledge that may eventually reach the public - and shape society; therefore, Frontiers only applies the most rigorous and unbiased reviews.

Frontiers revolutionizes research publishing by freely delivering the most outstanding research, evaluated with no bias from both the academic and social point of view. By applying the most advanced information technologies, Frontiers is catapulting scholarly publishing into a new generation.

\section{What are Frontiers Research Topics?}

Frontiers Research Topics are very popular trademarks of the Frontiers Journals Series: they are collections of at least ten articles, all centered on a particular subject. With their unique mix of varied contributions from Original Research to Review Articles, Frontiers Research Topics unify the most influential researchers, the latest key findings and historical advances in a hot research area! Find out more on how to host your own Frontiers Research Topic or contribute to one as an author by contacting the Frontiers Editorial Office: researchtopics@frontiersin.org 


\section{ETHNOPHARMACOLOGY IN CENTRAL AND EASTERN EUROPE IN THE CONTEXT OF GLOBAL RESEARCH DEVELOPMENTS}

Topic Editors:

Judit Hohmann, University of Szeged, Hungary

Anna Karolina Kiss, Medical University of Warsaw, Poland

Judith Maria Rollinger, University of Vienna, Austria

Atanas G. Atanasov, IGAB PAS, Poland; University of Vienna, Austria

Citation: Hohmann, J., Kiss, A. K., Rollinger, J. M., Atanasov, A. G., eds. (2019). Ethnopharmacology in Central and Eastern Europe in the Context of Global Research Developments. Lausanne: Frontiers Media.

doi: 10.3389/978-2-88945-890-5 


\section{Table of Contents}

06 Editorial: Ethnopharmacology in Central and Eastern Europe in the Context of Global Research Developments

Devesh Tewari, Judit Hohmann, Anna K. Kiss, Judith M. Rollinger and Atanas G. Atanasov

09 Biological Activity of Flavonoids and Rare Sesquiterpene Lactones Isolated From Centaurea ragusina $L$.

Ulrike Grienke, Sandra Radić Brkanac, Valerija Vujčić, Ernst Urban, Siniša Ivanković, Ranko Stojković, Judith M. Rollinger, Juran Kralj, Anamaria Brozovic and Marijana Radić Stojković

24 Berberine: Botanical Occurrence, Traditional Uses, Extraction Methods, and Relevance in Cardiovascular, Metabolic, Hepatic, and Renal Disorders Maria A. Neag, Andrei Mocan, Javier Echeverría, Raluca M. Pop, Corina I. Bocsan, Gianina Crişan and Anca D. Buzoianu

54 Googling the Guggul (Commiphora and Boswellia) for Prevention of Chronic Diseases

Ajaikumar B. Kunnumakkara, Kishore Banik, Devivasha Bordoloi, Choudhary Harsha, Bethsebie L. Sailo, Ganesan Padmavathi, Nand K. Roy, Subash C. Gupta and Bharat B. Aggarwal

73 Assessing the Quality and Potential Efficacy of Commercial Extracts of Rhodiola rosea L. by Analyzing the Salidroside and Rosavin Content and the Electrophysiological Activity in Hippocampal Long-Term Potentiation, a Synaptic Model of Memory

Wilfried Dimpfel, Leonie Schombert and Alexander G. Panossian

Vasculoprotective Effects of Pomegranate (Punica granatum L.)

Dongdong Wang, Cigdem Özen, Ibrahim M. Abu-Reidah, Sridevi Chigurupati, Jayanta Kumar Patra, Jarosław O. Horbanczuk, Artur Jóźwik, Nikolay T. Tzvetkov, Pavel Uhrin and Atanas G. Atanasov

99 An Ethnobotanical Study of Medicinal Plants in the Greek Islands of North Aegean Region

Evangelos Axiotis, Maria Halabalaki and Leandros A. Skaltsounis

105 Lycopene and Vascular Health

Ioana Mozos, Dana Stoian, Alexandru Caraba, Clemens Malainer, Jarostaw O. Horbańczuk and Atanas G. Atanasov

121 Protective Effect of Casperome ${ }^{\circledR}$, an Orally Bioavailable Frankincense Extract, on Lipopolysaccharide-Induced Systemic Inflammation in Mice Konstantin Loeser, Semjon Seemann, Stefanie König, Isabell Lenhardt, Mona Abdel-Tawab, Andreas Koeberle, Oliver Werz and Amelie Lupp

139 Extracts From Hypericum hircinum subsp. majus Exert Antifungal Activity Against a Panel of Sensitive and Drug-Resistant Clinical Strains.

Noemi Tocci, Daniele Perenzoni, Duilio lamonico, Francesca Fava, Tobias Weil and Fulvio Mattivi

149 Effects of Phytochemically Characterized Extracts From Syringa vulgaris and Isolated Secoiridoids on Mediators of Inflammation in a Human Neutrophil Model

Marta Woźniak, Barbara Michalak, Joanna Wyszomierska, Marta K. Dudek and Anna K. Kiss 
164 Greater Celandine's Ups and Downs-21 Centuries of Medicinal Uses of Chelidonium majus From the Viewpoint of Today's Pharmacology Sylwia Zielińska, Anna Jezierska-Domaradzka, Magdalena Wójciak-Kosior, Ireneusz Sowa, Adam Junka and Adam M. Matkowski

193 Evidence Supports Tradition: The in Vitro Effects of Roman Chamomile on Smooth Muscles

Zsolt Sándor, Javad Mottaghipisheh, Katalin Veres, Judit Hohmann,

Tímea Bencsik, Attila Horváth, Dezső Kelemen, Róbert Papp, Loránd Barthó and Dezső Csupor

204 Constituents of Propolis: Chrysin, Caffeic Acid, p-Coumaric Acid, and Ferulic Acid Induce PRODH/POX-Dependent Apoptosis in Human Tongue Squamous Cell Carcinoma Cell (CAL-27)

Katarzyna Celińska-Janowicz, Ilona Zaręba, Urszula Lazarek, Joanna Teul, Michat Tomczyk, Jerzy Pałka and Wojciech Miltyk

216 Botanical Provenance of Traditional Medicines From Carpathian Mountains at the Ukrainian-Polish Border

Weronika Kozlowska, Charles Wagner, Erin M. Moore, Adam Matkowski and Slavko Komarnytsky

233 Chemical Composition of Scrophularia lucida and the Effects on Tumor Invasiveness in Vitro

Verena Lewenhofer, Lisa Schweighofer, Tobias Ledermüller, Julia Eichsteininger, Hanspeter Kählig, Martin Zehl, Chi H. Nguyen, Georg Krupitza, Ali Özmen and Liselotte Krenn

242 Apigenin and Luteolin Attenuate the Breaching of MDA-MB231 Breast Cancer Spheroids Through the Lymph Endothelial Barrier in Vitro Junli Hong, Adryan Fristiohady, Chi H. Nguyen, Daniela Milovanovic, Nicole Huttary, Sigurd Krieger, Junqiang Hong, Silvana Geleff, Peter Birner, Walter Jäger, Ali Özmen, Liselotte Krenn and Georg Krupitza

252 Phytochemical Composition, Antioxidant, Antimicrobial and in Vivo Anti-inflammatory Activity of Traditionally Used Romanian Ajuga laxmannii (Murray) Benth. ("Nobleman's Beard" - Barba Împăratului) Anca Toiu, Andrei Mocan, Laurian Vlase, Alina E. Pârvu, Dan C. Vodnar, Ana-Maria Gheldiu, Cadmiel Moldovan and Ilioara Oniga

267 Traditional and Current Food Use of Wild Plants Listed in the Russian Pharmacopoeia

Alexander N. Shikov, Andrey N. Tsitsilin, Olga N. Pozharitskaya, Valery G. Makarov and Michael Heinrich

282 Bioactivity Potential of Prunus spinosa L. Flower Extracts: Phytochemical Profiling, Cellular Safety, Pro-inflammatory Enzymes Inhibition and Protective Effects Against Oxidative Stress In Vitro Anna Marchelak, Aleksandra Owczarek, Magdalena Matczak, Adam Pawlak, Joanna Kolodziejczyk-Czepas, Pawel Nowak and Monika A. Olszewska

297 Colocynth Extracts Prevent Epithelial to Mesenchymal Transition and Stemness of Breast Cancer Cells

Kaushik Chowdhury, Ankit Sharma, Suresh Kumar, Gyanesh K. Gunjan, Alo Nag and Chandi C. Mandal 
310 The Dietary Constituent Falcarindiol Promotes Cholesterol Efflux From THP-1 Macrophages by Increasing ABCA1 Gene Transcription and Protein Stability

Limei Wang, Veronika Palme, Nicole Schilcher, Angela Ladurner,

Elke H. Heiss, Herbert Stangl, Rudolf Bauer, Verena M. Dirsch and Atanas G. Atanasov

319 A Multidirectional Perspective for Novel Functional Products: In vitro Pharmacological Activities and In silico Studies on Ononis natrix subsp. hispanica

Serife Yerlikaya, Gokhan Zengin, Adriano Mollica, Mehmet C. Baloglu, Yasemin Celik Altunoglu and Abdurrahman Aktumsek

333 Veronica officinalis Product Authentication Using DNA Metabarcoding and HPLC-MS Reveals Widespread Adulteration With Veronica chamaedrys

Ancuta C. Raclariu, Andrei Mocan, Madalina O. Popa, Laurian Vlase, Mihael C. Ichim, Gianina Crisan, Anne K. Brysting and Hugo de Boer

346 Erratum: Veronica officinalis Product Authentication Using DNA Metabarcoding and HPLC-MS Reveals Widespread Adulteration With Veronica chamaedrys

Frontiers Production Office

347 Exploring Pharmacological Mechanisms of Lavender (Lavandula angustifolia) Essential Oil on Central Nervous System Targets

Víctor López, Birgitte Nielsen, Maite Solas, Maria J. Ramírez and Anna K. Jäger 


\title{
Editorial: Ethnopharmacology in Central and Eastern Europe in the Context of Global Research Developments
}

\author{
Devesh Tewari ${ }^{1}$, Judit Hohmann ${ }^{2}$, Anna K. Kiss ${ }^{3}$, Judith M. Rollinger ${ }^{4}$ and \\ Atanas G. Atanasov ${ }^{4,5,6 *}$ \\ ${ }^{1}$ Department of Pharmacognosy, School of Pharmaceutical Sciences, Lovely Professional University, Phagwara, India, \\ ${ }^{2}$ Department of Pharmacognosy, University of Szeged, Szeged, Hungary, ${ }^{3}$ Department of Pharmacognosy and Molecular \\ Basis of Phytotherapy, Medical University of Warsaw, Warsaw, Poland, ${ }^{4}$ Department of Pharmacognosy, University of Vienna, \\ Vienna, Austria, ${ }^{5}$ Institute of Genetics and Animal Breeding of the Polish Academy of Sciences, Magdalenka, Poland, \\ ${ }^{6}$ GLOBE Program Association (GLOBE-PA), Grandville, MI, United States
}

Keywords: pharmacognosy, phytochemistiy, pharmacology, ethnobotany, ethnopharmacology

OPEN ACCESS

Edited by:

Javier Echeverria,

Universidad de Santiago de Chile,

Chile

Reviewed by:

Michael Heinrich,

UCL School of Pharmacy,

United Kingdom

*Correspondence:

Atanas G. Atanasov

a.atanasov.mailbox@gmail.com

Specialty section:

This article was submitted to

Ethnopharmacology,

a section of the journal

Frontiers in Pharmacology

Received: 30 January 2019

Accepted: 19 March 2019

Published: 09 April 2019

Citation:

Tewari D, Hohmann J, Kiss AK,

Rollinger JM and Atanasov AG (2019)

Editorial: Ethnopharmacology in

Central and Eastern Europe in the

Context of Global Research

Developments.

Front. Pharmacol. 10:341.

doi: 10.3389/fphar.2019.00341
Editorial on the Research Topic

Ethnopharmacology in Central and Eastern Europe in the Context of Global Research Developments

Historically, medicinal plants have been a key element of healthcare, and are still widely used as alternative and complementary therapy (mainly in developed countries) or as a primary treatment (in most developing countries). Moreover, many of the modern pharmaceuticals were developed from molecules extracted from natural sources, and medicinal plants still today represent an important pool for the identification of novel drug leads.

The ethnopharmacological tradition in Central and Eastern Europe has a great historical significance and large parts of the Western world's knowledge for therapeutic properties of medicinal plants has its roots in the Greek and Roman cultures (e.g., with a reference to the influential works of Dioscorides, Pliny the Elder, and Galen). The German-speaking Central European areas also have played very important roles, with some influential medieval herbal book editions such as the Mainz Herbal (Herbarius Moguntinus, 1484) and The German Herbal (1485). Moreover, at the beginning of the nineteenth century modern drug discovery from plants started to played a key role in Central Europe, most notably the work of the German apothecary assistant Friedrich Sertürner, who isolated an analgesic and sleep-inducing agent from opium that was named morphium (morphine) after the Greek god of dreams, Morpheus. At the same time, it should be noted that on many occasions the rich ethnopharmacological knowledge of some East European countries and Russia remained localized and did not find its way to integrate with the Western world of herbal therapy traditions. The focus of this Research Topic is to make a special contribution to ethnopharmacology rooted in Central and Eastern Europe, on the context of global research developments in the area of ethnopharmacology, phytochemistry, molecular pharmacology of natural products, nutrigenomics, and other related disciplines. This special issue consists of 24 articles covering diverse topics related to the listed research fields.

Advances in screening methods and analytical equipment, increasing number of targets available for testing, and improved possibilities for optimization of natural leads using synthetic modification 
strategies substantially improved the process of modern drug discovery from natural sources (Atanasov et al., 2015). Hypericum is a well-known genus that is used for its medicinal properties. Tocci et al. evaluated five Hypericum species for their polyphenolic content, toxicological safety, and antifungal potential against some sensitive and drug resistant clinical fungal isolates. The authors have identified 52 compounds by LC-MS analysis and showed that $H$. hircinum subsp. majus infusion exerted broad antifungal activity against Aspergillus, Penicillium, and non-albicans Candida strains (Tocci et al.).

Ethnobotany has played an important role in new drug development and drug discovery (Kayser, 2018). The importance of the traditional use of medicinal plants on the Greek islands of the North Aegean Region was summarized in a Brief Research Report Article by Axiotis et al. In this report the authors present 109 wild plants from 52 families, and enlist their therapeutic uses, including uses in galenic preparations which were reported by local medical doctors and pharmacists (Axiotis et al.).

Medicinal plants and natural products are of potential importance in several age-related disorders, including memory impairment and dementia (Tewari et al., 2018). In this research topic Dimpfel et al. assessed the biological activity of some commercial extracts of the underground parts of Rhodiola rosea L. and its analytical markers, salidroside and rosavin, using longterm potentiation of synaptic transmission in hippocampus slices (a synaptic model of memory).

Loeser et al. evaluated the effect of Casperome ${ }^{\circledR}$, an orally bioavailable soy lecithin-based formulation of standardized frankincense extract on various systemic parameters and organ damages that were induced by severe systemic inflammation caused by intraperitoneal administration of lipopolysaccharides (a murine model of sepsis). The study revealed that the extract possesses anti-inflammatory, anti-oxidant, and hepatoprotective effects (Loeser et al.).

Zielińska et al. review older and recent data on pharmacology, phytochemistry, and clinical studies of Chelidonium majus L. along with controversies about this herb, its safety, and drug quality issues.

The study of Wozniak et al. evaluated the effects of phytochemically characterized extracts of various parts of traditionally used Syringa vulgaris L. (common lilac) on the pro-inflammatory functions of neutrophils. The authors also isolated active compounds, including neooleuropein from the extracts using bioassay-guided fractionation. It was found that neooleuropein acts through inhibition of cytokine production by attenuation of the MAP kinase pathways (Woźniak et al.).

Smooth muscle cells are a prominent target of diverse natural products with different bioactivities (Hnatyszyn et al., 2003; Uhrin et al., 2018). Sándor et al. assessed the relaxant effects or contracting activity of the hydroethanolic extract, fractions, phytoconstituents, and essential oil of Roman chamomile on smooth muscle preparations. The authors found that the essential oil of the plant possessed a good smooth muscle-relaxant effect (Sándor et al.).

Identification of the molecular mechanism of action of promising natural products is a vital step toward their clinical application (Butler, 2008; Tewari et al., 2018). In a mechanistic study, Celińska-Janowicz et al. studied the apoptosisinducing mechanisms of selected propolis components in tongue squamous cell carcinoma cells (CAL-27). In another work, Grienke et al. isolated several flavonoids and rare sesquiterpene lactones from the ethanolic extract of Centaurea ragusina L., a plant species endemic to Croatia. For the first time they identified ragusinin, and hemistepsin $\mathrm{A}$ from this genus. The authors further studied the mechanism underlining the ragusinin activity in cancer cells (Grienke et al.).

In their review article Kozlowska et al. summarize the herbal medicines used in the Beskid mountain ranges, which are located south of Krakow and Lviv, two important medieval centers of apothecary tradition in the area. In another interesting review Kunnumakkara et al. summarize potential benefits, traditional uses, chemistry, biological activities, and clinical trials of "guggul" from Commiphora and Boswellia.

Berberine is an important phytoconstituent of plants utilized in different traditional medicine systems, including the Chinese system of traditional medicine (Huang et al., 2011). In their work "Berberine: Botanical Occurrence, Traditional Uses, Extraction Methods, and Relevance in Cardiovascular, Metabolic, Hepatic, and Renal Disorders" Neag et al. review diverse biomedical information on berberine, including several chromatographic techniques for berberine extraction and quantification.

There is great interest of the scientific community toward the health benefits of food items used in the Mediterranean diet, which has a variety of health benefits (Sofi et al., 2010). Wang et al. review recent studies on pomegranate, mainly focusing on vasculoprotective effects attributed to diverse pomegranate phytoconstituents, including tannins, especially ellagitannins, and ellagic acid, among other compounds. Lycopene is a carotenoid which is responsible for different pigmentation in tomatoes, grapefruit, and watermelon, and it represents a compound with a potent antioxidant action (Horvitz et al., 2004). In their work, Mozos et al. review mechanisms of action of lycopene on the vascular system considering substantial epidemiological and experimental data, and clinical studies.

Breast cancer is the most common cancer in women and represents a global health burden both in the developed and developing countries. Hong et al. study apigenin and luteolin to better understand their mechanism of action in the context of breast cancer intravasation of the lymphatic barrier. Both of these natural compounds were evaluated in a three-dimensional assay system containing MDA-MB231 breast cancer spheroids and immortalized lymph endothelial cell monolayers (Hong et al.)

Toiu et al. evaluated the chemical composition, antimicrobial, antioxidant, and in vivo anti-inflammatory activity of various extracts of the aerial parts of an important plant species with traditional use in Romanian, Ajuga laxmannii (Murray) Benth.

In their work, Shikov et al. review 70 wild plant species that are referenced in the 11th edition of the State Pharmacopeia of the USSR and also discuss their health food value, mainly based on published Russian literature.

Marchelak et al. present a comprehensive study focused on extracts of the flowers of Prunus spinosa L. The authors 
profiled the phenolic contents of the extracts through UHPLC-PDA-ESI-MS ${ }^{3}$, and also studied several biological effects with relevance in the context of cardiovascular disease (Marchelak et al.).

Chowdhury et al. explore the anti-proliferative and antimetastatic potential of various extracts of the medicinal plant colocynth. The authors have found that ethanol and acetone extracts of colocynth fruit pulp exhibit promising activity in different cancer cell types, inhibiting viability, and cell migration in association with the modulation of the expression of diverse relevant genes and cellular pathways (Chowdhury et al.).

Ononis natrix is a member of the Fabaceae family and is a lessexplored plant species. In their work, Yerlikaya et al. give new insight on the chemical composition and biological activities of Ononis natrix subsp. hispanica.

Wang et al. identify and characterize the natural product falcarindiol as a novel inducer of macrophage cholesterol efflux, a process with a protective effect in atherosclerosis.

In their research paper, Raclariu et al. establish DNA metabarcoding and HPLC-MS techniques for the authentication of Veronica officinalis L. herbal products. The authors advocate for the use of an integrative approach for the detection of adulteration and substitution in the herbal products (Raclariu et al.).

López et al. study the effects of lavender essential oil on pharmacological targets that are involved in anti-depressive and anxiolytic effects, as well as on in vitro models of neurotoxicity.

\section{REFERENCES}

Atanasov, A. G., Waltenberger, B., Pferschy-Wenzig, E. M., Linder, T., Wawrosch, C., Uhrin, P., et al. (2015). Discovery and resupply of pharmacologically active plant-derived natural products: a review. Biotechnol. Adv. 33, 1582-1614. doi: 10.1016/j.biotechadv.2015.08.001

Butler, M. S. (2008). Natural products to drugs: natural product-derived compounds in clinical trials. Nat. Prod. Rep. 25, 475-516. doi: 10.1039/ b514294f

Hnatyszyn, O., Moscatelli, V., Garcia, J., Rondina, R., Costa, M., Arranz, C., et al. (2003). Argentinian plant extracts with relaxant effect on the smooth muscle of the corpus cavernosum of guinea pig. Phytomedicine 10, 669-674. doi: 10.1078/0944-7113-00261

Horvitz, M. A., Simon, P. W., and Tanumihardjo, S. A. (2004). Lycopene and $\beta$-carotene are bioavailable from lycopene 'red' carrots in humans. Eur. J. Clin. Nutr. 58, 803-811. doi: 10.1038/sj.ejcn. 1601880

Huang, Z. J., Zeng, Y., Lan, P., Sun, P. H., and Chen, W. M. (2011). Advances in structural modifications and biological activities of berberine: an active compound in traditional Chinese medicine. Mini Rev. Med. Chem. 11, 1122-1129. doi: 10.2174/138955711797655362

Kayser, O. (2018). Ethnobotany and medicinal plant biotechnology: from tradition to modern aspects of drug development. Planta Med. 84, 834-838. doi: 10.1055/a-0631-3876
Lewenhofer et al. performed an activity-guided identification of active principles from Scrophularia lucida L. using cancer cell models. The authors identified 14 substances including some very rare iridoids, like scrovalentinoside or koelzioside, and flavonoids such as nepitrin and homoplantaginin (Lewenhofer et al..)

In conclusion, this Research Topic includes a wide range of interdisciplinary research work contributing to the ultimate goals of augmenting the existing knowledge and fostering the scientific field of ethnopharmacology in Central and Eastern Europe, as well as globally. This research topic successfully gathered comprehensive interdisciplinary information in the field of ethnopharmacology from the field surveys to standardization and elucidation of molecular mechanisms of action of natural products. Moreover, with consideration of the existing challenges in the field of drug discovery form medicinal plants, this research topic provides up-to-date snapshot of current knowledge and paves the way for further chemical and biological assessment of diverse natural compounds with potential for future therapeutic development. We hope that this compendium will further inspire scientists from different research fields to make use of the gathered traditional medical knowledge in the search for superior future remedies from nature.

\section{AUTHOR CONTRIBUTIONS}

All authors listed have made a substantial, direct and intellectual contribution to the work, and approved it for publication.

Sofi, F., Abbate, R., Gensini, G. F., and Casini, A. (2010). Accruing evidence on benefits of adherence to the Mediterranean diet on health: an updated systematic review and meta-analysis. Am. J. Clin. Nutr. 92, 1189-1196. doi: 10.3945/ajcn.2010.29673

Tewari, D., Stankiewicz, A. M., Mocan, A., Sah, A. N., Tzvetkov, N. T., Huminiecki, L., et al. (2018). Ethnopharmacological approaches for dementia therapy and significance of natural products and herbal drugs. Front. Aging Neurosci. 10:3. doi: 10.3389/fnagi.2018.00003

Uhrin, P., Wang, D., Mocan, A., Waltenberger, B., Breuss, J. M., Tewari, D., et al. (2018). Vascular smooth muscle cell proliferation as a therapeutic target. Part 2: natural products inhibiting proliferation. Biotechnol. Adv. 36:1608-21. doi: 10.1016/j.biotechadv.2018.04.002

Conflict of Interest Statement: The authors declare that the research was conducted in the absence of any commercial or financial relationships that could be construed as a potential conflict of interest.

Copyright (C) 2019 Tewari, Hohmann, Kiss, Rollinger and Atanasov. This is an openaccess article distributed under the terms of the Creative Commons Attribution License (CC BY). The use, distribution or reproduction in other forums is permitted, provided the original author(s) and the copyright owner(s) are credited and that the original publication in this journal is cited, in accordance with accepted academic practice. No use, distribution or reproduction is permitted which does not comply with these terms. 


\section{OPEN ACCESS}

Edited by:

Atanas G. Atanasov, Institute of Genetics and Animal

Breeding (PAS), Poland

Reviewed by:

Adam Matkowski,

Wroclaw Medical University, Poland Pio Maria Furneri,

Università degli Studi di Catania, Italy

*Correspondence:

Anamaria Brozovic brozovic@irb.hr

Marijana Radić Stojković mradic@irb.hr

Specialty section: This article was submitted to

Ethnopharmacology,

a section of the journal Frontiers in Pharmacology

Received: 08 January 2018 Accepted: 06 August 2018 Published: 22 August 2018

Citation:

Grienke U, Radić Brkanac S, Vujčić V, Urban E, Ivanković S, Stojković R, Rollinger JM, Kralj J, Brozovic A and Radić Stojković M (2018) Biological Activity of Flavonoids and Rare Sesquiterpene Lactones Isolated From Centaurea ragusina $L$.

Front. Pharmacol. 9:972 doi: 10.3389/fphar.2018.00972

\section{Biological Activity of Flavonoids and Rare Sesquiterpene Lactones Isolated From Centaurea ragusina $\mathrm{L}$.}

\author{
Ulrike Grienke ${ }^{1}$, Sandra Radić Brkanac ${ }^{2}$, Valerija Vujčić ${ }^{2}$, Ernst Urban ${ }^{3}$, \\ Siniša Ivanković4, Ranko Stojković ${ }^{4}$, Judith M. Rollinger ${ }^{1}$, Juran Kralj ${ }^{5}$, \\ Anamaria Brozovic ${ }^{5 *}$ and Marijana Radić Stojković6*
}

'Department of Pharmacognosy, University of Vienna, Vienna, Austria, ${ }^{2}$ Department of Biology, Faculty of Science, University of Zagreb, Zagreb, Croatia, ${ }^{3}$ Department of Pharmaceutical Chemistry, University of Vienna, Vienna, Austria, ${ }^{4}$ Division of Molecular Medicine, Ruder Bošković Institute, Zagreb, Croatia, ${ }^{5}$ Division of Molecular Biology, Ruđer Bošković Institute, Zagreb, Croatia, ${ }^{6}$ Division of Organic Chemistry and Biochemistry, Ruđer Bošković Institute, Zagreb, Croatia

The endemic Croatian species Centaurea ragusina L., like other species from the genus Centaurea, has been traditionally used in Croatia as an antibacterial agent and for the treatment of gastrointestinal and urogenital disorders. In several chromatographic steps, three flavonoids and three sesquiterpene lactones (STLS) were isolated and identified from the most active fractions of the ethanol extract. Two STLs, one for which we created the trivial name ragusinin, and hemistepsin A are here reported for the first time as constituents of the genus Centaurea. All six compounds were screened for their effect on several tumor and one normal cell lines. Among them, ragusinin showed the best bioactivity and high specificity to affect tumor murine SCCVII, human HeLa and Caco-2 cell lines, but not the viability of normal V79 fibroblasts. Due to these characteristics the action of ragusinin was investigated in more detail. Since DNA is the primary target for many drugs with antibacterial and anticancer activity, we studied its interaction with ragusinin. Rather moderate binding affinity to DNA excluded it as the primary target of ragusinin. Due to the possibility of STL interaction with glutathione (GSH), the ubiquitous peptide that traps reactive compounds and other xenobiotics to prevent damage to vital proteins and nucleic acids, its role in deactivation of ragusinin was evaluated. Addition of the GSH precursor N-acetyl-cysteine potentiated the viability of HeLa cells, while the addition of GSH inhibitor L-buthionine sulfoximine decreased it. Moreover, pre-treatment of HeLa cells with the inhibitor of glutathione-S-transferase decreased their viability indicating the detoxifying role of GSH in ragusinin treated cells. Cell death, derived by an accumulation of cells in a G2 phase of the cell cylce, was shown to be independent of poly (ADP-ribose) polymerase and caspase-3 cleavage pointing toward an alternative cell death pathway.

Keywords: Centaurea, sesquiterpene lactones, DNA, cell viability, toxicity, glutathione 


\section{INTRODUCTION}

The genus Centaurea (Asteraceae) represents an attractive source for bioactive secondary metabolites such as sesquiterpene lactones (STLs), flavonoids, lignans, and their glycosides (Khammar and Djeddi, 2012). A number of therapeutic effects against microbial infections, gastrointestinal disorders, and urogenital ailments have been attributed to Centaurea species in Croatian folk medicine and worldwide (Pahlow, 1989; Ayad et al., 2012; Politeo et al., 2012).

Our recent study on the phytochemical and bioactive profile of non-volatile constituents of Centaurea ragusina L., an endemic Croatian halophytic species (Radić et al., 2013), indicated the strong potential for obtaining bioactive compounds from the leaf ethanol extract (CRE) (Vujčić et al., 2017).

In continuation of this previous study, the aim was to investigate the biological activity of all isolated compounds, namely the interaction with DNA, the antibacterial activity against Gram-positive (Staphylococcus aureus) and Gramnegative bacteria (Acinetobacter baumannii) (Lee et al., 2007; Ćurković-Perica et al., 2015) and anticancer activity against a panel of murine and human cancer cells.

Due to its high biological significance, DNA is the primary target for many drugs with antibacterial and anticancer activity. Small organic molecules can bind to DNA by means of a non-specific, electrostatic binding along the DNA backbone, a specific groove binding and intercalation or can form crosslinks with DNA strands and induce cleavage of the DNA backbone (Demeunynck et al., 2002; Sangeetha Gowda et al., 2014).

STLs are known to bind covalently to sulfhydryl groups of enzymes and other functional proteins by Michael type addition of their electrophilic $\alpha, \beta$-unsaturated carbonyl structures. It is believed that most of STL biological effects are due to their reaction with biological nucleophiles such as GSH (Kupchan et al., 1971; Picman, 1986; Schmidt, 1999). Among many roles of GSH in the cell, the most important one seems to be the removal of reactive species and elimination of xenobiotic compounds. The last one can be accomplished through conjugation with GSH followed by secretion of adducts from the cell (Boyland and Chasseaud, 1969).

Here, the aim was to study the activity of the most bioactive compound in more detail, which included the interaction with DNA as the potential primary target and the interaction with GSH and its impact on cytotoxicity, cell cycle and cell death.

\section{MATERIALS AND METHODS}

All safety precautions were taken when working with chemicals reagents used in the experiments.

\section{General Experimental Procedures}

$1 \mathrm{D}$ and 2D NMR experiments were performed on an Avance $500 \mathrm{MHz}$ instrument equipped with cryoprobe (Bruker, Billerica, MA, United States). The samples were measured in MeOD and DMSO-d6, respectively (calibrated to the residual non-deuterated solvent signals). HR-ESI-MS analyses were performed on a maXis HD ESI-Qq-TOF mass spectrometer (Bruker Daltonics, Bremen, Germany). The ESI ion source was operated as follows: capillary voltage: 2.0 to $4.5 \mathrm{kV}$ (individually

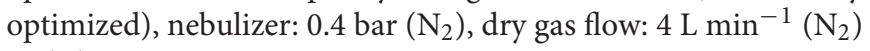
and dry temperature: $200^{\circ} \mathrm{C}$, scanning range, $m / z 50-1550$. For each isolated compound, fragment ion spectra of the $[\mathrm{M}+\mathrm{H}]^{+}$, the $[\mathrm{M}+\mathrm{Na}]^{+}$and either the $[\mathrm{M}-\mathrm{H}]{ }^{-}$or the $[\mathrm{M}+\mathrm{HCOO}]^{-}$ion were recorded. The sum formulas of the ions were determined using Bruker Compass DataAnalysis 4.2 based on the mass accuracy $(\Delta m / z \leq 2 \mathrm{ppm}$ for MS1 and $\leq 3 \mathrm{ppm}$ for MS/MS) and isotopic pattern matching (SmartFormula algorithm).

Column chromatography (CC) was performed using Merck silica gel $60(40-63 \mu \mathrm{m})$ and Pharmacia Sephadex LH-20 (20-100 $\mu \mathrm{m})$. The fractions obtained from all chromatographic steps were analyzed by TLC (mobile phase: $\mathrm{CH}_{2} \mathrm{Cl}_{2}$-EtOAc (85:15), $n$-hexane-EtOAc-CH $3 \mathrm{COOH}$ (6:3:1), or $n$-hexane-EtOAc $(8: 2)$; stationary phase: Merck silica gel $60 \mathrm{PF}_{254}$, detected with staining reagents vanillin/ $\mathrm{H}_{2} \mathrm{SO}_{4}$ at vis, $\mathrm{UV}_{254}$, and $\mathrm{UV}_{366}$ ). HPLC was performed on a Shimadzu UFLC-XR instrument (Kyoto, Japan) with a photodiode array detector (DAD). LC-parameters: stationary phase: Phenomenex Gemini-NX (C18), $150 \mathrm{~mm} \times 3.00 \mathrm{~mm}, 5 \mu \mathrm{m}$; temperature: $35^{\circ} \mathrm{C}$; mobile phase: water with $0.1 \%$ formic acid (A); acetonitrile (B); flow rate $0.4 \mathrm{~mL} / \mathrm{min}$; UV detection wavelength: $275 \mathrm{~nm}$; injection volume: $10 \mu \mathrm{L}$; gradient: $80 / 20 \mathrm{~A} / \mathrm{B}$ in 5 min to $70 / 30$ $\mathrm{A} / \mathrm{B}$, then within $20 \mathrm{~min}$ to $50 / 50 \mathrm{~A} / \mathrm{B}$ and within another $2 \mathrm{~min}$ to $2 / 98$, followed by a $5 \mathrm{~min}$ column wash $(2 \mathrm{~A} / 98 \mathrm{~B})$ and a re-equilibration period of $10 \mathrm{~min}$. All chemicals and solvents used were of analytical grade.

\section{Plant Material}

Centaurea ragusina L. plants (in vegetative phase) were collected in September, 2016, from two wild habitats - Katalinić brig $\left(43^{\circ} 30^{\prime} 03^{\prime \prime} \mathrm{N}, 16^{\circ} 26^{\prime} 40^{\prime \prime} \mathrm{E}, 363 \mathrm{~m}\right)$ and Sustipan $\left(43^{\circ} 30^{\prime} 04^{\prime \prime} \mathrm{N}\right.$, $16^{\circ} 25^{\prime} 35^{\prime \prime} \mathrm{E}, 754 \mathrm{~m}$ ), Split, Croatia and identified by M. Ruščić, Department of Biology, University of Split, Croatia. A voucher specimen (FSS-CR112016) is deposited at the above-mentioned department.

For extract preparation and isolation of pure compounds, lyophilized leaf materials from both locations were combined after confirmation of their comparable metabolite profile (Vujčić et al., 2017).

\section{Extraction and Isolation}

The dried ground leaves of C. ragusina L. (804.9 g) were macerated with $7 \mathrm{~L} \mathrm{EtOH} \mathrm{96 \%} \mathrm{(at} 22^{\circ} \mathrm{C}$ for 7 days). For an exhaustive extraction the procedure was repeated three times. The dried extract (CRE, $108.9 \mathrm{~g}$ ) was roughly fractionated by silica gel CC (Merck silica gel $60 \mathrm{PF} 254,510 \mathrm{~g} ; 5.5 \mathrm{~cm} \times 56 \mathrm{~cm}$ ) using a step gradient of $\mathrm{CH}_{2} \mathrm{Cl}_{2}$-EtOAc-MeOH $\left(\mathrm{CH}_{2} \mathrm{Cl}_{2}\right.$; $\mathrm{CH}_{2} \mathrm{Cl}_{2}$-EtOAc 98:2; 95:5; 90:10; 85:15; 80:20; 75:25; 65:35; 60:40; 55:45; 45:55; 35:65; 25:75; EtOAc; EtOAc-MeOH 80:20; 60:40; $40: 60 ; 20: 80 ; \mathrm{MeOH})$ to give twelve fractions (A1-12).

Fraction A6 (2.9 g) was further separated using silica gel CC (Merck silica gel $60 \mathrm{PF}_{254}, 213 \mathrm{~g} ; 3 \mathrm{~cm} \times 56 \mathrm{~cm}$ ) applying again a gradient system of $\mathrm{CH}_{2} \mathrm{Cl}_{2}$-EtOAc-MeOH to yield 25 fractions (B1-25). Fraction B11 (91.6 mg) was purified via Sephadex 
LH-20 CC (mobile phase: $\mathrm{MeOH}$ ) yielding eight fractions (C1-8). Fraction $\mathrm{C} 7$ was obtained as $17.5 \mathrm{mg}$ of compound $\mathbf{2}$ (oroxylin A). Also Fraction B12 (76.4 mg) was purified via Sephadex LH-20 CC (mobile phase: $\mathrm{MeOH}$ ) yielding 14 fractions (D1-14). Fraction D13 was obtained as $11.8 \mathrm{mg}$ of compound $\mathbf{1}$ (chrysin).

Fraction B19 (939.8 mg) was subjected to silica gel CC (Merck silica gel $60 \mathrm{PF}_{254}, 310 \mathrm{~g} ; 3.3 \mathrm{~cm} \times 63 \mathrm{~cm}$ ) eluting with the isocratic solvent system of $n$-hexane-EtOAc- $\mathrm{CH}_{3} \mathrm{COOH}$ (6:3:1), yielding 14 fractions (E1-14). Fraction E9 (26.9 mg) was further separated by means of a Sephadex LH-20 column (mobile phase: $\mathrm{MeOH}$ ) to give three fractions (F1-3). Fraction F2 was obtained as $21.8 \mathrm{mg}$ of compound 5 [(3aR,4S,6aR,8S,9aR,9bR)[dodecahydro-8-dihydroxy-3,6,9-tris(methylene)-2oxo-2(3H)azuleno[4,5-b]furanyl]-3-methyl-butanoate].

Fraction A8 (834.3 mg) was separated via a Sephadex LH-20 column (mobile phase: $\mathrm{MeOH}$ ) giving seven fractions (G1-7). Fraction G7 was obtained as $80.3 \mathrm{mg}$ of compound 3 (hispidulin). Fraction G4 (339.4 mg) was submitted to passage over a Sephadex LH-20 column (mobile phase: $\mathrm{CH}_{2} \mathrm{Cl}_{2}$ acetone, 85:15) to yield twelve fractions (H1-12). Fraction H10 (251.4 mg) was further separated using silica gel CC (Merck silica gel $\left.60 \mathrm{PF}_{254}, 150 \mathrm{~g} ; 1.5 \mathrm{~cm} \times 56 \mathrm{~cm}\right)$ applying again a gradient system of $n$-hexane-EtOAc- $\mathrm{CH}_{3} \mathrm{COOH}(6: 3: 1$ to $4: 5: 1)$ to yield ten fractions (I1-10). Fraction I9 (185.8 mg) was subjected to a Sephadex LH-20 column (mobile phase: $\mathrm{MeOH})$ resulting in three fractions (J1-3). Fraction J1 (17.8 mg) was purified by silica gel CC (Merck silica gel $60 \mathrm{PF}_{254}$, $50 \mathrm{~g} ; 1.5 \mathrm{~cm} \times 35 \mathrm{~cm}$ ) applying again a gradient system of $\mathrm{CH}_{2} \mathrm{Cl}_{2}$-EtOAc-MeOH to yield two fractions (K1-2). Fraction $\mathrm{K} 1$ was obtained as $3.4 \mathrm{mg}$ of compound 4 (deacylcynaropicrin). Fraction J2 (153.6 mg) was purified by preparative TLC (Merck silica gel $60 \mathrm{PF}_{254}, 20 \mathrm{~cm} \times 20 \mathrm{~cm}$ ) and a solvent system of $n$-hexane-EtOAc- $\mathrm{CH}_{3} \mathrm{COOH}(5: 4: 1)$ to yield three fractions (L1-3). Fraction L2 (115.7 mg) was separated via a Sephadex LH-20 column (mobile phase: $\mathrm{MeOH}$ ) giving two fractions (M1-2). Fraction M2 was obtained as $12.7 \mathrm{mg}$ of compound 6 (hemistepsin A).

The physical and spectroscopic data of compounds 1 to $\mathbf{6}$ agreed with those published previously for chrysin, oroxylin A, hispidulin, deacylcynaropicrin, [3aR,4S,6aR,8S,9aR,9bR)[dodecahydro-8-dihydroxy-3,6,9-tris(methylene)-2oxo-2(3H)azuleno[4,5-b]furanyl]-3-methyl-butanoate], and hemistepsin A (Miyase et al., 1985; Zdero et al., 1989; Jang et al., 1999; Nagao et al., 2002; Marques et al., 2010; Yang et al., 2013). Their purity was checked using TLC and LC-MS and revealed to be $>98 \%$ in all cases.

\section{Spectroscopic Experiments for the Determination of ctDNA Interactions}

The electronic absorption spectra (UV/Vis) were recorded on a Varian Cary 100 Bio spectrophotometer (Agilent, Santa Clara, CA, United States) and circular dichroism (CD) spectra on a JASCO J815 spectrophotometer (ABL\&E Handels GmbH, Wien, Austria) at $25^{\circ} \mathrm{C}$ using appropriate $1 \mathrm{~cm}$ path quartz cuvettes (Eriksson and Nordén, 2001). The calf thymus DNA (ctDNA) was purchased from Sigma-Aldrich. Isothermal titration calorimetry (ITC) experiments were performed on a MicroCal
VP-ITC microcalorimeter (MicroCal, Inc., Northampton, MA, United States) (Chaires, 2006). Origin 7.0 software, supplied by the manufacturer was used for data analysis. All additional data of these experiments are provided in the Supplementary Material.

\section{Antibacterial Assay}

Antibacterial activities of C. ragusina L. CRE extract, fractions and isolated compounds against Gram-negative A. baumannii Durn (Ćurković-Perica et al., 2015) and Gram-positive S. aureus ATCC 25923 were tested using modified Clinical and Laboratory Standards Institute (CLSI), broth microdilution (BD) using 2,3,5-triphenyltetrazolium chloride (TTC) (Lee et al., 2007). The TTC-BD were performed according to the guidelines of the CLSI using 96-well microplates (Clinical Laboratory Standards Institute [CLSI], 2007). The bacteria were grown on nutrient agar (Biolife, Milan, Italy) for $16 \mathrm{~h}$ at $36 \pm 0.1^{\circ} \mathrm{C}$ to obtain the cultures in log phase of growth. The bacterial biomass was then suspended in sterile $\mathrm{NaCl}(0.85 \% \mathrm{v} / \mathrm{v})$ to give turbidity equivalent to the McFarland 0.5 standard. Bacterial suspension $(0.1 \mathrm{~mL})$ was transferred to a tube containing $9.1 \mathrm{~mL}$ nutrient broth (Biolife) and $0.8 \mathrm{~mL} 0.05 \%$ TTC to give an inoculum density of $1 \times 10^{6}$ Colony Forming Units $(\mathrm{CFU}) / \mathrm{mL}$. Minimum inhibitory concentration (MIC) and minimum bactericidal concentration $(\mathrm{MBC})$ values were determined in triplicates. The final concentrations for MIC and MBC determination of samples were $1.9-4000 \mu \mathrm{g} / \mathrm{mL}$. Other data on antibacterial experiments are available in the Supplementary Material.

\section{Cytotoxicity Assays and Cell Death Analysis Crystal Violet (CV) Assay}

Murine melanoma (B16F10) cell lines, human colon carcinoma (Caco-2) and human breast carcinoma (MCF-7) cell lines were purchased from American Type Culture Collection (ATCC, Manassas, VA, United States), murine fibrosarcoma (FsaR) and murine squamous cell carcinoma (SCCVII) cell lines were obtained from BC Cancer Research Centre (Vancouver, Canada). Cells were grown in a humidified atmosphere of $5 \% \mathrm{CO}_{2}$, at $37{ }^{\circ} \mathrm{C}$ in Roswell Park Memorial Institute (RPMI) 1640 medium supplemented with $10 \%$ fetal bovine serum (FBS) (Sigma-Aldrich, St. Louis, MO, United States). As normal cell line, the V79 fibroblasts derived from hamster's lung tissue, were used. CV protocol (Ivanković et al., 2015) is described in Supplementary Material.

\section{[3-(4,5-Dimethylthiazol-2-yl)-2,5-Diphenyltetrazolium Bromide] Tetrazolium Reduction (MTT) Assay}

Ethacrynic acid (ETA; Sigma-Aldrich) was dissolved in DMSO (Sigma-Aldrich) and kept at $-20^{\circ} \mathrm{C}$. Buthionine sulfoximine (BSO; Sigma-Aldrich) and N-acetylcysteine (NAC; Sigma-Aldrich) were dissolved in water, 3-(4,5-dimethyl-2thiazolyl)-2,5-diphenyl-2H-tetrazolium bromide was purchased by Sigma-Aldrich and dissolved in phosphate-buffered saline and kept by $4^{\circ} \mathrm{C}$. Human cervical carcinoma HeLa cell line was obtained from cell culture bank (GIBCO BRL-Invitrogen, Waltham, MA, United States). The cells were grown as a 
monolayer culture in Dulbecco's modified Eagle's medium (DMEM; Sigma-Aldrich), supplemented with $10 \%$ (FBS; Sigma-Aldrich) in a humidified atmosphere of $5 \% \mathrm{CO}_{2}$ at $37^{\circ} \mathrm{C}$ and were sub-cultured every 3-4 days. Cytotoxic activity of the STL 5 was determined by MTT assay, an assay for assessing cell viability based on its metabolic activity, modified accordingly (Mickisch et al., 1990; also in Supplementary Material).

\section{Cell Cycle and Cell Death Analysis}

HeLa cells were seeded into tissue culture plates and treated with different concentrations of the compound during $72 \mathrm{~h}$. Thereafter, both adherent and floating cells were collected, washed with PBS and fixed overnight in $70 \%$ ethanol at $20^{\circ} \mathrm{C}$. Fixed cells were treated with RNase A $(0.1 \mathrm{mg} / \mathrm{mL}$, Sigma-Aldrich) for $1 \mathrm{~h}$ at room temperature and afterward stained with propidium iodide (PI; $50 \mu \mathrm{g} / \mathrm{mL}$, Sigma-Aldrich) for $30 \mathrm{~min}$ in the dark. In order to analyze the cell cycle progression, the DNA content and PI staining were detected by flow cytometry (FACS Calibur, BD Biosciences, San Jose, CA, United States). Data were analyzed with ModFit $\mathrm{LT}^{\mathrm{TM}}$ program (Verity Software House Inc., Topsham, ME, United States).

Twenty-four hours after the seeding, HeLa cells were treated with 2, 5, and $10 \mu \mathrm{M}$ of compound 5. After $48 \mathrm{~h}$, both adherent and floating cells were collected by centrifugation and then washed with PBS. The cell suspension was incubated with Annexin V (BD Biosciences; according to producer's protocol) and PI (5 $\mu \mathrm{g} / \mathrm{mL}$, Sigma-Aldrich). Upon $30 \mathrm{~min}$ incubation at room temperature in the dark, the viable, early apoptotic, late apoptotic/necrotic, and necrotic cell populations were detected and counted by flow cytometry (BD Biosciences). Data were analyzed with ModFit $\mathrm{LT}^{\mathrm{TM}}$ program (Verity Software House Inc.).

In addition, the specific markers of programmed cell death were determined, cleavage of Poly (ADP-ribose) polymerase-1 (PARP) and caspase-3, by western blot as described previously (Brozovic et al., 2013). In short, the $2 \mathrm{~h}$ incubation at room temperature with monoclonal anti-PARP (Santa Cruz Biotechnology) and polyclonal anti-caspase-3 (anti-Cas-3; Cell Signaling Technology, Danvers, MA, United States) antibodies was performed. After washing with $0.01 \%$ Tween 20 in PBS and incubation with the corresponding horseradish peroxidase-coupled secondary antibody (Amersham Pharmacia Biotech, Munich, Germany), proteins were visualized with ECL (Amersham Pharmacia Biotech) according to the manufacturer's protocol. All membranes were incubated with anti-extracellular-signal-regulated kinases $1 / 2$ (anti-ERK1/2) (Santa Cruz Biotechnology) antibody to confirm equal protein loading. ERK1/2 was used as loading controls since no changes in total ERK1/2 expression were detected upon exposure of cells to different drugs (Brozovic et al., 2004; Herraiz et al., 2011).

\section{Determination of Glutathione Function}

The function of intracellular GSH in cell response to STL 5 was investigated by MTT assay. HeLa cells were either pretreated overnight with a specific inhibitor, $0.001 \mathrm{mM}$ BSO or for $2 \mathrm{~h}$ with a precursor in GSH synthesis, $5 \mathrm{mM}$ NAC. Both compounds are frequently used in the manipulation of GSH level in the cells (Trachootham et al., 2009; Kannan et al., 2014). Upon pre-treatment with either BSO or NAC, different concentrations of STL 5 were added and the cytotoxicity effect of the compounds was determined $72 \mathrm{~h}$ later as described above.

The capacity of GSH to form the detoxification conjugates through enzymatic reaction with STL 5 was investigated by pretreatment of HeLa cells with of $5 \mu \mathrm{g} / \mathrm{mL}$ ETA for $2 \mathrm{~h}$ and then with different concentrations of STL 5 . The cell survival was examined $72 \mathrm{~h}$ after. The optimal concentrations of used modulators of GSH synthesis and glutathione S-transferase reaction were determined previously (Osmak and Eljuga, 1993; Brozovic et al., 2008; Brozovic et al., 2013).

\section{Statistical Analysis}

All results were evaluated using the software package Statistica 12.0 (StatSoft, Tulsa, OK, United States). Results were subjected to one-way ANOVA for comparison of means and significant differences were calculated according to Duncan's multiple range test. Data were considered statistically significant at $P<0.05$. Different letters indicate significant difference at $P<0.05$.

\section{RESULTS}

\section{Selection of Plant Material, Extraction, Fractionation, and First Bioactivity Assessment}

As a starting point for phytochemical investigations, an ethanol C. ragusina $\mathrm{L}$. leaf extract (CRE) was prepared to obtain a multicomponent mixture embracing a wide range of secondary metabolites (Figure 1).

In antibacterial assays, CRE showed moderate antibacterial effects against $S$. aureus with MIC of $500 \mu \mathrm{g} / \mathrm{mL}$ and MBC of $2000 \mu \mathrm{g} / \mathrm{mL}$, respectively, and weak activity against $A$. baumannii with MIC and MBC values both of $>4000 \mu \mathrm{g} / \mathrm{mL}$, respectively (Vujčić et al., 2017).

In contrast to the moderate antibacterial activity, CRE exhibited significant effects on all tested murine melanoma (B16F10), squamous cell carcinoma (SCCVII) fibrosarcoma (FsaR) cell lines and normal hamster fibroblasts (V79).

At a CRE concentration of $60 \mu \mathrm{g} / \mathrm{mL}$, the cell survival relative to the negative control was $\leq 10 \%$ for V79 fibroblasts, SCCVII and FsaR, respectively, and 21\% for the B16F10. CRE did not show selective cytotoxicity between murine cancer and normal cell lines, which may be a consequence of a cumulative effect of different bioactive compounds included in the crude extract.

For a more focused isolation process of the bioactive constituents of the crude extract, twelve fractions (A1-A12) obtained by separation of CRE via silica gel chromatography were retested in the mentioned cell lines, revealing A6 and A8 as fractions with the strongest cytotoxic activity. Both fractions (60 $\mu \mathrm{g} / \mathrm{mL})$ exhibited stronger cytotoxic activity on B16F10 $(<10 \%$ cell survival) and SCCVII $(\leq 20 \%$ cell survival) cell 


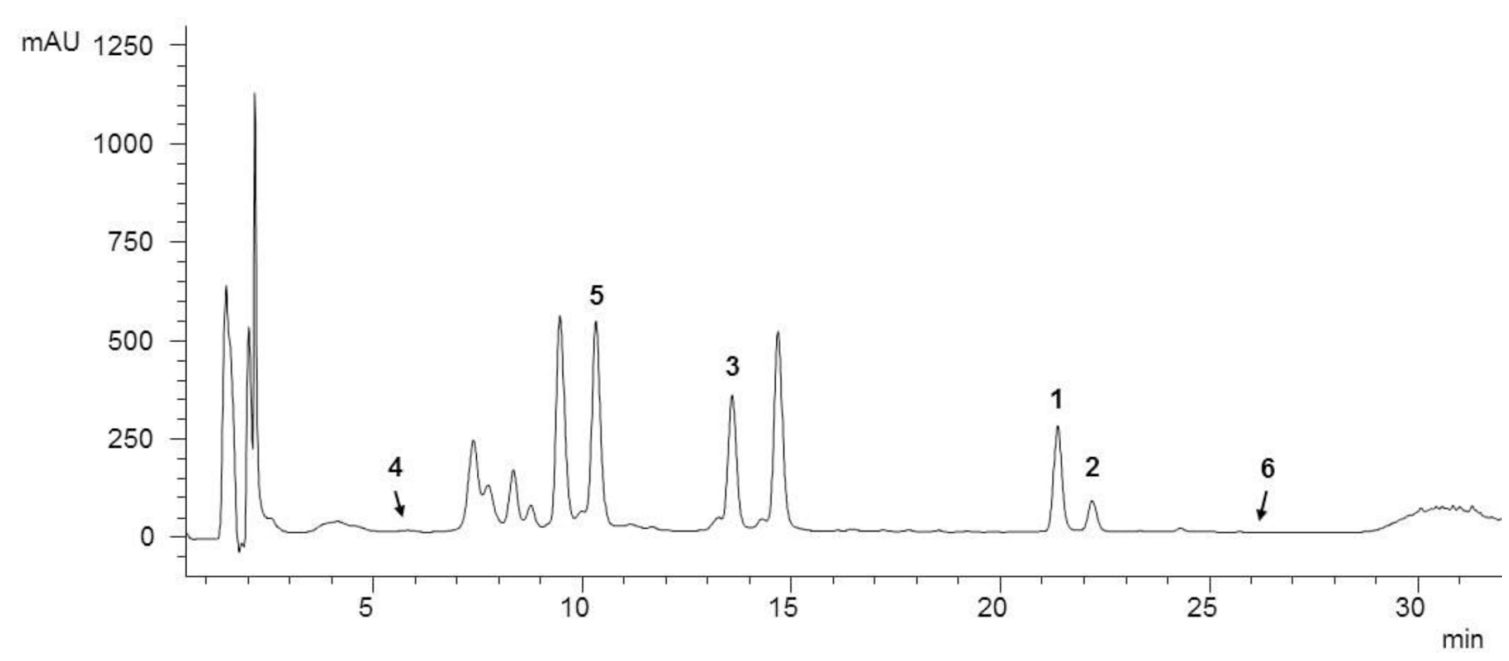

FIGURE 1 | HPLC analysis of the ethanol C. ragusina L. leaf extract (CRE) at $254 \mathrm{~nm}$ (for LC parameters see section "Experimental").

lines compared to the moderate activity observed toward FsaR and V79 cells ( $\leq 30 \%$ cell survival). Based on these results, fractions $\mathrm{A} 6$ and $\mathrm{A} 8$, were selected for further chromatographic separations.

\section{Isolation and Identification of Pure Compounds}

Six constituents were isolated from CRE fractions A6 and A8 by separation techniques including CC and preparative thin layer chromatography.

By using HR-ESI-MS analyses and NMR experiments, and by comparison with earlier studies (Miyase et al.,
1985; Zdero et al., 1989; Jang et al., 1999; Nagao et al., 2002; Marques et al., 2010; Yang et al., 2013), the isolates (Figure 2) were identified as chrysin (1), oroxylin A (2), hispidulin (3), deacylcynaropicrin (4), (3aR,4S,6aR,8S,9aR,9bR)[dodecahydro-8-dihydroxy-3,6,9-tris(methylene)-2oxo-

2(3H)-azuleno[4,5-b]furanyl]-3-methyl-butanoate (5), and hemistepsin A (6). Instead of using the complicated and long systematic name for compound $\mathbf{5}$, we created the trivial name ragusinin. The flavonoids (1-3) can be classified as flavones, whereby different substitution patterns with methoxy or hydroxy groups can be observed on C-6 and C- $4^{\prime}$. Compounds 4-6 are STLs belonging to the subtype of guajanolides.<smiles>O=c1cc(-c2ccc(F)cc2)oc2cc(O)cc(O)c12</smiles>

chrysin (1)<smiles>C=C1C[C@H]2C(=C)C[C@@H](O)C(=C)[C@H]2[C@H](O)C1C(=O)O</smiles>

deacylcynaropicrin (4)<smiles>COc1c(O)cc2oc(-c3ccccc3)cc(=O)c2c1O</smiles>

oroxylin A (2)

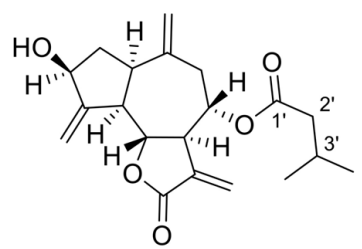

ragusinin (5)<smiles>COc1c(O)cc2oc(-c3ccc(O)cc3)cc(=O)c2c1O</smiles>

hispidulin (3)

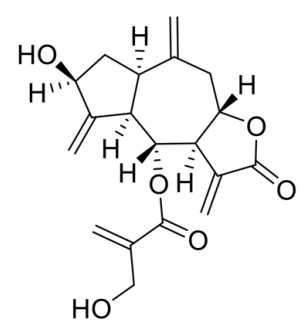

hemistepsin A (6)

FIGURE 2 | Chemical structures of isolated $C$. ragusina L. leaf constituents. 
Compound $\mathbf{1}$ is a frequently occurring flavone reported as a constituent of several Centaurea species (Mouffok et al., 2012). However, we are reporting here for the first time its isolation from C. ragusina L. Different bioactivities have been reported for compound 1. It suppresses inducible nitric oxide synthase, cyclooxygenase- 2 expression and inhibits NF- $\mathrm{kB}$ activation, which altogether leads to anti-inflammatory effects (Feng et al., 2014). Compound $\mathbf{1}$ is also reported as a tumor cell growth arrest compound, arresting C6 glioma cells in the G1 phase of the cell cycle either through activating p38-MAPK leading to the accumulation of $\mathrm{p} 21 \mathrm{Waf1} / \mathrm{Cip} 1$ protein or mediating the inhibition of proteasome activity (Weng et al., 2005). It also suppresses tumor growth of anaplastic thyroid cancer ATC cells both in vitro and in vivo (Yu et al., 2013).

Compound 2 is known as the main component of several medicinal plants including Oroxylum indicum (Krueger and Ganzera, 2012) and various Centaurea species. However, so far it has not been reported for C. ragusina L. Compound 2 was shown to activate caspase- 3 and caspase- 9 in human colon carcinoma HCT-116 cells and decrease tumor volume and weight in immunodeficient mice that were inoculated with HCT-116 cells (Hu et al., 2012). It also exhibits anti-inflammatory effects by decreasing pro-inflammatory cytokines mediated by estrogen receptor activity (Wang et al., 2013).

Compound 3 has been isolated from various Centaurea species, e.g., C. melitensis L. (Negrete et al., 1989), C. aspera L. (Ferreres et al., 1980), and C. jacea L. (Forgo et al., 2012), but never from C. ragusina $\mathrm{L}$. It is an important compound used in traditional Chinese medicine for the treatment of liver carcinoma (Gao et al., 2014). Besides an apoptotic effect on human liver cancer HepG2 cells, it was also shown that this effect is mediated via mitochondrial dysfunction (Gao et al., 2014). Furthermore, an anti-proliferative effect toward human lung cancer A-549 cells was reported for compound 3 (Zhang et al., 2012).

Compound 4 has already been reported as a constituent of C. ragusina L. (Mahmoud et al., 1986) and other Centaurea species with anti-inflammatory and cytotoxic activity (González et al., 1977; Sosa et al., 2011).

To the best of our knowledge, guajanolides 5 and $\mathbf{6}$ have never been identified as constituents from Centaurea species before. Whereas for $\mathbf{6}$ an antibacterial and cytotoxic activity toward human cell lines in the low $\mu \mathrm{M}$ range has previously been reported (Jang et al., 1999) compound 5 (ragusinin) with its isovalerate residue is a rare STL without reports on bioactivity.

\section{Study of Biological Activity (Interactions With DNA, Antibacterial and Cytotoxic Activity) of C. ragusina L. Constituents}

The DNA binding affinity of compounds is important to explore since the DNA represents a well-known target of several broadly used drugs and the binding to DNA is one of the common causes of cell death (Demeunynck et al., 2002; Sangeetha Gowda et al., 2014). In order to determine the binding affinity of the isolated compounds to ctDNA, UV/Vis spectroscopy and ITC (Bronowska, 2011) were employed (see section "Experimental" and "Supplementary Material for details"). CD was used for monitoring of conformational changes of ctDNA induced by small molecule binding and for gaining information about modes of interaction (Cantor and Schimmel, 1980; Johnson, 1994).

Among isolated STLs and flavonoids (Supplementary Material), only 5 exhibited significant changes in CD titrations (Supplementary Figure S4). Due to that reason, we decided to characterize the binding of DNA only with compound 5. UV/Vis spectroscopy was not applicable in the study of DNA-ragusinin interaction due to absorption of STLs at short wavelengths (210-220 nm). Therefore, the binding interaction of 5 with ctDNA was monitored by ITC.

The ITC experiment of 5 with ctDNA resulted in negative peaks indicating that the binding process was exothermic (Figure 3). The resulting values were fitted to a single-site binding model by the non-linear least square method yielding rather moderate binding constant $\left(\log K_{\mathrm{a}}=4.04\right)$. The stoichiometry (N) was fixed to 0.5 based on results from $\mathrm{CD}$ titration with ctDNA (the saturation of binding sites was reached at the ratio, $r=0.5$, Supplementary Material). The binding of $\mathbf{5}$ to ctDNA was characterized by a positive binding entropy $\left(T \Delta_{\mathrm{r}} \mathrm{S} / \mathrm{kJ}\right.$ $\left.\mathrm{mol}^{-1}=18.5\right)$ accompanied by smaller negative enthalpy $\left(\Delta_{\mathrm{r}} \mathrm{H} / \mathrm{kJ} \mathrm{mol} \mathrm{m}^{-1}=-4.6\right)$ revealing that its binding is entropically driven. In many cases, the groove binding is associated with positive (favorable) binding entropies due to the release of

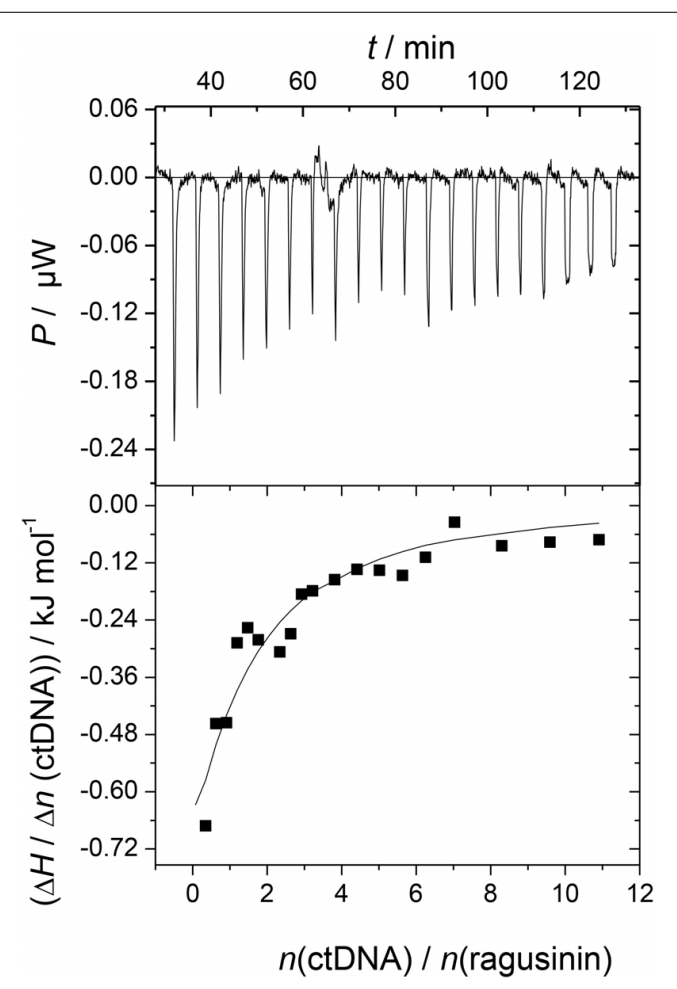

FIGURE 3 | Calorimetric titration of compound $\mathbf{5}\left(\mathrm{c}=3 \times 10^{-5} \mathrm{~mol} \mathrm{dm}^{-3}\right.$ ) with ctDNA $\left(c=1.5 \times 10^{-3} \mathrm{~mol} \mathrm{dm}^{-3}\right)$ in sodium cacodylate buffer $(\mathrm{pH} 7.0$, $\left.I=0.05 \mathrm{~mol} \mathrm{dm}^{-3} ; T=25^{\circ} \mathrm{C} ; \Delta_{\mathrm{r}} \mathrm{G} / \mathrm{kJ} \mathrm{mol}^{-1}=-23.0\right)$. The top panels represent the raw data from the single injection of ctDNA into a solution of $\mathbf{5}$ and the bottom panels show the experimental injection heats while the solid lines represent the calculated fit of the data. 


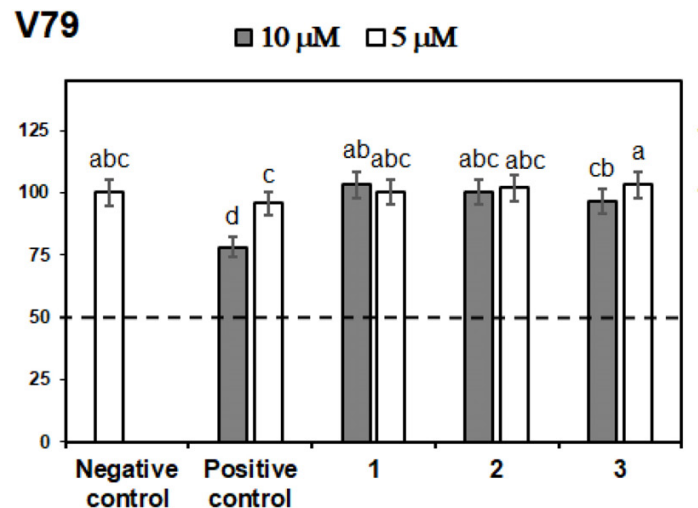

SCCVII

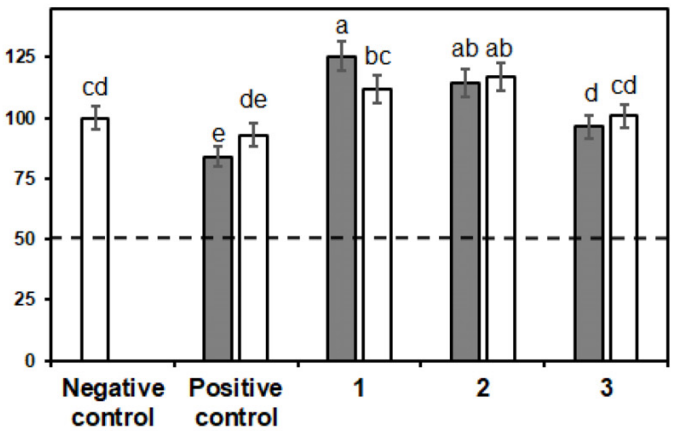

CaCo-2

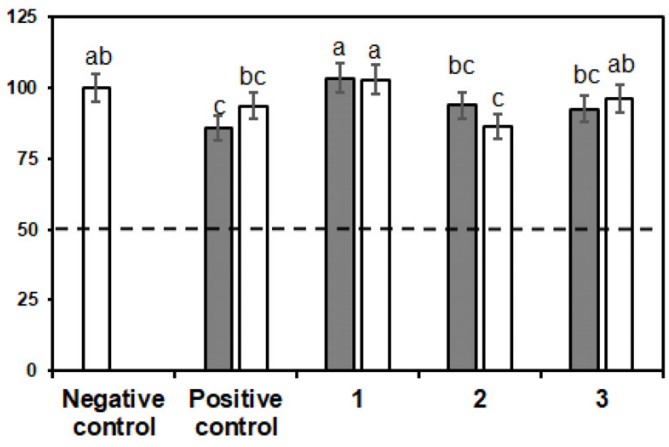

MCF-7

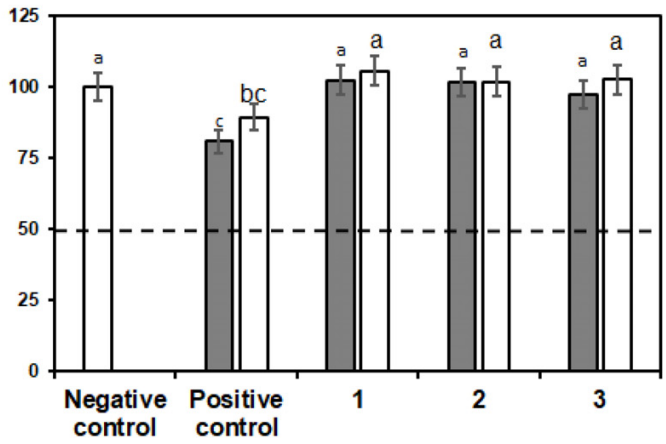

B16F10

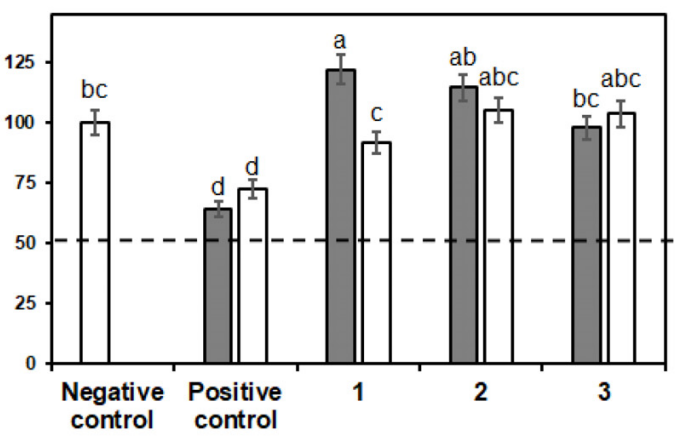

FsaR

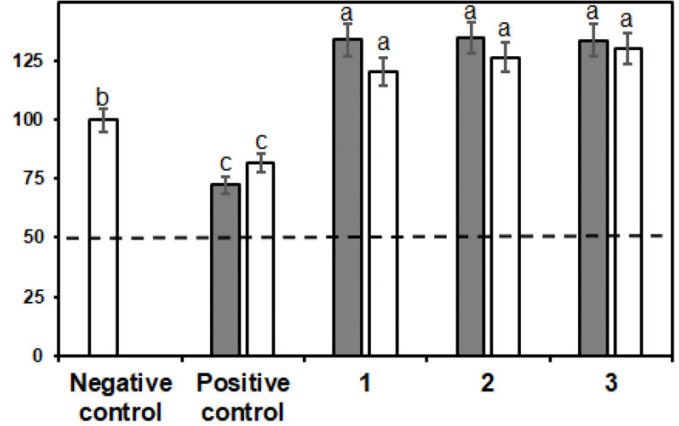

HeLa

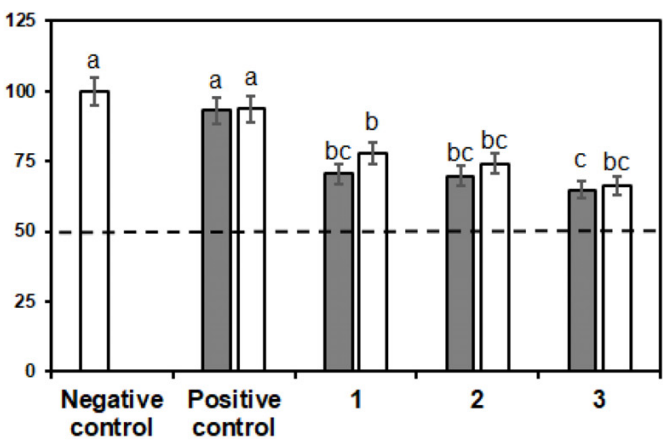

FIGURE 4 | Percentage of cell survival of rodent (V79, SCVII, B16F10, and FsaR) and human (Caco-2, HeLa, and MCF-7) cell lines after exposure to isolated flavonoids (compounds 1-3), at concentrations of $10 \mu \mathrm{M}$ (gray bars) and $5 \mu \mathrm{M}$ (white bars). Values represent mean of 3 replicates \pm SD. Different letters indicate significant difference at $p<0.05$. The dashed line indicates inhibition of cell growth by $50 \%$. The positive control is 5 -fluorouracil and the negative control are cells without the tested samples. 


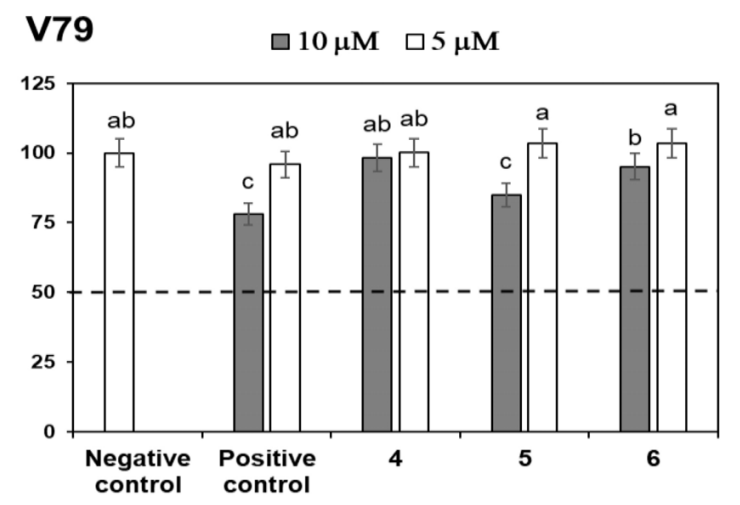

SccVII

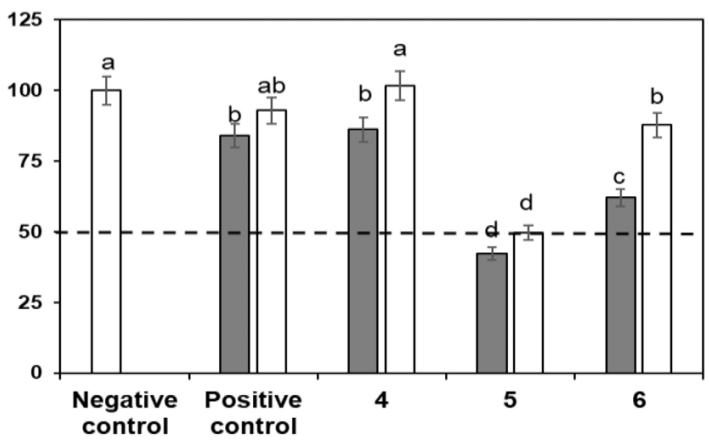

CaCo-2

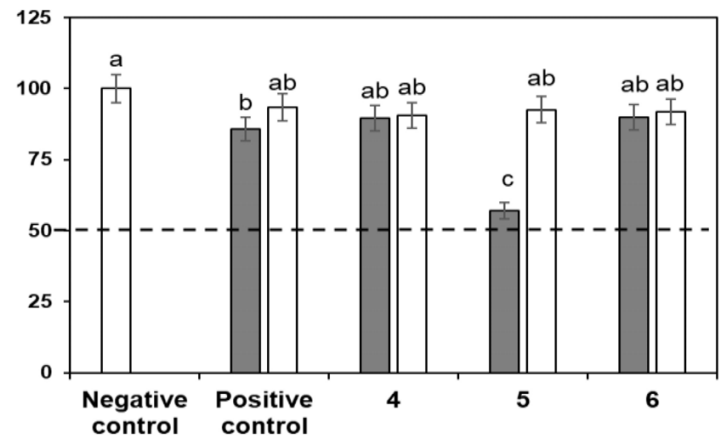

MCF-7

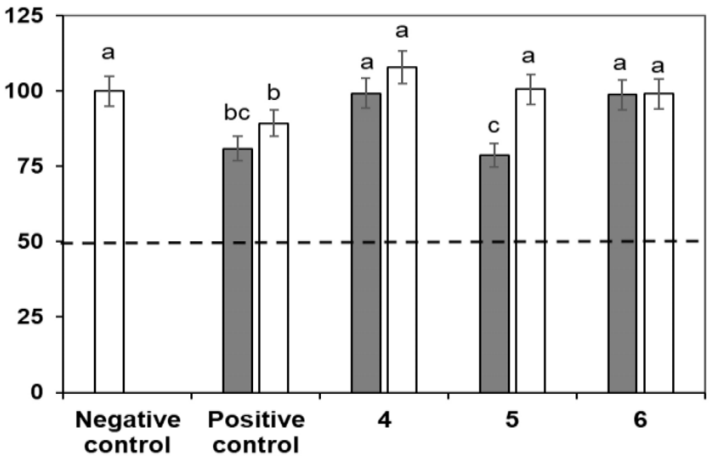

B16F10

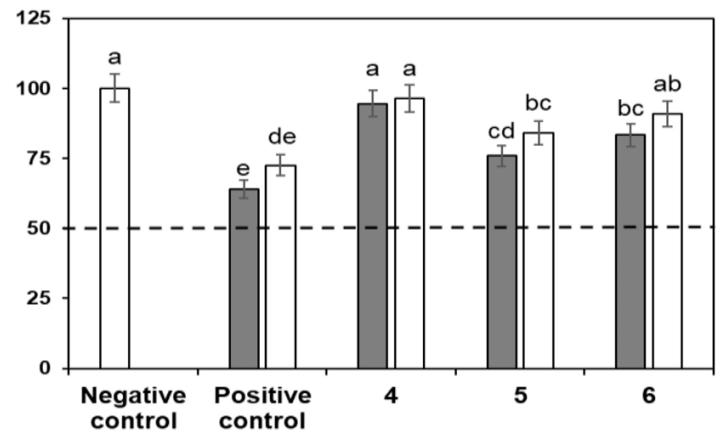

FsaR

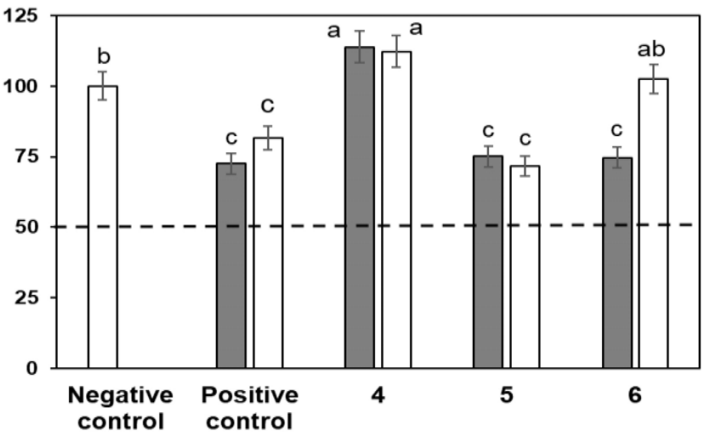

HeLa

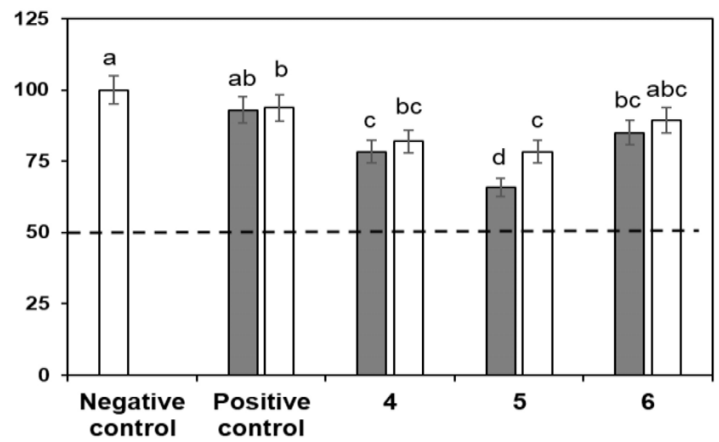

FIGURE 5 | Percentage of cell survival of murine (V79, SCVII, B16F10, and FsaR) and human (Caco-2, HeLa, and MCF-7) cell lines after exposure to deacylcynaropicrin (4), ragusinin (5) and hemistepsin A (6) at concentrations of $10 \mu \mathrm{M}$ (gray bars) and $5 \mu \mathrm{M}$ (white bars). Values represent means of three replicates $\pm \mathrm{SD}$. Different letters indicate significant difference at $p<0.05$. The dashed line indicates inhibition of cell growth by $50 \%$. The positive control is 5 -fluorouracil and the negative control are cells without the tested samples. 


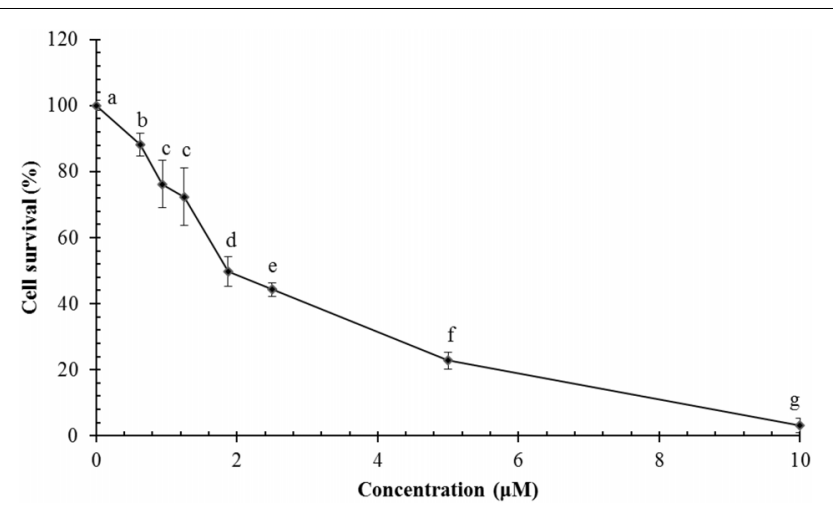

FIGURE 6 | Survival of HeLa cells following treatment with ragusinin (5). The cells were treated for $72 \mathrm{~h}$ with different doses of $\mathbf{5}$. The MTT assay was performed. Each point represents the mean of quadruplicates $\pm \mathrm{SD}$. Different letters indicate significant difference at $p<0.05$. The experiment is performed at least three times.

confined or interfacial water molecules to the bulk (Perozzo et al., 2004; Chaires, 2006).

Two STLs 5 and $\mathbf{6}$ were found to be the most active ones reducing the growth of $S$. aureus with a MIC value of $31.3 \mu \mathrm{g} / \mathrm{mL}$ (Supplementary Table S1). However, since the cytotoxic activity of the isolated compounds was more prominent than the antibacterial, we presented here cytotoxic activity in more detail while the data on antibacterial activity are available in the Supplementary Material.

Crystal Violet (CV) bioassay, which measures the DNA mass of living cells, was used for initial activity screening. The cytotoxic effect of the isolated compounds $\mathbf{1}$ to $\mathbf{6}$ was monitored with the $\mathrm{CV}$ test for $24 \mathrm{~h}$ at 5 and $10 \mu \mathrm{M}$ on a panel of three murine and three human cancer cell lines. 5-Fluorouracil was used as a positive control at equimolar concentrations as the studied compounds (Figures 4, 5). The isolated flavonoids 1 and 2 did not demonstrate cytotoxic activity against the majority of cancer cell lines at the applied concentrations. Only compound 3 exerted a noticeable cytotoxic effect against HeLa cells (Figure 4). Among the isolated STLs, 5 showed the most prominent cytotoxic activity. In particular, compound 5 reduced the cell survival of SCCVII cells to $42 \%$ at $10 \mu \mathrm{M}$ and $50 \%$ at $5 \mu \mathrm{M}$ concentration (Figure 5). It also exhibited promising activity against Caco-2 cells at $10 \mu \mathrm{M}$ and HeLa cells at both concentrations applied (Figure 5). On the other hand, 5 had weak (cell survival was $85 \%$ at $10 \mu \mathrm{M}$ ) or no activity (> $95 \%$ at $5 \mu \mathrm{M}$ ) against normal fibroblasts (V79). Moreover, while compound 4 did not show significant cytotoxic effects on any cell line at the applied concentrations, compound $\mathbf{6}$ showed noticeable effects against SCVII and FsaR cells at $10 \mu \mathrm{M}$.

\section{Cytotoxic Activity of Ragusinin (5) in HeLa Cell Line}

In order to determine the biological effect of the most active compound 5 (Figure 5) in more detail, we used MTT assay and HeLa cells as experimental model (Cimbora-Zovko et al., 2011).
Ragusinin decreased cell survival of HeLa cells in a concentration-dependent manner in comparison to untreated cells (Figure 6). The dose that killed $50 \%$ of the cell population ( $\mathrm{IC}_{50}$ value) after $72 \mathrm{~h}$ of continuous treatment with 5 was between 1.8 and $2.3 \mu \mathrm{M}$ (Figure 6).

To better understand the mechanism underlying the cell growth impairment by $\mathbf{5}$, the cell cycle progression was investigated. HeLa cells were treated with increasing doses of 5 during 48 h. As shown in Figure 7A, compound 5 triggers the accumulation of HeLa cells in the G2 phase of cell cycle. Moreover, a compound 5 induced dose-dependent increase of cells in the Sub G1 population indicates a ragusinin-triggered cell death (Figure 7A). The same was confirmed by treatment of cells with $10 \mu \mathrm{M}$ of 5 during 24-72 h. Time-dependent accumulation of HeLa cells in the G2 phase is detectable as well as time-increase of cells in the Sub G1 phase of the cell cycle (Figure 7B).

In order to determine the type of cell death triggered by $\mathbf{5}$, the cells were treated with increasing doses of the compound and $48 \mathrm{~h}$ later, FACS-Annexin V/PI staining was performed. Our results show a ragusinin-induced dose-dependent apoptosis (Figure 7C). We then performed Western blot analysis of specific cell death markers, PARP and caspase-3 cleavage, following treatment with 1.25 and $2.5 \mu \mathrm{M}$ of 5 for $24-72 \mathrm{~h}$. The obtained results were interesting since $\mathbf{5}$ did not induce PARP (Figure 7D) and caspase-3 cleavage (Figure 7E). The assumed caspase-independent cell death triggered by $\mathbf{5}$ was in addition confirmed by measuring the absence of caspase activity $3 / 7$ by Caspase-Glo $^{\circledR}$ 3/7 Assay (data not shown). The occurrence of caspase and PARP cleavage independent cell death indicates some alternative cell death pathway described in the literature to be triggered by a different type of cell stressors (Kroemer and Martin, 2005; Tait and Green, 2008). This is the first example of caspase independent cell death described for compound 5 .

\section{Role of Glutathione (GSH) in Protection of Cells From Ragusinin (5)}

We were further interested in the possible role of GSH as a protector of cells in ragusinin-induced cell death. For that

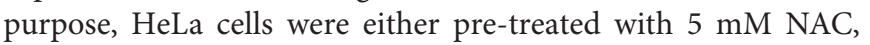
the precursor for GSH synthesis, for $2 \mathrm{~h}$ prior to treatment with compound 5 or overnight with a specific inhibitor of GSH synthesis, i.e., $0.001 \mathrm{mM}$ BSO. The conditions used were tested previously to be effective (Brozovic et al., 2008; Brozovic et al., 2014). The obtained data showed that an increased level of GSH protects HeLa cells from ragusinin's toxicity (Figure 8A). At the same time, depletion of GSH decreased cell survival of HeLa cells compared to cells treated with compound $\mathbf{5}$ only (Figure 8B).

Due to the fact that GSH instills several vital roles within a cell including antioxidation, maintenance of the redox state, modulation of the immune response, and detoxification of xenobiotics (Balendiran et al., 2004), we discussed first its possible role as an antioxidant. It is known that the cytotoxicity of the sesquiterpenes helenalin and cynaropicrin can be affected via generation of intracellular reactive oxygen species (ROS) (Cho et al., 2004; Jang et al., 2013). We examined that possibility for 

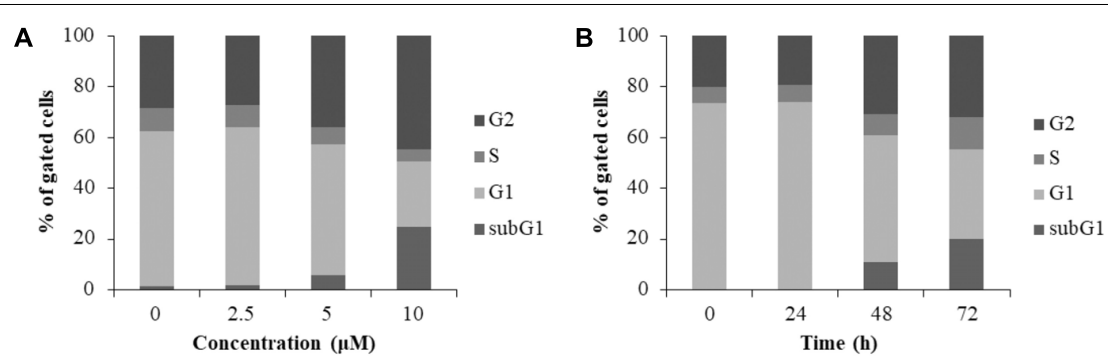

C
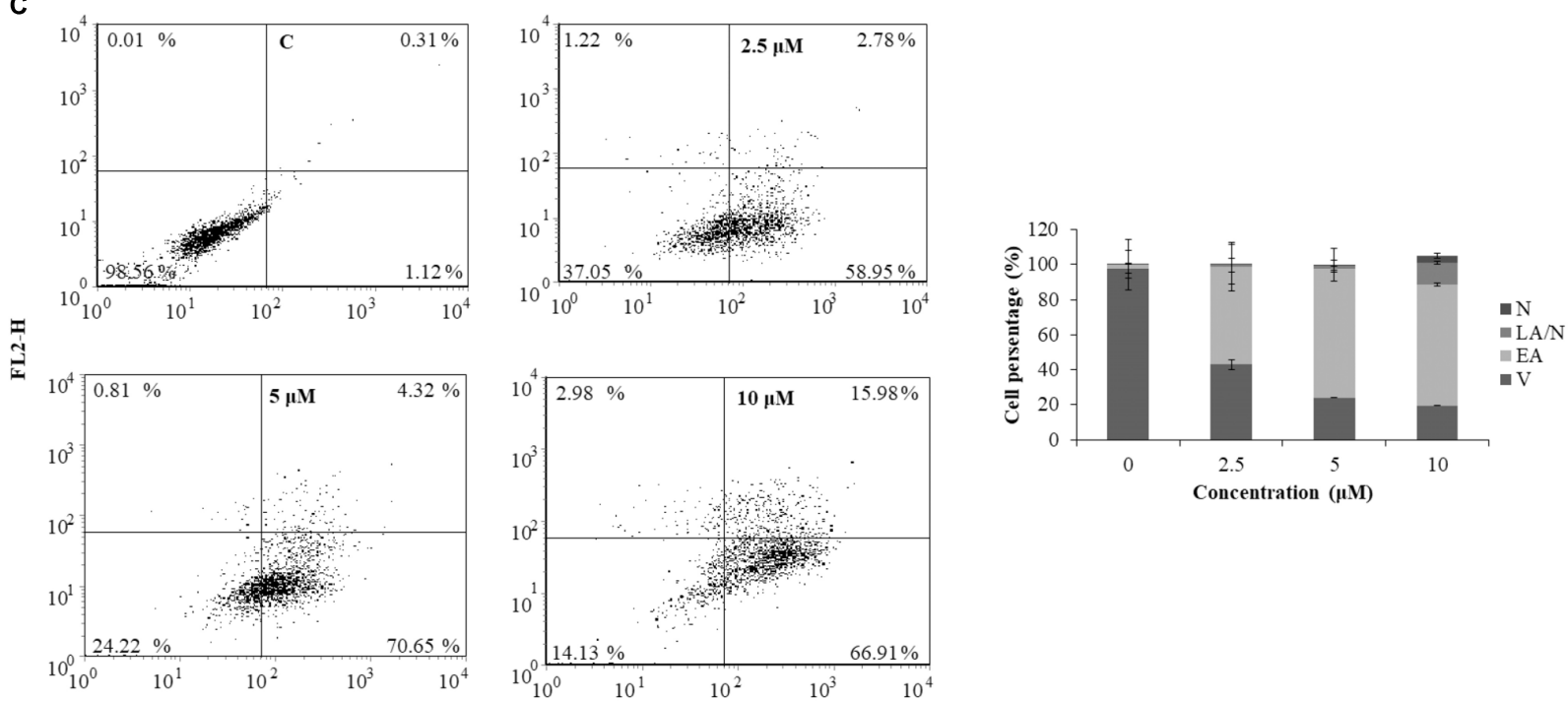

FL1-H

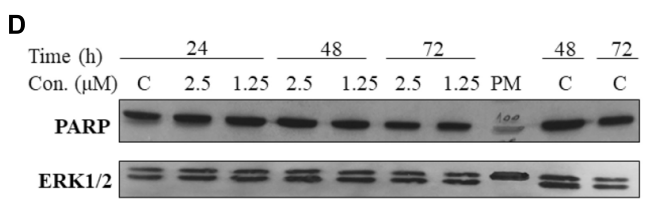

E

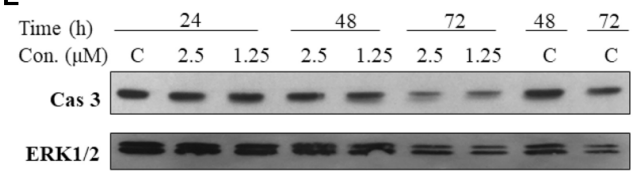

FIGURE 7 | Cell cycle progression and induction of cell death in HeLa cells following treatment with ragusinin (5). The cells were treated either with 2.5, 5, and $10 \mu \mathrm{M}$ of compound $\mathbf{5}$ for $48 \mathrm{~h}$ (A) or with $10 \mu \mathrm{M}$ during $24-72 \mathrm{~h}$ (B). Cell cycle analysis was performed by flow cytometry upon cell staining with PI. The representative data of three independent experiments which yielded similar results are shown. The cells were either non-treated or treated with $2.5,5$, and $10 \mu \mathrm{M}$ of 5. After $48 \mathrm{~h}$ and staining with Annexin $\mathrm{V}$ and $\mathrm{Pl}$, the cell death was measured by flow cytometry. The representative data of three independent experiments which yielded similar results are shown. The statistical analysis of data for three independent experiments are presented as means of percentage of viable $(\mathrm{V})$, early apoptotic (EA), late apoptotic/necrotic (LA/N), and necrotic (N) cell populations \pm standard deviation (C). 24-72 h after exposure to 1.25 and $2.5 \mu \mathrm{M}$ of $\mathbf{5}$, protein level of cleaved PARP (D) and activated (i.e., cleaved) caspase-3 (Cas 3) (E) and cleaved PARP was analyzed in whole cell extracts by Western blot analysis. ERK1/2 protein expression was used as internal protein loading control. The representative data of three independent experiments which yielded similar results are shown. Zero or C, non-treated cells; PM, protein marker PageRuler Prestained Protein Ladder (Thermo Fisher Scientific, United States). FL1-H/Annexin V-FITC,

FL2-H/Propidium iodide.

ragusinin by pre-treating HeLa cells either with tempol or trolox, two antioxidant compounds (Aliaga et al., 2003). Results showed that antioxidants, as well as salubrinal (Brozovic et al., 2013), i.e., an inhibitor of endoplasmic reticulum stress, had no impact on ragusinin's toxicity (Supplementary Material, in Supplementary Figure S6). Thus, we further explored the capability of 5 to reach functional protein targets in living cells without being deactivated by reaction with GSH. The combination treatment of HeLa cells with the well-known inhibitor of glutathione S-transferase (GST) ethacrynic acid (Osmak et al., 1998) (c=5 $\mathrm{g} / \mathrm{mL})$ decreased cell survival compared to cells treated with 5 only (Figure 9 ). The data imply the enzymatically regulated formation of a detoxification complex between GSH and $\mathbf{5}$.

\section{DISCUSSION}

Among the isolates ( 1 to $\mathbf{6}$ ) from the traditionally used herbal remedy $C$. ragusina $\mathrm{L}$. leaves, we discovered an interesting pharmacological profile for the rare guajanolides ragusinin (5) and hemistepsin A (6). Compound 5 was only once described as a constituent from the aerial parts of the Australian Helipterum 

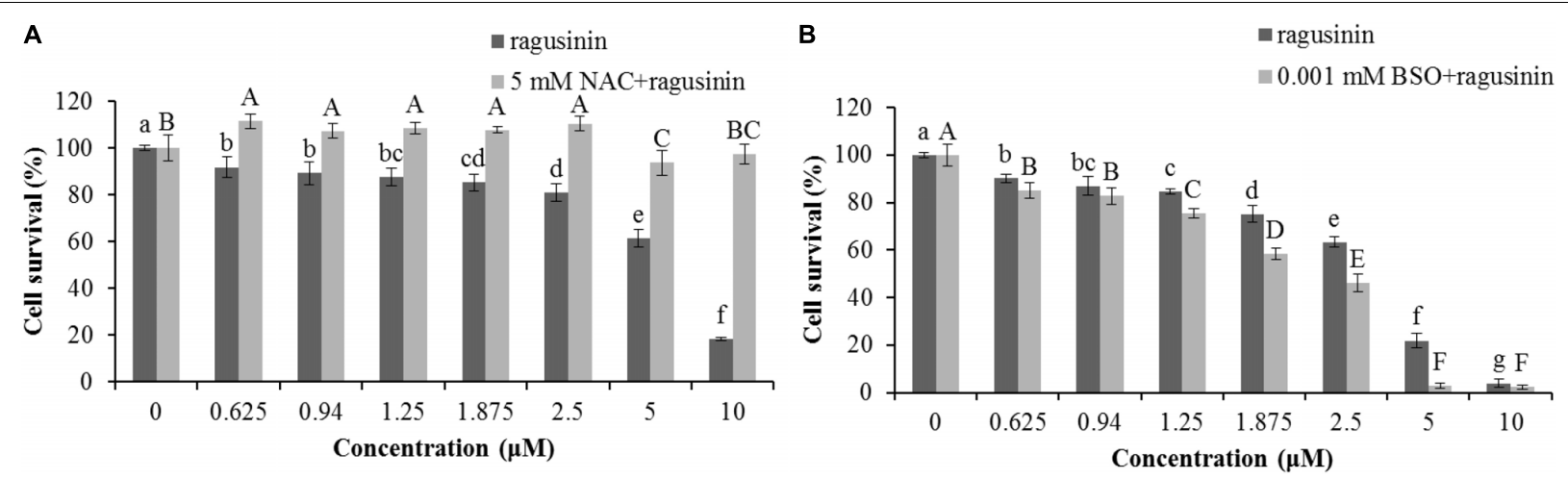

FIGURE 8 | The role of GSH in ragusinin's (5) toxicity. The cells were either pre-treated for $2 \mathrm{~h}$ with $5 \mathrm{mM}$ NAC (A) or overnight with $0.001 \mathrm{mM}$ BSO (B). After that, the cells were treated with different concentrations of $\mathbf{5 . 7 2} \mathrm{h}$ later, an MTT assay was performed. Bars represent the mean of quadruplicates \pm SD. Different letters indicate significant difference at $p<0.05$. All experiments were performed at least three times.

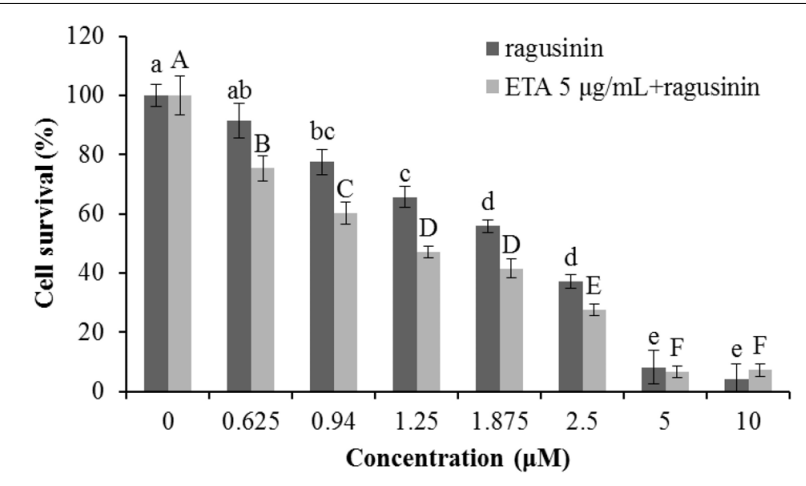

FIGURE 9 | The treatment of HeLa cells with ethacrynic acid (ETA). The cells were pre-treated with $5 \mu \mathrm{g} / \mathrm{mL}$ ETA. Two hours later the cells were treated with different concentrations of $\mathbf{5 . 7 2}$ h later an MTT assay was performed. Bars represent the mean of quadruplicates $\pm \mathrm{SD}$. Different letters indicate significant difference at $p<0.05$. All experiments were performed at least three times.

maryonii S. Moore (Zdero et al., 1989) and is characterized by an isovalerate residue in position C-9a; compound 6 has been described previously as a constituent from leaves and flowers of Hemisteptia lyrata Bunge (Jang et al., 1999).

Whereas both natural compounds showed no antibacterial activity against the Gram-negative A. baumannii, they exhibited moderate inhibitory activity against S. aureus ATTC 25923 (MIC value of $31.3 \mu \mathrm{g} / \mathrm{mL}$, Supplementary Material). For comparison, three STLs (13-acetylsolstitialin A, centaurepensin, and chlorojanerin) isolated from the aerial parts of Centaurea solstitialis L. ssp. solstitialis showed inhibitory activity against standard S. aureus with MIC values of $16 \mu \mathrm{g} / \mathrm{mL}$ (Özçelik et al., 2009). In line with these findings, we focused on a more prominent cytotoxic activity of the isolated compounds and the investigation of the mechanism of action of the most active compound 5 .

We investigated the binding of isolated compounds to DNA since studying the interactions of compounds with potential drug targets and the knowledge of their antiproliferative activity can help in forming the hypothesis about the mechanism of action of novel compounds.

The isolated flavonoids (1-3) exhibited only weak interactions with DNA which may explain the absence of cytotoxic effects against the majority of studied cell lines. The somewhat stronger cytotoxic activity of flavonoids against $\mathrm{HeLa}$ cells can be ascribed to interactions with biological targets other than DNA (Chen et al., 2012). Compound 3 with the highest number of hydroxy groups on the flavonoid skeleton exhibited the most pronounced cytotoxic activity. This finding is in agreement with literature data (Beutler et al., 1998; Jeong et al., 2007; Csupor-Löffler et al., 2009).

While there are numerous reports on DNA binding affinities with flavonoids (Kanakis et al., 2005; Rusak et al., 2010), there is little data on DNA binding interactions with sesquiterpenes (Vujčić et al., 2007). Regarding the structure of $\mathbf{5}$ and literature data (Gates, 2009; Chadwick et al., 2013), the interaction with DNA can be achieved via noncovalent binding to double-stranded DNA (dsDNA) or by alkylation of DNA nucleophiles through reaction with the $\alpha$-methylene- $\gamma$-lactone group. Several studies on the reactivity of STLs toward $\mathrm{OH}$ or N-nucleophiles (Atta-ur-Rahman, 2011) and CD changes which were not consistent with an alkylation effect (Agarwal et al., 2014) (Supplementary Figure S4) do not support the latter possibility. In addition, the - $\mathrm{SH}$ group was found to be much more susceptible to alkylation by sesquiterpenes than other nucleophiles (Gewirtz et al., 2007). A reasonable explanation based on our results from ITC and CD is noncovalent binding of 5 most probably inside a hydrophobic interior of the DNA groove. Due to its rather moderate binding affinity to ctDNA, it can be concluded that the cellular DNA is not 5's primary target in the living cell.

Among the isolated STLs, compound $\mathbf{5}$ showed the strongest cytotoxic activity, especially on murine SCVII as well as human Caco-2 and HeLa cell lines, while no cytotoxic effect on normal V79 fibroblasts was observed. Similar to the antibacterial activity, a correlation between the cytotoxic activity and the type and properties of the substituents on the central ring in the vicinity of the $\alpha$-methylene- $\gamma$-lactone group was observed. In comparison to less lipophilic substituents of sesquiterpenes, as observed for 


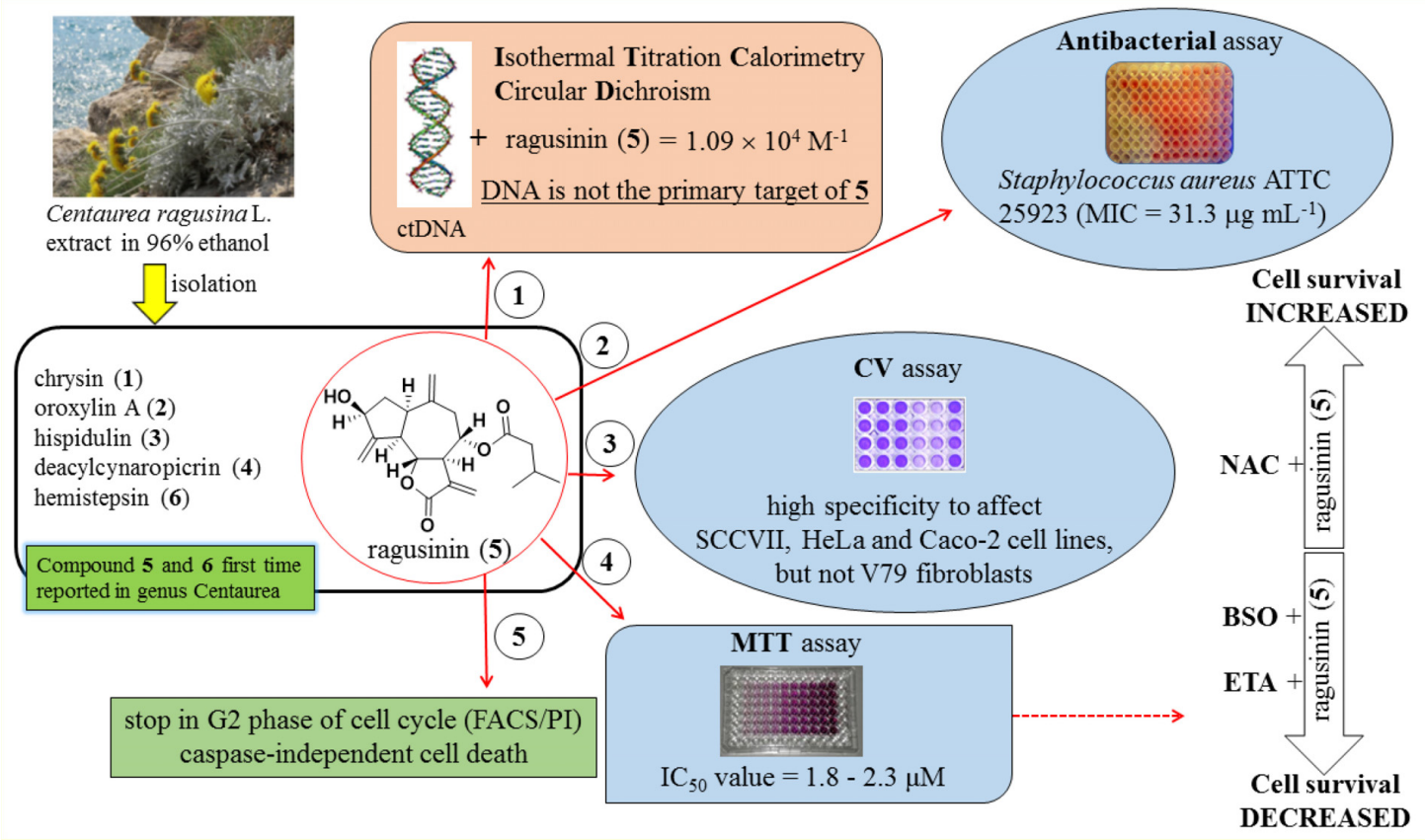

FIGURE 10 |A summary of the most significant results obtained in this study.

6 and especially 4, the hydrophobic character of the isovalerate residue of $\mathbf{5}$ most probably enables better penetration through the cell membrane and consequently better antibacterial and cytotoxic activity. In the literature, cytotoxic effects of various STLs have been explained by selective alkylation of growth regulatory macromolecules such as enzymes which control cell division and thus a variety of cellular functions (Lyss et al., 1998; Rasul et al., 2012).

Since compound 5 (ragusinin) showed the strongest activity compared to other isolates and since the biological activity of 5 was not described before we decided to investigate the mechanism of its toxicity in more details. The $\mathrm{IC}_{50}$ value of ragusinin was between 1.8 and $2.3 \mu \mathrm{M}$ (Figure 6). This is consistent with data from the literature, where compounds of similar chemical structure, i.e., sesquiterpene lactones such as helenalin (Gertsch et al., 2003), neoambrosin (Saeed et al., 2015), and damsin (Villagomez et al., 2013), are reported to be cytotoxic against various cell lines in the micromolar range. Further analysis revealed time-dependent accumulation of HeLa cells upon ragusinin treatment in the G2 phase and Sub G1 phase of the cell cycle (Figure 7B). But, the occurrence of caspase and PARP cleavage independent cell death indicates some alternative cell death pathway described in the literature to be triggered by a different type of cell stressors (Kroemer and Martin, 2005; Tait and Green, 2008). The phenomenon is interesting to follow up further, especially because it is known that helenalin, a STL isolated from Arnica montana, induces the same atypical form of cell death which does not include the activation of classical mediators of apoptosis (caspases, AIF, Omi/HtrA2, and Apaf/apoptosome) (Hoffmann et al., 2011).
In a series of noteworthy reports on the reactivity and kinetics of helenalin and helenanolide type STLs with GSH, it was demonstrated that at physiological $\mathrm{pH}$ helenalin reacts with GSH almost exclusively via its cyclopentenone structure while the $\alpha$-methylene- $\gamma$-lactone site is less reactive (Schmidt et al., 1999). Since the guajanolide 5 possesses one reactive site, i.e., the $\alpha$-methylene- $\gamma$-lactone group that can react with GSH, we decided to investigate the role of GSH in deactivation of 5 .

From the literature is known that GSH is one of the major endogenous antioxidants. In the cytoplasm, GSH is used as a substrate for glutathione peroxidase in the reduction of $\mathrm{H}_{2} \mathrm{O}_{2}$ and lipid hydroxyperoxides, a reaction that produces glutathione disulfide, the so-called oxidative form of GSH. Glutathione disulfide is rapidly reduced to GSH by glutathione reductase. This redox cycling of GSH plays a role in the maintenance of cellular redox homeostasis. GSH binds to endogenous and diverse xenobiotic electrophilic compounds either catalytically, through the action of glutathione S-transferase, or non-catalytically (Townsend and Tew, 2003; Brozovic et al., 2014). The formed GSH conjugates can be exported from cells, resulting in the loss of cellular GSH. Our data showed that variation of GSH amount in the cell induced by a specific precursor in GSH synthesis, NAC or a specific inhibitor of GSH synthesis, BSO had an impact on HeLa survival upon treatment with $\mathbf{5}$ (Figure 8). Similar results were obtained in human colon tumor cells upon BSO and helenalin treatment (Jordan et al., 1987). Moreover, we showed that GSH probably does not play a role as antioxidant (Supplementary Figure S6) but rather as molecule which form conjugates with 5 increasing in that way the survival of HeLa cells upon treatment with it (Figure 9). 


\section{CONCLUSION}

In this study, the most active compound has shown to be a sesquiterpene lactone, ragusinin (compound 5) whose biological activity has not been investigated so far. Currently, our knowledge about the mechanism of action of STLs is still limited. Several of them reached clinical trials due to their ability to trigger cell death in tumor but not in normal cells (Zhou and Zhang, 2008; Kawasaki et al., 2009; Crespo-Ortiz and Wei, 2012).

Here we are showing for the first time the biological activity of compound 5, ragusinin. Although ITC and CD results suggested that the cellular DNA is not the primary target of ragusinin in the living cell, these data represent valuable information since, to the best of found knowledge, STL - DNA interactions have not been communicated before.

The variation of the amount of GSH in the cell by using a specific precursor in GSH synthesis (NAC) or a specific inhibitor of GSH synthesis (BSO) showed the importance of GSH in the cell's response to 5. Moreover, it was shown that formation of GSH-ragusinin conjugates increased cell survival what implies the role in GSH detoxification rather than in stabilization of the cell redox system. Ragusinin induced G2 arrest followed by caspase-independent cell death.

Though the actual protein targets remain unclear at this stage of the investigation, we can confirm that compound $\mathbf{5}$ is deactivated by GSH resulting in a diminished cytotoxic effect.

A summary of the most significant results obtained in this study by using specified techniques is presented in Figure 10. In future studies, it will be interesting to investigate whether ragusinin-GSH conjugates have a biological activity or whether they are ejected from cells through so-called GSH pumps such as MRP1 and MRP2 (Homolya et al., 2003).

\section{AUTHOR CONTRIBUTIONS}

UG and JR isolated and characterized compounds, analyzed the data, and wrote a part of the manuscript. SRB and VV performed

\section{REFERENCES}

Agarwal, S., Jangir, D. K., Singh, P., and Mehrotra, R. (2014). Spectroscopic analysis of the interaction of lomustine with calf thymus DNA. J. Photochem. Photobiol. B 130, 281-286. doi: 10.1016/j.jphotobiol.2013.11.017

Aliaga, C., Lissi, E. A., Augusto, O., and Linares, E. (2003). Kinetics and mechanism of the reaction of a nitroxide radical (tempol) with a phenolic antioxidant. Free Radic. Res. 37, 225-230. doi: 10.1080/1071576031000081587

Atta-ur-Rahman. (ed.) (2011). "Studies in natural products chemistry", in Bioactive Natural Products (Amsterdam: Elsevier Science), 309-392.

Ayad, R., Ababsa, Z. E., Belfadel, F. Z., Akkal, S., León, F., Brouard, I., et al. (2012). Phytochemical and biological activity of Algerian Centaurea melitensis. Int. J. Med. Arom. Plants 2, 151-154.

Balendiran, G. K., Dabur, R., and Fraser, D. (2004). The role of glutathione in cancer. Cell Biochem. Funct. 22, 343-352. doi: 10.1002/cbf.1149

Beutler, J. A., Hamel, E., Vlietinck, A. J., Haemers, A., Rajan, P., Roitman, J. N., et al. (1998). Structure-activity requirements for flavone cytotoxicity and binding to tubulin. J. Med. Chem. 41, 2333-2338. doi: 10.1021/jm970842h

Boyland, E., and Chasseaud, L. F. (1969). The role of glutathione and glutathione S-transferases in mercapturic acid biosynthesis. Adv. Enzymol. Relat. Areas Mol. Biol. 32, 173-219. doi: 10.1002/9780470122778.ch5 antibacterial assays and processed the data. EU helped in NMR measurements and structure elucidation. RS and SI performed antiproliferative assay by $\mathrm{CV}$ method and processed the data. JK and $\mathrm{AB}$ performed antiproliferative assay by MTT method, flow cytometry and tests with GSH. AB designed part of the study, analyzed the data, and wrote a part of the manuscript. MRS designed the study, performed DNA binding study (UV/Vis, CD, and ITC titrations), analyzed the data, and wrote a part of the manuscript. All authors participated in the critical reading of the manuscript.

\section{FUNDING}

This research was funded by Croatian Science Foundation (Grants No. 1477 and IP-2016-06-1036) and by the European Union: the European Social Fund as part of the Human Resources Development 2007-2013, as part of project "HR.3.2.01-0290 Biological and phytochemical activity of Centaurea ragusina $\mathrm{L}$. (BioFitoCen)".

\section{ACKNOWLEDGMENTS}

The authors thank M. Ruščić (Department of Biology, University of Split, Croatia) for collecting and identifying C. ragusina L. plants from natural habitats, M. Zehl (Department of Pharmacognosy/Pharmaceutical Chemistry, University of Vienna, Austria) for HR-ESI-MS measurements, C. Draschl and C. Lechner (Department of Pharmacognosy, University of Vienna, Austria) for technical assistance.

\section{SUPPLEMENTARY MATERIAL}

The Supplementary Material for this article can be found online at: https://www.frontiersin.org/articles/10.3389/fphar. 2018.00972/full\#supplementary-material

Bronowska, A. K. (2011). "Thermodynamics of ligand-protein interactions: implications for molecular design", in Thermodynamics - Interaction Studies Solids, Liquids and Gases, ed. J. C. Moreno Piraj $\tilde{A}_{j n}$ (Rijeka: InTech), 2-48.

Brozovic, A., Fritz, G., Christmann, M., Zisowsky, J., Jaehde, U., Osmak, M., et al. (2004). Long term activation of SAPK/JNK, p38 kinase and fas-L expression by cisplatin is attenuated in human carcinoma cells that acquired drug resistance. Int. J. Cancer 112, 974-985. doi: 10.1002/ijc.20522

Brozovic, A., Majhen, D., Roje, V., Mikac, N., Jakopec, S., Fritz, G., et al. (2008). Alpha(v)beta(3) Integrin-mediated drug resistance in human laryngeal carcinoma cells is caused by glutathione-dependent elimination of druginduced reactive oxidative species. Mol. Pharmacol. 74, 298-306. doi: 10.1124/ mol.107.043836

Brozovic, A., Stojanovic, N., Ambriovic-Ristov, A., Brozovic Krijan, A., Polanc, S., and Osmak, M. (2014). 3-Acetyl-bis(2-chloro-4-nitrophenyl)triazene is a potent antitumor agent that induces oxidative stress and independently activates the stress-activated protein kinase/c-Jun NH2-terminal kinase pathway. Anticancer Drugs 25, 289-295. doi: 10.1097/CAD.0000000000000060

Brozovic, A., Vukovic, L., Polancac, D. S., Arany, I., Koberle, B., Fritz, G., et al. (2013). Endoplasmic reticulum stress is involved in the response of human laryngeal carcinoma cells to carboplatin but is absent in carboplatin-resistant cells. PLoS One 8:e76397. doi: 10.1371/journal.pone.0076397. 
Cantor, C. R. and Schimmel, P. R. (1980). Biophysical Chemistry, Vol. 3, San Francisco, CA: WH Freeman and Co.

Chadwick, M., Trewin, H., Gawthrop, F., and Wagstaff, C. (2013). Sesquiterpenoids lactones: benefits to plants and people. Int. J. Mol. Sci. 14, 12780-12805. doi: 10.3390/ijms140612780

Chaires, J. B. (2006). A thermodynamic signature for drug-DNA binding mode. Arch. Biochem. Biophys. 453, 26-31. doi: 10.1016/j.abb.2006.03.027

Chen, H., Yao, K., Nadas, J., Bode, A. M., Malakhova, M., Oi, N., et al. (2012). Prediction of molecular targets of cancer preventing flavonoid compounds using computational methods. PLoS One 7:e38261. doi: 10.1371/journal.pone. 0038261

Cho, J. Y., Kim, A. R., Jung, J. H., Chun, T., Rhee, M. H., and Yoo, E. S. (2004), Cytotoxic and pro-apoptotic activities of cynaropicrin, a sesquiterpene lactone, on the viability of leukocyte cancer cell lines. Eur. J. Pharmacol. 492, 85-94. doi: 10.1016/j.ejphar.2004.03.027

Cimbora-Zovko, T., Brozovic, A., Piantanida, I., Fritz, G., Virag, A., Alic, B. et al (2011). Synthesis and biological evaluation of 4-nitro-substituted 1,3-diaryltriazenes as a novel class of potent antitumor agents. Eur. J. Med. Chem. 46, 2971-2983. doi: 10.1016/j.ejmech.2011.04.024

Clinical and Laboratory Standards Institute [CLSI] (2007). Performance Standards for Antimicrobial Susceptibility Testing, 17th Informational Supplement. M100S17. Wayne, PA: CLSI

Crespo-Ortiz, M. P., and Wei, M. Q. (2012). Antitumor activity of artemisinin and its derivatives: from a well-known antimalarial agent to a potential anticancer drug. J. Biomed. Biotechnol. 2012:247597. doi: 10.1155/2012/247597.

Csupor-Löffler, B., Hajdú, Z., Zupkó, I., Réthy, B., Falkay, G., Forgo, P., et al. (2009). Antiproliferative effect of flavonoids and sesquiterpenoids from Achillea millefolium s.l. on cultured human tumor cell lines. Phytother. Res. 23, 672-676. doi: 10.1002/ptr.2697.

Ćurković-Perica, M., Barišić, I. G., Hrenović, J., and Tkalec, M. (2015) Antibacterial activity of Pinus pinaster bark extract and its components against multidrug-resistant clinical isolates of Acinetobacter baumannii. Croat. Chem. Acta 88, 133-137. doi: 10.5562/cca2548

Demeunynck, M., Bailly, C., and Wilson, W. D. (2002). DNA and RNA Binders: From Small Molecules to Drugs. Weinheim: Wiley-VCH. doi: 10.1002/ 3527601783

Eriksson, M., and Nordén, B. (2001). Linear and circular dichroism of drug-nucleic acid complexes. Methods Enzymol. 340, 68-98. doi: 10.1016/S0076-6879(01) 40418-6

Feng, X., Qin, H., Shi, Q., Zhang, Y., Zhou, F., Wu, H., et al. (2014). Chrysin attenuates inflammation by regulating M1/M2 status via activating PPARy. Biochem. Pharmacol. 8, 503-514. doi: 10.1016/j.bcp.2014.03.016.

Ferreres, F., Tomas, F., Guirado, A., and Tomas, F. A. (1980). Agliconas de flavonoides en la Centaurea aspera (Compositae). Afinidad 37, 337-338.

Forgo, P., Zupkó, I., Molnár, J., Vasas, A., Dombi, G., and Hohmann, J. (2012). Bioactivity-guided isolation of antiproliferative compounds from Centaurea jacea L. Fitoterapia 83, 921-925. doi: 10.1016/j.fitote.2012. 04.006

Gao, H., Wang, H., and Peng, J. (2014). Hispidulin induces apoptosis through mitochondrial dysfunction and inhibition of P13k/Akt signalling pathway in HepG2 cancer cells. Cell Biochem. Biophys. 69, 27-34. doi: 10.1007/s12013-013$9762-\mathrm{x}$

Gates, K. S. (2009). An overview of chemical processes that damage cellular DNA: spontaneous hydrolysis, alkylation, and reactions with radicals. Chem. Res. Toxicol. 22, 1747-1760. doi: 10.1021/tx900242k

Gertsch, J., Sticher, O., Schmidt, T., and Heilmann, J. (2003). Influence of helenanolide-type sesquiterpene lactones on gene transcription profiles in Jurkat $\mathrm{T}$ cells and human peripheral blood cells: anti-inflammatory and cytotoxic effects. Biochem. Pharmacol. 66, 2141-2153. doi: 10.1016/j.bcp.2003. 08.006

Gewirtz, D. A., Holt, S. E., and Grant, S. (2007). Apoptosis, Senescence and Cancer (Cancer Drug Discovery and Development) Berlin: Springer Science \& Business Media, 432-436.

González, A. G., Darias, V., Boada, J. N., and Feria, M. (1977). Cytostatic activity of sesquiterpene lactones from compositae of the Canary Islands. Arch. Farmacol. Toxicol. 3, 241-246.

Herraiz, C., Journé, F., Abdel-Malek, Z., Ghanem, G., Jiménez-Cervantes, C., and García-Borrón, J. C. (2011). Signaling from the human melanocortin 1 receptor to ERK1 and ERK2 mitogen-activated protein kinases involves transactivation of cKIT. Mol. Endocrinol. 25, 138-156. doi: 10.1210/me.2010-0217

Hoffmann, R., von Schwarzenberg, K., Lopez-Anton, N., Rudy, A., Wanner, G., Dirsch, V. M., et al. (2011). Helenalin bypasses Bcl-2-mediated cell death resistance by inhibiting NF-kappaB and promoting reactive oxygen species generation. Biochem. Pharmacol. 82, 453-463. doi: 10.1016/j.bcp.2011. 05.029

Homolya, L., Varadi, A., and Sarkadi, B. (2003). Multidrug resistance-associated proteins: export pumps for conjugates with glutathione, glucuronate or sulfate. Biofactors 17, 103-114. doi: 10.1002/biof.5520170111

Hu, R., Chen, N., Yao, J., Zhao, Q., Zhang, F., Li, Z. Y., et al. (2012). The role of Nrf2 and apoptotic signaling pathways in oroxylin A-mediated responses in HCT116 colorectal adenocarcinoma cells and xenograft tumors. Anticancer Drugs 23, 651-658. doi: 10.1097/CAD.0b013e3283512703

Ivanković, S., Stojković, R., Galić, Z., Galić, B., Ostojić, J., Marasović, M., et al. (2015). In vitro and in vivo antitumor activity of the halogenated boroxine dipotassium- trioxohydroxytetrafluorotriborate (K2[B3O3F4OH]). J. Enzyme Inhib. Med. Chem. 30, 354-359. doi: 10.3109/14756366.2014.926344

Jang, D. S., Yang, M. S., Ha, T. J., and Park, K. H. (1999). Hemistepsins with cytotoxic activity from Hemistepta lyrata. Planta Med. 65, 765-766. doi: 10. 1055/s-2006-960863

Jang, J. H., Iqbal, T., Min, K. J., Kim, S., Park, J. W., Son, E. I., et al. (2013). Helenalin-induced apoptosis is dependent on production of reactive oxygen species and independent of induction of endoplasmic reticulum stress in renal cell carcinoma. Toxicol. In Vitro 27, 588-596. doi: 10.1016/j.tiv.2012.10.014.

Jeong, J., Kang, S., Lee, I., Lee, J., Jung, H., and Choi, C. (2007). Antioxidant and chemosensitizing effects of flavonoids with hydroxy and/or methoxy groups and structure-activity relationship. J. Pharm. Pharmaceut. Sci. 10, 537-546. doi: 10.18433/J3KW2Z

Johnson, W. C. (1994). "CD of nucleic acids," in Circular Dichroism: Principles and Applications, eds K. Nakanishi, N. Berova, and R. W. Woody (New York, NY: VCH Publishers)

Jordan, J., Doherty, M. D., and Cohen, G. M. (1987). Effects of glutathione depletion on the cytotoxicity of agents toward a human colonic tumor-cell line. Br. J. Cancer 55, 627-631. doi: 10.1038/bjc.1987.127

Kanakis, C. D., Tarantilis, P. A., Polissiou, M. G., Diamantoglou, S., and TajmirRiahi, H. A. (2005). DNA interaction with naturally occurring antioxidant flavonoids quercetin, kaempferol, and delphinidin. J. Biomol. Struc. Dyn. 22, 719-724. doi: 10.1080/07391102.2005.10507038

Kannan, N., Nguyen, L. V., Makarem, M., Dong, Y., Shih, K., Eirew, P., et al. (2014). Glutathione-dependent and -independent oxidative stress-control mechanisms distinguish normal human mammary epithelial cell subsets. Proc. Natl. Acad. Sci. U.S.A. 111, 7789-7794. doi: 10.1073/pnas.1403813111

Kawasaki, B. T., Hurt, E. M., Kalathur, M., Duhagon, M. A., Milner, J. A., Kim, Y. S., et al. (2009). Effects of the sesquiterpene lactone parthenolide on prostate tumor-initiating cells: an integrated molecular profiling approach. Prostate 69, 827-837. doi: 10.1002/pros.20931

Khammar, A., and Djeddi, S. (2012). Pharmacological and biological properties of some Centaurea species. Eur. J. Sci. Res. 84, 398-416.

Kroemer, G., and Martin, S. J. (2005). Caspase-independent cell death. Nat. Med. 11, 725-730. doi: $10.1038 / \mathrm{nm} 1263$

Krueger, A., and Ganzera, M. (2012) Oroxylum indicum seeds - Analysis of flavonoids by HPLC-MS. J. Pharm. Biomed. Anal. 70, 553-556. doi: 10.1016/ j.jpba.2012.05.005

Kupchan, S. M., Eakin, M. A., and Thomas, A. M. (1971) Tumor inhibitors. 69. Structure-cytotoxicity relations among the sesquiterpene lactones. J. Med. Chem. 14, 1147-1152. doi: 10.1021/jm00294a001

Lee, D. D., Lee, E. Y., Jeong, S. H., and Chang, C. L. (2007). Evaluation of a colorimetric broth microdilution method for antimicrobial susceptibility testing using 2,3,5-triphenyltetrazolium chloride. Korean J. Clin. Microbiol. 10, 49-53.

Lyss, G., Knorre, A., Schmidt, T. J., Pahl, H. L., and Merfort, I. (1998). The antiinflammatory sesquiterpene lactone helenalin inhibits the transcription factor NF-kB by directly targeting p65. J. Biol. Chem. 273, 33508-33516. doi: 10.1074/ jbc.273.50.33508

Mahmoud, Z. F., Kasem, F. F., and Abdel Salam, N. A. (1986). Sesquiterpene lactones and flavonoids of Centaurea ragusina $\mathrm{L}$. subspecies ragusina growing in Egypt. Egypt. J. Pharm. Sci. 27, 283-289. 
Marques, M. R., Stuecker, C., Kichik, N., Tarrago, T., Giralt, E., Morel, A. F., et al. (2010). Flavonoids with prolyl oligopeptidase inhibitory activity isolated from Scutellaria racemosa Pers. Fitoterapia 81, 552-556. doi: 10.1016/j.fitote.2010.01. 018

Mickisch, G., Fajta, S., Keilhauer, G., Schlick, E., Tschada, R., and Alken, P. (1990). Chemosensitivity testing of primary human renal cell carcinoma by a tetrazolium based microculture assay (MTT). Urol. Res. 18, 131-136. doi: 10.1007/BF00302474

Miyase, T., Ueno, A., Noro, T., Kuroyanagi, K., and Fukushima S. (1985). Studies on sesquiterpene glycosides from Crepis japonica BENTH. Chem. Pharm. Bull. 33, 4451-4456. doi: 10.1248/cpb.33.4451

Mouffok, S., Haba, H., Lavaud, C., Long, C., and Benkhaled, M. (2012). Chemical constituents of Centaurea omphalotricha Coss. \& Durieu ex Batt. \& Trab. Rec. Nat. Prod. 6, 292-295. doi: 10.1016/j.apjtm.2016.04.016

Nagao, T., Abe, F., Kinjo, J., and Okabe, H. (2002). Antiproliferative constituents in plants 10. Flavones from the leaves of Lantana montevidensis BRIQ. and consideration of structure-activity relationship. Biol. Pharm. Bull. 25, 875-879. doi: $10.1248 /$ bpb. 25.875

Negrete, R. E., Backhouse, N., Prieto, P., Mejias, H., Camargo, R. C., Cassels, B. K., et al. (1989). Steroids, a lignan and a flavonoid from Centaurea melitensis L. Plantes Med. Phytother. 23, 293-304.

Osmak, M., and Eljuga, D. (1993). The characterization of two human cervical carcinoma HeLa sublines resistant to cisplatin. Res. Exp. Med. 193, 389-396. doi: 10.1007/BF02576247

Osmak, M, Brozovic, A., Ambriovic-Ristov, A., Hadzija, M., Pivcevic, B., and Smital, T. (1998). Inhibition of apoptosis is the cause of resistance to doxorubicin in human breast adenocarcinoma cells. Neoplasma 45, 223-230.

Özçelik, B., Gürbüz, I., Karaoglu, T., and Yeşilada, E. (2009). Antiviral and antimicrobial activities of three sesquiterpene lactones from Centaurea solstitialis L. ssp. solstitialis. Microbiol. Res. 164, 545-552. doi: 10.1016/j.micres. 2007.05.006

Pahlow, M. (1989). Velika Knjiga Ljekovitog Bilja. Ljubljana: Cankarjeva založba.

Perozzo, R., Folkers, G., and Scapozza, L. (2004). Thermodynamics of proteinligand interactions: history, presence, and future aspects. J. Recept. Signal Transduct. Res. 24, 1-52. doi: 10.1081/RRS- 120037896

Picman, A. K. (1986). Biological activities of sesquiterpene lactones. Biochem. Syst. Ecol. 14, 255-281. doi: 10.1016/0305-1978(86)90101-8

Politeo, O., Skočibušić, M., Carev, I., Burčul, F., Jerković, I., Sarolić, M., et al. (2012). Phytochemical profiles of volatile constituents from Centaurea ragusina leaves and flowers and their antimicrobial effects. Nat. Prod. Commun. 7, 1087-1090.

Radić, S., Štefanić, P. P., Lepeduš, H., Roje, V., and Pevalek-Kozlina, B. (2013). Salt tolerance of Centaurea ragusina $\mathrm{L}$. is associated with efficient osmotic adjustment and increased antioxidative capacity. Environ. Exp. Bot. 87, 39-48. doi: 10.1016/j.envexpbot.2012.11.002

Rasul, A., Parveen, S., and Ma, T. (2012). Costunolide: a novel anti-cancer sesquiterpene lactone. Bangladesh J. Pharmacol. 7, 6-13. doi: 10.3329/bjp.v7i1. 10066

Rusak, G., Piantanida, I., Mašić, L., Kapuralin, K., Durgo, K., and Kopjar, N. (2010). Spectrophotometric analysis of flavonoid-DNA interactions and DNA damaging/protecting and cytotoxic potential of flavonoids in human peripheral blood lymphocytes. Chem. Biol. Interact. 188, 181-189. doi: 10.1016/j.cbi.2010. 07.008 .

Saeed, M., Jacob, S., Sandjo, L. P., Sugimoto, Y., Khalid, H. E., Opatz, T., et al. (2015). Cytotoxicity of the sesquiterpene lactones neoambrosin and damsin from Ambrosia maritima against multidrug-resistant cancer cells. Front. Pharmacol. 6:267. doi: 10.3389/fphar.2015. 00267

Sangeetha Gowda, K. R., Mathew, B. B., Sudhamani, C. N., and Bhojya Naik, H. S. (2014). Mechanism of DNA binding and cleavage. J. Biomed. Biotechnol. 2, 1-9.

Schmidt, T. J. (1999). Toxic activities of sesquiterpene lactones:structural and biochemical aspects. Curr. Org. Chem. 3, 577-608.
Schmidt, T. J., Lyss, G., Pahl, H. L., and Merfort, I. (1999). Helenanolide type sesquiterpene lactones. Part 5: the role of glutathione addition under physiological conditions. Bioorg. Med. Chem. 7, 2849-2855. doi: 10.1016/ S0968-0896(99)00234-5

Sosa, A., Fusco, M. R., Rossomando, P., Juarez, A., Robles, S., Petenatti, E., et al. (2011). Anti-inflammatory properties from isolated compounds of Cyclolepis genistoides. Pharm. Biol. 49, 675-678. doi: 10.3109/13880200903431467

Tait, S. W., and Green, D. R. (2008). Caspase-independent cell death: leaving the set without the final cut. Oncogene 27, 6452-6461. doi: 10.1038/onc.2008.311

Townsend, D. M., and Tew, K. D. (2003). The role of glutathione-S-transferase in anti-cancer drug resistance. Oncogene 22, 7369-7375. doi: 10.1038/sj.onc. 1206940

Trachootham, D., Alexandre, J., and Huang, P. (2009). Targeting cancer cells by ROS-mediated mechanisms: a radical therapeutic approach? Nat. Rev. Drug Discov. 8, 579-591. doi: 10.1038/nrd2803

Villagomez, R., Rodrigo, G. C., Collado, I. G., Calzado, M. A., Munoz, E., Akesson, B. et al. (2013). Multiple anticancer effects of damsin and coronopilin isolated from Ambrosia arborescens on cell cultures. Anticancer Res. 33, 3799-3805.

Vujčić, M., Tufegdžić, S., Vujčić, Z., Gašić, M. J., and Sladić, D. (2007). Interactions of the anti-tumor sesquiterpene hydroquinone avarol with DNA in vitro. J. Serb. Chem. Soc. 72, 1265-1269. doi: 10.2298/JSC0712265V

Vujčić, V., Radić Brkanac, S., Radojčić Redovniković, I., Ivanković, S., Stojković, R., Žilić, I., et al. (2017). Phytochemical and bioactive potential of in vivo and in vitro grown plants of Centaurea ragusina $\mathrm{L}$. - detection of DNA/RNA active compounds in plant extracts via thermal denaturation and circular dichroism. Phytochem. Anal. 28, 584-592. doi: 10.1002/pca.2708

Wang, H., Guo, Y., Zhao, X., Li, H., Fan, G., Mao, H., et al. (2013). An estrogen receptor dependent mechanism of oroxylin a in the repression of inflammatory response. PLoS One 8:e69555. doi: 10.1371/journal.pone.0069555.

Weng, M. S., Ho, Y. S., and Lin, J. K. (2005). Chrysin induces G1 phase cell cycle arrest in C6 glioma cells through inducing p21Waf1/Cip1 expression: involvement of p38 mitogen-activated protein kinase. Biochem. Pharmacol. 69, 1815-1827. doi: 10.1016/j.bcp.2005.03.011

Yang, F., Jin, H., Pi, J., Jiang, J. H., Liu, L., Bai, H. H., Yang, P. H., et al. (2013). Antitumor activity evaluation of novel chrysin-organogermanium(IV) complex in MCF-7 cells. Bioorg. Med. Chem. Lett. 23, 5544-5555. doi: 10.1016/j.bmcl.2013. 08.055

Yu, X. M., Phan, T., Patel, P. N., Jaskula-Sztul, R., and Chen, H. (2013). Chrysin activates Notch 1 signaling and suppresses tumor growth of anaplastic thyroid carcinoma in vitro and in vivo. Cancer 119, 774-781. doi: 10.1002/cncr.27742

Zdero, C., Bohlmann, F., King, R. M., and Robinson, H. (1989). Sesquiterpene lactones and other constituents from Australian Helipterum species. Phytochemistry 28, 517-526. doi: 10.1016/0031-9422(89)80045-7

Zhang, C., Deng, Y., Wang, Y., and Mou, J. (2012). Inhibiting effects of flavonoids from Seriphidium santolium on human lung carcinoma cell line A-549. Chin. J. Exp. Tradit. Med. Formulae 18, 243-247.

Zhou, J., and Zhang, Y. (2008). Cancer stem cells: models, mechanisms and implications for improved treatment. Cell Cycle 7, 1360-1370. doi: 10.4161/cc. 7.10.5953

Conflict of Interest Statement: The authors declare that the research was conducted in the absence of any commercial or financial relationships that could be construed as a potential conflict of interest.

Copyright (C) 2018 Grienke, Radić Brkanac, Vujčić, Urban, Ivanković, Stojković, Rollinger, Kralj, Brozovic and Radić Stojković. This is an open-access article distributed under the terms of the Creative Commons Attribution License (CC BY). The use, distribution or reproduction in other forums is permitted, provided the original author(s) and the copyright owner(s) are credited and that the original publication in this journal is cited, in accordance with accepted academic practice. No use, distribution or reproduction is permitted which does not comply with these terms. 


\section{OPEN ACCESS}

Edited by:

Anna Karolina Kiss,

Medical University of Warsaw, Poland

Reviewed by:

Pinarosa Avato,

Università degli Studi di Bari Aldo

Moro, Italy

Sylwia Zielinska,

Wroclaw Medical University, Poland

*Correspondence:

Andrei Mocan

mocan.andrei@umfcluj.ro

Specialty section:

This article was submitted to

Ethnopharmacology,

a section of the journal

Frontiers in Pharmacology

Received: 21 December 2017

Accepted: 09 May 2018

Published: 21 August 2018

Citation:

Neag MA, Mocan A, Echeverría J, Pop RM, Bocsan Cl, Crişan G and Buzoianu AD (2018) Berberine:

Botanical Occurrence, Traditional Uses, Extraction Methods, and

Relevance in Cardiovascular.

Metabolic, Hepatic, and Renal Disorders. Front. Pharmacol. 9:557.

doi: 10.3389/fphar.2018.00557
Berberine: Botanical Occurrence, Traditional Uses, Extraction Methods, and Relevance in Cardiovascular, Metabolic, Hepatic, and Renal Disorders

\author{
Maria A. Neag ${ }^{1}$, Andrei Mocan ${ }^{2 *}$, Javier Echeverría ${ }^{3}$, Raluca M. Pop ${ }^{1}$, Corina I. Bocsan ${ }^{1}$, \\ Gianina Crişan ${ }^{2}$ and Anca D. Buzoianu ${ }^{1}$ \\ 'Department of Pharmacology, Toxicology and Clinical Pharmacology, "Iuliu Hatieganu" University of Medicine and Pharmacy, \\ Cluj-Napoca, Romania, ' 2 Department of Pharmaceutical Botany, "luliu Hatieganu" University of Medicine and Pharmacy, \\ Cluj-Napoca, Romania, ${ }^{3}$ Department of Environmental Sciences, Universidad de Santiago de Chile, Santiago de Chile, Chile
}

Berberine-containing plants have been traditionally used in different parts of the world for the treatment of inflammatory disorders, skin diseases, wound healing, reducing fevers, affections of eyes, treatment of tumors, digestive and respiratory diseases, and microbial pathologies. The physico-chemical properties of berberine contribute to the high diversity of extraction and detection methods. Considering its particularities this review describes various methods mentioned in the literature so far with reference to the most important factors influencing berberine extraction. Further, the common separation and detection methods like thin layer chromatography, high performance liquid chromatography, and mass spectrometry are discussed in order to give a complex overview of the existing methods. Additionally, many clinical and experimental studies suggest that berberine has several pharmacological properties, such as immunomodulatory, antioxidative, cardioprotective, hepatoprotective, and renoprotective effects. This review summarizes the main information about botanical occurrence, traditional uses, extraction methods, and pharmacological effects of berberine and berberine-containing plants.

Keywords: berberine, botanical occurrence, traditional uses, extraction methods, biological activities

\section{INTRODUCTION}

\section{Berberine}

Berberine(5,6-dihydro-9,10-dimethoxybenzo[g]-1,3-benzodioxolo[5,6-a] quinolizinium) Figure 1, is a nonbasic and quaternary benzylisoquinoline alkaloid, a relevant molecule in pharmacology and medicinal chemistry. Indeed, it is known as a very important natural alkaloid for the synthesis of several bioactive derivatives by means of condensation, modification, and substitution of functional groups in strategic positions for the design of new, selective, and powerful drugs (Chen et al., 2005). 


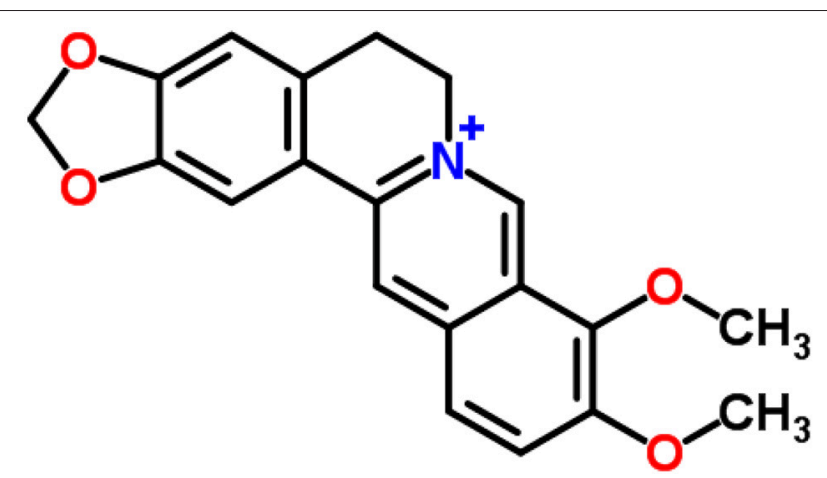

FIGURE 1 | Berberine structure (according to ChemSpider database).

\section{TRADITIONAL USE OF BERBERINE-CONTAINING SPECIES}

In the Berberidaceae family, the genus Berberis comprises of $\sim 450-500$ species, which represent the main natural source of berberine. Plants of this genus are used against inflammation, infectious diseases, diabetes, constipation, and other pathologies (Singh A. et al., 2010). The oldest evidence of using barberry fruit (Berberis vulgaris) as a blood purifying agent was written on the clay tablets in the library of Assyrian emperor Asurbanipal during 650 BC (Karimov, 1993). In Asia, the extensive use of the stem, stem bark, roots, and root bark of plants rich in berberine, particularly Berberis species, has more than 3000 years of history. Moreover, they have been used as raw material or as an important ingredient in Ayurvedic and traditional Chinese medicine (Birdsall, 1997; Kirtikar and Basu, 1998; Gupta and Tandon, 2004; Kulkarni and Dhir, 2010). In Ayurveda, Berberis species have been traditionally used for the treatment of a wide range of infections of the ear, eye, and mouth, for quick healing of wounds, curing hemorrhoids, indigestion and dysentery, or treatment of uterine and vaginal disorders. It has also been used to reduce obesity, and as an antidote for the treatment of scorpion sting or snakebite (Dev, 2006). Berberine extracts and decoctions are traditionally used for their activities against a variety of microorganisms including bacteria, viruses, fungi, protozoa, helminthes, in Ayurvedic, Chinese, and MiddleEastern folk medicines (Tang et al., 2009; Gu et al., 2010).

In Yunani medicine, Berberis asiatica has multiple uses, such as for the treatment of asthma, eye sores, jaundice, skin pigmentation, and toothache, as well as for favoring the elimination of inflammation and swelling, and for drying ulcers (Kirtikar and Basu, 1998). Decoction of the roots, and stem barks originating from Berberis aristata, B. chitria, and B. lycium (Indian Berberis species), have been used as domestic treatment of conjunctivitis or other ophthalmic diseases, enlarged liver and spleen, hemorrhages, jaundice, and skin diseases like ulcers (Rajasekaran and Kumar, 2009). On the other hand, the use of decoction of Indian barberry mixed with honey has also been reported for the treatment of jaundice. Additionally, it has been reported the use of decoction of Indian barberry and Emblic myrobalan mixed with honey in the cure of urinary disorders as painful micturition (Kirtikar and Basu, 1998). Numerous studies dealing with its antimicrobial and antiprotozoal activities against different types of infectious organisms (Vennerstrom et al., 1990; Stermitz et al., 2000; Bahar et al., 2011) have been assessed so far. Moreover, it has been used to treat diarrhea (Chen et al., 2014) and intestinal parasites since ancient times in China (Singh and Mahajan, 2013), and the Eastern hemisphere, while in China it is also used for treating diabetes (Li et al., 2004).

Nowadays, a significant number of dietary supplements based on plants containing berberine (Kataoka et al., 2008) are used for reducing fever, common cold, respiratory infections, and influenza (Fabricant and Farnsworth, 2001). Another reported use for berberine-containing plants is their application as an astringent agent to lower the tone of the skin. Also, positive effects were observed on the mucous membranes of the upper respiratory tract and gastrointestinal system with effects on the associated ailments (Chen et al., 2014; Yu et al., 2016).

In southern South America leaves and bark of species of the genus Berberis are used in traditional medicine administered for mountain sickness, infections, and fever (San Martín, 1983; Houghton and Manby, 1985; Anesini and Perez, 1993).

Furthermore, there are other genera which contain berberine. The genus Mahonia comprises of several species that contain berberine. Within them, M. aquifolium has been traditionally used for various skin conditions. Due to its main alkaloid (berberine), is known to be used in Asian medicine for its antimicrobial activity. Coptidis rhizoma (rhizomes of Coptis chinensis), another plant which contains berberine, is a famous herb very frequently used in traditional Chinese medicine for the elimination of toxins, "damp-heat syndromes", "purge fire", and to "clear heat in the liver" (Tang et al., 2009). Table 1 gathers a synthesis of the main traditional uses of species containing berberine.

\section{BOTANICAL SOURCES OF BERBERINE}

Berberine has been detected, isolated, and quantified from various plant families and genera including Annonaceae (Annickia, Coelocline, Rollinia, and Xylopia), Berberidaceae (Berberis, Caulophyllum, Jeffersonia, Mahonia, Nandina, and Sinopodophyllum), Menispermaceae (Tinospora), Papaveraceae (Argemone, Bocconia, Chelidonium, Corydalis, Eschscholzia, Glaucium, Hunnemannia, Macleaya, Papaver, and Sanguinaria), Ranunculaceae (Coptis, Hydrastis, and Xanthorhiza), and Rutaceae (Evodia, Phellodendron, and Zanthoxyllum) (Table 2). The genus Berberis is well-known as the most widely distributed natural source of berberine. The bark of $B$. vulgaris contains more than $8 \%$ of alkaloids, berberine being the major alkaloid (about 5\%) (Arayne et al., 2007).

Berberine is also widely present in barks, leaves, twigs, rhizomes, roots, and stems of several medicinal plants species, including Argemone mexicana (Etminan et al., 2005), Berberis aristata, $B$. aquifolium, $B$. heterophylla, B. beaniana, Coscinium fenestratum (Rojsanga and Gritsanapan, 2005), C. chinensis, C. japonica, C. rhizome, Hydratis canadensis (Imanshahidi and 
TABLE 1 | Traditional uses of berberine-containing species.

\begin{tabular}{|c|c|c|c|}
\hline Family & Scientific name & Traditional uses & References \\
\hline \multirow[t]{2}{*}{ Annonaceae } & $\begin{array}{l}\text { Annickia chlorantha (Oliv.) } \\
\text { Setten \& Maas (ex-Enantia } \\
\text { chlorantha Oliv.) }\end{array}$ & $\begin{array}{l}\text { Treat jaundice, hepatitis A, B, C, and D, } \\
\text { conjunctivitis, leishmaniasis, medicine for cuts } \\
\text { and infected wounds, sores and ulcers, } \\
\text { antipyretic for various fevers, tuberculosis, } \\
\text { vomiting of blood, urinary tract infections, } \\
\text { treatment of fatigue, rheumatism, treat malaria } \\
\text { symptoms, aches, wounds, boils, vomiting, } \\
\text { yellow bitter, chills, sore, spleen in children and } \\
\text { body pains, skin ailments, intercostal pain and } \\
\text { to promote conception, intestinal worms, } \\
\text { intestinal spasms, malaria and sexual asthenia, } \\
\text { treat coughs and wounds; rickettsia fever, treat } \\
\text { of sleeping sickness and dysentery, hemostatic } \\
\text { and rickettsia, treat yellow fever and typhoid } \\
\text { fever, treat diabetes, treat syphilis, and other } \\
\text { infectious diseases, poliomyelitis, treat } \\
\text { hypertension, treat HIV and prostate cancer }\end{array}$ & $\begin{array}{l}\text { Oliver, 1960; Sandberg, 1965; Bouquet, 1969; } \\
\text { Hamonniere et al., 1975; Onwuanibe, 1979; } \\
\text { Burkill, 1985; Gill and Akinwumi, 1986; Gbile } \\
\text { et al., 1988; Vennerstrom and Klayman, 1988; } \\
\text { Vennerstrom et al., 1990; Adjanohoun et al., } \\
\text { 1996; Nguimatsia et al., 1998; Kayode, 2006; } \\
\text { Odugbemi et al., 2007; Ehiagbonare and } \\
\text { Onyibe, 2008; Jiofack et al., 2008, 2009; } \\
\text { Kadiri, 2008; Ogbonna et al., 2008; } \\
\text { Olowokudejo et al., 2008; Betti and Lejoly, } \\
\text { 2009; Ndenecho, 2009; Adeyemi et al., 2010; } \\
\text { Noumi, 2010; Noumi and Anguessin, 2010; } \\
\text { Noumi and Yumdinguetmun, 2010; Bele et al., } \\
\text { 2011; Din et al., 2011; Ngono Ngane et al., } \\
\text { 2011; Oladunmoye and Kehinde, 2011; } \\
\text { Gbolade, 2012; Musuyu Muganza et al., 2012; } \\
\text { Tsabang et al., 2012; Betti et al., 2013; } \\
\text { Borokini et al., 2013; Fongod, 2014; Ishola } \\
\text { et al., 2014; Ohemu et al., 2014 }\end{array}$ \\
\hline & Annickia pilosa (Exell) Setten \& & Medicine for cuts & Versteegh and Sosef, 2007 \\
\hline
\end{tabular}

Maas (ex-Enantia pilosa Exell)

Annickia polycarpa (DC.)

Setten \& Maas ex I.M.Turner (ex-Enantia polycarpa (DC.)

Engl. \& Diels)

Rollinia mucosa (Jacq.) Baill. Xylopia polycarpa (DC.) Oliv.

Berberidaceae

Berberis actinacantha Mart. Berberis aquifolium Pursh

Berberis aristata DC.

Berberis asiatica Roxb. ex DC.
Treat cuts, antiseptic to treat sores, stomach ulcers, leprosy and ophthalmia, treatment of skin infections and sores, treat jaundice, and treat fever including malaria and to promote wound healing, against intestinal problems

Treat of tumors

Treat wounds, ulcers, leprosy, rheumatism, stomach and gall-bladder problems, eye diseases, for conception, diarrhea, malaria, fevers and sleeping disorders

Antipyretic

Skin conditions, treat eczema, acne, conjunctivitis and herpes, alleviate the symptoms of psoriasis, treat diarrhea and in higher doses to treat constipation, improvement of blood flow to the liver, stimulate intestinal secretions and bile flow, treat jaundice, hepatitis, cirrhosis and general digestive problems, treatment of gall bladder disease, hemorrhages and a few forms of cancer, fungal infections, dysentery, anti-inflammatory properties, stomach problems, sore womb following childbirth and/or menstruation

Treat allergies, metabolic disorders, ophthalmia, and other eye diseases, treat bleeding piles, anti-osteoporosis, treat skin diseases, menorrhagia, fever, diarrhea, dysentery, cholera, jaundice, ear and urinary tract infections, anti-bacterial, anti-fungal, anti-inflammatory, analgesic, anti-pyretic, jaundice, piles, malaria, laxative, anti-scorbutic, anti-diabetic, and anti-hepatopathic

Jaundice, diabetes mellitus, wound healing, asthma; drying unhealthy ulcers, anti-inflammatory, swelling, treat pneumococcal infections, eye (conjunctivitis) and ear diseases, rheumatism, fever, stomach disorders, skin disease (hyperpigmentation), malarial fever, laxative, teeth problems (toothache), and headache
Irvine, 1961; Bouquet and Debray, 1974; Ajali, 2000; Govindasamy et al., 2007; Versteegh and Sosef, 2007

Hartwell, 1982

Neuwinger, 1996

San Martín, 1983

King, 1898; Ritch-Krc et al., 1996

Bhattacharjee et al., 1980; Duke and Beckstrom-Sternberg, 1994; Küpeli et al., 2002; Acharya and Rokaya, 2005; Chhetri et al., 2005; Kunwar and Adhikari, 2005; Sharma et al., 2005; Joshi and Joshi, 2007; Meena et al., 2009; Shahid et al., 2009; Phondani et al., 2010; Saraf et al., 2010; Tiwary et al., 2010; Sati and Joshi, 2011; Yogesh et al., 2011

Watt, 1883; Kirtikar and Basu, 1933; Samhita, 1963; Hashmi and Hafiz, 1986; Bhandari et al., 2000; Shah and Khan, 2006; Uniyal et al., 2006; Uprety et al., 2010; Maithani et al., 2014 
TABLE 1 | Continued

\begin{tabular}{ll}
\hline Family & Scientific name \\
\hline $\begin{array}{l}\text { Berberis buxifolia Lam. } \\
\text { Berberis chitria Buch.-Ham. } \\
\text { ex Lindl. }\end{array}$ \\
Berberis darwinii Hook. \\
Berberis empetrifolia Lam. \\
Berberis integerrima Bunge.
\end{tabular}

Berberis jaeschkeana C. K. Schneid.

Berberis koreana Palib.

Berberis leschenaultia Wall. ex Wight \& Arn.

Berberis libanotica Ehrenb. ex C. K. Schneid.

Berberis lycium Royle

Berberis microphylla G. Forst. (ex-Berberis heterophylla Juss. ex Poir.)

Berberis oblonga (Regel) C. K. Schneid

Berberis petiolaris Wall. ex G. Don

Berberis pseudumbellata R. Parker

Berberis thunbergii DC.

Berberis tinctoria Lesch.

Berberis umbellata Wall. ex G. Don

Berberis vulgaris L.

Caulophyllum thalictroides (L.) Michaux

Jeffersonia diphylla (L.) Pers.
Traditional uses

Treat infections

Treat skin disease, jaundice, rheumatism, affection of eyes (household treatment for conjunctivitis, ophthalmic, bleeding piles), ulcers, skin diseases, enlarged liver and spleen

Antipyrectic, anti-inflammatory, treat stomach pains, indigestion, and colitis

Treat mountain sickness

Antipyretic, treat diabetes, bone fractures, rheumatism, radiculitis, heart pain, stomach aches, kidney stones, tuberculosis, chest pain, headaches, constipation, and wound

Treat eye diseases

Antipyretic, treat gastroenteritis, sore throats, and conjunctivitis

Antipyretic, cold and complications during post-natal period

Treat rheumatic and neuralgic diseases, anti-inflammatory, treat arthritis and muscular pain

Treat eye diseases, febrifuge, jaundice, diarrhoea, menorrhagia, piles, backache, dysentery, earache, fracture, eye ache, pimples, boils, wound healing, cough and throat pain, intestinal colic, diabetes, throat pain, scabies, bone fractures, sun blindness, against stomachache and intestinal problems

Febrifuge, anti-inflammatory and treat diarrhea

Heart tonic, treat neurasthenia, antipyretic, antidiarrheal, treat rheumatism, eye diseases and wounds of the mouth, jaundice, stomach aches, back pain and arthralgia

Treat malarial fever, diarrhea, conjunctivitis, and jaundice

Diuretic, treat jaundice, intestinal disorders, eye diseases, oxytocic and throat ache, stomach problems and ulcers

Anti-inflammatory

Antimicrobial for skin disease, jaundice, affection of eyes, treat menorrhagia, diarrhea, and rheumatism

Treating fever, jaundice, nausea, eye disorders and skin problems, tonic

Antiarrhythmic, sedative, anticancer, heal internal injuries, remove kidney stones, treat sore throat and fever

Menstrual cramps, relieve the pain of childbirth, promote prompt delivery, treat colics, cramps, hysteria, rheumatism, uterine stimulant, inducer of menstruation, and antispasmodic

Antispasmodic, diuretic, emetic, expectorant, treat diarrhea, dropsy, gravel and urinary problems, emetic, expectorant, treat sores, ulcers and inflamed parts

\section{References}

Anesini and Perez, 1993; Mølgaard et al., 2011 Watt, 1883; Kirtikar and Basu, 1933; Sir and Chopra, 1958

Montes and Wilkomirsky, 1987

San Martín, 1983

Khalmatov, 1964; Khodzhimatov, 1989;

Baharvand-Ahmadi et al., 2016

Kala, 2006

Ahn, 2003

Rajan and Sethuraman, 1992

El Beyrouthy et al., 2008; Esseily et al., 2012

Zaman and Khan, 1970; ul Haq and Hussain, 1993; Bushra et al., 2000; Kaur and Miani, 2001; Hamayun et al., 2003; Ahmed et al., 2004; Abbasi et al., 2005, 2009, 2010; Shah and Khan, 2006; Zabihullah et al., 2006;

Hussain et al., 2008; Sood et al., 2010

Muñoz, 2001

Khalmatov, 1964; Sezik et al., 2004; Pak, 2005

Karimov, 1993

Kala, 2006; Khan and Khatoon, 2007; Singh et al., 2009; Khan et al., 2016

Küpeli et al., 2002

Fyson, 1975; Satyavati et al., 1987

Singh et al., 2012

Tantaquidgeon, 1928; Chaudhury et al., 1980; Zovko Koncić et al., 2010

Castleman, 1991; Hutchens, 1992

Uphof, 1959; Duke and Ayensu, 1985; Foster and Duke, 1990; Coffey, 1993; Moerman, 1998; Lust, 2014 
TABLE 1 | Continued

\begin{tabular}{ll}
\hline Family & Scientific name \\
\hline $\begin{array}{l}\text { Mahonia fortunei (Lindl.) } \\
\text { Fedde } \\
\text { Mahonia napaulensis DC. }\end{array}$ \\
Nandina domestica Thunb. \\
\\
\\
Sinopodophyllum hexandrum \\
(Royle) T. S. Ying \\
Tinospora sinensis (Lour.) \\
Merr (ex-Tinospora cordifolia \\
(Willd.) Miers) \\
Papaveraceae \\
Argemone mexicana L. \\
(ex-Argemone alba F. Lestib.) \\
\end{tabular}

Argemone platyceras L.

Bocconia frutescens $\mathrm{L}$.

Chelidonium majus L.

Corydalis solida subsp. brachylova Corydalis solida subsp. slivenensis (Velen.) Hayek (ex-Corydalis slivenensis Velen.)

Corydalis solida subsp. tauri cola

Corydalis turtschaninovii Besser (ex-Corydalis ternata (Nakai) Nakai)
Traditional uses

Anticancer, febrifuge, antiodontalgic, treat testicular swelling and arthritic pain

Diuretic, demulcent, treat dysentery and inflammations of the eyes

Antitussive, astringent, febrifuge, stomachic and tonic, treat of fever in influenza, acute bronchitis, whooping cough, indigestion, acute gastro-enteritis, tooth abscess, pain in the bones, muscles and traumatic injuries, and antirheumatic

Regulate menstruation, promote the circulation of blood, treat amenorrhea, difficult labor and retention of dead fetus or placenta

Tonic, antiperiodic, anti-spasmodic, anti-inflammatory, antiarthritic, anti-allergic, anti-diabetic, improve the immune system, antistress, anti-leprotic and anti-malarial activities

Anthydropic, cathartic, diaphoretic, diuretic, demulcent, emetic, purgative, treat jaundice, skin ailments, colds, colics and wounds

Analgesic, antispasmodic, sedative, treat warts, cold sores, cutaneous affections, skin diseases, itches, treat cataracts, treat dropsy, jaundice, treat chronic skin diseases, expectorant, treat coughs and chest complaints, demulcent, emetic, expectorant, laxative and antidote to snake poisoning

Treat respiratory ailments as asthma, cough, bronchitis and pneumonia

Treat skin conditions (ulcers and eruptions) and respiratory tract infections (bronchistis and tuberculosis)

Treat ophthalmic diseases (remove films from the cornea of the eye), mild sedative, antispasmodic, relaxing the muscles of the bronchial tubes and intestines, treat warts, alterative, anodyne, antispasmodic, cholagogue, diaphoretic, diuretic, hydrogogue, narcotic, purgative, treat bronchitis, whooping cough, asthma, jaundice, gallstones and gallbladder pains, anticancer, analgesic, treat stomach ulcer, treat get rid of warts, ringworm and corns

Anodyne, antibacterial, antispasmodic, hallucinogenic, calm the nerves, sedative for insomnia, CNS stimulant, painkiller, treat painful menstruation, lowering the blood pressure, traumatic injury and lumbago

Treat memory dysfunction, treat gastric, duodenal ulcer, cardiac arrhythmia disease, rheumatism and dysmenorrhea

\section{References}

Duke and Ayensu, 1985; He and Mu, 2015

Chopra et al., 1986; Manandhar, 2002

Kariyone and Koiso, 1971; Duke and Ayensu, 1985; Fogarty, 1990

Kong et al., 2010

Singh et al., 2003

Smyth, 1903; Foster and Duke, 1990

Uphof, 1959; Pesman, 1962; Usher, 1974; Stuart and Smith, 1977; Emboden, 1979; Chopra et al., 1986; Coffey, 1993; Chevallier, 1996

Emes et al., 1994

Martinez, 1977, 1984

Launert, 1981; Grieve, 1984; Phillips and Foy, 1990; Phillips and Rix, 1991; Chevallier, 1996; Lust, 2014

Launert, 1981; Bown, 1995

Tang and Eisenbrand, 1992; Kamigauchi and Iwasa, 1994; Orhan et al., 2004; Houghton et al., 2006 
TABLE 1 | Continued

\begin{tabular}{l} 
Family Scientific name \\
\hline Eschscholzia californica \\
Cham. \\
\\
Glaucium corniculatum (L.) \\
Rud. subsp. corniculatum \\
Macleaya cordata (Willd.) \\
R.Br. \\
Macleaya microcarpa \\
(Maxim.) Fedde \\
Papaver dubium L. \\
Papaver dubium var. lecoquii \\
Papaver rhoeas L. var. \\
chelidonioides
\end{tabular}

Papaver hybridum L.

Ranunculaceae

Coptis chinensis Franch.

Coptis japonica (Thunb.) Makino

Coptis teeta Wall.

Hydrastis canadensis L.

Xanthorhiza simplicissima Marshall
Traditional uses

Sedative, diuretic, relieve pain, relax spasms, promote perspiration, treat nervous tension, anxiety, insomnia, urinary incontinence (especially in children), narcotic, relieve toothache, antispasmodic, analgesic and suppress the flow of milk in lactating women Reduce warts, antitusive, treat CNS disturbances, sedative, cooling, and mild laxative

Analgesic, antioedemic, carminative, depurative, diuretic, treat insect bites, and ringworm

Treat some skin diseases and inflammation

Sudorific, diuretic, expectorant and ophthalmia

Ailments in the elderly and children, mild pain reliever, treat irritable coughs, reduce nervous over-activity, anodyne, emollient, emmenagogue, expectorant, hypnotic, slightly narcotic, sedative, treat bronchial complaints and coughs, insomnia, poor digestion, nervous digestive disorders and minor painful conditions, treat jaundice, fevers, and anticancer

Treat dermatologic diseases, anti-infective, diuretic, sedative, and antitussive

Control of bacterial and viral infections, relax spasms, lower fevers, stimulate the circulation, treat diabetes mellitus, analgesic, locally anaesthetic, antibacterial, antipyretic, bitter, blood tonic, carminative, cholagogue, digestive, sedative, stomachic, vasodilator, treat diarrhoea, acute enteritis and dysentery, treat insomnia, fidget, delirium due to high fever, leukaemia and otitis media, treat conjunctivitis, skin problems (acne, boils, abscesses and burns whilst), mouth, tongue ulcers, swollen gums, and toothache

Control of bacterial and viral infections, relax spasms, lower fevers, stimulate the circulation, locally analgesic and anaesthetic, anti-inflammatory, stomachic, treat conjunctivitis, intestinal catarrh, dysentery, enteritis, high fevers, inflamed mouth and tongue

Control of bacterial and viral infections, relaxes spasms, lowers fevers and stimulate the circulation, locally analgesic, anaesthetic, ophthalmic and pectoral diseases, effective antibacterial, treat dysentery

Treat disorders of the digestive system and mucous membranes, treat constipation, antiperiodic, antiseptic, astringent, cholagogue, diuretic, laxative, stomachic, tonic, treat disorders affecting the ears, eyes, throat, nose, stomach, intestines, and vagina

Treat mouth ulcers, stomach ulcers, colds, jaundice, treat piles, and digestive disorders

\section{References}

Coffey, 1993; Bown, 1995; Chevallier, 1996; Moerman, 1998

Al-Douri, 2000; Al-Qura'n, 2009; Hayta et al., 2014

Grieve, 1984; Duke and Ayensu, 1985

Deng and Qin, 2010

Chopra et al., 1986

Uphof, 1959; Launert, 1981; Grieve, 1984; Duke and Ayensu, 1985; Phillips and Foy, 1990; Bown, 1995; Chevallier, 1996

Rivera Núñez and Obon de Castro, 1996; Ali et al., 2018

Uphof, 1959; Usher, 1974; Duke and Ayensu, 1985; Yeung, 1985; Bown, 1995

Kariyone and Koiso, 1971; Usher, 1974; Grieve, 1984; Bown, 1995

Stuart and Smith, 1977; Duke and Ayensu, 1985; Bown, 1995

Uphof, 1959; Weiner, 1980; Grieve, 1984; Mills, 1985; Foster and Duke, 1990; Coffey, 1993; Bown, 1995; Chevallier, 1996; Lust, 2014

Weiner, 1980; Foster and Duke, 1990; Moerman, 1998 
TABLE 1 | Continued

\begin{tabular}{|c|c|c|c|}
\hline Family & Scientific name & Traditional uses & References \\
\hline \multirow[t]{3}{*}{ Rutaceae } & $\begin{array}{l}\text { Phellodendron amurense } \\
\text { Rupr. }\end{array}$ & $\begin{array}{l}\text { Treat gastroenteritis, abdominal pain and } \\
\text { diarrhea, antiinflammator, immunostimulator } \\
\text { and treat cancer (antitumor activities) }\end{array}$ & Uchiyama et al., 1989; Park et al., 1999 \\
\hline & $\begin{array}{l}\text { - Phellodendron chinense C. } \\
\text { K. Schneid. } \\
\text { - Phellodendron chinense } \\
\text { var. glabriusculum C. K. } \\
\text { Schneid. } \\
\text { - (ex-Phellodendron wilsonii } \\
\text { Hayata \& Kaneh.) }\end{array}$ & $\begin{array}{l}\text { Act strongly on the kidneys, detoxicant for hot } \\
\text { damp conditions, treat meningitis, } \\
\text { conjunctivitis, antibacterial, antirheumatic, } \\
\text { aphrodisiac, bitter stomachic, cholagogue, } \\
\text { diuretic, expectorant, febrifuge, hypoglycaemic, } \\
\text { treat ophtalmia, skin, vasodilator and tonic, } \\
\text { treat acute diarrhoea, dysentery, jaundice, } \\
\text { vaginal infections (with Trichomonas vaginalis), } \\
\text { acute urinary tract infections, enteritis, boils, } \\
\text { abscesses, night sweats and skin diseases, } \\
\text { and expectorant }\end{array}$ & $\begin{array}{l}\text { Kariyone and Koiso, 1971; Usher, 1974; Stuart } \\
\text { and Smith, 1977; Grieve, 1984; Yeung, 1985; } \\
\text { Bown, 1995; Chevallier, } 1996\end{array}$ \\
\hline & $\begin{array}{l}\text { Zanthoxylum monophyllum } \\
\text { Tul. }\end{array}$ & Treat eye infections and dark vomitus & Hirschhorn, 1981; Eric Brussell, 2004 \\
\hline
\end{tabular}

Hosseinzadeh, 2008), Phellodendron amurense, P. chinense, Tinospora cordifolia (Khan et al., 2011), Xanthorhiza simplicissima (Bose et al., 1963; Knapp et al., 1967; Sato and Yamada, 1984; Steffens et al., 1985; Inbaraj et al., 2001; Liu et al., 2008a; Srinivasan et al., 2008; Vuddanda et al., 2010). Several researches found that berberine is widely distributed in the barks, roots, and stems of plants, nevertheless, bark and roots are richer in berberine compared to other plant parts (Andola et al., 2010a,b). In the Papaveraceae family, Chelidonium majus is another important herbal source of berberine (Tomè and Colombo, 1995). An important number of plants for medicinal use, such as Coptidis rhizoma and barberry, are the natural sources with the highest concentration of berberine. Barberries, such as Berberis aristata, B. aquifolium, B. asiatica, $B$. croatica, $B$. thunbergii, and $B$. vulgaris, are shrubs grown mainly in Asia and Europe, and their barks, fruits, leaves, and roots are often widely used as folk medicines (Imanshahidi and Hosseinzadeh, 2008; Kosalec et al., 2009; Andola et al., 2010c; Kulkarni and Dhir, 2010). Different research groups have reported that maximum berberine concentration accumulates in root (1.6-4.3\%) and in most of the Berberis species, plants that grow at low altitude contain more berberine compared to higher altitude plants (Chandra and Purohit, 1980; Mikage and Mouri, 1999; Andola et al., 2010a). However, a correlation could not be established within the results of berberine concentration regarding to species and season of the year (Srivastava et al., 2006a,c; Andola et al., 2010c; Singh et al., 2012). Comparative studies of berberine concentration contained in different species of the same genus have been reported, e.g., higher berberine content in B. asiatica (4.3\%) in comparison to B. lycium (4.0\%), and B. aristata (3.8\%). Meanwhile, Srivastava et al. (2004) documented a higher berberine content in root of $B$. aristata (2.8\%) compared with B. asiatica (2.4\%) (Andola et al., 2010a). Seasonal variation of berberine concentration has been reported, e.g., the maximum yield of berberine for B. pseudumbellata was obtained in the summer harvest, and was $2.8 \%$ in the roots and $1.8 \%$ for the stem bark, contrary to that reported in the roots of
B. aristata, where the berberine concentration (1.9\%) is higher for the winter harvest (Rashmi et al., 2009). These variations may be caused to multiple factors, among which stand out: (i) the intraspecific differences, (ii) location and/or, (iii) the analytical techniques used. Table 2 gathers a synthesis of the main species containing berberine.

\section{EXTRACTION METHODS}

Berberine, a quaternary protoberberine alkaloid (QPA) is one of the most widely distributed alkaloid of its class. Current studies suggest that isolation of the QPA alkaloids from their matrix can be performed using several methods. The principles behind these methods consist of the interconversion reaction between the protoberberine salt and the base. The salts are soluble in water, stable in acidic, and neutral media, while the base is soluble in organic solvents. Thus during the extraction procedure, the protoberberine salts are converted in their specific bases and further extracted in the organic solvents (Marek et al., 2003; Grycová et al., 2007).

In the case of berberine, the classical extraction techniques like maceration, percolation, Soxhlet, cold or hot continuous extraction are using different solvent systems like methanol, ethanol, chloroform, aqueous, and/or acidified mixtures. Berberine's sensitivity to light and heat is the major challenge for its extraction. Hence, exposure to high temperature and light could lead to berberine degradation and thus influencing its matrix recovery. In his study Babu et al. (2012) demonstrated that temperature represent a crucial factor in both extraction and drying treatments prior extraction. The yield of berberine content in $C$. fenestratum stem tissue samples was higher in case of samples dried under the constant shade with $4.6 \%$ weight/weight $(w / w)$ as compared to samples dried in oven at $65^{\circ} \mathrm{C}(1.32 \% w / w)$ or sun drying $(3.21 \% w / w)$. As well hot extraction procedure with methanol or ethanol at $50^{\circ} \mathrm{C}$ gave lower extraction yields when compared with methanol or ethanol cold extraction at $-20^{\circ} \mathrm{C}$. Thus, berberine content in 
TABLE 2 | Botanical sources of berberine.

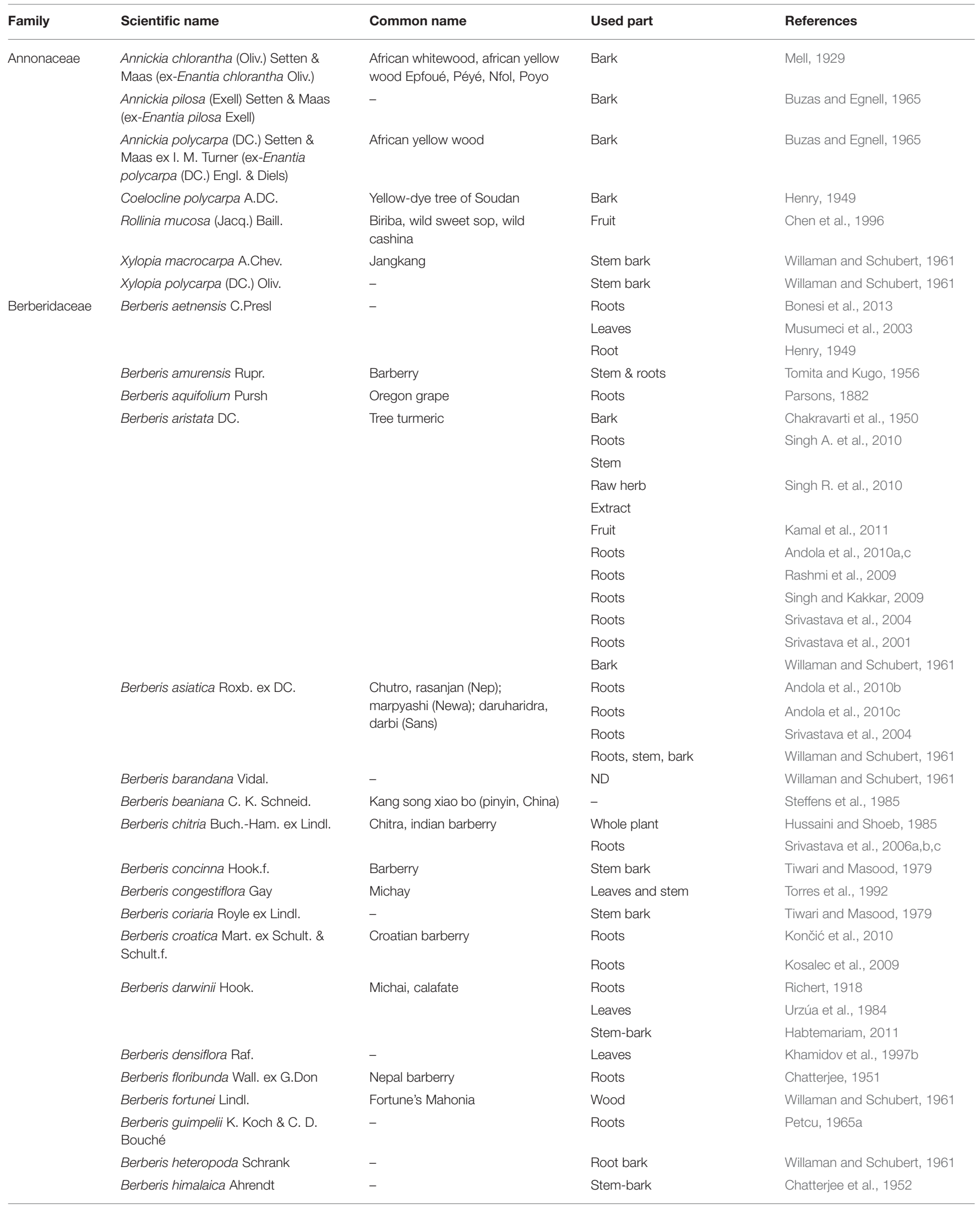


TABLE 2 | Continued

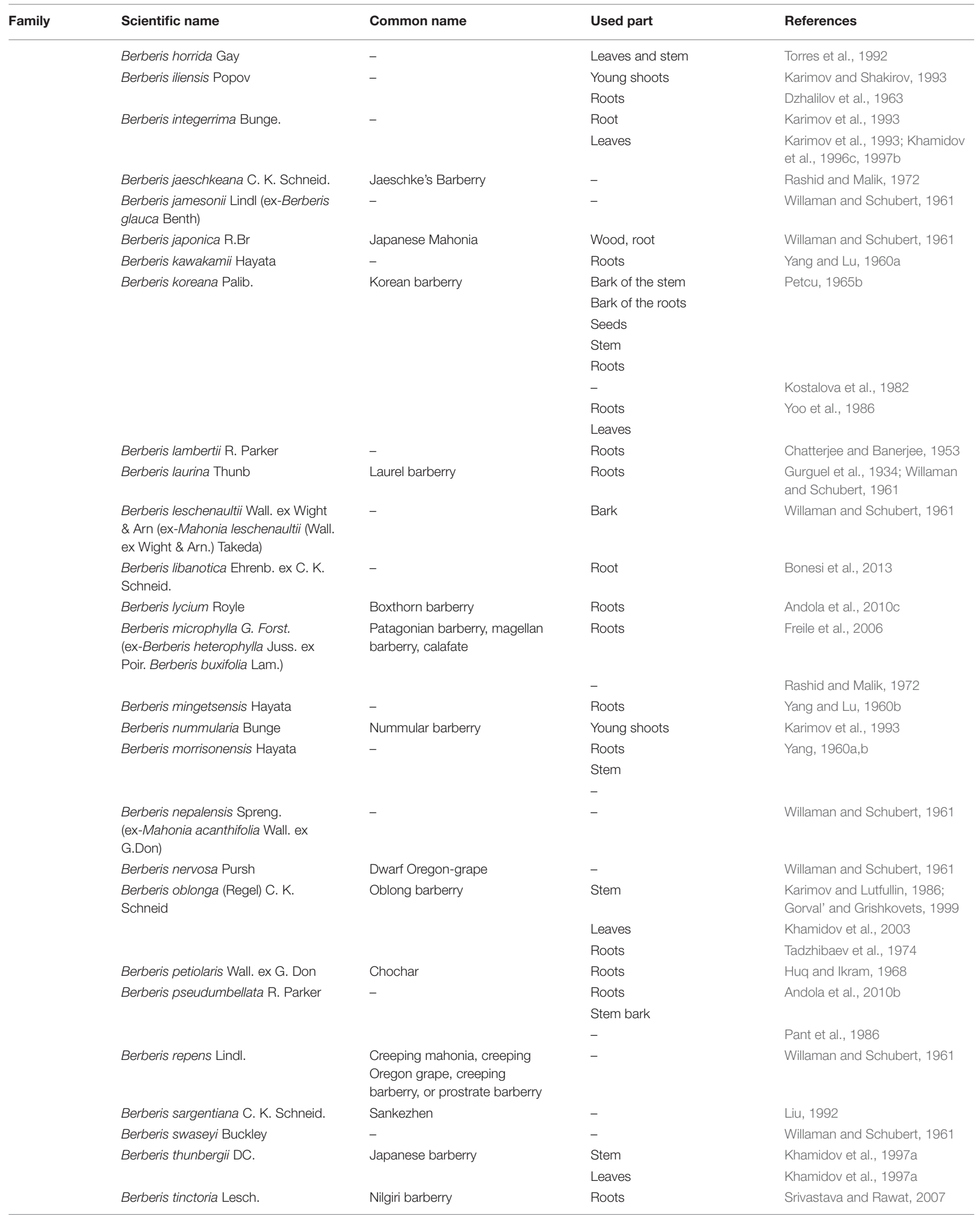


TABLE 2 | Continued

\begin{tabular}{|c|c|c|c|c|}
\hline Family & Scientific name & Common name & Used part & References \\
\hline & $\begin{array}{l}\text { Berberis trifolia (Cham. \& Schltdl.) } \\
\text { Schult. \& Schult.f. }\end{array}$ & - & Root, stem & Willaman and Schubert, 1961 \\
\hline & Berberis turcomanica Kar. ex Ledeb. & - & Leaves & Khamidov et al., 1996a,b,c \\
\hline & Berberis umbellata Wall. ex G.Don & Himalayan barberry & Roots & Singh et al., 2012 \\
\hline & Berberis vulgaris L. & Barberry & Stems and roots & $\begin{array}{l}\text { Imanshahidi and Hosseinzadeh, } \\
2008\end{array}$ \\
\hline & & & Roots & Končić et al., 2010 \\
\hline & & & Roots & Kosalec et al., 2009 \\
\hline & Berberis waziristanica Hieron. & - & Root bark & $\begin{array}{l}\text { Atta-ur-Rahma and Ahmad, } \\
1992\end{array}$ \\
\hline & $\begin{array}{l}\text { Caulophyllum thalictroides (L.) } \\
\text { Michaux (ex-Leontice thalictroides L.) }\end{array}$ & Blue cohosh & - & Willaman and Schubert, 1961 \\
\hline & Jeffersonia diphylla (L.) Pers. & Twinleaf & - & Willaman and Schubert, 1961 \\
\hline & Mahonia borealis Takeda & - & - & Willaman and Schubert, 1961 \\
\hline & Mahonia fortunei (Lindl.) Fedde & Fortune's Mahonia & wood & Willaman and Schubert, 1961 \\
\hline & $\begin{array}{l}\text { Mahonia napaulensis DC. (ex- } \\
\text { Mahonia griffithii; ex-Mahonia } \\
\text { manipurensis Takeda; Mahonia } \\
\text { sikkimensis Takeda) }\end{array}$ & Nepal Barberry & bark & Willaman and Schubert, 1961 \\
\hline & Mahonia simonsii Takeda & - & - & Willaman and Schubert, 1961 \\
\hline & Nandina domestica Thunb. & $\begin{array}{l}\text { Nandina, heavenly bamboo or } \\
\text { sacred bamboo }\end{array}$ & bark, root & Willaman and Schubert, 1961 \\
\hline & $\begin{array}{l}\text { Sinopodophyllum hexandrum (Royle) } \\
\text { T.S.Ying }\end{array}$ & $\begin{array}{l}\text { Himalayan May Apple, Indian } \\
\text { may apple }\end{array}$ & Root, rhizome & Willaman and Schubert, 1961 \\
\hline \multirow[t]{2}{*}{ Menispermaceae } & $\begin{array}{l}\text { Tinospora sinensis (Lour.) Merr. } \\
\text { (ex-Tinospora cordifolia) (Willd.) Miers }\end{array}$ & Gulbel, indian tinospora & Stem & Srinivasan et al., 2008 \\
\hline & & & - & Singh et al., 2003 \\
\hline \multirow[t]{16}{*}{ Papaveraceae } & $\begin{array}{l}\text { Argemone albiflora Hornem. } \\
\text { (ex-Argemone alba F.Lestib.) }\end{array}$ & $\begin{array}{l}\text { White prickly poppy, Bluestem } \\
\text { pricklypoppy }\end{array}$ & Aerial part and roots & Slavikova et al., 1960 \\
\hline & & & & Foote, 1932 \\
\hline & & & & Israilov and Yunusov, 1986 \\
\hline & Argemone hybrida R.Otto \& Verloove & - & Leaves and stem & Israilov and Yunusov, 1986 \\
\hline & Argemone mexicana L. & Prickly poppy & Apigeal parts, seeds & $\begin{array}{l}\text { Haisova and Slavik, 1975; } \\
\text { Israilov and Yunusov, 1986; } \\
\text { Fletcher et al., } 1993\end{array}$ \\
\hline & & & Leaves & Bapna et al., 2015 \\
\hline & & & Seeds & Fletcher et al., 1993 \\
\hline & & & - & Singh, 2014 \\
\hline & & & - & $\begin{array}{l}\text { Majumder et al., 1956; Hakim } \\
\text { et al., 1961; Misra et al., } 1961\end{array}$ \\
\hline & & & Superterranean parts & Slavikova and Slavik, 1955 \\
\hline & & & Roots & \\
\hline & & & - & $\begin{array}{l}\text { Santos and Adkilen, 1932; de } \\
\text { Almeida Costa, 1935; Misra } \\
\text { et al., 1961; Doepke et al., 1976; } \\
\text { Abou-Donia and El-Din, 1986; } \\
\text { Monforte-Gonzalez et al., } 2012\end{array}$ \\
\hline & & & Roots & $\begin{array}{l}\text { Pathak et al., 1985; Kukula-Koch } \\
\text { and Mroczek, } 2015\end{array}$ \\
\hline & & & Leaves and capsules & Schlotterbeck, 1902 \\
\hline & & & Whole plant & $\begin{array}{l}\text { Bose et al., 1963; Haisova and } \\
\text { Slavik, } 1975\end{array}$ \\
\hline & & & Latex & Santra and Saoji, 1971 \\
\hline
\end{tabular}


TABLE 2 | Continued

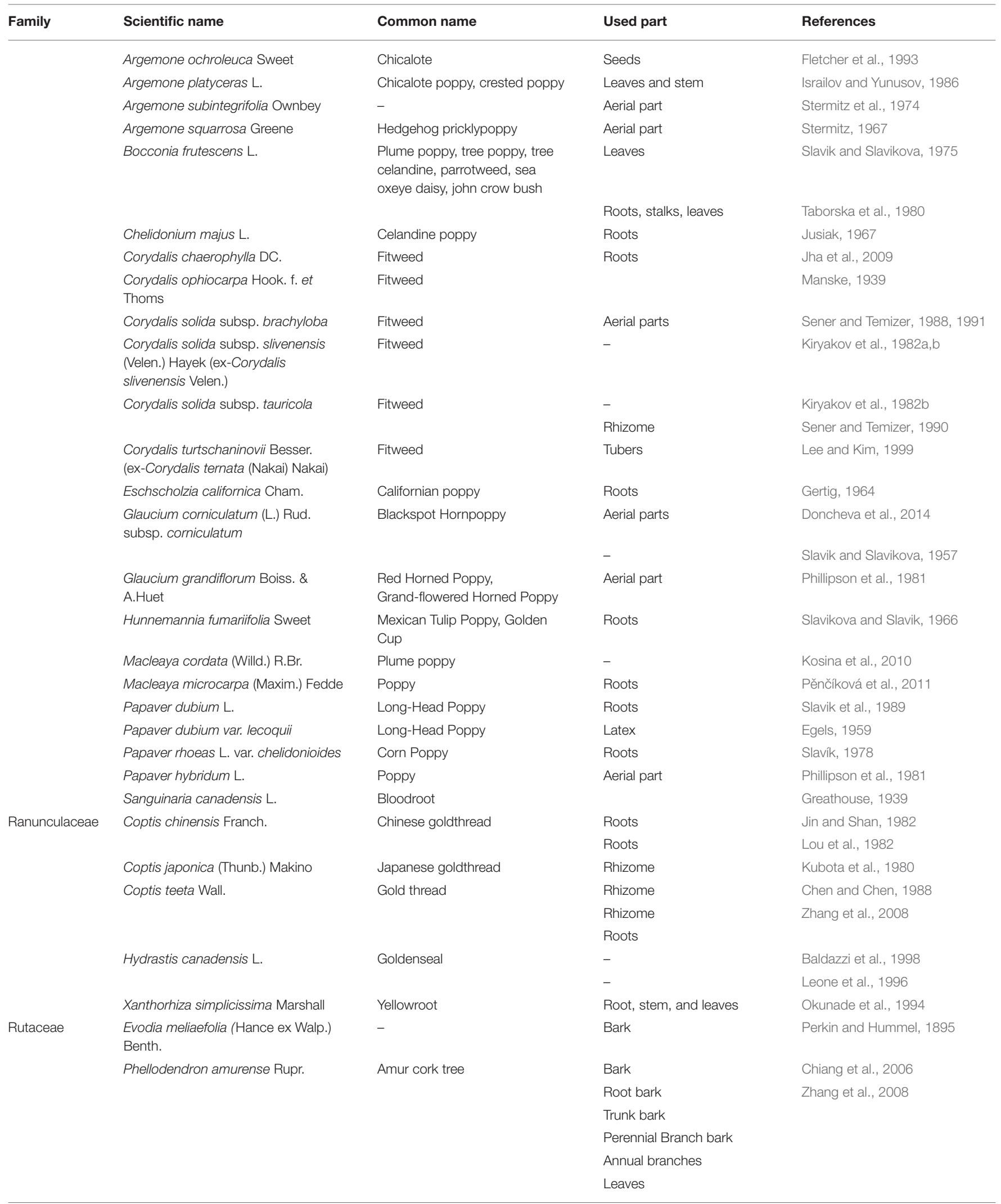


TABLE 2 | Continued

\begin{tabular}{|c|c|c|c|c|}
\hline Family & Scientific name & Common name & Used part & References \\
\hline & $\begin{array}{l}\text { Phellodendron chinense C. K. } \\
\text { Schneid. }\end{array}$ & Chinese cork tree & Bark & Chan et al., 2007 \\
\hline & $\begin{array}{l}\text { Phellodendron chinense var. } \\
\text { glabriusculum C. K. Schneid. } \\
\text { (ex-Phellodendron wilsonii Hayata \& } \\
\text { Kaneh.) }\end{array}$ & Chinese cork tree & $\begin{array}{l}\text { Bark, branch, leaf and } \\
\text { heartwood }\end{array}$ & Chen, 1981 \\
\hline & & & - & Tan et al., 2013 \\
\hline & & & Bark & Chen, 1982 \\
\hline & Phellodendron lavallei Dode & Lavalle corktree & Bark & Yavich et al., 1993 \\
\hline & $\begin{array}{l}\text { Zanthoxylum monophyllum (Lam.) P. } \\
\text { Wilson }\end{array}$ & Palo rubio & Stem and branches & Stermitz and Sharifi, 1977 \\
\hline & Zanthoxylum quinduense Tul. & - & - & Ladino and Suárez, 2010 \\
\hline
\end{tabular}

the shade-dried samples was $4.6 \%(w / w)$ for methanolic cold extraction and $1.29 \%(w / w)$ for methanolic hot extraction (Babu et al., 2012).

Along with extraction temperature, the choice of solvents is considered a critical step in berberine extraction as well (Figure 2). As seen in Table 3, methanol, ethanol, aqueous or acidified methanol or ethanol are the most used extraction solvents. The acidified solvents (usually with the addition of $0.5 \%$ of inorganic or organic acids) are used to combine with free base organic alkaloids and transform them in alkaloid salts with higher solubility (Teng and Choi, 2013). The effect of different inorganic acids like hydrochloric acid, phosphoric acid, nitric acid, and sulfuric acid as well as the effect of an organic acid like acetic acid were tested on berberine content and other alkaloids in rhizomes of Coptis chinensis Franch by Teng and Choi (2013). In this case, $0.34 \%$ phosphoric acid concentration was considered optimal. Moreover, when compared to other classical extraction techniques like reflux and Soxhlet extraction, the cold acid assisted extraction gave 1.1 times higher berberine yields.

Large solvent volumes and long extraction time represent other drawbacks of conventional extraction methods (Mokgadi et al., 2013). For example, Rojsanga and Gritsanapan (2005) used maceration process to extract $100 \mathrm{~g}$ of C. fenestratum plant material with a total volume of $3,200 \mathrm{~mL}$ solvent $(80 \%$ ethanol) over a period of $416 \mathrm{~h}$. Furthermore, in a different study, Rojsanga et al. (2006) used several classical extraction techniques like maceration, percolation, and Soxhlet extraction to extract the berberine from C. fenestratum stems. This time even if the extracted plant material was in a lower amount than the previous study (30 vs. $100 \mathrm{~g}$ ), large solvent volumes $(2,000 \mathrm{~mL}$ for maceration, $5,000 \mathrm{~mL}$ for percolation, and $600 \mathrm{~mL}$ for Soxhlet extraction) over long time periods (7 days for maceration and $72 \mathrm{~h}$ for Soxhlet extraction) were employed (Rojsanga and Gritsanapan, 2005; Rojsanga et al., 2006).

Large solvent volumes are characteristic for other conventional methods too. Shigwan et al. (2013) extracted berberine from Berberis aristata and B. tinctoria powdered stem bark $(800 \mathrm{~g})$ using hot extraction $\left(50^{\circ} \mathrm{C}\right.$ for $\left.3 \mathrm{~h}\right)$ with $2,500 \mathrm{~mL}$ methanol (Shigwan et al., 2013).
Even though conventional methods are widely used in berberine extraction, a number of other different methods have been developed lately. This led to an improved extraction efficiency, a decreased extraction time and solvents' volumes used in the extraction. Thus, ultrasound assisted solvent extraction (USE), microwave-assisted solvent extraction (MAE), ultrahigh pressure extraction (UPE), and supercritical fluid extractions (SFE), pressurized liquid extraction (PLE) have been successfully used as alternative extraction techniques with better results when compared with classical extraction methods.

Ultrasonically and microwave-assisted extraction are considered green, simple, efficient, and inexpensive techniques (Alupului et al., 2009).

Teng and Choi (2013) extracted berberine from Rhizome coptidis by optimized USE. Using response surface methodology, they identified that the optimal extraction conditions were $59 \%$ ethanol concentration, at $66.22^{\circ} \mathrm{C}$ within $46.57 \mathrm{~min}$. A decrease in the extraction time (39.81 min) was obtained by Chang (2013). He used the combination of ionic liquids solutions as green solvents with USE to extract berberine from Coptis chinensis in order to apply an environmentally friendly approach (Chang, 2013). Moreover, in their study, Xu et al. (2017) compared several extraction tehniques like USE, distillation, and Soxhlet extraction in order to establish an high-efficient method for phellodendrine, berberine, and palmatine extraction from fresh Phellodendron bark (Cortex phellodendri). In the case of berberine, the combination of simple or acidified solvent (water, ethanol, and methanol) with the adjustment of the specific setting characteristics to each extraction type enabled them to determine the highest extraction yield. They concluded that the use of USE and hydrochloric acid-acidified methanol were the most efficient in extracting berberine. The USE extraction yield was significantly higher when compared to distillation and Soxhlet extraction, with values of $\sim 100 \mathrm{mg} / \mathrm{g}$ toward 50 and $40 \mathrm{mg} / \mathrm{g}$ berberine, respectively (Xu et al., 2017).

The important reduction in organic solvent and extraction time determined the increasing interest in MAE, too. Lately, MAE was used as a green and cost-effective alternative to conventional methods. Using central composite design, Satija et al. (2015) successfully optimized the MAE parameters in terms 


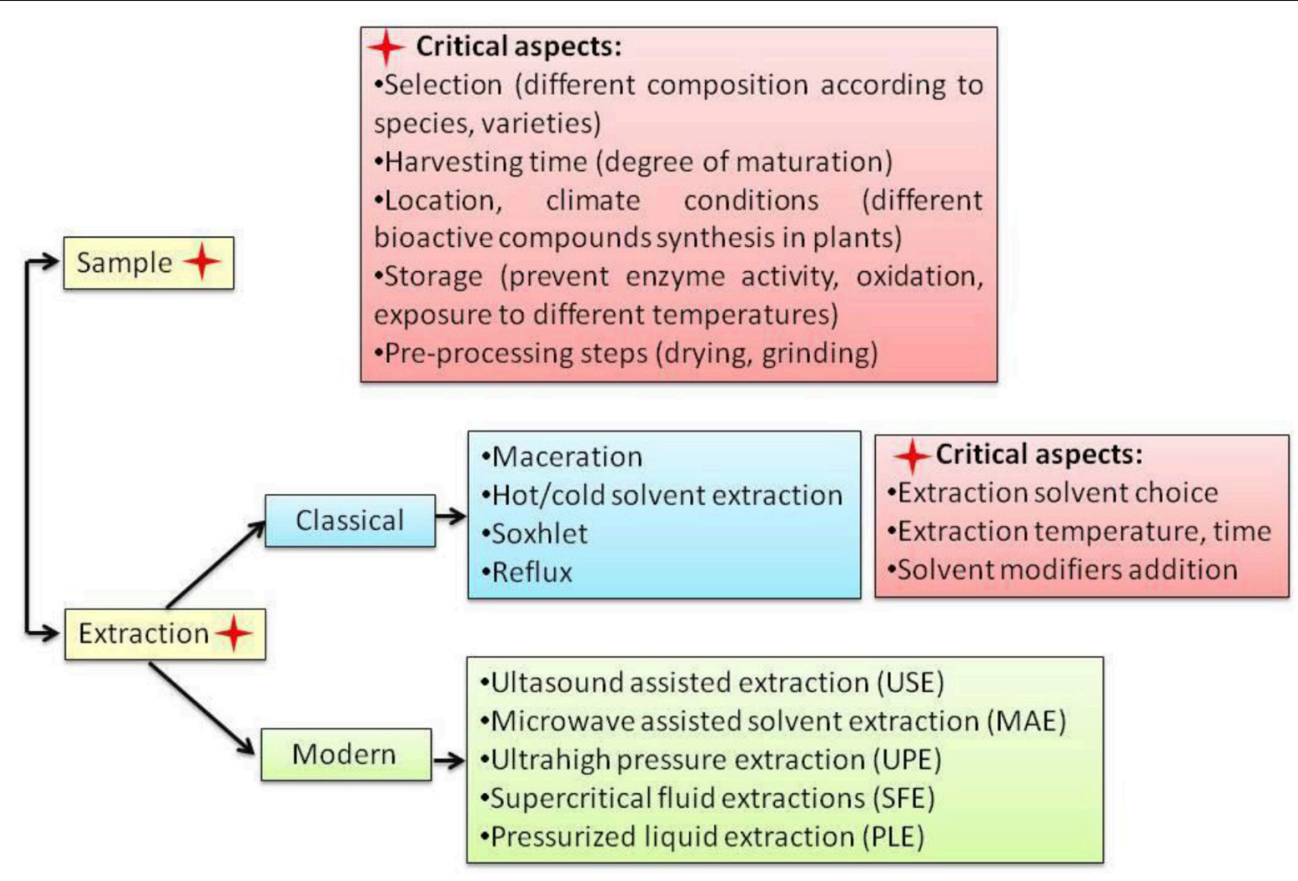

FIGURE 2 | Short view on berberine extraction methods.

of irradiation power, time, and solvent concentration to extract berberine form Tinospora cardifolia. They compared two classical extraction techniques like maceration and Soxhlet extraction with MAE under optimized conditions (60\% irradiation power, $80 \%$ ethanol concentration, and 3 min extraction time). The results showed that MAE extraction had the highest yield of berberine content with $1.66 \%(w / w)$ while Soxhlet and maceration had 1.04 and $0.28 \%(w / w)$, respectively. Their study is emphasizing the dramatic time reduction in case of MAE ( $3 \mathrm{~min}$ ) when compared with Soxhlet extraction $(3 \mathrm{~h})$ and maceration $(7$ days) together with solvent and energy consumption (Satija et al., 2015).

Another novel extraction technique considered to be environmentally friendly is UPE. The interest toward this extraction technique is increasing because it presents several advantages toward classical extraction techniques like increased extraction yields, higher quality of extracts, less extraction time, and decreased solvent consumption (Xi, 2015). These are achieved at room temperature by applying different pressure levels (from 100 to $600 \mathrm{MPa}$ ) between the interior (higher values) and the exterior of cells (lower values) in order to facilitate the transfer of the bioactive compounds through the plant matrices in the extraction solvent (Liu et al., 2006, 2013). In the study regarding berberine content in Cortex phellodendri, Guoping et al. (2012) made a comparison between UPE, MAE, USE, and heat reflux extraction techniques. They observed that the higher extraction yield and the lower extraction time was obtained in case of UPE with $7.7 \mathrm{mg} / \mathrm{g}$ and $2 \mathrm{~min}$ extraction time toward reflux, USE and MAE with $5.35 \mathrm{mg} / \mathrm{g}$ and $2 \mathrm{~h}, 5.61 \mathrm{mg} / \mathrm{g}$ and $1 \mathrm{~h}$. and $6 \mathrm{mg} / \mathrm{g}$ and $15 \mathrm{~min}$, respectively (Guoping et al., 2012).
Super critical fluid extraction is another environmentally friendly efficient technique used in phytochemical extraction. Because the extraction is performed in the absence of light and oxygen, the degradation of bioactive compounds is reduced. Also, the inert and non-toxic carbon dioxide used as a main extraction solvent in combination with various modifiers (e.g., methanol) and surfactants (e.g., Tween 80) at lower temperatures and relatively low pressure, allows the efficient extraction of bioactive compounds (Liu et al., 2006; Farías-Campomanes et al., 2015). In case of berberine extraction from the powdered rhizome of Coptis chinensis Franch, the highest recovery of berberine was obtained when 1,2-propanediol was used as a modifier of supercritical $\mathrm{CO}_{2}$ (Liu et al., 2006).

Pressurized liquid extraction, also known as pressurized fluid extraction, pressurized solvent extraction, and accelerated solvent extraction (ASE) is considered a green technology used for compounds extraction from plants (Mustafa and Turner, 2011). Compared with conventional methods, PLE increases the extraction yield, decreases time and solvent consumption, and protects sensitive compounds. In their study, Schieffer and Pfeiffer (2001) compared different extraction techniques like PLE, multiple USE, single USE, and Soxhlet extraction in order to extract berberine from goldenseal (Hydrastis canadensis). When compared in terms of extraction yield the results are comparable, $\sim 42 \mathrm{mg} / \mathrm{g}$ berberine, except single USE with slightly lower content (37 mg/g berberine). Big differences were observed in the extraction time, PLE requiring only $30 \mathrm{~min}$ for a single sample extraction compared to $2 \mathrm{~h}$ for multiple extraction techniques or $6 \mathrm{~h}$ for Soxhlet extraction (Schieffer and Pfeiffer, 2001). 
TABLE 3 | Extraction and detection methods for berberine in different herbal and biological matrixes.

\begin{tabular}{|c|c|}
\hline Sample (weight) & Extraction method \\
\hline $\begin{array}{l}\text { Dried stem powder } \\
\text { Coscinium fenestratum } \\
\text { (Gaertn.) }(1 \mathrm{~g})\end{array}$ & $\begin{array}{l}\text { Extraction solvents (ES): water, methanol-water (1:1. } \\
\text { v/v), and methanol } \\
\text { Sonication ( } 15 \mathrm{~min} \text {, room temperature) } \\
\text { Centrifugation ( } 2,800 \mathrm{rpm}, 15 \mathrm{~min}) \\
\text { Filtration and evaporation } \\
\text { Extracts resolubilization (methanol:water, } 9: 1 \mathrm{v} / \mathrm{v})\end{array}$ \\
\hline $\begin{array}{l}\text { C. fenestratum } \\
\text { (Gaertn.) (10 g) }\end{array}$ & $\begin{array}{l}\text { ES: methanol } \\
\text { Hot extraction: sample refluxed with ES for } 3 \mathrm{~h} \\
\text { Filtration and evaporation. } \\
\text { Extracts resolubilization (methanol) } \\
\text { Cold extraction: sample extraction with ES for } 24 \mathrm{~h} \\
\text { Filtration and evaporation. } \\
\text { Extracts resolubilization (methanol) }\end{array}$ \\
\hline $\begin{array}{l}\text { Dried C. fenestratum } \\
(0.1 \mathrm{~g})\end{array}$ & $\begin{array}{l}\text { ES: absolute methanol } \\
\text { Cold extraction: sample extraction at }-20^{\circ} \mathrm{C} \\
\text { Hot extraction: water bath sample extraction at } 50^{\circ} \mathrm{C} \\
\text { ES: absolute ethanol } \\
\text { Cold extraction sample extraction at }-20^{\circ} \mathrm{C} \\
\text { Hot extraction: water bath sample extraction at } 50^{\circ} \mathrm{C} \\
\text { Samples centrifugation }\left(10 \text { min at } 10^{\circ} \mathrm{C} \text { after cooling }\right. \\
\text { down) } \\
\text { Samples filtration }\end{array}$ \\
\hline C. fenestratum $(1,000 \mathrm{~g})$ & $\begin{array}{l}\text { ES: petroleum ether, chloroform, methanol ( } 1 \mathrm{~L} \text { each) } \\
\text { Soxhlet extraction: with each ES for } 3 \text { days at } \\
\left(30-40^{\circ} \mathrm{C}\right)\end{array}$ \\
\hline $\begin{array}{l}\text { Capsules (containing } \\
62.5 \mathrm{mg} \mathrm{C.} \\
\text { fenestratum) }\end{array}$ & $\begin{array}{l}\text { ES: methanol }(10 \mathrm{~mL}) \\
\text { Extraction for } 1 \mathrm{~h} \\
\text { Filtration and evaporation } \\
\text { Resolubilisation in methanol }(5 \mathrm{~mL})\end{array}$ \\
\hline
\end{tabular}

HPLC

Column: Luna C18, $150 \times 4.6 \mathrm{~mm}, 5 \mu \mathrm{m}$, Phenomenex Mobile Phases: (A) Potassium dihydrogen phosphate $(\mathrm{pH}-2.5)$ and $(\mathrm{B})$ Acetonitrile

Detection: $220 \mathrm{~nm}$

Flow: $1 \mathrm{~mL} / \mathrm{min}$

HPTLC

Adsorbent: Silica Gel 60F 254

Solvent system: n-Butanol: Glacial acetic acid: Water $(8: 1: 1, v / v / v)$

Detection: $350 \mathrm{~nm}$ for all measurements

UV-VIS

UV absorbance: $348 \mathrm{~nm}$
References

Akowuah et al., 2014

$4.6 \mathrm{~mm}$ Mobile Phases: Methanol/Deionized Water

(90:10, v/v) Flow: $0.5 \mathrm{~mL} / \mathrm{min}$, Temperature: $25^{\circ} \mathrm{C}$ UV

Adsorbent: Silica Gel GF 254 (2.5:1.5:1, v/v/v)

Column: C18, $250 \times 4.6 \mathrm{~mm}, 5 \mu \mathrm{m}$

Babu et al., 2012

Arawwawala and

Wickramaar, 2012

Jayaprakasam and

Ravi, 2014

Joshi and Kanaki, 2013
(Willd.), Tribulus terrestris (L.), Emblica officinalis (Gaertn.) (3g)

Cortex phellodendri (2g)

Rhizome of Coptis chinensis Franch $(1 \mathrm{~g})$
ES: chloroform

Dried sample trituration with ammonia solution

Drying at room temperature

Extraction with ES for $1 \mathrm{~h}$

Chloroform phase extraction with $5 \%$ sulfuric acid (x 3)

Basification of acid extract with sodium carbonate $(\mathrm{pH}$

-9)

Extraction of basified solution with chloroform $(x 3)$

Evaporation of chloroform phase ( temperature under

$\left.50^{\circ} \mathrm{C}\right)$

Residue solubilization with methanol

Ultrahigh pressure extraction (UPE)

Optimal parameters: ES: ethanol (69.1\%), liquid-solid ratio-31.3, extracting pressure-243.30 MPa, extraction time -2 min

\footnotetext{
Supercritical fluid extraction

Extraction time: up to $3 \mathrm{~h}$

Temperature: $60^{\circ} \mathrm{C}$

Pressure: from 200 to 500 bar

Flow-rate of carbon dioxide (gaseous state): $1 \mathrm{~L} / \mathrm{min}$

Flow-rate of modifier: $0.4 \mathrm{~mL} / \mathrm{min}$.

Organic solvent modifier systems:

ethanol-modified supercritical carbon dioxide,

methanol-modified supercritical carbon dioxide,
}

\section{HPLC}

Column: Hypersil ODS C18, $250 \times 4.6 \mathrm{~mm}, 5 \mu \mathrm{m}$

Mobile Phases: (A) $0.3 \%$ triethanolamine aqueous solution

$(\mathrm{pH}-3.5)$

Detection: $265 \mathrm{~nm}$

Temperature: $30^{\circ} \mathrm{C}$

Flow: $1 \mathrm{~mL} / \mathrm{min}$

HPLC

Column: Diamonsil C18, $250 \times 4.6 \mathrm{~mm}, 5 \mu \mathrm{m}$

Mobile Phases: $33 \mathrm{mM}$ Potassium dihydrogen

phosphate : acetonitrile $(70: 30, v / v)$

Detection: $345 \mathrm{~nm}$

Flow: $1 \mathrm{~mL} / \mathrm{min}$
Guoping et al., 2012

Liu et al., 2006 
TABLE 3 | Continued

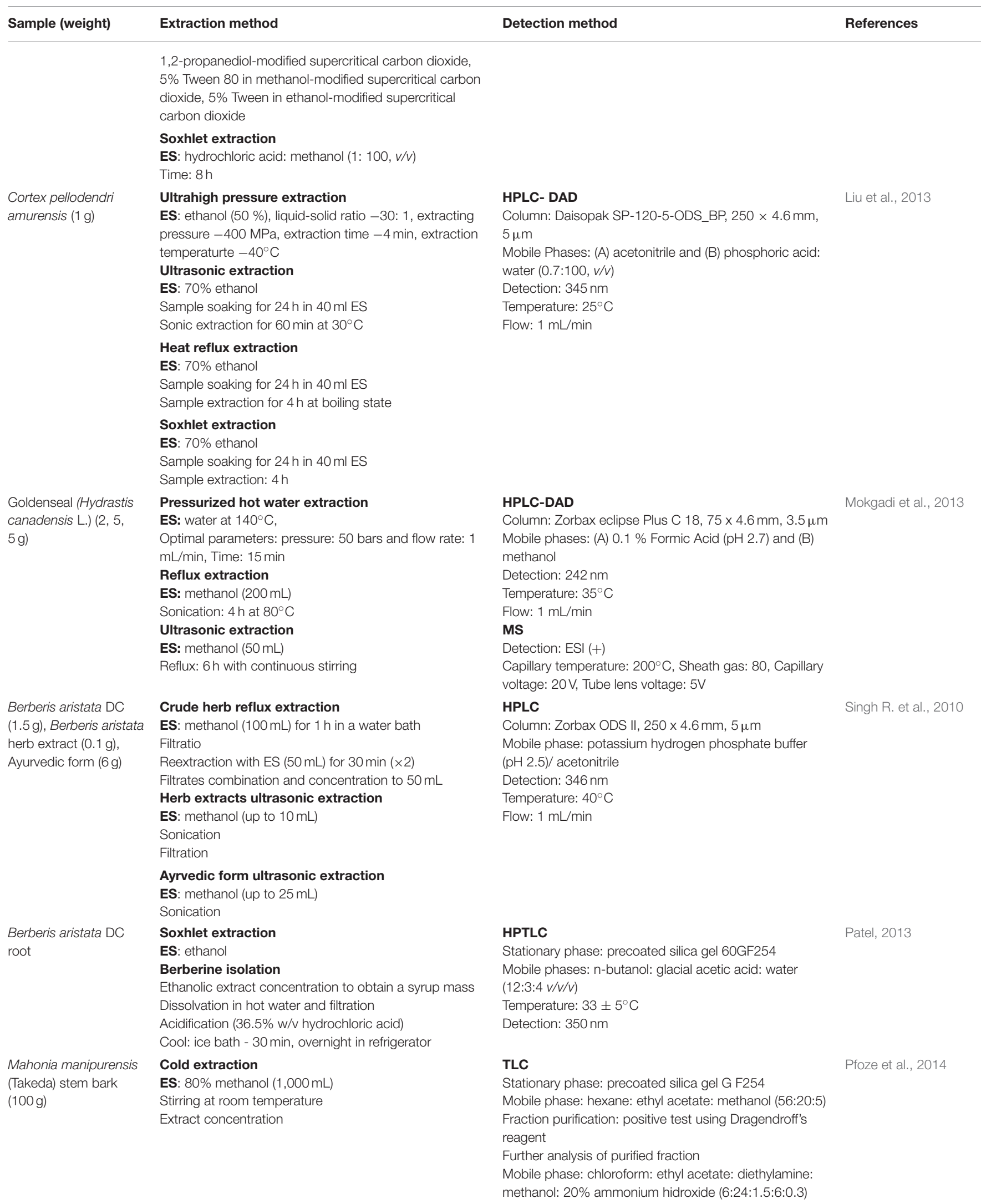


TABLE 3 | Continued

\begin{tabular}{|c|c|c|c|}
\hline Sample (weight) & Extraction method & Detection method & References \\
\hline & & $\begin{array}{l}\text { HPLC } \\
\text { Column: Water Symmetry C18, } 250 \times 4.6 \mathrm{~mm}, 5 \mu \mathrm{m} \\
\text { Mobile phase: methanol/ formic acid buffer }(0.1 \%, \mathrm{v} / \mathrm{V}) \\
\text { Detection: } 346 \mathrm{~nm} \\
\text { Flow: } 1 \mathrm{~mL} / \mathrm{min} \\
\text { UV-VIS } \\
\text { UV spectra: } 200-500 \mathrm{~nm} \\
\text { ESI-MS }\end{array}$ & \\
\hline $\begin{array}{l}\text { Coscinium fenestratum } \\
(100 \mathrm{~g})\end{array}$ & $\begin{array}{l}\text { Maceration } \\
\text { ES: } 80 \% \text { ethanol }(500 \mathrm{ml}), 160 \mathrm{~h} \\
\text { Shaken: } 80 \mathrm{~h} \text { ( } 200 \mathrm{rpm}), \text { stand: } 80 \mathrm{~h} \\
\text { Reextraction: } 48 \mathrm{~h} \text {, shaken: } 24 \mathrm{~h} \text {, stand: } 24 \mathrm{~h} \\
\text { Combined extracts concentration } \\
\text { Evaporation to dryness (dry extract) } \\
\text { Resolubilisation in } 80 \% \text { ethanol ( } 10 \mathrm{mg} \text { dry extract/mL) }\end{array}$ & $\begin{array}{l}\text { TLC } \\
\text { Stationary phase: Silica gel GF254 } \\
\text { Mobile phase: ethyl acetate : butanol : formic acid : } \\
\text { water (50:30:12:10); } \\
\text { Detection: } 366 \mathrm{~nm}\end{array}$ & $\begin{array}{l}\text { Rojsanga and } \\
\text { Gritsanapan, } 2005\end{array}$ \\
\hline Argemone mexicana & $\begin{array}{l}\text { Soxhlet extraction } \\
\text { ES: methanol } \\
\text { Evaporation to dryness } \\
\text { Resolubilisation in methanol (known concentration) }\end{array}$ & $\begin{array}{l}\text { HPTLC } \\
\text { Stationary phase: precoated silica gel 60F254 } \\
\text { Mobile phases: toluene: ethyl acetate }(9: 3, v / v) \text {. } \\
\text { Detection: } 266 \mathrm{~nm}\end{array}$ & Samal, 2013 \\
\hline $\begin{array}{l}\text { Tinospora cordifolia } \\
\text { (20 g) }\end{array}$ & $\begin{array}{l}\text { Microwave assisted extraction (MAE) } \\
\text { ES: } 80 \% \text { ethanol } \\
\text { Irradiation power: } 60 \% \text {, Extraction time: } 3 \mathrm{~min} \\
\text { Soxhlet extraction } \\
\text { ES: ethanol, for } 3 \mathrm{~h} \\
\text { Filtration } \\
\text { Concentration } \\
\text { Maceration } \\
\text { ES: ethanol }(200 \mathrm{~mL}), 7 \text { days, occasional stirring }\end{array}$ & $\begin{array}{l}\text { HPTLC } \\
\text { Mobile phases: methanol: acetic acid: water (8: 1: 1, } \\
\text { V/V/V). } \\
\text { Detection: } 366 \mathrm{~nm}\end{array}$ & Satija et al., 2015 \\
\hline $\begin{array}{l}\text { Berberis aristata, } \\
\text { Berberis tinctoria } \\
(800 \mathrm{~g})\end{array}$ & $\begin{array}{l}\text { Hot extraction } \\
\text { ES: methanol }(2.5 \mathrm{~L})(\mathrm{X} 2) \\
\text { Extraction time: } 3 \mathrm{~h} \\
\text { Temperature: } 50^{\circ} \mathrm{C} \\
\text { Extract concentration under vacuum }\end{array}$ & $\begin{array}{l}\text { HPLC } \\
\text { Column: Unisphere C18, } 150 \times 4.6 \mathrm{~mm}, 5 \mu \mathrm{m} \\
\text { Mobile phase: (A) } 0.1 \% \text { trifluoroacetic acid and (B) } \\
\text { acetonitrile }(60: 40, \mathrm{v} / \mathrm{v}) \\
\text { Detection: } 350 \mathrm{~nm} \\
\text { Temperature: } 30^{\circ} \mathrm{C} \\
\text { Flow: } 1 \mathrm{~mL} / \mathrm{min}\end{array}$ & Shigwan et al., 2013 \\
\hline $\begin{array}{l}\text { Coptis chinensis } \\
\text { Franch. (1g) }\end{array}$ & $\begin{array}{l}\text { Acid assisted extraction } \\
\text { ES: several inorganic acids (hydrochloric acid, } \\
\text { phosphoric acid, nitric acid, and sulfuric acid) and one } \\
\text { organic acid (acetic acid) } \\
\text { Extraction time: } 1-8 \mathrm{~h} \text {, Acid concentrations: } 0-1 \% \\
\text { Solvent to sample ratios: } 20-60 \mathrm{~mL} / \mathrm{g} \\
\text { Maceration at } 25^{\circ} \mathrm{C} \\
\text { Filtration } \\
\text { Dilution to } 100 \mathrm{~mL} \text { final volume } \\
\text { Soxhlet extraction } \\
\text { ES: } 50 \% \text { ethanol (100 mL), } 4 \mathrm{~h} \text { at } 70^{\circ} \mathrm{C} \\
\text { Extract evaporation to dryness } \\
\text { Resolubilization in } \mathrm{ES} \text { (up to } 100 \mathrm{~mL} \text { final volume) } \\
\text { Heating reflux extraction } \\
\text { ES: } 50 \% \text { ethanol } \\
\text { Soaked for } 1 \mathrm{~h} \\
\text { Extraction: } 4 \mathrm{~h} \text { at } 70^{\circ} \mathrm{C} \text { (heated water bath) } \\
\text { Filtration } \\
\text { Dilution (up to } 100 \mathrm{~mL} \text { final volume) }\end{array}$ & $\begin{array}{l}\text { HPLC } \\
\text { Column: XTerra C18, } 250 \times 4.6 \mathrm{~mm} \\
\text { Mobile phase: (A) acetonitrile and (B) } 25 \mathrm{mmol} / \mathrm{L} \\
\text { potassium dihydrogen phosphate, }(27: 75, \mathrm{~V} / \mathrm{V}) \\
\text { Detection: } 345 \mathrm{~nm} \\
\text { Temperature: } 30^{\circ} \mathrm{C}\end{array}$ & Teng and Choi, 2013 \\
\hline Rabbit plasma (100 $\mu \mathrm{l})$ & $\begin{array}{l}\text { Mixing } 100 \mu \mathrm{l} \text { sample with } 3 \% \text { formic acid in acetonitrile } \\
(200 \mu \mathrm{l}) \\
\text { Vortex: } 30 \mathrm{~s} \\
\text { Centrifugation: } 10 \mathrm{~min} \text { at } 4^{\circ} \mathrm{C} \\
\text { Evaporation of supernatant: under nitrogen stream at } \\
40^{\circ} \mathrm{C}\end{array}$ & $\begin{array}{l}\text { LC-ESI-MS } \\
\text { HPLC system } \\
\text { Column: Capcell Pakc } 18 \text { MG, } 100 \times 2.1 \mathrm{~mm}, 5 \mu \mathrm{m} \text { with } \\
\text { Security Guard C18, } 4 \times 2 \mathrm{~mm}, 5 \mu \mathrm{m} \\
\text { Mobile Phases: (A) } 0.4 \% \text { formic acid solution and (B) } 0.2 \\
\% \text { formic acid solution in methanol }(60: 40, v / v)\end{array}$ & Liu et al., 2011 \\
\hline
\end{tabular}


TABLE 3 | Continued

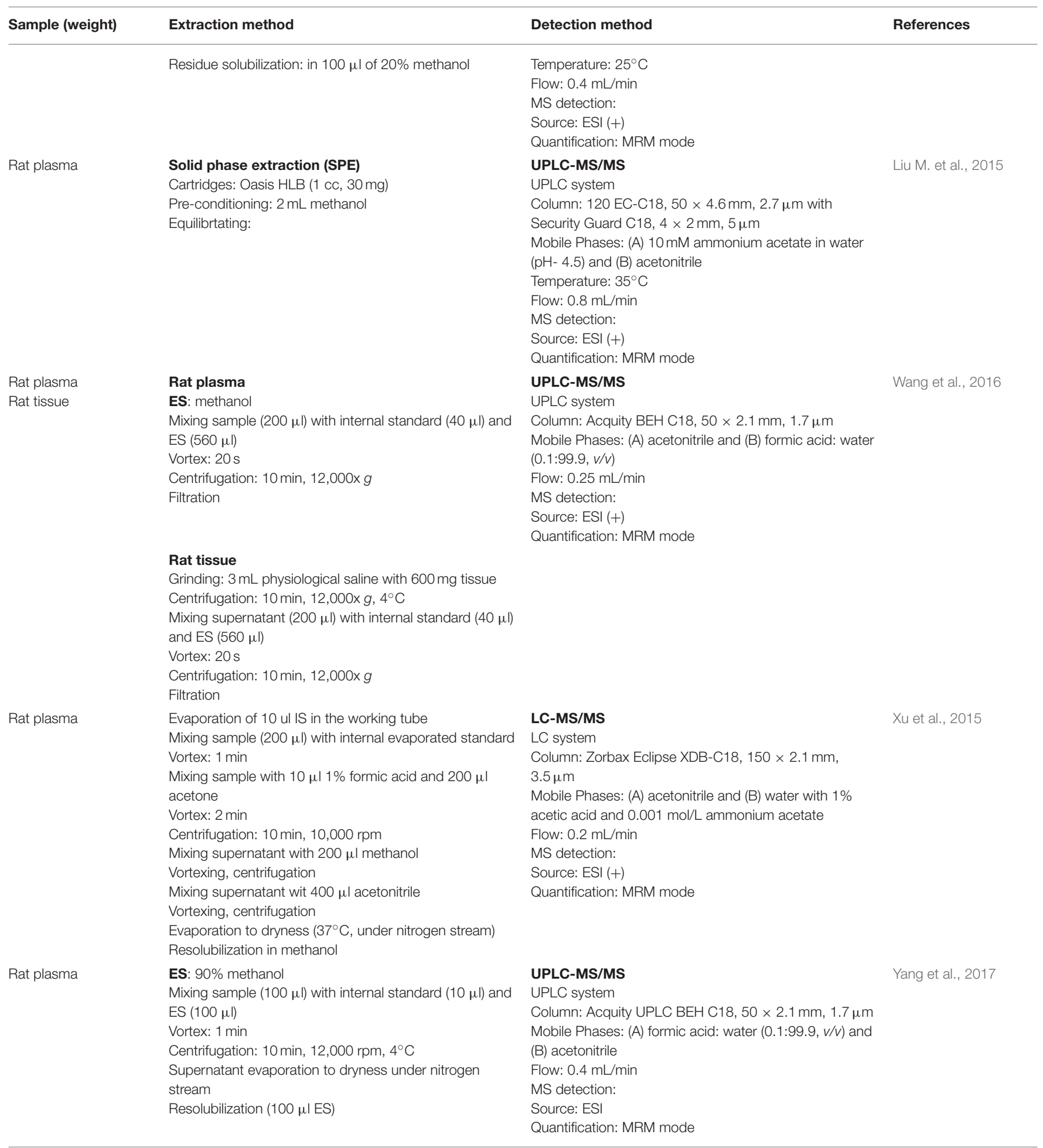

When referring to berberine extraction from biological samples, the extraction process is relatively simple and involves several steps like sample mixing with extraction solvents (e.g., methanol, acetone, acetonitrile), vortex, centrifugation followed by supernatant evaporation under nitrogen stream (Table 3). Other extraction techniques like solid phase extraction (SPE) can also be applied. 


\section{ANALYTICAL TECHNIQUES}

After extraction and purification, the separation and quantification of berberine are commonly resolved by chromatographic methods. According to literature studies, berberine determination in plants was predominantly performed using methods like UV spectrophotometry (Joshi and Kanaki, 2013), HPLC (Babu et al., 2012; Akowuah et al., 2014), HPTLC and TLC (Rojsanga and Gritsanapan, 2005; Arawwawala and Wickramaar, 2012; Samal, 2013), capillary electrophoresis (Du and Wang, 2010), while berberine content in biological fluids was mainly achieved by using LC-MS (Deng et al., 2008; Feng et al., 2010), UPLC-MS (Liu M. et al., 2015; Liu L. et al., 2016), UHPLC/Q-TOF-MS (Wu et al., 2015).

UV-Vis spectrophotometry can be considered as one of the most rapid detection methods for berberine quantitative analysis from plant extracts. Based on the Beer-Lambert law, berberine concentration can be determined according to its absorption maxima at $348 \mathrm{~nm}$. Joshi and Kanaki (2013) quantified berberine in Rasayana churna samples in the range of $2-20 \mu \mathrm{g} / \mathrm{mL}$, the interference with other compounds being avoided by the specific isolation of the alkaloid fraction (Joshi and Kanaki, 2013).

Next, high-performance liquid chromatography (HPLC) is a versatile, robust, and widely used technique for the qualitative and quantitative analysis of natural products (Sasidharan et al., 2011). This approach is widely used in berberine identification and quantification. Generally, the choices of stationary phase in berberine separation are variants of C18-based silica column (Table 3) with a mobile phase consisting of simple or acidified solvents like water, methanol, or acetonitrile, used as such or in combination with phosphate buffers. Normally, the identification and separation of berberine can be accomplished using either isocratic or gradient elution system. Berberine identification is further accomplished using high sensitivity UV or DAD (diode array detectors) detectors. For example, Shigwan et al. (2013) developed in his study a reverse phase HPLC method with photodiode array detection (PDA) to quantify berberine from Berberis aristata and B. tinctoria. They used a Unisphere-C18 column $(5 \mu \mathrm{m}, 4.6 \times 150 \mathrm{~mm})$ with an isocratic gradient of acidified water (with $0.1 \%$ trifluoroacetic acid) and acetonitrile $(60: 40, v / v)$ to elute berberine within $5 \mathrm{~min}$. The developed method was reproducible, validated, precise, and specific for berberine quantification (with a concentration range between 0.2 and $150 \mu \mathrm{g} / \mathrm{mL}$; Shigwan et al., 2013).

Two other commonly used techniques in berberine quantification are thin layer chromatography (TLC) and high performance thin layer chromatography (HTPLC). Sometimes, these methods are preferred over HPLC, offering the possibility of running several samples simultaneously along with the use of small amount of both samples and mobile phases (Samal, 2013). For these reasons, Samal (2013) used an HPTLC method to quantify berberine from $A$. mexicana $\mathrm{L}$. using toluene and ethyl acetate $(9: 3, v / v)$ as mobile phases, and a silica gel plate as stationary phase, they developed a simple, rapid, and cost-effective method for berberine quantification. The LOD $(0.120 \mu \mathrm{g})$ and LOQ $(0.362 \mu \mathrm{g})$ of the method are in accordance with high-quality requirements.
Following the same principles (small sample volume, high separation efficiency, and short analysis time), capillary electrophoresis (CE) was successfully used in berberine analysis. Du and Wang (2010) used CE with end-column electrochemiluminescence (ECL) detection for berberine analysis in both tablets and Rhizoma coptidis. Using a $4 \mathrm{~min}$ analysis time, a small sample volume $(3.3 \mathrm{~nL})$ and a LOD of $(5 \times$ $10^{-9} \mathrm{~g} / \mathrm{mL}$ ), the developed method proved to be highly sensitive and with good resolution (Du and Wang, 2010).

Besides UV, HPLC, HTPLC, TLC, and CE, other detection methods like liquid chromatography coupled with mass spectrometry (LC/MS) are currently employed to quantify berberine in biological fluids. Generally, it is considered a powerful technique for the analysis of complex samples because it offers rapid and accurate information about the structural composition of the compounds, especially when tandem mass spectrometry $\left(\mathrm{MS}^{\mathrm{n}}\right)$ is applied. For example, Xu et al. (2015) developed a sensitive an accurate LC-MS/MS method to determine berberine and other seven components in rat plasma using multiple reactions monitoring (MRM) mode. Compounds separation was optimized using six different types of reversephase columns, and two different mobile phases (methanolwater and acetonitrile-water with different additives). Additives like formic acid, acetic acid, and ammonium acetate were added in different concentrations as follows: $0.1,0.5,1$, and $2 \%$ for formic acid, $0.1,0.5,1$, and $2 \%$ for acetic acid and $0.0001,0.001$, $0.01 \mathrm{~mol} / \mathrm{L}$ for ammonium acetate. The method was also tested in terms of specificity, linearity, lower limit of quantification (LLOQ), precision, accuracy, and stability (Xu et al., 2015).

\section{ANTIOXIDANT EFFECT}

Under normal conditions, the body maintains a balance between the antioxidant and pro-oxidant agents (reactive oxygen species-ROS and reactive nitrogen species-RNS; Rahal et al., 2014).

The imbalance between pro and antioxidants occurs in case of increased oxidative stress (Bhattacharyya et al., 2014).

The oxidative stress builds up through several mechanisms: an increase in the production of reactive species, a decrease in the levels of enzymes involved in blocking the actions of pro-oxidant compounds, and/or the decrease in free radical scavengers (Pilch et al., 2014).

An experimental study demonstrated the effect of berberine on lipid peroxidation after inducing chemical carcinogenesis in small animals (rats). An increase in LPO (lipid peroxidation) was observed after carcinogenesis induction, but also its significant reversal after berberine administration $(30 \mathrm{mg} / \mathrm{kg})$. Berberine shows therefore at least partial antioxidant properties, due to its effect on lipid peroxidation (Thirupurasundari et al., 2009).

Other mechanisms involved in the antioxidant role of berberine are: ROS/RNS scavenging, binding of metals leading to the transformation/oxidation of certain substances, freeoxygen removal, reducing the destructiveness of superoxide ions and nitric oxide, or increasing the antioxidant effect of some endogenous substances. The antioxidant effect of berberine was comparable with that of vitamin C, a highly-potent antioxidant (Shirwaikar et al., 2006; Ahmed et al., 2015). 
The increase in blood sugar leads to oxidative stress not by generating oxygen reactive species but by impairing the antioxidant mechanisms. Administration of berberine to rats with diabetes mellitus increased the SOD (superoxide dismutase) activity and decreased the MDA (malondialdehyde) level (marker of lipid peroxidation). This antioxidant effect of berberine could explain the renal function improvement in diabetic nephropathy (Liu et al., 2008b).

The oxidative stress plays an important role in the pathogenesis of many diseases. The beneficial effect of berberine is presumed to reside mostly in its antioxidant role.

\section{CARDIOVASCULAR EFFECTS OF BERBERINE}

\section{Effect on Cardiac Contractility}

The beneficial effect of berberine in cardiac failure was demonstrated in a study on 51 patients diagnosed with NYHA (New York Heart Association) III/IV cardiac failure with low left ventricular ejection fraction (LVEF) and premature ventricular contractions and/or ventricular tachycardia. These patients received tablets containing $1.2 \mathrm{~g}$ berberine/day, together with conventional therapy (diuretics, ACEI-angiotensin-convertingenzyme inhibitors, digoxin, nitrates) for 2 weeks. An increase in LVEF was observed in all patients after this period, but also a decrease in the frequency and complexity of premature ventricular contractions. The magnitude of the beneficial effect was in direct proportion with the plasma concentration of berberine (Zeng, 1999).

\section{The Cardioprotective Effect During Ischemia}

Berberine can provide cardio-protection in ischemic conditions by playing various roles at different levels: modulation of AMPK (AMP-activated kinase) activity, AKT (protein kinase B) phosphorylation, modulation of the JAK/STAT (Janus kinase/signal transducers and activators of transcription) pathway and of GSK3 $\beta$ (glycogen synthase kinase $3 \beta$; Chang et al., 2016). AMPK is an important enzyme playing an essential role in cellular metabolism and offering protection in ischemic conditions by adjusting the carbohydrate and lipid metabolism, the function of cell organelles (mitochondria, endoplasmic reticulum) and the apoptosis (Zaha et al., 2016).

Berberine activates the PI3K (phosphoinositide 3kinase)/AKT pathway which is considered a compensatory mechanism limiting the pro-inflammatory processes and apoptotic events in the presence of aggressive factors. The activation of this pathway is associated with a reduction of the ischemic injury through the modulation of the TLR4 (toll-like receptor 4)-mediated signal transduction (Hua et al., 2007).

Several supporting data indicate that the JAK2/STAT3 signaling plays an important role in cardioprotection against ischemia-reperfusion injury (Mascareno et al., 2001).

GSK3 $\beta$ is a serine/threonine protein-kinase, an enzyme involved in reactions associated to important processes at the cellular level: metabolization, differentiation, proliferation, and apoptosis. Berberine inhibits this kinase, thereby exercising its cardioprotective effect (Park et al., 2014).

\section{EFFECTS ON THE ENDOTHELIUM}

Berberine induces endothelial relaxation by increasing NO production from arginine through the activity of eNOS (endothelial nitric oxide synthase) which is considered a key element in the vasodilation process. Besides increasing the NO level, it also up-regulates eNOS mRNA. Furthermore, berberine facilitates the phosphorylation of eNOS and its coupling to HSP 90 (heat shock proteins), which consequently increases NO production (Wang et al., 2009).

Moreover, berberine reduces endothelial contraction by reducing COX-2 expression. Any imbalance in COX 1 or 2 activity may alter the ratio between prothrombotic/antithrombotic and vasodilator/vasoconstrictor effects (Liu L. et al., 2015).

The beneficial effect of berberine on the TNF $\alpha$-induced endothelial contraction was also recorded, as well as an increase in the level of PI3K/AKT/eNOS mRNA (Xiao et al., 2014).

\section{THE ROLE OF BERBERINE IN ATHEROSCLEROSIS}

Atherogenesis is a consequence of high blood lipid levels and is associated with inflammatory changes in the vascular wall. Berberine interferes with this process by up-regulating the expression of SIRT1 (silent information regulator T1) and by inhibiting the expression of PPAR $\gamma$ (peroxisome proliferatoractivated receptor- $\gamma$ ). SIRT1 is a NAD-dependent deacetylase. The SIRT1 enzyme has many targets (PPAR $\gamma, \mathrm{p} 53)$, all playing different roles in atherogenesis (Chi et al., 2014).

\section{The Role of Berberine in Lipid Metabolism}

The effects of berberine on lipid metabolism are also the consequence of its effects on LDL cholesterol receptors. On one hand, these receptors are stabilized by an extracellular signalregulated kinase (ERK)-dependent pathway, and on the other, berberine increases the activity of LDL receptors through the JNK pathway (Cicero and Ertek, 2009).

Moreover, berberine has an effect on ACAT (cholesterol acyltransferases), a class of enzymes that transform cholesterol into esters, thus playing an essential role in maintaining cholesterol homeostasis in different tissues. There are two types of ACAT enzymes, ACAT1, and ACAT2. ACAT1 is a ubiquitous enzyme, while ACAT2 can be found only in hepatic cells and enterocytes. Berberine influences the activity of ACAT2 without an effect on ACAT1, therefore reducing the intestinal absorption of cholesterol and decreasing its plasmatic level (Chang et al., 2009; Wang et al., 2014).

The hypolipidemic effect of berberine is also a result of its action on PCSK9 (proprotein convertase subtilisin kexin 9). This enzyme can attach itself to LDL receptors, leading to a decrease in LDL metabolization and an increase in its blood level (Xiao et al., 2012). 
In a clinical trial, 63 patients with dyslipidemia were randomly divided in three groups. The first group was treated with berberine (1,000 $\mathrm{mg} /$ day), the second with simvastatin (20 $\mathrm{mg} /$ day) and the third with a combination of berberine and simvastatin. The authors reported a $23.8 \%$ reduction in LDL-C levels in patients treated with berberine, a $14.3 \%$ reduction in those treated with simvastatin and a $31.8 \%$ LDL-C reduction in the group treated with both simvastatin and berberine. This result demonstrates that berberine can be used alone or in association with simvastatin in the treatment of dyslipidemia (Kong et al., 2008).

\section{THE ROLE OF BERBERINE IN GLUCOSE METABOLISM}

Many studies demonstrated that berberine lowers blood sugar, through the following mechanisms:

- Inhibition of mitochondrial glucose oxidation and stimulation of glycolysis, and subsequently increased glucose metabolization (Yin et al., 2008a).

- Decreased ATP level through the inhibition of mitochondrial function in the liver, which may be the probable explanation of gluconeogenesis inhibition by berberine (Xia et al., 2011).

- Inhibition of DPP 4 (dipeptidyl peptidase-4), a ubiquitous serine protease responsible for cleaving certain peptides, such as the incretins GLP1 (glucagon-like peptide-1) and GIP (gastric inhibitory polypeptide); their role is to raise the insulin level in the context of hyperglycemia. The DPP4 inhibition will prolong the duration of action for these peptides, therefore improving overall glucose tolerance (Almasri et al., 2009; Seino et al., 2010).

Berberine has a beneficial effect in improving insulin resistance and glucose utilization in tissues by lowering the lipid (especially triglyceride) and plasma free fatty acids levels (Chen et al., 2011).

The effect of berberine (1,500 mg day) on glucose metabolism was also demonstrated in a pilot study enrolling 84 patients with type 2 diabetes mellitus. The effect, including on HbAlc, was comparable to that of metformin $(1,500 \mathrm{mg} /$ day $)$, one of the most widely used hypoglycemic drugs. In addition, berberine has a favorable influence on the lipid profile, unlike metformin, which has barely any effect (Yin et al., 2008b).

\section{HEPATOPROTECTIVE EFFECT OF BERBERINE}

The hepatoprotective effect of berberine was demonstrated on lab animals (mice), in which hepatotoxicity was induced by doxorubicin. Pretreatment with berberine significantly reduced both functional hepatic tests and histological damage (inflammatory cellular infiltrate, hepatocyte necrosis; Zhao et al., 2012).

The mechanism by which berberine reduces hepatotoxicity was also studied on $\mathrm{CCl}_{4}$ (carbon tetrachloride)-induced hepatotoxicity. Berberine lowers the oxidative and nitrosamine stress and also modulates the inflammatory response in the liver, with favorable effects on the changes occurring in the liver. Berberine prevents the decrease in SOD activity and the increase in lipid peroxidation and contributes to the reduction in TNF- $\alpha$, COX-2, and iNOS (inducible nitric oxide synthase) levels. The decrease in transaminase levels supports the hypothesis according to which berberine helps maintain the integrity of the hepatocellular membrane (Domitrović et al., 2011).

\section{NEPHROPROTECTIVE EFFECT OF BERBERINE}

The chronic kidney damage occurring in time in patients with HT (hypertension) and DM (diabetes mellitus) is well known; it is mainly due to the atherosclerosis of the renal artery, caused by inflammation and oxidative stress. The protective effect of berberine on kidneys was studied on 69 patients suffering from both HT and DM, with blood pressure and blood sugar levels controlled with conventional medication. The patients received $300 \mathrm{mg}$ berberine/day for 24 months, with 2-week interruptions every 5 months. The authors recorded lower CRP (C-reactive protein), MDA and SOD levels after treatment, but without significant changes in creatinine, arterial pressure, or glycaemia levels. These results support the renal protective effect of berberine through its anti-inflammatory and antioxidant effects (Dai et al., 2015).

Another animal study tested the renoprotective effect of berberine after administration of $\mathrm{HgCl}_{2}$ (mercury chloride). This substance induces hepato-renal damage by increasing the oxidative stress (increases lipid peroxidation and NO levels, and lowers glutathione and SOD levels as well as the activity of other protective enzymes). Administration of $\mathrm{HgCl}_{2}$ increased the AST (aspartate aminotransferase), ALT (alanine aminotransferase), and ALP (alkaline phosphatase) levels, compared to the control group. However, pretreatment with berberine lowered these enzymes significantly. In addition, both urea and creatinine levels were significantly increased in the $\mathrm{HgCl}_{2}$ group vs. the control group, and again pretreatment with berberine prevented these changes. Additionally, the authors recorded higher pro-oxidant and lower antioxidant levels in the intervention group. These data support the hepatic and renal protective effects of berberine. Other studies performed on animal models with $\mathrm{CCl}_{4-}$ induced hepatotoxicity demonstrated the same effect (Othman et al., 2014).

In addition, berberine can lower the nephrotoxicity caused by cisplatine. In an animal study, berberine was administered in progressive doses of $1,2,3 \mathrm{mg} / \mathrm{kg}$, orally, for 2 successive days, starting 2 days after cisplatine administration. After the last doses of berberine, the animals were sacrificed and the kidneys were examined by the pathologist. The results showed significant histological improvement and a reduction in NF$\mathrm{kB}$ (nuclear factor kappa-light-chain-enhancer of activated B cells), TNF $\alpha$, COX2 an iNOS levels, all of which support the anti-inflammatory effect of berberine (Domitrović et al., 2013). 


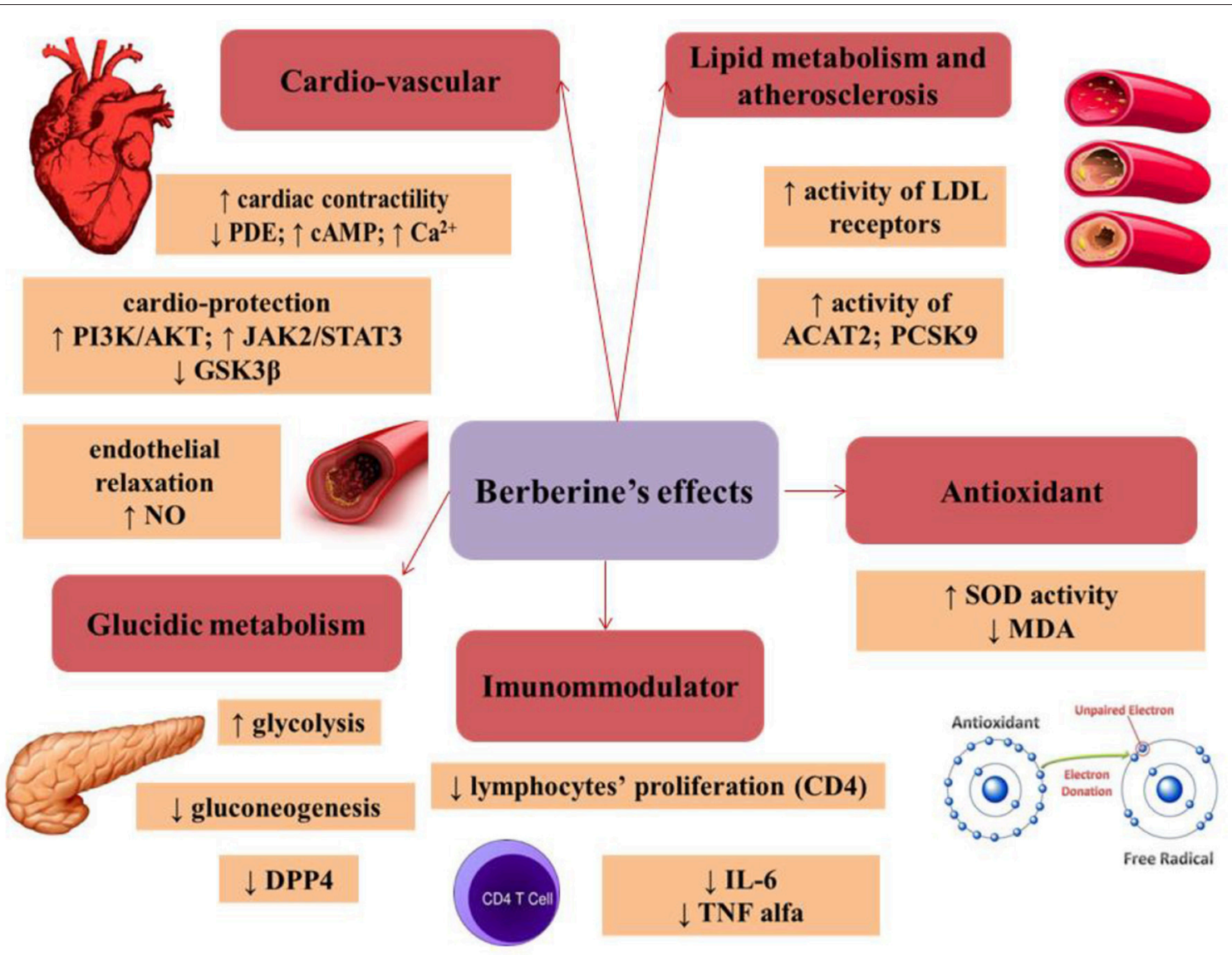

FIGURE 3 | Therapeutic effects of berberine in vivo. Mechanisms of berberine in regulation of metabolism, immunity and oxidative reactions. Phosphodiesterase (PDE), cyclic 3',5'-adenosine monophosphate (cAMP), phosphoinositide 3-kinase/protein kinase B (PI3K/AKT), Janus kinase/signal transducers and activators of transcription (JAK/STAT), glycogen synthase kinase $3 \beta$ (GSK3 $\beta$ ), superoxide dismutase (SOD), malondialdehyde (MDA), nitric oxide (NO), cholesterol acyltransferases (ACAT2), dipeptidyl peptidase-4 (DPP 4), proprotein convertase subtilisin kexin 9 (PCSK9).

\section{IMMUNOMODULATORY EFFECT OF BERBERINE}

The immunomodulatory effect of berberine was demonstrated in many experimental and clinical contexts.

In an experimental autoimmune myocarditis model, berberine contributed to mitigate the cardiac damage by: limiting the rise in anticardiac myosin antibodies, modulating the activity of certain STATs and blocking Th1 and Th2 cell differentiation, which play an important role in the pathogenesis of myocarditis (Liu X. et al., 2016).

Experimental autoimmune neuritis is an experimental animal model equivalent to the Guillain-Barre syndrome in humans. This neurologic syndrome is characterized by autoimmune injury of the peripheral nervous system. The beneficial effect of berberine on this animal model resided in its influence on cellular and humoral immunity through the inhibition of lymphocyte proliferation (especially CD4), and the decrease in pro-inflammatory cytokines (IL-6 and TNF $\alpha$; Li et al., 2014).

Experimental autoimmune encephalomyelitis is an established model of multiple sclerosis. Multiple sclerosis is a one of the most common diseases of the central nervous system (CNS) and involves neurodegenerative and inflammatory processes, and autoimmune demyelination (Ransohoff et al., 2015). The blood-brain barrier permeability and changes in matrix metalloproteinase (MMP) levels in the cerebrospinal fluid and brain were studied using this model (Ma et al., 2010). MMPs may be involved in demyelination and their activity in tissues depends on the balance between their level and their tissue inhibitors. MMP2 and MMP9 are the main endoproteinases involved in the migration of lymphocytes in CNS and in altering the BBB (blood brain barrier) (Avolio et al., 2003). Berberine has a beneficial effect in experimental autoimmune encephalomyelitis by inhibiting the activity of MMP9, reducing BBB permeability and, consecutively, by decreasing the inflammatory cellular infiltration of the CNS (Ma et al., 2010).

The current therapy used for inflammatory bowel diseases, including glucocorticoids and immunosuppressive agents, has a low level of safety. The effect of berberine was studied in combination with 5-ASA (5-aminosalicylic acid) vs. 5-ASA alone using an experimental animal model with DSS (dextran sulfate sodium)-induced colitis. The authors analyzed the level 
of proinflammatory cytokines in the animal gut. A decrease in COX2, IL6, and IL23 mRNA levels was observed in animals treated only with 5-ASA, whereas animals treated with both 5ASA and berberine had a reduction in mRNA levels for COX2, IL6, IL23 as well as for TNF alfa and IL12b. This beneficial effect could partially be attributed to the inhibition of NF-kB and the reduction in JAK2 phosphorylation (through the influence on the JAK/STAT pathway) by both 5-ASA and berberine (Li et al., 2015; Figure 3).

Another study demonstrated that berberine increases the corticosteroid level in rats with experimentally-induced colitis. This engendered the theory that its beneficial effect may also be attributed to the increase in endogenous glucocorticoid levels, compounds with well-known therapeutic effect in inflammatory bowel disease (Minaiyan et al., 2011).

\section{CONCLUSION}

A review of the available scientific literature shows that the traditional medical uses of berberine-containing plants have been evaluated by modern pharmacological studies. Different species of berberine-rich plants have multiple pharmacological and therapeutic actions, such as antioxidant and immunomodulatory effects, protective action on the cardiovascular system, liver and kidney, endothelial relaxation, regulator on glucose metabolism and atherosclerosis, which can all be explained by the presence of berberine as well as other phyto constituents (when dealing with berberinecontaining plant extracts). Moreover, the effects of berberine vary according to its origin (different plants or pharmaceutical

\section{REFERENCES}

Abbasi, A. M., Dastagir, G., Hussain, F., and Sanaullah, P. (2005). Ethnobotany and marketing of crude drug plants in district Haripur, Pakistan. Pak. J. Plant Sci. $11,103-114$.

Abbasi, A. M., Khan, M. A., Ahmad, M., Zafar, M., Jahan, S., and Sultana, S. (2010). Ethnopharmacological application of medicinal plants to cure skin diseases and in folk cosmetics among the tribal communities of North-West Frontier Province, Pakistan. J. Ethnopharmacol. 128, 322-335. doi: 10.1016/j.jep.2010.01.052

Abbasi, A. M., Khan, M. A., Ahmad, M., Zafar, M., Khan, H., Muhammad, N., et al. (2009). Medicinal plants used for the treatment of jaundice and hepatitis based on socio-economic documentation. Afr. J. Biotechnol. 8, 1643-1650.

Abou-Donia, A. H. A., and El-Din, A. A. S. (1986). Phytochemical study of Argemone mexicana L. grown in Egypt. Egypt. J. Pharm. Sci. 25, 1-5.

Acharya, K. P., and Rokaya, M. B. (2005). Ethnobotanical survey of medicinal plants traded in the streets of Kathmandu valley. Sci. World 3, 44-48.

Adeyemi, A. A., Gbolade, A. A., Moody, J. O., Ogbole, O. O., and Fasanya, M. T. (2010). Traditional anti-fever phytotherapies in Sagamu and Remo north districts in Ogun State, Nigeria. J. Herbs. Spices Med. Plants 16, 203-218. doi: 10.1080/10496475.2010.511075

Adjanohoun, J. E., Aboobakar, N., and Dramane, K. (1996). Traditional Medicine and Pharmacopoeia: Contribution to Ethnobotanical and Floristic Studies in Cameroon. Porto-Novo: Technical and Research Commission (STRC) of the Organization of African Unity.

Ahmed, E., Arshad, M., Ahmad, M., Saeed, M., and Ishaque, M. (2004). Ethnopharmacological survey of some medicinally important plants of products) and its concentration, depending on the very diverse extraction and detection techniques already described. Over time, modern extraction techniques were increasingly preferred to classical ones. Since classical methods are generally time- and solvent-consuming processes, modern extraction techniques such as USE, MAE, UPE, SFE, and PLE are seen as better alternatives to overcome these limitations. Furthermore, berberine, due to its antioxidant and anti-inflammatory effects, has several clinical applications in many disorders, from inflammatory conditions to the metabolic syndrome. However, there are some traditional uses that have not yet been completely elucidated, and further studies are needed. Therefore, extensive studies on the potential of plants containing berberine that have shown aforementioned pharmacological activities should go through additional in vitro and in vivo studies.

\section{AUTHOR CONTRIBUTIONS}

$\mathrm{MN}, \mathrm{AM}, \mathrm{JE}$, and RP have conceived and designed the structure of the manuscript, data collection, and drafting, as well as its revision. $\mathrm{CB}, \mathrm{GC}$, and $\mathrm{AB}$ have critically reviewed the manuscript. All authors have seen and agreed on the final version of the manuscript.

\section{ACKNOWLEDGMENTS}

This article was published under the frame of the internal grant no. 4945/15/08.03.2016 of the Iuliu Hatieganu University of Medicine and Pharmacy, Cluj-Napoca, Romania.
Galliyat Areas of NWFP, Pakistan. Asian J. Plant Sci. 3, 410-415. doi: 10.3923/ajps.2004.410.415

Ahmed, T., Gilani, A. U., Abdollahi, M., Daglia, M., Nabavi, S. F., and Nabavi, S. M. (2015). Berberine and neurodegeneration: a review of literature. Pharmacol. Rep. 67, 970-979. doi: 10.1016/j.pharep.2015.03.002

Ahn, D. K. (2003). Illustrated Book of Korean Medicinal Herbs. Seoul: Kyo-Hak Publishing, Kyohaksa.

Ajali, U. (2000). Antibacterial activity of Enantia polycarpa bark. Fitoterapia 71, 315-316. doi: 10.1016/S0367-326X(99)00153-7

Akowuah, G. A., Okechukwu, P. N., and Chiam, N. C. (2014). Evaluation of HPLC and spectrophotometric methods for analysis of bioactive constituent berberine in stem extracts of Coscinium fenestratum. Acta Chromatogr. 26, 243-254. doi: 10.1556/AChrom.26.2014.2.4

Al-Douri, N. A. (2000). A survey of medicinal plants and their traditional uses in Iraq. Pharm. Biol. 38, 74-79. doi: 10.1076/1388-0209(200001)3811-BFT074

Ali, M., Shah, S. Z., Khan, M. S., Naz, M. F. R., and Zafar, A. (2018). Ethnobotanical study on the weeds of wheat crop in district Swabi, Khyber Pakhtunkhwa, Pakistan. Int. J. Biosci. 12, 363-374. doi: 10.12692/ijb/12.1.363-374

Al-masri, I. M., Mohammad, M. K., and Tahaa, M. O. (2009). Inhibition of dipeptidyl peptidase IV (DPP IV) is one of the mechanisms explaining the hypoglycemic effect of berberine. J. Enzyme Inhib. Med. Chem. 24, 1061-1066. doi: 10.1080/14756360802610761

Al-Qura'n, S. (2009). Ethnopharmacological survey of wild medicinal plants in Showbak, Jordan. J. Ethnopharmacol. 123, 45-50. doi: 10.1016/j.jep.2009.02.031

Alupului, A., Calinescu, I., and Lavric, V. (2009). Ultrasonic vs. microwave extraction intensification of active principles from medicinal plants. Chem. Eng. Trans. 17, 1023-1028. doi: 10.3303/cet0917171 
Andola, H. C., Gaira, K. S., Rawal, R. S., Rawat, M. S., and Bhatt, I. D. (2010a). Habitat-dependent variations in berberine content of Berberis asiatica Roxb. ex. DC. in Kumaon, Western Himalaya. Chem. Biodivers. 7, 415-420. doi: 10.1002/cbdv.200900041

Andola, H. C., Rawal, R. S., Rawat, M. S. M., Bhatt, I. D., and Purohit, V. K. (2010b). Variations of berberine contents in Berberis pseudumbellata: a high value medicinal shrub of west Himalaya, India. Med. Plants Int. J. Phytomed. Relat. Ind. 2, 111-115. doi: 10.5958/j.0975-4261.2.2.017

Andola, H. C., Rawal, R. S., Rawat, M. S. M., Bhatt, I. D., and Purohit, V. K. (2010c). Analysis of berberine content using HPTLC fingerprinting of root and bark of three Himalayan Berberis species. Asian J. Biotechnol. 2, 239-245. doi: 10.3923/ajbkr.2010.239.245

Anesini, C., and Perez, C. (1993). Screening of plants used in Argentine folk medicine for antimicrobial activity. J. Ethnopharmacol. 39, 119-128. doi: 10.1016/0378-8741(93)90027-3

Arawwawala, L. D. A. M., and Wickramaar, W. A. N. (2012). Berberine content in Coscinium fenestratum (Gaertn.) Colebr grown in Sri Lanka. Pharmacologia 3, 679-682. doi: 10.5567/pharmacologia.2012.679.682

Arayne, M. S., Sultana, N., and Bahadur, S. S. (2007). The berberis story: Berberis vulgaris in therapeutics. Pak. J. Pharm. Sci. 20, 83-92.

Atta-ur-Rahma., and Ahmad, H. (1992). An aporphine-benzylisoquinoline alkaloid from Berberis waziristanica. Phytochemistry 31, 1835-1836. doi: 10.1016/0031-9422(92)83163-S

Avolio, C., Ruggieri, M., Giuliani, F., Liuzzi, G. M., Leante, R., Riccio, P., et al. (2003). Serum MMP-2 and MMP-9 are elevated in different multiple sclerosis subtypes. J. Neuroimmunol. 136, 46-53. doi: 10.1016/ S0165-5728(03)00006-7

Babu, N. H. R., Thriveni, H. N., and Vasudeva, R. (2012). Influence of drying methods and extraction procedures on the recovery of berberine content in Coscinium fenestratum. J. Nat. Prod. Plant Resour. 2, 540-544.

Bahar, M., Deng, Y., Zhu, X., He, S., Pandharkar, T., Drew, M. E., et al. (2011). Potent antiprotozoal activity of a novel semi-synthetic berberine derivative. Bioorg. Med. Chem. Lett. 21, 2606-2610. doi: 10.1016/j.bmcl.2011.01.101

Baharvand-Ahmadi, B., Bahmani, M., Tajeddini, P., Naghdi, N., and RafieianKopaei, M. (2016). An ethno-medicinal study of medicinal plants used for the treatment of diabetes. J. Nephropathol. 5, 44-50. doi: 10.15171/jnp.2016.08

Baldazzi, C., Leone, M. G., Casini, M. L., and Tita, B. (1998). Effects of the major alkaloid of Hydrastis canadensis L., berberine, on rabbit prostate strips. Phyther. Res. 12, 589-591. doi: 10.1002/(SICI)1099-1573(199812)12:8<589::AID-PTR347>3.0.CO;2-I

Bapna, S., Choudhary, P. K., Ramaiya, M., and Chowdhary, A. (2015). Antiplasmodial activity of Argemone mexicana: an in vivo and in vitro study. World J. Pharm. Res. 4, 1653-1663.

Bele, M. Y., Focho, D. A., Egbe, E. A., and Chuyong, B. G. (2011). Ethnobotanical survey of the uses Annonaceae around mount Cameroon. Afr. J. Plant Sci. 5, 237-247.

Betti, J. L., Caspa, R., Ambara, J., and Kourogue, R. L. (2013). Ethno-botanical study of plants used for treating malaria in a forest: savanna margin area, East region, Cameroon. Glob. J. Res. Med. Plants Indig. Med. 2, 692.

Betti, J. L., and Lejoly, J. (2009). Contribution to the knowledge of medicinal plants of the Dja Biosphere Reserve, Cameroon: plants used for treating jaundice. J. Med. Plants Res. 3, 1056-1065.

Bhandari, D. K., Nath, G., Ray, A. B., and Tewari, P. V. (2000). Antimicrobial activity of crude extracts from Berberis asiatica stem bark. Pharm. Biol. 38, 254-257. doi: 10.1076/1388-0209(200009)3841-AFT254

Bhattacharjee, S., Tiwari, K. C., Majumdar, R., and Misra, A. K. (1980). Folklore medicine from district Kamrup (Assam). Bull. Medic. Ethno. Bot. Res. 1, 447-460.

Bhattacharyya, A., Chattopadhyay, R., Mitra, S., and Crowe, S. E. (2014). Oxidative stress: an essential factor in the pathogenesis of gastrointestinal mucosal diseases. Physiol. Rev. 94, 329-354. doi: 10.1152/physrev.00040.2012

Birdsall, T. C. (1997). Berberine: therapeutic potential of an alkaloid found in several medicinal plants. Altern. Med. Rev. 2, 94-103.

Bonesi, M., Loizzo, M. R., Conforti, F., Passalacqua, N. G., Saab, A., Menichini, F., et al. (2013). Berberis aetnensis and B. libanotica: A comparative study on the chemical composition, inhibitory effect on key enzymes linked to Alzheimer's disease and antioxidant activity. J. Pharm. Pharmacol. 65, 1726-1735. doi: 10.1111/jphp.12172
Borokini, T. I., Clement, M., Dickson, N. J., and Edagbo, D. E. (2013). Ethnobiological survey of traditional medicine practice for fevers and headaches in Oyo State, Nigeria. Topclass J. Herb. Med. 2, 121-130.

Bose, B. C., Vijayvargiya, R., Saifi, A. Q., and Sharma, S. K. (1963). Chemical and pharmacological studies on Argemone mexicana. J. Pharm. Sci. 52, 1172-1175. doi: $10.1002 /$ jps.2600521216

Bouquet, A. (1969). Féticheurs et Médecines Traditionnelles du Congo (Brazzaville). Mém. O.R.S.T.O.M. (Paris: Office la Rech. Sci. Tech. outre-mer) 36, 282.

Bouquet, A., and Debray, M. (1974). Plantes Médicinales de la Côte d'Ivoire. Paris Off. la Rech. Sci. Tech. Paris: Outre Mer 231p. (Travaux Doc. I'ORSTOM no. 32) Illus., col. illus. Geog 5.

Bown, D. (1995). Encyclopaedia of Herbs and Their Uses. London: Dorling Kindersley London.

Burkill, H. M. (1985). The Useful Plants of West Tropical Africa. London: Royal Botanic Gardens, Kew.

Bushra, I., Kishwar, S., Qureshi, R. A., and Saddiqa, M. (2000). A checklist of plants of Bhogarmang, Siran Valley NWFP, Pakistan. Hamdard Med. 43, 62-76.

Buzas, A., and Egnell, C. (1965). On the presence of quinidine in addition to berberine alkaloids in the barks of Enantia pilosa and Enantia polycarpa (Annonaceae). Ann. Pharm. Fr. 23, 351.

Castleman, M. (1991). The Healing Herbs: The Ultimate Guide to the Curative Powers of Nature's Medicine. Emmaus: Rodale Press.

Chakravarti, K. K., Dhar, D. C., and Siddiqui, S. (1950). Alkaloidal constituents of the bark of Berberis aristata. J. Sci. Ind. Res. 9, 161-164.

Chan, C.-O., Chu, C.-C., Mok, D. K., and Chau, F.-T. (2007). Analysis of berberine and total alkaloid content in Cortex phellodendri by near infrared spectroscopy (NIRS) compared with high-performance liquid chromatography coupled with ultra-visible spectrometric detection. Anal. Chim. Acta 592, 121-131. doi: 10.1016/j.aca.2007.04.016

Chandra, P., and Purohit, A. N. (1980). Berberine contents and alkaloid profile of Berberis species from different altitudes. Biochem. Syst. Ecol. 8, 379-380. doi: 10.1016/0305-1978(80)90040-X

Chang, T.-Y., Li, B.-L., Chang, C. C., and Urano, Y. (2009). Acyl-coenzyme A:cholesterol acyltransferases. Am. J. Physiol. Endocrinol. Metab. 297, E1-E9. doi: 10.1152/ajpendo.90926.2008

Chang, W., Li, K., Guan, F., Yao, F., Yu, Y., Zhang, M., et al. (2016). Berberine pretreatment confers cardioprotection against ischemia-reperfusion injury in a rat model of type 2 diabetes. J. Cardiovasc. Pharmacol. Ther. 21, 486-494. doi: $10.1177 / 1074248415627873$

Chang, Y. (2013). Ultrasonic-assisted extraction of berberine in ionic liquid. Pharm. Eng. 33, 1-4.

Chatterjee, D. R. (1951). Plant alkaloids. I. Berberis floribunda. J. Indian Chem. Soc. $28,225-228$.

Chatterjee, R., and Banerjee, A. (1953). Plant alkaloids. V. Berberis lambertii. J. Indian Chem. Soc. 30, 705-707.

Chatterjee, R., Guha, M. P., and Das Gupta, A. K. (1952). Plant alkaloids. IV. Berberis himalaica and B. tinctoria. J. Indian Chem. Soc. 29, 921-924.

Chaudhury, R. H. N., Guha, A., Chaudhury, R., and Pal, D. C. (1980). Ethnobotanical uses of herbaria-2. J. Econ. Taxon. Bot. 1, 163-168.

Chen, A. H. (1981). Studies on the analysis of alkaloids of Phellodendron wilsonii Hay. et Kaneh. Kaneh. Kexue Fazhan Yuekan 9, 398-411.

Chen, A. H. (1982). Applied studies on the alkaloids of Phellodendron wilsonii Hay. et Kaneh. II. the alkaloid contents in Taiwan plants. Kexue Fazhan Yuekan 10, 279-286.

Chen, C., Yu, Z., Li, Y., Fichna, J., and Storr, M. (2014). Effects of berberine in the gastrointestinal tract - a review of actions and therapeutic implications. Am. J. Chin. Med. 42, 1053-1070. doi: 10.1142/S0192415X14500669

Chen, H. F., and Chen, C. M. (1988). Determination of berberine in crude and processed Chinese herb: Coptidis rhizoma and Phellodendri cortex. Zhonghua Yaoxue Zazhi 40, 259-264.

Chen, W. H., Pang, J. Y., Qin, Y., Peng, Q., Cai, Z., and Jiang, Z. H. (2005). Synthesis of linked berberine dimers and their remarkably enhanced DNA-binding affinities. Bioorg. Med. Chem. Lett. 15, 2689-2692. doi: 10.1016/j.bmcl.2004.10.098

Chen, Y., Wang, Y., Zhang, J., Sun, C., and Lopez, A. (2011). Berberine improves glucose homeostasis in streptozotocin-induced diabetic rats in association with multiple factors of insulin resistance. ISRN Endocrinol. 2011, 1-8. doi: $10.5402 / 2011 / 519371$ 
Chen, Y. Y., Chang, F. R., and Wu, Y. C. (1996). Isoquinoline alkaloids and lignans from Rollinia mucosa. J. Nat. Prod. 59, 904-906. doi: 10.1021/np960414z

Chevallier, A. (1996). The Encyclopedia of Medicinal Plants. London: Dorling Kindersley.

Chhetri, D. R., Parajuli, P., and Subba, G. C. (2005). Antidiabetic plants used by Sikkim and Darjeeling Himalayan tribes, India. J. Ethnopharmacol. 99, 199-202. doi: 10.1016/j.jep.2005.01.058

Chi, L., Peng, L., Pan, N., Hu, X., and Zhang, Y. (2014). The anti-atherogenic effects of berberine on foam cell formation are mediated through the upregulation of sirtuin 1. Int. J. Mol. Med. 34, 1087-1093. doi: 10.3892/ijmm.2014.1868

Chiang, Y. L., Su, C. R., Kuo, P. C., Damu, A. G., and Wu, T. S. (2006). Two isoquinolones from the roots of Phellodendron amurense var. Wilsonii. Heterocycles 68, 339-345. doi: 10.3987/COM-05-10598

Chopra, R. N., Nayar, S. I., and Chopra, I. C. (1986). Glossary of Indian Medicinal Plants (Including the Supplement). New Delhi: Canal of Scientific and Industrial Research.

Cicero, A., and Ertek, S. (2009). Berberine: metabolic and cardiovascular effects in preclinical and clinical trials. Nutr. Diet Suppl. 1, 1-10. doi: 10.2147/NDS.S6084

Coffey, T. (1993). The History and Folklore of North American Wildflowers. New York, NY: Facts on File Limited.

Dai, P., Wang, J., Lin, L., Zhang, Y., and Wang, Z. (2015). Renoprotective effects of berberine as adjuvant therapy for hypertensive patients with type 2 diabetes mellitus: evaluation via biochemical markers and color Doppler ultrasonography. Exp. Ther. Med. 10, 869-876. doi: 10.3892/etm.2015.2585

de Almeida Costa, O. (1935). (Mexican poppy) Argemone mexicana L. Rev. Flora Med. 1, 271-282.

Deng, A. J., and Qin, H. L. (2010). Cytotoxic dihydrobenzophenanthridine alkaloids from the roots of Macleaya microcarpa. Phytochemistry 71, 816-822. doi: 10.1016/j.phytochem.2010.02.007

Deng, Y., Liao, Q., Li, S., Bi, K., Pan, B., and Xie, Z. (2008). Simultaneous determination of berberine, palmatine and jatrorrhizine by liquid chromatography-tandem mass spectrometry in rat plasma and its application in a pharmacokinetic study after oral administration of coptis-evodia herb couple. J. Chromatogr. B Anal. Technol. Biomed. Life Sci. 863, 195-205. doi: 10.1016/j.jchromb.2007.12.028

Dev, S. (2006). A Selection of Prime Ayurvedic Plants Drugsancient- Modern Concordance. New Delhi: Anamaya Publishers.

Din, N., Dibong, S. D., Mpondo, E. M., Priso, R. J., Kwin, N. F., and Ngoye, A. (2011). Inventory and identification of plants used in the treatment of diabetes in douala town (Cameroon). Eur. J. Med. Plants 1, 60-73. doi: 10.9734/EJMP/2011/273

Doepke, W., Ulrich, H., and Jimenez, V. (1976). On the structure of a new alkaloid from Argemone mexicana. Z. Chem. 16, 54-55.

Domitrović, R., Cvijanović, O., Pernjak-Pugel, E., Škoda, M., Mikelić, L., and Crnčević-Orlić, Ž. (2013). Berberine exerts nephroprotective effect against cisplatin-induced kidney damage through inhibition of oxidative/nitrosative stress, inflammation, autophagy and apoptosis. Food Chem. Toxicol. 62, 397-406. doi: 10.1016/j.fct.2013.09.003

Domitrović, R., Jakovac, H., and Blagojević, G. (2011). Hepatoprotective activity of berberine is mediated by inhibition of TNF- $\alpha, \mathrm{COX}-2$, and iNOS expression in CCl4-intoxicated mice. Toxicology 280, 33-43. doi: 10.1016/j.tox.2010.11.005

Doncheva, T., Kostova, N., Yordanova, G., Saadi, H., Akrib, F., Dimitrov, D., et al. (2014). Comparison of alkaloid profile from Glaucium corniculatum (Papaveraceae) of Algerian and Bulgarian origin. Biochem. Syst. Ecol. 56, 278-280. doi: 10.1016/j.bse.2014.07.007

Du, J. X., and Wang, M. (2010). Capillary electrophoresis determination of berberine in pharmaceuticals with end-column electrochemiluminescence detection. J. Chinese Chem. Soc. 57, 696-700. doi: 10.1002/jccs.201000097

Duke, J. A., and Ayensu, E. S. (1985). Medicinal Plants of China. Algonac, MI: Reference Publications.

Duke, J. A., and Beckstrom-Sternberg, S. M. (1994). Dr. Duke's phytochemical and ethnobotanical databases. Available online at: http://www.ars-grin.gov/duke/ plants.html (Accessed January 15, 2017).

Dzhalilov, D. R., Goryaev, M. I., and Kruglykhina, G. K. (1963). Alkaloids from Berberis iliensis. I Izv. Akad. Nauk Kaz. SSR, Ser. Tekhn. i Khim. Nauk 3, 15-19.

Egels, W. (1959). Papaver dubium var. lecoquii, a berberine-containing poppy. Planta Med. 7, 92-102. doi: 10.1055/s-0028-1101592
Ehiagbonare, P. O., and Onyibe, J. (2008). Conservation studies on four medicinal taxa of Southern Nigeria. Sci. Res. Essays 3, 40-45.

El Beyrouthy, M., Arnold, N., Delelis-Dusollier, A., Dupont, F. (2008). Plants used as remedies antirheumatic and antineuralgic in the traditional medicine of Lebanon. J. Ethnopharmacol. 120, 315-334. doi: 10.1016/j.jep.2008.08.024

Emboden, W. (1979). Narcotic Plants. New York, NY: Collier.

Emes, M., Aguilar, A., Argueta, A., and Cano, L. (1994). Indigenous Medicinal Florae from México, Vol. II.

Eric Brussell, D. (2004). A medicinal plant collection from Montserrat, West Indies. Econ. Bot. 58, S203-S220. doi: 10.1663/00130001(2004)58[S203:AMPCFM]2.0.CO;2

Esseily, F., El Ezzy, M., Gali-Muhtasib, H., Safi, S., Esseily, J., Diab-Assaf, M., et al. (2012). The ethanol fraction from the stem of Berberis libanotica inhibits the viability of adult T cell leukemia. Minerva Biotecnol. 24, 129-133.

Etminan, M., Gill, S. S., and Samii, A. (2005). Intake of vitamin E, vitamin C, and carotenoids and the risk of Parkinson's disease: a meta-analysis. Lancet. Neurol. 4, 362-365. doi: 10.1016/S1474-4422(05)70097-1

Fabricant, D. S., and Farnsworth, N. R. (2001). The value of plants used in traditional medicine for drug discovery. Environ. Heal. Perspect. Suppl. 109:69. doi: 10.1289/ehp.01109s169

Farías-Campomanes, A. M., Rostagno, M. A., Coaquira-Quispe, J. J., and Meireles, M. A. A. (2015). Supercritical fluid extraction of polyphenols from lees: overall extraction curve, kinetic data and composition of the extracts. Bioresour. Bioprocess. 2, 45. doi: 10.1186/ s40643-015-0073-5

Feng, J., Xu, W., Tao, X., Wei, H., Cai, F., Jiang, B., et al. (2010). Simultaneous determination of baicalin, baicalein, wogonin, berberine, palmatine and jatrorrhizine in rat plasma by liquid chromatography-tandem mass spectrometry and application in pharmacokinetic studies after oral administration of traditional Chinese medicinal preparations containing Scutellaria-Coptis herb couple. J. Pharm. Biomed. Anal. 53, 591-598. doi: 10.1016/j.jpba.2010.04.002

Fletcher, M. T., Takken, G., Blaney, B. J., and Alberts, V. (1993). Isoquinoline alkaloids and keto-fatty acids of Argemone ochroleuca and A. mexicana (Mexican poppy) seed. I. An assay method and factors affecting their concentration. Aust. J. Agric. Res. 44, 265-275. doi: 10.1071/AR9930265

Fogarty, J. E. (1990). A Barefoot Doctor's Manual: The American Translation of the Official Chinese Paramedical Manual. Philadelphia, PA: Running Press Book Publishers.

Fongod, A. G. (2014). Ethnobotany, indigenous knowledge and unconscious preservation of the environment: An evaluation of indigenous knowledge in South and Southwest Regions of Cameroon. Int. J. Biodivers. Conserv. 6, 85-99. doi: 10.5897/IJBC2013.0637

Foote, P. A. (1932). The alkaloids of Argemone alba Lestib. J. Am. Pharm. Assoc. 21, 246-248.

Foster, S., and Duke, J. A. (1990). A Field Guide to Medicinal Plants: Eastern and Central North America. Boston, MA: Houghton Mifflin Company.

Freile, M., Giannini, F., Sortino, M., Zamora, M., Juarez, A., Zacchino, S., et al. (2006). Antifungal activity of aqueous extracts and of berberine isolated from Berberis heterophylla. Acta Farm. Bonaer. 25, 83-88.

Fyson, P. F. (1975). Flora of the Nilgiri and Pulney Hill-Tops. Dehra Dun: Bishen Singh Mahendra Pal Singh and Periodical Experts.

Gbile, Z. O., Soladoye, M. O., and Adesina, S. K. (1988). Plants in traditional medicine in West Africa. Monogr. Syst. Bot. Missouri Bot. Gard. 25, 343-349.

Gbolade, A. (2012). Ethnobotanical study of plants used in treating hypertension in Edo State of Nigeria. J. Ethnopharmacol. 144, 1-10. doi: 10.1016/j.jep.2012.07.018

Gertig, H. (1964). Alkaloids of Eschscholtzia californica. I. Isolation and thin-layer chromatography of alkaloid fractions from roots. Acta Pol. Pharm. 21, 59-64.

Gill, L. S., and Akinwumi, C. (1986). Nigerian folk medicine: practices and beliefs of the Ondo people. J. Ethnopharmacol. 18, 257-266. doi: 10.1016/0378-8741(86)90004-8

Gorval', L. M., and Grishkovets, V. I. (1999). Alkaloids of some species of the genus Berberis introduced into the Crimea. Chem. Nat. Compd. 35, 223-224. doi: 10.1007/BF02234944

Govindasamy, R., Simon, J., Puduri, V. S., Juliani, H. R., Asante-Dartey, J., Arthur, H., et al. (2007). Retailers and Wholesalers of African Herbal and Natural 
Products: Case Studies from Ghana and Rwanda. Issues New Crop. New Uses. Virginia ASHP, 332-337.

Greathouse, G. A. (1939). Alkaloids from Sanguinaria canadensis and their influence on growth of Phymatotrichum omnivorum. Plant Physiol. 14, 377. doi: $10.1104 /$ pp.14.2.377

Grieve, A. (1984). A Modern Herbal Penguin. Harmondsworth: Dover Publications Inc.

Grycová, L., Dostál, J., and Marek, R. (2007). Quaternary protoberberine alkaloids. Phytochemistry 68, 150-175. doi: 10.1016/j.phytochem.2006.10.004

Gu, Y., Zhang, Y., Shi, X., Li, X., Hong, J., Chen, J., et al. (2010). Effect of traditional Chinese medicine berberine on type 2 diabetes based on comprehensive metabonomics. Talanta 81, 766-772. doi: 10.1016/j.talanta.2010.01.015

Guoping, L., Jinhong, L., Shuai, H., Jian, C., and Zhongyi, Z. (2012). Optimization for ultrahigh pressure extraction of berberine from Cortex phellodendri by central composite design-response surface methodology. J. Med. Plants Res. 6, 3963-3970. doi: 10.5897/JMPR11.1092

Gupta, A. K., and Tandon, N. (2004). Rev. Indian Med. Plants, Vol 4. Delhi: ICMR.

Gurguel, L., de Costa, O. A., and da Silva, R. D. (1934). Berberis laurina. Anatomic, histologic and chemical study. Bol. Assoc. Bras. pharm. 15, 11-20.

Habtemariam, S. (2011). The therapeutic potential of Berberis darwinii stembark: quantification of berberine and in vitro evidence for Alzheimer's disease therapy. Nat. Prod. Commun. 6, 1089-1090.

Haisova, K., and Slavik, J. (1975). On the minor alkaloids from Argemone mexicana L. Collect Czech. Chem. Commun. 40, 1576-1578. doi: 10.1135/cccc19751576

Hakim, S. A., Mijovic, V., and Walker, J. (1961). Distribution of certain poppyfumaria alkaloids and a possible link with the incidence of glaucoma. Nature 189, 198-201. doi: 10.1038/189198a0

Hamayun, M., Khan, A., and Khan, M. A. (2003). Common medicinal folk recipes of District Buner, NWFP, Pakistan. Ethnobot. Leafl. 2003, 14.

Hamonniere, M., Leboeuf, A., and Paris, R. R. (1975). Alcaloïdes des annonacées: alcaloïdes de l'Enantia chlorantha. Plant. Med. Phytother. 9, 296-303.

Hartwell, J. L. (1982). Plants Used Against Cancer. Lawrence, MA: Quarterman Publications. Inc.

Hashmi, K., and Hafiz, A. (1986). In vivo antibacterial activity of Berberis asiatica. J. Pak. Med. Assoc. 36, 5.

Hayta, S., Polat, R., and Selvi, S. (2014). Traditional uses of medicinal plants in Elazig (Turkey). J. Ethnopharmacol. 154, 613-623. doi: 10.1016/j.jep.2014.04.026

He, J.-M., and Mu, Q. (2015). The medicinal uses of the genus Mahonia in traditional Chinese medicine: an ethnopharmacological, phytochemical and pharmacological review. J. Ethnopharmacol. 175, 668-683. doi: 10.1016/j.jep.2015.09.013

Henry, T. A. (1949). The Plant Alkaloids, 4th Edn. Philadelphia, PA: Blakiston.

Hirschhorn, H. H. (1981). Botanical remedies of South and Central America, and the Caribbean: an archival analysis. Part I. J. Ethnopharmacol. 4, 129-158. doi: 10.1016/0378-8741(81)90032-5

Houghton, P. J., and Manby, J. (1985). Medicinal plants of the Mapuche. J. Ethnopharmacol. 13, 89-103. doi: 10.1016/0378-8741(85)90063-7

Houghton, P. J., Ren, Y., and Howes, M.-J. (2006). Acetylcholinesterase inhibitors from plants and fungi. Nat. Prod. Rep. 23, 181-199. doi: 10.1039/b508966m

Hua, F., Ha, T., Ma, J., Li, Y., Kelley, J., Gao, X., et al. (2007). Protection against myocardial ischemia/reperfusion injury in TLR4-deficient mice is mediated through a phosphoinositide 3-kinase-dependent mechanism. J. Immunol. 178, 7317-7324. doi: 10.4049/jimmunol.178. 11.7317

Huq, M. E., and Ikram, M. (1968). Alkaloids of Berberis petiolaris. Sci. Res. 5, 75-76.

Hussain, K., Shahazad, A., and Zia-ul-Hussnain, S. (2008). An ethnobotanical survey of important wild medicinal plants of Hattar district Haripur, Pakistan. Ethnobot. Leafl. 2008, 5.

Hussaini, F. A., and Shoeb, A. (1985). Isoquinoline derived alkaloids from Berberis chitria. Phytochemistry 24, 633. doi: 10.1016/S0031-9422(00)80794-3

Hutchens, A. R. (1992). A Handbook of Native American Herbs: The Pocket Guide to 125 Medicinal Plants and Their Uses. Boston, MA: Shambhala Publications.

Imanshahidi, M., and Hosseinzadeh, H. (2008). Pharmacological and therapeutic effects of Berberis vulgaris and its active constituent, berberine. Phyther. Res. 22, 999-1012. doi: 10.1002/ptr.2399

Inbaraj, J. J., Kukielczak, B. M., Bilski, P., Sandvik, S. L., and Chignell, C. F. (2001). Photochemistry and photocytotoxicity of alkaloids from goldenseal
(Hydrastis canadensis L.) 1. Berberine. Chem. Res. Toxicol. 14, 1529-1534. doi: 10.1021/tx0155247

Irvine, F. R. (1961). Woody Plants of Ghana. London: Oxford University Press.

Ishola, I. O., Oreagba, I. A., Adeneye, A. A., Adirije, C., Oshikoya, K. A., and Ogunleye, O. O. (2014). Ethnopharmacological survey of herbal treatment of malaria in Lagos, Southwest Nigeria. J. Herb. Med. 4, 224-234. doi: 10.1016/j.hermed.2014.08.001

Israilov, I. A., and Yunusov, S. (1986). Alkaloids of four species of Argemone. Chem. Nat. Compd. 22, 189-192. doi: 10.1007/BF00598384

Jayaprakasam, R., and Ravi, T. K. (2014). Development and validation of HPTLC and RP-HPLC methods for the estimation of berberine in Coscinium fenestratum extract and its formulation. World J. Pharm. Res. 4, 206-218.

Jha, R. N., Pandey, M. B., Singh, A. K., Singh, S., and Singh, V. P. (2009). New alkaloids from Corydalis species. Nat. Prod. Res. 23, 250-255. doi: 10.1080/14786410801996390

Jin, C., and Shan, W. (1982). Quantitative determination of berberine in Coptis chinensis by TLC scanner method. Yaoxue Tongbao 17, 145-146.

Jiofack, T., Fokunang, C., Guedje, N., and Kemeuze, V. (2009). Ethnobotany and phytomedicine of the upper Nyong valley forest in Cameroon. Afr. J. Pharm. Pharmacol. 3, 144-150.

Jiofack, T., Fokunang, C., Kemeuze, V., Fongnzossie, E., Tsabang, N., Nkuinkeu, R., et al. (2008). Ethnobotany and phytopharmacopoea of the South-West ethnoecological region of Cameroon. J. Med. Plants Res. 2, 197-206.

Joshi, A. R., and Joshi, K. (2007). Ethnomedicinal plants used against skin diseases in some villages of Kali Gandaki, Bagmati and Tadi Likhu watersheds of Nepal. Ethnobot. Leafl. 2007, 27.

Joshi, H. R., and Kanaki, N. (2013). Quantitative analysis of berberine in an ayurvedic formulation-Rasayana churna by UV spectrophotometry. J. Pharm. Sci. Biosci. Res. 3, 32-34.

Jusiak, L. (1967). Separation of Chelidonium majus alkaloids by countercurrent cascade extraction. II. Acta Pol. Pharm. 24, 65-70.

Kadiri, A. B. (2008). Evaluation of medicinal herbal trade (Paraga) in Lagos State of Nigeria. Ethnobot. Leafl. 2008, 90.

Kala, C. P. (2006). Medicinal plants of the high altitude cold desert in India: diversity, distribution and traditional uses. Int. J. Biodivers. Sci. Manage. 2, 43-56. doi: $10.1080 / 17451590609618098$

Kamal, Y. T., Singh, M., Tamboli, E. T., Parveen, R., and Ahmad, S. (2011). Quantitative analysis of berberine in Berberis aristata fruits and in a traditional anti-inflammatory unani formulation by use of a validated HPLC method. Acta Chromatogr. 23, 157-168. doi: 10.1556/AChrom.21.2013.1.11

Kamigauchi, M., and Iwasa, K. (1994). "Corydalis spp.: in vitro culture and the biotransformation of protoberberines," in Medicinal and Aromatic Plants VI. Biotechnology in Agriculture and Forestry, Vol 26, ed Y. P. S. Bajaj (Berlin; Heidelberg: Springer), 93-105.

Karimov, A. (1993). Berberis alkaloids. Chem. Nat. Compd. 29, 415-438. doi: $10.1007 / \mathrm{BF} 00630564$

Karimov, A., and Lutfullin, K. L. (1986). Berberis alkaloids. 2'-Nmethylisotetrandrine from Berberis oblonga. Khimiya Prir. Soedin. 2, 249-251.

Karimov, A., Meliboev, S., Olimov, V., and Shakirov, R. (1993). Berberis alkaloids. XXX. Dynamics of alkaloid accumulation in Berberis integerrima and $B$. nummularia. Khimiya Prir. Soedin. 3, 472-473.

Karimov, A., and Shakirov, R. (1993). Berberis alkaloids. XX. Alkaloids of Berberis iliensis. Khimiya Prir. Soedin. 1, 83-84. doi: 10.1007/BF00631020

Kariyone, T., and Koiso, R. (1971). Atlas of Medicinal Plants. Osaka: Takeda Chemical Industries.

Kataoka, M., Tokuyama, E., Miyanaga, Y., and Uchida, T. (2008). The taste sensory evaluation of medicinal plants and Chinese medicines. Int. J. Pharm. 351, 36-44. doi: 10.1016/j.ijpharm.2007.09.017

Kaur, C., and Miani, S. (2001). Fruits and vegetables healthy foods for new millennium. Indian Hort. 45, 29-32.

Kayode, J. (2006). Conservation of indigenous medicinal botanicals in Ekiti State, Nigeria. J. Zhejiang Univ. Sci. B 7, 713-718. doi: 10.1631/jzus.2006.B0713

Khalmatov, K. (1964). Khalmatov, Wild-Growing Medicinal Plants of Uzbekistan [in Russian] Tashkent. Meditsina.

Khamidov, I., Faskhutdinov, M., Telezhenetskaya, M. V., Karimov, A., Levkovich, M. G., Abdullaev, N. D., et al. (1996a). Berberis alkaloids. XXXIV. Turcomanine, a new alkaloid from Berberis turcomanica. Khimiya Prir. Soedin. 1, 74-76. 
Khamidov, I., Karimov, A. K., Telezhenetskaya, M. V., and Tashkhodzhaev, B. (1996b). Berberis alkaloids. XXXV. Berberis turcomanica. Khimiya Prir. Soedin. $1,107-109$.

Khamidov, I. I., Aripova, S. F., Karimov, A., and Yusupov, M. M. (1997a). Berberis alkaloids. XL. An investigation of the alkaloids of Berberis thunbergii. Chem. Nat. Compd. 33, 599-599. doi: 10.1007/BF02254817

Khamidov, I. I., Aripova, S. F., and Karimov, A. K. (2003). Berberis alkaloids. XLI. Alkaloids from leaves of cultivated Berberis oblonga. Chem. Nat. Compd. 39, 407. doi: 10.1023/B:CONC.0000003429.41497.b6

Khamidov, I. I., Aripova, S. F., Telezhenetskaya, M. V., Karimov, A., and Dzhenberov, I. (1997b). Berberis alkaloids XXXIX. New alkaloids from B. densiflora. Chem. Nat. Comp. 33, 323-325. doi: 10.1007/BF02234886

Khamidov, I. I., Tashkhodzhaev, B., Aripova, S. F., Telezhenetskaya, M. V., and Karimov, A. K. (1996c). Berberis alkaloids. XXXVII. Study of the alkaloids of $B$. oblonga and B. integerrima. Crystal structure of 8trichloromethyldihydroberberine. Khimiya Prir. Soedin. 6, 889-893.

Khan, I., Najeebullah, S., Ali, M., and Shinwari, Z. K. (2016). Phytopharmacological and ethnomedicinal uses of the Genus Berberis (Berberidaceae): a review. Trop. J. Pharm. Res. 15, 2047-2057. doi: 10.4314/tjpr.v15i9.33

Khan, M. I., Sri Harsha, P. S. C., Giridhar, P., and Ravishankar, G. A. (2011). Berberine and lycopene profiling during the ontogeny of Tinospora cordifolia (Willd.) Miers ex Hook. F. \& Thoms fruit. Curr. Sci. 100, 1225-1231.

Khan, S. W., and Khatoon, S. (2007). Ethnobotanical studies on useful trees and shrubs of Haramosh and Bugrote valleys in Gilgit northern areas of Pakistan. Pak. J. Bot. 39, 699-710.

Khodzhimatov, M. (1989). Dikorastushchiye Lekarstvennuiye Rasteniya Tadzhikistana [Wild-Growing Medicinal Plants of Tadjikistan].

King, J. (1898). King's American Dispensatory. Cincinatti, OH: Ohio Valley Company.

Kirtikar, K., and Basu, B. (1933). Indian Medicinal Plants, I. Allahabad: Lalit Mohan Basu and Co.

Kirtikar, K. R., and Basu, B. D. (1998). Indian Medicinal Plants, Vol 1. Allahabad: CSIR publication.

Kiryakov, H. G., Daskalova, E., Georgieva, A., Kuzmanov, B., and Evstatieva, L. (1982a). Alkaloids from Corydalis solida (L.) Swarz. Folia Med. 24, 19-22.

Kiryakov, H. G., Iskrenova, E., Daskalova, E., Kuzmanov, B., and Evstatieva, L. (1982b). Alkaloids of Corydalis slivenensis. Planta Med. 44, 168-170. doi: 10.1055/s-2007-971432

Knapp, J. E., Hussein, F. T., Beal, J. L., Doskotch, R. W., and Tomimatsu, T. (1967). Isolation of two bisbenzylisoquinoline alkaloids from the rhizomes and roots of Xanthorhiza simplicissima. J. Pharm. Sci. 56, 139-141. doi: $10.1002 /$ jps.2600560129

Končić, M. Z., Kremer, D., Schühly, W., Brantner, A., Karlović, K., and Kalodera, Z. (2010). Chemical differentiation of Berberis croatica and B. vulgaris using HPLC fingerprinting. Croat. Chem. Acta 83, 451-456.

Kong, W. J., Wei, J., Zuo, Z. Y., Wang, Y. M., Song, D. Q., You, X. F., et al. (2008). Combination of simvastatin with berberine improves the lipid-lowering efficacy. Metabolism. 57, 1029-1037. doi: 10.1016/j.metabol.2008.01.037

Kong, Y., Xiao, J.-J., Meng, S.-C., Dong, X.-M., Ge, Y.-W., Wang, R.-F., et al. (2010). A new cytotoxic flavonoid from the fruit of Sinopodophyllum hexandrum. Fitoterapia 81, 367-370. doi: 10.1016/j.fitote.2009.11.003

Kosalec, I., Gregurek, B., Kremer, D., Zovko, M., Sanković, K., and Karlović, K. (2009). Croatian barberry (Berberis croatica Horvat): a new source of berberine? analysis and antimicrobial activity. World J. Microbiol. Biotechnol. 25, 145-150. doi: 10.1007/s11274-008-9860-x

Kosina, P., Gregorova, J., Gruz, J., Vacek, J., Kolar, M., Vogel, M., et al. (2010). Phytochemical and antimicrobial characterization of Macleaya cordata herb. Fitoterapia 81, 1006-1012. doi: 10.1016/j.fitote.2010.06.020

Kostalova, D., Brazdovicova, B., and Jin, H. Y. (1982). Alkaloids from the aboveground parts of Berberis koreana Palib. Farm. Obz. 51, 213-216.

Kubota, M., Katsunori, M., and Miyazawa, Y. (1980). Berberine contents in cultivated Coptis japonica Makino. Nagano-ken Eisei Kogai Kenkyusho Kenkyu Hokoku 2, 22-27.

Kukula-Koch, W., and Mroczek, T. (2015). Application of hydrostatic CCCTLC-HPLC-ESI-TOF-MS for the bioguided fractionation of anticholinesterase alkaloids from Argemone mexicana L. roots. Anal. Bioanal. Chem. 407, 2581-2589. doi: 10.1007/s00216-015-8468-x
Kulkarni, S. K., and Dhir, A. (2010). Berberine: a plant alkaloid with therapeutic potential for central nervous system disorders. Phyther. Res. 24, 317-324. doi: $10.1002 /$ ptr.2968

Kunwar, R. M., and Adhikari, N. (2005). Ethnomedicine of Dolpa district, Nepal: the plants, their vernacular names and uses. Lyonia 8, 43-49. doi: 10.1186/1746-4269-2-27

Küpeli, E., Koşar, M., Yeșilada, E., Hüsnü, K., and Başer, C. (2002). A comparative study on the anti-inflammatory, antinociceptive and antipyretic effects of isoquinoline alkaloids from the roots of Turkish Berberis species. Life Sci. 72, 645-657. doi: 10.1016/S0024-3205(02)02200-2

Ladino, O. J. P., and Suárez, L. E. C. (2010). Chemical constituents of the wood from Zanthoxylum quinduense Tul. (Rutaceae). Quim. Nova 33, 1019-1021. doi: 10.1590/S0100-40422010000500002

Launert, E. (1981). Edible and Medicinal Plants. London: Hamlyn.

Lee, H. Y., and Kim, C. W. (1999). Isolation and quantitative determination of berberine and coptisine from tubers of Corydalis ternata. Saengyak Hakhoechi $30,332-334$

Leone, M. G., Cometa, M. F., Palmery, M., and Saso, L. (1996). HPLC determination of the major alkaloids extracted from Hydrastis canadensis $\mathrm{L}$. Phyther. Res. 10, S45-S46.

Li, H., Li, X. L., Zhang, M., Xu, H., Wang, C. C., Wang, S., et al. (2014). Berberine ameliorates experimental autoimmune neuritis by suppressing both cellular and humoral immunity. Scand. J. Immunol. 79, 12-19. doi: 10.1111/sji.12123

Li, W. L., Zheng, H. C., Bukuru, J., and De Kimpe, N. (2004). Natural medicines used in the traditional Chinese medical system for therapy of diabetes mellitus. J. Ethnopharmacol. 92, 1-21. doi: 10.1016/j.jep.2003.12.031

Li, Y.-H., Zhang, M., Xiao, H.-T., Fu, H.-B., Ho, A., Lin, C.-Y., et al. (2015). Addition of berberine to 5 -aminosalicylic acid for treatment of dextran sulfate sodium-induced chronic colitis in C57BL/6 Mice. PLoS ONE 10:e0144101. doi: 10.1371/journal.pone.0144101

Liu, B., Li, W., Chang, Y., Dong, W., and Ni, L. (2006). Extraction of berberine from rhizome of Coptis chinensis Franch using supercritical fluid extraction. J. Pharm. Biomed. Anal. 41, 1056-1060. doi: 10.1016/j.jpba.2006.01.034

Liu, F., Li, Z., Shi, X., and Zhong, M. (2011). Determination of berberine, palmatine and jatrorrhizine in rabbit plasma by liquid chromatographyelectrospray ionization-mass spectrometry. J. Pharm. Biomed. Anal. 56, 1006-1015. doi: 10.1016/j.jpba.2011.08.001

Liu, J. (1992). Extraction of berbamine with water. Zhongguo Yaoxue Zazhi 27, 290-291

Liu, L., Liu, J., Huang, Z., Yu, X., Zhang, X., Dou, D., et al. (2015). Berberine improves endothelial function by inhibiting endoplasmic reticulum stress in the carotid arteries of spontaneously hypertensive rats. Biochem. Biophys. Res. Commun. 458, 796-801. doi: 10.1016/j.bbrc.2015.02.028

Liu, L., Wang, Z. B., Song, Y., Yang, J., Wu, L. J., Yang, B. Y., et al. (2016). Simultaneous determination of eight alkaloids in rat plasma by UHPLCMS/MS after oral administration of Coptis deltoidea C.Y. Cheng et Hsiao and Coptis chinensis Franch. Molecules 21, 1-15.

Liu, M., Su, X., Li, G., Zhao, G., and Zhao, L. (2015). Validated UPLCMS/MS method for simultaneous determination of simvastatin, simvastatin hydroxy acid and berberine in rat plasma: application to the drug-drug pharmacokinetic interaction study of simvastatin combined with berberine after oral administratio. J. Chromatogr. B Anal. Technol. Biomed. Life Sci. 1006, 8-15. doi: 10.1016/j.jchromb.2015.09.033

Liu, S., Chen, Y., Gu, L., Li, Y., Wang, B., Hao, J., et al. (2013). Effects of ultrahigh pressure extraction conditions on yields of berberine and palmatine from Cortex phellodendri amurensis. Anal. Methods 5, 4506. doi: 10.1039/c3ay40784e

Liu, W., Liu, P., Tao, S., Deng, Y., Li, X., Lan, T., et al. (2008b). Berberine inhibits aldose reductase and oxidative stress in rat mesangial cells cultured under high glucose. Arch. Biochem. Biophys. 475, 128-134. doi: 10.1016/j.abb.2008.04.022

Liu, W. H., Hei, Z. Q., Nie, H., Tang, F. T., Huang, H. Q., Li, X. J., et al. (2008a). Berberine ameliorates renal injury in streptozotocin-induced diabetic rats by suppression of both oxidative stress and aldose reductase. Chin. Med. J. 121, $706-712$.

Liu, X., Zhang, X., Ye, L., and Yuan, H. (2016). Protective mechanisms of berberine against experimental autoimmune myocarditis in a rat model. Biomed. Pharmacother. 79, 222-230. doi: 10.1016/j.biopha.2016.02.015

Lou, Y., Yuming, W., Yanfen, D., Jida, S., and Huang, L. (1982). Extractive spectrophotometric determination of berberine. Yaowu Fenxi Zazhi 2, 82-85. 
Lust, J. (2014). The Herb Book: The Most Complete Catalog of Herbs Ever Published. New York, NY: Courier Corporation.

Ma, X., Jiang, Y., Wu, A., Chen, X., Pi, R., Liu, M., et al. (2010). Berberine attenuates experimental autoimmune encephalomyelitis in C57 BL/6 mice. PLoS ONE 5:e13489. doi: 10.1371/journal.pone.0013489

Maithani, A., Parcha, V., and Kumar, D. (2014). Quantitative estimation of berberine content of Berberis asiatica from different altitude of Garhwal Himalaya. Asian J. Pharm. Clin. Res. 7, 165-167.

Majumder, B., Schindra, S. N., and Dutta, P. C. (1956). Occurrence of ceryl alcohol in Argemone mexicana. J. Indian Chem. Soc. 33, 351-352.

Manandhar, N. P. (2002). Plants and People of Nepal. Portland, OR: Timber Press.

Manske, R. H. F. (1939). The alkaloids of fumariaceous plants. XIX. Corydalis ophiocarpa Hook. f. et Thoms. Can. J. Res. Sect. B Chem. Sci. 17, 51-56. doi: $10.1139 /$ cjr39b-009

Marek, R., Seckárová, P., Hulová, D., Marek, J., Dostál, J., and Sklenár, V. (2003). Palmatine and berberine isolation artifacts. J. Nat. Prod. 66, 481-486. doi: $10.1021 / \mathrm{np} 0204996$

Martinez, M. (1984). Las Plantas Medicinales De México, 3rd Edn. Mexico City: CIESAS, Cuadernos de la Casa Chata.

Martinez, O. E. (1977). Flora de Veracruz, Fascículo 77. Riverside, CA: University of California.

Mascareno, E., El-Shafei, M., Maulik, N., Sato, M., Guo, Y., Das, D. K., et al. (2001). JAK/STAT signaling is associated with cardiac dysfunction during ischemia and reperfusion. Circulation 104, 325-329. doi: 10.1161/01.CIR.104.3.325

Meena, A. K., Bansal, P., and Kumar, S. (2009). Plants-herbal wealth as a potential source of ayurvedic drugs. Asian J. Tradit. Med. 4, 152-170.

Mell, C. D. (1929). Interesting sources of natural dyestuffs. Color 51, 619-820.

Mikage, M., and Mouri, C. (1999). Pharmacognostical studies of Berberis plants (Berberidaceae) from Nepal (1). Altitudinal, interspecific, and partial variations of berberine content in the barks. Sect. Title Pharm. 53, 249-254.

Mills, S. (1985). The Dictionary of Modern Herbalism: A Comprehensive Guide to Practical Herbal Therapy. Wellingborough: Inner Traditions/Bear \& Co.

Minaiyan, M., Ghannadi, A., Mahzouni, P., and Jaffari-Shirazi, E. (2011). Comparative study of Berberis vulgaris fruit extract and berberine chloride effects on acetic acid-induced colitis in rats. Iran. J. Pharm. Res. 10, 97-104.

Misra, P. S., Bhakuni, D. S., Sharma, V. N., and Kaul, K. N. (1961). Chemical constituents of Argemone mexicana. J. Sci. Ind. Res. 20, 186.

Moerman, D. E. (1998). Native American Ethnobotany. Portland, OR: Timber Press.

Mokgadi, J., Turner, C., and Torto, N. (2013). Pressurized hot water extraction of alkaloids in Goldenseal. Am. J. Anal. Chem. 4, 398-403. doi: 10.4236/ajac.2013.48050

Mølgaard, P., Holler, J. G., Asar, B., Liberna, I., Rosenbæk, L. B., Jebjerg, C. P., et al. (2011). Antimicrobial evaluation of Huilliche plant medicine used to treat wounds. J. Ethnopharmacol. 138, 219-227. doi: 10.1016/j.jep.2011.09.006

Monforte-Gonzalez, M., Cecilia, G. G., Jorge, R. P., Mildred, C. P., and VazquezFlota, F. (2012). Berberine and sanguinarine quantitation in Argemone mexicana L. (Papaveraceae) tissues by TLC-in situ fluorography. J. Planar Chromatogr. TLC 24, 358-360. doi: 10.1556/JPC.25.2012.4.14

Montes, M., and Wilkomirsky, T. (1987). Medicina Tradicional Chilena. Concepción: Editiorial de la Universidad de Concepción.

Muñoz, O. (2001). Plantas Medicinales de uso en Chile: Química y Farmacología. Editorial Universitaria.

Mustafa, A., and Turner, C. (2011). Pressurized liquid extraction as a green approach in food and herbal plants extraction: a review. Anal. Chim. Acta 703, 8-18. doi: 10.1016/j.aca.2011.07.018

Musumeci, R., Speciale, A., Costanzo, R., Annino, A., Ragusa, S., Rapisarda, A., et al. (2003). Berberis aetnensis C. Presl. extracts: antimicrobial properties and interaction with ciprofloxacin. Int. J. Antimicrob. Agents 22, 48-53. doi: 10.1016/S0924-8579(03)00085-2

Musuyu Muganza, D., Fruth, B. I., Nzunzu Lami J., Mesia, G. K., Kambu, O. K., Tona, G. L., et al. (2012). In vitro antiprotozoal and cytotoxic activity of 33 ethonopharmacologically selected medicinal plants from Democratic Republic of Congo. J. Ethnopharmacol. 141, 301-308. doi: 10.1016/j.jep.2012.02.035

Ndenecho, E. N. (2009). Herbalism and resources for the development of ethnopharmacology in Mount Cameroon region. Afr. J. Pharm. Pharmacol. 3, 78-86.
Neuwinger, H. D. (1996). African Ethnobotany: Poisons and Drugs: Chemistry, Pharmacology, Toxicology. London: CRC Press.

Ngono Ngane, R., Koanga Mogtomo, M., Tchinda Tiabou, A., Magnifouet Nana, H., Motso Chieffo, P. R., Mballa Bounou, Z., et al. (2011). Ethnobotanical survey of some Cameroonian plants used for treatment of viral diseases. Afr. J. Plant Sci. 5, 15-21.

Nguimatsia, F., Boustie, J., Baril, F., Amoros, M., and Girre, L. (1998). Les medicaments des pygmees Baka du Cameroun: moeurs therapeutiques, maladies et inventaire des plantes medicinales. Fitoterapia 69, 29-40.

Noumi, E. (2010). Ethno medicines used for treatment of prostatic disease in Foumban, Cameroon. Afr. J. Pharm. Pharmacol. 4, 793-805.

Noumi, E., and Anguessin, B. (2010). Insecticides and ethnomedicine of HIV/AIDS at Tokombere (Far North Cameroon). Int. J. Pharm. Biomed. Sci. 2, 20-28.

Noumi, E., and Yumdinguetmun, R. (2010). Plants and treatment of prostatic diseases in Foumban (West Region, Cameroon). Syllab. Rev. 2, 9-16.

Odugbemi, T. O., Akinsulire, O. R., Aibinu, I. E., and Fabeku, P. O. (2007). Medicinal plants useful for malaria therapy in Okeigbo, Ondo State, Southwest Nigeria. Afr. J. Tradit. Complement. Altern. Med. 4, 191-198.

Ogbonna, D. N., Sokari, T. G., and Agomuoh, A. A. (2008). Antimalarial activities of some selected traditional herbs from Southeastern Nigeria against Plasmodium species. Res. J. Parasitol. 3, 25-31. doi: 10.3923/jp.2008.25.31

Ohemu, T. L., Agunu, A., Olotu, P. N., Ajima, U., Dafam, D. G., and Azila, J. J. (2014). Ethnobotanical survey of medical plants used in the traditional treatment of viral infections in Jos, plateau state, Nigeria. Int. J. Med. Aromat. Plants 4, 74-81.

Okunade, A. L., Hufford, C. D., Richardson, M. D., Peterson, J. R., and Clar, A. M. (1994). Antimicrobial Properties of Alkaloids from Xanthorhiza simplicissima. J. Pharm. Sci. 83, 404-406. doi: 10.1002/jps.2600830327

Oladunmoye, M. K., and Kehinde, F. Y. (2011). Ethnobotanical survey of medicinal plants used in treating viral infections among Yoruba tribe of South Western Nigeria. Afr. J. Microbiol. Res. 5, 2991-3004. doi: 10.5897/AJMR10.004

Oliver, B. E. P. (1960). Medicinal Plants in Nigeria: Being a Course of Four Lectures. Pharmacy Department of the Nigerian College of Arts, Science and Technology, Ibadan.

Olowokudejo, J. D., Kadiri, A. B., and Travih, V. A. (2008). An ethnobotanical survey of herbal markets and medicinal plants in Lagos State of Nigeria. Ethnobot. Leafl. 2008, 116.

Onwuanibe, R. C. (1979). The philosophy of African medical practice. Afr. Issues 9, 25-28. doi: 10.2307/1166259

Orhan, I., Sener, B., Choudhary, M. I., and Khalid, A. (2004). Acetylcholinesterase and butyrylcholinesterase inhibitory activity of some Turkish medicinal plants. J. Ethnopharmacol. 91, 57-60. doi: 10.1016/j.jep.2003.11.016

Othman, M. S., Safwat, G., Aboulkhair, M., and Abdel Moneim, A. E. (2014). The potential effect of berberine in mercury-induced hepatorenal toxicity in albino rats. Food Chem. Toxicol. 69, 175-181. doi: 10.1016/j.fct.2014.04.012

Pak, V. (2005). Medicine plants of folk medicine used for treatment of gastrointestinal problems in Fergana valley. Korean Food Res. Inst. 18, 150-157.

Pant, N., Garg, H. S., and Bhakuni, K. (1986). Chemical constituents of B. pseudoumbellata. Fitoterapia 51, 427-428.

Park, D. W., Jiang, S., Liu, Y., Siegal, G. P., Inoki, K., Abraham, E., et al. (2014). GSK3 $\beta$-Dependent inhibition of AMPK potentiates activation of neutrophils and macrophages and enhances severity of acute lung injury. Am. J. Physiol. 307, L735-L745. doi: 10.1152/ajplung.00165.2014

Park, J.-I., Shim, J.-K., Do, J.-W., Kim, S.-Y., Seo, E.-K., Kwon, H.-J., et al. (1999). Immune-stimulating properties of polysaccharides from Phellodendri cortex (Hwangbek). Glycoconj. J. 16, 247-252. doi: 10.1023/A:10070845 06071

Parsons, H. B. (1882). Examination of the root of Berberis aquifolium, v. alpens, "oregon grape root." Pharm. J. 13, 46-48.

Patel, M. C. (2013). Isolation of berberine from Berberis aristata by an acid dye method and optimization of parameters. Int. J. Pharm. Sci. Rev. Res. 20, 187-189.

Pathak, N. K. R., Biswas, M., Seth, K. K., Dwivedi, S. P. D., and Pandey, V. B. (1985). Chemical investigation of Argemone mexicana. Pharmazie 40, 202.

Pěnčíková, K., Urbanová, J., Musil, P., Táborská, E., and Gregorová, J. (2011). Seasonal Variation of Bioactive Alkaloid Contents in Macleaya 
microcarpa (Maxim.) Fedde. Molecules 16, 3391-3401. doi: 10.3390/ molecules16043391

Perkin, A. G., and Hummel, J. J. (1895). XLV.-The colouring principle of Toddalia aculeata and Evodia meliaefolia. J. Chem. Soc. Trans. 67, 413-416. doi: 10.1039/CT8956700413

Pesman, M. W. (1962). Meet Flora Mexicana. Globe, AZ: D.S. King.

Petcu, P. (1965a). Der gehalt an alkaloiden und vitamin C in Berberis guimpelii. Planta Med. 13, 178-181. doi: 10.1055/s-0028-1100108

Petcu, P. (1965b). Phytochemical investigation of Berberis koreana. Farm. Bucharest, Rom. 13, 21-28.

Pfoze, N. L., Myrboh, B., Kumar, Y., and Rohman, R. (2014). Isolation of protoberberine alkaloids from stem bark of Mahonia manipurensis Takeda using RP-HPLC. J. Med. Plants Stud. 2, 48-57.

Phillips, R., and Foy, N. (1990). Herbs. London: Pan Books Ltd.

Phillips, R., and Rix, M. (1991). Perennials Vol. 1 and 2. London: Pan Books Ltd.

Phillipson, J. D., Gray, A. I., Askari, A. A. R., and Khalil, A. A. (1981). Alkaloids From Iraqi Species of Papaveraceae. J. Nat. Prod. 44, 296-307. doi: $10.1021 / \mathrm{np} 50015 \mathrm{a} 011$

Phondani, P. C., Maikhuri, R. K., Rawat, L. S., Farooquee, N. A., Kala, C. P., Vishvakarma, S. C. R., et al. (2010). Ethnobotanical uses of plants among the Bhotiya tribal communities of Niti Valley in Central Himalaya, India. Ethnobot. Res. Appl. 8, 233-244. doi: 10.17348/era.8.0.233-244

Pilch, W., Szygula, Z., Tyka, A. K., Palka, T., Tyka, A., Cison, T., et al. (2014). Disturbances in pro-oxidant-antioxidant balance after passive body overheating and after exercise in elevated ambient temperatures in athletes and untrained men. PLoS ONE 9:e85320. doi: 10.1371/journal.pone. 0085320

Rahal, A., Kumar, A., Singh, V., Yadav, B., Tiwari, R., Chakraborty, S., et al. (2014). Oxidative stress, prooxidants, and antioxidants: The interplay. Biomed Res. Int. (2014). doi: 10.1155/2014/761264

Rajan, S., and Sethuraman, M. (1992). Mahonia leschenaultii-a toda plant. Anc. Sci. Life 12, 242.

Rajasekaran, A., and Kumar, N. (2009). Rasont - A traditional crude drug prepared from Berberis sp and its uses. Indian, J. Tradit. Knowl. 8, 562-563.

Ransohoff, R. M., Hafler, D. A., and Lucchinetti, C. F. (2015). Multiple sclerosis - a quiet revolution. Nat. Rev. Neurol. 11, 134-142. doi: 10.1038/nrneurol.2015.14

Rashid, M. H., and Malik, M. N. (1972). Composition of alkaloids in some Berberis species. Pakistan J. For. 22, 43-47.

Rashmi, R. A., Pokhriyal, R., and Singh, Y. (2009). Quantitative Estimation of Berberine in Roots of Different provenances of Berberis aristata DC by HPLC and Study of their Antifungal Properties. Pharmacogn. Mag. 5, 355-358. doi: $10.4103 / 0973-1296.58566$

Richert, F. (1918). The extraction of berberine from "michai" (Berberis darwinii) and "calafate" (B. vuxifolia), in the Argentine. Rev. del Cent. Estud. Agron. y Vet. la Univ. Buenos Aires 11, 11-13.

Ritch-Krc, E. M., Thomas, S., Turner, N. J., and Towers, G. H. N. (1996). Carrier herbal medicine: traditional and contemporary plant use. J. Ethnopharmacol. 52, 85-94. doi: 10.1016/0378-8741(96)01392-X

Rivera Núñez, D., and Obon de Castro, C. (1996). Ethnopharmacology of Murcia, Actes du $2^{a}$ Colloque Européen d'Ethnopharmacologei et de la $11^{a}$ Conférence internationale d'Ethnomédecine (Heidelberg), 24.

Rojsanga, P., and Gritsanapan, W. (2005). Variation of Berberine Content in Coscinium fenestratum Stem in Thailand Market. Mahidol Univ. J. Pharm. Sci. 32, 66-70.

Rojsanga, P., Gritsanapan, W., and Suntornsuk, L. (2006). Determination of berberine content in the stem extracts of Coscinium fenestratum by TLC densitometry. Med. Princ. Pract. 15, 373-378. doi: 10.1159/000094272

Samal, P. K. (2013). HPTLC analysis of berberine in Argemone mexicana, L. J. Glob. Trends Pharm. Sci. 4, 1073-1076.

Samhita, S. (1963). Sutrasthanam Lakshadi Group. Ed K. K. Bhishagratna. Varanasi: Chaukhamba Sanskrit Sansthan.

San Martín, J. (1983). Medicinal plants in central Chile. Econ. Bot. 37, 216-227. doi: $10.1007 / \mathrm{BF} 02858788$

Sandberg, F. (1965). Etude sur les plantes medicinales et toxiques d'Afrique equatoriale. 1. Premier inventaire des plantes medicinales et toxiques de la region sudouest de la Republique Centrafricaine et de la region nord de la Republique du Congo/Brazzaville. Cah. la Maboké 3, 5-49.
Santos, A. C., and Adkilen, P. (1932). The alkaloids of Argemone mexicana. J. Am. Chem. Soc. 54, 2923-2924. doi: 10.1021/ja01346a037

Santra, D. K., and Saoji, A. N. (1971). Phytochemical study of Argemone mexicana latex. Curr. Sci. 40, 548-549.

Saraf, G., Mitra, A., Kumar, D., Mukherjee, S., and Basu, A. (2010). Role of nonconventional remedies in rural India. Int. J. Pharm. Life Sci. 1, 141-159.

Sasidharan, S., Chen, Y., Saravanan, D., Sundram, K. M., and Yoga Latha, L. (2011). Extraction, isolation and characterization of bioactive compounds from plants' extracts. Afr. J. Tradit. Complement. Altern. Med. 8, 1-10.

Sati, S. C., and Joshi, S. (2011). Aspects of antifungal potential of ethnobotanically known medicinal plants. Res. J. Med. Plants 5, 377-391. doi: 10.3923/rjmp.2011.377.391

Satija, S., Bansal, P., Dureja, H., and Garg, M. (2015). Microwave assisted extraction of Tinospora cordifolia and optimization through central composite design. J. Biol. Sci. 15, 106-115. doi: 10.3923/jbs.2015.106.115

Sato, F., and Yamada, Y. (1984). High berberine-producing cultures of Coptis japonica cells. Phytochemistry 23, 281-285. doi: 10.1016/S0031-9422(00)80318-0

Satyavati, G. V., Raina, M. K., and Sharma, M. (1987). Medicinal plants of India. New Delhi: Indian Council of Medical Research.

Schieffer, G. W., and Pfeiffer, K. (2001). Pressurized liquid extraction and multiple, ultrasonically-assisted extraction of hydrastine and berberine from Goldenseal (Hydrastis canadensis) with susequent HPLC assay. J. Liq. Chromatogr. Relat. Technol. 24, 2415-2427. doi: 10.1081/JLC-100105948

Schlotterbeck, J. O. (1902). Does Argemone mexicana contain morphine? J. Am. Chem. Soc. 24, 238-242. doi: 10.1021/ja02017a006

Seino, Y., Fukushima, M., and Yabe, D. (2010). GIP and GLP-1, the two incretin hormones: similarities and differences. J. Diabetes Investig. 1, 8-23. doi: 10.1111/j.2040-1124.2010.00022.x

Sener, B., and Temizer, H. (1988). Pharmacognosic investigations on Corydalis solida (L.) Swartz ssp. brachyloba (Boiss.) Cullen \& Davis. II. Alkaloids of Corydalis solida (L.) Swartz ssp. brachyloba (Boiss.) Cullen \& Davis. Gazi Univ. Eczac. Fak. Derg. 5, 9-11.

Sener, B., and Temizer, H. (1990). Chemical Studies on the Alkaloids from Corydalis solida subsp. tauricola. Planta Med. 56, 510-510. doi: $10.1055 / \mathrm{s}-2006-961052$

Sener, B., and Temizer, H. (1991). Chemical studies on the minor isoquinoline alkaloids from Corydalis solida subsp. brachyloba. J. Chem. Soc. Pakistan 13, 63-66.

Sezik, E., Yesilada, E., Shadidoyatov, H., Kulivey, Z., Nigmatullaev, A. M., Aripov, H. N., et al. (2004). Folk medicine in Uzbekistan: I. Toshkent, Djizzax, and Samarqand provinces. J. Ethnopharmacol. 92, 197-207. doi: 10.1016/j.jep.2004.02.016

Shah, G. M., and Khan, M. A. (2006). Common medicinal folk recipes of Siran valley, Mansehra, Pakistan. Ethnobot. Leafl. 2006, 5.

Shahid, M., Rahim, T., Shahzad, A., Latif, T. A., Fatma, T., Rashid, M., et al. (2009). Ethnobotanical studies on Berberis aristata DC. root extracts. African, J. Biotechnol. 8, 556-563.

Sharma, P. K., Chauhan, N. S., and Lal, B. (2005). Studies on plant associated indigenous knowledge among Malanis of Kullu district, Himachal Pradesh. Indian J. Trad. Knowl. 4, 403-408.

Shigwan, H., Saklani, A., Hamrapurkar, P. D., Mane, T., and Bhatt, P. (2013). HPLC method development and validation for quantification of berberine from Berberis aristata and Berberis tinctoria. Int. J. Appl. Sci. Eng. 11, 203-211.

Shirwaikar, A., Shirwaikar, A., Rajendran, K., and Punitha, I. S. R. (2006). In vitro antioxidant studies on the benzyl tetra isoquinoline alkaloid berberine. Biol. Pharm. Bull. 29, 1906-1910. doi: 10.1248/bpb.29.1906

Singh, A., Duggal, S., Kaur, N., and Singh, J. (2010). Berberine: Alkaloid with wide spectrum of pharmacological activities. J. Nat. Prod. 3, 64-75.

Singh, A., Lal, M., and Samant, S. S. (2009). Diversity, indigenous uses and conservation prioritization of medicinal plants in Lahaul valley, proposed Cold Desert Biosphere Reserve, India. Int. J. Biodivers. Sci. Manag. 5, 132-154. doi: 10.1080/17451590903230249

Singh, I. P., and Mahajan, S. (2013). Berberine and its derivatives: a patent review (2009-2012). Expert Opin. Ther. Pat. 23, 215-231. doi: $10.1517 / 13543776.2013 .746314$ 
Singh, J., and Kakkar, P. (2009). Antihyperglycemic and antioxidant effect of Berberis aristata root extract and its role in regulating carbohydrate metabolism in diabetic rats. J. Ethnopharmacol. 123, 22-26. doi: 10.1016/j.jep.2009.02.038

Singh, R., Katiyar, C., and Pasrija, A. (2010). Validated HPLC-UV method for the determination of berberine in raw herb Daruharidra (Berberis aristata DC), its extract, and in commercially marketed ayurvedic dosage forms. Int. J. Ayurveda Res. 1, 243. doi: 10.4103/0974-7788.76789

Singh, R., Tiwari, S. S., Srivastava, S., and Rawat, A. K. S. (2012). Botanical and phytochemical studies on roots of Berberis umbellata Wall. ex G. Don. Indian J. Nat. Prod. Resour. 3, 55-60.

Singh, S. (2014). Quantitative analysis of Berberine in Argemone mexicana Linn. (Papaveraceae) using HPLC and HPTLC. Adv. Plant Sci. 27, 209-211.

Singh, S. S., Pandey, S. C., Srivastava, S., Gupta, V. S., Patro, B., and Ghosh, A. C. (2003). Chemistry and medicinal properties of Tinospora cordifolia (Guduchi). Indian J. Pharmacol. 35, 83-91.

Sir, C. C., and Chopra, I. C. (1958). Indigenous Drugs of India. Kolkata: U.N.Dhar and Sons Private Limted.

Slavík, J. (1978). Characterization of alkaloids from the roots of Papaver rhoeas L. Collect. Czechoslov. Chem. Commun. 43, 316-319. doi: 10.1135/cccc19780316

Slavik, J., and Slavikova, L. (1957). Alkaloide der mohngewächse (Papaveraceae) VIII. Die alkaloide des roten hornmohns (Glaucium corniculatum CURT.). Collect. Czechoslov. Chem. Commun. 22, 279-285. doi: 10.1135/cccc19570279

Slavik, J., and Slavikova, L. (1975). Alkaloids of Papaveraceae. LIX. Alkaloids from the leaves of Bocconia frutescens. Collect. Czechoslov. Chem. Commun. 40, 3206-3210. doi: 10.1135/cccc19753206

Slavik, J., Slavikova, L., and Bochorakova, J. (1989). Alkaloids of the Papaveraceae. Part LXXXVIII. Alkaloids from Papaver rhoeas var. chelidonioides O. Kuntze, P. confine Jord., and P. dubium L. Collect. Czechoslov. Chem. Commun. 54, 1118-1125. doi: 10.1135/cccc19891118

Slavikova, L., and Slavik, J. (1955). Alkaloids of Papaveraceae. VII. Argemone mexicana. Chem. List. Pro Vedu a Prum. 49, 1546-1549.

Slavikova, L., and Slavik, J. (1966). Alkaloide der mohngewächse (Papaveraceae) XXXII. Über die alkaloide aus Hunnemannia fumariaefolia SWEET und über die konstitution des alkaloids HF 1. Collect. Czechoslov. Chem. Commun. 31, 1355-1362. doi: 10.1135/cccc19661355

Slavikova, L., Tschu, S., and Slavik, J. (1960). Alkaloids of Papaveraceae. XIV. Alkaloids of Argemone alba. Collect. Czechoslov. Chem. Commun. 25, 756-760. doi: $10.1135 / \operatorname{cccc} 19600756$

Smyth, B. B. (1903). Preliminary list of medicinal and economic kansas plants, with their reputed therapeutic properties. Trans. Kansas Acad. Sci. 18, 191-209. doi: $10.2307 / 3624794$

Sood, P., Modgil, R., and Sood, M. (2010). Physico-chemical and nutritional evaluation of indigenous wild fruit Kasmal, Berberis lycium Royle. Indian J. Nat. Prod. Resour. 1, 362-366.

Srinivasan, G. V., Unnikrishnan, K. P., Rema Shree, A. B., and Balachandran, I. (2008). HPLC estimation of berberine in Tinospora cordifolia and Tinospora sinensis. Indian J. Pharm. Sci. 70, 96-99. doi: 10.4103/0250-474X. 40341

Srivastava, S. K., Rai, V., Srivastava, M., Rawat, A. K. S., and Mehrotra, S. (2006a). Estimation of heavy metals in different Berberis species and its market samples. Environ. Monit. Assess. 116, 315-320. doi: 10.1007/s10661-006$7395-\mathrm{x}$

Srivastava, S. K., Rawat, A. K. S., Manjoosha, S., and Mehrotra, S. (2006c). Pharmacognostic Evaluation of the Roots of Berberis chitria Lindl. Nat. Prod. Sci. 12, 19-23.

Srivastava, S. K., Rawat, A. K. S., and Srivastava, M. (2006b). Pharmacognostic evaluation of the roots of Berberis chitria. Nat. Prod. Sci. 12, 19-23.

Srivastava, S. K., Sayyada, K., Singh Rawat, A. K., Mehrotra, S. (2001). Pharmacognostic evaluation of the root of Berberis aristata DC. Nat. Prod. Sci. 7, 102-106.

Srivastava, S. K., Singh Rawat, A. K., and Mehrotra, S. (2004). Pharmacognostic evaluation of the root of Berberis asiatica. Pharm. Biol. 42, 467-473. doi: $10.1080 / 13880200490886256$

Srivastava, S. K.., and Rawat, A. K. S. (2007). Pharmacognostic evaluation of the roots of Berberis tinctoria Lesch. Nat. Prod. Sci. 13, 27-32.

Steffens, P., Nagakura, N., and Zenk, M. H. (1985). Purification and characterization of the berberine bridge enzyme from Berberis beaniana cell cultures. Phytochemistry 24, 2577-2583. doi: 10.1016/S0031-9422(00)80672-X
Stermitz, F. (1967). Alkaloids of the Papaveraceae. V. Muramine and berberine from Argemone squarrosa. J. Pharm. Sci. 55, 760-762. doi: $10.1002 /$ jps. 2600560624

Stermitz, F. R., Lorenz, P., Tawara, J. N., Zenewicz, L. A., and Lewis, K. (2000). Synergy in a medicinal plant: antimicrobial action of berberine potentiated by 5 '-methoxyhydnocarpin, a multidrug pump inhibitor. Proc. Natl. Acad. Sci. U.S.A. 97, 1433-1437. doi: 10.1073/pnas.030540597

Stermitz, F. R., and Sharifi, I. A. (1977). Alkaloids of Zanthoxylum monophyllum and Z. punctatum. Phytochemistry 16, 2003-2006. doi: 10.1016/0031-9422(77)80113-1

Stermitz, F. R., Stermitz, J. R., Zanoni, T. A., and Gillespie, J. (1974). Alkaloids of Argemone subintegrifolia and A. munita. Phytochemistry 13, 1151-1153. doi: 10.1016/0031-9422(74)80089-0

Stuart, G. A., and Smith, F. P. (1977). Chinese Materia Medica: Vegetable Kingdom. Shanghai: Gordon Press Publishers.

Taborska, E., Frantisek, V., and Slavik, J. (1980). Alkaloids of the Papaveraceae. LXXI. Alkaloids from Bocconia frutescens L. Collect. Czechoslov. Chem. Commun. 45, 1301-1304. doi: 10.1135/cccc19801301

Tadzhibaev, M. M., Zatorskaya, I. N., Lutfullin, K. L., and Shakirov, T. T. (1974). Isolation of berberine. Khimiya Prir. Soedin. 10, 48-50. doi: $10.1007 / \mathrm{BF} 00568218$

Tan, E., Luo, S., Lin, S., Tan, R., Yu, W., Yi, Z., et al. (2013). Determination of five active ingredient in Phellodendron chinensis var. glabiusculum and $P$. chinense by HPLC. Zhongguo Shiyan Fangjixue Zazhi 19, 135-139.

Tang, J., Feng, Y., Tsao, S., Wang, N., Curtain, R., and Wang, Y. (2009). Berberine and Coptidis Rhizoma as novel antineoplastic agents: a review of traditional use and biomedical investigations. J. Ethnopharmacol. 126, 5-17. doi: $10.1016 /$ j.jep.2009.08.009

Tang, W., and Eisenbrand, G. (1992). "Corydalis turtschaninovii Bess. f. yanhusuo YH Chou et CC Hsü," in Chinese Drugs of Plant Origin (Berlin; Heidelberg: Springer), 377-393.

Tantaquidgeon, G. (1928). Mohegan medicinal practices, weather-lore and superstitions. SI-BAE Annu. Rep. 43, 264-270.

Teng, H., and Choi, O. (2013). Optimum extraction of bioactive alkaloid compounds from Rhizome coptidis (Coptis chinensis Franch.) using response surface methodology. Solvent Extr. Res. Dev. 20, 91-104. doi: $10.15261 /$ serdj.20.91

Thirupurasundari, C. J., Padmini, R., and Devaraj, S. N. (2009). Effect of berberine on the antioxidant status, ultrastructural modifications and protein bound carbohydrates in azoxymethane-induced colon cancer in rats. Chem. Biol. Interact. 177, 190-195. doi: 10.1016/j.cbi.2008. 09.027

Tiwari, K. P., and Masood, M. (1979). Chemical constituents of Berberis coriaria Royle. J. Indian Chem. Soc. 56, 310-311.

Tiwary, J. K., Ballabha, R., and Tiwari, P. (2010). Ethnopaediatrics in Garhwal Himalaya. Uttarakhand, India (Psychomedicine Medice). NY Sci. J. 3, 123-126.

Tomè, F., and Colombo, M. L. (1995). Distribution of alkaloids in Chelidonium majus and factors affecting their accumulation. Phytochemistry 40, 37-39. doi: 10.1016/0031-9422(95)00055-C

Tomita, M., and Kugo, T. (1956). Alkaloids of Berberidaceous plants - XIX: Alkaloids of B. tschonoskyana I. Isolation of bases. Yakugak Zasshi 79, 317-321. doi: 10.1248/yakushi1947.79.3_317

Torres, R., Villarroel, L., Urzua, A., and Fajardo, V. (1992). Constituents of Berberis congestiflora and Berberis horrida. Fitoterapia 63:376.

Tsabang, N., Fokou, P. V. T., Tchokouaha, L. R. Y., Noguem, B., BakarngaVia, I., Nguepi, M. S. D., et al. (2012). Ethnopharmacological survey of Annonaceae medicinal plants used to treat malaria in four areas of Cameroon. J. Ethnopharmacol. 139, 171-180. doi: 10.1016/j.jep.2011. 10.035

Uchiyama, T., Kamikawa, H., and Ogita, Z. (1989). Anti-ulcer effect of extract from Phellodendri cortex. Yakugaku zasshi J. Pharm. Soc. Japan 109, 672-676. doi: 10.1248/yakushi1947.109.9_672

ul Haq, I., and Hussain, M. (1993). Medicinal plants of Mansehra. Hamdard Med. $36,63-100$.

Uniyal, S. K., Singh, K. N., Jamwal, P., and Lal, B. (2006). Traditional use of medicinal plants among the tribal communities of Chhota Bhangal, Western Himalaya. J. Ethnobiol. Ethnomed. 2:14. doi: 10.1186/1746-4269-2-14

Uphof, J. C. (1959). Dictionary of Economic Plants, 2nd edn. Lehre. 
Uprety, Y., Asselin, H., Boon, E. K., Yadav, S., and Shrestha, K. K. (2010). Indigenous use and bio-efficacy of medicinal plants in the Rasuwa District, Central Nepal. J. Ethnobiol. Ethnomed. 6:3. doi: 10.1186/1746-4269-6-3

Urzúa, A., Torres, R., Villarroel, L., and Fajardo, V. (1984). Secondary metabolites of Berberis darwinii. Rev. Latinoam. Quim. 15, 27-29.

Usher, G. (1974). A Dictionary of Plants Used by Man. London: Constable and Company Ltd.

Vennerstrom, J. L., and Klayman, D. L. (1988). Protoberberine alkaloids as antimalarials. J. Med. Chem. 31, 1084-1087. doi: 10.1021/jm00401a006

Vennerstrom, J. L., Lovelace, J. K., Waits, V. B., Hanson, W. L., and Klayman, D. L. (1990). Berberine derivatives as antileishmanial drugs. Antimicrob. Agents Chemother. 34, 918-921. doi: 10.1128/AAC.34.5.918

Versteegh, C. P. C., and Sosef, M. S. M. (2007). Revision of the African genus Annickia (Annonaceae). Syst. Geogr. Plants 77, 91-118.

Vuddanda, P. R., Chakraborty, S., and Singh, S. (2010). Berberine: a potential phytochemical with multispectrum therapeutic activities. Expert Opin. Investig. Drugs 19, 1297-1307. doi: 10.1517/13543784.2010.517745

Wang, C., Li, J., Lv, X., Zhang, M., Song, Y., Chen, L., et al. (2009). Ameliorative effect of berberine on endothelial dysfunction in diabetic rats induced by high-fat diet and streptozotocin. Eur. J. Pharmacol. 620, 131-137. doi: 10.1016/j.ejphar.2009.07.027

Wang, W., Shen, Q., Liang, H., Hua, C., Liu, Y., Li, F., et al. (2016). Pharmacokinetic studies of novel berberine derivatives with ultra-performance liquid chromatography-tandem mass spectrometry. J. Chromatogr. B Anal. Technol. Biomed. Life Sci. 1031, 172-180. doi: 10.1016/j.jchromb.2016.07.038

Wang, Y., Yi, X., Ghanam, K., Zhang, S., Zhao, T., and Zhu, X. (2014). Berberine decreases cholesterol levels in rats through multiple mechanisms, including inhibition of cholesterol absorption. Metabolism 63, 1167-1177. doi: 10.1016/j.metabol.2014.05.013

Watt, G. (1883). Economic Products of India, Calcutta International Exhibition. Calcuta: Medicinal Products, Superintendent of Government Print.

Weiner, M. A. (1980). Earth Medicine-Earth Food: Plant Remedies, Drugs, and Natural Foods of the North American Indians. New York, NY: Macmillan.

Willaman, J. J., and Schubert, B. G. (1961). Alkaloid-Bearing Plants and Their Contained Alkaloids (No. 1234). Agricultural Research Service, US Department of Agriculture.

Wu, X., Li, Y., Wang, Q., Li, W., and Feng, Y. (2015). Effects of berberine and pomegranate seed oil on plasma phospholipid metabolites associated with risks of type 2 diabetes mellitus by U-HPLC/Q-TOF-MS. J. Chromatogr. B Anal. Technol. Biomed. Life Sci. 1007, 110-120. doi: 10.1016/j.jchromb.2015.11.008

$\mathrm{Xi}$, J. (2015). Ultrahigh pressure extraction of bioactive compounds from plants-a review. Crit. Rev. Food Sci. Nutr. 57, 1097-1106. doi: 10.1080/10408398.2013.874327

Xia, X., Yan, J., Shen, Y., Tang, K., Yin, J., Zhang, Y., et al. (2011). Berberine improves glucose metabolism in diabetic rats by inhibition of hepatic gluconeogenesis. PLOS ONE 6:e16556. doi: 10.1371/journal.pone.0016556

Xiao, H. B., Sun, Z. L., Zhang, H. B., and Zhang, D. S. (2012). Berberine inhibits dyslipidemia in C57BL/6 mice with lipopolysaccharide induced inflammation. Pharmacol. Rep. 64, 889-895. doi: 10.1016/S1734-1140(12)70883-6

Xiao, L., Xu, N., Guo, M., Guo, M., Lv, B, Tao, H., et al. (2014). Berberine protects endothelial progenitor cell from damage of TNF-alpha via the PI3K/AKT/eNOS signaling pathway. Eur. J. Pharmacol. 743, 11-16. doi: 10.1016/j.ejphar.2014.09.024

Xu, B., Li, P., and Zhang, G. (2015). Comparative pharmacokinetics of puerarin, daidzin, baicalin, glycyrrhizic acid, liquiritin, berberine, palmatine and jateorhizine by liquid chromatography-mass spectrometry after oral administration of Gegenqinlian decoction and active components alignmen. J. Chromatogr. B Anal. Technol. Biomed. Life Sci. 988, 33-44. doi: 10.1016/j.jchromb.2015.01.039

Xu, K., He, G., Qin, J., Cheng, X., He, H., Zhang, D., et al. (2017). High-efficient extraction of principal medicinal components from fresh Phellodendron bark (Cortex phellodendri). Saudi J. Biol. Sci. 25, 811-815. doi: 10.1016/j.sjbs.2017.10.008

Yang, L., Meng, X., Yu, X., and Kuang, H. (2017). Simultaneous determination of anemoside B4, phellodendrine, berberine, palmatine, obakunone, esculin, esculetin in rat plasma by UPLC-ESI-MS/MS and its application to a comparative pharmacokinetic study in normal and ulcerative colitis rats. J. Pharm. Biomed. Anal. 134, 43-52. doi: 10.1016/j.jpba.2016 11.021

Yang, T.-H. (1960a). Alkaloids of Berberidaceae. XXIX. Alkaloids of Mahonia lomariifolia and M. morrisonensis. Yakugaku Zasshi 80, 1304-1307. doi: 10.1248/yakushi1947.80.9_1304

Yang, T.-H. (1960b). Alkaloids of Berberidaceae. XXVIII. Alkaloids of Berberis morrisonensis. Yakugaku Zasshi 80, 1302-1304. doi: 10.1248/yakushi1947.80.9_1302

Yang, T.-H., and Lu, S.-T. (1960a). Alkaloids of berberidaceous plants. XXV. Alkaloids of Berberis kawakamii. 1. Yakugaku Zasshi 80, 847-849. doi: 10.1248/yakushi1947.80.6_847

Yang, T.-H., and Lu, S.-T. (1960b). Alkaloids of berberidaceous plants. XXVI. Alkaloids of Berberis mingetsensis. 1. Yakugaku Zasshi 80, 849-851. doi: 10.1248/yakushi1947.80.6_849

Yavich, P. A., Kakhtelidze, M. B., and Sarabunovich, A. G. (1993). Quantitative determination of berberine in Phellodendron lavallei bark. Farmatsiya 42, 49-50.

Yeung, H. (1985). Handbook of Chinese Herbs and Formulas, Vol. 1. Los Angeles, CA: Institute of Chinese Medicine.

Yin, J., Gao, Z., Liu, D., Liu, Z., and Ye, J. (2008a). Berberine improves glucose metabolism through induction of glycolysis. Am. J. Physiol. Endocrinol. Metab. 294, E148-E156. doi: 10.1152/ajpendo.00211.2007

Yin, J., Xing, H., and Ye, J. (2008b). Efficacy of berberine in patients with type 2 diabetes mellitus. Metabolism 57, 712-717. doi: 10.1016/j.metabol.2008.01.013

Yogesh, H. S., Chandrashekhar, V. M., Katti, H. R., Ganapaty, S., Raghavendra, H. L., Gowda, G. K., et al. (2011). Anti-osteoporotic activity of aqueous-methanol extract of Berberis aristata in ovariectomized rats. J. Ethnopharmacol. 134, 334-338. doi: 10.1016/j.jep.2010.12.013

Yoo, S. J., Lee, K. B., and Kwak, J. H. (1986). Studies on the seasonal variation of berberine contents in Berberis koreana. Saengyak Hakhoechi 17, 123-128.

Yu, C., Tan, S., Zhou, C., Zhu, C., Kang, X., Liu, S., et al. (2016). Berberine reduces uremia-associated intestinal mucosal barrier damage. Biol. Pharm. Bull. 39, 1787-1792. doi: 10.1248/bpb.b16-00280

Zabihullah, Q., Rashid, A., and Akhtar, N. (2006). Ethnobotanical survey in kot Manzaray Baba valley Malakand agency, Pakistan. Pak. J. Plant Sci. 12, 115-121.

Zaha, V. G., Qi, D., Su, K. N., Palmeri, M., Lee, H. Y., Hu, X., et al. (2016). AMPK is critical for mitochondrial function during reperfusion after myocardial ischemia. J. Mol. Cell. Cardiol. 91, 104-113. doi: 10.1016/j.yjmcc.2015. 12.032

Zaman, M. B., and Khan, M. S. (1970). Hundred drug plants of West Pakistan. Medicinal Plant Branch of Pakistan Forest Institute.

Zeng, X. (1999). Relationship between the clinical effects of berberine on severe congestive heart failure and its concentration in plasma studied by HPLC. Biomed. Chromatogr. 13, 442-444. doi: 10.1002/(SICI)1099-0801(199911)13:7<442::AID-BMC908>3.0.CO;2-A

Zhang, J., Cai, C. T., Cai, Z. Q., Liu, G. Z., Luo, Y., and Yang, Z. X. (2008). Variation patterns of Coptis teeta biomass and its major active compounds along an altitude gradient. J. Appl. Ecol. 19, 1455-1461.

Zhao, X., Zhang, J., Tong, N., Chen, Y., and Luo, Y. (2012). Protective effects of berberine on Doxorubicin-induced hepatotoxicity in mice. Biol. Pharm. Bull. 35, 796-800. doi: 10.1248/bpb.35.796

Zovko Koncić, Z., Kremer, D., Karlovć, K., and Kosalec, I. (2010). Evaluation of antioxidant activities and phenolic content of Berberis vulgaris L. and Berberis croatica Horvat. Food Chem. Toxicol. 48, 2176-2180. doi: 10.1016/j.fct.2010. 05.025

Conflict of Interest Statement: The authors declare that the research was conducted in the absence of any commercial or financial relationships that could be construed as a potential conflict of interest.

Copyright (c) 2018 Neag, Mocan, Echeverría, Pop, Bocsan, Crişan and Buzoianu. This is an open-access article distributed under the terms of the Creative Commons Attribution License (CC BY). The use, distribution or reproduction in other forums is permitted, provided the original author(s) and the copyright owner(s) are credited and that the original publication in this journal is cited, in accordance with accepted academic practice. No use, distribution or reproduction is permitted which does not comply with these terms. 


\section{Googling the Guggul (Commiphora and Boswellia) for Prevention of Chronic Diseases}

\section{OPEN ACCESS}

Edited by:

Atanas G. Atanasov,

Institute of Genetics and Animal

Breeding (PAS), Poland

Reviewed by:

Gokhan Zengin,

Selçuk University, Turkey

Ajay Bommareddy,

Wilkes University, United States

${ }^{*}$ Correspondence:

Ajaikumar B. Kunnumakkara kunnumakkara@iitg.ac.in

Bharat B. Aggarwal

bbaggarwal@gmail.com

Specialty section:

This article was submitted to

Ethnopharmacology,

a section of the journal

Frontiers in Pharmacology

Received: 15 January 2018

Accepted: 06 June 2018

Published: 06 August 2018

Citation:

Kunnumakkara $A B$, Banik K,

Bordoloi D, Harsha C, Sailo BL,

Padmavathi G, Roy NK, Gupta SC and Aggarwal BB (2018) Googling the

Guggul (Commiphora and Boswellia)

for Prevention of Chronic Diseases.

Front. Pharmacol. 9:686.

doi: 10.3389/fphar.2018.00686

\section{Ajaikumar B. Kunnumakkara ${ }^{1 *}$, Kishore Banik ${ }^{1}$, Devivasha Bordoloi ${ }^{1}$, Choudhary Harsha ${ }^{1}$, Bethsebie L. Sailo ${ }^{1}$, Ganesan Padmavathi ${ }^{1}$, Nand K. Roy ${ }^{1}$, Subash C. Gupta ${ }^{2}$ and Bharat B. Aggarwal ${ }^{3 *}$}

${ }^{1}$ Cancer Biology Laboratory, DBT-AIST International Laboratory for Advanced Biomedicine (DAILAB), Department of Biosciences and Bioengineering, Indian Institute of Technology Guwahati, Assam, India, ${ }^{2}$ Department of Biochemistry, Institute of Science, Banaras Hindu University, Varanasi, India, ${ }^{3}$ Inflammation Research Center, San Diego, CA, United States

Extensive research during last 2 decades has revealed that most drugs discovered today, although costs billions of dollars for discovery, and yet they are highly ineffective in their clinical response. For instance, the European Medicines Agency has approved 68 anti-cancer drugs, and out of which 39 has reached the market level with no indication of increased survival nor betterment of quality of life. Even when drugs did improve survival rate compared to available treatment strategies, most of these were found to be clinically insignificant. This is a fundamental problem with modern drug discovery which is based on thinking that most chronic diseases are caused by alteration of a single gene and thus most therapies are single gene-targeted therapies. However, extensive research has revealed that most chronic diseases are caused by multiple gene products. Although most drugs designed by man are mono-targeted therapies, however, those designed by "mother nature" and have been used for thousands of years, are "multitargeted" therapies. In this review, we examine two agents that have been around for thousands of years, namely "guggul" from Commiphora and Boswellia. Although we are all familiar with the search engine "google," this is another type of "guggul" that has been used for centuries and being explored for its various biological activities. The current review summarizes the traditional uses, chemistry, in vitro and in vivo biological activities, molecular targets, and clinical trials performed with these agents.

Keywords: guggul, guggulsterone, boswellia, boswellic acid, cancer, commiphora, chronic diseases

\section{INTRODUCTION}

Despite the remarkable advances made in the field of therapies for chronic diseases including cancer over the last few decades, they still present a major health burden and are the prime cause of death across the world. Most of the chronic illnesses are caused by the deregulation of multiple genes; however majority of the drugs approved by Food and Drug Administration (FDA) target single gene product or pathway only. This displays one of the major drawbacks of these synthetic drugs. In addition, these drugs are associated with different adverse side effects and hence not tolerable by patients (Siddiqui et al., 1984; Sarup et al., 2015; Kunnumakkara et al., 2017; Banik et al., 2018). Therefore, there is an urgent need to identify novel, safe, and multi-targeted agents for the prevention and treatment of these diseases (Bordoloi et al., 2016; Kunnumakkara et al., 2018). 
It has been well-evidenced that natural products are effective, multi-targeted, and extremely safe as they are the roots of many traditional systems of medicine such as Ayurveda, Unani, Siddha, traditional Chinese medicine etc. (Shishodia et al., 2008; Harsha et al., 2017). One such medicine of enormous use in Ayurveda is "Guggul." Guggul is the gum resin obtained from two different plants Commiphora and Boswellia, produced by drying the white sap of 15-20 years old tree for a year (Figure 1; Hanus et al., 2005).

The history of guggul goes as far back as 1700 BC. Ancient script on medicine and surgery; Sushrut Samhita, describes that guggul when taken orally can cure internal tumors, malignant sores, obesity, liver dysfunction, intestinal worms, leucoderma, sinus, and edema. It is also used as an Ayurvedic medicine for the prevention and treatment of various other diseases such as inflammatory bowel disease (IBD), ulcers, arthritis, cardiovascular diseases (CVDs), diabetes etc. (Shishodia et al., 2008). The main ingredients of guggul are guggulsterone (GS) and boswellic acid (BA) which are obtained from Commiphora and Boswellia respectively. It also contains a huge number of lignans and ketosterols, which contributes to the vivid health beneficiary effects of guggul (Arora et al., 1971, 1972; Kimura et al., 2001; Zhu et al., 2001; Francis et al., 2004).

According to Pubmed; "google," there are 449 publications on Commiphora, 519 on Boswellia, 207 on guggulsterone, 329 on boswellic acid, and 90 on guggul with earliest being in 1960 describing the "Antiarthritic and anti-inflammatory activity of the gum "guggul"; and in 1969 on "Analgesic effect of the gum resin from Boswellia serata." Some of the major species include Commiphora wightii (guggul), Commiphora mukul, Commiphora gileadensis, Boswellia serrata (salai guggul), Boswellia carterii, Boswellia sacra (source of frankincense \& gum resin), Boswellia ovalifoliolata, Boswellia dalzielii, Boswellia frerean, and Boswellia

\section{A Commiphora (Guggul)}
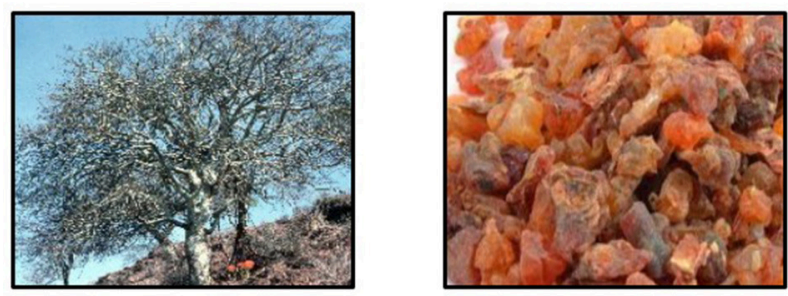

\section{B Boswellia (Salai Guggul)}
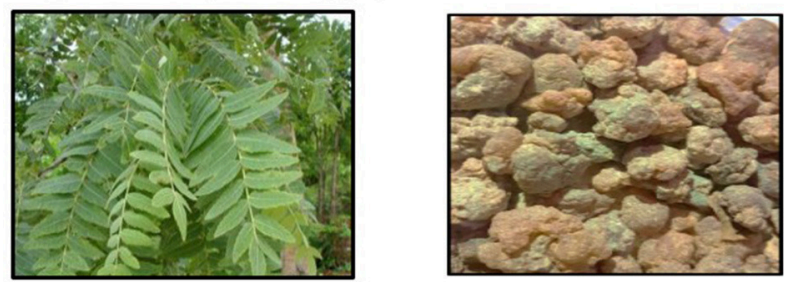

FIGURE 1 | (A) Commiphora (Mark W. Skinner/www.discoverlife.org) and Gum guggul (http://www.varionlife.com). (B) Boswellia (Pankaj Oudhia/www.discoverlife.org) and Salai guggul. thurifera. What is common among all these plants and their products is that all of them exhibit anti-inflammatory activities, although to a variable extent. The current review describes the traditional uses, chemistry, molecular targets, in vitro, in vivo and clinical studies of guggul isolated from Commiphora and Boswellia.

\section{SOURCE AND CHEMICAL CONSTITUENTS OF COMMIPHORA AND BOSWELLIA}

The guggul tree which belongs to the family Burseraceae, is mainly found in the dry regions of the Indian subcontinent mainly India, Pakistan and Bangladesh. The oleogum resin of C. mukul (guggul tree) is a yellowish substance that is tapped during winter and $\sim 700-900 \mathrm{~g}$ of resin is obtained from each tree (Deng, 2007; Shishodia et al., 2015; Yamada and Sugimoto, 2016). The guggul or balsam or the oleo gum resin is found in the balsam canals in the phloem of the large veins of leaf and base of the stem. It is a complicated mixture of minerals, gum, terpenes, sterols (Guggulsterol -I,-II,III,-IV,-V), essential oils, sterones (Z-, E-, M-guggulsterone, and dehydroguggulsterone-M), ferrulates, lignans, and flavanones. The ethyl acetate soluble fraction also known as guggulipid, consists of various bioactive components like diterpenoids, triterpenoids, steroids, lignans, and fatty tetrol esters. Based on the $\mathrm{pH}$ gradient, further fractionation yields $95 \%$ neutral, $4 \%$ acidic, and $1 \%$ basic fractions. The neutral fraction when subjected to further fractionation produces $88 \%$ non-ketonic and $12 \%$ ketonic fractions. A large number of steroids including the two isomers E-(cis-) and Z-(trans-) GS [4, 17(20)-pregnadiene3,16 -dione] were obtained from the ketonic fraction. Nearly $5 \%$ guggulipid and $2 \%$ gum guggul by weight is present in the GS (Figure 2A; Deng, 2007; Shishodia et al., 2008, 2015; Sarup et al., 2015).

Phenolics are common natural products found in plants and possess substantial antioxidant and anti-inflammatory effects. Various phenolic compounds such as hydroxybenzoic acid derivatives such as gallic acid, protocatechuic acid, gentisic acid, vanillic acid, p-hydroxy benzoic acid, syringic acid, ellagic acid, and cinnamic acid derivatives which include caffeic acid, chlorogenic acid, ferulic acid, sinapic acid (SA), and p-coumaric acid are largely present in plants. These phenolic compounds are predominantly available in guggul as well, which in part contributes to its immense biological function against diverse human chronic diseases (Hazra et al., 2018).

Guggulsterone is the only known antagonist of farnesoid $\mathrm{X}$ receptor (FXR). This FXR, also known as NR1H4 (nuclear receptor subfamily 1 , group $H$, member 4 ), is a bile acid receptor (BAR). Bioinformatics studies (molecular docking simulation) revealed that GS binds to FXR and nuclear factor-kappa B $(\mathrm{NF}-\kappa \mathrm{B})$ and it docks into two non-canonical binding sites of FXR, helix 1-loop-helix 2 loop and parts of helix-helix 8 including helix 8-loop-helix 9 (Meyer et al., 2005; Yang et al., 2014). Different bile acids and chenodeoxycholic acids act as natural ligand for FXR, whose expression is elevated in the liver and intestine. When FXR binds to its ligand, it gets activated 


\section{A Commiphora (Guggul)}

\section{1) Sterones}

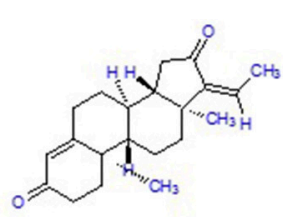

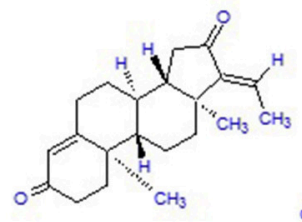

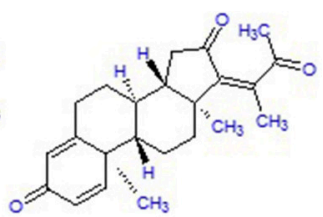

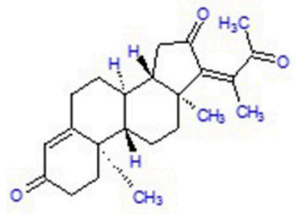

(Z)-Guggulsterone

(E)-Guggulsterone

Dehydroguggulsterone M

\section{Guggulsterone M}

2) Sterols

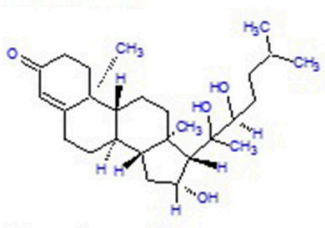

Guggulsterol I

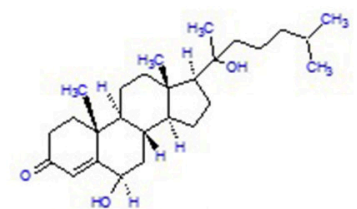

Guggulsterol III

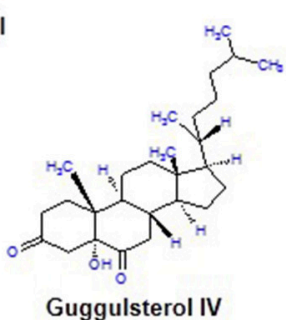

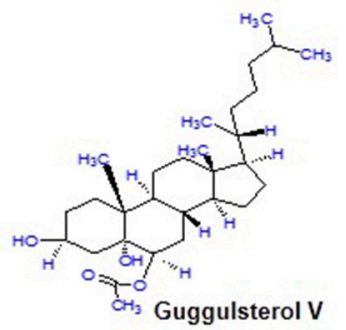

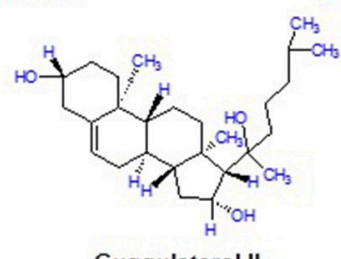

Guggulsterol II

Guggulsterol IV

\section{B Boswellia (Salai Guggul)}

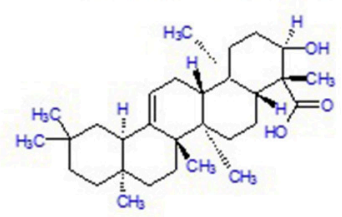

a-Boswellic Acid

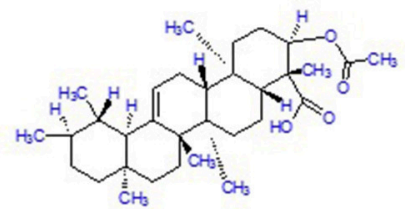

3-Acetyl- $\beta$-boswellic acid

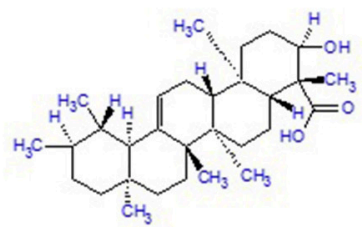

$\beta$ - Boswellic Acid

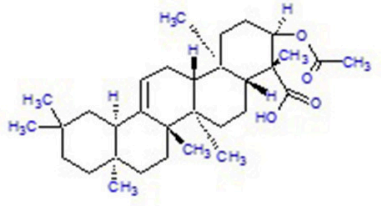

3-O-Acetyl-a-boswellic acid

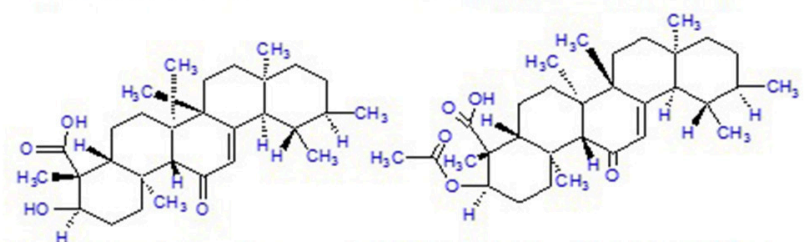

11-Keto-ß-boswellic acid

FIGURE 2 | Chemical constituents of Commiphora (guggul) and Boswellia (Salai guggul) (A) Commiphora (B) Boswellia.

and reaches the cell nucleus, where it forms a heterodimer with RXR. This heterodimer binds to the hormone response elements on DNA and regulates various genes. FXR activation downregulates cholesterol 7 alpha-hydroxylase (CYP7A1), the rate-limiting enzyme in bile acid synthesis from cholesterol by inducing the expression of small heterodimer partner (SHP) which in turn inhibits the transcription of CYP7A1 gene. While obeticholic acid, fexaramine, cafestol, and chenodeoxycholic acid act as agonist of FXR; GS, from the gum resin of guggul has also been confirmed to inhibit pro-inflammatory signals, together with transcription factor NF-кB (Sharma and Sharma, 1977; Urizar et al., 2002; Shishodia et al., 2008; Yamada and Sugimoto,
2016). Another study reported that the inhibitory activity of NF- $\kappa \mathrm{B}$ is due to the binding of GS to the RH domain of NF- $\kappa \mathrm{B}$ precursor protein $\mathrm{p} 105$ containing important sequences for DNA binding and dimerization (Khan et al., 2013).

B. serrata, commonly known as salai guggul, Indian olibanum, loban, or kundur, belongs to the Burseraceae family and is found in dry mountainous regions of India, Northern Africa, and the Middle East. Burseraceae family includes 17 genera and 600 species of plants. The genus Boswellia has 25 different species distributed throughout the tropical regions. B. serrata is one such medicinal plant which exhibits immense potential to combat various chronic disorders. The active pharmacological principle 
of the oleo gum resin from the trees of different Boswellia species is the BA (Büchele et al., 2003; Du et al., 2015; Roy et al., 2016). The gum resin of the Boswellia species mainly consists of mucus, resin acids, and volatile oil with different quantitative composition from species to species. The gum resin of salai guggul contains pentacyclic triterpenic acids, namely $\alpha$-boswellic acids, $\beta$-boswellic acids, $\gamma$-boswellic acid, acetyl- $\beta$ boswellic acid, 11-keto- $\beta$-boswellic acid (KBA), acetyl-11-keto$\beta$-boswellic acid (AKBA), and tetracyclic triterpenic acids like tirucallic acids viz 3-oxotirucallic acid, 3-hydroxytirucallic acid, and 3-acetoxytirucallic acid (Figure 2B). Other oleo gum resin compounds which display biological activities are: betulinic acid, lupenoic acid, epi-lupeol, isoincensole, isoincensole acetate and 1-ursene-2-diketone-incensole acetate along with few other terpenes that can be found in volatile oil (Du et al., 2015; Ammon, 2016; Roy et al., 2016).

\section{MOLECULAR TARGETS OF COMMIPHORA AND BOSWELLIA}

GS suppresses the physiological action of the FXR which is a nuclear hormone receptor that controls the synthesis and transport of bile acid (Sinal and Gonzalez, 2002; Urizar et al., 2002). However, it increases the transcription of bile salt export pump (BSEP) which is majorly involved in hepatic bile acid transport (Cui et al., 2003). Besides regulating transport of bile acid, GS is a potent anti-inflammatory agent which suppresses LPS-induced NO production (Meselhy, 2003). GS has also been reported to inhibit the activation of NF- $\kappa \mathrm{B}$ by suppressing the levels of receptor activator of NF- $\kappa$ B ligand (RANKL) (Ichikawa and Aggarwal, 2006). In 2004, Shishodia and group reported that GS suppressed the activation of NF- $\kappa \mathrm{B}$ and I $\kappa \mathrm{B}-\alpha$ kinase and exhibited antiproliferative activity by inhibiting c-Myc and cyclin D1. Furthermore, GS has also been found to exert antimetastatic effect through reducing the levels of MMP-9, COX2, and VEGF (Shishodia and Aggarwal, 2004). This group also reported that GS induced apoptosis by modulating the expression of anti-apoptotic genes, IAP1, XIAP, Bcl-2, cFLIP, Bfl-1/A1, and survivin (Shishodia and Aggarwal, 2004). Further, GS has also been found to induce tumor cell apoptosis by activating the apoptotic genes, caspase-3,-8,-9, and inducing the release of cytochrome c, cleavage of bid and PARP. This was controlled by activated mitogen-activated protein kinase 4 (MKK4) mediated upregulation of c-Jun N-terminal kinase (JNK) and suppression of Akt. The antiproliferative activity of GS was found to be supported by reduced levels of cyclin D1, cdc2, and simultaneous upregulation of cyclin-dependent kinase inhibitors p21 and p27 (Figure 3A; Shishodia et al., 2007).

Boswellic acid is known to inhibit leukotriene synthesis by inhibiting 5-lipoxygenase (5-LOX) (Safayhi et al., 1992, 1995; Ammon et al., 1993). This 5-LOX inhibitor has also been found to reduce the activity of human leukocyte elastase (HLE) in vitro (Safayhi et al., 1997). Suppression of these molecules contributes to anti-inflammatory action of Boswellia. Boswellia is also known to induce apoptosis in cancer cells. In 2007, Bhushan and group reported that a triterpenediol from $B$. serrata induced apoptosis in HL-60 cells through both intrinsic and extrinsic pathways (Bhushan et al., 2007). In the first case, the triterpenediol was found to disturb the mitochondrial membrane potential, reduce $\mathrm{Bcl}-2 / \mathrm{Bax}$ ratio and cause release of AIF, Smac/DIABLO, and cytochrome $\mathrm{c}$ from the mitochondria along with suppression of survivin and upregulation of caspases-3,-8, and-9, thereby leading to the cleavage of ICAD and PARP while in the second case, the oxidative stress generated in the cells due to excessive ROS and NO production triggered the activation of TNF-R1 and DR4 followed by activation of caspase-8. Another study in multiple myeloma cells also suggested that BA acetate induces apoptosis by upregulating death receptor proteins, DR4 and DR5 which subsequently leads to the activation of caspase- 8 followed by caspase-3 (Xia et al., 2005). The role of DR5-mediated pathway which involves activation of CAAT/enhancer binding protein homologous protein (CHOP) was reported in AKBA-mediated apoptosis of prostate cancer cells (Lu et al., 2008). Caspase-8 activation has also been reported in other BA-induced apoptosis studies (Liu et al., 2002a,b). In 2002, Park et al. hypothesized that $\mathrm{AKBA}$ contributed in the process of proliferation and apoptosis of tumors by inhibiting platelet-derived growth factor (PDGF)stimulated extracellular signal-regulated kinase 1 and 2 (ERK-1 and ERK-2) (Park et al., 2002b). BA mediated apoptosis has also been evident in cancer cells via activation of p21, an important cell cycle regulator protein (Glaser et al., 1999; Liu et al., 2006). Apart from this, AKBA has been found to interfere with IL-6induced STAT3 signaling via protein tyrosine phosphatase SHP-1 subsequently causing downregulation of cyclin D1, Bcl-2, Bcl$\mathrm{xL}, \mathrm{Mcl}-1$, and VEGF, thus impeding proliferation, survival and angiogenesis of multiple myeloma cells (Kunnumakkara et al., 2009). Moreover, BA has also been found to suppress metastatic growth factor, basic fibroblast growth factor (bFGF), chemokine receptor; CXCR4 and angiogenic factor; VEGFR 2 (Singh et al., 2007; Pang et al., 2009; Park et al., 2011a). Further, in vivo studies have unveiled that $\mathrm{BA}$ regulates proliferation and metastasis of cancer cells by downregulating other targets like COX-2, c-Myc, cyclin D1, MMP-9, VEGF, ICAM-1, Bcl-2, Bcl-xL, survivin, and cellular inhibitor of apoptosis protein 1 (IAP-1) (Park et al., 2011a,b; Yadav et al., 2012). Most of these genes are regulated by the transcription factor, NF- $\mathrm{B}$ which is also downregulated by BA (Syrovets et al., 2005a,b; Takada et al., 2006). Furthermore, BA has also been shown to regulate the activity of P-glycoprotein (Pgp) which is an important class of drug transporters (Weber et al., 2006). It is also an inhibitor of topoisomerases I and II in cancer cells (Hoernlein et al., 1999; Syrovets et al., 2000; Zhao et al., 2003). The anticancer activity of this potential compound also involves regulation of let-7 and miR-200 microRNA family (Figure 3B; Takahashi et al., 2012).

\section{THERAPEUTIC PROPERTIES OF GUGGUL}

Congregate evidences show guggul to be profoundly effective against diverse chronic diseases such as Alzheimer's disease, arthritis, cancer, pancreatitis, IBD, dermatitis, diabetes, infectious diseases, intestinal metaplasia, otitis media, respiratory diseases, asthma, psoriasis, gingivitis etc. Besides, it also 

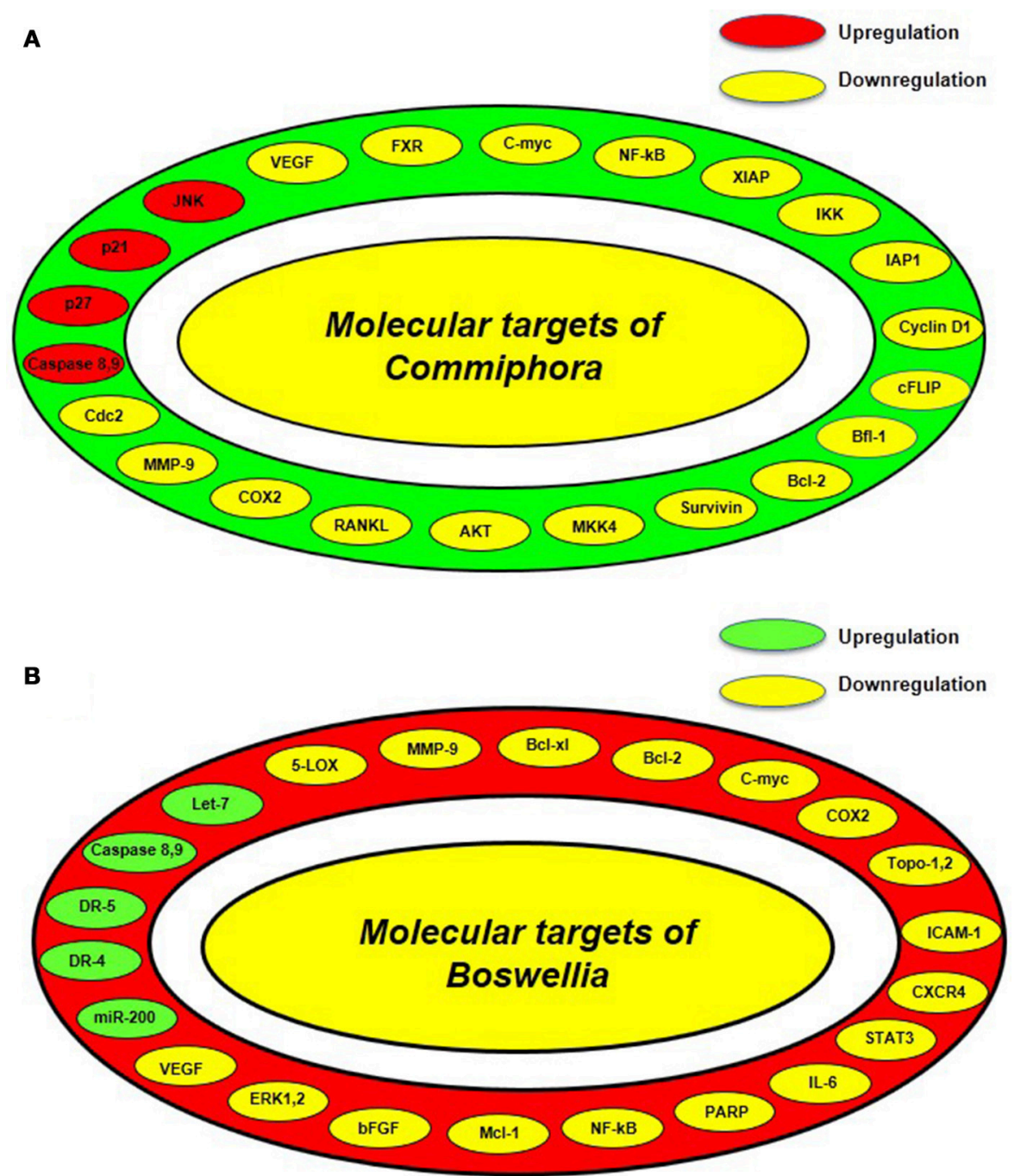

FIGURE 3 | Various molecular targets of guggul from Commiphora and Boswellia (A) Molecular targets of guggul from Commiphora includes Bcl2, B-cell lymphoma 2; CDC 2, cell division cycle kinase 2; C-FLIP, cellular caspase-8 (FLICE)-like inhibitory protein; COX, cyclooxygenase; FXR, farnesoid X receptor; IKK, IKB kinase; IAP, Inhibitors of apoptosis proteins; JNK, c-Jun N-terminal kinase; MKK4, mitogen-activated protein kinase kinase 4; MMP, matrix metalloproteinase; NF-kB, nuclear factor-kB; RANKL, Receptor activator of nuclear factor kappa-B ligand; VEGF, vascular endothelial growth factor; XIAP, $x$-linked inhibitor of apoptosis protein. (B) Molecular targets of guggul from Boswellia includes Bcl-2, B-cell lymphoma 2; Bcl-xL, B-cell lymphoma-extra-large; CXCR-4, C-X-C chemokine receptor type 4; DR, Death receptor; 5-LOX, 5-Lipoxygenase; MMP, Matrix metalloproteinase; NF-кB, nuclear factor-кB; Topo, Topoisomerase; ICAM-1, Intercellular adhesion molecule 1; STAT-3, Signal transducer and activator of transcription 3; IL-6, Interleukin 6; PARP, Poly ADP ribose polymerase; Mcl-1, Myeloid leukemia cell differentiation protein; bFGF, Basic fibroblast growth factor; ERK-1,-2, Extracellular signal-regulated kinases.

exerts hepatoprotective, neuroprotective, anti-inflammatory, anti-oxidant, cardioprotective, hypolipidemia, and thyroid stimulatory effect by targeting multiple signaling pathways (Table 1; Figure 4).

\section{IN VITRO STUDIES WITH COMMIPHORA AND BOSWELLIA AND THEIR ROLE IN DIFFERENT CHRONIC DISEASES}

Numerous in vitro studies have indicated the efficiency of guggul against diverse chronic diseases including cancer (Shishodia et al., 2007, 2008; Singh S. V. et al., 2007; Shah et al., 2012; Roy et al.,
2016). GS induced apoptosis in cancer cells via inhibition of NF- $\kappa$ B, activation of JNK and downregulation of Akt and antiapoptotic proteins (Shishodia and Aggarwal, 2004; Shishodia et al., 2007). Treatment with GS led to the inhibition of DNA synthesis and proliferation of leukemia cells via downregulation of cyclin D1, cdc2, and upregulation of p21 and p27 (Samudio et al., 2005; Shishodia et al., 2007). In addition, B. serrata gum resin displayed cytostatic and apoptosis-inducing effect against leukemia and brain tumor cells (Hostanska et al., 2002). Further, GS induced cell death in prostate cancer cells by reactive oxygen intermediate (ROI)-dependent activation of JNK, p38 MAPK and also activation of ERK1/2 (Singh S. V. et al., 2007; Xiao and Singh, 2008). Additionally, AKBA inhibited the proliferation and 
TABLE 1 | In vitro biological activities of guggul (Commiphora and Boswellia) against various chronic diseases.

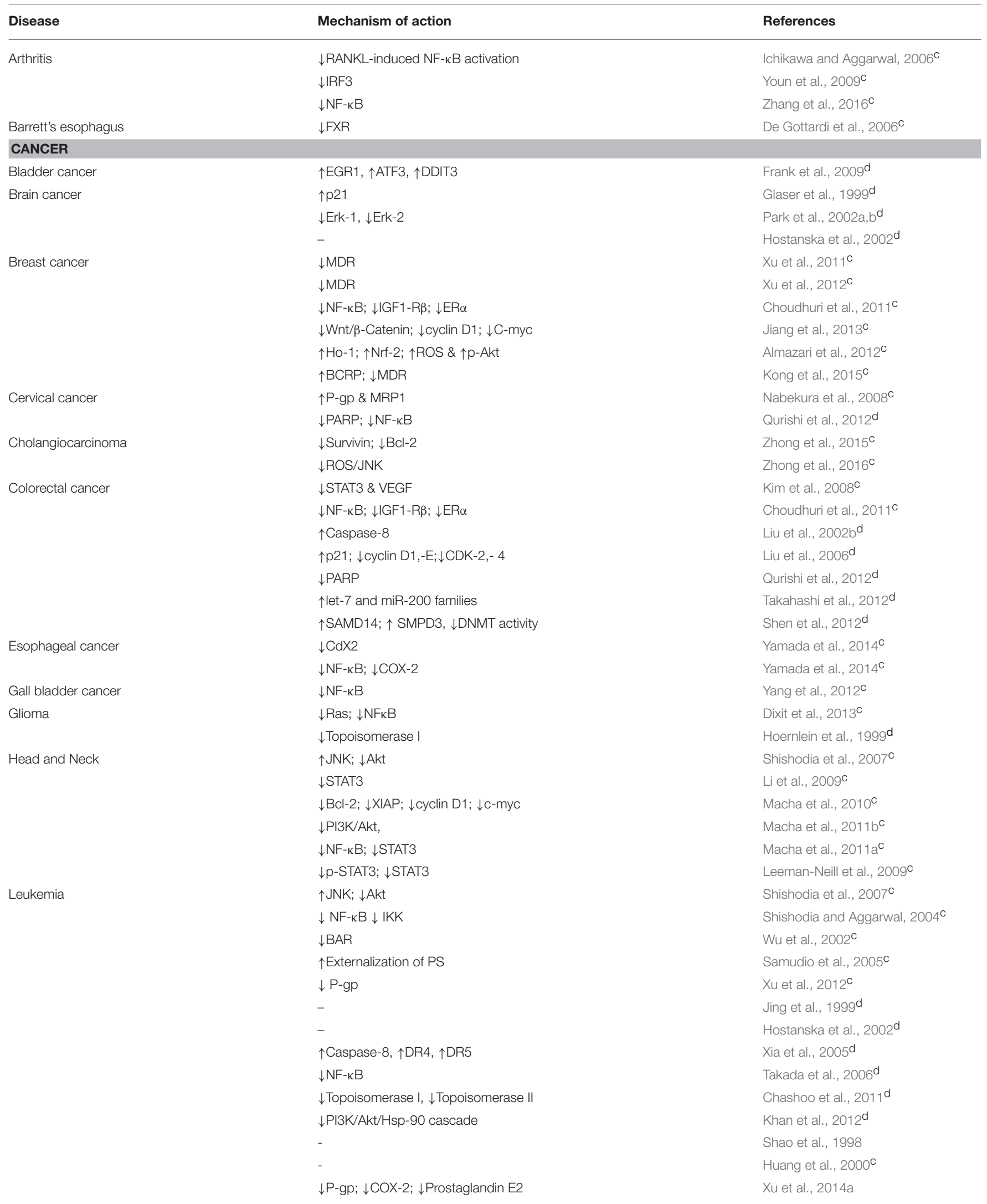


TABLE 1 | Continued

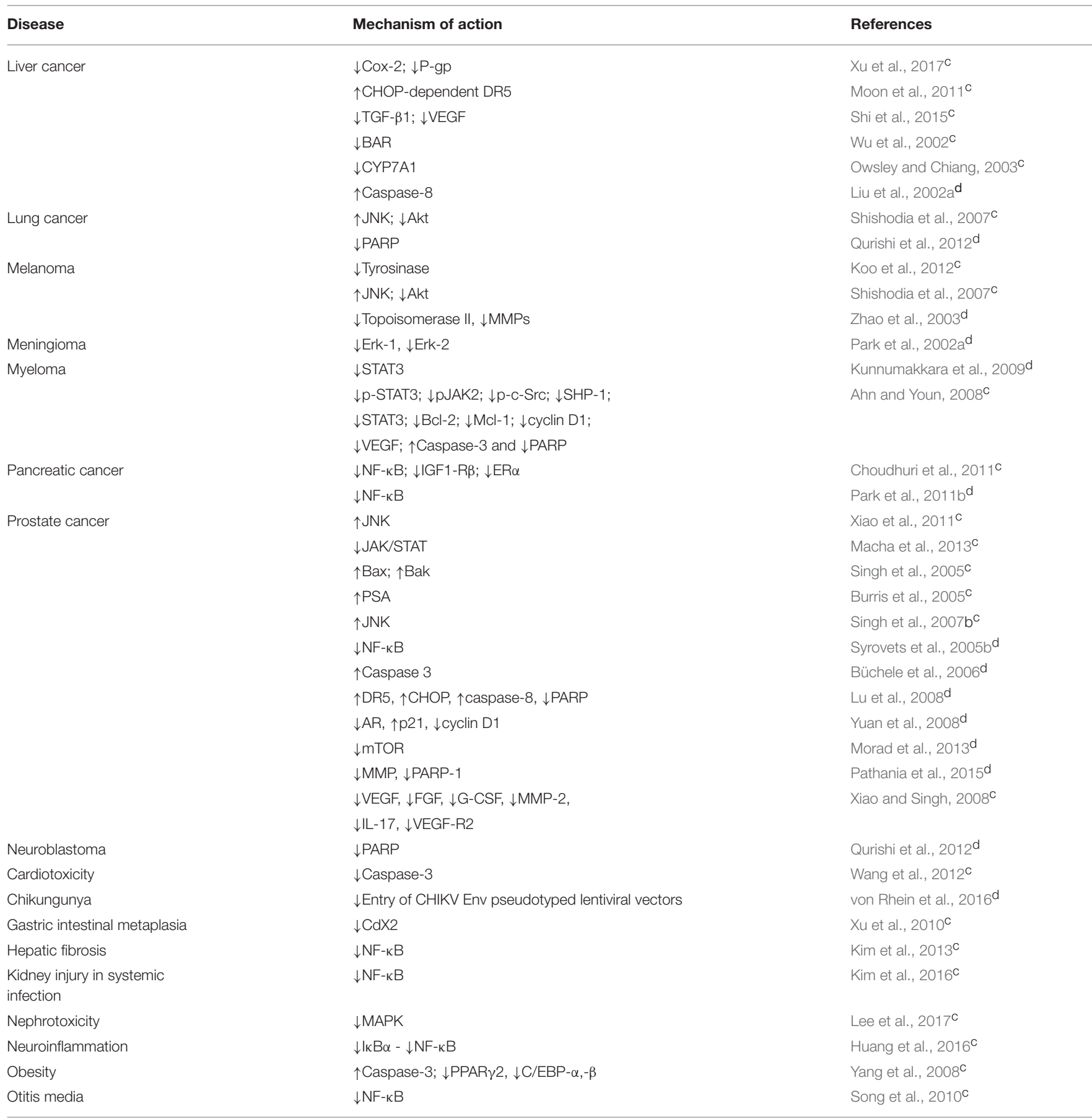

AR, Androgen receptor; ATF3, Activating transcription factor 3; BAR, Bile acid receptor; BCRP, Breast cancer resistance protein; C, Commiphora; C/EBP, CCAAT/enhancer binding protein; CDK, Cyclin dependent kinase; CdX2, Caudal-related homeobox 2; CHOP, CAAT/enhancer binding protein homologous protein; COX-2, Cyclooxygenase-2; CYP7A1, Cholesterol 7alpha-hydroxylase; d, Boswellia; DDIT3, DNA damage inducible transcript 3; DNMT, DNA methyl-transferase; DR, Death receptor; EGR1, Early growth response 1; ERa, Estrogen receptor alpha; FXR, Farnesoid X receptor; G-CSF, Granulocyte colony-stimulating factor; HO-1, Heme oxygenase-1; Hsp90, Heat shock protein 90; IGF1, Insulin-like growth factor 1; JNK, c-Jun N-terminal kinase; IL, Interleukin; IRF3, Interferon-regulatory factor 3; MAPK, Mitogen-activated protein kinase; MCL-1, myeloid leukemia cell differentiation protein; MDR, Multidrug resistance; MMP, Matrix metalloproteinases; MRP, Multidrug resistance protein; $m$ TOR, Mechanistic target of rapamycin; NF-kB, Nuclear factor kappa B; Nrf-2, The nuclear factor erythroid 2 (NFE2)-related factor 2; PARP, Poly ADP ribose polymerase; PI3K, Phosphatidylinositol-4,5-bisphosphate 3-kinase; PPAR, Peroxisome proliferator activated receptor; pRb, phosphorylated Retinoblastoma; PS, Phosphatidylserine; PSA, Prostate-specific antigen; RANKL, Receptor activator of nuclear factor kappa-B ligand; ROS, Reactive oxygen species; SAMD14, Sterile Alpha Motif Domain Containing 14; SHP-1, Src homology region 2 domain-containing phosphatase-1; SMPD3, Sphingomyelin phosphodiesterase 3; STAT, Signal transducer and activator of transcription 6; STAT3, Signal transducer and activator of transcription 3; TGF, Transforming growth factor; TLR-3, Toll-like receptor-3; TLR-4, Toll-like receptor-4; VEGF, Vascular endothelial growth factor; VEGFR2, Vascular endothelial growth factor receptor 2; XIAP, X-linked inhibitor of apoptosis protein. 


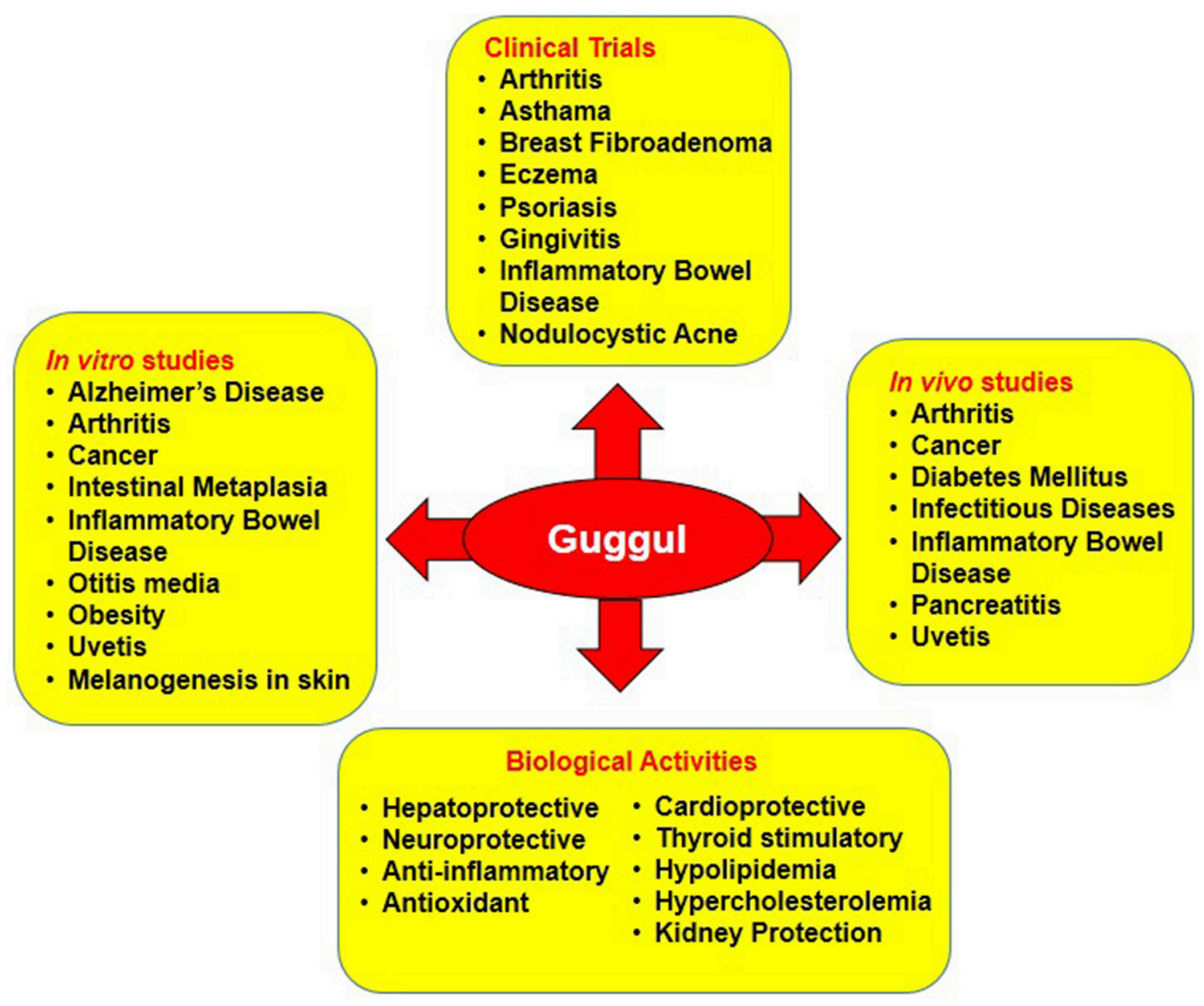

FIGURE 4 | In vitro, in vivo and clinical studies on the biological activities of guggul against diverse chronic diseases.

induced apoptosis in colon cancer cells through p21 and caspase8 dependent pathway (Liu et al., 2002b, 2006). Besides, it also modulated the expression of let-7, miR-200 families and their downstream targets in colon cancer cells (Takahashi et al., 2012).

Apart from cancer, the effect of guggul has been well proven against different inflammatory diseases such as rheumatoid arthritis, IBD, and various other diseases such as obesity, otitis media, uveitis etc. For example, treatment with GS downregulated RANKL induced osteoclastogenesis and blocked IL-1beta mediated production of chemokines and epithelial neutrophil activating peptide-78 (ENA-78), MMP-1,-3 via suppression of NF- $\kappa \mathrm{B}$, nuclear p50, and p65 subunit and I $\mathrm{B} \alpha$ degradation in rheumatoid arthritis (Ichikawa and Aggarwal, 2006; Kinne et al., 2007; Lee et al., 2008; Ammon, 2016). A study conducted by Cheon and group on IBD showed that GS inhibited IL-1beta- or lipopolysaccharide (LPS)- induced ICAM-1 expression, NF- $\kappa \mathrm{B}$ transcription activity and I $\mathrm{B}$ phosphorylation/degradation in human Caco-2 cells and rat nontransformed IEC-18 cells (Cheon et al., 2006). Further, treatment with GS alone showed increase in apoptosis and lipolysis and its combination with genistein resulted in increased cleavage of procaspase-3, PARP, expression of Bax, release of cyt-c and prevented lipid accumulation in maturing adipocytes resulting in inhibition of adipogenesis (Yang et al., 2007, 2008). GS exerted its effect against otitis media, the foremost cause of hearing impairment in children by inhibiting LPS-induced upregulation of TNF- $\alpha$ expression, COX-2 production and I $\kappa \mathrm{B} \alpha$ degradation (Ovesen and Ledet, 1992; Barrett et al., 2003; Song et al., 2010). In case of uveitis, treatment with GS inhibited LPS-induced expression of inflammatory proteins in human primary nonpigment ciliary epithelial cells (Kalariya et al., 2010).

As guggul is an FXR antagonist, it is used extensively as a cholesterol-lowering agent (Rizzo et al., 2006; Shah et al., 2012). GS eliminated the effect of chenodeoxycholic acid (CDCA), an FXR agonist on the expression of Cdx2 and MUC2 and thus prevented bile acid induced gastric intestinal metaplasia and carcinogenesis (Xu et al., 2010). Oswley and Chiang reported that GS antagonizes FXR induction of BSEP but activates pregnane $\mathrm{X}$ receptor to inhibit CYP7A1 gene (Owsley and Chiang, 2003). In addition, Commiphora and Boswellia showed potent cardioprotective as well as thyroid-stimulatory effects (Singh et al., 1982; Deng, 2007). For instance, GS inhibited DOX induced cytotoxicity, reduced apoptosis, and intracellular ROS and formation of MDA in DOX-treated H9C2 cells (Wang 
et al., 2012). In addition, triterpenes and prenylaromadendranetype diterpenes from the gum resin of $B$. carterii was shown to exert hepatoprotective effect against d-galactosamine-induced liver cell damage (Wang et al., 2013, 2016).

\section{IN VIVO STUDIES WITH COMMIPHORA AND BOSWELLIA AND THEIR ROLE IN DIFFERENT CHRONIC DISEASES}

Promising after effects of Commiphora and Boswellia against various chronic diseases in the in vitro setting has led to a handful of in vivo studies where the efficacy of guggul was evaluated in different experimental models of diverse chronic diseases such as cancer, inflammatory, cardiovascular, and metabolic diseases, atherosclerosis, asthma etc. (Table 2; Figure 4). Recently, several studies have reported the anti-tumor efficacy of guggul in different cancers such as cancers of breast, esophagus, head, and neck, pancreas, prostate etc. (An et al., 2009). For instance, AKBA was found to prevent intestinal tumorigenesis and exert chemopreventive effect via inhibition of wnt $/ \beta$-catenin and NF- $\mathrm{BB} / \mathrm{COX}-2$ signaling pathways (Liu et al., 2013; Wang R. et al., 2014). Another study showed AKBA to function via modulation of let-7 and miR-200 downstream genes in colorectal (CRC) tumors (Takahashi et al., 2012). In case of breast cancer, treatment with GS increased the chemosensitivity of MCF-7/DOX cells to doxorubicin in vivo through inhibition of Bcl-2 and Pgp (Xu et al., 2014b). In addition, GS suppressed esophageal tumor cell viability via inhibition of FXR and prevented the growth of esophageal cancer cells significantly in combination with amiloride in vivo (Guan et al., 2013, 2014). Furthermore, in case of glioma, cyano enone of methyl boswellates (CEMB), and 3- $\alpha$-propionyloxy- $\beta$ boswellic acid (POBA) significantly inhibited the tumor growth in murine models (Ravanan et al., 2011; Qurishi et al., 2013). Again, topical application of Boswellin (BE); B. serrata gum resin exudate inhibited skin inflammation, epidermal proliferation, and tumor promotion induced by 12-O-tetradecanoylphorbol13-acetate (TPA) in 7,12-dimethylbenz[a] anthracene (DMBA)initiated mice. Additionally, treatment with guggulipid was shown to cause reduced growth of HNSCC cells in vivo (LeemanNeill et al., 2009). Besides, GS enhanced the antitumor efficacy of gemcitabine in pancreatic cancer via modulation of Akt, NF$\kappa \mathrm{B}$, and apoptosis-related proteins (Ahn et al., 2012). In case of prostate cancer as well, guggul has been found to be highly effective in vivo (Syrovets et al., 2005b; Büchele et al., 2006; Pang et al., 2009; Pathania et al., 2015). Oral administration of guggulsterone prevented in vivo angiogenesis of prostate cancer cells through suppression of VEGF-VEGF-R2-Akt signaling (Xiao and Singh, 2008).

Apart from cancer, the efficacy of guggul was well proven in different inflammatory diseases such as arthritis, colitis, gastritis, IBD, pancreatitis, uveitis etc. (Sharma et al., 1989; Cheon et al., 2006; Xiao and Singh, 2008; Mencarelli et al., 2009; Kalariya et al., 2010; Kim et al., 2010, 2013; Dhaneshwar et al., 2013; Kang et al., 2013; Wang R. et al., 2014). In case of rheumatoid arthritis, treatment with guggul decreased the thickness of joint swelling, reduced the infiltration of leucocytes into the pleural cavity, suppressed the pro-inflammatory cytokines and increased betaglucuronidase activity in vivo (Sharma and Sharma, 1977; Reddy and Dhar, 1987; Sharma et al., 1989; Fan et al., 2005). In addition, guggul reduced the severity of IBD via inhibition of LPS- or IL-1beta-induced ICAM-1 gene expression and NF- $\kappa$ B activity (Krieglstein et al., 2001; Cheon et al., 2006; Mencarelli et al., 2009; Kim et al., 2010). Furthermore, administration of GS resulted in mitigation of histological damage, suppressed serum lipase levels, inhibition of infiltrations of neutrophils, and macrophages and decreased cytokine production in pancreatitis (Kim et al., 2015). Moreover, GS inhibited the expression of endotoxin-induced uveitis (EIU)-associated inflammatory markers such as MMP-2, $\mathrm{NO}$, and prostaglandin $\mathrm{E}_{2}\left(\mathrm{PGE}_{2}\right)$ (Kalariya et al., 2010).

Guggul exhibited profound cardioprotective effects as well in vivo (Chander et al., 2003). It decreased the lipid peroxide, creatine phosphokinase, phospholipase, xanthine oxidase activities, and total cholesterol level in the serum; increased superoxide dismutase (SOD), myocardial antioxidants, glutathione peroxidase (GSHPx), catalase (CAT); reduced glutathione (GSH), creatine-phosphokinase-MB (CK-MB), and lactate dehydrogenase (LDH) as well as reversed the cardiac damage induced by isoproterenol (Kaul and Kapoor, 1989; Batra et al., 2000; Ojha et al., 2011). The hypolipidemic effect of guggul has also been well studied in different animal models (Khanna et al., 1969; Dixit et al., 1980; Baldwa et al., 1981; Lata et al., 1991). Guggul diminished hyperlipidemia via inhibition of FXR activation. In high-fat-diet-fed mice, treatment with GS improved blood glucose in fasting condition, plasma insulin level, glucose tolerance, level of harmful lipids, phosphoenol pyruvate carboxykinase, glucose-6-phosphatase, and other proteins like glucose transporter-4, PPARc, and TNF- $\alpha$ (Satyavati et al., 1969; Singh et al., 1990; Urizar et al., 2002; Cui et al., 2003; Sharma et al., 2009; Tripathi, 2009). Further, C. opobalsamum, C. mukul, B. serrata, and B. ovalifoliolata species mitigated hepatic damage and displayed protective effect against lipid peroxidation and deviated serum enzymatic variables (Al-Howiriny et al., 2004; Y et al., 2006; Mahesh et al., 2014). In addition, GS reversed neuronal damage and memory deficits in mice by increasing glutathione level in the brains, antiacetylcholine esterase, and antioxidant activities (Saxena et al., 2007). Apart from these, administration of GS was found to increase thyroid function by enhancing iodine uptake, improved the activities of thyroid peroxidase, and protease and ameliorated hypothyroidism through its ability to increase thyroid hormone in vivo (Tripathi et al., 1975, 1984; Panda and Kar, 2005).

Taken together, these pre-clinical studies provide substantial evidence of the enormous potential of guggul as a multi-targeted agent for the prevention and treatment of different chronic diseases.

\section{CLINICAL STUDIES WITH COMMIPHORA AND BOSWELLIA AND THEIR ROLE IN DIFFERENT CHRONIC DISEASES}

Several clinical trials have been conducted to evaluate the effect of "guggul" from Commiphora and Boswellia on various chronic disorders. Human studies on guggul has 
TABLE 2 | In vivo biological activities of guggul (Commiphora and Boswellia) against various chronic diseases.

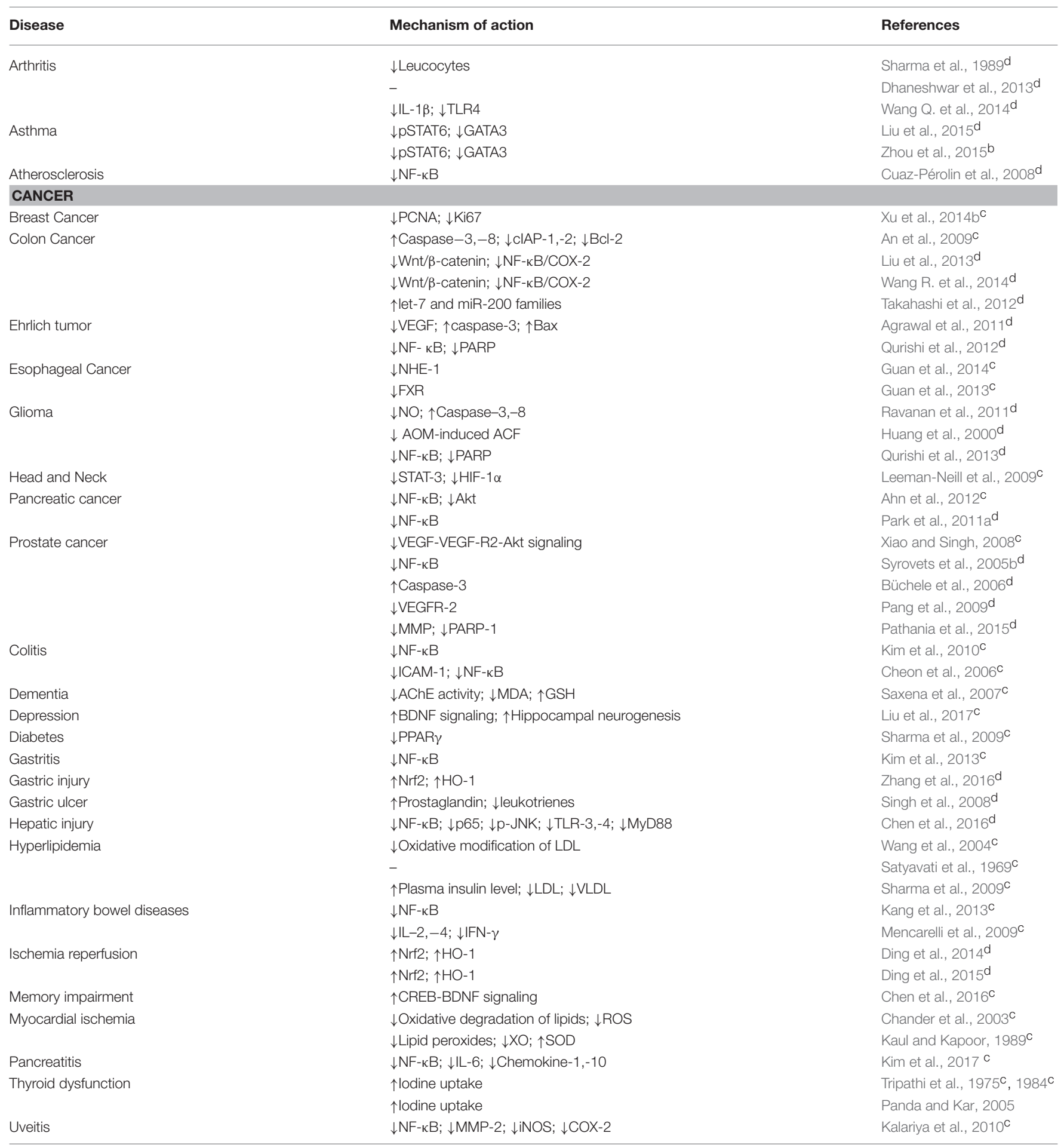

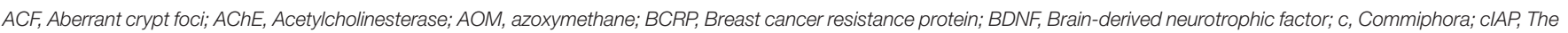

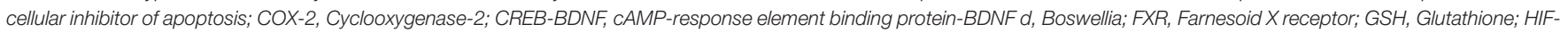

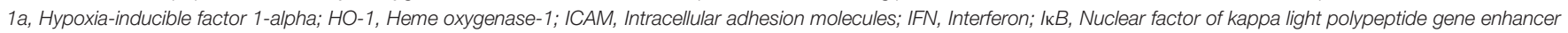

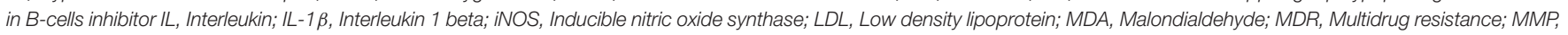

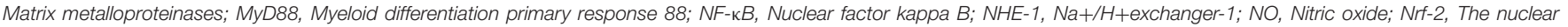

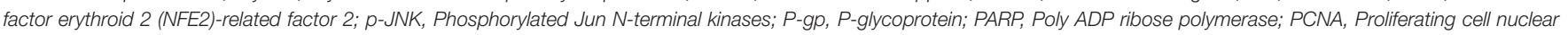

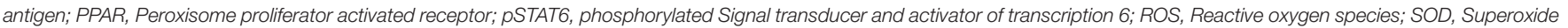

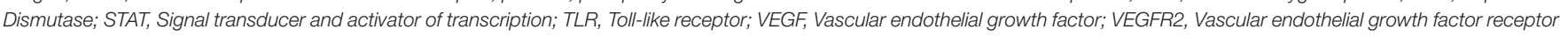
2; $X O$, Xanthine oxidase. 
been found to be effective against different diseases such as asthma, breast fibroadenoma, chronic kidney disease, colitis, Crohn's disease, fascioliasis, hepatitis C, hypercholesterolaemia, hyperlipidemia, metabolic syndrome, nodulocystic acne, arthritis, schistosomiasis, stress urinary incontinence etc. (Table 3; Figure 4).

\section{Arthritis}

Arthritis is mainly caused due to inflammation of joints, the tissues surrounding the joints and other connective tissues. Osteoarthritis is the most common form of arthritis which affects a wide range of people across all the places. As guggul has been reported to exhibit high affectivity against arthritis pre-clinically; hence, its effect was evaluated in the clinical setting as well. In one such study, 30 patients with arthritis were treated with gum guggul for 1 month which resulted in remarkable improvement in the total scores of Western Ontario and MacMaster Osteoarthritis Index and condition of the patients (Singh et al., 2003). Another study was conducted by Kimmatkar et al., to check the safety, tolerability, and efficacy of $B$. serrata extract in 30 patients with knee osteoarthritis. The patients receiving drug treatment reported a decrease in knee pain and swelling of the knee joint as well as increased knee flexion and walking distance (Kimmatkar et al., 2003).

\section{Asthma}

Asthma is a chronic multifactorial inflammatory disease of the respiratory tract and is one of the major health concern. Notably, Boswellia has been found to be effective in the treatment of this disease. In a clinical study, 40 patients having 23 males, and 17 females in the age range of 18-75 years, suffering from bronchial asthma were treated with $300 \mathrm{mg}$ of gum resin thrice daily for a period of 6 weeks. This led to improved prognosis in around $70 \%$ of the patients as various signs and symptoms of bronchial asthma like rhonchi, dyspnoea, and attacks disappeared upon treatment (Gupta et al., 1998).

\section{Breast Fibroadenomas}

Breast fibroadenoma accounts for the majority of breast lumps in young women. Boswellia was found to exert beneficial effect against breast fibroadenomas as evinced by a study conducted by Pasta and group. They showed that treatment with the combination of Boswellia, betaine, and myo-inositol resulted in decreased fibroadenoma dimension in young women without exerting any toxic effects. The combination also resulted in reduced fibroadenoma volume in $38.8 \%$ of the patients in the experimental group, whereas the same was observed only in $17.85 \%$ patients in the placebo group (Pasta et al., 2016).

\section{Cardiovascular Diseases (CVDs)}

CVDs, a group of diseases which involves the heart and the blood vessels is one of the most common causes of death across the globe. Notably, guggul presents a potent remedy for cardiovascular diseases. For example, Singh and group conducted a study to evaluate the cardioprotective benefits of guggul by enrolling 200 patients suffering from ischemic heart disease. The patients were treated with the combination of gum guggul and Inula racemosa for 6 months which resulted in the reduced levels of total cholesterol, triglyceride, and total blood lipids in the patients. It also restored the normal electrocardiogram (ECG) in $26 \%$ of the patients, showed improvement of ECG in 59\% of the patients and lessened the chest pain in $25 \%$ of the patients (Singh et al., 1993).

\section{Chronic Kidney Disease}

Chronic kidney disease (CKD) is a progressive disease where occurs due to enhanced inflammation and oxidative stress leading to reduced kidney function. Studies have indicated $B$. serrata in combination with Curcuma longa as an effective regimen to obtain reduced inflammation in patients with $\mathrm{CKD}$ which functioned via modulation of prostaglandin $\mathrm{E}_{2}\left(\mathrm{PGE}_{2}\right)$ (Shelmadine et al., 2017). Moreover, this regimen was found to be safe, well tolerated which also enhanced the levels of inflammatory cytokines in CKD patients (Moreillon et al., 2013).

\section{Diabetes Mellitus}

A large population of the world is affected by diabetes mellitus or type 2 diabetes. Several preclinical studies have shown that the gum resin of commiphora and boswellia are highly effective against this disease. In a clinical study conducted by Ahangarpour et al., it was observed that the treatment of patients with diabetes mellitus with B. serrata gum resin (900 mg daily for 6 weeks orally) resulted in decreased risk factors associated with this disease. Further, the treatment also helped in maintaining fructosamine levels, hepatic enzyme activities, and to bring lipid profiles close to normal levels in the patients (Ahangarpour et al., 2014).

\section{Eczema and Psoriasis}

Eczema, also known as dermatitis and psoriasis are caused mainly due to inflammation of the skin. Boswellia has been found to exert effectiveness against eczema and psoriasis. A group of scientists revealed that Boswellia-based cream lessens the use of topical corticosteroids and can diminish the grade of erythema and the skin superficial symptoms (Togni et al., 2015). Further, in a double blind study, the efficacy of a novel formulation of BA (Bosexil $^{\circledR}$ ) containing B. serrata resin extract and lecithin was evaluated against both psoriasis and eczema. Improvement in psoriasis, scales (70\% of cases), and erythema (50\% of cases) was observed with Bosexil ${ }^{\circledR}$ compared to placebo. In addition, when eczema patients were administrated with Bosexil ${ }^{\circledR}$ formulation, it showed improvement in both erythema (60\% of cases) and itch ( $60 \%$ of cases) of the patients without any case of waning (Togni et al., 2014).

\section{Fascioliasis}

Fascioliasis is a parasitic worm infection caused by the common liver fluke Fasciola hepatica and Fasciola gigantica. The formulation of myrhh, the gum resisn of Commiphora molmol was reported to be safe, well tolerated, and effective for the management of this disease. The formulated drug comprised of 8 parts of resin and 3.5 parts of volatile oils, all extracted from myrrh. They observed that 7 patients who were passing fasciola 
TABLE 3 | Clinical trials of guggul (Commiphora and Boswellia) against various chronic diseases.

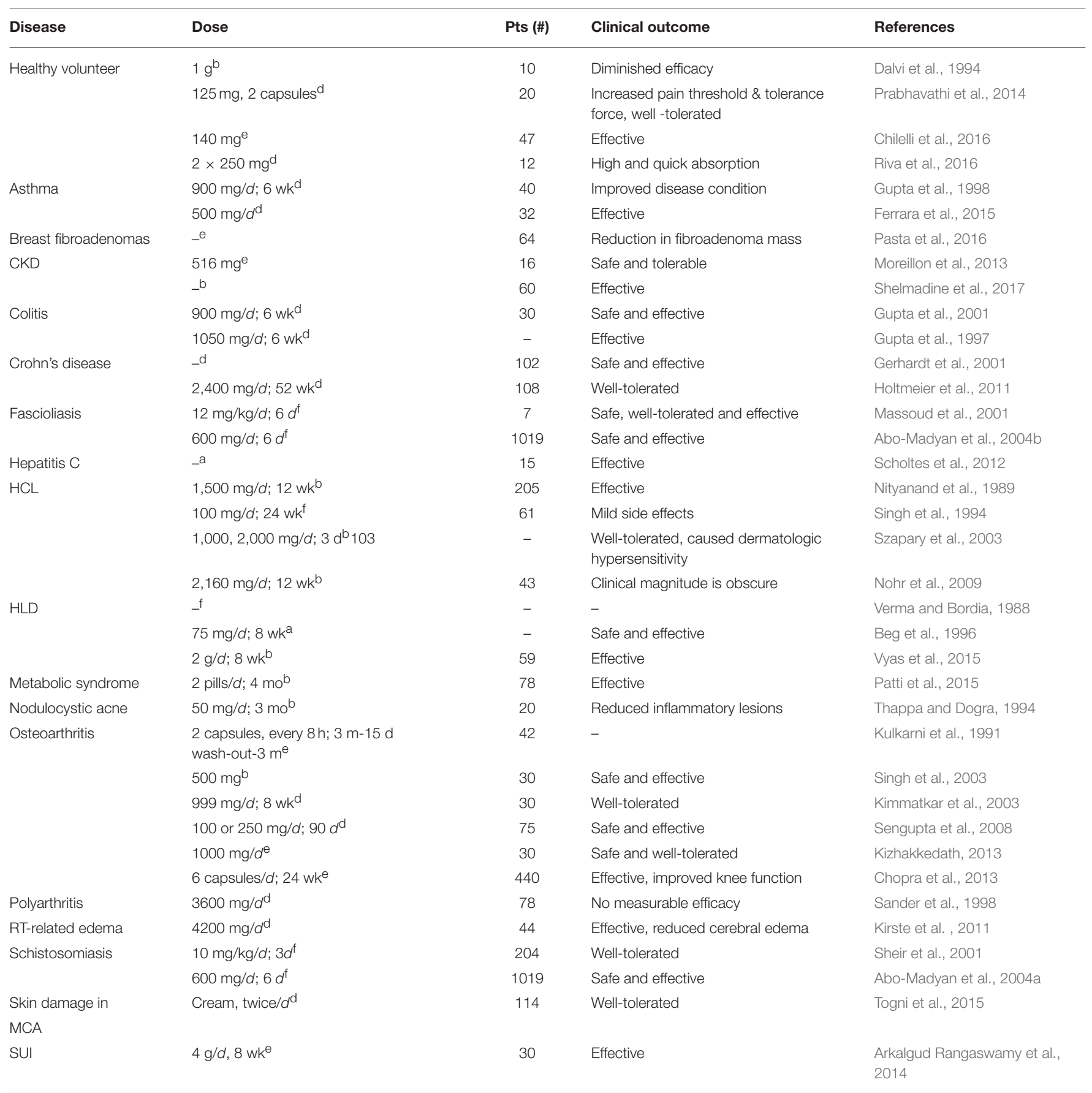

CKD, Chronic kidney disease; d, Day; HCL, Hypercholesterolemia; HLD, Hyperlipidemia; MCA, Mammary carcinoma; mo, Month; wk, Week; RT, Radiotherapy; SUI, Stress urinary incontinence; $a$, Gugglusterone; b, Guggul; $c$, Formulation of guggul; $d$, Boswellia; e, Formulation of Boswellia; $f$, Commiphora.

eggs in their stools displayed distinct improvement of the general condition, drop in the egg count, and improvement of all signs and symptoms with no adverse side effects after treatment with the drug (Massoud et al., 2001).

\section{Gingivitis}

Gingivitis, the inflammation of gingiva is a very common form of gum disease. Frankincense extract has been found to exhibit efficacy against gingivitis. A double blinded randomized placebo controlled trial was conducted among 75 female patients aged between 15 and 18 years with moderate plaque-induced gingivitis. Six groups were randomly formed based on the administration of $0.1 \mathrm{~g}$ of frankincense extract, $0.2 \mathrm{~g}$ of its powder, placebo, and whether the patients have undergone scaling and root planning (SRP) or not. Gingival index, plaque index, bleeding index, and probing pocket depth were measured on the 0,7 th, and 14 th days of the study. Detailed analysis of the data revealed that SRP along with the application of 
frankincense extract or powder might cause significant decrease in inflammatory indices in comparison to the groups without drug therapy and SRP (Khosravi Samani et al., 2011).

\section{Inflammatory Bowel Disease}

Different clinical studies with guggul have shown its efficacy against IBDs which include colitis and Crohn's disease. For instance, the gum resin of $B$. serrata was found to be effective in the treatment of chronic colitis with minimal side effects in a clinical study conducted by Gupta et al. In this study, the patients with chronic colitis were treated with gum resin from B. serrata at a dose of $900 \mathrm{mg}$ daily divided in three doses for 6 weeks. The treatment resulted in the improvement of stool properties, hemoglobin, serum iron, calcium, phosphorus, proteins, total leukocytes, and eosinophils in the patients (Gupta et al., 2001). Further, a double-blind, placebo-controlled, randomized, parallel study on 82 patients with Crohn's disease was conducted where patients were given a new B. serrata extract; Boswelan. In this trial, remission was observed in $59.9 \%$ of the actively treated patients. Additionally, this study also confirmed better tolerability of Boswelan in long-term treatment of Crohn's disease (Holtmeier et al., 2011). Furthermore, leukotrienes play an important role in inflammation of the colon in ulcerative colitis. Sallai guggul gum resin is known to be specific, non-redox, and non-competitive inhibitors of 5-LOX, a crucial enzyme of leukotriene biosynthesis. Patients with grade II and III ulcerative colitis were treated with $B$. serrata gum resin at a dose of $350 \mathrm{mg}$ thrice daily for 6 weeks. Stool properties, histopathology, and scan microscopy of rectal biopsies, blood parameters including hemoglobin, serum iron, calcium, phosphorus, proteins, total leukocytes, and eosinophils showed slightly better improvement in Boswellia treated patients (Gupta et al., 1997).

\section{Nodulocystic Acne}

Guggulipid is considered to be very effective in topical and oral complementary as well as an alternative medicine (CAM) for the treatment of acne (Magin et al., 2006). In a clinical study conducted by Thappa and Dogra, patients with nodulocystic acne were given guggulipid equivalent to $25 \mathrm{mg}$ GS for 3 months, which resulted in progressive reduction in lesions in majority of patients. However, patients with oily faces displayed better response to guggulipid (Thappa and Dogra, 1994).

Thus, these clinical studies well evince the potential effect of Commiphora and Boswellia on different chronic diseases. However, more studies are inevitable to establish them as cutting edge strategy for the treatment of diverse human diseases.

\section{REFERENCES}

Abo-Madyan, A. A., Morsy, T. A., and Motawea, S. M. (2004a). Efficacy of Myrrh in the treatment of schistosomiasis (haematobium and mansoni) in Ezbet ElBakly, Tamyia Center, El-Fayoum Governorate, Egypt. J. Egypt Soc. Parasitol. $34,423-446$.

Abo-Madyan, A. A., Morsy, T. A., Motawea, S. M., and Morsy, A. T. (2004b). Clinical trial of Mirazid in treatment of human fascioliasis, Ezbet El-Bakly (Tamyia Center) Al-Fayoum Governorate. J. Egypt. Soc. Parasitol. 34, 807-818.

\section{CONCLUSION}

Since ancient times, Commiphora and Boswellia are considered as important traditional medicinal plants which are used for the treatment of various ailments. Guggul isolated from Commiphora and Boswellia have immense therapeutic potential against several diseases and it has been well established by numerous in vitro, in vivo, and clinical studies. Guggul was used traditionally for the treatment of inflammation and hyperlipidemia, but with the extensive studies on guggul and associated molecular mechanisms unveiled newer insights of its use for the treatment of various other chronic diseases as well. Gum resin guggul possesses multiple pharmacological activities especially hypolipidemic, antiobesity, anti-inflammatory, anti-tumor effects, cardioprotective, neuroprotective, hepatoprotective, thyroid stimulatory effects etc. It effectively regulates different transcription factors, enzymes, cytokines, and anti-apoptotic proteins which are involved in inflammation, carcinogenesis, and other chronic diseases. Further, Commiphora in combination with other ayurvedic herbs is commercially available and marketed for the treatment and cure of arthritis, obesity and associated side effects of the disease. Many patents are also filed and approved to use guggul as a constituent of polyherbal formulations and cosmetics. Therefore, taking the medicinal importance and commercial use of guggul into consideration, it can be advocated to possess substantial therapeutic potential against diverse chronic disorders. However, more in vitro, in vivo, and well-designed clinical studies are required to validate the clinical usefulness of guggul and to obtain a potent herbal derived drug with enhanced efficacy, minimal side effects and strong disease combating properties.

\section{AUTHOR CONTRIBUTIONS}

$\mathrm{BA}$ and $\mathrm{AK}$ contributed to study design and writing of the manuscript. $\mathrm{KB}$ and $\mathrm{DB}$ carried out literature survey, writing and artwork. $\mathrm{CH}, \mathrm{BS}$, and NR contributed to the making of the tables and artwork. SG and GP performed proofreading of the manuscript.

\section{ACKNOWLEDGMENTS}

This work was supported by BT/529/NE/TBP/2013 grant awarded to AK by Department of Biotechnology (DBT), Government of India. The author KB acknowledges UGC, New Delhi, India for providing the fellowship. The author BS acknowledges DST-INSPIRE for providing her the fellowship.

Agrawal, S. S., Saraswati, S., Mathur, R., and Pandey, M. (2011). Antitumor properties of Boswellic acid against Ehrlich ascites cells bearing mouse. Food Chem. Toxicol. 49, 1924-1934. doi: 10.1016/j.fct.2011.04.007

Ahangarpour, A., Heidari, H., Fatemeh, R. A., Pakmehr, M., Shahbazian, H., Ahmadi, I., et al. (2014). Effect of B. serrate supplementation on blood lipid, hepatic enzymes and fructosamine levels in type2 diabetic patients. J. Diabetes Metab. Disord. 13:29. doi: 10.1186/2251-6581-13-29

Ahn, D. W., Seo, J. K., Lee, S. H., Hwang, J. H., Lee, J. K., Ryu, J. K., et al. (2012). Enhanced antitumor effect of combination therapy with 
gemcitabine and guggulsterone in pancreatic cancer. Pancreas 41, 1048-1057. doi: 10.1097/MPA.0b013e318249d62e

Ahn, S. I., and Youn, H. S. (2008). Guggulsterone suppresses the activation of NF$\mathrm{kB}$ and expression of COX-2 induced by toll-like receptor 2, 3, and 4 agonists. Food Sci. Biotechnol. 17, 1294-1298.

Al-Howiriny, T. A., Al-Sohaibani, M. O., Al-Said, M. S., Al-Yahya, M. A., El-Tahir, K. H., and Rafatullah, S. (2004). Hepatoprotective properties of Commiphora opobalsamum ("Balessan"), a traditional medicinal plant of Saudi Arabia. Drugs Exp. Clin. Res. 30, 213-220. doi: 10.1055/s-0034-1382426

Almazari, I., Park, J. M., Park, S. A., Suh, J. Y., Na, H. K., Cha, Y. N., et al. (2012). Guggulsterone induces heme oxygenase-1 expression through activation of Nrf2 in human mammary epithelial cells: PTEN as a putative target. Carcinogenesis 33, 368-376. doi: 10.1093/carcin/bgr259

Ammon, H. P. (2016). Boswellic acids and their role in chronic inflammatory diseases. Adv. Exp. Med. Biol. 928, 291-327. doi: 10.1007/978-3-319-41334-1_13

Ammon, H. P., Safayhi, H., Mack, T., and Sabieraj, J. (1993). Mechanism of antiinflammatory actions of curcumine and boswellic acids. J. Ethnopharmacol.38, 113-119. doi: 10.1016/0378-8741(93)90005-P

An, M. J., Cheon, J. H., Kim, S. W., Kim, E. S., Kim, T. I., and Kim, W. H. (2009). Guggulsterone induces apoptosis in colon cancer cells and inhibits tumor growth in murine colorectal cancer xenografts. Cancer Lett. 279, 93-100. doi: 10.1016/j.canlet.2009.01.026

Arkalgud Rangaswamy, P., Sultana, A., Rahman, K., and Nagapattinam, S. (2014). Efficacy of Boswellia serrata L. and Cyperus scariosus L. plus pelvic floor muscle training in stress incontinence in women of reproductive age. Complement. Ther. Clin. Pract. 20, 230-236. doi: 10.1016/j.ctcp.2014.08.003

Arora, R. B., Kapoor, V., Gupta, S. K., and Sharma, R. C. (1971). Isolation of a crystalline steroidal compound from C. mukul and its anti-inflammatory activity. Indian J. Exp. Biol. 9, 403-404.

Arora, R. B., Taneja, V., Sharma, R. C., and Gupta, S. K. (1972). Anti-inflammatory studies on a crystalline steroid isolated from C. mukul. Indian J. Med. Res. 60, 929-931.

Baldwa, V. S., Bhasin, V., Ranka, P. C., and Mathur, K. M. (1981). Effects of C. mukul (Guggul) in experimentally induced hyperlipemia and atherosclerosis. J. Assoc. Phys. India 29, 13-17.

Banik, K., Harsha, C., Bordoloi, D., Lalduhsaki Sailo, B., Sethi, G., Leong, H. C., et al. (2018). Therapeutic potential of gambogic acid, a caged xanthone, to target cancer. Cancer Lett. 416, 75-86. doi: 10.1016/j.canlet.2017.12.014

Barrett, T. Q., Kristiansen, L. H., and Ovesen, T. (2003). NF-kappaB in cultivated middle ear epithelium Int. J. Pediatr. Otorhinolaryngol. 67, 895-903. doi: 10.1016/S0165-5876(03)00137-X

Batra, S., Srivastava, S., Singh, K., Chander, R., Khanna, A. K., and Bhaduri, A. P. (2000). Syntheses and biological evaluation of 3- substituted amino-1-aryl6-hydroxy- hex-2-ene-1-ones as antioxidant and hypolipidemic agents. Bioorg. Med. Chem. 8, 2195-2209. doi: 10.1016/S0968-0896(00)00159-0

Beg, M., Singhal, K. C., and Afzaal, S. (1996). A study of effect of guggulsterone on hyperlipidemia of secondary glomerulopathy. Indian J. Physiol. Pharmacol. 40, 237-240.

Bhushan, S., Kumar, A., Malik, F., Andotra, S. S., Sethi, V. K., Kaur, I. P., et al. (2007). A triterpenediol from B. serrata induces apoptosis through both the intrinsic and extrinsic apoptotic pathways in human leukemia HL-60 cells. Apoptosis 12, 1911-1926. doi: 10.1007/s10495-007-0105-5

Bordoloi, D., Roy, N. K., Monisha, J., Padmavathi, G., and Kunnumakkara, A. B. (2016). Multi-targeted agents in cancer cell chemosensitization: what we learnt from curcumin thus far. Recent Pat. Anticancer. Drug Discov. 11, 67-97. doi: $10.2174 / 1574892810666151020101706$

Büchele, B., Zugmaier, W., and Simmet, T. (2003). Analysis of pentacyclic triterpenic acids from frankincense gum resins and related phytopharmaceuticals by high-performance liquid chromatography. Identification of lupeolic acid, a novel pentacyclic triterpene. J. Chromatogr. B. 791, 21-30. doi: 10.1016/S1570-0232(03)00160-0

Büchele, B., Zugmaier, W., Estrada, A., Genze, F., Syrovets, T., Paetz, C., et al. (2006). Characterization of 3alpha-acetyl-11-keto-alpha-boswellic acid, a pentacyclic triterpenoid inducing apoptosis in vitro and in vivo. Planta Med.72, 1285-1289. doi: 10.1055/s-2006-951680

Burris, T. P., Montrose, C., Houck, K. A., Osborne, H. E., Bocchinfuso, W. P., Yaden, B. C., et al. (2005). The hypolipidemic natural product guggulsterone is a promiscuous steroid receptor ligand. Mol. Pharmacol. 67, 948-954. doi: $10.1124 / \mathrm{mol} .104 .007054$

Chander, R., Rizvi, F., Khanna, A. K., and Pratap, R. (2003). Cardioprotective activity of synthetic guggulsterone ( $\mathrm{E}$ and Zisomers) in isoproterenol induced myocardial ischemia in rats: a comparative study. Indian J. Clin. Biochem. 18, 71-79. doi: 10.1007/BF02867370

Chashoo, G., Singh, S. K., Sharma, P. R., Mondhe, D. M., Hamid, A., Saxena, A., et al. (2011). A propionyloxy derivative of 11-keto- $\beta$-boswellic acid induces apoptosis in HL-60 cells mediated through topoisomerase I \& II inhibition. Chem. Biol. Interact. 189, 60-71. doi: 10.1016/j.cbi.2010.10.017

Chen, Z., Huang, C., and Ding, W. (2016). Z-guggulsterone improves the scopolamine-induced memory impairments through enhancement of the BDNF signal in C57BL/6J mice. Neurochem. Res. 41, 3322-3332. doi: 10.1007/s11064-016-2064-0

Cheon, J. H., Kim, J. S., Kim, J. M., Kim, N., Jung, H. C., and Song, I. S. (2006). Plant sterol guggulsterone inhibits nuclear factor-kappaB signaling in intestinal epithelial cells by blocking IkappaB kinase and ameliorates acute murine colitis. Inflamm. Bowel Dis. 12, 1152-1161. doi: 10.1097/01.mib.0000235830.94057.c6

Chilelli, N. C., Ragazzi, E., Valentini, R., Cosma, C., Ferraresso, S., Lapolla, A., and Sartore, G. (2016). Curcumin and Boswellia serrata modulate the glyco-oxidative status and lipo-oxidation in master athletes. Nutrients 8:E745. doi: 10.3390/nu8110745

Chopra, A., Saluja, M., Tillu, G., Sarmukkaddam, S., Venugopalan, A., Narsimulu, G., et al. (2013). Ayurvedic medicine offers a good alternative to glucosamine and celecoxib in the treatment of symptomatic knee osteoarthritis: a randomized, double-blind, controlled equivalence drug trial. Rheumatology 52, 1408-1417. doi: 10.1093/rheumatology/kes414

Choudhuri, R., Degraff, W., Gamson, J., Mitchell, J. B., and Cook, J. A. (2011). Guggulsterone-mediated enhancement of radiosensitivity in human tumor cell lines. Front. Oncol. 1:19. doi: 10.3389/fonc.2011.00019

Cuaz-Pérolin, C., Billiet, L., Baugé, E., Copin, C., Scott-Algara, D., Genze, F., et al. (2008). Antiinflammatory and antiatherogenic effects of the NF-kappaB inhibitor acetyl-11-keto-beta-boswellic acid in LPSchallenged ApoE-/- mice. Arterioscler. Thromb. Vasc. Biol. 28, 272-277. doi: 10.1161/ATVBAHA.107.155606

Cui, J., Huang, L., Zhao, A., Lew, J. L., Yu, J., Sahoo, S., et al. (2003). Guggulsterone is a farnesoid $\mathrm{X}$ receptor antagonist in coactivator association assays but acts to enhance transcription of bile salt export pump. J. Biol. Chem. 278, 10214-10220. doi: 10.1074/jbc.M209323200

Dalvi, S. S., Nayak, V. K., Pohujani, S. M., Desai, N. K., Kshirsagar, N. A., and Gupta, K. C. (1994). Effect of gugulipid on bioavailability of diltiazem and propranolol. J. Assoc. Physicians India 42, 454-455.

De Gottardi, A., Dumonceau, J. M., Bruttin, F., Vonlaufen, A., Morard, I., Spahr, L., et al. (2006). Expression of the bile acid receptor FXR in Barrett's esophagus and enhancement of apoptosis by guggulsterone in vitro. Mol. Cancer 5:48. doi: 10.1186/1476-4598-5-48

Deng, R. (2007). Therapeutic effects of guggul and its constituent guggulsterone: cardiovascular benefits. Cardiovasc. Drug Rev. 25, 375-390. doi: 10.1111/j.1527-3466.2007.00023.x

Dhaneshwar, S., Dipmala, P., Abhay, H., and Prashant, B. (2013). Diseasemodifying effect of anthraquinone prodrug with boswellic acid on collagenaseinduced osteoarthritis in Wistar rats. Inflamm. Allergy Drug Targets 12, 288-295. doi: 10.2174/18715281113129990002

Ding, Y., Chen, M., Wang, M., Li, Y., and Wen, A. (2015). Posttreatment with 11-Keto- $\beta$-boswellic acid ameliorates cerebral ischemia-reperfusion injury: Nrf2/HO-1 pathway as a potential mechanism. Mol. Neurobiol. 52, 1430-1439. doi: 10.1007/s12035-014-8929-9

Ding, Y., Chen, M., Wang, M., Wang, M., Zhang, T., Park, J., et al. (2014). Neuroprotection by acetyl-11-keto- $\beta$-Boswellic acid, in ischemic brain injury involves the Nrf2/HO-1 defense pathway. Sci. Rep. 4:7002. doi: 10.1038/srep07002

Dixit, D., Ghildiyal, R., Anto, N. P., Ghosh, S., Sharma, V., and Sen, E. (2013). Guggulsterone sensitizes glioblastoma cells to Sonic hedgehog inhibitor SANT-1 induced apoptosis in a Ras/NFkB dependent manner. Cancer Lett. 336, 347-358. doi: 10.1016/j.canlet.2013. 03.025

Dixit, V. P., Joshi, S., Sinha, R., Bharvava, S. K., and Varma, M. (1980). Hypolipidemic activity of guggal resin (C. mukul) and garlic (Alium sativum 
linn.) in dogs (Canis familiaris) and monkeys (Presbytis entellus entellus Dufresne). Biochem. Exp. Biol. 16, 421-424.

Du, Z., Liu, Z., Ning, Z., Liu, Y., Song, Z., Wang, C., et al. (2015). Prospects of boswellic acids as potential pharmaceutics. Planta Med. 81, 259-271. doi: 10.1055/s-0034-1396313

Fan, A. Y., Lao, L., Zhang, R. X., Zhou, A. N., Wang, L. B., Moudgil, K. D., et al. (2005). Effects of an acetone extract of B. carterii Birdw. (Burseraceae) gum resin on adjuvant-induced arthritis in lewis rats. J. Ethnopharmacol. 101, 104-109. doi: 10.1016/j.jep.2005.03.033

Ferrara, T., De Vincentiis, G., and Di Pierro, F. (2015). Functional study on Boswellia phytosome as complementary intervention in asthmatic patients. Eur. Rev. Med. Pharmacol. Sci. 19, 3757-3762.

Francis, J. A., Raja, S. N., and Nair, M. G. (2004). Bioactive terpenoids and guggulsteroids from $C$. mukul gum resin of potential anti-inflammatory interest. Chem. Biodiversity 1, 1842-1853. doi: 10.1002/cbdv.200490138

Frank, M. B., Yang, Q., Osban, J., Azzarello, J. T., Saban, M. R., Saban, R., et al. (2009). Frankincense oil derived from Boswellia carteri induces tumor cell specific cytotoxicity. BMC Complement. Altern. Med. 9:6. doi: 10.1186/1472-6882-9-6

Gerhardt, H., Seifert, F., Buvari, P., Vogelsang, H., and Repges, R. (2001). Therapy of active Crohn disease with Boswellia serrata extract H 15. Z. Gastroenterol. 39, 11-17. doi: 10.1055/s-2001-10708

Glaser, T., Winter, S., Groscurth, P., Safayhi, H., Sailer, E. R., Ammon, H. P., et al. (1999). Boswellic acids and malignant glioma: induction of apoptosis but no modulation of drug sensitivity. Br. J. Cancer. 80, 756-765. doi: $10.1038 /$ sj.bjc.6690419

Guan, B., Hoque, A., and Xu, X. (2014). Amiloride and guggulsterone suppression of esophageal cancer cell growth in vitro and in nude mouse xenografts. Front. Biol. 9, 75-81. doi: 10.1007/s11515-014-1289-z

Guan, B., Li, H., Yang, Z., Hoque, A., and Xu, X. (2013). Inhibition of farnesoid $\mathrm{X}$ receptor controls esophageal cancer cell growth in vitro and in nude mouse xenografts. Cancer 119, 1321-1329. doi: 10.1002/cncr.27910

Gupta, I., Gupta, V., Parihar, A., Gupta, S., Lüdtke, R., Safayhi, H., et al. (1998). Effects of $B$. serrata gum resin in patients with bronchial asthma: results of a double-blind, placebo-controlled, 6-week clinical study. Eur. J. Med. Res. 3, 511-514.

Gupta, I., Parihar, A., Malhotra, P., Gupta, S., Lüdtke, R., Safayhi, H., et al. (2001). Effects of gum resin of $B$. serrata in patients with chronic colitis. Planta Med. 67, 391-395. doi: 10.1055/s-2001-15802

Gupta, I., Parihar, A., Malhotra, P., Singh, G. B., Lüdtke, R., Safayhi, H., et al. (1997). Effects of B. serrata gum resin in patients with ulcerative colitis. Eur. J. Med. Res. 2, 37-43.

Hanus, L. O., Rezanka, T., Dembitsky, V. M., and Moussaieff, A. (2005). Myrrhcommiphora chemistry. Biomed. Papers 149, 3-28. doi: 10.5507/bp.2005.001

Harsha, C., Banik, K., Bordoloi, D., and Kunnumakkara, A. B. (2017). Antiulcer properties of fruits and vegetables: a mechanism based perspective. Food Chem. Toxicol. 108, 104-119. doi: 10.1016/j.fct.2017.07.023

Hazra, A. K., Sur, T. K., Chakraborty, B., and Seal, T. (2018). HPLC analysis of phenolic acids and antioxidant activity of some classical ayurvedic guggulu formulations. Int. J. Res. Ayurveda Pharm. 9, 112-117. doi: $10.7897 / 2277-4343.09122$

Hoernlein, R. F., Orlikowsky, T. H., Zehrer, C., Niethammer, D., Sailer, E. R., Simmet, T., et al. (1999). Acetyl-11-keto-beta-boswellic acid induces apoptosis in HL-60 and CCRF-CEM cells and inhibits topoisomerase I. J. Pharmacol. Exp. Ther. 288, 613-619.

Holtmeier, W., Zeuzem, S., Preiss, J., Kruis, W., Böhm, S., Maaser, C., et al. (2011). Randomized, placebo-controlled, double- blind trial of B. serrata in maintaining remission of Crohn's disease: good safety profile but lack of efficacy. Inflamm. Bowel Dis. 17, 573-582. doi: 10.1002/ibd.21345

Hostanska, K., Daum, G., and Saller, R. (2002). Cytostatic and apoptosis-inducing activity of boswellic acids toward malignant cell lines in vitro. Anticancer Res. $22,2853-2862$

Huang, C., Wang, J., Lu, X., Hu, W., Wu, F., Jiang, B., et al. (2016). Z-guggulsterone negatively controls microglia-mediated neuroinflammation via blocking ІкB$\alpha$-NF-kB signals. Neurosci. Lett. 619, 34-42. doi: 10.1016/j.neulet.2016.02.021

Huang, M. T., Badmaev, V., Ding, Y., Liu, Y., Xie, J. G., and Ho, C. T. (2000). Anti-tumor and anti-carcinogenic activities of triterpenoid, beta-boswellic acid. Biofactors 13, 225-230. doi: 10.1002/biof.5520130135
Ichikawa, H., and Aggarwal, B. B. (2006). Guggulsterone inhibits osteoclastogenesis induced by receptor activator of nuclear factor-kappaB ligand and by tumor cells by suppressing nuclear factor-kappaB activation. Clin. Cancer Res. 12, 662-668. doi: 10.1158/1078-0432.CCR-05-1749

Jiang, G., Xiao, X., Zeng, Y., Nagabhushanam, K., Majeed, M., and Xiao, D. (2013). Targeting beta-catenin signaling to induce apoptosis in human breast cancer cells by z-guggulsterone and Gugulipid extract of Ayurvedic medicine plant Commiphora mukul. BMC Complement. Altern. Med. 13:203. doi: 10.1186/1472-6882-13-203

Jing, Y., Nakajo, S., Xia, L., Nakaya, K., Fang, Q., Waxman, S., et al. (1999). Boswellic acid acetate induces differentiation and apoptosis in leukemia cell lines. Leuk. Res. 23, 43-50. doi: 10.1016/S0145-2126(98)00096-4

Kalariya, N. M., Shoeb, M., Reddy, A. B., Zhang, M., Van Kuijk, F. J., and Ramana, K. V. (2010). Prevention of endotoxin-induced uveitis in rats by plant sterol guggulsterone. Invest. Ophthalmol. Vis. Sci. 51, 5105-5113. doi: $10.1167 /$ iovs. $09-4873$

Kang, S. J., Kim, J. M., Koh, S. J., Kim, S. H., Im, J. P., Jung, H. C., et al. (2013). The guggulsterone derivative GG-52 inhibits NF- $\mathrm{kB}$ signaling in bone marrowderived dendritic cells and attenuates colitis in IL-10 knockout mice. Life Sci. 92, 1064-1071. doi: 10.1016/j.lfs.2013.04.003

Kaul, S., and Kapoor, N. K. (1989). Reversal of changes of lipid peroxide, xanthine oxidase and superoxide dismutase by cardio-protective drugs in isoproterenol induced myocardial necrosis in rats. Indian. J. Exp. Biol. 27, 625-627.

Khan, M. K., Ansari, I. A., and Khan, M. S. (2013), Dietary phytochemicals as potent chemotherapeutic agents against breast cancer: inhibition of NF-kappaB pathway via molecular interactions in rel homology domain of its precursor protein p105. Pharmacogn. Mag. 9, 51-57. doi: 10.4103/0973-1296.108140

Khan, S., Kaur, R., Shah, B. A., Malik, F., Kumar, A., Bhushan, S., et al. (2012). A novel cyano derivative of 11 -keto- $\beta$-boswellic acid causes apoptotic death by disrupting PI3K/AKT/Hsp-90 cascade, mitochondrial integrity, and other cell survival signaling events in HL-60 cells. Mol. Carcinog. 51, 679-695. doi: $10.1002 / \mathrm{mc} .20821$

Khanna, D. S., Agarwal, O. P., Gupta, S. K., and Arora, R. B. (1969). A biochemical approach to anti-atherosclerotic action of Commiphora-mukul: an Indian indigenous drug in Indian domestic pigs (Sus scrofa). Indian J. Med. Res. 57, 900-906.

Khosravi Samani, M., Mahmoodian, H., Moghadamnia, A., Poorsattar Bejeh Mir, A., and Chitsazan, M. (2011). The effect of Frankincense in the treatment of moderate plaque-induced gingivitis: a double blinded randomized clinical trial. Daru 19, 288-294.

Kim, B. H., Yoon, J. H., Yang, J. I., Myung, S. J., Lee, J. H., Jung, E. U., et al. (2013). Guggulsterone attenuates activation and survival of hepatic stellate cell by inhibiting nuclear factor kappa B activation and inducing apoptosis. J. Gastroenterol. Hepatol. 28, 1859-1868. doi: 10.1111/jgh.12314

Kim, D. G., Bae, G. S., Choi, S. B., Jo, I. J., Shin, J. Y., Lee, S. K., et al. (2015). Guggulsterone attenuates cerulein-induced acute pancreatitis via inhibition of ERK and JNK activation. Int. Immunopharmacol. 26, 194-202. doi: 10.1016/j.intimp.2015.03.030

Kim, D. G., Bae, G. S., Jo, I. J., Choi, S. B., Kim, M. J., Jeong, J. H., et al. (2016). Guggulsterone attenuated lipopolysaccharideinduced inflammatory responses in mouse inner medullary collecting duct-3 cells. Inflammation 39, 87-95. doi: 10.1007/s10753-0150226-x

Kim, E. S., Hong, S. Y., Lee, H. K., Kim, S. W., An, M. J., Kim, T. I., et al. (2008). Guggulsterone inhibits angiogenesis by blocking STAT3 and VEGF expression in colon cancer cells. Oncol. Rep. 20, 1321-1327. doi: 10.3892/or_00000147

Kim, J. M., Kang, H. W., Cha, M. Y., Yoo, D., Kim, N., Kim, I. K., et al. (2010). Novel guggulsterone derivative GG-52 inhibits NF-kappaB signaling in intestinal epithelial cells and attenuates acute murine colitis. Lab. Invest. 90, 1004-1015. doi: 10.1038/labinvest.2010.54

Kim, N., Park, J. M., Lee, S. H., Kim, B. H., Son, J. H., Ryu, J. K., et al. (2017). Effect of combinatory treatment with resveratrol and guggulsterone on mild acute pancreatitis in mice. Pancreas 46, 366-371. doi: 10.1097/MPA.0000000000000763

Kimmatkar, N., Thawani, V., Hingorani, L., and Khiyani, R. (2003). Efficacy and tolerability of $B$. serrata extract in treatment of osteoarthritis of kneea randomized double blind placebo controlled trial. Phytomedicine 10, 3-7. doi: $10.1078 / 094471103321648593$ 
Kimura, I., Yoshikawa, M., Kobayashi, S., Sugihara, Y., Suzuki, M., Oominami, H., et al. (2001). New triterpenes, myrrhanol A and myrrhanone A, from guggul-gum resins, and their potent antiinflammatory effect on adjuvantinduced air-pouch granuloma of mice. Bioorg. Med. Chem. Lett. 11, 985-989. doi: 10.1016/S0960-894X(01)00111-1

Kinne, R. W., Stuhlmüller, B., and Burmester, G. R. (2007). Cells of the synovium in rheumatoid arthritis Macrophages. Arthritis Res. Ther. 9:224. doi: $10.1186 / \operatorname{ar} 2333$

Kirste, S., Treier, M., Wehrle, S. J., Becker, G., Abdel-Tawab, M., Gerbeth, K., et al.(2011). Boswellia serrata acts on cerebral edema in patients irradiated for brain tumors: a prospective, randomized, placebo-controlled, double-blind pilot trial. Cancer 117, 3788-3795. doi: 10.1002/cncr.25945

Kizhakkedath, R. (2013).Clinical evaluation of a formulation containing Curcuma longa and Boswellia serrata extracts in the management of knee osteoarthritis. Mol. Med. Rep. 8, 1542-1548. doi: 10.3892/mmr.2013.1661

Kong, J. N., He, Q., Wang, G., Dasgupta, S., Dinkins, M. B., Zhu, G., et al. (2015). Guggulsterone and bexarotene induce secretion of exosome-associated breast cancer resistance protein and reduce doxorubicin resistance in MDA-MB-231 cells. Int. J. Cancer 137, 1610-1620. doi: 10.1002/ijc.29542

Koo, J. H., Rhee, K. S., Koh, H. W., Jang, H. Y., Park, B. H., and Park, J. W. (2012). Guggulsterone inhibits melanogenesis in B16 murine melanoma cells by downregulating tyrosinase expression. Int. J. Mol. Med. 30, 974-978. doi: 10.3892/ijmm.2012.1057

Krieglstein, C. F., Anthoni, C., Rijcken, E. J., Laukötter, M., Spiegel, H. U., Boden, S. E., et al. (2001). Acetyl-11-keto-beta-boswellic acid, a constituent of a herbal medicine from Boswellia serrata resin, attenuates experimental ileitis. Int. J. Colorectal Dis. 16, 88-95. doi: 10.1007/s003840100292

Kulkarni, R. R., Patki, P. S., Jog, V. P., Gandage, S. G., and Patwardhan, B. (1991). Treatment of osteoarthritis with a herbomineral formulation: a doubleblind, placebo-controlled, cross-over study. J. Ethnopharmacol. 33, 91-95. doi: 10.1016/0378-8741(91)90167-C

Kunnumakkara, A. B., Bordoloi, D., Harsha, C., Banik, K., Gupta, S. C., and Aggarwal, B. B. (2017). Curcumin mediates anticancer effects by modulating multiple cell signaling pathways. Clin. Sci. 131, 1781-1799. doi: 10.1042/CS20160935

Kunnumakkara, A. B., Nair, A. S., Sung, B., Pandey, M. K., and Aggarwal, B. B. (2009). Boswellic acid blocks signal transducers and activators of transcription 3 signaling, proliferation, and survival of multiple myeloma via the protein tyrosine phosphatase SHP-1. Mol. Cancer Res. 7, 118-128. doi: 10.1158/1541-7786.MCR-08-0154

Kunnumakkara, A. B., Sailo, B. L., Banik, K., Harsha, C., Prasad, S., Gupta, S. C., et al. (2018). Chronic diseases, inflammation, and spices: how are they linked? J. Transl. Med. 16:14. doi: 10.1186/s12967-018-1381-2

Lata, S., Saxena, K. K., Bhasin, V., Saxena, R. S., Kumar, A., and Srivastava, V. K. (1991). Beneficial effects of Allium sativum, Allium cepa and C. mukul on experimental hyperlipidemia and atherosclerosis - a comparative evaluation. J. Postgrad. Med. 37, 132-135.

Lee, D., Kim, T., Kim, K. H., Ham, J., Jang, T. S., Kang, K. S., et al. (2017). Evaluation of guggulsterone derivatives as novel kidney cell protective agents against cisplatin-induced nephrotoxicity. Bioorg. Med. Chem. Lett. 27, 3156-3161. doi: 10.1016/j.bmcl.2017.05.033

Lee, Y. R., Lee, J. H., Noh, E. M., Kim, E. K., Song, M. Y., Jung, W. S., et al. (2008). Guggulsterone blocks IL-1beta-mediated inflammatory responses by suppressing NF-kappaB activation in fibroblast-like synoviocytes. Life Sci. 82, 1203-1209. doi: 10.1016/j.lfs.2008.04.006

Leeman-Neill, R. J., Wheeler, S. E., Singh, S. V., Thomas, S. M., Seethala, R. R., Neill, D. B., et al. (2009). Guggulsterone enhances head and neck cancer therapies via inhibition of signal transducer and activator of transcription-3. Carcinogenesis 30, 1848-1856. doi: 10.1093/carcin/bgp211

Li, C., Zang, Y., Sen, M., Leeman-Neill, R. J., Man, D. S., Grandis, J. R., et al. (2009). Bortezomib up-regulates activated signal transducer and activator of transcription-3 and synergizes with inhibitors of signal transducer and activator of transcription 3 to promote head and neck squamous cell carcinoma cell death. Mol. Cancer Ther. 8, 2211-2220. doi: 10.1158/1535-7163.MCT-09-0327

Liu, F. G., Hu, W. F., Wang, J. L., Wang, P., Gong, Y., Tong, L. J., et al. (2017). Zguggulsterone produces antidepressant-like effects in mice through activation of the BDNF signaling pathway. Int. J. Neuropsychopharmacol. 20, 485-497. doi: $10.1093 /$ ijnp/pyx009
Liu, H. P., Gao, Z. H., Cui, S. X., Wang, Y., Li, B. Y., Lou, H. X., et al. (2013). Chemoprevention of intestinal adenomatous polyposis by acetyl-11keto-beta-boswellic acid in APC(Min/+) mice. Int. J. Cancer 132, 2667-2681. doi: $10.1002 /$ ijc. 27929

Liu, J. J., Huang, B., and Hooi, S. C. (2006). Acetyl-keto-beta-boswellic acid inhibits cellular proliferation through a p21-dependent pathway in colon cancer cells. Br. J. Pharmacol. 148, 1099-1107. doi: 10.1038/sj.bjp.0706817

Liu, J. J., Nilsson, A., Oredsson, S., Badmaev, V., and Duan, R. D. (2002a). Ketoand acetyl-keto-boswellic acids inhibit proliferation and induce apoptosis in Hep G2 cells via a caspase-8 dependent pathway. Int. J. Mol. Med. 10, 501-505. doi: 10.3892/ijmm.10.4.501

Liu, J. J., Nilsson, A., Oredsson, S., Badmaev, V., Zhao, W. Z., and Duan, R. D. (2002b). Boswellic acids trigger apoptosis via a pathway dependent on caspase-8 activation but independent on Fas/Fas ligand interaction in colon cancer HT-29 cells. Carcinogenesis 23, 2087-2093. doi: 10.1093/carcin/23.12.2087

Liu, Z., Liu, X., Sang, L., Liu, H., Xu, Q., and Liu, Z. (2015). Boswellic acid attenuates asthma phenotypes by downregulation of GATA3 via pSTAT6 inhibition in a murine model of asthma. Int. J. Clin. Exp. Pathol. 8, 236-243.

Lu, M., Xia, L., Hua, H., and Jing, Y. (2008). Acetyl-keto-beta-boswellic acid induces apoptosis through a death receptor 5-mediated pathway in prostate cancer cells. Cancer Res. 68, 1180-1186. doi: 10.1158/0008-5472.CAN-07-2978

Macha, M. A., Matta, A., Chauhan, S. S., Siu, K. W., and Ralhan, R. (2011a). Guggulsterone (GS) inhibits smokeless tobacco and nicotine-induced NF-кB and STAT3 pathways in head and neck cancer cells. Carcinogenesis 32, 368-380. doi: 10.1093/carcin/bgq278

Macha, M. A., Matta, A., Chauhan, S., Siu, K. M., and Ralhan, R. (2010). 14-3-3 zeta is a molecular target in guggulsterone induced apoptosis in head and neck cancer cells. BMC Cancer 10:655. doi: 10.1186/1471-2407-10-655

Macha, M. A., Matta, A., Chauhan, S., Siu, K. M., and Ralhan, R. (2011b). Guggulsterone targets smokeless tobacco induced PI3K/Akt pathway in head and neck cancer cells. PLOS ONE 6:e14728. doi: 10.1371/journal.pone.0014728

Macha, M. A., Rachagani, S., Gupta, S., Pai, P., Ponnusamy, M. P., Batra, S. K., et al. (2013). Guggulsterone decreases proliferation and metastatic behavior of pancreatic cancer cells by modulating JAK/STAT and Src/FAK signaling. Cancer Lett. 341, 166-177. doi: 10.1016/j.canlet.2013.07.037

Magin, P. J., Adams, J., Pond, C. D., and Smith, W. (2006). Topical and oral CAM in acne: a review of the empirical evidence and a consideration of its context. Complement. Ther. Med. 14, 62-76. doi: 10.1016/j.ctim.2005.10.007

Mahesh, B. U., Shrivastava, S., Pragada, R. R., Naidu, V. G., and Sistla, R. (2014). Antioxidant and hepatoprotective effects of Boswellia ovalifoliolata bark extracts. Chin. J. Nat. Med. 12, 663-671. doi: 10.1016/S1875-5364(14)60101-1

Massoud, A., El Sisi, S., Salama, O., and Massoud, A. (2001). Preliminary study of therapeutic efficacy of a new fasciolicidal drug derived from Commiphora molmol (myrrh). Am. J. Trop. Med. Hyg. 65, 96-99. doi: 10.4269/ajtmh.2001.65.96

Mencarelli, A., Renga, B., Palladino, G., Distrutti, E., and Fiorucci, S. (2009). The plant sterol guggulsterone attenuates inflammation and immune dysfunction in murine models of inflammatory bowel disease. Biochem. Pharmacol. 78, 1214-1223. doi: 10.1016/j.bcp.2009.06.026

Meselhy, M. R. (2003). Inhibition of LPS-induced NO production by the oleogum resin of Commiphora wightii and its constituents. Phytochemistry 62, 213-218. doi: 10.1016/S0031-9422(02)00388-6

Meyer, U., Costantino, G., Macchiarulo, A., and Pellicciari, R. (2005). Is antagonism of E/Z-guggulsterone at the farnesoid $\mathrm{X}$ receptor mediated by a noncanonical binding site? A molecular modeling study. J. Med. Chem. 48, 6948-6955. doi: 10.1021/jm0505056

Moon, D. O., Park, S. Y., Choi, Y. H., Ahn, J. S., and Kim, G. Y. (2011). Guggulsterone sensitizes hepatoma cells to TRAIL-induced apoptosis through the induction of CHOP-dependent DR5: involvement of ROS-dependent ER-stress. Biochem. Pharmacol. 82, 1641-1650. doi: 10.1016/j.bcp.2011. 08.019

Morad, S. A., Schmid, M., Buchele, B., Siehl, H. U., El Gafaary, M., Lunov, O., et al. (2013). A novel semisynthetic inhibitor of the FRB domain of mammalian target of rapamycin blocks proliferation and triggers apoptosis in chemoresistant prostate cancer cells. Mol. Pharmacol. 83, 531-541. doi: 10.1124/mol.112.081349

Moreillon, J. J., Bowden, R. G., Deike, E., Griggs, J., Wilson, R., Shelmadine, B., et al. (2013). The use of an anti-inflammatory supplement in patients 
with chronic kidney disease. J. Complement Integr. Med. 10, 143-152. doi: 10.1515/jcim-2012-0011

Nabekura, T., Yamaki, T., Ueno, K., and Kitagawa, S. (2008). Inhibition of P-glycoprotein and multidrug resistance protein 1 by dietary phytochemicals. Cancer Chemother. Pharmacol. 62, 867-873. doi: 10.1007/s00280-007-0676-4

Nityanand, S., Srivastava, J. S., and Asthana, O. P. (1989). Clinical trials with gugulipid. A new hypolipidaemic agent. J. Assoc. Physicians India 37, 323-328.

Nohr, L. A., Rasmussen, L. B., and Straand, J. (2009). Resin from the mukul myrrh tree, guggul, can it be used for treating hypercholesterolemia? A randomized, controlled study. Complement. Ther. Med. 17, 16-22. doi: 10.1016/j.ctim.2008.07.001

Ojha, S., Bhatia, J., Arora, S., Golechha, M., Kumari, S., and Arya, D. S. (2011). Cardioprotective effects of C. mukul against isoprenaline-induced cardiotoxicity: a biochemical and histopathological evaluation. J. Environ. Biol. $32,731-738$

Ovesen, T., and Ledet, T. (1992). Bacteria and endotoxin in middle ear fluid and the course of secretory otitis media. Clin. Otolaryngol. Allied Sci. 17, 531-534. doi: 10.1111/j.1365-2273.1992.tb01713.x

Owsley, E., and Chiang, J. Y. (2003). Guggulsterone antagonizes farnesoid X receptor induction of bile salt export pump but activates pregnane $\mathrm{X}$ receptor to inhibit cholesterol 7alpha-hydroxylase gene. Biochem. Biophys. Res. Commun. 304, 191-195. doi: 10.1016/S0006-291X(03)00551-5

Panda, S., and Kar, A. (2005). Guggulu (C. mukul) potentially ameliorates hypothyroidism in female mice. Phytother Res. 19, 78-80. doi: 10.1002/ptr.1602

Pang, X., Yi, Z., Zhang, X., Sung, B., Qu, W., Lian, X., et al. (2009). Acetyl-11-ketobeta-boswellic acid inhibits prostate tumor growth by suppressing vascular endothelial growth factor receptor 2-mediated angiogenesis. Cancer Res. 69, 5893-5900. doi: 10.1158/0008-5472.CAN-09-0755

Park, B., Prasad, S., Yadav, V., Sung, B., and Aggarwal, B. B. (2011a). Boswellic acid suppresses growth and metastasis of human pancreatic tumors in an orthotopic nude mouse model through modulation of multiple targets. PLoS ONE 6:e26943. doi: 10.1371/journal.pone.0026943

Park, B., Sung, B., Yadav, V. R., Cho, S. G., Liu, M., and Aggarwal, B. B. (2011b). Acetyl-11-keto- $\beta$-boswellic acid suppresses invasion of pancreatic cancer cells through the downregulation of CXCR4 chemokine receptor expression. Int. J. Cancer 129, 23-33. doi: 10.1002/ijc.25966

Park, Y. S., Lee, J. H., Bondar, J., Harwalkar, J. A., Safayhi, H., and Golubic, M. (2002a). Cytotoxic action of acetyl-11-keto-beta-boswellic acid (AKBA) on meningioma cells. Planta Med. 68, 397-401. doi: 10.1055/s-2002-32090

Park, Y. S., Lee, J. H., Harwalkar, J. A., Bondar, J., Safayhi, H., and Golubic, M. (2002b). Acetyl-11-keto-beta-boswellic acid (AKBA) is cytotoxic for meningioma cells and inhibits phosphorylation of the extracellularsignal regulated kinase 1 and 2. Adv. Exp. Med. Biol. 507, 387-393. doi: 10.1007/978-1-4615-0193-0_60

Pasta, V., Dinicola, S., Giuliani, A., Harrath, A. H., Alwasel, S. H., Tartaglia, F., et al. (2016). A randomized trial of Boswellia in association with betaine and myoinositol in the management of breast fibroadenomas. Eur. Rev. Med. Pharmacol. Sci. 20, 1860-1865.

Pathania, A. S., Wani, Z. A., Guru, S. K., Kumar, S., Bhushan, S., Korkaya, H., et al. (2015). The anti-angiogenic and cytotoxic effects of the boswellic acid analog BA145 are potentiated by autophagy inhibitors. Mol. Cancer. 14:6. doi: 10.1186/1476-4598-14-6

Patti, A. M., Al-Rasadi, K., Katsiki, N., Banerjee, Y., Nikolic, D., Vanella, L., et al. (2015). Effect of a natural supplement containing Curcuma longa, guggul, and chlorogenic acid in patients with metabolic syndrome. Angiology 66, 856-861. doi: $10.1177 / 0003319714568792$

Prabhavathi, K., Chandra, U. S., Soanker, R., and Rani, P. U. (2014). A randomized, double blind, placebo controlled, cross over study to evaluate the analgesic activity of Boswellia serrata in healthy volunteers using mechanical pain model. Indian J. Pharmacol. 46, 475-479. doi: 10.4103/0253-7613.140570

Qurishi, Y., Hamid, A., Sharma, P. R., Wani, Z. A., Mondhe, D. M., Singh, S. K., et al. (2012). PARP cleavage and perturbance in mitochondrial membrane potential by 3 - $\alpha$-propionyloxy- $\beta$-boswellic acid results in cancer cell death and tumor regression in murine models. Future Oncol. 8, 867-881. doi: $10.2217 /$ fon. 12.68

Qurishi, Y., Hamid, A., Sharma, P. R., Wani, Z. A., Mondhe, D. M., Singh, S. K., et al. (2013). NF-kB down-regulation and PARP cleavage by novel 3 - $\alpha$-butyryloxy- $\beta$-boswellic acid results in cancer cell specific apoptosis and in vivo tumor regression. Anticancer. Agents Med. Chem. 13, 777-790. doi: 10.2174/1871520611313050012

Ravanan, P., Singh, S. K., Rao, G. S., and Kondaiah, P. (2011). Growth inhibitory, apoptotic and anti-inflammatory activities displayed by a novel modified triterpenoid, cyano enone of methyl boswellates. J. Biosci. 36, 297-307. doi: 10.1007/s12038-011-9056-7

Reddy, G. K., and Dhar, S. C. (1987). Effect of a new non-steroidal antiinflammatory agent on lysosomal stability in adjuvant induced arthritis. Ital. J. Biochem. 36, 205-217.

Riva, A., Morazzoni, P., Artariam, C., Allegrinim, P., Meinsm, J., Savio Appendino, G., et al. (2016). A single-dose, randomized, cross-over, two-way, openlabel study for comparing the absorption of boswellic acids and its lecithin formulation. Phytomedicine 23, 1375-1382. doi: 10.1016/j.phymed.2016.07.009

Rizzo, G., Disante, M., Mencarelli, A., Renga, B., Gioiello, A., Pellicciari, R., et al. (2006). The farnesoid $\mathrm{X}$ receptor promotes adipocyte differentiation and regulates adipose cell function in vivo. Mol. Pharmacol. 70, 1164-1173. doi: 10.1124/mol.106.023820

Roy, N. K., Deka, A., Bordoloi, D., Mishra, S., Kumar, A. P., Sethi, G., et al. (2016). The potential role of boswellic acids in cancer prevention and treatment. Cancer Lett. 377, 74-86. doi: 10.1016/j.canlet.2016.04.017

Safayhi, H., Mack, T., Sabieraj, J., Anazodo, M. I., Subramanian, L. R., and Ammon, H. P. (1992). Boswellic acids: novel, specific, nonredox inhibitors of 5-lipoxygenase. J. Pharmacol. Exp. Ther. 261, 1143-1146.

Safayhi, H., Rall, B., Sailer, E. R., and Ammon, H. P. (1997). Inhibition by boswellic acids of human leukocyte elastase. J. Pharmacol. Exp. Ther. 281, 460-463.

Safayhi, H., Sailer, E. R., and Ammon, H. P. (1995). Mechanism of 5lipoxygenase inhibition by acetyl-11-keto-beta-boswellic acid. Mol. Pharmacol. $47,1212-1216$.

Samudio, I., Konopleva, M., Safe, S., McQueen, T., and Andreeff, M. (2005). Guggulsterones induce apoptosis and differentiation in acute myeloid leukemia: identification of isomer-specific antileukemic activities of the pregnadienedione structure. Mol. Cancer Ther. 4, 1982-1992. doi: 10.1158/1535-7163.MCT-05-0247

Sander, O., Herborn, G., and Rau, R. (1998). Is H15 (resin extract of Boswellia serrata, "incense") a useful supplement to established drug therapy of chronic polyarthritis? Results of a double-blind pilot study. Z. Rheumatol. 57, 11-16. doi: 10.1007/s003930050051

Sarup, P., Bala, S., and Kamboj, S. (2015). Pharmacology and Phytochemistry of Oleo-Gum Resin of Commiphora wightii (Guggulu). Scientifica 2015:138039. doi: $10.1155 / 2015 / 138039$

Satyavati, G. V., Dwarakanath, C., and Tripathi, S. N. (1969). Experimental studies on the hypocholesterolemic effect of C. mukul Engl. (Guggul). Indian J. Med. Res. 57, 1950-1962.

Saxena, G., Singh, S. P., Pal, R., Singh, S., Pratap, R., and Nath, C. (2007). Guggulipid, an extract of Commiphora whighitii with lipid- lowering properties, has protective effects against streptozotocin-induced memory deficits in mice. Pharmacol. Biochem. Behav. 86, 797-805. doi: 10.1016/j.pbb.2007.03.010

Scholtes, C., André, P., Trépo, C., Cornu, C., Remontet, L., Ecochard, R., et al. (2012). Farnesoid X receptor targeting for hepatitis C: study protocol for a proof-of-concept trial. Therapie 67, 423-427. doi: 10.2515/therapie/2012058

Sengupta, K., Alluri, K. V., Satish, A. R., Mishra, S., Golakoti, T., Sarma, K. V., et al. (2008). A double blind, randomized, placebo controlled study of the efficacy and safety of 5-Loxin for treatment of osteoarthritis of the knee. Arthritis Res. Ther. 10:R85. doi: 10.1186/ar2461

Shah, R., Gulati, V., and Palombo, E. A. (2012). Pharmacological properties of guggulsterones, the major active components of gum guggul. Phytother. Res. 26, 1594-1605. doi: 10.1002/ptr.4647

Shao, Y., Ho, C. T., Chin, C. K., Badmaev, V., Ma, W., and Huang, M. T. (1998). Inhibitory activity of boswellic acids from Boswellia serrata against human leukemia HL-60 cells in culture. Planta Med. 64, 328-331. doi: 10.1055/s-2006-957444

Sharma, B., Salunke, R., Srivastava, S., Majumder, C., and Roy, P. (2009). Effects of guggulsterone isolated from C. mukul in high fat diet induced diabetic rats. Food Chem. Toxicol. 47, 2631-2639. doi: 10.1016/j.fct.2009.07.021

Sharma, J. N., and Sharma, J. N. (1977). Comparison of the anti-inflammatory activity of C. mukul (an indigenous drug) with those of phenylbutazone and ibuprofen in experimental arthritis induced by mycobacterial adjuvant. Arzneimittelforschung 27, 1455-1457. 
Sharma, M. L., Bani, S., and Singh, G. B. (1989). Anti-arthritic activity of boswellic acids in bovine serum albumin (BSA)-induced arthritis. Int. J. Immunopharmacol. 11, 647-652. doi: 10.1016/0192-0561(89)90150-1

Sheir, Z., Nasr, A. A., Massoud, A., Salama, O., Badra, G. A., El-Shennawy, H., et al. (2001). A safe, effective, herbal antischistosomal therapy derived from myrrh. Am. J. Trop. Med. Hyg. 65, 700-704. doi: 10.4269/ajtmh.2001.65.700

Shelmadine, B. D., Bowden, R. G., Moreillon, J. J., Cooke, M. B., Yang, P., Deike, E., et al. (2017). A pilot study to examine the effects of an anti-inflammatory supplement on eicosanoid derivatives in patients with chronic kidney disease. J. Altern. Complement. Med. 23, 632-638. doi: 10.1089/acm.2016.0007

Shen, Y., Takahashi, M., Byun, H. M., Link, A., Sharma, N., Balaguer, F., et al. (2012). Boswellic acid induces epigenetic alterations by modulating DNAmethylation in colorectal cancer cells. Cancer Biol. Ther. 13, 542-552. doi: $10.4161 /$ cbt. 19604

Shi, J. J., Jia, X. L., Li, M., Yang, N., Li, Y. P., Zhang, X., et al. (2015). Guggulsterone induces apoptosis of human hepatocellular carcinoma cells through intrinsic mitochondrial pathway. World J. Gastroenterol. 21, 13277-13287. doi: 10.3748/wjg.v21.i47.13277

Shishodia, S., and Aggarwal, B. B. (2004). Guggulsterone inhibits NF-kappaB and IkappaBalpha kinase activation, suppresses expression of anti-apoptotic gene products, and enhances apoptosis. J. Biol. Chem. 279, 47148-47158. doi: 10.1074/jbc.M408093200

Shishodia, S., Azu, N., Rosenzweig, J. A., and Jackson, D. A. (2015). Guggulsterone for chemoprevention of cancer. Curr. Pharm. Des. 22, 294-306. doi: 10.2174/1381612822666151112153117

Shishodia, S., Harikumar, K. B., Dass, S., Ramawat, K. G., and Aggarwal, B. B. (2008). The guggul for chronic diseases: ancient medicine, modern targets. Anticancer Res. 28, 3647-3664.

Shishodia, S., Sethi, G., Ahn, K. S., and Aggarwal, B. B. (2007). Guggulsterone inhibits tumor cell proliferation, induces S-phase arrest, and promotes apoptosis through activation of c-Jun $\mathrm{N}$-terminal kinase, suppression of Akt pathway, and downregulation of antiapoptotic gene products. Biochem. Pharmacol. 74, 118-130. doi: 10.1016/j.bcp.2007.03.026

Siddiqui, M. M., Afaq, S. H., and Asif, M. (1984). Chemical standardization of 'Kundur'(Oleo-Gum-Resin of Boswellia serrata Roxb). Anc. Sci. Life. 4, 48-50.

Sinal, C. J., and Gonzalez, F. J. (2002). Guggulsterone: an old approach to a new problem. Trends Endocrinol. Metab. 13, 275-276. doi: 10.1016/S1043-2760(02)00640-9

Singh, A. K., Prasad, G. C., and Tripathi, S. N. (1982). In vitro studies on thyrogenic effect of C. mukul (guggulu). Anc. Sci. Life. 2, 23-28.

Singh, B. B., Mishra, L. C., Vinjamury, S. P., Aquilina, N., Singh, V. J., and Shepard, N. (2003). The effectiveness of C. mukul for osteoarthritis of the knee: an outcomes study. Altern. Ther. Health Med. 9, 74-79.

Singh, R. B., Niaz, M. A., and Ghosh, S. (1994). Hypolipidemic and antioxidant effects of Commiphora mukul as an adjunct to dietary therapy in patients with hypercholesterolemia. Cardiovasc. Drugs Ther. 8, 659-664. doi: $10.1007 / B F 00877420$

Singh, R. P., Singh, R., Ram, P., and Batliwala, P. G. (1993). Use of Pushkar- Guggul, an indigenous antiischemic combination, in the management of ischemic heart disease. Int. J. Pharmacol. 31, 147-160. doi: $10.3109 / 13880209309082932$

Singh, S. K., Bhusari, S., Singh, R., Saxena, A., Mondhe, D., and Qazi, G. N. (2007). Effect of acetyl 11-keto beta-boswellic acid on metastatic growth factor responsible for angiogenesis. Vascul. Pharmacol. 46, 333-337. doi: 10.1016/j.vph.2006.09.008

Singh, S. V., Choi, S., Zeng, Y., Hahm, E. R., and Xiao, D. (2007). Guggulsteroneinduced apoptosis in human prostate cancer cells is caused by reactive oxygen intermediate dependent activation of c-Jun NH2-terminal kinase. Cancer Res. 67, 7439-7449. doi: 10.1158/0008-5472.CAN-07-0120

Singh, S. V., Zeng, Y., Xiao, D., Vogel, V. G., Nelson, J. B., Dhir, R., et al. (2005). Caspase-dependent apoptosis induction by guggulsterone, a constituent of Ayurvedic medicinal plant Commiphora mukul, in PC-3 human prostate cancer cells is mediated by Bax and Bak. Mol. Cancer Ther. 4, 1747-1754. doi: 10.1158/1535-7163.MCT-05-0223

Singh, S., Khajuria, A., Taneja, S. C., Khajuria, R. K., Singh, J., Johri, R. K., et al. (2008). The gastric ulcer protective effect of boswellic acids, a leukotriene inhibitor from Boswellia serrata, in rats. Phytomedicine 15, 408-415. doi: 10.1016/j.phymed.2008.02.017
Singh, V., Kaul, S., Chander, R., and Kapoor, N. K. (1990). Stimulation of low density lipoprotein receptor activity in liver membrane of guggulsterone treated rats. Pharmacol. Res. 22, 37-44. doi: 10.1016/1043-6618(90)90741-U

Song, J. J., Kwon, S. K., Cho, C. G., Park, S. W., and Chae, S. W. (2010). Guggulsterone suppresses LPS induced inflammation of human middle ear epithelial cells (HMEEC). Int. J. Pediatr. Otorhinolaryngol. 74, 1384-1387. doi: 10.1016/j.ijporl.2010.09.012

Syrovets, T., Buchele, B., Gedig, E., Slupsky, J. R., and Simmet, T. (2000). Acetylboswellic acids are novel catalytic inhibitors of human topoisomerases I and II alpha. Mol. Pharmacol. 58, 71-81. doi: 10.1124/mol.58.1.71

Syrovets, T., Büchele, B., Krauss, C., Laumonnier, Y., and Simmet, T. (2005a). Acetyl-boswellic acids inhibit lipopolysaccharide-mediated TNF-alpha induction in monocytes by direct interaction with I kappa B kinases. $J$. Immunol. 174, 498-506. doi: 10.4049/jimmunol.174.1.498

Syrovets, T., Gschwend, J. E., Büchele, B., Laumonnier, Y., Zugmaier, W., Genze, F., et al. (2005b). Inhibition of IkappaB kinase activity by acetyl-boswellic acids promotes apoptosis in androgen-independent PC-3 prostate cancer cells in vitro and in vivo. J. Biol. Chem. 280, 6170-6180. doi: 10.1074/jbc.M409477200

Szapary, P. O., Wolfe, M. L., Bloedon, L. T., Cucchiara, A. J., DerMarderosian, A. H., Cirigliano, M. D., et al. (2003). Guggulipid for the treatment of hypercholesterolemia: a randomized controlled trial. JAMA 290, 765-772. doi: 10.1001/jama.290.6.765

Takada, Y., Ichikawa, H., Badmaev, V., and Aggarwal, B. B. (2006). Acetyl-11keto-beta-boswellic acid potentiates apoptosis, inhibits invasion, and abolishes osteoclastogenesis by suppressing NF-kappa B and NF-kappa B-regulated gene expression. J. Immunol. 176, 3127-3140. doi: 10.4049/jimmunol.176.5.3127

Takahashi, M., Sung, B., Shen, Y., Hur, K., Link, A., Boland, C. R., et al. (2012). Boswellic acid exerts antitumor effects in colorectal cancer cells by modulating expression of the let-7 and miR-200 microRNA family. Carcinogenesis 33, 2441-2449. doi: 10.1093/carcin/bgs286

Thappa, D. M., and Dogra, J. (1994). Nodulocystic acne: oral gugulipid versus tetracycline. J. Dermatol. 21, 729-731. doi: 10.1111/j.1346-8138.1994.tb03277.x

Togni, S., Maramaldi, G., Bonetta, A., Giacomelli, L., and Di Pierro, F. (2015). Clinical evaluation of safety and efficacy of Boswellia-based cream for prevention of adjuvant radiotherapy skin damage in mammary carcinoma: a randomized placebo controlled trial. Eur. Rev. Med. Pharmacol. Sci. 19, 1338-1344.

Togni, S., Maramaldi, G., Di Pierro, F., and Biondi, M. (2014). A cosmeceutical formulation based on boswellic acids for the treatment of erythematous eczema and psoriasis. Clin. Cosmet. Investig. Dermatol. 7, 321-327. doi: $10.2147 /$ CCID.S69240

Tripathi, S. N., Gupta, M., Sen, S. P., and Udupa, K. N. (1975). Effect of a ketosteroid of Commifora mukul $L$. on hypercholesterolemia and hyperlipidemia induced by neomercazole and cholesterol mixture in chicks. Indian J. Exp. Biol. $13,15-18$.

Tripathi, Y. B. (2009). BHUx: a patented polyherbal formulation to prevent hyperlipidemia and atherosclerosis. Recent Pat. Inflamm. Allergy Drug Discov. 3, 49-57. doi: 10.2174/187221309787158443

Tripathi, Y. B., Malhotra, O. P., and Tripathi, S. N. (1984). Thyroid-stimulating action of Z-guggulsterone obtained from C. mukul. Planta Med. 50, 78-80. doi: 10.1055/s-2007-969626

Urizar, N. L., Liverman, A. B., Dodds, D. T., Silva, F. V., Ordentlich, P., Yan, Y., et al. (2002). A natural product that lowers cholesterol as an antagonist ligand for FXR. Science 296, 1703-1706. doi: 10.1126/science.1072891

Verma, S. K., and Bordia, A. (1988). Effect of Commiphora mukul (gum guggulu) in patients of hyperlipidemia with special reference to HDL-cholesterol. Indian J. Med. Res. 87, 356-360.

von Rhein, C., Weidner, T., Hen,ß, L., Martin, J., Weber, C., Sliva, K., et al. (2016). Curcumin and Boswellia serrata gum resin extract inhibit chikungunya and vesicular stomatitis virus infections in vitro. Antiviral Res. 125, 51-57. doi: 10.1016/j.antiviral.2015.11.007

Vyas, K. Y., Bedarkar, P., Galib, R., and Prajapati, P. K. (2015). Comparative Antihyperlipidaemic activity of Navina (fresh) and Purāna (old) Guggulu. Anc. Sci. Life. 35, 101-109. doi: 10.4103/0257-7941.171672

Wang, Q., Pan, X., Wong, H. H., Wagner, C. A., Lahey, L. J., Robinson, W. H., et al. (2014). Oral and topical boswellic acid attenuates mouse osteoarthritis. Osteoarthr. Cartil. 22, 128-132. doi: 10.1016/j.joca.2013. 10.012 
Wang, R., Wang, Y., Gao, Z., and Qu, X. (2014). The comparative study of acetyl11-keto-beta-boswellic acid (AKBA) and aspirin in the prevention of intestinal adenomatous polyposis in APC(Min/+) mice. Drug Discov. Ther. 8, 25-32. doi: $10.5582 /$ ddt.8.25

Wang, W. C., Uen, Y. H., Chang, M. L., Cheah, K. P., Li, J. S., Yu, W. Y., et al. (2012). Protective effect of guggulsterone against cardiomyocyte injury induced by doxorubicin in vitro. BMC Complement. Altern. Med. 12:138. doi: 10.1186/1472-6882-12-138

Wang, X., Greilberger, J., Ledinski, G., Kager, G., Paigen, B., and Jürgens, G. (2004). The hypolipidemic natural product Commiphora mukul and its component guggulsterone inhibit oxidative modification of LDL. Atherosclerosis 172, 239-246. doi: 10.1016/j.atherosclerosis.2003.10.008

Wang, Y. G., Ma, Q. G., Tian, J., Ren, J., Wang, A. G., Ji, T. F., et al. (2016). Hepatoprotective triterpenes from the gum resin of Boswellia carterii. Fitoterapia 109, 266-273. doi: 10.1016/j.fitote.2015.12.018

Wang, Y. G., Ren, J., Wang, A. G., Yang, J. B., Ji, T. F., Ma, Q. G., et al. (2013). Hepatoprotective prenylaromadendrane-type diterpenes from the gum resin of Boswellia carterii. J. Nat. Prod. 76, 2074-2079. doi: 10.1021/np400526b

Weber, C. C., Reising, K., Müller, W. E., Schubert-Zsilavecz, M.,and Abdel-Tawab, M. (2006). Modulation of Pgp function by boswellic acids. Planta Med. 72, 507-513. doi: 10.1055/s-2006-931536

Wu, J., Xia, C., Meier, J., Li, S., Hu, X., and Lala, D. S. (2002). The hypolipidemic natural product guggulsterone acts as an antagonist of the bile acid receptor. Mol. Endocrinol. 16, 1590-1597. doi: 10.1210/mend.16.7.0894

Xia, L., Chen, D., Han, R., Fang, Q., Waxman, S., and Jing, Y. (2005). Boswellic acid acetate induces apoptosis through caspase-mediated pathways in myeloid leukemia cells. Mol. Cancer Ther. 4, 381-388. doi: 10.1158/1535-7163.MCT-03-0266

Xiao, D., and Singh, S. V. (2008). z-Guggulsterone, a constituent of Ayurvedic medicinal plant C. mukul, inhibits angiogenesis in vitro and in vivo. Mol. Cancer Ther. 7, 171-180. doi: 10.1158/1535-7163.MCT-07-0491

Xiao, D., Zeng, Y., Prakash, L., Badmaev, V., Majeed, M., and Singh, S. V. (2011). Reactive oxygen species-dependent apoptosis by gugulipid extract of Ayurvedic medicine plant Commiphora mukul in human prostate cancer cells is regulated by c-Jun N-terminal kinase. Mol. Pharmacol. 79, 499-507. doi: $10.1124 / \mathrm{mol} .110 .068551$

Xu, H. B., Fu, J., Huang, F., and Yu, J. (2017). Guggulsterone sensitized drug-resistant human hepatocarcinoma cells to doxorubicin through a Cox-2/P-gp dependent pathway. Eur. J. Pharmacol. 803, 57-64. doi: 10.1016/j.ejphar.2017.03.045

Xu, H. B., Li, L., and Liu, G. Q. (2011). Reversal of multidrug resistance by guggulsterone in drug-resistant MCF-7 cell lines. Chemotherapy 57, 62-70. doi: $10.1159 / 000321484$

Xu, H. B., Shen, Z. L., Fu, J., and Xu, L. Z. (2014a). Reversal of doxorubicin resistance by guggulsterone of Commiphora mukul in vivo. Phytomedicine 21, 1221-1229. doi: 10.1016/j.phymed.2014.06.003

Xu, H. B., Xu, L. Z., Li, L., Fu, J., and Mao, X. P. (2012). Reversion of P-glycoprotein-mediated multidrug resistance by guggulsterone in multidrug-resistant human cancer cell lines. Eur. J. Pharmacol. 694, 39-44. doi: 10.1016/j.ejphar.2012.06.046

Xu, H. B., Xu, L. Z., Mao, X. P., and Fu, J. (2014b). Guggulsterone of Commiphora mukul resin reverses drug resistance in imatinib-resistant leukemic cells by inhibiting cyclooxygenase-2 and P-glycoprotein. Phytomedicine 21, 1004-1009. doi: 10.1016/j.phymed.2014.02.014

Xu, Y., Watanabe, T., Tanigawa, T., Machida, H., Okazaki, H., Yamagami, H., et al. (2010). Bile acids induce $c d x 2$ expression through the farnesoid $\mathrm{x}$ receptor in gastric epithelial cells. J. Clin. Biochem. Nutr. 46, 81-86. doi: 10.3164/jcbn.09-71

Y, J., Kamath, J. V., and Asad, M. (2006). Effect of hexane extract of B. serrata oleo-gum resin on chemically induced liver damage. Pak. J. Pharm. Sci. 19, 129-133.

Yadav, V. R., Prasad, S., Sung, B., Gelovani, J. G., Guha, S., Krishnan, S., et al. (2012). Boswellic acid inhibits growth and metastasis of human colorectal cancer in orthotopic mouse model by downregulating inflammatory, proliferative, invasive and angiogenic biomarkers. Int. J. Cancer 130, 2176-2184. doi: 10.1002/ijc.26251
Yamada, T., and Sugimoto, K. (2016). Guggulsterone and its role in chronic diseases. Adv. Exp. Med. Biol. 929, 329-361. doi: 10.1007/978-3-319-41342-6_15

Yamada, T., Osawa, S., Ikuma, M., Kajimura, M., Sugimoto, M., Furuta, T., et al. (2014). Guggulsterone, a plant-derived inhibitor of NF-TB, suppresses CDX2 and COX-2 expression and reduces the viability of esophageal adenocarcinoma cells. Digestion 90, 208-217. doi: 10.1159/000365750

Yang, J. Y., Della-Fera, M. A., and Baile, C. A. (2008). Guggulsterone inhibits adipocyte differentiation and induces apoptosis in 3T3-L1 cells. Obesity 16, 16-22. doi: 10.1038/oby.2007.24

Yang, J. Y., Della-Fera, M. A., Rayalam, S., Ambati, S., and Baile, C. A. (2007). Enhanced pro-apoptotic and anti-adipogenic effects of genistein plus guggulsterone in 3T3-L1 adipocytes. Biofactors 30, 159-169. doi: 10.1002/biof.5520300303

Yang, L., Broderick, D., Jiang, Y., Hsu, V., and Maier, C. S. (2014). Conformational dynamics of human FXR-LBD ligand interactions studied by hydrogen/deuterium exchange mass spectrometry: insights into the antagonism of the hypolipidemic agent Z-guggulsterone. Biochim. Biophys. Acta 1844, 1684-1693. doi: 10.1016/j.bbapap.2014.06.007

Yang, M. H., Lee, K. T., Yang, S., Lee, J. K., Lee, K. H., Moon, I. H., et al. (2012). Guggulsterone enhances antitumor activity of gemcitabine in gallbladder cancer cells through suppression of NF-кB. J. Cancer Res. Clin. Oncol. 138, 1743-1751. doi: 10.1007/s00432-012-1254-7

Youn, H. S., Ahn, S. I., and Lee, B. Y. (2009). Guggulsterone suppresses the activation of transcription factor IRF3 induced by TLR3 or TLR4 agonists. Int. Immunopharmacol. 9, 108-112. doi: 10.1016/j.intimp.2008.10.012

Yuan, H. Q., Kong, F., Wang, X. L., Young, C. Y., Hu, X. Y., and Lou, H. X. (2008) Inhibitory effect of acetyl-11-keto-beta-boswellic acid on androgen receptor by interference of $\mathrm{Sp} 1$ binding activity in prostate cancer cells. Biochem. Pharmacol. 75, 2112-2121. doi: 10.1016/j.bcp.2008.03.005

Zhang, J. H., Shangguan, Z. S., Chen, C., Zhang, H. J., and Lin, Y. (2016). Antiinflammatory effects of guggulsterone on murine macrophage by inhibiting LPS-induced inflammatory cytokines in NF-кB signaling pathway. Drug Des. Devel. Ther. 10, 1829-1835. doi: 10.2147/DDDT.S104602

Zhao, W., Entschladen, F., Liu, H., Niggemann, B., Fang, Q., Zaenker, K. S., et al. (2003). Boswellic acid acetate induces differentiation and apoptosis in highly metastatic melanoma and fibrosarcoma cells. Cancer Detect. Prev. 27, 67-75. doi: 10.1016/S0361-090X(02)00170-8

Zhong, F., Tong, Z. T., Fan, L. L., Zha, L. X., Wang, F., Yao, M. Q., et al. (2016). Guggulsterone-induced apoptosis in cholangiocarcinoma cells through ROS/JNK signaling pathway. Am. J. Cancer Res. 6, 226-237.

Zhong, F., Yang, J., Tong, Z. T., Chen, L. L., Fan, L. L., Wang, F., et al. (2015). Guggulsterone inhibits human cholangiocarcinoma Sk-ChA1 and Mz-ChA-1 cell growth by inducing caspase-dependent apoptosis and downregulation of survivin and Bcl-2 expression. Oncol. Lett. 10, 1416-1422. doi: 10.3892/ol.2015.3391

Zhou, X., Cai, J. G., Zhu, W. W., Zhao, H. Y., Wang, K., and Zhang, X. F. (2015). Boswellic acid attenuates asthma phenotype by downregulation of GATA3 via nhibition of PSTAT6. Genet. Mol. Res. 14, 7463-7468. doi: 10.4238/2015.July.3.22

Zhu, N., Rafi, M. M., DiPaola, R. S., Xin, J., Chin, C. K., Badmaev, V., et al. (2001). Bioactive constituents from gum guggul (Commiphora wightii). Phytochemistry 56, 723-727. doi: 10.1016/S0031-9422(00)00485-4

Conflict of Interest Statement: The authors declare that the research was conducted in the absence of any commercial or financial relationships that could be construed as a potential conflict of interest.

Copyright (c) 2018 Kunnumakkara, Banik, Bordoloi, Harsha, Sailo, Padmavathi, Roy, Gupta and Aggarwal. This is an open-access article distributed under the terms of the Creative Commons Attribution License (CC BY). The use, distribution or reproduction in other forums is permitted, provided the original author(s) and the copyright owner(s) are credited and that the original publication in this journal is cited, in accordance with accepted academic practice. No use, distribution or reproduction is permitted which does not comply with these terms. 


\section{OPEN ACCESS}

Edited by:

Atanas G. Atanasov, Institute of Genetics and Animal Breeding (PAS), Poland

Reviewed by

Alexander N. Shikov,

St. Petersburg Institute of Pharmacy,

Russia

Milen I. Georgiev, Institute of Microbiology (BAS),

Bulgaria

*Correspondence:

Wilfried Dimpfe

w.dimpfe/@neurocode-ag.com

Alexander G. Panossian apanossian@europharmausa.com,

ap.phytomedicine@gmail.com

Specialty section:

This article was submitted to

Ethnopharmacology,

a section of the journal

Frontiers in Pharmacology

Received: 04 December 2017

Accepted: 11 April 2018

Published: 24 May 2018

Citation:

Dimpfel W, Schombert $L$ and Panossian AG (2018) Assessing

the Quality and Potential Efficacy of Commercial Extracts of Rhodiola rosea L. by Analyzing the Salidroside and Rosavin Content and the Electrophysiological Activity in Hippocampal Long-Term

Potentiation, a Synaptic Model of Memory. Front. Pharmacol. 9:425. doi: 10.3389/fphar.2018.00425

\section{Assessing the Quality and Potential Efficacy of Commercial Extracts of Rhodiola rosea L. by Analyzing the Salidroside and Rosavin Content and the Electrophysiological Activity in Hippocampal Long-Term Potentiation, a Synaptic Model of Memory}

\author{
Wilfried Dimpfel ${ }^{1 *}$, Leonie Schombert ${ }^{2}$ and Alexander G. Panossian ${ }^{3 *}$ \\ ${ }^{1}$ Department of Pharmacology, Justus Liebig University Giessen, Giessen, Germany, ${ }^{2}$ NeuroCode AG, Wetzlar, Germany, \\ ${ }^{3}$ EuroPharma USA Inc., Green Bay, WI, United States
}

Rhodiola rosea L. roots and rhizome extracts are active ingredients in adaptogenic herbal medicinal products (HMP) and dietary supplements for temporary relief of symptoms of stress, such as fatigue and weakness. $R$. rosea extract has a stimulating effect on the CNS, suggesting potential benefits on cognitive functions, memory, learning, and attention. The reproducible efficacy and quality of preparations of the underground parts of $R$. rosea depend on the highly variable content of the active markers, salidroside and rosavin, which affect the quality of HMP and dietary supplements. However, it is not clear which analytical markers are important for assessing the efficacy of $R$. rosea preparations intended for use in aging-induced mild cognitive disorders, such as attenuated memory, attention, and learning. Furthermore, the activity of various commercial $R$. rosea extracts has not been correlated with their content. Here, the biological activities of salidroside, rosavin, and seven commercial extracts of underground parts of $R$. rosea were assessed using a synaptic model of memory: long-term potentiation (LTP) of synaptic transmission in hippocampus slices. A high degree of variation in the content of all active markers was observed. One extract from China lacked rosavin, and there was even variation in the extracts from the Altai geographic region. In vitro, rosavin, salidroside and all tested $R$. rosea extracts potentiated electric stimulation of an intra-hippocampal electric circuit, which resulted in higher responses of the pyramidal cells in isolated hippocampus slices. Rosavin was more active at higher concentrations than salidroside; while, salidroside was more effective at lower concentrations. The highest content of both active markers was found in the extracts that were active at the lowest concentrations tested; while, some extracts contained some other compounds that presumably reduced the efficacy due to antagonistic interactions. Standardized content of active markers is necessary for 
the quality control of herbal preparations containing $R$. rosea extracts, but insufficient for assessment of their potential efficacy. Additional bioassays are needed to assure the reproducible pharmacological activity of $R$. rosea extracts; therefore, the LTP of synaptic transmission in hippocampus slices may serve as a validation tool for the quality control of $R$. rosea extracts.

Keywords: Rhodiola rosea, salidroside, rosavin, long-term potentiation, hippocampus, quality control, UPLC

\section{INTRODUCTION}

Rhodiola rosea L. [Crassulaceae, syn. Sedum rhodiola - DC. Sedum rosea - (L.) Scop cop, known as roseroot, rosenroot, golden root, arctic root, orpin rose, rhodiole rougeâtre] (Currier and Ampong-Nyarko, 2015) has a long history as a valuable medicinal plant and has appeared in the Materia Medica of several European countries (Panossian et al., 2010). Rhodiola rosea L. roots and rhizome extracts are active ingredients in adaptogenic herbal medicinal products (HMP) and dietary supplements for temporary relief of symptoms of stress, such as fatigue and weakness (Panossian and Wagner, 2005; Panossian and Wikman, 2009, 2010, 2014; EFSA, 2010; Panossian et al., 2010; European Medicine Authority [EMA], 2011). A growing body of evidence has indicated the extract's potential use in the prevention and treatment of stress- and age-related impairments of cognitive functions and mental disorders (Panossian et al., 2010, 2014; Panossian, 2013; Panossian and Gerbarg, 2016; Amsterdam and Panossian, 2016; Nabavi et al., 2016). The stimulating effects of $R$. rosea on the CNS were demonstrated long ago and suggested there were potential benefits on cognitive functions, memory, learning, and attention (Saratikov et al., 1965, 1978; Marina and Alekseeva, 1968; Kurkin and Zapesochnaya, 1986; Petkov et al., 1986; Marina et al., 1994; Saratikov and Krasnov, 2004). An active compound, named rhodioloside was isolated and identified as salidroside (Aksenova et al., 1968; Saratikov et al., 1968). A pilot study of rhodioloside (syn. salidroside) in 46 healthy human volunteers showed that $2.5 \mathrm{mg}$ salidroside increased attention in cognitive tests $1 \mathrm{~h}$ after a single dose was administered in $83 \%$ of subjects, compared with $54 \%$ of volunteers who were administered placebo (Aksenova et al., 1968). Further studies provided evidence that $R$. rosea and salidroside exhibit neuroprotective activity (Qu et al., 2009; Panossian et al., 2010, 2012; Jacob et al., 2013; Lee et al., 2013), suggesting they may be effective in treating neurodegenerative disorders, such as Alzheimer's disease (Nabavi et al., 2016).

Along with salidroside and its aglycone tyrosol (Figure 1), cinnamyl alcohol, glycosides, and rosavins (collective name of rosavin, rosarin, and rosin) also exhibited stress-protective (Barnaulov et al., 1986), stimulating, and neurotropic activities in rodents; reduced sleep induced by barbital, hexanal, and chloral hydrate in mice (Sokolov et al., 1985, 1990); increased locomotor activity in mice (Sokolov et al., 1990); and induced antidepressant-like effects in animal models of depression (Panossian et al., 2008). Salidroside is common for all species of Rhodiola, while phenylpropanoids, rosavin, rosarin, and rosin are specific only for $R$. rosea and $R$. sachalinensis (Kurkin et al., 1985; Nakamura et al., 2007; Booker et al., 2016b). Many publications have reported on the neuroprotective and neurotropic activity of salidroside (Sokolov et al., 1985, 1990; Barnaulov et al., 1986; Panossian et al., 2008; Cifani et al., 2010; Lee et al., 2013); while, there is limited evidence supporting the importance of rosavin, the major active marker (Sokolov et al., 1985, 1990; Panossian et al., 2008; Cifani et al., 2010; Marchev et al., 2017). Rosavin was inactive is rats during a behavioral test of binge eating; while, salidroside dose-dependently reduced or abolished binge eating for the period in which it was elicited (Cifani et al., 2010). In another study, salidroside was more effective than rosarin and rosin in inhibiting the expression of IL- $1 \beta$, and IL- 6 in microglial cells, while rosavin was not tested (Lee et al., 2013). Rosavin inhibited the expression of the TNF-related apoptosis-inducing ligand in concanavalin A activated Jurkat $\mathrm{T}$ cells, while salidroside was inactive and rosarin had an opposite effect (Marchev et al., 2017).

It is unclear which analytical markers are important for assessing the quality and efficacy of $R$. rosea herbal preparations intended for treating aging-induced mild cognitive disorders, such as attenuated memory, attention, and learning ability. Rhodiola preparations are usually standardized for salidroside (1\%) and rosavin (3\%). The content of active ingredients in herbal preparations depends on many factors, such as the geographic and climate zone it was grown in, which season and under what conditions it was harvested, and how it was dried, extracted, and prepared to give the final dosage form. For example, a high degree of inter-clonal variation was found for all tested constituents (salidroside, tyrosol, rosavin, rosarin, rosin, and cinnamyl alcohol) in six samples of $R$. rosea roots collected in various regions of Norway. The highest variation was found for salidroside and tyrosol, showing inter-clonal variations of 92.8 and $87.8 \%$, respectively (Hellum et al., 2010). Therefore, the preparations obtained by various producers can have quite different active dose levels. Furthermore, the contribution of these active markers to the overall activity of the total extracts was not systematically assessed. It was suggested that these phenolic compounds (rosavin, rosarin, rosin, salidroside/rhodioloside, and tyrosol) have no impact on activity of CYP450 enzymes and do not inhibit CYP3A4, CYP2D6, or CYP1A2 (Hellum et al., 2010; Xu et al., 2013; Thu et al., 2016a). The presence of minor amounts of herbacetin rhamnosides (rhodiosin and rhodionin) may presumably induce inhibition of CYP2D6 (Xu et al., 2013) in some commercial preparations of Rhodiola (Thu et al., 2016b, 2017).

It is a challenge to obtain reproducible efficacy and quality of HMP, particularly for preparations of the underground parts of $R$. rosea (Panossian et al., 2010; Ioset et al., 2011; Booker et al., 2016a). There may be unpredictable, complex interactions 


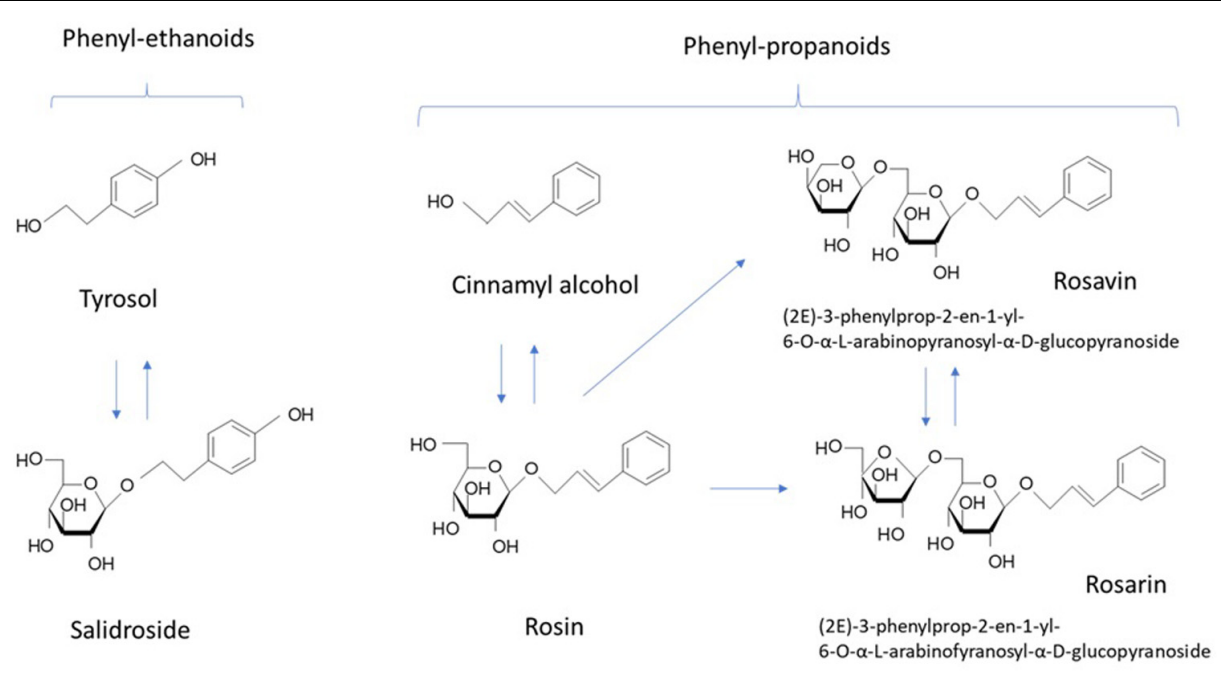

FIGURE 1 | Chemical structures of active markers of $R$. rosea extracts.

between the active constituents of the $R$. rosea extracts that affect the regulation of molecular networks playing an important role in cellular and physiological functions of human organisms (Panossian et al., 2014). The pharmacological activity of $R$. rosea crude extract is related to many compounds, such as salidroside, tyrosol, rosavin, and other phenolic compounds (Sokolov et al., 1985, 1990; Barnaulov et al., 1986; Panossian et al., 2008; Lee et al., 2013; Marchev et al., 2017). The batch to batch reproducible content of key active markers and the UPLC fingerprint are not a guaranty of reproducible efficacy and safety. Additional bioassays are required to assure reproducible pharmacological activity of HMP. These bioassays may serve as validation tools for the quality assurance of complex HMP where the total extract contains active pharmaceutical ingredients. In this context, assessment of the correlation between the content of active markers and pharmacological activity of HMP is important. Although the dose-response relationship of salidroside and rosavin and dietary supplements was studied (Cifani et al., 2010; Lee et al., 2013; Marchev et al., 2017), to the our best of our knowledge, the correlation between the content and biological activity of various commercial $R$. rosea extracts has not been investigated.

The aim of our study was to assess the biological activity of a selection of commercial extracts of the underground parts of $R$. rosea and their analytical markers, salidroside and rosavin, in a synaptic model of memory: the long-term potentiation (LTP) of synaptic transmission in the hippocampus (Bliss and Collingridge, 1993). An interesting result using this model was the ability of memantine, a substance used in the treatment of dementia, to increase the population spike amplitude in response to single stimuli (SS) and to increase LTP (Dimpfel, 1995). We used this method earlier for characterization of electrophysiological response of $R$. rosea in hippocampal slices and have demonstrated a concentration-dependent increase of the amplitude of the population spike (Dimpfel et al., 2016b). These results relate very well to previous clinical results where neurophysiological effects of $R$. rosea extract in healthy subjects were characterized (Dimpfel, 2014).

\section{MATERIALS AND METHODS}

\section{Test Samples}

Seven dry commercial extracts were obtained from different suppliers via the Internet. Our selection strategy was to compare Rhodiola extracts containing glycosides of cinnamyl alcohols (rosavins) with extracts containing only tyrosol and its glycoside salidroside. Some of the extracts were from plants grown in the Altai mountains of Siberia. The samples consisted of bulk powders, obtained from water. According to the manufacturers' certificates of analysis and origin, they were hydroalcoholic extracts of $R$. rosea roots and rhizome (harvest of 2015). Six extracts were preparations containing only root and rhizome powders, while one, SHR-5 contained maltodextrin as a carrier (for details see Supplementary Data S1 and Table 1). Our inclusion criteria were that products must be consumed as a solid dose or soft-gel manufactured item. Our exclusion criteria included ethanolic tinctures and raw materials including dried roots, rhizomes, and bulk tinctures. Rhodiola SHR-5 extract has been previously tested for efficacy and safety (Panossian and Wikman, 2014) and was included as a control (i.e., registered or licensed product). The samples of $R$. rosea $\mathrm{L}$. roots and rhizomes extracts were identified by thin-layer chromatography (TLC) and ultra-performance liquid chromatography (UPLC) using salidroside, tyrosol, rosavin, rosarin, rosin, and cinnamyl alcohol as reference standards (Supplementary Data S2). The voucher specimens were deposited in EuroPharma USA Inc.

\section{Reference Standards and Solvents}

Salidroside, tyrosol, rosavin, rosarin, rosin, and cinnamyl alcohol reference standards were purchased from Phytolab $\mathrm{GmbH} \&$ Co. KG (Vestenbergsgreuth, Germany) and used for 


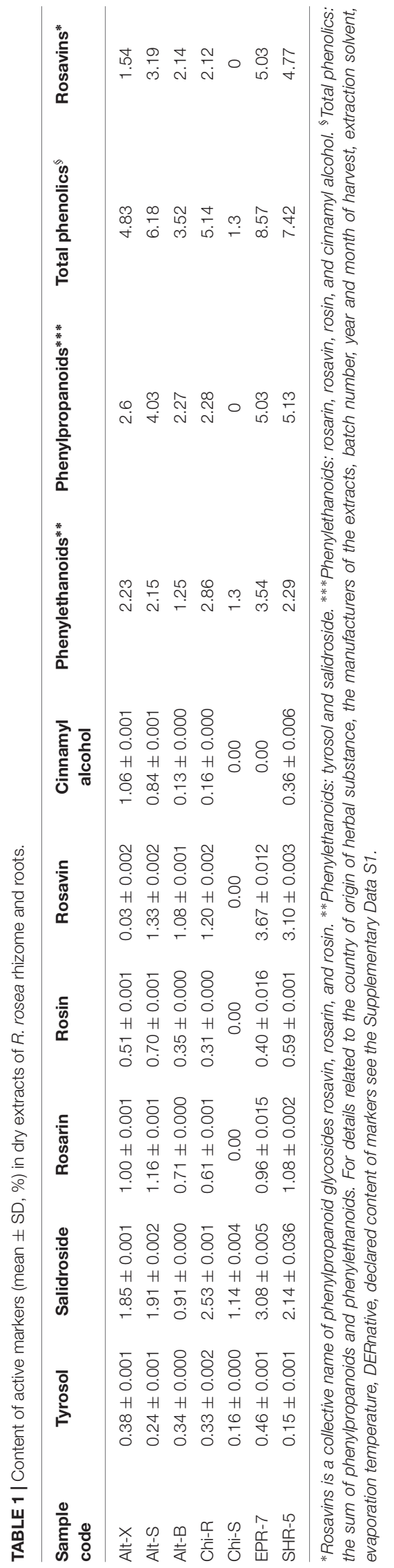

standard curve development. The solvents (water, methanol, and acetonitrile) used for extraction and chromatography were high performance liquid chromatographic grade (Waters Corporation, United States and Merck, Darmstadt, Germany).

\section{Preparation of the Analytical and Reference Standard Samples}

Powdered extract $(0.3 \mathrm{~g})$ was dissolved in $25 \mathrm{~mL}$ of solvent system water:acetonitrile (90:10) using an ultrasonic bath for $30 \mathrm{~min}$. It was then filtered through a $0.45 \mu \mathrm{m}$ pore size filter and analyzed by UPLC. Powdered extract $(1 \mathrm{~g})$ was dissolved in $10 \mathrm{~mL}$ of methanol using an ultrasonic bath for $30 \mathrm{~min}$ through a $00 \mathrm{H}$ filter. The filtrate was applied $(10 \mu \mathrm{L})$ to high performance TLC (HPTLC) plates. Stock solutions of the reference standard $(2 \mathrm{mg} / \mathrm{mL})$ in methanol was further diluted with methanol to $200 \mu \mathrm{g} / \mathrm{mL}, 20 \mu \mathrm{g} / \mathrm{mL}, 2 \mu \mathrm{g} / \mathrm{mL}, 200 \mathrm{ng} / \mathrm{mL}, 20 \mathrm{ng} / \mathrm{mL}$, and $2 \mathrm{ng} / \mathrm{mL}$.

\section{Analytical Methods}

The $R$. rosea extracts were analyzed with two basic chromatographic techniques - HPTLC and UPLC. Quantitative analysis of extracts was performed using a UPLC method, validated for linearity (Correlation coefficient $R>0.999$ ), repeatability and the levels 50,100, and 150\% (RSD < 5\%), intermediate precision at different days and analysts ( $\mathrm{RSD}<5 \%$ ), accuracy (recovery in the range from 90 to $110 \%$ ), selectivity (peak purity angle less than purity threshold with resolution $>2$ ), range from 80 to $120 \%$ and robustness (RSD $<2 \%$ ). Actual results are shown in tabulated form in the Supplementary Data S1, Table 2. The limits of detection (LOD) and quantification (LOQ) of UPLC methods were evaluated by calculations based upon the standard deviation of the response $(\sigma)$ and the slope $(S)$ of calibration curve and the following formulas: $\mathrm{LOD}=3.3 \sigma / \mathrm{S}$ and $\mathrm{LOQ}=10 \sigma / \mathrm{S}$. The specificity, generally defined as the ability of the UPLC methods to unequivocally assess the sample of interest in the presence of potential interferences, was evaluated in accordance with the new regulatory guideline (USP 25). In addition to the evaluation of the resolution between the sample peak and the next peak, a peak purity test based on photodiode array (PDA) detection was tested to demonstrate that the sample was pure with no co-eluting impurities. Specificity of the TLC method was based on the colors and $R_{\mathrm{f}}$ value of reference standards bands on the TLC plates visualized as described below.

\section{UPLC Method}

\section{Analytical instrumentation and chromatography details}

The UPLC fingerprints of the Rhodiola extracts were analyzed using a Waters Acquity UPLC system consisting of Quaternary Pumps Manager, Sample Manager, Column Manager, Photodiode Detector, and Empower 3 software (Waters Corporation, Milford, MA, United States). The UPLC column (Waters ACQUITY UPLC BEH C18, column; $100 \mathrm{~mm} \times 2.1 \mathrm{~mm}$ i.d., $1.8 \mu \mathrm{m}$, Waters Corporation, United States) solvent system was gradually increasing concentrations (2.5 to $100 \%$ in $14 \mathrm{~min}$ ) of acetonitrile in water with a flow rate of $0.6 \mathrm{~mL} / \mathrm{min}$ at $75^{\circ} \mathrm{C}$. The injection volume was $2 \mu \mathrm{L}$, detection was at $221 \mathrm{~nm}$ (phenylethanoids tyrosol and salidroside) and $252 \mathrm{~nm}$ 
TABLE 2 | Effective concentrations (\%) of Rhodiola extracts inducing single shock stimulation (SS) and theta burst stimulation (TBS) in the hippocampus slice preparation.

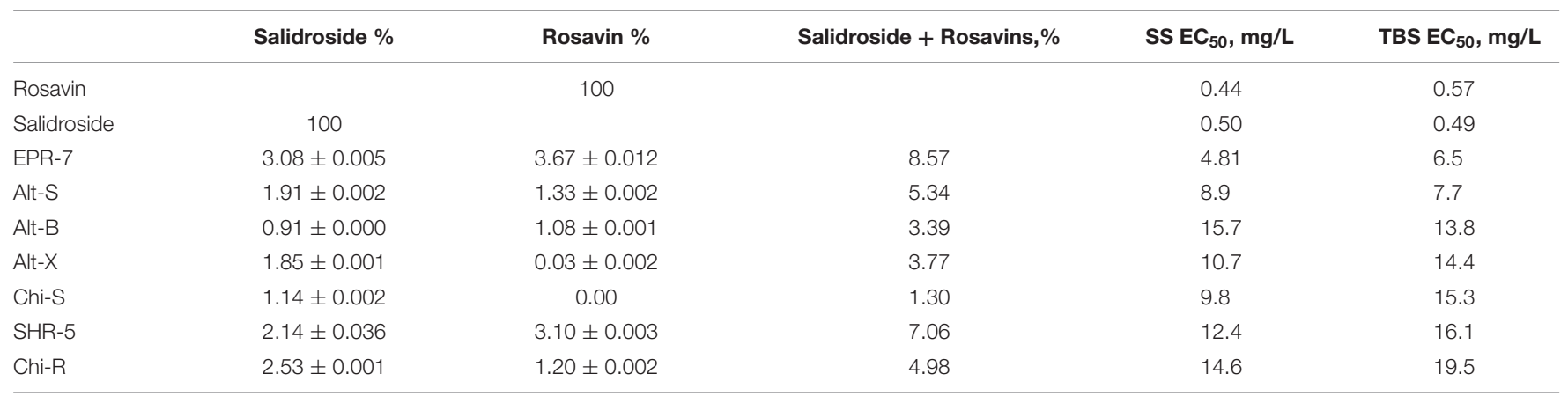

Rosavins is a collective name of phenylpropanoid glycosides rosavin, rosarin, and rosin.

(phenylpropanoids). All quantitative results were calculated per dry weight of the extracts.

\section{TLC Method}

Test solutions $(10 \mu \mathrm{L})$ were manually applied on HPTLC plates by a capillary. The solvent system used for HPTLC was ethyl acetate:methanol:water:acetic acid, 90:8:1:8. The bands on the silica gel 60 F254 pre-coated HPTLC plates were visualized by UV light at $254 \mathrm{~nm}$ and in the daylight after derivatization with anisaldehyde-sulfuric acid reagent (anisaldehyde:acetic acid:sulfuric acid:methanol, 0.5:10:5:85 $\mathrm{v} / \mathrm{v} / \mathrm{v} / \mathrm{v}$ ) at $105^{\circ} \mathrm{C}$ for $10 \mathrm{~min}$. The plates were documented using a "Reprostar" TLC/HPTLC imaging and documentation system for a UV system instrument (CAMAG, Switzerland). Images were captured under UV light at 254 and $366 \mathrm{~nm}$ prior to derivatization and in the daylight after derivatization.

\section{In Vitro Assay on Hippocampus Slices}

Hippocampus slices were obtained from 48 adult male SpragueDawley rats at the age of 40 days (Charles River Wiga, Sulzbach, Germany). Rats were kept under a reversed day/night cycle for 2 weeks prior to the start of the experiments to
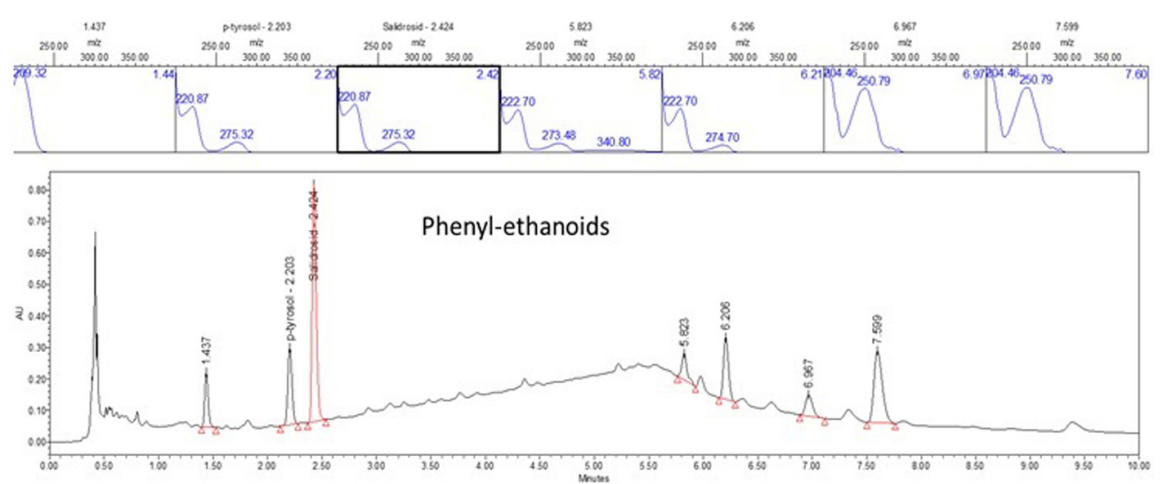

EurpPharma

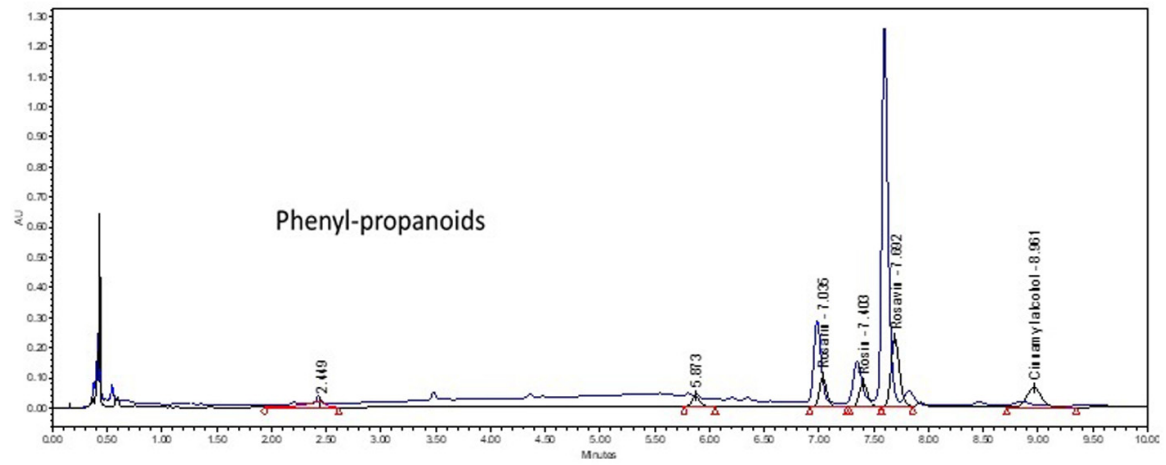

Europharma vS SHR-5

FIGURE 2 | UPLC-UV fingerprint of the R. rosea rhizome extracts EPR-7 and SHR-5: lower panel - overlay of chromatograms of EPR-7 and SHR-5 detected at $252 \mathrm{~nm}$, middle panel - EPR-7 detected at $221 \mathrm{~nm}$, upper panel - UV spectra of tyrosol, salidroside, rosarin, rosin, rosavin, and cinnamyl alcohol. 
allow recording of in vitro activity from slices during the active phase of their circadian rhythm (Dimpfel et al., 1994). Animals were exsanguinated under ether anesthesia, the brain was removed in total and the hippocampal formation was isolated under a microstereoscopic vision system. The midsection of the hippocampus was fixed to the table of a vibrating microtome (Rhema Labortechnik, Hofheim, Germany) using a cyanoacrylate adhesive, submerged in chilled bicarbonatebuffered saline [artificial cerebrospinal fluid (ACSF): $\mathrm{NaCl}$ : $124 \mathrm{mM}, \mathrm{KCl}: 5 \mathrm{mM}, \mathrm{CaCl}_{2}$ : $2 \mathrm{mM}, \mathrm{MgSO}_{4}: 2 \mathrm{mM}, \mathrm{NaHCO}_{3}$ : $26 \mathrm{mM}$, glucose: $10 \mathrm{mM}$ ], and cut into slices of $400 \mu \mathrm{m}$ thickness. All slices were pre-incubated for at least $1 \mathrm{~h}$ in Carbogen saturated ACSF (pH 7.4) in a pre-chamber before use (Dimpfel et al., 1991).

The stimulation of Schaffer Collaterals leads to release of glutamate, resulting in excitation of the postsynaptic pyramidal cells. The result of the electrical stimulation is recorded as a so-called population spike. The amplitude of the resulting population spike represents the number of recruited pyramidal cells. The response of the pyramidal cells to electric stimulation in the form of the amplitude of the population spike indicates activation as increase of the amplitude as reported earlier for Sideritis extract (Dimpfel et al., 2016a) or calming and sedating effects (attenuation of the amplitude). Of special interest is the response to theta burst stimulation (TBS) resulting in LTP, which relates to an increase of time- and space-dependent memory.

During the experiment, the slices were held and treated in a special super-fusion chamber (List Electronics, Darmstadt, Germany) (Haas et al., 1979) at $35^{\circ} \mathrm{C}$ (Schiff and Somjen, 1985). The preparation was super-fused with ACSF at 180-230 mL/h. Electrical stimulation $(200 \mu \mathrm{A}$ constant current pulses of $200 \mu \mathrm{s}$ pulse width) of the Schaffer Collaterals within the CA2 area and recording of extracellular field potentials from the pyramidal cell layer of CA1 (Dimpfel et al., 1991) was performed according to conventional electrophysiological methods using the "Labteam" Computer system "NeuroTool" software package (MediSyst $\mathrm{GmbH}$, Linden, Germany). Measurements were performed at 10 min intervals to avoid potentiation mechanisms. Four stimulations, each $20 \mathrm{~s}$ apart, were averaged for each time point. After obtaining three stable responses to SS, LTP was induced by applying a TBS. The mean amplitudes of three signals were averaged to give the mean of absolute voltage values (Microvolt) \pm standard error (SE) of the mean for four slices for one of the experimental conditions. Four slices were used per day.

\section{Statistical Analysis}

The results are reported as means \pm SD (standard deviation) or \pm SE for the indicated number of experiments. The significance of differences between samples and controls was determined with one-way independent measures ANOVA, followed by the post hoc Tukey's test for multiple comparisons. The correlations were evaluated using F-test. All calculations were performed using GraphPad (San Diego, CA, United States) Prism software (version 3.03) for Windows. GraphPad Prism was also used for supplemental graphs. All statistical tests were two-sided tests with $p$-values $<0.05$ regarded as significant (Supplementary Data S3). Wilcoxon-Mann-Whitney $U$ test was also used throughout all experimental data for comparison to results obtained by vehicle administration at the particular timing with respect to electrophysiological data (Supplementary Data S4).

\section{RESULTS}

\section{UPLC and HPTLC Metabolite Profiling}

Representative UPLC and HPTLC fingerprints of two $R$. rosea extracts, EPR-7 and the reference standard SHR-5, are shown in Figures 2, 3. They are almost identical except for the peaks corresponding to rosavins, which are higher on the chromatogram of EPR-7 (Figure 2) and the presence of some fluorescent compounds in SHR-5, which are absent in EPR-7 (Figure 3).

The contents of all active markers in all studied extracts (Table 1) were quite different, with a lack of rosavin, rosin, and rosarin in one extract from China. Even the extracts from the same geographic region - Altai (Russia) differed; e.g., the content of rosavin in Alt-X was 100 -fold lower (0.03\%) compared with SHR-5 (3.1\%) or EPR-7 (3.7\%), despite that all other phenyl- and ethyl propanoids were within common limits. This may have a significant impact on the activity of the Rhodiola Alt-X extract in T cell activation and apoptosis (Marchev et al., 2017). Overall, the highest content of rosavin and salidroside was in the Rhodiola EPR-7 extract.

\section{Electrophysiological Activity in a Synaptic Model of Memory: Hippocampal Long-Term Potentiation}

Figure 4 shows the concentration-dependent effects of salidroside and rosavin on pyramidal cell activity in terms

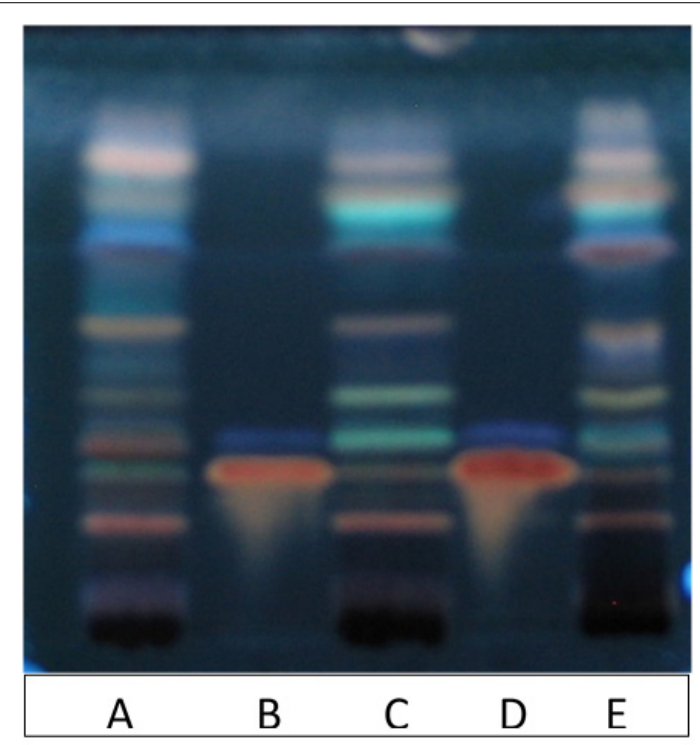

FIGURE 3 | High performance TLC (HPTLC) fingerprint of extracts exposed to UV $366 \mathrm{~nm}$, after derivatization with 10\% sulfuric acid in methanol. Track A EPR-7, track C - SHR-5, track E - Alt-S, track B and D - reference standards rosarin $R_{\mathrm{f}}=0.28$ and salidroside $R_{\mathrm{f}}=0.36$. Solvent system:

EtOAc:MeOH:H $\mathrm{H}_{2} \mathrm{O}: \mathrm{HCOOH}, 77: 13: 10: 2$. 
SS

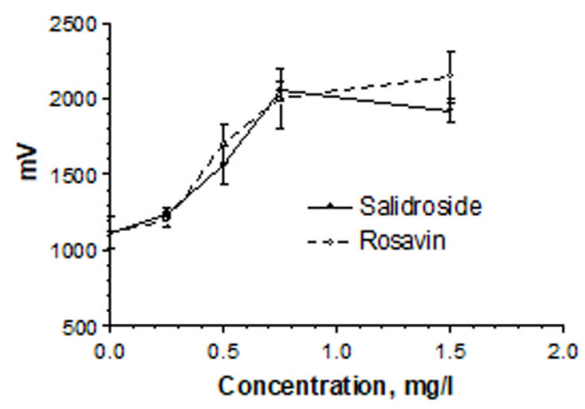

SS

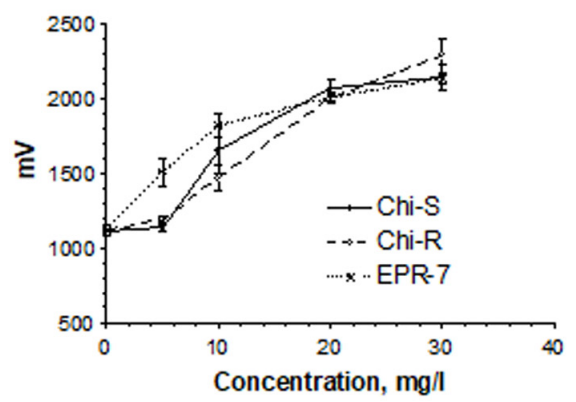

TBS

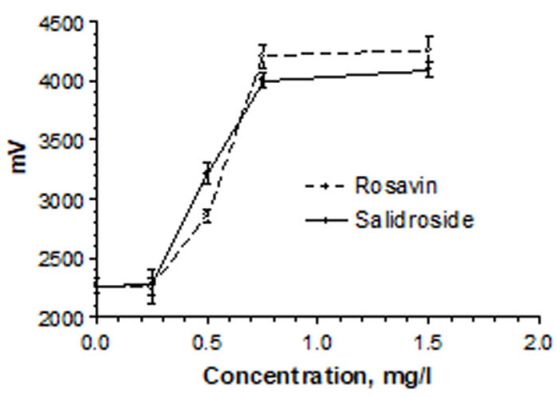

TBS

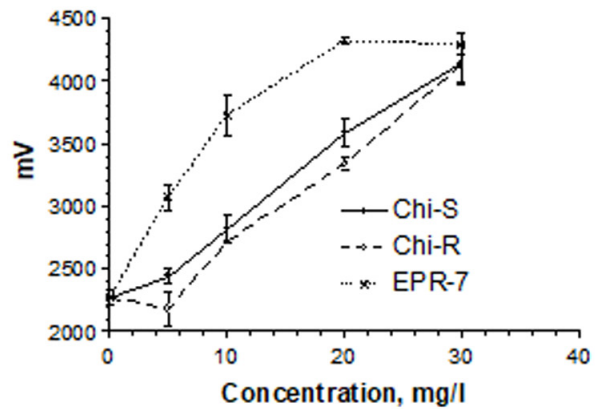

FIGURE 4 | Concentration-dependent effects of salidroside and rosavin on pyramidal cell activity in terms of changes of population spike amplitudes (millivolts). Results were obtained after a single stimuli (SS) or burst stimuli (TBS). Data represent the mean \pm SEM of $n=4$ slices (all concentrations). The details of statistical analysis see in Supplementary Data S3.

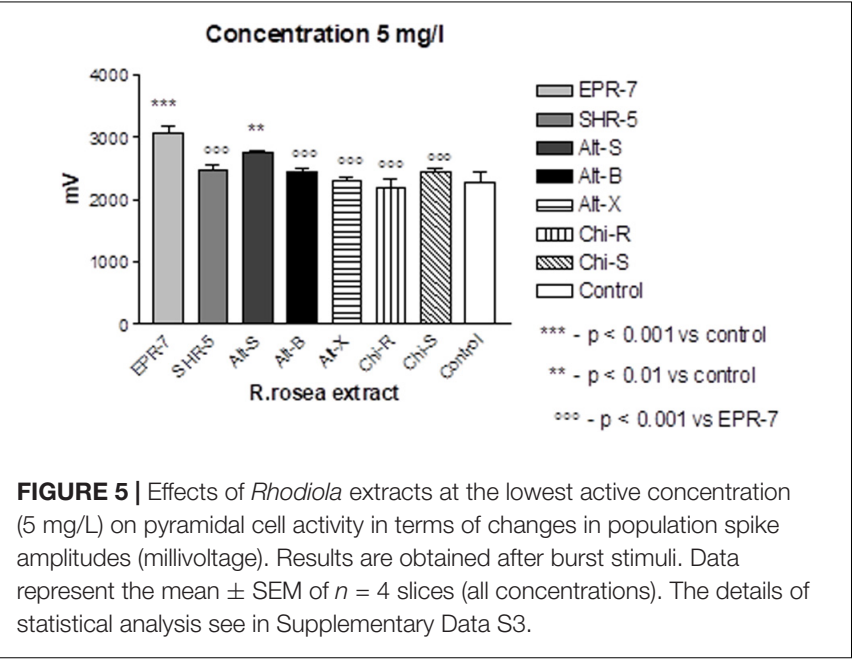

of changes of population spike amplitudes (millivolts) in hippocampus slices. In the presence of salidroside, the amplitudes of the population spike were enhanced in a concentrationdependent manner. During SS, amplitudes reached about $2.3 \mathrm{mV}$ and about $4.3 \mathrm{mV}$ during TBS. At $0.5 \mathrm{mg} / \mathrm{L}$, salidroside was more effective than rosavin in the TBS test, while at the higher concentrations of 0.75 and $1.5 \mathrm{mg} / \mathrm{L}$, the effect of rosavin was superior (Figure 4, upper part).
A comparison of the Rhodiola extract Chi-R, containing the phenyl ethanoids, tyrosol and salidroside, with the extracts containing both salidroside and rosavin, e.g., Chi-R vs. Rhodiola EPR-7, demonstrated that the content of salidroside in both extracts was almost the same 2.5-3.0\%, while the content of rosavin in EPR-7 was 3.5-fold higher than in Chi-R (Table 1). That is in line with results where the EPR-7 extract was as active as Chi-R at a 2.5-fold lower concentration (Figure 4, lower part, and Figure 5). Figure 5 shows that EPR-7 was the most active extract at $5 \mathrm{mg} / \mathrm{L}$, which corresponds to a concentration of rosavin of $0.18 \mathrm{mg} / \mathrm{L}(0.4 \mu \mathrm{M})$.

The $\mathrm{EC}_{50}$ values (Table 2) were calculated during SS and TBS. All results were fitted using a hyperbolic tangent function to give $\mathrm{EC}_{50}$ values (effective concentration to induce a halfmaximal effect). The lower the $\mathrm{EC}_{50}$ values, the less compound is needed to exert its pharmacological effect. The lower the effective concentration, the less side effects are expected. According to this bioassay, the EPR-7 extract had the lowest $\mathrm{EC}_{50}$ value among the seven Rhodiola extracts compared in this study (Table 2 and Figures 5, 6).

\section{DISCUSSION}

In this study, we compared the biological activity of various commercial extracts of $R$. rosea in a synaptic model of memory: 
ss

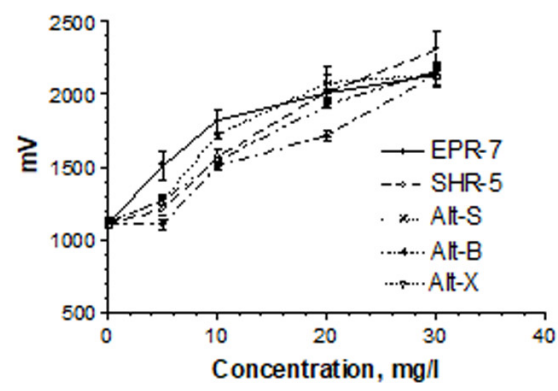

TBS

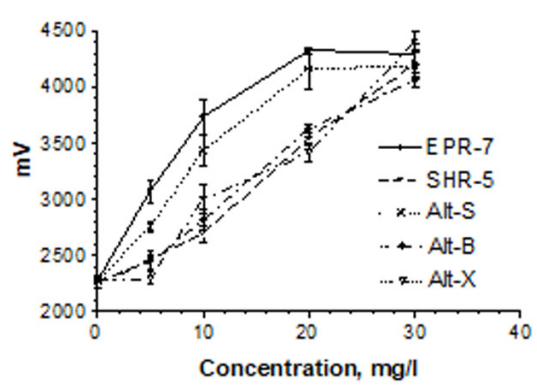

FIGURE 6 | Concentration-dependent effects of Rhodiola extracts on pyramidal cell activity in terms of changes in the population spike amplitudes (millivolts). Results were obtained after a single stimuli (SS) or burst stimuli (TBS). Data represent the mean \pm SEM of $n=4$ slices (all concentrations). The details of statistical analysis see in Supplementary Data S3.

the LTP of synaptic transmission in the hippocampus (Bliss and Collingridge, 1993). Synaptic transmission is critical in learning, memory, and functioning of the nervous system. As memories are thought to be determined by alteration of synaptic strength, a persistent increase in synaptic strength following high-frequency stimulation of a chemical synapse (LTP) is generally considered one of the major cellular mechanisms that triggers learning and memory.

When comparing the content of rosavin and/or salidroside in seven extracts with their $\mathrm{EC}_{50}$ values (Table 2), one can conclude that the EPR-7 extract, which has the highest content of both active markers, was active at the lowest concentrations. However, this correlation did not apply to other extracts, e.g., SHR-5, which presumably contained compounds that reduced the overall efficacy of the total extracts (Figure 3). Active compounds other than rosavin or salidroside might account for the efficacy of the extracts in this bioassay through synergistic or antagonistic modes of action. In this study, the brain slice is exposed directly to the samples, circumventing the blood-brain barrier. However, both preclinical and clinical studies have proven that Rhodiola extract exerts actions on the brain, suggesting enough compound passes the blood-brain barrier.

The efficacy of Rhodiola SHR-5 extract was demonstrated previously on healthy subjects (Spasov et al., 2000; Shevtsov et al., 2003; Dimpfel, 2014), subjects experiencing stress and fatigue (Darbinyan et al., 2000), and patients with chronic fatigue (Olsson et al., 2009) and major depressive disorder (Darbinyan et al., 2007; Mao et al., 2015). In a double blind, placebo-controlled study on 20 healthy subjects, Dimpfel demonstrated that a single dose administration of two capsules containing $200 \mathrm{mg} R$. rosea SHR-5 extract changed the spectral signature of electric brain activity in a stimulating way compared with placebo. The effect was regarded as a safe booster of mental activity during cognitive and emotional challenges. Rhodiola EPR-7 extract was also studied earlier in isolated skeletal muscle cells (Hernández-Santana et al., 2014), animals (Dimpfel et al., 2016b), and healthy human subjects (Shanely et al., 2014; Ahmed et al., 2015). Oral administration of $100 \mathrm{mg} / \mathrm{kg}$ of $R$. rosea root extract led to significant attenuation of $\alpha 1, \alpha 2, \beta 1, \beta 2, \delta$, and $\theta$ waves of the electropharmacograms, which are associated with the activation of dopamine, serotonin, glutamate, GABA, acetylcholine, and norepinephrine-mediated signaling pathways (Dimpfel et al., $2016 \mathrm{~b}$ ). The most affected were $\alpha 2$ (dopaminergic transmission CNS stimulating effect) and $\beta 1$ (glutaminergic transmission CNS stimulating effect) in the frontal cortex. The next strongest changes were seen in the striatum, and the weakest changes in the reticular formation. Spectral changes lasted up to $4 \mathrm{~h}$ after administration.

These results are in line with those of our recent publication, where we evaluated the effects of Rhodiola extract and salidroside on gene expression profiling in the T98G human neuroglia cell line (Panossian et al., 2014). The most significantly affected canonical pathways across the entire dataset, which contains the 1062 genes deregulated by Rhodiola and salidroside, were G-protein coupled receptor signaling, glutamate receptor signaling, ephrin receptor signaling, cAMP-mediated pathways, and dopamine signaling pathways associated with the expression of cell survival genes (Panossian et al., 2014). A meta-analysis on the putative antidepressant action of Rhodiola extract revealed it was effective on major depressive disorder (146 subjects) and stress-induced mild depression (714 individuals) (Amsterdam and Panossian, 2016). Rosavin was not included in that study. The results of our study are in line with a previous publication where the LTP effect of Rhodiola extract was tested in vitro in the hippocampal slice (Dimpfel et al., 2016b). A concentration of $5 \mathrm{mg} / \mathrm{L}$ induced a slight increase in the amplitude of the population spike and an increase in LTP. Further increases were observed by increasing the concentration up to $30 \mathrm{mg} / \mathrm{L}$. During TBS, amplitudes of more than $4 \mathrm{mV}$ were measured, indicating their effect on LTP.

\section{CONCLUSION}

In conclusion, rosavin, salidroside and various $R$. rosea extracts potentiated the in vitro electric stimulation of an intrahippocampal electric circuit, which resulted in higher responses 
of pyramidal cells in isolated hippocampus slices. Rosavin was more active in higher concentrations than salidroside; while, salidroside was more effective at lower concentrations. The highest content of both active markers was found in the extracts that were active at the lowest concentrations. Although, this correlation was not applicable to some extracts containing other compounds that presumably reduced the efficacy due to antagonistic interactions. The standardized content of active markers is necessary for the quality control of herbal preparations containing Rhodiola extracts, but insufficient for assessment of their potential efficacy. The application of bioassays should be required for adequate assessment of the quality and efficacy of $R$. rosea extracts.

\section{AVAILABILITY OF DATA AND MATERIAL}

The datasets used and/or analyzed during the current study are available from the corresponding author upon reasonable request.

\section{ETHICS STATEMENT}

The principles of laboratory animal care were followed in all trials and the local authority ("Regierungspräsidium" Giessen) responsible for animal care - was informed according to German Health Guidelines. Details of the acclimatization, housing conditions, and surgery have been reported (Dimpfel et al., 1991). Experiments were performed according to $\$ 4$ German Animal Protection Law (Tierschutzgesetz), which states, that animals are

\section{REFERENCES}

Ahmed, M., Henson, D. A., Sanderson, M. C., Nieman, D. C., Zubeldia, J. M., and Shanely, R. A. (2015). Rhodiola rosea exerts antiviral activity in athletes following a competitive marathon race. Front. Nutr. 2:24. doi: 10.3389/fnut. 2015.00024

Aksenova, R. A., Zotova, M. I., Nekhoda, M. F., and Cherdintsev, S. G. (1968). "Comparative characteristics of the stimulating and adaptogenic effects of Rhodiola rosea preparations," in Stimulants of the Central Nervous System, Vol. 2, ed. A. S. Saratikov (Tomsk: Tomsk University Press), 3-12.

Amsterdam, J. D., and Panossian, A. G. (2016). Rhodiola rosea L. as a putative botanical antidepressant. Phytomedicine 23, 770-783. doi: 10.1016/j.phymed. 2016.02.009

Barnaulov, O. D., Limarenko, A. Y., Kurkin, V. A., Zapesochnaya, G. G., and Shchavlinskij, A. N. (1986). A comparative evaluation of the biological activity of compounds isolated from species of Rhodiola. Khim. Pharm. Zh. 23, 1107-1112.

Bliss, T. V., and Collingridge, G. L. (1993). A synaptic model of memory: long-term potentiation in the hippocampus. Nature 361, 31-39. doi: 10.1038/361031a0

Booker, A., Jalil, B., Frommenwiler, D., Reich, E., Zhai, L., Kulic, Z., et al. (2016a). The authenticity and quality of Rhodiola rosea products. Phytomedicine 23, 754-762. doi: 10.1016/j.phymed.2015.10.006

Booker, A., Zhai, L., Gkouva, C., Li, S., and Heinrich, M. (2016b). From traditional resource to global commodities:-a comparison of Rhodiola species using NMR spectroscopy-metabolomics and HPTLC. Front. Pharmacol. 7:254. doi: 10.3389/fphar.2016.00254

Cifani, C., Micioni, Di Bonaventura, M. V., Vitale, G., Ruggieri, V., Ciccocioppo, R., et al. (2010). Effect of salidroside, active principle of Rhodiola rosea extract, on binge eating. Physiol. Behav. 101, 555-562. doi: 10.1016/j.physbeh.2010. 09.006 allowed to be killed for taking out organs for scientific purposes. Allowance to keep animals is renewed by governmental authority every 3 years.

\section{AUTHOR CONTRIBUTIONS}

AP planned the experiments and wrote the manuscript. LS performed the experiments. WD carried out data analysis and wrote a report on the results. All authors critically revised and approved the final version of the manuscript.

\section{FUNDING}

This work was supported by the EuroPharma USA. Sponsor of the research: Terry Lemerond, EuroPharma USA Inc.

\section{ACKNOWLEDGMENTS}

The authors acknowledge the support of EuroPharma USA Inc. for supplying the investigational agents and material support. The authors are grateful to all participants of the study.

\section{SUPPLEMENTARY MATERIAL}

The Supplementary Material for this article can be found online at: https://www.frontiersin.org/articles/10.3389/fphar. 2018.00425/full\#supplementary-material

Currier, A., and Ampong-Nyarko, K. (2015). Rhodiola Rosea. Boca Raton, FL: CRC Press.

Darbinyan, V., Aslanyan, G., Amroyan, E., Gabrielyan, E., Malmström, C., and Panossian, A. (2007). Clinical trial of Rhodiola rosea L. extract SHR-5 in the treatment of mild to moderate depression. Nord. J. Psychiatry 61, 343-348. doi: 10.1080/08039480701643290

Darbinyan, V., Kteyan, A., Panossian, A., Gabrielian, E., Wikman, G., and Wagner, H. (2000). Rhodiola rosea in stress induced fatigue-a double blind cross-over study of a standardized extract SHR-5 with a repeated low-dose regimen on the mental performance of healthy physicians during night duty. Phytomedicine 7, 365-371. doi: 10.1016/S0944-7113(00)80055-0

Dimpfel, W. (1995). Effects of memantine on synaptic transmission in the hippocampus in vitro. Arzneimittelforschung 45, 1-5.

Dimpfel, W. (2014). Neurophysiological effects of Rhodiola rosea extract containing capsules (A double-blind, randomized, placebo-controlled study). Int. J. Nutr. Food Sci. 3, 157-165.

Dimpfel, W., Dalhoff, B., Hofmann, W., and Schlüter, G. (1994). Electrically evoked potentials in the rat hippocampus slice in the presence of aminophylline alone and in combination with quinolones. Eur. Neuropsychopharmacol. 4, 151-156. doi: 10.1016/0924-977X(94)90009-4

Dimpfel, W., Schombert, L., and Feistel, B. (2016a). Ex vivo characterization of the action of sideritis extract using electrical activity in the hippocampus slice preparation. Pharmacol. Pharm. 7, 407-416. doi: 10.4236/pp.2016.710048

Dimpfel, W., Schombert, L., Vega-Morales, T., and Wiebe, J. (2016b). Neuropharmacological characterization of extracts from Rhodiola rosea, Oenothera paradoxa and Paullinia cupana in comparison to caffeine. Pharmacol. Pharm. 7, 290-303. doi: 10.4236/pp.2016.77036

Dimpfel, W., Spüler, M., Dalhoff, A., Hoffmann, W., and Schlüter, G. (1991). Hippocampal activity in the presence of quinolones and fenbufen in-vitro. Antimicrob. Agents Chemother. 6, 1142-1146. doi: 10.1128/AAC.35.6.1142 
EFSA Consolidated List of Article 13 Health Claims of the European Food Safety Authority [EFSA] (2010). Legal and Regulatory Framework for Herbal Medicines. Association of the European Self-Medication Industry (AESMI). Brussels, 151-158.

European Medicine Authority [EMA] (2011). Assessment report on Rhodiola rosea L., rhizoma et radix. London: European Medicines Agency.

Haas, H. L., Schaerer, B., and Vosmansky, M. (1979). A simple perfusion chamber for the study of nervous tissue slices in vitro. J. Neurosci. Methods 1, 323-325. doi: 10.1016/0165-0270(79)90021-9

Hellum, B. H., Tosse, A., Hoybakk, K., Thomsen, M., Rohloff, J., and Georg Nilsen, O. (2010). Potent in vitro inhibition of CYP3A4 and P-glycoprotein by Rhodiola rosea. Planta Med. 76, 331-338. doi: 10.1055/s-0029-1186153

Hernández-Santana, A., Pérez-López, V., Zubeldia, J. M., and Jiménez-delRio, M. (2014). A Rhodiola rosea root extract protects skeletal muscle cells against chemically induced oxidative stress by modulating heat shock protein 70 (HSP70) expression. Phytother Res. 28, 623-628. doi: 10.1002/ptr. 5046

Ioset, K. N., Nyberg, N. T., Van Diermen, D., Malnoe, P., Hostettmann, K., Shikov, A. N., et al. (2011). Metabolic profiling of Rhodiola rosea rhizomes by ${ }^{1}$ H NMR spectroscopy. Phytochem. Anal. 22, 158-165. doi: 10.1002/pca. 1262

Jacob, R., Nalini, G., and Chidambaranathan, N. (2013). Neuroprotective effect of Rhodiola rosea Linn against MPTP induced cognitive impairment and oxidative stress. Ann. Neurosci. 20, 47-51. doi: 10.5214/ans.0972.7531.200204

Kurkin, V. A., and Zapesochnaya, G. G. (1986). Chemical composition and pharmacological properties of Rhodiola rosea. Khim. Pharm. Zurnal. 20, 1231-1244.

Kurkin, V. A., Zapesochnaya, G. G., Shchavlinskii, A. N., Nukhi-movskii, E. L., and Vandyshev, V. V. (1985). Method of analysis of identity and quality of Rhodiola rosea rhizome. Khim. Farm. Zh. 19, 185-190.

Lee, Y., Jung, J.-C., Jang, S., Kim, J., Ali, Z., Khan, I. A., et al. (2013). Anti-Inflammatory and neuroprotective effects of constituents isolated from Rhodiola rosea. Evid. Based Complement. Alternat. Med. 2013:514049.

Mao, J. J., Xie, S. X., Zee, J., Soeller, I., Li, S. Q., Rockwell, K., et al. (2015). Rhodiola rose vs. sertraline for major depressive disorder: a randomized placebocontrolled trial. Phytomedicine 22, 394-399. doi: 10.1016/j.phymed.2015. 01.010

Marchev, A. S., Dimitrova, P., Koycheva, I. K., and Georgiev, M. I. (2017). Altered expression of TRAIL on mouse T cells via ERK phosphorylation by Rhodiola rosea L. and its marker compounds. Food Chem. Toxicol. 108(Pt B), 419-428. doi: 10.1016/j.fct.2017.02.009

Marina, T. F., and Alekseeva, L. P. (1968). "Effect of Rhodiola rosea extract on electroencephalograms"," in Stimulants of the Central Nervous System, ed. A. S. Saratikov (Tomsk: Tomsk University Press), 22-26.

Marina, T. F., Mikhaleva, L. K., and Suslov, N. I. (1994). "Comparative effects of para-tyrosol and Rhodiola extract on the central nervous system," in Proceedings of a Joint Plenary Session of Pathophysiologists and Pharmacologists of Siberia and the Far East on Mechanisms of the Development of Pathological Processes (Kemerovo: Kemerovo State University Press), 66-68.

Nabavi, S. F., Braidy, N., Orhan, I. E., Badiee, A., Daglia, M., and Nabai, S. M. (2016). Rhodiola rosea L. and Alzheimer's disease: from farm to pharmacy. Phytother. Res. 30, 532-539. doi: 10.1002/ptr.5569

Nakamura, S., Matsuda, H., Ninomiya, K., Morikawa, T., Yamaguti, K., et al. (2007). Bioactive constituents from Chinese natural medicines. XXVI. Chemical structures and hepatoprotective effects of constituents from roots of Rhodiola sachalinensis. Chem. Pharm. Bull. 55, 1505-1511. doi: 10.1248/cpb.55. 1505

Olsson, E. M. G., von Schéele, B., and Panossian, A. G. (2009). A randomized double-blind placebo controlled parallell group study of SHR-5 extract of Rhodiola rosea roots as treatment for patients with stress related fatigue. Planta Med. 75, 105-112. doi: 10.1055/s-0028- 1088346

Panossian, A., and Gerbarg, P. (2016). "Potential use of plant adaptogens in agerelated disorders," in Complementary, Alternative, and Integrative Interventions in Mental Health and Aging, eds H. Lavretsky, M. Sajatovic, and C. F. Reynolds III (New York: Oxford University Press), 197-211.

Panossian, A., Hamm, R., Wikman, G., and Efferth, T. (2014). Mechanism of action of Rhodiola, salidroside, tyrosol and triandrin in isolated neuroglial cells: an interactive pathway analysis of the downstream effects using RNA microarray data. Phytomedicine 21, 1325-1348. doi: 10.1016/j.phymed.2014. 07.008

Panossian, A., Nikoyan, N., Ohanyan, N., Hovhannisyan, A., Abrahamyan, H., Gabrielyan, E., et al. (2008). Comparative study of Rhodiola preparations on behavioral despair of rats. Phytomedicine 15, 84-91. doi: 10.1016/j.phymed. 2007.10.003

Panossian, A., and Wagner, H. (2005). Stimulating effect of adaptogens: an overview with particular reference to their efficacy following single dose administration. Phytother. Res. 19, 819-838. doi: 10.1002/ptr.1751

Panossian, A., and Wikman, G. (2009). Evidence-based efficacy of adaptogens in fatigue, and molecular mechanisms related to their stress-protective activity. Curr. Clin. Pharmacol. 4, 198-219. doi: 10.2174/1574884097893 75311

Panossian, A., and Wikman, G. (2010). Effects of adaptogens on the central nervous system and the molecular mechanisms associated with their stress-protective activity. Pharmaceuticals 3, 188-224. doi: 10.3390/ph301 0188

Panossian, A., and Wikman, G. (2014). "Evidence based efficacy and effectiveness of Rhodiola SHR-5 extract in treating stress- and age-associated disorders," in The Book Rhodiola rosea, eds C. Cuerrier and K. Ampong-Nyarko (Boca Raton, FL: CRC Press), 203-221.

Panossian, A., Wikman, G., Kaur, P., and Asea, A. (2012). Adaptogens stimulate neuropeptide $\mathrm{Y}$ and Hsp72 expression and release in neuroglia cells. Front. Neurosci. 6:6. doi: 10.3389/fnins.2012.00006

Panossian, A., Wikman, G., and Sarris, J. (2010). Rosenroot (Rhodiola rosea): traditional use, chemical composition, pharmacology and clinical efficacy. Phytomedicine 17, 481-493. doi: 10.1016/j.phymed.2010.02.002

Panossian, A. G. (2013). Adaptogens in mental and behavioral disorders. Psychiatr. Clin. North Am. 36, 49-64. doi: 10.1016/j.psc.2012.12.005

Petkov, V. D., Yonkov, D., Mosharoff, A., Kambourova, T., Alova, L., and Petkov, V. V. (1986). Effects of alcohol aqueous extract from Rhodiola rosea L. roots on learning and memory. Acta Physiol Pharmacol Bulg. 12, 3-16.

Qu, Z. Q., Zhou, Y., Zeng, Y. S., Li, Y., and Chung, P. (2009). Pretreatment with Rhodiola rosea extract reduces cognitive impairment induced by intracerebroventricular streptozotocin in rats: implication of anti-oxidative and neuroprotective effects. Biomed. Environ. Sci. 22, 318-326. doi: 10.1016/S08953988(09)60062-3

Saratikov, A. S., and Krasnov, E. A. (2004). Rhodiola rosea (Golden Root): A Valuable Medicinal Plant. Tomsk: Tomsk University Press.

Saratikov, A. S., Krasnov, E. A., Chnikina, L. A., Duvidson, L. M., Sotova, M. I., Marina, T. F., et al. (1968). Rhodiolosid, a new glycoside from Rhodiola rosea and its pharmacological properties. Pharmazie 23, 392-395.

Saratikov, A. S., Marina, T. F., and Fisanova, L. L. (1978). Mechanism of action of salidrozide on the metabolism of cerebral catecholamines. Vopr. Med. Khim. 5, 624-621.

Saratikov, A. S., Marina, T. F., and Kaliko, I. M. (1965). The stimulating effect of Rhodiola rosea on the higher brain structures. Vestn. Sibirsk. Otd. USSR Acad. Sci. 8, 120-125.

Schiff, S. J., and Somjen, G. G. (1985). The effects of temperature on synaptic transmission in hippocampal tissue slices. Brain Res. 345, 279-284. doi: 10.1016/ 0006-8993(85)91004-2

Shanely, R. A., Nieman, D. C., Zwetsloot, K. A., Knab, A. M., Imagita, H., Luo, B., et al. (2014). Evaluation of Rhodiola rosea supplementation on skeletal muscle damage and inflammation in runners following a competitive marathon. Brain Behav. Immun. 39, 204-210. doi: 10.1016/j.bbi.2013.09.005

Shevtsov, V. A., Zholus, B. I., Shervarly, V. I., Vol'skij, V. B., Korovin, Y. P., Khristich, M. P., et al. (2003). A randomized trial of two different doses of a SHR-5 Rhodiola roseaextract versus placebo and control of capacity for mental work. Phytomedicine 10, 95-105. doi: 10.1078/0944711033216 59780

Sokolov, S. Y., Boyko, V. P., Kurkin, V. A., Zapesochnaya, G. G., Rvantsova, N. V., and Grinenko, H. A. (1990). A comparative study of the stimulant property of certain phenylpropanoids. Khim. Pharm. Zh. 24, 66-68.

Sokolov, S. Y., Ivashin, V. M., Zapesochnaya, G. G., Kurkin, V. A., and Shavlinskiy, A. N. (1985). Study of neurotropic activity of new substances isolated from Rhodiola rosea. Khim. Pharm. Zh. 19, 1367-1371.

Spasov, A. A., Wikman, G. K., Mandrikov, V. B., Mironova, I. A., and Neumoin, V. V. (2000). A double-blind, placebo-controlled pilot study of the stimulating 
and adaptogenic effect of Rhodiola rosea SHR-5 extract on the fatigue of students caused by stress during an examination period with a repeated low-dose regimen. Phytomedicine 7, 85-89. doi: 10.1016/S0944-7113(00) 80078-1

Thu, O. K., Nilsen, O. G., and Hellum, B. (2016a). In vitro inhibition of cytochrome P-450 activities and quantification of constituents in a selection of commercial Rhodiola rosea products. Pharm. Biol. 54, 3249-3256. doi: 10.1080/13880209. 2016.1223145

Thu, O. K. F., Spigset, O., and Hellum, B. (2017). Noncompetitive inhibition of human CYP2C9 in vitro by a commercial Rhodiola rosea product. Pharmacol. Res. Perspect. 5:e00324. doi: 10.1002/pr p2.324

Thu, O. K., Spigset, O., Nilsen, O. G., and Hellum, B. (2016b). Effect of commercial Rhodiola rosea on CYP enzyme activity in humans. Eur. J. Clin. Pharmacol. 72, 295-300. doi: 10.1007/s00228-015-1988-7

Xu, W., Zhang, T., Wang, Z., Liu, T., Liu, Y., Cao, Z., et al. (2013). Two potent cytochrome P450 2D6 inhibitors found in Rhodiola rosea. Pharmazie 68, 974-976.
Conflict of Interest Statement: AP is currently a consultant to EuroPharma USA Inc., Founder of Phytomed AB (Sweden), and former Head of Research \& Development at the Swedish Herbal Institute, Gothenburg, Sweden. He is not a member of any pharmaceutical industry-sponsored advisory board or speaker's bureau and has no significant financial interest in any pharmaceutical company. WD and LS are not members of any pharmaceutical industry-sponsored advisory board or speaker's bureau, and have no significant financial interest in any pharmaceutical company. The samples of Rhodiola extracts used in the study were donated by the manufacturer EuroPharma USA Inc. EuroPharma USA Inc. had no other involvement in the study.

Copyright $\odot 2018$ Dimpfel, Schombert and Panossian. This is an open-access article distributed under the terms of the Creative Commons Attribution License (CC BY). The use, distribution or reproduction in other forums is permitted, provided the original author(s) and the copyright owner are credited and that the original publication in this journal is cited, in accordance with accepted academic practice. No use, distribution or reproduction is permitted which does not comply with these terms. 


\section{OPEN ACCESS}

Edited by:

Issy Laher,

University of British Columbia, Canada

Reviewed by:

Víctor López,

Universidad San Jorge, Spain

Roger Lyrio Santos,

Federal University of Espirito Santo,

Brazil

*Correspondence:

Pavel Uhrin

pavel.uhrin@meduniwien.ac.at

Atanas G. Atanasov

a.atanasov@ighz.pl

tPresent Address:

Ibrahim M. Abu-Reidah,

Industrial Chemistry Department,

Faculty of Sciences, Arab American University, Zababdeh-Jenin, Palestine

Specialty section:

This article was submitted to

Ethnopharmacology,

a section of the journal

Frontiers in Pharmacology

Received: 15 January 2018

Accepted: 04 May 2018

Published: 24 May 2018

Citation:

Wang D, Özen C, Abu-Reidah IM,

Chigurupati S, Patra JK,

Horbanczuk JO, Jóźwik A,

Tzvetkov NT. Uhrin P and

Atanasov AG (2018) Vasculoprotective

Effects of Pomegranate (Punica

granatum L.).

Front. Pharmacol. 9:544.

doi: 10.3389/fphar.2018.00544

\section{Vasculoprotective Effects of Pomegranate (Punica granatum L.)}

\author{
Dongdong Wang ${ }^{1,2,3}$, Cigdem Özen ${ }^{4}$, Ibrahim M. Abu-Reidah ${ }^{5 \dagger}$, Sridevi Chigurupati ${ }^{6}$, \\ Jayanta Kumar Patra ${ }^{7}$, Jarosław O. Horbanczuk ${ }^{1}$, Artur Jóźwik ${ }^{1}$, Nikolay T. Tzvetkov ${ }^{8,9}$, \\ Pavel Uhrin ${ }^{10 *}$ and Atanas G. Atanasov ${ }^{1,2,10 *}$ \\ ${ }^{1}$ Department of Molecular Biology, Institute of Genetics and Animal Breeding of the Polish Academy of Sciences, \\ Jastrzębiec, Poland, ${ }^{2}$ Department of Pharmacognosy, Faculty of Life Sciences, University of Vienna, Vienna, Austria, \\ ${ }^{3}$ Institute of Clinical Chemistry, University Hospital Zurich, University of Zurich, Zurich, Switzerland, ${ }^{4}$ Izmir International \\ Biomedicine and Genome Institute, Dokuz Eylul University, Health Campus Balcova, Izmir, Turkey, ${ }^{5}$ Department of Chemistry, \\ Faculty of Science, An-Najah National University, Nablus, Palestine, ${ }^{6}$ Department of Medicinal Chemistry and \\ Pharmacognosy, College of Pharmacy, Qassim University, Buraidah, Saudi Arabia, ${ }^{7}$ Research Institute of Biotechnology and \\ Medical Converged Science, Dongguk University-Seoul, Goyang, South Korea, ${ }^{8}$ Pharmaceutical Institute, University of \\ Bonn, Bonn, Germany, ${ }^{9}$ Department of Molecular Design and Biochemical Pharmacology, Institute of Molecular Biology \\ "Roumen Tsanev", Bulgarian Academy of Sciences, Sofia, Bulgaria, ${ }^{10}$ Department of Vascular Biology and Thrombosis \\ Research, Center for Physiology and Pharmacology, Medical University of Vienna, Vienna, Austria
}

Pomegranate (Punica granatum L.), one of the oldest known edible fruits, is nowadays broadly consumed throughout the world. Its fruits and seeds as well as other anatomical compartments (e.g., flowers and leaves) are rich in numerous bioactive compounds and therefore, the scientific interest in this plant has been constantly growing in recent years. It has been shown that pomegranate and its extracts exhibit potent antioxidative, antimicrobial, and anticarcinogenic properties. The present review summarizes some recent studies on pomegranate, highlighting mainly its vasculoprotective role attributed to the presence of hydrolyzable tannins ellagitannins and ellagic acid, as well as other compounds (e.g., anthocyanins and flavonoids). These in vitro and in vivo studies showed that substances derived from pomegranate reduce oxidative stress and platelet aggregation, diminish lipid uptake by macrophages, positively influence endothelial cell function, and are involved in blood pressure regulation. Clinical studies demonstrated that daily intake of pomegranate juice lessens hypertension and attenuates atherosclerosis in humans. Altogether, the reviewed studies point out the potential benefits of a broader use of pomegranate and its constituents as dietary supplements or as adjuvants in therapy of vascular diseases, such as hypertension, coronary artery disease, and peripheral artery disease.

\section{Keywords: pomegranate, antioxidant, blood pressure, cardiovascular disease, vasculoprotective}

\section{INTRODUCTION}

Pomegranate (Punica granatum L.), belonging to Punica L. genus, Punicaceae family, is an ancient fruit native to Central Asia in regions spanning from Iran and Turkmenistan to northern India as well as in the Mediterranean area and the Middle East (Holland et al., 2009). Archaeologists have found carbonized pomegranate exocarps originated from the Early Bronze Age (3000 BC), e.g., in Jericho and from the Late Bronze Age in Cyprus (Ward, 2003; Boncuk, 2014). Pomegranate has been highly appreciated since centuries by different cultures. For example, in Ancient Egypt it was not only a part of the supply of fruits for pharaoh's residence (at around 1600 B.C.), but pomegranate was also painted on walls and tombs to symbolize life after death (Ward, 2003; Boncuk, 2014). Pomegranate used to 
play an important role in different religions, including Zoroastrianism, Judaism, Buddhism, Christianity, and Islam (Langley, 2000; Jurenka, 2008). It was praised, e.g., by the Old Testament of the Bible as "a sacred fruit conferring powers of fertility, abundance, and good luck" (Jurenka, 2008). Besides being a part of the mythology and consumed as a fruit, pomegranate has been known for its medical use. For example, the Ebers papyrus originating from about $1550 \mathrm{BC}$ noted that the roots of the pomegranate tree were used to treat tapeworm parasites (Svenja, 2018). In addition, pomegranate was employed to treat diabetes by Indians (Saxena and Vikram, 2004) and to lessen tapeworm infestation also by Romans (Langley, 2000). The persisting significance of the medical use of pomegranate can be illustrated, for example, by the fact that in Great Britain the coats of arms of three royal colleague and the British Medical Association are decorated with the figure of this herb (Langley, 2000).

Besides native regions spreading from Iran to northern India and the Mediterranean area and the Middle East, pomegranate is nowadays cultivated in subtropical Africa as well as in California, Arizona, and Mexico, as this plant requires high exposure to sunlight during summer and temperature not lower than $\sim 12^{\circ} \mathrm{C}$ in winter (Levin, 2006; Holland et al., 2009). The pomegranate tree is about $2-3 \mathrm{~m}$ tall, glabrous, with multiple trunks and bushy appearance. The surface of the leaves is smooth and hairless, with a glossy appearance on the upper part of the leaf (Figure 1). The fruit ripens within 5-8 months after it has begun to form. During this process, the color of the external part of the fruit changes from yellow, green, or pink to fully red, pink, or deep purple (Figure 1). An edible juicy layer of a fruit varies in color from white to deep red (Holland et al., 2009).

Studies accomplished over the last several decades showed that pomegranate and its components exhibit potent antioxidative (Gil et al., 2000; Les et al., 2015), anti-inflammatory (Adams et al., 2006; Rasheed et al., 2009) as well as antibacterial, antimicrobial, and antifungal properties (Naz et al., 2007; Fawole et al., 2012; Elsherbiny et al., 2016; Wafa et al., 2017). In addition to these in vitro studies, in vivo and in vitro studies showed that pomegranate exhibits anti-hypertensive (Mohan et al., 2010; Dos Santos et al., 2016; Arun et al., 2017) and antiproliferative properties (Albrecht et al., 2004; Malik et al., 2005; Malik and Mukhtar, 2006). Pomegranate and its constituents have been tested for their use as adjuvant therapy for treatment of several forms of oncological diseases, mainly of prostate cancer (Lansky and Newman, 2007; Paller et al., 2013; Panth et al., 2017; Sharma et al., 2017). Furthermore, numerous pre-clinical studies have pointed out the beneficial effects of intake of pomegranate juice or pomegranate extracts in a variety of conditions. For example, such treatment improved sperm quality in mice (Türk et al., 2008), lowered amyloid deposition in a mouse model of Alzheimer's disease (Hartman et al., 2006), and lessened neuronal damage in a mouse neonatal hypoxic-ischemic brain injury model (Loren et al., 2005). In addition, single intraperitoneal injection with pomegranate extract applied to fishes that had been naturally infected with lymphocystis disease virus, stimulated their innate immune response, and reduced their mortality due to lymphocystis infection (Harikrishnan et al., 2010). In humans, oral administration of pomegranate extract enriched with ellagic acid is beneficial for minimizing ultraviolet-induced skin damage (Kasai et al., 2006), while hydro-alcoholic extracts of pomegranate have a significant antibacterial activity and are therefore useful for treatment of dental plaques (Menezes et al., 2009). Many studies also demonstrated potent vasculoprotective effects of pomegranate and its constituents, as presented below.

\section{BIOACTIVE CONSTITUENTS OF POMEGRANATE}

Bioactive substances of pomegranate include, for example, hydrolyzable tannins (gallotannins and ellagitannins), ellagic acid and its derivatives, gallic acid, anthocyanins/anthocyanidins, proanthocyanidins, flavonoids, vitamins, as well as sterols, lignans, saccharides, fatty acids, organic acids, terpenes, and terpenoids, among others. Ellagitannins and gallotannins together with ellagic acid and its derivatives are crucial bioactive compounds of pomegranate (Amakura et al., 2000a; Gil et al., 2000; Fischer et al., 2011a; Borges and Crozier, 2012; Brighenti et al., 2017). Furthermore, ellagitannins and gallotannins are hydrolyzed to ellagic acid and glucose or gallic acid and glucose, respectively (Arapitsas, 2012). In addition, pomegranate is a source of numerous (poly)phenolic compounds (Fischer et al., 2011a). Anthocyanins present in pomegranate comprise mainly delphinidin 3-glucoside, delphinidin 3,5-diglucoside, pelargonidin 3-glucoside, pelargonidin 3,5-diglucoside, cyanidin 3-glucoside, and cyanidin 3,5-diglucoside (Alighourchi et al., 2008; Fischer et al., 2013; Lantzouraki et al., 2015), and the characteristic colors of pomegranate fruits are attributed to them. Pomegranate seeds contain different fatty acids with the most represented punicic acid (Schubert et al., 1999; Kaufman and Wiesman, 2007; Pande and Akoh, 2009; Verardo et al., 2014; Górnaś and Rudzinska, 2016). Flavol-3-ols, flavonoid glycosides, phenolic acids, and hydrolyzable tannins represent main phenolic compounds in pomegranate seed residue (He et al., 2011). In pomegranate peel, gallic acid is a major phenolic constituent while kaempferol-3-O-glucoside is the most represented flavonoid (Ambigaipalan et al., 2016). Triterpenoids oleanolic acid and ursolic acid are present in pomegranate flower (Fu et al., 2014). Volatile components of pomegranate comprise monoterpenes, monoterpenoids, aldehydes, alcohols, and linear hydrocarbons monoterpenes, especially represented by alpha-terpinene, alpha-terpineol, and 3-carene (VázquezAraújo et al., 2011; Carbonell-Barrachina et al., 2012). An overview of compounds identified in pomegranate is outlined in Table 1.

\section{VASCULOPROTECTIVE EFFECTS OF VARIOUS PARTS OF POMEGRANATE REVEALED IN IN VITRO AND IN VIVO MODELS}

Many pomegranate-derived compounds exhibit a wide range of vasculoprotective effects. Various pomegranate parts 

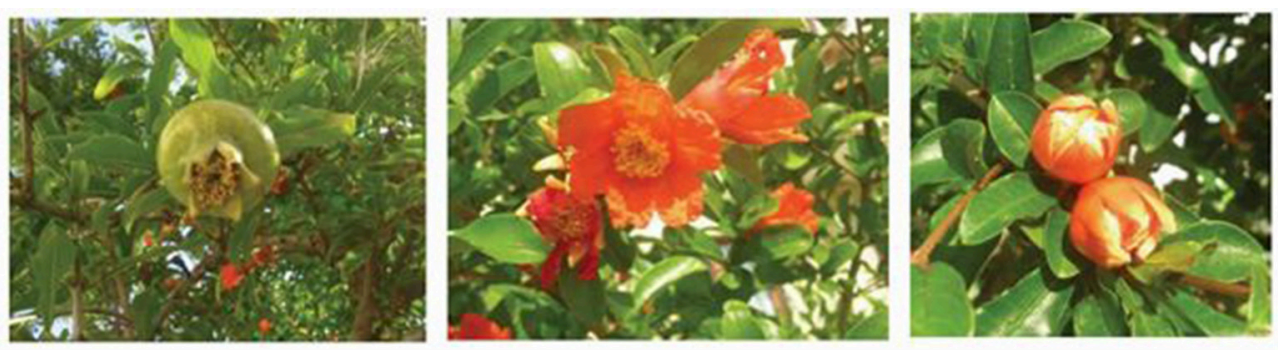

FIGURE 1 | Fruits, flowers, and leaves of Punica granatum L.

(components) have proven to reduce oxidative stress, lipid peroxidation, and generation of foam cells, to positively influence endothelial cell function (by increasing NO levels and lowering glucose levels), to attenuate platelet aggregation and diminish hypertension, thus altogether improving vascular function, as presented below. In addition, pomegranate and its components are protective against toxicity induced by chemicals or drugs (Table 2 and the text below).

\section{Pomegranate Juice and Extract}

In 2000s, Gil et al. in their pivotal study pointed out the strong antioxidant properties of pomegranate juice enriched with tannin punicalagin, anthocyanins, ellagic acid derivatives, as well as other phenolic substances. Using different analytical methods, the authors revealed potent antioxidant activities of pomegranate juice that were three times higher than the wellknown antioxidative properties of red wine or green tea (Gil et al., 2000). These findings were confirmed by subsequent studies that additionally pointed to vasculoprotective effects of pomegranate products, as presented below.

In a study involving mice as well as human volunteers, pomegranate juice intake attenuated oxidative stress (Aviram et al., 2000). More specifically, in apolipoprotein E-deficient mice, food supplementation with pomegranate juice reduced by $44 \%$ the size of atherosclerotic lesions and diminished the number of foam cells in such lesions (Aviram et al., 2000). In humans, intake of pomegranate juice diminished the susceptibility of low-density lipoproteins (LDLs) to aggregate and enhanced by up to $20 \%$ the activity of serum paraoxonase (Aviram et al., 2000), an esterase that is associated with high-density lipoproteins (HDLs) and can protect lipids against peroxidation (Chistiakov et al., 2017). Pomegranate juice also inhibited the oxidized LDL (oxLDL) uptake and cholesterol biosynthesis in a J774.A1 macrophagelike cell line (Fuhrman et al., 2005). A study utilizing diabetic mice model suggested that these protective effects might be due to the presence of unique complex sugars and/or phenolic sugars in pomegranate juice (Rozenberg et al., 2006). Another study involved high and low exercise lifestyle mimicking rats (high- and low-capacity runners) fed with pomegranate juice for 3 weeks (Rosenblat et al., 2015). While the effects were stronger in a group of low-capacity runners, the consumption of pomegranate juice decreased the cellular oxidation and increased the paraoxonase 2 activity in peritoneal macrophages from both animal groups when compared with non-treated cohorts of animals (Rosenblat et al., 2015).

In cultured human coronary artery endothelial cells exposed to high shear stress, pomegranate juice down-regulated the expression of redox sensitive genes ELK-1 and p-JUN and increased the expression of endothelial nitric oxide synthase (eNOS) (De Nigris et al., 2005) that is necessary for the proper functioning of blood endothelial cells (Vallance and Chan, 2001). In addition, an intake of pomegranate juice by LDL receptor-deficient mice fed with high-cholesterol diet, lessened progression of atherogenesis at different stages of the disease (De Nigris et al., 2005). Another in vitro study using cultured bovine pulmonary artery endothelial cells showed that the presence of even very low amounts of pomegranate juice in the cultivation medium protects the generated nitric oxide (NO) against its oxidative destruction (via an inhibition of a superoxide anionmediated disappearance of $\mathrm{NO}$, leading to an enhancement of the bioavailability of NO) (Ignarro et al., 2006). Another study of this group showed that presence of pomegranate juice in human coronary artery endothelial cells reverts down-regulation of the expression of eNOS caused by the addition of oxLDL (de Nigris et al., 2006). In a study accomplished in hypercholesterolemic mice, an intake of pomegranate juice enriched with punicalagin increased the eNOS expression and decelerated the progression of atherosclerosis, as well as enhanced nitrates levels (De Nigris et al., 2007). In pigs, an intake of the commercial pomegranate extract Pomanox ${ }^{\circledR}$ made from dried pomegranate skin or husk could reduce coronary endothelial dysfunction induced by hyperlipidemia (Vilahur et al., 2015). These beneficial effects included an activation of the protein kinase B (Akt)/eNOS pathway and an attenuation of vascular inflammation as well as of vascular damage induced by oxidative stress (Vilahur et al., 2015).

Furthermore, pomegranate juice attenuated the aggregation of human platelets exposed to collagen or arachidonic acid ex vivo (Aviram et al., 2000; Mattiello et al., 2009), by attenuating calcium mobilization, thromboxane A2 production, and hydrogen peroxide formation (Mattiello et al., 2009). These effects were assigned to the presence of polyphenols in pomegranate products (Mattiello et al., 2009). It was also shown that pomegranate fruit extract was active at a $2.0 \mu \mathrm{M}$ concentration that is possible to be achieved after polyphenolrich food intake by humans (Mattiello et al., 2009). On the other side, pomegranate seed oil inhibited cyclooxygenase (COX) 
TABLE 1 | List of compounds identified in pomegranate (Punica granatum L.).

\begin{tabular}{|c|c|c|}
\hline Pomegranate phytochemicals & Pomegranate part & References \\
\hline \multicolumn{3}{|l|}{ (1) ALKALOIDS } \\
\hline Caffeine & Peel $^{\star}$ & Elsherbiny et al., 2016 \\
\hline Peelletierine & Peel, bark & Neuhofer et al., 1993; Vidal et al., 2003 \\
\hline \multicolumn{3}{|l|}{ (2) ANTHOCYANINS/ANTHOCYANIDINS } \\
\hline Delphinidin glucosides and derivatives & Juice, peel & $\begin{array}{l}\text { Hernandez et al., 1999; Noda et al., 2002; } \\
\text { Alighourchi et al., 2008; Borges and Crozier, 2012; } \\
\text { Türkyilmaz, 2013; Ambigaipalan et al., 2016; Wafa } \\
\text { et al., } 2017\end{array}$ \\
\hline (Epi) afzelchin-delphinidin-3-O-hexoside & Seed & Ambigaipalan et al., 2017 \\
\hline Peonidin-3-O-(6"-O-acetyl)glucoside & Juice & Borges and Crozier, 2012 \\
\hline Vitisin A & Juice & Borges and Crozier, 2012 \\
\hline \multicolumn{3}{|l|}{ (3) ELLAGIC ACID AND DERIVATIVES } \\
\hline Ellagic acid & Juice, peel, seed, flower & $\begin{array}{l}\text { Amakura et al., 2000b; Gil et al., 2000; Wang et al., } \\
\text { 2004; Jain et al., 2011; Wafa et al., } 2017\end{array}$ \\
\hline Ellagic acid glucosides and derivatives & Juice, peel & Gil et al., 2000; Wafa et al., 2017 \\
\hline \multicolumn{3}{|l|}{ (4) FATTY ACIDS } \\
\hline $\begin{array}{l}\text { Arachidic acid, behenic acid, docosadienoic acid, } \\
\text { eicosapentaenoic acid, erucic acid, gondoic acid, lignoceric } \\
\text { acid, linoleic acid, linolelaidic acid, linolenic acid, myristic acid, } \\
\text { margaric acid, nervonic acid, oleic acid, palmitic acid, }\end{array}$ & Seed & $\begin{array}{l}\text { Hopkins and Chisholm, 1968; Schubert et al., 1999; } \\
\text { Kaufman and Wiesman, 2007; Pande and Akoh, } \\
\text { 2009; Elfalleh et al., 2011; Verardo et al., 2014; } \\
\text { Siano et al., } 2016\end{array}$ \\
\hline
\end{tabular}

palmitoleic acid, punicic acid, stearic acid, cis-vaccenic acid

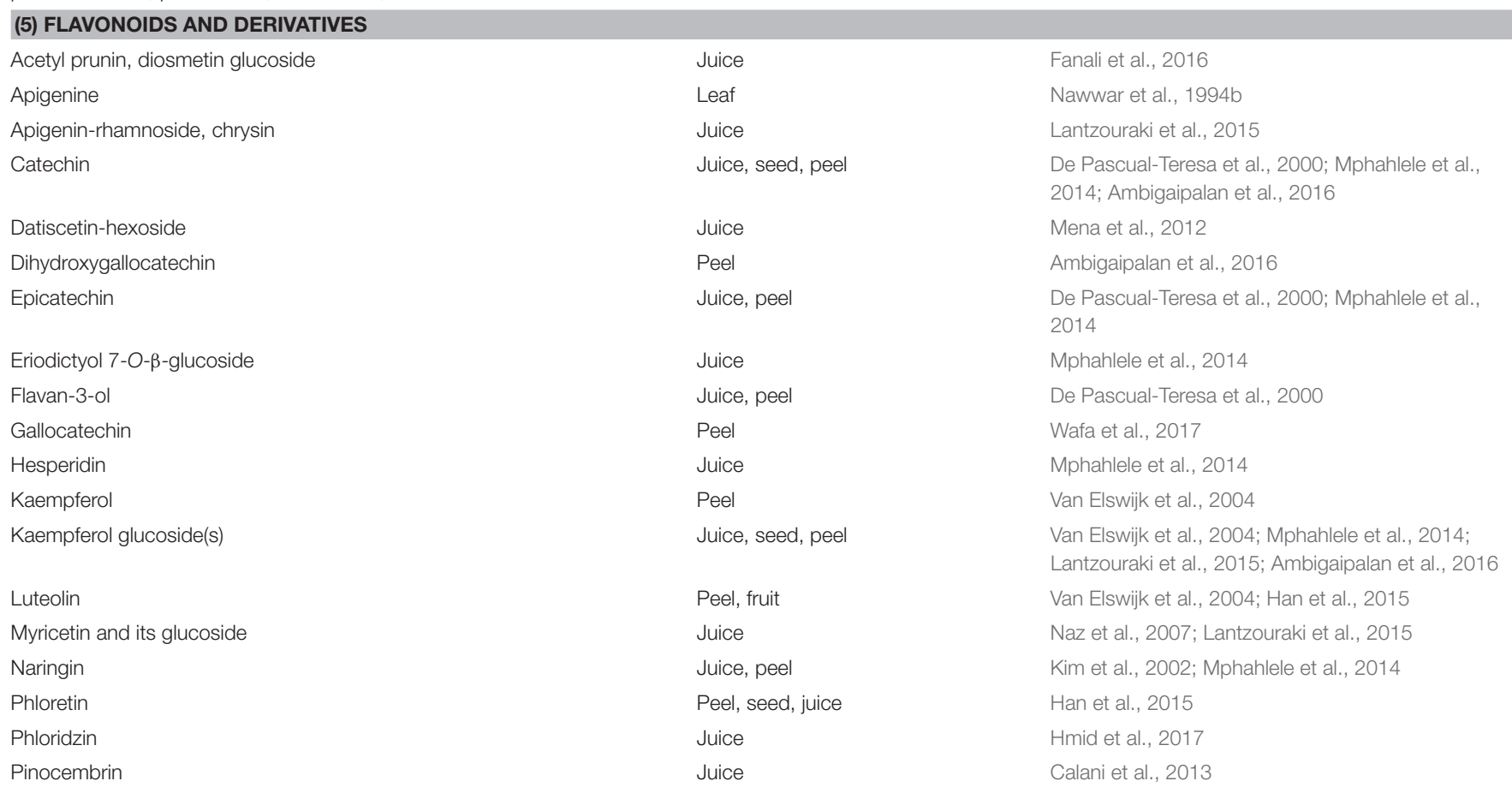


TABLE 1 | Continued

\begin{tabular}{|c|c|c|}
\hline Pomegranate phytochemicals & Pomegranate part & References \\
\hline Quercetin and its derivatives & Juice, seed, peel & $\begin{array}{l}\text { Artik, 1998; Naz et al., 2007; Borges and Crozier, } \\
\text { 2012; Han et al., 2015; Lantzouraki et al., 2015; } \\
\text { Ambigaipalan et al., } 2016\end{array}$ \\
\hline Rutin & Juice, peel & Artik, 1998; Mphahlele et al., 2014 \\
\hline Taxifolin and its glycosides & Peel, seed, juice & Calani et al., 2013; Han et al., 2015 \\
\hline \multicolumn{3}{|l|}{ (6) LIGNANS } \\
\hline \multicolumn{3}{|l|}{ (7) ORGANIC ACIDS } \\
\hline Citric acid & Juice & $\begin{array}{l}\text { Poyrazoglu et al., 2002; Carbonell-Barrachina et al., } \\
\text { 2012; Gundogdu and Yilmaz, 2012; Legua et al., } \\
\text { 2012; Lantzouraki et al., } 2015\end{array}$ \\
\hline Fumaric acid & Juice & $\begin{array}{l}\text { Poyrazoglu et al., 2002; Gundogdu and Yilmaz, } \\
2012\end{array}$ \\
\hline Methylmalonic acid & Juice & Alper et al., 2011 \\
\hline Oxalic acid & Juice & Legua et al., 2012 \\
\hline Quinic acid & Juice, peel & $\begin{array}{l}\text { Artik, 1998; Amakura et al., 2000a; Ehling and Cole, } \\
2011\end{array}$ \\
\hline Succinic acid & Juice & Poyrazoglu et al., 2002; Alper et al., 2011 \\
\hline Tartaric acid & Juice & $\begin{array}{l}\text { Poyrazoglu et al., 2002; Ehling and Cole, 2011; } \\
\text { Legua et al., } 2012\end{array}$ \\
\hline Uronic acid & Peel & Hasnaoui et al., 2014 \\
\hline \multicolumn{3}{|l|}{ (8) OTHER PHENOLIC COMPOUNDS } \\
\hline 3-Hydroxytyrosol & Peel & Elsherbiny et al., 2016 \\
\hline Benzaldehyde & Peel & Hadrich et al., 2014 \\
\hline 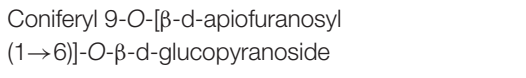 & Seed & Wang et al., 2004 \\
\hline Cyanidin-pentoside-hexoside & Fruit & Fischer et al., 2011a \\
\hline Ethyl cinnamate & Juice & Cadwallader et al., 2010 \\
\hline Ferulic acid and its hexoside & Juice & Lantzouraki et al., 2015 \\
\hline Gallic acid & Juice, seed, peel & $\begin{array}{l}\text { Amakura et al., 2000b; Huang et al., 2005a; Jain } \\
\text { et al., 2011; Mphahlele et al., 2014; Ambigaipalan } \\
\text { et al., 2016; Fanali et al., } 2016\end{array}$ \\
\hline Protocatechuic acid & Juice, seed, peel & Ambigaipalan et al., 2016; Fanali et al., 2016 \\
\hline$p$-Coumaric acid & Juice, peel, seed & $\begin{array}{l}\text { Artik, 1998; Amakura et al., 2000a; Ambigaipalan } \\
\text { et al., } 2017\end{array}$ \\
\hline Salycilic acid & Peel & Elsherbiny et al., 2016 \\
\hline Sesamin, 4-hydroxysesamin & Peel & Jiang et al., 2012 \\
\hline Vanillic acid & Juice & Mena et al., 2012 \\
\hline \multicolumn{3}{|l|}{ (9) PROANTHOCYANIDINS } \\
\hline Procyanidin dimer B2 and B3 & Peel & Ambigaipalan et al., 2016 \\
\hline $\begin{array}{l}\text { Arabinose, xylose, galactose, glucose, mannose, } \\
\text { rhamnose }\end{array}$ & Peel & Hasnaoui et al., 2014 \\
\hline
\end{tabular}


TABLE 1 | Continued

Pomegranate phytochemicals

\section{(10) SACCHARIDES}

Glucose, fructose, maltose, sucrose

\section{(11) STEROLS}

\section{$\beta$-Sitosteryl acetate}

Avenasterol, $\Delta 7$-avenasterol, campesterol,

cycloartenol, $\Delta 7$-stigmasterol, stigmasterol,

$\beta$-sitosterol

Camestero

Daucosterol

Stigmasterol

(12) TANNINS (GALLOTANNINS AND ELLAGITANNINS AND THEIR DERIVATIVES)

1,2,3-Tri-O-galloyl- $\beta-{ }^{4} \mathrm{C} 1$-glucose

2-O-Galloylpunicalin

3,3'-Di-O-methylellagic acid

$3,3^{\prime}, 4^{\prime}$-Tri-O-methylellagic acid

Castalagin

Castalin

Casuarinin (Galloyl-bis-HHDP-hexoside)

Corilagin (Galloyl-HHDP-hexoside)

Epicatechin gallate

Flavogallonic acid

Gallagic acid

Gallagyldilacton

Granatin A/B

Lagerstannin C (Galloyl-HHDP-gluconic)

Pedunculagin I (bis-HHDP-hexoside)

Pentagalloylglucopyranose

Punicacortein A, B, C, and D

Punicafolin

Punicalagin (HHDP-gallagyl-hexoside)

Punicalin $\alpha$ and $\beta$

Punicatannin C

Punigluconin (Digalloyl-HHDP-glucoside)

Tellimagrandin

Tergallagic acid-O-glucoside

Valoneic acid bilactone

\section{(13) TERPENES AND TERPENOIDS}

3-Carene, $\alpha$-terpinene, $\alpha$-terpineol, eugenol

Asiatic acid

Betulinol, 24-methylenecycloartenol, cycloartenol,

squalene

Camphor

Eugenol

Maslinic acid

Oleanolic acid

$\alpha / \beta$-Pinene, limonene, terpineol, $\beta$-farnesene, $\beta$-caryophyllene, bisabolene
Pomegranate part

Juice

Peel

Seed

Seed

Seed

Seed

Leaf

Juice

Seed

Seed

Juice, peel

Fruit, juice

Peel

Peel, leaf

Peel

Peel

Peel, juice

Peel

Peel

Peel

Juice, peel

Seed

Peel, bark

Leaf

Juice, peel, leaf

Peel, juice, leaf

Flower

Peel

Peel

Juice

Juice

Juice

Flower

Seed

Peel

Juice

Flower

Flower

Juice
References

Carbonell-Barrachina et al., 2012; Legua et al., 2012; Vegara et al., 2014; Conidi et al., 2017

Jiang et al., 2012

Górnaś and Rudzinska, 2016

Abd El Wahab et al., 1998

Wang et al., 2004

Abd El Wahab et al., 1998

Nawwar et al., 1994a

Borges and Crozier, 2012

Wang et al., 2004

Wang et al., 2004

Fischer et al., 2011a

Fischer et al., 2011b

Satomi et al., 1993; Ambigaipalan et al., 2016

Satomi et al., 1993; Nawwar et al., 1994a;

Ambigaipalan et al., 2016

Ambigaipalan et al., 2016

Jiang et al., 2012

Tzulker et al., 2007

Satomi et al., 1993; Anibal et al., 2013

Tanaka et al., 1990; Wafa et al., 2017

Wafa et al., 2017

Satomi et al., 1993; Lantzouraki et al., 2015; Wafa et al., 2017

He et al., 2011

Tanaka et al., 1986a; Anibal et al., 2013

Nawwar et al., 1994a

Tanaka et al., 1986b; Jain et al., 2011; Anibal et al., 2013; Lantzouraki et al., 2015

Tanaka et al., 1986b; Tzulker et al., 2007; Jain et al., 2011; Wafa et al., 2017

Yuan et al., 2013

Wafa et al., 2017

Satomi et al., 1993

Borges and Crozier, 2012

Fischer et al., 2011a,b

Carbonell-Barrachina et al., 2012

Batta and Rangaswami, 1973

Verardo et al., 2014

Hadrich et al., 2014

Carbonell-Barrachina et al., 2012

Batta and Rangaswami, 1973

Huang et al., 2005b; Fu et al., 2014

Vázquez-Araújo et al., 2011 
TABLE 1 | Continued

\begin{tabular}{|c|c|c|}
\hline Pomegranate phytochemicals & Pomegranate part & References \\
\hline 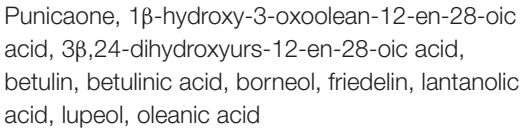 & Peel & Jiang et al., 2012 \\
\hline Ursolic acid & Seed, flower & $\begin{array}{l}\text { Ahmed et al., 1995; Huang et al., 2005a; Fu et al., } \\
2014\end{array}$ \\
\hline \multicolumn{3}{|l|}{ (14) VITAMINS } \\
\hline Vitamin C & Juice & Dumlu and Gurkan, 2007 \\
\hline \multicolumn{3}{|l|}{ (15) XANTONOIDS } \\
\hline Mangiferin & Peel & Elsherbiny et al., 2016 \\
\hline
\end{tabular}

${ }^{\star}$ Peel (pericarp, rind, and hull are synonyms).

TABLE 2 | Vasculoprotective effects of pomegranate determined in in vitro and in vivo pre-clinical studies.

\begin{tabular}{|c|c|c|}
\hline Vasculoprotective effects & Pomegranate part & References \\
\hline Antioxidative properties in vitro and in vivo & Juice, fruit extract, peel extract & $\begin{array}{l}\text { Gil et al., 2000; Les et al., 2015; Delgado et al., } \\
2016\end{array}$ \\
\hline $\begin{array}{l}\text { Suppression of peroxidation of plasma lipids, induction of serum } \\
\text { paraoxonase activity, lowering lipid uptake by macrophages, and } \\
\text { diminishing development of atherosclerosis in mice }\end{array}$ & Juice, fruit extract & $\begin{array}{l}\text { Aviram et al., 2000; Fuhrman et al., 2005; Rosenblat } \\
\text { et al., 2015; Mollazadeh et al., } 2016\end{array}$ \\
\hline $\begin{array}{l}\text { Improvement of endothelial cell function in vitro, in mice and pigs } \\
\text { [due to an activation of the protein kinase B (Akt)/eNOS pathway, } \\
\text { an inhibition of a superoxide anion-mediated disappearance of } \\
\mathrm{NO} \text {, and reduction of vascular inflammation] }\end{array}$ & Juice, fruit extract & $\begin{array}{l}\text { De Nigris et al., 2005; de Nigris et al., 2006; De } \\
\text { Nigris et al., 2007; Ignarro et al., 2006; Vilahur et al., } \\
2015\end{array}$ \\
\hline $\begin{array}{l}\text { Reduction the collagen- and arachidonic acid-induced platelet } \\
\text { aggregation ex vivo }\end{array}$ & Juice, fruit extract & Aviram et al., 2000; Mattiello et al., 2009 \\
\hline $\begin{array}{l}\text { Reduction in activity of angiotensin-converting enzyme (ACE); } \\
\text { decrease in mean arterial blood pressure in rats }\end{array}$ & Juice, fruit extract, peel extract & $\begin{array}{l}\text { Mohan et al., 2010; Dos Santos et al., 2016; Arun } \\
\text { et al., } 2017\end{array}$ \\
\hline $\begin{array}{l}\text { Lessening cardiac toxicity induced by drugs or smoking } \\
\text { (diminishing lipid peroxidation and increasing levels of antioxidant } \\
\text { enzymes) }\end{array}$ & Juice, fruit extract & Jadeja et al., 2010; Al Hariri et al., 2016 \\
\hline $\begin{array}{l}\text { Reduction of blood glucose levels in a variety of mouse and rat } \\
\text { models (effects mediated via upregulation of PPAR- } \gamma \text { leading to an } \\
\text { increase in insulin sensitivity) }\end{array}$ & Seed-, flower-, and peel-extract & $\begin{array}{l}\text { Das et al., 2001; Huang et al., 2005a; Li et al., } \\
\text { 2005; Vroegrijk et al., 2011; Salwe et al., } 2015\end{array}$ \\
\hline $\begin{array}{l}\text { Lowering fatty acid, triglycerides and total cholesterol plasma } \\
\text { levels as well as cardiac triglycerides (in Zucker diabetic fatty rats) }\end{array}$ & Flower extract & Huang et al., 2005b \\
\hline
\end{tabular}

(Schubert et al., 1999), the key enzyme catalyzing the conversion of arachidonic acid to prostaglandin (PGI2) (Grosser et al., 2006). The latter substance is known as a potent vasoprotective factor inhibiting platelet adhesion and thrombus formation on endothelium (Weiss and Turitto, 1979). In addition, feeding of rats with pomegranate extract diminished in colonic mucosa levels of COX-2, prostaglandin E2 (PGE2) as well as inducible nitric oxide synthase (iNOS) (Larrosa et al., 2010b).

Some other works investigated how pomegranate affects arterial hypertension, an important risk factor for cardiovascular diseases (Pickering, 1972). For example, in a study involved the use of Wistar rats in which diabetes was induced by streptozotocin administration, and the animals were additionally challenged by a subcutaneous administration of angiotensin II to induce hypertension, a prolonged administration of pomegranate juice (for 4 weeks) reduced activity of angiotensin converting enzyme (ACE), as well as decreased mean arterial blood pressure in comparison with non-treated animals (Mohan et al., 2010).

Pomegranate fruit extracts were also studied regarding their protective effect against cardiac toxicity induced by drugs or smoking. For example, detrimental effects of a cardiotoxic drug isoproterenol (known to cause a cardiac necrosis leading to a myocardial infarction) were reduced upon pre-treatment of rats with pomegranate juice for 30 consecutive days before isoproterenol treatment (Jadeja et al., 2010). Such pre-treatment significantly lessened an increase in the heart weight, infarction size, plasma marker enzymes, lipid peroxidation levels as well as levels of $\mathrm{Ca}^{2+}$ ATPase (Jadeja et al., 2010). The protective effects of pomegranate juice intake were also demonstrated in a study using rats in which a cardiac hypertrophy was induced by cigarette smoke exposure (Al Hariri et al., 2016). 


\section{Pomegranate Seed Oil}

Pomegranate seeds comprise about $3 \%$ of the pomegranate weight and contain about $12-20 \%$ seed oil (Lansky and Newman, 2007) that is rich in fatty acids and contains mainly punicic acid (Kaufman and Wiesman, 2007; Verardo et al., 2014; Górnaś and Rudzinska, 2016).

In rats with streptozotocin-induced diabetes, oral feeding with seed extracts significantly reduced blood glucose levels (Das et al., 2001). In mice, an intake of pomegranate seed oil counteracted their obesity induced by a high-fat diet by enhancing peripheral insulin sensitivity (Vroegrijk et al., 2011). Oral treatment of the above cited diabetic rats with pomegranate seed oil significantly decreased peroxidation of plasma lipids (Mollazadeh et al., 2016). In addition, such treatment diminished malondialdehyde content in homogenates from the heart and kidney tissues, and reduced triglyceride levels in treated animals in comparison to the control cohort (Mollazadeh et al., 2016).

\section{Pomegranate Flower, Peel, and Leaf Extracts}

A 6-week oral administration of pomegranate flower extracts suppressed plasma glucose levels in Zucker diabetic fatty rats following their exposure to glucose-loading. In addition, such treatment in these animals increased cardiac peroxisome proliferator-activated receptor gamma (PPAR- $\gamma$ ) mRNA expression as well as restored the down-regulated cardiac glucose transporter (GLUT)-4 mRNA, altogether improving insulin sensitivity (Huang et al., 2005a). These beneficial effects were assigned mainly to the presence of gallic acid (Huang et al., 2005a). A long-term treatment of Zucker diabetic fatty rats with pomegranate flower extracts was cardioprotective, as it lowered their fatty acid-, triglycerides-, and total cholesterol plasma levels as well as reduced the cardiac triglycerides content (Huang et al., 2005b). In another study, oral administration of pomegranate flower extracts decreased plasma glucose levels in non-fasted diabetic rats (but not in fasted-diabetic rats or in normal rats). This study also showed that pomegranate flower extracts inhibit $\alpha$-glucosidase (a key enzyme for carbohydrate digestion in intestines) and administration of pomegranate flower extracts may improve postprandial hyperglycemia in type 2 diabetes, and altogether diminish the risk of cardiovascular dysfunctions ( $\mathrm{Li}$ et al., 2005). In mice fed with a high-fat diet to induce obesity, treatment with pomegranate leaf extract decreased body weight, energy intake as well as total cholesterol, triglyceride, and glucose levels (Lei et al., 2007). Administration of hydroalcoholic peel or leaf extracts of pomegranate for 28 days decreased blood glucose levels in a Wister rat model of diabetes induced by streptozotocin (Salwe et al., 2015). Hydroalcoholic peel extracts of pomegranate were also tested in spontaneously hypertensive ovariectomized female rats (an animal model for menopause characterized by an increase in the superoxide anion levels; Delgado et al., 2016). Such treatment diminished elevation of superoxide anion levels and lessened oxidative stress in this animal model (Delgado et al., 2016). Treatment of spontaneously hypertensive rats of different ages for 30 days with pomegranate peel extracts, significantly reduced systolic blood pressure, ACE activity, oxidative stress as well as vascular remodeling (Dos Santos et al., 2016). A recent in vitro study showed that pomegranate peel methanolic extracts potently scavenge superoxide and hydroxyl radicals, protect LDL against oxidation and suppress ACE activity (Arun et al., 2017). Altogether, these studies demonstrated that also the non-edible parts of pomegranate-peel and leaves-exhibit vasculoprotective effects.

\section{VASCULOPROTECTIVE EFFECTS OF PURE COMPOUNDS DERIVED FROM POMEGRANATE}

Studies presented above showed the numerous vasculoprotective effects of different parts of the pomegranate. It was suggested that many of these protective effects are caused by the presence of hydrolyzable tannins (ellagitannins and gallotannins), their derivative ellagic acid, or their common metabolites urolithins (Table 3 and the text below).

Pomegranate ellagitannins and a single high molecular weight ellagitannin punicalagin, attenuated the inflammatory cell signaling in colon cancer cells (Adams et al., 2006). Punicalagin and gallic acid induced in isolated macrophages the expression of paraoxonase 2 (Shiner et al., 2007). These substances also reduced oxidative stress in macrophages via activation of transcription factors PPAR- $\gamma$ and activator protein 1 (AP-1; Shiner et al., 2007).

Single components (e.g., punicalin, punicalagin, ellagic acid, and gallic acid) isolated from pomegranate fruit suppressed the formation of advanced glycation end products (AGEs, known to contribute to a number of diseases including diabetic complications and arteriosclerosis) from bovine serum albumin and sugar in antiglycation assays in vitro (Kumagai et al., 2015). Pomegranate fruit extracts also reduced the accumulation of AGEs in mice fed with a high-fat and high-sucrose diet (Kumagai et al., 2015). In addition, punicalagin and ellagic acid inhibited lipid metabolism in mouse and human adipocytes ex vivo (Les et al., 2017).

Effects of ellagic acid on reactive oxygen species (ROS) generation were also investigated in endothelial cells. Pretreatment of HUVECs with ellagic acid attenuated ROS production and prevented eNOS downregulation induced by oxLDL (Lee et al., 2010; Ou et al., 2010). Ex vivo, ellagic acid stimulated vasorelaxation of the rat thoracic aorta via an endothelium-dependent mechanism and an inhibition of calcium influx (Yilmaz and Usta, 2013). Nevertheless, as ellagitannins and ellagic acid in vivo metabolize into urolithins that enter systemic circulation (Cerdáet al., 2005; Larrosa et al., 2010a), researchers also studied how these metabolites affect the vascular function.

The antioxidant properties of different urolithins were evaluated in a cell-based assay and the results showed that urolithin $\mathrm{C}$ and $\mathrm{D}$ were more potent antioxidants than the parental substance ellagic acid and punicalagin (Bialonska et al., 
TABLE 3 | Vasculoprotective effects of pomegranate-derived substances or their metabolites, as determined in vitro and in vivo pre-clinical studies.

\begin{tabular}{|c|c|c|}
\hline Vasculoprotective effects & Vasculoprotective substances & References \\
\hline $\begin{array}{l}\text { Induction of paraoxonase } 2 \text { and reduction in oxidative } \\
\text { stress in isolated macrophages }\end{array}$ & Punicalagin, gallic acid & Shiner et al., 2007 \\
\hline $\begin{array}{l}\text { Attenuation of reactive oxygen species (ROS) generation } \\
\text { and prevention of eNOS downregulation induced by } \\
\text { oxLDL in HUVECs. Stimulation of vasorelaxation of the } \\
\text { rat thoracic aorta ex vivo, via an endothelium-dependent } \\
\text { mechanism and through an inhibition of calcium influx }\end{array}$ & Ellagic acid & $\begin{array}{l}\text { Lee et al., 2010; Ou et al., 2010; Yilmaz } \\
\text { and Usta, } 2013\end{array}$ \\
\hline $\begin{array}{l}\text { Suppression of formation of advanced glycation end } \\
\text { products (AGEs) in vitro and in mice }\end{array}$ & Punicalin, punicalagin, ellagic acid, gallic acid & Kumagai et al., 2015 \\
\hline Inhibition of lipid metabolism in adipocytes & Punicalagin, ellagic acid & Les et al., 2017 \\
\hline Antioxidative properties in a cell-based assay in vitro & Urolithins & Bialonska et al., 2009 \\
\hline $\begin{array}{l}\text { Inhibition of adhesion of monocytes to endothelial cells, } \\
\text { of secretion of a cellular adhesion molecule (VCAM-1) } \\
\text { and pro-inflammatory cytokine (IL-6). Decrease in the } \\
\text { accumulation of cholesterol in THP-1-derived } \\
\text { macrophages }\end{array}$ & Ellagic acid, urolithin A glucuronide, other urolithins & $\begin{array}{l}\text { Gimenez-Bastida et al., 2012; Mele et al., } \\
2016\end{array}$ \\
\hline $\begin{array}{l}\text { Attenuation of endothelial dysfunction induced by oxLDL } \\
\text { in cultured human artery endothelial cells, partly by } \\
\text { counteracting eNOS-dependent decrease in NO } \\
\text { production. Reduction in myocardial } \\
\text { ischemia/reperfusion injury and myocardial infarct size in } \\
\text { vivo }\end{array}$ & Urolithin A & Han et al., 2016; Tang et al., 2017 \\
\hline Anti-hypertensive effects of sweetie juice in humans & Naringin & Reshef et al., 2005 \\
\hline $\begin{array}{l}\text { Amelioration of glucose tolerance and diminishing } \\
\text { obesity-related inflammation via activation of PPAR- } \gamma \\
\text { and }-\alpha\end{array}$ & Puninic acid & Hontecillas et al., 2009 \\
\hline
\end{tabular}

2009). Nonetheless, although an in vitro antioxidant potential of urolithin A was relatively low in comparison with other urolithins, its plasma concentrations was the highest among them (Bialonska et al., 2009). In a subsequent study, urolithin A glucuronide inhibited adhesion of monocytes to endothelial cells in the micromolar range $(5-15 \mu \mathrm{M})$, suggesting that the beneficial effects of pomegranate intake on the vasculature might be partly mediated by urolithin A glucuronide (GimenezBastida et al., 2012). In addition, a recent in vitro study showed potent anti-atherogenic properties of ellagic acid and some urolithins (Mele et al., 2016). All these compounds reduced the adhesion of THP-1 derived macrophages to HUVECs and diminished secretion of soluble vascular cell adhesion molecule-1 (VCAM-1) and inflammatory interleukin-6 (IL-6) (Mele et al., 2016). In a study utilizing cultured human artery endothelial cells, urolithin A attenuated endothelial dysfunction induced by oxLDL (Han et al., 2016). These effects were partly mediated by counteracting eNOS-dependent decrease in NO production (Han et al., 2016). In addition, urolithin A reduced the expression of intracellular adhesion molecule1 (ICAM-1) and monocyte chemotactic protein 1 (MCP1), upon adhesion of THP-1 cells to the endothelial cells. Urolithin A also suppressed the expression of tumor necrosis factor- $\alpha$ (TNF- $\alpha)$, IL- 6 and endothelin-1, increased PPAR$\gamma$ mRNA expression, and downregulated phosphorylation of the extracellular signal-regulated protein kinase $1 / 2(\mathrm{ERK} 1 / 2)$ (Han et al., 2016). In another study, urolithin A inhibited heme peroxidases [myeloperoxidase (MPO) and lactoperoxidase
(LPO)] more effectively than its parent compound ellagic acid (Saha et al., 2016). Animal experiments using C57BL/6 mice revealed potent anti-inflammatory properties of urolithin $\mathrm{A}$, as it efficiently reduced phorbol myristate acetate (PMA)induced mouse ear edema formation (Saha et al., 2016). Urolithin A also lessened myocardial ischemia/reperfusion injury and reduced myocardial infarct size in mice via the phosphoinositide 3-kinase/Akt (PI3K/Akt) pathway (Tang et al., 2017).

Altogether, the studies presented above pointed to beneficial vascular effects of urolithins and especially metabolite urolithin A. Additional information on metabolic fate and health effects of ellagitanins and urolithins can be found in several recent reviews (Garcia-Muñoz and Vaillant, 2014; Lipinska et al., 2014; Landete et al., 2016; Tomas-Barberan et al., 2017).

In addition to the above presented effects of hydrolyzable tannins (ellagitannins and gallotannins), their derivative ellagic acid, or their common metabolites urolithins, but also other substances were shown to contribute to beneficial effects of pomegranate products. These include (poly)phenolic compounds anthocyanins (Alighourchi et al., 2008; Fischer et al., 2011a) and flavonoids (Sudheesh and Vijayalakshmi, 2005; Ricci et al., 2006), as well as fatty acids (Kaufman and Wiesman, 2007). For example, anthocyanins exhibit antiinflammatory activities (Vendrame and Klimis-Zacas, 2015). Flavonoid naringin abundantly present in pomegranate juice (Mphahlele et al., 2014) is considered to contribute (together 
with flavonoid naritutin) to the anti-hypertensive effects of sweetie juice in humans (Reshef et al., 2005). Puninic acid was shown to ameliorate glucose tolerance and diminish obesity-related inflammation via an activation of PPAR- $\gamma$ and $\alpha$ (Hontecillas et al., 2009). Quercetin present in juice, seed, and peel of pomegranate (Artik, 1998; Borges and Crozier, 2012; Ambigaipalan et al., 2016) is known to mediate endothelium-dependent vasodilatation via stimulation of both the NO/cyclic guanylyl monophosphate (cGMP) pathway and endothelium-derived hyperpolarizing factor (EDHF) (Khoo et al., 2010).

\section{CLINICAL STUDIES ON POMEGRANATE IN THE CONTEXT OF CARDIOVASCULAR DISEASES}

Many clinical studies investigating the effects of pomegranate in the context of CVDs were performed in the last two decades. These works profusely demonstrated the vasculoprotective properties of pomegranate products (Table 4). Nevertheless, some of these studies pointed to the fact that when applying pomegranate for a longer period or in high amounts, certain possible side effects of such treatment (mainly diarrhea) might occur (Paller et al., 2013).

In hypertensive patients, daily consumption of pomegranate juice for 2 weeks reduced the activity of ACE by $36 \%$ as well as diminished systolic blood pressure by $5 \%$ (Aviram and Dornfeld, 2001). The same group also reported that a long-duration intake of pomegranate juice (for 3 years) by patients with carotid artery stenosis significantly reduced their blood pressure, LDL oxidation and common carotid intimamedia thickness (Aviram et al., 2004). A 4-week consumption of pomegranate juice reduced significantly blood pressure in a cohort of 51 healthy women (without significantly changing serum ACE activity; Lynn et al., 2012). Another study involving 13 hypersensitive men demonstrated that intake of pomegranate juice lowered blood pressure (Asgary et al., 2013). However, in these patients the levels of some clinical parameters, such as serum concentrations of C-reactive protein (CRP), E-selectin, VCAM-1, ICAM-1, and IL-6 remained unchanged (Asgary et al., 2013). A subsequent study involving 21 hypertensive patients showed that consumption of pomegranate juice significantly reduced systolic as well as diastolic blood pressure (Asgary et al., 2014). In addition, a double blind, randomized, placebo controlled pilot study revealed that the pomegranate peel hydroalcoholic extract reduced cardiovascular risk factors in obese women with dyslipidemia (Haghighian et al., 2016).

Although a meta-analysis evaluating the effects of pomegranate consumption on CRP concentrations did not reveal a significant correlation between these parameters (Sahebkar et al., 2016), the effects of pomegranate consumption on blood pressure regulation in accomplished animal and human studies seem to be clinically relevant. In a recent review it was concluded that both pomegranate juice and seed oil can effectively lower blood pressure (Asgary et al., 2017). Another recently accomplished meta-analysis came to the same conclusions, as intake of pomegranate juice decreased levels of systolic blood pressure regardless of the duration and dose of the juice consumed in the evaluated studies, whereas doses more than $240 \mathrm{cc}$ (eight ounces) exhibited a borderline significant effect in reducing of a diastolic blood pressure (Sahebkar et al., 2017). The authors of this metaanalysis determined a constant benefit of pomegranate juice intake on blood pressure, which may be considered clinically relevant. Additional information of how pomegranate affects

TABLE 4 | Outcome of clinical studies involving intake of pomegranate juice or peel hydro alcoholic extract.

Type of the study/Number of probands
Daily consumption of pomegranate juice for 2 weeks by
hypertensive patients $(N=10)$
A long-duration intake of pomegranate juice (for 3 years) by
patients with carotid artery stenosis ( $N=19)$
A 4-week consumption of pomegranate juice by healthy
women ( $N=51)$
Intake of pomegranate juice by hypersensitive men $(N=13)$

Consumption of pomegranate juice by hypertensive patients $(N=21)$

Intake of pomegranate peel hydro alcoholic extract by obese women with dyslipidemia $(N=38)$

A meta-analysis focusing on effects of pomegranate consumption on CRP

A meta-analysis focusing on blood pressure lowering effects of intake of pomegranate juice
Clinical outcome

References

Reduction in ACE activity by $36 \%$ and of a systolic blood pressure by $5 \%$

Reduction in systolic blood pressure by $12 \%$, decrease in common carotid intima-media thickness up to $30 \%$

A mild, but significant reduction in blood pressure (without significantly changing serum ACE activity)

Decrease in blood pressure while other parameters (serum concentrations of CRP, E-selectin, VCAM-1, ICAM-1, and IL-6) remain unchanged

Significant reduction in systolic as well as diastolic blood pressure

Significant reduction in systolic blood pressure

Aviram and Dornfeld, 2001

Aviram et al., 2004

Lynn et al., 2012

Asgary et al., 2013

Asgary et al., 2014

Haghighian et al., 2016

No significant correlation between pomegranate consumption and CRP levels

Decrease in systolic blood pressure levels (regardless of the duration and dose of the juice consumed in the evaluated studies). A borderline significant effect in reducing of diastolic blood pressure by doses higher than 240 cc (eight ounces)
Sahebkar et al., 2016

Sahebkar et al., 2017 
vasculature can be found in some other reviews (Lansky and Newman, 2007; Aviram and Rosenblat, 2013; Hyson, 2015; Zheng et al., 2017). In addition to many described beneficial effects of pomegranete on endothelial function, pomegranate juice was also found to enhance the inhibitory effect of NO on vascular smooth muscle cell proliferation (Ignarro et al., 2006). This aspect might be clinically relevant and a subject of further studies, as vascular smooth muscle cell proliferation plays an important role in the development and progression of atherosclerosis and restenosis (Uhrin et al., 2018; Wang et al., 2018).

\section{CONCLUSION}

Pomegranate, an ancient and highly distinctive fruit, is a rich source of natural bioactive constituents. Various studies showed that pomegranate and its products exhibit protective effects on the cardiovascular system. These vasculoprotective effects include diminishing of oxidative stress, positive influencing macrophage-, endothelial cell-, and platelet function, lowering lipid oxidation, reducing blood glucose levels, vasodilatory effects as well as decreasing blood pressure via an inhibition of ACE activity. In light

\section{REFERENCES}

Abd El Wahab, S. M., El Fiki, N. M., Mostafa, S. F., and Hassan, A. E. B. (1998). Characterization of certain steroid hormones in Punica granatum L. seeds. Bull. Facul. Pharm. (Cairo Univ.) 36, 11-15.

Adams, L. S., Seeram, N. P., Aggarwal, B. B., Takada, Y., Sand, D., and Heber, D. (2006). Pomegranate juice, total pomegranate ellagitannins, and punicalagin suppress inflammatory cell signaling in colon cancer cells. J. Agric. Food Chem. 54, 980-985. doi: 10.1021/jf052005r

Ahmed, R., Ifzal, S. M., Saifuddin, A., and Nazeer, M. (1995). Studies on Punica granatum-l isolation and identification of some constituents from the seeds of Punica granatum. Pak. J. Pharm. Sci. 8, 69-71.

Albrecht, M., Jiang, W., Kumi-Diaka, J., Lansky, E. P., Gommersall, L. M., Patel, A., et al. (2004). Pomegranate extracts potently suppress proliferation, xenograft growth, and invasion of human prostate cancer cells. J. Med. Food 7, 274-283. doi: 10.1089/jmf.2004.7.274

Al Hariri, M., Zibara, K., Farhat, W., Hashem, Y., Soudani, N., Al Ibrahim, F., et al. (2016). Cigarette smoking-induced cardiac hypertrophy, vascular inflammation and injury are attenuated by antioxidant supplementation in an animal model. Front. Pharmacol. 7:397. doi: 10.3389/fphar.2016.00397

Alighourchi, H., Barzegar, M., and Abbasi, S. (2008). Anthocyanins characterization of 15 Iranian pomegranate (Punica granatum L.) varieties and their variation after cold storage and pasteurization. Eur. Food Res. Technol. 227, 881-887. doi: 10.1007/s00217-007-0799-1

Alper, N., Onsekizoglu, P., and Acar, J. (2011). Effects of various clarification treatments on phenolic compounds and organic acid compositions of pomegranate (Punica granatum L.) juice. J. Food Process. Preserv. 35, 313-319. doi: $10.1111 / j .1745-4549.2009 .00458 . x$

Amakura, Y., Okada, M., Tsuji, S., and Tonogai, Y. (2000a). Determination of phenolic acids in fruit juices by isocratic column liquid chromatography. $J$. Chromatogr. A 891, 183-188. doi: 10.1016/S0021-9673(00)00625-7

Amakura, Y., Okada, M., Tsuji, S., and Tonogai, Y. (2000b). High-performance liquid chromatographic determination with photodiode array detection of ellagic acid in fresh and processed fruits. J. Chromatogr. A 896, 87-93. doi: 10.1016/S0021-9673(00)00414-3

Ambigaipalan, P., De Camargo, A. C., and Shahidi, F. (2016). Phenolic compounds of pomegranate byproducts (outer skin, mesocarp, divider of the altogether promising outcome of numerous preclinical and clinical studies, pomegranate is advocated to be used as a dietary supplement for prevention and treatment of cardiovascular diseases, thus representing a supplementary non-pharmacological therapy for cardiovascular diseases.

\section{AUTHOR CONTRIBUTIONS}

DW, CÖ, IA-R, SC, JP, PU, and AA wrote the first draft of the manuscript. JH and AJ prepared Tables 2-4 during the revision and NT improved the revised version of the manuscript.

\section{ACKNOWLEDGMENTS}

The authors are grateful to the Polish KNOW (Leading National Research Centre) Scientific Consortium Healthy Animal-Safe Food, decision of Ministry of Science and Higher Education (No. 05-1/KNOW2/2015), the Homing grant from Foundation for Polish Sciences, the Peter und Traudl Engelhorn Foundation for the promotion of Life Sciences for financial support. The funding agency did not have any role in writing or submitting this review for publication.

membrane) and their antioxidant activities. J. Agric. Food Chem. 64, 6584-6604. doi: 10.1021/acs.jafc.6b02950

Ambigaipalan, P., de Camargo, A. C., and Shahidi, F. (2017). Identification of phenolic antioxidants and bioactives of pomegranate seeds following juice extraction using HPLC-DAD-ESI-MSn. Food Chem. 221, 1883-1894. doi: $10.1016 /$ j.foodchem.2016.10.058

Anibal, P. C., Peixoto, I. T., Foglio, M. A., and Höfling, J. F. (2013). Antifungal activity of the ethanolic extracts of Punica granatum L. and evaluation of the morphological and structural modifications of its compounds upon the cells of Candida spp. Braz. J. Microbiol. 44, 839-848. doi: 10.1590/S1517-83822013005000060

Arapitsas, P. (2012). Hydrolyzable tannin analysis in food. Food Chem. 135, 1708-1717. doi: 10.1016/j.foodchem.2012.05.096

Artik, N. (1998). Determination of phenolic compounds in pomegranate juice by using HPLC. Fruit Process. 8, 492-499.

Arun, K. B., Jayamurthy, P., Anusha, C. V., Mahesh, S. K., and Nisha, P. (2017). Studies on activity guided fractionation of pomegranate peel extracts and its effect on antidiabetic and cardiovascular protection properties. J. Food Process. Preserv. 41:e13108. doi: 10.1111/jfpp.13108

Asgary, S., Keshvari, M., Sahebkar, A., Hashemi, M., and Rafieian-Kopaei, M. (2013). Clinical investigation of the acute effects of pomegranate juice on blood pressure and endothelial function in hypertensive individuals. ARYA Atheroscler. 9, 326-331.

Asgary, S., Keshvari, M., Sahebkar, A., and Sarrafzadegan, N. (2017). Pomegranate consumption and blood pressure: a review. Curr. Pharm. Des. 23, 1042-1050. doi: 10.2174/1381612822666161010103339

Asgary, S., Sahebkar, A., Afshani, M. R., Keshvari, M., Haghjooyjavanmard, S., and Rafieian-Kopaei, M. (2014). Clinical evaluation of blood pressure lowering, endothelial function improving, hypolipidemic and anti-inflammatory effects of pomegranate juice in hypertensive subjects. Phytother. Res. 28, 193-199. doi: $10.1002 /$ ptr.4977

Aviram, M., and Dornfeld, L. (2001). Pomegranate juice consumption inhibits serum angiotensin converting enzyme activity and reduces systolic blood pressure. Atherosclerosis 158, 195-198. doi: 10.1016/S0021-9150(01)00412-9

Aviram, M., Dornfeld, L., Rosenblat, M., Volkova, N., Kaplan, M., Coleman, R., et al. (2000). Pomegranate juice consumption reduces oxidative stress, atherogenic modifications to LDL, and platelet aggregation: studies in humans 
and in atherosclerotic apolipoprotein E-deficient mice. Am. J. Clin. Nutr. 71, 1062-1076. doi: $10.1093 /$ ajcn/71.5.1062

Aviram, M., and Rosenblat, M. (2013). Pomegranate for your cardiovascular health. Rambam Maimonides Med. J. 4:e0013. doi: 10.5041/RMMJ.10113

Aviram, M., Rosenblat, M., Gaitini, D., Nitecki, S., Hoffman, A., Dornfeld, L., et al. (2004). Pomegranate juice consumption for 3 years by patients with carotid artery stenosis reduces common carotid intima-media thickness, blood pressure and LDL oxidation. Clin. Nutr. 23, 423-433. doi: 10.1016/j.clnu.2003.10.002

Batta, A. K., and Rangaswami, S. (1973). Crystalline chemical components of some vegetable drugs. Phytochemistry 12, 214-216. doi: 10.1016/S0031-9422(00)84654-3

Bialonska, D., Kasimsetty, S. G., Khan, S. I., and Ferreira, D. (2009). Urolithins, intestinal microbial metabolites of pomegranate ellagitannins, exhibit potent antioxidant activity in a cell-based assay. J. Agric. Food Chem. 57, 10181-10186. doi: 10.1021/jf9025794

Boncuk, M. (2014). Word Origin, Nar, Pomegranete. Available online at: http:// maviboncuk.blogspot.ch/2014/08/word-origin-nar-pomegranete.html?m=0 (accsessed April 24, 2018).

Bonzanini, F., Bruni, R., Palla, G., Serlataite, N., and Caligiani, A. (2009). Identification and distribution of lignans in Punica granatum L. fruit endocarp, pulp, seeds, wood knots and commercial juices by GC-MS. Food Chem. 117, 745-749. doi: 10.1016/j.foodchem.2009.04.057

Borges, G., and Crozier, A. (2012). HPLC-PDA-MS fingerprinting to assess the authenticity of pomegranate beverages. Food Chem. 135, 1863-1867. doi: $10.1016 /$ j.foodchem.2012.05.108

Brighenti, V., Groothuis, S. F., Prencipe, F. P., Amir, R., Benvenuti, S., and Pellati, F. (2017). Metabolite fingerprinting of Punica granatum L. (pomegranate) polyphenols by means of high-performance liquid chromatography with diode array and electrospray ionization-mass spectrometry detection. J. Chromatogr. A 1480, 20-31. doi: 10.1016/j.chroma.2016.12.017

Cadwallader, K. R., Tamamoto, L. C., and Sajuti, S. C. (2010). “Aroma components of fresh and stored pomegranate (punica granatum L.) juice," in Flavors in Noncarbonated Beverages, eds N. C. Da Costa and R. J. Cannon (Washington, DC: American Chemical Society), 93-101. doi: 10.1021/bk-2010-1036.ch007

Calani, L., Beghè, D., Mena, P., Del Rio, D., Bruni, R., Fabbri, A., et al. (2013). UltraHPLC-MS(n) (poly)phenolic profiling and chemometric analysis of juices from ancient Punica granatum L. cultivars: a nontargeted approach. J. Agric. Food Chem. 61, 5600-5609. doi: 10.1021/jf400387c

Carbonell-Barrachina, A. A., Calín-Sánchez, A., Bagatar, B., Hernández, F., Legua, P., Martínez-Font, R., et al. (2012). Potential of Spanish sour-sweet pomegranates (cultivar C25) for the juice industry. Food Sci. Technol. Int. 18, 129-138. doi: 10.1177/1082013211414783

Cerdá, B., Periago, P., Espín, J. C., and Tomás-Barberán, F. A. (2005). Identification of urolithin a as a metabolite produced by human colon microflora from ellagic acid and related compounds. J. Agric. Food Chem. 53, 5571-5576. doi: 10.1021/jf050384i

Chistiakov, D. A., Melnichenko, A. A., Orekhov, A. N., and Bobryshev, Y. V. (2017). Paraoxonase and atherosclerosis-related cardiovascular diseases. Biochimie 132, 19-27. doi: 10.1016/j.biochi.2016.10.010

Conidi, C., Cassano, A., Caiazzo, F., and Drioli, E. (2017). Separation and purification of phenolic compounds from pomegranate juice by ultrafiltration and nanofiltration membranes. J. Food Eng. 195, 1-13. doi: 10.1016/j.jfoodeng.2016.09.017

Das, A. K., Mandal, S. C., Banerjee, S. K., Sinha, S., Saha, B. P., and Pal, M. (2001). Studies on the hypoglycaemic activity of Punica granatum seed in streptozotocin induced diabetic rats. Phytother. Res. 15, 628-629. doi: $10.1002 /$ ptr.740

Delgado, N. T., Rouver, W. D., Freitas-Lima, L. C., De Paula, T. D., Duarte, A., Silva, J. F., et al. (2016). Pomegranate extract enhances endothelium-dependent coronary relaxation in isolated perfused hearts from spontaneously hypertensive ovariectomized rats. Front. Pharmacol. 7:522. doi: $10.3389 /$ fphar.2016.00522

de Nigris, F., Williams-Ignarro, S., Botti, C., Sica, V., Ignarro, L. J., and Napoli, C. (2006). Pomegranate juice reduces oxidized low-density lipoprotein downregulation of endothelial nitric oxide synthase in human coronary endothelial cells. Nitric Oxide 15, 259-263. doi: 10.1016/j.niox.2005. 12.004 de Nigris, F., Williams-Ignarro, S., Lerman, L. O., Crimi, E., Botti, C., Mansueto, G., et al. (2005). Beneficial effects of pomegranate juice on oxidation-sensitive genes and endothelial nitric oxide synthase activity at sites of perturbed shear stress. Proc. Natl. Acad. Sci. U.S.A. 102, 4896-4901. doi: 10.1073/pnas.0500998102

De Nigris, F., Williams-Ignarro, S., Sica, V., Lerman, L. O., D’armiento, F. P., Byrns, R. E., et al. (2007). Effects of a pomegranate fruit extract rich in punicalagin on oxidation-sensitive genes and eNOS activity at sites of perturbed shear stress and atherogenesis. Cardiovasc. Res. 73, 414-423. doi: 10.1016/j.cardiores.2006.08.021

De Pascual-Teresa, S., Santos-Buelga, C., and Rivas-Gonzalo, J. C. (2000). Quantitative analysis of flavan-3-ols in Spanish foodstuffs and beverages. J. Agric. Food Chem. 48, 5331-5337. doi: 10.1021/jf000549h

Dos Santos, R. L., Dellacqua, L. O., Delgado, N. T., Rouver, W. N., Podratz, P. L., Lima, L. C., et al. (2016). Pomegranate peel extract attenuates oxidative stress by decreasing coronary angiotensin-converting enzyme (ACE) activity in hypertensive female rats. J. Toxicol. Environ. Health A 79, 998-1007. doi: $10.1080 / 15287394.2016 .1213690$

Dumlu, M. U., and Gürkan, E. (2007). Elemental and nutritional analysis of Punica granatum from Turkey. J. Med. Food 10, 392-395. doi: 10.1089/jmf.2006.295

Ehling, S., and Cole, S. (2011). Analysis of organic acids in fruit juices by liquid chromatography-mass spectrometry: an enhanced tool for authenticity testing. J. Agric. Food Chem. 59, 2229-2234. doi: 10.1021/jf104527e

Elfalleh, W., Ying, M., Nasri, N., Sheng-Hua, H., Guasmi, F., and Ferchichi, A. (2011). Fatty acids from Tunisian and Chinese pomegranate (Punica granatum L.) seeds. Int. J. Food Sci. Nutr. 62, 200-206. doi: 10.3109/09637486.2010.526932

Elsherbiny, E. A., Amin, B. H., and Baka, Z. A. (2016). Efficiency of pomegranate (Punica granatum L.) peels extract as a high potential natural tool towards Fusarium dry rot on potato tubers. Postharvest Biol. Technol. 111, 256-263. doi: 10.1016/j.postharvbio.2015.09.019

Fanali, C., Belluomo, M. G., Cirilli, M., Cristofori, V., Zecchini, M., Cacciola, F., et al. (2016). Antioxidant activity evaluation and HPLC-photodiode array/MS polyphenols analysis of pomegranate juice from selected italian cultivars: a comparative study. Electrophoresis 37, 1947-1955. doi: 10.1002/elps.201500501

Fawole, O. A., Makunga, N. P., and Opara, U. L. (2012). Antibacterial, antioxidant and tyrosinase-inhibition activities of pomegranate fruit peel methanolic extract. BMC Complement. Altern. Med. 12:200. doi: 10.1186/1472-6882-12-200

Fischer, U. A., Carle, R., and Kammerer, D. R. (2011a). Identification and quantification of phenolic compounds from pomegranate (Punica granatum L.) peel, mesocarp, aril and differently produced juices by HPLC-DAD-ESI/MS(n). Food Chem. 127, 807-821. doi: 10.1016/j.foodchem.2010.12.156

Fischer, U. A., Carle, R., and Kammerer, D. R. (2013). Thermal stability of anthocyanins and colourless phenolics in pomegranate (Punica granatum L.) juices and model solutions. Food Chem. 138, 1800-1809. doi: $10.1016 /$ j.foodchem.2012.10.072

Fischer, U. A., Dettmann, J. S., Carle, R., and Kammerer, D. R. (2011b). Impact of processing and storage on the phenolic profiles and contents of pomegranate (Punica granatum L.) juices. Eur. Food Res. Technol. 233, 797-816. doi: 10.1007/s00217-011-1560-3

Fu, Q. J., Zhang, L. H., Cheng, N. N., Jia, M., and Zhang, Y. H. (2014). Extraction optimization of oleanolic and ursolic acids from pomegranate (Punica granatum L.) flowers. Food Bioprod Process 92, 321-327. doi: $10.1016 /$ j.fbp. 2012.12 .006

Fuhrman, B., Volkova, N., and Aviram, M. (2005). Pomegranate juice inhibits oxidized LDL uptake and cholesterol biosynthesis in macrophages. J. Nutr. Biochem. 16, 570-576. doi: 10.1016/j.jnutbio.2005.02.009

Garcia-Muñoz, C., and Vaillant, F. (2014). Metabolic fate of ellagitannins: implications for health, and research perspectives for innovative functional foods. Crit. Rev. Food Sci. Nutr. 54, 1584-1598. doi: $10.1080 / 10408398.2011 .644643$

Gil, M. I., Tomás-Barberán, F. A., Hess-Pierce, B., Holcroft, D. M., and Kader, A. A. (2000). Antioxidant activity of pomegranate juice and its relationship with phenolic composition and processing. J. Agric. Food Chem. 48, 4581-4589. doi: $10.1021 /$ jf000404a

Giménez-Bastida, J. A., González-Sarrías, A., Larrosa, M., Tomas-Barberan, F., Espin, J. C., and Garcia-Conesa, M. T. (2012). Ellagitannin metabolites, urolithin A glucuronide and its aglycone urolithin A, ameliorate 
TNF-alpha-induced inflammation and associated molecular markers in human aortic endothelial cells. Mol. Nutr. Food Res. 56, 784-796. doi: $10.1002 / \mathrm{mnfr} .201100677$

Górnaś, P., and Rudzinska, M. (2016). Seeds recovered from industry by-products of nine fruit species with a high potential utility as a source of unconventional oil for biodiesel and cosmetic and pharmaceutical sectors. Ind. Crops Prod. 83, 329-338. doi: 10.1016/j.indcrop.2016.01.021

Grosser, T., Fries, S., and Fitzgerald, G. A. (2006). Biological basis for the cardiovascular consequences of COX-2 inhibition: therapeutic challenges and opportunities. J. Clin. Invest. 116, 4-15. doi: 10.1172/JCI27291

Gundogdu, M., and Yilmaz, H. (2012). Organic acid, phenolic profile and antioxidant capacities of pomegranate (Punica granatum L.) cultivars and selected genotypes. Sci Hortic 143, 38-42. doi: 10.1016/j.scienta.2012.05.029

Hadrich, F., Cher, S., Gargouri, Y. T., and Adel, S. (2014). Antioxidant and lipase inhibitory activities and essential oil composition of pomegranate peel extracts. J. Oleo Sci. 63, 515-525. doi: 10.5650/jos.ess 13163

Haghighian, M. K., Rafraf, M., Moghaddam, A., Hemmati, S., Jafarabadi, M. A., and Gargari, B. P. (2016). Pomegranate (Punica granatum L.) peel hydro alcoholic extract ameliorates cardiovascular risk factors in obese women with dyslipidemia: a double blind, randomized, placebo controlled pilot study. Eur. J. Integr. Med. 8, 676-682. doi: 10.1016/j.eujim.2016.06.010

Han, L., Yuan, Z., Feng, L., and Yin, Y. (2015). Changes in the composition and contents of pomegranate polyphenols during fruit development. Acta Hortic. 1089, 53-61. doi: 10.17660/ActaHortic.2015.1089.5

Han, Q. A., Yan, C., Wang, L., Li, G., Xu, Y., and Xia, X. (2016). Urolithin A attenuates ox-LDL-induced endothelial dysfunction partly by modulating microRNA-27 and ERK/PPAR-gamma pathway. Mol. Nutr. Food Res. 60, 1933-1943. doi: 10.1002/mnfr.201500827

Harikrishnan, R., Heo, J., Balasundaram, C., Kim, M. C., Kim, J. S., Han, Y. J., et al. (2010). Effect of Punica granatum solvent extracts on immune system and disease resistance in Paralichthys olivaceus against lymphocystis disease virus (LDV). Fish Shellfish Immunol. 29, 668-673. doi: 10.1016/j.fsi.2010.07.006

Hartman, R. E., Shah, A., Fagan, A. M., Schwetye, K. E., Parsadanian, M., Schulman, R. N., et al. (2006). Pomegranate juice decreases amyloid load and improves behavior in a mouse model of Alzheimer's disease. Neurobiol. Dis. 24, 506-515. doi: 10.1016/j.nbd.2006.08.006

Hasnaoui, N., Wathelet, B., and Jiménez-Araujo, A. (2014). Valorization of pomegranate peel from 12 cultivars: dietary fibre composition, antioxidant capacity and functional properties. Food Chem. 160, 196-203. doi: 10.1016/j.foodchem.2014.03.089

He, L., Xu, H. G., Liu, X., He, W. H., Yuan, F., Hou, Z. Q., et al. (2011). Identification of phenolic compounds from pomegranate (Punica granatum L.) seed residues and investigation into their antioxidant capacities by HPLC-ABTS(+) assay. Food Res. Int. 44, 1161-1167. doi: $10.1016 /$ j.foodres.2010.05.023

Hernandez, F., Melgarejo, P., Tomas-Barberan, F. A., and Artes, F. (1999). Evolution of juice anthocyanins during ripening of new selected pomegranate (Punica granatum) clones Eur. Food Res. Technol. 210, 39-42.

Hmid, I., Elothmani, D., Hanine, H., Oukabli, A., and Mehinagic, E. (2017). Comparative study of phenolic compounds and their antioxidant attributes of eighteen pomegranate (Punica granatum L.) cultivars grown in Morocco. Arab. J. Chem. 10, S2675-S2684. doi: 10.1016/j.arabjc.2013.10.011

Holland, D., Hatib, K., and Bar-Ya'akov, I. (2009). "Pomegranate: botany, horticulture, breeding," in Horticultural Reviews, ed J. Janick (New Jersey, NJ: John Wiley \& Sons, Inc.), 127-191.

Hontecillas, R., O'shea, M., Einerhand, A., Diguardo, M., and Bassaganya-Riera, J. (2009). Activation of PPAR gamma and alpha by punicic acid ameliorates glucose tolerance and suppresses obesity-related inflammation. J. Am. Coll. Nutr. 28, 184-195. doi: 10.1080/07315724.2009.10719770

Hopkins, C. Y., and Chisholm, M. J. (1968). A survey of the conjugated fatty acids of seed oils. J. Am. Oil Chem. Soc. 45, 176-182. doi: 10.1007/BF02915346

Huang, T. H., Peng, G., Kota, B. P., Li, G. Q., Yamahara, J., Roufogalis, B. D., et al. (2005a). Anti-diabetic action of Punica granatum flower extract: activation of PPAR-gamma and identification of an active component. Toxicol. Appl. Pharmacol. 207, 160-169. doi: 10.1016/j.taap.2004. 12.009

Huang, T. H., Peng, G., Kota, B. P., Li, G. Q., Yamahara, J., Roufogalis, B. D., et al. (2005b). Pomegranate flower improves cardiac lipid metabolism in a diabetic rat model: role of lowering circulating lipids. Br. J. Pharmacol. 145, 767-774. doi: 10.1038/sj.bjp.0706245

Hyson, D. A. (2015). A review and critical analysis of the scientific literature related to $100 \%$ fruit juice and human health. Adv. Nutr. 6, 37-51. doi: 10.3945/an.114.005728

Ignarro, L. J., Byrns, R. E., Sumi, D., De Nigris, F., and Napoli, C. (2006). Pomegranate juice protects nitric oxide against oxidative destruction and enhances the biological actions of nitric oxide. Nitric Oxide 15, 93-102. doi: 10.1016/j.niox.2006.03.001

Jadeja, R. N., Thounaojam, M. C., Patel, D. K., Devkar, R. V., and Ramachandran, A. V. (2010). Pomegranate (Punica granatum L.) juice supplementation attenuates isoproterenol-induced cardiac necrosis in rats. Cardiovasc. Toxicol. 10, 174-180. doi: 10.1007/s12012-010-9076-9

Jain, V., Murugananthan, G., Deepak, M., Viswanatha, G. L., and Manohar, D. (2011). Isolation and standardization of various phytochemical constituents from methanolic extracts of fruit rinds of Punica granatum. Chin. J. Nat. Med. 9, 414-420. doi: 10.3724/SP.J.1009.2011.00414

Jiang, H.-Z., Ma, Q.-Y., Fan, H.-J., Liang, W.-J., Huang, S.-Z., Dai, H.-F., et al. (2012). Fatty acid synthase inhibitors isolated from Punica granatum L. J. Braz. Chem. Soc. 23, 889-893. doi: 10.1590/S0103-50532012000500014

Jurenka, J. S. (2008). Therapeutic applications of pomegranate (Punica granatum L.): a review. Altern. Med. Rev. 13, 128-144.

Kasai, K., Yoshimura, M., Koga, T., Arii, M., and Kawasaki, S. (2006). Effects of oral administration of ellagic acid-rich pomegranate extract on ultravioletinduced pigmentation in the human skin. J. Nutr. Sci. Vitaminol. 52, 383-388. doi: $10.3177 /$ jnsv.52.383

Kaufman, M., and Wiesman, Z. (2007). Pomegranate oil analysis with emphasis on MALDI-TOF/MS triacylglycerol fingerprinting. J. Agric. Food Chem. 55, 10405-10413. doi: 10.1021/jf072741q

Khoo, N. K., White, C. R., Pozzo-Miller, L., Zhou, F., Constance, C., Inoue, T., et al. (2010). Dietary flavonoid quercetin stimulates vasorelaxation in aortic vessels. Free Radic. Biol. Med. 49, 339-347. doi: 10.1016/j.freeradbiomed.2010.04.022

Kim, N. D., Mehta, R., Yu, W., Neeman, I., Livney, T., Amichay, A., et al. (2002). Chemopreventive and adjuvant therapeutic potential of pomegranate (Punica granatum) for human breast cancer. Breast Cancer Res. Treat. 71, 203-217. doi: 10.1023/A:1014405730585

Kumagai, Y., Nakatani, S., Onodera, H., Nagatomo, A., Nishida, N., Matsuura, Y., et al. (2015). Anti-glycation effects of pomegranate (Punica granatum L.) fruit extract and its components in vivo and in vitro. J. Agric. Food Chem. 63, 7760-7764. doi: 10.1021/acs.jafc.5b02766

Landete, J. M., Arqués, J., Medina, M., Gaya, P., De Las Rivas, B., and Muñoz, R. (2016). Bioactivation of phytoestrogens: intestinal bacteria and health. Crit. Rev. Food Sci. Nutr. 56, 1826-1843. doi: 10.1080/10408398.2013.789823

Langley, P. (2000). Why a pomegranate? BMJ 321, 1153-1154. doi: $10.1136 / \mathrm{bmj} .321 .7269 .1153$

Lansky, E. P., and Newman, R. A. (2007). Punica granatum (pomegranate) and its potential for prevention and treatment of inflammation and cancer. J. Ethnopharmacol. 109, 177-206. doi: 10.1016/j.jep.2006. 09.006

Lantzouraki, D. Z., Sinanoglou, V. J., Zoumpoulakis, P. G., Glamoclija, J., Ciric, A., Sokovic, M., et al. (2015). Antiradical-antimicrobial activity and phenolic profile of pomegranate (Punica granatum L.) juices from different cultivars: a comparative study. RSC Adv. 5, 2602-2614. doi: 10.1039/C4RA11795F

Larrosa, M., García-Conesa, M. T., Espín, J. C., and Tomás-Barberán, F. A. (2010a). Ellagitannins, ellagic acid and vascular health. Mol. Aspects Med. 31, 513-539. doi: 10.1016/j.mam.2010.09.005

Larrosa, M., González-Sarrías, A., Yáñez-Gascón, M. J., Selma, M. V., Azorín-Ortuño, M., Toti, S., et al. (2010b). Anti-inflammatory properties of a pomegranate extract and its metabolite urolithin-A in a colitis rat model and the effect of colon inflammation on phenolic metabolism. J. Nutr. Biochem. 21, 717-725. doi: 10.1016/j.jnutbio.2009. 04.012

Lee, W. J., Ou, H. C., Hsu, W. C., Chou, M. M., Tseng, J. J., Hsu, S. L., et al. (2010). Ellagic acid inhibits oxidized LDL-mediated LOX-1 expression, ROS generation, and inflammation in human endothelial cells. J. Vasc. Surg. 52, 1290-1300. doi: 10.1016/j.jvs.2010.04.085

Legua, P., Melgarejo, P., Martinez, J. J., Martinez, R., and Hernandez, F. (2012). Evaluation of Spanish pomegranate juices: organic 
acids, sugars, and anthocyanins. Int. J. Food Prop. 15, 481-494. doi: $10.1080 / 10942912.2010 .491931$

Lei, F., Zhang, X. N., Wang, W., Xing, D. M., Xie, W. D., Su, H., et al. (2007). Evidence of anti-obesity effects of the pomegranate leaf extract in high-fat diet induced obese mice. Int. J. Obes. (Lond). 31, 1023-1029. doi: $10.1038 /$ sj.ijo. 0803502

Les, F., Carpene, C., Arbones-Mainar, J. M., Decaunes, P., Valero, M. S., and Lopez, V. (2017). Pomegranate juice and its main polyphenols exhibit direct effects on amine oxidases from human adipose tissue and inhibit lipid metabolism in adipocytes. J. Funct. Foods 33, 323-331. doi: 10.1016/j.jff.2017.04.006

Les, F., Prieto, J. M., Arbonés-Mainar, J. M., Valero, M. S., and López, V. (2015). Bioactive properties of commercialised pomegranate (Punica granatum) juice; antioxidant, antiproliferative and enzyme inhibiting activities. Food Funct. 6, 2049-2057. doi: 10.1039/C5FO00426H

Levin, G. M. (2006). Pomegranate Roads: A Soviet Botanist's Exile from Eden. Edited by B. L. Baer (Forestville, CA: Floreat Press), 15-183.

Li, Y., Wen, S., Kota, B. P., Peng, G., Li, G. Q., Yamahara, J., et al. (2005). Punica granatum flower extract, a potent alpha-glucosidase inhibitor, improves postprandial hyperglycemia in Zucker diabetic fatty rats. J. Ethnopharmacol. 99, 239-244. doi: 10.1016/j.jep.2005.02.030

Lipinska, L., Klewicka, E., and Sójka, M. (2014). The structure, occurrence and biological activity of ellagitannins: a general review. Acta Sci. Pol. Technol. Aliment. 13, 289-299. doi: 10.17306/J.AFS.2014.3.7

Loren, D. J., Seeram, N. P., Schulman, R. N., and Holtzman, D. M. (2005). Maternal dietary supplementation with pomegranate juice is neuroprotective in an animal model of neonatal hypoxic-ischemic brain injury. Pediatr. Res. 57, 858-864. doi: 10.1203/01.PDR.0000157722.07810.15

Lynn, A., Hamadeh, H., Leung, W. C., Russell, J. M., and Barker, M. E. (2012). Effects of pomegranate juice supplementation on pulse wave velocity and blood pressure in healthy young and middle-aged men and women. Plant Foods Hum. Nutr. 67, 309-314. doi: 10.1007/s11130-012-0295-z

Malik, A., Afaq, F., Sarfaraz, S., Adhami, V. M., Syed, D. N., and Mukhtar, H. (2005). Pomegranate fruit juice for chemoprevention and chemotherapy of prostate cancer. Proc. Natl. Acad. Sci. U.S.A. 102, 14813-14818. doi: $10.1073 /$ pnas. 0505870102

Malik, A., and Mukhtar, H. (2006). Prostate cancer prevention through pomegranate fruit. Cell Cycle 5, 371-373. doi: 10.4161/cc.5.4.2486

Mattiello, T., Trifirò, E., Jotti, G. S., and Pulcinelli, F. M. (2009). Effects of pomegranate juice and extract polyphenols on platelet function. J. Med. Food 12, 334-339. doi: 10.1089/jmf.2007.0640

Mele, L., Mena, P., Piemontese, A., Marino, V., López-Gutiérrez, N., Bernini, F., et al. (2016). Antiatherogenic effects of ellagic acid and urolithins in vitro. Arch. Biochem. Biophys. 599, 42-50. doi: 10.1016/j.abb.2016.02.017

Mena, P., Calani, L., Dall'asta, C., Galaverna, G., García-Viguera, C., Bruni, R., et al. (2012). Rapid and comprehensive evaluation of (poly)phenolic compounds in pomegranate (Punica granatum L.) juice by UHPLC-MSn. Molecules 17, 14821-14840. doi: 10.3390/molecules171214821

Menezes, S. M., Cordeiro, L. N., and Viana, G. S. (2009). Punica granatum (pomegranate) extract is active against dental plaque. J. Herb. Pharmacother. 6, 79-92. doi: 10.1080/J157v06n02_07

Mohan, M., Waghulde, H., and Kasture, S. (2010). Effect of pomegranate juice on angiotensin II-induced hypertension in diabetic Wistar rats. Phytother. Res. 24(Suppl 2), S196-203. doi: 10.1002/ptr.3090

Mollazadeh, H., Sadeghnia, H. R., Hoseini, A., Farzadnia, M., and Boroushaki, M. T. (2016). Effects of pomegranate seed oil on oxidative stress markers, serum biochemical parameters and pathological findings in kidney and heart of streptozotocin-induced diabetic rats. Ren. Fail. 38, 1256-1266. doi: 10.1080/0886022X.2016.1207053

Mphahlele, R. R., Stander, M. A., Fawole, O. A., and Opara, U. L. (2014). Effect of fruit maturity and growing location on the postharvest contents of flavonoids, phenolic acids, vitamin $\mathrm{C}$ and antioxidant activity of pomegranate juice (cv. Wonderful). Sci. Hortic. 179, 36-45. doi: 10.1016/j.scienta.2014.09.007

Nawwar, M. A. M., Hussein, S. A. H., and Merfort, I. (1994a). NMR spectral analysis of polyphenols from Punica granatum. Phytochemistry 36, 793-798. doi: 10.1016/S0031-9422(00)89820-9

Nawwar, M. A. M., Hussein, S. A. M., and Merfort, I. (1994b). Leaf phenolics of Punica granatum L. Phytochemistry 37, 1175-1177. doi: $10.1016 /$ S0031-9422(00)89552-7
Naz, S., Siddiqi, R., Ahmad, S., Rasool, S. A., and Sayeed, S. A. (2007). Antibacterial activity directed isolation of compounds from Punica granatum. J. Food Sci. 72, M341-345. doi: 10.1111/j.1750-3841.2007.00533.x

Neuhofer, H., Witte, L., Gorunovic, M., and Czygan, F. C. (1993). Alkaloids in the bark of Punica granatum L. (pomegranate) from Yugoslavia. Pharmazie 48, 389-391.

Noda, Y., Kaneyuki, T., Mori, A., and Packer, L. (2002). Antioxidant activities of pomegranate fruit extract and its anthocyanidins: delphinidin, cyanidin, and pelargonidin. J. Agric. Food Chem. 50, 166-171. doi: 10.1021/jf0108765

Ou, H. C., Lee, W. J., Lee, S. D., Huang, C. Y., Chiu, T. H., Tsai, K. L., et al. (2010). Ellagic acid protects endothelial cells from oxidized low-density lipoproteininduced apoptosis by modulating the PI3K/Akt/eNOS pathway. Toxicol. Appl. Pharmacol. 248, 134-143. doi: 10.1016/j.taap.2010.07.025

Paller, C. J., Ye, X., Wozniak, P. J., Gillespie, B. K., Sieber, P. R., Greengold, R. H., et al. (2013). A randomized phase II study of pomegranate extract for men with rising PSA following initial therapy for localized prostate cancer. Prostate Cancer Prostatic Dis. 16, 50-55. doi: 10.1038/pcan.2012.20

Pande, G., and Akoh, C. C. (2009). Antioxidant capacity and lipid characterization of six Georgia-grown pomegranate cultivars. J. Agric. Food Chem. 57, 9427-9436. doi: 10.1021/jf901880p

Panth, N., Manandhar, B., and Paudel, K. R. (2017). Anticancer activity of Punica granatum (pomegranate): a review. Phytother. Res. 31, 568-578. doi: $10.1002 /$ ptr. 5784

Pérez-Ramírez, I. F., Reynoso-Camacho, R., Saura-Calixto, F., and PérezJiménez, J. (2018). Comprehensive characterization of extractable and nonextractable phenolic compounds by high-performance liquid chromatography-electrospray ionization-quadrupole time-of-flight of a grape/pomegranate pomace dietary supplement. J. Agric. Food Chem. 66, 661-673. doi: 10.1021/acs.jafc.7b05901

Pickering, G. (1972). Hypertension. Definitions, natural histories and consequences. Am. J. Med. 52, 570-583. doi: 10.1016/0002-9343(72)90 049-6

Poyrazoglu, E., Gökmen, V., and Artık, N. (2002). Organic acids and phenolic compounds in pomegranates (Punica granatum L.) grown in Turkey. J. Food Compos. Anal. 15, 567-575. doi: 10.1016/S0889-1575(02)91071-9

Rasheed, Z., Akhtar, N., Anbazhagan, A. N., Ramamurthy, S., Shukla, M., and Haqqi, T. M. (2009). Polyphenol-rich pomegranate fruit extract (POMx) suppresses PMACI-induced expression of pro-inflammatory cytokines by inhibiting the activation of MAP Kinases and NF-kappaB in human KU812 cells. J. Inflamm. (Lond). 6:1. doi: 10.1186/1476-9255-6-1

Reshef, N., Hayari, Y., Goren, C., Boaz, M., Madar, Z., and Knobler, H. (2005). Antihypertensive effect of sweetie fruit in patients with stage I hypertension. Am. J. Hypertens. 18, 1360-1363. doi: 10.1016/j.amjhyper.2005.05.021

Ricci, D., Giamperi, L., Bucchini, A., and Fraternale, D. (2006). Antioxidant activity of Punica granatum fruits. Fitoterapia 77, 310-312. doi: 10.1016/j.fitote.2006.01.008

Rosenblat, M., Volkova, N., Abassi, Z., Britton, S. L., Koch, L. G., and Aviram, M. (2015). High intrinsic aerobic capacity and pomegranate juice are protective against macrophage atherogenecity: studies in high- vs. lowcapacity runner (HCR vs. LCR) rats. J. Nutr. Biochem. 26, 1015-1021. doi: 10.1016/j.jnutbio.2015.04.001

Rozenberg, O., Howell, A., and Aviram, M. (2006). Pomegranate juice sugar fraction reduces macrophage oxidative state, whereas white grape juice sugar fraction increases it. Atherosclerosis 188, 68-76. doi: 10.1016/j.atherosclerosis.2005.10.027

Saha, P., Yeoh, B. S., Singh, R., Chandrasekar, B., Vemula, P. K., Haribabu, B., et al. (2016). Gut microbiota conversion of dietary ellagic acid into bioactive phytoceutical urolithin A inhibits heme peroxidases. PLoS ONE 11:e0156811. doi: 10.1371/journal.pone.0156811

Sahebkar, A., Ferri, C., Giorgini, P., Bo, S., Nachtigal, P., and Grassi, D. (2017). Effects of pomegranate juice on blood pressure: a systematic review and meta-analysis of randomized controlled trials. Pharmacol. Res. 115, 149-161. doi: $10.1016 /$ j.phrs.2016.11.018

Sahebkar, A., Gurban, C., Serban, A., Andrica, F., and Serban, M. C. (2016). Effects of supplementation with pomegranate juice on plasma C-reactive protein concentrations: a systematic review and metaanalysis of randomized controlled trials. Phytomedicine 23, 1095-1102. doi: 10.1016/j.phymed.2015.12.008 
Salwe, K. J., Sachdev, D. O., Bahurupi, Y., and Kumarappan, M. (2015). Evaluation of antidiabetic, hypolipedimic and antioxidant activity of hydroalcoholic extract of leaves and fruit peel of Punica granatum in male Wistar albino rats. J. Nat. Sci. Biol. Med. 6, 56-62. doi: 10.4103/0976-9668.149085

Satomi, H., Umemura, K., Ueno, A., Hatano, T., Okuda, T., and Noro, T. (1993). Carbonic anhydrase inhibitors from the pericarps of Punica granatum L. Biol. Pharm. Bull. 16, 787-790. doi: 10.1248/bpb.16.787

Saxena, A., and Vikram, N. K. (2004). Role of selected Indian plants in management of type 2 diabetes: a review. J. Altern. Complement. Med. 10, 369-378. doi: 10.1089/107555304323062365

Schubert, S. Y., Lansky, E. P., and Neeman, I. (1999). Antioxidant and eicosanoid enzyme inhibition properties of pomegranate seed oil and fermented juice flavonoids. J. Ethnopharmacol. 66, 11-17. doi: 10.1016/S0378-8741(98)00222-0

Sharma, P., McClees, S. F., and Afaq, F. (2017). Pomegranate for prevention and treatment of cancer: an update. Molecules 22:177. doi: 10.3390/molecules22010177

Shiner, M., Fuhrman, B., and Aviram, M. (2007). Macrophage paraoxonase 2 (PON2) expression is up-regulated by pomegranate juice phenolic antioxidants via PPAR gamma and AP-1 pathway activation. Atherosclerosis 195, 313-321. doi: 10.1016/j.atherosclerosis.2007.01.007

Siano, F., Straccia, M. C., Paolucci, M., Fasulo, G., Boscaino, F., and Volpe, M. G. (2016). Physico-chemical properties and fatty acid composition of pomegranate, cherry and pumpkin seed oils. J. Sci. Food Agric. 96, 1730-1735. doi: $10.1002 /$ jsfa.7279

Sudheesh, S., and Vijayalakshmi, N. R. (2005). Flavonoids from Punica granatum-potential antiperoxidative agents. Fitoterapia 76, 181-186. doi: 10.1016/j.fitote.2004.11.002

Svenja (2018). Tapeworms in Time: Ancient Egypt and the Ebers Papyrus. Available online at: https://diagnost-x.de/ancient-egypt-and-the-ebers-papyrus/ (accsessed April 24, 2018).

Tanaka, T., Nonaka, G. I., and Nishioka, I. (1986a). Tannins and related compounds. XLI. Isolation and characterization of novel ellagitannins, punicacorteins A, B, C and D, and punigluconin from the bark of Punica granatum L. Chem. Pharm. Bull. 34, 656-663. doi: 10.1248/cpb.34.656

Tanaka, T., Nonaka, G. I., and Nishioka, I. (1990). Tannins and related compounds. C. Reaction of dehydrohexahydroxydiphenic acid esters with bases, and its application to the structure determination of pomegranate tannins, granatins a and b. Chem Pharm Bull 38, 9424-9428. doi: 10.1248/cpb.38.2424

Tanaka, T., Nonaka, G., and Nishioka, I. (1986b). Tannins and related compounds. $\mathrm{XL}$. Revision of the structures of punicalin and punicalagin, and isolation and characterization of 2-O-galloylpunicalin from the bark of Punica granatum L. Chem. Pharm. Bull. 34 650-655. doi: 10.1248/cpb.34.650

Tang, L., Mo, Y., Li, Y., Zhong, Y., He, S., Zhang, Y., et al. (2017). Urolithin A alleviates myocardial ischemia/reperfusion injury via PI3K/Akt pathway. Biochem. Biophys. Res. Commun. 486, 774-780. doi: 10.1016/j.bbrc.2017.03.119

Tomas-Barberan, F. A., Gonzalez-Sarrias, A., Garcia-Villalba, R., Nunez-Sanchez, M. A., Selma, M. V., Garcia-Conesa, M. T., et al. (2017). Urolithins, the rescue of "old" metabolites to understand a "new" concept: Metabotypes as a nexus among phenolic metabolism, microbiota dysbiosis, and host health status. Mol. Nutr. Food Res. 61. doi: 10.1002/mnfr.201500901

Türk, G., Sönmez, M., Aydin, M., Yüce, A., Gür, S., Yüksel, M., et al. (2008). Effects of pomegranate juice consumption on sperm quality, spermatogenic cell density, antioxidant activity and testosterone level in male rats. Clin. Nutr. 27, 289-296. doi: 10.1016/j.clnu.2007.12.006

Türkyilmaz, M. (2013). Anthocyanin and organic acid profiles of pomegranate (Punica granatum L.) juices from registered varieties in Turkey. Int J Food Sci Technol 48, 2086-2095.

Tzulker, R., Glazer, I., Bar-Ilan, I., Holland, D., Aviram, M., and Amir, R. (2007). Antioxidant activity, polyphenol content, and related compounds in different fruit juices and homogenates prepared from 29 different pomegranate accessions. J. Agric. Food Chem. 55, 9559-9570. doi: 10.1021/jf071413n

Uhrin, P., Wang, D., Mocan, A., Waltenberger, B., Breuss, J. M., Tewari, D., et al. (2018). Vascular smooth muscle cell proliferation as a therapeutic target. Part 2: Natural products inhibiting proliferation. Biotechnol. Adv. [Epub ahead of print]. doi: 10.1016/j.biotechadv.2018.04.002

Vallance, P., and Chan, N. (2001). Endothelial function and nitric oxide: clinical relevance. Heart 85, 342-350. doi: 10.1136/heart.85.3.342

Van Elswijk, D. A., Schobel, U. P., Lansky, E. P., Irth, H., and Van Der Greef, J. (2004). Rapid dereplication of estrogenic compounds in pomegranate (Punica granatum) using on-line biochemical detection coupled to mass spectrometry. Phytochemistry 65, 233-241. doi: 10.1016/j.phytochem.2003.07.001

Vázquez-Araújo, L., Chambers, E., Adhikari, K., and Carbonell-Barrachina, A. A. (2011). Physico-chemical and sensory properties of pomegranate juices with pomegranate albedo and carpellar membranes homogenate. LWT - Food Science and Technology 44, 2119-2125. doi: 10.1016/j.lwt.2011.07.014

Vegara, S., Martí, N., Lorente, J., Coll, L., Streitenberger, S., Valero, M., et al. (2014). Chemical guide parameters for Punica granatum cv. 'Mollar' fruit juices processed at industrial scale. Food Chem. 147, 203-208. doi: 10.1016/j.foodchem.2013.09.122

Vendrame, S., and Klimis-Zacas, D. (2015). Anti-inflammatory effect of anthocyanins via modulation of nuclear factor-kappaB and mitogenactivated protein kinase signaling cascades. Nutr. Rev. 73, 348-358. doi: 10.1093/nutrit/nuu066

Verardo, V., Garcia-Salas, P., Baldi, E., Segura-Carretero, A., Fernandez-Gutierrez, A., and Caboni, M. F. (2014). Pomegranate seeds as a source of nutraceutical oil naturally rich in bioactive lipids. Food Res. Int. 65(Pt C), 445-452. doi: 10.1016/j.foodres.2014.04.044

Vidal, A., Fallarero, A., Peña, B. R., Medina, M. E., Gra, B., Rivera, F., et al. (2003). Studies on the toxicity of Punica granatum L. (Punicaceae) whole fruit extracts. J. Ethnopharmacol. 89, 295-300. doi: 10.1016/j.jep.2003. 09.001

Vilahur, G., Padró, T., Casaní, L., Mendieta, G., López, J. A., Streitenberger, S., et al. (2015). Polyphenol-enriched diet prevents coronary endothelial dysfunction by activating the Akt/eNOS pathway. Rev. Esp. Cardiol. (Engl. Ed). 68, 216-225. doi: 10.1016/j.recesp.2014.03.023

Vroegrijk, I. O., Van Diepen, J. A., Van Den Berg, S., Westbroek, I., Keizer, H. Gambelli, L., et al. (2011). Pomegranate seed oil, a rich source of punicic acid, prevents diet-induced obesity and insulin resistance in mice. Food Chem. Toxicol. 49, 1426-1430. doi: 10.1016/j.fct.2011.03.037

Wafa, B. A., Makni, M., Ammar, S., Khannous, L., Hassana, A. B., Bouaziz, M., et al. (2017). Antimicrobial effect of the Tunisian Nana variety Punica granatum L. extracts against Salmonella enterica (serovars Kentucky and Enteritidis) isolated from chicken meat and phenolic composition of its peel extract. Int. J. Food Microbiol. 241, 123-131. doi: 10.1016/j.ijfoodmicro.2016. 10.007

Wang, D., Uhrin, P., Mocan, A., Waltenberger, B., Breuss, J. M., Tewari, D., et al. (2018). Vascular smooth muscle cell proliferation as a therapeutic target. Part 1: molecular targets and pathways. Biotechnol. $A d v$. doi: 10.1016/j.biotechadv.2018.04.006. [Epub ahead of print].

Wang, R. F., Xie, W. D., Zhang, Z., Xing, D. M., Ding, Y., Wang, W., et al. (2004). Bioactive compounds from the seeds of Punica granatum (pomegranate). J. Nat. Prod. 67, 2096-2098. doi: 10.1021/np0498051

Ward, C. (2003). Pomegranates in eastern Mediterranean contexts during the Late Bronze Age. World Archaeol. 34, 529-541. doi: 10.1080/0043824021000026495

Weiss, H. J., and Turitto, V. T. (1979). Prostacyclin (prostaglandin I2, PGI2) inhibits platelet adhesion and thrombus formation on subendothelium. Blood $53,244-250$.

Yilmaz, B., and Usta, C. (2013). Ellagic acid-induced endothelium-dependent and endothelium-independent vasorelaxation in rat thoracic aortic rings and the underlying mechanism. Phytother. Res. 27, 285-289. doi: 10.1002/ptr.4716

Yuan, T., Wan, C., Ma, H., and Seeram, N. P. (2013). New phenolics from the flowers of Punica granatum and their in vitro alpha-glucosidase inhibitory activities. Planta Med. 79, 1674-1679. doi: 10.1055/s-0033-1350925

Zheng, J., Zhou, Y., Li, S., Zhang, P., Zhou, T., Xu, D. P., et al. (2017). Effects and mechanisms of fruit and vegetable juices on cardiovascular diseases. Int. J. Mol. Sci. 18, 1-15. doi: 10.3390/ijms18030555

Conflict of Interest Statement: The authors declare that the research was conducted in the absence of any commercial or financial relationships that could be construed as a potential conflict of interest.

Copyright (c) 2018 Wang, Özen, Abu-Reidah, Chigurupati, Patra, Horbanczuk, Jóźwik, Tzvetkov, Uhrin and Atanasov. This is an open-access article distributed under the terms of the Creative Commons Attribution License (CC BY). The use, distribution or reproduction in other forums is permitted, provided the original author(s) and the copyright owner are credited and that the original publication in this journal is cited, in accordance with accepted academic practice. No use, distribution or reproduction is permitted which does not comply with these terms. 


\title{
An Ethnobotanical Study of Medicinal Plants in the Greek Islands of North Aegean Region
}

\author{
Evangelos Axiotis, Maria Halabalaki* and Leandros A. Skaltsounis
}

Department of Pharmacognosy and Natural Products Chemistry, Faculty of Pharmacy, National and Kapodistrian University of Athens, Athens, Greece

Greek islands of the North Aegean Region are a group of nine inhabited islands (Lemnos, Agios Efstratios, Lesvos, Chios, Psara, Oinousses, Samos, Ikaria, and Fourni) located in the northern part of the Aegean Sea, close to Asia Minor. Each island of this region can be considered autonomous in terms of culture and biodiversity. With this work we try to evaluate the status of the traditional uses of medicinal plants in this region. Endemic and endangered species such as Sideritis sipylea Boiss., Origanum sipyleum L., Thymus sipyleus Boiss., Pistacia lentiscus L., Verbascum ikaricum Murb., are still used by locals to treat different ailments. Moreover, the use of some species for the treatment of specific diseases has been reported for the first time. We report about 109

OPEN ACCESS

Edited by:

Atanas G. Atanasov,

Institute of Genetics and Animal

Breeding (PAS), Poland

Reviewed by:

Sevser Sahpaz,

Lille University, France

Ligia Salgueiro,

University of Coimbra, Portugal

${ }^{*}$ Correspondence:

Maria Halabalak

mariaha/@pharm.uoa.gr

Specialty section:

This article was submitted to

Ethnopharmacology,

a section of the journal

Frontiers in Pharmacology

Received: 08 December 2017

Accepted: 09 April 2018

Published: 23 May 2018

Citation:

Axiotis E, Halabalaki $M$ and

Skaltsounis LA (2018) An

Ethnobotanical Study of Medicinal

Plants in the Greek Islands of North

Aegean Region.

Front. Pharmacol. 9:409.

doi: 10.3389/fphar.2018.00409 wild plants of medicinal importance, from 52 families, listing their uses for therapeutic purposes and galenic preparations provided by local medical doctors and pharmacists. The information we include was derived from literature sources and additionally collected through semi-structured interviews conducted on 200 informants (100 men and 100 women). Additionally, informant consensus factor (FIC) and UV value were calculated for the medicinal plants in the current study in relation with the diseases treated. This research confirms the importance of the medicinal plants and the diffusion of their use in traditional medicine within this region. This ethnopharmacological survey is a fundamental step for the preservation of the local knowledge both for further scientific research and for the protection of endangered and endemic medicinal plants.

Keywords: ethnopharmacology, traditional medicine, Near East Greek islands, North Aegean Sea, ethnobotany

\section{INTRODUCTION}

Greece holds a unique position with respect to the number of plant species and subspecies compared to other areas of the globe. This depends on the variety of habitats, as well as on geological history, climate conditions, and geographical position within the Mediterranean. Thus, it is noted for its high plant species diversity (5800 species and 1893 subspecies) and endemism (22.2\% of all species present with 1278 species and 452 subspecies) (Davis, 1965-1986; Tutin et al., 1968-1980; Strid, 1986; Strid and Tan, 1991, 1997, 2002; Georgiou and Delipetrou, 2010).

The Northeast Aegean islands offer a unique ecosystem with significant "hotspots" for various plant diversification responses and endemism. This depends on the fact that Aegean Sea is an archipelago of continental islands placed on the conjunction of Europe, Asia, and Africa (Gogou et al., 2016). The available "ecological space" with "environmental heterogeneity" in addition with the "land-bridge system" with the continents determined a high floristic diversity 
and endemism (Kallimanis et al., 2011). Several botanical studies have been conducted on these islands (Christodoulakis, 1986, 1996; Panitsa et al., 1994, 2006; Panitsa and Tzanoudakis, 1998, 2001; Snogerup et al., 2001; Bazos, 2005). However, there is limited information regarding the medicinal plants and traditional plant remedies in this area.

Nowadays the use of plants and traditional medicine plays an important role for the discovery of new pharmacological agents. Ethnopharmacology represents a multidimensional approach, shaped by tradition and science that can improve our knowledge of plant use and local meaning of health and disease (World Health Organization [WHO], 2002). The aim of this study is threefold: to present a complete list of the medicinal plants used in traditional medicine in the islands of Northeast Aegean Sea, preserve information about their use and, lastly, highlight the use in traditional medicine of endemic and endangered plant species, in order to prevent their extinction.

\section{MATERIALS AND METHODS}

\section{Study Area}

The current study was conducted on the islands and islets of the northeast edge of the Aegean Sea and is dominated by the islands of Lemnos, Agios Efstratios, Lesvos, Chios, Psara, Oinousses, Samos, Ikaria, and Fournoi (Figure 1). The geographical coordinates of the islands are represented in Supplementary Table 4 . The region covers an area of $3835 \mathrm{~km}^{2}$ and has approximately 2500 vascular plant species.

The terrain of the islands is 33\% mountainous, 35\% hilly, and $32 \%$ flat. The surface area and the maximum elevation ranged from $40 \mathrm{~km}^{2}$ for Psara and Fourni to $1633 \mathrm{~km}^{2}$ for Lesvos and from 0 to $1433 \mathrm{~m}$ of height (Kerketea mt, Samos), respectively (Higgins and Higgins, 1996). The islands can be grouped in two phytogeographical zones among the 13 existing in Greece (Supplementary Figure 1); the zone of the Northern Aegean (NAe) with Lemnos and Agios Efstratios and the zone of the Eastern Aegean (EAe) with Lesvos, Psara, Chios, Oinousses, Samos, Ikaria and Fournoi, with specific climatic differences (Rauh, 1949). The palaeography and the geological events that separated these islands from Anatolian mainland, certain environmental parameters (temperature and humidity) and habitat diversity in well-defined fragmented areas, are among the causes of the high endemism, floral richness and diversification in quantity and quality of many secondary metabolites that characterize the pharmacological properties of many species of the study area (Panitsa et al., 2010).

\section{Methodology}

The information summarized in the present paper was compiled from 15 randomly selected villages from the islands of the surveyed area (Figure 1). The methodology followed in the field surveys is based on Fujita et al. (1995). The data was collected through semi-structured interviews performed with local people (Martin, 1995). After explaining the purpose of our research, a questionnaire in Greek was administered to the informants including questions about their age, education, and interest in traditional medicine. A total of 200 people was surveyed, 100 women and 100 men, with an average age of 40. The informants with knowledge about medicinal plants were questioned multiple times, and, during the interviews, local names of the plants, utilized parts, preparation methods and traditional cultivation techniques were recorded. The majority of these skillful informants were medical doctors, pharmacists, and farmers. All the results are summarized in Supplementary Table 1 . The data acquired for each plant includes family, botanical name, local name in Greek, locality, voucher number, parts used and their preparation, therapeutic effect and ailments treated. The plant families were listed in alphabetical order.

Field trips were conducted with the interviewees to collect specimens of the plants (with the exception of the endangered ones). A photographic archive of the observed species was created to help the identification. The specimens were collected and herborized by the department's herbarium with a specific voucher number. Furthermore, we examined the therapeutic effect and preparations of each plant based on the informants' feedback and on literature records.

For the analysis of the use of the medicinal plants against specific diseases, we used informant consensus factor (FIC), summarized in Supplementary Table 3 (Trotter and Logan, 1986; Heinrich, 2000). Moreover, the Use Value (UV) was calculated to demonstrate the relative importance of the species known locally, summarized in Supplementary Table 1. The plant species scientific names were verified according to the Plant List and to the International Plant Name Index. Information that was not possible to confirm was not recorded. Moreover, in Supplementary Table 1, we reported some of the most important bibliographic references for plant species and ailments treated that were not mentioned by the informants (Snogerup and Snogerup, 1987, 1993; Panitsa et al., 1994, 2006, 2010; Snogerup et al., 2001; Bergmeier et al., 2003; Bazos, 2005; BourbonnaisSpear et al., 2006; Saliaris, 2008; Axiotis and Axiotis, 2012; Strid, 2016).

\section{RESULTS AND DISCUSSION}

In the context of the current survey numerous medicinal plants were ethnopharmacologicaly investigated (in Supplementary Table 1). The interviews indicated that 109 wild plant species from 52 different families are being used for medicinal purposes. Lamiaceae family is represented by the highest number of species (17), such as the endemic and endangered Sideritis sipylea Boiss. Asteraceae family is represented by 12 species. Brassicaceae and Rosaceae by 5 species. The rest of the plant families are shown in Supplementary Figure 2. Most of the plant parts were used to treat different diseases and they were mostly stored in glass bottles as homemade dry powders obtained by crushing down well-dried plant materials; the most frequently used parts are leaves $(22,8 \%)$, roots $(12,78 \%)$, flowers $(11,41 \%)$, essential oils $(7,30 \%)$, fruits $(6,84 \%)$, and barks $(5 \%)$. The most common preparation is the decoction. 


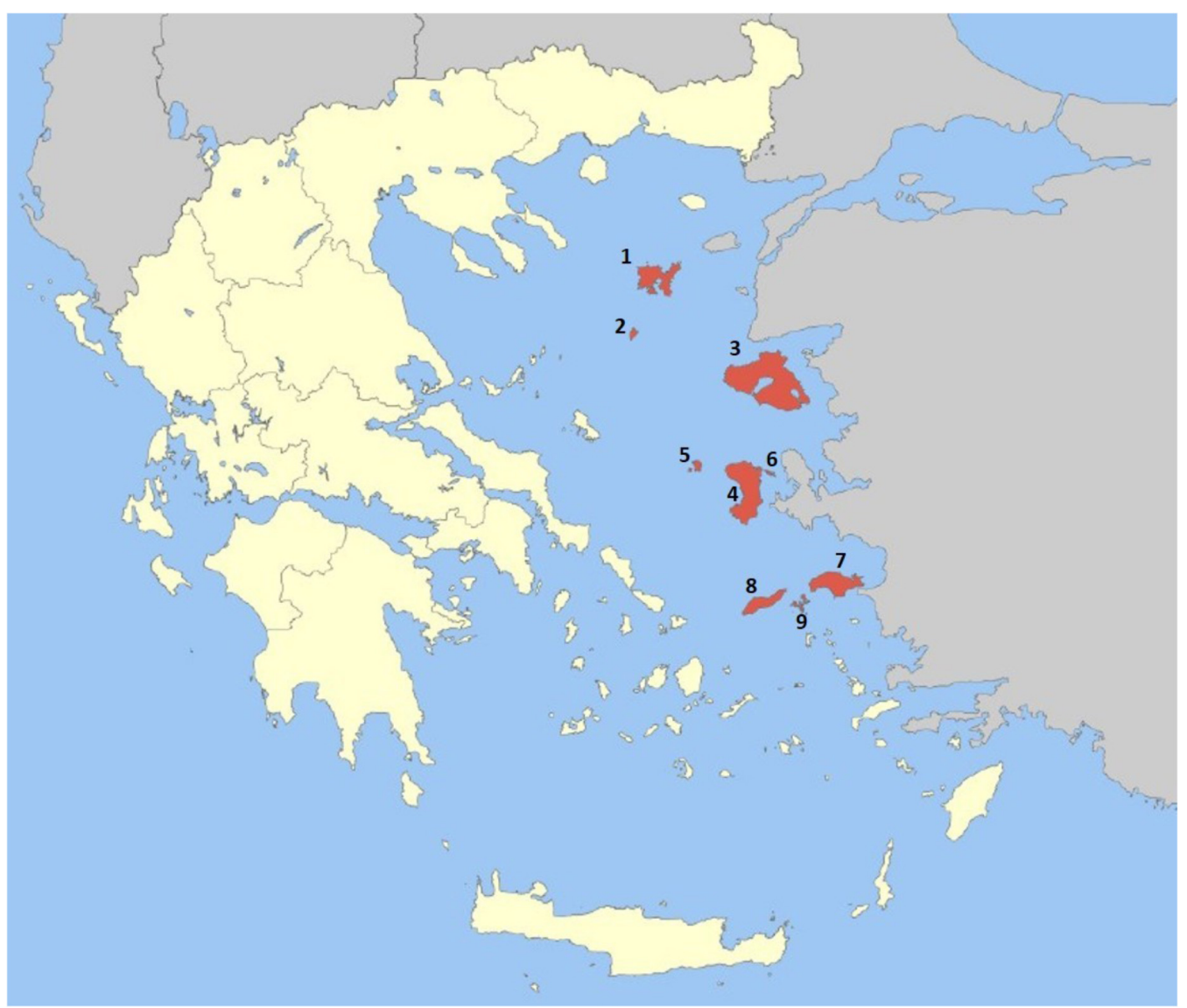

FIGURE 1 | Geographical location of the study area (Islands in red). Collection sites of the information are as follows. (1) Lemnos, (2) Agios Efstratios, (3) Lesvos, (4) Chios, (5) Psara, (6) Oinousses, (7) Samos, (8) Ikaria, (9) Fourni (http://www.pvaigaiou.gov.gr).

Interestingly, Hypericum perforatum L. and Verbascum ikaricum Murb. are kept in olive oil and used as solutions for wounds and sun burns. Pharmacists from Lesvos, Chios and Samos, still use these preparations as traditional therapy for skin wound healing in combination with modern treatments. Furthermore, clinicians from Lesvos use the powder of the root of Alkanna tinctoria for skin regeneration after injuries. They observed a combined antibacterial and antipruritic action, which reduces the healing time of the wound. It is important to note that Sideritis sipylea Boiss., Origanum sipyleum L., Thymus sipyleus Boiss., which are endemic and endangered plants are mostly used by the local people of Lesvos, Chios, and Ikaria with many curative purposes, especially for the infections of the respiratory and gastrointestinal tract as indicated from the UV values (Supplementary Table 1).

Furthermore, the resin of Pistacia lentiscus L., unique product globally of the Chios island is widely used against diarrhea, as expectorant and for ulcer healing. The use of the water extract of the roots of Anthyllis hermanniae L. as an efficient natural remedy against kidney stones in Lesvos and Lemnos, is reported here for the first time. It was also observed that local people, especially farmers, use the same name for different plant species. For example, Cistus creticus L. and Cistus salvifolius L. are both called "Aksistaros"; Origanum vulgare L. and the endemic and endangered Origanum sipyleum L. are both called "Rigani." This lack of distinction by the local population exacerbates the risks for the endangered species.

Very interesting is the distribution of ailments treated versus plant species used. As it is presented in Figure 2, locals are choosing herbal remedies mainly for antimicrobial activity, gastrointestinal disorders, respiratory, inflammatory, cardiovascular diseases, and skin burns. In Supplementary Table 3, FIC values of categories of ailments are summarized. Hormonal diseases have the highest FIC value $(0,722)$, while the lowest belongs to the cardiovascular diseases $(0,161)$. Moreover, among the species recorded (Supplementary Table 1), the highest UV were calculated for Matricaria chamomilla L. (0.75) and Lavandula stoechas L. (0.64). From the endemic species the highest UV is recorded for Sideritis sipylea Boiss. (0.31).

Essential oils are also common in the region, mainly derived from aromatic plants of Lamiaceae family and they are produced by water or steam distillation. The oils are usually used as drops directly on the skin or in galenic formulations, for skin infections or burns. In some cases, they are used for stomach pain (Anthemis sp.) or even for urinary infections (Sideritis sipylea Boiss.). The most common plants used for producing essential oils are Salvia 


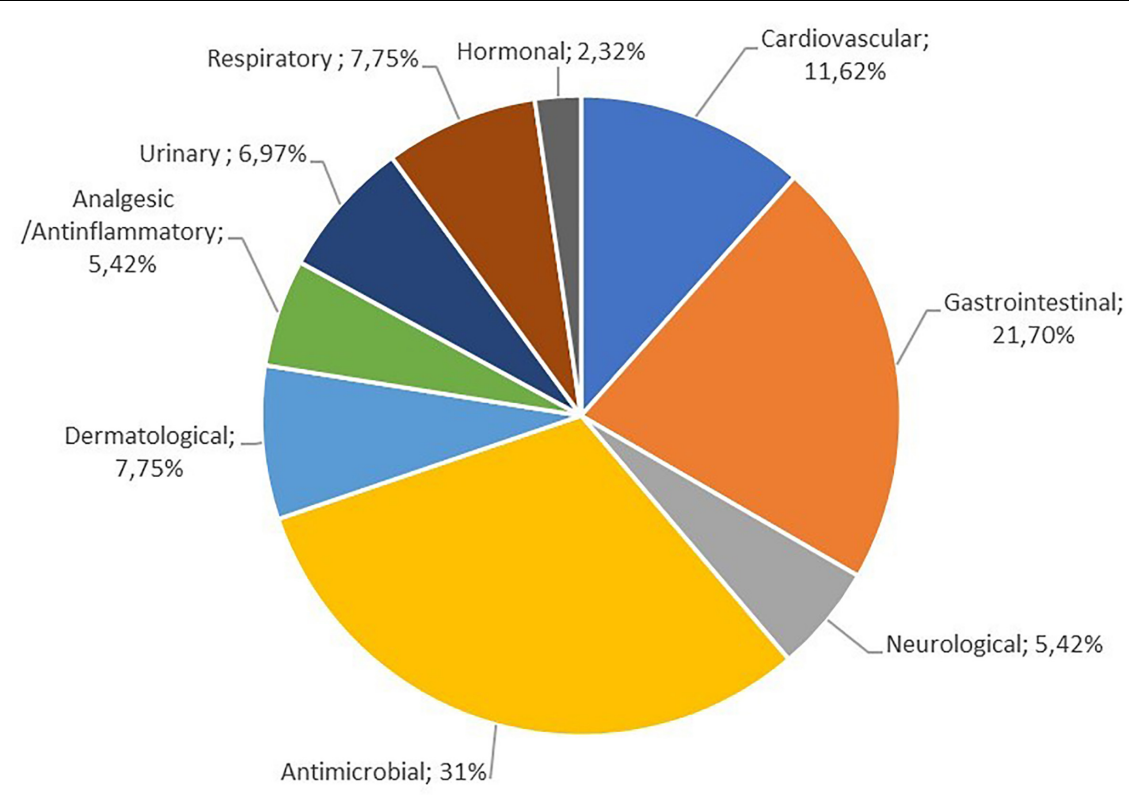

FIGURE 2 | Distribution of ailments treated versus plant species used.

fruticosa Miller, Salvia officinalis L., Origanum vulgare L., Sideritis sipylea Boiss., and Lavandula stoechas L. Furthermore, it has been reported a traditional method for the production of rose oil of the flower petals of Rosa damascena Mill. in a small village of Lesvos. The oil is used for external burns or skin inflammations.

The pharmacological properties of medicinal plants presented in the current study has been also verified by published data, in several cases. For example, the essential oil of Laurus nobilis L., used as anti-inflammatory is reported by Sayyah et al. (2003). The expectorant effect of Cistus creticus L. and the use of Mentha spicata L. in cases of common cold have been reported by Fakir et al. (2009). Tonic effects of Rosmarinus officinalis L. and the diuretic effects of Raphanus raphanistrum L. have been reported by Bruni et al. (1997). The essential oil of Sideritis species presents an important antimicrobial and anti-inflammatory activity and it has been previously reported by Aligiannis et al. (2001).

Generally, these plants are distributed mainly in Lesvos, Chios, Samos, and Ikaria and the majority are wild. A serious threat for some of these plants, is that they are collected in large amounts for the local markets; some of them, such as Sideritis sipylea Boiss., Origanum sipyleum L., Thymus sipyleus Boiss., are in fact listed in the book of the Red List of Threatened Species. Many species, such as Origanum vulgare L. and Paeonia mascula (L.) Mill. subsp. mascula are not yet listed but their population is in a critical condition. Endemic medicinal plants of the research area are also annotated in Supplementary Table 2.

A very important issue is the increased use of medicinal plants in these islands which has led to heavy pressure on the native populations of many species. The local population must acknowledge their endangerment and should cultivate them, in order to protect their populations from extinction. Sideritis sipylea Boiss. is one of the over harvested species. Habitat loss and deforestation in Lesvos, Chios, and Samos resulted in a general degradation of the ecosystems that could lead to the extinction of many species.

In conclusion, from this brief report is clear that medicinal plants are an important element of indigenous medical system of Northeast Aegean islands. In spite of the fact that the local population has access to modern medicines, many people continue to depend, at least for the treatment of some diseases, on herbal remedies. These therapies represent for many local doctors a low-cost alternative. The population of many endemic species such as Sideritis sipylea Boiss., Origanum sipyleum L., are in a critical condition due to unsustainable harvesting techniques, habitat destruction, and absence of cultivation. The endemic species of the area have medicinal properties dependent on the metabolites which respond to environmental stimuli that may be absent under culture conditions. Because of this it is very important to educate local people about respecting their natural habitats and strengthen the legislation against the illegal trade of endemic and endangered plants in the local and national market.

\section{AUTHOR CONTRIBUTIONS}

EA contributed to the study conception and design of the methodology. Moreover, he was responsible for the analysis and the interpretation of data. $\mathrm{MH}$ contributed substantially to supervising the work and drafting the manuscript. LS contributed to the critical and final revision of the manuscript.

\section{FUNDING}

This research received specific grant from North Aegean Region, Greece NSRF 2014-2020 (National Strategic Reference Framework). 


\section{ACKNOWLEDGMENTS}

The authors thank all the local people who shared their knowledge of plants and Dr. Makis Axiotis for the valuable information provided to the present study.

\section{REFERENCES}

Akin, M., Aktumsek, A., and Nostro, A. (2010). Antibacterial activity and composition of the essential oils of Eucalyptus camaldulensis Dehn. and Myrtus communis L., growing in Northern Cyprus. Afr. J. Biotechnol. 9, 531-535. doi: 10.5897/AJB09.1562

Aligiannis, N., Kalpoutzakis, E., Chinou, I. B., Mitakou, S., Gikas, E., and Tsabropoulos, A. (2001). Composition and antimicrobial activity of the essential oil of five taxa of Sideritis from Greece. J. Agric. Food Chem. 49, 811-815. doi: 10.1021/jf001018w

Aubert, P., Guinobert, I., Guilbot, A., and Neunlist, M. (2016). Antispasmodic and spasmolytic activity of Melissa officinalis EPS upon mice gastrointestinal tract: an ex vivo pilot study. Planta Med. 82, S1-S381. doi: 10.1055/s-00361596945

Axiotis, M., and Axiotis, E. (2012). Medicinal Plants of Greece. The Research in Lesvos Island. Athens: Endelexeia Editions.

Bahmani, M., Shirzad, H., Rafieian, S., and Rafieian-Kopaei, M. (2015). Silybum marianum: beyond hepatoprotection. J. Evid. Complementary Altern. Med. 20, 292-301. doi: 10.1177/2156587215571116

Bazos, I. (2005). Study of the Flora and Vegetation of Lesvos. Ph.D. thesis, University of Athens, Athens.

Bergmeier, E., Dimopoulos, F., and Dimopoulos, P. (2003). The vegetation of islets in the Aegean and the relation between the occurrence of islet specialists, island size and grazing. Phytoenologia 33, 447-474. doi: 10.1127/0340-269X/2003/ 0033-0447

Bourbonnais-Spear, N., Poissant, J., Cal, V., and Arnason, J. (2006). Culturally important plants from southern Belize: domestication by Q'eqchi' Maya healers and conservation. Ambio 35, 138-140. doi: 10.1579/0044-7447(2006)35[138: CIPFSB]2.0.CO;2

Bruni, A., Ballero, M., and Polli, F. (1997). Quantitative ethnopharmacological study of the Campidano Valley and Urzulei district, Sardinia, Italy. J. Ethnopharmacol. 57, 97-124. doi: 10.1016/S0378-8741(97)00 055-X

Cayci, M. K., and Dayioglu, H. (2009). Hypericum perforatum extracts healed gastric lesions induced by hypothermic restraint stress in Wistar rats. Saudi Med. J. 30, 750-754.

Christodoulakis, D. (1986). Flora and Vegetation of Samos. Ph.D. thesis, University of Patras, Patras.

Christodoulakis, D. (1996). The flora of Ikaria (Greece, E. Aegean Islands). Phyton $36,63-91$.

Dadalioglu, I., and Evrendilek, G. A. (2004). Chemical compositions and antibacterial effects of essential oils of Turkish oregano (Origanum minutiflorum), bay laurel (Laurus nobilis), Spanish lavender (Lavandula stoechas L.), and fennel (Foeniculum vulgare) on common foodborne pathogens. J. Agric. Food Chem. 52, 8255-8260. doi: 10.1021/jf049033e

Davis, P. H. (1965-1986). Flora of Turkey and the East Aegean Islands, Vol. 10. Edinburgh: Edinburgh University Press.

Fakir, H., Korkmaz, M., and Güller, B. (2009). Medicinal plant diversity of Western Mediterranean region in Turkey. J. Appl. Biol. Sci. 3, 30-40.

Friedman, M. (2014). Chemistry and multibeneficial bioactivities of carvacrol (4isopropyl-2-methylphenol), a component of essential oils produced by aromatic plants and spices. J. Agric. Food Chem. 62, 7652-7670. doi: 10.1021/jf50 23862

Fujita, T., Sezik, E., Tabata, M., Yesilada, E., Honda, G., Takeda, Y., et al. (1995). Traditional medicine in Turkey vii. Folk medicine in middle and west Black Sea regions. Econ. Bot. 49, 406-422. doi: 10.1007/BF02863092

Gambarana, C., Tolu, P. L., Masi, F., Rinaldi, M., Giachetti, D., Morazzoni, P., et al. (2001). A study of antidepressant activity of Hypericum perforatum on animal models. Pharmacopsychiatry 34, S42-S44. doi: 10.1055/s-200115515

\section{SUPPLEMENTARY MATERIAL}

The Supplementary Material for this article can be found online at: https://www.frontiersin.org/articles/10.3389/fphar. 2018.00409/full\#supplementary-material

Gasparetto, J. C., Martins, C. A., Hayashi, S. S., Otuky, M. F., and Pontarolo, R. (2011). Ethnobotanical and scientific aspects of Malva sylvestris L.: a millennial herbal medicine. J. Pharm. Pharmacol. 64, 172-189. doi: 10.111/j.2042-7158. 2011.01383.x

Georgiou, K., and Delipetrou, P. (2010). Patterns and traits of the endemic plants of Greece. Bot. J. Linn. Soc. 162, 130-422. doi: 10.111/j.1095-8339.2010.01025.x

Gogou, A., Triantaphyllou, M., Xoplaki, E., Izdebski, A., Parinos, C., Dimiza, M., et al. (2016). Climate variability and socio-environmental changes in the northern Aegean (NE Mediterranean) during the last 1500 years. Quat. Sci. Rev. 136, 209-228. doi: 10.1016/j.quascirev.2016. 01.009

Heinrich, M. (2000). Ethnobotany and its role in drug development. Phytother. Res. 14, 479-488. doi: 10.1002/1099-1573(200011)14:7<479::AID-PTR958>3. $0 . \mathrm{CO} ; 2-2$

Hentschel, C., Dressler, S., and Hahn, E. G. (1995). Fumaria officinalis (fumitory) Clinical applications. Fortschr. Med. 113, 291-292.

Higgins, M. D., and Higgins, R. (1996). A Geological Companion to Greece and the Aegean. Ithaca, NY: Cornell University Press.

Kallimanis, A. S., Panitsa, M., Bergmeier, E., and Dimopoulos, P. (2011). Examining the relationship between total species richness and single island palaeo- and neo-endemics. Acta Ecol. Int. J. Ecol. 37, 65-70. doi: 10.1016/j.actao.2010. 11.008

Khan, S., and Sharma, N. R. (2016). Antifungal potential of ethanol extracts of Allium sativum and Allium ampeloprasum. Asian J. Pharm. Clin. Res. 10, 1-4.

Koksal, E., Bursal, E., Dikici, E., Tozoglu, F., and Gulcin, I. (2011). Antioxidant activity of Melissa officinalis leaves. J. Med. Plants Res. 5, 217-222.

Lattanzio, F., Greco, E., Carretta, D., Cervellati, R., Govoni, P., and Speroni, E. (2011). In vivo anti-inflammatory effect of Rosa canina L. extract. J. Ethnopharmacol. 137, 880-885. doi: 10.1016/j.jep.2011.07.006

Lone, B. A., Chishti, M. Z., Bhat, F. A., Tak, H., Bandh, S. A., and Khan, A. (2017). Evaluation of anthelmintic, antimicrobial and antioxidant activity of Chenopodium album. Trop. Anim. Health Prod. 49, 1597-1605. doi: 10.1007/ s11250-017-1364-y

Longaray Delamare, A. P., Moschen-Pistorello, I. T., Artico, L., Atti-Serafini, L., and Echeverrigaray, S. (2007). Antibacterial activity of the essential oils of Salvia Officinalis L. and Salvia triloba L., cultivated in South Brazil. Food Chem. 100, 603-608. doi: 10.1016/j.foodchem.2005.09.078

Martin, G. J. (1995). Ethnobotany: A Methods Manual. London: Chapman and Hall.

Meral, G. E., Konyalioglu, S., and Ozturk, B. (2002). Essential oil composition and antioxidant activity of endemic Ziziphora taurica subsp. cleonioides. Fitoterapia 716-718. doi: 10.1016/S0367-326X(02)00244-7

Mirzaei, A., Delaviz, H., Mirzaei, M., and Tolooei, M. (2015). The effects of Medicago sativa and Allium porrum on iron overload in rats. Glob. J. Health Sci. 7, 137-142. doi: 10.5539/gjhs.v7n7p137

Panitsa, M., Dimopoulos, P., Iatrou, G., and Tzanoudakis, D. (1994). Contribution to the study of the Greek flora: flora and vegetation of the Enousses (Oinousses) islands (E. Aegean area). Flora 189, 69-78. doi: 10.1016/S0367-2530(17) 30570-4

Panitsa, M., Trigas, P., Iatrou, G., and Sfenthourakis, S. (2010). Factors affecting plant species richness and endemism on land-bridge islands - An example from the East Aegean archipelago. Acta Oecologica 36, 431-437. doi: 10.1016/j.actao. 2010.04.004

Panitsa, M., and Tzanoudakis, D. (1998). Contribution to the study of the Greek flora: flora and vegetation of the E. Aegean islands Agathonisi and Pharmakonisi. Willdenowia 28, 95-116. doi: 10.3372/wi.28.2809

Panitsa, M., and Tzanoudakis, D. (2001). A floristic investigation of the islet groups Arki and Lipsi (East Aegean area, Greece). Folia Geobot. 36, 265-279. doi 10.1007/BF02803180

Panitsa, M., Tzanoudakis, D., Triantis, K. A., and Sfenthourakis, S. (2006). Patterns of species richness on very small islands: the plants of the 
Aegean archipelago. J. Biogeogr. 33, 1223-1234. doi: 10.1111/j.1365-2699.2006. 01481.x

Rauh, W. (1949). Klimatologie und Vegetations verhältnisse der Athos-Halbinsel und der ostägeischen Inseln Lemnos, Hag. Evstratios, Mytilene und Chios. Heidelberg: Heidelberger, 511-615.

Saliaris, P. (2008). Natura Chia. A Journey to the Flora and Fauna of Chios island. Chios: Egeas Editions.

Sayyah, M., Saroukhani, G., Peirovi, A., and Kamalinejad, M. (2003). Analgesic and antinflammatory activity of the leaf essential oil of Laurus nobilis Linn. Phytother. Res. 17, 733-736. doi: 10.1002/ptr.1197

Shara, M., and Stohs, S. J. (2015). Efficacy and safety of white willow bark (Salix alba) extracts. Phytother. Res. 29, 1112-1116. doi: 10.1002/ptr.5377

Singh, J., Dubeyd, A. K., and Tripathi, N. N. (1994). Antifungal activity of Mentha spicata. Pharm. Biol. 32, 314-319. doi: 10.3109/13880209409083009

Snogerup, S., and Snogerup, B. (1987). Repeated floristical observations on islets in the Aegean. Plant Syst. Evol. 155, 143-164. doi: 10.1007/BF00936296

Snogerup, S., and Snogerup, B. (1993). Additions to the flora of Samos, Greece. Flora Medit. 3, 211-222.

Snogerup, S., Snogerup, B., Phitos, D., and Kamari, G. (2001). The flora of Chios island (Greece). Bot. Chron. 14, 5-197.

Soni, P., Siddiqui, A. A., and Soni, V. (2012). Pharmacological properties of Datura stramonium L. as a potential medicinal tree: an overview. Asian Pac. J. Trop. Biomed. 2, 1002-1008. doi: 10.1016/S2221-1691(13)60014-3

Strid, A. (1986). Mountain Flora of Greece, Vol. 1. Cambridge: Cambridge University Press.

Strid, A. (2016). Atlas of the Aegean Flora. Part 1: Text \& plates. Part 2: Maps. Berlin: Botanic Garden and Botanical Museum Berlin.

Strid, A., and Tan, K. (1991). Mountain Flora of Greece, Vol. 2. Edinburgh: Edinburgh University Press.

Strid, A., and Tan, K. (1997). Flora Hellenica 1. Königstein: Koeltz Scientific Books. Strid, A., and Tan, K. (2002). Flora Hellenica 2. Ruggell: Koeltz Scientific Books.

Süntar, I., Koca, U., Keleş, H., and Akkol, E. K. (2011). Wound healing activity of Rubus sanctus Schreber (Rosaceae): preclinical study in animal models," Evid. Based Complement. Alternat. Med. 2011:816156. doi: 10.1093/ecam/nep137

Tapondjou, A. L., Adler, C., Fontem, D. A., Bouda, H., and Reichmuth, C. (2005). Bioactivities of cymol and essential oils of Cupressus sempervirens and Eucalyptus saligna against Sitophilus zeamais Motschulsky and Tribolium confusum du Val. J. Stored Prod. Res. 41, 91-102. doi: 10.1016/j.jspr.2004. 01.004

Tomczyk, M., and Latté, K. P. (2009). Potentilla - A review of its phytochemical and pharmacological profile. J. Ethnopharmacol. 122, 184-204. doi: 10.1016/j. jep.2008.12.022
Trotter, R. T., and Logan, M. H. (1986). "Informant consensus: a new approach for identifying potentially effective medicinal plants," in Plants in Indigenous Medicine and Diet, Behavioural Approaches, ed. N. L. Etkin (New York, NY: Redgrave Publishing Company).

Tunalier, Z., Kosar, M., Ozturk, N., and Baser, H. C. (2004). Antioxidant properties and phenolic composition of Sideritis species. Chem. Nat. Compd. 40, 206-210. doi: 10.1023/B:CONC.0000039124.83109.ac

Tutin, T. G., Heywood, V. H., Burges, N. A., Moore, D. M., Valentine, D. H., Walters, S. M., et al. (1968-1980). Flora Europaea, Vol. 2. Cambridge: Cambridge University Press.

Wang, Y., Xiang, L., Wang, C., Tang, C., and He, X. (2013). Antidiabetic and antioxidant effects and phytochemicals of mulberry fruit (Morus alba L.) polyphenol enhanced extract. PLoS One 8:e71144. doi: 10.1371/journal.pone. 0071144

Wittschier, N., Faller, G., and Hensel, A. (2009). Aqueous extracts and polysaccharides from liquorice roots (Glycyrrhiza glabra L.) inhibit adhesion of Helicobacter pylori to human gastric mucosa. J. Ethnopharmacol. 125, 218-223. doi: 10.1016/j.jep.2009.07.009

Woelk, H., Burkard, G., and Grinwald, J. (1994). Benefits and risks of the hypericum extract LI 160: drug monitoring study with 3250 patients. J. Geriatr. Psychiatry Neurol. 4(Suppl.1), S34-S38. doi: 10.1177/089198879400700110

World Health Organization [WHO] (2002). WHO Traditional Medicinal Strategy 2002-2005. Geneva: World Health Organization.

Yayalacı, Y., Celik, I., and Bat , B. (2014). Hepatoprotective and Antioxidant activity of linden (Tilia platyphyllos L.) infusion against ethanol-induced oxidative stress in rats. J. Membr. Biol. 247, 181-188. doi: 10.1007/s00232-0139622-Z

Yildirim, A., Mavi, A., and Kara, A. A. (2001). Determination of antioxidant and antimicrobial activities of Rumex crispus L. extracts. J. Agric. Food Chem. 49, 4083-4089. doi: 10.1021/jf0103572

Conflict of Interest Statement: The authors declare that the research was conducted in the absence of any commercial or financial relationships that could be construed as a potential conflict of interest.

Copyright (c) 2018 Axiotis, Halabalaki and Skaltsounis. This is an open-access article distributed under the terms of the Creative Commons Attribution License (CC BY). The use, distribution or reproduction in other forums is permitted, provided the original author(s) and the copyright owner are credited and that the original publication in this journal is cited, in accordance with accepted academic practice. No use, distribution or reproduction is permitted which does not comply with these terms. 


\title{
Lycopene and Vascular Health
}

\author{
Ioana Mozos ${ }^{1,2 \star}$, Dana Stoian ${ }^{3}$, Alexandru Caraba ${ }^{4}$, Clemens Malainer $^{5}$, \\ Jarosław O. Horbańczuk ${ }^{6}$ and Atanas G. Atanasov ${ }^{6,7 *}$
}

${ }^{1}$ Department of Functional Sciences, "Victor Babes" University of Medicine and Pharmacy, Timișoara, Romania, ${ }^{2}$ Center for Translational Research and Systems Medicine, "Victor Babes" University of Medicine and Pharmacy, Timișoara, Romania, ${ }^{3}$ 2nd Department of Internal Medicine, "Victor Babes" University of Medicine and Pharmacy, Timișoara, Romania, ${ }^{4} 1$ st Department of Internal Medicine, "Victor Babes" University of Medicine and Pharmacy, Timișoara, Romania, ${ }^{5}$ Independent Researcher, Vienna, Austria, ${ }^{6}$ Institute of Genetics and Animal Breeding, Polish Academy of Sciences, Magdalenka, Poland, ${ }^{7}$ Department of Pharmacognosy, Faculty of Life Sciences, University of Vienna, Vienna, Austria

\section{OPEN ACCESS}

Edited by:

Thomas Efferth,

Johannes Gutenberg-Universität

Mainz, Germany

Reviewed by:

Ali Hussein Eid,

American University of Beirut,

Lebanon

Miriam Ferrer,

Cambridge Nutraceuticals,

United Kingdom

*Correspondence:

Ioana Mozos

ioana_mozos@yahoo.com

Atanas G. Atanasov

a.atanasov.mailbox@gmail.com

Specialty section:

This article was submitted to

Ethnopharmacology,

a section of the journal

Frontiers in Pharmacology

Received: 20 September 2017

Accepted: 30 April 2018

Published: 23 May 2018

Citation:

Mozos I, Stoian D, Caraba A,

Malainer C, Horbańczuk JO and Atanasov AG (2018) Lycopene and

Vascular Health

Front. Pharmacol. 9:521.

doi: 10.3389/fphar.2018.00521
Lycopene is a lipophilic, unsaturated carotenoid, found in red-colored fruits and vegetables, including tomatoes, watermelon, papaya, red grapefruits, and guava. The present work provides an up to date overview of mechanisms linking lycopene in the human diet and vascular changes, considering epidemiological data, clinical studies, and experimental data. Lycopene may improve vascular function and contributes to the primary and secondary prevention of cardiovascular disorders. The main activity profile of lycopene includes antiatherosclerotic, antioxidant, anti-inflammatory, antihypertensive, antiplatelet, anti-apoptotic, and protective endothelial effects, the ability to improve the metabolic profile, and reduce arterial stiffness. In this context, lycopene has been shown in numerous studies to exert a favorable effect in patients with subclinical atherosclerosis, metabolic syndrome, hypertension, peripheral vascular disease, stroke and several other cardiovascular disorders, although the obtained results are sometimes inconsistent, which warrants further studies focusing on its bioactivity.

Keywords: lycopene, arterial stiffness, endothelial function, intima-media thickness, cardiovascular risk

\section{INTRODUCTION}

Cardiovascular disorders (CVD) are the leading mortality cause worldwide and prophylactic measures to combat it deserve special attention. Atherosclerosis is, in most cases, the main manifestation of CVD, and its progression may be clinical silent for a long time, up to a certain moment, when it directly leads into a severe adverse event (e.g., heart attack or stroke). Beneficial lifestyle changes are important and most cost-effective components of prevention or treatment of cardiovascular disorders. Compounds occurring in plants often display diverse bioactivities with therapeutic potential and different plants and plant-derived compounds have a long history of being reported to exhibit effects counteracting CVD (Atanasov et al., 2015; Waltenberger et al., 2016). Among lifestyle factors, a healthy diet is considered a cornerstone of cardiovascular disease prevention, and the inclusion of sufficient fruits and vegetables in the diet is regarded as especially important (Böhm, 2012; Piepoli et al., 2016). The prevalence of cardiovascular disorders is remarkably unevenly distributed in developed countries, and some areas, e.g., Southern Europe, seem to be protected by having significantly less prevalence of the disease. This effect has often been attributed to dietary factors, as e.g., the Mediterranean diet, with a lot of vegetables, including tomatoes, tomato products, and olive oil (Müller-Nordhorn et al., 2008; Krasinska et al., 2017). Tomatoes, tomato sauce, and watermelon are important sources of lycopene and may be a surrogate 
for the Mediterranean diet to some degree (Sesso et al., 2003; Burton-Freeman and Sesso, 2014; Naz et al., 2014).

Dietary lycopene is considered to confer cardiovascular benefits, as e.g., consuming at least 7 servings/week of lycopene-based products significantly decreased cardiovascular risk within seven years in postmenopausal women, free from prior cardiovascular disorders and cancer (Sesso et al., 2003). A negative correlation between serum lycopene concentration and cardiovascular mortality was also found in a follow-up study with a large Japanese cohort (Ito et al., 2006). Many studies about the relationship between lycopene and cardiovascular risk have been conducted and, although, some of the results are inconsistent, overall dietary lycopene intake and highserum concentration of lycopene, significantly reduced the risk of major cardiovascular events (Cheng et al., 2017; Song et al., 2017). Not all outcomes were positive, as exemplified in the Physicians Health Study, including 499 patients with cardiovascular disorders, not revealing any association of higher plasma lycopene and CVD (Müller et al., 2016). Within the Kuopio Ischaemic Heart Disease Risk Factor (KIHD), no relationship was reported between low serum lycopene and an increased CVD mortality and sudden cardiac death, respectively, in Finnish, middle-aged men (Karppi et al., 2012; Müller et al., 2016).

Vascular health depends on endothelial function, arterial stiffness, and the presence of atherosclerotic plaques. The following section will discuss the different methods to measure vascular health. Endothelial dysfunction enables development of the atherosclerotic plaque. The flow mediated dilatation of the brachial artery was traditionally used to assess endothelial function for a long period, but forearm plethysmography and reactive hyperemia-peripheral arterial tonometry have become the gold standards for assessing endothelial vascular function (Kim et al., 2011; Gajendragadkar et al., 2014). While hyperemia-peripheral arterial tonometry is less operatordependent and non-invasive, while being as reliable as the traditional method, forearm plethysmography additionally provides mechanistic information related to nitric oxide synthesis, is a marker for cardiovascular risk, and can improve risk prediction (Kim et al., 2011; Gajendragadkar et al., 2014).

An increased arterial stiffness is one of the first structural and functional changes of the vessel wall and is mainly caused by arteriosclerosis, atherosclerosis and vessel wall calcification (Cavalcante et al., 2011). Arterial stiffness is associated with cardiovascular risk and predicts cardiovascular disorders and mortality (Vlachopoulos et al., 2010; Mozos et al., 2017c). Pulse wave velocity (PWV) and augmentation indices are simple, noninvasive, inexpensive and validated methods used to assess arterial stiffness and as screening methods for the detection of pre-clinical cardiovascular disorders (Vlachopoulos et al., 2010; Mozos et al., 2017a).

Carotid intima-media thickness (IMT) assessed non-invasively by B-mode ultrasonography, is a simple, validated and safe method used to measure the extent of subclinical atherosclerosis (Dwyer et al., 2004; Wood and Johnson, 2004; Hosseini et al., 2017; Kim and Youn, 2017). IMT is defined by the measurement of the dimension of the intima and media of the arterial wall, whereas a value $>0.9 \mathrm{~mm}$ is considered abnormal (Cooney et al., 2015; Piepoli et al., 2016). Although IMT has been considered as a surrogate measure of cardiovascular risk, predicting cardiovascular events, especially myocardial infarction and stroke (O'Leary et al., 1999; Touboul, 2015; Piepoli et al., 2016; Hosseini et al., 2017; Pleskovic et al., 2017), both the American and the European guidelines on cardiovascular disease prevention do not recommend the systematic measurement of IMT to improve risk assessment (Goff et al., 2014; Piepoli et al., 2016). Major concerns of IMT include lack of standardization, its high variability and low intra-individual reproducibility (Piepoli et al., 2016). Additionally, a trained sonographer is required to limit variability of the results due to the patient or medical equipment (Touboul, 2015). There are also methods available that can better predict cardiovascular events than IMT, most prominently MRI (magnetic resonance imaging) that can be used to monitor atherosclerotic plaques. Several methods are stronger predictor of myocardial infarction than IMT, such as monitoring atherosclerotic plaques and exploring the carotid wall by MRI (because the adventitia is also included in the measurement of the wall thickness; Zhang et al., 2014). However, the resources needed to access wall thickness by MRI are manifold higher than using ultrasonography, but especially for patients with abnormal findings in IMT screenings, a subsequent MRI scan might be useful to provide a more accurate description of the atherosclerotic plaque and the risk of major cardiovascular events (Zhang et al., 2014).

Despite the inevitable influence of aging, vascular changes are at least partially reversible, and dietary changes may improve vascular function. Lycopene has several advantages, such as almost no adverse reactions, its wide availability and low cost (Gao et al., 2016). The present review aims to give a current overview of the mechanisms linking lycopene in the human diet and vascular changes, considering epidemiological data, clinical observational, retrospective, intervention and randomized studies, dietary and biomarker studies, in vitro and in vivo research and discussing preventive benefits of lycopene intake in context of cardiovascular disease prevention.

\section{LYCOPENE}

Lycopene is a lipophilic, biologically active, unsaturated, acyclic carotenoid, with the chemical formula $\mathrm{C}_{40} \mathrm{H}_{56}$ (Figure 1). In plants it is considered as an important intermediate of carotenoid synthesis, but in human nutrition lycopene plays no role as provitamin A precursor due to the absence of appropriate enzymes. Plant lycopene is usually present as all-trans isomer. Isomerization of the all-trans isomer into the more bioavailable cis-isomer, occurs under acidic conditions (e.g., gastric acid), due to exposure to light and thermal energy. Lycopene can be found in tomatoes, watermelon, papaya, red grapefruits, apricots, and guava, and gives their red color (Kong et al., 2010; Gajendragadkar et al., 2014; Mozos et al., 2017c). Lycopene content increases during different stages of ripening of fruits, e.g., for tomatoes there is a steady increase in lycopene content from the breaker to the red stage (Saini et al., 2017). Watermelon 
pulp can also be used for lycopene extraction (Oberoi and Soqi, 2017) and is a rich source of cis-isomeric lycopene, abundant in higher concentrations than in tomatoes (Naz et al., 2014). Interestingly also the fungal plant pathogen Blakeslea trispora has been recognized as a commercial source to produce lycopene (Mantzouridou and Tsimidou, 2008). Several foods high in lycopene content are classified as functional foods (Naz et al., 2014). Tomato juice, paste, puree, ketchup, sauce or soup represent lycopene sources with improved bioavailability due to thermal treatment, but also because processing releases lycopene from the fibrous cell structure matrix (Basu and Imrhan, 2007; Thies et al., 2012; Burton-Freeman and Sesso, 2014).

Not only lycopene intake counts, but also its serum concentration may influence cardiovascular risk. Low serum and adipose tissue lycopene levels were correlated with early atherosclerosis, and major acute coronary and cerebrovascular events, and were found to be more reliable in risk assessment than the daily intake of lycopene (Agarwal and Rao, 2000; Rissanen et al., 2001; Kim et al., 2011). Oxidative stress and inflammation are responsible for a reduced level of antioxidants (Kim et al., 2010). Smoking is a potent oxidative stressor, able to impair arterial elasticity and endothelial function (Kim et al., 2010; Mozos et al., 2017b), and lycopene was the only major serum carotenoid able to reduce the atherosclerotic risk in current and former smokers according to the Rotterdam Study (KlipsteinGrobusch et al., 2000).

\section{CARDIOVASCULAR PROTECTIVE MECHANISMS}

Lycopene has several cardiovascular beneficial effects, such as an antioxidative, antiinflammatory, anti-atherogenic, cardioprotective, and antiplatelet effect, improving endothelial function (nitric oxide bioavailability and blood flow), the metabolic profile (by impairing cholesterol synthesis) and blood pressure control (Figure 2) (Klipstein-Grobusch et al., 2000; Heber and Lu, 2002; Ahuja et al., 2006; Basu and Imrhan, 2007; Verghese et al., 2008; Kim et al., 2010; Kong et al., 2010; Riccioni et al., 2010; Ried and Fakler, 2011; Böhm, 2012; Wolak and Paran, 2013; Gajendragadkar et al., 2014; Naz et al., 2014; Müller et al., 2016; Cheng et al., 2017; Milani et al., 2017).

\section{Antioxidative and Protective Endothelial Effects}

Lycopene is considered an effective singlet oxygen quencher in the carotenoids group (Kong et al., 2010; Viuda-Martos et al., 2014). It is a much more potent antioxidant than alphatocopherol $(10 \times$ more potent) or beta-carotene (twice as potent) (Kim et al., 2010; Kong et al., 2010). Lycopene modulates also the production of antioxidant enzymes, such as superoxide dismutase and catalase (Böhm, 2012; Pereira et al., 2017). Lycopene can also scavenge peroxynitrite, resulting oxidized lycopene products (Pisoschi and Pop, 2015).

Oxidative stress causes endothelial dysfunction due to uncoupling of the nitric oxide synthase and oxidative injury of the endothelial cells (Mozos and Luca, 2017). Both are associated with inflammation. By reducing oxidative stress and reactive oxygen species, lycopene increases the bioavailability of nitric oxide (NO), improves endothelium-dependent vasodilation and reduces protein, lipids, DNA, and mitochondrial damage (Hollman et al., 2011; Naz et al., 2014; Nakamura et al., 2017; Abdel-Daim et al., 2018).

Endothelial NO enables vasodilation, inhibits platelet functions, and adhesion and transmigration of white blood cells, and reduces smooth muscle cell proliferation (Opatrilova et al., 2017). Watermelon supplementation, due to L-citrulline content, increases plasma L-arginine, enabling NO production (Figueroa et al., 2017), because NO is synthesized from L-arginine by NO synthase in virtually all cell types (Jobgen et al., 2006). Lycopene supplementation improved endothelial mediated vasodilation in cardiovascular disease patients, but not in healthy controls (Gajendragadkar et al., 2014), suggesting the importance of lycopene in secondary cardiovascular prevention (Costa-Rodrigues et al., 2018).

In summary, lycopene scavenges both reactive oxygen and nitrogen species, increases the production of antioxidant enzymes and protects the endothelial cells from oxidative damage.

\section{Anti-inflammatory Effect}

Inflammation is related to atherosclerosis, arterial stiffness, and major cardiovascular events. The anti-inflammatory role of lycopene was demonstrated by several studies (Hung et al., 2008; Kim et al., 2010; Xu et al., 2012; He et al., 2016). Hung et al. revealed that lycopene can inhibit TNF-alpha induced NF-kappa $B$ activation, expression of intracellular adhesion molecule-1 (ICAM-1), and interaction between monocytes and endothelial cells, which might explain the cardiovascular benefits of lycopene (Hung et al., 2008). In a different study with Korean women, lycopene levels were found to correlate with cytokines, but no correlation with acute phase reactants was found, probably due to lycopene's inhibitory effect on the formation of oxidized LDL (Kim et al., 2010). Xu et al. found an inverse association of lycopene with vascular cell adhesion protein 1 (VCAM-1), which enable adhesion of monocytes to the endothelial cells, but could not verify any association between serum lycopene concentration and atherosclerosis in their study as suggested by earlier studies (Xu et al., 2012). In the same context, Gianetti et al. reported no significant correlations between plasma lycopene and soluble adhesion molecules (Gianetti et al., 2002). Lycopene obtained from red guava exerts several anti-inflammatory effects besides modulation of inflammatory mediators, such as inhibition of leukocyte mobilization, stabilization of mast cells, and inhibition of genes which expression is involved in inflammation (Vasconcelos et al., 2017).

Lycopene can also reduce the secretion of metalloproteinases by macrophages and inhibit $\mathrm{T}$ lymphocyte activation (Thies et al., 2017). Recently, lycopene was found as an effective antiglycation agent, able to reduce the synthesis of advanced glycation end-products (AGE), downregulating the expression of their receptors (RAGE), which further contributes to vessel protection (Tabrez et al., 2015; Thies et al., 2017). 


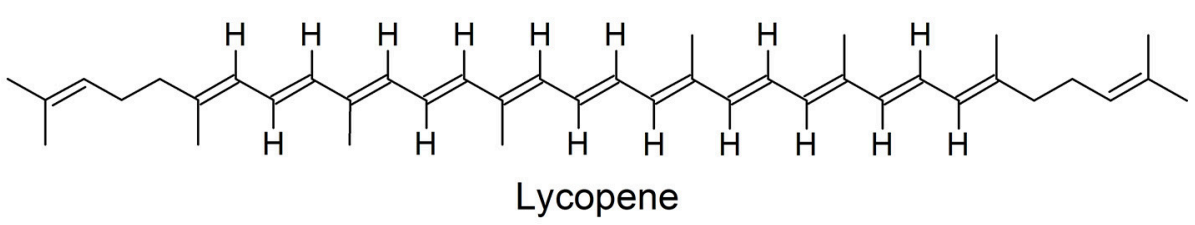

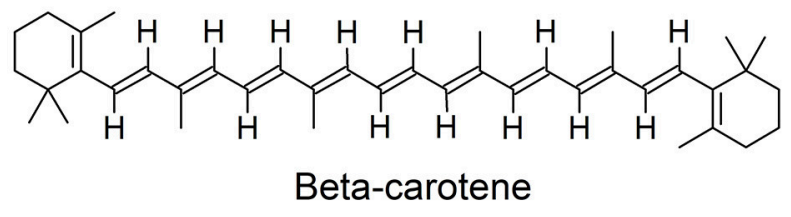

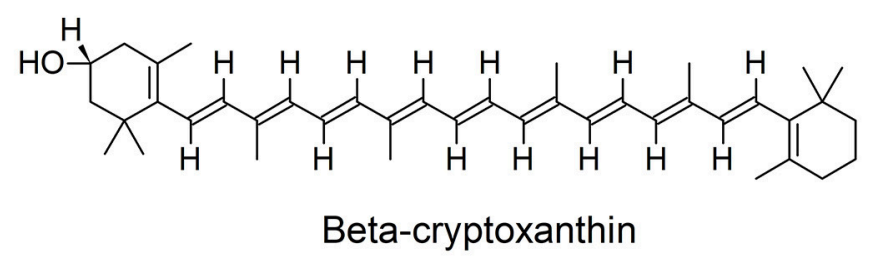<smiles>C/C=C/C=C/C(C)=C/C=C/C=C/C=C/C=C/C=C(C)/C=C/C1C(C)=CC(O)CC1(C)C</smiles>

Lutein

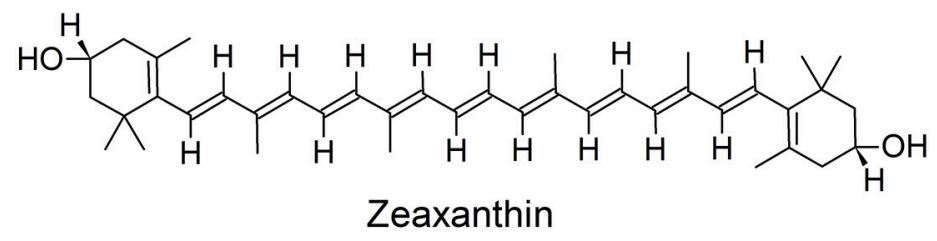

FIGURE 1 | Chemical structures of lycopene and other carotenoids.

Tomato products reduced oxidative stress related to postprandial lipemia and the associated inflammatory reaction in a study including normal weight participants (BurtonFreeman et al., 2012). He et al. reported the benefits of lycopene in preventing transplant vasculopathy, demonstrating that intimal hyperplasia and smooth muscle cell proliferation were reduced by the administration of lycopene and the infiltration of inflammatory cells in allograft vessels was reduced in an animal model (He et al., 2016). Lycopene can ameliorate allograft atherosclerosis via downregulating Rho-associated kinases and regulating the expression of key factors through $\mathrm{NO} / \mathrm{cGMP}$ pathways (He et al., 2016). On the other hand, the benefits of the tomato-rich diet were not directly related to the antiinflammatory effect according to a randomized study including 103 apparently healthy volunteers, after $300 \mathrm{~g}$ tomatoes daily for 1 month or placebo (Blum et al., 2007).
Watermelon was shown to reduce levels of inflammation by downregulation of the proinflammatory mediator cyclooxygenase 2 (COX-2), impairing prostaglandin E2 and I2 production, which reduces the progression of cardiovascular disorders (Sellers et al., 2010; Hong et al., 2015). Watermelon powder supplementation exerts an anti-inflammatory effect similar to COX-2 inhibitors or conventional non-steroidal anti-inflammatory drugs (Hong et al., 2015).

High mobility group box 1 (HMGB1), a non-histone DNA binding protein, produced by necrotic and immune cells, exposed to pro-inflammatory signals, has an important proinflammatory effect by attracting and activating inflammatory cells and mediators and binding to RAGE and toll-like receptors, related to fatal outcomes (Lee et al., 2012). Lee et al. demonstrated that lycopene inhibits adhesion molecules expression, which impair HMGB1-induced monocyte adhesion 


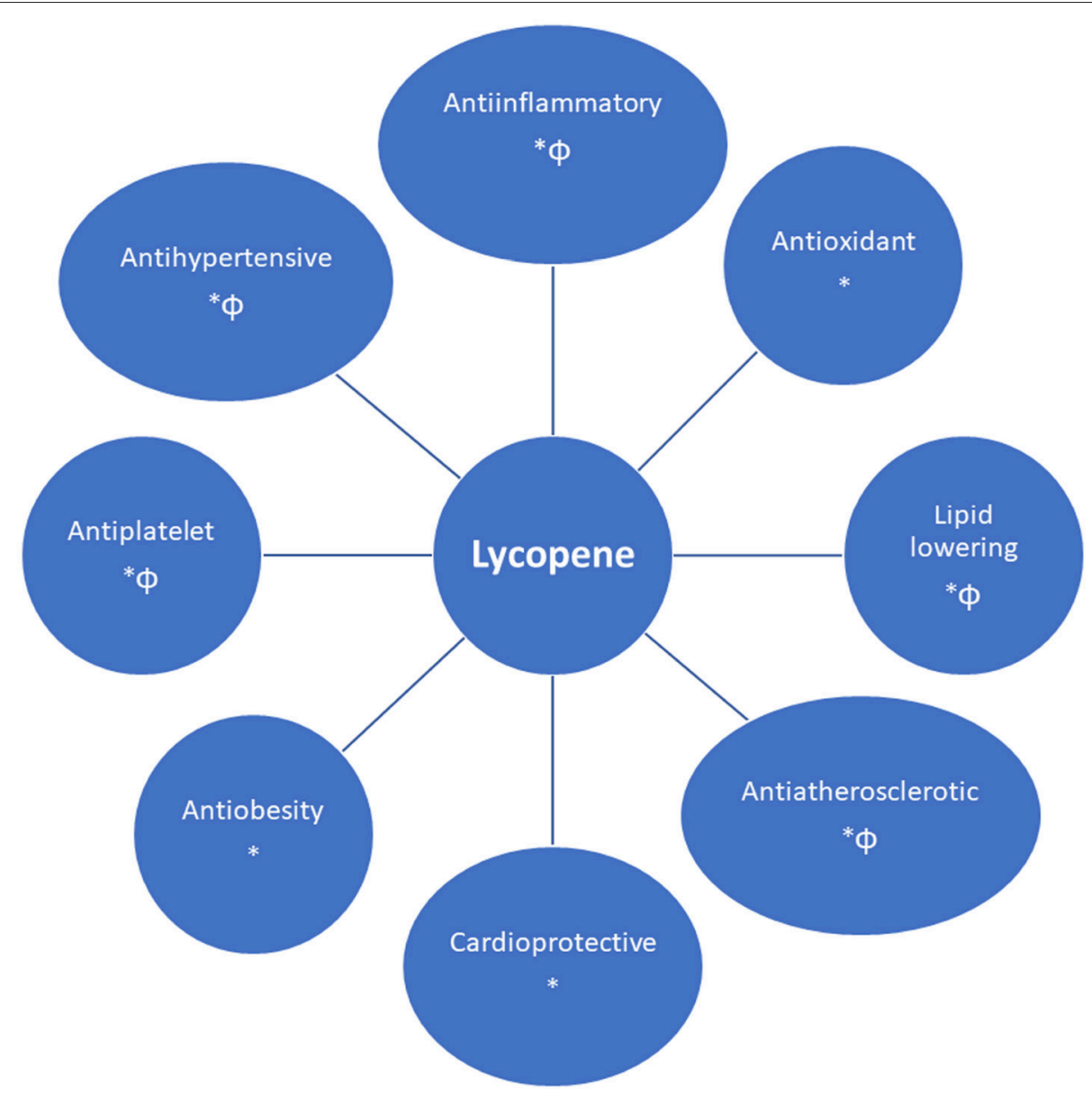

FIGURE 2 | Cardiovascular benefits of lycopene [supported by in vivo (*) and/or in vitro ( $\Phi)$ findings].

and transmigration (Lee et al., 2012). Lycopene has been also shown to inhibit lipopolysaccharide-induced HMGB1 release and HMGB1-mediated secretion of TNF-alpha and secretory phospholipase A2 (Lee et al., 2012).

Oxysterols, the result of cholesterol auto-oxidation, accumulate in the subendothelial arterial layer, exerting oxidative and pro-inflammatory roles and favoring the atherosclerotic process (Palozza et al., 2011). Lycopene impairs oxysterol-induced pro-inflammatory cytokines production in human macrophages and oxysterol-induced ROS production, limiting the formation of atherosclerotic plaque (Palozza et al., 2011).

Lycopene exerts a cardioprotective effect against atrazine induced cardiac injury due to its anti-inflammatory effect, by blocking the NF-kappa B pathway and NO production (Li et al., 2017).

Considering the mentioned anti-inflammatory mechanisms (Figure 3), including decrease of adhesion molecules, proinflammatory cytokines, inhibition of leukocyte migration and genes involved in inflammation, impaired monocyteendothelium interaction, T lymphocytes activation and synthesis of AGE and RAGE and downregulation of cyclooxygenase 2, lycopene can be useful in the therapy for vascular inflammatory disorders.

\section{Modulation of Lipids}

Lycopene is transported in the circulation by lipoproteins and is actively taken up into adipocytes (McEneny et al., 2013). Lycopene is a regulator of cholesterol levels by inhibition of HMG-CoA reductase (like statins) and down-regulation of proprotein convertase subtilisin/kexin type 9 mRNA synthesis. These results suggest that lycopene supplementation could be especially beneficial for patients with statin intolerance (Sultan Alvi et al., 2017). Changes in hepatic gene expression, such as reduced expression in fatty acid synthase, responsible for fatty acid storage, were described after watermelon powder consumption in rats (Hong et al., 2015). Carbonic anhydrase III and adenylate kinase 2 were involved in the lipid-lowering and antioxidant effects of tomatoes (Hsu et al., 2008). On the other hand, NO stimulates fatty acid oxidation and lipolysis in adipose cells (Jobgen et al., 2006; Hong et al., 2015).

Lycopene is not able to increase HDL cholesterol, but was shown to improve the LDL/HDL ratio, HDL functionality and reduced the accumulation of cholesterol in the rabbit aorta, 


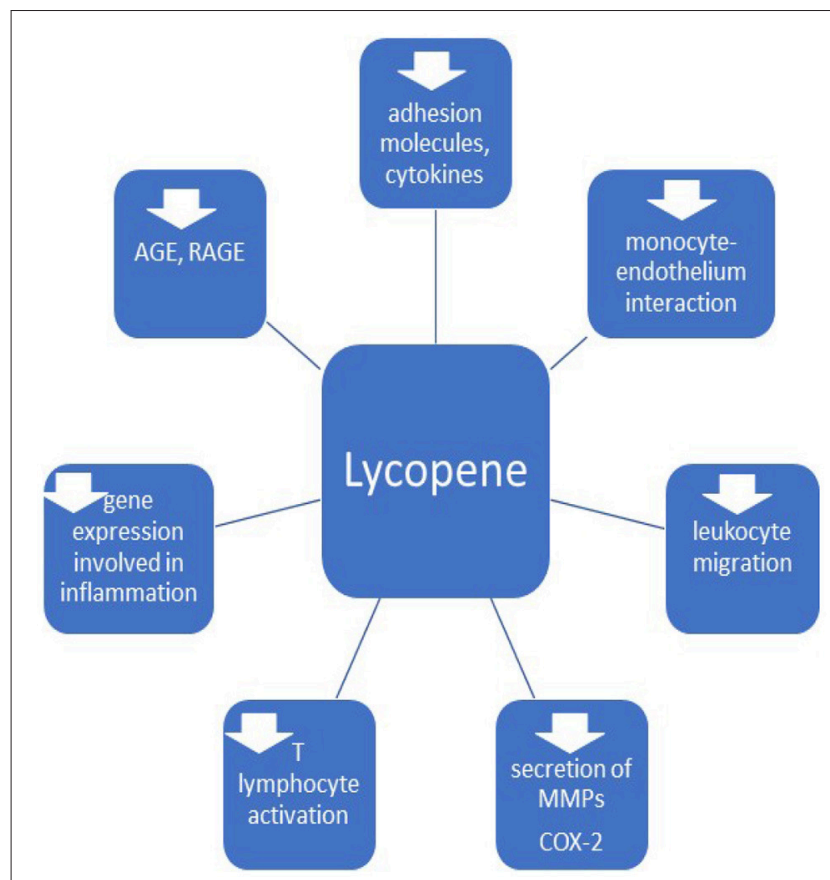

FIGURE 3 | Anti-inflammatory effects of lycopene. MMPs, matrix metalloproteinases; COX-2, cyclooxigenase 2; AGE, advanced glycation end-products; RAGE, receptors of AGE.

underlining the beneficial effects of lycopene during initial stages of atherosclerosis (Lorenz et al., 2012; Thies et al., 2017). However, a recent meta-analysis found that significant reductions in total and LDL cholesterol were revealed only at doses of, at least, $25 \mathrm{mg}$ lycopene/day in human subjects, and the effects were comparable with low-dose statin medication, without concomitant side effects (Ried and Fakler, 2011; Lorenz et al., 2012). Fecal cholesterol excretion increases with levels of dietary lycopene due to decreased intestinal cholesterol absorption. This can be explained by the insight that increased fecal excretion impairs the enterohepatic circuit of bile acids, thus increasing the conversion of cholesterol to bile acids (Verghese et al., 2008). The lipid lowering properties of lycopene involve also an increased activity of LDL receptors in macrophages (Li et al., 2015; Cheng et al., 2017).

Lycopene can lower synthesis of dysfunctional HDL, modulating HDL functionality toward an antiatherogenic phenotype, with a low serum amyloid A level and beneficial changes of the activity of HDL remodeling enzymes (cholesterol ester transfer protein and lecithin cholesterol acyl transferase) (McEneny et al., 2013). A hypotriglyceridemic effect of tomato juice was seen only in subjects with initial high serum triglyceride levels (Li et al., 2015).

Lycopen can also regulate the hepatic lipid metabolism and counteracts the hepatic steatosis induced by a high-fat diet, due to sirtuins (SIRT1) induction and activation, being able to suppresses lipogenesis, to stimulate lipid catabolism in the liver and skeletal muscles and lipid mobilization in the white adipose tissue (Lomb et al., 2010; Li et al., 2015). Another mechanism able to ameliorate liver steatosis, by lycopene, was described in mice and is related to decrease of fatty acid binding protein 7 due to binding to microRNA-21 (Ahn et al., 2012; Li et al., 2015).

In summary, lycopene has lipid lowering properties, reducing the total and LDL cholesterol, triglyceride level, LDL oxidation, and synthesis of dysfunctional HDL.

\section{Anti-aggregative Effect}

Platelets are involved in the pathogenesis of the atherosclerotic plaque, development of acute thrombotic events and restenosis after endovascular procedures (Krasinska et al., 2017; O’Kennedy et al., 2017). Another cardiovascular beneficial effect of lycopene, protecting against myocardial infarction and stroke, is its antiplatelet activity, which is concentration dependent, and was demonstrated in vivo and in vitro (Sawardekar et al., 2016). Several mechanisms were considered in explaining the reversible antiplatelet effect of lycopene, such as the interaction with thromboxane, thrombin, collagen, von Willebrand factor, Pselectin and inflammatory mediators, the influence on calcium and cyclic guanosine monophosphate signaling and ADPmediated aggregation (Sawardekar et al., 2016; Krasinska et al., 2017). It was noticed that lycopene can potentiate the antiplatelet effect of aspirin, which requires low lycopene diet in patients on secondary prophylaxis with aspirin due to the potential bleeding risk (Sawardekar et al., 2016). On the other hand, in high cardiovascular risk, aspirin (ASP) resistant patients, or those with ASP contraindications, high risk of complications after antiplatelet therapy or hyperactive platelets (obese, sedentary, hypertensive, diabetic, aging patients, and smokers), lycopene could have an important contribution in cardiovascular prophylaxis (Sawardekar et al., 2016; Krasinska et al., 2017; O'Kennedy et al., 2017).

\section{Antihypertensive Effect}

Lycopene has antihypertensive effects due to inhibition of the angiotensin converting enzyme (ACE) and due to its antioxidant effect, reducing oxidative stress induced by angiotensin-II and indirectly enhancing production of nitric oxide in the endothelium ( $\mathrm{Li}$ and $\mathrm{Xu}, 2013$; Belovic et al., 2016; Khan et al., 2016; Han and Liu, 2017). A study including 8,556 adult overweight and obese participants demonstrated association of lycopene and lycopene/uric acid ratio with lower prevalence of hypertension (Han and Liu, 2017). Paran et al. reported a decrease in both systolic and diastolic blood pressure in 54 patients with moderate hypertension, treated with ACE inhibitors or calcium channel blockers, after 6 weeks of tomato extract supplementation, suggesting a cause-effect relationship (Paran et al., 2009). Li et al. concluded, in a metanalysis, that lycopene supplementation (more than $12 \mathrm{mg} /$ day) might significantly reduce systolic, but not diastolic blood pressure, in prehypertensive or hypertensive patients ( $\mathrm{Li}$ and $\mathrm{Xu}, 2013$ ).

Angiotensin II induces, besides direct vasoconstriction and oxidative stress, also vascular smooth muscle cells phenotypic transformation and production of inflammatory cytokines (Ren et al., 2017), and lycopene might impair the mentioned pathways, as well. 


\section{Anti-atherosclerotic Mechanisms}

Besides improving endothelial function, oxidative stress (preventing oxidation of LDL) and metabolic profile, the antiinflammatory and antiplatelet effect, lycopene has several other anti-atherosclerotic contributions (Figure 4), such as inhibition of vascular smooth muscle cell (VSMC) proliferation and foam cell formation (Napolitano et al., 2007; Wang et al., 2014). In general, not all studies confirm the relationship between lycopene and early atherosclerosis (Kim et al., 2010).

Contractile VSMC change to a proliferative and migratory phenotype during the atherosclerotic process, enabling migration of VSMC into the intima and production of the extracellular matrix of the plaque (Karagiannis et al., 2013). Such changes in phenotype are called "phenotypic modulation" (Manabe and Nagai, 2003) and play an important role in vascular remodeling, not only due to atherosclerosis, but also in hypertension and diabetic macroangiopathy (Ren et al., 2017). Lycopene can suppress VSMCs proliferation, due to inhibiting G1 phase cells entry into the $S$ phase of the cell cycle, related to its antioxidative effect (Chen et al., 2010), and not due to the inhibition of matrix metalloproteinases (Lo et al., 2007). Minimally-oxidized LDL can induce phenotypic modulation of VSMC (Karagiannis et al., 2013), and synthesis of oxidized LDL may be impaired by lycopene (Kim et al., 2010). Lycopene may block VSMC migration and proliferation also by direct binding to plateletderived growth factor (PDGF) and inhibiting PDGF-signaling (Lo et al., 2007), or due to it antioxidant effect, considering that reactive oxygen species accelerate the switch from the contractile to the synthetic phenotype (Sung et al., 2005).

Lycopene has also barrier integrity activity in the endothelial membrane, by blocking the activation of CD14 and Toll

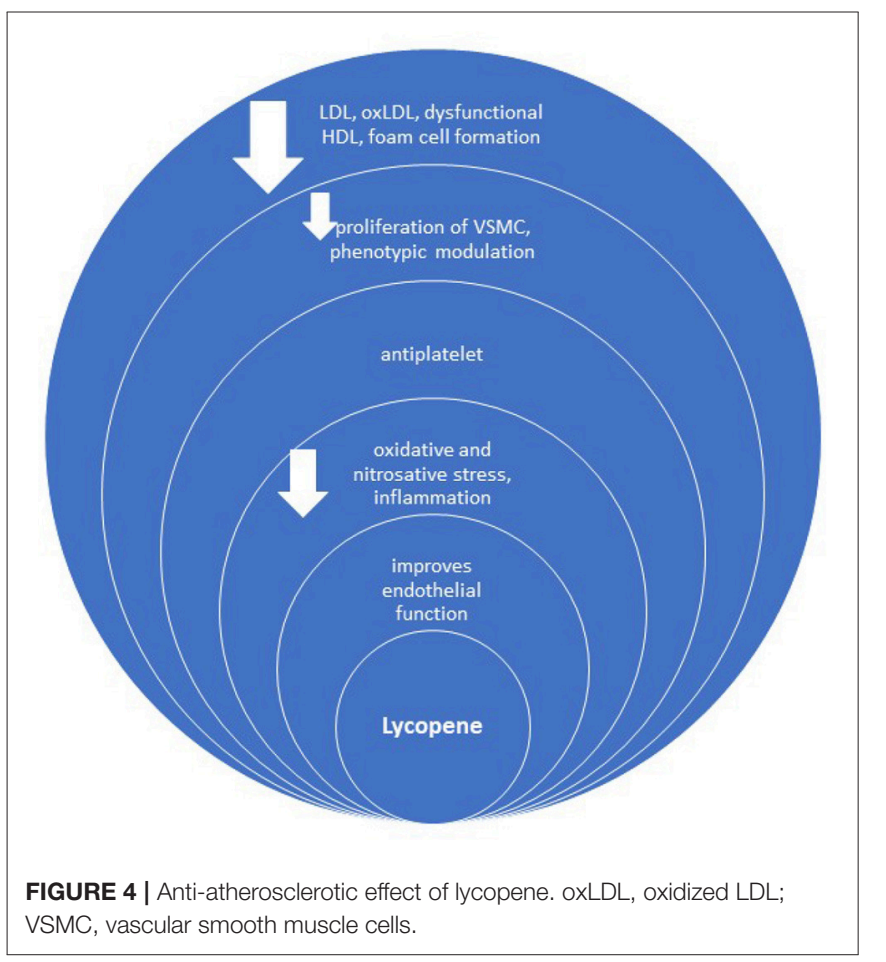

like receptor-4 expression (Bae and Bae, 2011) and impairs the apoptosis of endothelial cells in vitro, by disrupting the upregulation of p53 and caspase 3 mRNA (Tang et al., 2009).

\section{Cardioprotective Effects}

Recent studies emphasized the cardioprotective effects of lycopene. An experimental study including rats with isoproterenol induced myocardial infarction, demonstrated improved ECG findings (shorter QT and RR intervals and QRS complexes, reduced ST segment elevation) if lycopene was previously administrated, related to its cell membrane stabilizing and antioxidant properties (Aman et al., 2012). Supplementation of lycopene in the same study also prevented alteration in hemodynamic parameters (systolic, diastolic, and mean blood pressure), biochemical and inflammatory markers, apoptotic changes, and reduced the size of myocardial infarction due to the antioxidant and anti-inflammatory properties of lycopene (Aman et al., 2012; Wong et al., 2017).

Tomato and lycopene supplementation attenuated cardiacremodeling and improved diastolic dysfunction after myocardial infarction according to a study including male Wistar rats, enabled by impairing type I and type III collagen content in the left ventricle, reduced cardiomyocyte death, expression of miRNAs and by the anti-inflammatory effect and reduction of oxidative stress (Pereira et al., 2017). Wang et al. administered lycopene in infarcted rats for 28 days, revealing an increased ejection fraction compared to controls, associated with decreased collagen fraction in the peri-infarct zone, due to inhibition of p38 mitogen-activated protein kinase and matrix metalloproteinase 9 expression (Wang et al., 2014). Lycopene effect on interstitial collagen accumulation was not confirmed by Anjos Ferreira et al., in a study including male Wistar rats and testing the effect of lycopene on doxorubicin-induced cardiotoxicity (Anjos Ferreira et al., 2007). The anti-inflammatory effect of lycopene has also a contribution in reversing ventricular remodeling, by inhibiting the nuclear factor- $\kappa \mathrm{B}$ signaling pathway (He et al., 2015).

Lycopene might also serve as a cardioprotective agent against several drugs. The cardioprotective effect of lycopene was demonstrated in the case of tulathromycin, a macrolide antibiotic, and diclofenac sodium, a non-steroidal antiinflammatory drug, by Abdel-Daim et al., in a study including Swiss albino mice, and was attributed to its antioxidant activity (Abdel-Daim et al., 2018). Lycopene can also protect against the cardiotoxicity induced by doxorubicin (Karimi et al., 2005; Anjos Ferreira et al., 2007; Abushouk et al., 2017), isoproterenol (Aman et al., 2012; Mohamadin et al., 2012), and atrazine (Li et al., 2017).

Concluding, lycopene supplementation is beneficial for early and late prognosis in myocardial infarction. It may reduce myocardial infarction size and related electrocardiographic, hemodynamic, and biochemical changes (Aman et al., 2012). Lycopene also reverses ventricular extracellular matrix remodeling after an acute coronary event, by inhibiting myocardial fibrosis and preventing apoptosis and necrosis of cardiomyocytes, its anti-inflammatory effect and the ability to increase left ventricular function, preventing development of heart failure and increasing survival rates (Aman et al., 2012; 
Wang et al., 2014; He et al., 2015). Considering difficulties and high cost of early revascularization and significant side effects of cardiovascular drugs, lycopene could represent a safe and effective option in controlling post infarction ventricular remodeling (Wang et al., 2014).

\section{Lycopene and Endoplasmic Reticulum Stress (ERS)}

The endoplasmic reticulum has several functions related to intracellular calcium storage, lipid and protein synthesis, and modification (Schönthal, 2012). Disturbance of those functions causes "endoplasmic reticulum stress" (ERS) involved in myocardial ischemia/reperfusion injury (Gao et al., 2016). The cell reacts to ERS by initiating an "unfolded protein response," including several mechanisms, enabling cellular adaptation and survival or, in severe cases, apoptosis (Schönthal, 2012).

Lycopene protects the cardiomyocytes by relieving ERS and preventing apoptosis, through stimulation of adenosine 5monophosphate-activated proteinkinase, the transcription factor CHOP (C/EBP homologous protein), p-JNK and Caspase pathways (Xu et al., 2015; Gao et al., 2016). However, high lycopene levels might be toxic to the cardiomyocytes (Gao et al., 2016).

\section{PRECLINICAL STUDIES}

Lycopene supplementation for 4 weeks strongly reduced total and LDL serum cholesterol and the amount of cholesterol in the aorta, but the surface lipid accumulation in the aorta and IMT were not significantly reduced and no impairment of vasoreactivity or increase of pro-oxidant parameters were detected in New Zealand White rabbits after either a standard or a high-cholesterol diet (Lorenz et al., 2012). Although lycopene suppressed cholesterol uptake and intestinal absorption and increased fecal cholesterol excretion in rabbits, it did not decrease the expression of HMG-CoA reductase (Lorenz et al., 2012).

$\mathrm{Hu}$ et al. reported anti-atherosclerotic effects for both lycopene and fluvastatin, in the aorta, in rabbits, after a high-fat diet (Hu et al., 2008; He et al., 2009). Verghese et al. also revealed a significant decrease in the atherosclerotic plaque formation with the consumption of lycopene in a study including New Zealand male rabbits, receiving a high cholesterol diet. Further observed effects include an improved serum lipid profile as well as reduction in total cholesterol, LDL and triglycerides (Verghese et al., 2008). Hsu reported, besides reduction in total and LDL cholesterol and plasma malondialdehyde (MDA) levels, also an increase in HDL cholesterol in hamsters fed a $9 \%$ tomato paste containing $0.2 \%$ cholesterol, after 8 weeks (Hsu et al., 2008).

Watanabe Heritable Hyperlipidemic rabbits were used in a study by Frederiksen et al. demonstrating that lycopene did not influence cholesterol and triacylglycerol levels, lipoprotein fractions, oxidation of lipids, and aortic atherosclerosis evaluated biochemically and by microscopy (Frederiksen et al., 2007). The lack of response of lycopene in Watanabe Heritable Hyperlipidemic rabbits is, probably, related to their defective LDL receptors (Tanazawa et al., 1980; Lorenz et al., 2012), which would suggest the involvement of these receptors in the cardiovascular benefits of lycopene (Perera and Yen, 2007). Considering the bloodstream transport of lycopene in LDL particles, functional LDL receptors enable cardiovascular benefits of lycopene (Lorenz et al., 2012). Bansal et al. reported a beneficial cardioprotective effect of lycopene, due to the reduction of oxidative stress and myocardial injury, in an experimental model of myocardial ischemia-reperfusion injury in adult male albino Wistar rats (Bansal et al., 2006). Lycopene protects also endothelial progenitor cells, necessary to replace the injured vascular endothelium and for angiogenesis, in a microenvironment of advanced glycation end products (AGEs), which act as damage-causing agents (Zeng et al., 2017). Lycopene improved cell proliferation and regulated protective mechanisms of AGEs-induced autophagy in endothelial progenitor cells from diabetic rats, suggesting that supplementation with this compound might be a new therapeutic option for diabetic vascular complications (Zeng et al., 2017).

\section{CLINICAL RESEARCH}

\section{Endothelial Function}

Both cross-sectional and supplementation studies emphasized the benefits of tomato products on vascular function, mainly due to the antioxidative effects of lycopene (Kim et al., 2010; Thies et al., 2012; Xaplanteris et al., 2012; Gajendragadkar et al., 2014). However, several articles failed to show improvement of endothelial function after tomato consumption (Stangl et al., 2011; Table 1).

Gajendragadkar et al. concluded that lycopene supplementation can improve endothelial function in patients with cardiovascular disorders, but not in age-matched healthy volunteers (Gajendragadkar et al., 2014). Forearm responses to intraarterial infusions of acetylcholine were assessed using venous plethysmography, which resulted in an improvement by $53 \%$ of endothelium-dependent vasodilatation (EDV) in patients with cardiovascular disorders post-lycopene (Gajendragadkar et al., 2014). Endothelial function was improved regardless of traditional risk factors or inflammatory markers, and, even a modest increase in serum lycopene, further impaired endothelial function in atherosclerotic patients (Gajendragadkar et al., 2014). One conclusion of the mentioned study was that lycopene affects especially smaller vessels, such as resistance arteries, rather than larger vessels, as measured arterial stiffness remained unaltered in all study participants (Gajendragadkar et al., 2014).

\section{Pulse Wave Velocity (PWV)}

Kim et al. reported an independent, inverse association between circulating lycopene and brachial pulse wave velocity in 264 healthy women, regardless of age, body mass index, smoking and drinking habits, menopause, blood pressure, beta-carotene, alpha-tocopherol, markers of oxidative stress, and inflammation (Kim et al., 2010; Table 1). Reduced oxidative LDL changes may have an important contribution to arterial stiffness reduction due to lycopene (Kim et al., 2010). Another study, including 126 healthy men revealed the benefits of lycopene on oxidative stress and endothelial dysfunction, especially in subjects with an 
TABLE 1 | Effects of lycopene on endothelial function and arterial stiffness.

\begin{tabular}{|c|c|c|c|}
\hline Number of participants & Methodology & Results, conclusions & References \\
\hline $\begin{array}{l}36 \text { statin treated cardiovascular } \\
\text { patients (mean age: } 67-68 \\
\text { years) and } 36 \text { healthy volunteers } \\
\text { (mean age: } 61-68 \text { years) }\end{array}$ & $\begin{array}{l}\text { Double-blind trial: } 7 \text { mg lycopene or placebo } \\
\text { daily for } 2 \text { months. }\end{array}$ & $\begin{array}{l}\text { Lycopene supplementation improved } \\
\text { endothelial function in patients with } \\
\text { cardiovascular disorders on optimal } \\
\text { secondary prevention, but not in healthy } \\
\text { persons. }\end{array}$ & $\begin{array}{l}\text { Gajendragadkar et al., } \\
2014\end{array}$ \\
\hline $\begin{array}{l}225 \text { overweight volunteers, aged } \\
\text { 40-65 years }\end{array}$ & $\begin{array}{l}\text { Participants were randomly assigned into } 1 \text { of } 3 \\
\text { dietary intervention groups: control diet (low } \\
\text { tomato content), a high-tomato-content diet, or } \\
\text { a control diet with addition of lycopene } \\
\text { capsules ( } 10 \mathrm{mg} / \mathrm{d} \text { ) for } 12 \text { wk. Collected blood } \\
\text { samples were tested for carotenoid and lipid } \\
\text { profiles and inflammatory markers. Arterial } \\
\text { stiffness and dietary intake were also } \\
\text { monitored. }\end{array}$ & $\begin{array}{l}\text { A relatively high daily consumption of } \\
\text { tomato-based products (32-50 mg } \\
\text { lycopene/day) or lycopene supplements } \\
\text { (10 mg/day) was ineffective in reducing } \\
\text { conventional cardiovascular risk markers, } \\
\text { inflammatory markers, markers of insulin } \\
\text { resistance and sensitivity, lipid profile and } \\
\text { arterial stiffness in moderately overweight, } \\
\text { healthy, middle-aged individuals. }\end{array}$ & Thies et al., 2012 \\
\hline $\begin{array}{l}25 \text { study participants, mean age: } \\
27 \pm 8 \text { years }\end{array}$ & $\begin{array}{l}\text { Randomized, intervention model, crossover } \\
\text { assignment. The participants consumed } \\
\text { high-fat meals containing processed tomato } \\
\text { products or non-tomato alternative. }\end{array}$ & $\begin{array}{l}\text { Tomato products attenuate postprandial } \\
\text { lipemia-induced oxidative stress and } \\
\text { inflammatory response, with a modest } \\
\text { improved flow-mediated dilatation (FMD). }\end{array}$ & $\begin{array}{l}\text { Burton-Freeman et al., } \\
2012\end{array}$ \\
\hline 19 volunteers, $39 \pm 13$ years & $\begin{array}{l}\text { Randomized, single-blind, crossover } \\
\text { assignment. }\end{array}$ & $\begin{array}{l}\text { Daily tomato paste consumption exerts a } \\
\text { beneficial midterm effect on endothelial } \\
\text { function. }\end{array}$ & Xaplanteris et al., 2012 \\
\hline 299 Korean men & $\begin{array}{l}\text { Subgrouped according to the number of } \\
\text { metabolic syndrome risk factors; brachial-ankle } \\
\text { pulse wave velocity (PWV), oxidative stress and } \\
\text { antioxidants (including lycopene) were } \\
\text { measured. }\end{array}$ & $\begin{array}{l}\text { An inverse correlation was found between } \\
\text { PWV and serum lycopene, considering } \\
\text { blood pressure, insulin resistance and } \\
\text { oxidative stress. }\end{array}$ & Yeo et al., 2011 \\
\hline $\begin{array}{l}19 \text { healthy non-smoking } \\
\text { postmenopausal women }\end{array}$ & $\begin{array}{l}\text { Administration of } 70 \mathrm{~g} \text { tomato puree. } \\
\text { Endothelial-dependent FMD and } \\
\text { endothelial-independent nitro-mediated dilation } \\
\text { of the brachial artery were measured with } \\
\text { high-resolution ultrasound. }\end{array}$ & $\begin{array}{l}\text { Acute and long-term ( } 7 \mathrm{~d} \text { ) intake of tomato } \\
\text { products, despite a significant increase in } \\
\text { plasma lycopene had no effect on } \\
\text { endothelial function. }\end{array}$ & Stangl et al., 2011 \\
\hline 126 healthy men & $\begin{array}{l}\text { Administration of placebo/ } 6 \mathrm{mg} / 15 \mathrm{mg} \\
\text { lycopene daily for } 8 \text { weeks. Endothelial function } \\
\text { was assessed by reactive hyperemia peripheral } \\
\text { arterial tonometry. Plasma superoxide } \\
\text { dismutase was used to assess oxidative stress. }\end{array}$ & $\begin{array}{l}\text { An inverse correlation between serum } \\
\text { lycopene levels and arterial stiffness was } \\
\text { found. An increased serum lycopene } \\
\text { decreases oxidative stress, which might } \\
\text { influence endothelial function. }\end{array}$ & Kim et al., 2011 \\
\hline 264 healthy women, $31-75$ years & $\begin{array}{l}\text { The relationship between serum lycopene and } \\
\text { brachial-ankle pulse wave velocity was } \\
\text { assessed. }\end{array}$ & $\begin{array}{l}\text { An independent, inverse relationship } \\
\text { between circulating lycopene and brachial } \\
\text { PWV was observed. }\end{array}$ & Kim et al., 2010 \\
\hline
\end{tabular}

impaired endothelial function (Kim et al., 2011). After $15 \mathrm{mg} /$ day lycopene supplementation, for 8 weeks, Kim et al. reported a decrease of systolic blood pressure and high sensitivity $\mathrm{C}$ reactive protein (Kim et al., 2011).

The main biological mechanism by which lycopene reduces the risk and mortality of the metabolic syndrome, include the antioxidant, anti-inflammatory and antiobesity effects, the ability to improve endothelial function, glycemic control, insulin sensitivity and lipid profile (Tsitsimpikou et al., 2014; Li et al., 2015; Han et al., 2016a,b). An inverse relationship was found by Yeo et al. between lycopene level and brachial-ankle pulse wave velocity (Yeo et al., 2011). PWV was significantly higher in patients with metabolic syndrome, with lower than median serum lycopene values $(\leq 0.0294 \mathrm{mmol} / \mathrm{l})$ compared to patients without metabolic syndrome; no statistically significant differences between the 2 groups were found when lycopene levels were high (Fantin et al., 2010; Yeo et al., 2011).
Higher serum carotenoid levels were associated not just with a lower prevalence of the metabolic syndrome, but also with fewer abnormal metabolic syndrome components. A significant association between lycopene and the metabolic syndrome was described only for normal-weight and overweight participants, but not in obese patients, according to a study enrolling 13,196 subjects, probably related to an increased oxidative stress and decreased antioxidant ability, due to sequestration of lycopene in the adipose tissue and more important inflammation in obese (Han et al., 2016b). On the other hand, daily tomato juice intake reduced waist circumference, cholesterol, and monocyte chemotactic protein-1 (inflammatory adipokine) and increased adiponectin (anti-inflammatory adipokine) levels in 30 young, healthy Taiwanese females (Li et al., 2015). Lycopene was shown to impair pro-inflammatory cytokine production, such as IL6 , IL- $1 \mathrm{~b}$, and TNF- $\alpha$, preventing insulin resistance (Gouranton et al., 2011). 
L-arginine or L-citrulline supplementation modulate the arginine-NO pathway, enabled by multiple cyclic guanosine$3^{\prime}, 5^{\prime}$-monophosphate-dependent pathways, with important prophylactic and therapeutic contributions in the metabolic syndrome (Jobgen et al., 2006).

A large study, including 225 middle-aged, overweight volunteers reported no changes of conventional cardiovascular risk factors, inflammatory tests, insulin resistance and sensitivity, lipid profile, oxidized LDL, von Willebrand factor, and arterial stiffness after high daily intake of lycopene, despite good compliance (Thies et al., 2012). One week of lycopene supplementation increased plasma lycopene, but not biomarkers of vascular oxidative stress and inflammation, or biomarkers of nitric oxide (plasma nitrate/nitrite) in healthy, active subjects, suggesting that the participants already possessed a robust antioxidant capacity and lycopene provided no additional benefit (Denniss et al., 2008).

\section{Intima-Media Thickness (IMT)}

Several studies reported an association between serum lycopene levels and intima-media thickness (Gianetti et al., 2002; Riccioni et al., 2009, 2011; Karppi et al., 2013; Zou et al., 2014), while other authors reported no association (Dwyer et al., 2004; Table 2). Zou et al. revealed a decrease in carotid artery intimamedia thickness (IMT) after 12 months of lutein and lycopene supplementation (20 mg each) in 144 Chinese patients with subclinical atherosclerosis, demonstrating more effective results after the intake of both lutein and lycopene compared to lutein alone (Zou et al., 2014).

High serum levels of lycopene, alpha and beta-carotene were associated with a slow IMT progression during 7 years in a study including 840 middle-aged men from Eastern Finland (Karppi et al., 2013). The association between lycopene level and IMT was mentioned in the scientific literature also for elderly Finish subjects (Karppi et al., 2011).

Higher carotenoids levels (lutein, zeaxanthin, and betacryptoxanthin; Figure 1) were correlated with reduced IMT progression over 18 months, in a study with 573 middle-aged participants, free of cardiovascular symptoms at baseline. Betacarotene and lycopene levels were not significantly associated with IMT progression (Dwyer et al., 2004).

An inverse correlation was found in women between lycopene levels and IMT, independent of conventional risk factors, in a large study including 1,111 subjects (McQuillan et al., 2001).

\section{WHY CONFLICTING RESULTS?}

Several studies revealed the anti-atherosclerotic effect of lycopene (McQuillan et al., 2001; Gianetti et al., 2002; Hu et al., 2008; Verghese et al., 2008; Riccioni et al., 2009; Kim et al., 2010; Gajendragadkar et al., 2014), but there are also studies available that report conflicting results related to the vascular effects of lycopene (McQuillan et al., 2001; Dwyer et al., 2004; Stangl et al., 2011; Thies et al., 2012). The possible reasons for this obvious discrepancy are manifold and include methodological differences in the study designs, such as different lycopene sources, the use of food-frequency questionnaires, different intervention times, the methodology used to assess vascular function, measurement of blood, adipose or dietary lycopene. Besides those, the use of unstandardized amounts of tomato food products, different modes of delivery, misclassification of overall tomato intake, combination of lycopene with other antioxidants, different processing procedures or eating behavior influenced by cultural and temporal patterns among different individuals, may influence the results (Sesso et al., 2003; Kong et al., 2010; Thies et al., 2012; Gajendragadkar et al., 2014). Other carotenoids extracted from tomatoes could be also partially responsible for the effects attributed to lycopene (Rao, 2002). This is underlined by a study that could not find beneficial effects for lycopene supplementation alone, but beneficial effects upon supplementation with tomato-based products (Sesso et al., 2003). Some studies did not consider dietary intake at all (Yeo et al., 2011). The interaction flavanone metabolites-lycopene is difficult to assess, considering the rapid metabolization of the mentioned metabolites (Habauzit et al., 2015). Duration of treatment, dose and bioavailability of lycopene, and vascular endpoint were also different in the studies published on this topic and might have influenced obtained results. Several factors influence the bioavailability of lycopene, such as season, the processing of tomatoes, their origin, dimensions, shape, and the way they are consumed (Gajendragadkar et al., 2014; Gammone et al., 2015). Absorption of lycopene may be reduced by diets rich in fibers and in elderly people (Kong et al., 2010) and is increased in the presence of oil.

The isomerization of lycopene is another source of variability. Fresh tomatoes contain lycopene in all-trans form (Shi and Le Maguer, 2000). Several factors, including high temperatures, light, oxygen, acids, and metal ions enable isomerization of lycopene (Kong et al., 2010). Lycopene degradation occurs during thermal processing, mainly isomerization of all- trans to cis forms and oxidation (Shi and Le Maguer, 2000). Dehydrated and powdered tomatoes have poor lycopene stability, depending of storage in a hermetically sealed atmosphere, and a significant increase of cis-isomers, giving the highest bioavailability of lycopene and higher ability to be incorporated in lipoproteins (Shi and Le Maguer, 2000; Kong et al., 2010). Uptake of cis lycopene is significantly higher than all trans-isomers (Kong et al., 2010).

Lycopene is very bioavailable in the presence of oil, especially in monounsaturated oils, other dietary fats and processed tomato products (Shi and Le Maguer, 2000; Basu and Imrhan, 2007; Kong et al., 2010; Gajendragadkar et al., 2014). Lycopene can increase the antioxidant properties of vitamin C, E, polyphenols and beta-carotene in a synergistic way (Kong et al., 2010; Karppi et al., 2013). Supplementation with tomatoes, containing lycopene (red tomatoes) or not (yellow tomatoes), showed a better antioxidant effect than lycopene alone, probably due to the synergistic effects of naturally occurring secondary metabolites in tomatoes (Basu and Imrhan, 2007; Gitenay et al., 2007). Generally, supplementation with whole fruits is often more beneficial than supplementing single food constituents: Watermelons contain, besides lycopene, hundreds of different compounds, including L-citrulline and ascorbic acid, both of which improve the L-arginine/NO pathway, endothelial function, 
TABLE 2 | Lycopene and intima-media thickness (IMT).

\begin{tabular}{|c|c|}
\hline Number of participants & Methodology \\
\hline $\begin{array}{l}144 \text { subjects, aged } 45-68 \text { years, } \\
\text { with subclinical atherosclerosis }\end{array}$ & $\begin{array}{l}20 \text { mg lutein }(n=48), 20 \mathrm{mg} \text { lutein }+20 \mathrm{mg} \\
\text { lycopene }(n=48) \text { or placebo }(n=48) \text { were } \\
\text { administrated for } 12 \text { months; carotid artery } \\
\text { intima-media thickness (IMT) was measured } \\
\text { using Doppler ultrasonography. }\end{array}$ \\
\hline $\begin{array}{l}840 \text { middle-aged men from } \\
\text { Eastern Finland }\end{array}$ & $\begin{array}{l}\text { Ultrasonography of the common carotid } \\
\text { arteries, serum levels of carotenoids }\end{array}$ \\
\hline
\end{tabular}

Eastern Finland

\author{
1,212 elderly men from Eastern \\ Finland
}

120 subjects without history of symptomatic carotid artery

disease

640 participants with

asymptomatic carotid

atherosclerosis

573 middle-aged women and men from an occupational cohort

\author{
11 healthy controls, 11 patients \\ with uncomplicated \\ hypertension, 11 with essential \\ hypertension and peripheral \\ vascular disease \\ 1,111 subjects, aged 27-77 \\ years
} arteries was performed, lipid profile and risk factors were assessed at baseline and determined at baseline. habit and body mass index; IMT, adhesion molecules, LDL and antioxidants (including lycopene) were measured. vitamins ( $A, C$, and $E)$, lycopene, alpha and
B-mode ultrasound (IMT of the common carotid artery); plasma levels of carotenoids

\begin{abstract}
Ultrasonic measurement of common carotid artery IMT, serum profile analysis of cholesterol (total and LDL), triglycerides and lycopene. Carotid ultrasound investigation was performed; medical history and laboratory data were collected.
\end{abstract}

Results, conclusions

Lutein and lycopene supplementation significantly increased the serum concentration of lutein and lycopene with a decrease in carotid artery IMT.

7-year change in maximum intima media thickness was inversely associated with serum levels of lycopene, alpha and beta-carotene, respectively. Elevated serum levels of carotenoids may have anti-atherosclerotic effect.

High plasma concentrations of lycopene, alpha-carotene and beta-cryptoxanthin are related to decreased carotid atherosclerosis in elderly patients.

Carotid atherosclerosis was associated with lower plasma lycopene levels.

Participants with IMT $\geq 0.8 \mathrm{~mm}$ had significantly lower concentrations of vitamin $\mathrm{A}$ and $\mathrm{E}$, lycopene, and beta-carotene compared to participants with no evidence of carotid atherosclerosis.

Ultrasound examination of the common carotid 18-month follow-up. Plasma antioxidants were

Patients were matched for age, sex, smoking

Dietary vitamin intake, fasting plasma levels of beta-carotene, bilateral 18-month change in IMT was inversely related to serum levels of some measured carotenoids, not including lycopene, regardless of cardiac risk factors and high-sensitivity C-reactive protein.

A statistically significant correlation was found between lycopene and IMT, independent of LDL, creatinine clearance, and plasma insulin. No significant correlation was found between lycopene and soluble adhesion molecules

There was an inverse association between carotid artery IMT and plasma lycopene in women, but not in men.
References

Zou et al., 2014

Karppi et al., 2013

Karppi et al., 2011

Riccioni et al., 2011

Riccioni et al., 2011

Dwyer et al., 2004

Gianetti et al., 2002

McQuillan et al., 2001 aortic systolic blood pressure, reduce arterial stiffness, and improve glycemic control, thus acting synergistic with lycopene (Wu et al., 2007; Figueroa et al., 2013, 2017). Grapefruits also include in their composition not just lycopene but also flavonoids, with several benefits, such as the anti-inflammatory and anti-atherogenic effect, improving vascular reactivity, reducing insulin resistance, decreasing arterial stiffness, LDL cholesterol, and blood pressure (Habauzit et al., 2015). These synergistic effects hamper assessment of quantitative and qualitative effects of lycopene as a dietary factor.

Several studies included healthy participants or subjects with different disorders and cardiovascular risk factors (Kong et al., 2010; Thies et al., 2012; Gajendragadkar et al., 2014). Enrolling volunteers with established elevated risk markers for cardiovascular disorders may increase the probability of detecting changes, especially in short time studies (Thies et al., 2012). Also, several other uncontrolled or unidentified lifestyle factors or dietary constituents associated with cardiovascular disorders, may provide alternative explanations for the different study results (Sesso et al., 2003). Genetic factors remain unconsidered at all in all of the reviewed publications, although they are reported to strongly influence circulating concentrations of lycopene in different ethnicities (Zubair et al., 2015). Furthermore, plasma, adipose, and dietary carotenoids are not sufficiently correlated to be interchangeably (Sesso et al., 2003).

\section{FUTURE RESEARCH DIRECTIONS}

Most of the studies considered only tomatoes and tomato products as lycopene source. It will be the aim of future human intervention studies to include other lycopene containing fruits such as watermelon, papaya, red grapefruits, and guava, and consider synergistic effects with other components and their importance in primary and secondary cardiovascular prophylaxis. 
Benefits of lycopene should be especially considered in patients with high cardiovascular risk, statin intolerance, borderline hypertension, aspirin resistance, hyperactive platelets, vascular inflammatory diseases, metabolic syndrome and coronary heart disease, and its inclusion in combination therapies for the mentioned disorders, should be approached. Further mechanistic research is needed to identify new targets for prevention and complementary treatment of cardiovascular disorders.

\section{CONCLUSIONS}

The present review supports the importance of lycopene in improving vascular function and in the primary and secondary prevention of cardiovascular disorders. The demonstrated effects of lycopene in view of cardiovascular health comprise its general antioxidant and anti-inflammatory abilities, the antiplatelet, antiapoptotic and antihypertensive properties, the ability to improve endothelial function, the metabolic profile and ventricular remodeling, reduction of arterial stiffness as well as reduction of size of atherosclerotic plaque. Lycopene exerts favorable effects in patients with subclinical atherosclerosis, metabolic syndrome, hypertension, peripheral vascular disease, and several other cardiovascular disorders, but sometimes conflicting results were obtained. Clearly, more and better-designed studies will be

\section{REFERENCES}

Abdel-Daim, M. M., Eltaysh, R., Hassan, A., and Mousa, S. A. (2018). Lycopene attenuates tulathromycin and diclofenac sodium-induced cardiotoxicity in mice. Int. J. Mol. Sci. 19:e344. doi: 10.3390/ijms19020344

Abushouk, A. I., Ismail, A., Salem, A. M. A., Afifi, A. M., and AbdelDaim, M. M. (2017). Cardioprotective mechanisms of phytochemicals against doxorubicin-induced cardiotoxicity. Biomed. Pharmacother. 90, 935-946. doi: 10.1016/j.biopha.2017.04.033

Agarwal, S., and Rao, A. V. (2000). Tomato lycopene and its role in human health and chronic diseases. Can. Med. Assoc. J. 163, 739-744.

Ahn, J., Lee, H., Jung, C. H., and Ha, T. (2012). Lycopene inhibits hepatic steatosis via microRNA-21-induced downregulation of fatty acid-binding protein 7 in mice fed a high-fat diet. Mol. Nutr. Food Res. 56:1665-1674. doi: 10.1002/mnfr.201200182

Ahuja, K. D., Pittaway, J. K., and Ball, M. J. (2006). Effects of olive oil and tomato lycopene combination on serum lycopene, lipid profile, and lipid oxidation. Nutrition 22, 259-265. doi: 10.1016/j.nut.2005.07.015

Aman, U., Vaibhav, P., and Balaraman, R. (2012). Tomato lycopene attenuates myocardial infarction induced by isoproterenol: electrocardiographic, biochemical and anti-apoptotic study. Asian Pac. J. Trop. Biomed. 2, 345-351. doi: 10.1016/S2221-1691(12)60054-9

Anjos Ferreira, A. L., Russell, R. M., Rocha, N., Placido Ladeira, M. S., Favero Salvadori, D. M., Oliveira Nascimento, M. C., et al. (2007). Effect of lycopene on doxorubicin-induced cardiotoxicity: an echocardiographic, histological and morphometrical assessment. Basic Clin. Pharmacol. Toxicol. 101, 16-24. doi: 10.1111/j.1742-7843.2007.00070.x

Atanasov, A. G., Waltenberger, B., Pferschy-Wenzig, E. M., Linder, T., Wawrosch, C., Uhrin, P., et al. (2015). Discovery and resupply of pharmacologically active plant-derived natural products: a review. Biotechnol. Adv. 33, 1582-1614. doi: 10.1016/j.biotechadv.2015.08.001

Bae, J. W., and Bae, J. S. (2011). Barrier protective effects of lycopene in human endothelial cells. Inflamm. Res. 60:751-758. doi: 10.1007/s00011-011-0330-9

Bansal, P., Gupta, S. K., Ojha, S. K., Nandave, M., Mittal, R., Kumari, S., et al. (2006). Cardioprotective effect of lycopene in the experimental model necessary to improve our understanding of the positive effects of lycopene on vascular health and to elucidate the involved mechanisms on a molecular level.

Future cardiovascular disease prevention strategies might include lycopene-enriched products, lycopene supplementation and new combinations including lycopene. Future studies focused on dietary lycopene and its synergistic effects with other dietary components in different study populations, with elevated cardiovascular risk, are highly warranted and might enable development of functional foods useful in prevention and complementary treatment of cardiovascular disorders.

\section{AUTHOR CONTRIBUTIONS}

IM is the author of the first draft of the manuscript. DS, AC, CM, $\mathrm{JH}$, and AA contributed toward revising the paper and agree to be accountable for all aspects of the work. All authors agreed on the finally submitted version of the manuscript.

\section{ACKNOWLEDGMENTS}

The authors acknowledge the support by the Polish KNOW (Leading National Research Centre) Scientific Consortium Healthy Animal-Safe Food, decision of Ministry of Science and Higher Education No. 05-1/KNOW2/2015. of myocardial ischemia-reperfusion injury. Mol. Cell. Biochem. 289, 1-9. doi: 10.1007/s11010-006-9141-7

Basu, A., and Imrhan, V. (2007). Tomatoes versus lycopene in oxidative stress and carcinogenesis: conclusions from clinical trials. Eur. J. Clin. Nutr. 61, 295-303. doi: $10.1038 /$ sj.ejcn. 1602510

Belovic, M. M., Girones-Vilaplana, A., Moreno, D. A., Milovanovic, I. L. J., Novakovic, A. R., Karaman, M. A., et al. (2016). Tomato (Solanum Lycopersicum L.) processing main product (juice) and by-product (pomace) bioactivity potential measured as antioxidant activity and angiotensinconverting enzyme inhibition. J. Food Process. Preserv. 40, 1229-1237. doi: $10.1111 /$ jfpp. 12707

Blum, A., Monir, M., Khazim, K., Peleg, A., and Blum, N. (2007). Tomato-rich (Mediterranean) diet does not modify inflammatory markers. Clin. Invest. Med. 30, E70-E74. doi: 10.25011/cim.v30i2.982

Böhm, V. (2012). Lycopene and heart health. Mol. Nutr. Food Res. 56, 296-303. doi: $10.1002 / \mathrm{mnfr} .201100281$

Burton-Freeman, B., and Sesso, H. D. (2014). Whole food versus supplement: comparing the clinical evidence of tomato intake and lycopene supplementation on cardiovascular risk factors. Adv. Nutr. 5, 457-485. doi: 10.3945/an.114.005231

Burton-Freeman, B., Talbot, J., Park, E., Krishnankutty, S., and Edirisinghe, I. (2012). Protective activity of processed tomato products on postprandial oxidation and inflammation: a clinical trial in healthy weight men and women. Mol. Nutr. Food Res. 56, 622-631. doi: 10.1002/mnfr.201100649

Cavalcante, J. L., Lima, J. A., Redheuil, A., and Al-Mallah, A. H. (2011). Aortic stiffness: current understanding and future directions. J. Am. Coll. Cardiol. 57, 1511-1522. doi: 10.1016/j.jacc.2010.12.017

Chen, L. P., He, S. Y., Zheng, H., and Dai, Y. L. (2010). Effects and mechanisms of lycopene on the proliferation of vascular smooth muscle cells. Chin. J. Nat. Med. 8, 218-222. doi: 10.3724/SP.J.1009.2010.00218

Cheng, H. M., Koutsidis, G., Lodge, J. K., Ashor, A. W., Siervo, M., and Lara, J. (2017). Lycopene and tomato and risk of cardiovascular diseases: a systematic review and meta-analysis of epiedemiological evidence. Crit. Rev. Food Sci. Nutr. 11, 1-18. doi: 10.1080/10408398.2017.13 62630 
Cooney, M., Cooney, M. T., Maher, V., Khan, B., Leong, T., and Graham, I. (2015). Improvement in the estimation of cardiovascular risk by carotid intima-medial thickness: a report from the Dublin Cardiohealth station study. Prev. Med. Rep. 2, 725-729. doi: 10.1016/j.pmedr.2015.08.004

Costa-Rodrigues, J., Pinho, O., and Monteiro, P. R. R. (2018). Can lycopene be considered an effective protection against cardiovascular disease? Food Chem. 245, 1148-1153. doi: 10.1016/j.foodchem.2017.11.055

Denniss, S. G., Haffner, T. D., Kroetsch, J. T., Davidson, S. R., Rush, J. W., and Hughson, R. (2008). Effect of short-term lycopene supplementation and postprandial dyslipidemia on plasma antioxidants and biomarkers of endothelial health in young, healthy individuals. Vasc. Health Risk Manag. 4, 213-222. doi: 10.2147/vhrm.2008.04.01.213

Dwyer, J. H., Paul-Labrador, M. J., Fan, J., Shircore, A. M., Merz, C. N., and Dwyer, K. M. (2004). Progression of carotid intima-media thickness and plasma antioxidants: the Los Angeles Atherosclerosis Study. Arterioscler. Thromb. Vasc. Biol. 24, 313-319. doi: 10.1161/01.ATV.0000109955.80818.8a

Fantin, F., Di Francesco, V., Rossi, A., Giuliano, K., Marino, F., Cazzadori, M., et al. (2010). Abdominal obesity and subclinical vascular damage in the elderly. J. Hypertens. 28, 333-339. doi: 10.1097/HJH.0b013e328333d23c

Figueroa, A., Wong, A., Hooshmand, S., and Sanchez-Gonzalez, M. A. (2013). Effects of watermelon supplementation on arterial stiffness and wave reflection amplitude in postmenopausal women. Menopause 20, 573-577. doi: 10.1097/GME.0b013e3182733794

Figueroa, A., Wong, A., Jaime, S. J., and Gonzales, J. U. (2017). Influence of L-citrulline and watermelon supplementation on vascular function and exercise performance. Curr. Opin. Clin. Nutr. Metab. Care 20, 92-98. doi: 10.1097/MCO.0000000000000340

Frederiksen, H., Rasmussen, S. E., Schroder, M., Bysted, A., Jakobsen, J., Frandsen, H., et al. (2007). Dietary supplementation with an extract of lycopene-rich tomatoes does not reduce atherosclerosis in Watanabe Heritable Hyperlipidemic rabbits. Br. J. Nutr. 97, 6-10. doi: 10.1017/S0007114507210153

Gajendragadkar, P. R., Hubsch, A., Mäki-Petäjä, K. M., Serg, M., Wilkinson, I. B., and Cheriyan, J. (2014). Effects of oral lycopene supplementation on vascular function in patients with cardiovascular disease and healthy volunteers: a randomised controlled trial. PLOS ONE 9:e99070. doi: 10.1371/journal.pone.0099070

Gammone, M. A., Riccioni, G., and D’Orazio, N. (2015). Carotenoids: potential allies of cardiovascular health? Food Nutr. Res. 59:26762. doi: $10.3402 /$ fnr.v59.26762

Gao, Y., Jia, P., Shu, W., and Jia, D. (2016). The protective effect of lycopene on hypoxia/reoxygenation-induced endoplasmic reticulum stress in H9C2 cardiomyocytes. Eur. J. Pharmacol. 774, 71-79. doi: 10.1016/j.ejphar.2016.02.005

Gianetti, J., Pedrinelli, R., Petrucci, R., Lazzerini, G., De Caterina, M., Bellomo, G., et al. (2002). Inverse association between carotid intima-media thickness and the antioxidant lycopene in atherosclerosis. Am. Heart J. 143, 467-474. doi: $10.1067 / \mathrm{mhj} .2002 .120776$

Gitenay, D., Lyan, B., Rambeau, M., Mazur, A., and Rock, E. (2007). Comparison of lycopene and tomato effects on biomarkers of oxidative stress in vitamin $\mathrm{E}$ deficient rats. Eur. J. Nutr. 46, 468-475. doi: 10.1007/s00394-007-0687-2

Goff, D. C. Jr, Lloyd-Jones, D. M., Bennett, G., Coady, S., D’Agostino, R. B., sr, Gibbons, R., et al. (2014). 2013 ACC/AHA guideline on the assessment of cardiovascular risk: a report of the American College of Cardiology/American Heart Association Task Force on Practice Guidelines. J. Am. Coll. Cardiol. 63(25 Pt B), 2935-2959. doi: 10.1016/j.jacc.2013.11.005

Gouranton, E., Thabuis, C., Riollet, C., Malezet-Desmoulins, C., El Yazidi, C., Amiot, M. J., et al. (2011). Lycopene inhibits proinflammatory cytokine and chemokine expression in adipose tissue. J. Nutr. Biochem. 22, 642-648. doi: 10.1016/j.jnutbio.2010.04.016

Habauzit, V., Verny, M. A., Milenkovic, D., Barber-Chamoux, N., Mazur, A., Dubray, C., et al. (2015). Flavanones protect from arterial stiffness in postmenopausal women consuming grapefruit juice for $6 \mathrm{mo}$ : a randomized, controlled, crossover trial. Am. J. Clin. Nutr. 102, 66-74. doi: 10.3945/ajcn.114.104646

Han, G. M., and Liu, P. (2017). Higher serum lycopene is associated with reduced prevalence of hypertension in overweight or obese adults. Eur. J. Integr. Med. 13, 34-40. doi: 10.1016/j.eujim.2017.07.002
Han, G. M., Meza, J. L., Soliman, G. A., Islam, K. M., and Watanabe-Galloway, S. (2016a). Higher levels of serum lycopene are associated with reduced mortality in individuals with metabolic syndrome. Nutr. Res. 36, 402-407. doi: 10.1016/j.nutres.2016.01.003

Han, G. M., Soliman, G. A., Meza, J. L., Islam, K. M., and WatanabeGalloway, S. (2016b). The influence of BMI on the association between serum lycopene and the metabolic syndrome. Br. J. Nutr. 115, 1292-1300. doi: 10.1017/S0007114516000179

He, Q., Kong, X., Wu, G., Ren, P., Tang, H., Hao, F., et al. (2009). Metabolomic analysis of the response of growing pigs to dietary L-arginine supplementation. Amino Acids 37, 199-208. doi: 10.1007/s00726-008-0192-9

He, Q., Zhou, W., Xiong, C., Tan, G., and Chen, M. (2015). Lycopene attenuates inflammation and apoptosis in post-myocardial infarction remodeling by inhibiting the nuclear factor-kappaB signaling pathway. Mol. Med. Rep. 11, 374-378. doi: 10.3892/mmr.2014.2676

He, Y., Xia, P., Jin, H., Zhang, Y., Chen, B., and Xu, Z. (2016). Lycopene ameliorates transplant arteriosclerosis in vascular allograft transplantation by regulating the NO/cGMP pathways and Pho-associated kinases expression.Oxid. Med. Cell Longev. 2016:3128280. doi: 10.1155/2016/3128280

Heber, D., and Lu, Q. Y. (2002). Overview of mechanisms of action of lycopene. Exp. Biol. Med. 227, 920-923. doi: 10.1177/153537020222701013

Hollman, P. C. H., Cassidy, A., Comte, B., Heinonen, M., Richelle, M., Richling, E., et al. (2011). The biological relevance of direct antioxidant effects of polyphenols for cardiovascular health in humans is not established. J. Nutr. 141, 989S-1009S. doi: 10.3945/jn.110.131490

Hong, M. Y., Hartig, N., Kaufman, K., Hooshmand, S., Figueroa, A., and Kern, M. (2015). Watermelon consumption improves inflammation and antioxidant capacity in rats fed an atherogenic diet. Nutr. Res. 5, 251-258. doi: 10.1016/j.nutres.2014.12.005

Hosseini, B., Saedisomeolia, A., and Skilton, M. R. (2017). Association between micronutrients intake/status and carotid intima media thickness: a systematic review. J. Acad. Nutr. Diet. 117, 69-82. doi: 10.1016/j.jand.2016.09.031

Hsu, Y. M., Lai, C. H., Chang, C. Y., Fan, C. T., Chen, C. T., and Wu, C. H. (2008). Characterizing the lipid-lowering effects and antioxidant mechanisms of tomato paste. Biosci. Biotechnol. Biochem. 72, 677-685. doi: 10.1271/bbb.70402

Hu, M. Y., Li, Y. L., Jiang, C. H., Liu, Z. Q., Qu, S. L., and Huang, Y. M. (2008). Comparison of lycopene and fluvastatin effects on atherosclerosis induced by a high-fat diet in rabbits. Nutrition 24, 1030-1038. doi: 10.1016/j.nut.2008.05.006

Hung, C. F., Huang, T. F., Chen, B. H., Shieh, J. M., Wu, P. H., and Wu, W. B. (2008). Lycopene inhibits TNF-alpha-induced endothelial ICAM1 expression and monocyte-endothelial adhesion. Eur. J. Pharmacol. 586, 275-282. doi: 10.1016/j.ejphar.2008.03.001

Ito, Y., Kurata, M., Suzuki, K., Hamajima, N., Hishida, H., and Aoki, K. (2006). Cardiovascular disease mortality and serum carotenoid levels: a Japanese population-based follow-up study. J. Epidemiol. 16, 154-160. doi: 10.2188 /jea.16.154

Jobgen, W. S., Fried, S. K., Fu, W. J., Meininger, C. J., and Wu, G. (2006). Regulatory role for the arginine-nitric oxide pathway in metabolism of energy substrates. $J$. Nutr. Biochem. 17:571-588. doi: 10.1016/j.jnutbio.2005.12.001

Karagiannis, G. S., Weile, J., Bader, G. D., and Minta, J. (2013). Integrative pathway dissection of molecular mechanisms of moxLDL-induced vascular smooth muscle phenotype transformation. BMC Cardiovasc. Disord. 13:4. doi: 10.1186/1471-2261-13-4

Karimi, G., Ramezani, M., and Abdi, A. (2005). Protective effects of lycopene and tomato extract against doxorubicin-induced cardiotoxicity. Phytother. Res. 19, 912-914. doi: 10.1002/ptr.1746

Karppi, J., Kurl, S., Laukkanen, J. A., Rissanen, T. H., and Kauhanen, J. (2011). Plasma carotenoids are related to intima-media thickness of the carotid artery wall in men from eastern Finland. J. Intern. Med. 270 , 478-485. doi: 10.1111/j.1365-2796.2011.02401.x

Karppi, J., Kurl, S., Ronkainen, K., Kauhanen, J., and Laukkanen, J. A. (2013). Serum carotenoids reduce progression of early atherosclerosis in the carotid artery wall among Eastern Finnish men. PLoS ONE 8:e64107. doi: 10.1371/journal.pone.0064107

Karppi, J., Laukkanen, J. A., Mäkikallio, T. H., and Kurl, S. (2012). Low serum lycopene and $\beta$-carotene increase risk of acute myocardial infarction in men. Eur. J. Public Health 22, 835-840. doi: 10.1093/eurpub/ckr174 
Khan, N. I., Noori, S., and Mahboob, T. (2016). Efficacy of lycopene on modulation of renal antioxidant enzymes, ACE and ACE gene expression in hyperlipidaemic rats. J. Renin. Angiotensin Aldosterone Syst. 17:1470320316664611. doi: 10.1177/1470320316664611

Kim, G. H., and Youn, H. J. (2017). Is carotid artery ultrasound still useful method for evaluation of atherosclerosis? Korean Circ. J. 47, 1-8. doi: 10.4070/kcj.2016.0232

Kim, J. Y., Paik, J. K., Kim, O. Y., Park, H. W., Lee, J. H., et al. (2011). Effects of lycopene supplementation on oxidative stress and markers of endothelial function in healthy men. Atherosclerosis 215, 189-195. doi: 10.1016/j.atherosclerosis.2010.11.036

Kim, O. Y., Yoe, H. Y., Kim, H. J., Park, J. Y., Kim, J. Y., Lee, S. H., et al. (2010). Independent inverse relationship between serum lycopene concentration and arterial stiffness. Atherosclerosis 208, 581-586. doi: 10.1016/j.atherosclerosis.2009.08.009

Klipstein-Grobusch, K., Launer, L. J., Geleijnse, J. M., Boeing, H., Hofman, A., and Witteman, J. C. (2000). Serum carotenoids and atherosclerosis. The Rotterdam study. Atherosclerosis 148, 49-56. doi: 10.1016/S0021-9150(99)00221-X

Kong, K. W., Khoo, H. E., Prasad, K. N., Ismail, A., Tan, C. P., and Rajab, N. F. (2010). Revealing the power of the natural red pigment lycopene. Molecules 15, 959-987. doi: 10.3390/molecules15020959

Krasinska, B., Osinaka, A., Osinski, M., Krasinska, A., Rzymski, P., Tykarshi, A., et al. (2017). Standardised tomato extract as an alternative to acetylsalicylic acid in patients with primary hypertension and high cardiovascular risk - a randomized, controlled trial. Arch. Med. Sci. doi: 10.5114/aoms.2017. 69864

Lee, W., Ku, S. K., Bae, J. W., and Bae, J. S. (2012). Inhibitory effects of lycopene on HMGB1-mediated pro-inflammatory responses in both cellular and animal models. Food Chem. Toxicol. 50, 1826-1833. doi: 10.1016/j.fct.2012.03.003

Li, X. N., Lin, J., Xia, J., Qin, L., Zhu, S. Y., and Li, J. L. (2017). Lycopene mitigates atrazine-induced cardiac inflammation via blocking the NF-kB pathway and NO production. J. Functs Foods 29, 208-216. doi: 10.1016/j.jff.2016.12.029

$\mathrm{Li}, \mathrm{X}$., and $\mathrm{Xu}, \mathrm{J}$. (2013). Lycopene supplement and blood pressure: an updated meta-analysis of intervention trials. Nutrients 5, 3696-3712. doi: $10.3390 /$ nu5093696

Li, Y. F., Chang, Y. Y., Huang, H. C., Wu, Y. C., Yang, M. D., and Chao, P. M. (2015). Tomato juice supplementation in young women reduces inflammatory adipokine levels independently of body fat reduction. Nutrition 31, 691-696. doi: 10.1016/j.nut.2014.11.008

Lo, H. M., Hung, C. F., Tseng, Y. L., Chen, B. H., Jian, J. S., and Wu, W. B. (2007). Lycopene binds PDGF-BB and inhibits PDGF-BB-induced intracellular signaling transduction pathway in rat smooth muscle cells. Biochem. Pharmacol. 74, 54-63. doi: 10.1016/j.bcp.2007.03.017

Lomb, D. J., Laurent, G., and Haigis, M. C. (2010). Sirtuins regulate key aspects of lipid metabolism. Biochim. Biophys. Acta 1804:1652-1657. doi: 10.1016/j.bbapap.2009.11.021

Lorenz, M., Fechner, M., Kalkowski, J., Fröhlich, K., Trautmann, A., Böhm, V., et al. (2012). Effects of lycopene on the initial state of atherosclerosis in New Zeeland White (NZW) rabbits. PLoS ONE 7:e30808. doi: 10.1371/journal.pone.0030808

Manabe, I., and Nagai, R. (2003). Regulation of smooth muscle phenotype. Curr. Atheroscler. Rep. 5, 214-222. doi: 10.1007/s11883-003-0027-9

Mantzouridou, F., and Tsimidou, M. Z. (2008). Lycopene formation in Blakeslea trispora. Chemical aspects of a bioprocess. Trends Food Sci. Technol. 19, 363-371. doi: 10.1016/j.tifs.2008.01.003

McEneny, J., Wade, L., Young, I. S., Masson, L., Duthie, G., McGinty, A., et al. (2013). Lycopene intervention reduces inflammation and improves HDL functionality in moderately overweight middle-aged individuals. J. Nutr. Biochem. 24, 163-168. doi: 10.1016/j.jnutbio.2012.03.015

McQuillan, B. M., Hung, J., Beilby, J. P., Nidorf, M., and Thompson, P. L. (2001). Antioxidant vitamins and the risk of carotid atherosclerosis. Perth Carotid Ultrasound Disease Assessment study (CUDAS). J. Am. Coll. Cardiol. 38, 1788-1794. doi: 10.1016/S0735-1097(01)01676-X

Milani, A., Basirnejad, M., Shahbazi, S., and Bolhassani, A. (2017). Carotenoids: biochemistry, pharmacology and treatment. Br. J. Pharmacol. 174, 1290-1324. doi: 10.1111/bph.13625

Mohamadin, A. M., Elberry, A. A., Mariee, A. D., Morsy, G. M., and AlAbbasi, F. A. (2012). Lycopene attenuates oxidative stress and heart lysosomal damage in isoproterenol induced cardiotoxicity in rats: a biochemical study. Pathophysiology 19, 121-130. doi: 10.1016/j.pathophys.2012.04.005

Mozos, I., Borzak, G., Caraba, A., and Mihaescu, R. (2017a). Arterial stiffness in hematologic malignancies. Onco. Targets. Ther. 10, 1381-1388. doi: 10.2147/OTT.S126852

Mozos, I., and Luca, C. T. (2017). Crosstalk between oxidative and nitrosative stress and arterial stiffness. Curr. Vasc. Pharmacol. 15, 446-456. doi: 10.2174/1570161115666170201115428

Mozos, I., Maidana, J. P., Stoian, D., and Stehlik, M. (2017b). Gender differences of arterial stiffness and arterial age in smokers. Int. J. Environ. Res. Public Health 14:565. doi: 10.3390/ijerph14060565

Mozos, I., Stoian, D., and Luca, C. T. (2017c). Crosstalk between vitamins A, B12, D, K, C and E status and arterial stiffness. Dis. Markers 10, 1381-1388. doi: $10.1155 / 2017 / 8784971$

Müller, L., Caris-Veyrat, C., Lowe, G., and Böhm, V. (2016). Lycopene and its antioxidant role in the prevention of cardiovascular diseases - A critical review. Crit. Rev. Sci. Nutr. 56, 1868-1879. doi: 10.1080/10408398.2013.801827

Müller-Nordhorn, J., Binting, S., Roll, S., and Willich, S. N. (2008). An update on regional variation in cardiovascular mortality within Europe. Eur. Heart J. 29, 1316-1326. doi: 10.1093/eurheartj/ehm604

Nakamura, A., Itaki, C., Saito, A., Yonezawa, T., Aizawa, K., Hirai, A., et al. (2017). Possible benefits of tomato juice consumption: a pilot study on irradiation human lymphocytes from healthy donors. Nutr. J. 16:27. doi: 10.1186/s12937-017-0248-3

Napolitano, M., De Pascale, C., Wheeler-Jones, C., Botham, K. M., and Bravo, E. (2007). Effects of lycopene on the induction of foam cell formation by modified LDL. Am. J. Physiol. Endocrinol. Metab. 293, E1820-E1827. doi: 10.1152/ajpendo.00315.2007

Naz, A., Butt, M. S., Sultan, M. T., Qayyum, M. M., and Niaz, R. S. (2014). Watermelon lycopene and allied health claims. EXCLI J. 13, 650-660.

Oberoi, D. P. S., and Sogi, D. S. (2017). Utilization of watermelon pulp for lycopene extraction by response surface methodology. Food Chem. 232, 316-321. doi: 10.1016/j.foodchem.2017.04.038

O'Kennedy, N., Raederstorff, D., and Duttaroy, A. K. (2017). Fruitflow ${ }^{\mathrm{R}}$ : the first European Food Safety Authority-approved natural cardio-protective functional ingredient. Eur. J. Nutr. 56, 461-482. doi: 10.1007/s00394-016-1265-2

O'Leary, D. H., Polak, J. F., Kronmal, R. A., Manolio, T. A., Burke, G. L., Wolfson, S. K., et al. (1999). Carotid-artery intima and media thickness as a risk factor for myocardial infarction and stroke in older adults. Cardiovascular health study collaborative research group. N. Engl. J. Med. 340, 14-22. doi: 10.1056/NEJM199901073400103

Opatrilova, R., Kubatka, P., Caprnda, M., Büsselberg, D., Krasnik, V., Veselz, P., et al. (2017). Nitric oxide in the pathophysiology of retinopathy: evidences from preclinical and clinical research. Acta Ophthalmol. 96, 222-231. doi: 10.1111/aos.13384

Palozza, P., Simone, R., Catalano, A., Monego, G., Barini, A., Mele, M. C., et al. (2011). Lycopene prevention of oxysterol-induced proinflammatory cytokine cascade in human macrophages: inhibition of NF- $\mathrm{KB}$ nuclear binding and increase in PPAR $\gamma$ expression. J. Nutr. Biochem. 22, 259-268. doi: 10.1016/j.jnutbio.2010.02.003

Paran, E., Novack, V., Engelhard, Y. N., and Hazan-Halevy, I. (2009). The effects of natural antioxidants from tomato extract in treated but uncontrolled hypertensive patients. Cardiovasc. Drugs Ther. 23, 145-151. doi: 10.1007/s10557-008-6155-2

Pereira, B. L. B., Reis, P. P., Severino, F. E., Felix, T. F., Braz, M. G., Nogueira, F. R., et al. (2017). Tomato (Lycopersicon esculentum) or lycopene supplementation attenuates ventricular remodeling after myocardial infarction through different mechanistic pathways. J. Nutr. Biochem. 46:117-124. doi: 10.1016/j.jnutbio.2017.05.010

Perera, C. O., and Yen, G. M. (2007). Functional properties of carotenoids in human health. Int. J. Food Propert. 10, 201-230. doi: 10.1080/10942910601045271

Piepoli, M. F., Hoes, A. W., Agewall, S., Albus, C., Brotons, C., Catapano, A. L., et al. (2016). European Guidelines on cardiovascular disease prevention in clinical practice. The Sixth Joint Task Force of the European Society of Cardiology and Other Societies on Cardiovascular Disease Prevention in Clinical Practice (constituted by representatives of 10 societies and by invited experts). Developed with the special contribution of the European Association 
for Cardiovascular Prevention and Rehabilitation (EACPR). Eur. Heart J. 37, 2315-2381. doi: 10.1093/eurheartj/ehw106

Pisoschi, A. M., and Pop, A. (2015). The role of antioxidants in the chemistry of oxidative stress: a review. Eur. J. Med. Chem. 97:55-74. doi: 10.1016/j.ejmech.2015.04.040

Pleskovic, A., Letonja, M. S., Vujkovac, A. C., Nikolajevic Starcevic, J., Caprnda, M., Curilla, E., et al. (2017). Matrix metalloproteinase-3 gene polymorphism (rs3025058) affects markers atherosclerosis in type 2 diabetes mellitus. VASA 19, 1-7. doi: 10.1024/0301-1526/a000637

Rao, A. V. (2002). Lycopene, tomatoes, and the prevention of coronary heart disease. Exp. Biol. Med. 227, 908-913. doi: 10.1177/153537020222701011

Ren, X. S., Tong, Y., Ling, L., Chen, D., Sun, H. J., and Zhou, H. (2017). NLRP3 gene deletion attenuates angiotensin ii-induced phenotypic transformation of vascular smooth muscle cells and vascular remodeling. Cell. Physiol. Biochem. 44, 2269-2280. doi: 10.1159/000486061

Riccioni, G., Orazio, D. N., Palumbo, N., Bucciarelli, V., Ilio Ed, Bazzano, L. A., et al. (2009). Relationship between plasma antioxidant concentrations and carotid intima-media thickness: the Asymptomatic Carotid Atherosclerotic Disease in Manfredonia Study. Eur. J. Cardiovasc. Prev. Rehabil. 16, 351-357. doi: 10.1097/HJR.0b013e328325d807

Riccioni, G., D’Orazio, N., Speranza, L., Di Ilio, E., Glaude, M., Bucciarelli, V., et al. (2010). Carotenoids and asymptomatic carotid atherosclerosis. J. Biol. Regul. Homeost. Agents 24, 447-452.

Riccioni, G., Scotti, L., Di Ilio, E., Bucciarelli, V., Ballone, E., De Girolamo, M., et al. (2011). Lycopene and preclinical carotid atherosclerosis. J. Biol. Regul. Homeost. Agents 25, 435-441.

Ried, K., and Fakler, P. (2011). Protective effect of lycopene on serum cholesterol and blood pressure: meta-analyses of intervention trials. Maturitas 68, 299-310. doi: 10.1016/j.maturitas.2010.11.018

Rissanen, T. H., Voutilainen, S., Nyyssönen, K., Lakka, T. A., Sivenius, J., Salonen, R., et al. (2001). Low serum lycopene concentration is associated with an excess incidence of acute coronary events and stroke: the Kuopio ischaemic heart disease risk factor study. Br. J. Nutr. 85, 749-754. doi: 10.1079/BJN2001357

Saini, R. K., Zamany, A. J., and Keum, Y. S. (2017). Ripening improves the content of carotenoid, alpha-tocopherol, and polyunsaturated fatty acids in tomato (Solanum lycopersicum L.) fruits. 3 Biotech 7:43. doi: 10.1007/s13205-017-0666-0

Sawardekar, S. B., Patel, T. C., and Uchil, D. (2016). Comparative evaluation of antiplatelet effect of lycopene with aspirin and the effect of their combination on platelet aggregation: an in vitro study. Indian J. Pharmacol. 48, 26-31. doi: $10.4103 / 0253-7613.174428$

Schönthal, A. H. (2012). Endoplasmic reticulum stress: its role in disease and novel prospects for therapy. Scientifica 2012:857516 doi: 10.6064/2012/857516

Sellers, R. S., Radi, Z. A., and Khan, N. K. (2010). Pathophysiology of cyclooxygenases in cardiovascular homeostasis. Vet. Pathol. 47, 601-613. doi: 10.1177/0300985810364389

Sesso, H. D., Liu, S., Gaziano, J. M., and Buring, J. E. (2003). Dietary lycopene, tomato-based food products and cardiovascular disease in women. J. Nutr. 133, 2336-2341. doi: 10.1093/jn/133.7.2336

Shi, J., and Le Maguer, M. (2000). Lycopene in tomatoes: chemical and physical properties affected by food processing. Crit. Rev. Biotechnol. 20, 293-334. doi: $10.1080 / 07388550091144212$

Song, B., Liu, K., Gao, Y., Zhao, L., Fang, H., Li, Y., et al. (2017). Lycopene and risk of cardiovascular diseases: a meta-analysis of observational studies. Mol. Nutr. Food Res. 61:1601009. doi: 10.1002/mnfr.201601009

Stangl, V., Kuhn, C., Hentschel, S., Jochmann, N., Jacob, C., Böhm, V., Fröhlich, K., et al. (2011). Lack of effects of tomato products on endothelial function in human subjects: results of a randomised, placebo-controlled cross-over study. Br. J. Nutr. 105, 263-267. doi: 10.1017/S0007114510003284

Sultan Alvi, S., Ansari, I. A., Khan, I., Iqbal, J., and Khan, M. S. (2017). Potential role of lycopene in targeting proprotein convertase subtilisin/kexin type-9 to combat hypercholesterolemia. Free Radic. Biol. Med. 108, 394-403. doi: 10.1016/j.freeradbiomed.2017.04.012

Sung, H. J., Eskin, S. G., Sakurai, Y., Yee, A., Kataoka, N., and McIntire, L. V. (2005). Oxidative stress produced with cell migration increases synthetic phenotype of vascular smooth muscle cells. Ann. Biomed. Eng. 33, 1546-1554. doi: 10.1007/s10439-005-7545-2
Tabrez, S., Al-Shali, K. Z., and Ahmad, S. (2015). Lycopene powers the inhibition of glycation-induced diabetic nephropathy: a novel approach to halt the AGERAGE axis menace. Biofactors 41, 372-381. doi: 10.1002/biof.1238

Tanazawa, K., Shimada, Y., Kuroda, M., Tsujita, Y., Arai, M., and Watanabe, H. (1980). WHHL-rabbit: a low density lipoprotein receptor-deficient animal model for familial hypercholesterolemia. FEBS Lett. 118, 81-84. doi: 10.1016/0014-5793(80)81223-3

Tang, X., Yang, X., Peng, Y., and Lin, J. (2009). Protective effects of lycopene against $\mathrm{H} 2 \mathrm{O} 2$-induced oxidative injury and apoptosis in human endothelial cells. Cardiovasc. Drugs Ther. 23, 439-498. doi: 10.1007/s10557-009-6206-3

Thies, F., Masson, L. F., Rudd, A., Vaughan, N., Tsang, K., Brittenden, J., et al. (2012). Effect of a tomato-rich diet on markers of cardiovascular disease risk in moderately overweight, disease-free, middle-aged adults: a randomized controlled trial. Am. J. Clin. Nutr. 95, 1013-1022. doi: 10.3945/ajcn.111.026286

Thies, F., Mills, L. M., Moir, S., and Masson, L. F. (2017). Cardiovascular benefits of lycopene: fantasy or reality? Proc. Nutr. Soc. 76, 122-129. doi: $10.1017 /$ S0029665116000744

Touboul, P. J. (2015). Intima-media thickness of carotid arteries. Front. Neurol. Neurosci. 36, 31-39. doi: 10.1159/000366234

Tsitsimpikou, C., Tsarouhas, K., Kioukia-Fougia, N., Skondra, C., Fragkiadaki, P., Papalexis, P., et al. (2014). Dietary supplementation with tomato-juice in patients with metabolic syndrome: a suggestion to alleviate detrimental clinical factors. Food Chem. Toxicol. 74, 9-13. doi: 10.1016/j.fct.2014.08.014

Vasconcelos, A. G., Amorim, A. D. G. N., Dos Santos, R. C., Souza, J. M. T., de Souza, L. K. M., Araújo, T. S. L., et al. (2017). Lycopene rich extract from red guava (Psidium guajava L.) displays anti-inflammatory and antioxidant profile by reducing suggestive hallmarks of acute inflammatory response in mice. Food Res. Int. 99(Pt 2), 959-968. doi: 10.1016/j.foodres.2017.01.017

Verghese, M., Richardson, J. E., Boateng, J., Shackelford, L. A., Howard, C., Walker, L. T., et al. (2008). Dietary lycopene has a protective effect on cardiovascular disease in New Zeeland male rabbits. J. Biol. Sci. 8, 268-277. doi: $10.3923 /$ jbs.2008.268.277

Viuda-Martos, M., Sanchez-Zapata, E., Sayas-Barberá, E., Sendra, E., PérezÁlvarez, J. A., and Fernández-López, J. (2014). Tomato and tomato byproducts. Human health benefits of lycopene and its application to meat products: a review. Crit. Rev. Food Sci. Nutr. 54, 1032-1049. doi: 10.1080/10408398.2011.623799

Vlachopoulos, C., Aznaouridis, K., and Stefanadis, C. (2010). Prediction of cardiovascular events and all-cause mortality with arterial stiffness. A systematic review and meta-analysis. J. Am. Coll. Cardiol. 55, 1318-1327. doi: 10.1016/j.jacc.2009.10.061

Waltenberger, B., Mocan, A., Šmejkal, K., Heiss, E. H., and Atanasov, A. G. (2016). Natural products to counteract the epidemic of cardiovascular and metabolic disorders. Molecules 21:807. doi: 10.3390/molecules 21060807

Wang, X., Lv, H., Gu, Y., Wang, X., Cao, H., Tang, Y., et al. (2014). Protective effect of lycopene on cardiac function and myocardial fibrosis after acute myocardial infarction in rats via the modulation of p38 and MMP-9. J. Mol. Histol. 45, 113-120. doi: 10.1007/s10735-013-9535-2

Wolak, T., and Paran, E. (2013). Can carotenoids attenuate vascular aging? Vascul. Pharmacol. 59, 63-66. doi: 10.1016/j.vph.2013.07.006

Wong, Z. W., Thanikachalam, P. V., and Ramamurthy, S. (2017). Molecular understanding of the protective role of natural products on isoproterenolinduced myocardial infarction: a review. Biomed. Pharmacother. 94, 1145-1166. doi: 10.1016/j.biopha.2017.08.009

Wood, N., and Johnson, R. B. (2004). The relationship between tomato intake and congestive heart failure risk in periodontitis subjects. J. Clin. Periodontol. 31, 574-580. doi: 10.1111/j.1600-051X.2004.00531.X

Wu, G., Collins, J. K., Perkins-Veazie, P., Siddiq, M., Dolan, K. D., Kelly, K. A., et al. (2007). Dietary supplementation with watermenlon pomace juice enhances arginine availability and ameliorates the metabolic syndrome in Zucker diabetic fatty rats. J. Nutr. 137, 2680-2685. doi: 10.1093/jn/137.12.2680

Xaplanteris, P., Pietri, P., Terentes-Printzios, D., Kardara, D., Alexopoulos, N., Aznaouridis, K., et al. (2012). Tomato paste supplemen-tation improves endothelial dynamics and reduces plasma total oxida-tive status in healthy subjects. Nutr. Res. 32, 390-394. doi: 10.1016/j.nutres.2012.03.011

Xu, J., Hu, H., Chen, B., Yue, R., Zhou, Z., Liu, Y., et al. (2015). Lycopene protects against hypoxia/reoxygenation injury by alleviating er stress induced 
apoptosis in neonatal mouse cardiomyocytes. PLoS ONE 10:e136443. doi: 10.1371/journal.pone.0136443

Xu, X. R., Zou, Z. Y., Huang, Y. M., Xiao, X., Ma, L., and Lin, X. M. (2012). Serum carotenoids in relation to risk factors for development of atherosclerosis. Clin. Biochem. 45, 1357-1361. doi: 10.1016/j.clinbiochem.2012.07.101

Yeo, H. Y., Kim, O. Y., Lim, H. H., Kim, J. Y., and Lee, J. H. (2011). Association of serum lycopene and brachial-ankle pulse wave velocity with metabolic syndrome. Metab. Clin. Exp. 60, 537-543. doi: 10.1016/j.metabol.2010.05.003

Zeng, Y. C., Peng, L. S., Zou, L., Huang, S. F., Xie, Y., Mu, G. P., et al. (2017). Protective effect and mechanism of lycopene on endothelial progenitor cells (EPCs) from type 2 diabetes mellitus rats. Biomed. Pharmacother. 92, 86-94, doi: 10.1016/j.biopha.2017.05.018

Zhang, Y., Guallar, E., Qiao, Y., and Wasserman, B. A. (2014). Is carotid intimamedia thickness as predictive as other noninvasive techniques for the detection of coronary artery disease? Arterioscler. Thromb. Vasc. Biol. 34, 1341-1345. doi: 10.1161/ATVBAHA.113.302075

Zou, Z. Y., Xu, X. R., Lin, X. M., Zhang, H. B., Xiao, X., Ouyang, L., et al. (2014). Effects of lutein and lycopene on carotid intimamedia thickness in Chinesesubjects with subclinical atherosclerosis: a randomised, double-blind, placebo-controlled trial. Br. J. Nutr. 111, 474-480. doi: 10.1017/S0007114513002730

Zubair, N., Kooperberg, C., Liu, J., Di, C., Peters, U., and Neuhouser, M. L. (2015). Genetic variation predicts serum lycopene concentrations in a multiethnic population of postmenopausal women. J. Nutr. 145, 187-192. doi: 10.3945/jn.114.2 02150

Conflict of Interest Statement: The authors declare that the research was conducted in the absence of any commercial or financial relationships that could be construed as a potential conflict of interest.

Copyright (C) 2018 Mozos, Stoian, Caraba, Malainer, Horbańczuk and Atanasov. This is an open-access article distributed under the terms of the Creative Commons Attribution License (CC BY). The use, distribution or reproduction in other forums is permitted, provided the original author(s) and the copyright owner are credited and that the original publication in this journal is cited, in accordance with accepted academic practice. No use, distribution or reproduction is permitted which does not comply with these terms. 


\section{OPEN ACCESS}

Edited by:

Judith Maria Rollinger,

Universität Wien, Austria

Reviewed by:

Wentzel Christoffel Gelderblom,

Cape Peninsula University

of Technology, South Africa

Subhalakshmi Ghosh,

Independent Researcher, India

*Correspondence:

Amelie Lupp

Amelie.Lupp@med.uni-jena.de

Specialty section:

This article was submitted to

Ethnopharmacology,

a section of the journal

Frontiers in Pharmacology

Received: 12 January 2018

Accepted: 04 April 2018

Published: 20 April 2018

Citation:

Loeser K, Seemann S, König S,

Lenhardt I, Abdel-Tawab M,

Koeberle A, Werz O and Lupp A

(2018) Protective Effect

of Casperome ${ }^{\circledR}$

an Orally Bioavailable Frankincense Extract, on Lipopolysaccharide-

Induced Systemic Inflammation

in Mice. Front. Pharmacol. 9:387.

doi: 10.3389/fphar.2018.00387

\section{Protective Effect of Casperome ${ }^{\circledR}$, an Orally Bioavailable Frankincense Extract, on Lipopolysaccharide- Induced Systemic Inflammation in Mice}

\author{
Konstantin Loeser 1,2, Semjon Seemann ${ }^{1}$, Stefanie König ${ }^{2}$, Isabell Lenhardt', \\ Mona Abdel-Tawab ${ }^{3}$, Andreas Koeberle ${ }^{2}$, Oliver Werz ${ }^{2}$ and Amelie Lupp ${ }^{1 *}$ \\ ${ }^{1}$ Institute of Pharmacology and Toxicology, Jena University Hospital, Jena, Germany, ${ }^{2}$ Chair of Pharmaceutical Medicinal \\ Chemistry, Institute of Pharmacy, Friedrich Schiller University Jena, Jena, Germany, ${ }^{3}$ Central Laboratory of German \\ Pharmacists, Eschborn, Germany
}

Introduction: Despite recent advances in critical care, sepsis remains a crucial cause of morbidity and mortality in intensive care units. Therefore, the identification of new therapeutic strategies is of great importance. Since ancient times, frankincense is used in traditional medicine for the treatment of chronic inflammatory disorders such as rheumatoid arthritis. Thus, the present study intends to evaluate if Casperome ${ }^{\circledR}$ (Casp), an orally bioavailable soy lecithin-based formulation of standardized frankincense extract, is able to ameliorate systemic effects and organ damages induced by severe systemic inflammation using a murine model of sepsis, i.e., intraperitoneal administration of lipopolysaccharides (LPS).

Methods: Male 60-day-old mice were assigned to six treatment groups: (1) control, (2) LPS, (3) soy lecithin (blank lecithin without frankincense extract), (4) Casp, (5) soy lecithin plus LPS, or (6) Casp plus LPS. Soy lecithin and Casp were given $3 \mathrm{~h}$ prior to LPS treatment; $24 \mathrm{~h}$ after LPS administration, animals were sacrificed and health status and serum cytokine levels were evaluated. Additionally, parameters representing liver damage or liver function and indicating oxidative stress in different organs were determined. Furthermore, markers for apoptosis and immune cell redistribution were assessed by immunohistochemistry in liver and spleen.

Results: LPS treatment caused a decrease in body temperature, blood glucose levels, liver glycogen content, and biotransformation capacity along with an increase in serum cytokine levels and oxidative stress in various organs. Additionally, apoptotic processes were increased in spleen besides a pronounced immune cell infiltration in both liver and spleen. Pretreatment with Casp significantly improved health status, blood glucose values, and body temperature of the animals, while serum levels of pro-inflammatory cytokines and oxidative stress in all organs tested were significantly diminished. Finally, apoptotic processes in spleen, liver glycogen loss, and immune cell infiltration in liver and spleen were distinctly reduced. Casp also appears to induce various cytochrome 
P450 isoforms, thus causing re-establishment of liver biotransformation capacity in LPStreated mice.

Conclusion: Casp displayed anti-inflammatory, anti-oxidative, and hepatoprotective effects. Thus, orally bioavailable frankincense extracts may serve as a new supportive treatment option in acute systemic inflammation and accompanied liver dysfunction.

Keywords: frankincense, lipopolysaccharides, systemic inflammation, cytokines, oxidative stress, liver function

\section{INTRODUCTION}

Despite recent advances in critical care, sepsis, septic shock, and subsequent multi-organ failure remain an important cause of morbidity and mortality in intensive care units (Engel et al., 2007; Martin, 2012; Gaieski et al., 2013). About $20-25 \%$ of patients with sepsis and associated organ failure display early impaired liver function, which in severe cases can result in acute liver failure (Kramer et al., 2007; Koch et al., 2011; Nesseler et al., 2012; Horvatits et al., 2013; Yan et al., 2014). Such liver dysfunction seems to be mediated to a considerable extent by pro-inflammatory cytokines such as TNF- $\alpha$, IL- 6 , and IL- 8 that are liberated by resident macrophages of the liver, the so-called Kupffer cells (Nesseler et al., 2012; Yan et al., 2014). Moreover, it has been shown that the severity of liver damage (even more than the impairment of lung or kidney function) is of major consequence for overall patient outcome (Kramer et al., 2007; Koch et al., 2011; Nesseler et al., 2012; Horvatits et al., 2013; Yan et al., 2014). However, although extensive research efforts have been made, no specific therapy exists so far for the treatment of sepsis and of sepsis-associated liver dysfunction (Nesseler et al., 2012; Horvatits et al., 2013). Therefore, the identification of new therapeutic strategies is of great clinical importance (Yan et al., 2014).

Extracts of frankincense gum resins from different Boswellia species have been used for centuries in traditional medicine, e.g., in Indian Ayurveda medicine, in China, and in several African countries, to treat infectious diseases and chronic inflammatory disorders. But also in Europe, frankincense extracts are commonly and increasingly used, and several clinical trials have been conducted to demonstrate their therapeutic benefit (Abdel-Tawab et al., 2011). Recent data from both animal experiments and clinical trials revealed that gum resin extracts from Boswellia species not only exert beneficial effects in various inflammatory conditions such as, e.g., rheumatoid arthritis, osteoarthritis, ulcerative colitis, Crohn's disease, multiple sclerosis, or bronchial asthma, but also in hyperlipidemia, obesity, and cancer (see e.g., Poeckel and Werz, 2006; Moussaieff and Mechoulam, 2009; Abdel-Tawab et al.,

Abbreviations: ALAT, alanine aminotransferase; BROD, benzyloxyresorufinO-debenzylation; b.wt., body weight; Casp, Casperome ${ }^{\circledR}$; CD, cluster of differentiation; COX, cyclooxygenase; CSS, clinical severity score; CYP, cytochrome P450; ECOD, ethoxycoumarin-O-deethylation; EMND, ethylmorphine- $N$-demethylation; EROD, ethoxyresorufin-O-deethylation; GSH, reduced glutathione; GSSG, oxidized glutathione; IL, interleukin; iNOS, inducible nitric oxide synthase; LPO, lipid peroxides; LPS, lipopolysaccharides; MROD, methoxyresorufin-O-demethylation; NO, nitric oxide; Phyto, soy lecithin; PROD, pentoxyresorufin- $O$-depentylation; TBARS, thiobarbituric acid reactive substances; TNF, tumor necrosis factor.
2011; Du et al., 2015; Stürner et al., 2017). Additionally, in 2002 the European Medicines Agency classified Boswellia serrata extract as an "orphan drug" for the treatment of peritumoral brain edema. The main active principles of these extracts are pentacyclic triterpenes, termed boswellic acids, which are unique to Boswellia species (Ammon, 2006). Among others, there are four important $\beta$-configurated boswellic acid derivatives: 3-Oacetyl-11-keto- $\beta$-boswellic acid (AKBA), 3-O-acetyl- $\beta$-boswellic acid (A $\mathrm{BBA}), 11$-keto- $\beta$-boswellic acid (KBA), and $\beta$-boswellic acid $(\beta-B A)$.

The exact molecular basis for the pharmacological actions of boswellic acids in chronic inflammatory conditions still remains to be resolved. As possible target structures microsomal prostaglandin $\mathrm{E}_{2}$ synthase-1, 5-lipoxygenase, human leukocyte elastase, cathepsin G, topoisomerase I and II, as well as members of the IKB kinase/NFKB and MAPK signaling pathways have been identified (Syrovets et al., 2005; Poeckel and Werz, 2006; Takada et al., 2006; Cuaz-Pérolin et al., 2008; Moussaieff and Mechoulam, 2009; Siemoneit et al., 2009, 2011; Ammon, 2010; Abdel-Tawab et al., 2011). Additionally, using precipitation experiments with immobilized boswellic acids, LPS has recently been identified as a possible direct molecular interaction partner of distinct boswellic acids and their synthetic derivatives (Henkel et al., 2012). The authors were also able to show that distinct boswellic acids are able to inhibit LPS-mediated effects in a series of cell-based assays via direct binding to LPS. The experiments revealed clearcut structure-activity relationships and $\beta$-BA was found to be the most potent derivative. These findings are further supported by the fact that boswellic acids as well as frankincense resin extracts reduce LPS-induced NO and proinflammatory cytokine production in macrophages both in vitro and in vivo (Syrovets et al., 2000; Pandey et al., 2005; Gayathri et al., 2007; Sharma et al., 2016), thereby exerting hepato- and renoprotective effects (Pandey et al., 2005).

To the best of our knowledge, neither individual boswellic acids nor frankincense extracts have been evaluated for their effects in acute systemic inflammatory conditions before. Therefore, the aim of the present study was to investigate for the first time the impact of Casp, an orally bioavailable soy lecithin-based delivery form of standardized frankincense extract, in a murine model of acute systemic inflammation, i.e., the intraperitoneal administration of LPS. The LPS model was chosen based on the assumption of a direct interaction between boswellic acids, especially $\beta$-BA and LPS (Henkel et al., 2012). Additionally, in comparison to other sepsis animal models such as peritoneal contamination and infection with (human) stool bacteria (PCI) or cecal ligation and puncture (CLP), the LPS model has several essential advantages such 
as technical ease and high reproducibility (Seemann et al., 2017; Stortz et al., 2017). Furthermore, the LPS model has been proven to be most suitable for studying effects of new therapies on acute systemic inflammation (Seemann et al., 2017). Casp is composed of Boswellia serrata extract and soy lecithin in a 1:1 ratio, with about half part of microcrystalline cellulose added to improve physical stability. The formulation is standardized to contain $\geq 25 \%$ of triterpenoic acids by HPLC. Compared with poorly bioavailable non-formulated frankincense extracts, Casp provides a quicker absorption as well as considerably higher plasma and tissue levels of the most important boswellic acids, including $\beta$-BA (Hüsch et al., 2013; Riva et al., 2016). Since in rats maximum plasma levels were reached after $180 \mathrm{~min}$ (Hüsch et al., 2013), in the present study Casp was administered to the mice $3 \mathrm{~h}$ before LPS challenge; $24 \mathrm{~h}$ after LPS administration, animals of the different treatment groups (soy lecithin alone; Casp alone; soy lecithin plus LPS; Casp plus LPS) were evaluated in comparison to control mice and to LPS-only-treated animals with regard to general condition, body temperature, blood glucose values, and serum cytokine concentrations. Additionally, parameters of oxidative stress were determined in different organs and immune cell distribution in two organs decisively involved in sepsis pathogenesis, the liver and spleen. Since liver function is of importance for overall patient outcome, liver glycogen content and biotransformation capacity, representing two important liver function parameters, were also assessed. Finally, to further substantiate the animal studies, cell-based experiments using human peripheral blood mononuclear cells (PBMCs) were performed to confirm the suppressive effect of Casp on cytokine release in vitro.

\section{MATERIALS AND METHODS}

\section{Animals and Experimental Procedure}

The study was conducted under the license of the Thuringian Animal Protection Committee (approval number: 02-011/12). The principles of laboratory animal care and the German Law on the Protection of Animals as well as the Directive 2010/63/EU were followed. Male adult C57BL/6N mice (60-days-old, b.wt. 25-30 g; Charles River Laboratories, Sulzfeld, Germany) were used and the animals were housed in plastic cages under standardized conditions (light-dark cycle 12/12 h, temperature $22 \pm 2{ }^{\circ} \mathrm{C}$, humidity $50 \pm 10 \%$, pellet diet Altromin 1316, water ad libitum).

A total of 48 mice were randomly divided into six groups ( $n=8$ each): control (vehicle-treated animals; $0.9 \% \mathrm{NaCl}$, used for dissolution of LPS), LPS, soy lecithin, Casp, soy lecithin plus LPS, and Casp plus LPS. LPS (Escherichia coli 0111:B4, Sigma-Aldrich, Steinheim, Germany) was dissolved in $0.9 \%$ $\mathrm{NaCl}$ and injected intraperitoneally at a dosage of $5 \mathrm{mg} / \mathrm{kg}$ b.wt. With this dosage, a medium-grade non-lethal systemic inflammation was intended to allow for detection of possible beneficial effects of co-administered drugs (Seemann and Lupp, 2015, 2016; Seemann et al., 2017). Thus, no antibiotic treatment or fluid resuscitation of the animals was necessary. Soy lecithin and Casp (kindly donated by Indena S.p.A., Milano, Italy) were given orally at a dosage of $80 \mathrm{mg} / \mathrm{kg}$ b.wt. (equivalent to the proportion of soy lecithin in Casp) or $240 \mathrm{mg} / \mathrm{kg}$ b.wt., respectively, $3 \mathrm{~h}$ before LPS challenge to ensure maximum plasma levels of boswellic acids at the time of LPS challenge (Hüsch et al., 2013). Based on previous findings (Seemann et al., 2017), animals were sacrificed $24 \mathrm{~h}$ after LPS challenge when plasma levels of pro-inflammatory cytokines are still elevated and changes in immune cell distribution and liver function are already detectable. Twenty-four hours after LPS treatment, body temperature was measured and the mice's health status was assessed by using the CSS described previously (Gonnert et al., 2011). Afterward, mice were sacrificed with an overdose of isoflurane, decapitated, and bled completely. Whole blood was collected in a tube (S Monovette ${ }^{\circledR} 1.2 \mathrm{ml}$ Z Clotting Activator/Serum, Sarstedt, Nümbrecht, Germany) for clotting. Blood glucose levels were determined using a droplet of the whole blood with a commercially available blood glucose meter and respective test strips (BG Star ${ }^{\circledR}$, SanofiAventis, Frankfurt, Germany). After $30 \mathrm{~min}$, clotted blood was centrifuged at $2000 \times g$ for $10 \mathrm{~min}$ to obtain serum which was used for ELISA and enzymatic activity measurements. Additionally, brain, thymus, heart, lung, spleen, kidneys, liver, and adrenals were removed, weighed, and either fixed in 10\% buffered formaldehyde or snap-frozen in liquid nitrogen for biochemical analysis. For histological analyses, formalin-fixed organ samples were embedded in paraffin blocks $(n=8$ animals for each treatment group).

\section{Interleukin (IL)-6, IL-10, Tumor Necrosis Factor (TNF)- $\alpha$, and Alanine Aminotransferase (ALAT) Assays}

Serum levels of inflammatory and liver damage markers were quantified with the following commercially available reagents: mouse TNF- $\alpha$ Quantikine ELISA Kit (R\&D Systems, Minneapolis, MN, United States), IL-6 Mouse ELISA Kit (Thermo Scientific, Rockford, IL, United States), IL-10 Mouse ELISA Kit (Thermo Scientific), and EnzyChrom ${ }^{\text {TM }}$ Alanine Transaminase Assay Kit (BioAssay Systems, Hayward, CA, United States).

\section{Oxidative Status in the Tissues}

To determine tissue content of GSH and GSSG, samples were homogenized with 11 volumes of $0.2 \mathrm{M}$ sodium phosphate buffer [5 mM ethylenediaminetetraacetic acid (EDTA); $\mathrm{pH}$ 8.0] and 4 volumes of $25 \%$ metaphosphoric acid. After centrifugation $\left(12,000 \times g, 4^{\circ} \mathrm{C}, 30 \mathrm{~min}\right), \mathrm{GSH}$ was measured photometrically in the supernatants (Ellman, 1959) and GSSG fluorometrically (Hissin and Hilf, 1976). To assess the tissue content of LPO as TBARS, tissue samples were homogenized with 19 volumes of icecold saline and analyzed fluorometrically, as previously described (Yagi, 1987).

\section{Liver Biotransformation Capacity}

Biotransformation capacity was assessed in the $9000 \times g$ supernatants of the livers. To obtain $9000 \times g$ supernatants, livers 
were homogenized with $0.1 \mathrm{M}$ sodium phosphate buffer $(\mathrm{pH} 7.4)$ $(1: 3, w / v)$ and subsequently centrifuged at $9000 \times g$ for $20 \mathrm{~min}$ at $4^{\circ} \mathrm{C}$. The protein content of these fractions was determined using a modified Biuret method (Klinger and Müller, 1974). For determination of CYP enzyme activities, the following model reactions for different $\mathrm{CYP}$ isoforms were performed: ECOD [CYP1A, 2A, 2B, 2C, 2E (Aitio, 1978)], EROD [CYP1A (Pohl and Fouts, 1980)], MROD [CYP1A2 (Lubet et al., 1985)], PROD [CYP2B (Lubet et al., 1985)], BROD [CYP2A, 2B, 2C, 3A (Lubet et al., 1985)], and EMND [CYP3A (Kleeberg and Klinger, 1982)]. The amount of metabolite formed in these model reactions was normalized to the protein content of the respective $9000 \times g$ supernatants and expressed as $\left(\mathrm{pmol} \times \mathrm{mg} \operatorname{protein}^{-1} \times \mathrm{min}^{-1}\right)$.

\section{Histopathology and Immunohistochemistry}

Samples for histopathology and immunohistochemistry were prepared by cutting $4-\mu \mathrm{m}$ sections from the paraffin blocks and floating these onto positively charged slides. Immunostaining was performed by an indirect peroxidase-labeling method, as described previously (Kaemmerer et al., 2017). Briefly, sections were de-waxed, microwaved in $10 \mathrm{mM}$ citric acid ( $\mathrm{pH} \mathrm{6.0)} \mathrm{for}$ $16 \mathrm{~min}$ at $600 \mathrm{~W}$, and incubated with the respective primary antibodies (Table 1 ) at $4^{\circ} \mathrm{C}$ overnight. Detection of the primary antibodies was performed using either biotinylated goat antirabbit, horse anti-mouse or rabbit anti-goat IgGs, followed by incubation with peroxidase-conjugated avidin (Vector ABC "Elite" Kit, Vector, Burlingame, CA, United States). Binding of the primary antibody was visualized using 3-amino-9-ethylcarbazole (AEC) in acetate buffer (BioGenex, San Ramon, CA, United States). Sections were then rinsed, counterstained with Mayer's hematoxylin (Sigma-Aldrich, Steinheim, Germany), and mounted in VectaMount ${ }^{\mathrm{TM}}$ Mounting Medium (Vector Laboratories, Burlingame, CA, United States). All immunohistochemical stainings were evaluated by two independent blinded investigators. To detect liver glycogen content, periodic-acid-Schiff staining (PAS; periodic acid, Schiff's reagent: Sigma-Aldrich, Steinheim, Germany) was performed, and to obtain a histological overview, hematoxylin and eosin staining (HE) of livers and spleens was conducted, using standard protocols (McManus, 1948; Fischer et al., 2008). Identification of the specific cell types was based on their microscopic features along with the relative location of the cells in the respective tissues.

\section{Cell Culture Experiments Cells}

Peripheral blood (Institute for Transfusion Medicine, Jena University Hospital, Jena, Germany) was collected from healthy adult donors who had not taken any anti-inflammatory drugs during the last 10 days. The protocols for experiments with human monocytes were approved by the ethical committee of the Friedrich Schiller University Jena, Jena, Germany, on March 19, 2014; approval number: 4025-02/14. All methods were performed in accordance with the relevant guidelines and regulations. Leukocyte concentrates were centrifuged $(4000 \times g$, $20 \mathrm{~min}, 20^{\circ} \mathrm{C}$ ) and PBMCs were freshly isolated by dextran sedimentation and centrifugation on lymphocyte separation medium (Histopaque ${ }^{\circledR}-1077$, Sigma-Aldrich, Steinheim, Germany). Resulting PBMCs were seeded in RPMI 1640 (Sigma-Aldrich, Steinheim, Germany) supplemented with 10\% heat inactivated FCS, $100 \mathrm{U} / \mathrm{ml}$ penicillin, and $100 \mu \mathrm{g} / \mathrm{ml}$ streptomycin in cell culture flasks (Greiner Bio-one, Nuertingen, Germany) for $1-1.5 \mathrm{~h}$ at $37^{\circ} \mathrm{C}, 5 \% \mathrm{CO}_{2}$. Adherent monocytes were washed twice with PBS and finally harvested by cell scraping.

\section{Cytokine Measurement}

In order to determine the effect of Casp on cytokine release, $1.5 \times 10^{6}$ monocytes/ml in RPMI 1640 (supplemented with $5 \%$ heat inactivated FCS, $100 \mathrm{U} / \mathrm{ml}$ penicillin, and $100 \mu \mathrm{g} / \mathrm{ml}$ streptomycin) were pre-incubated for $30 \mathrm{~min}$ with vehicle (0.2\% DMSO) or $30 \mu \mathrm{g} / \mathrm{ml}$ Casp after allowing for monocyte adhesion for $1.5 \mathrm{~h}$ at $37^{\circ} \mathrm{C}$ and $5 \% \mathrm{CO}_{2}$. Cells were stimulated with $10 \mathrm{ng} / \mathrm{ml}$ LPS for distinct intervals (TNF- $\alpha$, IL-8: $4 \mathrm{~h}$; IL-6, IL-10: $18 \mathrm{~h}$ ), and cytokines released into the medium were measured using specific Quantikine ELISA Kits (R\&D Systems, Minneapolis, MN, United States). The glucocorticoid dexamethasone ( $1 \mu \mathrm{M}$; Sigma-Aldrich, Steinheim, Germany) was used as positive control.

\section{MTT Assay}

To exclude cytotoxic effects of Casp, monocytes were diluted to $2 \times 10^{6} / \mathrm{ml}$ in RPMI 1640 (containing 5\% heat inactivated FCS, $100 \mathrm{U} / \mathrm{ml}$ penicillin, and $100 \mu \mathrm{g} / \mathrm{ml}$ streptomycin) and seeded in a 96-well plate. Monocytes were allowed to adhere for $1.5 \mathrm{~h}$ at $37^{\circ} \mathrm{C}$ and $5 \% \mathrm{CO}_{2}$ prior to treatment with vehicle $(0.5 \%$ DMSO $)$ or extract $(30 \mu \mathrm{g} / \mathrm{ml})$ in $100 \mu \mathrm{l}$ of medium for $24 \mathrm{~h}$. Afterward, cells were incubated with thiazolyl blue tetrazolium bromide solution (MTT, $5 \mathrm{mg} / \mathrm{ml}$ PBS; SigmaAldrich, Steinheim, Germany) until blue staining of the control. Formazan formation was stopped by adding $100 \mu$ l SDS-lysis buffer $(10 \%$, w/v in $20 \mathrm{mM} \mathrm{HCl}, \mathrm{pH} 4.5)$ and the well plates were shaken at room temperature overnight. Finally, absorbance was measured at $570 \mathrm{~nm}$ with a Multiskan ${ }^{\mathrm{TM}}$ microplate spectrophotometer (Thermo Scientific, Ulm, Germany). The pan-protein kinase inhibitor staurosporine ( $1 \mu \mathrm{M}$; Calbiochem, La Jolla, CA, United States) was used as positive (cytotoxic) control.

\section{Statistical Analysis}

For statistical analysis, the IBM SPSS statistics program (version 22.0) was used. In all cases, animal experiments were performed with eight mice per treatment group. Statistical significance was determined by using the non-parametric Kruskal-Wallis test, followed by the Mann-Whitney $U$-test with Holm-Bonferroni correction. A $p$-value $\leq 0.05$ was considered statistically significant. Data are given as mean \pm standard error of the mean (SEM).

Cell culture experiments were conducted in triplicate. Data are given as means \pm SEM. Statistically significant differences in comparison to LPS-stimulated control cells were determined by one-way ANOVA plus Bonferroni post hoc test and are denoted as follows: ${ }^{*} p \leq 0.05 ;{ }^{* *} p \leq 0.01 ;{ }^{* * *} p \leq 0.001$. 
TABLE 1 | Primary antibodies used for immunohistochemistry.

\begin{tabular}{|c|c|c|c|c|}
\hline Antibody & Type, Catalog-No. & Manufacturer & Dilution & Host \\
\hline CD3 & Monoclonal, ab16669 & Abcam & $1: 400$ & Rabbit \\
\hline CD68 & Monoclonal, ab955 & Abcam & $1: 500$ & Mouse \\
\hline Cleaved caspase-3 & Monoclonal, 9661 & Cell Signaling Technology & $1: 600$ & Rabbit \\
\hline CYP1A & Polyclonal & Daiichi Pure Chemicals & $1: 5000$ & Goat \\
\hline CYP2B & Polyclonal & Daiichi Pure Chemicals & $1: 5000$ & Goat \\
\hline CYP3A & Polyclonal & Daiichi Pure Chemicals & $1: 5000$ & Goat \\
\hline $\mathrm{F} 4 / 80$ & Monoclonal, MCA497G & Bio-Rad Laboratories & $1: 200$ & Rat \\
\hline iNOS & Polyclonal, sc-651 & Santa Cruz Biotechnology & $1: 500$ & Rabbit \\
\hline TNF- $\alpha$ & Monoclonal, sc-52746 & Santa Cruz Biotechnology & $1: 500$ & Mouse \\
\hline
\end{tabular}

\section{RESULTS}

\section{Health Status, Body Temperature, and Blood Glucose Values}

To examine the systemic influence of Casp on LPS-induced systemic inflammation, health status as assessed by the CSS, body temperature, blood sugar level, and body and organ weight was determined. Twenty-four hours after treatment, the health status of LPS-challenged mice was distinctly impaired (Figure 1A). Additionally, body temperature was significantly decreased by an average of $2.6^{\circ} \mathrm{C}$ (mean control value: $37.2^{\circ} \mathrm{C}$ ). Blood glucose levels were even diminished by more than $50 \%$ when compared with control animals (Figures 1B,C). In all cases, Casp was able to significantly ameliorate LPS-induced effects, whereas soy lecithin (solvent control, devoid of frankincense) showed no protective impact. Mice receiving soy lecithin or Casp alone displayed no relevant differences to the control group.

In comparison to controls, LPS challenge caused an average decrease in b.wt. by 14\% (Figure 1D) and in liver and thymus weights by 12 and $25 \%$, respectively, whereas spleen and adrenal weights were increased by $23 \%$ (Figures 1E,F). Weights of brain, kidney, and heart, in contrast, remained unaffected (data not shown). Treatment with soy lecithin or Casp alone caused a decrease in spleen weights by 15 and $22 \%(p=0.071 ; p=0.007)$ and in thymus weights by 21 and $29 \%(p=0.066 ; p=0.016)$, respectively (Figure 1E), with no overt histological changes in these organs. While administration of soy lecithin had no effect on LPS-mediated changes in body and organ weights, Casp was able to significantly return the LPS-induced increase in spleen and adrenal weights to control values (Figures 1E,F). Thymus weights, in contrast, remained significantly reduced not only by additional soy lecithin treatment, but also by co-administration of Casp by 39 and $34 \%(p=0.006 ; p=0.007)$, respectively, which was not statistically different from the weight reduction observed after sole LPS treatment ( $p=0.167 ; p=0.264$; data not shown).

\section{Serum Cytokine and ALAT Levels}

To evaluate the impact of Casp on LPS-induced alterations in circulating cytokine levels, serum concentrations of the proinflammatory cytokines TNF- $\alpha$ and IL-6 as well as of the anti-inflammatory cytokine IL-10 were measured at sacrifice (Figures 2A-C). Compared to control animals, administration of
LPS caused an elevation of serum TNF- $\alpha$, IL- 6 , and IL-10 values by about 120,670 , and $70 \%$, respectively, whereas sole treatment with soy lecithin or Casp had no effect. Co-administration of Casp was able to reduce the LPS-induced increase in serum TNF$\alpha$ and IL- 6 levels by about 30 and 50\%, respectively, whereas IL-10 values were slightly further elevated. Co-administration of soy lecithin, in contrast, had no influence on LPS effects.

As a parameter indicating liver damage, serum ALAT levels were determined (Figure 2D). Here, LPS treatment caused a distinct increase by about $140 \%$ compared to controls. Sole soy lecithin or Casp administration, in contrast, had no influence on serum ALAT concentration and no effect of soy lecithin cotreatment on LPS-induced liver damage was noted. In contrast, after co-administration of Casp significantly lower ALAT values were observed compared to LPS-only-treated mice.

\section{Oxidative Stress in Different Organs}

Since oxidative stress plays a major role in systemic inflammation, the impact of Casp on LPS-induced changes in the oxidative status was evaluated in different organs. For this purpose, tissue levels of lipid peroxidation products as well as the tissue content of GSH and GSSG were determined. After LPS challenge, tissue content of lipid peroxidation products was increased by 12 , $20,24,45,60$, and $170 \%$ in brain, heart, lung, spleen, kidney, and liver, respectively, suggesting considerably elevated oxidative stress in these organs (Figures 3A-E). Soy lecithin administration alone caused a decrease in LPO levels only in liver by about 30\%, compared to control levels. Similarly, only in heart LPO were decreased by about $20 \%$ after sole Casp treatment. Compared to LPS-only-treated animals, combined soy lecithin and LPS administration reduced LPO levels in lung, heart, and spleen, but increased the values in liver. In contrast, co-treatment with Casp significantly attenuated LPS-mediated elevation of LPO toward control in all organs investigated, thus indicating a noticeable additional antioxidant capacity of Boswellia serrata extract (Figures 3A-E). Regarding glutathione status, increases in GSH, GSSG, and total glutathione concentrations by about 10 , 20 , and $60 \%$ were noticed in kidney, lung, and spleen after LPS treatment (Figure 3F), respectively, while liver levels decreased by $10 \%$ (representing the primary organ of glutathione synthesis; data not shown). There was, however, no major change in the GSH/GSSG ratio in all tissues. In brain and heart, no influence of LPS administration on glutathione concentrations was observed. 

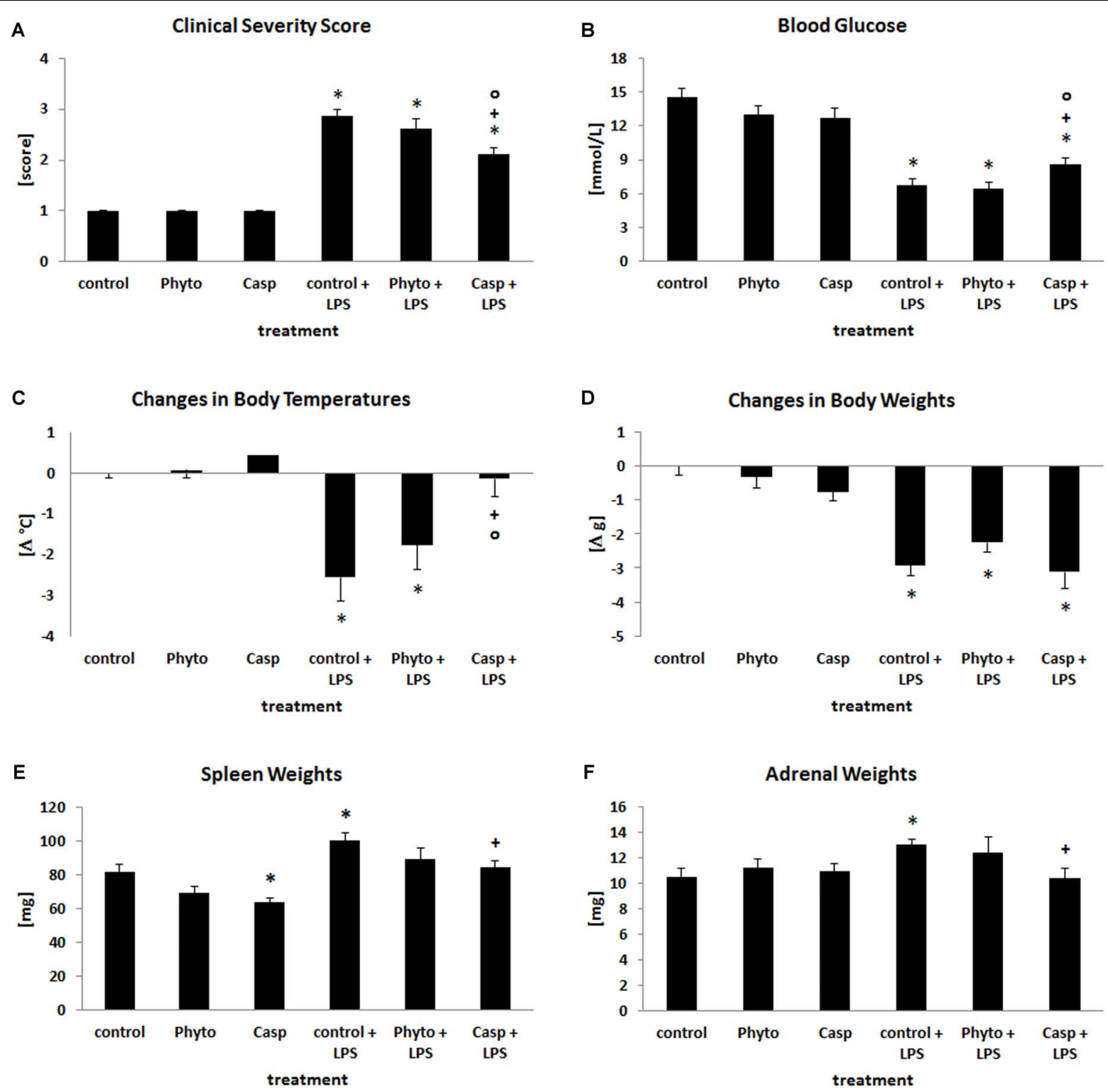

FIGURE 1 | Clinical severity score (A), blood glucose values (B), changes in body temperatures (C), changes in b.wt. (D), spleen (E), and adrenal weights (F). Mice were administered either vehicle (control), soy lecithin (Phyto), Casp, LPS (control + LPS), soy lecithin + LPS, or Casp plus LPS (Casp + LPS). Data are given as means \pm SEM, $n=8$ for each group. *, significantly different from controls; +, significantly different from LPS-treated animals; o, significantly different from soy lecithin plus LPS-treated animals ( $\rho \leq 0.05$; Mann-Whitney $U$-test followed by Holm-Bonferroni correction).

After additional administration of Casp to LPS-treated animals only in spleens a normalization of the glutathione status could be achieved (Figure 3F). In all other organs no relevant significant impact of either soy lecithin or Casp on LPS-mediated effects on tissue glutathione levels was observed.

\section{Spleen: Apoptosis, Immune Cell Redistribution, and TNF- $\alpha$ Expression}

Since apoptotic processes are of substantial importance in the course of systemic inflammation and spleen function is crucial for the removal of damaged red and white blood cells and of bacteria from the blood stream, cleaved caspase-3 expression was assessed in spleens by means of immunohistochemistry as a marker of apoptotic processes. Examination of spleens from LPS-only-treated mice revealed a markedly enhanced expression of cleaved caspase-3, especially in the white pulp, compared to controls and to soy lecithin ${ }^{\circledR}$ - or Casp-onlytreated animals (Figures 4A,B). Besides being increasingly present in spleno- and lymphocytes, strong immunostaining was noticed especially in tingible body macrophages, which were much more frequently present in the white pulp of spleens from LPS-challenged mice than in the other treatment groups (arrowheads in Figure 4B). While no difference in 

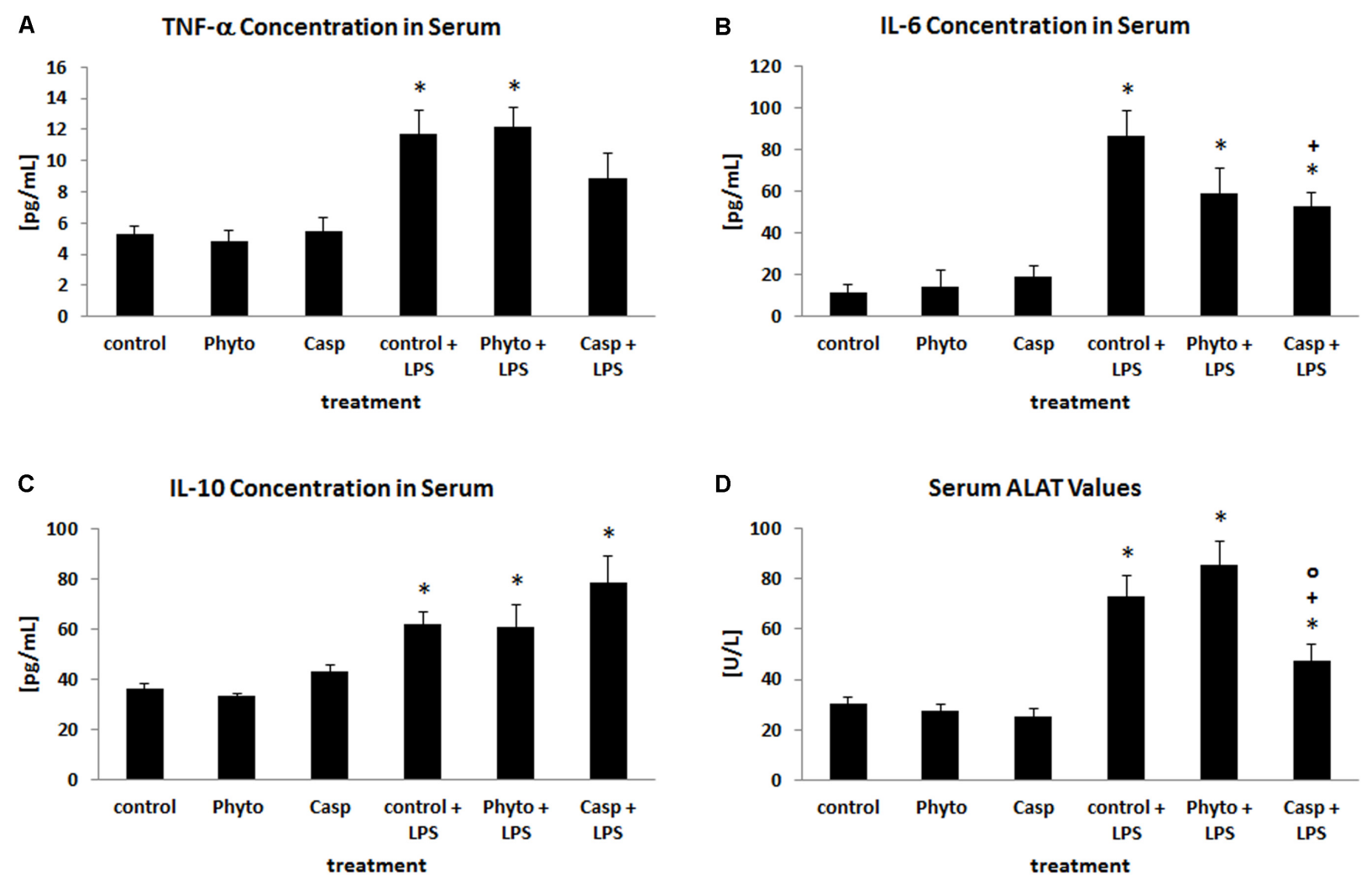

FIGURE 2 | Serum concentrations of TNF- $\alpha$ (A), IL-6 (B), IL-10 (C), and ALAT (D). Mice were administered either vehicle (control), soy lecithin (Phyto), Casp, LPS (control + LPS), soy lecithin + LPS, or Casp plus LPS (Casp + LPS). Data are given as means \pm SEM, $n=8$ for each group. *, significantly different from controls; +, significantly different from LPS-treated animals; o, significantly different from soy lecithin plus LPS-treated animals $(p \leq 0.05$; Mann-Whitney $U$-test followed by Holm-Bonferroni correction).

cleaved caspase-3 expression was detected between LPS-only and soy lecithin-plus-LPS-treated mice, co-administration of Casp yielded a clear-cut reduction in cleaved-casepase-3 expression in spleen, thus indicating an anti-apoptotic effect (Figure 4C; NB: in Figure 4 and Figures 5-8 photomicrographs from soy lecithin- or Casp-only-treated mice and from soy lecithin plus LPS-treated mice are not depicted separately since they did not differ from controls or from LPS-only-treated mice, respectively).

To assess the effects of Casp on LPS-induced changes in immune cell distribution in the spleen, immunostainings for CD3 and CD68 as markers for T-lymphocytes and monocytes/macrophages, respectively, were performed. In control animals and in soy lecithin- or Casp-only-treated mice, CD3 + cells were abundantly present in the periarteriolar sheaths of the white pulp (Figure 4D). In contrast, after LPS challenge the splenic tissue was nearly devoid of CD3+ cells (Figure 4E). This effect was partially reversed after co-treatment with Casp (Figure 4F), but not with soy lecithin, indicating a specific immunomodulatory action of the frankincense extract. In contrast to the massive emigration of CD3+ positive cells from the splenic tissue after LPS administration, a considerable immigration of CD68+ cells was detected in spleens after LPS treatment of the animals when compared to controls or to soy lecithin- or Casp-only-treated mice (Figures 4G,H). These cells were mainly observed in the red pulp, but an increased infiltration of CD68+ cells was also noticed in the white pulp. Again, this effect was nearly completely reversed after co-administration of Casp, but not of soy lecithin (Figure 4I).

Tumor necrosis factor- $\alpha$ is mainly secreted by macrophages. Due to the massive immigration of CD68+ cells into the spleens after LPS treatment, TNF- $\alpha$ expression was additionally assessed in spleen tissue by immunohistochemistry. These investigations revealed TNF- $\alpha$ expression patterns, which were very similar to those seen in the CD68 immunostaining: In comparison to control or to soy lecithin- or Casp-only treatment, LPSchallenge caused a massive immigration of TNF- $\alpha$-positive cells into the splenic tissue, which was almost completely abolished by co-treatment of the mice with Casp (Figures 5A-C), whereas co-administration of soy lecithin showed no effect.

Not only LPS, but also pro-inflammatory cytokines such as TNF- $\alpha$ are known to induce iNOS expression in immune cells such as neutrophils and monocytes/macrophages via the $\mathrm{NF} \kappa \mathrm{B}$ pathway. Besides giving rise to cytotoxic peroxynitrite, excess NO production is critically involved in the course of systemic inflammation, since it contributes to hypotension, cardiodepression, vascular hyporeactivity, and ultimately septic shock. Therefore, in the present investigation, iNOS expression 

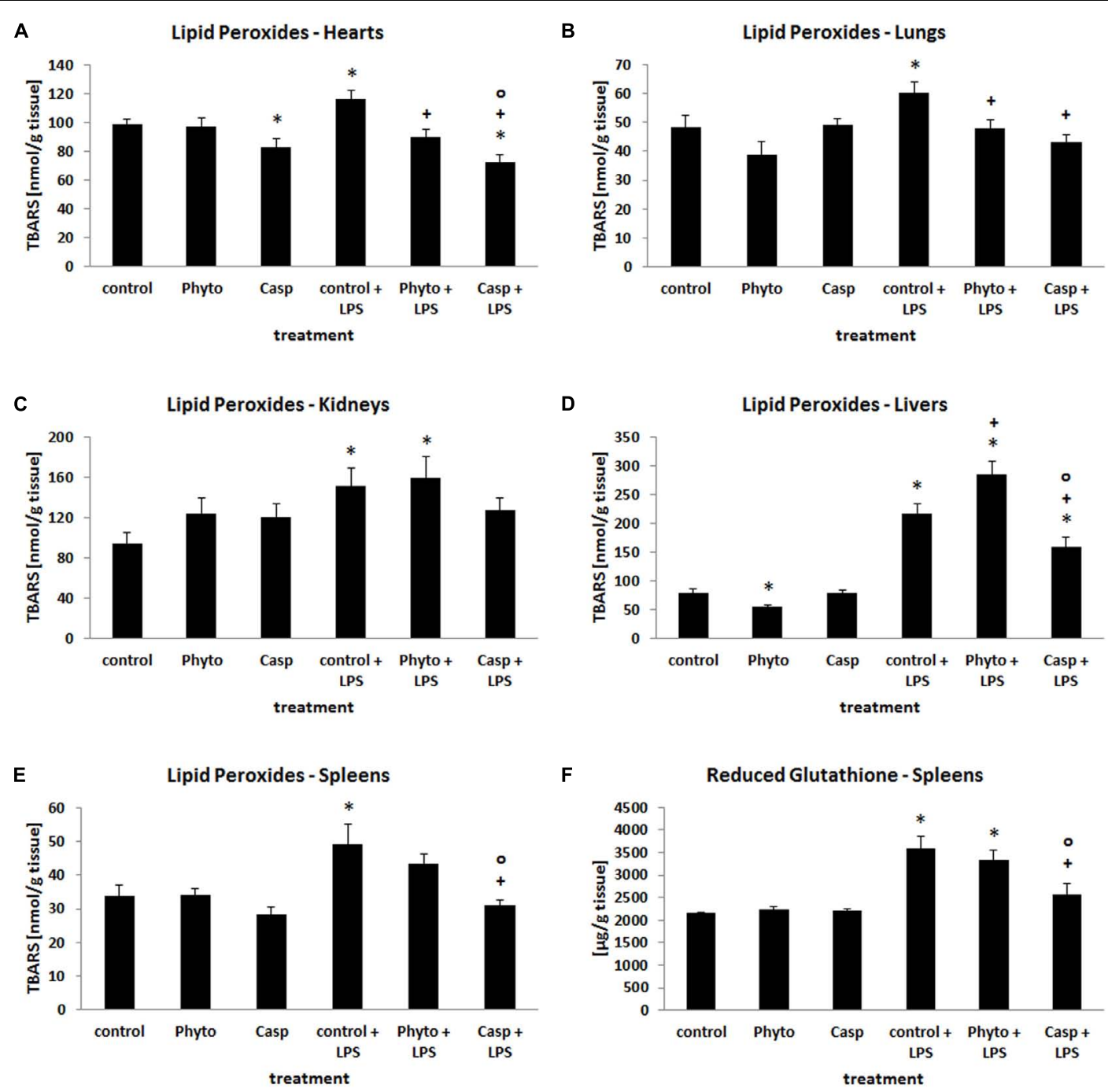

FIGURE 3 | Tissue content of LPO in hearts (A), lungs (B), kidneys (C), livers (D), and spleens (E), and concentration of GSH in spleen tissue (F) as a measure of oxidative stress in the organs. Mice were administered either vehicle (control), soy lecithin (Phyto), Casp, LPS (control + LPS), soy lecithin + LPS, or Casp plus LPS (Casp + LPS). Data are given as means \pm SEM, $n=8$ for each group. * , significantly different from controls; + , significantly different from LPS-treated animals; o, significantly different from soy lecithin plus LPS-treated animals ( $p \leq 0.05$; Mann-Whitney U-test followed by Holm-Bonferroni correction).

was also assessed in spleen and liver tissue. Following LPS treatment, a massive immigration of iNOS-positive neutrophils (and occasionally also of macrophages) was detected in the red and occasionally also in the white pulp of the spleens from LPS-only-treated animals in comparison to control and soy lecithin- or Casp-only-treated mice (Figures 5D,E). Also here, co-administration of Casp, but not of soy lecithin, distinctly ameliorated this effect (Figure 5F).

\section{Liver: Immune Cell Immigration, Glycogen Content, and Biotransformation Capacity}

In parallel to the investigations in the spleens, immunostainings for different immune cell markers and for TNF- $\alpha$ and iNOS were also performed in the livers. Here, in contrast to the spleens, an increased presence of CD3 + T-lymphocytes was observed after LPS treatment of the animals (Figures 6A,B). Additionally, liver expression of the macrophage marker F4/80 was distinctly higher in LPS-challenged mice in comparison to controls, soy lecithin- or Casp-only-treated animals (Figures 6D,E). Likewise, TNF- $\alpha$ expression was strongly enhanced in monocytes/macrophages and also in sinusoidal endothelial cells after LPS administration (Figures 7D,E). Additionally, and as in the spleens, LPS challenge yielded a massive appearance of iNOS-positive neutrophils in the livers (Figures 7A,B). All these effects were significantly less pronounced after pre-administration of Casp (Figures 6C,F, 7C,F), whereas soy lecithin had no impact on LPS-induced changes. 

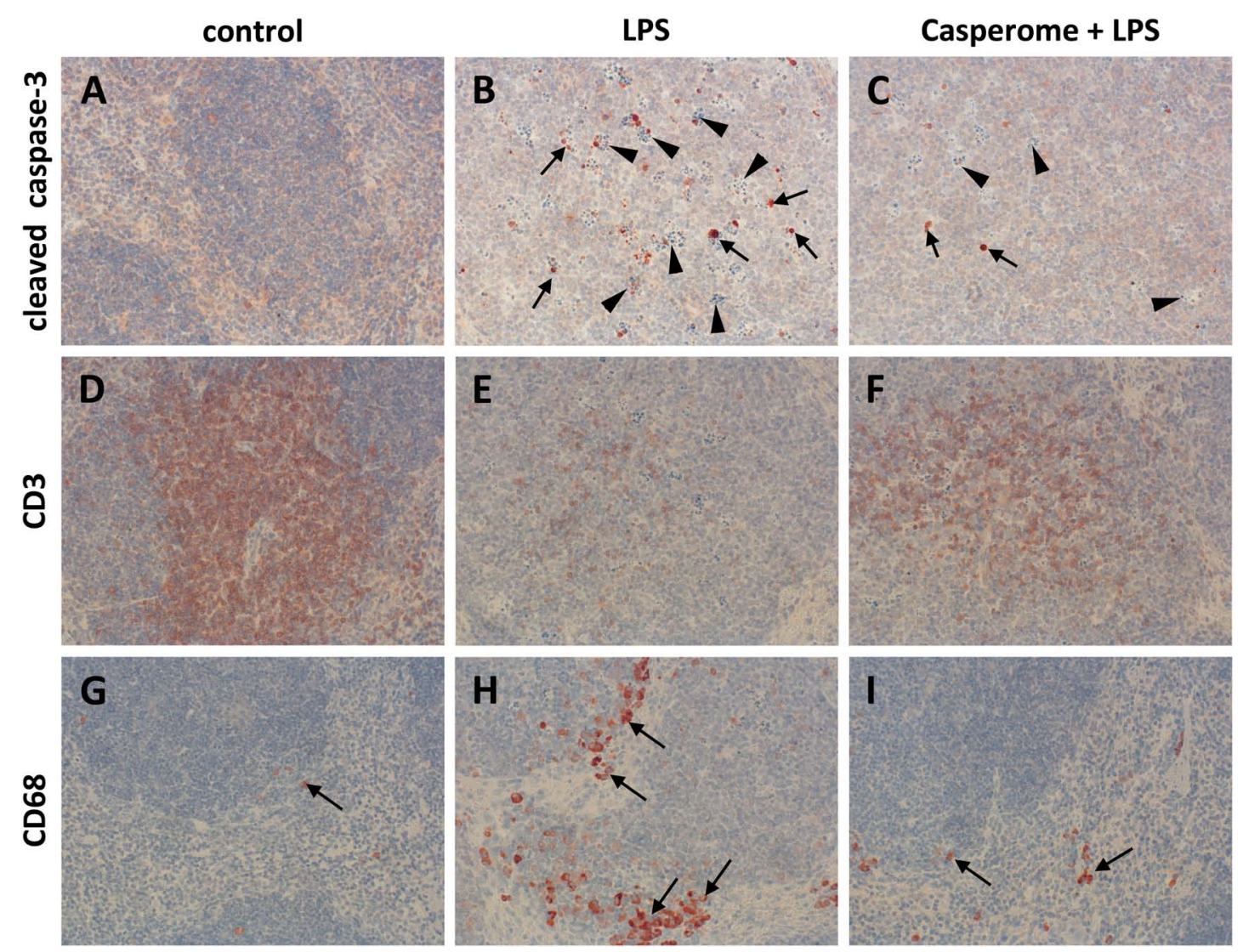

FIGURE 4 | Cleaved caspase-3 (A-C), CD3 (D-F), and CD68 (G-I) expression in the spleens of control (A,D,G), LPS (B,E,H), or Casp plus LPS (C,F,I)-treated mice. Mice were administered either vehicle (control), soy lecithin, Casp, LPS (control + LPS), soy lecithin + LPS, or Casp plus LPS (Casp + LPS). Representative photomicrographs from one of eight different tissue samples from control mice, LPS-treated, and LPS plus Casp-treated animals are shown. Photomicrographs from soy lecithin- or Casp-only-treated mice and from soy lecithin plus LPS-treated mice are not depicted separately since they did not differ from controls or LPS-only-treated mice, respectively. Immunohistochemistry (red-brown color), counterstaining with hematoxylin; original magnification: $\times 400$. Arrows in B and C mark cleaved caspase-3-positive apoptotic cells, arrowheads indicate tingible body macrophages; arrows in $\mathbf{G}, \mathbf{H}$, and I mark CD68-positive monocytes/macrophages.

Since liver function is crucial for overall patient outcome in severe systemic inflammation, liver glycogen content and biotransformation capacity were assessed as representative parameters. In comparison to livers from control, soy lecithin- or Casp-only-treated mice, LPS-challenge caused almost complete glycogen depletion. This effect was partially reversed by additional administration of Casp but not of soy lecithin (Figures 7G-I).

Biotransformation capacity was evaluated by assessing the expression levels of three different CYP isoforms and by measuring CYP-dependent monooxygenase activities by means of specific model reactions. All three CYP isoforms (CYP1A1, CYP2B1, and CYP3A2) assessed were predominantly expressed in the hepatocytes located around the central veins (Figure 8). In comparison to controls and soy lecithin-only-treated mice, LPS administration produced a strong reduction in CYP isoforms expression. In Casp-only-treated animals, in contrast, a strong increase in CYP expression was observed, which was most clearly visible in the pericentral regions of the liver lobules. Whereas co-administration of soy lecithin did not show any visible influence on LPS-mediated effects, additional treatment with Casp was able to restore CYP expression levels completely (Figure 8). Also in the CYP model reactions a distinct decrease in enzyme activities was observed after LPS administration in comparison to controls (Figure 9). In contrast, after sole soy lecithin and especially after sole Casp treatment distinct increases in CYP activities were observed, suggesting a noticeable CYP-inducing capacity of Casp (Figure 9). Whereas additional soy lecithin treatment in some instances slightly ameliorated the LPS effects, biotransformation capacity was completely restored back to control values after supplementary Casp administration (Figure 9).

\section{Effects of Casperome ${ }^{\oplus}$ in Human Primary Monocytes}

In order to investigate anti-inflammatory effects of Casp in a human in vitro model, the influence of the extract on LPS-induced cytokine release from human monocytes was 


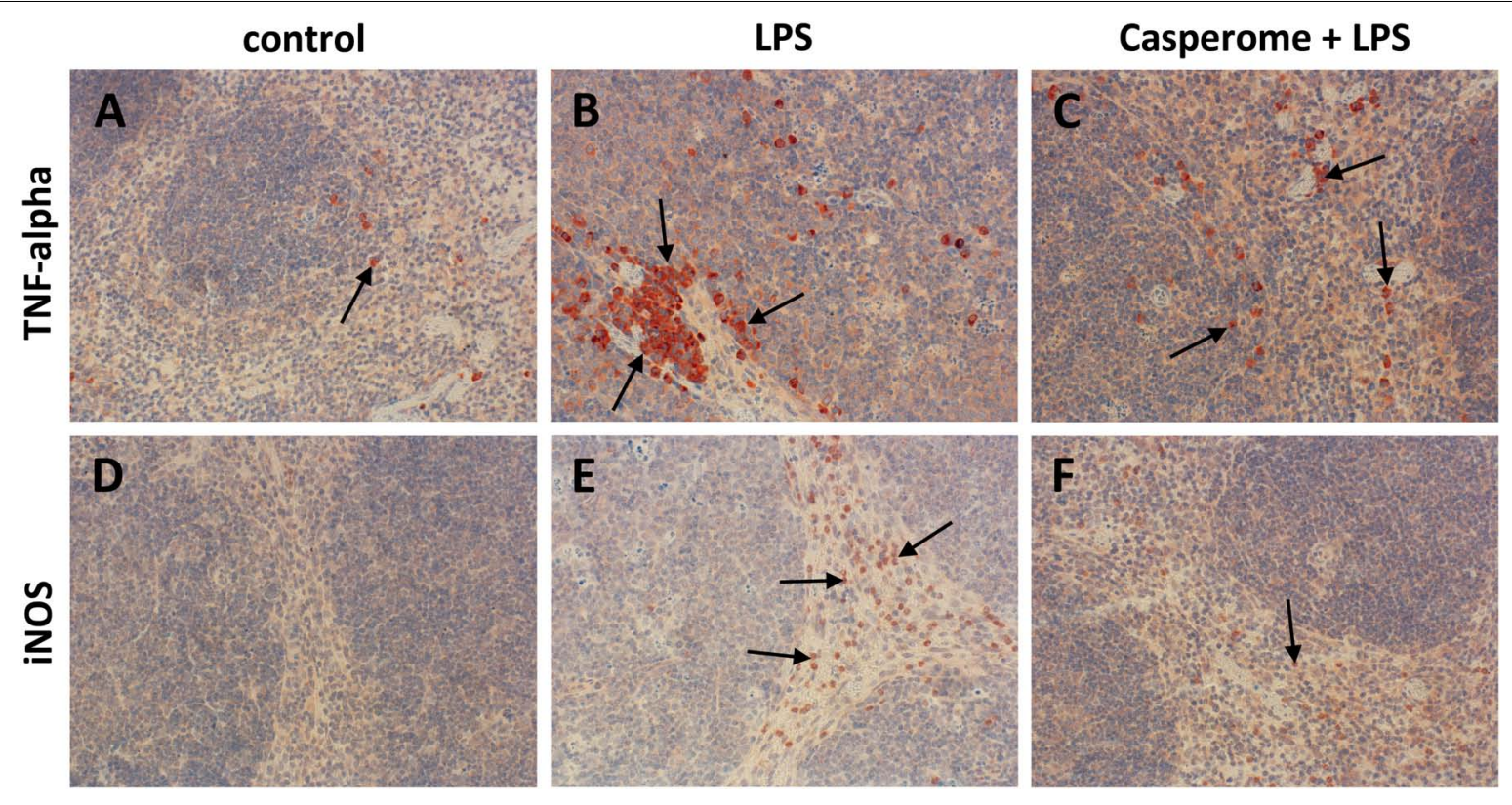

FIGURE $\mathbf{5}$ | TNF- $\alpha$ (A-C) and iNOS (D-F) expression in the spleens of control (A,D), LPS (B,E), or Casp plus LPS (C,F)-treated mice. Mice were administered either vehicle (control), soy lecithin, Casp, LPS (control + LPS), soy lecithin + LPS, or Casp plus LPS (Casp + LPS). Representative photomicrographs from one of eight different tissue samples from control mice, LPS-treated, and LPS plus Casp-treated animals are shown. Photomicrographs from soy lecithin- or Casp-only-treated mice and soy lecithin plus LPS-treated mice are not depicted separately since they did not differ from controls or LPS-only-treated mice, respectively. Immunohistochemistry (red-brown color), counterstaining with hematoxylin; original magnification: $\times 400$. Arrows in A-C mark TNF- $\alpha$-positive monocytes/macrophages, arrows in $\mathbf{E}$ and $\mathbf{F}$ indicate iNOS-positive neutrophils, respectively.
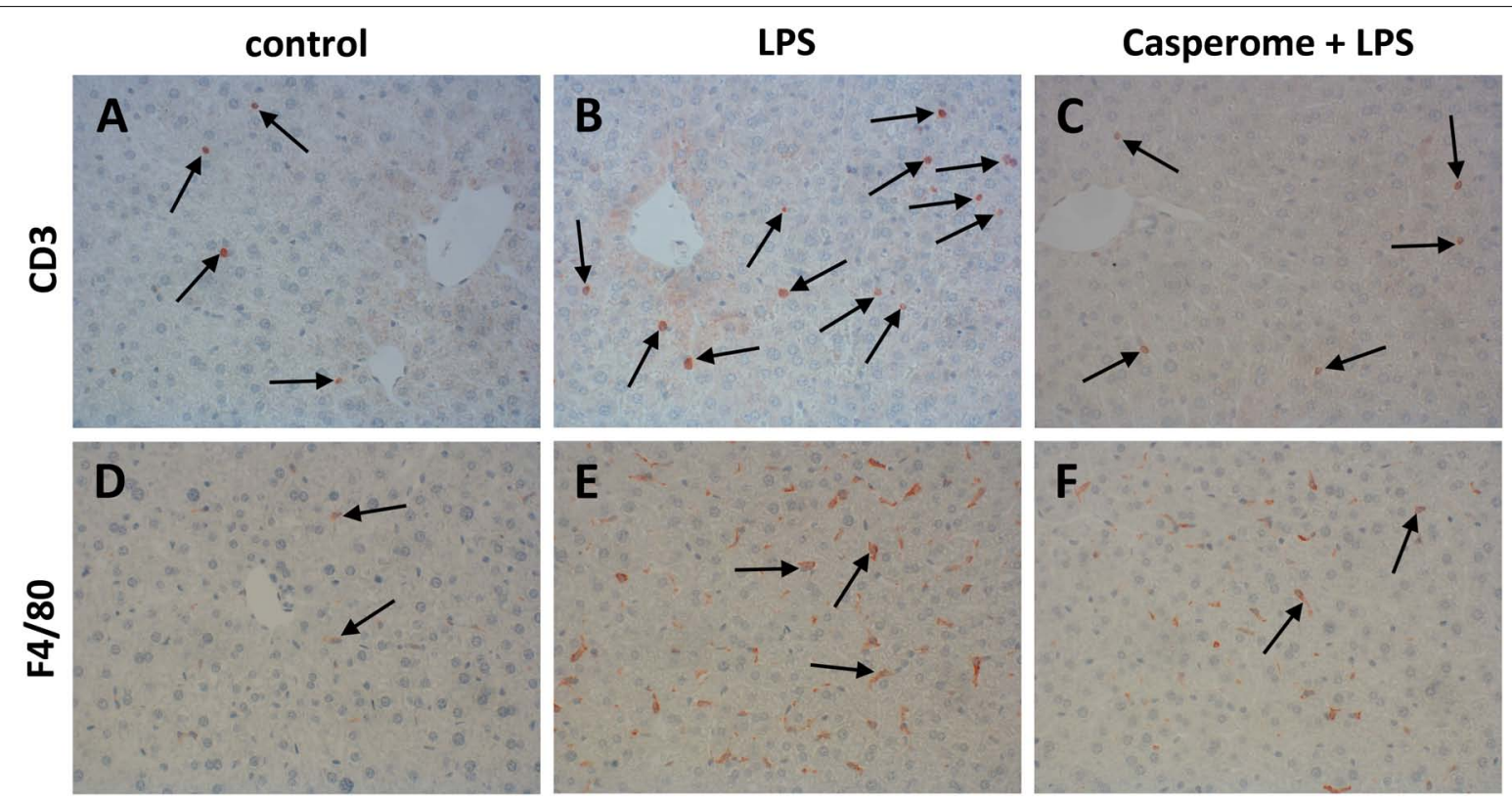

FIGURE 6 | CD3 (A-C) and F4/80 (D-F) expression in the spleens of control (A,D), LPS (B,E), or Casp plus LPS (C,F)-treated mice. Mice were administered either vehicle (control), soy lecithin, Casp, LPS (control + LPS), soy lecithin + LPS, or Casp plus LPS (Casp + LPS). Representative photomicrographs from one of eight different tissue samples from control mice, LPS-treated, and LPS plus Casp-treated animals are shown. Photomicrographs from soy lecithin- or Casp-only-treated mice and soy lecithin plus LPS-treated mice are not depicted separately since they did not differ from controls or LPS-only-treated mice, respectively.

Immunohistochemistry (red-brown color), counterstaining with hematoxylin; original magnification: $\times 400$. Arrows in $\mathbf{A}-\mathbf{C}$ mark CD3-positive T-lymphocytes, arrows in $\mathbf{D}-\mathbf{F}$ indicate F4/80-positive macrophages, respectively. 

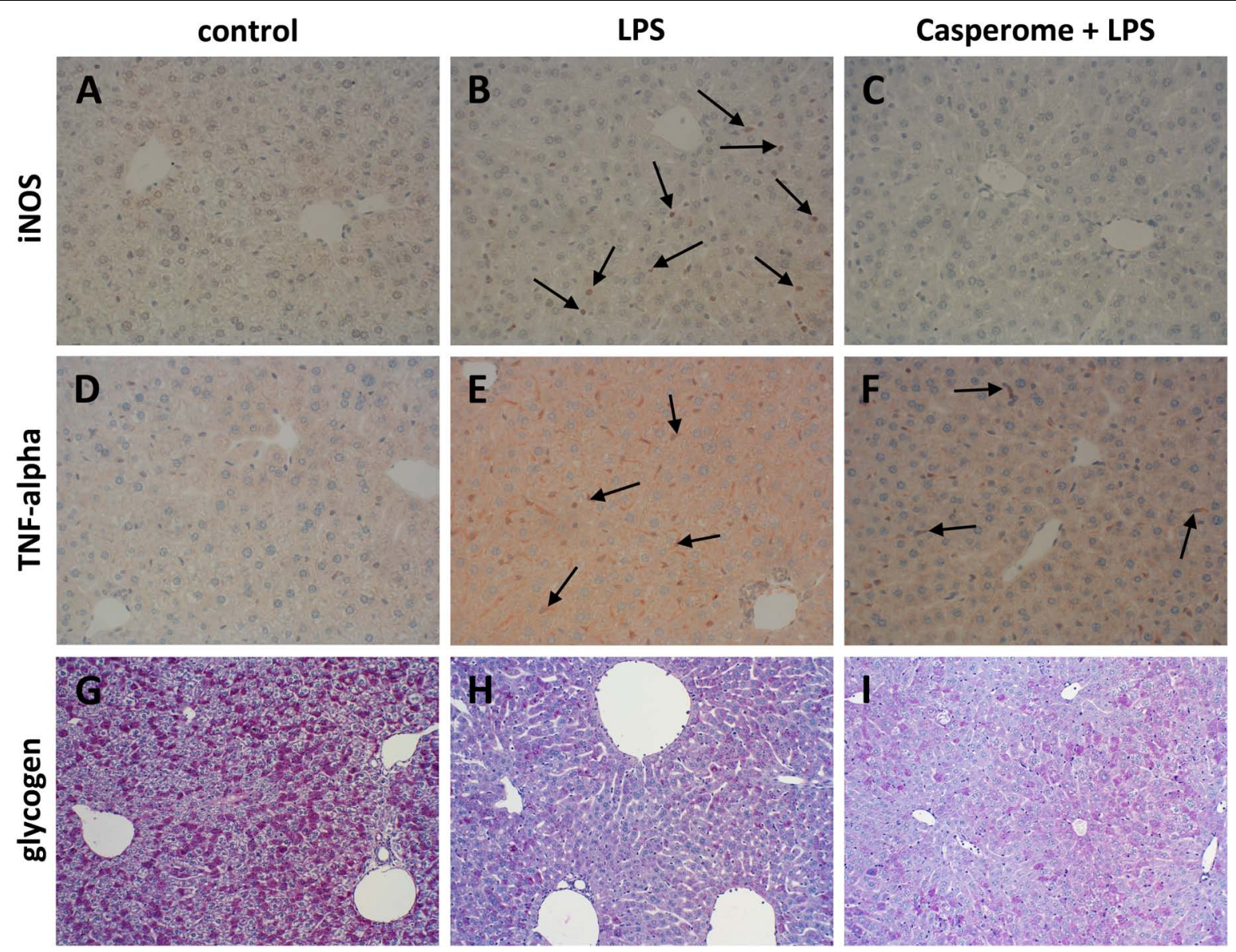

FIGURE 7 | iNOS (A-C), TNF- $\alpha$ expression (D-F), and glycogen content (G-I) in the livers of control (A,D,G), LPS (B,E,H), or Casp plus LPS (C,F,I)-treated mice. Mice were administered either vehicle (control), soy lecithin, Casp, LPS (control + LPS), soy lecithin + LPS, or Casp plus LPS (Casp + LPS). Representative photomicrographs from one of eight different tissue samples from control mice, LPS-treated, and LPS plus Casp-treated animals are shown. Photomicrographs from soy lecithin- or Casp-only-treated mice and soy lecithin plus LPS-treated mice are not depicted separately since they did not differ from controls or LPS-only-treated mice, respectively. A-F: Immunohistochemistry (red-brown color), counterstaining with hematoxylin; G-I: periodic acid-Schiff staining; original magnification: $\times 400$. Arrows in $\mathbf{B}$ mark iNOS-positive neutrophils, arrows in $\mathbf{E}$ and $\mathbf{F}$ indicate TNF- $\alpha$-positive monocytes/macrophages.

tested in comparison to the glucocorticoid dexamethasone. After pre-incubation with the test compounds, freshly isolated human primary monocytes were stimulated with $10 \mathrm{ng} / \mathrm{ml}$ LPS to elicit cytokine release (assessed by ELISA). At a concentration of $30 \mu \mathrm{g} / \mathrm{ml}$, Casp decreased the levels of all pro-inflammatory cytokines measured. Thus, TNF- $\alpha$ release was reduced by $32 \%$ with similar efficiency as for dexamethasone (30\% reduction). Even stronger effects of Casp were detected for release of IL-6 and the chemokine IL-8 (Figure 10A). Additionally, Casp and dexamethasone blocked the release of the anti-inflammatory cytokine IL-10. To exclude any cytotoxicity being responsible for diminished cytokine release, Casp was evaluated in a cell viability (MTT) assay. As shown in Figure 10B, $30 \mu \mathrm{g} / \mathrm{ml}$ Casp produced no detrimental impact on monocyte viability after $24 \mathrm{~h}$ incubation in comparison to control, whereas staurosporine reduced cell viability to about 54\% of control values. Thus, these data support Casp as a potent anti-inflammatory agent without cytotoxic effects.

\section{DISCUSSION}

Since the beneficial effects of frankincense in the treatment of chronic inflammatory diseases have already been well documented (see e.g., Poeckel and Werz, 2006; Moussaieff and Mechoulam, 2009; Abdel-Tawab et al., 2011; Du et al., 2015), the aim of the present study was to assess possible protective effects of frankincense on acute systemic inflammation for the first time. Because of the well-documented influence of the boswellic acids on cytokine production by monocytes/macrophages (Syrovets et al., 2000; Pandey et al., 2005; Gayathri et al., 2007; Sharma et al., 2016), we focused on cytokine plasma levels, immune cell redistribution, and oxidative stress as well as liver function. The latter parameter is known to be critical for overall patient outcome during systemic inflammation and has been shown to be negatively influenced by pro-inflammatory cytokines (Morgan, 2001; Novotny et al., 2007; Jacob et al., 2009; Nesseler et al., 2012).

After LPS challenge, reduced b.wt. of the animals, decreased blood glucose levels, and almost complete glycogen depletion of 

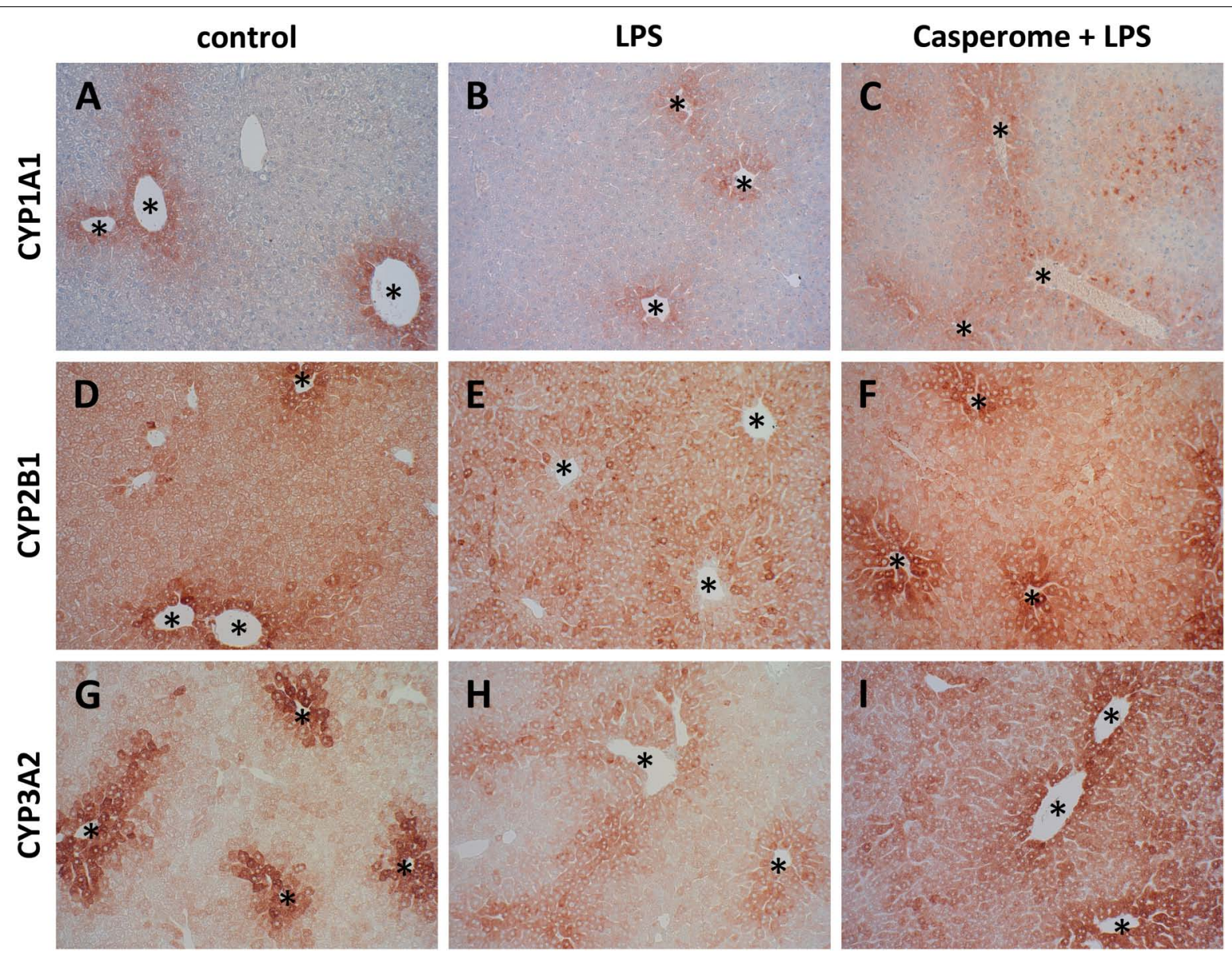

FIGURE 8 | CYP 1A1 (A-C), CYP2B1 (D-F), and CYP3A2 (G-I) expression in the livers of control (A,D,G), LPS (B,E,H), or Casp plus LPS (C,F,I)-treated mice. Mice were administered either vehicle (control), soy lecithin, Casp, LPS (control + LPS), soy lecithin + LPS, or Casp plus LPS (Casp + LPS). Representative photomicrographs from one of eight different tissue samples from control mice, LPS-treated, and LPS plus Casp-treated animals are shown. Photomicrographs from soy lecithin- or Casp-only-treated mice and soy lecithin plus LPS-treated mice are not depicted separately since they did not differ from controls or LPS-only-treated mice, respectively. Immunohistochemistry (red-brown color), counterstaining with hematoxylin; original magnification: $\times 400$. CYP expression was mainly confined to the hepatocytes around the central veins (asterisks).

the livers were observed, which is in line with literature data (Raetzsch et al., 2009; Santos et al., 2013; Seemann and Lupp, 2015, 2016; Seemann et al., 2017). On the one hand, these effects could be explained by the distinctly reduced health status of the animals, mirrored by low CSS scores, which may have led to reduced food intake. On the other hand, it has been shown that (partially mediated via increased TNF- $\alpha$ expression) LPS is able to reduce gluconeogenesis (Goto et al., 2001; Raetzsch et al., 2009; Santos et al., 2013) and to increase glycogenolysis (Casteleijn et al., 1988). Presumably by direct binding of the boswellic acids to LPS and thus blunting TLR4, MyD88, and NFKB signaling (and, consequently, TNF- $\alpha$ release and COX-2 induction), but probably also by a direct interaction with $\mathrm{NF \kappa B}$ and COX-2, co-administration of Casp significantly increased blood glucose values and liver glycogen content compared to the LPS-only treatment group. Additionally, general health status of the animals was significantly improved after co-treatment with Casp with increased food consumption, lower levels of circulating pro-inflammatory cytokines, a lower severity of the inflammatory processes, and a normalization of the body temperatures, compared to the LPS-only-treated animals.

In the present investigation, markedly increased serum levels of the pro-inflammatory cytokines TNF- $\alpha$ and IL- 6 were observed in LPS-treated animals, as expected from literature data (Seemann and Lupp, 2015, 2016; Seemann et al., 2017). These increased levels were distinctly reduced after Casp co-treatment of the mice. Thus, the reported suppression of pro-inflammatory cytokine release from monocytes/macrophages by boswellic acids (Syrovets et al., 2000; Pandey et al., 2005; Gayathri et al., 2007; Sharma et al., 2016) could be reproduced also with the frankincense extract Casp in vivo in acute systemic inflammation. These results were further confirmed by cellbased assays using human primary monocytes, which revealed a distinct reduction of the LPS-induced TNF- $\alpha$, IL- 6 , and IL-8 release after Casp treatment. In contrast to the suppressive effects on pro-inflammatory cytokines, murine serum levels of the anti-inflammatory IL-10 were slightly enhanced by Casp co-treatment, whereas in the cell-based assay IL-10 release was 


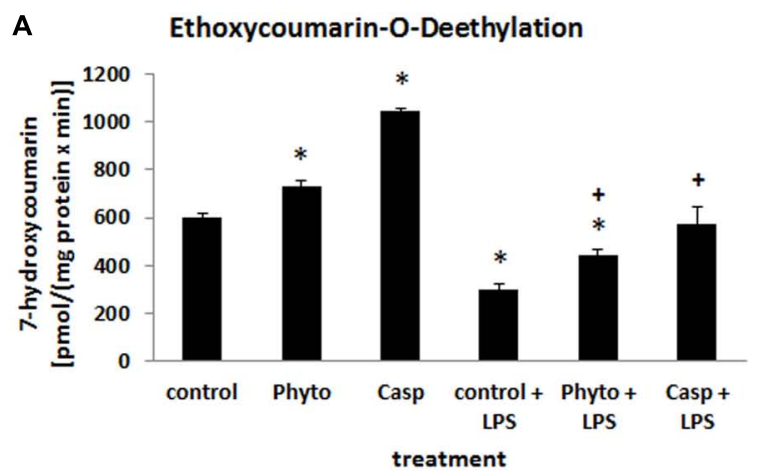

C

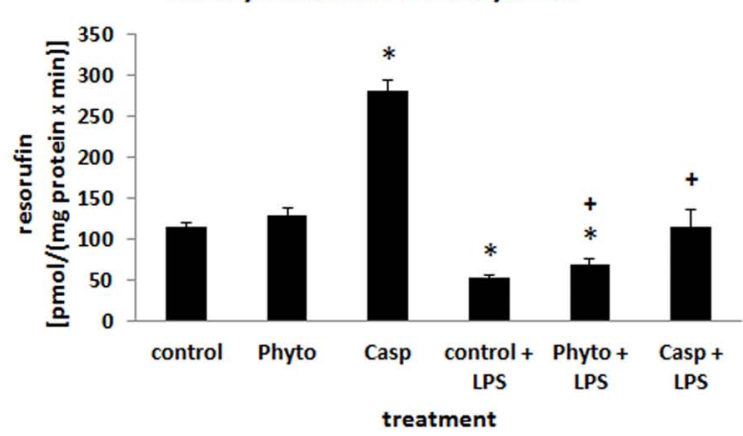

E

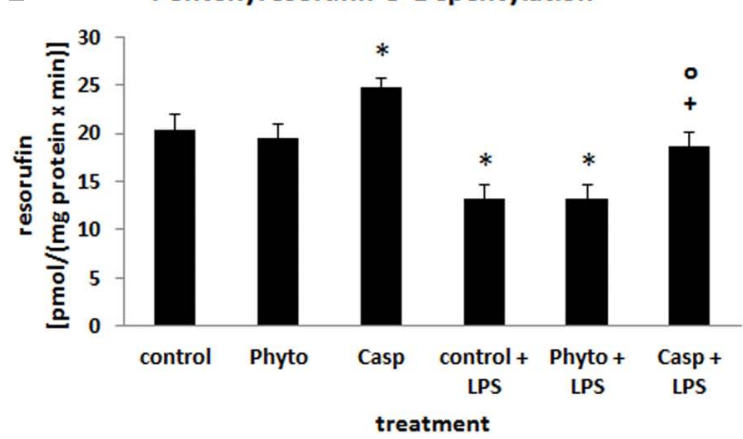

B

Benzyloxyresorufin-O-Debenzylation

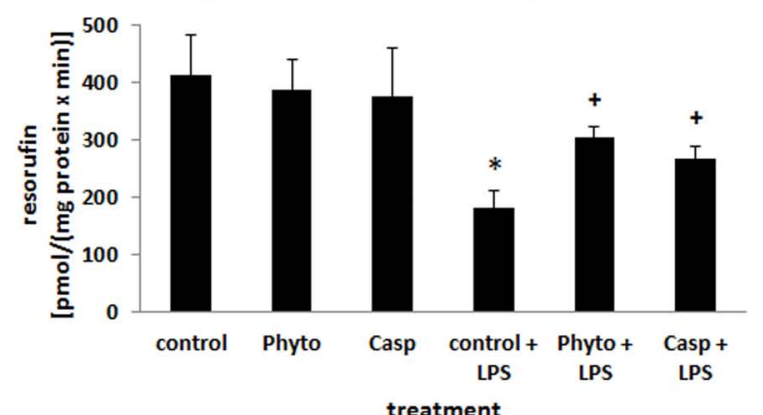

D

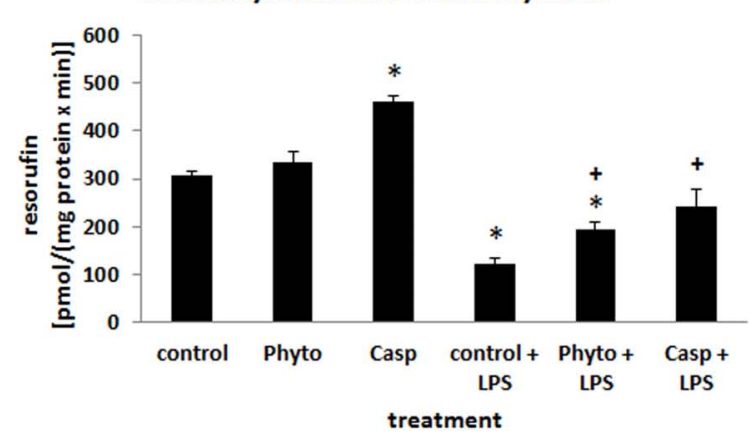

$\mathbf{F}$

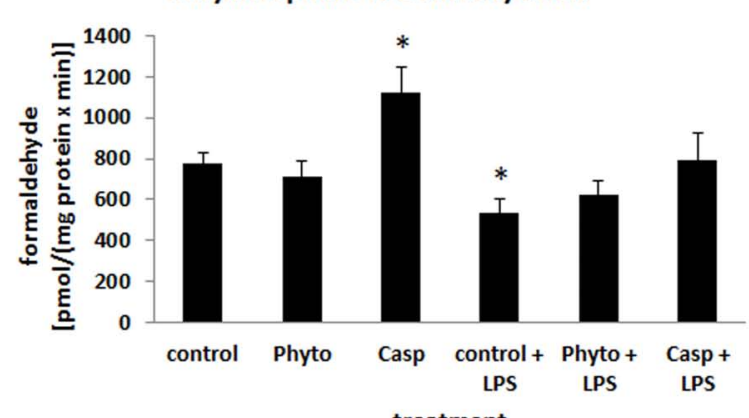

treatment

FIGURE 9 | Biotransformation capacity as determined by a panel of model reactions for different CYP isoforms. Mice were administered either vehicle (control), soy lecithin (Phyto), Casp, LPS (control + LPS), soy lecithin + LPS, or Casp plus LPS (Casp + LPS). ECOD (A), BROD (B), EROD (C), MROD (D), PROD (E), and EMND (F) were determined in the $9000 \times g$ supernatants of the livers. Data are given as means $\pm \mathrm{SEM}, n=8$ for each group. *, significantly different from controls; +, significantly different from LPS-treated animals; o, significantly different from soy lecithin plus LPS-treated animals $(p \leq 0.05$; Mann-Whitney $U$-test followed by Holm-Bonferroni correction).

strongly decreased. This discrepancy may be due to the fact that in vivo IL-10 is not only released by monocytes/macrophages but also by many other immune cells such as $\mathrm{TH}_{2}$ - or Treglymphocytes. IL-10 released from these cells has a central role in limiting immune responses to pathogens to protect the organism from damage due to exaggerated inflammatory processes (Saraiva and O'Garra, 2010). Thus, in addition to the inhibitory effects seen on cytokine production by monocytes and macrophages, stimulation of IL-10 release from these other immune cells may have also contributed to the overall better outcome observed after co-treatment with Casp, compared to LPS-only-treated mice.
In parallel to the influence of Casp on pro-inflammatory and anti-inflammatory cytokines, also immune cell redistribution was distinctly reduced by the frankincense extract. In spleens, LPS caused a massive appearance of iNOS-positive neutrophils and of CD68/iNOS/TNF- $\alpha$-positive monocytes/macrophages, but a depletion of CD3+ T-lymphocytes, whereas in livers immigration of iNOS positive neutrophils as well as CD3+ cells was observed. All these effects were distinctly ameliorated after co-administration of Casp. Especially the influence on iNOS expression may be of importance, since increased iNOS expression and, most importantly, immigration of iNOS-positive 

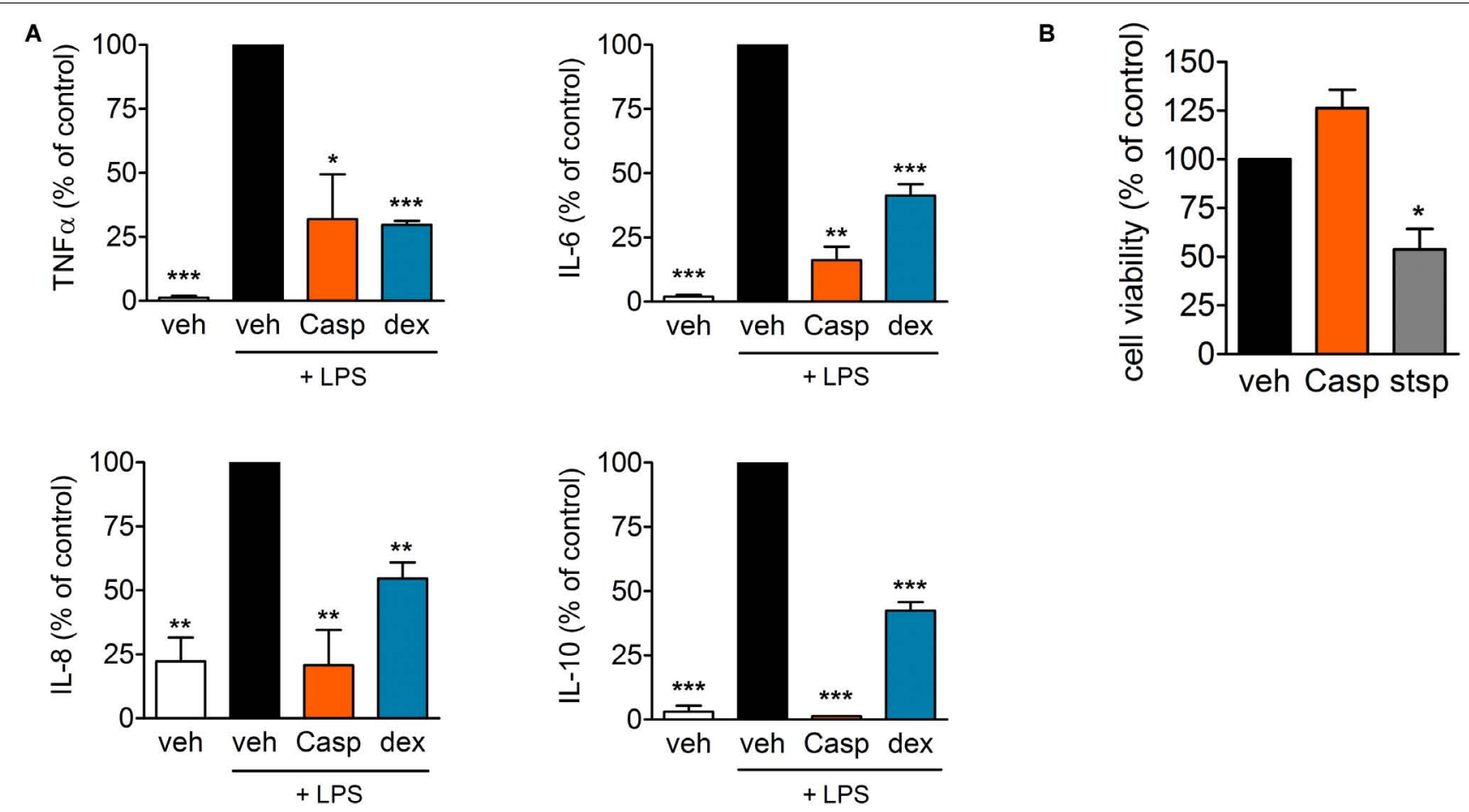

FIGURE 10 | Effect of Casp on cytokine release and cytotoxicity in human monocytes. (A) Human primary monocytes were pre-incubated with vehicle or compounds (Casp; dexamethasone, dex) for 30 min prior to stimulation with LPS for 4 (TNF- $\alpha$, IL-8) or 18 h (IL-6, IL-10). Formed cytokines were measured by ELISA. Data are means \pm SEM; $n=3 ; * * * p<0.001 ; * * p<0.01 ; * p<0.05$; inhibitor vs. stimulated control (100\%), one-way ANOVA plus Bonferroni test. (B) Effect of Casp on cell viability. Intact human monocytes were incubated with compounds (Casp; staurosporine, stsp) or vehicle for $24 \mathrm{~h}$ and cell viability was analyzed by MTT assay. Data are means \pm SEM; $n=3$; * $p<0.05$; inhibitor vs. vehicle control (100\%), one-way ANOVA plus Bonferroni test.

neutrophils, but also of iNOS-positive macrophages, into the tissues has been shown to be critical for the course of the disease and overall outcome (Kirkeboen and Strand, 1999; Tsukahara et al., 2001; Farley et al., 2006; Wang et al., 2012). Finally, in parallel to the positive influence on TNF- $\alpha$ serum values, Casp was also able to ameliorate LPS-induced increases in TNF- $\alpha$ expression by monocytes/macrophages and sinusoidal endothelial cells in liver tissue.

In contrast to average b.wt., spleen weights were distinctly enhanced after LPS treatment. The occurrence of splenomegaly in response to severe systemic inflammation has been shown also in humans (Arismendi-Morillo et al., 2004) and has been correlated with, among others, TNF- $\alpha$ - and IL-6-mediated elevation of serum high-mobility group box 1 (HMGB1) levels in spleen tissue (Valdés-Ferrer et al., 2013). The increase in volume has been demonstrated for both red and white pulp and has been shown to be due to hyperemia and enhanced immigration of white blood cells into the splenic tissue, especially neutrophils and macrophages (Valdés-Ferrer et al., 2013; Seemann et al., 2017). As mentioned above, an increased presence of iNOS-positive neutrophils and CD68/iNOS/TNF- $\alpha$-positive monocytes/macrophages was observed in the spleens after LPS challenge also in the present investigation. Additionally, a distinct rise in apoptotic processes was also noted, demonstrated by increased cleaved caspase- 3 stainings, and mirrored by increased presence of tingible body macrophages in spleens after LPS treatment. Probably, there is an increased need for degradation of nonfunctional erythrocytes and white blood cells as a result of systemic inflammation. In contrast to these processes, massive emigration of CD3 + T-lymphocytes from spleens was observed after LPS challenge, which is in line with previous investigations (Valdés-Ferrer et al., 2013; Seemann et al., 2017). Since the present investigation demonstrated increased immigration of CD3+ cells into liver tissue, these cells may leave the spleen to enter other organs in response to systemic inflammation. Probably by suppressing increased levels of pro-inflammatory cytokines, spleen weights were significantly decreased back to normal values after co-administration of Casp. Additionally, less neutrophils and macrophages were present in spleen tissue while apoptotic processes as well as presence of tingible body macrophages were distinctly reduced in comparison to LPS-onlytreated animals. Furthermore, emigration of CD3 + cells from spleen into other organs was significantly attenuated, suggesting a noticeable immunomodulatory and anti-inflammatory action of the frankincense extract.

Lipopolysaccharides-only-treated animals also showed adrenal hypertrophy, which may be explained by activation of the hypothalamic-pituitary-adrenocortical axis in response to the inflammatory event (Prigent et al., 2004; Yang et al., 2007; Zhang et al., 2014), but also by stimulation of adrenal medullary catecholamine synthesis to stabilize cardiovascular function (Flierl et al., 2008). Probably by decreasing pro-inflammatory 
cytokine levels, thus exerting anti-inflammatory effects, Casp was evidently able to counteract these effects, since adrenal weights were significantly returned back to normal values in our investigation.

In contrast to spleen and adrenals, thymus weights were distinctly reduced after LPS treatment. As in spleen, this effect may be due to a massive emigration of T-lymphocytes into other organs, but also to increased apoptotic processes as has been shown in other sepsis animal experiments before (Hiramatsu et al., 1997, Seemann and Lupp, 2015). There was, however, also a comparable decrease in thymus weights and also a reduction in spleen weights after sole soy lecithin or Casp treatment. These effects remain unclear since no overt histological changes were noticed in the histological stainings of both organs after sole soy lecithin or Casp administration. The effects of Casp on the weights of these two immune organs could be, however, an expression of the known immunomodulatory properties of the boswellic acids (see e.g., Poeckel and Werz, 2006; Moussaieff and Mechoulam, 2009; Abdel-Tawab et al., 2011; Stürner et al., 2017), which in our mouse model evidently helped the animals to better cope with the LPS-induced systemic inflammation. After combined treatment with LPS, clear-cut protective effects were seen on all parameters tested, including spleen weights, plasma levels of pro- and anti-inflammatory cytokines, and immune cell redistribution, and there was no additional negative influence on thymus weights. Nevertheless, in future experiments, the effects of Casp (but also of soy lecithin) on the immune system in systemic inflammation should be elaborated in more detail in order to elucidate the underlying mechanisms of the protective findings of the present investigation more precisely and to exclude any adverse effects.

Lipopolysaccharides can lead to enhanced generation of reactive oxygen (ROS) and nitrogen species (RNS), both directly and via increased pro-inflammatory cytokine expression. ROS are the result of elevated NADPH-oxidase expression in neutrophils and monocytes/macrophages after LPS challenge (Kim et al., 2007), but may also be caused by increased mitochondrial leakage (Shoji et al., 1995; Galley, 2011). RNSs are generated both via enhanced NADPH-oxidase and iNOS expression in neutrophils and monocytes/macrophages. ROS/RNS are neutralized, among others, by the glutathione system, which represents an important anti-oxidative mechanism of the body to protect cellular macromolecules such as DNA, lipids, and proteins from chemical damage and degradation (Hayes and McLellan, 1999). In the present investigation, a distinct decrease in glutathione content in livers was observed after LPS treatment of the animals. This glutathione loss may either be caused by LPS-induced liver cell damage, as indicated by increased serum ALAT values, and subsequent impairment of glutathione synthesis, but may also be caused by intensified export to other organs, such as kidneys, lungs, and spleens. This latter hypothesis is supported by the observation that in these organs, and in contrast to liver, an increase in glutathione content was observed after LPS treatment. Lipid peroxidation products are the result of excessive oxidative stress which has overwhelmed the overall anti-oxidative capacity of the body and has led to a degradation of membrane lipids (Ayala et al., 2014). In the present investigation, corresponding to elevated ALAT values, a strong increase in lipid peroxidation products was seen especially in livers after LPS treatment, but also in all other organs tested. Casp caused a marked reduction in hepatic LPO content when given in addition to LPS, presumably by direct binding to LPS, thereby preventing increased expression of NADPH-oxidase, iNOS, and pro-inflammatory cytokines, but probably also due to a direct radical scavenging capacity (Afsar et al., 2012; AlHarrasi et al., 2013). In all other organs investigated, levels were even reverted back to normal values. Altogether, these effects suggest a noticeable anti-oxidative capacity of the frankincense extract.

Biotransformation capacity is of essential importance for the detoxification and elimination of endogenous substances, as well as exogenously administered therapies, and the CYP system represents one of the key enzyme systems involved in phase I metabolism of xenobiotics (Nebert and Russell, 2002). It is well known from the literature that the pro-inflammatory cytokines TNF- $\alpha$, IL-1 $\beta$, IL- 6 , interferon- $\alpha$, and interferon- $\gamma$, as well as NO cause a strong downregulation especially of the CYP system but also of other biotransformation enzymes (Morgan, 2001; Aitken et al., 2006). Consequently, in sepsis patients CYP-mediated drug metabolism is markedly impaired (Carcillo et al., 2003; Jacob et al., 2009). Since sepsis patients usually are concomitantly treated with several drugs, such as, e.g., antibiotics, reduction in CYP activity may lead to an accumulation of these substances and enhanced side effects of these multiple medications, which may have additional negative impact on the health status of these patients (Carcillo et al., 2003). In the present investigation, Casp turned out to be a strong inducer of all CYP enzymes tested. Since no other comparable in vivo data are available so far for either the frankincense extract or individual boswellic acids, this effect remains to be further elucidated. In the only in vitro study performed so far on liver microsomes, an inhibitory action of frankincense extract and various boswellic acids on different CYP enzymes has been shown (Frank and Unger, 2006). However, in vitro experiments using microsomes do not allow for prediction of potential drug effects as CYP inducer or inhibitor in vivo beyond a mere interaction, since for CYP induction intact cells are required. Based on the CYP-inducing capacity and the decreased levels of pro-inflammatory cytokines and NO, we postulate that Casp may be able to restore both CYP enzymes expression and activities back to normal values, which would suggest a therapeutic use of frankincense extract in the treatment of sepsis patients.

Besides the distinct beneficial effects of Casp on most of the parameters tested, in some instances a significant protective influence on LPS-mediated changes was also observed with the phospholipid soy lecithin. Thus, soy lecithin was able to cause a slight reduction in elevated LPO levels in lungs, hearts, and spleens and an improvement of CYP-mediated biotransformation capacity in some of the model reactions. However, in all these cases, the effect of Casp (i.e., the combination of soy lecithin and frankincense extract) exceeded that of soy lecithin alone. Protective effects of phospholipidrich lipid solutions in sepsis have been reported both in humans and in animal experiments (Read et al., 1995; 
Goldfarb et al., 2003; Gordon et al., 2005). The exact mechanism underlying these effects still remains to be elucidated. However, an increased binding of LPS by lipoproteins generated from these lipids and thus prevention of interaction of the LPS/LPS-binding protein complex with Toll-like receptors has been suggested (Read et al., 1995; Goldfarb et al., 2003; Gordon et al., 2005).

\section{CONCLUSION}

In summary, the present investigation shows for the first time that the orally bioavailable frankincense extract Casp is able to prevent acute LPS-induced systemic inflammation in mice by exerting anti-inflammatory, anti-oxidative, and hepatoprotective effects. Casp may therefore serve as a new prophylactically acting supportive treatment option in systemic inflammatory conditions, especially when accompanied by impaired liver function. It would be of further interest to determine whether Casp also exerts protective effects when given later in the course of the disease and/or using other sepsis models. Additionally, individual boswellic acids should be tested to find out, which of the different components of frankincense extract may indeed be responsible for the beneficial effects of Casp in acute systemic inflammation.

\section{AVAILABILITY OF DATA AND MATERIALS}

The materials used and raw data supporting the conclusions of the present manuscript will be available from the corresponding author without undue reservation on reasonable request to any qualified researcher.

\section{REFERENCES}

Abdel-Tawab, M., Werz, O., and Schubert-Zsilavecz, M. (2011). Boswellia serrata: an overall assessment of in vitro, preclinical, pharmacokinetic and clinical data. Clin. Pharmacokinet. 50, 349-369. doi: 10.2165/11586800-00000000000000

Afsar, V., Reddy, Y. M., and Saritha, K. V. (2012). In vitro antioxidant activity and antiinflammatory activity of methanolic leaf extract of Boswellia serrata. Int. J. Life Sci. Biotechnol. Pharma Res. 1, 15-23.

Aitio, A. (1978). A simple and sensitive assay of 7-ethoxycoumarin deethylation. Anal. Biochem. 85, 488-491. doi: 10.1016/0003-2697(78)90245-2

Aitken, A. E., Richardson, T. A., and Morgan, E. T. (2006). Regulation of drug-metabolizing enzymes and transporters in inflammation. Annu. Rev. Pharmacol. Toxicol. 46, 123-149. doi: 10.1146/annurev.pharmtox.46.120604. 141059

Al-Harrasi, A., Ali, L., Ceniviva, E., Al-Rawahi, A., Hussain, J., Hussain, H., et al. (2013). Antiglycation and antioxidant activities and HPTLC analysis of Boswellia sacra oleogum resin: the sacred frankincense. Trop. J. Pharm. Res. 12, 597-602. doi: 10.4314/tjpr.v12i4.23

Ammon, H. P. T. (2006). Boswellic acids in chronic inflammatory diseases. Planta Med. 72, 1100-1116. doi: 10.1055/s-2006-947227

Ammon, H. P. T. (2010). Modulation of the immune system by Boswellia serrata extracts and boswellic acids. Phytomedicine 17, 862-867. doi: 10.1016/j.phymed. 2010.03.003

Arismendi-Morillo, G. J., Briceño-García, A. E., Romero-Amaro, Z. R., FernándezAbreu, M. C., and Girón-Piña, H. E. (2004). Acute non-specific splenitis as indicator of systemic infection. Assessment of 71 autopsy cases. Invest. Clin. $45,131-135$

\section{AUTHOR CONTRIBUTIONS}

$\mathrm{KL}$, OW, and $\mathrm{AL}$ conceived and designed the experiments. MA-T and OW provided the compounds. AK, OW, and AL supervised the experiments. KL, SS, IL, SK, and AL performed the experiments. KL, SK, and AL analyzed the data. AL interpreted the data. AL wrote the manuscript. KL, SS, IL, SK, MA-T, $\mathrm{AK}, \mathrm{OW}$, and $\mathrm{AL}$ critically revised the manuscript. KL, SS, IL, SK, MA-T, AK, OW, and AL gave the final approval to the version to be published. All authors have read and approved the final version of the manuscript. Each of the authors acknowledges that he or she participated sufficiently in the work to take public responsibility for its content and each of the authors agreed to be accountable for all aspects of the work in ensuring that questions related to the accuracy or integrity of any part of the work are appropriately investigated and resolved.

\section{FUNDING}

This work was partially funded by the Free State of Thuringia and the European Social Fund (2016 FGR 0045).

\section{ACKNOWLEDGMENTS}

The paper was presented in part as an abstract at the 7th International Congress "Sepsis and Multiorgan Dysfunction" Weimar Sepsis Update, September 9-11, 2015; Infection 43, S21 (Abstract No. 086).

Ayala, A., Muñoz, M. F., and Argüelles, S. (2014). Lipid peroxidation: production, metabolism, and signaling mechanisms of malondialdehyde and 4-hydroxy-2nonenal. Oxid. Med. Cell. Longev. 2014:360438. doi: 10.1155/2014/360438

Carcillo, J. A., Doughty, L., Kofos, D., Frye, R. F., Kaplan, S., Sasser, H., et al. (2003). Cytochrome P450 mediated-drug metabolism is reduced in children with sepsis-induced multiple organ failure. Intensive Care Med. 29, 980-984. doi: 10.1007/s00134-003-1758-3

Casteleijn, E., Kuiper, J., Van Rooij, H. C. J., Kamps, J. A. A. M., Koster, J. F., and Van Berkel, T. J. C. (1988). Endotoxin stimulates glycogenolysis in the liver by means of intercellular communication. J. Biol. Chem. 263, 6953-6955.

Cuaz-Pérolin, C., Billiet, L., Baugé, E., Copin, C., Scott-Algara, D., Genze, F., et al. (2008). Antiinflammatory and antiatherogenic effects of the NF-к B inhibitor acetyl-11-keto- $\beta$-boswellic acid in LPS-challenged ApoE-/- mice. Arterioscler. Thromb. Vasc. Biol. 28, 272-277. doi: 10.1161/ATVBAHA.107. 155606

Du, Z., Liu, Z., Ning, Z., Liu, Y., Song, Z., Wang, C., et al. (2015). Prospects of boswellic acids as potential pharmaceutics. Planta Med. 81, 259-271. doi: 10.1055/s-0034-1396313

Ellman, G. L. (1959). Tissue sulfhydryl groups. Arch. Biochem. Biophys. 82, 70-77. doi: 10.1016/0003-9861(59)90090-6

Engel, C., Brunkhorst, F. M., Bone, H. G., Brunkhorst, R., Gerlach, H., Grond, S., et al. (2007). Epidemiology of sepsis in Germany: results from a national prospective multicenter study. Intensive Care Med. 33, 606-618. doi: 10.1007/ s00134-006-0517-7

Farley, K. S., Wang, L. F., Razavi, H. M., Law, C., Rohan, M., McCormack, D. G., et al. (2006). Effects of macrophage inducible nitric oxide synthase in murine septic lung injury. Am. J. Physiol. Lung Cell. Mol. Physiol. 290, L1164-L1172. doi: 10.1152/ajplung.00248.2005 
Fischer, A. H., Jacobson, K. A., Rose, J., and Zeller, R. (2008). Hematoxylin and eosin staining of tissue and cell sections. CSH Protoc. 2008:pdb.rot4986. doi: $10.1101 /$ pdb.prot4986

Flierl, M. A., Rittirsch, D., Huber-Lang, M., Sarma, J. V., and Ward, P. A. (2008). Catecholamines - Crafty weapons in the inflammatory arsenal of immune/inflammatory cells or opening Pandora's box? Mol. Med. 14, 195-204.

Frank, A., and Unger, M. (2006). Analysis of frankincense from various Boswellia species with inhibitory activity on human drug metabolizing cytochrome P450 enzymes using liquid chromatography mass spectrometry after automated online extraction. J. Chromatogr. A 1112, 255-262. doi: 10.1016/j.chroma.2005. 11.116

Gaieski, D. F., Edwards, J. M., Kallan, M. J., and Carr, B. G. (2013). Benchmarking the incidence and mortality of severe sepsis in the United States. Crit. Care Med. 41, 1167-1174. doi: 10.1097/CCM.0b013e31827c09f8

Galley, H. F. (2011). Oxidative stress and mitochondrial dysfunction in sepsis. $B r$. J. Anaesth. 107, 57-64. doi: 10.1093/bja/aer093

Gayathri, B., Manjula, N., Vinaykumar, K. S., Lakshmi, B. S., and Balakrishnan, A. (2007). Pure compound from Boswellia serrata extract exhibits antiinflammatory property in human PBMCs and mouse macrophages through inhibition of TNF- $\alpha$, IL-1 $\beta$, NO and MAP kinases. Int. Immunopharmacol. 7 , 473-482. doi: 10.1016/j.intimp.2006.12.003

Goldfarb, R. D., Parker, T. S., Levine, D. M., Glock, D., Akhter, I., Alkhudari, A., et al. (2003). Protein-free phospholipid emulsion treatment improved cardiopulmonary function and survival in porcine sepsis. Am. J. Physiol. Regul. Integr. Comp. Physiol. 284, R550-R557. doi: 10.1152/ajpregu.00285. 2002

Gonnert, F. A., Recknagel, P., Seidel, M., Jbeily, N., Dahlke, K., Bockmeyer, C. L., et al. (2011). Characteristics of clinical sepsis reflected in a reliable and reproducible rodent sepsis model. J. Surg. Res. 170, e123-e134. doi: 10.1016/j. jss.2011.05.019

Gordon, B. R., Parker, T. S., Levine, D. M., Feuerbach, F., Saal, S. D., Sloan, B. J., et al. (2005). Neutralization of endotoxin by a phospholipid emulsion in healthy volunteers. J. Infect. Dis. 191, 1515-1522. doi: 10.1086/428908

Goto, M., Yoshioka, T., Battelino, T., Ravindranath, T., and Zeller, P. (2001). TNF decreases gluconeogenesis in hepatocytes isolated from 10-day-old rats. Pediatr. Res. 49, 552-557. doi: 10.1203/00006450-200104000-00018

Hayes, J. D., and McLellan, L. I. (1999). Glutathione and glutathionedependent enzymes represent a co-ordinately regulated defence against oxidative stress. Free Radic. Res. 31, 273-300. doi: 10.1080/10715769900 300851

Henkel, A., Kather, N., Mönch, B., Northoff, H., Jauch, J., and Werz, O. (2012). Boswellic acids from frankincense inhibit lipopolysaccharides functionally through direct molecular interference. Biochem. Pharmacol. 82, 115-121. doi: $10.1016 /$ j.bcp.2011.09.026

Hiramatsu, M., Hotchkiss, R. S., Karl, I. E., and Buchman, T. G. (1997). Cecal ligation and puncture (CLP) induces apoptosis in thymus, spleen, lung, and gut by an endotoxin and TNF-independent pathway. Shock 7, 247-253. doi: 10.1097/00024382-199704000-00002

Hissin, P. J., and Hilf, R. (1976). A fluorometric method for determination of oxidized and reduced glutathione in tissues. Anal. Biochem. 74, 214-226. doi: 10.1016/0003-2697(76)90326-2

Horvatits, T., Trauner, M., and Fuhrmann, V. (2013). Hypoxic liver injury and cholestasis in critically ill patients. Curr. Opin. Crit. Care 19, 128-132. doi: 10.1097/MCC.0b013e32835ec9e6

Hüsch, J., Bohnet, J., Fricker, G., Skarke, C., Artaria, C., Appendino, G., et al. (2013). Enhanced absorption of boswellic acids by a lecithin delivery form (Phytosome $\left({ }^{\circledR}\right)$ ) of Boswellia extract. Fitoterapia 84, 89-98. doi: 10.1016/j.fitote. 2012.10.002

Jacob, A., Zhou, M., Wu, R., and Wang, P. (2009). The role of hepatic cytochrome P-450 in sepsis. Int. J. Clin. Exp. Med. 2, 203-211.

Kaemmerer, D., Sänger, J., Arsenic, R., D’Haese, J. G., Neumann, J., SchmittGraeff, A., et al. (2017). Evaluation of somatostatin, CXCR4 chemokine and endothelin A receptor expression in a large set of paragangliomas. Oncotarget 8, 89958-89969. doi: 10.18632/oncotarget.21194

Kim, Y. S., Morgan, M. J., Choksi, S., and Liu, Z. G. (2007). TNF-induced activation of the Nox1 NADPH oxidase and its role in the induction of necrotic cell death. Mol. Cell 26, 675-687. doi: 10.1016/j.molcel.2007.04.021
Kirkeboen, K. A., and Strand, O. A. (1999). The role of nitric oxide in sepsis an overview. Acta Anaesthesiol. Scand. 43, 275-288. doi: 10.1034/j.1399-6576. 1999.430307.x

Kleeberg, U., and Klinger, W. (1982). Sensitive formaldehyde determination with Nash's reagent and a 'tryptophan reaction'. J. Pharmacol. Methods 8, 19-31. doi: 10.1016/0160-5402(82)90004-3

Klinger, W., and Müller, D. (1974). The influence of age on the protein concentration in serum, liver and kidney of rats determined by various methods. Z. Versuchstierkd. 16, 149-153.

Koch, A., Horn, A., Dückers, H., Yagmur, E., Sanson, E., Bruensing, J., et al. (2011). Increased liver stiffness denotes hepatic dysfunction and mortality risk in critically ill non-cirrhotic patients at a medical ICU. Crit. Care 15:R266. doi: $10.1186 / \mathrm{cc} 10543$

Kramer, L., Jordan, B., Druml, W., Bauer, P., Metnitz, P. G. H., Austrian Epidemiologic Study on Intensive Care et al. (2007). Incidence and prognosis of early hepatic dysfunction in critically ill patients - A prospective multicenter study. Crit. Care Med. 35, 1099-1104. doi: 10.1097/01.CCM.0000259462. 97164.A0

Lubet, R. A., Mayer, R. T., Cameron, J. W., Nims, R. W., Burke, M. D., Wolff, T., et al. (1985). Dealkylation of pentoxyresorufin: a rapid and sensitive assay for measuring induction of cytochrome(s) P-450 by phenobarbital and other xenobiotics in the rat. Arch. Biochem. Biophys. 238, 43-48. doi: 10.1016/00039861(85)90138-9

Martin, G. S. (2012). Sepsis, severe sepsis and septic shock: changes in incidence, pathogens and outcomes. Expert Rev. Anti Infect. Ther. 10, 701-706. doi: $10.1586 /$ eri. 12.50

McManus, J. F. (1948). Histological and histochemical uses of periodic acid. Stain Technol. 23, 99-108. doi: 10.3109/10520294809106232

Morgan, E. T. (2001). Regulation of cytochrome p450 by inflammatory mediators: why and how? Drug Metab. Dispos. 29, 207-212.

Moussaieff, A., and Mechoulam, R. (2009). Boswellia resin: from religious ceremonies to medical uses; a review of in-vitro, in-vivo and clinical trials. J. Pharm. Pharmacol. 61, 1281-1293. doi: 10.1211/jpp/61.10.0003

Nebert, D. W., and Russell, D. W. (2002). Clinical importance of the cytochrome P450. Lancet 360, 1155-1162. doi: 10.1016/S0140-6736(02)11203-7

Nesseler, N., Launey, Y., Aninat, C., Morel, F., Mallédant, Y., and Seguin, P. (2012). Clinical review: the liver in sepsis. Crit. Care 16, 235-243. doi: 10.1186/cc11381

Novotny, A. R., Emmanuel, K., Maier, S., Westerholt, A., Weighardt, H., Stadler, J., et al. (2007). Cytochrome P450 activity mirrors nitric oxide levels in postoperative sepsis: predictive indicators of lethal outcome. Surgery 141, 376-384. doi: 10.1016/j.surg.2006.08.011

Pandey, R. S., Singh, B. K., and Tripathi, Y. B. (2005). Extract of gum resins of Boswellia serrata L. inhibits lipopolysaccharide induced nitric oxide production in rat macrophages along with hypolipidemic property. Ind. J. Exp. Biol. 43, 509-516.

Poeckel, D., and Werz, O. (2006). Boswellic acids: biological actions and molecular targets. Curr. Med. Chem. 13, 3359-3369. doi: 10.2174/092986706779010333

Pohl, R. J., and Fouts, J. R. (1980). A rapid method for assaying the metabolism of 7-ethoxyresorufin by microsomal subcellular fractions. Anal. Biochem. 107, 150-155. doi: 10.1016/0003-2697(80)90505-9

Prigent, H., Maxime, V., and Annane, D. (2004). Science review: mechanisms of impaired adrenal function in sepsis and molecular actions of glucocorticoids. Crit. Care 8, 243-252. doi: 10.1186/cc2878

Raetzsch, C. F., Brooks, N. L., McKee Alderman, J., Moore, K. S., Hosick, P. A., Klebanov, S., et al. (2009). LPS inhibition of glucose production through the TLR4, MYD88, NF(B pathway. Hepatology 50, 592-600. doi: 10.1002/hep. 22999

Read, T. E., Grunfeld, C., Zindaba, I., Kumwenda, L., Calhoun, M. C., Kane, J. P., et al. (1995). Triglyceride-rich lipoproteins prevent septic death in rats. J. Exp. Med. 182, 267-272. doi: 10.1084/jem.182.1.267

Riva, A., Morazzoni, P., Artaria, C., Allegrini, P., Meins, J., Savio, D., et al. (2016). A single-dose, randomized, cross-over, two-way, open-label study for comparing the absorption of boswellic acids and its lecithin formulation. Phytomedicine 23, 1375-1382. doi: 10.1016/j.phymed.2016.07.009

Santos, G. A., Moura, R. F., Vitorino, D. C., Roman, E. A., Torsoni, A. S., Velloso, L. A., et al. (2013). Hypothalamic AMPK activation blocks lipopolysaccharide inhibition of glucose production in mice liver. Mol. Cell. Endocrinol. 381, 88-96. doi: 10.1016/j.mce.2013.07.018 
Saraiva, M., and O'Garra, A. (2010). The regulation of IL-10 production by immune cells. Nat. Rev. Immunol. 10, 170-181. doi: 10.1038/nri2711

Seemann, S., and Lupp, A. (2015). Administration of a CXCL12 analog in endotoxemia is associated with anti-inflammatory, anti-oxidative and cytoprotective effects in vivo. PLoS One 10:e0138389. doi: 10.1371/journal.pone. 0138389

Seemann, S., and Lupp, A. (2016). Administration of AMD3100 in endotoxemia is associated with pro-inflammatory, pro-oxidative, and pro-apoptotic effects in vivo. J. Biomed. Sci. 23:68. doi: 10.1186/s12929-016-0286-8

Seemann, S., Zohles, F., and Lupp, A. (2017). Comprehensive comparison of three different animal models for systemic inflammation. J. Biomed. Sci. 24:60. doi: 10.1186/s12929-017-0370-8

Sharma, S., Gupta, S., Khajuria, V., Bhagat, A., Ahmed, Z., and Shah, B. A. (2016). Analogues of boswellic acids as inhibitors of pro-inflammatory cytokines TNF$\alpha$ and IL-6. Bioorg. Med. Chem. Lett. 26, 695-698. doi: 10.1016/j.bmcl.2015. 11.035

Shoji, Y., Uedono, Y., Ishikura, H., Takeyama, N., and Tanaka, T. (1995). DNA damage induced by tumour necrosis factor- $\alpha$ in L929 cells is mediated by mitochondrial oxygen radical formation. Immunology 84, 543-548.

Siemoneit, U., Koeberle, A., Rossi, A., Dehm, F., Verhoff, M., Reckel, S., et al. (2011). Inhibition of microsomal prostaglandin E2 synthase-1 as a molecular basis for the anti-inflammatory actions of boswellic acids from frankincense. Br. J. Pharmacol. 162, 147-162. doi: 10.1111/j.1476-5381.2010.01020.x

Siemoneit, U., Pergola, C., Jazzar, B., Northoff, H., Skarke, C., Jauch, J., et al. (2009). On the interference of boswellic acids with 5-lipoxygenase: mechanistic studies in vitro and pharmacological relevance. Eur. J. Pharmacol. 606, 246-254. doi: 10.1016/j.ejphar.2009.01.044

Stortz, J. A., Raymond, S. L., Mira, J. C., Moldawer, L. L., Mohr, A. M., and Efron, P. A. (2017). Murine models of sepsis and trauma: can we bridge the gap? ILAR J. 58, 90-105. doi: 10.1093/ilar/ilx007

Stürner, K. H., Stellmann, J. P., Dörr, J., Paul, F., Friede, T., Schammler, S., et al. (2017). A standardized frankincense extract reduces disease activity in relapsing-remitting multiple sclerosis (the SABA phase IIa trial). J. Neurol. Neurosurg. Psychiatry 89, 1-9. doi: 10.1136/jnnp-2017-317101

Syrovets, T., Büchele, B., Gedig, E., Slupsky, J. R., and Simmet, T. (2000). Acetylboswellic acids are novel catalytic inhibitors of human topoisomerases I and II $\alpha$. Mol. Pharmacol. 58, 71-81. doi: 10.1124/mol.58.1.71

Syrovets, T., Gschwend, J. E., Büchele, B., Laumonnier, Y., Zugmaier, W., Genze, F., et al. (2005). Inhibition of IкB kinase activity by acetyl-boswellic acids promotes apoptosis in androgen-independent PC-3 prostate cancer cells in vitro and in vivo. J. Biol. Chem. 280, 6170-6180. doi: 10.1074/jbc.M409477200

Takada, Y., Ichikawa, H., Badmaev, V., and Aggarwal, B. B. (2006). Acetyl-11keto- $\beta$-boswellic acid potentiates apoptosis, inhibits invasion, and abolishes

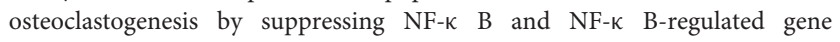
expression. J. Immunol. 176, 3127-3140. doi: 10.4049/jimmunol.176.5.3127

Tsukahara, Y., Morisaki, T., Kojima, M., Uchiyama, A., and Tanaka, M. (2001). iNOS expression by activated neutrophils from patients with sepsis. ANZ J. Surg. 71, 15-20. doi: 10.1046/j.1440-1622.2001.02025.x

Valdés-Ferrer, S. I., Rosas-Ballina, M., Olofsson, P. S., Lu, B., Dancho, M. E., Ochani, M., et al. (2013). HMGB1 mediates splenomegaly and expansion of splenic CD11b+ Ly-6Chigh inflammatory monocytes in murine sepsis survivors. J. Intern. Med. 274, 381-390. doi: 10.1111/joim.12104

Wang, L., Taneja, R., Razavi, H. M., Law, C., Gillis, C., and Mehta, S. (2012). Specific role of neutrophil inducible nitric oxide synthase in murine sepsis-induced lung injury in vivo. Shock 37, 539-547. doi: 10.1097/SHK.0b013e31824dcb5a

Yagi, K. (1987). Lipid peroxides and human diseases. Chem. Phys. Lipids 45, 337-351. doi: 10.1016/0009-3084(87)90071-5

Yan, J., Li, S., and Li, S. (2014). The role of the liver in sepsis. Int. Rev. Immunol. 33, 498-510. doi: 10.3109/08830185.2014.889129

Yang, Y., Liu, L., Zhao, B., Li, M. Q., Wu, B., Yan, Z., et al. (2007). Relationship between adrenal function and prognosis in patients with severe sepsis. Chin. Med. J. 120, 1578-1582.

Zhang, G., Dong, G., Zhao, X., Wang, M., and Li, C. S. (2014). Prognostic significance of hypothalamic-pituitary-adrenal axis hormones in early sepsis: a study performed in the emergency department. Intensive Care Med. 40, 1499-1508. doi: 10.1007/s00134-014-3468-4

Conflict of Interest Statement: The authors declare that the research was conducted in the absence of any commercial or financial relationships that could be construed as a potential conflict of interest.

Copyright (c) 2018 Loeser, Seemann, König, Lenhardt, Abdel-Tawab, Koeberle, Werz and Lupp. This is an open-access article distributed under the terms of the Creative Commons Attribution License (CC BY). The use, distribution or reproduction in other forums is permitted, provided the original author(s) and the copyright owner are credited and that the original publication in this journal is cited, in accordance with accepted academic practice. No use, distribution or reproduction is permitted which does not comply with these terms. 


\section{OPEN ACCESS}

Edited by:

Atanas G. Atanasov, Institute of Genetics and Animal Breeding (PAS), Poland

Reviewed by:

Letizia Angiolella,

Sapienza Università di Roma, Italy Kannan R. R. Rengasamy,

Alagappa University, India

${ }^{*}$ Correspondence: Tobias Weil tobias.weil@fmach.it

Fulvio Mattivi

fulvio.mattivi@unitn.it;

fulvio.mattivi@fmach.it

Specialty section: This article was submitted to

Ethnopharmacology a section of the journal Frontiers in Pharmacology

Received: 15 December 2017 Accepted: 03 April 2018 Published: 20 April 2018

Citation: Tocci N, Perenzoni $D$, lamonico $D$, Fava F, Weil T and Mattivi F (2018) Extracts From Hypericum hircinum subsp. majus Exert Antifungal Activity Against a Panel of Sensitive and Drug-Resistant Clinical Strains.

Front. Pharmacol. 9:382. doi: 10.3389/fphar.2018.00382

\section{Extracts From Hypericum hircinum subsp. majus Exert Antifungal Activity Against a Panel of Sensitive and Drug-Resistant Clinical Strains.}

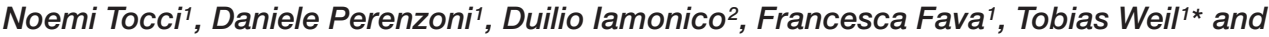 \\ Fulvio Mattivi ${ }^{1,3 *}$ \\ ${ }^{1}$ Research and Innovation Centre, Fondazione Edmund Mach, San Michele all'Adige, Italy, ${ }^{2}$ Laboratory of Phytogeography \\ and Applied Geobotany, Section Environment and Landscape, Department of Planning, Design, and Technology of \\ Architecture, Sapienza University of Rome, Rome, Italy, ${ }^{3}$ Centre Agriculture Food Environment, University of Trento, \\ Trento, Italy
}

During the last two decades incidences of fungal infections dramatically increased and the often accompanying failure of available antifungal therapies represents a substantial clinical problem. The urgent need for novel antimycotics called particular attention to the study of natural products. The genus Hypericum includes many species that are used in the traditional medicine to treat pathological states like inflammations and infections caused by fungi. However, despite the diffused use of Hypericum-based products the antifungal potential of the genus is still poorly investigated. In this study five Hypericum species autochthonous of Central and Eastern Europe were evaluated regarding their polyphenolic content, their toxicological safety and their antifungal potential against a broad panel of clinical fungal isolates. LC-MS analysis led to the identification and quantification of 52 compounds, revealing that Hypericum extracts are rich sources of flavonols, benzoates and cinnamates, and of flavan-3-ols. An in-depth screen of the biological activity of crude extracts clearly unveiled $H$. hircinum subsp. majus as a promising candidate species for the search of novel antifungals. H. hircinum is diffused in the Mediterranean basin from Spain to Turkey where it is traditionally used to prepare a herbal tea indicated for the treatment of respiratory tract disorders, several of which are caused by fungi. Noteworthy, the infusion of $H$. hircinum subsp. majus excreted broad antifungal activity against Penicillium, Aspergillus and non-albicans Candida isolates comprising strains both sensitive and resistant to fluconazole. Additionally, it showed no cytotoxicity on human cells and the chemical characterization of the $\mathrm{H}$. hircinum subsp. majus infusion revealed high amounts of the metabolite hyperoside. These results scientifically support the traditional use of $H$. hircinum extracts for the treatment of respiratory tract infections and suggest the presence of exploitable antifungal principles for further investigations aimed at developing novel antifungal therapies.

Keywords: Hypericum, antifungal, Candida, cytotoxicity, LC-MS, phenolic compounds

Abbreviations: CFU, colony forming units; GM, geometric mean; LC-MS, liquid chromatography-mass spectrometry; MIC, minimal inhibitory concentration. 


\section{INTRODUCTION}

The rising incidence of fungal infections is an emerging serious health problem, especially in cases of immune compromised patients caused either by diseases like AIDS, or by cancer therapies and prolonged antibiotic treatments (Lass-Flörl, 2009; Weil et al., 2017). Estimates of global mortality rates suggest that fungi are responsible for more deaths than either tuberculosis or malaria (Brown et al., 2012). Millions more suffer from severe illness caused by chronic or allergic fungal infections. Candidiasis is the most prevalent fungal disease worldwide difficult and extremely costly to treat (World Health Organization [WHO], 2014). The available antifungal drugs like polyenes, pyrimidines, azoles, and echinocandins often provoke significant toxicity including fever, nausea and vomiting, dose-limiting nephrotoxicity, liver toxicity, and controversial interactions with co-administered drugs (Dismukes, 2000; Zager, 2000). Another crucial problem is represented by the increasing rate of drug resistance that invalidates the clinical treatments. The alarming gap in the anti-fungal pipeline makes the discovery of new active compounds a priority for the scientific community. The strong request of new classes of compounds has renewed the interest in the screening of natural products (Abreu et al., 2012; Atanasov et al., 2016). Especially, ethnobotany represents an important guide in the search of novel antifungal agents. Due to their ability to synthesize a limitless variety of chemical structures, plants have represented a source of bioactive remedies for centuries (Arif et al., 2009). Despite of this, it is estimated that only a small proportion of plants are used by humans. Therefore, bioactivity screening programs and research on new plant-derived compounds are of prime importance for the search of novel leads. Plants belonging to the genus Hypericum, are characteristic components of the European flora. In particular, Europe and the Mediterranean basin represent a hot spot of diversity for Hypericum species (Nürk and Crockett, 2011). The best characterized species is H. perforatum L., which was already described by Hippocrates as a remedy against possession in the Ancient Greece (Dugoua et al., 2006). During the centuries it has been used for the treatment of various pathological states, like neurological disorders, wounds, inflammations, and infections. The use of $H$. perforatum extracts is so popular that this species is considered the most important medicinal plant of the 20th century (Gurib-Fakim, 2006). The German Commission E approved its usage for several indications, including treatment and post-therapy of acute and contused injuries, myalgia and first-degree burns as well as dyspeptic complaints (Arsic et al., 2010). In the Balkan Countries it is considered as one of the oldest remedies and in the Serbian folk medicine it is indicated for the treatment of cuts, burns, hemorrhoids and also as an antiseptic, for liver and stomach complaints, diarrhea, and gastric ulcers. Its sale represents a huge part of the overall herbal market (Crockett et al., 2011), exceeding $\$ 570$ million/annum worldwide (Ayan et al., 2006). Regarding its antifungal potential, inhibitory properties against Candida spp., (Tocci et al., 2013) and fungal plant pathogens (Crockett et al., 2011) have been reported. Nevertheless, a growing number of scientific evidences underlines the importance to investigate other Hypericum species that might exert a more promising antifungal activity (Radulović et al., 2007; Stojanovic et al., 2013). In this regard the species $H$. carinatum, $H$. myrianthum, $H$. linoides (Barros et al., 2013), H. garciae (Tocci et al., 2018a) have been shown to have the potential to inhibit the growth of Candida spp. Cryptococcus neoformans and Rhodotorula mucilaginosa growth.

In Central and Eastern Europe several different Hypericum species co-occur and the area is considered a significant source of plant material for the market of herbal products (Raclariu et al., 2017).

Amongst others, H. maculatum Crantz subsp. maculatum, $H$. hircinum L. s. lat., $H$. hirsutum L., and $H$. montanum L., as plants used to treat wounds, skin inflammations and burns (Menkovic et al., 2011), as well as infections of the respiratory (Sagratini et al., 2008) and urinary tract (SarićKundalić et al., 2010). These pathologies are often caused by fungi, with Candida as the main causative agent and by dermatophytes in the case of external infections (Borgers et al., 2005; Johnson, 2012; Ritchie and Eltahawy, 2014). To screen for new candidates suitable for the development of novel antimycotic solutions, we studied extracts deriving from the aerial parts of several Hypericum taxa (H. perforatum L., H. maculatum Crantz subsp. maculatum, $H$. hircinum L. s.lat., $H$. hirsutum L., and $H$. montanum L.) with respect to their chemical profile, their antifungal activity against a broad panel of clinical fungal isolates of the human gut and their toxicological safety on human skin fibroblasts and peripheral blood mononuclear cells (PBMCs).

\section{MATERIALS AND METHODS}

\section{Plant Material}

Samples of $H$. hirsutum subsp. hirsutum, $H$. hircinum subsp. majus, $H$. maculatum, $H$. montanum, and $H$. perforatum have been collected during spring-summer of 2015 in the Alpine region (Northern-Eastern Italy). Species were identified according to the most recent Flora of Italy (Pignatti, 1982, 2017), nomenclature follows Bartolucci et al. (2018) and type specimens were deposited in the Department of Food Quality and Nutrition at the Fondazione Edmund Mach in San Michele all'Adige, Trento, Italy (Reference number: NT0001 H. perforatum, NT0002 H. montanum, NT0003 H. maculatum, NT0004 H. hirsutum, NT0005 H. hircinum subsp. majus).

\section{Preparation of Crude Plant Extracts}

For each species, $500 \mathrm{mg}$ of dried plant biomass, represented by stems and leaves, were powdered by grinding and extracted in the dark with methanol $(\mathrm{drug} /$ solvent ratio $=1: 20 \mathrm{w} / \mathrm{v})$ by maceration $(3 \times 24 \mathrm{~h})$. $H$. hircinum subsp. majus was also subjected to extractions with boiling water $\left(100^{\circ} \mathrm{C}\right)$ for $20 \mathrm{~min}$, and ethanol $80 \%$ by maceration for $24 \mathrm{~h}$. The obtained extracts were evaporated to dryness, weighed and stored at $-20^{\circ} \mathrm{C}$ until analysis. 


\section{Preparation of Samples for Chemical Analysis}

Prior to analysis methanolic extracts were resuspended in $50 \%$ methanol/water and filtered using sterile $(0.2 \mu \mathrm{m})$ PTFE filters.

\section{LC-MS Analysis}

Analysis of phenolic metabolites was performed using a Waters Acquity UPLC system (Milford, MA, United States) which consists of a binary pump, an online vacuum degasser, an autosampler and a column compartment as previously described (Vrhovsek et al., 2012). Phenolic compounds were separated at a temperature of $40^{\circ} \mathrm{C}$ on a Waters Acquity HSS T3 column $1.8 \mu \mathrm{m}, 150 \mathrm{~mm} \times 2.1 \mathrm{~mm}$ (Milford, MA, United States). The mobile phase was composed of component A $(0.1 \%$ formic acid in water) and component $\mathrm{B}$ ( $0.1 \%$ formic acid in acetonitrile). Acetonitrile was of LC-MS grade and was purchased from Sigma-Aldrich (St. Louis, MO, United States). Pure standards of hypericin from Hypericum perforatum $\sim 95 \%$ (HPLC), hyperoside and hyperforin were purchased from Sigma-Aldrich (St. Louis, MO, United States). Milli-Q water was used for the chromatography. The flow was set to $0.4 \mathrm{~mL} / \mathrm{min}$, and the gradient profile was: $0 \mathrm{~min}$, $5 \% \mathrm{~B}$; from 0 to $3 \mathrm{~min}$, linear gradient to $20 \% \mathrm{~B}$; from 3 to $4.3 \mathrm{~min}$, isocratic $20 \% \mathrm{~B}$; from 4.3 to $9 \mathrm{~min}$, linear gradient to $45 \% \mathrm{~B}$; from 9 to $11 \mathrm{~min}$, linear gradient to $100 \% \mathrm{~B}$; from 11 to $13 \mathrm{~min}$, wash at $100 \% \mathrm{~B}$; from 13.01 to $15 \mathrm{~min}$, the re-equilibrated to the initial conditions of $5 \%$ B. Analysis of hypericin, hyperforin and hyperoside was performed using the same Waters Acquity UPLC system (Milford, MA, United States) and conditions, by means of a different gradient. The gradient profile in this case was: $0 \mathrm{~min}$, $10 \% \mathrm{~B}$; from 0 to $4,5 \mathrm{~min}$, linear gradient to $100 \% \mathrm{~B}$; from 4,5 to $12 \mathrm{~min}$, isocratic $100 \% \mathrm{~B}$; finally the column was reequilibrated to the initial conditions of $10 \% \mathrm{~B}$ for $4 \mathrm{~min}$. MRM transitions for hypericin, hyperforin and hyperoside are reported in Supplementary Table S1. Each sample was analyzed in triplicate. Samples were kept at $6^{\circ} \mathrm{C}$ during the analysis. Mass spectrometry detection for both methods was performed on a Waters Xevo TQMS (Milford, MA, United States) instrument equipped with an electrospray (ESI) source. Capillary voltage was $3.5 \mathrm{kV}$ in positive mode and $-2.5 \mathrm{kV}$ in negative mode; the source was kept at $150^{\circ} \mathrm{C}$; the desolvation temperature was $500^{\circ} \mathrm{C}$; cone gas flow, $50 \mathrm{~L} / \mathrm{h}$; and desolvation gas flow, $800 \mathrm{~L} / \mathrm{h}$. Further MS parameters are reported in Vrhovsek et al., 2012.

\section{Data Analysis}

Quantification was done using Waters MassLynx 4.1 and TargetLynx software.

\section{Microorganisms and Media}

For antifungal susceptibility testing, the reference strain C. albicans ATCC MYA-2876 from the American Type Culture Collection (ATCC), Rockville, MD, United States was used. The human gut clinical isolates, C. albicans YN7, C. parapsilosis YB1,
C. parapsilosis YB3 (Di Paola et al., unpublished), C. albicans YHS254, C. albicans YHS89, C. lusitaniae YHS217, C. lusitaniae YHS72, C. parapsilosis YHS133, C. parapsilosis YHS312, C. parapsilosis YHS301, Aspergillus glaucus YHS165, Penicillium paneum YHS245 (Strati et al., 2016), C. glabrata MFB004, C. tropicalis MFB035-1, C. tropicalis RTT037-3 (Strati et al., unpublished) were tested for their susceptibility against the applied drugs. All strains were grown on Sabouraud agarized medium for $48 \mathrm{~h}$ at $30^{\circ} \mathrm{C}$ before testing.

\section{Antifungal Susceptibility Testing}

Candida strains were tested for their susceptibility to Hypericum spp. extracts (7 dilution series, ranging from 500 to $8 \mu \mathrm{g} / \mathrm{ml}$ ) following the European Committee for Antimicrobial Susceptibility Testing protocol (EUCAST DEFINITIVE DOCUMENT EDef 7.2 Revision; European Committee for Antimicrobial Susceptibility Testing, 2012). Briefly, cells were grown in RPMI1640 medium supplied with $2.0 \%$ glucose, counted and inoculated at a concentration of $1-5 \times 10^{5} \mathrm{CFU} / \mathrm{ml}$. $\mathrm{MIC}_{50}$ and $\mathrm{MIC}_{90}$ values were determined at $530 \mathrm{~nm}$ using a spectrophotometer, as the lowest concentration of the drug that resulted in $\geq 50 \%$ and $\geq 90 \%$ inhibition of growth relative to the growth control after $48 \mathrm{~h}$ incubation.

\section{PBMC Isolation}

Peripheral blood mononuclear cells were obtained from buffy coat of healthy donors (as approved by the Ethical Committee of the local health centre, Azienda Provinciale per I Servizi Sanitari, Provincia Autonoma di Trento) within $4 \mathrm{~h}$ from sample collection. PBMC isolation was performed using Lympholite (CEDARLANE) density gradient (1.077 g/liter, $\mathrm{pH}$ 6,9), according to manufacturer's instructions, and incubated in complete nutrient RPMI 1640 medium (Euroclone) supplemented with glutamine (2 mol/L), 10\% fetal calf serum (FCS) and antibiotics (10000 U/ml Penicillin, $10000 \mu \mathrm{g} / \mathrm{ml}$ Streptomycin) (Biological Industries), at $37^{\circ} \mathrm{C}$ and $5 \% \mathrm{CO} 2$.

\section{Cytotoxicity Assay}

The cytotoxicity of $H$. hircinum subsp. majus infusion, methanolic and hydro-alcoholic extracts was tested using the WST-8 (4-[3-(2-methoxy-4-nitrophenyl)-2-(4-nitrophenyl)-2H5-tetrazolio]-1,3-benzene disulfonate sodium salt) conversion assay. The cytotoxicity of the extracts was evaluated on two normal cell lines, namely PBMC and human foreskin fibroblast Hs27. Cells were seeded in 96-well culture plates at concentration of 5000 cells/well and incubated for $24 \mathrm{~h}$ before treatment with $H$. hircinum subsp. majus extracts. Plant extracts were diluted in DMSO and added to a final concentration of $500 \mu \mathrm{g} / \mathrm{ml}$ (final volume of DMSO $0,5 \%$ $\mathrm{v} / \mathrm{v})$. To measure the cytotoxic effects $10 \mu \mathrm{l}$ of Cell Counting Kit-8 (Sigma-Aldrich) (containing the WST-8 solution) were added to each well and incubated for 2-4 h for fibroblasts and PBMCs, respectively, at $37^{\circ} \mathrm{C}$ before reading the absorbance at $450 \mathrm{~nm}$. 
TABLE 1 | Analysis of phenolic compounds in the crude extracts of Hypericum species.

\begin{tabular}{|c|c|c|c|c|c|}
\hline & H. hirc majus & H. hirs & H. mac & H. mont & H. perf \\
\hline & \multicolumn{5}{|c|}{$\mu \mathrm{g} / \mathrm{g}$ extract } \\
\hline Benzoates and cinnamates & $427,04 \pm 47,1$ & $45,9 \pm 3,65$ & $243,22 \pm 1,14$ & $390,72 \pm 6,17$ & $112,6 \pm 10,5$ \\
\hline 2,6-di-OH-benzoic acid & nd* & $\mathrm{nd} *$ & $\mathrm{nd} d^{*}$ & nd* & $\mathrm{nd}^{*}$ \\
\hline 3,5-dihydroxybenzoic acid & $1,78 \pm 0,013$ & $1,98 \pm 0,012$ & $1,47 \pm 0,013$ & $0,98 \pm 0,009$ & $0,96 \pm 0,016$ \\
\hline Caffeic acid & nd* & nd* & nd* & nd* & $\mathrm{nd} *$ \\
\hline Cinnamic acid & $\mathrm{nd}^{*}$ & $n d^{*}$ & $n d^{*}$ & $\mathrm{nd}^{*}$ & $\mathrm{nd}^{*}$ \\
\hline Ellagic acid & $5,83 \pm 0,073$ & $3,29 \pm 0,088$ & $7,22 \pm 0,114$ & $6,25 \pm 0,10$ & $2,18 \pm 0,054$ \\
\hline Ferulic acid & $0,06 \pm 0,003$ & $0,38 \pm 0,003$ & $0,54 \pm 0,012$ & $\mathrm{nd}^{*}$ & $0,26 \pm 0,006$ \\
\hline Gallic acid & $0,70 \pm 0,028$ & $1,07 \pm 0,008$ & $0,51 \pm 0,013$ & $0,73 \pm 0,032$ & $0,42 \pm 0,0013$ \\
\hline$p$-coumaric acid & $0,08 \pm 0,001$ & $0,6 \pm 0,019$ & $0,26 \pm 0,009$ & $0,25 \pm 0,002$ & $0,23 \pm 0,007$ \\
\hline p-OH-benzoic acid & $\mathrm{nd}^{*}$ & $n d^{*}$ & $\mathrm{nd} d^{*}$ & $n d^{*}$ & $\mathrm{nd}^{*}$ \\
\hline Vanillic acid & $\mathrm{nd}^{*}$ & $\mathrm{nd} *$ & $\mathrm{nd} *$ & $n d^{*}$ & $\mathrm{nd}^{*}$ \\
\hline Chlorogenic acid & $323,03 \pm 4,946$ & $8,51 \pm 0,450$ & nd* & nd* & $18,92 \pm 0,949$ \\
\hline Cryptochlorogenic acid & $0,58 \pm 0,009$ & $0,12 \pm 0,007$ & $1,32 \pm 0,048$ & $4,44 \pm 0,025$ & $0,72 \pm 0,01$ \\
\hline Neochlorogenic acid & $95,07 \pm 0,194$ & $29,95 \pm 0,032$ & $231,90 \pm 0,112$ & $378,07 \pm 0,449$ & $88,91 \pm 0,204$ \\
\hline Flavonols & $9393,64 \pm 50,3$ & $7470,05 \pm 28,01$ & $6555,52 \pm 30,21$ & $5284,31 \pm 13,25$ & $7189,19 \pm 47,26$ \\
\hline Hyperoside & $9306 \pm 385$ & $7430 \pm 211$ & $6240 \pm 488$ & $4940 \pm 66,3$ & $6880 \pm 469$ \\
\hline Quercetin & $2,28 \pm 0,043$ & $9,67 \pm 0,063$ & $45,44 \pm 0,159$ & $14,35 \pm 0,085$ & $5,13 \pm 0,011$ \\
\hline Quercetin-3-arabinoside & $\mathrm{nd}^{*}$ & $0,19 \pm 0,005$ & $32,40 \pm 0,099$ & $23,19 \pm 0,047$ & $132,86 \pm 1,522$ \\
\hline Quercetin-3-glucoside & $43,56 \pm 0,536$ & $18,12 \pm 0,529$ & $226,89 \pm 1,948$ & $235,33 \pm 1,712$ & $14,28 \pm 0,479$ \\
\hline Quercetin-3-glucuronide & $28,77 \pm 0,212$ & $\mathrm{nd} *$ & $\mathrm{nd} d^{*}$ & $\mathrm{nd} *$ & $2,54 \pm 0,02$ \\
\hline Quercetin-3-sulfate & $11,91 \pm 0,026$ & $2,22 \pm 0,018$ & $4,64 \pm 0,024$ & $0,69 \pm 0,004$ & $34,82 \pm 0,024$ \\
\hline Rutin & $\mathrm{nd}^{*}$ & $2,47 \pm 0,031$ & $3,19 \pm 0,015$ & $0,86 \pm 0,006$ & $118,89 \pm 0,197$ \\
\hline Kaempferol & $0,025 \pm 0,001$ & $1,73 \pm 0,007$ & $0,43 \pm 0,005$ & $0,11 \pm 0,001$ & $0,19 \pm 0,001$ \\
\hline Kaempferol-3-glucuronide & $0,48 \pm 0,0151$ & $n d^{*}$ & $n d^{*}$ & $\mathrm{nd}^{*}$ & $0,05 \pm 0,001$ \\
\hline Kaempferol -3-glucoside & $0,38 \pm 0,012$ & $2,38 \pm 0,071$ & $2,16 \pm 0,038$ & $1,18 \pm 0,031$ & $0,33 \pm 0,009$ \\
\hline Syringetin-3-glucoside & $n d^{*}$ & $n d^{*}$ & $n d^{*}$ & $\mathrm{nd}^{*}$ & nd* \\
\hline Iso-rhamnetin & $\mathrm{nd}^{*}$ & $n d^{*}$ & $0,13 \pm 0,003$ & $n d^{*}$ & $\mathrm{nd}^{*}$ \\
\hline Iso-rhamnetine-3-glucoside & $\mathrm{nd}^{*}$ & $0,08 \pm 0,004$ & $\mathrm{nd}^{*}$ & $n d^{*}$ & $\mathrm{nd}^{*}$ \\
\hline Myricetin & $0,23 \pm 0,004$ & $3,19 \pm 0,014$ & $0,24 \pm 0,003$ & $0,29 \pm 0,004$ & $0,10 \pm 0,002$ \\
\hline Flavones & $0,65 \pm 0,036$ & $2,215 \pm 0,016$ & $1,175 \pm 0,006$ & $3,035 \pm 0,014$ & $0,45 \pm 0,010$ \\
\hline Luteolin & trace & $1,93 \pm 0,011$ & $0,79 \pm 0,004$ & $2,52 \pm 0,009$ & $0,22 \pm 0,006$ \\
\hline Luteolin 7-O-glucoside & $0,65 \pm 0,036$ & $0,28 \pm 0,005$ & $0,38 \pm 0,002$ & $0,51 \pm 0,005$ & $0,18 \pm 0,004$ \\
\hline Flavanones & $0,44 \pm 0,009$ & $1,43 \pm 0,031$ & $0,58 \pm 0,018$ & $2,3 \pm 0,009$ & $0,82 \pm 0,016$ \\
\hline sNaringenin & $0,05 \pm 0,004$ & $0,18 \pm 0,006$ & nd* & $0,07 \pm 0,002$ & $0,10 \pm 0,004$ \\
\hline Naringenin-7-glucoside & $0,39 \pm 0,005$ & $1,25 \pm 0,025$ & $0,58 \pm 0,018$ & $2,23 \pm 0,007$ & $0,72 \pm 0,012$ \\
\hline Chalcones & $0,11 \pm 0,006$ & $0,42 \pm 0,005$ & $0,32 \pm 0,008$ & $\mathrm{nd} *$ & $0,31 \pm 0,005$ \\
\hline Phlorizin & $0,11 \pm 0,006$ & $0,42 \pm 0,005$ & $0,32 \pm 0,008$ & nd* & $0,31 \pm 0,005$ \\
\hline Coumarins & $0,23 \pm 0,0032$ & $0,07 \pm 0,002$ & $0,14 \pm 0,004$ & $0,55 \pm 0,005$ & $0,22 \pm 0,002$ \\
\hline Esculin & $0,23 \pm 0,0032$ & $0,07 \pm 0,002$ & $0,14 \pm 0,004$ & $0,55 \pm 0,005$ & $0,22 \pm 0,002$ \\
\hline Flavan-3-ols & $53,12 \pm 1,24$ & $74,12 \pm 3,12$ & $100,09 \pm 4,81$ & $58,28 \pm 1,53$ & $59,47 \pm 2,47$ \\
\hline Catechin & $21,94 \pm 0,609$ & $5,25 \pm 0,034$ & $3,68 \pm 0,114$ & $1,79 \pm 0,048$ & $4,21 \pm 0,161$ \\
\hline Epicatechin & $19,28 \pm 0,212$ & $40,21 \pm 0,258$ & $68,52 \pm 0,029$ & $53,61 \pm 0,166$ & $43,76 \pm 0,17$ \\
\hline Epigallocatechin & $3,31 \pm 0,030$ & $0,42 \pm 0,014$ & $0,52 \pm 0,008$ & $1,44 \pm 0,031$ & $0,82 \pm 0,025$ \\
\hline Gallocatechin & $2,21 \pm 0,032$ & $n d^{*}$ & $\mathrm{nd} *$ & $\mathrm{nd}^{*}$ & $\mathrm{nd}^{*}$ \\
\hline Procyanidin $\mathrm{B}_{1}$ & $1,37 \pm 0,007$ & $0,94 \pm 0,025$ & $0,89 \pm 0,018$ & $\mathrm{nd}^{*}$ & $0,42 \pm 0,005$ \\
\hline Procyanidin $\mathrm{B}_{2}$ & $5,01 \pm 0,091$ & $27,3 \pm 0,521$ & $26,48 \pm 0,770$ & $1,44 \pm 0,287$ & $10,26 \pm 0,111$ \\
\hline Stilbenoids & $0,06 \pm 0,001$ & $0,91 \pm 0,015$ & $2,24 \pm 0,009$ & $0,99 \pm 0,008$ & $3,32 \pm 0,052$ \\
\hline cis-piceid & $0,06 \pm 0,001$ & $0,91 \pm 0,015$ & $2,24 \pm 0,009$ & $0,99 \pm 0,008$ & $3,32 \pm 0,052$ \\
\hline Phloroglucinols & $n d^{*}$ & $n d^{*}$ & $n d^{*}$ & $20 \pm 1,91$ & $8050 \pm 565$ \\
\hline Hyperforin & nd* & $\mathrm{nd*}$ & $\mathrm{nd} *$ & $20 \pm 1,91$ & $8050 \pm 565$ \\
\hline Naphtodianthrones & $n d^{*}$ & $30 \pm 1,79$ & $20 \pm 1,71$ & $40 \pm 2,67$ & $150 \pm 25,1$ \\
\hline Hypericin & $\mathrm{nd}^{*}$ & $30 \pm 1,79$ & $20 \pm 1,71$ & $40 \pm 2,67$ & $150 \pm 25,1$ \\
\hline
\end{tabular}

H. hirs majus, H. hircinum subsp. majus; H. hirs, H. hirsutum; H. mac, H. maculatum; H. mon, H. montanum; H. perf, H. perforatum. * ${ }^{n d, ~ n o t ~ d e t e c t e d . ~}$ 
The results were analyzed by Student's $t$-test. The data are expressed as the percentage of viability and standard error of the mean.

\section{Microscopy}

To estimate the impact of plant extracts on cell morphology and health, cultures were visualized and recorded using an inverted microscope Motic AE31 equipped with a camera Moticam 580 $5.0 \mathrm{MP}$.

\section{RESULTS}

\section{Extract Composition}

The extracts have been chemically characterized by the identification and quantification of 52 low molecular weight polyphenols belonging to the classes: flavonols, benzoates and cinnamates, chalcones, flavan-3-ols, flavones, stilbens, coumarins, phloroglucinols, and naphtodianthrones (Table 1). LC-MS analysis revealed that species were characterized by different content of the detected metabolites, yet in all of the extracts, quercetin derivatives represented the most prevalent group of compounds.

Quercetin and quercetin glycosides ranged from 5,21 mg/g in $H$. montanum to $9,4 \mathrm{mg} / \mathrm{g}$ in $H$. hircinum subsp. majus, with hyperoside being the major compounds in the extracts representing the $45,48 \%$ of the detected compounds in H. perforatum and more than the $99 \%$ in the other Hypericum species. Quercetin-3-glucoside was abundant in $H$. maculatum and in H. montanum, while quercetin 3-rhamnoside was highly present in $H$. perforatum (132,9 $\mu \mathrm{g} / \mathrm{g})$. Quercetin-3-glucuronide only occurred in $H$. hircinum subsp. majus $(28,8 \mu \mathrm{g} / \mathrm{g})$ and in $H$. perforatum (10-fold less concentrated). Chlorogenic acid derivatives (chlorogenic acid, neochlorogenic acid, and cryptochlorogenic acid) ranged from $38 \mu \mathrm{g} / \mathrm{g}$ for $\mathrm{H}$. hirsutum and $H$. perforatum to $456,7 \mu \mathrm{g} / \mathrm{g}$ in $H$. hircinum subsp. majus. Also the flavan-3-ols were relatively abundant ranging from $53,12 \mu \mathrm{g} / \mathrm{g}$ in $H$. hircinum subsp. majus to $100,12 \mu \mathrm{g} / \mathrm{g}$ in $H$. maculatum, with epicatechin being the principal compound of this class (from $19 \mu \mathrm{g} / \mathrm{g}$ in $H$. hircinum subsp. majus up to $68,5 \mu \mathrm{g} / \mathrm{g}$ in $H$. maculatum).

\section{Antifungal Activity of Hypericum Crude Extracts}

Hypericum extracts were screened against a panel of clinical Candida isolates (Table 2 and Supplementary Table S2) and the results are hereafter given as GM of MIC values. The screening unveiled that $H$. hircinum subsp. majus crude methanolic extract had a strong anti-Candida activity against all tested fungal strains, with inhibition properties against C. parapsilosis $\left(\mathrm{MIC}_{50}\right.$ $53,5 \mu \mathrm{g} / \mathrm{ml})$ in the same magnitude of fluconazole $\left(\mathrm{MIC}_{50}\right.$ $22,63 \mu \mathrm{g} / \mathrm{ml})$.

All extracts exerted antifungal properties against C. albicans and C. lusitaniae while only $H$. hircinum subsp. majus was also active against C. tropicalis and C. glabrata $\left(\mathrm{MIC}_{50} 353,55\right.$ and $16 \mu \mathrm{g} / \mathrm{ml}$, respectively).

Also, with respect to the to the $\mathrm{MIC}_{90}$ values, $H$. hircinum subsp. majus extract showed higher inhibitory properties than the other tested extracts (Supplementary Table S2).

TABLE 2 | Antifungal activity of Hypericum species.

\begin{tabular}{|c|c|c|c|c|c|c|c|c|c|c|c|c|}
\hline Species (strain no.) & \multicolumn{2}{|c|}{ H. hircinum } & \multicolumn{2}{|c|}{ H. maculatum } & \multicolumn{2}{|c|}{ H. montanum } & \multicolumn{2}{|c|}{ H. perforatum } & \multicolumn{2}{|c|}{ H. hirsutum } & \multicolumn{2}{|c|}{ Fluconazole } \\
\hline C. parapsilosis (2) & 32 & 32 & 500 & 500 & $>500$ & $>500$ & $\geq 125$ & $125-500$ & $\geq 500$ & 500 & 48 & $32-64$ \\
\hline C. tropicalis (2) & 353,55 & $>500$ & $>500$ & $>500$ & $>500$ & $>500$ & $>500$ & $>500$ & $>500$ & $>500$ & $>64$ & $>64$ \\
\hline C. Iusitaniae (1) & 16 & 125 & 64 & 64 & 125 & 125 & 125 & 125 & 64 & 64 & 0,5 & 0,5 \\
\hline
\end{tabular}

Data are expressed as GM in $\mu \mathrm{g} / \mathrm{ml}$.

TABLE 3 | Antifungal activity of $H$. hircinum subsp. majus.

\begin{tabular}{|c|c|c|c|c|c|c|c|c|}
\hline Species (strain no.) & \multicolumn{2}{|c|}{ Methanol } & \multicolumn{2}{|c|}{ Ethanol $80 \%$} & \multicolumn{2}{|c|}{ Infusion } & \multicolumn{2}{|c|}{ Fluconazole } \\
\hline C. tropicalis (2) & 353,55 & $>500$ & 353,55 & $>500$ & 177 & $>500$ & $>64$ & $>64$ \\
\hline C. parapsilosis (5) & 53,5 & $\geq 250$ & 250 & $\geq 500$ & 95,18 & $\geq 300$ & $\geq 60$ & $>64$ \\
\hline C. Iusitaniae (2) & 16 & 89,4 & 63,25 & 250 & 32 & 177 & 0,35 & 1,5 \\
\hline Penicillium paneum (1) & $>500$ & $>500$ & $>500$ & $>500$ & 64 & 500 & 0,13 & 4 \\
\hline
\end{tabular}

Data are expressed as GM in $\mu \mathrm{g} / \mathrm{ml}$. 


\section{Antifungal Activity of $\boldsymbol{H}$. hircinum subsp. majus Extracts}

The antifungal activity of $H$. hircinum subsp. majus was further investigated against a broader panel of Candida, including fluconazole resistant strains, and other fungi (Table 3 and Supplementary Table S3). In addition to crude methanolic extract, infusion and hydroalcoholic extracts have been tested. Notably, all $H$. hircinum subsp. majus extracts showed antifungal properties and, in particular a remarkable activity against fluconazole resistant $C$. parapsilosis and C. tropicalis strains. The methanolic extract showed the most pronounced cytotoxic properties. Yet, in general the antifungal activity of the infusion outcompeted those of the hydroalcoholic extract, also with respect to the $\mathrm{MIC}_{90}$ values.

\section{Cytotoxicity of $\boldsymbol{H}$. hircinum subsp. majus Extracts}

The cytototoxicity of $H$. hircinum subsp. majus extracts was tested on PBMC and human skin fibroblast Hs27 cell lines by using the WST- 8 conversion assay. The extracts were tested at a dose of $500 \mu \mathrm{g} / \mathrm{ml}$, which corresponds to a 1 - (for C. tropicalis) to 10 -fold higher concentration than those showing inhibition of $50 \%$ of Candida growth. As shown in Figure 1, only the plant infusion showed no cytotoxic effect on both human cell lines, while treatment with methanolic and hydroalcoholic extracts inhibited cell viability by $50 \%$ for $\mathrm{Hs} 27$ cell line and by $65-70 \%$ for PBMCs.

The effect of $H$. hircinum subsp. majus on human cells was also observed microscopically (Figure 2). The imagines confirmed the cytotoxicity data. Fibroblast treated with plant infusion showed a normal phenotype, while under treatment with methanolic and hydro-alcoholic extracts the cell morphology was markedly altered. The same alteration was observed for PBMCs (data not shown).

\section{Chemical Composition of $\boldsymbol{H}$. hircinum susbps. majus Infusion}

The chemical composition of the aqueous extract of $H$. hircinum subsp. majus is reported in Table 4. The analysis revealed that the infusion process allowed the extraction of higher amounts of phenols in comparison to the extraction by maceration in methanol. The aqueous extract was mainly characterized by derivatives of quercetin $(24,18 \mathrm{mg} / \mathrm{g})$, of chlorogenic acid $(4,51 \mathrm{mg} / \mathrm{g})$ and by flavan-3-ols $(2,26 \mathrm{mg} / \mathrm{g})$. Hyperoside was the most abundant compound, representing the $2,23 \%$ of the extract. Abundant were also quercetin-3-glucoside $(1,65 \mathrm{mg} / \mathrm{g})$, neochlorogenic acid $(2,61 \mathrm{mg} / \mathrm{g})$, chlorogenic acid $(1,68 \mathrm{mg} / \mathrm{g})$, procyanidin B2 $(0,89 \mathrm{mg} / \mathrm{g})$, epicatechin $(0,59 \mathrm{mg} / \mathrm{g})$ and procyanidin $\mathrm{B} 1(0,40 \mathrm{mg} / \mathrm{g})$.

\section{DISCUSSION}

Plant-based treatments of the traditional medicine could bring forth new solutions for antifungal therapies. In particular polyphenol-rich plants represent an import source of bioactive products for human health (Marchelak et al., 2017) including antifungal treatments. Among polyphenols, flavan-3-ols, flavonols and tannins show a high antimicrobial potential (Daglia, 2012). Flavan-3-ols isolated from green and black tea exert important anti-Candida activity (Hirasawa and Takada, 2004; Sitheeque et al., 2009), ellagitannins obtained from Punica granatum inhibit the growth of plant pathogenic fungi (Glazer et al., 2012), the flavonols myricitrin and fisetin are considered effective agents against Candida glabrata and Cryptococcus neoformans, respectively (Salazar-Aranda et al., 2015; Reis et al., 2016). The mechanisms of action of phenols against Candida have been intensively studied and include inactivation of enzyme production (Evensen and Braun, 2009) and anti-biofilm effects (Evensen and Braun, 2009; Shahzad et al., 2014). Species
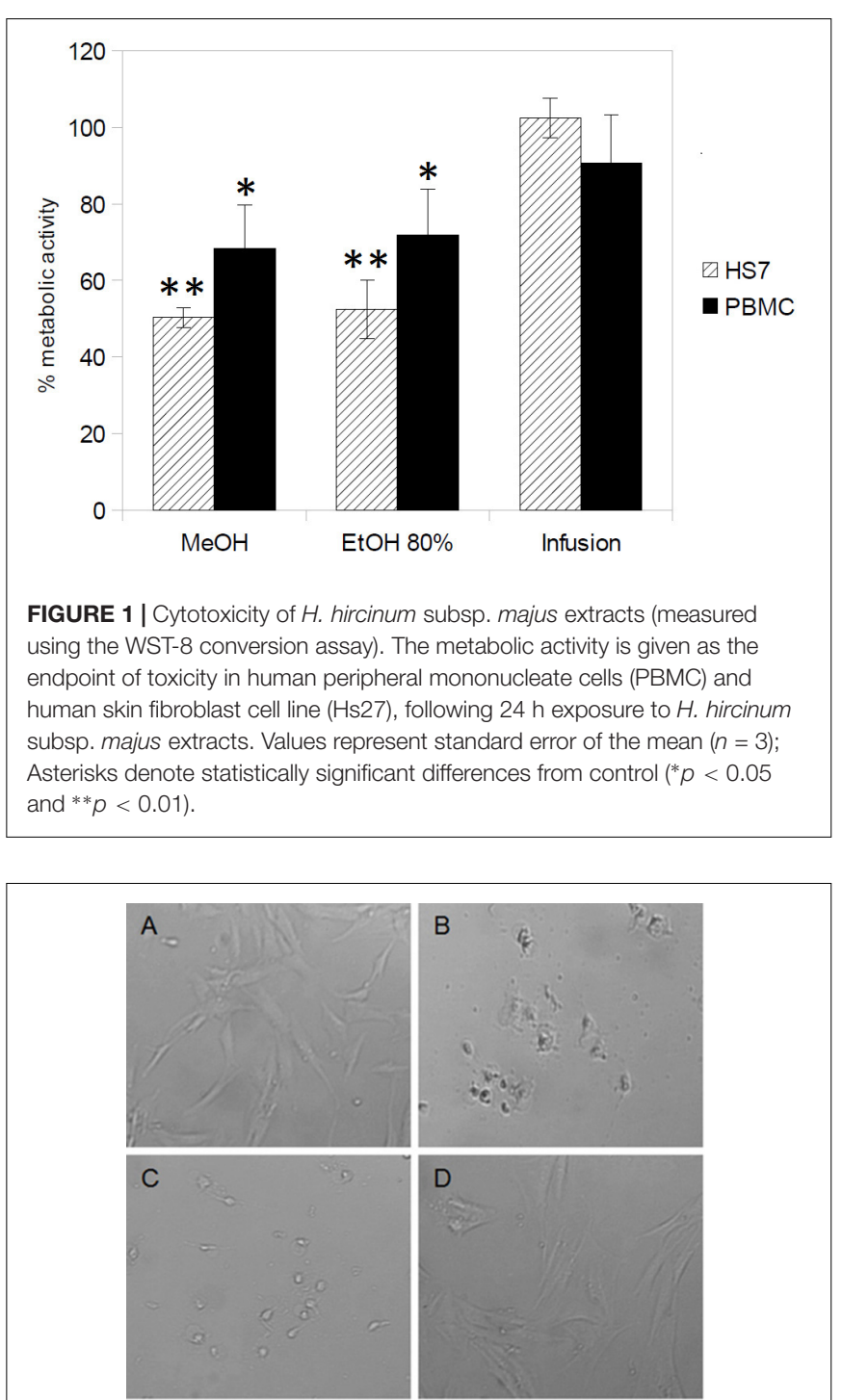

FIGURE 2 | Morphology change of normal fibroblast Hs27 after $24 \mathrm{~h}$ treatment with $H$. hircinum subsp. majus extracts (observed under inverted microscope, magnification 100 ): (A) control (DMSO), (B) methanolic extract, (C) hydroalcoholic extract, and (D) infusion. 
belonging to the Hypericum genus are able to synthesize a broad array of such bioactive phenolic compounds, mainly as products of the polyketide metabolism (Tocci et al., 2018b), and therefore might contain novel antifungal remedies.

Here we report new data on the inhibitory activity of extracts deriving from $H$. montanum, H. maculatum, $H$. hirsutum, and $H$. hircinum subsp. majus and additional data on the activity of $H$. perforatum on the growth of clinical isolates of Candida spp. The crude extracts obtained from H. maculatum, $H$. montanum, $H$. hirsutum, and $H$. perforatum showed a moderate antifungal activity against $C$. albicans, in the range of 250-500 $\mu \mathrm{g} / \mathrm{ml}$, good activity against C. lusitaniae (64$125 \mu \mathrm{g} / \mathrm{ml}$ ) and no or only weak activity against C. parapsilosis, C. tropicalis and C. glabrata. Our data are in accordance with those of Radulovic et al., 2007, showing that extracts of $H$. hirsutum, $H$. maculatum, and $H$. perforatum exerted comparable antifungal activity against $C$. albicans using the disk diffusion method assay. Discordant data are reported in Cecchini et al., 2007 which found no activity of extracts obtained from $H$. hirsutum or H. montanum against C. albicans. Most interesting, the antifungal screening of crude extracts unveiled the promising anti-Candida properties of H. hircinum subsp. majus. The test showed that extracts obtained from this species are able to inhibit $50 \%$ of growth of $C$. albicans at concentrations that are fourfold to eightfold lower than other Hypericum extracts and with a more pronounced activity against C. parapsilosis and C. lusitaniae. Moreover H. hircinum methanolic extract was the only treatment able to inhibit the growth of $C$. tropicalis and C. glabrata. These results are important especially in the light of the increasing incidence of infections caused by emerging Candida pathogens like C. glabrata, C. parapsilosis, C. tropicalis, and C. krusei, which are less susceptible to the commonly used antifungal drugs (Tortorano et al., 2006; Papon et al., 2013). Previous data on the antifungal activity of $H$. hircinum are scarce and limited to susceptibility tests against just a single reference strain of C. albicans. Using the disk diffusion test method Pistelli et al., 2000 found no antifungal activity against the tested strain, while Cecchini et al., 2007 reported a higher activity of H. hircinum subsp. majus in comparison to other Hypericum species.

From a chemical point of view, the extracts applied as treatments in our tests have been deeply characterized by investigating the presence of 52 phenolic compounds. In general, the most abundant classes of detected compounds were flavonols, benzoate and cinnamates (chlorogenic acid and neochlorogenic acid), and flavan-3-ols. The major metabolite in all the extracts was the flavonol hyperoside, ranging from $4940 \pm 66,3 \mu \mathrm{g} / \mathrm{g}$ in $H$. montanum to $9306 \pm 385 \mu \mathrm{g} / \mathrm{g}$ in $H$. hircinum subsp. majus, and very abundant were the neochlorogenic acid and the flavan-3-ol epicatechin. Hyperoside is known for its anticancer, antiinflammatory and antimicrobial properties (Ogawa et al., 2014). The extract of $H$. hircinum subsp. majus, showed several chemical peculiarities. It contained the highest amount of hyperoside, higher amounts of chlorogenic acid, quercetin-3-glucuronide, kampferol-3-glucuronide, catechin, epigallogatechin, gallocatechin, and no traces of the phloroglucinol hyperforin and of the naphtodianthrone hypericin.

In the light of its pronounced growth inhibition properties against Candida spp. and considering the ethnobotanical knowledge available for this species, $H$. hircinum subsp. majus has been further subjected to a deeper antifungal screen.

TABLE 4 | Analysis of phenolic compounds in the infusion of $H$. hircinum subsp. majus.

\begin{tabular}{|c|c|c|c|}
\hline Compound & Amount ( $\mu \mathrm{g} / \mathrm{g}$ extract) & Compound & Amount ( $\mu \mathrm{g} / \mathrm{g}$ extract) \\
\hline 2,6-di-OH-benzoic acid & $n d^{* *}$ & Kaempferol & $1,08 \pm 0,15$ \\
\hline 3,5-dihydroxybenzoic acid & $7,08 \pm 0,79$ & Kaempferol -3-glucoside & $7,89 \pm 1,09$ \\
\hline Caffeic acid & $44,62 \pm 4,97$ & Kaempferol-3-glucuronide & $104,41 \pm 14,49$ \\
\hline Coniferyl alcohol & nd* & Iso-rhamnetin & $0,78 \pm 0,11$ \\
\hline Gallic acid & $15,74 \pm 2,18$ & Iso-rhamnetine-3-glucoside & $\mathrm{nd}^{*}$ \\
\hline Cinnamic acid & $6,10 \pm 0,85$ & Syringetin-3-glucoside & $3,39 \pm 0,46$ \\
\hline$p$-coumaric acid & $\mathrm{nd}^{*}$ & Myricetin & $7,68 \pm 1,07$ \\
\hline p-OH-benzoic acid & $8,97 \pm 1,24$ & Luteolin & $3,51 \pm 0,22$ \\
\hline Vanillic acid & $1,29 \pm 0,18$ & Luteolin 7-O-glucoside & $1,77 \pm 0,24$ \\
\hline Cryptochlorogenic acid & $229,7 \pm 31,88$ & Naringenin & nd* \\
\hline Chlorogenic acid & $1683 \pm 233,7$ & Esculin & $21,28 \pm 2,95$ \\
\hline Neochlorogenic acid & $2615 \pm 362,9$ & Phlorizin & $6,57 \pm 0,91$ \\
\hline Hyperoside & $22203 \pm 380$ & Catechin & $282,2 \pm 2,71$ \\
\hline Quercetin & $164,38 \pm 15,52$ & Epicatechin & $586,51 \pm 68,05$ \\
\hline Quercetin-3-arabinoside & $\mathrm{nd} *$ & Epigallocatechin & $55,77 \pm 7,74$ \\
\hline Quercetin-3-glucoside & $1647 \pm 65,03$ & Gallocatechin & $48,92 \pm 9,72$ \\
\hline Quercetin-3-glucuronide & $104,41 \pm 14,92$ & Procyanidin $\mathrm{B}_{1}$ & $401,6 \pm 55,74$ \\
\hline Quercetin-3-sulfate & $\mathrm{nd}^{*}$ & Procyanidin $\mathrm{B}_{2}$ & $886,16 \pm 108,77$ \\
\hline Rutin & nd* & cis-piceid & $1,37 \pm 0,19$ \\
\hline
\end{tabular}

Data are means of three independent experiments, *nd, not detected. 
H. hircinum is a shrub native to the areas of the Mediterranean Basin comprising Greece and Turkey (Euro+Med, 2006). The infusion of its aerial part is applied in the traditional medicine to treat chronic catarrhal affections, asthma, sore throats and cough (Pieroni et al., 2004). Recent scientific evidences indicate that fungi of the phyla Basidiomycetes and Ascomycetes (e.g., Bjerkandera, Aspergillus, Candida, and Saccharomyces) are causative agents of these pathologies (Raza et al., 2017).

To investigate whether the beneficial effect of $H$. hircinum treatments may be attributed to its antifungal activity, we studied, for the first time, the activity of extracts obtained from the aerial parts of the plant with a hydroalcoholic solution and, in accordance with the traditional preparations, by infusion in boiling water. Additionally, we characterized the chemical profile. The assay was performed on a broad panel of clinical Candida strains, a strain of Aspergillus glaucus and a strain of Penicillium paneum (Table 3 and Supplementary Table S3). The extract obtained from the herbal tea inhibited $50 \%$ of the growth of all tested fungi, showing strong activity against both fluconazole sensitive and resistant Candida strains and moderate activity against Aspergillus and Penicillium strains. The chemical profile revealed an enrichment in the polyphenol content in comparison with the crude methanolic extract. Particularly abundant (content higher than $40 \mu \mathrm{g} / \mathrm{g}$ extract) were caffeic acid, chlorogenic, neochlorogenic and cryptochlorogenic acid, quercetin, quercetin-3-glucoside, quercetin-3-glucuronide, kaempferol-3-glucuronide, all the flavan-3-ols investigated and extraordinary high levels of hyperoside were detected. Even if the bioactivity may be due to the synergistic action of the phytocomplex, it appears reasonable to exclude caffeic acid, chlorogenic acid, quercetin-3-glucuronide, kaempferol-3glucuronide, and gallocatechin as secondary metabolites to which the antifungal activity may be attributed, since these compounds are not present in the other Hypericum extracts showing growth inhibitory properties on Candida strains. Also, cryptochlorogenic acid, neochlorogenic acid quercetin, quercetin-3-glucoside, epicatechin, have been detected in all the crude extract, but they were less present in $H$. hircinum subsp. majus and thus their presence cannot be considered correlated to the bioactivity. According to our data a positive correlation between the amount of catechin, epigallocatechin, procyanidin $B_{1}$ and hyperoside and the antifungal activity exerted can be individuated. The anti-Candida properties of flavan-3ols, and in particular of catechin and epigallocatechin, are well known and documented (Hirasawa and Takada, 2004; Saito et al., 2013). Nevertheless, our data suggest that the hyperoside, being detected in such high amounts in the extract obtained by the infusion of $H$. hircinum subsp. majus in water, may play a fundamental role in the observed fungal growth inhibition properties. The hypothesis is supported by studies proposing hyperoside as a lead for the development of new fungicides ( $\mathrm{Li}$ et al., 2005).

Finally, the aqueous extract of $H$. hircinum subsp. majus showed another promising characteristic exploitable for the development of a new antifungal therapy. When tested at high concentrations on human PBMCs and human skin fibroblast Hs27cell lines, no cytotoxicity was detected.

\section{CONCLUSION}

Our work reports a comprehensive phenolic profile of five European Hypericum species, highlighting the presence of important exploitable bioactive metabolites. The infusion of $H$. hircinum subsp. majus used in the popular medicine for the treatment of respiratory tract affections has been chemically and biologically investigated for the first time. The chemical analysis unveiled a new source of the bioactive metabolite hyperoside that might be involved in the observed bioactivity of the extract. The biological tests suggest that the beneficial effects of the traditional remedies based on $H$. hircinum could be attributed to its antiseptic, and in particular to its antifungal properties against fungal pathogens of the respiratory tract. Moreover, the lack of cytotoxicity indicates the presence of exploitable and promising antimycotic principles for the development of an antifungal therapy with limited cytotoxic side effects. Hence it encourages future studies to assess the effective therapeutic properties of $H$. hircinum subsp. majus with particular attention to the treatment of fungal-associated pathologies of the respiratory tract.

\section{ETHICS STATEMENT}

PBMCs were obtained from buffy coats prepared from blood donations collected at the Centro Trasfusionale c/o Ospedale Santa Chiara, Trento, after donor's signed informed consent. Study procedures were approved by the Comitato Etico per le Sperimentazioni Cliniche, Azienda Provinciale per i Servizi Sanitari di Trento on the 12th February 2015.

\section{AUTHOR CONTRIBUTIONS}

NT, TW, and FM conceived the study and wrote the manuscript with contributions from all authors. NT, DP, FF, and TW performed the experiments. DI identified the species. NT, DP, and TW analyzed the data. NT drafted the manuscript. All authors approved the final manuscript.

\section{FUNDING}

This work was supported by the Project ADP 2016, funded by the Autonomous Province of Trento, Italy.

\section{SUPPLEMENTARY MATERIAL}

The Supplementary Material for this article can be found online at: https://www.frontiersin.org/articles/10.3389/fphar.2018. 00382/full\#supplementary-material 


\section{REFERENCES}

Abreu, A. C., McBain, A. J., and Simões, M. (2012). Plants as sources of new antimicrobials and resistance-modifying agents. Nat. Prod. Rep. 29, 1007-1021. doi: $10.1039 / \mathrm{c} 2 \mathrm{np} 20035 \mathrm{j}$

Arif, T., Bhosale, J. D., Kumar, N., Mandal, T. K., Bendre, R. S., Lavekar, G. S., et al. (2009). Natural products-antifungal agents derived from plants. J. Asian Nat. Prod. Res. 11, 621-638. doi: 10.1080/10286020902942350

Arsic, I., Žugic, A., Antic, D. R., Zdunic, G., Dekanski, D., Markovic, G., et al. (2010). Hypericum perforatum L. Hypericaceae/Guttiferae sunflower, olive and palm oil extracts attenuate cold restraint stress - induced gastric lesions. Molecules 15, 6688-6698. doi: 10.3390/molecules 15106688

Atanasov, A. G., Waltenberger, B., Eva-Maria Pferschy-Wenzig, T. L., Wawrosch, C., Uhrin, P., Temml, V., et al. (2016). Discovery and resupply of pharmacologically active plant- derived natural products: a review. Biotechnol. Adv. 33, 1582-1614. doi: 10.1016/j.biotechadv.2015.08.001.Discovery

Ayan, A. K., Kizilkaya, R., Cirak, C., and Kevseroglu, K. (2006). Heavy metal contents of St. John's wort (Hypericum perforatum L.) Growing in Northern Turkey. J. Plant Sci. 1, 182-186. doi: 10.3923/jps.2006.182.186

Barros, F. M., Pippi, B., Dresch, R. R., Dauber, B., Luciano, S. C., Apel, M. A., et al. (2013). Antifungal and antichemotactic activities and quantification of phenolic compounds in lipophilic extracts of Hypericum spp. native to South Brazil. Ind. Crops Prod. 44, 294-299. doi: 10.1016/j.indcrop.2012.11.017

Bartolucci, F., Peruzzi, L., Galasso, G., Albano, A., Alessandrini, A., Ardenghi, N. M. G., et al. (2018). An updated checklist of the vascular flora native to Italy. Plant Biosyst. 152, 179-303. doi: 10.1080/11263504.2017.1419996

Borgers, M., Degreef, H., and Cauwenbergh, G. (2005). Fungal infections of the skin: infection process and antimycotic therapy. Curr. Drug Targets 6, 849-862. doi: $10.2174 / 138945005774912726$

Brown, G. D., Denning, D. W., Gow, N. A., Levitz, S. M., Netea, M. G., and White, T. C. (2012). Hidden killers: human fungal infections. Sci. Transl. Med. 4:165rv13. doi: 10.1126/scitranslmed.3004404

Cecchini, C., Cresci, A., Coman, M. M., Ricciutelli, M., Sagratini, G., Vittori, S., et al. (2007). Antimicrobial activity of seven Hypericum entities from central Italy. Planta Med. 73, 564-566.

Crockett, S. L., Poller, B., Tabanca, N., Pferschy-Wenzig, E. M., Kunert, O., Wedge, D. E., et al. (2011). Bioactive xanthones from the roots of Hypericum perforatum (common St John's wort). J. Sci. Food Agric. 91, 428-434. doi: 10.1002/jsfa.4202

Daglia, M. (2012). Polyphenols as antimicrobial agents. Curr. Opin. Biotechnol. 23, 174-181. doi: 10.1016/j.copbio.2011.08.007

Dismukes, W. E. (2000). Introduction to antifungal drugs. Clin. Inf. Dis. 30, 653-657. doi: 10.1086/313748

Dugoua, J.-J., Mills, E., Perri, D., and Koren, G. (2006). Safety and efficacy of St. John's wort (hypericum) during pregnancy and lactation. Can. J. Clin. Pharmacol. 13, e268-76.

Euro+Med (2006). Euro+Med Plant Base the Information Resource for Euro-Mediterranean Plant Diversity. Available at: http://ww2.bgbm.org/ EuroPlusMed/ [accessed December 15, 2017].

European Committee for Antimicrobial Susceptibility Testing (2012). Method for the Determination of Broth Dilution Minimum Inhibitory Concentrations of Antifungal Agents for Yeasts. EUCAST DEFINITIVE DOCUMENT EDef 7. 2 Revision. Brussels: European Commission.

Evensen, N. A., and Braun, P. C. (2009). The effects of tea polyphenols on Candida albicans: inhibition of biofilm formation and proteasome inactivation. Can. J. Microbiol. 55, 1033-1039. doi: 10.1139/w09-058

Glazer, I., Masaphy, S., Marciano, P., Bar-Ilan, I., Holland, D., Kerem, Z., et al. (2012). Partial identification of antifungal compounds from Punica granatum peel extracts. J. Agric. Food Chem. 60, 4841-4848. doi: 10.1021/jf300330y

Gurib-Fakim, A. (2006). Medicinal plants: traditions of yesterday and drugs of tomorrow. Mol. Aspects Med. 27, 1-93. doi: 10.1016/j.mam.2005.07.008

Hirasawa, M., and Takada, K. (2004). Multiple effects of green tea catechin on the antifungal activity of antimycotics against Candida albicans. J. Antimicrob. Chem. 53, 225-229. doi: 10.1093/jac/dkh046

Johnson, D. C. (2012). Chronic candidal bronchitis: a consecutive series. Open Respir. Med. J. 6, 145-149. doi: 10.2174/1874306401206010145

Lass-Flörl, C. (2009). The changing face of epidemiology of invasive fungal disease in Europe. Mycoses 52, 197-205. doi: 10.1111/j.1439-0507.2009. 01691.x
Li, S., Zhang, Z., Cain, A., Wang, B., Long, M., and Taylor, J. (2005). Antifungal activity of camptothecin, trifolin, and hyperoside isolated from Camptotheca acuminata. J. Agric. Food Chem. 53, 32-37. doi: 10.1021/jf0484780

Marchelak, A., Owczarek, A., Matczak, M., Pawlak, A., Kolodziejczyk-Czepas, J., Nowak, P., et al. (2017). Bioactivity potential of Prunus spinosa L. Flower extracts: phytochemical profiling, cellular safety, pro-inflammatory enzymes inhibition and protective effects against oxidative stress in vitro. Front. Pharmacol. 8:680. doi: 10.3389/fphar.2017.00680

Menkovic, N., Savikin, K., Tasic, S., Zdunic, G., Stesevic, D., Milosavljevic, S., et al. (2011). Ethnobotanical study on traditional uses of wild medicinal plants in Prokletije Mountains (Montenegro). J. Ethnopharmacol. 133, 97-107. doi: 10.1016/j.jep.2010.09.008

Nürk, N. M., and Crockett, S. L. (2011). Morphological and phytochemical diversity among Hypericum species of the Mediterranean Basin. Med. Aromat. Plant Sci. Biotechnol. 5, 14-28.

Ogawa, H., Fujimura, M., Ohkura, N., and Makimura, K. (2014). It is time to call attention to the clinical significance of fungal colonization in chronic cough. Allergol. Int. 63, 611-612. doi: 10.2332/allergolint. 14-LE-0691

Papon, N., Courdavault, V., Clastre, M., and Bennett, R. J. (2013). Emerging and emerged pathogenic candida species: beyond the Candida albicans paradigm. PLoS Pathog. 9:e1003550. doi: 10.1371/journal.ppat.1003550

Pieroni, A., Quave, C. L., and Santoro, R. F. (2004). Folk pharmaceutical knowledge in the territory of the Dolomiti Lucane, inland southern Italy. J. Ethnopharmacol. 95, 373-384. doi: 10.1016/j.jep.2004.08.012

Pignatti, S. (1982). Flora d'Italia. Bologna: Edagricole.

Pignatti, S. (2017). Flora d'Italia, 2nd Edn. Bologna: Edagricole.

Pistelli, L., Bertoli, A., Zucconelli, S., Morelli, I., Panizzi, L., and Menichini, F. (2000). Antimicrobial activity of crude extracts and pure compounds of Hypericum hircinum. Fitoterapia 71, S138-S140. doi: 10.1016/S0367-326X(00) 00189-1

Raclariu, A. C., Paltinean, R., Vlase, L., Labarre, A., Manzanilla, V., Ichim, M. C., et al. (2017). Comparative authentication of Hypericum perforatum herbal products using DNA metabarcoding, TLC and HPLC-MS. Sci. Rep. 7:1291. doi: 10.1038/s41598-017-01389-w

Radulović, N., Stankov-Jovanović, V., Stojanović, G., Šmelcerović, A., Spiteller, M., and Asakawa, Y. (2007). Screening of in vitro antimicrobial and antioxidant activity of nine Hypericum species from the Balkans. Food Chem. 103, 15-21. doi: 10.1016/j.foodchem.2006.05.062

Raza, A., Xu, X., Sun, H., Tang, J., and Ouyang, Z. (2017). Pharmacological activities and pharmacokinetic study of hyperoside: a short review. Trop. J. Pharm. Res. 16, 483-489. doi: 10.4314/tjpr.v16i2.30

Reis, M. P. C., Carvalho, C. R. C., Andrade, F. A., Fernandes, O. D. F. L., Arruda, W., and Silva, M. R. R. (2016). Fisetin as a promising antifungal agent against Cryptococcus neoformans species complex. J. Appl. Microbiol. 121, 373-379. doi: 10.1111/jam.13155

Ritchie, T., and Eltahawy, E. (2014). Diagnosis and management of fungal urinary tract infections. Curr. Bladder Dysfunct. Rep. 9, 161-166. doi: 10.1007/s11884014-0238-7

Sagratini, G., Ricciutelli, M., Vittori, S., Öztürk, N., Öztürk, Y., and Maggi, F. (2008). Phytochemical and antioxidant analysis of eight Hypericum taxa from Central Italy. Fitoterapia 79, 210-213. doi: 10.1016/j.fitote.2007.11.011

Saito, H., Tamura, M., Imai, K., Ishigami, T., and Ochiai, K. (2013). Catechin inhibits Candida albicans dimorphism by disrupting Cek1 phosphorylation and cAMP synthesis. Microb. Pathog. 56, 16-20. doi: 10.1016/j.micpath.2013. 01.002

Salazar-Aranda, R., Granados-Guzmán, G., Pérez-Meseguer, J., González, G. M., and de Torres, N. W. (2015). Activity of polyphenolic compounds against Candida glabrata. Molecules 20, 17903-17912. doi: 10.3390/molecules 201017903

Sarić-Kundalić, B., Dobes, C., Klatte-Asselmeyer, V., and Saukel, J. (2010). Ethnobotanical study on medicinal use of wild and cultivated plants in middle, south and west Bosnia and Herzegovina. J. Ethnopharmacol. 131, 33-55. doi: 10.1016/j.jep.2010.05.061

Shahzad, M., Sherry, L., Rajendran, R., Edwards, C. A., Combet, E., and Ramage, G. (2014). Inhibitory effect of plant (poly) phenolics on growth and biofilm formation by Candida albicans. Proc. Nutr. Soc. 73:E28. doi: 10.1017/ S0029665114000421 
Sitheeque, M. A. M., Panagoda, G. J., Yau, J., Amarakoon, A. M. T., Udagama, U. R. N., and Samaranayake, L. P. (2009). Antifungal activity of black tea polyphenols (catechins and theaflavins) against Candida species. Chemotherapy 55, 189-196. doi: 10.1159/000216836

Stojanovic, G., Dordevic, A., and Smelcerovic, A. (2013). Do other Hypericum species have medical potential as St. John's Wort (Hypericum perforatum)? Curr. Med. Chem. 20, 2273-2295. doi: 10.2174/0929867311320180001

Strati, F., Di Paola, M., Stefanini, I., Albanese, D., Rizzetto, L., Lionetti, P., et al. (2016). Age and gender affect the composition of fungal population of the human gastrointestinal tract. Front. Microbiol. 7:1277. doi: 10.3389/fmicb.2016. 01227

Tocci, N., Gaid, M., Kaftan, F., Belkheir, A. K., Belhadj, I., Liu, B., et al. (2018b). Exodermis and endodermis are the sites of xanthone biosynthesis in Hypericum perforatum roots. New Phytol. 217, 1099-1112. doi: 10.1111/nph. 14929

Tocci, N., Weil, T., Perenzoni, D., Narduzzi, L., Madriñán, S., Crockett, S., et al. (2018a). Phenolic profile, chemical relationship and antifungal activity of Andean Hypericum species. Ind. Crops Prod. 112, 32-37. doi: 10.1016/j.indcrop. 2017.10.030

Tocci, N., Simonetti, G., D’Auria, F. D., Panella, S., Palamara, A. T., Ferrari, F., et al. (2013). Chemical composition and antifungal activity of Hypericum perforatum subsp. angustifolium roots from wild plants and plants grown under controlled conditions. Plant Biosyst. 147, 557-562. doi: 10.1080/11263504.2013. 806964

Tortorano, A. M., Kibbler, C., Peman, J., Bernhardt, H., Klingspor, L., and Grillot, R. (2006). Candidaemia in Europe: epidemiology and resistance. Int. J. Antimicrob. Agents 27, 359-366. doi: 10.1016/j.ijantimicag.2006. 01.002
Vrhovsek, U., Masuero, D., Gasperotti, M., Franceschi, P., Caputi, L., Viola, R., et al. (2012). A versatile targeted metabolomics method for the rapid quantification of multiple classes of phenolics in fruits and beverages. J. Agric. Food Chem. 60, 8831-8840. doi: 10.1021/jf2051569

Weil, T., Santamaría, R., Lee, W., Rung, J., Tocci, N., Abbey, D., et al. (2017). Adaptive mistranslation accelerates the evolution of fluconazole resistance and induces major genomic and gene expression alterations in Candida albicans. mSphere 2, e00167-17.

World Health Organization [WHO] (2014). Antimicrobial Resistance Global Report on Surveillance 2014. Geneva: WHO Press.

Zager, R. A. (2000). Polyene antibiotics: relative degrees of in vitro cytotoxicity and potential effects on tubule phospholipid and ceramide content. Am. J. Kidney Dis. 36, 238-249. doi: 10.1053/ajkd.2000.8967

Conflict of Interest Statement: The authors declare that the research was conducted in the absence of any commercial or financial relationships that could be construed as a potential conflict of interest.

The reviewer LA declared a shared affiliation, though no other collaboration, with one of the authors DI to the handling Editor.

Copyright (c) 2018 Tocci, Perenzoni, Iamonico, Fava, Weil and Mattivi. This is an open-access article distributed under the terms of the Creative Commons Attribution License (CC BY). The use, distribution or reproduction in other forums is permitted, provided the original author(s) and the copyright owner are credited and that the original publication in this journal is cited, in accordance with accepted academic practice. No use, distribution or reproduction is permitted which does not comply with these terms. 


\section{OPEN ACCESS}

Edited by:

Luc Pieters,

University of Antwerp, Belgium

Reviewed by:

Esperanza Bas Infante,

University of Miami, United States

Xian-Wen Yang,

Third Institute of Oceanography, State

Oceanic Administration, China

${ }^{*}$ Correspondence:

Anna K. Kiss

akiss@wum.edu.p

Specialty section:

This article was submitted to

Ethnopharmacology,

a section of the journal

Frontiers in Pharmacology

Received: 01 February 2018

Accepted: 26 March 2018

Published: 11 April 2018

Citation:

Woźniak M, Michalak B, Wyszomierska J, Dudek MK and Kiss AK (2018) Effects of

Phytochemically Characterized

Extracts From Syringa vulgaris and Isolated Secoiridoids on Mediators of Inflammation in a Human Neutrophil

Model. Front. Pharmacol. 9:349

doi: 10.3389/fphar.2018.00349

\section{Effects of Phytochemically Characterized Extracts From Syringa vulgaris and Isolated Secoiridoids on Mediators of Inflammation in a Human Neutrophil Model}

\author{
Marta Woźniak ${ }^{1}$, Barbara Michalak ${ }^{1}$, Joanna Wyszomierska ${ }^{1}$, Marta K. Dudek ${ }^{2}$ and \\ Anna K. Kiss ${ }^{1 *}$ \\ ${ }^{1}$ Department of Pharmacognosy and Molecular Basis of Phytotherapy, Medical University of Warsaw, Warsaw, Poland, \\ ${ }^{2}$ Centre of Molecular and Macromolecular Studies of Polish Academy of Sciences, Lodz, Poland
}

Aim of the study: The aim of the present study was to investigate the effects of phytochemically characterized extracts connected with the traditional use (infusions and ethanolic extracts) of different parts of Syringa vulgaris (common lilac) on the pro-inflammatory functions of neutrophils. Active compounds were isolated from the most promising extract(s) using bioassay-guided fractionation, and their activity and molecular mechanisms of action were determined.

Methods: The extracts were characterized using a HPLC-DAD- MS $^{\text {n }}$ method. The effects on ROS, MMP-9, TNF- $\alpha$, IL-8, and MCP-1 production by neutrophils were measured using luminol-dependent chemiluminescence and enzyme-linked immunosorbent assay (ELISA) methods. The effects on p38MAPK, ERK1/2, JNK phosphorylation, and NF-kB p65 translocation were determined using western blots.

Results: The major compounds detected in the extracts and infusions belong to structural groups, including caffeic acid derivatives, flavonoids, and iridoids. All extracts and infusions were able to significantly reduce ROS and IL-8 production. Bioassay-guided fractionation led to the isolation of the following secoiridoids: 2"-epiframeroside, oleonuezhenide, oleuropein, ligstroside, neooleuropein, hydroxyframoside, and framoside. Neooleuropein appeared to be the most active compound in the inhibition of cytokine production by attenuating the MAP kinase pathways.

Conclusion: The present study demonstrated that common lilac, which is a traditionally used medicinal plant in Europe, is a valuable source of active compounds, especially neooleuropein.

Keywords: Syringa vulgaris, Oleaceae, secoiridoids, neooleuropein, pro-inflammatory mediators, neutrophils 


\section{INTRODUCTION}

The Oleaceae family encompasses 25 genera and $\sim 600$ species. Members of this family include trees, shrubs, or woody climbers with almost worldwide distribution from northern temperate to southern subtropical regions, with the exception of the Antarctic. Genera include Olea (20 spp.), Forsythia (7-11 spp.), Fraxinus (40-70 spp.), Syringa (20-30 spp.), Osmanthus (1530 spp.), Jasminum (200-300 spp.), Chionanthus (80-100 spp.), and Ligustrum (40-50 spp.) (Jensen et al., 2002; Evans, 2009). In Central-Eastern Europe, only a few representatives of the Oleaceae family are found: Fraxinus exelsior L., Ligustrum vulgare L., Syringa vulgaris L., and Forsythia sp. Moreover, Olea (leaves) and Fraxinus (leaves) have monographs in the European Pharmacopoeia as well as European Agency EMEA monographs. The Syringa genus is distributed in Eurasia, which is subdivided into western (Central and Southeast Europe) and eastern zones (Central to East Asia) (Wallander and Albert, 2000). Most of the species are found in Asia, while only $S$. vulgaris and S. josikaea are found in Europe (Jensen et al., 2002; Lendvay et al., 2016). S. vulgaris L., known as the common lilac, is native to the Balkan peninsula and is widely cultivated as an ornamental plant in Europe. In Poland, it has been naturalized since the sixteenth century, where it is cultivated but also found in the wild (Witkowska-Zuk, 2013). In European ethnopharmacology, the common lilac inflorescence or leaves in the form of an infusion or alcoholic extract were used to treat gout, rheumatism and muscle/joint aches (Fournier, 1948; Hanlidou et al., 2010; Jarić et al., 2015; Sõukand and Pieroni, 2016). In Poland, Bulgaria, Italy, and Greece, the flowers, leaves, bark, or fruits in the forms of infusions, decoctions, or alcoholic extracts were used as antipyretics and to treat cold, cough, etc. (Fournier, 1948; Kuznicka, 1986; Kuzniewski and AugustynPuziewicz, 1986; Anioł-Kwiatkowska et al., 1993; Leporatti and Ivancheva, 2003; Hanlidou et al., 2010). Locally, different parts of the plant are used to treat toothaches, gastrointestinal disorders, skin wounds, and other ailments (Kuznicka, 1986; Kuzniewski and Augustyn-Puziewicz, 1986; Anioł-Kwiatkowska et al., 1993; Agelet and Vallès, 2003; Hanlidou et al., 2010; Papp et al., 2014). Interestingly, in North American materia medica (pharmacognosy) textbooks, the antipyretic use of the leaves, flowers, and fruits of the common lilac was mentioned (Lyle, 1897; Remington and Woods, 1918), and native Americans used to chew the bark or leaves for treating a sore mouth (Moerman, 1998). The Asian species are also used in traditional medicine to treat bronchial disease [Syringa reticulata (Blume) H. Hara leaves], lung and heart diseases (Syringa pinnatifolia Hemsl. stem), tooth pain (Syringa vetulina Kom. Bark), and as antipyretics (Syringa afghanica C.K. Schneid. leaves) (Park et al., 1999; Takenaka et al., 2002; Machida et al., 2009; Su et al., 2016).

Scientific data supporting the traditional use of $S$. vulgaris are connected with its antioxidant, anti-inflammatory, antinociceptive and antipyretic properties (Bálinet, 1971; Tóth et al., 2015, 2016; Dudek et al., 2017). Moreover, information about the single compounds responsible for each of these activities is scarce.
The aim of the present study was to investigate the effects of phytochemically characterized (HPLC-DAD-MS ${ }^{\mathrm{n}}$ ) extracts connected with the traditional use (infusions and ethanolic extracts) of different parts of the common lilac (bark, fruits, leaves and flowers) on the pro-inflammatory functions of neutrophils, such as reactive oxygen species (ROS) production, metalloproteinase-9 (MMP-9), interleukin 8 (IL-8), and tumor necrosis factor (TNF- $\alpha$ ) release. We isolated active compounds from the most promising extracts using bioassay-guided fractionation. Finally, we focused on the activity and molecular mechanisms of action of the isolated compounds that were able to regulate neutrophil inflammation. The biological activities of single compounds were compared with oleuropein and ligstroside, characteristic compounds from the Oleacea family (Jensen et al., 2002), and with positive control clarithromycin, a macrolide antibiotic, which has been shown to inhibit the pro-inflammatory function of neutrophils (Simpson et al., 2008; Cervin et al., 2009).

\section{MATERIALS AND METHODS Chemicals and General Experimental Procedures}

Hanks' balanced salt solution (HBSS), RPMI 1640, f-MLP (formyl-Met-Leu-Phenylalanine), LPS (from Escherichia coli 0111:B4), HEPES solution, and L-glutamine were purchased from Sigma-Aldrich Chemie GmbH (Germany). Fetal bovine serum (FBS) and phosphate-buffered saline (PBS) were purchased from Gibco (USA). Ficoll Hypaque gradient (LSM 1077) was obtained from PAA Laboratories, GmbH (Austria). Human Quantikine ELISA Kits were purchased from R\&D System (USA). Anti- pp38 (\#9211), ppJNK (\#9251), pJNK (\#9252), pERK1/2 (\#9102), NFKB(p65) (\#8242S), and $\beta$ actin (\#4967) antibodies were purchased from Cell Signaling Technology (USA). Anti- ppERK1/2 (\#V8031) was purchased from Promega (USA) and anti-p38 (\#SC-535) from Santa Cruz Biotechnology (USA). Propidium iodide was purchased from BD Biosciences (USA).

NMR spectra were recorded on a Bruker Advance III 600 $\mathrm{MHz}$ spectrometer (Bruker Biospin, Germany) in methanol$d_{4}$. Preparative HPLC was performed with a Shimadzu LC20AP instrument (Japan) using a Zorbax SB-C18 column $(150 \times 21.2 \mathrm{~mm}, 5 \mu \mathrm{m})$ (Agilent, USA) at a flow rate of $20.0 \mathrm{~mL} / \mathrm{min}$. TLC was performed on Merck silica gel $60 \mathrm{~F}$ $254(0.25 \mathrm{~mm})$ plates with a dichloromethane/methanol/formic acid/water (80:25:1.5:4; v/v/v/v) solvent system. Chromatograms were visualized by spraying with sulfuric acid (5\% in methanol) followed by heating at $105^{\circ} \mathrm{C}$ for $10 \mathrm{~min}$. All solvents used for chromatography were of gradient grade. HPLC-DAD-MS ${ }^{\mathrm{n}}$ analysis was performed on a UHPLC-3000 RS system (Dionex, Germany) with DAD detection and an AmaZon SL ion trap mass spectrometer with an ESI interface (Bruker Daltonik GmbH, Germany). Separation was performed on a Zorbax SB-C18 column $(150 \times 2.1 \mathrm{~mm}, 1.9 \mu \mathrm{m})$ (Agilent, USA). The mobile phase consisted of $0.1 \% \mathrm{HCOOH}$ in water $(\mathrm{A})$ and $0.1 \% \mathrm{HCOOH}$ in $\mathrm{MeCN}$ (B) using the following gradients: 0-60 min, 5-40\% 
B. The LC eluate was introduced into the ESI interface without splitting, and compounds were analyzed in negative ion mode with the following settings: nebulizer pressure of $40 \mathrm{psi}$; drying gas flow rate of $9 \mathrm{~L} / \mathrm{min}$; nitrogen gas temperature of $300^{\circ} \mathrm{C}$; and a capillary voltage of $4.5 \mathrm{kV}$. The mass scan ranged from 100 to $2,200 \mathrm{~m} / \mathrm{z}$. UV spectra were recorded in the range of $200-400 \mathrm{~nm}$.

The absorbance and luminescence were measured using a BioTek microplate reader (Highland Park, USA). Flow cytometry was performed using a BD FACSCalibur apparatus (BD Biosciences, USA).

\section{Plant Material}

Bark from the branches, leaves, flowers, and fruits of S. vulgaris L. were collected in May and August (fruits) 2016, from a native plant growing in Legionowo, Mazovian district, Poland $\left(52^{\circ} 23^{\prime} 17.647^{\prime \prime} \mathrm{N} ; 20^{\circ} 55^{\prime} 53.171^{\prime \prime} \mathrm{E}\right)$. The plant material was authenticated according to Flora Europaea (Tutin et al., 1972) by Anna K. Kiss. A voucher specimen (no. SV052015) has been deposited in the Plant Collection, Department of Pharmacognosy and Molecular Basis of Phytotherapy, Medical University of Warsaw, Poland (Figure 1S).

\section{Extract Preparation and Phytochemical Characterization by HPLC-DAD-MS/MS Infusions (Aqueous Extracts)}

Air-dried plant materials $(5 \mathrm{~g})$ were poured with boiling water $(250 \mathrm{~mL})$, covered, and allowed to stand for $15 \mathrm{~min}$. Extracts were then filtered and lyophilized, resulting in the following aqueous extract masses: bark- $0.52 \mathrm{~g}$, fruit- $0.48 \mathrm{~g}$, flower- $1.69 \mathrm{~g}$, and leaf$1.04 \mathrm{~g}$.

\section{Ethanolic Extracts}

The air-dried plant materials $(2 \mathrm{~g})$ were extracted with $60 \%$ ethanol $(\mathrm{v} / \mathrm{v}, 1: 20)$ in a water bath $\left(70^{\circ} \mathrm{C}\right)$ for $1 \mathrm{~h}$. Then, extracts were filtered, the ethanol was evaporated, and the water residues were lyophilized, resulting in the following ethanolic extract masses: bark- $0.25 \mathrm{~g}$, fruit- $0.21 \mathrm{~g}$, flower- $0.58 \mathrm{~g}$ and leaf- $0.51 \mathrm{~g}$.

The extracts were characterized using an HPLC-DAD-MS/MS method. The presence of substances in extracts was confirmed by comparing the retention times and spectra (UV, MS, MS/MS) with standards and/or published data.

\section{Bioassay-Guided Isolation of Active Compounds From Leaf Extract}

The air-dried plant material $(100 \mathrm{~g})$ was crushed and extracted three times with $75 \%$ methanol $(1: 10)$ at $70^{\circ} \mathrm{C}$ for $1 \mathrm{~h}$ each time (3 times). The methanol from combined extracts was evaporated under reduced pressure, and the aqueous residue was lyophilized to yield $40 \mathrm{~g}$ of extract. The crude extract was subjected to Diaion HP-20 (Supelco) column chromatography $(45 \times 5 \mathrm{~cm})$ and eluted with an $\mathrm{H}_{2} \mathrm{O}-\mathrm{MeOH}$ gradient $(100: 0 \rightarrow 0: 100$, v/v) in 5 steps of $1.5 \mathrm{~L}$ each to obtain 35 fractions, which were pooled into 6 main fractions (F1-F6) based on their TLC and HPLC profiles. The activity of the fractions toward the inhibition of IL- 8 and TNF$\alpha$ was tested at a concentration of $50 \mu \mathrm{g} / \mathrm{mL}$. Active fraction F6 $(2.5 \mathrm{~g})$ was subjected to further separation on a Sephadex LH20 (Pharmacia) column $(25 \times 3 \mathrm{~cm})$ with $\mathrm{H}_{2} \mathrm{O}-\mathrm{MeOH}(50: 50$, $\mathrm{v} / \mathrm{v})$ to obtain 20 fractions of $50 \mathrm{~mL}$ each, which were pooled into 6 main fractions (F6_1-F6_6) based on their TLC and HPLC profiles. From fraction F6_2 (235 mg), compounds I (75 mg; Rt $=11.3-12.3 \mathrm{~min}$.) and II (13 mg; Rt = 13.4-13.9); from fraction F6_3 (633 mg), compounds III (78 mg; Rt $=8.3-8.9 \mathrm{~min})$ and IV (90 mg; Rt = 11.9-12.7 min), from fraction F6_4 (243 mg), compounds V (5 mg; Rt = 12.0-12.3 min), VI (23 mg; Rt = 15.4$16.0 \mathrm{~min})$ and VII (7 mg; Rt $=19.0-19.5 \mathrm{~min})$ were isolated using preparative HPLC with a gradient of $0.1 \% \mathrm{HCOOH}$ in $\mathrm{H}_{2} \mathrm{O}-0.1 \%$ $\mathrm{HCOOH}$ in $\mathrm{MeCN}(80: 20 \rightarrow 40: 60)$ over $60 \mathrm{~min}$.

\section{Preparation of Solutions of Extracts, Fractions, and Compounds for Bioassay}

Tested extracts were dissolved in PBS or HBSS $(1 \mathrm{mg} / \mathrm{mL})$. Fractions and compounds were dissolved in DMSO $(10 \mathrm{mg} / \mathrm{mL}$ or $10 \mathrm{mM}$ stock solutions) and then diluted with RPMI 1640 medium. The extracts were tested in a concentration range of $25-100 \mu \mathrm{g} / \mathrm{mL}$. Compounds were tested at concentrations of $10-50 \mu \mathrm{M}$. The concentration of DMSO used $(<0.1 \%)$ did not influence the assays.

\section{Isolation of Human Neutrophils}

Peripheral venous blood was taken from healthy human donors (18-35 years old) in the Warsaw Blood Donation Centre. Donors did not smoke or take any medications. They were clinically recognized to be healthy and a routine laboratory tests showed all values to be within the normal ranges. The study conformed to the principles of the Declaration of Helsinki.

Neutrophils were isolated by dextran sedimentation and centrifugation in a Ficoll Hypaque gradient (Böyum, 1968) and then re-suspended in HBSS buffer or RPMI 1640 medium.

\section{Cytotoxicity}

Cytotoxicity was determined by a standard flow cytometric probe using propidium iodide (PI) staining. After $24 \mathrm{~h}$ of incubation with extracts/compounds, the neutrophils were harvested and centrifuged (1500 RPM; $10 \mathrm{~min} ; 4^{\circ} \mathrm{C}$ ), washed once with cold PBS and re-suspended in $400 \mu \mathrm{L}$ of PBS. Five microliters of PI $(50 \mu \mathrm{g} / \mathrm{mL})$ solution was added to the cell suspensions. After 15 min of incubation at room temperature, cells were analyzed by flow cytometry, and 10,000 events were recorded per sample. Cells that displayed high permeability to PI were expressed as a percentage of $\mathrm{PI}(+)$ cells.

\section{ROS Production}

Neutrophils $\left(3.5 \times 10^{5}\right)$ were incubated in HBSS buffer with $50 \mu \mathrm{L}$ of the tested extracts and $50 \mu \mathrm{L}$ of luminol $(20 \mathrm{mM})$ in a 96-well plate. ROS production was initiated by the addition of f-MLP $(0.1 \mu \mathrm{g} / \mathrm{mL})$ to obtain a total volume of $200 \mu \mathrm{L} /$ well. Chemiluminescence changes were measured over $40 \mathrm{~min}$ at intervals of $2 \mathrm{~min}$ in a microplate reader. The background chemiluminescence produced by non-stimulated cells was also determined. The percentage of inhibition was calculated by comparison to the control without the tested extracts at the maximum luminescence. 


\section{IL-8, MMP-9, MCP-1, and TNF- $\alpha$ Production}

Neutrophils $\left(2 \times 10^{6}\right)$ were cultured in 24-well plates in RPMI 1640 medium with $10 \%$ FBS, $10 \mathrm{mM}$ HEPES, and $2 \mathrm{mM} \mathrm{L}$ glutamine in the presence or absence of LPS $(100 \mathrm{ng} / \mathrm{mL})$ for $24 \mathrm{~h}$ at $37^{\circ} \mathrm{C}$ with $5 \% \mathrm{CO}_{2}$ in the presence or absence of test extracts/fractions/compounds. After $24 \mathrm{~h}$, the neutrophils were harvested and centrifuged (2000 RPM; $10 \mathrm{~min} ; 4^{\circ} \mathrm{C}$ ). The amount of released cytokines was measured by enzyme-linked immunosorbent assay (ELISA) following the manufacturer's instructions (BD Biosciences, USA). The effects on IL-8, MMP$9, \mathrm{MCP}-1$, and TNF- $\alpha$ production were calculated by comparing the percentages of the released agents to the stimulated control, which lacked the test extracts/fractions/compounds.

\section{Western Blotting}

Neutrophils $\left(4 \times 10^{6}\right)$ were suspended in RPMI 1640 medium and incubated for $40 \mathrm{~min}$ at $37^{\circ} \mathrm{C}$ in the presence or absence of LPS $(100 \mathrm{ng} / \mathrm{mL})$ and in the presence or absence of the test compounds. They were then centrifuged at 1500 RPM for $10 \mathrm{~min}$ at $4^{\circ} \mathrm{C}$. Cells were lysed in ice-cold buffer containing PBS, $5 \mathrm{mM}$ EDTA, 1\% Triton X-100, phosphatase and protease inhibitors, and the resulting lysates were centrifuged at 8,000 RPM for $15 \mathrm{~min}$ at $4^{\circ} \mathrm{C}$. The proteins were separated by $12 \%$ SDS-PAGE. Proteins were transferred to nitrocellulose filters and immunoblotted with rabbit anti-p38MAPK, anti-ERK1/2, and anti-JNK at 1:1000 dilutions and a rabbit anti-actin polyclonal antibody at a 1:2000 dilution. Peroxidase-conjugated affinipure goat anti-rabbit antibody was used as a secondary antibody at a dilution of 1:10000. Finally, the blots were incubated with chemiluminescent substrate for the detection of HRP (ThermoScientific, USA) for 10-15 min. Western blots were quantified using the ImageJ 1.38 software after densitometric scanning of the bands.

\section{Statistical Analysis}

The results were expressed as the mean \pm SEM of three independent experiments performed at least in duplicate. All analyses were performed using Statistica 9 software. The statistical significance of the differences between means was established by ANOVA with Dunnett's or Tukey's post hoc test. $P$-values below 0.05 were considered statistically significant.

\section{RESULTS}

\section{Phytochemical Characterization and Comparison of S. vulgaris From Bark, Fruit, Flower and Leaf Infusions and Ethanolic Extracts}

The phytochemical analysis of S. vulgaris bark, fruit, flower and leaf infusions and ethanolic extracts was performed using a HPLC-DAD-MS/MS method, which allowed for the identification or partial identification of 64 compounds (Table 1 and Figure 1) from the following groups: phenylethanoids (compounds 27, 31, 36, 39, and 42), rare phenylethanoids esterified with an oleoside 11-methyl ester (compounds 43, 49,
50, and 61), flavonoids (compounds 32, 33, and 35), other phenolic compounds $(1-3,5-14,17,18$, and 24), lignans (compounds 21 and 37), iridoids (compounds 34 and 41), secoiridoids (compounds $4,15,16,20,26,28-30,38,40,44-$ 46, 48, 51-57, 60, 62, and 63), together with eight unknown compounds. Compounds 1, 2, 5, 7, 14-16, 27-29, 34, 36, 38, $41,43-46,48-50,52-54,61$ were previously isolated as pure compounds from S. vulgaris leaves, flowers, and bark (Kurkin et al., 1989, 1990; Damtoft et al., 1995; Kikuchi et al., 2010; Dudek et al., 2017). However, this is a first comprehensive phytochemical analysis of a broad range of compounds from different parts of the plant. Significant differences were observed in the phytochemical profiles of different plant parts (Table 1 and Figure 1). In bark extracts, the major compounds were as follows: syringin $\left(14 ; \mathrm{Rt}=16.9 \mathrm{~min}, \mathrm{~m} / z\right.$ $\left.417[\mathrm{M}-\mathrm{H}+\mathrm{HCOOH}]^{-}\right)$, demethyloleuropein $\left(\mathbf{2 8} ; \mathrm{Rt}=28.2 \mathrm{~min}, \mathrm{~m} / \mathrm{z} 525[\mathrm{M}-\mathrm{H}]^{-}\right)$, forsythoside B (31; Rt $\left.=30.0 \mathrm{~min}, \mathrm{~m} / z 755[\mathrm{M}-\mathrm{H}]^{-}\right)$, acteoside (36; Rt $=31.4 \mathrm{~min}, \mathrm{~m} / z 623[\mathrm{M}-\mathrm{H}]^{-}$) and oleuropein (46; Rt $\left.=38.3 \mathrm{~min}, \mathrm{~m} / z 539[\mathrm{M}-\mathrm{H}]^{-}\right)$. In fruit extracts, the major compounds were as follows: nuzhenide (38; $\mathrm{Rt}=33.0 \mathrm{~min}, \mathrm{~m} / \mathrm{z}$ $\left.685[\mathrm{M}-\mathrm{H}]^{-}\right)$and an oleonuezhenide isomer $(55 ; \mathrm{Rt}=43.3 \mathrm{~min}$, $\left.\mathrm{m} / z 1071[\mathrm{M}-\mathrm{H}]^{-}\right)$. In flower extracts, the major compounds were as follows: caffeoylglucaric acid $(\mathbf{5} ; \mathrm{Rt}=10.1 \mathrm{~min}, \mathrm{~m} / \mathrm{z} 371$ $\left.[\mathrm{M}-\mathrm{H}]^{-}\right)$, quercetin rutinoside $(32 ; \mathrm{Rt}=30.1 \mathrm{~min}, \mathrm{~m} / \mathrm{z} 609[\mathrm{M}-$ $\mathrm{H}]^{-}$), acteoside $\left(36 ; \mathrm{Rt}=31.4 \mathrm{~min}, \mathrm{~m} / z 623[\mathrm{M}-\mathrm{H}]^{-}\right)$, oleuropein (46; Rt $\left.=38.3 \mathrm{~min}, \mathrm{~m} / z 539[\mathrm{M}-\mathrm{H}]^{-}\right)$, oleoacteoside (49; Rt $=40.1 \mathrm{~min}, \mathrm{~m} / \mathrm{z} 1009[\mathrm{M}-\mathrm{H}]^{-}$) and oleonuezhenide (60; Rt $\left.=45.4 \mathrm{~min}, \mathrm{~m} / z 1071[\mathrm{M}-\mathrm{H}]^{-}\right)$. In leaf extracts, the major compounds were as follows: secologanoside $(\mathbf{1 6} ; \mathrm{Rt}=17.3 \mathrm{~min}$, $\left.\mathrm{m} / z 389[\mathrm{M}-\mathrm{H}]^{-}\right)$, quercetin rutinoside $(32 ; \mathrm{Rt}=30.1 \mathrm{~min}, \mathrm{~m} / \mathrm{z}$ $\left.609[\mathrm{M}-\mathrm{H}]^{-}\right)$, syringalactone B $(34 ; \mathrm{Rt}=30.5 \mathrm{~min}, \mathrm{~m} / z 525[\mathrm{M}-$ $\mathrm{H}]^{-}$), oleuropein $\left(46 ; \mathrm{Rt}=38.3 \mathrm{~min}, \mathrm{~m} / z 539[\mathrm{M}-\mathrm{H}]^{-}\right)$, 2" $2^{\prime \prime}$-epiframeroside $\left(53 ; \mathrm{Rt}=42.6 \mathrm{~min}, \mathrm{~m} / z 601[\mathrm{M}-\mathrm{H}]^{-}\right)$, and ligstroside (54; Rt $\left.=43.1 \mathrm{~min}, \mathrm{~m} / z 523[\mathrm{M}-\mathrm{H}]^{-}\right)$. The phytochemical profiles of the infusions and ethanolic extracts were similar, and only quantitative differences were observed.

\section{Effect of Bark, Fruit, Flower and Leaf Infusions and Ethanolic Extracts of S. vulgaris on the Pro-inflammatory Function of Stimulated Neutrophils}

In all concentrations of the tested extracts, no significant reduction in membrane integrity was observed in comparison to the control using a propidium iodide assay (Figures 2A,B). Activation of neutrophils by f-MLP results in degranulation and a significant release of ROS compared to the untreated control (Figures 2C,D). Additionally, stimulation with LPS resulted in a significant release of proteinase MMP-9, chemokine IL-8, and cytokine TNF- $\alpha$ (Figure 3). Incubation of stimulated neutrophils with extracts at concentration ranges of $25-100 \mu \mathrm{g} / \mathrm{mL}$ resulted in a statistically significant and dose dependent reduction of ROS production by all extracts over all tested concentrations. There was no statistically significant difference between infusions and ethanolic extracts (Figures 2C,D).

All infusions and extracts were able to inhibit the release of IL-8 in a dose-dependent manner (Figures $3 \mathbf{A}, \mathbf{B}$ ). The effect 
TABLE 1 | Retention time, UV, and MS/MS data of the compounds present in extracts and infusions from S. vulgaris.

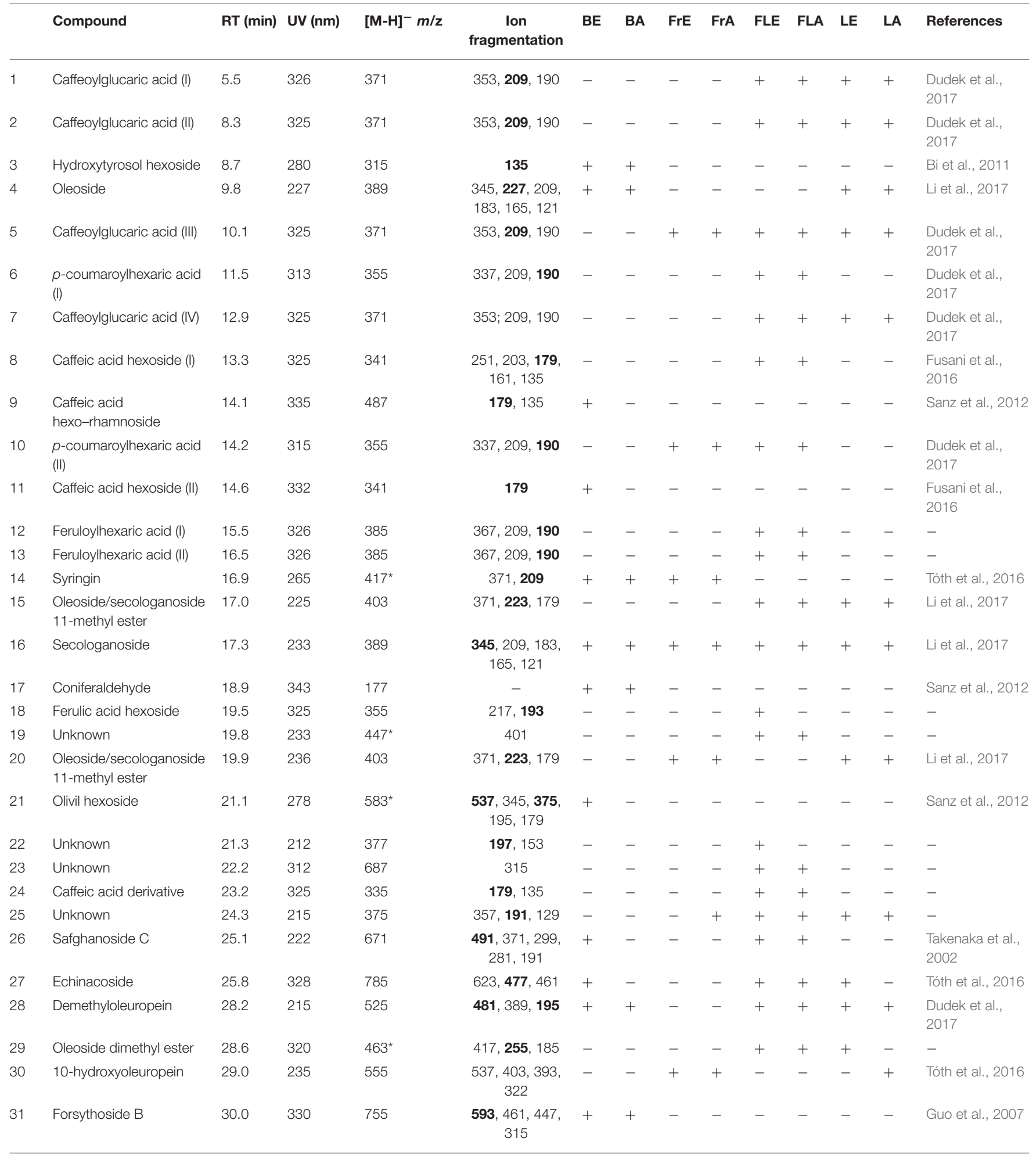


TABLE 1 | Continued

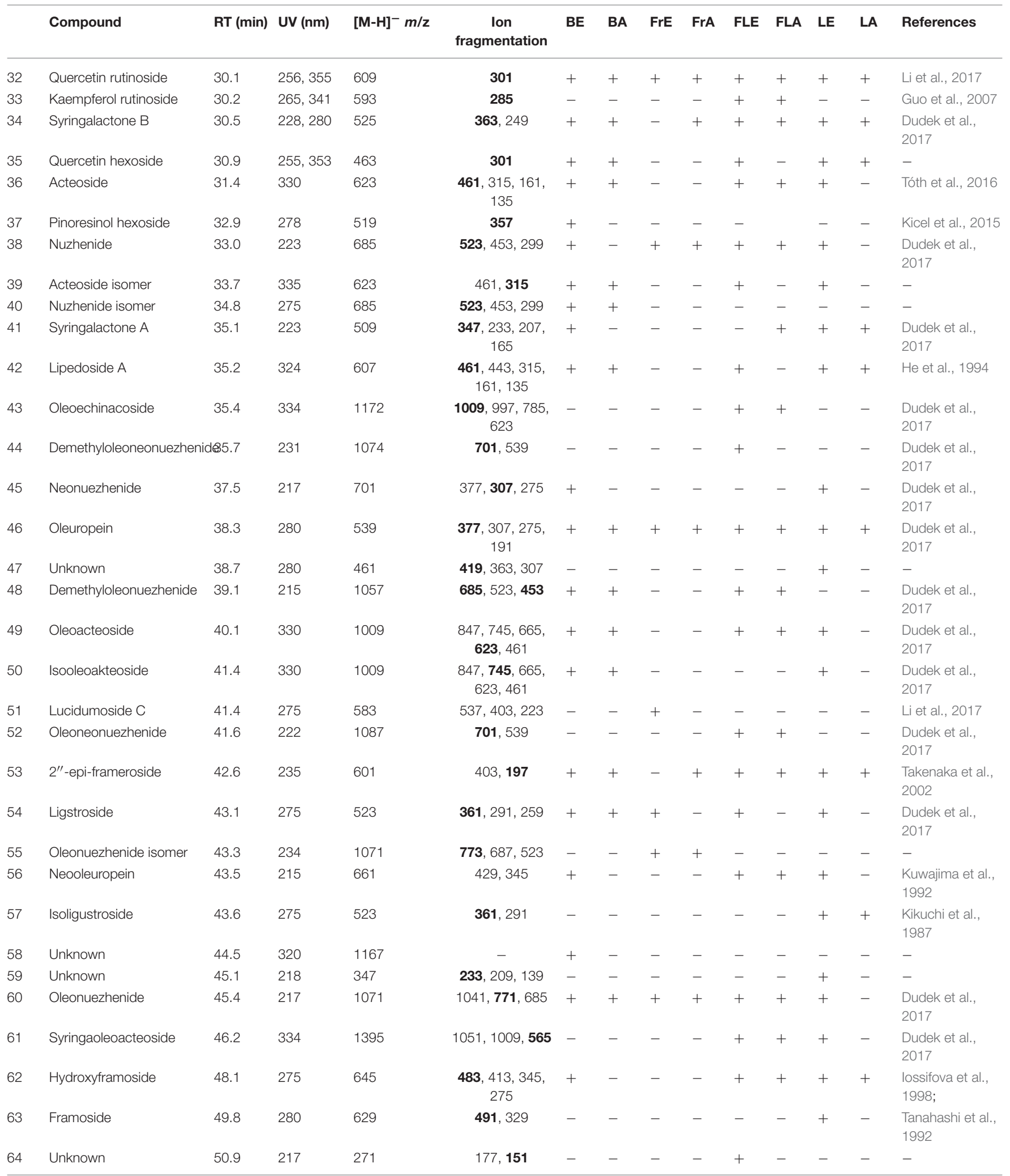

${ }^{*}[\mathrm{M}-\mathrm{H}+\mathrm{HCOOH}]^{-}$

$\boldsymbol{E}$, ethanolic extract; $\boldsymbol{A}$, infusion; B, bark; $\boldsymbol{F r}$, fruit; $\boldsymbol{F} \mathbf{L}$, flower; $\mathbf{L}$, leaf. Bold, most abundant fragmentation ion. 


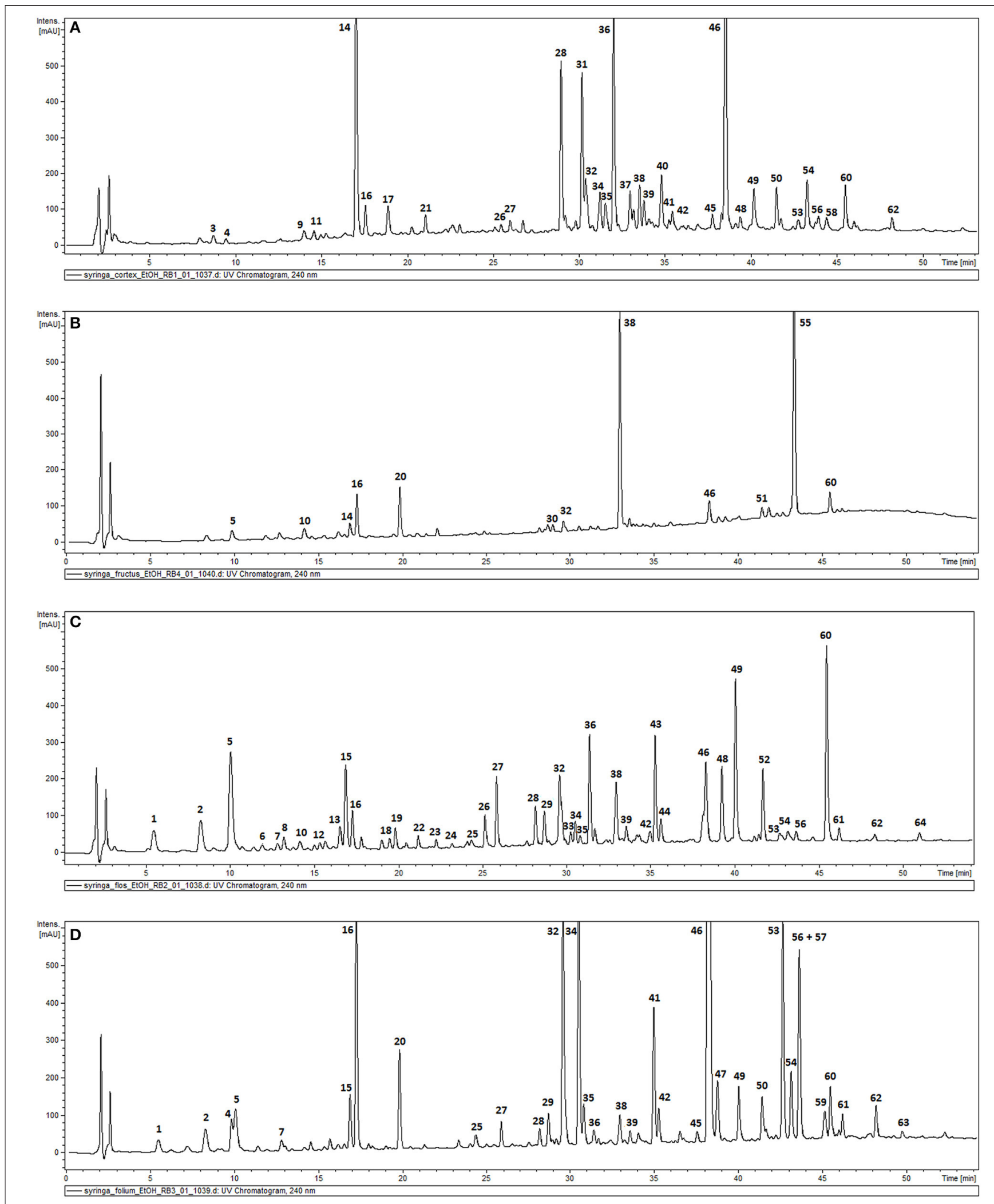

FIGURE 1 | HPLC-DAD chromatograms of the ethanolic extracts of S. vulgaris bark (A); fruit (B); flower (C); leaf (D) recorded at $240 \mathrm{~nm}$. 


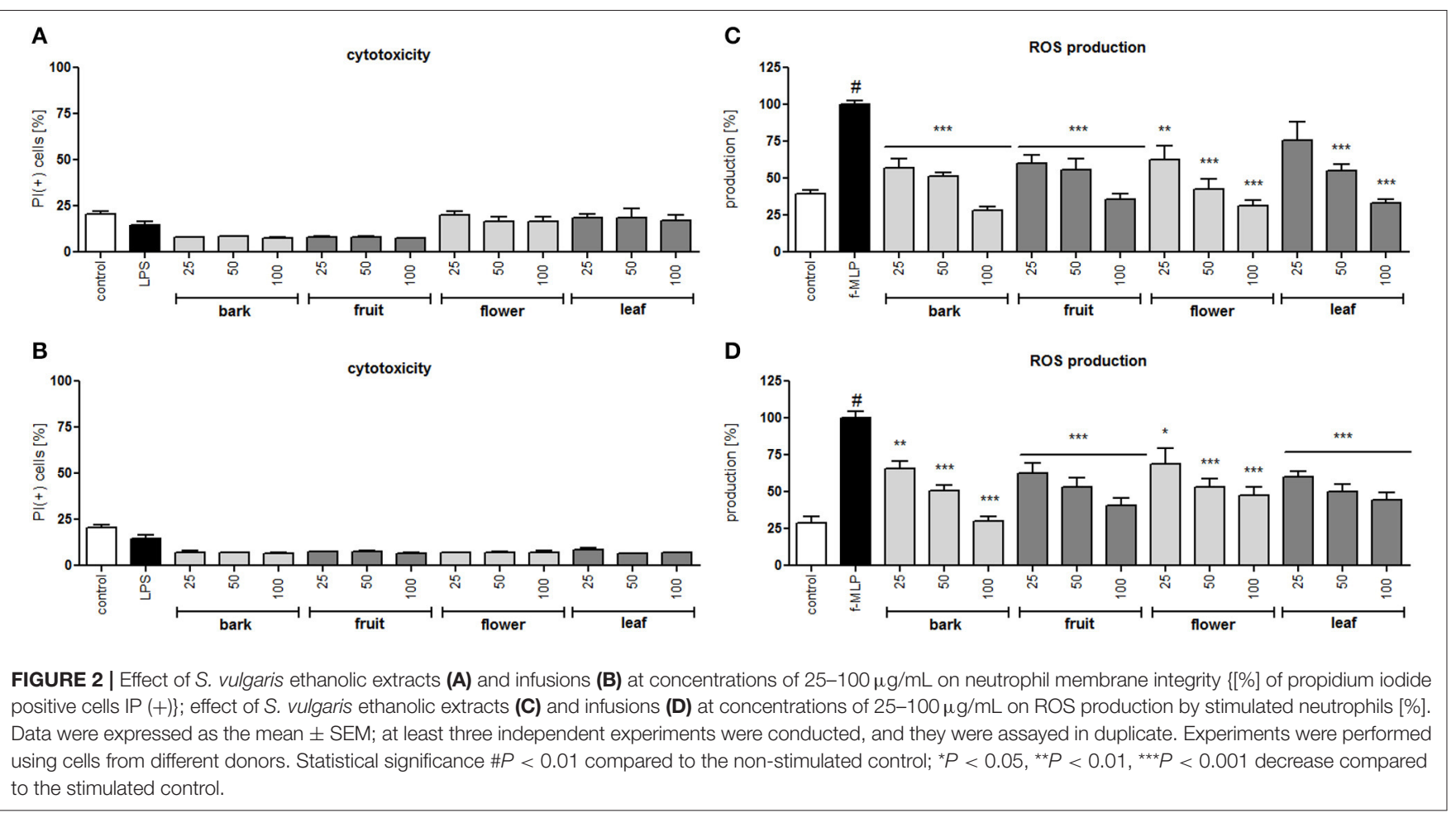

was significant $(p<0.001)$ for all concentrations tested of the flower infusion and flower and leaf extracts. The effect on TNF$\alpha$ production/release was less pronounced and was statically significant only at the highest concentration of $100 \mu \mathrm{g} / \mathrm{mL}$ for bark and leaf ethanolic extracts and the flower infusion (Figures 3C,D). MMP-9 release was inhibited the most by bark and fruit ethanolic extracts (Figures 3E,F). In general, ethanolic extracts were slightly more active than infusions, and flower and leaf preparations were more active than bark and fruit preparations. In a previous study, we isolated 29 compounds that were hydroxycinnamoyl and secoiridoid derivatives (Dudek et al., 2017) from the flower extract with moderate antiinflammatory activity. In this study, we performed a bioassayguided isolation of active compounds from the leaf extract.

\section{Bioassay-Guided Isolation of Active Compounds From Leaf Extract}

The crude alcoholic leaf extract was fractionated using a Diaion HP-20 and eluted with a 5-step $\mathrm{H}_{2} \mathrm{O}-\mathrm{MeOH}$ gradient $(100: 0 \rightarrow 0: 100)$ to obtain six main fractions (F1-F6) based on their TLC and HPLC profiles. The activities of the fractions were evaluated based on IL- 8 inhibition and TNF$\alpha$ release. Only fraction F6 inhibited the IL- 8 release at a concentration of $50 \mu \mathrm{g} / \mathrm{mL}$ in a statistically significant manner $(p<0.001)$. Interestingly, fraction F2 stimulated TNF- $\alpha$ production, while fractions F5 and F6 decreased the production of this cytokine (Figure 4). Fraction 5 was rich in oleuropein. Fraction 6, which contained several compounds, was subject to further fractionation on a Sephadex $\mathrm{LH}-20$ with $\mathrm{H}_{2} \mathrm{O}$ $\mathrm{MeOH}$ (50:50) and then preparative chromatography was used to isolate the following seven compounds: $2^{\prime \prime}$-epiframeroside (I), oleonuezhenide (II), oleuropein (III), ligstroside (IV), neooleuropein (V), hydroxyframoside (VI), and framoside (VII) (Figure 4). The structures of these compounds (Figure 5) were confirmed based on their ${ }^{1} \mathrm{H}$ and ${ }^{13} \mathrm{C}$ NMR spectra, which were compared with reference data (Fukuyama et al., 1987; Damtoft et al., 1992; Kuwajima et al., 1992; Tanahashi et al., 1992; Iossifova et al., 1998; Konno et al., 1998; Takenaka et al., 2002). Analysis of the extracted ion intensity for single compounds in each extract confirmed that $2^{\prime \prime}$-epiframeroside (I), oleuropein (III), ligstroside (IV), neooleuropein (V), hydroxyframoside (VI), and framoside (VII) were the major compounds present in leaf extract. Only oleonuezhenide (II) was present in a higher concentration in the flower extract (Figure 2S).

\section{Effect on Cytokine/Chemokine Production, MAP Kinases and NF- $k$ B Activation}

Lipopolysaccharide stimulation of human neutrophils resulted in the induction of the production/release of chemokines IL8 (neutrophil chemotactic factor) and MCP-1 (monocyte chemoattractant protein 1) along with cytokine TNF- $\alpha$ in comparison to cells that were not stimulated. Isolated compounds I, II, V, VI, and VII were tested at concentrations of 10,25 , and $50 \mu \mathrm{M}$, and their activity was compared with oleuropein and ligstroside, the characteristic compounds of the Oleacea family that were also isolated from active fraction F6, and with the positive control clarithromycin, a macrolide antibiotic which has been shown to inhibit the pro-inflammatory function of neutrophils. At all concentrations of the tested compounds, no significant reduction in membrane integrity 


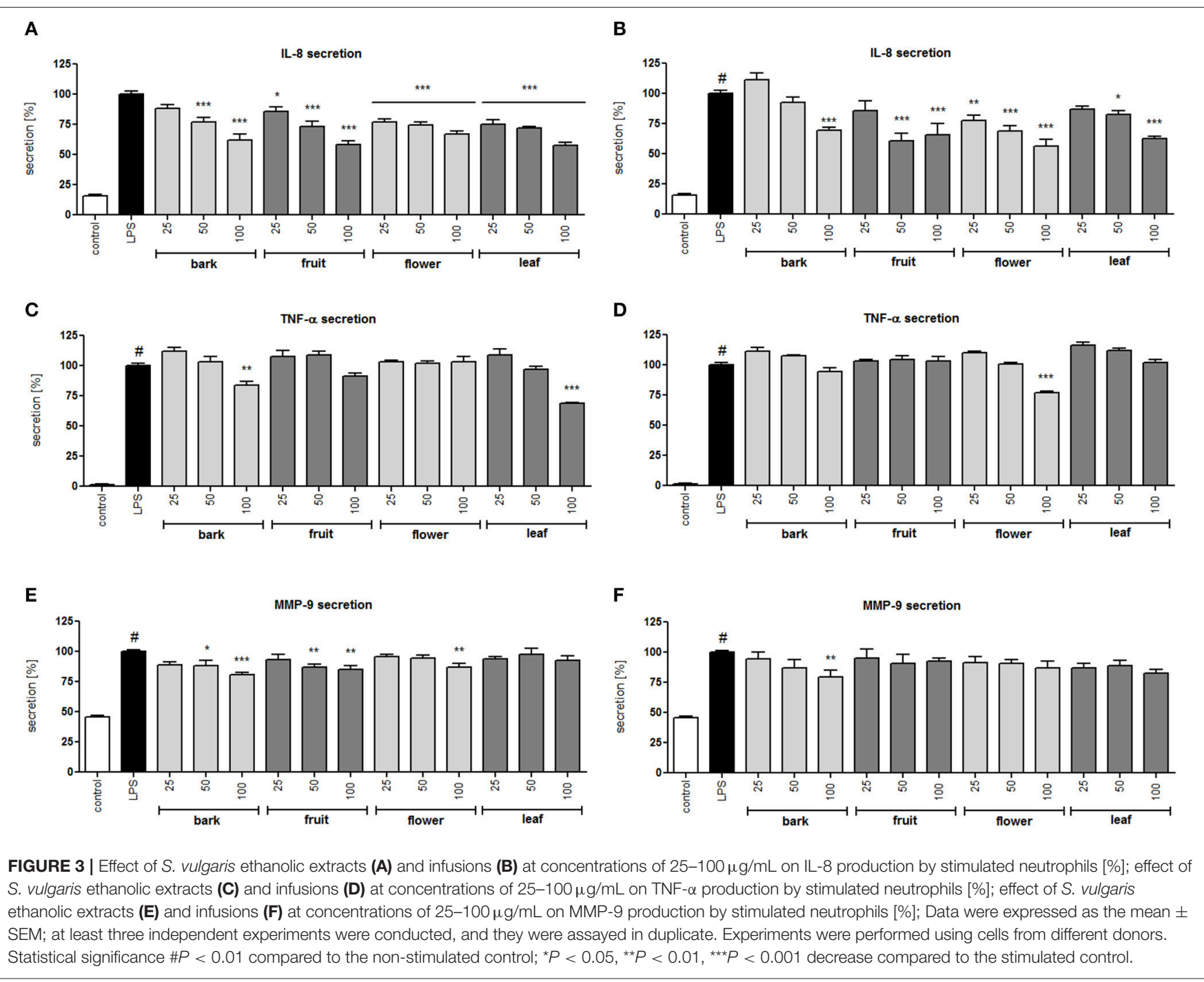

was observed in comparison to the control using a propidium iodide assay (Figure 6A). Neooleuropein was the most active compound toward inhibiting IL-8 secretion (Figure 6B); at concentrations of 25 and $50 \mu \mathrm{M}$, the release was reduced to 50.2\% $(p<0.001)$ and $31.4 \%(p<0.001)$ in comparison with LPS-stimulated cells (100\% of release), respectively. The effect of neooleuropein at $50 \mu \mathrm{M}$ was more significant than the effects of $2^{\prime \prime}$-epiframeroside $(p<0.001)$, oleonuezhenide $(p<0.001)$, ligstroside $(p<0.001)$, and oleuropein $(p<0.001)$ at the same concentration. Framoside and neooleuropein were the most active in reduction of TNF- $\alpha$ release at all concentrations tested (Figure 6C). The effect of framoside at $50 \mu \mathrm{M}$ was more significant than the effect of $2^{\prime \prime}$-epiframeroside $(p<0.001)$, oleonuezhenide $(p<0.001)$, and ligstroside $(p<0.001)$ at the same concentration. Additionally, neooleuropein was statistically more active than the positive control clarithromycin $(p<0.05)$. All isolated compounds except oleonuezhenide significantly inhibited the release of MCP-1 from stimulated neutrophils (Figure 6D). However, only neooleuropein was active at all concentrations tested (Figure 6D). The effect of neooleuropein at $10 \mu \mathrm{M}$ was more significant than the effects of framoside $(p<0.05)$ and hydroxyframoside $(p<0.05)$; at $25 \mu \mathrm{M}$, the effect was more significant than the effects of framoside $(p<$ $0.01)$, hydroxyframoside $(p<0.01)$ and oleonuezhenide $(p<$ 0.01 ), while at $50 \mu \mathrm{M}$, the effect was more significant than the effect of oleonuezhenide $(p<0.001)$ and the positive control clarithromycin $(p<0.01)$ at the same concentration. In general, neooleuropein was as effective or more effective in comparison with oleuropein and clarithromycin in reducing the release of pro-inflammatory agents.

The molecular mechanism of the observed effect was related to MAP kinases and NF- $k B$ activation. Lipopolysaccharide stimulation of human neutrophils resulted in the rapid phosphorylation of proteins, including p38 MAPK, p42/44 extracellular signal-regulated kinase (ERK), and c-Jun $\mathrm{NH}_{2}$ terminal kinase (JNK), as well as the translocation of NF- $k$ Bp65 from the cytoplasm to the nucleus. Our data show that the LPS-induced phosphorylation of p38 MAPK and ERK1/2 

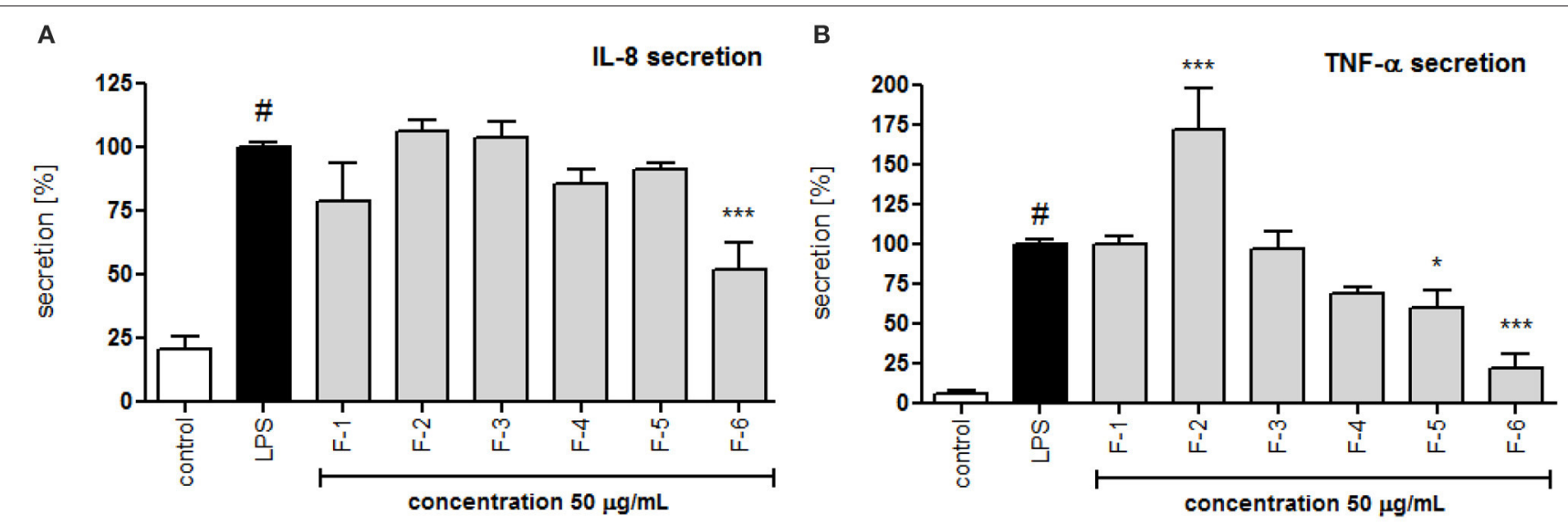

C

Syringa vulgaris leaf extract $(40 \mathrm{~g})$

Diaion HP-20

H2O-MeOH gradient (0-100\%)

\begin{tabular}{|l|l|l|l|l|l|}
\hline$F 1$ & $F 2$ & $F 3$ & $F 4$ & $F 5$ & $F 6$ \\
\hline
\end{tabular}

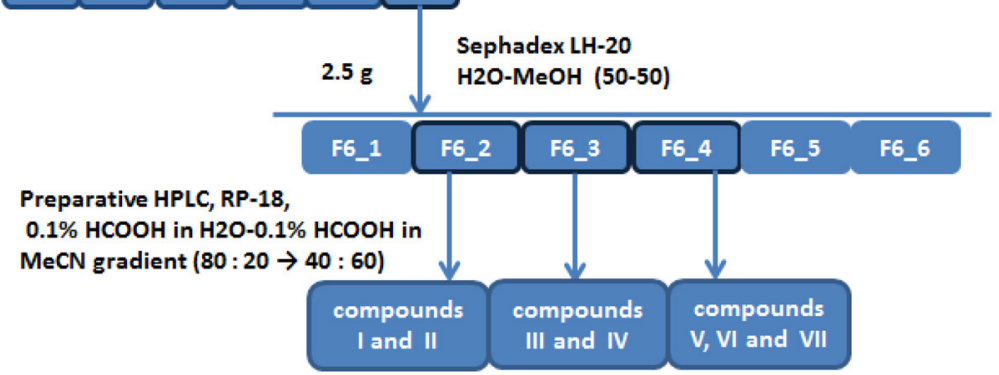

FIGURE 4 | Effect of S. vulgaris leaf extract fractions on IL-8 production by stimulated neutrophils [\%] (A) on TNF- $\alpha$ production by stimulated neutrophils [\%] (B); scheme of fractionation and compounds isolation from leaf extract (C). Data were expressed as the mean \pm SEM; at least three independent experiments were conducted, and they were assayed in duplicate. Experiments were performed using cells from different donors. Statistical significance \#P < 0.01 compared to the non-stimulated control; ${ }^{\star} P<0.05,{ }^{\star \star} P<0.01,{ }^{\star \star \star} P<0.001$ decrease compared to the stimulated control.

was decreased by neooleuropein and oleuropein, while JNK phosphorylation was decreased by all tested compounds at $50 \mu \mathrm{M}$ (Figure 7A). The inhibition of the translocation of NF- $k$ B-p65 from the cytoplasm to the nucleus was observed most significantly for oleuropein; however, the activity of neooleuropein was evident but more modest (Figure 7B).

\section{DISCUSSION}

S. vulgaris preparations have been traditionally used in Europe to treat several ailments connected with inflammation and, together with Asian Syringa sp., appear to be an interesting source of diverse bioactive structures ( $\mathrm{Su}$ et al., 2015). The aim of the present study was to investigate the effects of phytochemically characterized (HPLC-DAD-MS ${ }^{\mathrm{n}}$ ) extracts that have been used in traditional medicine (infusions and ethanolic extracts) from different parts of the common lilac (bark, fruits, leaves, and flowers) on the pro-inflammatory functions of neutrophils and to isolate the most active compounds using bioassay-guided fractionation. Finally, we focused on the activity and molecular mechanisms of action of the isolated compounds able to regulate neutrophil inflammation.

For the first time, we provided a comprehensive phytochemical analysis of common lilac and a comparison of different parts such as bark, fruit, flower, and leaf infusions/extracts (Table 1, Figure 1). We were able to identify or partially identify 64 compounds, and we also demonstrated significant differences in the phytochemical profiles of different parts of the plant (Figure 1). In general, the major compounds in S. vulgaris are as follows: caffeoylglucaric acid (5), syringin (14), secologanoside (15), demethyloleuropein (28), forsythoside B (31), quercetin rutinoside (32), syringalactone B (34), acteoside (36), nuzhenide (38), oleuropein (46), oleoacteoside (49), $2^{\prime \prime}$-epi-frameroside (53), ligstroside (54), oleonuezhenide isomer (55), and oleonuezhenide (60). The presence of phenyl ethanoids $31,36,49$, and secoiridoids $28,38,46,53,54,55,60$ as major compounds seems to be specific to this species, while lignans are more characteristic of S. pinnatifolia (Su et al., 2016), and 


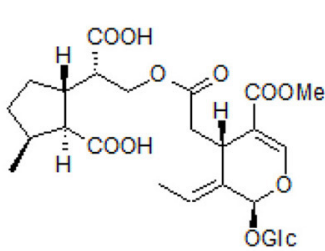

2"-epiframeroside (I)<smiles>C/C=C1\C(C(=O)C(=O)OCCc2ccc(O)cc2)OC=C(OC)C1OC</smiles>

ligstroside (IV)

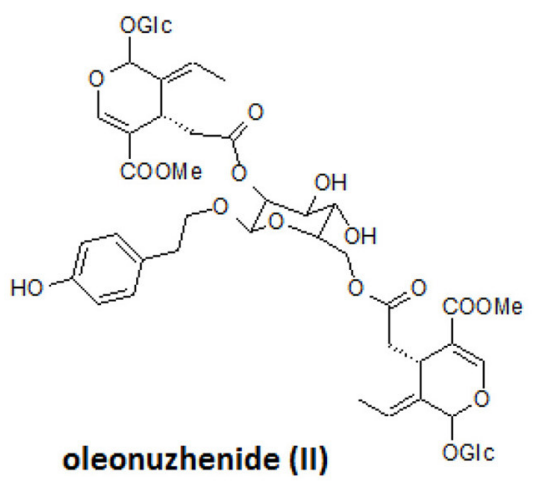

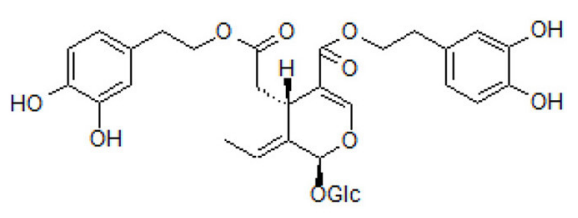

neooleuropein (V)<smiles>C/C=C1\C(C(=O)OCCc2ccc(O)c(O)c2)OC=C(OC)C1OC</smiles>

oleuropein (III)<smiles>C/C=C1C(=C/C(=O)OCCc2ccc(O)c(O)c2)\C(=O)OCC\1C(=O)OCCc1ccc(O)cc1</smiles>

hydroxyframoside (VI)

neooleuropein (V)

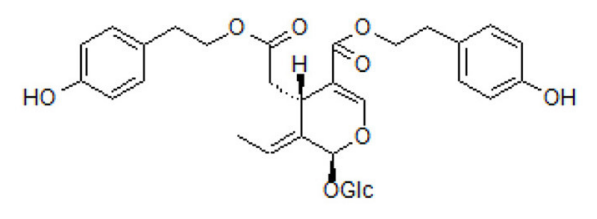

framoside (VII)

\section{GLc- glucose}

FIGURE 5 | Structures of isolated compounds.

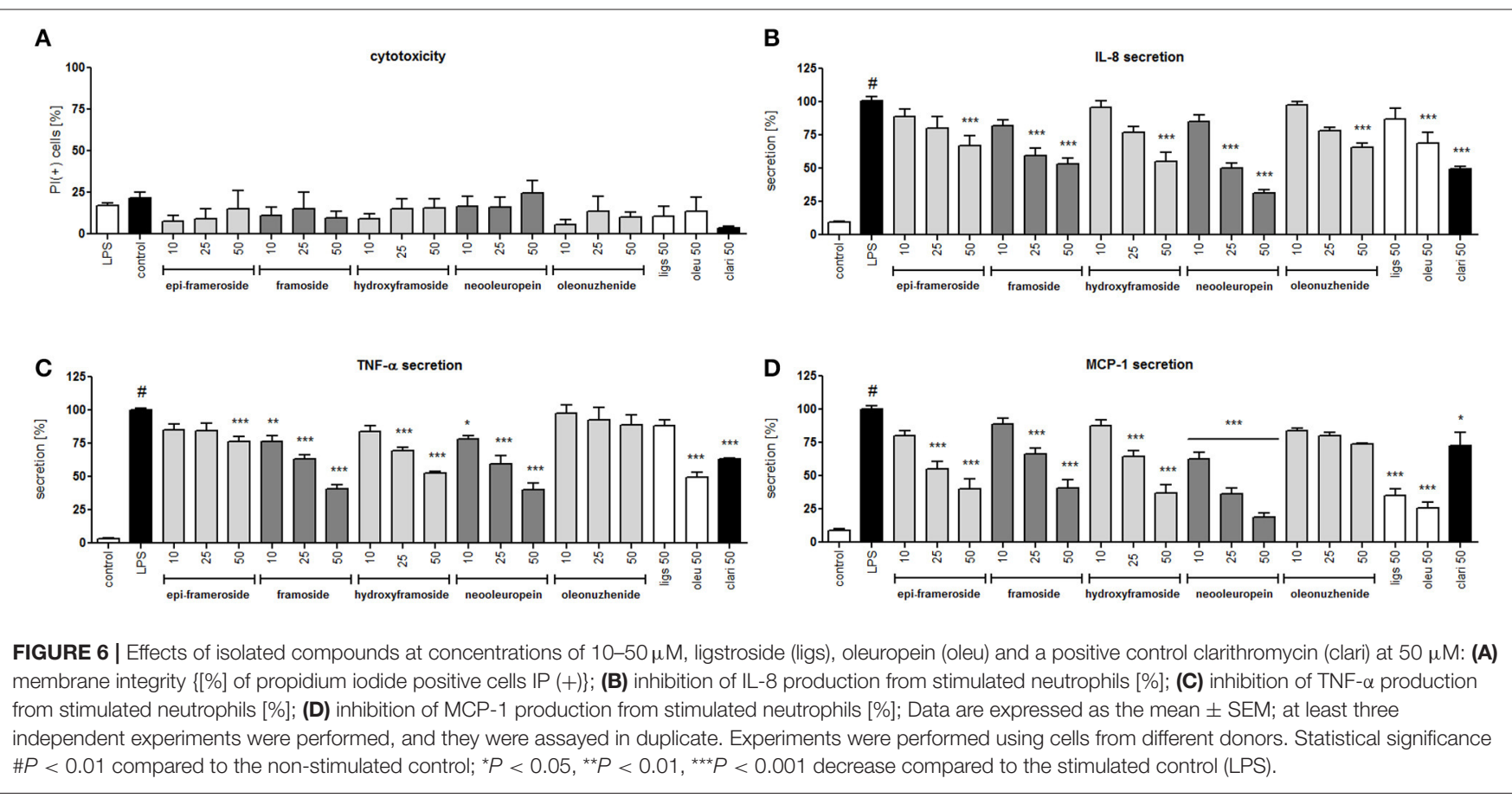



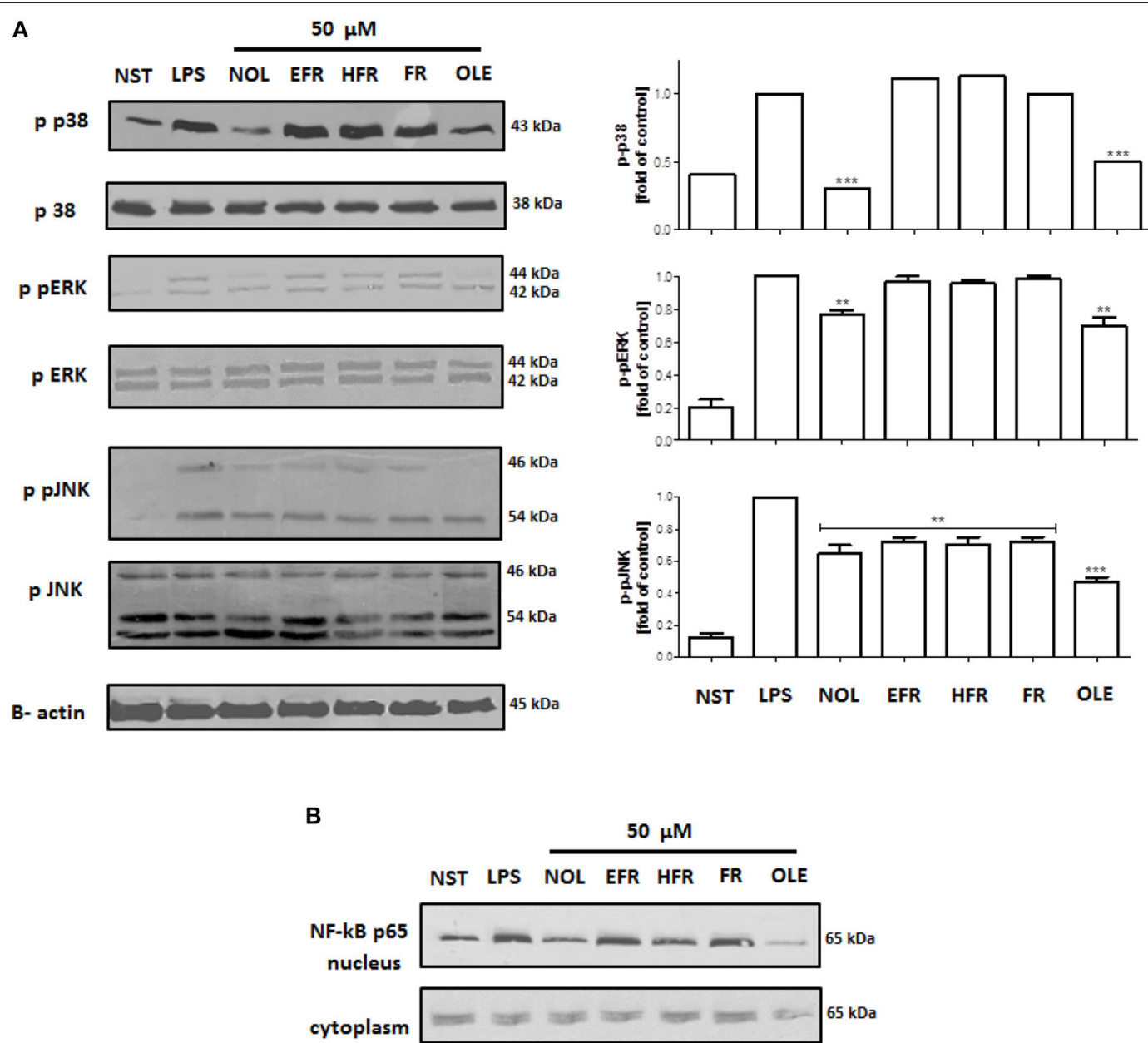

FIGURE 7 | Effects of compounds at $50 \mu \mathrm{M}$ on the phosphorylation of p38 MAPK, p42/44 ERK and JNK in LPS-activated human neutrophils (A); and NF-kB p65 translocation to the nucleus (B). Phosphorylation of p38, p42/44 ERK and JNK, and NF-kB p65 translocation were analyzed by an immunoblot assay using antibodies against the phosphorylated form and based on total protein. Representative images are shown (left A,B). Western blots for p38 MAPK, p42/44 ERK and JNK were quantified using the ImageJ 1.38 software after densitometric scanning of the bands of at least two independent experiments. Statistical significance ${ }^{\star \star} P<0.01$, ${ }^{\star \star \star} P<0.001$ decrease compared to the stimulated control (right A). NST- not stimulated cells, LPS- stimulated cells, neooleuropein (NOL), $2^{\prime \prime}$-epiframeroside (EFR), hydroxyframoside (HFR), framoside (FR), oleuropein (OLE).

secoiridoids named safghanosides are more characteristic of $S$. afghanica (Takenaka et al., 2002). S. reticulata contains similar phenyl ethanoids and secoiridoids as $S$. vulgaris; however, the presence of the iridoid syringapicroside and its derivatives in $S$. reticulata may distinguish both species phytochemically (Kikuchi et al., 1989; Machida et al., 2002).

In the neutrophil model of the pro-inflammatory state, all extracts and infusions were able to significantly reduce ROS production (Figures 2C,D). This is probably related to the presence of compounds that contain caffeic acid, $p$ coumaric acid, hydroxytyrosol or tyrosol phenolic moieties, which display antioxidant properties (Bi et al., 2011; Dudek et al., 2017). All extracts and infusions were especially active in decreasing IL-8 production (Figures $3 \mathbf{A}, \mathbf{B}$ ) and, to a lesser extent, MMP-9 and TNF $\alpha$ release (Figures 3C-F). However, the leaf extract fractionation revealed that fraction F2 increased TNF $\alpha$ production, while fractions F5 and F6 significantly decreased the production of this cytokine (Figure 4). A similar phenomenon was observed for Echinacea purpurea extracts (Todd et al., 2015). Further bioassay-guided fractionation of fraction F6 led to the isolation of the following secoiridoids: 2"-epiframeroside, oleonuezhenide, oleuropein, ligstroside, neooleuropein, hydroxyframoside, and framoside (Figure 5). We compared the anti-inflammatory activity of $2^{\prime \prime}$-epiframeroside, oleonuezhenide, neooleuropein, hydroxyframoside, and framoside with oleuropein and ligstroside, two well-known compounds that are widespread in the Oleaceae family (Jensen et al., 2002). Apart from oleonuezhenide, a dimeric secoiridoid, all compounds exhibited significant inhibition of pro-inflammatory cytokine and chemokine release/production. 
$2^{\prime \prime}$-epiframeroside primarily inhibited MCP-1 release, while neooleuropein appeared as the most active compound (Figure 6). Neooleuropein inhibited the secretion of IL-8 more significantly than oleuropein (Figure 6B) and secretion of TNF- $\alpha$ and MCP-1 more significantly than the positive control clarithromycin (Figures 6C,D).

The mechanism of LPS stimulation of human neutrophils is connected with a functional response through the activation of mitogen-activated protein kinases (MAPKs): p38 kinase, p42/44 extracellular signal-regulated kinase (ERK) and c-Jun $\mathrm{NH}_{2}$-terminal kinases (JNKs) (Nick et al., 1999; Arndt et al., 2004; Simard et al., 2015). Neooleuropein and oleuropein significantly inhibited the phosphorylation of the MAP kinases ERK1/2, p38 and JNK after LPS stimulation (Figure 7A), as well as the translocation of NF- $k \mathrm{~B}$ p65 to the nucleus (Figure 7B). Neooleuropein was more active toward the inhibition of p38 phosphorylation, while oleuropein more significantly inhibited NF- $k$ B p65 translocation, and all tested compounds inhibited JNK phosphorylation (Figures 7A,B). The activation of p38 MAPK in neutrophils is connected with the synthesis of TNF$\alpha$ and IL-8 (Nick et al., 1999). ERK activation also led to the elevated expression of pro-inflammatory cytokines in human neutrophils (Simard et al., 2015). In our study, neooleuropein was the most active compound in inhibiting p38 phosphorylation, which correlated with a significant inhibition of IL-8 production. However, oleuropein was more active in NF- $k$ B inhibition, which has been shown to affect cytokine gene expression in human neutrophils, although it has a less marked effect on IL-8 gene expression (Cloutier et al., 2007). The inhibition of MCP-1 production by all tested compounds was correlated with the inhibition of JNK phosphorylation, which has been strictly correlated with MCP-1 expression but not IL-8 or TNF$\alpha$ expression (Arndt et al., 2004). Interestingly, framoside was able to significantly decrease the TNF- $\alpha$ and IL-8 secretion (Figures 6B,C) without affecting MAPKs nor NF- $k$ B activation (Figure 7). It appears that framoside, the most lipophilic compounds, displays a different mode of action. As in human neutrophils other pathways such as PI3K are also involved in IL8 production and acts downstream of p38 MAPK (Fortin et al., 2011).

The observed significant inhibition of TNF- $\alpha$ and chemokine (IL-8 and MCP-1) production is of special interest for treating inflammatory diseases such as rheumatic arthritis, respiratory diseases, and arteriosclerosis. The pro-inflammatory effect of TNF- $\alpha$ mainly results from its capacity to stimulate the expression of adhesive molecules in endothelial cells and promote neutrophil attachment to the vascular endothelium in addition to their degranulation and pro-oxidative activity (Witko-Sarsat et al., 2000). MCP-1 (CCL2) is able to stimulate

\section{REFERENCES}

Agelet, A., and Vallès, J. (2003). Studies on pharmaceutical ethnobotany in the region of Pallars (Pyrenees, Catalonia, Iberian Peninsula). Part II. New or very rare use of previously known medicinal plants. J. Ethnopharmacol. 84, 211-227. doi: 10.1016/S0378-8741(02)00319-7 chemotaxis of monocytes and cellular events associated with chemotaxis and integrin expression. At high concentrations, MCP-1 elicits a respiratory burst leading to the generation of ROS (Palomino and Marti, 2015). IL-8 (CXCL8), one of the most important cytokines produced by neutrophils, mediates chemotaxis, releases granule enzymes, and promotes integrin expression and adhesion to endothelial cells (Gabrilovich, 2013). Although the anti-inflammatory and antioxidant activity of oleuropein and ligstroside was intensively studied, this is the first report concerning the biological activity of $2^{\prime \prime}$-epiframeroside, neooleuropein, hydroxyframoside, and framoside. In particular, neooleuropein appears to an interesting compound for further in vitro and in vivo study.

\section{CONCLUSIONS}

The present study demonstrated that the common lilac, which has been traditionally used in Europe as a medicinal plant, is a valuable source of active compounds, especially neooleuropein, for further research regarding their use in treating inflammatory diseases that result from the excessive activation of neutrophils. The observed decreases in the production of cytokines, such as TNF- $\alpha$, IL- 8, MCP-1, depend on the inhibition of the phosphorylation of MAP kinases.

\section{AUTHOR CONTRIBUTIONS}

MW, BM, MD, JW, and AK: performed the experiments; MW, $\mathrm{BM}, \mathrm{MD}$, and $\mathrm{AK}$ : carried out data analysis; $\mathrm{AK}$ : planned the experiments; AK: wrote the manuscript and supervised all work. All authors revised and approved the final version of the manuscript.

\section{FUNDING}

This study was financially supported by research grant 2015/17/B/NZ7/03086 from the Polish National Science Center. This project was carried out with the use of CePT infrastructure financed by the European Union's European Regional Development Fund within the Operational Program Innovative economy for 2007-2013. We would like to thank Monika Marciniak and Roman Rudecki for their technical assistance.

\section{SUPPLEMENTARY MATERIAL}

The Supplementary Material for this article can be found online at: https://www.frontiersin.org/articles/10.3389/fphar. 2018.00349/full\#supplementary-material 
Bálinet, G. A. (1971). Pharmakologische untersuchungen des blattextraktes von Syringa vulgaris. Planta Med. 19, 215-221. doi: 10.1055/s-0028-1099634

Bi, X., Li, W., Sasaki, T., Li, Q., Mitsuhata, N., Asada, Y., et al. (2011). Secoiridoid glucosides and related compounds from Syringa reticulata and their antioxidant activities. Bioorg. Med. Chem. Lett. 21, 6426-6429. doi: 10.1016/j.bmcl.2011.08.089

Böyum, A. (1968). A one-stage procedure for isolation of granulocytes and lymphocytes from human blood. General sedimentation properties of white blood cells in a $1 \mathrm{~g}$ gravity field. Scand. J. Clin. Lab. Invest. Suppl. 97, 51-76.

Cervin, A., Wallwork, B., Mackay-Sim, A., Coman, W. B., and Greiff, L. (2009). Effects of long-term clarithromycin treatment on lavage-fluid markers of inflammation in chronic rhinosinusitis. Clin. Physiol. Funct. Imaging 29, 136-142. doi: 10.1111/j.1475-097X.2008.00848.x

Cloutier, A., Ear, T., Blais-Charron, E., Dubois, C. M., and McDonald, P. P. (2007). Differential involvement of NF-kappaB and MAP kinase pathways in the generation of inflammatory cytokines by human neutrophils. J. Leukoc. Biol. 81, 567-577. doi: 10.1189/jlb.0806536

Damtoft, S., Franzyk, H., and Jensen, S. R. (1992). Excelsioside, a secoiridoid glucoside from Fraxinus excelsior. Phytochemistry 31, 4197-4201. doi: 10.1016/0031-9422(92)80442-H

Damtoft, S., Franzyk, H., and Jensen, S. R. (1995). Biosynthesis of iridoids in Syringa and Fraxinus: carbocyclic iridoids precursors. Phytochemistry 40, 785-792. doi: 10.1016/0031-9422(95)00210-X

Dudek, M. K., Michalak, B., Wozniak, M., Czerwinska, M. E., Filipek, A., Granica, S., et al. (2017). Hydroxycinnamoyl derivatives and secoiridoid glycoside derivatives from Syringa vulgaris flowers and their effects on the pro-inflammatory responses of human neutrophils. Fitoterapia 121, 194-205. doi: $10.1016 /$ j.fitote.2017.07.008

Evans, W. C. (2009). Trease and Evans Pharmacognosy. Philadelphia, PA: Saunders Elsevier, 35. 506

Fortin, C. F., Cloutier, A., Ear, T., Sylvain-Prévost, S., Mayer, T. Z., Bouchelaghem, R., et al. (2011). A class IA PI3K controls inflammatory cytokine production in human neutrophils. Eur. J. Immunol. 41, 1709-1719. doi: 10.1002/eji.201040945

Fournier, P. (1948). Le Livre des Plantes Médicinales et Vénéneuses [The Book of Medicinal and Toxic Plants, (Vol. II)]. Paris: Paul Chevalier Éditeur 421.

Fukuyama, Y., Koshino, K., Hasegawat, T., Yamadat, T., and Nakagawa, K. (1987). New secoiridoid glucosides from Ligustrum japonicum. Planta Med. 53, 427-431. doi: 10.1055/s-2006-962764

Fusani, P., Piwowarski, J. P., Zidorn, C., Kiss, A. K., Scartezzini, F., and Granica, S. (2016). Seasonal variation in secondary metabolites of edible shoots of Buck's beard [Aruncus dioicus (Walter) Fernald (Rosaceae)]. Food Chem. 202, 23-30. doi: 10.1016/j.foodchem.2016.01.103

Gabrilovich, D. (2013). The Neutrophils. New Outlook for Old Cells. London: Imperial College Press. 191-193.

Guo, H., Liu, A.-H., Ye, M., Yang, M., and Guo, D.-A. (2007). Characterization of phenolic compounds in the fruits of Forsythia suspensa by highperformance liquid chromatography coupled with electrospray ionization tandem mass spectrometry. Rapid Commun. Mass Spectrom. 21, 715-729. doi: $10.1002 / \mathrm{rcm} .2875$

Hanlidou, E., Karousou, R., Kleftoyanni, V., and Kokkini, S. (2010). The herbal market of Thessaloniki (N Greece) and its relation to the ethnobotanical tradition. J. Ethnopharmacol. 128, 412-418. doi: 10.1016/j.jep.2004.01.007

He, Z. D., Ueda, S., Akaji, M., Fujita, T., Inoue, K., and Yang, C. R. (1994). Monoterpenoid and phenylethanoid glycosides from Ligustrum pedunculare. Phytochemistry 36, 709-716. doi: 10.1016/S0031-9422(00)89802-7

Iossifova, T., Vogler, B., and Kostova, I. (1998). Secoiridoid glucosides from fraxinus ornus bark. Phytochemistry 49, 1329-1716. doi: 10.1016/S0031-9422(98)00097-1

Jarić, S., Mačukanović-Jocić, M., Djurdjević, L., Mitrović, M., Kostić, O., KaradŽić, B., et al. (2015). An ethnobotanical survey of traditionally used plants on Suva planina mountain (south-eastern Serbia). J. Ethnopharmacol. 175, 93-108. doi: 10.1016/j.jep.2015.09.002

Jensen, S. R., Franzyk, H., and Wallander, E. (2002). Chemotaxonomy of the Oleaceae: iridoids as taxonomic markers. Phytochemistry 60, 213-231. doi: $10.1016 /$ S0031-9422(02)00102-4

Kicel, A., Owczarek, A., Michel, P., Skalicka-Wozniak, K., Kiss, A. K., and Olszewska, M. A. (2015). Application of HPCCC, UHPLC-PDA-ESI-MS3 and HPLC-PDA methods for rapid, one-step preparative separation and quantification of rutin in Forsythia flowers. Ind. Crops Prod. 76, 86-94. doi: 10.1016/j.indcrop.2015.06.019

Kurkin, V. A., Zapesochnaya, G. G., and Grinenko, N. A. (1990). Iridoids of the bark of Syringa vulgaris. Chem. Nat. Compd. 26, 592-593. doi: $10.1007 / \mathrm{bf} 00601302$

Kikuchi, M., Yamauchi, Y., Takahashi, Y., and Sugiyama, M. (1989). Studies on the constituents of syringa species. VIII. Isolation and Structures of Phenylpropanoid Glycosides from the Leaves of Syringa reticulata (BLUME) HARA. Yakugaku zasshi 109, 366-371. doi: 10.1248/yakushi1947.109.6_366

Kikuchi, M., Yaoita, Y., Mano, N., and Kikuchi, M. (2010). Glycosides from the leaves of Syringa vulgaris and their growth inhibitory activity against human cancer cell lines. Japan Soc. Pharm. 64, 104-105.

Kikuchi, M., Yamauchi, Y., Yanase, C., and Nagaoka, I. (1987). Structures of new secoiridoid glycosides from the leaves of Syringa vulgaris Linn. Yakugaku zasshi 107, 245-248. doi: 10.1248/yakushi1947.107.3_245

Konno, K., Yasui, H., and Hirayama, C. (1998). Glycine protects against strong protein denaturing activity of oleuropein, a phenolic compound in privet leaves. J. Chem. Ecol. 24, 735-751. doi: 10.1023/A:1022350521287

Kurkin, V. A., Zapesochnaya, G. G., Grinenko, N. A., and Zolotarev, B. M. (1989). Phenolic compounds of the bark of Syringa vulgaris. Chem. Nat. Compd. 25, 499-500. doi: 10.1007/BF00597667

Kuwajima, H., Morita, M., Takaishi, K., Inouea, K., Fujita, T., He, Z.D., et al. (1992). Secoiridoid, coumarin and secoiridoid-coumarin glucosides from Fraxinus chinensis. Phytochemistry 31, 1277-1280. doi: 10.1016/0031-9422(92)80277-L

Kuznicka, B. (1986). Historia Leków Naturalnych. Zródła do Dziejów Etnofarmacji Polskiej. [History of Natural Drugs. History of Polish Ethnopharmacy]. Polish Academy of Sciences. Warsaw: Institute of the History of Science, Education, and Technology. 257

Kuzniewski, E., and Augustyn-Puziewicz, J. (1986). Przewodnik Ziołolecznictwa Ludowego [Traditional Medicine Guide]. Warsaw: Panstwowe Wydawnictwo Naukowe.

Lendvay, B., Kadereit, J. W., Westberg, E., Cornejo, C., Pedryc, A., and Höhn, M. (2016). Phylogeography of Syringa josikaea (Oleaceae): early pleistocene divergence from east asian relatives and survival in small populations in the carpathians. Biol. J. Linn. Soc. 119, 689-703. doi: 10.1111/bij.12499

Leporatti, M. L., and Ivancheva, S. (2003). Preliminary comparative analysis of medicinal plants used in the traditional medicine of Bulgaria and Italy. $J$. Ethnopharmacol. 87, 123-142. doi: 10.1016/S0378-8741(03)00047-3

Li, H., Yao, W., Liu, Q., Xu, J., Bao, B., Shan, M., et al (2017). Application of UHPLC-ESI-Q-TOF-MS to identify multiple constituents in processed products of the herbal medicine ligustri lucidi fructus. Molecules. 22:E689. doi: $10.3390 /$ molecules22050689

Lyle, T. J. (1897). Physio-Medical Therapeutics, Materia Medica and Pharmacy. London: The National Association of Medical Herbalists of Great Britain, Ltd.

Machida, K., Kaneko, A., Hosogai, T., Kakuda, R., Yaoita, Y., and Kikuchi, M. (2002). Studies on the constituents of Syringa species. X. Five new iridoid glycosides from the leaves of Syringa reticulata (Blume) Hara. Chem. Pharm. Bull. 50, 493-497. doi: 10.1248/cpb.50.493

Machida, K., Ohkawa, N., Ohsawa, A., and Kikuchi, M. (2009). Two new phenolic glycosides from Syringa reticulate. J. Nat. Med. 63, 192-194. doi: 10.1007/s11418-008-0295-3

Moerman, D. E. (1998). Native America Ethnobotany. London: Timber Press. 549.

Nick, J. A., Avdi, N. J., Young, S. K., Lehman, L. A., McDonald, P. P., Frasch, S. C., et al. (1999). Selective activation and functional significance of p38a mitogen-activated protein kinase in lipopolysaccharide-stimulated neutrophils. J. Clin. Invest. 103, 851-858. doi: 10.1172/JCI5257

Palomino, D. C., and Marti, L. C. (2015). Chemokines and immunity. Einstein 13 , 469-473. doi: 10.1590/S1679-45082015RB3438

Papp, N., Birkás-Frendl, K., Bencsik, T., Stranczinger, S., and Czégényi, D. (2014). Survey of traditional beliefs in the Hungarian Csángó and Székely ethnomedicine in Transylvania, Romania. Rev. Bras. Farmacogn. 24, 141-152. doi: 10.1016/j.bjp.2014.03.005

Park, H.-J., Lee, M.-S., Lee, K.-T., Sohn, I.-C., Han, Y.-N., and Miyamota, K. (1999). Studies on constituents with cytotoxic activity from the stem bark of Syringa velutina. Chem. Pharm. Bull. 47, 1029-1031. doi: 10.1248/cpb. 47.1029 
Remington, J. P., and Woods, H. C. (Editors) (1918). The Dispensatory of the United State of America. The Southwest School of Botanical Medicine. Available online at: http://www.swsbm.com. 134.

Sanz, M., Fernández de Simón, B., Cadahía, E., Esteruelas, E., Mu-oz, A. M., Hernández, T., et al. (2012). LC-DAD/ESI-MS/MS study of phenolic compounds in ash (Fraxinus excelsior L. and F. americana L.) heartwood. Effect of toasting intensity at cooperage. J. Mass. Spectrom. 47, 905-918. doi: 10.1002/jms.3040

Simard, F. A., Cloutier, A., Ear, T., Vardhan, H., and McDonald, P. P. (2015). MEKindependent ERK activation in human neutrophils and its impact on functional responses. J. Leukoc. Biol. 98, 565-573. doi: 10.1189/jlb.2MA1214-599R

Simpson, J. L., Powell, H., Boyle, M. J., Scott, R. J., and Gibson, P. G. (2008). Clarithromycin targets neutrophilic airway inflammation in refractory asthma. Am. J. Respir. Crit. Care Med. 177, 148-155. doi: 10.1164/rccm.200707$1134 \mathrm{OC}$

Sõukand, R., and Pieroni, A. (2016). The importance of a border: medical, veterinary, and wild food ethnobotany of the Hutsuls living on the Romanian and Ukrainian sides of Bukovina. J. Ethnopharmacol. 185, 17-40. doi: 10.1016/j.jep.2016.03.009

Su, G., Cao, Y., Li, C., Yu, X., Gao, X., Tu, P., et al. (2015). Phytochemical and pharmacological progress on the genus Syringa. Chem. Cent. J. 9:2. doi: 10.1186/s13065-015-0079-2

Su, G., Zhang, R., Yang, X., Bai, R., Yin, X., Gao, X., et al. (2016). Lignans from the stem bark of Syringa pinnatifolia. Fitoterapia 114, 63-68. doi: 10.1016/j.fitote.2016.08.011

Takenaka, Y., Okazaki, N., Tanahashi, T., Nagakura, N., and Nishi T. (2002). Secoiridoid and iridoid glucosides from Syringa afghanica. Phytochemistry 59, 779-787. doi: 10.1016/S0031-9422(02)00024-9

Tanahashi, T., Watanabe, H., Itoh, A., Nagakura, N., Inoue, K., Ono, M., et al. (1992). Five secoiridoid glucosides from Fraxinus formosana. Phytochemistry 36, 709-716. doi: 10.1016/0031-9422(92)80118-X

Todd, D. A., Gulledge, T. V., Britton, E. R., Oberhofer, M., Leyte-Lugo, M., Moody, A. N., et al. (2015). Ethanolic Echinacea purpurea extracts contain a mixture of cytokine-suppressive and cytokine-inducing compounds, including some that originate from endophytic bacteria. PLoS ONE 10:e0124276. doi: 10.1371/journal.pone. 0124276

Tóth, G., Barabás, C., Tóth, A., Kéry, Á., Béni, S., Boldizsár, I., et al. (2016). Characterization of antioxidant phenolics in Syringa vulgaris L. flowers and fruits by HPLC-DAD-ESI-MS. Biomed. Chromatogr. 30, 923-932. doi: $10.1002 / b m c .3630$

Tutin, T. G., Heywood, V. H., Burges, N. A., Moore, D. M., Valentine, S. M., Walters, S. M., et al. (1972). Flora Europeae. London: Cambridge University Press. 3, 54.

Tóth, G., Barabás, C., Tóth, A., Kéry, Á., Béni, S., Boldizsár, I., et al. (2015). Phenolic profile, antioxidant and antinociceptive properties of Syringa vulgaris. Planta Med. 81:1435. doi: 10.1055/s-0035-1565418

Wallander, E., and Albert, V. A. (2000). Phylogeny and classification of Oleaceae based on rps16 and trnL-F sequence data. Am. J. Bot. 87, 1827-1841. doi: $10.2307 / 2656836$

Witko-Sarsat, V., Rieu, P., Descamps-Latscha, B., Lesavre, P., and HalbwachsMecarelli, L. (2000). Neutrophils: molecules, functions and pathophysiological aspects. Lab. Invest. 80, 617-653. doi: 10.1038/labinvest.3780067

Witkowska-Zuk, L. (2013). Rośliny Leśne [Plants of the Forests]. Warsaw: Multico Oficyna Wydawnicza. 137.

Conflict of Interest Statement: The authors declare that the research was conducted in the absence of any commercial or financial relationships that could be construed as a potential conflict of interest.

Copyright (๐ 2018 Woźniak, Michalak, Wyszomierska, Dudek and Kiss. This is an open-access article distributed under the terms of the Creative Commons Attribution License (CC BY). The use, distribution or reproduction in other forums is permitted, provided the original author(s) and the copyright owner are credited and that the original publication in this journal is cited, in accordance with accepted academic practice. No use, distribution or reproduction is permitted which does not comply with these terms. 


\section{OPEN ACCESS}

Edited by:

Anna Karolina Kiss, Medical University of Warsaw, Poland

Reviewed by:

Francesco Paolo Busardò, Sapienza Università di Roma, Italy Robert Nawrot, Adam Mickiewicz University in

Poznań, Poland

Suzana Popovic,

University of Kragujevac, Serbia

*Correspondence:

Adam M. Matkowski

pharmaceutical.biology@wp.eu

Specialty section:

This article was submitted to

Ethnopharmacology,

a section of the journal

Frontiers in Pharmacology

Received: 15 December 2017

Accepted: 15 March 2018

Published: 11 April 2018

Citation:

Zielińska S, Jezierska-Domaradzka A Wójciak-Kosior M, Sowa I, Junka A and Matkowski AM (2018) Greater Celandine's Ups and Downs-21

Centuries of Medicinal Uses of

Chelidonium majus From the Viewpoint of Today's Pharmacology. Front. Pharmacol. 9:299. doi: 10.3389/fphar.2018.00299

\section{Greater Celandine's Ups and Downs-21 Centuries of Medicinal Uses of Chelidonium majus From the Viewpoint of Today's Pharmacology}

\author{
Sylwia Zielińska ${ }^{1}$, Anna Jezierska-Domaradzka ${ }^{1,2}$, Magdalena Wójciak-Kosior ${ }^{3}$, \\ Ireneusz Sowa ${ }^{3}$, Adam Junka ${ }^{4}$ and Adam M. Matkowski ${ }^{1,2 *}$ \\ ${ }^{1}$ Pharmaceutical Biology and Botany, Wrocław Medical University, Wrocław, Poland, ${ }^{2}$ Botanical Garden of Medicinal Plants, \\ Wrocław Medical University, Wrocław, Poland, ${ }^{3}$ Analytical Chemistry, Medical University of Lublin, Lublin, Poland, \\ ${ }^{4}$ Pharmaceutical Microbiology and Parasitology, Wrocław Medical University, Wrocław, Poland
}

As antique as Dioscorides era are the first records on using Chelidonium as a remedy to several sicknesses. Inspired by the "signatura rerum" principle and an apparent ancient folk tradition, various indications were given, such as anti-jaundice and cholagogue, pain-relieving, and quite often mentioned-ophthalmological problems. Central and Eastern European folk medicine has always been using this herb extensively. In this region, the plant is known under many unique vernacular names, especially in Slavonic languages, associated or not with old Greek relation to "chelidon" - the swallow. Typically for Papaveroidae subfamily, yellow-colored latex is produced in abundance and leaks intensely upon injury. Major pharmacologically relevant components, most of which were first isolated over a century ago, are isoquinoline alkaloids-berberine, chelerythrine, chelidonine, coptisine, sanguinarine. Modern pharmacology took interest in this herb but it has not ended up in gaining an officially approved and evidence-based herbal medicine status. On the contrary, the number of relevant studies and publications tended to drop. Recently, some controversial reports and sometimes insufficiently proven studies appeared, suggesting anticancer properties. Anticancer potential was in line with anecdotical knowledge spread in East European countries, however, in the absence of directly-acting cytostatic compounds, some other mechanisms might be involved. Other properties that could boost the interest in this herb are antimicrobial and antiviral activities. Being a common synanthropic weed or ruderal plant, C. majus spreads in all temperate Eurasia and acclimates well to North America. Little is known about the natural variation of bioactive metabolites, including several aforementioned isoquinoline alkaloids. In this review, we put together older and recent literature data on phytochemistry, pharmacology, and clinical studies on C. majus aiming at a critical evaluation of state-of-the-art from the viewpoint of historical and folk indications. The controversies around this herb, the safety and drug quality issues and a prospective role in phytotherapy are discussed as well.

Keywords: isoquinoline alkaloids, chelidonine, chelerythrine, cytotoxic, anti-inflammatory, anti-microbial 


\section{INTRODUCTION}

"No less extraordinary is the property of the herb Celandine; which, it is said, if any man shall have this herb, with the heart of a Mole, he shall overcome all his enemies, all matters in suit, and shall put away all debate," and "if before named herb be put upon the head of a sick man, if he shall die, he shall sing anon with a loud voice, if not, he shall weep"; and "it bringeth the business begun to an end," so wrote Albertus Magnus in thirteenth century A.D. (Best and Brightman, 1999). Nowadays, mankind would definitely benefit from such a miraculous remedy. Unfortunately, such claims about Chelidonium majus L.- the Greater Celandine, have not been verified according to the modern evidence-based approach (but no data on rigorous testing toward such properties actually exist in the literature). However, through years of investigations, many other properties ascribed to this inconspicuous but characteristic plant have been confirmed or re-discovered. Several others could not be positively confirmed. Despite the widespread use in folk medicine and in official phytotherapy, both in Europe and in Traditional Chinese Medicine, the celandine herb did not join the most popular herbal remedies such as chamomile, valerian, St. John's wort or ginseng. It has been listed in pharmacopeias and sold in pharmacies in parallel to the spontaneous collection by people seeking drugs against gastrointestinal disorders, cancer, infections, but especially against warts and any skin protuberances. This was the reason to combine the available historical and ethnobotanical data with the state-of-the art in pharmacology of C. majus and its components in the present review (Supplementary Figure 1). To date, only a couple of papers have provided review of pharmacological and phytochemical knowledge with the EMA assessment report from 2011 (European Medicines Agency, 2011) and the review (Gilca et al., 2010), being the most recent and comprehensive ones. Biswas (2013) has published a short review with overview of selected bioactivities but only covering a fraction of available literature and suggesting future directions of research. Similar approach to review the C. majus properties was used by Arora and Sharma (2013) who summarized some activities based on selected literature and included pharmacognostic characteristics. Gilca et al. (2010) has classified the pharmacological evidence into the categories related to traditional usage, also from the viewpoint of the TCM, such as anti-infectious, spasmolytic, gastric and hepatic, and anticancer. They also listed traditional indications that had not been confirmed by modern research, such as diuretic, anti-edema, expectorant and antitussive, pulmonary, and ophthalmological diseases. Some current information is also available in herbal books and compendia (Wichtl, 2004) or in the Internet. The latter source is of course difficult to verify.

Therefore, we chose to include a possibly high number of available literature, by selecting records from database search (PubMed, Scopus, Google Scholar) with the term "chelidonium" or "celandine" and manually eliminating papers pertaining to field botany, ecology and other aspects not relevant to medicinal use of this plant. Some references that have been reviewed earlier (e.g., in Colombo and Bosisio, 1996) were also not cited directly if the information was redundant. Some information about the historical applications and folk medicine in Central and Eastern Europe were obtained from sources available in local libraries. In particular, we describe the phytochemical composition of various parts of the plant, the methods used for obtaining extracts and analysis of the herbal material. Further, we critically summarize the most credible research on bioactivity and clinical efficacy of various products and substances from $C$. majus. In addition, the highly debated and controversial issue of the patented, apparently semi-synthetic drug NSC-631570 (Ukrain ${ }^{\circledR}$ ) promoted mainly as a cancer cure was discussed (Ernst and Schmidt, 2005). With this review, we hope to encourage more research and attract interest to this quite common but not always adequately respected weed.

\section{BOTANICAL DESCRIPTION}

C. majus L. (Papaveraceae ${ }^{1}$ ) is a short-lived hemicryptophyte. It has up to $1 \mathrm{~m}$ high stem, branched and sparsely pubescent. The alternately placed leaves are light bluish at the bottom and green at the top. The basal leaves are long-petioled, with the obovated in contour, pinnatosected leaflets with 5-7 lobed segments. The apical leaves are short-petioled, with 3-lobed leaflet. From April to October the plant produces umbellate inflorescences with 26 flowers, which have 4 bright yellow petals and two whitish, early dropping sepals. The fruit is an elongated $(3 \mathrm{~cm})$, podshaped, multiseeded capsule, dehiscent with two valves. The seeds are shiny, ovate and dark brown or black, with elaiosomes. The underground part is a short tap root with numerous and elongated lateral roots. The whole plant contains yellow to orange latex. C. majus grows in the lowlands and foothills in leafy forests, in brushwood, parks, gardens, on the roadsides and around buildings. It prefers moist soils rich in nitrogen and organic matter (Zarzycki et al., 2002).

\section{Distribution Area}

C. majus is native in Europe, western and central part of Asia and in northern Africa. It occurs from Portugal in the West, to Central, Eastern to Northern Europe. The Asian range covers Turkey, Iran, Kazakhstan, Mongolia, Caucasus, and Siberia. In North America it is an introduced plant.

\section{Taxonomy and Nomenclature}

Until the mid-twentieth century the genus Chelidonium L. was monotypic with C. majus L. as the only species. In 1982 Krahulcowa based on cytotaxonomic study of C. majus L. sensu lato, proposed to divide the genus into two microspecies. She proposed C. majus L. $(2 \mathrm{n}=12)$ distributed in Europe, Siberia, and China and a new species C. asiaticum (Hara) Krahulcova $(2 \mathrm{n}=10)$, an East Asian vicariant (Krahulcová, 1982). Aside from the difference in chromosome numbers and distribution area, C. asiaticum slightly differs morphologically from C. majus. It is more hairy, with narrower and sharper leaf lobes. Within C. majus she distinguished European C. majus L. subsp. majus with more laciniate lobes of leaves, and C. majus.

${ }^{1}$ The Plant List. Available online at: http://www.theplantlist.org/1.1/browse/A/ Papaveraceae/Chelidonium/ (Accessed Sept 29, 2017). 
subsp. grandiflorum (DC.) Printz, in South Siberia and China (Krahulcová, 1982).

(Krahulcová, 1982) Name of the genus derives from Greek ( $\chi \varepsilon \lambda \iota \delta o ́ v \iota o v)$ with chelidon $(\chi \varepsilon \lambda \iota \delta o ́ v)$ meaning swallow (a bird) for the plant usually blooms simultaneously with arrival of these birds. The specific epithet majus in Latin means bigger.

Common English name: Greater celandine.

The name celandine originates from Medieval Latin word celidonia, a phonetic variant of Latin chelidonia, which was recorded by Pliny. Similarly, the German name, Schöllkraut, comes from Schellkraut (Bauhin, 1651), which is derived from Latin-Greek chelidonium (Waniakowa, 2015).

\section{Common and Folk Names in Some European Languages}

Albanian-latrapeci, bar saraleku; Belarussian-padtynnik, barodaunik (wart herb); Bosnian-rosopas; Bulgarian-zmiysko mlako; Croatian-zmijino mlijeko, rosopas; Czechvlaštovičník, celadona, celduně, cen dalie, dravnicovina, hadí mlíc, krkavník; Dutch-stinkende gouwe; EnglishTetterwort, devils's milk, rock poppy; French-grande chélidoine Éclair, herbe aux boucs, herbe a l'hirondelle; German-Schöllkraut, Gilbkraut, Goldwurz, Schwalbenkraut, Warzenkraut; Italian-celidonia, cinerognola, Montenegrinrusopas, rusa; Polish-glistnik jaskólcze-ziele, celidonia, cyndalia, cencylja, glistewnik, gliśnik, niebospad, złoty groszek, złotnik, zółte ziele, zółte kwiatki, roztopaść; Ukrainianhladyshnyk, hnystnyk, zhovtyi molochay, smetannyk, chystotil; Romanian: rostopascăa Russian-chistotel; Rusyn-rostopast'; Serbian-rusopas, rusa, rusomača; Spanish-Golondrinera, Hierba de las verrugas (wart herb).

\section{Chelidonium majus IN FOLK MEDICINE History of Usage}

C. majus has been known as medicinal species since the very Antiquity. Medicinal properties of C. majus were described by Dioscorides and Pliny the Elder in the first century AD. Dioscorides in De Materia Medica states that celandine begins to blossom when the swallows arrive and withers when they depart. He also refers to a lore saying that swallows restore sight to their blind nestlings with use of celandine (Osbaldeston and Wood, 2000). Pliny the Elder repeats these accounts in Naturalis Historiae (Jones, 1966).

The foremost medicinal use of celandine, described since the ancient times until the sixteenth century, was treating visual impairment and eye diseases. For such conditions, Dioscorides advised to use herb juice, boiled with honey in a brass vessel. The juice could also be dried in the shade and the resulting small pellets were ingredients of other medicinal products. According to Dioscurides celandine soaked in wine together with anise fruits was helpful in treating jaundice and dermatologic disorders such as herpes. Besides, chewing on a root relieved toothache (Osbaldeston and Wood, 2000). Pliny advised a kind of eye lotion, which takes its name, chelidonia, from the name of the plant (Jones, 1966).
Celandine was an admired medicinal plant during the Middle Ages, mostly used to cure eye diseases, for throat cleansing, treatment of ulcers and skin eczema as well as against colic and jaundice (Mayer et al., 2003). In 1080, French monk and physician Odo Magdunensis wrote De viribus herbarum, also known as Macer floridus, a botanical poem describing medical effects of 77 plants. One of the chapters of $M$. floridus deals with medicinal application of celandine in "visual impairment as well as skin and liver conditions" (Mayer et al., 2003). Hildegard of Bingen wrote about celandine in her work Liber subtilitatum diversarum naturarum creaturarum created during 1150-1160 A.D. and finally published in 1533. Hildegard recommended celandine juice to enhance sight and juice mixed with tallow as a cure for skin ulcer (Mayer et al., 2003). Moreover, celandine would be a strong aphrodisiacum, but causing infertility on ladies. Breathing the smell of the plant by spouses prevents arguments. Celandine was also recommended by to treat jaundice and against hair overgrowth (Czekański, 2007).

Since the sixteenth century, according to Paracelsus's signature doctrine, celandine was used to treat jaundice and liver diseases (Rostanski, 1997). C. majus was described in comprehensive botanical medical works of scholars such as: Joannes Minoritanus, Marcello Vergili, Hieronymus Bock, Leonhard Fuchs, Pierandrea Matthioli, and Adam Lonicer. The authors refer to antique sources and recommend using celandine to treat eye and skin conditions. According to Lonicer, to cure various dermatologic diseases, known in those days as "leprosy," the juice of the root of celandine had to be applied on the skin, conjointly with drinking the juice mixed with syrup of common fumitory (Fumaria officinalis) twice a day for 9 days (Mayer et al., 2003). At the turn of the sixteenth and seventeenth century two large works on herbs and their applications were written in Poland. The first is Herbarz Polski by Marcin of Urzędowo, printed in 1595 and the second is Herbal by Simon Syrenius, published in 1613. Marcin of Urzędowo described, based on works of Dioscorides, uses of celandine to treat eye diseases, jaundice, wounds, toothache, and colic. To cure sight impairment, herb juice boiled with honey or pellets made of juice dried in shade should be used. Cataract, on the other hand, should be treated with juice draining from a broken stem or root of the plant. In case of jaundice, root of celandine should be boiled in white wine and the decoction should be drunk for a few days. The crashed root with wine was drunk to treat colic and applied on wounds. Applying a piece of root of celandine to an aching tooth, relieved the pain (Marcin of Urzędowo, 1595).

Simon Syrenius, described celandine-based recipes used in eye diseases. The main ingredient of such medicines is fresh celandine juice. As one of the few authors, Syrenius considered the juice irritative and therefore recommended mixing it with small amounts of vinegar, milk, or rose water. He also advised drinking a decoction of celandine roots cooked in wine with anise or a mixture of powdered root with vinegar before bedtime to treat jaundice, colic, and stomach ache. In case of toothache he advised to rub teeth with powdered root with vinegar. Body ulcers and scabs on the head skin can be cured with a salve of powdered root mixed with pork fat and vinegar. Alternatively, the 
powdered root alone could be put directly on the ulcers. Syrenius also describes the diaphoretic and diuretic effects of the herb along with the roots or the root itself. The root boiled in wine has a diuretic effect. As diaphoretic remedy Syrenius recommended taking dry bath of celandine herb, which would cause extensive sweating and expulsion of excess water from the body as well as drinking decoction from roots boiled in rose vinegar or white wine with great water dock (Rumex hydrolapathum). In addition, the celandine could be used to dye the hair yellow, and to lighten the freckles and hyperpigmentation on the face (Syrenius, 1613).The British Flora Medica (Barton and Castle, 1845) cites the traditional applications of $C$. majus in treatment of jaundice, visceral obstructions, fevers, dropsies, scrofula, syphilitic affliction, gout, cataract, ophtalmia, and specks as described previously by Dioscorides and Galen.

In countries, where C. majus is the native species, it became one of the most widespread drug of folk herbal medicine. The scope of its applications in folk medicine shows high similarity among many regions of Central and Eastern Europe. It is worth to mention its prevalent application to treat warts, eczema and other skin diseases, gastrointestinal parasites, jaundice, and liver complaints, inflammatory eye infections and other diseases, including cancer.

\section{Skin Diseases}

In folk medicine, $C$. majus was most commonly used to remove warts. Herb juice or latex were used most frequently for this purpose, however, use of leaves and flowers was also noted. In Poland it was common to rub the fresh juice from the broken stem of the celandine onto warts (De Verdmon, 1936; Kuźniewski and Augustyn-Puziewicz, 1999; Kujawska et al., 2016). In the Bieszczady Mountains and in the Podkarpacie Region (S-E Poland) the juice was used directly on warts, or they were first scrubbed off and then the juice was applied on the wound (Szary, 2013). A cataplasm made of flowers that was supposed to be changed every few days was used in the Kielce Region (Central Poland) (Szot-Radziszewska, 2012). Occasionally, fresh leaves were also used (Kujawska et al., 2016). The juice of the aerial part of the plant was used in the Ukrainian Carpathians (Szary, 2010, 2013) and in Russia (Zevin et al., 1997). The herb juice was applied to treat warts also in the Balkan countries (Redžič, 2007; Tiţa et al., 2009; Menković et al., 2011; Mustafa et al., 2012; Koleva et al., 2015) as well as in Central Italy (Menale et al., 2006) and Great Britain (Barton and Castle, 1845). Other dermatologic conditions were also treated. All around Poland it was common to apply fresh leaves or juice on wounds. In Podolia (Ukraine) corns were treated by rubbing with a root of celandine and by application of fresh leaves. After a week, corns softened and ruptured. A salve from celandine, olive oil, fir resin and beeswax was a remedy for pustules (Kujawska et al., 2016). In the Bieszczady mountains (Polish-Slovakian-Ukrainian frontier), juice of celandine was applied to eczema and cuts, and decoction of root was used for baths and rinse for dermatologic conditions (Szary, 2013). In the Rzeszowszczyzna (S-E Poland) region leaves were applied to ulcers to stimulate picking up and rupture (Wdowiak and Bielecka-Grzela, 2013). The juice was also used to lighten freckles (Kuźniewski and Augustyn-Puziewicz,
1999). In Russia the juice of aerial parts was used in the treatment of skin wounds, skin irritation, allergic rashes and dermatitis, leaves, and flowers in the treatment of boils (Mamedov et al., 2004). The aerial parts of the plant were used by the people of Montenegro to cure blisters, rashes, and scabies (Menković et al., 2011). In Central Serbia, juice was applied directly on skin in skin eruptions, psoriasis and eczema (Jarić et al., 2007).

\section{Liver Diseases}

C. majus is one of the best-known folk medicine remedy for jaundice and liver diseases, such as inflammation, spastic conditions, and gallstones. In Poland, infusion made of young celandine leaves was used as a cholagogue and to regulate action of the digestive tract (Kuźniewski and Augustyn-Puziewicz, 1999). Jacques (De Verdmon, 1936) in case of jaundice advised infusion made of half of a teaspoon celandine per cup. All around Poland it was common to bath children with jaundice in celandine and to give celandine infusion to drink (Kujawska et al., 2016). In the Bieszczady and in the Ukrainian Carpathians, herb infusion was drunk to relieve liver conditions (Szary, 2010). In Western Ukraine, infusion was used as relaxant in colic attacks (Szot-Radziszewska, 2007). Also in Balkan countries, celandine was employed in the treatment of liver disorders. In the Albanian Alps to treat hepatitis a decoction of fresh aerial parts has been drunk with sugar in small portions-half coffee cup (Pieroni et al., 2005). In Serbia celandine was used internally for inflammation of the gallbladder, bile duct, jaundice, and hepatitis (Jarić et al., 2007; Šavikin et al., 2013). The use of celandine is similar in Gollak region in Kosovo (Mustafa et al., 2012), in the Prokletije Mountains (Menković et al., 2011) and in Zagori in Epirus, North-West Greece (Vokou et al., 1993).

\section{Against Digestive Tract Parasites}

Polish name of C. majus "glistnik" (roundworm herb) comes from a common traditional usage of this plant to expel roundworms. For this reason decoction of the herb had to be drunk for 12 days (De Verdmon, 1936). In the Bieszczady Mountains children were bathed in decoction of the celandine herb and were given celandine infusion to drink (Szary, 2013). Decoction of seeds was also used in the Kielce Region (SzotRadziszewska, 2012). In western Ukraine, infusion of the herb was prepared (Szot-Radziszewska, 2007).

\section{Eye Diseases}

Contrary to ancient phytotherapy, celandine was rarely used to treat eye conditions in folk medicine. Wdowiak (2015) reports that in the Podolia Region (Ukraine) drops of celandine juice mixed with vodka were put into eyes. In west Ukraine a tincture made from celandine was applied. Moreover, a popular belief among people of the Podolia Region, as well as the Lubelszczyzna and Podkarpacie Regions (Eastern Poland) said that feces of a swallow can cause sight loss, if they fall into the eye, which can be only cured by C. majus. In the small town Giby, of PolishLithuanian-Belarusian borderland, the celandine pollen was used against eye infections (Kujawska et al., 2017). 


\section{Other Usage}

People of S-W Romania and Zagori in Greece were applying celandine as diuretic (Vokou et al., 1993; Tița et al., 2009). In the central Serbia and in Podolia (Ukraine), celandine was considered a remedy for gout. Occasionally, it was used as tonic and stimulant of cardiac functions, also increasing blood pressure (Vokou et al., 1993). In the Bieszczady Mountains people incensed aching teeth with the smoke from the burning herb (Szary, 2015). In Poland, juice was used internally to cure hydropsy (Kujawska et al., 2016). In Romania it was esteemed as an antidote for snake venom (Tiţa et al., 2009). People of Russian descent, called Russlanddeutschen, living in S-W Germany used celandine as depurative (Pieroni and Gray, 2008). Among the Hutsuls living on the Ukrainian side of Bukovina (SW Ukraine), tea from aerial parts of celandine was employed in the treatment of cancer (Sǒukand and Pieroni, 2016). In Bosnia and Herzegovina it was used to cure cancer of lungs (Redžič, 2007). In veterinary treatments, herb decoction was given to cows suffering from inflammation of the udder, in case of dermatologic conditions animals were rubbed with leaves. Furthermore, cows were given root to eat to cause vomit to relieve bloat. Besides, herb overcooked in milk was applied to ulcers (Kujawska et al., 2016). In the Bieszczady Mountains celandine was a symbol of purification of living world from threatening death, it was used as talismans to protect from demons (Szary, 2015). Dried herb was used to incense the interiors of huts to deter flies and mosquitos as well as during plague and other epidemics. Grains soaked in celandine juice were used as fish and bird poison (Szary, 2013).

\section{PHYTOCHEMISTRY}

For the therapeutic purposes, dried herb of C. majus is used (European Pharmacopeia). In some regions (Central and Eastern Europe) roots are also exploited. European Pharmacopeia calls for total alkaloid content as chelidonine [1], assayed spectrophotometrically with additional TLC screening and microscopic authentication.

\section{Alkaloids}

Pharmacologically relevant substances of C. majus are isoquinoline alkaloids (Figures 1-7, Table 1). These are the components of latex produced in all plant parts, but flowers. Latex is stored in special secretory cells called laticifers. Presence of articulate laticifers with yellowish content is also used as an authentication microscopic mark in powdered herb by pharmacopoeial monographs. The composition of latex is plant organ specific (Tomē and Colombo, 1995; Sowa et al., 2018). Generally, five groups of alkaloids were found in C. majus. These are the derivatives of phenanthridine (3,4-benzoisoquinoline), protoberberine, protopine [37], quinolizidine, aporphine (Kadan et al., 1990, 1992; Pavao and Pinto, 1995; Táborská et al., 1995; Petruczynik et al., 2002; Nečas et al., 2005; Sārközi et al., 2006; Zhou et al., 2012; Kędzia et al., 2013; Grosso et al., 2014; Poormazaheri et al., 2017). More than forty alkaloids of different types were isolated and identified from C. majus (Figures 1-7). Major phenanthridine derivatives that were found in aerial and underground parts are (+)-chelidonine [1], chelerythrine [9], (Kadan et al., 1990; Sārközi et al., 2006; Zhou et al., 2012).

Protoberberine derivatives that accumulate in higher amounts are coptisine [31], berberine [28], stylopine [33] (Slavik and Slavikowa, 1977; Fulde and Wichtl, 1994; Shafiee and Jafarabadi, 1998; Sārközi et al., 2006). Aporphine alkaloids like corydine [39] also appear (Slavik and Slavikowa, 1977; Shafiee and Jafarabadi, 1998; Kopytko et al., 2005). Two protopines were found in C. majus, allocryptopine [36] and protopine [37] (Fulde and Wichtl, 1994; Shafiee and Jafarabadi, 1998; Kopytko et al., 2005). Sparteine [38] is the only representative of quinolizidine alkaloids (Kopytko et al., 2005) but no other publications report its presence. Moreover, new unusual turkiyenine-type alkaloid named (-)-turkiyenine [42] was found in C. majus from Turkey (Kadan et al., 1990).

Alkaloid content in different plant organs was found to be unstable (Kustrak et al., 1982; Tomē and Colombo, 1995). Daily variations were probably due to the alkaloid degradation rather than translocation, because of similar time-course of the compounds accumulation in all plant parts. Significant increase of sanguinarine [12], chelerythrine [9], chelidonine [1], and coptisine [31] was observed during the day, with the highest content in the evening, whereupon the alkaloids diminished during the night (Tomē and Colombo, 1995). Day light seems to be the crucial factor influencing alkaloid biosynthesis in C. majus, especially in underground parts of the plants (Kustrak et al., 1982; Tomē and Colombo, 1995). Diurnal changes of alkaloid content seem to be less dependent on temperature, what was observed during winter time, when the alkaloid content was low and stable, due to the reduced metabolism and the senescence of the aerial parts. According to Tomē and Colombo (1995) total content of alkaloids in leaves was lower than in underground parts. In latex the content was 32 times higher than in leaves and 9 times higher than in roots. These results suggest that the amount of alkaloids in plant organs depend on the number of laticifers in which they are stored. Moreover, the number of laticifers is probably organ specific. Laticifers in C. majus are unbranched (without anastomoses), articulated with perforated transverse cells (Hagel et al., 2008). Articulated laticifers develop from multiple cells. The structures form longitudinal rows composed of series of superimposed cells with perforated end walls. The type of laticifers can differ even within the same plant family. In another Papaveraceae species-opium poppy, the perforation of lateral walls leads to the formation of anastomoses (connections) between adjacent laticifer elements, unlike that of Greater celandine (Hagel et al., 2008). From wounded laticifers, a matrix emerges with various organic substances suspended in it. This excretion is called latex and depending on the plant species, it contains proteins, organic acids, alkaloids, sterols, tannins, and mucilage. The growth and development of laticifers runs close to the surrounding phloem, which affects the composition of latex. A major site of alkaloid accumulation in the protoplast of laticifer cells are vesicular organelles, that had been found in opium poppy between early 70's and 80's of the last century (Dickenson and Fairbairn, 1975; Roberts et al., 1983). Unfortunately, research on the site of alkaloid biosynthesis in Greater celandine has 


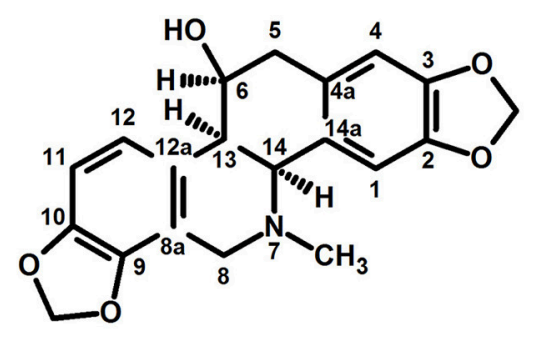

1. (+)-chelidonine<smiles>CN1Cc2c(ccc3c2OCO3)C2C(O)C(O)c3cc4c(cc3C21)OCO4</smiles>

3. 10-hydroxychelidonine<smiles>CN1Cc2cc3c(cc2C2C(O)Cc4cc5c(cc4[C@H]21)OCO5)OCO3</smiles>

5. isochelidonine

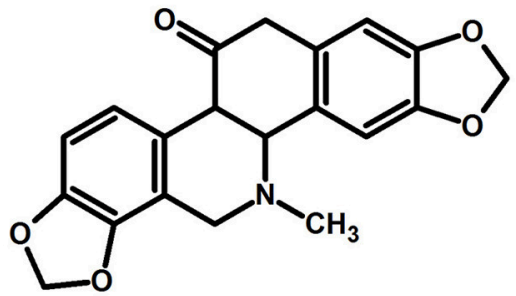

7. oxychelidonine<smiles>CN1Cc2c(ccc(C=O)c2C=O)[C@@H]2[C@@H](O)Cc3cc4c(cc3[C@H]21)OCO4</smiles>

2. alfa (+)-homochelidonine<smiles></smiles>

4. 10-hydroxy-homochelidonine<smiles>O[C@H]1Cc2cc3c(cc2[C@H]2NCc4c(ccc5c4OCO5)[C@@H]12)OCO3</smiles>

6. norchelidonine

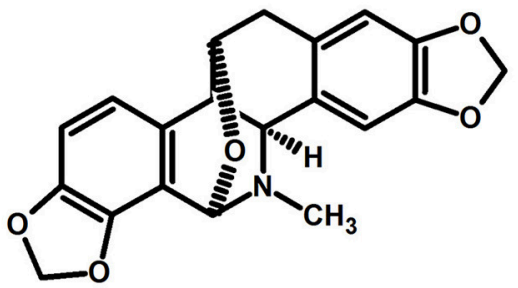

8. dihydrochelidonine

FIGURE 1 | Structures of phenanthridine (3,4-benzoisoquinoline) alkaloids -chelidonine [1] derivatives.

not been continued since then. Recent reports concern protein determination in latex and confirm its complex composition (for more information see the separate "Protein subsection" underneath).

The presence and number of laticifers is due to the physiological functions of aerial parts and underground parts of the plant, such as responses to environmental factors, defense against herbivory, metabolic reserves, and energy store (Agrawal and Konno, 2009). Coptisine [31] was found mostly in fruits and herb (Sārközi et al., 2006; Kędzia et al., 2013; Sowa et al., 2018), whereas berberine [28] showed no significant difference between aerial parts and roots (Tomē and Colombo, 1995). Larger amounts of phenanthridine alkaloids like chelidonine [1], chelerythrine [9], sanguinarine [12] were observed in roots rather 


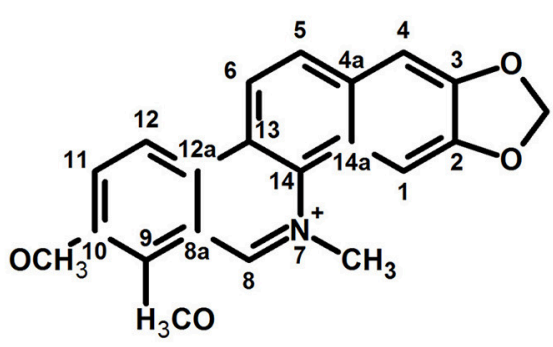<smiles>COc1ccc2c(c1OC)CN(C)c1c-2ccc2cc3c(cc12)OCO3</smiles>

10. dihydrochelerythrine

9. chelerythrine<smiles>COc1c(C)ccc2c1C(O)N(C)c1c-2ccc2cc3c(cc12)OCO3</smiles><smiles>C[n+]1cc2c3c(ccc2c2ccc4cc5c(cc4c21)OCO5)OCO3</smiles>

12. sanguinarine (y-chelerythrine)

10a. 8-hydroxydihydrochelerythrine<smiles>COc1ccc2c(cnc3c4cc5c(cc4ccc23)OCO5)c1C</smiles><smiles>CN1Cc2c(ccc3c2OCO3)-c2ccc3cc4c(cc3c21)OCO4</smiles>

14. dihydrosanguinarine

11. norchelerythrine<smiles>Cn1c(=O)c2c3c(ccc2c2ccc4cc5c(cc4c21)OCO5)OCO3</smiles><smiles>CN1c2c(ccc3cc4c(cc23)OCO4)-c2ccc3c(c2C1O)OCO3</smiles>

14a. 8-hydroxydihydrosanguinarine

13. oxysanguinarine<smiles>O=c1[nH]c2c(c3ccc4c(c13)OCO4)CCc1cc3c(cc1-2)OCO3</smiles><smiles>c1cc2c(ccc3c4cc5c(cc4ccc23)OCO5)c2c1OCO2</smiles>

15. N-demethyl-9,10-dihydrooxysanguinarine

than in aerial parts of the plants (Tomē and Colombo, 1995; Sowa et al., 2018).

Total alkaloid content (\%) in in vitro shoots and embryos expressed as chelidonine [1] was 1.53 and $1.58 \%$, respectively (Cirić et al., 2008).

\section{Phenolic Compounds}

Several flavonoids were found in aerial parts of C. majus in low amounts. Four diglycosides and five monoglycosides were identified as derivatives of kaempferol, quercetin, and isorhamnetin (kaempferol-3-O-rutinoside, 


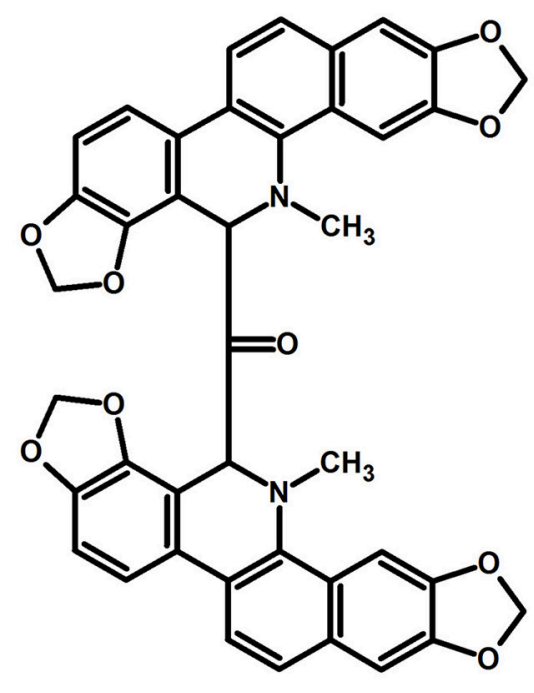

17. chelidimerine<smiles>COc1cc2c(cc1OC)-c1ccc3cc4c(cc3c1N(C)C2)OCO4</smiles>

19. dihydronitidine<smiles>COc1cc2c(=O)n(C)c3cc4c(cc3c2cc1OC)OCO4</smiles>

21. oxynitidine<smiles>CN1Cc2c(ccc3c2OCO3)C2C(O)=C(O)c3cc4c(cc3C21)OCO4</smiles>

18. chelamine

20. nitidine<smiles></smiles>

22. chelamidine

FIGURE 3 | Further structures of minor phenanthridine (3,4-benzoisoquinoline) alkaloids.

quercetin-3-O-rutinoside, isorhamnetin-3-O-glucoside). The identification was based on the mass spectra of the compounds (Grosso et al., 2014). In stems, leaves, and flowers, 5 '-methoxyflavonol [43] and 6'- methoxy-flavonol [44] were also detected (Stancic-Rotaru et al., 2003).

Hydroxycinnamic acids (caffeic, p-coumaric, ferulic) and their derivatives ((-)-2-(E)-caffeoyl- $\mathrm{D}$-glyceric acid [46], (-)-4(E)-caffeoyl-L-threonic acid [47], (-)-2-(E)-caffeoyl- ${ }_{-}$-threonic acid lactone, (+)-(E)-caffeoyl- ${ }_{-}-$malic acid [48]), as well as hydroxybenzoic acids (genistic, p-hydroxybenzoic) were identified in aerial parts (Hahn and Nahrstedt, 1993; Wojdyło et al., 2007). Later, another three hydroxycinnamic acids were identified using HPLC-DAD-ESI/MS: caffeoyl threonic acid, caffeoyl glyceric acid, caffeoylmalic acid (Grosso et al., 2014), that have been detected previously by Hahn and Nahrstedt (1993). Two caffeoyl acid derivatives isomers with precursor ions at $\mathrm{m} / \mathrm{s}$ 359 corresponding to rosmarinic acid were also found in aerial parts (Grosso et al., 2014). 


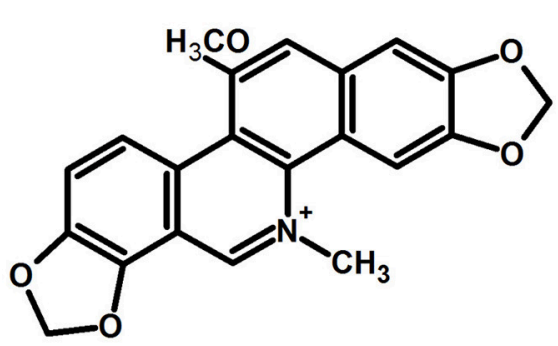

23. chelerubine<smiles>COc1ccc2c(c1O)C=[N+](C)C1c3cc4c(cc3C=C(C)C21)OCO4</smiles>

25. chelelutine<smiles>COc1ccc2c(c1OC)C(OC)N(C)c1c-2ccc2cc3c(cc12)OCO3</smiles><smiles></smiles>

24. dihydrochelerubine<smiles>COc1cc2cc3c(cc2c2c1CN(C)c1c-2ccc(C)c1OC)OCO3</smiles>

26. dihydrochelelutine

\section{7. angoline}

FIGURE 4 | Further structures of minor phenanthridine (3,4-benzoisoquinoline) alkaloids.

\section{Proteins}

A phytocystatin-chelidocystatin was one of the first proteins isolated from latex and characterized (Rogelja et al., 1998). Cystatins, a class of thiol protease inhibitors are involved in defense and stress-response mechanisms and could also contribute to the antimicrobial and antiviral activity of $C$. majus latex (Benchabane et al., 2010). Whether or not the presence of cystatin is relevant to the medicinal properties and such traditional folk uses as anti-warts is yet to be found out.

In a series of papers, Nawrot et al. (2007a,b, 2008, 2013, 2014, 2016, 2017a,b) described a number of proteins from root and leaf latex. Proteomic analysis using LC-ESI-MS/MS system revealed the presence of three categories of proteins according to their functions: proteins involved in disease and defenses responses (i.e., superoxide dismutases, lactoylglutathione lyases), nucleic acid binding proteins (i.e., glycine-rich proteins, nucleic acid binding, DNA-binding, or RNA-binding proteins), and these that are involved in general metabolism (acyl-CoA binding protein, malate dehydrogenase, flavodoxin-like quinone reductase, ubiquitin, polyubiquitin, serine/threonine protein kinases, rubber elongation factor). A total of 21 proteins were identified in C. majus latex, although in several cases the identification was based on correlation between experimental and the theoretical $\mathrm{p} I /$ molecular mass, due to their low score results. The results shown less complexity of latex proteins in this species compared to opium poppy. Their contribution to the 


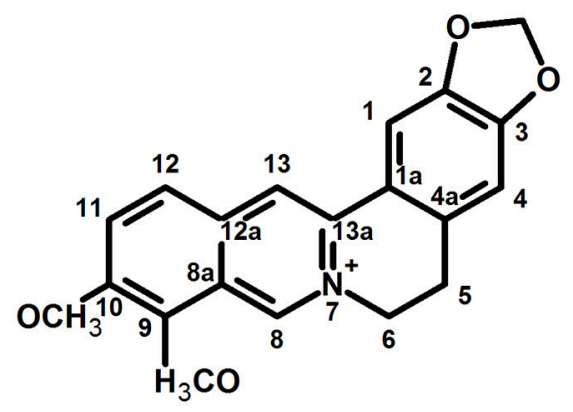<smiles>COc1ccc2c(c1C(C)=O)CN1CCc3cc4c(cc3C1=C2)OCO4</smiles>

28. berberine<smiles>COC(=O)O[Na]</smiles>

29. dihydroberberine

30. tetrahydroberberine<smiles></smiles>

31. coptisine<smiles>c1cc2c(c3c1CC1c4cc5c(cc4CCN1C3)OCO5)OCO2</smiles>

32. dihydrocoptisine

33. stylopine<smiles>Cc1c2[n+](cc3c4c(ccc13)OCO4)CCc1cc3c(cc1-2)OCO3</smiles>

34. corysamine<smiles>COc1ccc2c(c1OC)CN1CCc3cc4c(cc3C1C2)OCO4</smiles>

35. canadine

FIGURE 5 | Structures of protoberberine alkaloids

traditional use of C. majus as antiviral and antimicrobial remedy has to be further explored and may, in combination with highly active alkaloids, render unique synergistic effects providing a multifaceted tool for combat against troublesome infections. In living plants, these proteins are probably also serving as a chemical defense against pathogens (Nawrot et al., 2007a,b, 


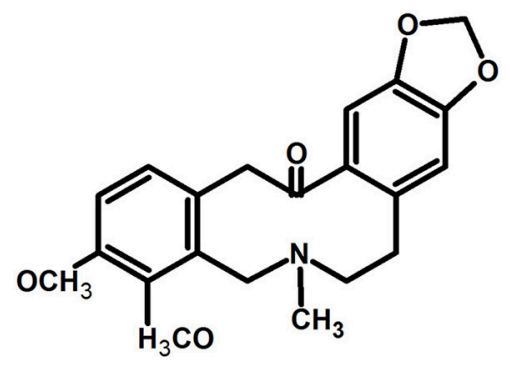

36. allocryptopine

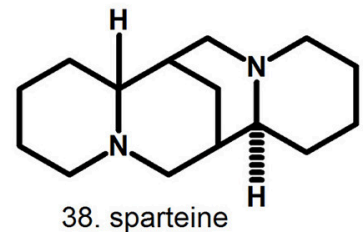<smiles>CN1CCc2cc(C=O)c(O)c3c2[C@H]1Cc1ccc(C=O)c(C=O)c1-3</smiles>

39. corydine<smiles>C[C@]12Cc3ccc(C=O)c(O)c3-c3c(O)c(C=O)cc(c31)CC[N+]2(C)C</smiles>

41. magnoflorine<smiles>CN1CCc2cc3c(cc2C(=O)Cc2ccc4c(c2C1)OCO4)OCO3</smiles>

37. protopine<smiles>CC(=O)c1cc2c3c(c1O)-c1c(ccc(C=O)c1C=O)C[C@H]3NCC2</smiles>

40. norcorydine

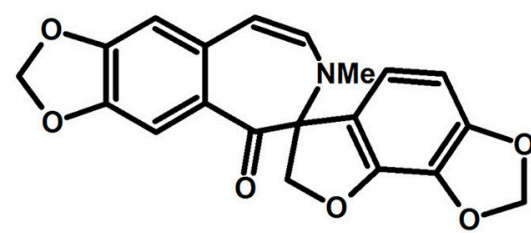

42. (-)-turkiyenine

FIGURE 6 | Structures of aporphine, protopine alkaloids, and non-isoquinoline alkaloids.

2014, 2017a,b). Protein-bound polysaccharide (CM-AIa) bearing immunomodulatory and cytotoxic activity was isolated by Song et al. (2002).

\section{Other Compounds}

Organic acids: chelidonic [45], malic, citric, succinic, (Kopytko et al., 2005); Biogenic amines: histamine, methyloamine, tyramine (Kwasniewski, 1958); choline in fruits (Kwasniewski,
1958); essential oil constituents (Hansel et al., 1992; Kohlmünzer, 2000) triterpenoids (Hahn and Nahrstedt, 1993; Deng et al., 2016); saponins (Kwasniewski, 1958; Kopytko et al., 2005); Resin (Hahn and Nahrstedt, 1993); vitamins A, C, nicotinic acid (Hahn and Nahrstedt, 1993; Kopytko et al., 2005).

Flowers contain xanthophyll pigments like lutein, violaxanthin, flavoxanthin, chrysanthemoxanthin (Horváth et al., 2010). 


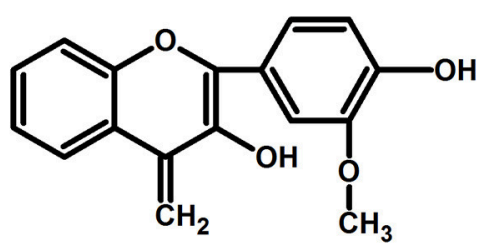

43. 5'-methoxy-flavonol<smiles>O=C(O)c1cc(=O)cc(C(=O)O)o1</smiles>

45. chelidonic acid

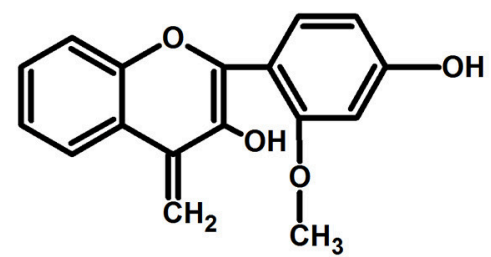

44. 6'-methoxy-flavonol

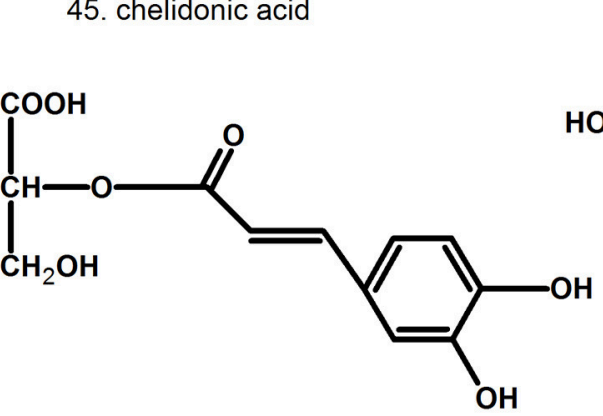<smiles>O=C(/C=C/c1ccc(O)c(O)c1)OCC(O)C(O)C(=O)O</smiles>

46. (-)-2-(E)-caffeoyl-D-glyceric acid

47. (-)-4-(E)-caffeoyl-L-threonic acid

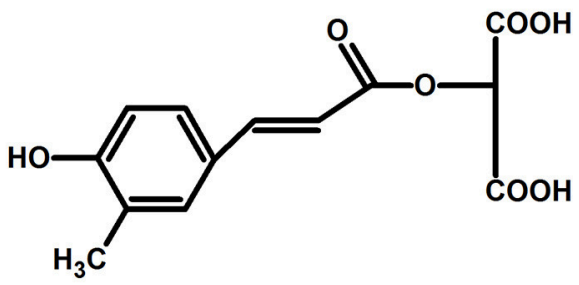

48. (+)-( E)-caffeoyl-L-malic acid

FIGURE 7 | Structures of miscellaneous compounds.

\section{METHODS FOR ANALYSIS OF ACTIVE COMPONENTS FROM Chelidonium majus}

\section{Alkaloids}

First isolation of Chelidonium alkaloids was achieved in nineteenth century with an important contribution from $\mathrm{Mr}$. Emanuel Merck's company at Darmstadt (Henschke, 1888; Schmidt, 1888) and obtaining and characterization of pure compounds (chelidonine [1], chelerythrine [9], protopine [37]) was successful in the following years (Selle, 1890; Wintgen and
Schmidt, 1901). Alkaloids of C. majus occur as salts or bases depending on $\mathrm{pH}$ of medium; thus, their extraction was mostly carried out in acidic condition to convert all compounds to water-soluble salts. Methanol or ethanol often with addition of water (Bugatti et al., 1987; Han et al., 1991; Koriem et al., 2013) and hydrochloric (Kursinszki et al., 2006; Gu et al., 2010) or acetic acid (Paulsen et al., 2015) were used as extractants. The isolation from plant material was also achieved with the use of pure acidified water; further, the solution was alkalized with ammonia or sodium hydroxide to obtain base forms 
TABLE 1 | Content [\%] of pharmacologically relevant constituents in aerial parts and roots of Chelidonium majus.

\begin{tabular}{|c|c|c|c|}
\hline \multirow[t]{2}{*}{ Compound } & \multicolumn{2}{|c|}{ Content [\% of dry mass] } & \multirow[t]{2}{*}{ References } \\
\hline & Aerial parts & Roots & \\
\hline \multirow[t]{2}{*}{ Chelidonine } & & & $\begin{array}{l}\text { Bugatti et al., 1987; Fulde and Wichtl, 1994; Niu and He, 1994; Kedzia et al., 2003; Sārközi et al., } \\
\text { 2006; Gañán et al., 2016; Sowa et al., } 2018\end{array}$ \\
\hline & $t-0.3$ & $t-1.51$ & \\
\hline Chelerythrine & $t-0.3$ & $t-0.77$ & $\begin{array}{l}\text { Bugatti et al., 1987; Fulde and Wichtl, 1994; Niu and He, 1994; Shafiee and Jafarabadi, 1998; } \\
\text { Sārközi et al., 2006; Gañán et al., 2016; Sowa et al., } 2018\end{array}$ \\
\hline Sanguinarine & $t-0.1$ & $0.1-0.4$ & $\begin{array}{l}\text { Bugatti et al., 1987; Fulde and Wichtl, 1994; Kedzia et al., 2003; Sārközi et al., 2006; Sowa et al., } \\
2018\end{array}$ \\
\hline Berberine & $t-0.1$ & $\mathrm{t}-0.1$ & $\begin{array}{l}\text { Bugatti et al., 1987; Fulde and Wichtl, 1994; Kedzia et al., 2003; Sārközi et al., 2006; Sowa et al., } \\
2018\end{array}$ \\
\hline Coptisine & $t-1.0$ & $\mathrm{t}-0.3$ & Fulde and Wichtl, 1994; Kedzia et al., 2003; Sārközi et al., 2006; Sowa et al., 2018 \\
\hline $\begin{array}{l}\text { Flavonols (quercetin, } \\
\text { kaempferol and } \\
\text { isorhamnetin } \\
\text { glycosides) }\end{array}$ & 0.8 & & Grosso et al., 2014 \\
\hline hydroxycinnamic acids & 0.03 & & Grosso et al., 2014 \\
\hline xanthophylls & 0.03; 1.36 (flowers) & & Horváth et al., 2010 \\
\hline
\end{tabular}

$t$, traces $(<0.08 \%)$.

followed by liquid-liquid extraction with organic solvents such as dichloromethane, butanol or chloroform (Sārközi et al., 2006; Sárközi et al., 2007; Migas et al., 2012; Jesionek et al., 2016; Bogucka-Kocka and Zalewski, 2017). The isolation was conducted by percolation (Capistrano et al., 2015), maceration (Koriem et al., 2013; Borghini et al., 2015), heating under reflux (Gu et al., 2010; Yao et al., 2011; Seidler-Łozykowska et al., 2016), in water bath (Sārközi et al., 2006) or Soxhlet apparatus (Bugatti et al., 1987; Stuppner and Ganzera, 1995) as well as ultrasound assisted (UAE) (Kursinszki et al., 2006; Sárközi et al., 2007; Paulsen et al., 2015; Jesionek et al., 2016) or microwave energy (MAE) (Then et al., 2000; Zhou et al., 2012). Supercritical fluid extraction (SFE) (Then et al., 2000) or SFE combined with enhanced solvent extraction (ESE) and low pressure solvent extraction with water (LPSE) (Gañán et al., 2016) was also applied. Moreover, in situ solvent formation microextraction (ISFME) with the use of ion-pairing agent (KPF6) and a water-miscible ionic liquid ([C6MIM][Br]) which formed a hydrophobic ionic liquid extraction phase ([C6MIM]PF6) for the pre-concentration of sanguinarine [12] and chelerythrine [9] was elaborated by $\mathrm{Wu}$ and $\mathrm{Du}$ (2012). After extraction, the solution was usually filtrated through a $0.22-\mu \mathrm{m}$ membrane ( $\mathrm{Gu}$ et al., 2010) or additionally purified using solid phase extraction (SPE) on C18 cartridge (Stuppner and Ganzera, 1995; Kursinszki et al., 2006; Sārközi et al., 2006). Sārközi et al. (2006) developed ion-pair SPE with n-heptanesulfonic acid (HS). Dried residue obtained after extraction was dissolved in methanol with $0.05 \mathrm{M}$ hydrochloric acid, diluted with $0.05 \mathrm{M}$ aqueous solution of HS and loaded on an SPE C18 microcolumn. Further, 70\% HS $(0.05 \mathrm{M})$ in methanol was used to remove the matrix and $5 \%$ HS $(0.05 \mathrm{M})$ in methanol to elute analytes. The examples of conditions used to isolate alkaloids from C. majus are presented in Supplementary Table 1.

\section{Thin Layer Chromatography (TLC)}

TLC was mostly employed for screening purposes or for qualitative analysis of alkaloid composition in C. majus extracts. This technique has been largely limited to the screening and multiple sample fingerprinting, but usually does not enable highsensitivity or high-resolution insight into the minor components of the phytochemical profile. Despite of being less intensively modernized in comparison to column-based techniques like (U)HPLC, this method is still favored when cost-effectiveness and simplicity of sample preparation is important, for example in herbal industries and educational use. Some recent developments in mass spectrometry hyphenation in form of matrix transfer or DART (direct analysis in real time) used already in studying of other species (Móricz et al., 2018) should be useful also for C. majus alkaloids.

Silica was the most common stationary phase, often impregnated with salts (Ni, $\mathrm{Zn}, \mathrm{Cr}, \mathrm{Co})$ to improve the selectivity (Wagner et al., 1984; Then et al., 2000; Waksmundzka-Hajnos et al., 2000; Sārközi et al., 2006; Sárközi et al., 2007; Petruczynik et al., 2008; Jesionek et al., 2016) Silica modified with octadecyl (C-18) and cyanopropyl (CN) groups and aqueous solutions of methanol or isopropanol with ammonia or diethylamine which prevented tailing of chromatographic bands were sporadically applied (Petruczynik et al., 2007). Petruczynik et al. (2008) combined various types of stationary phases e.g., cyanopropyl silica with silica or octadecyl silica to obtain adsorbent gradient.

Apart from the routine isocratic elution with solvent mixtures, using different modes of gradient elution improved the separation, e.g., as a two-dimensional or unidimensional multiple development (Szumiło and Flieger, 1999; Waksmundzka-Hajnos et al., 2000; Migas et al., 2012), bivariant multiple development (Bogucka-Kocka and Zalewski, 2017), or stepwise gradient elution (Matysik and Jusiak, 1990; Waksmundzka-Hajnos et al., 
2000; Bogucka-Kocka and Zalewski, 2017). TLC separation of alkaloids was supported by use of magnetic field (Malinowska et al., 2017) or forced flow of mobile phase (OPLCoverpressure layer chromatography or optimum performance laminar chromatography; Pothier et al., 1993; Malinowska et al., 2005).

TLC was also applied for direct bioautography (TLC-DB) to test the antibacterial activity of $C$. majus extracts (Sárközi et al., 2007; Móricz et al., 2015; Jesionek et al., 2016) combined with densitometry for quantitative analysis (Then et al., 2000; Sārközi et al., 2006) and it proved useful as preparative technique for isolation of alkaloids (Waksmundzka-Hajnos et al., 2002; Koriem et al., 2013).

\section{High Performance Liquid Chromatography (HPLC)}

High performance liquid chromatography (HPLC) and ultrafast liquid chromatography (UFLC) have been the most often applied analytical techniques. For many years, separation of C. majus extracts was mostly carried out in reversed phase (RP) system using long (150 or $250 \mathrm{~mm}$ ) C18 columns with $4.6 \mathrm{~mm}$ of diameter and $5 \mu \mathrm{m}$ of particle size (Niu and He, 1994; Petruczynik et al., 2002; Kursinszki et al., 2006; Borghini et al., 2015; Paulsen et al., 2015; Gañán et al., 2016). More recently, adsorbents with smaller particles $(\leq 3 \mu \mathrm{m})$ or shorter columns (Prosen and Pendry, 2016; Seidler-Łozykowska et al., 2016) were also applied to achieve shorter separation and saving solvents. Mobile phases are usually composed of water and acetonitrile or/and methanol with various additives e.g., ammonium formate/acetate (Borghini et al., 2015; Seidler-Łozykowska et al., 2016), organic amines (triethyl-, tetrabutylamine) (Paulsen et al., 2015), ion-pair reagents (sodiumdodecylsulfate, alkylsulfonic acids) (Gañán et al., 2016). The additives were necessary to reduce peak tailing forming as a results of interaction of alkaloid cationic forms with residual silanol group of stationary phase. Eluents were acidified with acetic or formic acid to $\mathrm{pH}<4$ to avoid the co-occurrence of ionic and uncharged forms.

Normal-phase (NP) chromatography set ups were used rather seldom in analysis of alkaloids. Here, silica columns was eluted with sodium acetate in methanol, dioxane and acetic acid mixture (Bugatti et al., 1987) or chloroform and methanol with trifluoroacetic acid (Rey et al., 1993) Also, cyanopropyl stationary phase was eluted with acetonitrile, tetrahydrofuran, dioxane or methanol with phosphate buffer and octane-1-sulfonic acid sodium salt or di-(2-ethyl hexyl) orthophosphoric acid (HDEHP) (Petruczynik et al., 2002).

Alkaloids have a strong absorption in UV region and have ability to fluorescence; thus both spectrophotometric and fluorescence detector ( $\mathrm{Wu}$ and $\mathrm{Du}, 2012)$ were applied. Additionally, ESI-MS and/or NMR were coupled with HPLC to confirm the identity and structure elucidation of new alkaloids (Paulsen et al., 2015). Moreover, preparative separation of particular alkaloids from C. majus extract using silica gel and sequentially elution with petroleum ether, ethyl acetate and methanol (Yao et al., 2011) or $\mathrm{CaCO}_{3}$ and different compositions of toluene-hexane and acetone-hexane mixtures (Horváth et al., 2010) was performed with use of LC system.

\section{Capillary Electrophoresis CE}

$\mathrm{CE}$ method with spectrophotometric (DAD) and fluorescence detection (UV-LEDIF ultraviolet light-emitting diode-induced native fluorescence or LED-fluorescence) was also used for C. majus analysis (Stuppner and Ganzera, 1995; Ševčik et al., 2000; Kulp et al., 2011; Zhou et al., 2012; Kulp and Bragina, 2013); however, this technique has not yet won high popularity and it is also limited to charged or polar compounds. Recently, Sun et al. (2015) elaborated the microchip variant of CE with laser-induced fluorescence detection and $50 \%$ formamide as a run buffer and this technique was applied for separation of chelerythrine [9] and sanguinarine [12]. The examples of application the separation techniques in analysis of alkaloids from C. majus are presented in Supplementary Table 2.

\section{Spectrophotometry}

Spectrophotometric method given in European Pharmacopeia (monograph of Greater celandine) may be useful to estimate of total alkaloids in C. majus extract. The sample is mixed with sulphuric and chromotropic acid, and heated $10 \mathrm{~min}$. at $100^{\circ} \mathrm{C}$ in water-bath. After cooling to $20^{\circ} \mathrm{C}$, the absorption of sample is measured at $570 \mathrm{~nm}$ and the amount of alkaloid is expressed as chelidonine [1]. This approach was applied by (Then et al., 2000; Seidler-Łozykowska et al., 2016). However, for research purposes, a total-content approach should be discouraged as too inaccurate and sometimes misleading about contribution of each component of an actual alkaloid profile.

In summary, most of the published preparative and analytical approaches were rather routine and typical for phytochemical studies. However, the efficiency of standard methods often appeared to be insufficient. The properties that influenced the separation and analysis processes depend mostly on the tertiary or quaternary character, oxygenation and secondary cyclization pattern. Among the most important features are thermo- and photo-sensitivity, so the procedures should be carried out in mild conditions which has not always been considered. Thus, due to the diversity in physicochemical properties, even among compounds from a single subclass, various specialized modifications were applied which in most cases significantly improved the separation.

Another issue that should be addressed in future analytical studies is that numerous minor alkaloids have been frequently missed or overlooked whereas some of them have profound activity. The focus should be on comprehensive qualitative and quantitative profiling under non-destructive conditions that would reveal the full phytochemical complexity and allow to understand the intraspecific and environmental variation of C. majus.

\section{Other Components}

\section{Carotenoids}

Carotenoids were determined in aerial parts (Varzaru et al., 2015) and flowers (Horváth et al., 2010) of greater celandine. The components were hydrolyzed with alcoholic solution of 
potassium hydroxide and extracts were analyzed on RP-HPLC using C18 column in isocratic mode using $13 \%$ of water in acetone (Varzaru et al., 2015) or in gradient mode with mixture of three eluents: $12 \%(\mathrm{v} / \mathrm{v})$ water in methanol (A), methanol (B), and $50 \%(\mathrm{v} / \mathrm{v})$ acetone in methanol $(\mathrm{C})$ in different proportions (Horváth et al., 2010).

\section{Phenolic Compounds}

Grosso et al. (2014) tested different combinations: time, temperature type of extraction, and eluent composition and used Box-Behnken design to establish the effective extraction conditions for phenolic compounds. $76.8 \%$ of methanol, $150.0 \mathrm{~min}$ and $60^{\circ} \mathrm{C}$ were found to be optimal. Further, hydroxycinnamic acids and flavonoids were characterized by HPLC-DAD-ESI/MS ${ }^{n}(\mathrm{C} 18$ column: $150 \times 1.0 \mathrm{~mm}, 3 \mu \mathrm{m})$ and quantified using RP-HPLC DAD (C18 column: $250 \times 4.6 \mathrm{~mm}$, $5 \mu \mathrm{m})$. For both methods, gradient elution with mobile phase composed of $1 \%$ acetic acid in water and methanol was applied (Grosso et al., 2014). Hence, the method for phenolic analysis is similar to some which were used for alkaloids and there would be reasonable to include phenolic compounds in any future HPLC or LC-MS profiling of this herb.

\section{Proteins}

The composition of proteins in C. majus milky sap was studied by Nawrot et al. (2007a,b, 2014, 2017a,b). The compounds were pre-separated using two-dimensional gel electrophoresis and further, HPLC on BEH C18 column $(100 \mathrm{~mm} \times 100 \mu \mathrm{m}, 1.7 \mu \mathrm{m}$ particle diameter) (Nawrot et al., 2017b) or nano-HPLC on $75 \mu \mathrm{m}$ analytical column (Nawrot et al., 2007a, 2014, 2017a) and gradient elution with mobile phase containing $0.1 \%(\mathrm{v} / \mathrm{v})$ formic acid in water and in acetonitrile were performed. Proteins were identified by tandem mass spectrometry (MS/MS).

\section{Micro and Macro-elements}

Mineral composition of greater celandine herb and root, aqueous solutions (infusion, decoction) and alcoholic extracts were determined with the use of inductively coupled plasma atomic emission spectrometry (Then et al., 2000; Sárközi et al., 2005).

\section{PHARMACOLOGICAL ACTIVITIES AND CLINICAL EVIDENCE}

Obviously enough, the great majority of published pharmacological properties of $C$. majus pertains to the complex mixture or individual alkaloids. Recently, more and more attention has been also paid to proteins from latex, that could significantly contribute to the observed activities. Other compounds, such as various phenolics and chelidonic acid were rarely considered. The versatile pharmacological activities of coptisine [31], one of the major constituents of $C$. majus as well as the less abundant berberine [28], have been widely described in the literature but usually as compounds obtained from other sources, such as Coptis (Ranunculaceae) (Tan et al., 2016) or Berberidaceae (Imanshahidi and Hosseinzadeh, 2008).

However, these compounds are also contributing significantly to many of the pharmacological properties of $C$. majus.
From the growing evidence obtained both in vitro and in vivo, with several examples of ex vivo studies on isolated organs, it is clear that four types of medicinal properties are predominating: antimicrobial and antiviral, hepatic and gastric, anti-inflammatory, and finally anticancer. Several other activites have been also reported but less extensively.

\section{Activites in Vitro and in Vivo Antibacterial and Antifungal}

The antimicrobial activity of C. majus is attributed mostly to the alkaloids and flavonoids (Zuo et al., 2008). This kind of activity was reported already in the early research on chelidonium alkaloids, e.g., by Stickl (1928) who proved the bactericidal properties against Gram-positive strains (Staphylococcus aureus and Bacillus anthracis) with chelerythrine [9] and sanguinarine [12] being more potent than chelidonine [1] and berberine [28].

In experiments with multidrug resistant bacteria existing in surgical wounds and infections of critically ill patients, C. majus ethanol extract affected Gram-positive bacteria. Ethanolic extracts of C. majus also showed antimicrobial activity against Bacillus cereus, E. coli, Pseudomonas aeruginosa, S. aureus, (Kokoska et al., 2002). The complex composition of alkaloids can manifest wide spectrum of antimicrobial activity, arising from different chemical structures of the compounds. Hence, antimicrobial activity of $C$. majus was also tested with a use of different solvent extraction. Antibacterial and antifungal tests were performed using $96 \%$ methanol extracts from leaves and petioles of plants grown in nature as well as in vitro shoots and embryos (Cirić et al., 2008). Methanolic extracts were examined against Gram-positive (Bacillus subtilis, Micrococcus luteus, Sarcinia lutea, and S. aureus), Gram-negative bacteria (E. coli, Proteus mirabilis, Salmonella enteritidis), plant pathogensAgrobacterium rhizogenes, A. tumefaciens), and clinically isolated C. albicans. Both, in vivo and in vitro derived plant material extracts showed similar bioactivity, with a slight advantage of in vitro shoots. Only few extracts were equally active $(80 \mathrm{mg} / \mathrm{ml})$ against E. coli, S. enteritidis, and C. albicans, when compared to the commercially available antibacterial and antifungal drugs (streptomycin, bifonazole, respectively), while the rest of them showed low or no activity (Cirić et al., 2008).

Apart from plant extracts, as it was previously summarized by Kedzia and Hołderna-Kedzia (2013), individually tested compounds showed different antimicrobial activity. Chelerythrine [9] and sanguinarine [12] were significantly more potent than chelidonine [1] against Gram-positive (S. aureus, $S$. epidermidis, B. subtilis, B. anthracis), Gram-negative ( $P$. aeruginosa, E. coli, Klebsiella pneumoniae, Salmonella gallinarum, S. typhi, S. paratyphi, Proteus vulgaris, Shigella flexneri), and acidfast mycobacteria (Mycobacterium tuberculosis, M. smegmatis). Moreover, chelerythrine [9] exhibited antimicrobial activity against $S$. aureus and $M$. smegmatis, while chelerythrine [9] derivatives: 8-hydroxydihydrosanguinarine [14a], 8hydroxydihydrochelerythrine [10a], dihydrosanguinarine [10], and dihydrochelerythrine [14] were active against methicillin-resistant $S$. aureus $\left(\mathrm{MIC}_{50}=0.49,0.98,23.4\right.$, $46.9 \mu \mathrm{g} / \mathrm{ml}$, respectively). The strongest activity was presented by 8 -hydroxydihydrosanguinarine at $\mathrm{MIC}_{90}=1.95 \mu \mathrm{g} / \mathrm{ml}$, 
comparable to that of vancomycin $(2.23 \mu \mathrm{g} / \mathrm{ml})$ (Zuo et al., 2008). In the ethanol extract of $C$. majus aerial parts there are several other alkaloids that show potent antimicrobial inhibitory effect. For instance, berberine [28] was effective against Gramnegative bacteria-Vibrio cholerae and E. coli. It damaged bacterial fimbria, thereby inhibited adhesion to the mucosal surface (Imanshahidi and Hosseinzadeh, 2008; Wongbutdee, 2009).

Enzymes included in latex, like extracellular peroxidases, DNases, and lectin-like-active glycoproteins can also exhibit antimicrobial activity. Moreover, chelidocystatines protect plant against pests and are among the components of latex which presumably contribute to removal of warts resulting from human papilloma virus infection (Rogelja et al., 1998; Song et al., 2002; Gerenčer et al., 2006; Nawrot et al., 2007b; Cirić et al., 2008).

The antifungal activity was tested with the use of plant latex, and different solvent extracts from aerial and underground parts like ethanol/methanol ether, chloroform, acetone, and water (Kokoska et al., 2002; Kedzia et al., 2003). Chelerythrine [9], sanguinarine [12], chelidonine [1], and their derivatives were also individually tested against large number of human and plant pathogenic fungi, i.e., Aspergillus fumigatus, A. niger, Candida sp. Cladosporium herbarum, Cryptococcus neoformans, Epidermophyton floccosum, Fusarium sp. Keratinomyces ajelloi, Microsporum sp. Penicillium notatum, Rhodotorula rubra, Scopulariopsis brevicaulis, Torlopsis utilis, Trichophyton sp. The effect of $C$. majus extracts on pathogenic fungi was significantly weaker compared to the effect on pathogenic bacteria. For example, the MIC of ethanol and methanol extracts was ranging between 1.5 and $8 \mathrm{mg} / \mathrm{ml}$ against the most resistant bacteria, whereas the MIC of ethanol herb extract against the most pathogenic fungi was $20-40 \mathrm{mg} / \mathrm{ml}$ (Pepeljnjak et al., 2003). Chelerythrine [9], sanguinarine [12] and their derivatives (e.g., 8 -hydroxydihydrochelerythrine [10a]) were up to several times stronger than chelidonine [1] (Ma et al., 2000; Meng et al., 2009; Kedzia and Hołderna-Kedzia, 2013). Chelerythrine [9] was also able to inhibit spore germination of several plant pathogenic fungi: Sphaerulina juglandis, Septoria microspora, Fusarium oxysporum, and Curvularia lunata. It suggests the actual biological-antipathogenic function of isoquinoline alkaloids in the plant (Wei et al., 2017). However, no particular structural feature seems to be pinpointed as a determinant of antimicrobial potency despite some variation in activity toward different strains between individual alkaloids. It is rather the combination of them and other compounds targeting multiple sites of action that result in the observed final effect. This hypothesis still needs experimental verification for potential synergies or other interactions.

\section{Antiviral}

The glycosaminoglycan present in the latex inhibits intracellular human immunodeficiency virus HIV viral migration and blocks reverse transcriptase (Gerenčer et al., 2006). Moreover, individually tested five C. majus alkaloids: chelidonine [1], chelerythrine [9], sanguinarine [12], coptisine [31], and berberine [28] were able to inhibit the development of HIV1. The first two decreased the activity of the virus reverse transcriptase at the concentrations $150-200 \mu \mathrm{g} / \mathrm{ml}$, while sanguinarine [12], berberine [28], and coptisine [31] were already active at concentrations of $50-150 \mu \mathrm{g} / \mathrm{ml}$ (Tan et al., 1991).

The chloroform extract in the concentration of $35 \mu \mathrm{g} / \mathrm{ml}$ decreased the number of adenoviruses responsible for inducing acute fevelitis of the upper respiratory tract and conjunctiva in humans (Kéry et al., 1987). The experiments with animals showed that ethanol extract of $C$. majus inhibited encephalomyocarditis virus in $45 \%$ of experimental mice, whereas berberine [28] tested in the concentration range between 20 and $125 \mu \mathrm{g} / \mathrm{ml}$ inhibited influenza virus type A and $B$ in chicken embryos with 33-99.97\% efficiency (data previously reviewed by Kedzia et al., 2003). These results were presented only once, and from that time have never been confirmed or repeated.

\section{Antiprotozoal}

Herb and root water extracts, as well as sanguinarine [12] (2$4 \mu \mathrm{g} / \mathrm{ml}$ ) were highly effective in the treatment of trichomoniasis caused by Trichomonas vaginalis. After 8-10 days of treatment there was no protozoa detected in genitals of nearly $64 \%$ young girl patients. Sanguinarine [12] was also found to inhibit the development of Entamoeba histolytica, responsible for the hepatic abscess (Kozicka and Radomanski, 1963; Vychkanova et al., 1969).

\section{Liver and Biliary Tract}

One of the most widespread and repeatedly mentioned indications of C. majus, both in European/Mediterranean and East Asian (TCM) tradition, was for various liver complaints. It may date back to "signatura rerum" rule from coloration of the latex and flowers but obviously must have been verified by observations. Nowadays, even though this indication has been supported by just a few in vitro and in vivo studies, caution is necessary for alleged hepatotoxicity. Also, clinical evidence is not sufficient to ultimately recommend this herb and galenic preparations thereof (European Medicines Agency, 2011).

Some of the hepatoprotective and choleretic/cholagogue activity could be more aptly attributed to the presence of hydroxycinnamic (caffeic) acids esters which have been quite frequently overlooked in alkaloid-focused studies (Weiskirchen, 2016).

The question whether the supposed stimulation of bile flow is caused only by cholagogue activity or also by increasing bile production or excretion was first addressed by Rentz (1947). Comparison of guinea pigs and rats (that do not have gall bladder and did not respond to the treatment) reaction to C. majus tincture suggested only cholecystokinetic mechanism of action, attributed by the author to the stimulation of smooth musculature by berberine [28]. However, the tincture composition was unknown.

Vahlensieck et al. (1995) indicated using isolated rat livers that beside the earlier reports on cholecystokinetic action, also increase of bile production contributes to the final outcome. The activity was not very high, reaching $20 \%$ increase by perfusion with C. majus extract. The activity of alkaloid and polyphenol 
fractions separately were only about half of that. It suggests an additive action of the complex mixture of all active constituents.

The antispasmodic activity of $C$. majus extract was tested in trials based on acetylcholine ( $\mathrm{ACh}$ )-induced contraction in isolated rat ileal smooth muscle (Boegge et al., 1996). The extract was found to be moderate antagonist $\left(12.7 \% ; 2.0 \times 10^{-4} \mathrm{~g} / \mathrm{ml}\right.$ organ bath) against (ACh)-induced contraction compared to caffeoylmalic acid [48] $\left(6.9 \% ; 2.5 \times 10^{-5} \mathrm{~g} / \mathrm{ml}\right)$ and coptisine [31] $\left(16.5 \% ; 1.0 \times 10^{-5} \mathrm{~g} / \mathrm{ml}\right)$. Also, individual alkaloids, i.e., chelidonine [1], stylopine [33], and coptisine [31] have been tested for relaxant activity on ileum smooth muscles (Hiller et al., 1998). Among them, chelidonine [1] and stylopine [33] showed papaverine-like musculotropic action, whereas coptisine [31] was ineffective in $\mathrm{BaCl} 2$ stimulation model. In carbachol and electric field induced contractions, all three alkaloids and ethanolic extracts were effective.

In a different contraction model-isolated and perfused porcine uterus, the commercial alcoholic extract exhibited twophase response, initially stimulating very strong contractions followed by a longer relaxation period (Kuenzel et al., 2013). These properties were suggested as potentially useful in supporting artificial insemination or facilitating fertilization by acceleration of sperm movement toward fallopian tubes. However, this indication was rather unknown in traditional usage and would be a novel application of C. majus.

\section{Cytoprotective}

The macerated ethanol extract from juice expressed from pulped fresh plant material (according to the homeopathic recipes) was able to counteract carbon tetrachloride induced hepatotoxicity (Mitra et al., 1996). The effects of extract administration included reduced cell necrosis, absence of fibrosis, and lower lipid accumulation. Here again, no reliable data on the composition of the tested extract was available. C. majus primary tincture (German Homeopathic Pharmacopeia), diluted $100 \mathrm{x}$ and $1,000 \mathrm{x}$ was significantly effective against cadmiuminduced hepatoxicity in HepG2 and primary rat hepatocyte models (Gebhardt, 2009). The activity was stronger than the proprietary compound preparation and similar to the recognized hepatoprotective herb-Silybum marianum. The putative mechanisms of cytoprotective activity was associated to oxidative stress relieve as demonstrated by improvement of several parameters such as lipid peroxidation, intracellular Reactive Oxygen Species, reduced glutathione (GSH) level, as well as diminished apoptosis symptoms (nuclei fragmentation, cytochrome $\mathrm{C}$ release, caspase 3 activation). Hepatotoxicity caused by cadmium was also ameliorated in vivo in mice and ex vivo in hepatocyte cultures. In mice, administration of 50 or 75 $\mathrm{mg} / \mathrm{kg}$ body weight chelidonine [1], also in form of nanocapsules, improved histopathological picture of livers damaged by $\mathrm{Cd}$ treatment. Also, biochemical parameters such as ALT, AST, and ALP activities were lowered to the levels intermediate between control animals and Cd treated ones. Moreover, the expression of cell death related genes $\mathrm{Bax}$ and $\mathrm{Bcl}-2$ was modulated to the levels closer to the Cd untreated animals. In all tested hepatotoxicity parameters, nanoencapsulated chelidonine [1] was more efficient. It was corroborated with the ca. 1/3 higher distribution of chelidonine [1] from nanoformulation into the liver tissues. The putative mechanisms relate to alleviation of oxidative stress as revealed by improvement of antioxidant status (lower lipid peroxidation, higher GSH level, and SOD and CAT activities) and various cell death and inflammation markers (decreased protein level of TNF- $\alpha$, IL-6, NFKB, p65, cas-3, iNOS) (Paul et al., 2013).

Notwithstanding, the literature evidence supporting beneficial properties in hepatobiliary disorders from both in vitro, in vivo, or ex vivo studies is still less abundant than case reports on liver toxicity. This, quite surprising disparity, should motivate pharmacologists and clinical researchers to do further and more insightful studies to explain the mechanisms of action and pinpoint the most active constituents or their combinations.

\section{Antiproliferative, Pro-apoptotic, and Cytotoxicity to Cancer Cell Lines}

As in case of liver and biliary tract disorders, the antitumor properties have been indicated since antiquity. Today, this kind of activity belongs to the most intensely investigated. Unlike the hepatoprotective properties, this kind of properties has been quite well-documented in a high number of studies. Mostly, some of the major alkaloids are expected to be able to cause cell death or stop proliferation of cancerous cells. This is based on the ability of berberine [28], chelerythrine [9], sanguinarine [12], and to some extent also other alkaloids to intercalate DNA that should interfere with replication and cell division (Philchenkov et al., 2008; Basu et al., 2013; Noureini et al., 2017). However, other mechanisms have been also discovered, albeit most studies used in vitro experiments on cell lines. Chelidonine [1] appeared to exert its cytostatic activity through interactions with microtubules and thereby causing cell cycle arrest (Panzer et al., 2001; Havelek et al., 2016a).

The selected examples of cell line-based studies on cytotoxic properties of $C$. majus and its major alkaloids are summarized in Table 2.

In more detail, quite many different, but mostly human cell lines were used as model systems (for references see Table 2), representing leukemias (Jurkat with several modifications to study certain cell death mechanisms), Raji, MT-4, MOLT-4, HL60, U-937, HEL-92.1.7, CCRF/CEM, CCRF/ADR5000), colon carcinomas (Caco-2, HT-29, HCT116, SW480), breast cancer (MCF-7, MDA-MB231), pancreatic cancer (human PANC1, murine PANC02), lung cancer ((A549, H460), prostate cancer (DU-145), cervical cancer (HeLa), ovarian carcinoma (A2780), liver cancer (HepG2), gastric cancer (SGC-7901), vulvar squamous cell carcinoma (A431), oesophageal squamous carcinoma (WHCO5), and mouse melanoma (B16F10). Noncancerous lines, such as lung fibroblasts (MRC-5, WI-38), skin fibroblasts (Hs27) immortalized cells from mice (3T3), green monkey (Vero), humans (293N3S, HS-27, HaCaT), or SV-40 transformed bronchial epithelium (BEAS-2B) were also used.

From most of the published mechanistic studies a clear distinction can be established between mechanisms of action of chelidonine [1] and sanguinarine [12]/berberine [28]/chelerythrine [9].

Sanguinarine [12], chelerythrine [9], and berberine [28] possess strong affinity to binding G-quadruplex in telomeres 
TABLE 2 | Influence of C. majus and its alkaloids on various cell lines in vitro.

\begin{tabular}{ll}
\hline Cell line & Tested extracts/compounds \\
\hline HaCaT-human keratinocyte & Dry extract--0.68\% alkaloids, pure \\
& berberine [28], chelidonine [1], \\
& chelerythrine [9], hydrastinine, \\
& sanguinarine [12] \\
WHCO5 -squamous esophageal & Chelidonine [1] 10-134 $\mu \mathrm{M}$
\end{tabular}

cancer, HeLa, Vero, 293Graham, HS-27 -transformed human foreskin fibroblasts

DU-145-human prostate carcinoma from brain metastasis,

MCF-7-human breast

adenocarcinoma, A549-human lung

carcinoma, HepG2-human

hepatocellular carcinoma, HT-29

human rectosigmoid adenocarcinoma

Raji-human lymphoma

Murine Nk/Ly lymphoma

Murine (L1210) and human (HL-60) leukemia

Human T cell lymphoblastic leukemia

HL-60

HepG2

HeLa (human cervical cancer), PBMC (peripheral blood mononuclear cells)

HepG2, PBMC

B16F10-murine melanoma, MCF-7-, 3T3-murine embryonal fibroblasts

HeLa, HepG2, Caco2-human colon adenocarcinoma, human $T$ cell leukemia-standard CCRF-CEM and multidrug resistant CCRF/ADR5000
Chelidonine [1] and stylopine [33]

C. majus $40 \%$ ethanol (vodka) extract $10-200 \mu \mathrm{g} / \mathrm{ml}$, equimolar mix of chelerythrine [9] and sanguinarine [12] $0.2-10 \mu \mathrm{g} / \mathrm{ml}$

Chelidonine [1], chelerythrine [9], coptisine [31], sanguinarine [12] $1-40 \mu \mathrm{g} / \mathrm{ml}$

$\mathrm{MeOH}$ extract, $10-100 \mu \mathrm{g} / \mathrm{ml}$

Chelidonine [1], sanguinarine [12] $1-10 \mu \mathrm{g} / \mathrm{mL}$

Chelerythrine [9]

Chelidonine [1] 0.1-100 $\mu \mathrm{M}$

Chelidonine [1] from ethanole extract isolated via MTT cytotoxicity guides chromatography, test conc. $22.5-37.5 \mu \mathrm{g} / \mathrm{ml}$

Chelidonine [1], nano-encapsulated chelidonine [1]

Mixture of allocryptopine, chelidonine [1], protopine, sanguinarine [12], stylopine [33] (all $3.3 \mu \mathrm{g} / \mathrm{ml}$ )

$\mathrm{MeOH}$ extract from Ch. herba (HPLC tested) $5-4,000 \mu \mathrm{g} / \mathrm{ml}$ and pure chelidonine [1] 5-500 $\mu \mathrm{M}$ (concentrations of extract and chelidonine [1] didn't match in the experiments)
Main observed outcome/response

References

Antiproliferative activity with $\mathrm{IC}_{50}$ lowest for sanguinarine $(2.26 \mu \mathrm{M})$, extract (as chelidonine) ca. $5.68 \mu \mathrm{M}$, chelidonine and chelerythrine ca. $28 \mu \mathrm{M}$, low activity of berberine and hydrastinine;

Cytotoxic to all types of cell lines, little specific to malignant cells (HeLa, WHCO5), mitotic arrest, slippage, upregulated cyclin B1 and cdc2 activity, activation of SAPK/JNK cascade, disruption of tubular network;

Chelidonine cytotoxic to DU-145, MCF-7, and HT-29 (IC 50 18.4, 8.30, $5.90 \mu \mathrm{M}$, respectively) whereas stylopine active against DU-145 and MCF-7 only ( $\mathrm{IC}_{50} 13.9$ and $16.6 \mu \mathrm{M}$, respectively);

Vavrečková et al., 1996

Panzer et al., 2001

Lee et al., 2007

Complete cell kill by extract above $10 \mu \mathrm{g} / \mathrm{ml}$ (strongest among 61 tested traditional Russian and Siberian medicinal plant species) and by alkaloid mix above $5 \mu \mathrm{g} / \mathrm{ml}$

Chelerythrine and sanguinarine strong cytotoxic (LD50 4, 25 and $6.2 \mu \mathrm{M}$, respectively), low activity of chelidonine and coptisine; strong correlation to DNA intercalation and genotoxicity (Comet assay)

Cytotoxicity by apoptosis induction (DAPI staining), HL-60 cells more susceptible with $100 \%$ growth inhibition at $75 \mu \mathrm{g} / \mathrm{ml}$ (ca. $70 \%$ in L1210);

Sanguinarine stronger cytotoxic $\left(\mathrm{IC}_{50} \mathrm{ca} .2 .5 \mu \mathrm{M}, 100 \%\right.$ reached at $1.5 \mu \mathrm{M}$ ) than chelidonine (maximum $60 \%$ cell toxicity at a highest dose). Apoptosis induction comparable in two alkaloids (microscopy and cas-3 and Bax expression), but low genotoxicity of chelidonine; chelidonine but not sangunarine induced G2/M arrest;

Cytotoxicity with $\mathrm{IC}_{50}$ of $2.6 \mu \mathrm{M}$, cell cycle arrest in $\mathrm{G} 1$, apoptosis induction (annexin, cas-8 activity) and necrosis;

ca. $50 \%$ cell toxicity, dose independent; apoptosis and autophagy induction; decrease of telomerase activity and expression of hTERT subunit of telomerase (at $0.1 \mu \mathrm{M}$ ); long term treatment with $0.1 \mu \mathrm{M}$ induced senescence.

Selectively cytotoxic to HeLa cells (LD 50 ca. $85 \mu \mathrm{M})$; apoptosis induction (microscopy, annexin staining), cell cycle arrest $\left(G_{0}-G_{1}\right)$, upregulation of p38, p53, cas-3, cas-9, Bax, APAF-1, down-regulation of PI3K, AKT, JAK3, STAT3, Bcl proteins, corroborating with RT-PCR;

HepG2 selective apoptosis promotion and cell cycle arrest $\left(G_{2} / M\right)$, modulation of apoptosis-associated genes transcription and protein levels; stronger effect of chelidonine in nanoformulation (e.g., $\mathrm{IC}_{50}$ in MTT assay was 2.91 vs. $5.45 \mu \mathrm{M}$ ), supported by its higher bioavailability in mice;

Cytotoxic (up to 55\%) to all lines, slightly selective to mouse melanoma; changes in alkaloid conc. in the medium and cell lysated was measured but no correlation of the cellular uptake to the toxicity was found;

Cytotoxicity selective against some lines (most susceptible CCRF-CEM, moderate HepG2 and HeLa, resistant Caco2 and CCRF-ADR5000 to extract but not chelidonine) and apoptosis promotion; chelidonine much more effective than extract; enhanced doxorubicine toxicity in resistant cells, inhibition of ABC transporters; a number of cell death, efflux pumps, metabolism related genes were modulated-down regulation of MDR, upregulation of cell death and cell cycle control genes;
Spiridonov et al., 2005

Kaminskyy et al., 2006

Nadova et al., 2008

Philchenkov et al., 2008

Vrba et al., 2008

Noureini and Wink, 2009

Paul et al., 2013

Paul et al., 2013

Kulp et al., 2011; Kulp 2013

El-Readi et al., 2013 and Bragina, 
TABLE 2 | Continued

\begin{tabular}{ll}
\hline Cell line & Tested extracts/compounds \\
\hline MCF-7 & $\begin{array}{l}\text { Chelidonine [1] } 0.25-250 \mu \mathrm{M}, \\
\text { berberine [28] } 1-1,000 \mu \mathrm{M}\end{array}$
\end{tabular}

HepG2

PANC-1-human pancreatic cancer, PANC02-mouse pacreas cancer, HT-29-colon cancer, MDA-MB-231 - human mammary gland cancer, BEAS-28-human bronchial eppithelium (non-cancerous), 3T3, PC-EM-primary endometrial cancer cultures (from surgery)

MDA-MB-231

A431-human squamous cells epidermoid carcinoma

Human lung carcinoma (A549, H460), colon carcinoma (HCT116, SW480), breast (MCF-7, MDA-MB231), PBMC

Jurkat E6.1 mutant-human T cell leukemia, MOLT4-human T cell lymphoblastic leukemia,

U937-human histiocytic lymphoma, HEL92.1.7-human erythroleukemia, Raji-human Burkitt lymphoma, HL-60, A2780-human ovarian endometrioid adenocarcinoma, A549, human primary lung fibroblast (MRC-5 and WI-38), PBMC

SGC-7901 human gastric carcinoma
Dichloromathane, ethanol, 50\% ethanol, water extracts from C. herba (characterized by NMR and HPLC), diluted 1:501

$80 \%$ ethanol extract and $n$-hexane defatted ethanol extract $1-1,000 \mu \mathrm{g} / \mathrm{ml}$ (standardized by HPLC), Ukrain ${ }^{\circledR}$

Chelidonine [1] 0.3-10 $\mu \mathrm{M}$

C. majus (parts used undefined) water extract, no standardization, 50-500 $\mu \mathrm{g} / \mathrm{ml}$

C. majus whole plants, dried ethanol extract, standardized for alkaloids and polyphenols, $16-500 \mu \mathrm{g} / \mathrm{ml}$

Chelidonine [1], homochelidonine [1] $1-20 \mu \mathrm{M}$

Chelidonine [1] 5-160 $\mu \mathrm{M}$
Main observed outcome/response

Strong chelidonine cytotoxicity (LD50 ca. 8 vs. $54 \mu \mathrm{M}$ berberine); apoptosis dominating at lower $(<5 \mu \mathrm{M})$ and autophagy at higher conc.; senescence induction at $0.05 \mu \mathrm{M}$ for 33 days; telomerase suppression by chelidonine via down regulation of hTERT expression and inhibition of enzyme activity - interaction with G-DNA excluded;

Inhibition of cell proliferation, highest by ethanolic extract, followed by dichloromethane (no activity of water extract); activation of genes (whole genome microarray) related to drug metabolism, oxidative stress and cellular damage; no correlation to the alkaloid content;

Cytotoxic to cancer lines ( $\left.\mathrm{IC}_{50} 19.4-57.8 \mu \mathrm{g} / \mathrm{ml}\right)$, with MDA-MB-231 significantly more resistant than other lines; lower cytotoxicity in 3T3 and PC-EM cells; pilot in vivo results of anti-metastatic effect against transplanted PANC02 in mice but no significant effect on tumor growth;

Inhibition of collagen-stimulated cell migration and invasion (but not fibronectin-induced); mechanisms include suppression of actin cytoskeleton reorganization, inhibition of integrin signaling via suppression of ILK association with other components of IPP complex essential for adhesion;

Increase of cas-3 activity, three-fold increase of apoptotic cells; modulation of cell death related genes - mRNA down regulation of $\mathrm{Bcl}-2$, survivin and $\mathrm{Mcl}-1$, upregulation of p21 and Bax; increase of p38, MEK, and ERK phosphorylation (activation), inhibition of NFKB activation;

Selectively and differently toxic to cancerous cells, non-toxic to PBMC. $I_{50}$ between 44 and $143 \mu \mathrm{g} / \mathrm{ml}$, depending on cell line, also synergistic activity with doxorubicin in its lower (1-2 $\mu \mathrm{M})$ concentrations, antagonistic in higher dox doses; apoptosis induction (annexin, microscopy); G2/M arrest; inhibition of cell migration by scratch assay;

Cytotoxic effect differing between cell lines $\left(\mathrm{IC}_{50}\right.$ 1.8-5.0 $\left.\mu \mathrm{M}\right)$, chelidonine stronger than homochelidonine, strongest effect toward MRC-5, and mutated Jurkat cells; induction of apoptosis with involvement of mitochondrial pathway; biphasic cell cycle arrest (G1, G2/M) by homochelidonine, but only G2/M by chelidonine; increase in $\mathrm{H} 3$ histone phosphorylation, activation of checkpoint kinases, inhibition of cell adhesion, interference with tubular skeleton, nuclei fragmentation;

Cytotoxic with $\mathrm{IC}_{50}$ at $23.13 \mu \mathrm{M}$; apoptosis inducing (microscopy); increase of histone $\mathrm{H} 3$ phosphorylation, time-dependent regulation of mitotic slippage associated protein levels-BubR1 (a checkpoint kinase), Cdk-1, cyclin B1; increase of cas-3 expression; $\mathrm{G}_{2} / \mathrm{M}$ arrest; microtubule polymerization inhibition causing mitotic catestrophe;

Different toxicity $\left(\mathrm{IC}_{50}=2.5,3.0,8.0,54.0\right.$, and $120.0 \mu \mathrm{M}$ for chelerythrine, sanguinarine, chelidonie, berberine, and papaverine); strong inhibition of telomerase and hTERT expression by chelerythrine and sanguinarine, but only of hTERT expression by chelidonine; strong binding of chelerythrine and sanguinarine to telomeric G quadruplex, confirmed by docking;

\section{References}

Noureini et al., 2017

Orland et al., 2014

Kim et al., 2015

Park et al., 2011

Deljanin et al., 2016

Havelek et al., 2016a,b

Qu et al., 2016

Noureini et al., 2017 chelerythrine [9]--2-25 $\mu \mathrm{M}$, chelidonine [1]--5-100 $\mu \mathrm{M}$, papaverine, sanguinarine [12]--2-25 $\mu \mathrm{M}$

(Continued) 
TABLE 2 | Continued

\begin{tabular}{|c|c|c|c|}
\hline Cell line & Tested extracts/compounds & Main observed outcome/response & References \\
\hline \multicolumn{4}{|c|}{ REPORTS WHERE UKRAIN ${ }^{\circledR}$ WAS USED AS AN ACTIVE SUBSTANCE } \\
\hline $\begin{array}{l}\text { Jurkat - various modifications (A3, } \\
\text { J16, Cas-9 DN expressing, cas-8 and } \\
\text { FADD-negative, CD95/TRAIL } \\
\text { resistant A3, Bcl-2 overexpressing, } \\
\text { cFLIP-L expressing) }\end{array}$ & $\begin{array}{l}\text { Ukrain }{ }^{\circledR}(5-50 \mu \mathrm{g} / \mathrm{ml}) \text { and alkaloid } \\
\text { standards }\end{array}$ & $\begin{array}{l}\text { Ukrain }{ }^{\circledR} \text { disproved from being a derivative-just mixture of native } \\
\text { alkaloids by mass spectrometry; strong apoptosis induction by } \\
\left.\text { Ukrain }{ }^{\circledR} \text { and activation of caspases ( } 3 \text { and } 8\right) \text {; apoptosis mediated } \\
\text { by mitochondrial pathway; similar activity observed for } \\
\text { chelidonine, chelerythrine, and sanguinarine; }\end{array}$ & $\begin{array}{l}\text { Habermehl } \\
\text { et al., } 2006\end{array}$ \\
\hline $\begin{array}{l}\text { Ewing sarcoma cell lines } \\
\text { (CADO-ES-1, VH-64, STA-ET-1, } \\
\text { STA-ET-2.1) }\end{array}$ & $\begin{array}{l}\text { Ukrain }{ }^{\circledR} 0.05-50 \mu \mathrm{M}, \text { C. majus } \\
\text { alcohol extract (commercial, } \\
\text { standardized to } 1.2 \mathrm{mg} / \mathrm{g} \text { chelidonine } \\
[1])--0.5-500 \mu \mathrm{M} \text { chelidonine [1] }\end{array}$ & $\begin{array}{l}\text { Different cytostatic activity depending on the target line: STA-ET-1 } \\
\text { and } \mathrm{VH} 64 \text { more sensitive (mean } \mathrm{Gl}_{50} \text { was } 11.9 \mu \mathrm{M} \text { for Ukrain }{ }^{\circledR} \\
\text { and } 12.3 \mu \mathrm{M} \text { for extract) }\end{array}$ & $\begin{array}{l}\text { Lanvers- } \\
\text { Kaminsky } \\
\text { et al., } 2006\end{array}$ \\
\hline human glioblastoma T60, T63 & Ukrain ${ }^{\circledR}$ & $\begin{array}{l}\text { Significant inhibition of cell proliferation at } 10 \mu \mathrm{M} \text {, aopoptosis } \\
\text { induction, increase of glial fibrillary acidic protein-crucial for lower } \\
\text { malignancy adn tumor growth suppression; }\end{array}$ & $\begin{array}{l}\text { Gagliano } \\
\text { et al., } 2006 \text {, } \\
2007\end{array}$ \\
\hline $\begin{array}{l}\text { Caki-1, ACHN-renal clear cell } \\
(\text { Caki-1, ACHN) and papillary (Caki-2) } \\
\text { renal metastatic carcinoma }\end{array}$ & Ukrain $^{\circledR}$ & $\begin{array}{l}\text { Reduced cell proliferation }(10 \mu \mathrm{M}), \text { modulation of malignant } \\
\text { phenotype and invasiveness, by inhibition of MMP activity and } \\
\text { downregulation of secreted and upregulation of intracellular } \\
\text { SPARC protein levels; }\end{array}$ & $\begin{array}{l}\text { Gagliano } \\
\text { et al., } 2011\end{array}$ \\
\hline $\begin{array}{l}\text { Pancreatic ductal adenocarcinoma } \\
\text { (HPAF-II, HPAC, PL45) }\end{array}$ & Ukrain $^{\circledR}$ & $\begin{array}{l}\text { Reduced cell proliferation }(10 \mu \mathrm{M}) \text {, retaining of epithelial } \\
\text { phenotype; decreased invasiveness by inhibition of MMP activity } \\
\text { and downregulation of secreted and upregulation of intracellular } \\
\text { SPARC protein levels; modification of mitotic spindle microtubules; }\end{array}$ & $\begin{array}{l}\text { Funel et al., } \\
2010 ; \\
\text { Gagliano } \\
\text { et al., } 2012\end{array}$ \\
\hline $\begin{array}{l}\text { Murine (4T07, TUBO) or human } \\
\text { (SKBR-3) breast cancer, 3T3 }\end{array}$ & Ukrain $^{\circledR}$ & $\begin{array}{l}\text { Moderate (13-30\%) cell death induction specific for cancerous } \\
\text { lines. Inhibition of proliferation regain potential; induction of } \\
\text { apoptosis (annexin, caspase-3 activity); verified by an in vivo } \\
\text { experiment-diminished tumor growth in mice; }\end{array}$ & $\begin{array}{l}\text { Bozeman } \\
\text { et al., } 2012\end{array}$ \\
\hline $\begin{array}{l}\text { 4T1 - murine mammary gland cancer, } \\
\text { B16F10 }\end{array}$ & $\begin{array}{l}\text { Ukrain }{ }^{\circledR} \text {, in combination with several } \\
\text { cytostatic drugs }\end{array}$ & $\begin{array}{l}\mathrm{IC}_{50} \text { was } 40 \mu \mathrm{M}(4 \mathrm{~T} 1) \text { and } 76 \mu \mathrm{M} \text { (B16F10), synergistic effect with } \\
\text { bortezomid; }\end{array}$ & $\begin{array}{l}\text { Savran et al., } \\
2014\end{array}$ \\
\hline
\end{tabular}

which leads to blocking telomerase activity in fast proliferating cells (Noureini et al., 2017).

Unlike the quarternary alkaloids, chelidonine [1] is only a weak DNA intercalating agent and does not induce lethal mutations or DNA damage. Its mechanism of action is suggested to rely on interactions with spindle microtubules leading to cell cyle arrest and mitotic catastrophe, inhibition of $\mathrm{ABC}$ transporters thus abolishing multidrug resistance and finally modulation of gene transcription (telomerase, cell death-related, cell cycle-controlling). These properties combined with the stronger-acting intercalating alkaloids can make the whole alkaloid fraction a unique multifaceted agent targeting cancer cells.

For example, El-Readi et al. (2013) demonstrated complex interaction of chelidonine [1] and alkaloid-rich extract with several signaling pathways, including those responsible for cell cycle, cell death, and proliferation. Using microarrays confirmed by RT-PCR data, it was shown that a set of genes associated with multidrug resistance (e.g., $\mathrm{ABC}$ transporters and $\mathrm{CYP}$ ) were significantly downregulated (from $50 \%$ in case of CYP3A4 to $99 \%$ in ABCG2). On the other hand, caspase- 3 and 8 genes were upregulated (up to 27 -fold). These results explain the strong augmenting of doxorubicin toxicity to resistant Caco2 and CCRF/ADR5000 cells. Upon low-dose treatment with chelidonine [1] $(20 \mu \mathrm{M})$, the $\mathrm{LC}_{50}$ decreased from $3.67 \mu \mathrm{M}$ (Caco-2) and $32.16 \mu \mathrm{M}$ (CCRF/ADR5000) to 0.42 and $7.4 \mu \mathrm{M}$, respectively. Similar results were obtained with an adequate concentration of extract $(5 \mu \mathrm{g} / \mathrm{ml})$ containing (in decreasing order) chelidonine [1], coptisine [31], stylopine [33], and protopine [37] as four major compounds and smaller amounts of other alkaloids. In the more resistant CCRF/ADR5000 cells the effect of extract was even stronger than pure chelidonine [1], suggesting that some of the other alkaloids are more efficient Pgp inhibitors. Induction of apoptosis was the major mechanism of cytotoxicity, also supported by increase of caspase activity and annexin staining.

These results support the potential of chelidonine [1] (and at least some other C. majus alkaloids) as ideal agents to overcome MDR as targeting both transport proteins, metabolic enzymes and pro-apoptotic pathways.

The interference of chelidonine [1] with the cell proliferation apparatus was also demonstrated using planarian stem cell model (Isolani et al., 2012) in which the gene expression of $\mathrm{mcm} 2$ (essential DNA replication factor) or inx-11 (a gap-junction protein essential for regeneration) was decreased by $20 \mu \mathrm{M}$ of the alkaloid. The stem cells were affected but not differentiated populations such as neuronal or intestinal.

Quite a particular story pertains to the mentioned earlier patented product referred to as a semisynthetic derivative of alkaloids - a trimeric structure with thiophosphoric acid moiety connecting alkaloid molecules. It has been mentioned in the literature as NSC-631570 or Ukrain ${ }^{\circledR}$. However, one of the major doubts regarding this product is its chemical identity (Panzer et al., 2000; Habermehl et al., 2006; Jesionek et al., 
2016). Despite the earlier manufacturer claims, it couldn't be positively confirmed that it is indeed a thiophosphoric acid complex and at least in some batches, it appeared as a rich mixture of native alkaloids, that have been identified using reliable chromatographic and spectroscopic methods.

Several papers additionally report cytotoxic activity of Ukrain ${ }^{\circledR}$ on other cell lines such as prostate cancer (LNCaP, PC-3), glioblastoma (T60, T63, primary cancer line), pancreatic ductal adenocarcinoma (HPAF-II, HPAC, PL45, renal clear cell or papillary cell-derived lines (Caki-1, Caki-2, ACHN), melanoma (B16F10) and murine (TUBO, 4T1) or human (SKBR-3) breast cancer (see Table 2 for respective references).

However, due to the conflicting data about the chemical identity of this product, the previous results would have to be repeated or reinterpreted. If indeed Ukrain ${ }^{\circledR}$ is just a version of alkaloid mix, all the research done using it as an active substance would extend the scope of anticancer properties of C. majus native alkaloids. One of the early interesting findings is radiosensitizing influence of Ukrain ${ }^{\circledR}$ (Cordes et al., 2002, 2003) and it should be verified using non-modified alkaloid-rich extract and individual compounds to find out which of them or a combination would be responsible for this property. Also, other studies reporting Ukrain ${ }^{\circledR}$ as an active principle need reevaluation with native alkaloids as probably constituting major component in the patented preparation. Moreover, Ukrain ${ }^{\circledR}$ remains the only preparation from $C$. majus that has a vast literature documenting its anti-cancer and also other properties in clinical (see the respective section in the present paper), pre-clinical and in vivo studies.

\section{Anti-inflammatory and Immunomodulating}

Some of the versatile traditional uses of $C$. majus can be explained, as in many other herbs, by anti-inflammatory potential targeting various pathways in the organism as well as modulation of immune response. Both have been confirmed in many studies using in vitro cellular models, as well as in vivo.

The ability to inhibit inflammation or, in some cases, to stimulate immune response and mitigate excessive reactiveness can contribute to the postulated anticancer properties and improve symptoms of gastric disorders as well.

Schneider et al. (2016) used human colon cell line (NCM460) to check anti-inflammatory action of composite preparations containing C. majus marketed and popular in Europe and any of the individual components. C. majus extract was among the most potent in a couple of checked parameters, such as inhibition of IL-8, MCP, and I-TAC secretion, thus contributing significantly to the overall and apparently synergistic combination of active principles. This activity was likely to influence the clinical outcome mentioned below (Abdel-Aziz et al., 2017).

Mostly, the total extracts or isolated alkaloids were tested, but in our opinion, other components such as hydroxycinnamic derivatives, flavonoids and chelidonic acid are likely to contribute significantly. Chelidonic acid [45] was efficient in mouse models of ovoalbumin-elicited allergic rhinitis (Oh et al., 2011) and ulcerative colitis (Kim et al., 2012). This compound also attenuated inflammatory responses by reducing levels and gene expression of several mediators and enzymes in colon tissues (COX-2, HIF1 $\left.\alpha, \mathrm{PGE}_{2}\right)$ and in allergic mice (IL-4, IL-1 $\beta, \mathrm{COX}-2$, caspase-1, and increase of IFN- $\gamma$ ). In human mast cell line HMC-1 stimulated for inflammatory response by the phorbol ester (TPA) and calcium ionophore A23187, chelidonic acid [45] inhibited IL-6 expression by blocking NFKB (Shin et al., 2011).

Stylopine [33] added to the cell culture of in lipopolysaccharide-stimulated RAW264.7 macrophages was inhibiting production of several proinflammatory molecules such as nitric oxide, PGE 2 , TNF- $\alpha$, IL-1 $\beta$, and IL-6 (Jang et al., 2004). Also, iNOS and COX-2 protein levels were lowered. However, the cyclooxygenase activity inhibition was not selective. Chelidonine [1] and 8-hydroxydihydrosanguinarine [14a] in the same model inhibited NO production and iNOS and COX-2 gene transcription (Park et al., 2011). These results suggest the underlying inhibition of $\mathrm{NF \kappa B}$ as potential mechanism but it wasn't investigated in this study. However, in HCT-1 colon cancer cell line treated with chelidonine [1], the $\mathrm{NF} \kappa \mathrm{B}$ activation was blocked by inhibition of I $\mathrm{B} \alpha$ degradation and nuclear translocation of p65 (an NFאB subunit) as well as mitogen-activated protein kinase pathway activation by blocking c-Jun N-terminal kinase and p38 phosphorylation (Zhang et al., 2018).

Alkaloid fraction and sanguinarine [12] were efficient against carrageenan-induced rat paw edema but chelerythrine [9] showed lower activity (Lanfeld et al., 1981). However, in the later study by Mikołajczak et al. (2015), various fractions of water extract at relatively high doses of $200 \mathrm{mg} / \mathrm{kg}$ body weight failed to alleviate the inflammation in the similar model. The crude water extract treatment actually aggravated the paw inflammation. Conversely, the extracts containing mainly coptisine [31] and chelidonine [1] were effective in hot plate test for antinociceptive properties that suggests a supramedullary way of action.

Chelerythrine [9] inhibited inflammatory and pain reaction in several in vivo and cell models employed by Niu et al. (2011). In vivo, i.p administration of the alkaloid (1-5 mg/kg) alleviated mouse ear edema, rat paw edema, and abdominal constriction (pain reaction). Also, the isolated peritoneal macrophages upon treatment with $0.0001-1 \mu \mathrm{g} / \mathrm{ml}$ chelerythrine [9] had dose dependently reduced $\mathrm{PGE}_{2}$ and COX-2 expression.

In $\mathrm{NC} / \mathrm{Nga}$ mice model for atopic dermatitis induced by DNCB (1-chloro-2,4-dinitrobenzene), the hydroethanolic extract from aerial parts alleviated several measures of dermatitis such as itching behavior and skin severity (Yang et al., 2011). Interestingly, the oral administration at doses of $200 \mathrm{mg}$ and 400 $\mathrm{mg} / \mathrm{kg}$ were even more efficient than topical application as 1 and $2 \%$ smear. The IgE levels were put back to control values upon the higher dose oral administration and reduced by about $50 \%$ after topical treatment. Also, IL-4 and TNF- $\alpha$ serum levels were significantly reduced but remained higher than in control animals.

In an animal model of ovoalbumine-provoked asthma, chelidonine [1] suppressed eosinophile-mediated inflammation. The activity at the doses of 1 and $5 \mathrm{mg} / \mathrm{kg}$ body weight was similar to $0.5 \mathrm{mg} / \mathrm{kg}$ body weight dexamethasone. Among the several monitored parameters such as different pro-inflammatory cell population counts in bronchoalveolar lovage fluid and lungs, IgE, and cytokine protein and transcripts levels, some were inhibited even stronger than by dexamethasone (total BALF cells, Gr-1+/CD11b+ cells, IL-4), whereas others were inhibited either 
similarly or weaker than the standard drug. It suggests a specific mechanism involving STAT6 and Foxp3 transcription pathways (Kim et al., 2015).Yet another inflammation-based condition absent from list of traditional indications, in which C. majus was found to be efficient is arthritis (Lee et al., 2007). In the mice model of collagen-induced arthritis, aqueous-methanol extract at the oral doses of 40 and $400 \mathrm{mg} / \mathrm{kg}$ body weight, suppressed progress of joint damage as well as a set of studied inflammationrelated cellular and biochemical parameters. In the higher dose regime, the incidence of arthritis decreased from 100 to $<40 \%$ during 4 weeks. Cell invasion into lymph nodes, spleen, thymus and synovial fluid was inhibited in all organs, but most marked effect was in lymph nodes and joints. Among the tested $\mathrm{T}$ cells populations, there was an insignificant decrease of CD4+, $\mathrm{CD} 8+$, and $\mathrm{CD} 3 \mathrm{e}+$ cells, but a quite remarkable decrease of CD19+ B cells, almost to the level of non-arthritic animals. On the other hand, the number of regulatory CD4+CD25+ $\mathrm{T}$ cells increased significantly that suggests mobilizing adaptive response of the immune system to counteract and balance the excessive inflammatory processes. Suppression of inflammation mediators such as IL-6, TNF $\alpha$, IFN $\gamma$ was observed as well as lowered level of IgG and IgM but the latter only upon the higher dose treatment. However, the extract was not standardized an no particular compound or phytochemical class could be pinpointed as determining this potent anti-arthritic action.

A couple of reports indicate a potential of C. majus against Alzheimer's progression due to significant inhibition of acetylcholinesterase (AChE) without influence on butyrylcholinesterase (BuChE), which is a desired profile for potential drug-likeliness. The extract inhibited AChE in vitro by $98 \%$ at a concentration of $200 \mu \mathrm{g} / \mathrm{ml}$ and BuChE by only $13 \%$. The isolated alkaloid 8-hydroxydihydrochelerythrine [10a] was the most active and AChE-selective ( $\mathrm{IC}_{50} 0.61 \mu \mathrm{M}$ and selectivity index 56.7) (Cho et al., 2006). In another study, chelidonine [1] and other active alkaloids were completely unselective with similar $\mathrm{IC}_{50}$ values against $\mathrm{AChE}$ and BuChE (Cahlíková et al., 2010). Interestingly, the specific AChE inhibiting activity by coptisine [31]-rich extract was discovered in vivo in a herbivore insect Lymantria dispar in which this activity contributes to killing the pest (Zou et al., 2017). By this property the plant is protecting itself from herbivore attack and it would be a feasible biological explanation of the therapeutic potential existence in the wild growing species.

The analgetic properties of alkaloids, mentioned before (Mikołajczak et al., 2015) can be explained by the observations of interaction with glycine transporters (Shin et al., 2003; Jursky and Baliova, 2011). The water extract inhibited the glycine-activated and enhanced glutamate-activated ion current in isolated rat periaqueductal gray (PAG) neurons studied by patch-clamp technique (Shin et al., 2003). Chelerythrine [9] and sanguinarine [12] selectively inhibited GlyT1 (but not GlyT2) in the micromolar concentrations $(5-10 \mu \mathrm{M})$ while berberine [28] showed no inhibition in transfected HEK293T cells. GlyT1 inhibition was time-dependent, noncompetitive and increased with glycine concentration. Interestingly, chelerythrine [9] effect was reversible while sanguinarine [12] persisted through washing out (Jursky and Baliova, 2011).

\section{Clinical Studies}

Quite typically for many traditionally used medicinal plants, the clinical evidence of efficacy remains scarce and C. majus is no exception. Therefore, the relatively numerous pharmacological studies need rigorous verification by properly designed and supervised clinical studies. At present, most of the allegedly curative properties toward some important complaints remain unconfirmed, even if there is strong pre-clinical evidence. The millennia long tradition of use becomes confirmed in a significant part even if some mythical indications turned out to be invalid or perhaps have been misunderstood or distorted when passed through generations of practitioners and authors.

Several clinical studies exist on the compound preparation STW-5 containing $10 \%$ of C. majus herb extract (Drug-toExtract Ratio $=1: 3$ ) as one of nine ingredients. The gastric disorders are the main indications and was evidenced both clinically and pharmacologically (Von Arnim et al., 2007; AbdelAziz et al., 2017). Certainly, there is no proof that C. majus was the essential ingredient in this preparation but the functional dyspepsia/postprandial distress syndrome it seems to be one of the most active (Abdel-Aziz et al., 2017) influencing acid regulation and antrum contraction as well as moderate mitigation of inflammatory reactions. The mechanisms behind these effects were also studied in vitro using cell lines showing antiinflammatory activity (Schneider et al., 2016). IFN- $\gamma$ dependent stat 1 phosphorylation was postulated as a putative mechanism of action in which $C$. majus extract was among the averagely active, but apparently the whole composition had superior properties suggesting synergistic rather than additive effect.

A series of older clinical trials were performed on a product containing C. majus extract to test the efficacy in patients with bile tract and gall bladder complaints and gall stones. These studies, reviewed in detail in the EMA report, suggest significant improvement of many clinical parameters. These included: subjective complaints (pain attacks, feeling of fullness), physician's examination (sonography of gall bladder and liver, liver palpation, meteorism, jaundice) and laboratory tests (bilirubin, transaminases, blood sedimentation). The patient conditions in which the preparation containing C. majus alkaloids (in daily doses of ca. $0.2 \mathrm{mg}$ alkaloid sum as chelidonine [1]) was administered with positive outcome were for example: cholelithiasis, cholangitis/cholecystitis, postcholecystectomy, and alcohol toxic liver damage. In the entire area of gastrointestinal/hepatic complaints, one can estimate the number of human subjects involved in to date published clinical literature as exceeding 1,500.

An impressive number of studies suggest antitumor properties of the apparently semisynthetic product-Ukrain ${ }^{\circledR}$ that showed efficiency in several in vitro studies on an assortment of neoplastic and non-transformed cell lines (Capistrano et al., 2015) but it by large failed to demonstrate clinically relevant activity in humans. Several case reports have been published that suggest its beneficial action in a range of malignancies, such as melanoma, metastatic breast cancer, various carcinomas.

The randomized clinical trials (RCTs) using Ukrain ${ }^{\circledR}$ were reviewed over a decade ago (Ernst and Schmidt, 2005) with a conclusion that despite the intensive publishing activity of several 
groups testing Ukrain ${ }^{\circledR}$ against miscellaneous malignacies with very promising outcome, most of these data are full of shortcomings that prevent unequivocal credibility. Since then, only a few more studies have been realized but it is still far from definite resolving of this issue, especially in the situation that the chemical analyses, some of which were even co-authored by the inventor (Jesionek et al., 2016) proved that it is not the compound which was initially claimed.

Most of the RTCs published were full of inconsistencies or even appeared unreliable due to the insufficient information regarding the methodology. Despite the spectacular results in such malignancies as pancreatic or terminal colorectal cancer, serious doubts remain about the evidence provided in some of these trials. Some of the studies are so poorly documented that it would undermine the validity of the results. For example, missing or suspect randomization method, missing methods for tumor dimensions measuring, unclear protocols, subjective outcome evaluation, lack of proper statistics. Also, a great majority of the clinical trials and case reports were published in one journal. Nonetheless, these few reports on Ukrain ${ }^{\circledR}$ remain the only available clinical data focusing specifically on C. majus and strongly suggesting extraordinary antitumor potential.

Since the potential conflicts of interest, reliability of the clinical and chemical data of this product have been disputed repeatedly (Farrugia and Slevin, 2000, 2001; Nowicky, 2001; Ernst and Schmidt, 2005), more studies are indeed mandatory also in this aspect of $C$. majus as a source of highly potent substances with multidirectional mechanisms of action. Nonetheless, it should be borne in mind that approval of an extract/mixture based drug for clinical practice in such a sensitive field as oncology is highly improbable at the moment, and most likely it would remain so in the near future. Thus, further studies are mandatory that would focus on using individual alkaloids as lead structures for drugs that would target multiple anticancer mechanisms. On the other hand, clinical data on efficacy of various $C$. majus products used in complementary or adjuvant therapy may facilitate the tedious process of drug development without changing the existing paradigm of chemotherapy based on combination of chemically defined substances. Quite differently from the relatively safe (with limitations described below) gastrointestinal and cutaneous indications, the cautious policies of EMA and other agencies demonstrated in case of declared anticancer actions are reasonable and should be continued.

\section{Toxicology and Safety Issues}

Repeatedly, studies and case reports occur that suggest hepatic injury/hepatotoxicity. It is especially important because one of the main indications of $C$. majus relates to liver and biliary tract disorders due to its cholagogue and hepatoprotective activities. The incidence of hepatotoxic cases and the possible clinical importance and safety issues have been reviewed recently (Pantano et al., 2017). Specifically, the results from animal studies are ambiguous and suggest a rather complex mixture of various mechanisms, such as inducing or alleviating oxidative stress or modulation of hepatic enzymes such as $\mathrm{MAO}$ and SOD, or slowing down mitochondrial respiration.
The mitochondrial toxicity was related to DNA intercalating properties of sanguinarine [12] and chelerythrine [9].

In humans, numerous reports have been recorded since the 1990's. The main symptoms included cholestasis and mild to severe liver impairments with quite well documented causality in a majority of cases. In total, over 50 such cases have been reported from Europe, mostly from Germany (Etxenagusia et al., 2000; Stickel et al., 2003).

However, no certain constituent has been directly linked to the toxicity of the herb. On the contrary, it has been suggested that drug interactions rather than intrinsic toxicity are responsible for reported cases. Also, individual hypersensitivity or allergy has to be considered. Latex contained in the fresh plant is also possibly more toxic or allergenic than the dried material (EMA report, 2011).

In an in vitro study on HepG2 cells treated with various solvent extracts (Orland et al., 2014), the biotransformation and toxicity-related gene expression was enhanced, but the dichloromethane extract richest in chelidonine [1] and total alkaloids was the weakest inducer and least cytotoxic, whereas ethanolic extracts containing more coptisine [31] and sanguinarine [12] were more cytotoxic. However, the in vivo relevance of these results is uncertain. In rats, the high doses (up to $3 \mathrm{~g} / \mathrm{kg}$ body weight; Mazzanti et al., 2009) did not elicit any symptoms of hepatic injury and did not alter liver function.

Despite proven interaction of sanguinarine [12] and other alkaloids with DNA, no genotoxicity was observed using well established methods such as Ames tests or in vivo DNA damage (EMA report, 2011).

Nonetheless, uncontrolled internal use of unstandardized preparations should be discouraged and appropriate pharmacovigilance measures should be implemented to prevent unnecessary complications following C. majus administration. The comprehensive pharmacotoxicological investigation is also needed and should encompass establishing toxic doses range of various forms and preparations as well as individual constituents.

The European Medicine Agency therefore published the following recommendation:

"Two possible therapeutic indications were proposed for the monograph:

Traditional herbal medicinal product:

1) For symptomatic relief of digestive disorders such as dyspepsia and flatulence (oral intake)

2) For treatment of warts, callus and corns (cutaneous use)" (EMA, 2011).

However, based on the reported undesirable effects, including cases of liver damage, special precautions are necessary, especially during pregnancy and lactation, people suffering from liver diseases or taking liver-damaging drugs.

\section{CONCLUSIONS AND FUTURE OUTLOOKS}

Much effort has been put into the recognition of bioactive compounds contained in the extracts of C. majus and the mechanisms of their action. Numerous reports have been 
published about its effectiveness in treatment of different medical complaints, such as gastrointenstinal and hepatobiliary disorders or cutaneous ailments caused by a microbial or viral infection. Next to the huge number of scientific reports indicating the beneficial effect of $C$. majus, information about its potential toxic properties emerged. The use of herbal remedy carries the risk of adverse effects, which is why it is important to know the raw material. Official recommendations do not exclude the use of $C$. majus as traditional herbal medicinal product. Therefore, further research on the mechanisms of action during treatment should be carried out. It is also important to control the phytochemical composition of the raw material both in conventional growing conditions and in vitro cultures. The more so, because the potential risk of carcinogenesis and hepatotoxicity is still not well documented.

Finally, we conclude that the millenia long history of Chelidonium in folk, traditional, and official medicine is far from coming to the end. On the contrary, recent years witnessed a revival of advanced pharmacological and mechanistic approaches using both native complexes and individual components in discovery of the therapeutic potential of this herb. We are quite convinced that in the near future, at least some of the already known and evidence-based properties should and would find their place in officially recognized therapeutic procedures. Moreover, new discoveries should broaden the scope of traditional usage and deliver better preparations, especially combining different classes of active molecules such as proteins, alkaloids, chelidonic acid, and perhaps also polyphenols. To achieve it, much more joined and interdisciplinary efforts are necessary. Further, the plant's diversity on different levels must be thoroughly evaluated and optimal combination of the complex profile should be established for various target applications. As one of the oldest proverbs from Aristotle's book, also referring to C. majus original Greek name, "one swallow does not make a spring," even if we observe a lot of "first swallows," we urgently need more, to ensure rational exploitation of the huge potential hidden in the inconspicuous and common weed.

\section{REFERENCES}

Abdel-Aziz, H., Kelber, O., Lorkowski, G., and Storr, M. (2017). Evaluating the multitarget effects of combinations through multistep clustering of pharmacological data: the example of the commercial preparation iberogast. Planta Med. 83, 1130-1140. doi: 10.1055/s-0043-116852

Agrawal, A. A., and Konno, K. (2009). Latex: a model for understanding mechanisms, ecology, and evolution of plant defence against herbivory. Annu. Rev. Ecol. Evol. Syst. 40, 311-331. doi: 10.1146/annurev.ecolsys.110308.120307

Arora, D., and Sharma, A. (2013). A review on phytochemical and pharmacological potential of genus Chelidonium. Pharmacogn. J. 5, 184-190. doi: 10.1016/j.phcgj.2013.07.006

Barton, B. H., and Castle, T. (1845). The British Flora Medica, or History of the Medicinal Plants of Great Britain. London: E. Cox.

Basu, P., Bhowmik, D., and Suresh Kumar, G. (2013). The benzophenanthridine alkaloid chelerythrine binds to DNA by intercalation: photophysical aspects and thermodynamic results of iminium versus alkanolamine interaction. J. Photoch. Photobiol. B, Biol. 129, 57-68. doi: 10.1016/j.jphotobiol. 2013.09.011

\section{AUTHOR CONTRIBUTIONS}

SZ: Wrote phytochemical and bioactivity parts of the manuscript and critically reviewed and corrected the final manuscript; AJ-D: Wrote the ethno botanical and historical section of the manuscript; $\mathrm{MW}-\mathrm{K}$ and IS: Contributed to the phytochemical and analytical parts of the manuscript; AJ: Contributed to the antimicrobial activity section of the manuscript; AM: Developed the entire concept of this review, wrote bioactivity and pharmacology sections, and critically read and corrected all parts of the paper.

\section{FUNDING}

The scientific activity of Botanical Garden of Medicinal Plants is supported by the Ministry of Science and Higher Education of Poland, grant No. 215259/E-394/SPUB/2-16/1. AM and SZ get support from the Wroclaw Medical University grant No. ST.D030.17.028.

\section{ACKNOWLEDGMENTS}

Assistance in graphical abstract preparation by Miss Hanna Zielinska is kindly acknowledged. The support of Wroclaw Medical University is acknowledged.

\section{SUPPLEMENTARY MATERIAL}

The Supplementary Material for this article can be found online at: https://www.frontiersin.org/articles/10.3389/fphar. 2018.00299/full\#supplementary-material

Supplementary Figure 1 | Chelidonium majus has been highly valued by ancient physicians. Nowadays, it is still attracting researchers' and clinicians' attention for its unique composition of the yellow latex, rich in alkaloids and proteins.

Supplementary Table 1 | The examples of conditions used to isolate alkaloids from C. majus.

Supplementary Table 2 | The examples of application the separation techniques in analysis of alkaloids from C. majus.
Bauhin, J. (1651). Historia Plantarum Uniuersalis: Noua et Absolutissima Cum Consensu et Dissensu Circa eas. 3, Vol. 483. Ebrodunum.

Benchabane, M., Schlüter, U., Vorster, J., Goulet, M. C., and Michaud, D. (2010). Plant cystatins. Biochimie 92, 1657-1666. doi: 10.1016/j.biochi.2010.06.006

Best, M. R., and Brightman, F. H. (1999). The Book of Secrets of Albertus Magnus. York Beach, ME: Weiser Books.

Biswas, S. J. (2013). Chelidonium majus: a review on pharmacological activities and clinical effects. Global J. Res. Med. Plants Indigen. Med. 2, 238-245.

Boegge, S. C., Kesper, S., Verspohl, E. J., and Nahrstedt, A. (1996). Reduction of ACh-induced contraction of rat isolated ileum by coptisine, $(+)$-caffeoylmalic acid, Chelidonium majus, and Corydalis lutea extracts. Planta Med. 62, 173-174. doi: 10.1055/s-2006-957845

Bogucka-Kocka, A., and Zalewski, D. (2017). Qualitative and quantitative determination of main alkaloids of Chelidonium majus L. using thin-layer chromatographic-densitometric method. Acta Chromatogr. 29, 385-397. doi: 10.1556/1326.2017.29.3.09

Borghini, A., Pietra, D., di Trapani, C., Madau, P., Lubinu, G., and Bianucci, A. M. (2015). Data mining as a predictive model for Chelidonium majus extracts production. Ind. Crops Prod. 64, 25-32. doi: 10.1016/j.indcrop.2014.10.066 
Bozeman, E. N., Srivatsan, S., Mohammadi, H., Daniels, D., Shashidharamurthy, R., and Selvaraj, P. (2012). Ukrain ${ }^{\circledR}$, a plant derived semi-synthetic compound, exerts antitumor effects against murine and human breast cancer and induce protective antitumor immunity in mice. Exp. Oncol. 34, 340-347.

Bugatti, C., Colombo, M. L., and Tomè, F. (1987). High-performance liquid chromatographic separation of quaternary alkaloids of Chelidonium majus L. Roots. J. Chromatogr. A, 393, 312-316. doi: 10.1016/S0021-9673(01) 94228-1

Cahlíková, L., Opletal, L., Kurfürst, M., Macáková, K., Kulhánková, A., and Hostálková, A. (2010). Acetylcholinesterase and butyrylcholinesterase inhibitory compounds from Chelidonium majus (Papaveraceae). Nat. Prod. Commun. 5, 1751-1754.

Capistrano, I. R., Wouters, A., Lardon, F., Gravekamp, C., Apers, S., and Pieters, L. (2015). In vitro and in vivo investigations on the antitumour activity of Chelidonium majus. Phytomedicine 22, 1279-1287. doi: 10.1016/j.phymed.2015.10.013

Cho, K. M., Yoo, I. D., and Kim, W. G. (2006). 8-hydroxydihydrochelerythrine and 8-hydroxydihydrosanguinarine with a potent acetylcholinesterase inhibitory activity from Chelidonium majus L. Biol. Pharm. Bull. 29, 2317-2320. doi: $10.1248 / \mathrm{bpb} .29 .2317$

Cirić, A., Vinterhalter, B., Šavikin-Fodulović, K., Soković, M., and Vinterhalter, D. (2008). Chemical analysis and antimicrobial activity of methanol extracts of celandine (Chelidonium majus L.) plants growing in nature and cultured in vitro. Arch. Biol. Sci. 60, 7-8. doi: 10.2298/ABS0801169C

Colombo, M. L., and Bosisio, E. (1996). Pharmacological activities of Chelidonium majus L. (Papaveraceae). Pharmacol. Res. 33, 127-134.

Cordes, N., Plasswilm, L., Bamberg, M., and Rodemann, H. P. (2002). Ukrain ${ }^{\circledR}$, an alkaloid thiophosphoric acid derivative of Chelidonium majus L. protects human fibroblasts but not human tumour cells in vitro against ionizing radiation. Int. J. Radiat. Biol. 78, 17-27. doi: 10.1080/09553000110 089991

Cordes, N., Blaese, M. A., Plasswilm, L., Rodemann, H. P., and Van Beuningen, D. (2003). Fibronectin and laminin increase resistance to ionizing radiation and the cytotoxic drug Ukrain ${ }^{\circledR}$ in human tumour and normal cells in vitro. Int. J. Radiat. Biol. 79, 709-720. doi: 10.1080/09553000310001610240

Czekański, M. (2007). Zioła św. Hildegardy. Lecznicze Właściwości ziół według opisu św. Kraków: Hildegardy z Bingen. Wydawnictwo M.

De Verdmon, L. J. (1936). "Jaskólcze ziele," in Kuracja roslinna (Warszawa: Wydawnictwo M. Arcta), 267-268.

Deljanin, M., Nikolic, M., Baskic, D., Todorovic, D., Djurdjevic, P., Zaric, M., et al. (2016). Chelidonium majus crude extract inhibits migration and induces cell cycle arrest and apoptosis in tumor cell lines. J. Ethnopharmacol. 190, 362-371. doi: 10.1016/j.jep.2016.06.056

Deng, A. J., Zhang, Z. H., Li, Q., Ma, L., and Qin, H. L. (2016). Two new hopanetype triterpenes from the aerial part of Chelidonium majus. Phytochem. Lett. 17, 75-78. doi: 10.1016/j.phytol.2016.07.009

Dickenson, P. B., and Fairbairn, J. W. (1975). The ultrastructure of the alkaloidal vesicles of Papaver somniferum latex. Ann. Bot. 39, 707-712.

El-Readi, M. Z., Eid, S., Ashour, M. L., Tahrani, A., and Wink, M. (2013). Modulation of multidrug resistance in cancer cells by chelidonine and Chelidonium majus alkaloids. Phytomedicine 20, 282-294. doi: 10.1016/j.phymed.2012.11.005

Ernst, E., and Schmidt, K. (2005). Ukrain ${ }^{\circledR}$ - a new cancer cure? A systematic review of randomised clinical trials. BCM Cancer 5:69. doi: 10.1186/1471-2407-5-69

Etxenagusia, M. A., Anda, M., González-Mahave, I., Fernández, E., and Fernández de Corrès, L. (2000). Contact dermatitis from Chelidonium majus (greater celandine). Contact Derm. 43:47.

European Medicines Agency (2011). Assessment Report on Chelidonium majus L., Herba. London.

Farrugia, D., and Slevin, M. L. (2000). Alternative cancer cures. Lancet Oncol, 1, 204-205. doi: 10.1016/S1470-2045(00)00147-9

Farrugia, D., and Slevin, M. L. (2001). Clinical testing of Ukrain ${ }^{\circledR}$ - Authors' reply. Lancet Oncol. 2:10. doi: 10.1016/S1470-2045(00)00189-3

Fulde, G., and Wichtl, M. (1994). Analytic von Schöllkraut. Deutsch Apoth Ztg. 134, 1031-1035.

Funel, N., Costa, F., Pettinari, L., Taddeo, A., Sala, A., Chiriva-Internati, M., et al. (2010). Ukrain affects pancreas cancer cell phenotype in vitro by targeting
MMP-9 and Intra-/Extracellular SPARC expression. Pancreatology 10, 545-552. doi: $10.1159 / 000266127$

Gagliano, N., Moscheni, C., Torri, C., Magnani, I., Bertelli, A. A., Nowicky, W., et al. (2006). Effect of Ukrain on matrix metalloproteinase-2 and Secreted Protein Acidic and Rich in Cysteine (SPARC) expression in human glioblastoma cells. Anti Cancer Drugs 17, 189-194. doi: 10.1097/00001813-200602000-00010

Gagliano, N., Moscheni, C., Torri, C., Donetti, E., Magnani, I., Costa, F., et al. (2007). Ukrain modulates glial fibrillary acidic protein, but not connexin 43 expression, and induces apoptosis in human cultured glioblastoma cells. Anti Cancer Drugs 18, 669-676. doi: 10.1097/CAD.0b013e32808bf9ec

Gagliano, N., Pettinari, L., Aureli, M., Martinelli, C., Colombo, E., Costa, F., et al. (2011). Malignant phenotype of renal cell carcinoma cells is switched by Ukrain administration in vitro. Anti Cancer Drugs 22, 749-762. doi: 10.1097/CAD.0b013e328346c7f7

Gagliano, N., Volpari, T., Clerici, M., Pettinari, L., Barajon, I., Portinaro, N., et al. (2012). Pancreatic cancer cells retain the epithelial-related phenotype and modify mitotic spindle microtubules after the administration of ukrain in vitro. Anti Cancer Drugs 23, 935-946. doi: 10.1097/CAD.0b013e32835507bc

Gañán, N. A., Dias, A. M. A., Bombaldi, F., Zygadlo, J. A., Brignole, E. A., De Sousa, H. C., et al. (2016). Alkaloids from Chelidonium majus L.: fractionated supercritical CO2 extraction with co-solvents. Sep. Purif. Technol. 165, 199-207. doi: 10.1016/j.seppur.2016.04.006

Gebhardt, R. (2009). Prevention of cadmium-induced toxicity in liver-derived cells by the combination preparation Hepeel ${ }^{\circledR}$. Environ. Toxicol. Pharmacol. 27, 402-409. doi: 10.1016/j.etap.2009.01.006

Gerenčer, M., Turecek, P. L., Kistner, O., Mitterer, A., Savidis-Dacho, H., and Barrett, N. P. (2006). In vitro and in vivo anti-retroviral activity of the substance purified from the aqueous extract of Chelidonium majus L. Antivir. Res. 72, 153-156. doi: 10.1016/j.antiviral.2006.03.008

Gilca, M., Gaman, L., Panait, E., Stoian, I., and Atanasiu, V. (2010). Chelidonium majus-an integrative review: traditional knowledge versus modern findings. Forschende Komplementärmedizin. 17, 241-248. doi: 10.1159/000321397

Grosso, C., Ferreres, F., Gil-Izquierdo, A., Valentão, P., Sampaio, M., Lima, J., et al. (2014). Box-Behnken factorial design to obtain a phenolic-rich extract from the aerial parts of Chelidonium majus L. Talanta 130, 128-136. doi: 10.1016/j.talanta.2014.06.043

Gu, Y., Qian, D., Duan, J. A., Wang, Z., Guo, J., Tang, Y., et al. (2010). Simultaneous determination of seven main alkaloids of Chelidonium majus L. by ultraperformance LC with photodiode-array detection. J. Sep. Sci. 33, 1004-1009. doi: 10.1002/jssc. 200900690

Habermehl, D., Kammerer, B., Handrick, R., Eldh, T., Gruber, C., Cordes, N., et al. (2006). Proapoptotic activity of Ukrain ${ }^{\circledR}$ is based on Chelidonium majus L. alkaloids and mediated via a mitochondrial death pathway. BMC Cancer 6:14. doi: 10.1186/1471-2407-6-14

Hagel, J. M., Yeung, E. C., and Facchini, P. J. (2008). Got milk? The secret life of laticifers. Trends Plant Sci. 13, 631-639. doi: 10.1016/j.tplants.2008.09.005

Hahn, R., and Nahrstedt, A. (1993). Hydroxycinnamic acid derivatives, caffeoylmalic and new caffeoylaldonic acid esters, from Chelidonium majus. Planta Med. 59, 71-75. doi: 10.1055/s-2006-959608

Han, L. F., Nowicky, W., and Gutmann, V. (1991). Reversed-phase highperformance liquid chromatographic separation of tertiary and quaternary alkaloids from Chelidonium majus L. J. Chromatogr. A 543, 123-128. doi: 10.1016/S0021-9673(01)95760-7

Hansel, R., Keller, K., Rimpler, H., and Schneider, G. (1992). Hangers Handbuch der Pharmazeutischen Praxis. Berlin; Heilderberg: Springer-Verlag.

Havelek, R., Seifrtova, M., Kralovec, K., Habartova, K., Cahlikova, L., and Rezacova, M. (2016a). Chelidonine and homochelidonine induce cell death through cell cycle checkpoints and MAP kinase pathways. Nat. Prod. Commun. 12, 1419-1430.

Havelek, R., Seifrtova, M., Kralovec, K., Krocova, E., Tejkalova, V., Novotny, I., et al. (2016b). Comparative cytotoxicity of chelidonine and homochelidonine, the dimethoxy analogues isolated from Chelidonium majus L. (Papaveraceae), against human leukemic and lung carcinoma cells. Phytomedicine 23, 253-266. doi: 10.1016/j.phymed.2016.01.001

Henschke, A. (1888). Über das Chelidonin. Arch. Pharm. 226, 624-644. doi: 10.1002/ardp.18882261404

Hiller, K. O., Ghorbani, M., and Schlicher, H. (1998). Antispasmodic and relaxant activity of chelidonine, protopine, coptisine, and Chelidonium 
majus extracts on isolated guinea-pig ileum. Planta Med. 64, 758-760. doi: 10.1055/s-2006-957576

Horváth, G., Molnár, P., Farkas, Á., Szabó, L. G., Turcsi, E., and Deli, J. (2010). Separation and identification of carotenoids in flowers of Chelidonium majus L. and Inflorescences of Solidago canadensis L. Chromatographia. 71, 103-108. doi: 10.1365/s10337-010-1510-4

Imanshahidi, M., and Hosseinzadeh, H. (2008). Pharmacological and therapeutic effects of Berberis vulgaris and its active constituent, Berberine - review article. Phytother. Res. 22, 999-1012. doi: 10.1002/ptr.2399

Isolani, M. E., Pietra, D., Balestrini, L., Borghini, A., Deri, P., Imbriani, M., et al. (2012). The in vivo effect of chelidonine on the stem cell system of planarians. Eur. J. Pharmacol. 686, 1-7. doi: 10.1016/j.ejphar.2012.03.036

Jang, S. I., Kim, B. H., Lee, W. Y., An, S. J., Choi, H. G., Jeon, B. H., et al. (2004). Stylopine from Chelidonium majus inhibits LPS-induced inflammatory mediators in RAW 264.7 cells. Arch. Pharm. Res. 27, 923-929. doi: 10.1007/BF02975845

Jarić S., Popović Z., Mačukanović-Jocić M., Djurdjević L., Mijatović M., KaradŽić B., et al. (2007). An ethnobotanical study on the usage of wild medicinal herbs from Kopaonik Mountain (Central Serbia). J. Ethnopharmacol. 111, 160-175. doi: 10.1016/j.jep.2006.11.007

Jesionek, W., Fornal, E., Majer-Dziedzic, B., Móricz, Á. M., Nowicky, W., and Choma, I. M. (2016). Investigation of the composition and antibacterial activity of Ukrain ${ }^{\mathrm{TM}}$ drug using liquid chromatography techniques. J. Chromatogr. A 1429, 340-347. doi: 10.1016/j.chroma.2015.12.015

Jones, W. H. S. (1966). Pliny Natural History with an English Translation in Ten Volumes, Vol. VII, Libri XXIV-XXVII. London: Harvard University Press.

Jursky, F., and Baliova, M. (2011). Differential effect of the benzophenanthridine alkaloids sanguinarine and chelerythrine on glycine transporters. Neurochem. Int. 58, 641-647. doi: 10.1016/j.neuint.2011.02.001

Kadan, G., Gözler, T., and Hesse, M. (1992). (+)-Norchelidonine from Chelidonium majus. Planta Med. 58:477. doi: 10.1055/s-2006-961523

Kadan, G., Gözler, T., and Shamma, M. (1990). (-)-Turkiyenine, a new alkaloid from Chelidonium majus. J. Nat. Prod. 53, 531-532. doi: 10.1021/np50068a046

Kaminskyy, V., Lootsik, M., and Stoika, R. (2006). Correlation of the cytotoxic activity of four different alkaloids, from Chelidonium majus (greater celandine), with their DNA intercalating properties and ability to induce breaks in the DNA of NK/Ly murine lymphoma cells. Open Life Sci. 1, 2-15. doi: 10.2478/s11535-006-0001-y

Kéry, A., Horváth, J., Nász, I., Verzár-Petri, G., Kulcsár, G., and Dán, P. (1987). Antiviral alkaloid in Chelidonium majus L. Acta Pharm. Hung. 57, 19-25.

Kedzia, B., Hołderna-Kedzia, E., Gozdzicka-Józefiak, A., and Buchwald, W. (2003). The antimicrobial activity of Chelidonium majus L. Post. Fitoter. 4, 236-243.

Kedzia, B., and Hołderna-Kedzia, E. (2013). The effect of alkaloids and other groups of plant compounds on bacteria and fungi. Post. Fitoter. 1, 8-16.

Kędzia, B., Łozykowska, K., and Gryszczynska, A. (2013). Chemical composition and contents of biological active substances in Chelidonium majus L. Post. Fitoter. 3, 174-181

Kim, D. S., Kim, S. J., Kim, M. C., Jeon, Y. D., Um, J., and Hong, S. H. (2012). The therapeutic effect of chelidonic acid on ulcerative colitis. Biol. Pharm. Bull. 35, 666-671. doi: 10.1248/bpb.35.666

Kim, S. H., Hong, J. H., and Lee, Y. C. (2015). Chelidonine, a principal isoquinoline alkaloid of Chelidonium majus, attenuates eosinophilic airway inflammation by suppressing IL-4 and eotaxin-2 expression in asthmatic mice. Pharmacol. Rep. 67, 1168-1177. doi: 10.1016/j.pharep.2015.04.013

Kohlmünzer, S. (2000). Farmakognozja. Warszawa: Wyd Lek PZWL, 450-451.

Kokoska, L., Polesny, Z., Rada, V., Nepovim, A., and Vanek, T. (2002). Screening of some Siberian medicinal plants for antimicrobial activity. J. Ethnopharmacol. 82, 51-53. doi: 10.1016/S0378-8741(02)00143-5

Koleva, V., Dragoeva, A., Nanova, Z., Koynova, T., and Dashev, G. (2015). An ethnobotanical study on current status of some medicinal plants used in Bulgaria. Int. J. Curr. Microbiol. App. Sci. 4, 297-305.

Kopytko, Y. F., Dargaeva, T. D., Sokolskaya, T. A., Grodnitskaya, E. I., and Kopnin, A. A. (2005). New methods for the quality control of a homeopathic matrix tincture of greater celandine. Pharm. Chem. J. 39, 603-609. doi: 10.1007/s11094-006-0028-4

Koriem, K. M., Arbid, M. S., and Asaad, G. F. (2013). Chelidonium majus leaves methanol extract and its chelidonine alkaloid ingredient reduce cadmium-induced nephrotoxicity in rats. J. Nat. Med. 67, 159-167. doi: $10.1007 /$ s11418-012-0667-6
Kozicka, A., and Radomanski, T. (1963). Próby Leczenia Rzesistkowicy u Dziewczynek Przy Pomocy Wyciagów z Glistnika Jaskótcze Ziele - Chelidonium majus, Vol. 18, Lublin: Annales Universitatis Mariae Curie-Sklodowska.

Krahulcová, A. (1982). Cytotaxonomic Study of Chelidonium majus L. s. 1. Folia Geobot. Phytotax. 17, 238-270.

Kuenzel, J., Geisler, K., Strahl, O., Grundtner, P., Beckmann, M. W., and Dittrich, R. (2013). Chelidonium majus and its effects on uterine contractility in a perfusion model. Eur. J. Obstet. Gynecol. Reprod. Biol. 169, 213-217. doi: 10.1016/j.ejogrb.2013.03.014

Kujawska, M., Klepacki, P., and Łuczaj, Ł. (2017). Fischer's Plants in folk beliefs and customs: a previously unknown contribution to the ethnobotany of the Polish-Lithuanian-Belarusian borderland. J. Ethnobiol. Ethnomed. 13:20. doi: 10.1186/s13002-017-0149-8

Kujawska, M., Łuczaj, Ł., Sosnowska, J., and Klepacki, P. (2016). Rośliny w Wierzeniach i Zwyczajach Ludowych. Słownik Adama Fischera. Prace Materiały Etnograficzne, Tom XXXVII, Wrocław, 367-368.

Kulp, M., Bragina, O., Kogerman, P., and Kaljurand, M. (2011). Capillary electrophoresis with led-induced native fluorescence detection for determination of isoquinoline alkaloids and their cytotoxicity in extracts of Chelidonium majus L. J. Chromatog. A. 1218, 5298-5304. doi: 10.1016/j.chroma.2011.06.016

Kulp, M., and Bragina, O. (2013). Capillary electrophoretic study of the synergistic biological effects of alkaloids from Chelidonium majus L. in normal and cancer cells. Anal. Bioanal. Chem. 405, 3391-3397. doi: 10.1007/s00216-0136755-y

Kustrak, D., Petrićić, J., and Kalodera, Z. (1982). Seasonal changes of alkaloid contents in celandine (Chelidonium majus L.). Acta Pharm. Jugosl. 32, 225-230.

Kursinszki, L., Sárközi, Á., Kéry, Á., and Szöke, É. (2006). Improved RP-HPLC method for analysis of isoquinoline alkaloids in extracts of Chelidonium majus. Chromatographia S63, 131-135. doi: 10.1365/s10337-006-0808-8

Kuźniewski, E., and Augustyn-Puziewicz, J. (1999). Przyroda Apteka. Wrocław: Wydawnictwo Dolnoślaskie.

Kwasniewski, V. (1958). Untersuchungen über die nichtalkaloidschen Inhaltstoffe des Schöllkrauts (Chelidonium majus L.) Pharmazie 13, 3363-3364.

Lanfeld, J., Kroutil, M., Mársalek, E., Slavík, J., Preininger, V., and Simánek, V. (1981). Antiinflammatory activity of quaternary benzophenanthridine alkaloids from Chelidonium majus. Planta Med. 43, 161-165. doi: 10.1055/s-2007-971493

Lanvers-Kaminsky, C., Nolting, D. M., Köster, J., Schröder, A., Sandkötter, J., and Boos, J. (2006). In-vitro toxicity of Ukrain against human Ewing tumor cell lines. Anti Cancer Drugs 17, 1025-1030. doi: 10.1097/01.cad.0000231467.61911.50

Lee, Y. C., Kim, S. H., Roh, S. S., Choi, H. Y., and Seo, Y. B. (2007). Suppressive effects of Chelidonium majus methanol extract in knee joint, regional lymph nodes, and spleen on collagen-induced arthritis in mice. J. Ethnopharmacol. 112, 40-48. doi: 10.1016/j.jep.2007.01.033

Ma, W. G., Fukushi, Y., Tahava, S., and Osawa, T. (2000). Fungitoxic alkaloids from Hokkaido Papaveraceae. Fitoterapia 71, 527-534. doi: 10.1016/S0367-326X(00)00193-3

Malinowska, I., Gadzikowska, M., Waksmundzka- Hajnos, M., and Kramek, A. (2005). Mobile-phase velocity - a tool for separation of Alkaloids by OPLC. J. Planar. Chromat. 18, 176-180. doi: 10.1556/JPC.18.2005.3.1

Malinowska, I., Studzinski, M., Malinowski, H., and Gadzikowska, M. (2017). Retention and separation changes of ternary and quaternary Alkaloids from Chelidonium majus L. by TLC under the influence of external magnetic field. Chromatographia 80, 923-930. doi: 10.1007/s10337-017-3293-3

Mamedov, N., Gardner, Z., and Craker, L. E. (2004). Medicinal plants used in Russia and Central Asia for the treatment of selected skin conditions. J. Herbs Spices Med. Plants 11, 191-222. doi: 10.1300/J044v11n01_07

Marcin of Urzędowo (1595), Herbarz Polski, to iest o Przyrodzeniu zioł y Drzew Rozmaitych, y innych rzeczy do Lekarzy Nalezacych, Ksiegi Dwoje, w Krakowie w Drukarni Lazarzowey, 90-91.

Matysik, G., and Jusiak, L. (1990). Stepwise gradient in thin-layer chromatography of Chelidonium alkaloids. J. Chromatogr. A 518, 273-276. doi: 10.1016/S0021-9673(01)93188-7

Mayer, J. G., Uehleke, B., and Saum, K. (2003). Handbuch der Klosterheilkunde. Muenchen: Verlag Zabert Sandmann.

Mazzanti, G., Di Sotto, A., Franchitto, A., Mammola, C. L., Mariani, P., Mastrangelo, S., et al. (2009). Chelidonium majus is not hepatotoxic in Wistar 
rats, in a 4 weeks feeding experiment. J. Ethnopharmacol. 126, 518-524. doi: 10.1016/j.jep.2009.09.004

Menale, B., Amato, G., Di Prisco, C., and Muoio, R. (2006). Traditional uses of plants in North-Western Molise (Central Italy). Delpinoa 48, 29-36.

Meng, F., Zuo, G., Hao, X., Wang, G., Xiao, H., Zhang, J., et al. (2009). Antifungal activity of the benzo [c] phenanthridine alkaloids from Chelidonium majus Linn against resistant clinical yeast isolates. J. Ethnopharmacol. 125, 494-496. doi: 10.1016/j.jep.2009.07.029

Menković, N., Savikin, K., Tasić, S., Zdunić, G., Stesević, D., Milosavljević, S., et al. (2011). Ethnobotanical study on traditional uses of wild medicinal plants in Prokletije Mountains (Montenegro). J. Ethnopharmacol. 133, 97-107. doi: 10.1016/j.jep.2010.09.008

Migas, P., Heyka, M., Pobłocka-Olech, L., and Krauze-Baranowska, M. (2012). BMD-TLC - the useful technique for quantitative analysis of chelidonine, chelerithrine and berberine in herbal drugs. J. Planar. Chromatogr. 25, 439-444. doi: 10.1556/JPC.25.2012.5.9

Mikołajczak, P. Ł., Kedzia, B., Ozarowski, M., Kujawski, R., Bogacz, A., Bartkowiak-Wieczorek, J., et al. (2015). Evaluation of anti-inflammatory and analgesic activities of extracts from herb of Chelidonium majus L. Cent. Eur. J. Immunol. 40, 400-410. doi: 10.5114/ceji.2015.54607

Mitra, S., Sur, R. K., Roy, A., and Mukherjee, A. S. (1996). Effect of Chelidonium majus L. on experimental hepatic tissue injury. Phytother Res. 10, 354-356.

Móricz, Á. M., Fornal, E., Jesionek, W., Majer-Dziedzic, B., and Choma, I. M. (2015). Effect-directed isolation and identification of antibacterial Chelidonium majus L. Alkaloids. Chromatographia 78, 707-716. doi: 10.1007/s10337-015-2870-6

Móricz, Á. M., Ott, P. G., Yüce, I., Darcsi, A., Béni, S., and Morlock, G. E. (2018). Effect-directed analysis via hyphenated high-performance thin-layer chromatography for bioanalytical profiling of sunflower leaves. J. Chromatogr. A. 19, 213-220. doi: 10.1016/j.chroma.2017.12.034

Mustafa, B., Hajdari, A., Pajazita, Q., Syla, B., Quave, C. L., and Pieroni, A. (2012). An ethnobotanical survey of the Gollak region, Kosovo. Genet. Resour. Crop. Evol. 59, 739-754. doi: 10.1007/s10722-011-9715-4

Nadova, S., Miadokova, E., Alfoldiova, L., Kopaskova, M., Hasplova, K., Hudecova, A., et al. (2008). Potential antioxidant activity, cytotoxic and apoptosis-inducing effects of Chelidonium majus L. extract on leukemia cells. Neuroendocrinol. Lett. 29, 649-652.

Nawrot, R., Barylski, J., Lippmann, R., Altschmied, L., and Mock, H. P. (2016). Combination of transcriptomic and proteomic approaches helps to unravel the protein composition of Chelidonium majus L. milky sap. Planta 244, 1055-1064. doi: 10.1007/s00425-016-2566-7

Nawrot, R., Kalinowski, A., and Gozdzicka-Jozefiak, A. (2007a). Proteomic analysis of Chelidonium majus milky sap using two-dimensional gel electrophoresis and tandem mass spectrometry. Phytochemistry 68, 1612-1622. doi: 10.1016/j.phytochem.2007.03.039

Nawrot, R., Lesniewicz, K., Pienkowska, J., and Gozdzicka-Jozefiak, A. (2007b). A novel extracellular peroxidase and nucleases from a milky sap of Chelidonium majus. Fitoterapia 78, 496-501. doi: 10.1016/j.fitote.2007.04.012

Nawrot, R., Tomaszewski, L., Czerwoniec, A., and Gozdzicka-Józefiak, A. (2013). Identification of a coding sequence and structure modeling of a Glycine-Rich RNA-Binding Protein (CmGRP1) from Chelidonium majus L. Plant Mol. Biol. Rep. 31, 470-476. doi: 10.1007/s11105-012-0510-y

Nawrot, R., Wołun-Cholewa, M., and Gozdzicka-Józefiak, A. (2008). Nucleases isolated from Chelidonium majus L. milky sap can induce apoptosis in human cervical carcinoma HeLa cells but not in Chinese Hamster Ovary CHO cells. Folia Histochem. Cytobiol. 46, 79-83. doi: 10.2478/v10042-008-0011-x

Nawrot, R., Zauber, H., and Schulze, W. X. (2014). Global proteomic analysis of Chelidonium majus and Corydalis cava (Papaveraceae) extracts revealed similar defense-related protein compositions. Fitoterapia 94, 77-87. doi: 10.1016/j.fitote.2014.01.015

Nawrot, R., Jozefiak, D., Sip, A., Kuzma, D., Musidlak, O., Gozdzicka-Józefiak, A., et al. (2017a). Isolation and characterization of a non-specific lipid transfer protein from Chelidonium majus L. latex. Int. J. Biol. Macromol. 104(Pt A), 554-563. doi: 10.1016/j.ijbiomac.2017.06.057

Nawrot, R., Lippmann, R., Matros, A., Musidlak, O., Nowicki, G., and Mock, H. P. (2017b). Proteomic comparison of Chelidonium majus L. latex in different phases of plant development. Plant Physiol. Biochem. 112, 312-325. doi: 10.1016/j.plaphy.2017.01.010
Nečas, M., Dostál, J., Kejnovská, I., Vorlíčková, M., and Slavík, J. (2005). Molecular and crystal structures of $(+)$-homochelidonine, (+)-chelamine, and (-)norchelidonine. J. Mol. Struct. 734, 1-6. doi: 10.1016/j.molstruc.2004.08.006

Niu, C.-Q., and He, L.-Y. (1994). Determination of isoquinoline alkaloids in Chelidonium majus L. by ion-pair high-performance liquid chromatography. J. Chromatogr. A 542, 193-199.

Niu, X. F., Zhou, P., Li, W. F., and Xu, H. B. (2011). Effects of chelerythrine, a specific inhibitor of cyclooxygenase-2, on acute inflammation in mice. Fitoterapia 82, 620-625. doi: 10.1016/j.fitote.2011.01.020

Noureini, S. K., Esmaeili, H., Abachi, F., Khiali, S., Islam, B., Kuta, M., et al. (2017). Selectivity of major isoquinoline alkaloids from Chelidonium majus towards telomeric G-quadruplex: a study using a transition-FRET (t-FRET) assay. Biochim. Biophys. Acta. 1861, 2020-2030. doi: 10.1016/j.bbagen.2017.05.002

Noureini, S. K., and Wink, M. (2009). Transcriptional down regulation of hTERT and senescence induction in HepG2 cells by chelidonine. World J. Gastroenterol. 15, 3603-3610. doi: 10.3748/wjg.15.3603

Nowicky, W. (2001). Clinical testing of Ukrain ${ }^{\circledR}$. Lancet Oncol. 2:10. doi: 10.1016/S1470-2045(00)00188-1

Oh, H.-A., Kim, H.-M., and Jeong, H.-J. (2011). Beneficial effects of chelidonic acid on a model of allergic rhinitis. Int. Immunopharmacol. 11, 39-45. doi: 10.1016/j.intimp.2010.10.002

Orland, A., Knapp, K., König, G. M., Ulrich-Merzenich, G., and Knöß, W. (2014). Combining metabolomic analysis and microarray gene expression analysis in the characterization of the medicinal plant Chelidonium majus L. Phytomedicine 21, 1587-1596. doi: 10.1016/j.phymed.2014.07.012

Osbaldeston, T. A., and Wood, R. P. A. (2000). Dioscorides de Materia Medica, Being a Herbal with Many other Materials Written in Greek in the First Century of the Common Era. An Indexed Version in Modern English; Book 2. Johannesburg: Ibidis Press, 352-355.

Pantano, F., Mannocchi, G., Marinelli, E., Gentili, S., Graziano, S., Busardò, F. P., et al. (2017). Hepatotoxicity induced by greater celandine (Chelidonium majus L.): a review of the literature. Eur. Rev. Med. Pharmacol. Sci. 21, 46-52.

Panzer, A., Joubert, A. M., Eloff, J. N., Albrecht, C. F., Erasmus, E., and Seegers, J. C. (2000). Chemical analyses of Ukrain ${ }^{\circledR}(T M)$, a semi-synthetic Chelidonium majus alkaloid derivative, fail to confirm its trimeric structure. Cancer Lett. 160, 237-241. doi: 10.1016/S0304-3835(00)00595-4

Panzer, A., Joubert, A. M., Bianchi, P. C., and Seegers, J. C. (2001). The antimitotic effects of Ukrain ${ }^{\circledR}(\mathrm{TM})$, a Chelidonium majus alkaloid derivative, are reversible in vitro. Cancer Lett. 150, 85-92. doi: 10.1016/S0304-3835(99) 00375-4

Park, J. E., Cuong, T. D., Hung, T. M., Lee, I., Na, M., Kim, J. C., et al. (2011). Alkaloids from Chelidonium majus and their inhibitory effects on LPS-induced NO production in RAW264.7 cells. Bioorg. Med. Chem. Lett. 21, 6960-6963. doi: 10.1016/j.bmcl.2011.09.128

Paul, A., Das, j., Das, S., Samadder, A., and Khuda-Bukhsh, A. R. (2013). Poly (lactide-coglycolide) nano-encapsulation of chelidonine, an active bioingredient of greater celandine (Chelidonium majus), enhances its ameliorative potential against cadmium induced oxidative stress and hepatic injury in mice. Envir. Toxicol. Phar. 36, 937-947. doi: 10.1016/j.etap.2013.08.008

Paulsen, J., Yahyazadeh, M., Hänsel, S., Kleinwächter, M., Ibrom, K., and Selmar, D. (2015). 13,14-Dihydrocoptisine - The genuine alkaloid from Chelidonium majus. Phytochemistry 111, 149-153. doi: 10.1016/j.phytochem.2015.01.006

Pavao, M. L., and Pinto, R. E. (1995). Densitometric assays for the evaluation of water soluble alkaloids from Chelidonium majus L. (Papaveraceae) roots in the Azores, along one year cycle. Arquipelago 13, 85-91.

Pepeljnjak, S., Kosalec, I., Kalodera, Z., and Kustrak, D. (2003). "Natural antimycotics from Croatia plants" in Plant- Derived Antimycotics. Current Trends and Future Prospects, eds M. Rai and D. Mares (New York, NY: Food Products Press), 49-79.

Petruczynik, A., Gadzikowska, M., and Waksmundzka-Hajnos, M. (2002). Optimization of the separation of some Chelidonium maius L. alkaloids by reversed phase high-performance liquid chromatography using cyanopropyl bonded stationary phase. Acta Pol. Pharm. 59, 61-64.

Petruczynik, A., Waksmundzka-Hajnos, M., Michniowski, T., Plech, T., Tuzimski, T., Hajnos, M. L., et al. (2007). Thin-Layer Chromatography of Alkaloids on Cyanopropyl Bonded Stationary Phases. Part I. J. Chromatogr. Sci. 45, 447-454. doi: $10.1093 /$ chromsci/45.7.447 
Petruczynik, A., Waksmundzka-Hajnos, M., Plech, T., Tuzimski, T., Hajnos, M. Ł., Józwiak, G., et al. (2008). TLC of Alkaloids on Cyanopropyl Bonded Stationary Phases. Part II. Connection with RP18 and Silica Plates. J. Chromatogr. Sci. 46, 291-297. doi: 10.1093/chromsci/46.4.291

Philchenkov, A., Kaminskyy, V., Zavelevich, M., and Stoika, R. (2008). Apoptogenic activity of two benzophenanthridine alkaloids from Chelidonium majus L. does not correlate with their DNA damaging effects. Toxicol. In Vitro 22, 287-295. doi: 10.1016/j.tiv.2007.08.023

Pieroni, A., Dibra, B., Grishaj, G., Grishaj, I., and Maçai, S. (2005). Traditional pytotherapy of the Albanians of Lepushe, Northern Albanian Alps. Fitoterapia 76, 379- 399. doi: 10.1016/j.fitote.2005.03.015

Pieroni, A., and Gray, C. (2008). Herbal and Food Folk Medicines of the Russlanddeutschen living in Künzelsau/ Taläcker, South-Western Germany. Phytother. Res. 22, 889-901. doi: 10.1002/ptr.2410

Poormazaheri, H., Baghban, K., Khosravi, D. N., Naghavi, M., Kalantar, E., Mohammadkhani, E., et al. (2017). High-content analysis of chelidonine and berberine from Iranian Chelidonium majus L. ecotypes in different ontogenetical stages using various methods of extraction. J. Agric. Sci. Technol. 19, 1381-1391.

Pothier, J., Galand, N., Tivollier, P., and Viel, C. (1993). Separation of quaternary alkaloids in plant extracts by OPLC. J. Planar. Chromatogr. 6, 220-222.

Prosen, H., and Pendry, B. (2016). Determination of shelf life of Chelidonium majus, Sambucus nigra, Thymus vulgaris and Thymus serpyllum herbal tinctures by various stability-indicating tests. Phytochem. Lett. 16, 311-323. doi: 10.1016/j.phytol.2016.05.012

Qu, Z., Zou, X., Zhang, X., Sheng, J., Wang, Y., Wang, Y., et al. (2016). Chelidonine induces mitotic slippage and apoptotic-like death in SGC-7901 human gastric carcinoma cells. Mol. Med. Rep. 13, 1336-1344. doi: 10.3892/mmr.2015.4683

Redžič, S. S. (2007). The ecological aspect of ethnobotany and ethnopharmacology of population in Bosnia and Herzegovina. Coll. Antropol. 31, 869-890.

Rentz, E. (1947). Zum Wirkungsmechanismus einiger bei Leber- und Gallenleiden angewandter pflanzlicher Mittel (Berberis, Chelidonium, Chelone). Naun Schmiedebergs Arch. 205, 332-339.

Rey, J. P., Levesque, J., and Pousset, J. L. (1993). Analytical studies of dl-stylopine in Chelidonium majus L. using high-performance liquid chromatography. J. Chromatogr. A 641, 180-183

Roberts, M. F., McCarthy, D., Kutchan, T. M., and Coscia, C. J. (1983). Localization of enzymes and alkaloidal metabolites in Papaver latex. Arch. Biochem. Biophys. 222, 599-609. doi: 10.1016/0003-9861(83)90558-1

Rogelj, B., Popoviča, T., Ritonjaa, A., Strukeljb, B., and Brzina, J. (1998). Chelidocystatin, a novel phytocystatin from Chelidonium majus. Phytochemistry 49, 1645-1649. doi: 10.1016/S0031-9422(98)00281-7

Rostanski, K. (1997). Szymon Syreniusz i jego dzieło. Wiadomości Botaniczne 41, 7-12.

Sārközi, Ā., Janicsāk, G., Kursinszki, L., and Kery, A. (2006). Alkaloid composition of Chelidonium majus L. studied by different chromatographic techniques. Chromatographia S63, 81-86. doi: 10.1365/s10337-006-0728-7

Sárközi, Á., Móricz, Á. M., Ott, P. G., Tyihák, E., and Kéry, Á. (2007). Investigation of Chelidonium alkaloids by use of a complex bioautographic system. J. Planar Chromat. 19, 267-272. doi: 10.1556/JPC.19.2006.4.2

Sárközi, Á., Then, M., and Szentmihályi, K. (2005). Mineral element content of greater celandine (Chelidonium majus L.). Acta Alimentaria 34, 113-120. doi: 10.1556/AAlim.34.2005.2.3

Savikin, K., Zdunić, G., Menković, N., Zivković, J., Cujić, N., Tereščenko, M., et al. (2013). Ethnobotanical study on traditional use of medicinal plants in South-Western Serbia, Zlatibor district. J. Ethnopharmacol. 146, 803-810. doi: 10.1016/j.jep.2013.02.006

Savran, B., Yerlikaya, A., Erdogan, E., and Genç, O. (2014). Anticancer agent Ukrain and bortezomib combination is synergistic in $4 \mathrm{~T} 1$ breast cancer cells. Anti. Canc. Agent Med. Chem. 14, 466-472. doi: 10.2174/18715206113139990318

Schmidt, E. (1888). Über Papaveraceen-Alkaloide. Arch Pharm. 226, 622-623. doi: 10.1002/ardp.18882261403

Schneider, M., Efferth, T., and Abdel - Aziz, H. (2016). Anti-inflammatory effects of herbal preparations STW5 and STW5-II in cytokine-challenged normal human colon cells. Front. Pharmacol. 7:393. doi: 10.3389/fphar.2016.00393

Seidler-Łozykowska, K., Kedzia, B., Bocianowski, J., Gryszczynska, A., Łowicki, Z., Opala, B., et al. (2016). Content of alkaloids and flavonoids in celandine
(Chelidonium majus L.) herb at the selected developmental phases. Acta Sci. Pol. Hortorum. Cultus 15, 161-172.

Selle, F. (1890). Über Papaveraceen alkaloide. Über die Alkaloide von Chelidonium majus. Arch. Pharm. 228, 441-462. doi: 10.1002/ardp.18902280905

Sevčik, J., Vicar, J., Ulrichová, J., Válka, I., Lemr, K., and Simánek, V. (2000). Capillary electrophoretic determination of sanguinarine and chelerythrine in plant extracts and pharmaceutical preparations. J. Chromatogr. A 866, 293-298. doi: 10.1016/S0021-9673(99)01126-7

Shafiee, A., and Jafarabadi, A. H. (1998). Corydine and norcorydine from the roots of Chelidonium majus. Planta Med. 64:489. doi: 10.1055/s-2006-957498

Shin, M. C., Jang, M. H., Chang, H. K., Lim, S., Han, S. M., Park, H. J., et al. (2003). Modulation of Chelidonii herba on glycine-activated and glutamate-activated ion currents in rat periaqueductal gray neurons. Clin. Chim. Acta. 337, 93-101. doi: 10.1016/j.cccn.2003.07.002

Shin, H. J., Kim, H. L., Kim, S. J., Chung, W. S., Kim, S. S., and Um, J. Y. (2011). Inhibitory effects of chelidonic acid on IL- 6 production by blocking NF- $\kappa B$ and caspase-1 in HMC-1 cells. Immunopharmacol. Immunotoxicol. 33, 614-619. doi: $10.3109 / 08923973.2011 .552508$

Slavik, J., and Slavikowa, J. (1977). Minor alkaloids from Chelidonium majus L. Collect. Czechoslov. Chem. Commun. 42, 2686-2693. doi: $10.1135 / \operatorname{cccc} 19772686$

Song, J. Y., Yang, H. O., Pyo, S. N., Jung, I. S., Yi, S. Y., and Yun, Y. S. (2002). Immunomodulatory activity of protein-bound polysaccharide extracted from Chelidonium majus. Arch. Pharm. Res. 25, 158-164. doi: 10.1007/BF02976557

Sǒukand, R., and Pieroni, A. (2016). The importance of a border: medical, veterinary, and wild food ethnobotany of the Hutsuls living on the Romanian and Ukrainian sides of Bukovina. J. Ethnopharmacol. 185, 17-40. doi: 10.1016/j.jep.2016.03.009

Sowa, I., Zielinska, S., Sawicki, J., Bogucka-Kocka, A., Staniak, M., BartusiakSzczesniak, E., et al. (2018). Systematic evaluation of chromatographic parameters for isoquinoline alkaloids on XB-C18 core-shell column using different mobile phase compositions. J. Anal. Methods Chem. 2018:9624327. doi: 10.1155/2018/9624327

Spiridonov, N. A., Konovalov, D. A., and Arkhipov, V. V. (2005). Cytotoxicity of some Russian ethnomedicinal plants and plant compounds. Phytother. Res. 19, 428-432. doi: 10.1002/ptr.1616

Stancic-Rotaru, M., Mititelu, M., Crasmaru, M., and Balaban, D. (2003). Spectroanalytical profile of flavonoids from Chelidonium majus L. Rom Biotechnol. Lett. 8, 1093-1100.

Stickel, F., Pöschl, G., Seitz, H. K., Waldherr, R., Hahn, E. G., and Schuppan, D. (2003). Acute hepatitis induced by greater celandine (Chelidonium majus). Scand. J. Gastroenterol. 38, 565-568. doi: 10.1080/00365520310000942

Stickl, O. (1928). Die bactericide Wirkung der Extrakte und Alkaloide des Schöllkrautes (Chelidonium maius) auf grampositive pathogene Mikroorganismen. Zeitschrift für Hygiene und Infektionskrankheiten. 108, 567-577. doi: 10.1007/BF02201625

Stuppner, H., and Ganzera, M. (1995). Application of $\beta$-cyclodextrin for the analysis of the main alkaloids from Chelidonium majus by capillary electrophoresis. J. Chromatogr. A 717, 271-277. doi: 10.1016/0021-9673(95)00643-1

Sun, Y., Li, Y., Zeng, J., Lu, Q., and Li, P. C. H. (2015). Microchip electrophoretic separation and fluorescence detection of chelerythrine and sanguinarine in medicinal plants. Talanta 142, 90-96. doi: 10.1016/j.talanta.2015.04.008

Szary, A. (2010). Flora Bieszczadów w Magiczno - Religijnej Swiadomości Łemków, Bojków i Hucułów, Bieszczad nr 16, Wyd. Ustrzyki Dolne: Towarzystwo Opieki nad Zabytkami oddział Bieszczadzki.

Szumiło, H. and Flieger, J. (1999). Application of differently modified silica gel in the TLC Analysis of alkaloids, J. Planar Chromatogr. 12, 466-470.

Szary, A. (2013). Tajemnice Bieszczadzkich Roślin Wczoraj i dziś. Rzeszów: Wyd. Carpathia.

Szary, A. (2015). Bieszczadzkie Motywy Roślinne Miedzy Swiatem Zywych a Kraina Zmartych. Rzeszów: Wyd. Carpathia.

Szot-Radziszewska, E. (2007). Lecznictwo ludowe Ukrainców na Przełomie XIX $i$ XX wieku, Analecta 16/1-2.

Szot-Radziszewska, E. (2012). "Swiat roślin w ludowych praktykach leczniczych na Kielecczyznie,” in Lek roślinny - historia i współczesność, T. 1, eds B. PłonkaSyroka, A. Syroka and K. Sudoł (Wrocław: Oficyna Wydawnicza Arboretum), $65-82$. 
Táborská, E., Bochoráková, H., Dostál, J., and Paulová, H. (1995). The greater celandine (Chelidonium majus L.) review of present knowledge. Ceska a Slovenska Farmacie, 44, 71-75.

Tan, G. T., Pezzuto, J. M., and Kinghorn, A. D. (1991). Evaluation of natural products as inhibitors of human immunodeficiency virus type 1 (HIV-1) reverse transcriptase. J. Nat. Prod. 54, 143-154.

Tan, H.-L., Chan, K.-G., Pusparajah, P., Duangjai, A., Saokaew, S., Mehmood Khan, T., et al. (2016). Rhizoma Coptidis: a potential cardiovascular protective agent. Front. Pharmacol. 7:362. doi: 10.3389/fphar.2016.00362

Then, M., Szentmihályi, K., Sárközi, A., Illés, V., and Forgács, E. (2000). Effect of sample handling on alkaloid and mineral content of aqueous extracts of greater celandine (Chelidonium majus L.). J. Chromatogr. A. 889, 69-74. doi: 10.1016/S0021-9673(00)00236-3

Tita, I., Mogoşanu, G. D., and Tita, M. G. (2009). Ethnobotanical inventory of medicinal plants fom the sout-west of Romania. Farmacia 57, 141-156.

Tomē, F., and Colombo, M. L. (1995). Distribution of alkaloids in Chelidonium majus and factors affecting their accumulation. Phytochemistry 40, 37-39. doi: 10.1016/0031-9422(95)00055-C

Vahlensieck, U., Hahn, R., Winterhoff, H., Gumbinger, H. G., Nahrstedt, A., and Kemper, F. H. (1995). The effect of Chelidonium majus herb extract on choleresis in the isolated perfused rat liver. Planta Med. 61, 267-270. doi: 10.1055/s-2006-958070

Varzaru, I., Untea, A. E., and Van, I. (2015). Determination of bioactive compounds with benefic potential on health in several medicinal plants. Romanian Biotechnol. Lett. 20:5.

Vavrecková, C., Gawlik, I., and Müller, K. (1996). Benzophenanthridine alkaloids of Chelidonium majus; II. Potent inhibitory action against the growth of human keratinocytes. Planta Med. 62, 491-494. doi: 10.1055/s-2006957955

Vokou, D., Katradi, K., and Kokkini, S. (1993). Ethnobotanical survey of Zagori (Epirus, Greece), a renowned centre of folk medicine in the past. J. Ethnopharmacol. 39, 187-196. doi: 10.1016/0378-8741(93)90035-4

Von Arnim, U., Peitz, U., Vinson, B., Gundermann, K. J., and Malfertheiner, P. (2007). STW 5, a phytopharmacon for patients with functional dyspepsia: results of a multicenter, placebo-controlled double-blind study. J. Gastroenterol. 102, 1268-1275. doi: 10.1111/j.1572-0241.2006.01183.x

Vrba, J., Dolezel, P., Vicar, J., Modrianský, M., and Ulrichová, J. (2008). Chelerythrine and dihydrochelerythrine induce G1 phase arrest and bimodal cell death in human leukemia HL-60 cells. Toxicol. In Vitro 22, 1008-1017. doi: 10.1016/j.tiv.2008.02.007

Vychkanova, S. A., Rubinchyk, M. A., Adgina, V. V., and Fedorchenko, T. S. (1969). Izucheniyye khimioterapevtycheskogo deystviya sangvinaryna. Farmakolog. Toksikol. (Moskva) 31, 325-328.

Wagner, H., Bladt, S., and Zgainski, E. M. (1984). Plant Drug Analysis-A Thin Layer Chromatography Atlas. Berlin; Heidelberg: Springer-Verlag.

Waksmundzka-Hajnos, M., Gadzikowska, M., and Golkiewicz, W. (2000). Special modes of development of Chelidonium majus L. alkaloids in systems of the type polar adsorbent-multicomponent mobile phase by TLC. J. Planar Chromat. 13, 205-209.

Waksmundzka-Hajnos, M., Gadzikowska, M., and Hajnos, M. Ł. (2002). Strategy for Preparative Separation of Quaternary Alkaloids from Chelidonium majus L. by Thin-Layer Chromatography. J. Planar Chromatogr. 15, 289-293. doi: 10.1556/JPC.15.2002.4.9

Waniakowa, J. (2015). "Osobliwe procesy fonetyczne w gwarowych wyrazach zapozyczonych na przykładzie nazw roślin (Peculiar Phonetic Processes in Dialectal Borrowings Exemplified by Plant Names)," in Prirodzený vývin jazyka a jazykové kontakty. Jazykovedné štúdie, XXXII, eds K. Bálleková, L. Králik, G. Múcsková (Bratislava: Veda), 203-210.

Wdowiak, L., and Bielecka-Grzela, S. (2013). "Leki roslinne i inne remedia w leczeniu bolaków, skuł, i gruc," in Lek roślinny - Historia i Współczesność, T. 2, eds B. Płonka-Syroka, A. Syroka, and K. Sudoł (Wrocław: Oficyna Wydawnicza Arboretum), 189.
Wdowiak, L. (2015). "Srodki roślinne i inne remedia stosowane w chorobach oczu na ziemiach polskich w czasach zaborów," in Lek Roślinny - Historia i Współczesność, T. 4, eds B. Płonka-Syroka and A. Syroka (Wrocław: Wydawnictwo Quaestio), 117-130.

Wei, Q., Zhao, M., and Li, X. (2017). Extraction of chelerythrine and its effects on pathogenic fungus spore germination. Pharmacogn. Mag. 13:600. doi: $10.4103 /$ pm.pm_545_16

Weiskirchen, R. (2016). Hepatoprotective and anti-fibrotic agents: it's time to take the next step. Front. Pharmacol. 6:303. doi: 10.3389/fphar.2015.00303

Wichtl, M. (2004). "Chelidonii herba," in Herbal Drugs and Phytopharmaceuticals: A Handbook for Practice on a Scientific Basis, ed M. Wichtl (Stuttgart: CRC Press), 130-133.

Wintgen, M., and Schmidt, E. (1901). Ueber die Alkaloide von Chelidonium majus. Arch. Pharm. 239, 438-451. doi: 10.1002/ardp.19012390606

Wojdyło, A., Oszmianski, J., and Czemerys, R. (2007). Antioxidant activity and phenolic compounds in 32 selected herbs. Food Chem. 105, 940-949. doi: 10.1016/j.foodchem.2007.04.038

Wongbutdee, J. (2009). Physiological effects of berberine - Review article. Thai Pharma Health. Sci. J. 4, 78-83.

$\mathrm{Wu}, \mathrm{H}$., and $\mathrm{Du}, \mathrm{L}$. (2012). Ionic liquid-liquid phase microextraction for the sensitive determination of sanguinarine and chelerythrine in Chinese herbal medicines and human urine. J. Liq. Chromatogr. Rel Technol 35, 1662-1675. doi: 10.1080/10826076.2011.621156

Yang, G., Lee, K., Lee, M. H., Kim, S. H., Ham, I. H., and Choi, H. Y. (2011). Inhibitory effects of Chelidonium majus extract on atopic dermatitislike skin lesions in NC/Nga mice. J. Ethnopharmacol. 138, 398-403. doi: 10.1016/j.jep.2011.09.028

Yao, J., Li, X., Shen, J., Pan, X., Hao, G., xu, Y., et al. (2011). Isolation of bioactive components from Chelidonium majus L. with activity against Trichodina sp. Aquaculture 318, 235-238. doi: 10.1016/j.aquaculture.2011.04.035

Zarzycki, K., Trzcinska-Tacik, H., Rózanski, W., Szelag, Z., Wołek, J., and Korzeniak, U. (2002). "Ecological indicator values of vascular plants of Poland," in Biodiversity of Poland, Vol. 2., eds Z. Mirek and W. Szafer (Krakow: Institute of Botany, Polish Academy of Sciences), 184.

Zevin, I. V., Altman, N., and Zevin, L. V. (1997). A Russian Herbal: Traditional Remedies for Health and Healing. Rochester: Healing Art Press.

Zhang, Z. H., Mi, C., Wang, K. S., Wang, Z., Li, M. Y., Zuo, H. X., et al. (2018). Chelidonine inhibits TNF- $\alpha$-induced inflammation by suppressing the NF- $\mathrm{B}$ pathways in HCT116 cells. Phytother. Res. 32, 65-75. doi: 10.1002/ptr.5948

Zhou, Q., Liu, Y., Wang, X., and Di, X. (2012). Microwave-assisted extraction in combination with capillary electrophoresis for rapid determination of isoquinoline alkaloids in Chelidonium majus L. Talanta 99, 932-938. doi: 10.1016/j.talanta.2012.07.061

Zou, C., Lv, C., Wang, Y., Cao, C., and Zhang, G. (2017). Larvicidal activity and insecticidal mechanism of Chelidonium majus on Lymantria dispar. Pestic. Biochem. Physiol. 142, 123-132. doi: 10.1016/j.pestbp.2017.04.009

Zuo, G. Y., Meng, F. Y., Hao, X. Y., Zhang, Y. L., Wang, G. C., and Xu, G. L. (2008). Antibacterila alkaloids from Chelidonium majus Linn (Papaveraceae) against clinical isolates of methicillin-resistant Staphylococcus aureus. J. Pharm. Pharmaceut. Sci. 11, 90-94. doi: 10.18433/J3D30Q

Conflict of Interest Statement: The authors declare that the research was conducted in the absence of any commercial or financial relationships that could be construed as a potential conflict of interest.

Copyright (c) 2018 Zielińska, Jezierska-Domaradzka, Wójciak-Kosior, Sowa, Junka and Matkowski. This is an open-access article distributed under the terms of the Creative Commons Attribution License (CC BY). The use, distribution or reproduction in other forums is permitted, provided the original author $(s)$ and the copyright owner are credited and that the original publication in this journal is cited, in accordance with accepted academic practice. No use, distribution or reproduction is permitted which does not comply with these terms. 


\section{OPEN ACCESS}

Edited by:

Luc Pieters,

University of Antwerp, Belgium

Reviewed by:

Christian W. Gruber,

Medizinische Universität Wien, Austria Juliana Montani Raimundo,

Universidade Federal do Rio de Janeiro, Brazi

Liselotte Krenn,

Universität Wien, Austria

*Correspondence: Dezső Csupor csupor.dezso@pharm.u-szeged.hu; csupor.dezso@pharmacognosy.hu

${ }^{t}$ These authors have contributed equally to this work as first authors.

Specialty section: This article was submitted to Ethnopharmacology,

a section of the journal Frontiers in Pharmacology

Received: 18 December 2017 Accepted: 20 March 2018 Published: 06 April 2018

Citation:

Sándor Z, Mottaghipisheh J, Veres K, Hohmann J, Bencsik T, Horváth A, Kelemen D, Papp R,

Barthó L and Csupor D (2018) Evidence Supports Tradition: The in Vitro Effects of Roman Chamomile on Smooth Muscles.

Front. Pharmacol. 9:323. doi: 10.3389/fphar.2018.00323

\section{Evidence Supports Tradition: The in Vitro Effects of Roman Chamomile on Smooth Muscles}

\author{
Zsolt Sándor ${ }^{1 \dagger}$, Javad Mottaghipisheh ${ }^{2 \dagger}$, Katalin Veres ${ }^{2}$, Judit Hohmann ${ }^{2}$, \\ Tímea Bencsik ${ }^{3}$, Attila Horváth ${ }^{2}$, Dezső Kelemen ${ }^{4}$, Róbert Papp ${ }^{4}$, Loránd Barthó ${ }^{1}$ and \\ Dezső Csupor ${ }^{2,5 *}$ \\ ${ }^{1}$ Department of Pharmacology and Pharmacotherapy, University of Pécs, Medical School, Pécs, Hungary, \\ ${ }^{2}$ Department of Pharmacognosy, University of Szeged, Szeged, Hungary, ${ }^{3}$ Department of Pharmacognosy, University of \\ Pécs, Pécs, Hungary, ${ }^{4}$ Department of Surgery, Clinical Center, University of Pécs, Medical School, Pécs, Hungary, \\ ${ }^{5}$ Interdisciplinary Centre for Natural Products, University of Szeged, Szeged, Hungary
}

The dried flowers of Chamaemelum nobile (L.) All. have been used in traditional medicine for different conditions related to the spasm of the gastrointestinal system. However, there have been no experimental studies to support the smooth muscle relaxant effect of this plant. The aim of our research was to assess the effects of the hydroethanolic extract of Roman chamomile, its fractions, four of its flavonoids (apigenin, luteolin, hispidulin, and eupafolin), and its essential oil on smooth muscles. The phytochemical compositions of the extract and its fractions were characterized and quantified by HPLC-DAD, the essential oil was characterized by GC and GC-MS. Neuronally mediated and smooth muscle effects were tested in isolated organ bath experiments on guinea pig, rat, and human smooth muscle preparations. The crude herbal extract induced an immediate, moderate, and transient contraction of guinea pig ileum via the activation of cholinergic neurons of the gut wall. Purinoceptor and serotonin receptor antagonists did not influence this effect. The more sustained relaxant effect of the extract, measured after pre-contraction of the preparations, was remarkable and was not affected by an adrenergic beta receptor antagonist. The smooth muscle-relaxant activity was found to be associated with the flavonoid content of the fractions. The essential oil showed only the relaxant effect, but no contracting activity. The smooth muscle-relaxant effect was also detected on rat gastrointestinal tissues, as well as on strip preparations of human small intestine. These results suggest that Roman chamomile extract has a direct and prolonged smooth muscle-relaxant effect on guinea pig ileum which is related to its flavonoid content. In some preparations, a transient stimulation of enteric cholinergic motoneurons was also detected. The essential oil also had a remarkable smooth muscle relaxant effect in this setting. Similar relaxant effects were also detected on other visceral preparations, including human jejunum. This is the first report on the activity of Roman chamomile on smooth muscles that may reassure the rationale of the traditional use of this plant in spasmodic gastrointestinal disorders.

Keywords: Roman chamomile, Chamaemelum nobile, Asteraceae, organ bath experiment, gastrointestinal preparations, spasmolytic effect, contractile action 


\section{INTRODUCTION}

Chamaemelum nobile (L.) All. (Asteraceae), widely known as Roman chamomile, is a perennial herb native to SouthWestern Europe, but it is cultivated as a medicinal plant all over Europe and in Africa as well. Dried flowers of the cultivated, double-flowered variety of the species are official in the European Pharmacopoeia (European Pharmacopoeia, 2008). Incorporating the plant in traditional herbal medicinal products has been acknowledged by the European Medicines Agency. The comminuted herbal substance (as tea) and a liquid extract of the plant (extraction solvent: ethanol 70\% $v / v$ ) may be used for the symptomatic treatment of mild, spasmodic gastrointestinal complaints including bloating and flatulence. The British Herbal Pharmacopoeia indicates its use as carminative, anti-emetic, antispasmodic, and sedative (British Herbal Pharmacopoeia, 1971).

Roman chamomile (RC) has been used as a medicinal plant from the middle ages. The cultivation of the plant started in England in the 16th century (Hiller and Melzig, 1999). The double variety of the flower, which now serves as the main commercial drug, has certainly been known since the 18th century (Evans, 1989). The plant gained the name "nobile" (Latin, noble) to illustrate its superior therapeutic efficacy over Matricaria recutita L. (German chamomile) (Hiller and Melzig, 1999). The plant was first listed in the Pharmacopoeia of Württemberg (1741) as a carminative, painkiller, diuretic, and digestive aid (Lukacs, 1990). In the folk medicine of different regions of Europe, RC has been used for numerous conditions, including dyspepsia, flatulence, nausea and vomiting, anorexia, vomiting of pregnancy, dysmenorrhoea, and specifically for gastrointestinal cramps and flatulent dyspepsia associated with mental stress (Augustin et al., 1948; Rápóti and Romváry, 1974; Melegari et al., 1988; Rossi et al., 1988; Bradley, 1992). In the Mediterranean region, $\mathrm{RC}$ tea is consumed to improve appetite and also after meal to prevent indigestion (Rivera and Obon, 1995; Menendez-Baceta et al., 2014; Alarcün et al., 2015). Traditional use of RC is largely related to its supposed smooth muscle-relaxant activity.

The majority of the secondary metabolites described from the plant belong to the aliphatic esters (essential oil) (Fauconnier et al., 1996), sesquiterpene lactones (Bisset, 1994) and flavonoids (Herisset et al., 1971, 1973; Abou-Zied and Rizk, 1973; Pietta et al., 1991). The polysaccharide content of the dried flower is noteworthy, 3.9\% (Lukacs, 1990). The supposed smooth muscle-relaxant activity of the plant might be attributed to its flavonoid content. Apigenin and luteolin possess remarkable smooth muscle relaxant effects on guinea pig ileum (LemmensGruber et al., 2006).

Although several studies on the bioactivities of RC are available, the majority of these studies were carried out using the essential oil, which is not used medicinally, or the observed activities are not related to the traditional use of the plant. Several studies demonstrate the antimicrobial effects of RC essential oil against different bacterial and fungal strains (Hänsel et al., 1993; Piccaglia et al., 1993; Chao et al., 2000; Bail et al., 2009), and antifungal activity was demonstrated also for the aqueous extracts of RC (Magro et al., 2006). The anti-inflammatory capacity and heat shock protein modulating effects of the flavonoids apigenin and quercetin, as well as the anti-inflammatory activities of $\alpha$-bisabolol, guajazulene, and chamazulene have been reported in preclinical studies (Viola et al., 1995; Baghalian et al., 2008, 2011; Hernández-Ceruelos et al., 2010). The polysaccharides of RC exerted antiphlogistic effect in vivo (Lukacs, 1990).

Although the use of RC extract for gastrointestinal problems seems to be related to its presumptive smooth muscle-relaxant effect, interestingly no in vitro or in vivo studies have been carried out so far to assess this bioactivity. However, in an in vitro study an aqueous extract of $C$. nobile was demonstrated to induce a vasorelaxant effect through the NO-cGMP pathway or possibly through a combination of $\mathrm{Ca}^{2+}$ channel inhibition plus NO-modulating and phosphodiesterase inhibitory mechanisms. After the oral administration of RC aqueous extract, significant hypotensive effect was observed in an animal study on spontaneously hypertensive rats (Zeggwagh et al., 2009), which may be related to the flavonoid content of the plant (Jouad et al., 2001). The clinical efficacy of orally applied preparations has not been studied yet, only the effects of external application (Schrader et al., 1997) and aromatherapeutic use (Wilkinson et al., 1999) have been reported.

The aim of the current study was to assess the effects of a hydroethanolic RC extract, its fractions and essential oil on gastrointestinal and urogenital smooth muscles, including preparations of human jejunum, in order to clarify the rationale for the use of this plant for smooth muscle relaxation. Experiments were carried out with an extract conforming to the monograph of the European Medicines Agency (see in section "Preparation of Herbal Extracts and Isolation of Reference Standards"), and also with the fractions of this extract and with the essential oil of the plant.

\section{MATERIALS AND METHODS}

\section{Plant Material}

Roman chamomile flowers were purchased from Pál Bobvos (Hungary). The identity of the plant material was confirmed according to the requirements of the European Pharmacopoeia. A voucher specimen is stored for verification purposes in the herbarium of the Department of Pharmacognosy, University of Szeged. RC essential oil was purchased from Aromax Ltd. (Hungary).

\section{Chemicals}

For the pharmacological experiments the following drugs were used: atropine sulfate (Sigma), $\alpha, \beta$-methylene ATP lithium salt (Tocris), capsaicin, histamine dihydrochloride, indomethacin, isoprenaline hydrochloride, $\mathrm{N}^{\mathrm{G}}$-nitro-L-arginine, papaverine hydrochloride, prostaglandin $\mathrm{F}_{2 \alpha}$ (Sigma), methysergide (Sandoz), ( \pm )-propranolol hydrochloride (Sigma), pyridoxalphosphate-6-azophenyl-2', $4^{\prime}$-disulfonic acid tetrasodium salt (PPADS), tetrodotoxin (Tocris), 8-amino -7-chloro-2,3-dihydro-1,4-benzodioxan-5-carboxylic acid, $1^{\prime}$ butyl-4'-piperidinylmethyl ester (SB204070), N-(1-azabicyclo 
[2.2.2] oct-3-yl)-6-chloro-4-methyl-3-oxo-3,4-dihydro-2H-1,4benzoxazine-8-carboxamide hydrochloride (Y25130) (all from Tocris). For dissolving and diluting of these drugs the following solvents were used: $96 \%$ ethanol for capsaicin, indomethacin, and prostaglandin $\mathrm{F}_{2 \alpha}$, DMSO for SB204070 and isotonic $\mathrm{NaCl}$ solution for the rest of the drugs. Solutions in organic solvents were administered at a maximum of $1 \mu \mathrm{l} / \mathrm{ml}$, aqueous solutions at 1 or $3 \mu \mathrm{l} / \mathrm{ml}$ bathing solution.

Millipore Direct-Q UV3 clarifier (Molsheim, France) was used to produce purified water for the HPLC measurements. Methanol and ethanol (LiChrosolv ${ }^{\circledR}$ HPLC grade) was obtained from Merck (Darmstadt, Germany). For chromatographic separation, polyamide (ICN Polyamide for Column Chromatography), Sephadex LH-20 (25-100 $\mu \mathrm{m}$, Pharmacia Fine Chemicals), $\mathrm{SiO}_{2}$ (Silica gel $60 \mathrm{G}, 15 \mu \mathrm{m}$, Merck), and $\mathrm{SiO}_{2}$ plates $(20 \mathrm{~cm} \times 20 \mathrm{~cm}$ Silica gel $60 \mathrm{~F}_{254}$, Merck) were used.

\section{Preparation of Herbal Extracts and Isolation of Reference Standards}

For the pharmacological experiments, RC crude extract was prepared according to the description of the European Medicines Agency monograph (EMA-HMPC, 2012). Ten grams of plant material was extracted with $70 \% \mathrm{EtOH}(3 \times 100 \mathrm{ml})$ via ultrasonic bath, evaporated in vacuum and lyophilized (yield, 3.169\%). Part of the RC crude extract was fractionated to gain RC extract fractions with different compositions for further experiments. Vacuum liquid chromatography on polyamide with elution by $\mathrm{MeOH}-\mathrm{H}_{2} \mathrm{O}(20: 80,40: 60,60: 40,80: 20,100: 0)$ was used to gain fractions from the crude extract as follows: F20, F40, F60, F80, and F100.

From the methanolic extract of $200 \mathrm{~g}$ RC flowers, four marker compounds (used as reference standards in further experiments) were isolated using medium pressure liquid chromatography $\left(\mathrm{SiO}_{2}\right.$ as stationary phase), gel chromatography (Sephadex LH-20), preparative HPLC (RP), and preparative TLC $\left(\mathrm{SiO}_{2}\right)$. The purity and identity of these compounds were analyzed by NMR. NMR spectra were recorded in MeOD on a Bruker Avance DRX 500 spectrometer (Bruker, Fallanden, Switzerland) at $500 \mathrm{MHz}\left({ }^{1} \mathrm{H}\right)$ or $125 \mathrm{MHz}\left({ }^{13} \mathrm{C}\right)$.

\section{HPLC Experiments}

HPLC experiments were carried out on a Shimadzu LC-20AD Liquid Chromatograph (SPD-M20A diode array detector, CBM-20A controller, SIL-20AC $\mathrm{AT}_{\mathrm{T}}$ autosampler, DGU-20A $5 \mathrm{R}$ degasser unit, CTO-20AC column oven) using a Kinetex $5 \mu \mathrm{m}$ C-18 100A $(150 \mathrm{~mm} \times 4.6 \mathrm{~mm})$ with a gradient of $0,01 \%$ trifluoroacetic acid in $\mathrm{H}_{2} \mathrm{O}(\mathrm{A})$ and acetonitrile (B) as follows: 0-5 $\min 25 \% \mathrm{~B}, 14 \mathrm{~min} 28 \% \mathrm{~B}, 15 \mathrm{~min} 70 \% \mathrm{~B}, 16 \mathrm{~min}$ $70 \% \mathrm{~B}, 16.5 \mathrm{~min} 25 \% \mathrm{~B}$, and $20 \mathrm{~min} 25 \% \mathrm{~B}$. The flow was $1.2 \mathrm{ml} / \mathrm{min}$, column oven temperature was $55^{\circ} \mathrm{C}$. Detection was carried out within the range of 190-800 nm. For quantification, chromatograms were integrated at $344 \mathrm{~nm}$. The reference standards and the evaporated extracts were dissolved in $\mathrm{MeOH}$, filtered through a PTFE syringe filter and injected in volumes of 5 or $10 \mu \mathrm{l}$. Calibration curves were established for all the four reference standards.

\section{GC and GC-MS Experiments}

The GC analysis was carried out with an HP 5890 Series II gas chromatograph (FID), using a $30 \mathrm{~m} \times 0.35 \mathrm{~mm} \times 0.25 \mu \mathrm{m}$ HP-5 fused silica capillary column. The temperature program ranged from $60^{\circ} \mathrm{C}$ to $210^{\circ} \mathrm{C}$ at $3^{\circ} \mathrm{C} \mathrm{min}^{-1}$, and from $210^{\circ} \mathrm{C}$ to $250^{\circ} \mathrm{C}(2 \mathrm{~min}$ hold $)$ at $5^{\circ} \mathrm{C} \mathrm{min}^{-1}$. The detector and injector temperature was set to $250^{\circ} \mathrm{C}$ and the carrier gas was $\mathrm{N}_{2}$, with split sample introduction. Quantities of the individual components of the essential oil were expressed as the percent of the peak area relative to the total peak area from the GC/FID analysis.

The GC-MS analysis was performed with a Finninan GCQ ion trap bench-top mass spectrometer. All conditions were as above except that the carrier gas was $\mathrm{He}$ at a linear velocity of $31.9 \mathrm{~cm} \mathrm{~s}^{-1}$ and the capillary column was DB-5MS $(30 \mathrm{~m} \times 0.25 \mathrm{~mm} \times 0.25 \mu \mathrm{m})$. The positive ion electron ionization mode was used, with ionization energy of $70 \mathrm{eV}$, and the mass range of 40-400 amu.

Identification of the compounds was based on comparisons with published MS data (Adams, 2007) and with a computer library search (the database was delivered together with the instrument) and also by comparing their retention indices with literature values (Adams, 2007). Retention indices were calculated against C8-C32 n-alkanes on a CB-5 MS column (Kovats, 1965). A mixture of aliphatic hydrocarbons was injected in hexane (Sigma-Aldrich, St. Louis, MO, United States) by using the same temperature program that was used for analyzing the essential oil.

\section{Guinea Pig and Rat Preparations}

Guinea pigs (short-haired, colored, 350-450 g) or Wistar rats (220-300 g) of either sex were killed by a blow to the head and exsanguination. Three centimeter segments of the ileum or distal colon were placed into the organ bath in a vertical position, under a constant tension of $7 \mathrm{mN}$. Longitudinal strip preparations (approximately $2-3 \mathrm{~cm}$ in length) of guinea pig urinary bladder or rat gastric fundus were fixed under a tension of $5 \mathrm{mN}$.

This study was carried out in accordance with University guidelines. The protocol was approved by the Animal Welfare Committee, University of Pécs (registration number, BA02/2000$1 / 2012$ ) with the understanding that isolated organ studies after stunning the animal cannot be regarded as animal experiments.

\section{Organ Bath Experiments}

Smooth muscle preparations were used in a traditional organ bath arrangement. The preparations were suspended in KrebsHenseleit solution of 5 or $7 \mathrm{ml}$, kept at $37^{\circ} \mathrm{C}$, and aerated with a mixture of $95 \% \mathrm{O}_{2}$ and $5 \% \mathrm{CO}_{2}$. The solution contained $119 \mathrm{mM} \mathrm{NaCl}, 25 \mathrm{mM} \mathrm{NaHCO}, 2.5 \mathrm{mM} \mathrm{KCl}, 1.5 \mathrm{mM}$ $\mathrm{MgSO}_{4}, 2.5 \mathrm{mM} \mathrm{CaCl}_{2}, 1.2 \mathrm{mM} \mathrm{KH} \mathrm{PO}_{4}$, and $11 \mathrm{mM}$ glucose $(\mathrm{pH}=7.4)$. Movements of the preparations were recorded with isotonic transducers (Hugo Sachs Elektronik-Harvard Apparatus, March, Germany) on ink writers or stored online on a personal computer.

The guinea pig ileum is a preparation of a low spontaneous tone. In part of the experiments the effects of RC extract, 
its fractions, essential oil, or flavonoids were studied at basal tone. All experiments commenced with determining the maximal longitudinal spasm by adding histamine $(10 \mu \mathrm{M})$ to the organ bath for $2 \mathrm{~min}$. This response served as reference for determining the relative amplitude of any contractile effect that the samples to be tested might have had.

In another set of experiments the possible relaxant effect of the materials was tested. For this reason, a tonic, approximately halfmaximal contraction of the ileum was provoked by a moderate concentration of histamine $(0.5 \mu \mathrm{M}$, added for $15 \mathrm{~min})$. In preliminary experiments, no pretreatment was used. In the bulk of the experiments, however, the muscarinic receptor antagonist atropine, as well as tetrodotoxin, an inhibitor of nerve axonal conduction (blocker of voltage-sensitive $\mathrm{Na}^{+}$channels) was added prior to the histamine administration. This was done to avoid the interference with a possible contractile effect of the material to be examined, i.e., to create a methodologically clear situation.

All experiments commenced after an equilibration period of $45 \mathrm{~min}$. Relaxant effects were studied on pre-contracted preparations (see below). Maximal spasm of the tissue was evoked to serve as a basis for the comparisons to evaluate the responses obtained by our test materials. The materials examined were administered in a single concentration (i.e., in a non-cumulative manner).

\section{Human Jejunal Preparations}

Macroscopically intact segments of human jejunum, removed during the surgical treatment of pancreatic cancer were used. Strips of either longitudinal or circular orientation were prepared (the mucosa and submucosa were removed) and fixed as described above, under a tension of $10 \mathrm{mN}$. Freeze-dried extracts of RC, as well as the essential oil of the plant were mixed in and further diluted with DMSO. The amounts of DMSO administered to the jejunum preparations are indicated in the Section "Results".

This study was carried out in accordance with the Declaration of Helsinki and the guidelines set by the Research Ethics Committee, Scientific Council of the Ministry of Health, Hungary. This Committee agreed to the use of discarded human tissue for the experimental work. The protocol was approved by the ETT-TUKEB (Scientific Council of the Ministry of Health, Research Ethical Committee) [No. 17861-0/20101018EKU (749/PI/10)]. All subjects gave written informed consent in accordance with the Declaration of Helsinki.

\section{Statistical Analysis}

Results of the pharmacological experiments are given as mean \pm SEM, with $N$ denoting the number of preparations used for testing efficacy. At most 2 preparations from the same animal were used for each type of experiment. Results are provided in relative values, where $100 \%$ contraction means maximal spasm of the preparations from the basal tone and, in pre-contracted preparations, $100 \%$ relaxation means relaxation of the preparation to the level before adding the precontracting agent. The Mann-Whitney $U$ test was used for the comparisons of two independent groups, more than two independent samples were compared by the Kruskal-Wallis test, and two dependent samples were compared by the Wilcoxon test. The statistical program GraphPad Prism 5 was used throughout. A probability of $P<0.05$ or less indicated statistical significance.

\section{RESULTS}

\section{Chemical Characterization of RC Extracts}

In the HPLC retention time range of 4-9 $\mathrm{min}$, four characteristic peaks were detected in the crude RC extract and its fractions F40-F100. The corresponding components were isolated from the plant material and were identified as the flavonoids apigenin, eupafolin, hispidulin, and luteolin by ${ }^{1} \mathrm{H}$ and ${ }^{13} \mathrm{C}$ NMR experiments (see Supplementary Figures S1-S6). These compounds were further used as reference standards to characterize the RC extracts. The identification of reference compounds in different extracts was based on matching the retention times and UV spectra. Retention times of luteolin, eupafolin, apigenin, and hispidulin were 4.5, 5.0, 7.5, and $8.5 \mathrm{~min}$, respectively (Figure 1). The baseline separation of these compounds allowed their reliable quantification in different extracts.

The crude RC extract contained eupafolin as the main flavonoid, followed by luteolin, hispidulin, and apigenin (Table 1). The fractionation on polyamide resulted in subfractions F20-F100 with different compositions, as demonstrated by the differences in their flavonoid content. In F20, the quantities of flavonoids were below the level of quantification. The flavonoid content of the fractions increased with increasing $\mathrm{MeOH}$ content of the eluting solvent. The highest flavonoid levels were measured in F80, except for luteolin and apigenin, which were mainly concentrated in F100.

\section{Chemical Characterization of RC Essential Oil}

Based on their retention times and mass spectrometric data, methallyl angelate, 3-methyl pentyl angelate, and 3-methylamyl isobutyrate were identified as the major constituents of RC essential oil (19.0, 18.2, and 10.4\%, respectively) (Table 2). The identified components comprised $97 \%$ of the essential oil. Previous articles reported isobutyl angelate as the major constituent of RC essential oil (21.6-38.5\%), followed by 2-methylbutyl angelate (11.6-20.3\%) (Antonelli and Fabbri, 1998; Farkas et al., 2003; Omidbaigi et al., 2003, 2004; Bail et al., 2009), and propyl tiglate (10.8-13.1\%) (Omidbaigi et al., 2003, 2004) or isobutyl isobutyrate (3.3\%) (Bail et al., 2009) or 2-butenyl angelate (7.9-8.4\%) (Antonelli and Fabbri, 1998) or 2-methyl-2propenyl angelate (9.1\%) (Farkas et al., 2003). However, several commercial samples have similar compositions to the sample analyzed by us, with methallyl angelate and 3-methyl pentyl angelate as the major components.

\section{Effects on Guinea Pig lleum}

The crude RC extract induced a transient longitudinal contraction on guinea pig ileum preparations (Figure 2). 


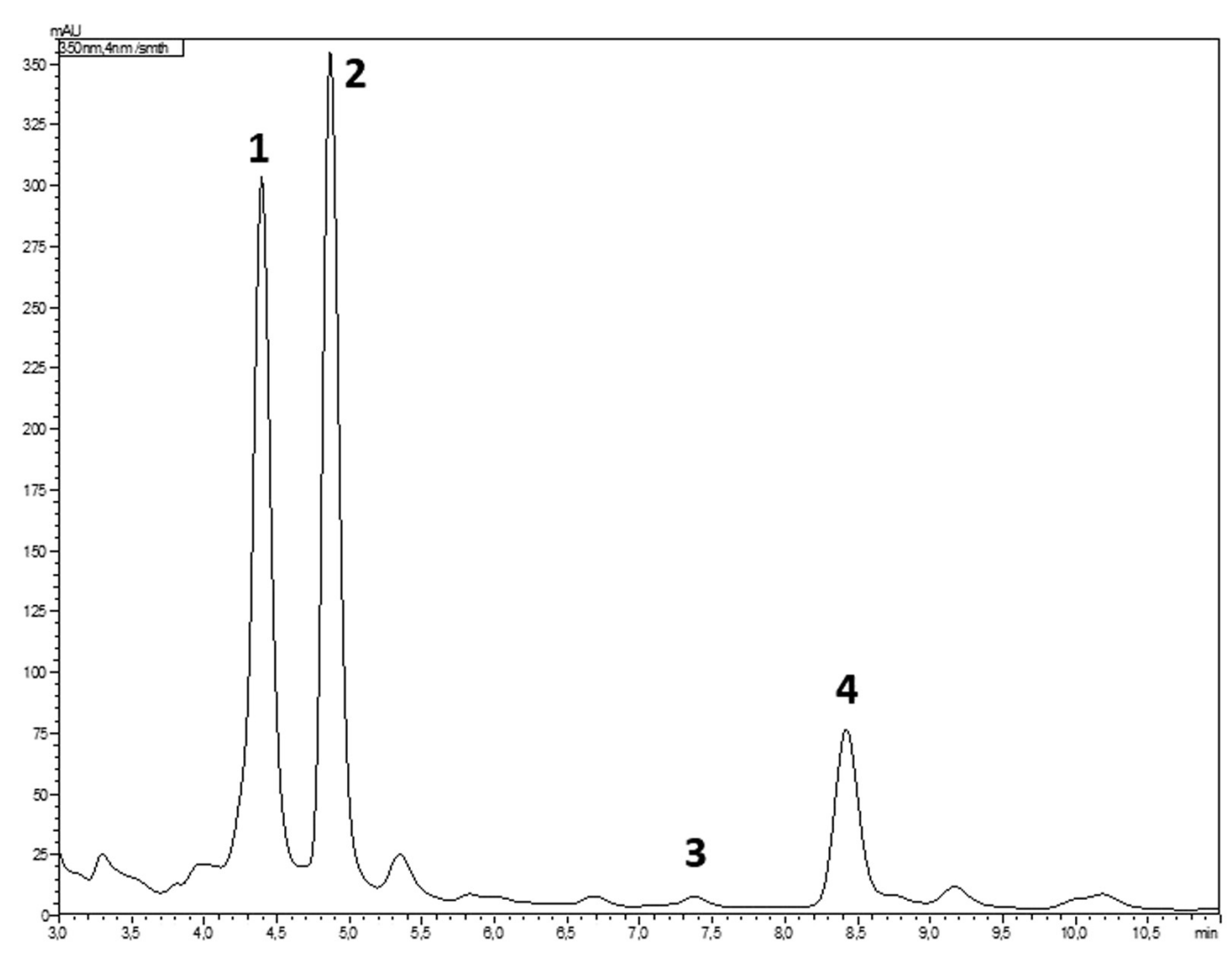

FIGURE 1 | HPLC chromatogram of the crude RC extract with the peaks of luteolin (1), eupafolin (2), apigenin (3), and hispidulin (4) (344 nm).

TABLE 1 | Flavonoid content of RC crude extract and its fractions as determined by HPLC.

\begin{tabular}{|c|c|c|c|c|}
\hline Sample & Luteolin (mg/g extract) & Eupafolin (mg/g extract) & Apigenin (mg/g extract) & Hispidulin (mg/g extract) \\
\hline Crude extract & $4.617 \pm 0.616$ & $18.756 \pm 2.121$ & $0.298 \pm 0.027$ & $1.584 \pm 0.181$ \\
\hline $\mathrm{F} 20$ & Not detected & Not detected & Not detected & Not detected \\
\hline $\mathrm{F} 40$ & $0.578 \pm 0.001$ & $1.800 \pm 0.001$ & $0.179 \pm 0.001$ & $0.231 \pm<0.001$ \\
\hline F60 & $1.904 \pm 0.001$ & $62.591 \pm 0.025$ & $0.151 \pm<0.001$ & $5.951 \pm 0.004$ \\
\hline F80 & $22.605 \pm 0.001$ & $223.488 \pm 0.036$ & $0.859 \pm<0.001$ & $17.060 \pm 0.006$ \\
\hline F100 & $55.305 \pm 0.002$ & $150.206 \pm 0.005$ & $2.055 \pm<0.001$ & $4.983 \pm<0.001$ \\
\hline
\end{tabular}

The amplitudes of the contractions were related to the maximal longitudinal spasm evoked with $10 \mu \mathrm{M}$ of histamine at the beginning of the experiments. The threshold concentration for this effect was equal to or below $20 \mu \mathrm{g} / \mathrm{ml}$ of the extract (which was the lowest concentration tested) and reached a plateau with 60 and $200 \mu \mathrm{g} / \mathrm{ml}$. Quantitative results were as follows (expressed as \% of the maximal spasm): $18.8 \pm 3.1 \%$ at $20 \mu \mathrm{g} / \mathrm{ml}(N=6), 40.1 \pm 3.3 \%$ at $60 \mu \mathrm{g} / \mathrm{ml}(N=11)$, and $36.3 \pm 4.9 \%$ at $200 \mu \mathrm{g} / \mathrm{ml}(N=7)$. A second administration of the same concentration after a 40-min washout period usually had a qualitatively similar effect. Yet, because of variable reproducibility, we examined the effects on separate preparations with only one administration of the extract. The solvent of the extract (DMSO; 0.3 or $1 \mu \mathrm{l} / \mathrm{ml}$ ) caused no or minimal contraction (on average, 0 and $2 \%$, respectively; $N=12)$.
The $60 \mu \mathrm{g} / \mathrm{ml}$ concentration of the extract was used for pharmacological analysis. Both atropine $(0.5 \mu \mathrm{M})$, an antagonist of acetylcholine at the muscarinic receptors and tetrodotoxin $(0.5 \mu \mathrm{M})$, an inhibitor of voltage-sensitive $\mathrm{Na}^{+}$channels (hence, of neuronal axonal conduction) inhibited the contractile effect of RC crude extract. In contrast, the purinoceptor antagonist PPADS $(50 \mu \mathrm{M})$ or a combination of serotonin (5-HT) receptor antagonists methysergide $(0.3 \mu \mathrm{M}), \operatorname{SB} 204070(1 \mu \mathrm{M})$ and Y25130 (1 $\mu \mathrm{M})$ failed to influence the contractile effect of the extract (Table 3). This combination of 5-HT antagonists is suitable for blocking the contractile effect of 5-HT (Sandor et al., 2016).

The functional blockade of capsaicin-sensitive neurons did not inhibit the contractile effect of the RC extract (Barthó et al., 2004) as compared to time-matched, solvent-treated controls (Table 3). The cyclooxygenase inhibitor indomethacin $(3 \mu \mathrm{M})$ 
TABLE 2 | Chemical composition of RC essential oil.

\begin{tabular}{|c|c|c|}
\hline Compounds $^{a}$ & $\mathbf{R} \mathbf{I}^{\mathbf{b}}$ & $\%$ in samples \\
\hline Methyl tiglate & 867 & $\operatorname{tr}$ \\
\hline$n$-Hexanol & 875 & 1.1 \\
\hline 2-Methylbutyl acetate & 897 & 0.4 \\
\hline Isobutyl isobutyrate & 925 & 0.8 \\
\hline Acetonyl acetone & 932 & 0.7 \\
\hline$\alpha$-Pinene & 935 & 2.4 \\
\hline Camphene + allyl methacrylate & 958 & 0.6 \\
\hline Thuja-2,4(10)-diene & 960 & 1.1 \\
\hline Isoamyl propionate & 966 & $\operatorname{tr}$ \\
\hline$\beta$-Pinene & 969 & 0.3 \\
\hline Myrcene & 973 & 0.6 \\
\hline Propyl angelate & 993 & 1.1 \\
\hline Isobutyl 2-methylbutyrate & 998 & $\operatorname{tr}$ \\
\hline Isoamyl isobutyrate & 1,004 & 1.5 \\
\hline 2-Methylbutyl isobutyrate & 1,006 & 2.7 \\
\hline 1,8-Cineol & 1,035 & $\operatorname{tr}$ \\
\hline Isoamyl methacrylate & 1,037 & 1.1 \\
\hline Isobutyl angelate & 1,058 & 4.9 \\
\hline Methallyl angelate & 1,068 & 19.0 \\
\hline 2-Butenyl angelate & 1,119 & $\operatorname{tr}$ \\
\hline 3-Methylamyl isobutyrate & 1,122 & 10.4 \\
\hline 3-Methylamyl methacrylate & 1,150 & 6.6 \\
\hline trans-Pinocarveol + isoamyl angelate & 1,153 & 8.6 \\
\hline 2-Methylbutyl angelate & 1,168 & 8.3 \\
\hline Pinocarvone & 1,177 & 3.9 \\
\hline Prenyl angelate & 1,213 & 1.5 \\
\hline Myrtenal & 1,217 & 1.2 \\
\hline 3-Methyl pentyl angelate & 1,264 & 18.2 \\
\hline Identified components & & 97.0 \\
\hline
\end{tabular}

${ }^{a}$ Compounds listed in sequence of elution from a DB-5 MS column. ${ }^{b}$ Retention indices calculated against C8 to C32 n-alkanes on a DB-5MS column. tr, in traces.

moderately but significantly inhibited the contraction in response to the RC extract.

Fractions of the RC extract were also tested for contracting activity. Fraction F20 induced a moderate contraction (approximately 20\% of the maximum) (Table 4). Similar results were obtained with F40, F60, F80, and F100, although the extent of contraction tended to decline with F60, F80, and F100.

Our experiments revealed the smooth muscle relaxing activity of RC extract, fractions and essential oil in this experimental setting. On histamine-precontracted preparations (treated with $0.5 \mu \mathrm{M}$ histamine for $15 \mathrm{~min}$, in the presence of atropine and tetrodotoxin, both $0.5 \mu \mathrm{M}$ ), concentration-dependent relaxation was observed in response to treatment with $\mathrm{RC}$ crude extract $(60-200 \mu \mathrm{g} / \mathrm{ml})$ (Figure 3 and Table 5). The highest concentration tested induced full relaxation. The relaxation detected with the $20 \mu \mathrm{g} / \mathrm{ml}$ test sample did not exceed the changes evoked by the solvent itself ( $1 \mu \mathrm{l} / \mathrm{ml}$ DMSO; see below). In several cases the relaxation was preceded by a little contraction. The relaxation induced by the $60 \mu \mathrm{g} / \mathrm{ml}$ extract was not significantly altered by the adrenergic $\beta$-receptor antagonist propranolol ( $1 \mu \mathrm{M} ; 51.3 \pm 7.8 \%$ relaxation, $N=6$ ) or by the NO synthase inhibitor $\mathrm{N}^{\mathrm{G}}$-nitro-L-arginine $(100 \mu \mathrm{M} ; 47.4 \pm 7.4 \%$ relaxation,

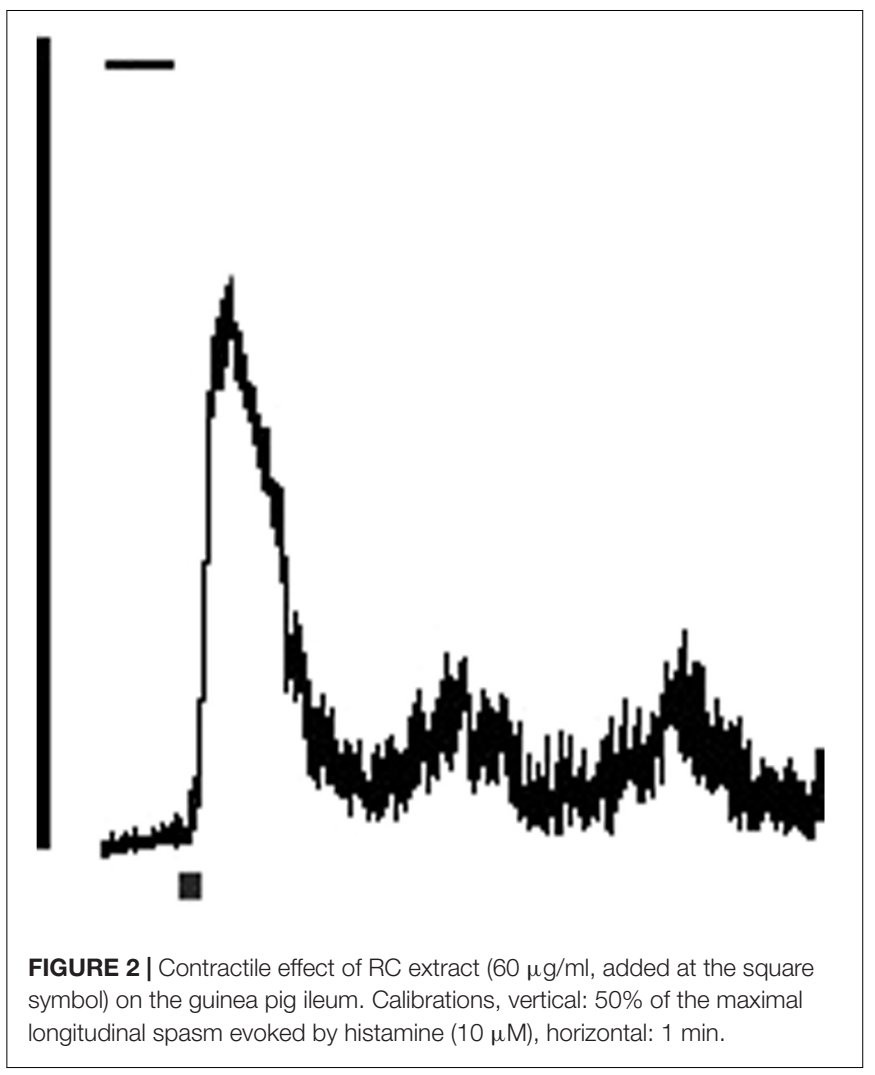

TABLE 3 | Effects of drugs on the contractile response to $\mathrm{RC}$ crude extract $(60 \mu \mathrm{g} / \mathrm{ml})$ on guinea pig small intestine (mean \pm SEM).

\begin{tabular}{|c|c|c|}
\hline Pretreatment & Contraction ( $\%$ of maximal spasm) & $\boldsymbol{N}$ \\
\hline No pretreatment (control) & $40.1 \pm 3.3$ & 11 \\
\hline Tetrodotoxin $(0.5 \mu \mathrm{M})$ & $15.0 \pm 1.8^{*}$ & 6 \\
\hline Atropine $(0.5 \mu \mathrm{M})$ & $8.2 \pm 2.0^{*}$ & 6 \\
\hline PPADS $(50 \mu \mathrm{M})$ & $49.7 \pm 2.7$ & 9 \\
\hline 5-HT receptor antagonists $\#$ & $52.1 \pm 3.0$ & 11 \\
\hline Solvent for capsaicin & $51.1 \pm 5.2$ & 7 \\
\hline Capsaicin \& & $46.9 \pm 6.0$ & 9 \\
\hline Solvent for indomethacin & $46.4 \pm 4.0$ & 6 \\
\hline Indomethacin (3 $\mu \mathrm{M})$ & $31.2 \pm 5.6^{*}$ & 10 \\
\hline
\end{tabular}

Values significantly different from the respective control group are indicated by asterisks (*). "methysergide (0.3 $\mu \mathrm{M})$, SB204070 (1 $\mu \mathrm{M})$ and Y25130 (1 $\mu \mathrm{M})$. $\& 10 \mu \mathrm{M}$ of capsaicin for $10 \mathrm{~min}$, followed by a 60 -min washout period.

$N=10)$ (both compared with the group indicated in Table 5). The solvent DMSO $(0.3$ or $1 \mu \mathrm{l} / \mathrm{ml})$ had a slight relaxant effect in this experimental arrangement (histamine-precontracted ileum pretreated with atropine and tetrodotoxin); it amounted to $3.0 \pm 1.6 \%$ at $0.3 \mu \mathrm{l} / \mathrm{ml}$ and $7.9 \pm 1.4 \%$ at $1 \mu \mathrm{l} / \mathrm{ml} ; N=11$ and 17 , respectively.

On histamine-precontracted, atropine- and tetrodotoxintreated ileum, different fractions of RC extract showed distinct relaxant effects (Table 6). F20 and F40 (60 or $200 \mu \mathrm{g} / \mathrm{ml})$ practically produced no relaxation $(N=5$ for both concentrations). This observation refers to the potential role of flavonoids in the relaxant effect, and experiments with 


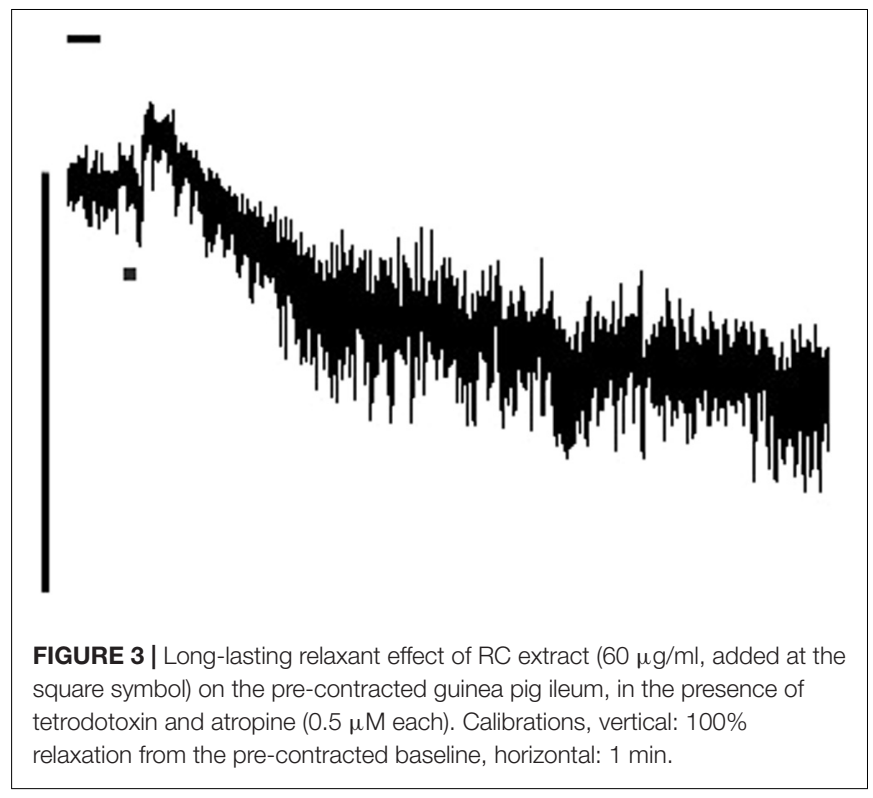

TABLE 4 | Contractile effects of RC extract fractions on guinea pig ileum (longitudinally oriented preparations, mean \pm SEM).

\begin{tabular}{|c|c|c|}
\hline Bath concentration & Contraction ( $\%$ of the maximal spasm) & $N$ \\
\hline \multicolumn{3}{|l|}{ F20 } \\
\hline $2 \mu \mathrm{g} / \mathrm{ml}$ & $5.2 \pm 2.4 \%$ & 5 \\
\hline $20 \mu \mathrm{g} / \mathrm{ml}$ & $18.2 \pm 6.1 \%$ & 5 \\
\hline $60 \mu \mathrm{g} / \mathrm{ml}$ & $18.5 \pm 4.4 \%$ & 6 \\
\hline $200 \mu \mathrm{g} / \mathrm{ml}$ & $22.2 \pm 3.1 \% *$ & 5 \\
\hline \multicolumn{3}{|l|}{ F40 } \\
\hline $2 \mu \mathrm{g} / \mathrm{ml}$ & $2.5 \pm 1.2 \%$ & 5 \\
\hline $20 \mu \mathrm{g} / \mathrm{ml}$ & $18.2 \pm 5.9 \%$ & 5 \\
\hline $60 \mu \mathrm{g} / \mathrm{ml}$ & $15.5 \pm 3.3 \%$ & 5 \\
\hline $200 \mu \mathrm{g} / \mathrm{ml}$ & $22.8 \pm 8.4 \% *$ & 5 \\
\hline \multicolumn{3}{|l|}{ F60 } \\
\hline $2 \mu \mathrm{g} / \mathrm{ml}$ & $8.4 \pm 1.3 \%$ & 6 \\
\hline $20 \mu \mathrm{g} / \mathrm{ml}$ & $21.8 \pm 6.4 \%^{*}$ & 5 \\
\hline $60 \mu \mathrm{g} / \mathrm{ml}$ & $20.9 \pm 8.2 \% *$ & 5 \\
\hline $200 \mu \mathrm{g} / \mathrm{ml}$ & $4.0 \pm 2.7 \%$ & 6 \\
\hline \multicolumn{3}{|l|}{ F80 } \\
\hline $2 \mu \mathrm{g} / \mathrm{ml}$ & $0.4 \pm 0.4 \%$ & 5 \\
\hline $20 \mu \mathrm{g} / \mathrm{ml}$ & $13.0 \pm 3.2 \%$ & 5 \\
\hline $60 \mu \mathrm{g} / \mathrm{ml}$ & $12.0 \pm 4.0 \%$ & 5 \\
\hline $200 \mu \mathrm{g} / \mathrm{ml}$ & $2.5 \pm 1.5 \%$ & 5 \\
\hline \multicolumn{3}{|l|}{ F100 } \\
\hline $20 \mu \mathrm{g} / \mathrm{ml}$ & $12.4 \pm 2.1 \%$ & 6 \\
\hline $60 \mu \mathrm{g} / \mathrm{ml}$ & $26.4 \pm 5.8 \% *$ & 5 \\
\hline $200 \mu \mathrm{g} / \mathrm{ml}$ & $15.1 \pm 2.7 \%$ & 6 \\
\hline
\end{tabular}

Asterisks denote values significantly different from a pooled group of solvent effects (Kruskal-Wallis test for several unrelated samples).

four flavonoids isolated from the plant material reassured this hypothesis. All the flavonoids exerted a dual effect on the guinea pig ileum, i.e., a short-lived contraction at basal tone and a long-lasting relaxation on the histamine-pre-contracted, atropine- and tetrodotoxin-pretreated preparations (Table 7). At
TABLE 5 | Relaxing effect of RC crude extract and essential oil on the pre-contracted ileum.

\begin{tabular}{lcc}
\hline $\begin{array}{l}\text { Bath concentration of } \\
\text { RC crude extract }\end{array}$ & Relaxation (\% of the maximum) & N \\
\hline $20 \mu \mathrm{g} / \mathrm{ml}$ & $18 \pm 5.2 \%$ & 6 \\
$60 \mu \mathrm{g} / \mathrm{ml}$ & $76.2 \pm 8.5 \% *$ & 9 \\
$200 \mu \mathrm{g} / \mathrm{ml}$ & $100 \% *$ & 5 \\
\hline Bath concentration of & Relaxation \% & $\mathbf{N}$ \\
$\mathbf{R C}$ essential oil & & \\
\hline $0.1 \mu \mathrm{g} / \mathrm{ml}$ & $12.8 \pm 3.5 \%$ & 6 \\
$1 \mu \mathrm{g} / \mathrm{ml}$ & $30.8 \pm 5.9 \% *$ & 9 \\
$10 \mu \mathrm{g} / \mathrm{ml}$ & $69.7 \pm 5.6 \% *$ & 6
\end{tabular}

Atropine and tetrodotoxin (0.5 $\mu \mathrm{M}$ each) were present in the medium in order to inhibit the contractile effect of the $R C$ extract. Tonic submaximal contraction was evoked with histamine (0.5 $\mu \mathrm{M}$ for $15 \mathrm{~min})$. Values are given in \%, where 100\% means full relaxation reaching the pre-histamine values (mean \pm SEM). Asterisks denote values significantly different from a pooled group of solvent effects $(0.3$ or $1 \mu \mathrm{l} / \mathrm{ml}$ of DMSO, see text) (Kruskal-Wallis test for several unrelated samples).

TABLE 6 | Relaxant effects of RC extract fractions on pre-contracted guinea pig ileum (mean \pm SEM).

\begin{tabular}{lcc}
\hline Bath concentration & Relaxation \% & N \\
\hline F60 & & \\
$20 \mu \mathrm{g} / \mathrm{ml}$ & $18.7 \pm 5.7 \%$ & 5 \\
$60 \mu \mathrm{g} / \mathrm{ml}$ & $96.0 \pm 3.0 \% *$ & 6 \\
$200 \mu \mathrm{g} / \mathrm{ml}$ & $93.5 \pm 4.9 \% *$ & 6 \\
$\mathbf{F 8 0}$ & & \\
$20 \mu \mathrm{g} / \mathrm{ml}$ & $47.2 \pm 7.7 \%$ & 5 \\
$60 \mu \mathrm{g} / \mathrm{ml}$ & $93.0 \pm 5.9 \% *$ & 6 \\
$200 \mu \mathrm{g} / \mathrm{ml}$ & $100 \% *$ & 4 \\
$\mathbf{F 1 0 0}$ & & 4 \\
$6 \mu \mathrm{g} / \mathrm{ml}$ & $12.5 \%$ & 5 \\
$20 \mu \mathrm{g} / \mathrm{ml}$ & $61 \pm 13.7 \%$ & 6 \\
$60 \mu \mathrm{g} / \mathrm{ml}$ & $69.4 \pm 7.5 \% *$ & 4 \\
$200 \mu \mathrm{g} / \mathrm{ml}$ & $100 \% *$ & \\
\hline
\end{tabular}

$100 \%$ relaxation denotes that tone returns to baseline. Asterisks denote values significantly different from a pooled group of solvent effects (0.3 or $1 \mu \mathrm{l} / \mathrm{ml} \mathrm{DMSO})$ (Kruskal-Wallis test for several unrelated samples).

a concentration of $1 \mu \mathrm{M}$, the flavonoids did not exert contractile activities, however, at $10 \mu \mathrm{M}$, a slight effect $(34.6 \pm 7.4 \%$ for hispidulin, $32.0 \pm 3.1 \%$ for luteolin, $27.1 \pm 4.2 \%$ for eupafolin, and $20.5 \pm 5.1 \%$ for apigenin, $N=5$ each) was observed. The relaxant activities at $2 \mu \mathrm{M}$ ranged between $18.2 \pm 5.4 \%$ and $24.2 \pm 3.7 \%$, whereas at $20 \mu \mathrm{M}$ between $64.5 \pm 4.1 \%$ and $81.9 \pm 5.3 \%$.

The essential oil of $\mathrm{RC}(0.1,1,10$, or $30 \mu \mathrm{g} / \mathrm{ml})$ showed no contractile effect on the ileum $(N=6-8)$. RC oil $(1$ or $10 \mu \mathrm{g} / \mathrm{ml})$ induced considerable relaxation on histamine-precontracted, atropine- and tetrodotoxin-pretreated preparations (Table 5). Similar results were obtained on preparations without atropine and tetrodotoxin pretreatment $(N=6-8$, data not shown).

Papaverine was used as positive control. As with other experiments for studying relaxation, the drug was administered to histamine-precontracted, atropine- and tetrodotoxin-treated 
TABLE 7 | Relaxant effects of flavonoids on pre-contracted guinea pig ileum (mean \pm SEM).

\begin{tabular}{lll}
\hline Bath concentration & Relaxation $\%$ & N \\
\hline Hispidulin & & 5 \\
$2 \mu \mathrm{M}$ & $19.4 \pm 3.5 \%$ & 6 \\
$20 \mu \mathrm{M}$ & $64.5 \pm 4.1 \%$ & \\
Luteolin & & 5 \\
$2 \mu \mathrm{M}$ & $19.6 \pm 1.6 \%$ & 6 \\
$20 \mu \mathrm{M}$ & $80.0 \pm-4.5 \%$ & \\
Eupafolin & & 5 \\
$2 \mu \mathrm{M}$ & $18.2 \pm 5.4 \%$ & 6 \\
$20 \mu \mathrm{M}$ & $68.7 \pm 6.8 \%$ & \\
Apigenin & & 5 \\
$2 \mu \mathrm{M}$ & $24.2 \pm 3.7 \%$ & 6 \\
$20 \mu \mathrm{M}$ & $81.9 \pm 5.3 \%$ & \\
\hline
\end{tabular}

$100 \%$ relaxation denotes that tone returns to baseline. The solvent contained a maximum of $10 \%$ DMSO and had no relaxant effect in the volumes used.

TABLE 8 | Contractile effect of RC extract on guinea pig urinary bladder strip, without pretreatment and following treatment with capsaicin or its solvent (mean \pm SEM).

\begin{tabular}{lcc}
\hline $\begin{array}{l}\text { Bath concentration of } \\
\text { RC crude extract }\end{array}$ & Contraction (\% of the maximal spasm) & $\mathbf{N}$ \\
\hline $20 \mu \mathrm{g} / \mathrm{ml}$ No pretreatment & $8.0 \pm 4.2 \%$ & 7 \\
$200 \mu \mathrm{g} / \mathrm{ml}$ No pretreatment & $20.0 \pm 5.1 \%$ & 7 \\
$200 \mu \mathrm{g} / \mathrm{ml}$ Ethanol & $21.6 \pm 4.9 \%$ & 8 \\
pretreatment & & 8 \\
$200 \mu \mathrm{g} / \mathrm{ml}$ Capsaicin & $9.1 \pm 1.3 \% *$ & \\
$(10 \mu \mathrm{M})$ pretreatment & & \\
\end{tabular}

\#Solvent and time control for capsaicin. \&Capsaicin was added and incubated for $10 \mathrm{~min}$. This was followed by a 90-min washout period. * Significantly different from the ethanol control.

ileum preparations, in a non-cumulative manner. The relaxant responses obtained were as follows, $0.3 \mu \mathrm{M}$ : $19.7 \pm 4.7 \%(N=6)$; $1 \mu \mathrm{M}: 29.1 \pm 3.5 \%(N=6) ; 3 \mu \mathrm{M}: 30.1 \pm 4.8 \%(N=7) ; 10 \mu \mathrm{M}$ : $92.9 \pm 4.2 \%(N=6)$, where, as also elsewhere in this paper, relaxation to the pre-histamine baseline was taken as $100 \%$.

\section{Effects on Guinea Pig Urinary Bladder}

The effects of the RC extract and the essential oil were tested on guinea pig urinary bladder strips as well. RC crude extract evoked concentration-dependent contraction at 20 and $200 \mu \mathrm{g} / \mathrm{ml}$ (Table 8 and Figure 4 for $200 \mu \mathrm{g} / \mathrm{ml}$ ). The solvent of the extract had no contractile effect on the bladder $(N=4)$. The effect of the $200 \mu \mathrm{g} / \mathrm{ml}$ extract was diminished (approximately halved) by in vitro capsaicin pretreatment (Table 8). Contractile responses were compared to the maximal spasm evoked by $100 \mathrm{mM}$ of $\mathrm{KCl}$ at the end of the experiment.

To study the relaxant effect of the crude extract, the bladder strips were sub-maximally pre-contracted with histamine (1-3 $\mu \mathrm{M})$. Atropine and tetrodotoxin were present in the bathing fluid. Since excitatory purinergic mechanisms are present in the bladder, $\alpha, \beta$-methylene ATP desensitization $(10+10 \mu \mathrm{M}$ for 10 min each) was also performed. RC crude extract had no

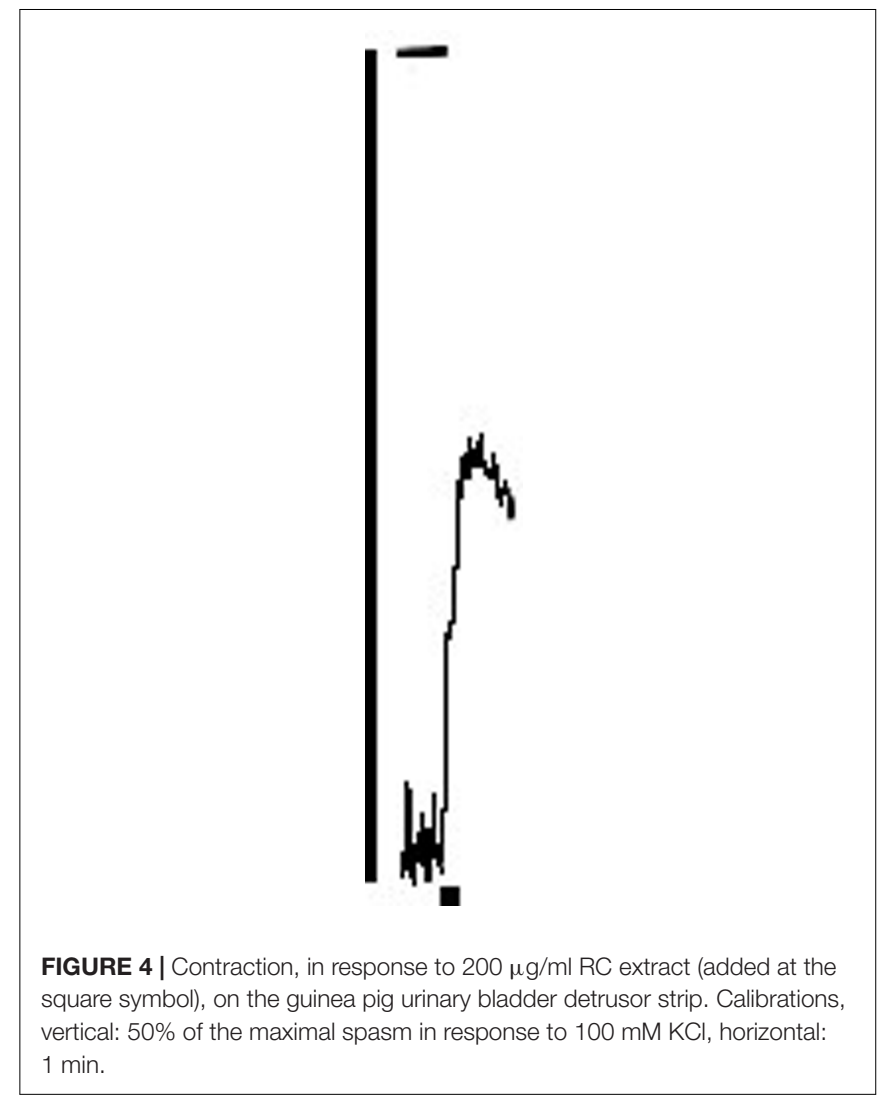

effect at a concentration of $20 \mu \mathrm{g} / \mathrm{ml}$, whereas a concentration of $200 \mu \mathrm{g} / \mathrm{ml}$ induced $58.3 \pm 4.6 \%$ relaxation $(N=7$ and 8 , respectively). The solvent itself ( $1 \mu \mathrm{l} / \mathrm{ml}$ DMSO) exerted no effect.

Roman chamomile essential oil caused no contraction in a concentration of $10 \mu \mathrm{g} / \mathrm{ml}$. Similarly to the extract, RC oil exerted a moderate relaxing effect on the pre-contracted bladder: $17.9 \pm 6.1 \%$ and $34.1 \pm 5.8 \%$ relaxation was evoked with 1 and $10 \mu \mathrm{g} / \mathrm{ml}, N=7$ and 6 , respectively.

\section{Effects on Rat Gastrointestinal Preparations}

The relaxant effect of $\mathrm{RC}$ essential oil was confirmed on longitudinally oriented preparations of the rat gastrointestinal tract. Rat whole ileum and distal colon preparations are characterized by high intrinsic tone, therefore no precontraction was needed. RC oil $(10 \mu \mathrm{g} / \mathrm{ml})$ induced relaxation $(41.4 \pm 7.4 \%$ and $21.8 \pm 3.7 \%, N=7$ and 10 , respectively, on these preparations, where $100 \%$ means the relaxant response to $3 \mu \mathrm{M}$ isoprenaline, given at the end of the experiment). A concentration of $1 \mu \mathrm{g} / \mathrm{ml}$ was barely effective. No contractile effect was observed. Rat stomach fundus strips were pre-contracted with $0.1-0.3 \mu \mathrm{M}$ acetylcholine. RC oil produced relaxation (on average 44 and $70.3 \%$ relaxation in response to concentrations of 1 and $10 \mu \mathrm{l} / \mathrm{ml}, N=4$ each, where $100 \%$ means relaxation to the pre-acetylcholine level). 


\section{Effects on Human Jejunal Preparations}

In untreated longitudinally oriented human jejunal preparations RC crude extract induced transient contraction amounting to $12.4 \pm 3.5 \%$ and $30.5 \pm 0.6 \%$ at 20 and $200 \mu \mathrm{g} / \mathrm{ml}$ concentrations, respectively $(N=8)$. Atropine- and tetrodotoxin-pretreated preparations were pre-contracted with PGF2 $\alpha$ ( 1 or $2 \mu \mathrm{M})$. RC extract showed a concentration-dependent relaxant effect $(25.8 \pm 4.3 \%$ at $20 \mu \mathrm{g} / \mathrm{ml}, N=8 ; 68.5 \pm 8.5 \%$ at $60 \mu \mathrm{g} / \mathrm{ml}$, $N=7 ; 50.8 \pm 3.7 \%$ at $200 \mu \mathrm{g} / \mathrm{ml}, N=9)$. RC essential oil produced no contraction. A lasting relaxant effect was detected on pre-contracted preparations (in the presence of atropine and tetrodotoxin). The relaxant effect reached $26.9 \pm 5.2 \%$ at $1 \mu \mathrm{g} / \mathrm{ml}$ and $81.4 \pm 8.2 \%$ at $10 \mu \mathrm{g} / \mathrm{ml}$ concentrations $(N=8$ and 7 , respectively).

In untreated circularly oriented human jejunal preparations the RC crude extract evoked transient contraction $(43.4 \pm 10 \%$ at $200 \mu \mathrm{g} / \mathrm{ml}, N=5$ ), while the $20 \mu \mathrm{g} / \mathrm{ml}$ concentration sample had a negligible effect ( $2.8 \%$ contraction on 5 preparations). RC extract induced relaxation in $\mathrm{PGF}_{2 \alpha}$-precontracted preparations (in the presence of atropine and tetrodotoxin). This effect reached $45 \%$ with $60 \mu \mathrm{g} / \mathrm{ml}$ concentration $(N=4)$, while a concentration of $200 \mu \mathrm{g} / \mathrm{ml}$ produced full relaxation $(N=6)$, where a relaxation to the pre-prostaglandin level was taken as $100 \%$.

The solvent itself $(1 \mu \mathrm{l} / \mathrm{ml}$ DMSO) did not evoke either contraction or relaxation on this preparation $(N=4-6)$.

\section{DISCUSSION}

The experiments presented here have been carried out with RC, which has been used traditionally for the symptomatic treatment of mild, spasmodic and other gastrointestinal complaints, however, the presumed smooth muscle-relaxant effect has not been confirmed experimentally. The aim of our study was to investigate the effect of a traditionally used extract of the plant, its fractions and the essential oil of the plant, as well as of four of its flavonoid components.

The phytochemical analysis of the extract revealed the presence of flavonoids in the plant, four of which (apigenin, luteolin, eupafolin, and hispidulin) were isolated and identified. The hydroethanolic extract was fractionated on polyamide to gain fractions with different flavonoid content in order to examine the role of these compounds in the effect on smooth muscles. The flavonoid content of the extract and fractions was analyzed by HPLC. The flavonoid content of the extract was fractionated successfully, fractions F80, F100 (and to some extent F60) containing higher amounts of flavonoids than the crude extracts. The flavonoid pattern of fractions also differed. The essential oil analyzed by us belongs to the chemotype characterized by the predominance of methallyl angelate.

Pharmacological experiments were carried out with the hydroethanolic extract, its fractions (F20, F40, F60, F80, and F100), four flavonoids (apigenin, eupafolin, hispidulin, and luteolin) and the essential oil of the plant on different smooth muscles in vitro. The hydroethanolic extract of RC has both stimulatory and relaxant effects on guinea pig ileum. The moderate, transient stimulatory activity results from the activation of cholinergic neurons, as confirmed by its inhibition by tetrodotoxin (an inhibitor of neuronal voltage-sensitive $\mathrm{Na}^{+}$channels) and atropine (antagonist on muscarinic receptors for acetylcholine). Although capsaicin, a stimulant of a certain class of sensory receptors (through which it evokes a "local efferent" response) shows a similar cholinergic, neurogenic effect in guinea pig small intestine preparations (see Barthó et al., 2004 for review), a functional blockade of capsaicin-sensitive nerve endings by capsaicin pretreatment failed to inhibit the contractile action of RC extract. The contractile action of RC extract was moderately, yet significantly reduced by the cyclooxygenase inhibitor indomethacin, which may indicate a modulatory role of endogenous prostanoids. Other pharmacological inhibitors tested had no contraction-reducing effect, therefore it is proposed that neither endogenous serotonin, nor PPADSsensitive purinergic mechanisms play a role in the excitatory action of RC extract. It should be noted that both serotonin and ATP or the $\mathrm{P}_{2 \mathrm{X}}$ receptor agonist $\alpha, \beta$-methylene ATP are able to evoke cholinergic contractions in guinea pig ileum (Benko et al., 2005; Barthó et al., 2006; Sandor et al., 2016 for recent data).

Following the transient and mild-to-moderate stimulant effect, the RC crude extract exhibited a sustained relaxant effect on the guinea pig ileum in the presence of tetrodotoxin and atropine, and preliminary experiments indicated a similar relaxant effect also in the absence of atropine and tetrodotoxin. Yet, atropine and tetrodotoxin were included into these experiments for creating a methodologically clear situation, where an initial, neuronally mediated excitatory action would hardly interfere with the relaxant one. The concentrations of the extract causing relaxation were roughly the same as those causing contraction. Based on these data we propose that the site of action for the relaxant effect of RC is on the smooth muscle itself. Neither the adrenergic $\beta$-receptor antagonist propranolol nor the $\mathrm{NO}$ synthase inhibitor $\mathrm{N}^{\mathrm{G}}$ nitro-L-arginine significantly reduced the relaxant effect of the RC extract, hence, no evidence was found for these mechanisms to be involved in the relaxant response. In fact, a direct relaxant effect of the extract was demonstrated on all gastrointestinal preparations tested, including human and rat gastrointestinal preparations, as well as in guinea pig bladder. In preparations with high intrinsic tone (rat ileum and rat colon), relaxant activity was practically the only response to be seen.

Different RC extract fractions evoked both contraction and relaxation on guinea pig ileum. While there was a clear-cut tendency correlation between the flavonoid content and the relaxant effect, in case of contractile action such correlation was not observed (on the contrary, fractions with lower flavonoid content exerted slightly higher contractile activities). Fractions with high flavonoid content (F60, F80, and F100) had remarkable relaxant effects, whereas fractions with no (F20) or low (F40) flavonoid content exerted no such activity. The pure flavonoids of the extract showed dual effects in the guinea pig ileum, much similarly to the crude extract. Moreover, there was no substantial 
difference between the potencies of the flavonoids. This means that any of these flavonoids may contribute to the sustained relaxant and the transient contractile effect of the extract. The essential oil of RC caused no contraction on the test preparations; nevertheless, a consistent relaxant effect was detected. This seems to indicate that (i) chemical components responsible for the stimulant effect may be absent in the essential oil; (ii) it may be that several types of compounds present in the extract are responsible for the smooth muscle-relaxant action. This point needs to be clarified in subsequent experiments.

The contraction of guinea pig bladder in response to the $\mathrm{RC}$ extract proved to be special, in that it was reduced by half following in vitro capsaicin pretreatment. This indicates that capsaicin-sensitive sensory nerves of the bladder wall are in some way involved in the excitatory effect of the RC extract (see Barthó et al., 2004). Nevertheless, the RC extract and oil also induced bladder relaxation in pre-contracted preparations.

Roman chamomile extract was effective in human jejunal preparations as well. Both an excitatory and a tetrodotoxinand atropine-resistant relaxing effect were demonstrated, again, the smooth muscle relaxant one being more sustained than the excitatory one. Preliminary experiments have shown that this early contraction is reduced by atropine. Due to limited access to human tissue, no further analysis of these responses could be performed.

In terms of possible medical uses it may be noted that a combination of nerve-mediated, mild-to moderate excitatory effect and a smooth muscle-relaxant action may even be advantageous for some gastrointestinal problems, e.g., diminished peristaltic activity, while for spasms in the stomach or large intestine it is obviously the smooth muscle relaxing activity that offers benefits.

\section{CONCLUSION}

Our results support the overall smooth muscle relaxant effect of a hydroethanolic RC extract and of the essential oil of RC. The

\section{REFERENCES}

Abou-Zied, E., and Rizk, A. (1973). Phytochemical investigation of Anthemis nobilis L. growing in Egypt. Qual. Plant. Mater. Veg. 2, 141-144. doi: 10.1007/ BF01100682

Adams, R. P. (2007). Identification of Essential Oil Components by Gas Chromatography/Mass Spectroscopy. Carol Stream, IL: Allured Pub. Corp.

Alarcün, R., Pardo-de-Santayana, M., Priestley, C., and Morales, R. (2015). Author's accepted manuscript. J. Ethnopharmacol. 176, 207-224. doi: 10.1016/j.jep.2015. 10.022

Antonelli, A., and Fabbri, C. (1998). Study on Roman chamomile Chamaemelum nobile L. all.) oil. J. Essent. Oil Res. 10, 571-574. doi: 10.1080/10412905.1998. 9700974

Augustin, B., Javorka, S., and Giovannini, R. R. P. (1948). Magyar Gyógynövények [Hungarian Herbal Drugs. New Delhi: Ministry of Agriculture, 299-300.

Baghalian, K., Abdoshah, S., Khalighi-Sigaroodi, F., and Paknejad, F. (2011). Physiological and phytochemical response to drought stress of German chamomile (Matricaria recutita L.). Plant Physiol. Biochem. 49, 201-207. doi: 10.1016/j.plaphy.2010.11.010 predominant effect of the extract, its fractions and flavonoids is relaxation, being more sustained than the transient contraction observed in some cases. This activity is in correlation with the flavonoid content of the extract. Since the components of the essential oil are partly extracted with alcohols, the constituents of the oil also contribute to the overall effect of the hydroethanolic RC extract. The extract used in our experiments was prepared in accordance with the European Medicines Agency monograph for this plant, therefore our study contributes to the body of evidence relating the traditional use of this plant.

\section{AUTHOR CONTRIBUTIONS}

$\mathrm{ZS}, \mathrm{DK}, \mathrm{TB}$, and RP carried out the organ bath experiments. JM, $\mathrm{KV}$, and $\mathrm{AH}$ performed the phytochemical studies. $\mathrm{JH}, \mathrm{LB}$, and DC designed and co-ordinated the experiments and wrote the manuscript.

\section{FUNDING}

Financial support from the National Research, Development and Innovation Office (OTKA K115796), Economic Development and Innovation Operative Programme GINOP-2.3.2-15-201600012, and János Bolyai Research Scholarship of the Hungarian Academy of Sciences are gratefully acknowledged. This study was also supported by the Faculty of Medicine, University of Pécs.

\section{SUPPLEMENTARY MATERIAL}

The Supplementary Material for this article can be found online at: https://www.frontiersin.org/articles/10.3389/fphar. 2018.00323/full\#supplementary-material

Baghalian, K., Haghiry, A., Naghavi, M. R., and Mohammadi, A. (2008). Effect of saline irrigation water on agronomical and phytochemical characters of chamomile (Matricaria recutita L.). Sci. Hortic. 116, 437-441. doi: 10.1016/j. scienta.2008.02.014

Bail, S., Buchbauer, G., Jirovetz, L., Denkova, Z., Slavchev, A., Stoyanova, A., et al. (2009). Antimicrobial activities of Roman chamomile oil from France and its main compounds. J. Essent. Oil Res. 21, 283-286. doi: 10.1080/10412905.2009. 9700171

Barthó, L., Benkó, R., Patacchini, R., Pethö, G., Holzer-Petsche, U., Holzer, P., et al. (2004). Effects of capsaicin on visceral smooth muscle: a valuable tool for sensory neurotransmitter identification. Eur. J. Pharmacol. 500, 143-157. doi: 10.1016/j.ejphar.2004.07.020

Barthó, L., Undi, S., Benkó, R., Wolf, M., Lázár, Z., Lénárd, L., et al. (2006). Multiple motor effects of ATP and their inhibition by P purinoceptor antagonist, pyridoxalphosphate-6-azophenyl-2',4'-disulphonic acid in the small intestine of the guinea-pig. Basic Clin. Pharmacol. Toxicol. 98, 488-495. doi: 10.1111/j. 1742-7843.2006.pto_369.x

Benko, R., Undi, S., Wolf, M., and Bartho, L. (2005). Effects of acute administration of and tachyphylaxis to alpha,beta-methylene ATP in the guinea-pig small intestine. Basic Clin. Pharmacol. Toxicol. 97, 369-373. doi: 10.1111/j.1742-7843. 2005.pto_117.x 
Bisset, N. (1994). Herbal Drugs and Phytopharmaceuticals. Stuttgart: Medpharm Scientific Publishers GmbH, 140-142.

Bradley, P. (1992). British Herbal Compendium. Bristol: British Herbal Medicine Association, 191-193.

British Herbal Pharmacopoeia (1971). British Herbal Pharmacopoeia. London: British Herbal Medicine Association.

Chao, S. C., Young, D. G., and Oberg, C. J. (2000). Screening for inhibitory activity of essential oils on selected bacteria, fungi and viruses. J. Essent. Oil Res. 12, 639-649. doi: 10.1080/10412905.2000.9712177

EMA-HMPC (2012). Community Herbal Monograph on Chamaemelum nobile (L.) All., Flos. London: EMA-HMPC.

European Pharmacopoeia (2008). European Pharmacopoeia, 6th Edn. Strasbourg: Council of Europe.

Evans, W. (1989). Trease and Evan's Pharmacognosy, 13th Edn. London: Bailliere Tindall.

Farkas, P., Hollá, M., Vaverková, S., Stahlová, B., Tekel, J., and Havránek, E. (2003). Composition of the essential oil from the flowerheads of Chamaemelum nobile (L.) All. (Asteraceae) cultivated in Slovak Republic. J. Essent. Oil Res. 15, 83-85. doi: 10.1080/10412905.2003.9712073

Fauconnier, M. L., Jaziri, M., Homes, J., Shimomura, K., and Marlier, M. (1996). "Anthemis nobilis L. (Roman Chamomile): in vitro culture, micropropagation, and the production of essential oils," in Medicinal and Aromatic Plants IX. Biotechnology in Agriculture and Forestry, ed. Y. P. S. Bajaj (Berlin and Heidelberg: Springer), 37.

Hänsel, R., Keller, K., and Rimpler, H. S. G. (1993). Hagers Handbuch der Pharmazeutischen Praxis Drogen A-D, 5th Edn. Berlin: Springer-Verlag.

Herisset, A., Chaumont, J. P., and Paris, R. R. (1973). Flavonoids of Roman camomille (Anthemis nobilis), simple variety. Plant Med. Phytother. 7, 234-240.

Herisset, A., Paris, R. R., and Chaumont, C. J. (1971). Flavonoids of Roman camomile (Anthemis nobilis). Plant Med. Phytother. 5, 234-239.

Hernández-Ceruelos, A., Madrigal-Santillán, E., Morales-González, J. A., Chamorro-Cevallos, G., Cassani-Galindo, M., and Madrigal-Bujaidar, E. (2010). Antigenotoxic effect of Chamomilla recutita (L.) rauschert essential oil in mouse spermatogonial cells, and determination of its antioxidant capacity in vitro. Int. J. Mol. Sci. 11, 3793-3802. doi: 10.3390/ijms111 03793

Hiller, K., and Melzig, M. (1999). Lexikon der Arzneipflanzen und Drogen. Heidelberg: Spektrum Akademischer Verlag GmbH.

Jouad, H., Lacaille-Dubois, M. A., Lyoussi, B., and Eddouks, M. (2001). Effects of the flavonoids extracted from Spergularia purpurea Pers. on arterial blood pressure and renal function in normal and hypertensive rats. J. Ethnopharmacol. 76, 159-163. doi: 10.1016/S0378-8741(01)00209-4

Kovats, E. (1965). "Gas chromatographic characterization of organic substances in the retention index system," in Advances in Chromatography, Vol. 1, eds J. C. Giddings and R. A. Keller (New York, NY: Marcel Dekker, Inc.), 229-247.

Lemmens-Gruber, R., Marchart, E., Rawnduzi, P., Engel, N., Benedek, B., and Kopp, B. (2006). Investigation of the spasmolytic activity of the flavonoid fraction of Achillea millefolium s.l. on isolated guinea-pig ilea. Arzneimittelforschung 56, 582-588. doi: 10.1055/s-0031- 1296755

Lukacs, M. (1990). The Phytochemical Investigation of Anthemis nobilis L. Ph.D. thesis, Szent-Györgyi Albert Medical Universtiy, Szeged.

Magro, A., Carolino, M., Bastos, M., and Mexia, A. (2006). Efficacy of plant extracts against stored-products fungi. Rev. Iberoam. Micol. 23, 176-178. doi: 10.1016/ S1130-1406(06)70039-0

Melegari, M., Albasini, A., Pecorari, P., Vampa, G., Rinaldi, M., Rossi, T., et al. (1988). Chemical characteristics and pharmacological properties of the essential oils of Anthemis nobilis. Fitoterapia 59, 449-455.
Menendez-Baceta, G., Aceituno-Mata, L., Molina, M., Reyes-García, V., Tardío, J., and Pardo-de-Santayana, M. (2014). Medicinal plants traditionally used in the northwest of the Basque Country (Biscay and Alava), Iberian Peninsula. J. Ethnopharmacol. 152, 113-134. doi: 10.1016/j.jep.2013.12.038

Omidbaigi, R., Sefldkon, F., and Kazemi, F. (2003). Roman chamomile oil: comparison between hydro-distillation and supercritical fluid extraction. J. Essent. Oil Bear. Plants 6, 191-194. doi: 10.1080/0972-060X.2003.1064 3350

Omidbaigi, R., Sefidkon, F., and Kazemi, F. (2004). Influence of drying methods on the essential oil content and composition of Roman chamomile. Flavour Fragr. J. 19, 196-198. doi: 10.1002/ffj.1340

Piccaglia, R., Marotti, M., Giovanelli, E., Deans, S. G., and Eaglesham, E. (1993). Antibacterial and antioxidant properties of Mediterranean aromatic plants. Ind. Crops Prod. 2, 47-50. doi: 10.1016/0926-6690(93)90010-7

Pietta, P., Mauri, P., Bruno, A., Rava, A., Manera, E., and Ceva, P. (1991). Identification of flavonoids from Ginkgo biloba L., Anthemis nobilis L. and Equisetum arvense L. by high-performance liquid chromatography with diodearray UV detection. J. Chromatogr. A 553, 223-231. doi: 10.1016/S00219673(01)88492-2

Rápóti, J., and Romváry, V. (1974). Gyógyító Növények [Medicinal Plants], 4th Edn. Budapest: Medicina Kiadó.

Rivera, D., and Obon, C. (1995). The ethnopharmacology of Madeira and Porto Santo Islands, a review. J. Ethnopharmacol. 46, 73-93. doi: 10.1016/03788741(95)01239-A

Rossi, T., Melegari, M., Bianchi, A., Albasini, A., and Vampa, G. (1988). Sedative, anti-inflammatory and anti-diuretic effects induced in rats by essential oils of varieties of Anthemis nobilis: a comparative study. Pharmacol. Res. Commun. 20(Suppl. 5), 71-74. doi: 10.1016/S0031-6989(88)80844-0

Sandor, Z. I., Bencsik, T., Dekany, A., and Bartho, L. (2016). Serotonin or the mucosa do not mediate the motor effect of allyl isothiocyanate in the guinea-pig small intestine. Pharmacology 98, 199-203. doi: 10.1159/000447427

Schrader, A., Eckey, H., and Rohr, M. (1997). Examination of the activity of irradiation-lowering substances on the human skin exemplified with various chamomile extracts. SOFW J. 123, 3-11.

Viola, H., Wasowski, C., Levi de Stein, M., Wolfman, C., Silveira, R., Dajas, F., et al. (1995). Apigenin, a component of Matricaria recutita flowers, is a central benzodiazepine receptors-ligand with anxiolytic effects. Planta Med. 61, 213-216. doi: 10.1055/s-2006-958058

Wilkinson, S., Aldridge, J., Salmon, I., Cain, E., and Wilson, B. (1999). An evaluation of aromatherapy massage in palliative care. Palliat. Med. 13, 409-417. doi: 10.1191/026921699678148345

Zeggwagh, N. A., Moufid, A., Michel, J. B., and Eddouks, M. (2009). Hypotensive effect of Chamaemelum nobile aqueous extract in spontaneously hypertensive rats. Clin. Exp. Hypertens. 31, 440-450. doi: 10.1080/106419609028 25453

Conflict of Interest Statement: The authors declare that the research was conducted in the absence of any commercial or financial relationships that could be construed as a potential conflict of interest.

Copyright (C) 2018 Sándor, Mottaghipisheh, Veres, Hohmann, Bencsik, Horváth, Kelemen, Papp, Barthó and Csupor. This is an open-access article distributed under the terms of the Creative Commons Attribution License (CC BY). The use, distribution or reproduction in other forums is permitted, provided the original author(s) and the copyright owner are credited and that the original publication in this journal is cited, in accordance with accepted academic practice. No use, distribution or reproduction is permitted which does not comply with these terms. 


\section{OPEN ACCESS}

Edited by:

Anna Karolina Kiss,

Medical University of Warsaw, Poland

Reviewed by:

Antonietta Rossi,

University of Naples Federico II, Italy Kai Wang,

Institute of Apiculture Research

(CAAS), China

*Correspondence:

Katarzyna Celińska-Janowicz ksiemionow2@student.umb.edu.p

Specialty section:

This article was submitted to

Ethnopharmacology,

a section of the journal

Frontiers in Pharmacology

Received: 13 January 2018 Accepted: 22 March 2018

Published: 06 April 2018

Citation:

Celińska-Janowicz K, Zaręba I, Lazarek U, Teul J, Tomczyk M,

Patka J and Miltyk W (2018) Constituents of Propolis: Chrysin, Caffeic Acid, p-Coumaric Acid, and Ferulic Acid Induce PRODH/POX-Dependent Apoptosis in Human Tongue Squamous Cell Carcinoma Cell (CAL-27).

Front. Pharmacol. 9:336. doi: 10.3389/fphar.2018.00336

\section{Constituents of Propolis: Chrysin, Caffeic Acid, $p$-Coumaric Acid, and Ferulic Acid Induce PRODH/POX-Dependent Apoptosis in Human Tongue Squamous Cell Carcinoma Cell (CAL-27)}

Katarzyna Celińska-Janowicz ${ }^{\text {* }}$, Ilona Zaręba ${ }^{2}$, Urszula Lazarek ${ }^{1}$, Joanna Teul', Michał Tomczyk ${ }^{3}$, Jerzy Pałka ${ }^{2}$ and Wojciech Miltyk ${ }^{1}$

'Department of Pharmaceutical Analysis, Faculty of Pharmacy, Medical University of Białystok, Białystok, Poland, ${ }^{2}$ Department of Medicinal Chemistry, Faculty of Pharmacy, Medical University of Bialystok, Białystok, Poland, ${ }^{3}$ Department of Pharmacognosy, Faculty of Pharmacy, Medical University of Białystok, Białystok, Poland

Propolis evokes several therapeutic properties, including anticancer activity. These activities are attributed to the action of polyphenols. Previously it has been demonstrated, that one of the most abundant polyphenolic compounds in ethanolic extracts of propolis are chrysin, caffeic acid, p-coumaric acid, and ferulic acid. Although their pro-apoptotic activity on human tongue squamous cell carcinoma cells (CAL-27) was established previously, the detailed mechanism of this process remains unclear. Considering the crucial role of proline metabolism and proline dehydrogenase/proline oxidase (PRODH/POX) in the regulation of cancer cell survival/apoptosis, we studied these processes in polyphenol-treated CAL-27 cells. All studied polyphenols evoked anti-proliferative activity, accompanied by increased PRODH/POX, P53, active caspases-3 and -9 expressions and decreased collagen biosynthesis, prolidase activity and proline concentration in CAL-27 cells. These data suggest that polyphenols of propolis induce PRODH/POX-dependent apoptosis through up-regulation of mitochondrial proline degradation and down-regulation of proline utilization for collagen biosynthesis.

Keywords: proline, proline oxidase, propolis, flavonoids, phenolic acids, collagen, SCC

\section{INTRODUCTION}

Historically, propolis has been a key element of healthcare, and it products are still widely used as an alternative and complementary therapy or often as a primary treatment. Ethnopharmacology proved that propolis has medicinal properties, setting out directions for further research. Studies on the biological activity of propolis conducted in recent years revealed a wide spectrum of its activities. Propolis has many therapeutic properties, including antibacterial, antifungal, antiviral, cytotoxic, antioxidant, anti-inflammatory as well as immunomodulatory activity (Bankova et al., 2014). 
The chemical composition of propolis is related to its region of origin, however, some compounds occur in a comparable amount in all propolis samples (Popova et al., 2015, 2017a,b; Graikou et al., 2016). To broadly known propolis components belong among others: diterpenes, lignans, sesquiterpenes, acetophenones (Bankova, 2005), flavonoids and phenolic acid esters (Bankova et al., 2000) which are responsible for numerous of its biological activities (Kubiliene et al., 2015; Turan et al., 2015; Yildirim et al., 2016).

Recently, the interest is focused on anticancer properties of propolis. In cancer cells treated with propolis significant changes in various intracellular amino acids and their metabolites were found, among which proline was proved to significantly affect cell viability. The increase in proline concentration was observed in numerous cancer cell lines. The phenomenon was related to cancer survival (Surazynski et al., 2008a) and poor patient prognosis. However, proline degradation by $\mathrm{PRODH} / \mathrm{POX}$ was found to induce apoptosis in several cancer cell lines (Sadowska et al., 2013; Kononczuk et al., 2015a). Therefore, compounds that modulate intracellular proline metabolism are of great importance as potential anticancer factors.

An important source of proline is collagen. The final products of collagen degradation in lysosomes are imidodipeptides (containing C-terminal proline) that are further hydrolyzed to amino acids by cytoplasmic imidodipeptidase-prolidase [E.C. 3.4.13.9]. Therefore, prolidase plays an important role in the regulation of proline-dependent functions. One of the regulatory role of prolidase is providing proline for collagen re-synthesis. Alternatively, proline is degraded into pyrroline-5-carboxylic acid (P5C) in mitochondria by PRODH/POX. The conversion generates superoxide anion that may contribute to ROS-dependent induction of apoptosis (Liu et al., 2006; Phang et al., 2008a,b). Several studies established the function of PRODH/POX as a tumor suppressor gene. Therefore, the inhibition of its activity is correlated with a cancer progression. In several cancer cells, as cancer of kidney or cancers of digestive tract, the expression of PRODH/POX is very low or not detected (Phang and Liu, 2012).

Proline can be also synthesized either from ornithine by ornithine aminotransferase (OAT) or from glutamic acid by $\mathrm{P} 5 \mathrm{C}$ synthase, then $\mathrm{P} 5 \mathrm{C}$ might be converted to proline by $\mathrm{P} 5 \mathrm{C}$ reductase (PYCR), leading to increase in proline concentration. These pathways are therefore considered in studied on prolinedependent responses of cancer cells.

The squamous cell carcinoma (SCC) is one of the most frequent cancers of the oral cavity cancer [oral squamous cell carcinoma (OSCC)]. It is characterized as highly invasive with the ability to give early and extensive lymph nodes metastases (Carvalho et al., 2004). The cancer treatment is primarily focused on the resection of a tumor. Afterward, the radiotherapy and the chemotherapy are followed. Up to date, therapeutic protocols do not offer to the patients with advanced SCC, effective therapeutic options. The lack of any advanced alternative pharmacotherapy of SCC is a problem of modern medicine (Pilch and Bouquot, 2005).
Several studies revealed the connection between the overexpression of oral cancer overexpressed 1 protein (ORAOV1) and the occurrence of more aggressive tumors in esophagus (Zhai et al., 2014). Additionally, ORAOV-1overexpressed cell lines, among others CAL-27 cell line, are marked with a higher intracellular proline concentration and stronger resistance on treatment (Jiang et al., 2008, 2010). It was confirmed that ORAOV1 influences PYCR activity. Hence, the increase in intracellular proline level was observed and consequently the promotion of tumor progression (Togashi et al., 2014). Hence, the effect of chosen polyphenols on proline metabolism may be a relevant issue in the apoptosis induction (Phang et al., 2012).

The purpose of the study was to identify mechanisms of cytotoxic activity of selected propolis components considering their effect on the proline metabolism in CAL-27 cell line. Previous analysis of the commercially available, standardized preparations of propolis allowed to identify a few of the most abundant polyphenols as chrysin, caffeic acid, p-coumaric acid, and ferulic acid (Maciejewicz et al., 2001; Markiewicz-Żukowska et al., 2012; Czyżewska et al., 2015, 2016). The study revealed the proapoptotic effect of these compounds on CAL-27 cell line (Czyżewska et al., 2016), however, its molecular mechanism still remains unclear. In this report, we present their effect on the apoptosis in tongue squamous cell carcinoma (CAL-27) and the mechanism of the activity.

\section{MATERIALS AND METHODS}

\section{Cell Culture}

The studies were performed on tongue squamous cell line (CAL27). The cell line was purchased in ATCC (CRL-2095 TM, American Type Culture Collection, Manassas, VA, United States). Cell culture was maintained in Dulbecco's modified Eagle's medium (Gibco, United States) with 10\% fetal bovine serum (FBS) (Gibco, United States) and $50 \mathrm{U} / \mathrm{mL}$ penicillin, $50 \mu \mathrm{g} / \mathrm{mL}$ streptomycin (Gibco, United States) and incubated at $37^{\circ} \mathrm{C}$ in $5 \% \mathrm{CO}_{2}$.

\section{Polyphenols Solutions}

Chrysin was purchased from Roth (Karlsruhe, Germany), $p$-coumaric acid, caffeic acid, and ferulic acid were purchased from Sigma-Aldrich (Steinheim, Germany). All chemicals and reagents used in this study were of analytical grade (purity $\geq 99 \%$ ). Solutions were prepared directly before adding to cell cultures in appropriate concentrations.

\section{Cell Viability Assay}

Cytotoxicity effects of studied polyphenols on CAL-27 cells was determined using methyl thiazolyl tetrazolium (MTT) according to modified Carmichael's method. MTT assay bases on the conversion of yellow tetrazolium bromide MTT solution to the purple formazan derivatives, by the mitochondrial enzyme succinate dehydrogenase,occurred in viable cells. Cells $\left(10^{5} / \mathrm{mL}\right)$ were cultured on 96 -well plates to obtain $70 \%$ of confluency. Then cells were incubated with studied polyphenols 
for $24 \mathrm{~h}$. Polyphenolic compounds were added as DMSO solution prepared directly before assay. The final concentration of DMSO did not exceed $0.1 \%$ in each well. The tested polyphenols were used at the following concentrations: chrysin - 5, 25, 50, $80 \mu \mathrm{g} / \mathrm{mL}$; ferulic acid - 50, 100, $150 \mu \mathrm{g} / \mathrm{mL}$, caffeic acid - 65, 130, $190 \mu \mathrm{g} / \mathrm{mL}$; and $p$-coumaric acid - 70, 140, $210 \mu \mathrm{g} / \mathrm{mL}$. The concentrations correspond to the following molar concentrations: chrysin - 0.020, 0.098, 0.216, $0.315 \mathrm{mM}$; caffeic acid - 0.361, 0.722, $1.055 \mathrm{mM}$; ferulic acid - 0.257, 0.515, $0.772 \mathrm{mM}$; and $p$-coumaric acid - 0.426, 0.853, $1.279 \mathrm{mM}$. Cisplatin (CDDP) (Sigma-Aldrich, Germany) $(10 \mu \mathrm{M})$ was used as a positive control. The selection of polyphenols concentration was made on the grounds of previously received data (Czyżewska et al., 2016). Then followed the incubation for $24 \mathrm{~h}$, then the liquid was removed and cells were washed with PBS. Afterward, $50 \mu \mathrm{L}$ of MTT solution (1.0 mg of MTT per $1 \mathrm{~mL}$ of PBS) was added to each well and incubated for $1 \mathrm{~h}$. To dissolve formazan derivatives dimethyl sulfoxide was added after MTT solution removing. The absorbance was measured at $570 \mathrm{~nm}$ wavelength using an Asys UVN 340 microplate reader (Biogenet, Józefów, Poland). For the treated cells, amount of viable cells was expressed as the percentage of control.

\section{Proliferation Assay}

The proliferation of studied cells was evaluated as [methyl${ }^{3} \mathrm{H}$ ]thymidine (Hartman Analytic GmbH, Braunschweig, Germany) incorporation into DNA of cells treated with studied polyphenols. In 24-well tissue culture dishes, cells were plated at $1 \times 10^{5}$ cells/well, using $1 \mathrm{~mL}$ of growth medium in each well. Various concentrations of polyphenols were added to the culture wells. Cisplatin $(10 \mu \mathrm{M})$ was used as a positive control. After incubation for $24 \mathrm{~h}$ at $37^{\circ} \mathrm{C}, 0.5 \mu \mathrm{Ci} / \mathrm{mL}$ of [methyl${ }^{3} \mathrm{H}$ ] thymidine $(6.7 \mathrm{Ci} / \mathrm{mmol})$ was added to the wells and cultures were incubated at $37^{\circ} \mathrm{C}$ for $4 \mathrm{~h}$. Thereafter, the cells were washed with $0.05 \mathrm{M}$ Tris- $\mathrm{HCl}$ and with 5\% TCA. Then followed cell lysis in $0.1 \mathrm{M} \mathrm{NaOH}$ containing $1 \%$ sodium dodecyl sulfate (SDS). Radioactivity was measured in Liquid Scintillation Analyzer Tri-Carb 2810 TR (Perkin-Elmer, United States) after adjustment of $2 \mathrm{~mL}$ of scintillation liquid (Ultima Gold XR, Perkin-Elmer, Waltham, MA, United States) to each sample.

\section{Western Immunoblot}

To determine the expression of proteins involved in apoptosis process and proline metabolism western immunoblot was performed. Goat monoclonal anti-PRODH/POX antibody (\#EB11136) was purchased from EverestBiotech (United Kingdom). Rabbit anti-caspase-3 (\#9665), rabbit anti-cleaved-caspase-3 (\#9664), rabbit anti-caspase-9 (\#9508), and mouse anti-cleaved-caspase-9 (\#7237) antibodies were purchased from Cell Signaling (United States). Purified mouse anti-p53 antibody (\#554169) was acquired from Becton, Dickinson and Company (B\&D) (United States). Monoclonal anti- $\beta$-actin antibody produced in mouse was obtained from Sigma-Aldrich (\#A2228, United States). Secondary polyclonal anti-goat, anti-rabbit and anti-mouse antibodies labeled with horseradish peroxidase were purchased in Sigma-Aldrich, Corp. (United States).
The Laemmli method was used to perform SDS-PAGE electrophoresis (Laemmli, 1970). The equal amounts of cell supernatants (15 $\mu \mathrm{g}$ of protein) were analyzed. Semi-dry electrotransfer was performed after electrophoresis. For this procedure, Multiphor II Electrophoresis System Multiphor (GE Healthcare Bio-Sciences AB, Sweden) was used. Subsequently, the sequence of incubation with primary and secondary antibodies and were performed. Afterward, membranes were incubated with Amersham ECL Western Blotting Detection Reagent (GE Healthcare Life Science, United Kingdom), and chemiluminescence was analyzed with BioSpectrum Imaging System UVP (UltraViolet Products, Ltd., United Kingdom). The densitometry of bands was quantified by ImageJ software.

\section{Intracellular Proline Concentration}

After $24 \mathrm{~h}$ incubation with chosen polyphenols, cells were scratched and suspended in a cold mixture of water and methanol $(v / v$ 80:20). After then a cycle of frost and defrost followed. Lastly, the cell solution was sonicated and preserved at $-80^{\circ} \mathrm{C}$ until further analysis. The used method allowed to determine intracellular concentration of proline and to compare treated cells with untreated ones.

The intracellular level of proline was determined using HPLC system (Agilent Technologies, Germany) connected to QTOF mass spectrometry detector (Agilent Technologies, Germany). Electrospray ionization (ESI) was used as an ion source. The analysis was performed on a HILIC column (Luna HILIC, Phenomenex, United States). Samples were injected in a volume of $2 \mu \mathrm{L}$. The procedure required maintenance at $40^{\circ} \mathrm{C}$. The system was operated in both positive and negative mode at flow rate $1 \mathrm{~mL} / \mathrm{min}$ with solvent $\mathrm{A}$ - water with $10 \mathrm{mM}$ ammonium formate, and solvent B - acetonitrile/water (9:1, $v: v)$ with $10 \mathrm{mM}$ ammonium formate. A mobile phase was as follows: $100 \% \mathrm{~B}$ during $1.5 \mathrm{~min}$ in isocratic mode, at $1.5 \mathrm{~min}$ the gradient mode started from $100 \%$ B to $70 \%$ B in $5.5 \mathrm{~min}$, then $40 \% \mathrm{~B}$ in $6.0 \mathrm{~min}$, maintained $40 \% \mathrm{~B}$ during $1 \mathrm{~min}$ and returned to starting conditions in $0.5 \mathrm{~min}$, keeping the re-equilibration until $10 \mathrm{~min}$. The detector operated in full scan mode. During analyses, reference masses were delivered continuously to obtain accurate mass measurements. Reference masses were at $\mathrm{m} / z 121.0509$ (protonated purine) and $\mathrm{m} / z$ 922.0098 [HP-921]. Compound identification was performed monitoring monoisotopic ions of proline and the internal standard (Proline-d3, \#791261, Sigma-Aldrich, United States). The concentration range of proline to which calibration curve was registered was as followed: 6.32-101.12 [ $\mu \mathrm{M}]$. Each of sample was analyzed in triplicates. To further calculations the average value of signals was used. Obtained concentrations were normalized to the internal standard and to the amount of protein in a sample.

\section{Vitality Assay}

Vitality assay describes changes in the intracellular level of (reduced) thiols such as glutathione which exists in two forms: reduced - GSH and - oxidized state (GSSG). The decrease in GSH levels is an early signal of cell death caused either by the direct GSH oxidation promoted by radicals or 
by the export of GSH through an ATP-dependent plasma membrane transport system. The assay was performed using NucleoCounter ${ }^{\circledR}$ NC-3000 ${ }^{\mathrm{TM}}$ system (ChemoMetec, Denmark) according to manufacturer's protocol. Cells incubated with studied concentrations of polyphenols were detached from the plates, washed and stained with Solution 5 (containing three various reagents: VitaBright-48, propidium iodide, and acridine orange). Cisplatin (10 $\mu \mathrm{M})$ was used as a positive control. Thereafter, the intensity of fluorescence was measured using NucleoCounter ${ }^{\circledR}$ NC-3000 ${ }^{\mathrm{TM}}$.

\section{Determination of Prolidase Activity}

The activity of prolidase was determined according to the method of Myara et al. (1982). It is colorimetric assay using Chinard's reagent. To perform the assay cells were detached and scraped off using Cell Lysis Buffer (Cell Signaling, United States). N-Benzyloxycarbonyl-L-proline (CbzPro) (Bachem, United States) $(5 \mathrm{mM})$ was used as a positive control. Then samples were sonicated for $3 \mathrm{~s} \times 10 \mathrm{~s}$ at $0^{\circ} \mathrm{C}$ and then centrifuged at $16000 \times g$ for $15 \mathrm{~min}$. Afterward, the cell pellet was discarded. Supernatants were used for protein determination according to Lowry method and evaluation of prolidase activity. The activation of prolidase requires incubation of cell extract supernatant with $\mathrm{Mn}$ (II), due to this fact the $100 \mu \mathrm{L}$ of each sample was mixed with $100 \mu \mathrm{L}$ of $50 \mathrm{mM}$ HEPES ( $\mathrm{pH}$ 7.8) containing $\mathrm{MnCl}_{2}$ to achieve the final concentration $1 \mathrm{mM}$ of $\mathrm{MnCl}_{2}$. Incubation lasts for $24 \mathrm{~h}$ at $37^{\circ} \mathrm{C}$ then the prolidase reaction was initiated by adding $100 \mu \mathrm{L}$ of the activation mixture which was $94 \mathrm{mM}$ glycyl-proline (Gly-Pro). After adding of Gly-Pro samples were incubated for $1 \mathrm{~h}$ at $37^{\circ} \mathrm{C}$. The reaction was terminated by addition $1 \mathrm{~mL}$ of $0.45 \mathrm{M}$ trichloroacetic acid. Parallel blank tubes contained trichloroacetic acid was added at time "zero." After the samples centrifugation $(10.000 \times g$ for $15 \mathrm{~min})$ the amount of released proline was determined by adding trichloroacetic acid/supernatant to the mixture of glacial acetic acid: Chinard's reagent. Then followed incubation for $10 \mathrm{~min}$ at $90^{\circ} \mathrm{C}$. The determination of proline was based on the measurement of absorbance at $515 \mathrm{~nm}$. Prolidase activity was expressed as nanomoles of proline released per minute per milligram of protein.

\section{Collagen Biosynthesis}

Collagen biosynthesis was measured as the incorporation of radioactive proline into proteins. CAL-27 were incubated with various concentrations of polyphenols and with the $5 \mu \mathrm{Ci} / \mathrm{mL}$ of $5\left[{ }^{3} \mathrm{H}\right]$-proline (Hartman Analytic $\mathrm{GmbH}$, Germany) for 24 h. 2-Metoxyestradiol (MOE) (Sigma-Aldrich, United States) (10 $\mu \mathrm{M})$ was used as a positive control. The amount of incorporated $5\left[{ }^{3} \mathrm{H}\right]$-proline into collagen was detected after its digesting by Clostridium histolyticum collagenase (SigmaAldrich, United States) according to the method of Peterkofsky and Diegelmann (1971).

\section{Statistical Analysis}

Statistical analysis was conducted using GraphPad PRISM v. 5 (GraphPad Software, Inc., San Diego, CA, United States).
For results from all experiments, the mean values for at least six measuring points \pm standard error of the mean (SEM) were determined. The statistical significances were calculated using one-way ANOVA with Dunnett's multiple comparison tests. Differences at $p<0.001$ were accepted as statistically significant.

\section{RESULTS}

\section{Studied Polyphenols Significantly Reduce the Viability of CAL-27 Cancer Cells}

The influence of chosen polyphenols (chrysin, caffeic acid, $p$-coumaric acid, ferulic acid) on the viability of human tongue squamous cell carcinoma cell line (CAL-27) was determined with MTT assay. The studied polyphenols ability to induce the cytotoxic effect in CAL-27 was observed for all polyphenolic compounds (Figure 1). The use of chrysin resulted in a reduction of cell survival to $82,62,32$, and $29 \%$. In the case of caffeic acid, decreased CAL-27 cells survival to 81,62 , and $61 \%$ was observed. The use of ferulic acid decreased cell survival to 85,65 , and 50\% compared to controls whereas in the case of $p$-coumaric acid, the viability of CAL-27 cells was reduced to 82,80 , and $73 \%$ compared to controls $(100 \%)$. To evaluate whether polyphenols cytotoxicity was a result of their influence on cell DNA biosynthesis the capability of intracellular thymidine incorporation was assessed.

\section{The Inhibition of DNA Biosynthesis After Polyphenols Application}

The effects of chrysin, caffeic acid, $p$-coumaric acid, ferulic acid on DNA biosynthesis in CAL-27 cells were evaluated as $\left[{ }^{3} \mathrm{H}\right]$ thymidine incorporation into viable cells. The downregulation of DNA biosynthesis in CAL-27 cells was observed for all polyphenolic compounds (Figure 2). The obtained results show that the addition of chrysin resulted in a reduction in DNA biosynthesis by $46,80,83$, and $83 \%$ whereas the application of caffeic acid leads to a reduction of DNA biosynthesis by 14, 31 , and $52 \%$. Moreover, the similar situation was observed after adding ferulic acid where the DNA biosynthesis was reduced by $16,46,53$, and $71 \%$. In the case of using $p$-coumaric acid, DNA biosynthesis was also reduced by 67,78 , and $83 \%$. These findings of indicate that all polyphenols are inhibitors of DNA biosynthesis in CAL-27 SCC cells, and the intensity of this process depends on the concentration of the compound used.

To evaluate whether chosen compounds lead to the cell death through the apoptosis pathway involved proline metabolism regulation the western immunoblot assay was performed. For further analysis polyphenols were used at the lowest doses that contributed to noticeable cytotoxic effects. Applied concentrations were as follows: chrysin $-5 \mu \mathrm{g} / \mathrm{mL}$, caffeic acid $65 \mu \mathrm{g} / \mathrm{mL}$, ferulic acid - $50 \mu \mathrm{g} / \mathrm{mL}$, and $p$-coumaric acid $70 \mu \mathrm{g} / \mathrm{mL}$. 

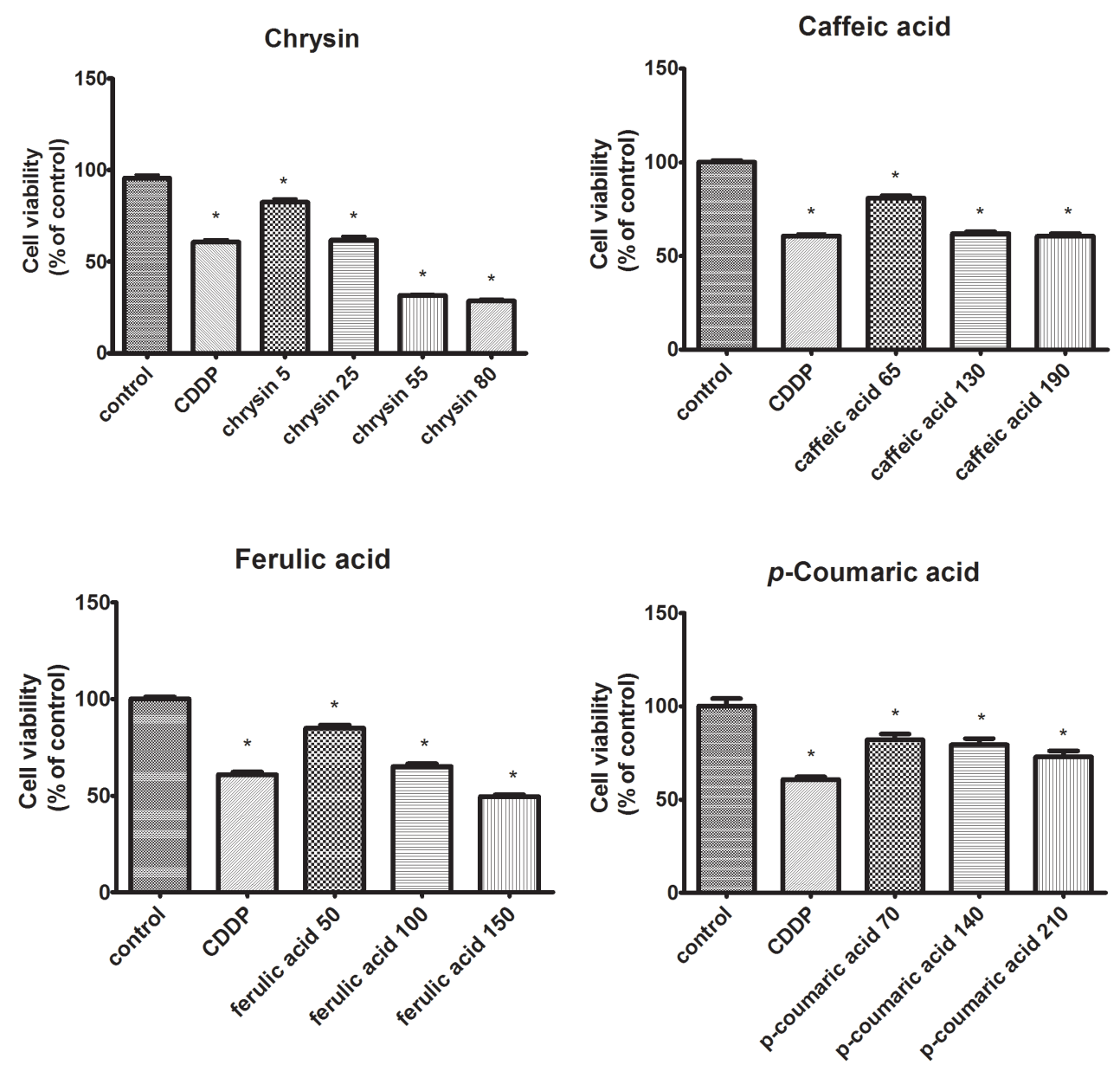

FIGURE 1 | The cytotoxic effects of polyphenolic compounds on CAL-27 cells. The cells were treated with a specified concentration [ $\mu \mathrm{g} / \mathrm{mL}$ ] of respective components for $24 \mathrm{~h}$ and cytotoxic effects were determined by MTT assay. Cisplatin (CDDP) (10 $\mu \mathrm{M})$ was used as a positive control. The values represent mean \pm SEM of two independent experiments conducted six times $(n=12)$. The stars indicate that value were significantly different $\left({ }^{*} p<0.001\right)$ compared with control.

\section{Selected Polyphenols Activate p53, PRODH/POX, Caspase 9 and 3 in CAL-27 Cell Line}

The study was conducted applying Western Immunoblot method described in chapter 2.5. CAL-27 cells were subjected to $24 \mathrm{~h}$ exposure to selected concentrations of polyphenols: chrysin (5 $\mu \mathrm{g} / \mathrm{mL})$, caffeic acid $(65 \mu \mathrm{g} / \mathrm{mL})$, ferulic acid $(50 \mu \mathrm{g} / \mathrm{mL})$, and $p$-coumaric acid $(70 \mu \mathrm{g} / \mathrm{mL})$. To evaluate the course of apoptosis process the caspases expression were assessed. The findings showed the reduction of expression of total caspase 3 in CAL-27 cells incubated with all of the tested polyphenols. The largest reduction in the expression of the total caspase 3 form compared to the control was observed in cells incubated with chrysin $(5 \mu \mathrm{g} / \mathrm{mL})$ and ferulic acid $(50 \mu \mathrm{g} / \mathrm{mL})$ (Figure 3A). Additionally, the obtained data showed reduced expression of total caspase 9 in CAL-27 cells incubated with all polyphenols tested. The most significant reduction in the expression of the total caspase 9 form was observed in cells incubated with chrysin $(5 \mu \mathrm{g} / \mathrm{mL})$ and ferulic acid $(50 \mu \mathrm{g} / \mathrm{mL})$ (Figure 3C). Moreover, obtained results revealed an increase in cleaved caspases 9 and 3 expressions in CAL-27 cells after incubation with each of used polyphenols (Figure 3). The highest increase in expression of active caspase 9 compared to control was observed in CAL27 cells exposed to caffeic acid $(65 \mu \mathrm{g} / \mathrm{mL})$ and $p$-coumaric acid $(70 \mu \mathrm{g} / \mathrm{mL})$ (Figure 3D). Furthermore, the data revealed increased expression of the active form of caspase 3 in CAL-27 cells incubated with each of the polyphenols tested. The most noticeable increase in expression of active caspase 3 in relation to control was observed in the case of CAL-27 cells exposed to chrysin $(5 \mu \mathrm{g} / \mathrm{mL})$ and $p$-coumaric acid $(70 \mu \mathrm{g} / \mathrm{mL})$ (Figure 3B). The following results confirm the pro-apoptotic effect of the polyphenols. These compounds stimulate the expression of the active forms of the initiator caspase 9 involved in the activation of the intrinsic pathway of apoptosis and the executive caspase 3. The initiation of apoptosis through the activation of the p53 protein is accompanied by the induction of proline oxidase (PRODH/POX) expression. It is a mitochondrial enzyme that catalyzes the conversion of proline to pyrroline-5-carboxylate while transferring free electrons to cytochrome $c$ resulting in 

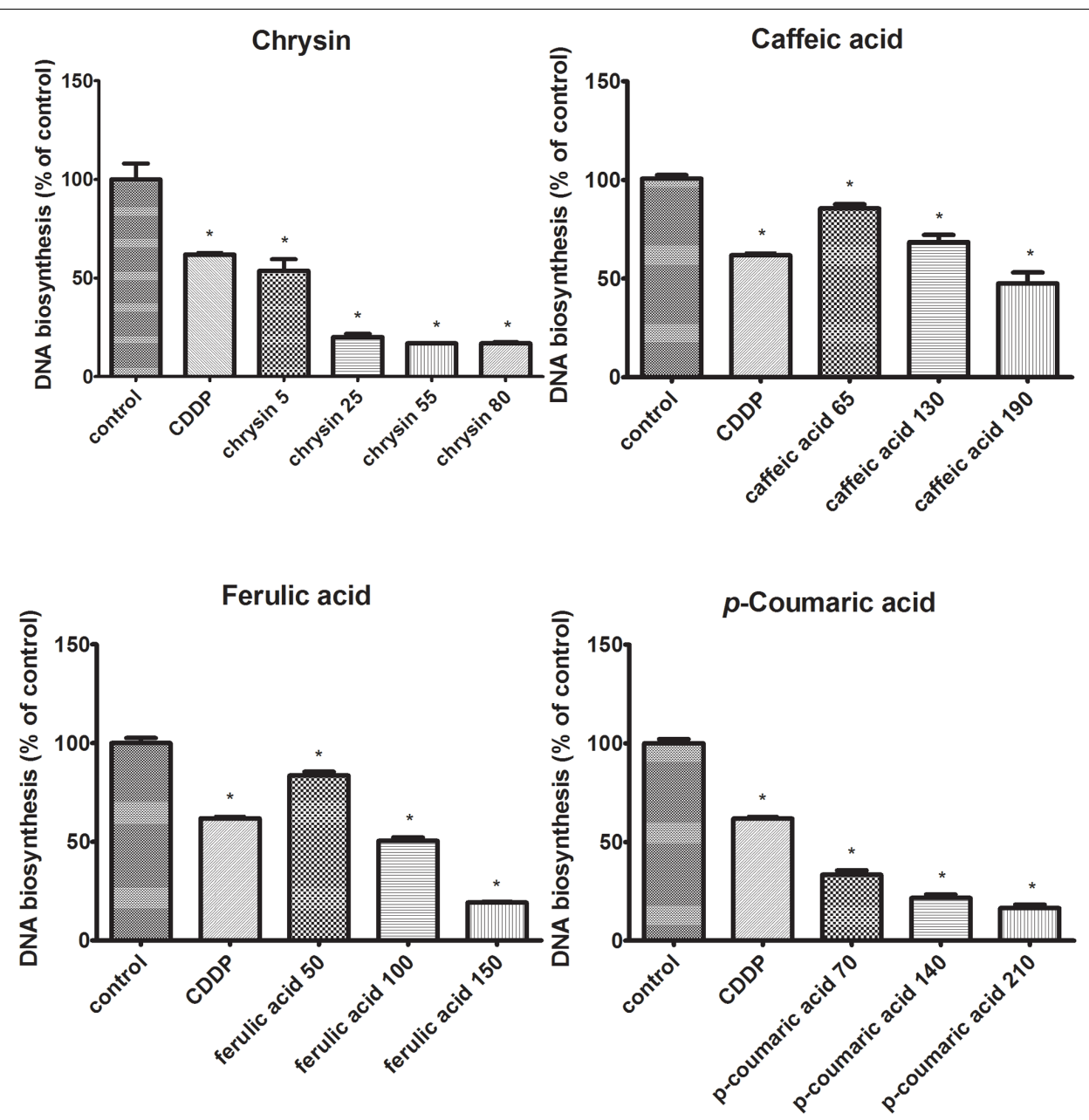

FIGURE 2 | Decreasing effects of polyphenolic compounds on DNA biosynthesis in CAL-27 cells. The cells were incubated with specified concentration [ $\mu \mathrm{g} / \mathrm{mL}$ ] of respective polyphenols for $24 \mathrm{~h}$ and $\left[{ }^{3} \mathrm{H}\right]$-thymidine incorporation into $\mathrm{CAL}-27$ was measured in scintillation counter. Cisplatin (10 $\left.\mu \mathrm{M}\right)$ was used as a positive control. The values represent mean \pm SEM of two independent experiments conducted three times $(n=6)$. The stars indicate that value were significantly different $\left({ }^{*} p<0.001\right)$ compared with control.

apoptosis. Therefore, to further examination whether observed apoptosis involved the changes in $\mathrm{PRODH} / \mathrm{POX}$ activity, the western immunoblot for PRODH/POX and p53 (the most potent inducer of this protein expression) was conducted. Received outcomes demonstrated the increase in PRODH/POX expression in all studied cases (Figure $3 F$ ). The obtained results coincide with the p53 expression that was increased in all samples. Nonetheless, the lowest increase was observed in CAL-27 cells previously incubated with ferulic acid (Figures 3E,F).

\section{The Proapoptotic Effect of Chrysin, Caffeic Acid, $p$-Coumaric Acid, and Ferulic Acid}

To confirm induction of apoptosis by the polyphenols the vitality assay was performed. The cells were subjected to $24 \mathrm{~h}$ exposure to tested polyphenols in the following concentrations: chrysin $(5 \mu \mathrm{g} / \mathrm{mL})$, caffeic acid $(65 \mu \mathrm{g} / \mathrm{mL})$, ferulic acid $(50 \mu \mathrm{g} / \mathrm{mL})$, and $p$-coumaric acid $(70 \mu \mathrm{g} / \mathrm{mL})$. The obtained outcomes revealed a significant decrease in cellular GSH concentration in treated cells compared to untreated control (Figure 4). The most noticeable increase in the cell population with the low level of GSH was observed in CAL-27 cells treated with chrysin. The loss of GSH is broadly known to be directly related to the apoptosis progression and the assay confirmed our data received from MTT assay.

The performed assays confirmed the apoptosis induction in treated cells. To estimate whether chosen compounds leads to apoptosis through the disruption of proline metabolism, further studies were conducted.

\section{Chrysin, Caffeic Acid, p-Coumaric Acid, and Ferulic Acid Affect the Cellular Metabolism of Proline}

Proline transformations affect the regulation of cellular metabolism and apoptosis. The effect of proline on the metabolism of tumor cells seems to be particularly important, 
A

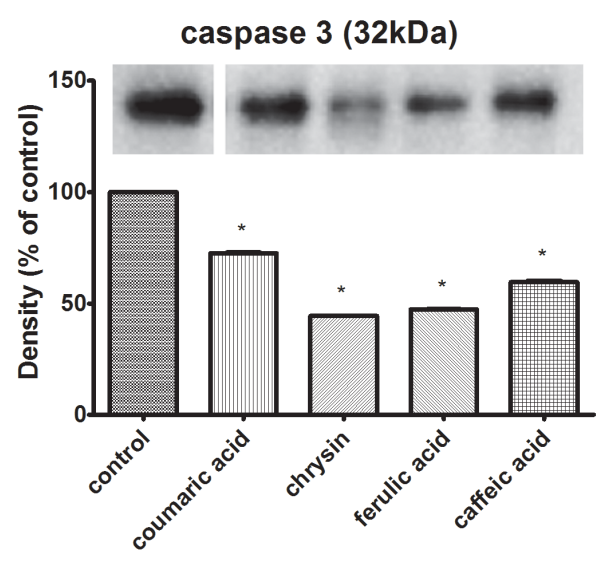

C

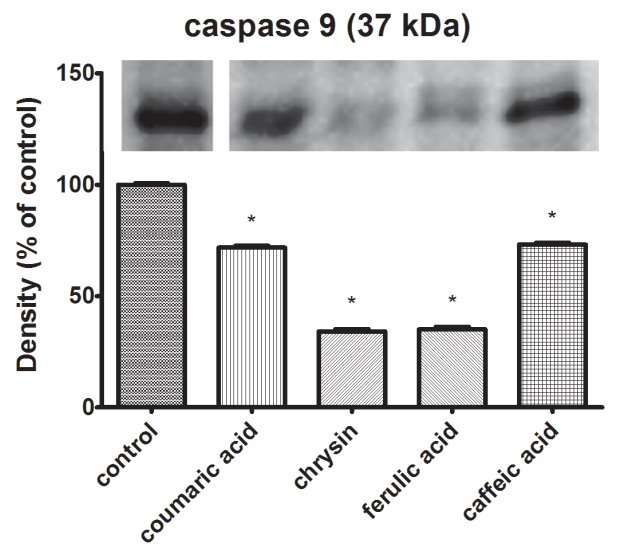

E

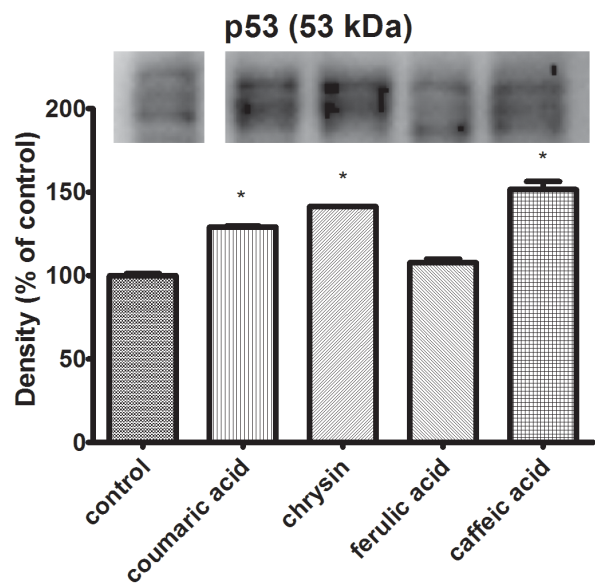

B

cleaved-caspase 3 (19

and $17 \mathrm{kDa}$ )

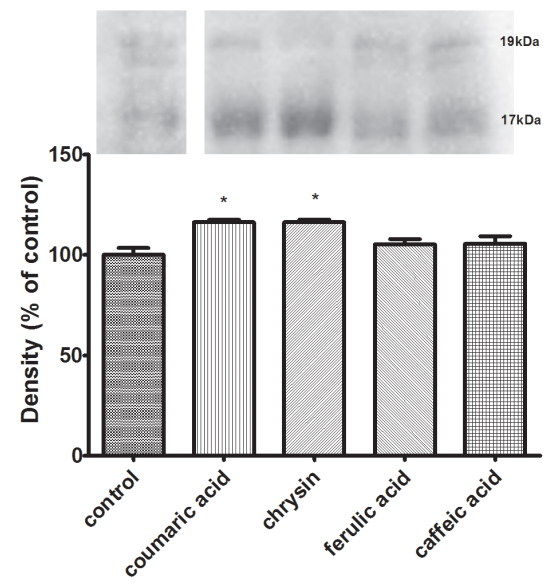

D

cleaved-caspase 9

(37 kDa)

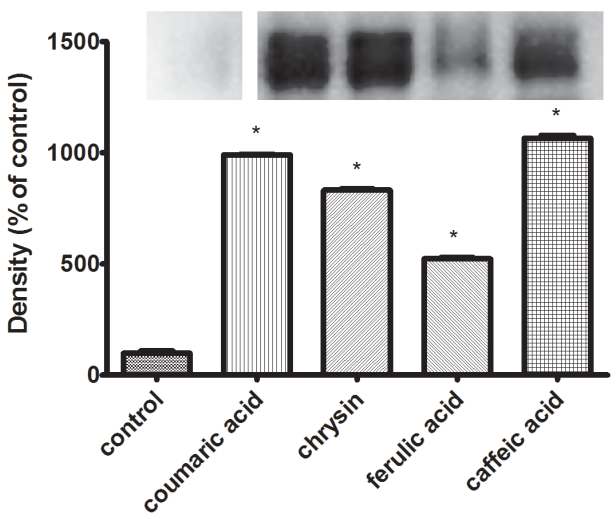

$\mathbf{F}$

PRODH/POX (75 kDa)

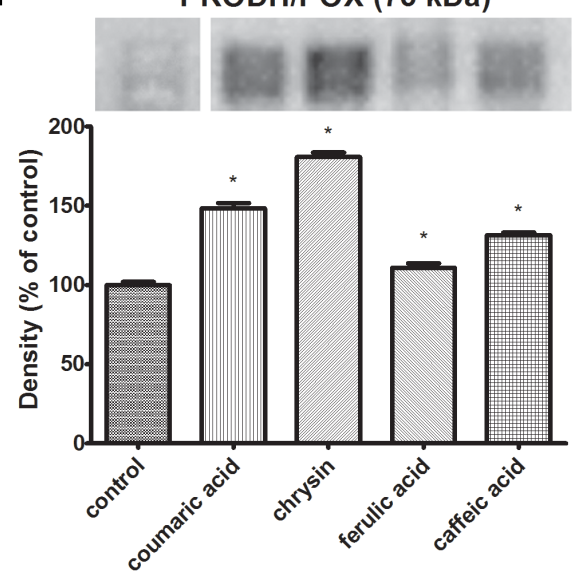

FIGURE 3 | Expression of un-cleaved caspase-3 (A), cleaved-caspase-3 (B), un-cleaved caspase-9 (C), cleaved-caspase-9 (D), PRODH/POX (F) and p53 (E), in CAL-27 cells and the effect of: $p$-coumaric acid, chrysin, ferulic acid, and caffeic acid on the process. $\beta$-Actin was used as a control (data contained in

Supplementary Presentation 1). The values represent mean \pm SEM of six pooled cell homogenates extracts from three independent experiments $(n=18)$. The stars indicate which values were significantly different $(* p<0.001)$ compared with control.

where a significant increase in the concentration of this amino acid is observed in comparison with normal cells. The high concentration of intracellular proline is associated with an enhanced ability of tumor cells to metastasize. The performed studies showed the pro-apoptotic activity of all tested polyphenols. The next stage of the study was to determine 


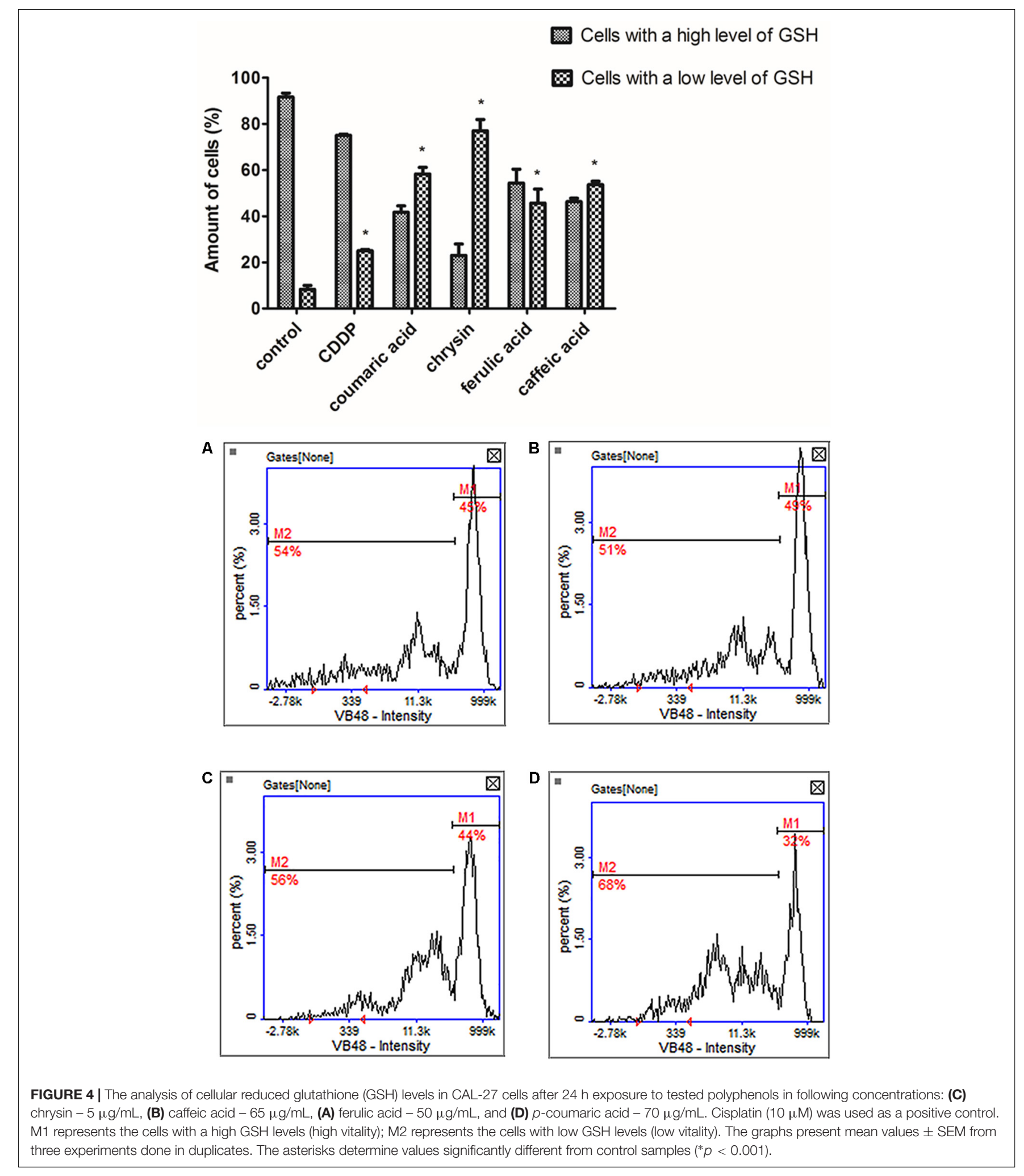

the effect of the tested polyphenols on the proline level in CAL-27 SCC cells. For evaluation, if discerned changes might induce the adjustments in proline metabolism and hence in its intracellular concentration, the HPLC-QTOF system was used. Data acquired after chromatographic analysis displayed a significant decrease in the intracellular proline concentration in case of CAL-27 cells treated with all studied polyphenols (Figure 5). 


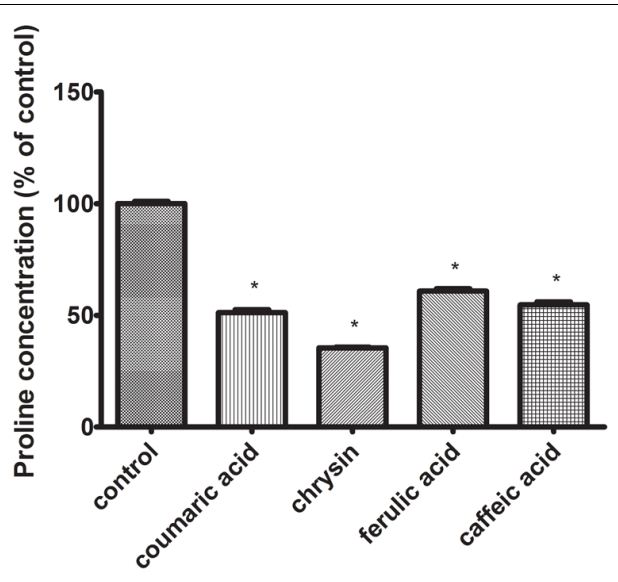

FIGURE 5 | The effect of $24 \mathrm{~h}$ exposure to $p$-coumaric acid $(70 \mu \mathrm{g} / \mathrm{mL})$, chrysin $(5 \mu \mathrm{g} / \mathrm{mL})$, ferulic acid $(50 \mu \mathrm{g} / \mathrm{mL})$, and caffeic acid $(65 \mu \mathrm{g} / \mathrm{mL})$ on intracellular proline concentration in CAL-27 cell culture. The mean values \pm SEM from three experiments done in duplicates are presented $(n=6)$. The stars indicate which values were significantly different $\left.{ }^{*} p<0.001\right)$ compared with control.

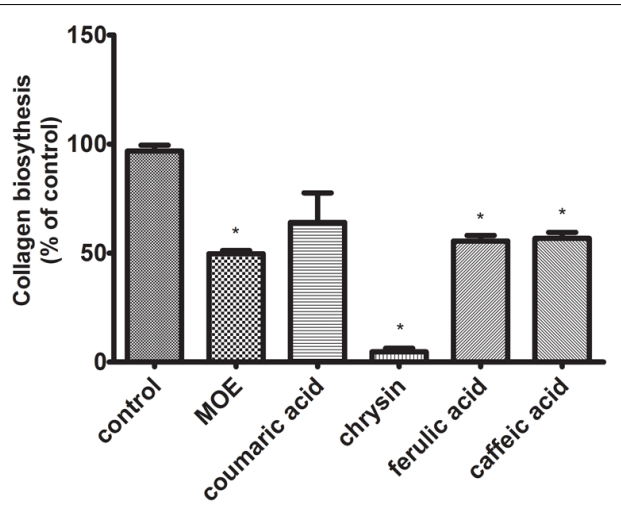

FIGURE 6 | Collagen biosynthesis measured as $5\left[{ }^{3} \mathrm{H}\right]$-proline incorporation into proteins susceptible to the action of bacterial collagenase in CAL-27 cell culture incubated for $24 \mathrm{~h}$ with polyphenols in following concentrations: chrysin $-5 \mu \mathrm{g} / \mathrm{mL}$, caffeic acid - $65 \mu \mathrm{g} / \mathrm{mL}$, ferulic acid - $50 \mu \mathrm{g} / \mathrm{mL}$, and p-coumaric acid - $70 \mu \mathrm{g} / \mathrm{mL}$. 2-Metoxyestradiol (MOE) $(10 \mu \mathrm{M})$ was used as a positive control. The values represent mean \pm SEM of two independent experiments conducted three times $(n=6)$. The stars indicate values significantly different from control $(* p<0.001)$.

\section{The Decline of Collagen Biosynthesis in the Cells Treated With Polyphenols}

Collagen is a most abundant protein in the extracellular matrix (ECM), where in addition to supporting functions, it participates in the modulation of cellular signals by interacting with integrin receptors. It affects a number of cell processes, including regulation of gene expression, growth, differentiation, and metabolism of cells. It has been observed that in cancer cells, this mechanism is disrupted due to the increased activity of metalloproteinases (MMPs), contributing to the increased degradation of collagen. The biosynthesis level of this protein may reflect changes in metabolic processes in living cells. Since

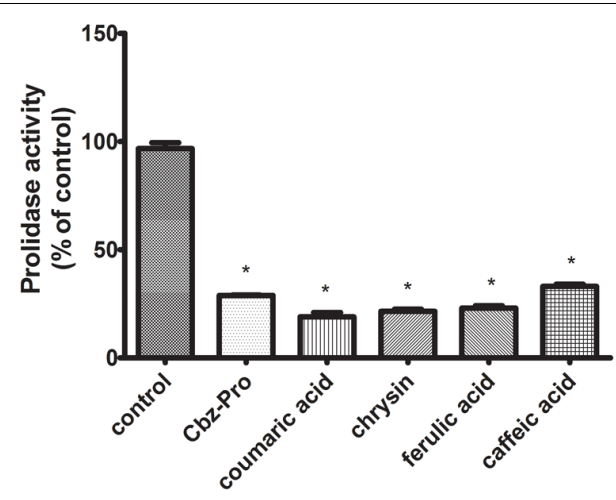

FIGURE 7 | Prolidase activity in CAL-27 cells treated for $24 \mathrm{~h}$ with chosen polyphenols (chrysin - $5 \mu \mathrm{g} / \mathrm{mL}$, caffeic acid - $65 \mu \mathrm{g} / \mathrm{mL}$, ferulic acid $50 \mu \mathrm{g} / \mathrm{mL}$, and $p$-coumaric acid - $70 \mu \mathrm{g} / \mathrm{mL}$ ). N-Benzyloxycarbonyl-L-proline (Cbz-Pro) (5 mM) was used as a positive control. The mean values $\pm \mathrm{SEM}$ from three experiments done in duplicates $(n=6)$ are presented. Asterisks indicate differences between studied cells compared to control untreated cells at $* p<0.001$.

collagen is reported to be the essential source of proline further experiment was designed to assess the possible disorders of collagen biosynthesis after exposure to studied polyphenols. The assay was performed according to the Peterkofsky's method. Obtained data revealed the decline of collagen biosynthesis in the cells treated with all tested compounds (Figure 6). The reduction of collagen biosynthesis by $36 \%$ - p-coumaric acid, $95 \%$ - chrysin, $44 \%$ - ferulic acid, and $43 \%$ - caffeic acid, compared to control $(100 \%)$ was observed. The most noticeable decrease in collagen biosynthesis was observed in the cells incubated with chrysin.

\section{Inhibition of Prolidase Activity by Tested Polyphenols}

Prolidase [E.C. 3.4.13.9] is a key enzyme involved in the process of collagen biosynthesis. This enzyme is involved in the breakdown of imidopeptides that are degradation products of collagen fibers. In the last stage of collagen degradation, prolidase catalyzes the reaction of the release of proline and hydroxyproline imidopeptides. The released proline can then be included in the collagen biosynthesis or other metabolic pathways, e.g., related to the process of apoptosis or the neoangiogenesis process. Since proline is the major constituent of collagen (it represents the main source of the aminoacids), their metabolism is directly connected. Due to this fact, prolidase plays a crucial role in the recycling of proline for collagen biosynthesis. In CAL-27 cells incubated with test polyphenols (chrysin $-5 \mu \mathrm{g} / \mathrm{mL}$, caffeic acid $65 \mu \mathrm{g} / \mathrm{mL}$, ferulic acid $-50 \mu \mathrm{g} / \mathrm{mL}$, $p$-coumaric acid $-70 \mu \mathrm{g} / \mathrm{mL}$ ), a reduction in the activity of prolidase was observed, by $81 \%$ p-coumaric acid, $78 \%$ - chrysin, $77 \%$ - ferulic acid, $66 \%$ - caffeic acid in relation to the control (Figure 7).

\section{DISCUSSION}

The SCC is one of the most frequent cancers of the oral cavity. It is associated with high invasiveness and ability to induce 
early and extensive lymph nodes metastases (Carvalho et al., 2004). The lack of advanced pharmacotherapy of SCC requires searching for new effective pharmacological strategies (Pilch and Bouquot, 2005). In this study, we found that selected polyphenols of propolis induced apoptosis in CAL-27 cells through upregulation of proline metabolism.

Recent studies revealed the enhanced progression of the SCC due to up-regulation of proline level induced by amplified genes expression of ORAOV1 and P5C-reductase (Togashi et al., 2014). Therefore, we considered proline metabolism as a target for potential treatment of SCC. In fact, proline bearing reducing potential is considered as a stress molecule. Its metabolism plays an important role in maintaining energetic and redox balance in cells. Free cytoplasmic proline may up-regulate transcriptional activity of HIF- $1 \alpha$ that is considered as prosurvival and inflammatory transcription factor since it induces expression of COX-2, VEGF, TNF- $\alpha$, IL-1, NF-кB (Surazynski et al., 2008a).

Proline could be removed from the cytoplasm by incorporation into collagen. Collagen biosynthesis may serve as a sink for proline. Up-regulation of collagen biosynthesis can remove reducing potential, but it may contribute to tissue cirrhosis usually accompanying prolonged inflammation (Priest and Davies, 1969; Davis and Cowie, 1990). Interesting is a fact of significant alterations in collagen content in SCC tissue. Agarwal and Ballabh indicated a definite alteration in the distribution of collagen type IV with its significant loss in poorly-differentiated SCC tissue (Agarwal and Ballabh, 2013), related to increased metastasis. On the other hand, proline is generated from imidodipeptides (derived from collagen degradation products) by imidopeptidase prolidase. Therefore, the enzyme activity represents an important factor in the regulation of intracellular proline concentration. However, other mechanisms that contribute to generation as well as, utilization of intracellular proline are as well-important.

A critical pathway of proline utilization is mitochondrial degradation by proline oxidase - PRODH/POX. During this process ROS and/or ATP are generated. PRODH/POX is widely distributed in living organisms and is responsible for a number of regulatory processes such as redox homeostasis, osmotic adaptation, cell signaling, and oxidative stress. Recent data provided evidence that enzyme plays a crucial role in inhibition of carcinogenesis and tumor growth. It may induce apoptosis in both intrinsic and extrinsic ways in cancer cells (Zareba and Palka, 2016). Unfortunately, the enzyme is often down-regulated in cancer cells leading to inhibited apoptosis (Kononczuk et al., 2015a). Our results indicated almost undetectable expression of PRODH/POX in CAL-27 cells. Therefore, we analyzed propolis compounds as factors inducing PRODH/POX expression and decreasing proline level. We suggest that polyphenols contained in propolis induced the enzyme activity, leading to induction of apoptosis in CAL-27 cells.

We found that studied polyphenols decreased in a dosedependent manner the viability of CAL-27 cells. Moreover, the treatment inhibited DNA biosynthesis in these cells. The decrease in cell number was related to induction of apoptosis. We observed a significant increase in expression of active/cleaved caspases -3 and -9 and increase in the amount of the cells with low level of reduced glutathione (GSH) in polyphenol-treated CAL-27 cells. Considering the important role of PRODH/POX in apoptosis (Phang, 2017), its expression was evaluated. The polyphenols induced expression of PRODH/POX in CAL-27 cells. Cell growth arrest related to apoptosis is induced by p53. The transcription factor is the most potent inducer of PRODH/POX expression (Nagano et al., 2017). In fact, p53 expression was also induced by studied polyphenols in SCC cells.

PRODH/POX-dependent utilization of proline in polyphenoltreated CAL-27 cells was confirmed by LC-MS analysis of proline concentration. Since proline could be utilized in collagen, the biosynthesis of this protein was determined. All tested compounds significantly inhibited the process. It suggests that observed decrease in proline concentration was related to its metabolism by PRODH/POX. The decrease of collagen biosynthesis in polyphenol-treated CAL-27 cells may result from inhibition of prolidase by polyphenols. The enzyme plays an important role in collagen biosynthesis (Palka and Phang, 1997; Surazynski et al., 2008b; Kononczuk et al., 2015a). We observed inhibition of prolidase activity in the cells treated with studied compounds (however to various degree). We suggest, that decrease in proline level by propolis compounds result from proline degradation in mitochondria by induced PRODH/POX and not by utilization of this amino acid for collagen biosynthesis. The significant role of PRODH/POX in apoptosis induction we described previously (Kononczuk et al., 2015b). Stimulation of PRODH/POX expression by Eptifibatide, $\alpha \operatorname{IIb} \beta 3$-integrin inhibitor, lead to strong proapoptotic effect in MCF-7 breast cancer cells.

The present study provides for the first time evidence that compounds of propolis (chrysin, caffeic acid, $p$-coumaric acid, and ferulic acid, of which the most potential effect on CAL27 cells growth inhibition was attributed to chrysin) contribute to the induction of $\mathrm{PRODH} / \mathrm{POX}$-dependent apoptosis in CAL-27 cells. Therefore, PRODH/POX may represent a novel target for the propolis-induced anticancer activity in SCC cells.

\section{AUTHOR CONTRIBUTIONS}

KC-J, UL, and WM: conceived and designed the experiments. KC-J, IZ, and JT: performed the experiments. KC-J, JT, JP, and WM: analyzed the data. KC-J, IZ, JT, UL, and MT: contributed reagents/materials/analysis tools. KC-J, MT, and WM: wrote the paper.

\section{FUNDING}

The work was supported by the Medical University of Białystok (Poland) (Grant Nos. N/ST/MN/16/002/2227, N/ST/MN/17/ 003/2227, and N/ST/ZB/17001/2227). The study was partially 
funded by the National Science Center NCN (Project No. 2014/13/B/NZ7/02196). The project was conducted in part with the use of equipment purchased by the Medical University of Białystok (Poland) as part of the OP DEP 2007-2013, Priority Axis I.3, contract No. POPW.01.03.00-20-008/09.

\section{REFERENCES}

Agarwal, P., and Ballabh, R. (2013). Expression of type IV collagen in different histological grades of oral squamous cell carcinoma: an immunohistochemical study. J. Cancer Res. Ther. 9, 272-275. doi: 10.4103/0973-1482. 113382

Bankova, V. (2005). Recent trends and important developments in propolis research. Evid. Based Complement. Alternat. Med. 2, 29-32. doi: 10.1093/ecam/ neh059

Bankova, V., de Castro, S. L., and Marcucci, M. C. (2000). Propolis: recent advances in chemistry and plant origin. Apidologie 31, 3-15. doi: 10.1051/apido:20 00102

Bankova, V., Popova, M., and Trusheva, B. (2014). Propolis volatile compounds: chemical diversity and biological activity: a review. Chem. Cent. J. 5, 2-8. doi: 10.1186/1752-153X-8-28

Carvalho, A. L., Singh, B., Spiro, R. H., Kowalski, L. P., and Shah, J. P. (2004). Cancer of the oral cavity: a comparison between institutions in a developing and a developed nation. Head Neck 26, 31-38. doi: 10.1002/hed.10354

Czyżewska, U., Kononczuk, J., Teul, J., Drạgowski, P., Pawlak-Morka, R., Surażyński, A., et al. (2015). Verification of chemical composition of commercially available propolis extracts by gas chromatography-mass spectrometry analysis. J. Med. Food 18, 584-591. doi: 10.1089/jmf.2014.0069

Czyżewska, U., Siemionow, K., Zareba, I., and Miltyk, W. (2016). Proapoptotic activity of propolis and their components on human tongue squamous cell carcinoma cell line (CAL-27). PLoS One 11:e0157091. doi: 10.1371/journal. pone.0157091

Davis, J. M. G., and Cowie, H. A. (1990). The relationship between fibrosis and cancer in experimental- animals exposed to asbestos and other fibers. Environ. Health Perspect. 88, 305-309. doi: 10.1289/ehp.9088305

Graikou, K., Popova, M., Gortzi, O., Bankova, V., and Chinou, I. (2016). Characterization and biological evaluation of selected Mediterranean propolis samples. Is it a new type? LWT Food Sci. Technol. 65, 261-267. doi: 10.3390/ molecules22071159

Jiang, L., Zeng, X., Wang, Z., Ji, N., Zhou, Y., Liu, X. T., et al. (2010). Oral cancer overexpressed 1 (ORAOV1) regulates cell cycle and apoptosis in cervical cancer HeLa cells. Mol. Cancer 9:20. doi: 10.1186/1476-4598-9-20

Jiang, L., Zeng, X., Yang, H. S., Wang, Z., Shen, J., Bai, J. P., et al. (2008). Oral cancer overexpressed 1 (ORAOV1): a regulator for the cell growth and tumor angiogenesis in oral squamous cell carcinoma. Inter. J. Cancer 123, 1779-1786. doi: $10.1002 /$ ijc. 23734

Kononczuk, J., Czyzewska, U., Moczydlowska, J., Surazynski, A., Palka, J., and Miltyk, W. (2015a). Proline oxidase (POX) as a target for cancer therapy. Curr. Drug Targets 16, 1464-1469.

Kononczuk, J., Surazynski, A., Czyzewska, U., Prokop, I., Tomczyk, M., Palka, J., et al. (2015b). alpha(IIb)beta(3)-integrin ligands: abciximab and eptifibatide as proapoptotic factors in MCF- 7 human Breast cancer cells. Curr. Drug Targets 16, 1429-1437.

Kubiliene, L., Laugaliene, V., Pavilonis, A., Maruska, A., Majiene, D., Barcauskaite, K., et al. (2015). Alternative preparation of propolis extracts: comparison of their composition and biological activities. BMC Complement. Altern. Med. 15:156. doi: 10.1186/s12906-015-0677-5

Laemmli, U. K. (1970). Cleavage of structural proteins during the assembly of the head of bacteriophage T4. Nature 227, 680-685.

Liu, Y., Borchert, G. L., Surazynski, A., Hu, C. A., and Phang, J. M. (2006). Proline oxidase activates both intrinsic and extrinsic pathways for apoptosis: the role of ROS/superoxides, NFAT and MEK/ERK signaling. Oncogene 25, 5640-5647. doi: 10.1038/sj.onc.1209564

Maciejewicz, W., Daniewski, M., and Bal, K. (2001). GC-MS identification of the flavonoid aglycones isolated from propolis. Chromatographia 53, 343-346. doi: 10.1007/BF02490438

\section{SUPPLEMENTARY MATERIAL}

The Supplementary Material for this article can be found online at: https://www.frontiersin.org/articles/10.3389/fphar. 2018.00336/full\#supplementary-material

Markiewicz-Żukowska, R., Car, H., Naliwajko, S. K., Sawicka, D., Szynaka, B., Chyczewski, L., et al. (2012). Ethanolic extract of propolis, chrysin, CAPE inhibit human astroglia cells. Adv. Med. Sci. 57, 208-216. doi: 10.2478/v10039012-0042-6

Myara, I., Charpentier, C., and Lemonnier, A. (1982). Optimal conditions for prolidase assay by proline colorimetric determination: application to imidopeptiduria. Clin. Chim. Acta 125, 193-205.

Nagano, T., Nakashima, A., Onishi, K., Kawai, K., Awai, Y., Kinugasa, M., et al. (2017). Proline dehydrogenase promotes senescence through the generation of reactive oxygen species. J. Cell. Sci. 130, 1413-1420. doi: 10.1242/jcs. 196469

Palka, J. A., and Phang, J. M. (1997). Prolidase activity in fibroblasts is regulated by interaction of extracellular matrix with cell surface integrin receptors. J. Cell. Biochem. 67, 166-175.

Peterkofsky, B., and Diegelmann, R. (1971). Use of a mixture of proteinase-free collagenases for specific assay of radioactive collagen in presence of other proteins. Biochemistry 10, 19-47.

Phang, J. M. (2017). Proline metabolism in cell regulation and cancer biology: recent advances and hypotheses. Antioxid. Redox Signal. doi: 10.1089/ars.2017. 7350 [Epub ahead of print].

Phang, J. M., Donald, S. P., Pandhare, J., and Liu, Y. (2008a). The metabolism of proline, a stress substrate, modulates carcinogenic pathways. Amino Acids 35, 681-690. doi: 10.1007/s00726-008-0063-4

Phang, J. M., and Liu, W. (2012). Proline metabolism and cancer. Front. Biosci. 17, 1835-1845.

Phang, J. M., Liu, W., Hancock, C., and Christian, K. J. (2012). The proline regulatory axis and cancer. Front. Oncol. 2:60. doi: 10.3389/fonc.2012.00060

Phang, J. M., Pandhare, J., and Liu, Y. M. (2008b). The metabolism of proline as microenvironmental stress substrate. J. Nutr. 138, 2008S-2015S.

Pilch, B. Z., and Bouquot, J. (2005). "Squamous cell carcinoma," in World Health Organization Classification of Tumours Pathology \& Genetics Head and Neck Tumours, eds L. Barnes, J. W. Eveson, P. Reichart, and D. Sidransky (Lyon: IARC Press), 168-175.

Popova, M., Giannopoulou, E., Skalicka-Woźniak, K., Graikou, K., Widelski, J., Bankova, V., et al. (2017a). Characterization and biological evaluation of propolis from Poland. Molecules 22:1159. doi: 10.3390/molecules22 071159

Popova, M., Lyoussi, B., Aazza, S., Antunes, D., Bankova, V., and Miguel, G. (2015). Antioxidant and alpha-glucosidase inhibitory properties and chemical profiles of moroccan propolis. Nat. Prod. Commun. 10, 1961-1964.

Popova, M., Trusheva, B., and Bankova, V. (2017b). Content of biologically active compounds in Bulgarian propolis: a basis for its standardization. Bulg. Chem. Commun. 49, 115-120.

Priest, R. E., and Davies, L. M. (1969). Cellular proliferation and synthesis of collagen. Labour Invest. 21, 138-142.

Sadowska, A., Prokopiuk, S., Miltyk, W., Surazynski, A., Kononczuk, J., Sawicka, D., et al. (2013). Metronidazole affects breast cancer cell lines. Adv. Med. Sci. 58, 90-95. doi: 10.2478/v10039-012-0070-2

Surazynski, A., Donald, S. P., Cooper, S. K., Whiteside, M. A., Salnikow, K., Liu, Y., et al. (2008a). Extracellular matrix and HIF-1 signaling: the role of prolidase. Int. J. Cancer 122, 1435-1440. doi: 10.1002/ijc.23263

Surazynski, A., Miltyk, W., Palka, J., and Phang, J. M. (2008b). Prolidase-dependent regulation of collagen biosynthesis. Amino Acids 35, 731-738. doi: 10.1007/ s00726-008-0051-8

Togashi, Y., Arao, T., Kato, H., Matsumoto, K., Terashima, M., Hayashi, H., et al. (2014). Frequent amplification of ORAOV1 gene in esophageal squamous cell cancer promotes an aggressive phenotype via proline metabolism and ROS production. Oncotarget 5, 2962-2973. doi: 10.18632/oncotarget.1561

Turan, I., Demir, S., Misir, S., Kilinc, K., Mentese, A., Aliyazicioglu, Y., et al. (2015). Cytotoxic effect of Turkish propolis on liver, colon, breast, cervix and prostate cancer cell lines. Trop. J. Pharm. Res. 14, 777-782. doi: 10.4314/tjpr.v14i5.5 
Yildirim, A., Duran, G. G., Duran, N., Jenedi, K., Bolgul, B. S., Miraloglu, M., et al. (2016). Antiviral activity of hatay propolis against replication of herpes simplex virus Type 1 and type 2. Med. Sci. Monit. 22, 422-430. doi: 10.12659/MSM. 897282

Zareba, I., and Palka, J. (2016). Prolidase-proline dehydrogenase/proline oxidasecollagen biosynthesis axis as a potential interface of apoptosis/autophagy. Biofactors 42, 341-348. doi: 10.1002/biof.1283

Zhai, C., Li, Y., Mascarenhas, C., Lin, Q., Li, K., Vyrides, I., et al. (2014). The function of ORAOV1/LTO1, a gene that is overexpressed frequently in cancer: essential roles in the function and biogenesis of the ribosome. Oncogene 33, 484-494. doi: 10.1038/onc.2012.604
Conflict of Interest Statement: The authors declare that the research was conducted in the absence of any commercial or financial relationships that could be construed as a potential conflict of interest.

Copyright $\odot 2018$ Celińska-Janowicz, Zaręba, Lazarek, Teul, Tomczyk, Pałka and Miltyk. This is an open-access article distributed under the terms of the Creative Commons Attribution License (CC BY). The use, distribution or reproduction in other forums is permitted, provided the original author(s) and the copyright owner are credited and that the original publication in this journal is cited, in accordance with accepted academic practice. No use, distribution or reproduction is permitted which does not comply with these terms. 


\title{
Botanical Provenance of Traditional Medicines From Carpathian Mountains at the Ukrainian-Polish Border
}

\author{
Weronika Kozlowska ${ }^{1}$, Charles Wagner ${ }^{2,3}$, Erin M. Moore ${ }^{2,4}$, Adam Matkowski ${ }^{1}$ and \\ Slavko Komarnytsky ${ }^{2,5 *}$
}

'Department of Pharmaceutical Biology, Medical University of Wroclaw, Wroclaw, Poland, ${ }^{2}$ Plants for Human Health Institute, North Carolina State University, Kannapolis, NC, United States, ${ }^{3}$ Department of Plant and Microbial Biology, North Carolina State University, Raleigh, NC, United States, ${ }^{4}$ Department of Biology, Catawba College, Salisbury, NC, United States,

${ }^{5}$ Department of Food, Bioprocessing \& Nutrition Sciences, North Carolina State University, Raleigh, NC, United States

\section{OPEN ACCESS}

Edited by:

Atanas G. Atanasov Institute of Genetics and Animal

Breeding (PAS), Poland

Reviewed by: Kannan R. R. Rengasamy, Department of Biotechnology, Alagappa University, India Víctor López,

Universidad San Jorge, Spain

*Correspondence:

Slavko Komarnytsky

komarnytsky@ncsu.edu

Specialty section: This article was submitted to

Ethnopharmacology a section of the journal Frontiers in Pharmacology

Received: 17 December 2017 Accepted: 14 March 2018 Published: 05 April 2018

Citation: Kozlowska W, Wagner C, Moore EM, Matkowski A and Komarnytsky S (2018) Botanical Provenance of

Traditional Medicines From Carpathian Mountains at the Ukrainian-Polish Border. Front. Pharmacol. 9:295. doi: 10.3389/fphar.2018.00295
Plants were an essential part of foraging for food and health, and for centuries remained the only medicines available to people from the remote mountain regions. Their correct botanical provenance is an essential basis for understanding the ethnic cultures, as well as for chemical identification of the novel bioactive molecules with therapeutic effects. This work describes the use of herbal medicines in the Beskid mountain ranges located south of Krakow and Lviv, two influential medieval centers of apothecary tradition in the region. Local botanical remedies shared by Boyko, Lemko, and Gorale ethnic groups were a part of the medieval European system of medicine, used according to their Dioscoridean and Galenic qualities. Within the context of ethnic plant medicine and botanical classification, this review identified strong preferences for local use of St John's-wort (Hypericum perforatum L.), wormwood (Artemisia absinthium L.), garlic (Allium sativum L.), gentian (Gentiana lutea L.), lovage (Levisticum officinale W.D.J. Koch), and lesser periwinkle (Vinca minor L.). While Ukrainian ethnic groups favored the use of guilder-rose (Viburnum opulus L.) and yarrow (Achillea millefolium L.), Polish inhabitants especially valued angelica (Angelica archangelica L.) and carline thistle (Carlina acaulis L.). The region also holds a strong potential for collection, cultivation, and manufacture of medicinal plants and plant-based natural specialty ingredients for the food, health and cosmetic industries, in part due to high degree of biodiversity and ecological preservation. Many of these products, including whole food nutritional supplements, will soon complement conventional medicines in prevention and treatment of diseases, while adding value to agriculture and local economies.

Keywords: ethnobotany, traditional knowledge, herbal texts, bioactive compounds, functional foods, folk remedies

\section{INTRODUCTION}

The terms Beskids or Beskid Mountains (Beschad Alpes Poloniae) denote the Carpathian mountain ranges that historically separated kingdoms of Poland from Ruthenia (Ukraine) and Hungary as described in the 1269 Hungarian deed (Papee, 1891). The Beskids are approximately $600 \mathrm{~km}$ in length stretching from Moravian Gate in the west (Czech Republic) to Vyshkovsky Pass in the 
east (Ukraine) along the modern Polish-Slovak border, but are rather narrow (50-70 km in width). Gorale, Lemko, Boyko, and Rusyn ethnic groups of mixed Slavic, Vlah (Aromanian) and Thraco-Celtic origins primarily populate these areas (Figure 1). During tenth-fourteenth centuries, Beskids formed a transborder region of limited political and economic affiliation, defined by a line of defensive border settlements (La: ultra indagines, Ua: zasiky, Pl: bron) advancing slowly toward the mountain ridges along the Spis-Borzhava axis in the south and Myslenice-SanokDolyna axis in the north (Halaga, 1961). Turka on the route to Uzhok Pass and Wietrzno-Bobrka on the route to Dukla Pass were typical examples of such defensive hillforts (Ua: gorodysche, $\mathrm{Pl}$ : grodzisko). In the fourteenth century, continuous colonization of the Beskids was described as of Ruthenus, Ruthenus-Valachus, Ruthenos seu Valachos, or Valachus origin (Hoshko, 1983). The ongoing debate whether these terms identified a proper ethnic affiliation of the migrants or their rustic (agricultural) vs. valachian (pastoral) background has not been settled (Stavrovsky, 1967). The transborder status, however, combined with severe geographical and socioeconomic isolation, resulted in selftreatment as a primary choice of both medicinal and spiritual healing. Even at the beginning of the twentieth century, one medical doctor served on average 9,000-17,000 inhabitants (Otchet, 1915), and a doctor's reputation and recognition was well below that of a local pharmacist or a traditional healer.

The use of plants and their preparations in European medicine, drawing upon the traditions of both the classical and Arab civilizations, was formalized by works of Hippocrates of Kos (Corpus Hippocraticum), Dioscorides (De Materia Medica), Pliny (Naturalis Historiae), Avicenna (Liber Canonis), and Galen of Pergamon (Hidden Drugs, survived only in Arabic translation as Al-Adwiya al-Maktuma) as the science of using fresh plants and dry herbs to improve human health (Cruse, 1999). Prior to 900-1200 AD, however, no written records described traditional herbal treatments used by Celtic, Slavic, and Germanic tribes that bordered the Roman Empire. First medieval manuscripts that partially documented the use of herbs in these areas were largely translations from earlier
Latin works that incorporated local variations in methods of collection, preparation, and use (Singer, 1927), including Bald's Leechbook and Old English herbals (Anglo-Saxon, tenth-eleventh century), Regimen Sanitatis (Italian, tenth century), Physicians of Myddvai (Welsh, fourteenth century), and various editions of [H]ortus Sanitatis (1491) based on the German Herbarius Maguntie impressus of 1484 and Herbarius zu Teutsch of 1485. The latter monographs were intended to treat of cheap and homely remedies for the use of the poor, as most herbs listed in these books were native or garden plants, while very few exotic remedies were described in details.

A glimpse of medieval Slavic herbal tradition could be found in the tenth-eleventh century manuscripts Izborniki (Anthologies, transcribed in 1073 and 1076 for Prince Svyatoslav of Kyiv from manuscripts that had belonged to Tsar Simeon of Bulgaria) that described medicinal uses of several plants, including henbane (Hyoscyamus niger), hemlock (Conium maculatum), and wormwood (Artemisia absinthium). Subsequent herbal manuscripts (Ua: travnyk, zilejnyk, vertograd, Pl: zielnik) from sixteenth to eighteenth centuries often combined traditional herbal medicines with irrational magical applications. For example, while the seventeenth century vertograd described management of fever and pain with cabbage (Brassica oleracea) and beet (Beta vulgaris) leaves, and infection diseases with onion (Allium cepa) and garlic (Allium sativum), it also prescribed a mixture of horse and goat manure to cure alopecia in 3 days (Krylov, 1985). Nonetheless, these manuscripts served during the late Middle Ages as veritable manuals of practical herbal medicine. Their text and images were usually mechanically transcribed from earlier works of the most varied provenance, while the successive owners often enriched the contents with personal observations and experiences.

\section{OLDEST HERBALS PRINTED IN THE CARPATHIAN FORELAND}

Foundation of Prague University in 1348 and Krakow University in 1364 provided diverse educational opportunities for wealthy

\section{A}

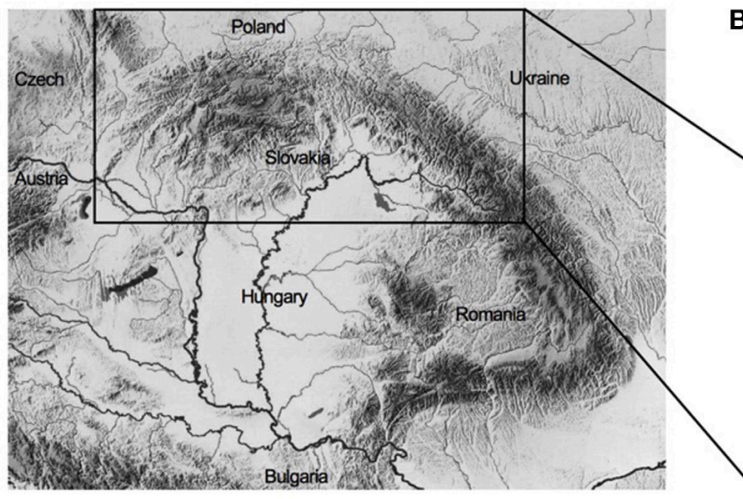

B
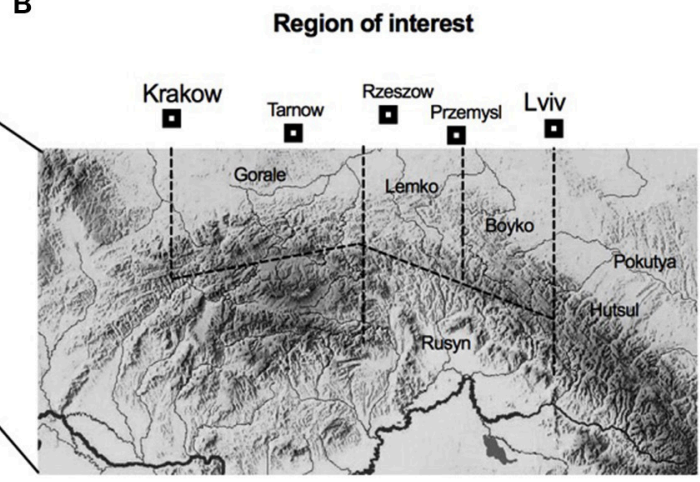

FIGURE 1 | Study area that includes Beskin ridges of the Carpathin Mountains: (A) Schematic map of the mountain region that serves as a border between Ukraine, Poland, Slovakia, Hungary, and Romania, and (B) Schematic mountain areas inhibited by Gorale, Lemko, Boyko, Rusyn, and Hutsul ethinc groups. 
local families of Polish and Ruthenian descent, and led to the expansion of local medicinal and apothecary tradition. Krakow, and later Lviv, quickly developed into two influential medieval centers of apothecary, with former also serving as an important publishing site of printed herbal texts.

Here, six major monographs describing the use of medicinal plants were published between 1532 and 1613 (Table 1). The first one was a Latin reprint of De Herbarum Virtutibus (Naples, 1477) with plants names translated into Polish for the first time by Simon de Lowicz in 1532. Its significance lies in the fact that it was the first document of such length to indicate a renewed interest on this subject and to reveal direct influence of Dioscorides and Pliny. Even though there was no evidence of experimentation, and whether or not the author actually ever attempted to use any of the herbs of which he wrote, this herbal had a tremendous influence on medical and botanical literature from the early Middle Ages (Flood, 1976). One testimonial to the wider influence Macer had on subsequent medical and botanical commentators was a large number of translations of the herbal into vernacular languages and dialects. Its popularity was likely partially due to the selection of predominantly native European plants (39) or medicinal herbs cultivated in the gardens (25) over the exotic plants (13) that were difficult to procure.

Stefan Falimirz (Falimir, a Ruthenian courtier to Jan Tenczynski, the Duke of Podola in Krasnik) compiled various Latin [H]ortus Sanitatis manuscripts, translated them into Polish, and released a first popular encyclopedia of traditional medicines entitled $O$ ziolach $i$ o mocy ich (On herbs and their power) in Krakow (1534). The subjects covered herbs and herbal medicines (including the first use of Polish term wodka meaning "little water" to describe high proof spirit distilled from grain), medicinal properties of animals and minerals, obstetrics, surgery, and were profusely illustrated with over 550 mostly original woodcuts. Plants were described in a set of 261 articles on European and 69 exotic herbal remedies. Descriptions of plants and their use, however, were mostly translations from the previous sources and likely unknown to the author (Elbanowski, 2014). This became very clear when compared to other chapters describing animals, where Stefan provided extensive and original details on local fishes, a subject apparently of great familiarity to the author. Specifically, Stefan stated, "Because we live in Poland, there is a need to know characteristics and use of Polish fishes, as we use them regularly. The ones that help, the ones that harm, the good, the better, and the worst." This statement for the first time indicated the emerging perception of inadequacy of contemporary Latin manuscripts that lacked local knowledge and tradition.

Hieronim Spiczynski of Wielun (a wealthy Polish councilman in Krakow) attempted an adaptation of Stefan Falimirz's work by publishing $O$ ziolach tutecznych y zamorskich y o mocy ich (On local and overseas herbs and their power, 1542) with improved woodcuts. The monograph contained similar and often identical descriptions of several medical guides to practical use of herbal remedies. The catalog of herbs was focused on local plants listed under Polish and Latin names, with a shorter list of exotic plants identified only in Latin. Compared to other herbals, it contained narrower descriptions of plant species with no mention of their origin or occurrence.

Marcin Siennik (Merten Heüwrecher, a wealthy merchant of German origin in Krakow) compiled another adaptation of Falimirz's herbal 26 years later as Herbarz to jest ziol tutecznych, postronnych $i$ zamorskich opisanie (Herbal as description of local, foreign and overseas plants, 1568). Quick comparison among these editions revealed minor changes in the order of the chapters, woodcuts, and plant names. Siennik held neither a professional title nor a degree, and seemingly edited books solely to make income in accordance with publisher's instructions. The only freedom that he enjoyed was expanding the list of plant nicknames, to which he contributed many new names, however both descriptions and uses of various remedies were transcribed intact, together with all previous errors and omissions. The

TABLE 1 | Medieval herbals printed in the Carpathian foothills in the fifteenth-seventeenth centuries.

\begin{tabular}{|c|c|c|c|c|c|c|}
\hline Front page & 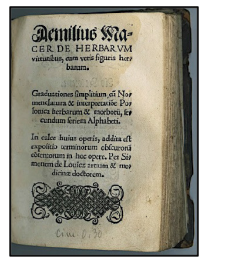 & 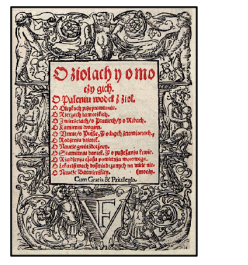 & 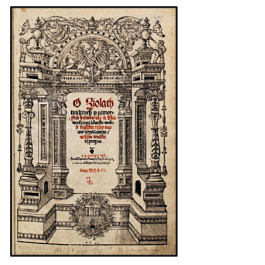 & 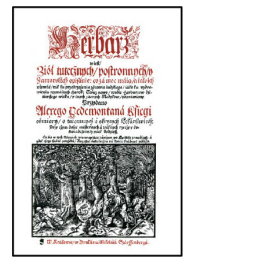 & 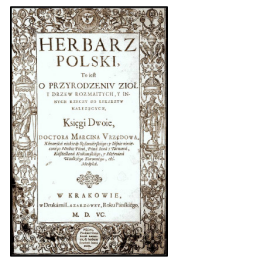 & 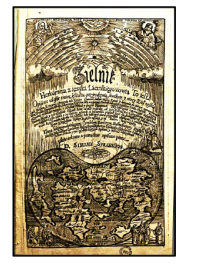 \\
\hline Author & $\begin{array}{l}\text { Simon de Lowicz } \\
(-1538)\end{array}$ & $\begin{array}{l}\text { Stefan Falimirz } \\
(-1550)\end{array}$ & $\begin{array}{l}\text { Hieronymus Spiczynski } \\
(-1550)\end{array}$ & $\begin{array}{l}\text { Marcin Siennik } \\
(-1588)\end{array}$ & $\begin{array}{l}\text { Marcin de Urzedow } \\
(-1573)\end{array}$ & $\begin{array}{l}\text { Simon Syrenius } \\
(-1611)\end{array}$ \\
\hline Title & $\begin{array}{l}\text { Aemilius Macer, De } \\
\text { herbarum virtutibus } \\
\text { (Krakow, 1532) }\end{array}$ & $\begin{array}{l}\text { Hortus sanitatis, } \\
\text { O ziolach i o mocy ich } \\
\text { (Krakow, 1534) }\end{array}$ & $\begin{array}{l}\text { O ziolach tutecznych i } \\
\text { zamorskich i o mocy } \\
\text { ich } \\
\text { (Krakow, 1542) }\end{array}$ & $\begin{array}{l}\text { Herbarz to jest zioł } \\
\text { tutecznych, } \\
\text { postronnych i } \\
\text { zamorskich opisanie... } \\
\text { (Krakow, 1568) }\end{array}$ & $\begin{array}{l}\text { Herbarz polski, to jest o } \\
\text { przyrodzeniu ziol i dzew } \\
\text { rozmaitych... } \\
\text { (Krakow, 1595) }\end{array}$ & $\begin{array}{l}\text { Zielnik herbarzem z } \\
\text { iezyka lacinskiego } \\
\text { zowia... } \\
\text { (Krakow, 1613) }\end{array}$ \\
\hline Notes & $\begin{array}{l}2,756 \text { verse lines } \\
77 \text { plants } \\
13 \text { exotic } \\
\text { Added Polish names }\end{array}$ & $\begin{array}{l}433 \text { pages } \\
329 \text { plants } \\
69 \text { exotic } \\
\text { Translated to Polish }\end{array}$ & $\begin{array}{l}\text { Adapted from Falimirz } \\
\text { Sorted by Polish names }\end{array}$ & $\begin{array}{l}\text { Adapted from Falimirz } \\
\text { Added German names }\end{array}$ & $\begin{array}{l}488 \text { pages } \\
477 \text { plants total } \\
105 \text { exotic } \\
\text { New compilation }\end{array}$ & $\begin{array}{l}1,540 \text { pages } \\
765 \text { plants total }\end{array}$ \\
\hline
\end{tabular}


resulting monograph was published under a new name, with modified woodcuts and an expanded index including German names (Rostafinski, 1888).

Marcin de Urzedow (a Polish Catholic priest born in Urzedow near Lublin, and a graduate of Krakow University) compiled Herbarz polski, to jest o przyrodzeniu ziol $i$ dzew rozmaitych (Polish Herbal, 1595) that was printed after his death and included descriptions of 372 local plant remedies and 105 exotic herbs. Plants were listed in Latin alphabetical order and were heavily influenced by independent translations from Dioscorides Materia medica and Fuchs Cometraii insignes. Among those, only 259 chapters described wild native plants characteristic of the Polish countryside. The woodcuts, however, were the same ones used in Siennik herbal to depict 240 local herbs-and for this reason, the rest of the herbal remedies were illustrated by woodcuts that were repeated or omitted altogether. The individual reports lacked a particular order of presentation, with some chapters organized by location, description and use, while others discussed herbal properties prior to their looks and origins (Rostafinski, 1888).

Szymon Syrenius (Syreniusz, Syrenski) of Oswiencim, a graduate of Krakow and Padua Universities, moved to Lviv and established his medical office there in 1578 . He spent next 30 years collecting botanical flora from the northern slopes of Beskids mountain ranges, including trips to more distant regions of Podolia and Pokuttya. Here he compiled the most extensive Polish herbal to date-Zielnik herbarzem z iezyka lacinskiego zowia (Polish herbal, 1613), printed 2 years subsequent to his death. Similarly to previously described monographs, this volume was largely based on translations of Dioscorides Materia medica and its adaptation by Petri Matthioli, known as Senensis midici, commentarii in sex libros (1565), including the original woodcuts from Matthioli's work (Rostanski, 1997). The volume provided detailed records of 765 plants, mostly from Southern and Central Europe. The information was presented in a systematic way, including plant description, area of distribution, collection and processing techniques, and medicinal properties, followed by an index of Latin, Polish, and German names.

Finally, a fairly unknown Old-Eastern-Yiddish remedy book (refue-bukh) with the Hebrew title of Seyfer derekh ets ha-khayim was printed in Poland in 1613. While the exact printing place of the book is unknown, as is the name of the author, Krakow was one of the possible places of its origin (Geller, 2009). Apart from a richly decorated title page, the book contains no illustrations, so technically it was not an herbal but rather a self-help botanical guide. It described 87 remedies of plant, animal, and mineral origin, with many Polish words used to identify plant names. Most botanical terms and folk remedies were referenced to the tradition of Hippocrates, Dioscorides, and Avicenna, therefore it is not clear whether this book was an original compilation of the classical sources, or a more recent adaptation of one of the herbals similar to those listed above. To this point, we would like to highlight the unknown author's experience at the Padua medical school to which he refers in the beginning of his book (Geller, 2009)-a striking parallel to the educational background of his contemporaries Marcin de Urzedow and Szymon Syrenius. This may suggest a Padua-Krakow axis as a primary route for introduction and adaptation of antique botanical knowledge into the Carpathian region. Indeed, over the course of the sixteenth century, approximately 1,400 Polish and Ruthenian students, including nearly all physicians to the Polish royal family, attended Padua University (Theodorescu, 1989). Jan Zamoyski, Polsih Crown Chancellor and graduate of Padua University, founded Zamojski Academy in 1594 and shortly after that, a Jesuit Collegium was founded in Lviv (1608), which later became Lviv Academy (1661). Together with Krakow University, these institutions rapidly became the major centers of intellectual and spiritual life in the region.

\section{ON RELEVANCE OF PRINTED HERBALS TO TRADITIONAL BOTANICAL KNOWLEDGE}

The sixteenth century scholars discovered a critical obstacle in sorting and presenting botanical knowledge that had accumulated over the centuries in antique natural and medical literature, and had been translated to various languages on multiple independent occasions. The same plants were often given different names based on the place of their origin. To make things worse, the ancient texts and names of old herbal remedies were often distorted due to evolution of the language and inadequate translation or transcribing, especially when detailed descriptions of target plants were absent from the original texts. This notion did not escape the attention of herbal editors, as stated by Marcin de Urzedow in the introduction to his work: "We are no longer satisfied by knowledge of garden and field plants, but search for new ones in the wild, in forests, on mountains and cliffs, and even places where mountain goats reached seldom." For this particular reason, it was very difficult to define and isolate local traditional knowledge in these monographs, as medicinal use of many ancient herbs was fully adopted and integrated into local folk knowledge. There was, however, a speculative evidence of an opposite flow of knowledge about medicinal plants from Slavic into Greco-Roman tradition: Slavic knowledge of medicinal plants supported the hypothesis of Tomaschek that the majority of so-called Dacian synonyms interpolated into the Dioscorides text from the Viennese codex are of Slavic origin (Grmek, 1959).

\section{PHARMACOPEIAS THAT REGULATED APOTHECARY TRADITION IN THE REGION (FIFTEENTH-NINETEENTH CENTURIES)}

The official Polish and Polish-Latin scientific literature concerned with herbal medicines became comparatively extensive between fifteenth and seventeenth centuries. The first place among earlier manuscripts from the region belongs to the volume entitled Antidotarium seu Vocabularium medicum, passim cum nominibus herbarum Germanis et Polonis rubro adscriptis (1419), currently kept at the Jagiellonian Library in Krakow. It was likely written by an unknown physician or monk of German origin who worked in Poland and had first hand knowledge of Polish plants (Magowska, 2004). This pharmacological work was 
a direct adaptation from Antidotarium parvum, a pharmacopeialike manuscript written by an unknown author in Salerno between eleventh and thirteenth centuries. Different editions of this work included 110-170 recipes from antiquity and early medieval writers, which were annotated with German and Polish names. We also cannot rule out the possibility that some chapters were borrowed from Antidotarium magnum, another work of unclear origin from the twelfth century, which may have been initiated by Constantinus Africanus and contained 1,200 recipes (Prioreschi, 2003).

Jan Stanko (Johannes Stanconis) of Luban near Wroclaw, a graduate of Padua University and a royal physician to Polish King Kazimierz Jagiellonczyk (Lisowski, 2007), compiled a botanical and medical glossary entitled Incipit Antibolomenum Benedicti Parthi in 1472 (now kept at the Archives of the Krakow Cathedral Chapter). He listed 20,000 scientific and medicinal terms of Latin, Greek, Arabic, Italian and other origins, translated into German, Polish, and Czech languages. The Antibolomenum served as an apothecary index identifying 433 domestic and 90 foreign plants, 219 domestic species of animals and the minerals used as the ingredients of the apothecary remedies. While useful for quick identification of medicinal ingredients and their equivalents, the manuscript was never published, and had no major impact on the development of the natural sciences at the time.

The first printed pharmacopeia that had significant impact on the apothecary tradition of Poland was compiled by a German physician Johann Placotomus (Breitschneider) under the title of Pharmacopoeia in compendium redacta and published in 1560. This book was developed on the basis of Dispensatorium written by Valerius Cordus (1546). The work included information on individual materia medica (simples) and complex recipes for powders, pills, syrups, decoctions, ointments, and oils. It also included recipes and instructions for preparation of theriacs-panacea-type medical concoctions as general antidotes against poisons and infectious diseases. Another pharmacopeia was compiled by Polish physician and apothecary in Krakow, Jan Woyna of Jasienica under the title of Pharmacopoea Cracoviensis and published in 1683, in compilation with two other works, Experemintorum medicochymicorum and Observationes medicochirurgico by Daniel Matthia. This monograph was rather short; although it listed circa 1,500 apothecary ingredients in alphabetical order, the descriptions were very brief or nonexistent. Instead, multiple cross-references to other, mostly foreign pharmacopeias and dispensatoria, were provided. The only exceptions were made for new recipes developed by the author, in which case more detailed information on ingredients and methods of preparation were given. Certain new recipes were very complex, for example a novel preparation named Spiritus Castorei Cracoviensis consisted of four herbs, Castorei (locally sourced Castor fiber), Anacardini (American Anacardium occidentale), Asa (Asa foetida from Persia or Afghanistan), and Storacis (Styrax liquidum from Asia Minor). All four monographs, however, were private publications without any official character or certification.

In spite of the fact that unification and standardization of apothecary tradition in Europe were long overdue, the first legally enforced government pharmacopeia was published only in 1698 as Dispensatorium Brandenburgicum. This monograph was released in Latin language, contained descriptions of over 1,000 apothecary ingredients, including 906 complex mixtures. The most demanding recipe was that of Theriaca Andromachi that comprised a mixture of 71 individual components. Duke of Warsaw Frederick Augustus I made Dispensatorium a required apothecary regulatory document for all Polish lands in 1714 under a modified title. Following the first partition of Poland, the Carpathian foothills were divided between Poland and Austria, and two new pharmcopeias were published to regulate the apothecary practices including Pharmacopoea Regni Poloniae (1817) and Pharmacopoea Austrica (1820) (Table 2). These monographs contained descriptive notes on prescribed medicines and were intended to register approved and established remedies (for the physician), and to provide formula and appropriate methods of preparation (for the pharmacist).

\section{HANDWRITTEN HERBAL MANUSCRIPTS FROM THE REGION (SIXTEENTH-EIGHTEENTH CENTURIES)}

The beginning of the seventeenth century marked a turning point in the use of botanicals for medicine and folk healing. The practices of trained physicians and apothecaries became chiefly summarized and regulated by pharmacopeias, while the local knowledge of healers and witchcraft practitioners was mainly restricted to oral tradition and handwritten manuscripts. While the second sources often originated from the first, they were much less consistent. These manuscripts typically borrowed from multiple printed sources, original or translated, and accreted with remarks and personal observations of the successive owners. Thus, while printed herbals recommended carline thistle (Carlina acaulis) to protect children from the evil enchantments of the "old women," the same "old women" often used this plant to aid in apotropaic and love magic (Ostling, 2014).

One of the earliest examples of such manuscripts, recorded somewhere in Grand Duchy of Lithuania and self-described as "a translation from Latin and Polish books," was created in the mid sixteenth century (designated here as Pushkarev). If the dating is correct, this manuscript was translated at the same time as Hortus sanitatis books known as "vertograds" were printed in Moscow (1534) and Kolomna (1588) (Novoselski and Pushkarev, 1977). The section of the manuscript that describes herbal tradition is entitled Knigi lechebnya ot mnogih lekarev sobranye o koreniah $i$ o zeliah (Medicinal books collected from many doctors). Other sections contained discussions on hygiene, Galenic commentary on Hippocrates, notes and quotations from Avicenna and other medieval physicians, exerts from the pseudo-Aristotle tractates, descriptions of common medicinal practices such as bloodletting, and magical spells. Large numbers of spelling errors, incorrect interpretations and misrepresentations, all point to the fact that the manuscript was written not for personal use, but rather for sale by a professional transcriber with little knowledge on the subject. Extensive references to old Ruthenian terms zhuravly (cranes), zubry (buffalo), tryascia (malaria), tsybula (onion), 
TABLE 2 | Pharmacopeias that regulated regional apothecary practices in the sixteenth-nineteenth centuries.

\begin{tabular}{|c|c|c|c|c|c|}
\hline Front page & 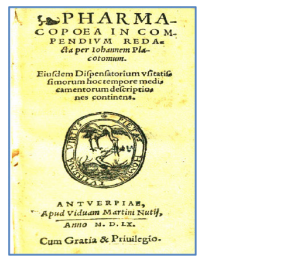 & 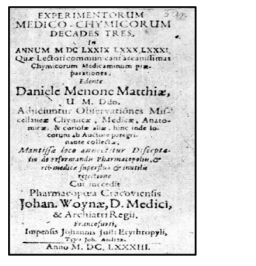 & 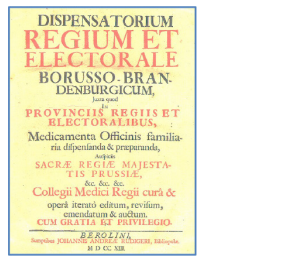 & 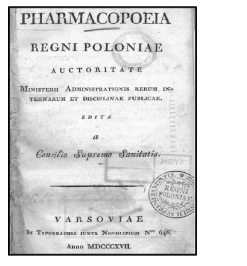 & 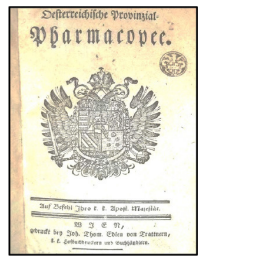 \\
\hline Author & $\begin{array}{l}\text { Johann Placotomus } \\
(1514-1574)\end{array}$ & $\begin{array}{l}\text { Jan Woyna } \\
(1605-1693)\end{array}$ & Prussian Magistrate & $\begin{array}{l}\text { Polish Supreme } \\
\text { Health Council }\end{array}$ & $\begin{array}{l}\text { Austrian Pharmaceutical } \\
\text { College }\end{array}$ \\
\hline Title & $\begin{array}{l}\text { Pharmacopoea in } \\
\text { compendium redacta per } \\
\text { Johannem Placotomum } \\
\text { (Antwerp, 1560) }\end{array}$ & $\begin{array}{l}\text { Pharmacopoea } \\
\text { Cracoviensis (Frankfurt } \\
\text { am Main, 1683) }\end{array}$ & $\begin{array}{l}\text { Dispensatorium Regium et } \\
\text { Electo-rale Borusso- } \\
\text { Brandenburgicum } \\
\text { (1714) }\end{array}$ & $\begin{array}{l}\text { Pharmacopoea Regni } \\
\text { Poloniae (1817) }\end{array}$ & $\begin{array}{l}\text { Pharmacopoea Austrica } \\
\text { (1820) }\end{array}$ \\
\hline Notes & $\begin{array}{l}256 \text { pages } \\
502 \text { plants total }\end{array}$ & $\begin{array}{l}147 \text { pages } \\
1,500 \text { recipes }\end{array}$ & & $\begin{array}{l}305 \text { ingredients } \\
357 \text { mixtures }\end{array}$ & \\
\hline
\end{tabular}

pevne (probably), and many other words suggested Volhynia or Carpathian forelands as possible places of its origin.

The compilation of such manuscripts with no reference to the original sources was very common among Eastern Slavic groups during sixteenth-eighteenth centuries. In his Russkije vrachebniki work, a bibliographer of the history of Russian medicine listed 186 such manuscripts, most from the seventeenth century (Zmejev, 1895). As these manuscripts were transcribed in time, each subsequent version underwent a complex change based on the local needs and traditions, with a progressively shifting emphasis onto local and easily collectible plants, and away from their exotic and expensive alternatives. It is therefore critical in future works to establish which particular plants and beliefs were incorporated into Slavic folk healing manuscripts from European sources, which knowledge specifically reflects oral local tradition that was fixated in these works, and which part of the tradition was an original contribution of local ethnic groups. To achieve this goal, we would need to determine and investigate independent parts of handwritten herbal manuscripts (i.e., chapter groups) that originated from the common source. Another prevalent feature of these manuscripts that separated them from printed herbals was the lack of plant illustrations or prints. Otherwise, the plant uses described in these manuscripts were correct and targeted either a function of the human body (medicine), social life (relationships and feelings), household issues, or superstition beliefs (Ippolitova, 2008). With time, these descriptions became a part of the oral local tradition, and many of medicinal prescriptions and uses were eventually transferred to other unrelated plants.

A Ruthenian handwritten manuscript from the late sixteenth century, also likely a translation from a Polish source, was found in the State Archive of Bucharest (Syrku, 1883) (designated here as Syrku). Some of the plant descriptions from this manuscript, for example, plantain (Plantago major), St. Benedict's thistle (Cnicus benedictus), Norwegian angelica (Archangelica officinalis), and lesser burnet (Pimpinella saxifraga) were very close if not identical to other subsequent manuscripts, showing continuation of handwritten tradition in time (Nimchuk, 1976). One of them, a Ruthenian herbal manuscript Kniha lechebnaya o mnogih lekarstv, dated to the mid seventeenth century, survived in the Swidzinski collection from Sanok area (Peredrijenko, 1984) (designated here as Swidzinski). This manuscript was a compilation of various texts, legends, poems, and prayers in addition to herbal tradition. The territory to which the manuscript was localized, had formed a border line between two ethnic groups of Ruthenian origin, Boyko in the east and Lemko in the west (Falkowski and Pasznycki, 1935). Both ethnic groups still maintain a deep tradition in collecting and consuming a variety of wild plants. Among those, berries, mushrooms and hazelnuts are most popular. Several reorganizations, including the deletions of nonessential magical sections, and additions of important material about the habitat and preparation of the plant, were evident from this manuscript. We must therefore postulate, that the original knowledge about the medicinal properties of the herbs that was fixated in the earlier printed herbals, became amorphous and open to interpretation when it entered oral and written tradition of common people in the seventeenth-eighteenth centuries.

\section{ETHNOGRAPHIC STUDIES OF THE CARPATHIAN FOOTHILLS (NINETEENTH-TWENTIETH CENTURIES)}

While political and economic transformations took place at the lowlands of Poland and Ukraine, the people who inhabited the Carpathian ridges of the Beskid Mountains saw little change and depended mostly on cheap and readily available traditional herbal medicines for the poorest. By that time, the combined knowledge of printed herbals and handwritten manuscripts was deeply rooted in the oral tradition of folk remedies (Figure 2). This fusion was so profound, that most of the ethnographic studies of the region that took place in nineteenth and early twentieth century recorded and transcribed traditional folk medicines as a part of local cultural and ethnographic "heritage." The rise 


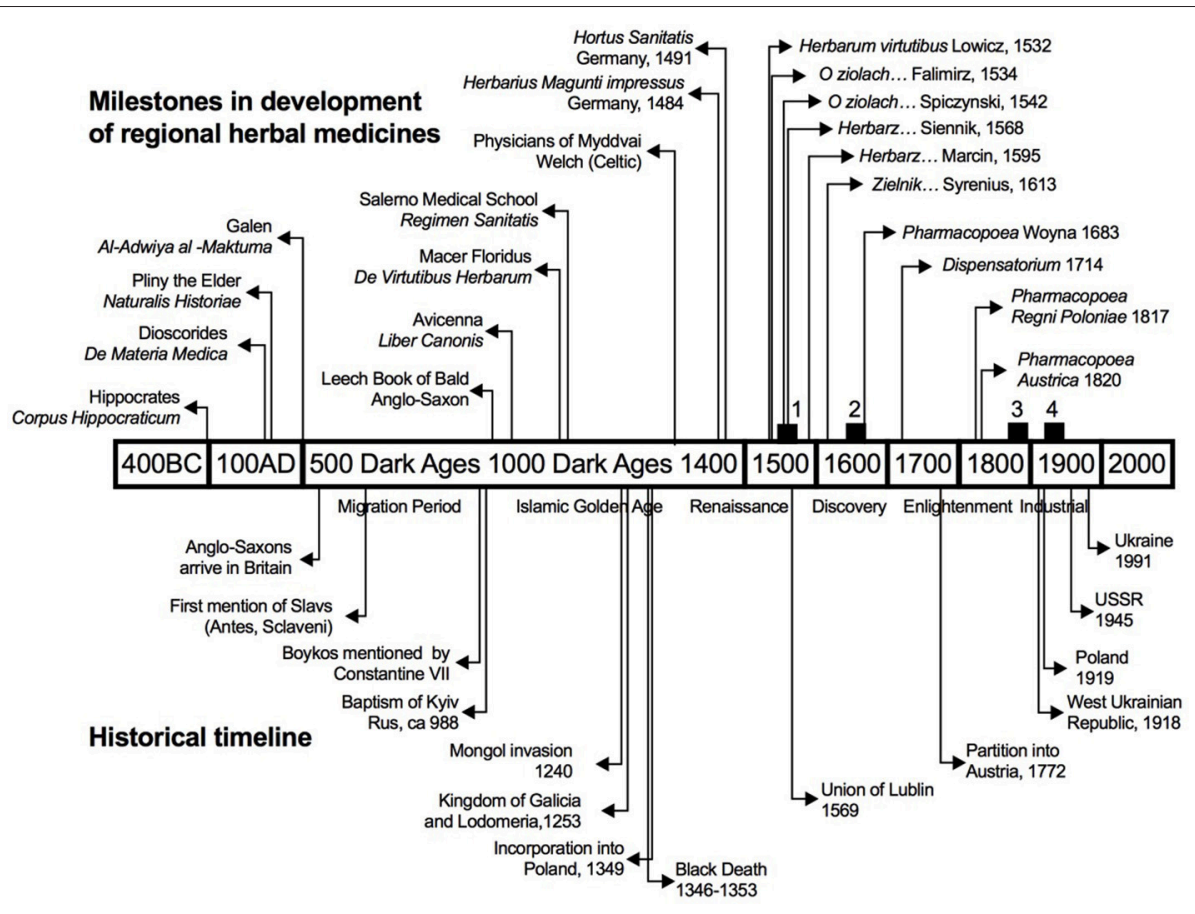

FIGURE 2 | Major historical and publishing milestones that define development of regional herbal texts and botanical manuscripts. Black squares indicate the emergence of handwritten herbal mauscripts including Pushkarev (1) and Swidzinski (2), as well as ethnobotanical surveys of Rostafinski (3) and Fisher (4) discussed in this study.

of alternative medicines in these areas could be interpreted as one of many signs of poor development and often of an economic decline of the region. The inaccessibility and sometime active rejection of medicine, the acceptance of simplistic medical notions, and the reliance on imaginary observations indicated a loss of confidence in empirical observations and a pessimistic view of the capacity of men to overcome external difficulties by reason (Prioreschi, 2000).

Early ethnographic studies in the region were general descriptive compilations of oral traditional medicines recorded by Ukrainian and Polish enthusiasts without further analysis or comparison to the previous written works on the subject. Danylo Chomych Lepki described Boyko women from mountain areas who treated skin disorders in babies with herbal baths that included a mixture of pasqueflower (Pulsatilla patens), cornflower (Centaurea cyanus), rue (Ruta graveolens) and lesser periwinkle (Vinca minor), as well as the use of infusions from the bark of rowan (Sorbus aucuparia) and mezereon (Daphne mezereum) in love magic (Lepkij, 1884). Oskar Kolberg, a Polish ethnographer, observed the traditional use of thyme (Thymus serpyllum), milk thistle (Silybum marianum), scentless chamomile (Anthemis arvensis), chamomile (Matricaria chamomilla), wormwood (Artemisia absinthium), galipot (Picea alba), a mixture of water and burned aspen (Populus tremula) for treatment of osteomyelitis, and a fermented mixture of oat hulls and goose feces for treatment of abscesses among the inhabitants of the Carpathian foothills (Kolberg, 1888). Ivan Kuziw, a Greek-Catholic priest from Boyko villages Likot and Dydiowa, noted an unusual method of making infusions of valerian roots. They were placed in glass bottle, covered with alcohol, and baked inside a breadloaf (Kuziw, 1889). Julian Talko-Hryncewicz described 360 medicinal plants used in Ukrainian lowlands (Talko-Hryncewicz, 1893). Ivan Franko, an Ukrainian poet and ethnographer of Boyko origin, published several collections of his ethnographic observations of Boyko people, including their use of herbal medicines in magic and protection (Franko, 1898), including blessed chalk, salt, field poppy (Papaver rhoeas), zillya troyan (a mixture of Levisticum officinale, Vinca minor, and Ocimum basilicum), and garlic mustard (Alliaria petiolata).

Volodymyr Szuchewycz, an Ukrainian ethnographer and teacher residing in Lviv, observed extensive use of traditional herbal medicines by Hutsuls (a neighboring ethnic group of Ruthenian origin to the east of Boykos) and described some of the remedies in the 5th volume of his monograph (Szuchewycz, 1908), including the widely used comfrey (Symphytum officinale), yarrow (Achillea millefolium), marsh woundwort (Stachys palustris), mistletoe (Viscum album), and yellow gentian (Gentiana lutea). Josef Schneider, a Polish ethnographer born in Stebnyk, documented the use of herbal remedies by Boyko people from the Dolyna region (Schneider, 1912). He noted use of bath prepared with aspen (Populus tremula) and periwinkle (Vinca minor) for treatment of various skin disorders, baked onion (Allium cepa) for alleviation of abscess, green cones from spruce (Picea abies) for syphilis, flower petals for leg pain, and decoction of beet (Beta vulgaris) 
leaves for ringworm. Jan Falkowski, a Polish ethnographer, described treatment of the Polish plait (plica polonica, irreversibly entangled moist damaged hair) with chorne zillya (Pulsatilla vulgaris). Tinctures made with yellow gentian (Gentiana lutea) were a popular remedy against cholera. Special attention was paid to ashes of burned medicinal plants. Cataracts were treated by burning periwinkle (Vinca minor) and using the ashes to wash the eyes. Burn wounds were treated with ashes from burned wool or black ligules of sedge (Schoenus nigricans). Open cut wounds were treated with juice from yarrow (Achillea millefolium) (Falkowski and Pasznycki, 1935). Several more recent ethnographical monographs provided comparative analysis of previously published traditional knowledge and cultural differences of Boyko people, including limited data on Boyko herbal remedies. As such, they are not discussed here (Kyrchiv, 1978; Boltarovych, 1980; Hoshko, 1983). Throughout the twentieth century, there was a steady growth of published books on Ukrainian medicinal plants that also contained fragmented information on the topic from Beskids. Three of them had a particular influence on the folk knowledge of the local medicinal plants (Nosal and Nosal, 1962; Komedar, 1971; Tovstuha, 1990).

Gorale from Silesian Beskids used sap from crushed goldmoss stonecrop (Sedum acre) to treat ulcers. Crude, crushed herb was applied for heel pain or, if mixed with fat, into the ear to reduce earache. In Sacz Beskids it was also used internally to treat flu or urinary tract diseases. Its relaxant and emetic properties were used in food poisoning. Sedum maximum leaves were applied externally crude or crushed on ulcers, bruises or edema. Sap of its leaves was mixed with barley flour and as a plaster applied to slow healing wounds (Tylkowa, 1989). On the border of Sandomierz Basin and Central Beskidian Piedmont, wound healing properties of Sedum were also known. It was applied in cataplasms or ointments, when fried with Thymus serpyllum in unsalted pork fat. Additionally, Gorale used its decoction in mouth rinsing as a gum strengthening agent or as a remedy to "destroy the scurvy impurities." Among Gorales, several different plants had the dialect name "nine forces" but the most famous one was carline thistle (Carlina acaulis). The Silesian Beskids inhabitants used it both internally and externally. Its root boiled with milk or water and drank once a day on empty stomach was used in kidney and bladder disease. Leaves were put on ulcers because of its properties of getting pus out. Moreover, herb infusion was used internally to treat colic and stitching pain (Tylkowa, 1989).

It is critical to note that historical handwritten manuscripts and ethnographic studies reported equal if not larger use of verbal spells (Ua: zagovor, Pl: zamawianie), charm heals, and whispering prayers to treat diseases and cast magical incantations (Figure 3A). This knowledge was often culture and language specific, and most likely truly represented the original healing tradition that predated Roman and Greek therapies. The herbal remedies, however, were either substituted in their entirety by the knowledge gathered from translation of mostly Roman medicine, directly or via Italy and Germany, or were so similar that they could not be distinguished. This overlap though was never complete due to endemic plant species in the region, which were unknown to Greeks and Romans, and therefore could provide an exciting opportunity to observe pre-Christian folk healing traditions from the Carpathian Mountains. Guelder rose (Viburnum opulus) could be of particular interest in this matter as it was largely unused by the Western cultures. Similarly, the endemic species of Arran whitebeam (Sorbus arranensis), unknown in Roman healing tradition, was used as an antimicrobial remedy in the Scottish Highlands (Wagner et al., 2017). Previously, it was reported that the Bulgarian flora comprises 3,900 species, $12.8 \%$ of which are endemics (Petrova, 2005). Assuming a similar ratio of endemic plant species in the Ukrainian flora $(6,086$

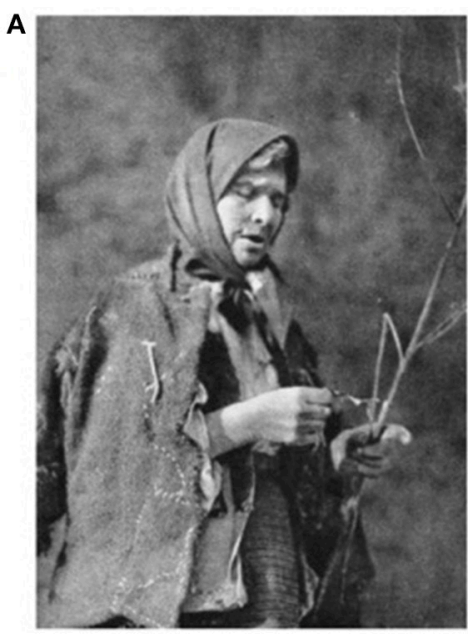

B Herbs Import 2014

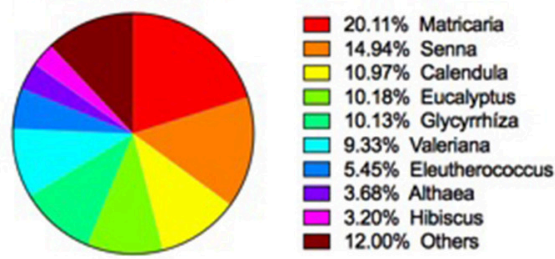

C Herbs Export 2014

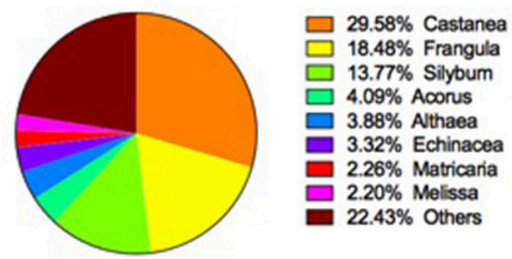

FIGURE 3 | Botanical provenance of medicinal plants from the Carpathian Mountains: (A) Traditional healer from Eastern Carpathians as documented by Henryk Gasiorowski at the beginning of the twentieth century, and botanical profiles of major (B) imported and (C) exported medicinal plants from Ukraine in 2014 (after Nykytyuk, 2015). 
species), we could expect at least 600 endemic species, of which at least 200 species could potentially contain biologically active components and be used medicinally (Konischuk et al., 2016).

\section{SURVEYS THAT DOCUMENTED TRADITIONAL USES OF PLANTS IN THE REGION}

Apart from the observational ethnographic studies, we are also aware of two attempts to collect and analyze ethnographical data on local use of herbal remedies in the Beskid region by mailed or published questionnaires of Rostafinski in 1883 (Köhler, 2015) and Fischer in 1929-1934 (Kujawska et al., 2015), both addressed to Polish speakers. Nearly 370 individuals replied to the first enquiry and 290 filecards were collected in the second call from the geographical region of our interest. Rostafinski's informants returned nearly 800 records of plants used by traditional societies for medical purposes. The most extensive version consisted of 70 questions concerning about 130 species. It was formulated in greater detail and asked specifically what names people used for cultivated plants, medicinal remedies (curative, ceremonial and magic plants), mushrooms, and timber. The information on the medical use of various plants obtained by Rostafinski showed that traditional homemade remedies had not yet been substituted by chemical medicines prepared by pharmacists at the time of the survey (Köhler, 2015).

Fischer's survey reported 179 plant taxa from the Galicia region. However, only 87 (49\%) of them were used as medicines and targeted predominantly respiratory (24), digestive (24), and skin (23) illnesses. The remaining herbs (53 or $30 \%$ ) were used for blessing during various religious ceremonies (Kujawska et al., 2015). The species that achieved the highest use were yarrow (Achillea millefolium), garlic (Allium sativum), periwinkle (Vinca minor), St. John's wort (Hypericum perforatum), and juniper (Juniperus communis), which was strictly in line with previous observations across various centuries. Two plants, yarrow (Achillea millefolium) and coltsfoot (Tussilago farfara) were also recognized as the most versatile remedies with multiple pharmacological indications.

\section{COMPARATIVE ANALYSIS OF MEDICINAL PLANTS FOR WOUND AND SKIN APPLICATIONS}

To illustrate our assumption that the original Carpathian herbal remedies were either substituted by knowledge gathered from classical medicinal monographs or were identical to them, we attempted a direct comparison of descriptions of herbal medicines used to treat wounds among printed herbals and subsequent traditional folk knowledge in the region. Falimirz's herbal (1534) listed 63 plants that were used for wound healing out of 246 plants shown in Table 3 (25\%). Among the herbal references analyzed, Falimirz's plant list matches $70 \%$ of plants described in the Welsh (Celtic) Physicians of
Myddvai manuscript, even though both works are separated by a hundred years and 2,000 km-suggesting that both authors used similar, if not identical, sources for transcribing these monographs. This striking similarity could be explained in part by the fact that medieval Celtic physicians (Scottish, Irish, and Welsh) often received education at the University of Padua, similar to their Ruthenian and Polish counterparts (Scottish Historical review, 1906). Subsequent handwritten manuscripts and surveys reported both smaller numbers of total medicinal plants, as well as those used for wound healing. Only 3 plant species are recommended to treat wounds across all 6 sources studied, including derevij (Achillea millefolium), babka (Plantago maior) and shalvia (Salvia officinalis), but at least 14 plants are shared among the majority of the sources. Comfrey root (Symphytum officinale) was used on multiple occasions to heal wounds associated with bone fractures, minced in animal fat. The type of fat used in this ointment varied depending on the location: in Silesian Beskids, pork fat was preffered, in Zywiec Beskids, goose lard was believed to possess the best curative properties, while in Sacz Beskids they were both considered as effective. Another treatment that remained largerly uniform among the discussed regions was application of galipot of conifer trees to wound healing ointments after heating it with bee wax and butter.

\section{MODERN PRODUCTION AND USE OF MEDICINAL HERBS IN THE REGION}

Worldwide, around 20,000 plant species are used for their medicinal properties, including 180 herbs that are recognized as medicinal by modern medicine. The annual market need for herbal and botanical raw materials is estimated at 600,000 tons, with sales reaching $\$ 7.5$ billion in the United States alone, and $\$ 60$ billion worlwide. Cultivation of medicinal plants remains a fast growing and sustainable section of agriculture, with profits margins exceeding those of grains and other food crops 2 to 50 -fold due to development of value-addded products and parallel processing streams of raw materials and waste products. Low costs of intitial investment into growing, processing, and packaging medicinal herbs in Eastern Europe $(<\$ 10,000)$ is also a very attractive factor, however it is partially offset by lack of modern cultivation practices and delayed returns due to intial 2 to 3-year establishment of annual crop harvest (Mirzoyeva, 2013). Russia remains the largest producer of medicinal plants in the region, with Poland (cultivated herbs) and Bulgaria (wild collected herbs) in the second place. Ukrainian market of medicinal plants is rather small, with annual exports (averaging 3,000 tons) exceeding imports (averaging 2,000 tons) in most years (Nykytyuk, 2015). Botanical imports are largerly dominated by chamomile (Matricaria chamomilla) and senna (Senna alexandrina), while chestnut (Castanea sativa), buckthorn (Frangula alnus), and milk thistle (Silybum marianum) are leading exports in recent years (Figures 3B,C). This is a drastic difference from 1920s, when the same region was one of the world leaders in growing, processing, and exporting medicinal plants, predominantly for 
TABLE 3 | Medicinal plants listed in Falimirz's Herbal recipes (1534) and their occurrences in manuscripts and regional surveys, including Myddvai (M, 1382), Lowicz (L, 1532), Pushkarev (P, 1550), Swidzinski (S, 1650), Talko-Hrynkiewicz (T, 1893), and Fischer (F, 1934).

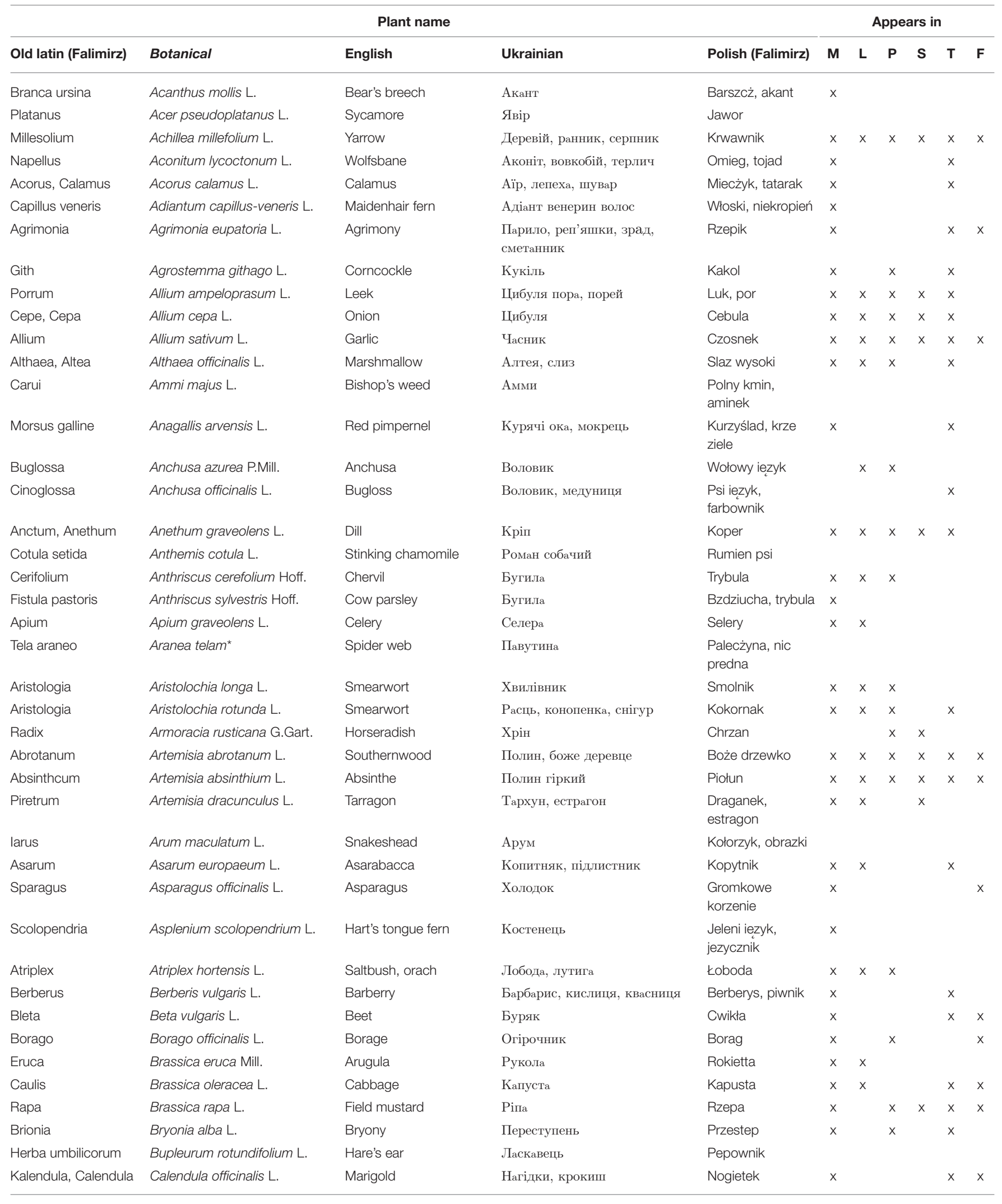


TABLE 3 | Continued

\begin{tabular}{|c|c|c|c|c|c|c|c|c|c|c|}
\hline \multicolumn{5}{|c|}{ Plant name } & \multicolumn{6}{|c|}{ Appears in } \\
\hline Old latin (Falimirz) & Botanical & English & Ukrainian & Polish (Falimirz) & $\mathbf{M}$ & $\mathbf{L}$ & $\mathbf{P}$ & $\mathbf{S}$ & $\mathbf{T}$ & $\mathbf{F}$ \\
\hline Soldanella & Calystegia soldanella R.Br. & Morning glory & Плетуха & Urdzik & & & & & & \\
\hline Canapus & Cannabis sativa L. & Hemp & Конопля & Konoṕ & $x$ & & $x$ & $x$ & $x$ & $x$ \\
\hline Bursa pastoris & Capsella bursa-pastoris Md. & Shepherd's purse & Грицики, зозульник, ріжух & Tobołki, tasznik & $x$ & & & & $x$ & \\
\hline Herba victorialis & Carlina acaulis L. & Carlina & Дев'ятисил, відкласник & Dziewesił & & & & $x$ & $x$ & $x$ \\
\hline Cartamus & Carthamus tinctorius L. & Safflower & Сафлор, шафран & Krokosz & & & & & & $x$ \\
\hline Scabiesa & Centaurea scabiosa L. & Knapweed & Волошка, блевит, сонце & $\begin{array}{l}\text { Dryjak, chaber, } \\
\text { blawatek }\end{array}$ & $x$ & & & & $x$ & \\
\hline Centaurea & Centaurium erythraea Rafn. & Centaury & Золототисячник, цинторія & $\begin{array}{l}\text { Centuria, } \\
\text { tysiacznik }\end{array}$ & $x$ & $x$ & $x$ & & $x$ & $x$ \\
\hline Os de corde cervi & Cervus elaphus* & Red deer bone & Кістка оленя & $\begin{array}{l}\text { Kosć z serca } \\
\text { jeleniego }\end{array}$ & & & & & & \\
\hline Celidonis, Chelidonia & Chelidonium majus L. & Celandine & Чистотіл, гладишник & $\begin{array}{l}\text { Złotnik, glistnik, } \\
\text { jaskolcze }\end{array}$ & $x$ & $x$ & & & $x$ & $x$ \\
\hline Gira solis & Chondrilla juncea L. & Devil's grass & Хондрила ситниковидна & Skocżek & & & & & & \\
\hline Cicorea & Cichorium intybus L. & Chicory & $\begin{array}{l}\text { Цикорій, петрові батоги, } \\
\text { старівник }\end{array}$ & Podrożnik & & & & & $x$ & \\
\hline Kamphora & Cinnamomum camphora J.P. & Camphor laurel & Камфорний лавр & Balsamowiec & $x$ & & & & & \\
\hline Calamentum & Clinopodium nepeta Kuntze & Lesser calamint & Каламінт & Miętka kamienna & $x$ & & & & & \\
\hline Pes corinnus & Clinopodium vulgare L. & Wild basil & Пахучка & Sturzesz, czyscica & & & & & & \\
\hline Hermodactilus & Colchicum autumnale L. & Autumn crocus & Пізньоцвіт, зимовник & Paluchy, zimowit & & & & & & \\
\hline Cicuta & Conium maculatum L. & Hemlock & Болиголов, блекот, булав & $\begin{array}{l}\text { Swinia wesz, } \\
\text { szczwół }\end{array}$ & $x$ & $x$ & & & $x$ & \\
\hline Lilium convalium & Convallaria majalis L. & Lily of the valley & Конвалія & Lanka, konwalia & $x$ & & & & $x$ & \\
\hline Coriandrum & Coriandrum sativum L. & Coriander & Коріандр & $\begin{array}{l}\text { Koriander, } \\
\text { kolendra }\end{array}$ & $x$ & $x$ & & & & $x$ \\
\hline Pallacium leporis & Crepis tectorum L. & Hawksbeard & Скерда & $\begin{array}{l}\text { Pałac zaieczi, } \\
\text { pepawa }\end{array}$ & & & & & & $x$ \\
\hline Melon & Cucumis melo L. & Maskmelon & Диня & Melon & & & $x$ & & $x$ & \\
\hline Cucumer & Cucumis sativus L. & Cucumber & Огірок & Ogurek & $x$ & & $x$ & & $x$ & \\
\hline Cucurbita & Cucurbita pepo L. & Squash & Гарбуз & Bania, dynia & & & & & & $x$ \\
\hline Amcos, Cyminum & Cuminum cyminum L. & Cumin & Кмин & Kmin & $x$ & $x$ & & & $x$ & \\
\hline Cuscuta, Epitimum & Cuscuta epilinum Weihe & Flax dodder & Повитиця, перстенець & $\begin{array}{l}\text { Kania przedza, } \\
\text { kanianka }\end{array}$ & & & & & & \\
\hline Panis porcinus & Cyclamen purpurascens Mil. & Alpine cyclamen & Цикламен & Obraski & $x$ & & & & & \\
\hline Squinantum & Cymbopogon schonanthus S. & Camel grass & Цимбопогон & Palczatka & & & & & & \\
\hline Ciperus, Cyperus & Cyperus longus L. & Galilgale & Смикавець & Cibora & $x$ & $x$ & & & & \\
\hline Laurcola & Daphne mezereum L. & February Daphne & Вовчі ягоди & $\begin{array}{l}\text { Wilcże łyko, } \\
\text { wawrzynek }\end{array}$ & $x$ & & & & $x$ & \\
\hline Pastinaca, Daucus & Daucus carota L. & Carrot & Морква & Marchew & $x$ & & $x$ & & $x$ & $x$ \\
\hline Staphisagria & Delphinium consolida Gray & Larkspur & Дельфіній, сокирки & Ostróżka & $x$ & & & & $x$ & \\
\hline Gariosili & Dianthus caryophyllus L. & Carnation & Гвоздика & Gwozdzika & & & & & & $x$ \\
\hline Filius ante patrem & Dianthus deltoides L. & Maiden pink & Гвоздика & Goździk & & & & & & $x$ \\
\hline Virga pastoris & Dipsacus fullonum L. & Wild teasel & Ворсянка, будяк, чишаки & $\begin{array}{l}\text { Polna szcżeć, } \\
\text { drapacz }\end{array}$ & & & & & $x$ & $x$ \\
\hline Poligonia & Dipsacus sylvestris L. & Fuller's teasel & Ворсянка, будяки & Szcżotki, szczeć & & & & & $x$ & \\
\hline Squilla & Drimia maritima (L.) Stearn & Squill & Дримія & Czebula zamorzka & & & & & & \\
\hline Filix & Dryopteris filix-mas Schott & Fern & Щитник, папороть & Paproć & $x$ & & & & & \\
\hline Cauda equina & Equisetum arvense L. & Horsetail & Хвощ, падиволос & Przesika, skrzyp & & & & & & $x$ \\
\hline Iringus & Eryngium campestre L. & Eryngo & Миколайчики & Mikołaiek & $x$ & & & & $x$ & \\
\hline Eupatorium & Eupatorium cannabinum L. & Hemp-agrimony & Сідач, давник, вовчки & Szadziecz & $x$ & & & & $x$ & $x$ \\
\hline Esula minor & Euphorbia esula L. & Green spurge & Молочай гострий & Sosnka mniejsza & & & & & & \\
\hline Titimallus & Euphorbia helioscopia L. & Sun spurge & Молочай, самозелень & Romanowo ziele & & & & & & \\
\hline
\end{tabular}


TABLE 3 | Continued

\begin{tabular}{|c|c|c|c|c|c|c|c|c|c|c|}
\hline \multicolumn{5}{|c|}{ Plant name } & \multicolumn{6}{|c|}{ Appears in } \\
\hline Old latin (Falimirz) & Botanical & English & Ukrainian & Polish (Falimirz) & $\mathbf{M}$ & $\mathbf{L}$ & $\mathbf{P}$ & $\mathbf{S}$ & $\mathbf{T}$ & $\mathbf{F}$ \\
\hline Catapucia & Euphorbia lathyris L. & Spurge & Молочай & $\begin{array}{l}\text { Skocżek, } \\
\text { ostromlecz }\end{array}$ & $x$ & & & & $x$ & \\
\hline Eufrasia & Euphrasia rostkoviana Hayn & Eyebright & Очанка & Swiecżki & $x$ & & & & & \\
\hline Stercus & Feces $^{\star}$ & Feces & Кал & Layno, vykal & $x$ & & & & & \\
\hline Elitropium & Filipendula ulmaria Maxim. & Meadowsweet & Гадючник & Wiazówka & $x$ & & & & & \\
\hline Filipendula & Filipendula vulgaris Moen. & Dropwort & Лабазник, балабан & $\begin{array}{l}\text { Orzeszki, } \\
\text { wiąówka }\end{array}$ & $x$ & & & & & \\
\hline Feniculus & Foeniculum vulgare Mill. & Fennel & Фенхель & Włoski kopr, fenkuł & $x$ & $x$ & & & & \\
\hline Fragaria & Fragaria vesca L. & Strawberry & Суниці, ягода & Pozimki, poziomka & $x$ & & & & $x$ & \\
\hline Frarimus & Fraxinus excelsior L. & Ash & Ясен & Jesion & $x$ & & & & & $x$ \\
\hline Fumus terre & Fumaria officinalis L. & Fumitory & Рутка, сплавник & Dymnica & $x$ & & & & $x$ & \\
\hline Fungus & Fungi generaliter ${ }^{*}$ & Mushroom & Гриб & Grzyb & $x$ & & & & & \\
\hline Camepiteos & Galium mollugo L. & Hedge bedstraw & Підмаренник, брочник & Przytulia & & & & & & $x$ \\
\hline Genesta & Genista pilosa L. & Broom & Дрік & Janowiec & & & & & $x$ & \\
\hline Genciana & Gentiana punctate L. & Spotted gentian & Тирлич, горичка & Goricżka & & & $x$ & & $x$ & $x$ \\
\hline Pes columbinus & Geranium columbinum L. & Longstalk cranesbill & Герань, кучеряве & Gołebia noga & & & & & $x$ & \\
\hline Gariossilata & Geum urbanum L. & Herb Bennet & $\begin{array}{l}\text { Гравілат, ребник, чистець, } \\
\text { підойма }\end{array}$ & Kuklik & $x$ & & & & $x$ & $x$ \\
\hline Edera terrestris & Glechoma hederacea L. & Ground ivy & Розхідник, собача м'ята & Bluszcz & $x$ & & & & & \\
\hline Liquiricium & Glycyrrhiza glabra L. & Liquorice & Локриця & Lakricija & $x$ & & & & $x$ & \\
\hline Gramen & Gramen generaliter & Hay & Сіно & Trawa & $x$ & & & & & \\
\hline Palma christi & Gymnadenia conopsea R.Br. & Fragrant orchid & Билинець комарниковий & $\begin{array}{l}\text { Dłoń Kristowa, } \\
\text { golka }\end{array}$ & $x$ & & & & & \\
\hline Sticados citrinum & Helichrysum arenarium L. & Dwarf everlast & Цмин, жовтяниця & Koczenki, kocanki & & & & & $x$ & \\
\hline Sticados arabicum & Helichrysum stoechas L. & Shrubby everlast & Цмин арабський & Koczenki z Arabiji & & & & & & \\
\hline Elleborus niger & Helleborus niger L. & Black hellebore & Чемерник & Cżemierzycza & $x$ & $x$ & $x$ & & $x$ & \\
\hline Samsucus & Hibiscus syriacus L. & Hibiscus & Гібіскус & Wielki slaz & & & & & & \\
\hline Auricula, Pilosella & Hieracium pilosella L. & Mousear hawkweed & Нечуйвітер, волосник & $\begin{array}{l}\text { Niedospiałek, } \\
\text { kosmaczek }\end{array}$ & $x$ & & & & $x$ & \\
\hline Luppulus, Velubilis & Humulus lupulus L. & Hops & Хмільь & Chmiel, pawey & $x$ & & & & & \\
\hline lusquiamus & Hyoscyamus niger L. & Golden henbane & Блекота, люлян, немиця & Bielon, lulek & $x$ & $x$ & & & $x$ & \\
\hline Iperico & Hypericum perforatum L. & St John's wort & Звіробій, кровка & $\begin{array}{l}\text { Jana ziele, } \\
\text { dziurawiec }\end{array}$ & $x$ & & $x$ & & $x$ & $x$ \\
\hline Isopus & Hyssopus officinalis L. & Hyssop & Гісоп, юсипок & Izop, hyzop & $x$ & $x$ & & & $x$ & $\mathrm{x}$ \\
\hline Enula campana & Inula helenium L. & Elecampane & Оман, дивосил, галаґан, велике & Oman & $x$ & $x$ & $x$ & & $x$ & $x$ \\
\hline Ircos & Iris germanica L. & Iris & Півники & Kosaciecz & & & & & & \\
\hline Iris illirica & Iris illyrica Tomm. & Illyrian iris & Півники & Fijołkowy korzeń & & & & & $x$ & \\
\hline Gladiollus & Iris pseudacorus L. & Yellow iris & Півники, косатень & Miecżyk & $x$ & & $x$ & & & \\
\hline Sandix & Isatis tinctoria L. & Woad & Вайда & Urzet & & & & & & \\
\hline luniperus & Juniperus communis L. & Juniper & Ялівець & Jałowiecz & $x$ & & $x$ & & $x$ & $x$ \\
\hline Savina, Sabina & Juniperus sabina L. & Savin juniper & Ялівець, женепин & Sawina, jałowiec & $x$ & $x$ & & & $x$ & \\
\hline Lactuca & Lactuca sativa L. & Lettuce & Салат & Sałata & $x$ & $x$ & & & $x$ & \\
\hline Siler montanum & Laserpitium siler L. & Laserwort & Розрив-трава & Czarnogłow & & & & & & \\
\hline Lavendula & Lavandula angustifolia Mill. & Lavender & Лаванда & Lavenda & $x$ & & & & $x$ & \\
\hline Lenticula & Lemna minor L. & Duckweed & Ряска & Rzasa & $x$ & & & & $x$ & \\
\hline Lens & Lens culinaris Medikus & Lentil & Сочевиця & Socżowicza & $x$ & & & & & \\
\hline Nasturtium ortulanum & Lepidium sativum L. & Garden cress & Жеруха, хріниця & $\begin{array}{l}\text { Rzeżucha, } \\
\text { pieprzyca }\end{array}$ & & $x$ & $x$ & & $x$ & \\
\hline Oculus bouis & Leucanthemum vulgare Lam. & Ox-eye daisy & Королиця & Jastrun, zlocen & $x$ & & & & $x$ & \\
\hline Ligusticum, Leuisticus & Levisticum officinale Koch & Lovage & Любисток & Lubczyk & $x$ & $x$ & $x$ & & $x$ & \\
\hline
\end{tabular}


TABLE 3 | Continued

\begin{tabular}{|c|c|c|c|c|c|c|c|c|c|c|}
\hline \multicolumn{5}{|c|}{ Plant name } & \multicolumn{6}{|c|}{ Appears in } \\
\hline Old latin (Falimirz) & Botanical & English & Ukrainian & Polish (Falimirz) & $\mathbf{M}$ & $\mathbf{L}$ & $\mathbf{P}$ & $\mathbf{S}$ & $\mathbf{T}$ & $\mathbf{F}$ \\
\hline Lilium & Lilium candidum L. & Madonna lily & Лілія біла & Lilija & $x$ & $x$ & $x$ & & $x$ & \\
\hline Assodillus, Narciscus & Lilium martagon L. & Martagon Lily & Лілія лісова, маслянка & $\begin{array}{l}\text { Złotogłow, } \\
\text { powojek }\end{array}$ & & & & & & \\
\hline Linaria & Linaria vulgaris Mill. & Toadflax & Льонок, медовики, чистець & $\begin{array}{l}\text { Matki Bożey len, } \\
\text { Inica }\end{array}$ & & & & & $x$ & \\
\hline Grana solis & Lithospermum officinale L. & Gromwell & Горобейник & $\begin{array}{l}\text { Wroble proso, } \\
\text { nawrot }\end{array}$ & $x$ & & & & & $x$ \\
\hline Matrisilva & Lonicera caprifolium L. & Italian honeysuckle & Жимолость, козий листок & Marsylia, powoj & $x$ & & & & $x$ & \\
\hline Spica celtica & Lycopodium clavatum L. & Clubmoss & Плавун, диреч & Widłak, babimur & $x$ & & & & $x$ & $x$ \\
\hline $\begin{array}{l}\text { Kantarides, } \\
\text { Cantharides }\end{array}$ & Lytta vesicatoria $L$. & Spanish fly & Шпанська мушка & Pryszczel & & & & & & \\
\hline Malva & Malva sylvestris L. & Mallow & $\begin{array}{l}\text { Калачики, проскурник, } \\
\text { слизівник }\end{array}$ & Slaz & $x$ & $x$ & $x$ & & $x$ & $x$ \\
\hline Mandragora & Mandragora officinarum L. & Mandrakes & Мандрагора & Pokrzyk & $x$ & & & & & \\
\hline Epatica & Marchantia polymorpha L. & Liverwort & Маршанція (мох) & Watrobnt ziele & $x$ & & & & & \\
\hline Maiorana & Marrubium vulgare L. & Horehound & Шандра & Szanta & $x$ & & & & $x$ & \\
\hline Camomilla & Matricaria chamomilla L. & Chamomile & Ромашка, роман, рум'янок & Rumien, rumianek & $x$ & $x$ & & & $x$ & $x$ \\
\hline Paritaria & Melampyrum pretense L. & Broomrape & Перестріч, братики & $\begin{array}{l}\text { Nocz y dzień, } \\
\text { pszeniec }\end{array}$ & $x$ & & & & $x$ & $x$ \\
\hline Mellilotum & Melilotus officinalis (L.) Pall. & Melilot & Буркун & Nostrzyk, melot & $x$ & & & & $x$ & \\
\hline Mellisa & Melissa officinalis L. & Lemon balm & Меліса, маточник, медовник & $\begin{array}{l}\text { Miodunka, } \\
\text { rojownik }\end{array}$ & $x$ & $x$ & $x$ & & $x$ & \\
\hline Piperita & Mentha balsamea Wild. & Peppermint & М'ята перцева & Pieprzycżka & $x$ & & & & & \\
\hline Menta & Mentha spicata L. & Spearmint & М'ята & Miętka & $x$ & $x$ & $x$ & & $x$ & $x$ \\
\hline Pulegium & Mentha pulegium L. & Pennyroyal & М'ята блошина & Mieta poley & $x$ & $x$ & $x$ & & $x$ & $x$ \\
\hline Mercurialis & Mercurialis annua L. & Mercury & Переліска & Szcżyr & $x$ & & $x$ & & & \\
\hline Spicanardi & Nardostachys jatamansi DC. & Spikenard & Нард & Nard & & & & & & \\
\hline Nasturcium aquaticum & Nasturtium officinale Aiton. & Watercress & Настурція, жеруха & $\begin{array}{l}\text { Wodna rzeżucha, } \\
\text { rukiew }\end{array}$ & $x$ & & $x$ & & $x$ & \\
\hline Nigella & Nigella sativa L. & Black caraway & Чорнушка & $\begin{array}{l}\text { Kakolica, } \\
\text { czarnuszka }\end{array}$ & $x$ & & & & $x$ & $x$ \\
\hline Ungula cababellina & Nuphar lutea (L.) Sm. & Yellow waterlily & Глечики жовті & $\begin{array}{l}\text { Końskie kopyto, } \\
\text { grazel }\end{array}$ & & & & & & \\
\hline Nenufar & Nymphaea alba L. & Water Lily & Латаття & Grzybienie & $x$ & & & & $x$ & \\
\hline Baselicon & Ocimum basilicum L. & Basil & Васильки, базилі́к & Bazylia & $x$ & & & & $x$ & \\
\hline Turbit & Operculina turpethum Mans & Turpeth & Кручені паничі & Wilec & & & & & & \\
\hline Satirion & Orchis morio et mascula L. & Military orchid & Зозулинець & $\begin{array}{l}\text { Lisie jayka, } \\
\text { storczyk }\end{array}$ & $x$ & & & & $x$ & \\
\hline Diptamus & Origanum dictamnus L. & Cretan dittany & Материнка, ясинець & Trzemdała & $x$ & & $x$ & & $x$ & \\
\hline Origanum & Origanum vulgare L. & Oregano & Материнка, душинка & $\begin{array}{l}\text { Lebiodka, } \\
\text { majeranek }\end{array}$ & $x$ & $x$ & $x$ & $x$ & $x$ & $x$ \\
\hline Os mundi & Osmunda regalis L. & Royal fern & Осмунда & $\begin{array}{l}\text { Stnisowe proso, } \\
\text { dlugosz }\end{array}$ & $x$ & & & & & \\
\hline Pionia & Paeonia officinalis L. & Peony & Півонія & Piwonija & $x$ & $x$ & & & $x$ & $x$ \\
\hline Opium, papaver & Papaver somniferum L. & Opium poppy & Мак опійний & Mak lekarski & $x$ & $x$ & $x$ & $x$ & $x$ & \\
\hline Oculus corvi & Paris quadrifolia L. & True lover's knot & Вороняче око, ранник, бешезник & $\begin{array}{l}\text { Wronie oko, } \\
\text { czworolist }\end{array}$ & & & & & $x$ & \\
\hline Pastinaca domestica & Pastinaca sativa L. & Parsnip & Пастернак, Пастярник & Pasternak & $x$ & $x$ & $x$ & & $x$ & \\
\hline Serpentaria & Persicaria bistorta Samp. & Bistort & Гірчак, ракові шийки & Wezownik, rdest & $x$ & & $x$ & $x$ & & $\mathrm{x}$ \\
\hline Persicaria & Persicaria maculosa Gray & Lady's thumb & Гірчак, реплик & Rdest & $x$ & & $x$ & & & \\
\hline Petrosilinum & Petroselinum sativum Fuss & Parsley & Петрушка & Piotruszka & $x$ & & $x$ & $x$ & $x$ & $x$ \\
\hline Paucedamum & Peucedanum officinale L. & Hog's fennel & Смовдь руська & $\begin{array}{l}\text { Wszywy kopr, } \\
\text { gorysz }\end{array}$ & $x$ & & & & & \\
\hline
\end{tabular}


TABLE 3 | Continued

\begin{tabular}{|c|c|c|c|c|c|c|c|c|c|c|}
\hline \multicolumn{5}{|c|}{ Plant name } & \multicolumn{6}{|c|}{ Appears in } \\
\hline Old latin (Falimirz) & Botanical & English & Ukrainian & Polish (Falimirz) & $\mathbf{M}$ & $\mathbf{L}$ & $\mathbf{P}$ & $\mathbf{S}$ & $\mathbf{T}$ & $\mathbf{F}$ \\
\hline Ostrus ostrucium & Peucedanum ostruthium Koch & Masterwort & Смовдь & Gorysz & & $x$ & & & & \\
\hline Meu & Peucedanum palustre Monch & Milk parsley & Горичник & Olesznik, gorysz & & & & & & \\
\hline Alkakenge & Physalis alkekengi L. & Bladder cherry & Фізаліс, сердечник & $\begin{array}{l}\text { Michunki, } \\
\text { zydowska wisznia }\end{array}$ & & & & & $x$ & \\
\hline Pipinella & Pimpinella saxifraga L. & Saxifrage & Бедринець, бедрич & Biedrzeniecz & $x$ & & $x$ & $x$ & $x$ & \\
\hline Pinea & Pinus silvestris L. & Pine & Сосна & Sosna & $\mathrm{x}$ & & & & $x$ & $x$ \\
\hline Pistacca sistica & Pistacia terebinthus L. & Pistacia & Терпентинне дерево & Pistacja, terebint & & & & & & \\
\hline Pes milui & Plantago coronopus L. & Miinutina & Подорожник перистий & Kania noga & $x$ & & & & & \\
\hline Amoglossa, Psilium & Plantago maior L. & Plantain & Бабка, припутник & Babka & $\mathrm{x}$ & $x$ & $x$ & $x$ & $x$ & $\mathrm{x}$ \\
\hline Lingua avis & Polygala vulgaris L. & Tufted milkwort & Китятки & Krzyżownica & & & & & & \\
\hline Lingua passerina & Polygonum aviculare L. & Knotgrass & Спориш & Sporzysz, rdest & & & & & $x$ & \\
\hline Aaron & Polygonum bistorta Samp. & Bistort & Гірчак зміїний, рачки, криве & $\begin{array}{l}\text { Weżownik, rakowe } \\
\text { szyjki }\end{array}$ & & & $x$ & & $x$ & \\
\hline Polipodium & Polypodium vulgare L. & Polypody & БаГатоніжка, слодишка & Paprotka & $\mathrm{x}$ & & & & $x$ & \\
\hline Populus & Populus nigra L. & Black poplar & Тополя & Topola & & & & & $x$ & \\
\hline Portulata & Portulaca oleracea L. & Purslane & Портулак & $\begin{array}{l}\text { Kurza noga, } \\
\text { portulaka }\end{array}$ & $x$ & $x$ & & & $x$ & \\
\hline Consolida, Tormentilla & Potentilla erecta Uspen. & Tormentil, septfoil & Перстач, Калган & $\begin{array}{l}\text { Pieciornik, kurze } \\
\text { ziele }\end{array}$ & $x$ & & $x$ & & $x$ & $x$ \\
\hline Pentassilon & Potentilla reptans L. & Cinquefoil & Перстач & Pięciornik & $\mathrm{x}$ & & & & & \\
\hline Herba paralisis & Primula veris L. & Cowslip & Первоцвіт, баранці & $\begin{array}{l}\text { Paraliżowe, } \\
\text { pierwiosnek }\end{array}$ & $x$ & & & & $x$ & \\
\hline Simphicum & Prunella vulgaris L. & Self-heal & Суховершки & Sylfion, glowienka & $\mathrm{x}$ & & & & $x$ & \\
\hline Persicus & Prunus persica (L.) Batsch & Peach & Персик & Brzoskiwia & $\mathrm{x}$ & & & & & $x$ \\
\hline Pulicari & Pulicaria vulgaris Gaertn. & Fleabane & Блошниця & Płesznik & $x$ & & & & & \\
\hline Pirula & Pyrola minor L. & Lesser wintergreen & Грушанка & Gruszyczka & $x$ & & & & & \\
\hline Lilialis & Pyrola rotundifolia L. & Wintergreen & Грушанка & Gruszyczka & & & & & $x$ & \\
\hline Galla & Galla* & Oak (galls) & Чорнильні горішки & Gallas & $x$ & & $x$ & & & \\
\hline Quercus & Quercus robur L. & Oak & Дуб & Dab & $x$ & & $x$ & & $x$ & \\
\hline Flammula & Ranunculus flammula L. & Spearwort & Жовтець & Jaskier & & & & & $x$ & \\
\hline Raffanus & Raphanus raphanistrum L. & Radish & Редька & Rzodkiew & $\mathrm{x}$ & & $x$ & & $x$ & \\
\hline Rosa & Rosa canina L. & Rose & Шипшина, свербивус & Roża & $x$ & $x$ & $x$ & $x$ & $x$ & \\
\hline Rozmarinus & Rosmarinus officinalis L. & Rosemary & Розмарин & Rozmarin & $x$ & & & & $x$ & \\
\hline Rubea tinctorum & Rubia tinctorum L. & Madder & Марена & Marzana & $x$ & & & & $x$ & \\
\hline Rubus & Rubus plicatus Weihe & Blackberry & Ожина & Jeżyny & $\mathrm{x}$ & & & & $x$ & \\
\hline Ribes & Rubus idaeus L. & Raspberry & Малина & Maliny & $x$ & & & & $x$ & \\
\hline Acetoja, Lapathum & Rumex acetosa L. & Sorrel, dock & Щавель & Szcżaw & $x$ & $x$ & $x$ & & $x$ & $x$ \\
\hline Lappacium acutum & Rumex confertus Wild. & Bitter dock & Щавель кінський & Kobyliszcżaw & $x$ & & $x$ & & & \\
\hline Ruta & Ruta graveolens L. & Rue & Рута & Ruta & $x$ & $x$ & $x$ & & $x$ & $x$ \\
\hline Salix & Salix alba L. & Willow & Верба & Wirzba & $\mathrm{x}$ & & $x$ & $x$ & $x$ & \\
\hline Ambrosiana & Salvia viridisL. & Annual clary & Шавлія & Szałwia & $x$ & $x$ & & & & \\
\hline Salvia & Salvia officinalis L. & Sage & Шавлія & Szałwija & $x$ & $x$ & $x$ & $x$ & $x$ & $x$ \\
\hline Galletricum & Salvia sclarea L. & Clary sage & Шавлія мускатна & $\begin{array}{l}\text { Szałwia } \\
\text { muszkatołowa }\end{array}$ & $\mathrm{x}$ & & & & & \\
\hline Sambucus & Sambucus ebulus L. & Elderberry & Бузина, хобза & $\mathrm{Bez}$ & $x$ & & $x$ & & $x$ & \\
\hline Ebulus & Sambucus nigra L. & Elderberry & Бузина & Chebd, bez czarny & $x$ & & & & & $x$ \\
\hline Sapo & Saponaria officinalis L. & Soapwart & Мильнянка & Mydło, mydlnica & & & & & $x$ & \\
\hline Saturegia & Satureja hortensis L. & Savory & Чабер & Czabr & $x$ & & & & $x$ & \\
\hline Costus & Saussurea costus Lipsch. & Costus & Сосюрея & Saussurea & & $x$ & & & & \\
\hline Saxifraga & Saxifraga granulata L. & Saxifrage & Ломикамінь & $\begin{array}{l}\text { Lamikamień, } \\
\text { skalnica }\end{array}$ & $x$ & & $x$ & & & \\
\hline
\end{tabular}


TABLE 3 | Continued

\begin{tabular}{|c|c|c|c|c|c|c|c|c|c|c|}
\hline \multicolumn{5}{|c|}{ Plant name } & \multicolumn{6}{|c|}{ Appears in } \\
\hline Old latin (Falimirz) & Botanical & English & Ukrainian & Polish (Falimirz) & $\mathbf{M}$ & $\mathbf{L}$ & $\mathbf{P}$ & $\mathbf{S}$ & $\mathbf{T}$ & $\mathbf{F}$ \\
\hline Febrifuga & Scrophularia nodosa L. & Figwort & Ранник, старовина & Trędownik & $x$ & & & & $x$ & \\
\hline Pellicinus & Securigera varia Lassen & Crownvetch & В'язіль & Wilcży groch & & & & & & \\
\hline Semperviva & Sedum acre L. & Stonecrop & Очиток, оливник & Roschodnik & $x$ & & $x$ & & $x$ & $x$ \\
\hline Senecion & Senecio vulgaris L. & Groundsel & Жовтозілля, дідик & Przymiot, starzec & $x$ & & & & $x$ & \\
\hline Herba sulonum & Serratula tinctoria L. & Saw-wort & Серпуха & $\begin{array}{l}\text { Jeleni trunk, } \\
\text { sierpik }\end{array}$ & & & $x$ & & & \\
\hline Sinapis & Sinapis alba L. & Mustard & Гірчиця & Gorcżycza & $x$ & $x$ & $x$ & & & $x$ \\
\hline Olus & Smyrnium olusatrum L. & Alexanders & Смирня & Gir, przewłoka & $x$ & & & & & \\
\hline Perfeliata & Smyrnium perfoliatum L. & Perfoliate Alexanders & Смирня & Pepkowe ziele & $x$ & & & & & \\
\hline Solatrum & Solanum nigrum L. & Black nightshade & Паслін, натинник & Psianki & $x$ & & & & & \\
\hline Virga aurea & Solidago virgaurea L. & Goldenrod & Золотушник & $\begin{array}{l}\text { Weżowe ziele, } \\
\text { nawloc }\end{array}$ & $x$ & & & & & \\
\hline Endivia & Sonchus oleraceus L. & Sowthistle & Жовтий осот & Mlecż & $x$ & & $x$ & & & \\
\hline Spinachia & Spinacia oleracea L. & Spinach & Шпинат & Szpinak & & & & & & \\
\hline Spongia marina & Spongia officinalis L. & Bath sponge & Губка & Morzkie bdły & & & & & & \\
\hline Betonica & Stachys officinalis Trevis. & Betony & Буквиця, чистець & Bukwica & $x$ & $x$ & $x$ & & $x$ & $\mathrm{x}$ \\
\hline Premorsa & Succisa pratensis Moench & Devil's bit & Комонник & $\begin{array}{l}\text { Cartowo ziobro, } \\
\text { komonica }\end{array}$ & $x$ & & & & & \\
\hline Consolida maior minor & Symphytum officinale L. & Comfrey & Живокіст, гавйиз & $\begin{array}{l}\text { Kostywał, } \\
\text { zywokost }\end{array}$ & $x$ & & & & $x$ & $x$ \\
\hline Tenacetum & Tanacetum vulgare $\mathrm{L}$. & Tansy & Пижмо & Wrotycż & $x$ & & & & $x$ & $x$ \\
\hline Rostrum porcinum & Taraxacum officinale L. & Dandelion & Кульбаба & Pepawa, mniszek & $x$ & & & & $x$ & \\
\hline Edera arborea & Taxus baccata L. & English yew & Тис, негній-дерево & Cis & $x$ & & & & $x$ & \\
\hline Serpillum & Thymus serpyllum L. & Thyme & Чебрець, материнка & Macierza duszka & $x$ & & $x$ & & $x$ & $\mathrm{x}$ \\
\hline Timus & Thymus vulgaris L. & Thyme & Материнка & $\begin{array}{l}\text { Dziecielina, } \\
\text { macierzanka }\end{array}$ & $x$ & & & & & \\
\hline Tribulus & Tribulus terrestris L. & Goats-head & Якірці & $\begin{array}{l}\text { Oseth, } \\
\text { buzdyganek }\end{array}$ & & & & & & \\
\hline Trifolium & Trifolium pretense L. & Red clover & Конюшина, троян & Konik, chwast & $x$ & & & & $x$ & $x$ \\
\hline Matricaria & Triplerospermum inodorum L. & False mayweed & Триреберник & Maruna & & & & & & \\
\hline Spatula fetida & Typha latifolia L. & Cattail & Рогіз & Pałki & $x$ & & & & & \\
\hline Urtica & Urtica dioica L. & Nettle & Кропива & Pokrzywa & $x$ & $x$ & $x$ & $x$ & $x$ & $x$ \\
\hline Usnea & Usnea barbata L. & Olds man beard & Уснея (лишайник) & Mech, porost & & & & & & \\
\hline Moracelsi & Vaccinium oxycoccos L. & Cranberry & Журавлина & Zorawiny & & & & & $x$ & \\
\hline Valeriana & Valeriana officinalis L. & Valerian & Валер'яна, оделен & Kozłek & $x$ & & & & $x$ & $x$ \\
\hline Elleborus albus & Veratrum album L. & White hellebore & Чемериця & Czemierzycza & $x$ & $x$ & & & $x$ & $x$ \\
\hline Tapsus barbatus & Verbascum thapsus L. & Mullein & Дивина & Dziewanna & $x$ & & $x$ & & $x$ & $x$ \\
\hline Verbena & Verbena officinalis L. & Vervain & Вербена & $\begin{array}{l}\text { Koszyszcżko, } \\
\text { werbena }\end{array}$ & $x$ & $x$ & $x$ & & $x$ & \\
\hline Camedreos & Veronica chamaedrys L. & Speedwell & Вероніка & Przetacznik & $x$ & & & & $x$ & \\
\hline Viole & Viola odorata L. & Violet & Фіалка & Fijołki & $x$ & $x$ & $x$ & $x$ & $x$ & $x$ \\
\hline Viscus & Viscum album L. & Mistletoe & Омела & Jemioła & $x$ & & $x$ & & & \\
\hline Agnus castus & Vitex agnus-castus L. & Chaste tree & Витекс & Włoska wirzba & $x$ & & & & & \\
\hline Passula (uva) & Vitis vinifera L. & Raisins & Родзинки & $\begin{array}{l}\text { Greckie wino, } \\
\text { rozynka }\end{array}$ & $x$ & & & & & \\
\hline
\end{tabular}

${ }^{*}$ Natural substances of non-botanical origin.

German markets (Onipko, 2010). A current well-demonstrated, long-term trend toward natural medicine and consumers is indicative of increasing interest in botanical products that support whole-body health rather than focus on a specific health condition, representing a key opportunity to adequately tap the potential of agriculture- and forest-grown medicinal and aromatic plants of the Carpathian Mountains and their foreland. 


\section{CONCLUSIONS}

The first printed herbals published in the Carpathian forelands were early compilations of transcribed Greek and Roman works on the subject, and set a continuous trend of ethnic use of local botanical remedies according to their Dioscorides and Galenic qualities. The Padua-Krakow axis was identified as a key putative factor in introduction of this knowledge to Ruthenian and Polish medical students during the fifteenthsixteenth centuries. Subsequently, this tradition was incorporated into oral and written legacy of Boyko, Lemko, and Gorale ethnic groups, and fused with Slavic use of charm and prayer heals to treat diseases and cast magical incantations. However, the traditional use of several endemic plant species in the region could provide an exciting opportunity to observe preChristian folk healing traditions from the Beskid Mountains. The study also highlighted the vast potential for growing and processing medicinal plants in the region, and cited low

\section{REFERENCES}

Boltarovych, Z. (1980). Narodne Likuvannya Ukrajintsiv Karpat Kintsya XIXpochatku XX st. Kyiv: Naukova dumka.

Cruse, J. M. (1999). History of medicine: the metamorphosis of scientific medicine in the ever-present past. Am. J. Med. Sci. 318, 171-180. doi: 10.1016/S0002-9629(15)40609-3

Elbanowski, A. (2014). Obraz Nowego Swiata w staropolskiej literaturze botanicznej i przyrodniczo-lekarskiej. Acta Bot. Silesiaca 10, 207-234.

Falkowski, J., and Pasznycki, B. (1935). Na Pograniczu Lemkowsko-Bojkowskiem. Lviv: Towarzystwo ludoznawcze.

Flood, B. P. (1976). The medieval herbal tradition of Macer Floridus. Pharm. Hist. $18,62-66$.

Franko, I. (1898). "Lyudovi viruvannya na Pidgiryu," in Etnografichnyj zbirnyk, ed I. Franko (Lviv: NTS), 160-218.

Geller, E. (2009). A new portrait of early seventeenth century Polish jewry in an unlnown Eastern yiddish remedy book. Eur. Jud. J. New Eur. 42, 62-79. doi: $10.3167 /$ ej.2009.420208

Grmek, M. (1959). Ancient Slavic medicine. J. Hist. Med. Allied Sci. 14, 18-40. doi: 10.1093/jhmas/XIV.1.18

Halaga, O. (1961). Hospodarsko-Spolocensky vyvoj Sarisa a Spisa ako Pohranicia v 12-13 Storoci. Kosice: Nove obzory.

Hoshko, Y. (1983). Boykivschyna Istoryko-Etnografichne Doslidzhennya. Kyiv: Naukova dumka.

Ippolitova, A. (2008). Russkije Rukopisnyje Travniki XVII-XVIII Vekov. Moscow: Indrik.

Köhler, P. (2015). Jozef Rostafinski's ethnobotanical enquiry of 1883 concerning Polish vernacular names and uses of Plants. Arch. Nat. Hist. 42, 140-152. doi: 10.3366/anh.2015.0286

Kolberg, O. (1888). Pokucie: Obraz Etnograficzny. Krakow: Nakladem autora.

Komedar, V. (1971). Likarski Roslyny Karpart. Uzhgorod: Karpaty.

Konischuk, V., Bobryk, I., Bulgakov, V., and Skakalska, O. (2016). Osoblyvosti zberezhennya likarskych roslyn Ukrajiny. Agroecol. J. 2, 79-84.

Krylov, Y. (1985). Udivitelnyj Mir Lekarstv. Moscow: Znanie.

Kujawska, M., Łuczaj, Ł., and Typek, J. (2015). Fischer's Lexicon of Slavic beliefs and customs: a previously unknown contribution to the ethnobotany of Ukraine and Poland. J. Ethnobiol. Ethnomed. 11, 85. doi: 10.1186/s13002-015-0073-8

Kuziw, I. (1889). Zhyttya-Buttya, Zvychai i Obychai girskogo Narodu. Zorya.

Kyrchiv, R. (1978). Etnografichne Doslidzhennya Boykivschyny. Kyiv: Naukova dumka.

Lepkij, D. (1884). Pro narodni Zabobony. Zorya. 5, 105-124. awareness among farming communities, inadequate processing capacities, price risks, and non-availability of planting material as critical constraints that need to be addressed.

\section{AUTHOR CONTRIBUTIONS}

WK drafted ethnobiology sections of the manuscript. CW drafted and contributed knowledge on classical and Celtic medicines. EM drafted and contributed knowledge on skin and wound healing applications. AM and SK conceived, designed, and wrote the manuscript. All authors read and approved the manuscript.

\section{ACKNOWLEDGMENTS}

This work was supported in part by NCSU faculty start-up funds (SK), PHHI seed grant 2015-2017 (SK), and UGPN Research Collaboration funds 2015 and 2016 (SK).

Lisowski, W. (2007). Doktor Jan Stanko. Skalpel 2, 28-31.

Magowska, A. (2004). History of Polish pharmacopoeias. Poznan: Nakladem autora. Mirzoyeva, T. (2013). Perspektyvy rozvytku likarskogo roslynnyctva. NVNUB 181, 176-181.

Nimchuk, V. (1976). Mova ukrajinskogo travnyka XVI stolittya. Movoznavstvo 5, 43-55.

Nosal, M., and Nosal, I. (1962). Likarski Roslyny i Sposoby yikh Zastosuvannia v Narodi. Kyiv: Zdorovya.

Novoselski, A., and Pushkarev, L. (1977). Drevneruskij Lechebnik, Redkije Istochniki po Istorii Rossii. Moscow: AN USSR.

Nykytyuk, Y. (2015). Rozvytok zovnishnoi torgivli likaskoyu roslynnoyu syrovynoyu. NVUNU 4, 62-66.

Otchet (1915). Otchet ob Sostojanii Narodnogo Zdravija i Organizacii Vrachebnoj Pomoschi v Rosii. Sankt-Petersburg: Trud.

Onipko, T. (2010). Vyrobnychi Galuzi Spozhyvchoi Kooperatsiji Ukrajiny $v$ Konteksti Novoi Ekonomichnoi Polityky (1921-1928). Poltava: Poltava Economic University.

Ostling, M. (2014). Witches' Herbs on Trial. Folklore 125, 179-201.

Papee, F. (1891). Skole $i$ Tucholszczyzna: Monografia Historyczna. Lwow: Gubrynowicz i Schmidt.

Peredrijenko, V. (1984). Likarski ta Hospodarski Porandyky XVIII st. Kyiv: Naukova dumka.

Petrova, A. (2005). Current State of Bulgarian Biodiversity-Problems and Perspectives. Sofia: Bulgarian Bioplatform.

Prioreschi, P. (2000). Alternative medicine in ancient and medieval history. Med. Hypotheses 55, 319-325. doi: 10.1054/mehy. 2000.1061

Prioreschi, P. (2003). A History of medicine: V. Medieval Medicine. Omaha: Horatius Press.

Rostafinski, J. (1888). Pamietnik. Polska Akademia Umiejetności Wydział.

Rostanski, K. (1997). Szymon Syreniusz i jego dzielo. Wiadomosci Bot. 41, 7-12.

Schneider, J. (1912). Z zycia gorali nadlomnickich. Lud Lviv 18, 176-193.

Singer, C. (1927). The Herbal in Antiquity and its Transmission to later Ages. J. Hell. Stud. 47, 1-52. doi: 10.2307/625251

Stavrovsky, E. (1967). Slovensko-Polsko-Ukrajinske Pohranicie do 18 Storocia. Bratislava: Slovenske pedagogicke nakladatelstvo.

Syrku, P. (1883). Otryvok malorusskogo prostonarodnogo lechebnogo travnika i dva zagovora. Filol. Zap. 22, 1-12.

Szuchewycz, W. (1908). Huculszczyzna. Lviv: NTS.

Talko-Hryncewicz, J. (1893). Zarys lecznictwa ludowego na Rusi Poludniowej. Krakow: Academia Umiejetnosci. 
Theodorescu, R. (1989). The beginnings of higher education in Eastern Europe: paduan internationalism. High. Educ. Eur. 14, 54-58. doi: 10.1080/037977289 0140108

Tovstuha, Y. (1990). Fitoterapiya. Kyiv: Zdorovya.

Tylkowa, D. (1989). Medycyna ludowa w Kulturze wsi Karpat Polskich: Tradycja $i$ Współczesność. Wroclaw: Zaklad Narodowy Ossolinskich.

Wagner, C. S., De Gezelle, J., Robertson, M., Robertson, K., Wilson, M., and Komarnytsky, S. (2017). Antibacterial activity of medicinal plants from The Physicians of Myddvai, a 14th century Welsh medical manuscript. J. Ethnopharmacol. 203, 171-181. doi: 10.1016/j.jep.2017.03.039

Zmejev, L. (1895). Russkie vrachebniki. Saint Peterburg: Pammiatniki Drevniej Pismennosti.
Conflict of Interest Statement: The authors declare that the research was conducted in the absence of any commercial or financial relationships that could be construed as a potential conflict of interest.

Copyright (c) 2018 Kozlowska, Wagner, Moore, Matkowski and Komarnytsky. This is an open-access article distributed under the terms of the Creative Commons Attribution License (CC BY). The use, distribution or reproduction in other forums is permitted, provided the original author(s) and the copyright owner are credited and that the original publication in this journal is cited, in accordance with accepted academic practice. No use, distribution or reproduction is permitted which does not comply with these terms. 


\section{OPEN ACCESS}

Edited by: Judit Hohmann,

University of Szeged, Hungary

Reviewed by:

Didem Sohretoglu,

Hacettepe University, Turkey

Gabor Vasas,

University of Debrecen, Hungary

*Correspondence:

Ali Özmen

aozmen@adu.edu.tr

Liselotte Krenn

liselotte.krenn@univie.ac.at

Specialty section:

This article was submitted to

Ethnopharmacology,

a section of the journal

Frontiers in Pharmacology

Received: 04 October 2017

Accepted: 15 March 2018

Published: 03 April 2018

Citation:

Lewenhofer V, Schweighofer L, Ledermüller $T$, Eichsteininger $J$, Kählig $\mathrm{H}$, Zehl $\mathrm{M}$, Nguyen $\mathrm{CH}$, Krupitza G, Özmen A and Krenn L

(2018) Chemical Composition of Scrophularia lucida and the Effects on Tumor Invasiveness in Vitro.

Front. Pharmacol. 9:304 doi: 10.3389/fphar.2018.00304

\section{Chemical Composition of Scrophularia lucida and the Effects on Tumor Invasiveness in Vitro}

\author{
Verena Lewenhofer ${ }^{1}$, Lisa Schweighofer ${ }^{1}$, Tobias Ledermüller ${ }^{1}$, Julia Eichsteininger ${ }^{1,2}$, \\ Hanspeter Kählig ${ }^{3}$, Martin Zehl1,4, Chi H. Nguyen ${ }^{2,5,6}$, Georg Krupitza ${ }^{2}$, Ali Özmen ${ }^{7 *}$ and \\ Liselotte Krenn ${ }^{1 *}$
}

\begin{abstract}
${ }^{1}$ Department of Pharmacognosy, Faculty of Life Sciences, University of Vienna, Vienna, Austria, ${ }^{2}$ Clinical Institute of Pathology, Medical University of Vienna, Vienna, Austria, ${ }^{3}$ Department of Organic Chemistry, Faculty of Chemistry, University of Vienna, Vienna, Austria, ${ }^{4}$ Department of Analytical Chemistry, Faculty of Chemistry, University of Vienna, Vienna, Austria, ${ }^{5}$ Department of Clinical Pharmacy and Diagnostics, Faculty of Life Sciences, University of Vienna, Vienna, Austria, ${ }^{6}$ Department of Medicine I and Comprehensive Cancer Center, Medical University of Vienna, Vienna, Austria,

${ }^{7}$ Department of Biology, Faculty of Science and Art, Adnan Menderes University, Aydin, Turkey
\end{abstract}

A detannified methanolic extract of Scrophularia lucida L. attenuated the formation of cancer cell-induced circular chemorepellent induced defects (CCIDs) in the lymph endothelial cell barrier, which resemble entry ports for the intravasating tumor into the vasculature as a prerequisite for lymph node metastasis. Therefore, the composition of this extract was studied in an activity-guided approach. Since no data on the secondary metabolites of this plant were available, first phytochemical data were collected in the course of the fractionation of the extract. The study resulted in the identification of 14 substances, among them very rare iridoids, such as scrovalentinoside or koelzioside, and several flavonoids (e.g., nepitrin and homoplantaginin). One of the latter group, 2 "-O-acetyl-homoplantaginin, is a new natural compound. In the most active fraction, the flavonoid hispidulin was identified as major component and the assay of the pure compound confirmed a contribution of hispidulin to the CCID-inhibitory effects of $S$. lucida. The activity of the two major iridoids in this assay was less compared to hispidulin.

\footnotetext{
Keywords: Scrophularia lucida L., iridoids, phenolic compounds, 2"-O-acetyl-homoplantaginin, hispidulin, intravasation, circular chemorepellent-induced defects (CCID) assay
}

\section{INTRODUCTION}

Species of the genus Scrophularia have been applied for centuries in traditional medicine. The most important one among those, Scrophularia ningpoensis Hemsl., is widely used in traditional Chinese medicine for a broad spectrum of inflammatory diseases (De Santos Galíndez et al., 2002; Tong et al., 2006; Li et al., 2009) and listed in the Chinese Pharmacopeia against febrile diseases with excessive thirst or eruptions, constipation, cough due to exhaustion, conjunctivits, skin disorders, and others (Stöger, 2017). The use in the 
treatment of inflammatory disorders, wound healing, skin ailments, and health disturbances has been reported for numerous species such as Scrophularia auriculata L. or Scrophularia canina L. from the Mediterranean area (Giner et al., 2000; Guarrera and Lucia, 2007; Passalacqua et al., 2007) as well as for Scrophularia variegata M. Bieb. and Scrophularia striata Boiss. in traditional Iranian medicine (Azadmehr et al., 2013, 2015). A traditional use as an antipyretic and anti-inflammatory agent was described for Scrophularia oldhamii Oliv. and as a diuretic for Scrophularia grossheimii Schischkin (De Santos Galíndez et al., 2002). In Turkish traditional medicine, the species Scrophularia nodosa L. is well-known as a diuretic, to treat hemorrhoids, and also for wound healing, eruptive skin diseases, eczema, psoriasis, and pruritus (Baytop, 1999; Stevenson et al., 2002; Crisan et al., 2009). A use in these indications is also conveyed for the Turkish species Scrophularia depauperata Boiss., Scrophularia cryptophila Boiss. et. Heldr. Boiss., and Scrophularia floribunda Boiss. et. Bal. (Ozbilgin et al., 2014).

Recently, the activity of various extracts from several Scrophularia species has been studied in different cancer models: weak cytotoxic activity of an ethanolic extract from S. striata via induction of apoptosis and $\mathrm{G}_{2} / \mathrm{M}$ phase arrest was shown in Jurkat human leukemia cells (Azadmehr et al., 2013). In WEHI 164 fibrosarcoma cells, a methanolic extract of this plant exhibited a significant and dose-dependent reduction of the activity of matrix metalloproteinases (Hajiaghaee et al., 2007), which play a role in tissue remodeling and tumor invasion. An apolar fraction from a methanolic extract of Scrophularia orientalis L. significantly reduced the viability of neuroblastoma cells due to a loss of mitochondrial membrane integrity (Lange et al., 2016). In MCF-7 breast cancer cells, weak cytotoxicity of an ethanolic extract from $S$. variegata was reported (Azadmehr et al., 2015). A dichloromethane extract from Scrophularia oxysepala caused significant cytotoxic and apoptotic effects in MCF-7 cells by influencing the expression of p53, caspase 3, and c-myc, and the cleavage of PARP (Valiyari et al., 2013). The anti-tumor activity of $S$. oxysepala in breast cancer was confirmed in vivo in an allograft model in mice inoculated with mammary carcinoma $4 \mathrm{~T} 1$ cells. Animals treated with 50 or $100 \mathrm{mg} / \mathrm{kg}$ of an ethanolic extract developed significantly smaller tumors in size and weight (Baradaran et al., 2017). In a study of five Scrophularia species, S. floribunda and Scrophularia lucida L. displayed the highest cytotoxic activity against HL-60 promyeloic leukemia cells. These extracts were also tested in a three-dimensional co-culture cell model consisting of MCF-7 breast cancer spheroids placed on top of telomerase-immortalized human lymph endothelial cell (LEC) monolayers. There, tumor spheroids cause the formation of large cell-free areas in the lymph endothelial barrier, so-called "circular chemorepellent induced defects" (CCIDs), through which tumor cells penetrate the vasculature. This assay mimics intravasation which is an early step within the metastatic cascade resembling the pathological situation in rodents and humans (Madlener et al., 2010; Vonach et al., 2011; Viola et al., 2013). A detannified methanolic extract of $S$. lucida L. was the most active one in suppressing CCID formation (Giessrigl et al., 2012).

The lack of any information about the chemical composition of $S$. lucida prompted us to perform an activity-guided search for the active principle of this species contributing to the inhibitory effect in the CCID assay.

\section{EXPERIMENTAL}

\section{General Experimental Procedures}

Hispidulin was obtained from Sigma-Aldrich. All other flavonoids for dereplication were on stock at the Department of Pharmacognosy, University of Vienna.

For TLC, silica gel plates or HPTLC plates RP-8 (Merck, Germany) and the mobile phases (1) $\mathrm{CHCl}_{3}-\mathrm{CH}_{3} \mathrm{COOH}_{\text {conc.- }}$ $\mathrm{MeOH}-\mathrm{H}_{2} \mathrm{O} \quad(60: 32: 12: 18)$, (2) EtOAc-HCOOH conc. $-\mathrm{CH}_{3}$ $\mathrm{COOH}_{\text {conc }} \cdot-\mathrm{H}_{2} \mathrm{O} \quad(100: 11: 11: 20)$, (3) EtOAc-MeOH$\mathrm{HCOOH}_{\text {conc. }}-\mathrm{H}_{2} \mathrm{O}$ (75:15:4:4), or (4) $\mathrm{MeOH}-\mathrm{H}_{2} \mathrm{O}$ (90:10) were used.

A Shimadzu LC-20AD pump, an SPD M20A diode array detector, a CTO-20AC column oven, and a SIL 20AC HT auto-sampler (Kyoto, Japan) served for analytical HPLC on a Luna $5 \mu \mathrm{C}-18(250 \mathrm{~mm} \times 4.6 \mathrm{~mm})$ column (Phenomenex, United States) at $25^{\circ} \mathrm{C}$ and a flow rate of $1 \mathrm{~mL} / \mathrm{min}$. Mobile phase 1 consisted of (A) aqueous formic acid ( $\mathrm{pH} \mathrm{3)}$ and (B) acetonitrile-aqueous formic acid $(\mathrm{pH} 3 ; 80+2)$ and the gradient program 1 was $5 \%$ B (0 min), 20\% B (15 min), 20\% B (25 min), $50 \% \mathrm{~B}(65 \mathrm{~min})$, and $95 \% \mathrm{~B}(80 \mathrm{~min})$. Mobile phase 2 was (A) aqueous formic acid ( $\mathrm{pH} 3$ ) and (B) acetonitrile and the gradient 2 increased from $5 \% \mathrm{~B}(0 \mathrm{~min})$ to $95 \% \mathrm{~B}(60 \mathrm{~min})$. UV data were recorded from 190 to $400 \mathrm{~nm}$. All solvents of analytical or HPLC-grade (acetonitrile, Chromanorm; methanol, LiChrosolv) were obtained from VWR International (West Chester, PA, United States) and $\mathrm{CH}_{3} \mathrm{COOH}_{\text {conc }}$ (Rotichrom) from Carl Roth (Karlsruhe, Germany).

NMR spectra were recorded on an Avance III $600 \mathrm{MHz}$ NMR spectrometer (Bruker BioSpin, Germany) using a $5 \mathrm{~mm}$ broadband observe probe (BBFO Smart probe) with $z$-axis gradients and automatic tuning and matching accessory; resonance frequency for ${ }^{1} \mathrm{H}$ NMR: $600.13 \mathrm{MHz}$, for ${ }^{13} \mathrm{C} \mathrm{NMR}$ : $150.90 \mathrm{MHz}$. The measurements were performed for solutions in fully deuterated DMSO or methanol at 298 K. Standard $1 \mathrm{D}$ and 2D experiments like double quantum filtered COSY, TOCSY, NOESY, HSQC, and HMBC were used as supplied by the manufacturer. Chemical shifts were referenced internally to the residual, non-deuterated solvent signal for ${ }^{1} \mathrm{H}(\delta=2.50 \mathrm{ppm}$ for DMSO, $\delta=3.31 \mathrm{ppm}$ for methanol) or to the carbon signal of the solvent for ${ }^{13} \mathrm{C}(\delta=39.52$ for DMSO, $\delta=49.00 \mathrm{ppm}$ for methanol). The analysis of the ${ }^{1} \mathrm{H}$ NMR multiplett structures was assisted by spin simulations using DAISY within the Topspin Software (Bruker BioSpin).

LC-MS analyses were carried out on an UltiMate 3000 RSLCseries system (Dionex, Germering, Germany) coupled to a 3D quadrupole ion trap mass spectrometer via an orthogonal ESI source (HCT, Bruker Daltonics, Bremen, Germany). The HPLC parameters were as described above. The eluate flow was split 1:4 before entering the ESI ion source, which was operated as follows: capillary voltage: $+3.5 /-3.7 \mathrm{kV}$, nebulizer: 26 psi $\left(\mathrm{N}_{2}\right)$, dry gas flow: $9 \mathrm{~L} / \mathrm{min}\left(\mathrm{N}_{2}\right)$, and dry temperature: $340^{\circ} \mathrm{C}$. Positive and negative ion mode multistage mass spectra up to $\mathrm{MS}^{4}$ were 
obtained in automated data-dependent acquisition mode using helium as collision gas, an isolation window of $\Delta m / z=4$, and a fragmentation amplitude of $1.0 \mathrm{~V}$. In addition, compounds 13 and 14 were measured on the HCT instrument by direct infusion after addition of ammonium acetate as additive to generate $\left[\mathrm{M}+\mathrm{NH}_{4}\right]^{+}$ions. HRESIMS spectra of compound 3 were recorded on a maXis HD ESI-Qq-TOF mass spectrometer (Bruker Daltonics) by direct infusion. The sum formulas of the detected ions were determined using Bruker Compass DataAnalysis 4.2 based on the mass accuracy $(\Delta m / z \leq 2 \mathrm{ppm})$ and isotopic pattern matching (SmartFormula algorithm).

\section{Plant Material}

Aerial parts of S. lucida were collected on April 20th 2012, along the Mediterranean coast east of Dalyan and south of Ortaca (latitude: 36.808838, longitude: 28.735583). The material was authenticated by Dr. Özkan Eren, Department of Biology, Adnan Menderes University, using the serial "Flora of Turkey and the East Aegean Islands" (Davis, 1968-1985). Voucher specimen (Herbarium code: AYDN No.: 2601) in duplicates was deposited in the herbarium of Department of Biology, Adnan Menderes University.

\section{Extraction and Isolation}

The freeze-dried and pulverized material of S. lucida $(1.8 \mathrm{~kg})$ was extracted with methanol at the ratio of 1:10 at room temperature by maceration on a shaker overnight. The combined solutions were evaporated at $40^{\circ} \mathrm{C}$ resulting in $158 \mathrm{~g}$ crude methanol extract; $140 \mathrm{~g}$ of the extract were fractionated in four portions, whereby each portion was dissolved in $300 \mathrm{~mL} \mathrm{MeOH}-\mathrm{H}_{2} \mathrm{O}$ (10:1) and partitioned three times with $300 \mathrm{~mL}$ petroleum ether, each, to remove chlorophyll, waxes, and fatty matters. The $\mathrm{MeOH}-\mathrm{H}_{2} \mathrm{O}$ phase was then diluted with $300 \mathrm{~mL} \mathrm{H}_{2} \mathrm{O}$ and partitioned with three times $600 \mathrm{~mL}$ chloroform. After washing the organic layer with $1 \%$ aqueous $\mathrm{NaCl}$ solution and evaporation, $15.6 \mathrm{~g}$ chloroform fraction $(\mathrm{CF})$ was obtained. From the remaining $\mathrm{MeOH}-\mathrm{H}_{2} \mathrm{O}$ fraction, $\mathrm{MeOH}$ was removed and the aqueous residue partitioned with three times $280 \mathrm{~mL}$ ethyl acetate, resulting in $12.3 \mathrm{~g}$ ethyl acetate fraction (EF) and $110.2 \mathrm{~g}$ aqueous fraction (AF) after evaporation.

Ten grams of EF were separated by column chromatography (CC; $3 \mathrm{~cm} \times 60 \mathrm{~cm}$ ) on Sephadex LH-20 under elution with 50\% $\mathrm{MeOH}$ yielding 15 combined fractions $(\mathrm{a}-\mathrm{o})$. In fractions $\mathrm{k}$ and 1, compound $\mathbf{1}$ was identified by TLC and LC-DAD. Fractions e $(1 \mathrm{~g})$ and $\mathrm{g}(580 \mathrm{mg})$ were further fractionated on the same stationary phase under elution with $30 \% \mathrm{MeOH}$, resulting in fractions e1-e22 and g1-g19. From fraction e18, $46.5 \mathrm{mg}$ of compound 2 precipitated. Solid phase extraction (Megabond Elut C18 cartridge; $12 \mathrm{~mL}$; Agilent, Santa Clara, CA, United States; sequential elution with $20,30,40$, and $50 \% \mathrm{MeOH}$ ) of fraction e22 resulted in the isolation of compound $3(7 \mathrm{mg})$ and the identification of compound 4. A major component from fraction g6 (63.2 mg) was finally purified by another CC on Sephadex LH-20 under elution with $\mathrm{H}_{2} \mathrm{O}$ to yield $27 \mathrm{mg}$ compound 5. In fraction g10, compounds 6 and 7 were identified, and in fraction g19, compound 8 was confirmed.

\section{Treatment of MCF7/LEC model with methanol extract and its fractions}

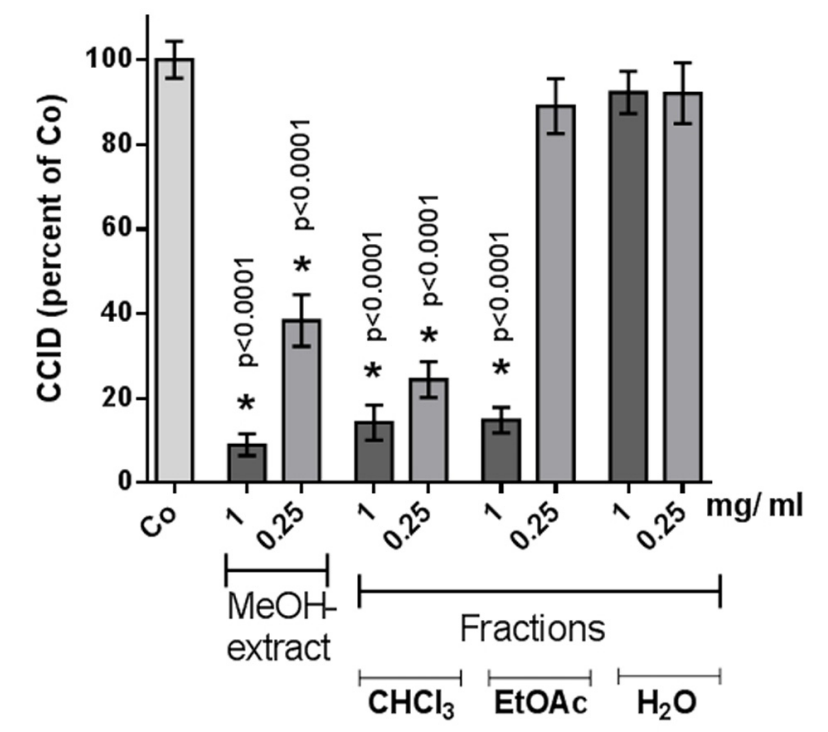

FIGURE 1 | MCF-7 spheroids pre-treated for 30 min with solvent (Co) or indicated concentration of different fractions, placed on top of LEC monolayers, and co-cultivated for $4 \mathrm{~h}$ when CCIDs were analyzed. Three independent experiments with at least 15 replicates were analyzed. Error bars indicate means \pm SEM and asterisks significance ( $t$-test).

A portion of CF (3.9 g) was submitted to $\mathrm{CC}$ on silica and elution with $\mathrm{CHCl}_{3}-\mathrm{MeOH}-\mathrm{H}_{2} \mathrm{O}$ (75:15:1.5), resulting in 25 fractions, five of which (A-E) were further investigated. In fraction A $(62.5 \mathrm{mg})$, compound 9 was identified as the major component. In fraction B $(150.8 \mathrm{mg})$, three major compounds (10-12) besides 8 were detected and tentatively identified by LC-MS. From fraction C (150.9 mg), compound 13 (144.7 mg) and from fraction D (630.5 $\mathrm{mg})$, compound 14 (532 $\mathrm{mg}$ ) were obtained. Fraction E (125.9 mg) contained a complex mixture of easy decomposable substances and attempts to isolate those by CC were not successful.

Astragalin (1): Rf in TLC: system $1=0.50$; system $2=0.74$.

Homoplantaginin (2): yellowish amorphous powder; ${ }^{1} \mathrm{H}-\mathrm{NMR}$ and ${ }^{13} \mathrm{C}-\mathrm{NMR}$ data: see Table 1; + ESIMS $m / z \quad 463.1 \quad[\mathrm{M}+\mathrm{H}]^{+} ; \quad+$ ESIMS $^{2} \quad(463.1 \rightarrow)$ $\mathrm{m} / z 301.3(100) ;+\mathrm{ESIMS}^{3}(463.1 \rightarrow 301.3 \rightarrow) \mathrm{m} / z 286.3$ $(100) ;+$ ESIMS $^{4}(463.1 \rightarrow 301.3 \rightarrow 286.3 \rightarrow) \mathrm{m} / z 240.1(10)$, 186.2 (28), 168.3 (100), 167.1 (11), 121.5 (25), and 120.5 (14); - ESIMS $m / z 461.0[\mathrm{M}-\mathrm{H}]^{-}$; - ESIMS $^{2}(461.0 \rightarrow) \mathrm{m} / z 446.2$ (45), 299.2 (100), 298.2 (17), 297.2 (16), 285.2 (20), 284.2 (73), and $283.2(57) ;-$ ESIMS $^{3}(461.0 \rightarrow 299.2 \rightarrow) \mathrm{m} / z 284.2(100)$; and - ESIMS $^{3}(461.0 \rightarrow 284.2 \rightarrow) \mathrm{m} / z 255.2(100), 239.1(10)$, 227.1 (51), 211.1 (21), 183.1 (11), and 137.2 (11).

$\mathbf{2}^{\prime \prime}$-O-acetyl-homoplantaginin (3): yellowish amorphous powder; ${ }^{1} \mathrm{H}-\mathrm{NMR}$ and ${ }^{13} \mathrm{C}-\mathrm{NMR}$ data: see Table $\mathbf{1} ;+$ ESIMS $m / z$ $505.0[\mathrm{M}+\mathrm{H}]^{+} ;+\mathrm{ESIMS}^{2}(505.0 \rightarrow) \mathrm{m} / z 301.3(100) ;+$ ESIMS $^{3}$ $(505.0 \rightarrow 301.3 \rightarrow) \mathrm{m} / z 286.3(100) ;+\mathrm{ESIMS}^{4}(505.0 \rightarrow 301.3$ $\rightarrow 286.3 \rightarrow) m / z 186.1(18), 168.3(100), 140.2(20)$, and 121.4 


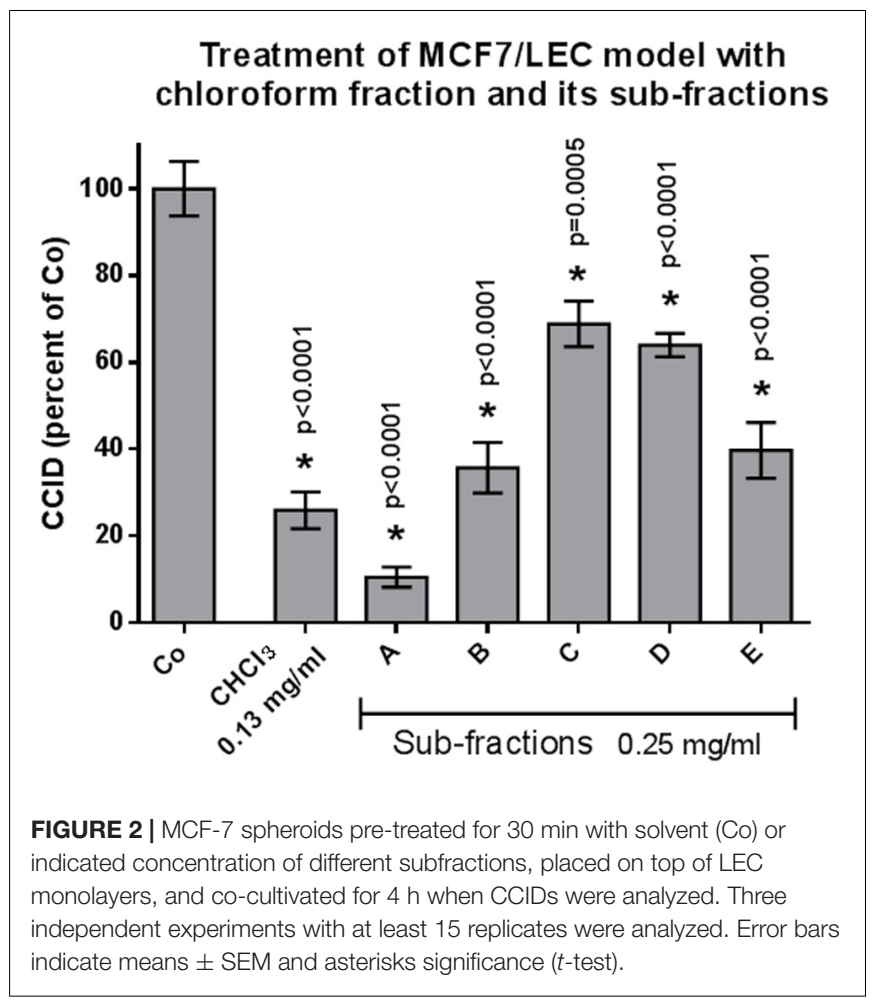

(11); -ESIMS m/z 503.0 [M-H] ${ }^{-}$; - ESIMS $^{2}(503.0 \rightarrow) \mathrm{m} / z$ 488.2 (9), 443.2 (10), 299.2 (100), 298.3 (20), 297.3 (20), 284.2 $(45)$, and $283.2(36) ;-$ ESIMS $^{3}(503.0 \rightarrow 299.2) \mathrm{m} / z 284.2$ (100); and - ESIMS $^{4}(503.0 \rightarrow 299.2 \rightarrow 284.2 \rightarrow) \mathrm{m} / z$ 256.1 (59), 255.1 (100), 239.1 (24), 229.0 (28), 228.1 (20), 227.1 (23), 216.0 (27), 211.2 (23), 200.2 (24), 199.2 (38), 183.0 (12), 166.1 (17), 163.1 (13), 150.2 (13), and 141.2 (13). +HRESIMS $m / z 505.1340$ $[\mathrm{M}+\mathrm{H}]^{+}\left(\right.$calcd for $\left.\mathrm{C}_{24} \mathrm{H}_{25} \mathrm{O}_{12}{ }^{+}, m / z 505.1341, \Delta=0.2 \mathrm{ppm}\right)$, 527.1159 $[\mathrm{M}+\mathrm{Na}]^{+}$(calcd for $\mathrm{C}_{24} \mathrm{H}_{24} \mathrm{O}_{12} \mathrm{Na}^{+}, m / z$ 527.1160, $\Delta=0.2 \mathrm{ppm})$.

Nepitrin (4): Rf in TLC: system $2=0.63$. LC-DAD data: see Supplementary Material.

Verbascoside (5): whitish amorphous powder; LC-DAD-data: see Supplementary Material; +ESIMS $m / z 642.2\left[\mathrm{M}+\mathrm{NH}_{4}\right]^{+}$, $625.1[\mathrm{M}+\mathrm{H}]^{+}$; + ESIMS $^{2}(642.2 \rightarrow) \mathrm{m} / z 479.1(18), 471.1$ (74), and $325.0(100) ;+$ ESIMS $^{3}(642.2 \rightarrow 471.1 \rightarrow) \mathrm{m} / z 453.2(11)$, 325.0 (100), 309.0 (11), and $162.9(54) ;+$ ESIMS $^{3}(642.2 \rightarrow 325.0$ $\rightarrow) \mathrm{m} / z 162.9(100) ;+\mathrm{ESIMS}^{4}(642.2 \rightarrow 325.0 \rightarrow 162.9 \rightarrow)$ $m / z 162.9$ (16), 144.9 (100), and 134.9 (23); -ESIMS $m / z 623.2$ $[\mathrm{M}-\mathrm{H}]^{-} ;-\mathrm{ESIMS}^{2}(623.2 \rightarrow) \mathrm{m} / z$ 461.1 (100); and - ESIMS $^{3}$ $(623.2 \rightarrow 461.1 \rightarrow) \mathrm{m} / z 315.0(43), 160.8$ (12), and 134.9 (100).

Kaempferol-3-O-rutinoside (6): Rf in TLC: system $2=0.55$. LC-DAD data: see Supplementary Material.

Rutin (7): Rf in TLC: system $2=0.48$. LC-DAD data: see Supplementary Material.

Luteolin-7-O-glucoside (8): Rf in TLC: system $2=0.71$. LCDAD data: see Supplementary Material.

Hispidulin (9): yellowish amorphous powder; LC-DAD data: see Supplementary Material; +ESIMS $m / z$ 301.1 $[\mathrm{M}+\mathrm{H}]^{+}$; + ESIMS $^{2}(301.1 \rightarrow) m / z 286.0(100) ;+$ ESIMS $^{3}(301.1 \rightarrow$
$286.0 \rightarrow) \mathrm{m} / z 256.8(16), 185.9(17), 167.9(100), 140.0(14), 122.0$ (38), 120.9 (30), 119.0 (24), and 112.0 (23); -ESIMS $m / z 299.0$ $[\mathrm{M}-\mathrm{H}]^{-} ;-$ESIMS $^{2}(299.0 \rightarrow) \mathrm{m} / z 283.9(100)$; and - ESIMS $^{3}$ $(299.0 \rightarrow 283.9 \rightarrow) \mathrm{m} / z 266.8$ (17), 255.8 (56), 254.9 (29), 238.8 (22), 227.7 (64), 226.8 (74), 213.8 (43), 211.8 (55), 210.8 (18), 199.8 (77), 198.9 (10), 198.0 (10), 185.7 (32), 183.7 (28), 182.9 (17), 177.7 (11), 163.8 (24), 149.7 (21), 136.8 (100), and 129.8 (11).

Buergeriside C1 isomer (10): +ESIMS $\mathrm{m} / \mathrm{z} \quad 307.1$ $\left[\mathrm{M}-\mathrm{H}_{2} \mathrm{O}+\mathrm{H}\right]^{+}, \quad 347.1[\mathrm{M}+\mathrm{Na}]^{+} ;$ESIMS $^{2}(307.1 \rightarrow)$ $\mathrm{m} / z 160.9(100) ;+$ ESIMS $^{3}(307.1 \rightarrow 160.9 \rightarrow) \mathrm{m} / z 132.9$

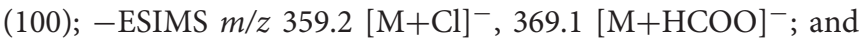
- ESIMS $^{2}(369.1 \rightarrow$ ) $m / z 323.0$ (48), 262.9 (26), 218.8 (34), 190.8 (12), 176.8 (100), and 144.9 (27).

$\begin{array}{lllll}\text { Buergeriside } & \mathrm{Cl} & \text { isomer (11):+ESIMS } \mathrm{m} / \mathrm{z} & 307.1\end{array}$ $\left[\mathrm{M}-\mathrm{H}_{2} \mathrm{O}+\mathrm{H}\right]^{+}, \quad 347.1[\mathrm{M}+\mathrm{Na}]^{+} ; \quad+$ ESIMS $^{2}(307.1 \rightarrow)$ $\mathrm{m} / z 161.0(100) ;+$ ESIMS $^{3}(307.1 \rightarrow 161.0 \rightarrow) \mathrm{m} / z 133.0$

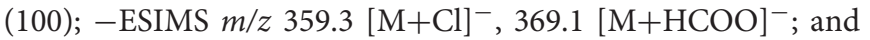
- ESIMS $^{2}(369.1 \rightarrow$ ) $m / z 323.9$ (22), 323.0 (74), 236.8 (12), 219.7 (15), 218.8 (28), 190.8 (36), 176.9 (43), 145.9 (11), 144.8 (100), and $126.9(7)$.

6-O-(2"-Acetyl-3" , $^{\prime \prime}$-O-di-trans-cinnamoyl- $\alpha$-L-rhamnopyranosyl-catalpol (12): +ESIMS $m / z 833.3[\mathrm{M}+\mathrm{Na}]^{+}$; + ESIMS $^{2}$ $(833.3 \rightarrow) \mathrm{m} / z$ 803.2 (11), 773.2 (35), 751.3 (100), 495.1 (30); - ESIMS $m / z 845.5[\mathrm{M}+\mathrm{Cl}]^{-}, 855.3\left[\mathrm{M}+\mathrm{HCOO}^{-}\right.$; - ESIMS $^{2}$ $(855.3 \rightarrow) m / z$ 810.2 (65), 809.3 (100), and 533.2 (14); and - ESIMS $^{3}(855.3 \rightarrow 809.3 \rightarrow$ ) $m / z 767.2(36), 750.2(28), 749.2$ (37), 662.1 (39), 661.2 (100), 648.1 (41), 647.2 (36), 629.2 (11), 620.2 (16), 619.2 (21), 618.3 (12), 617.0 (17), 533.1 (16), 353.0 (11), 311.0 (18), and 293.0 (11).

Koelzioside (13): whitish amorphous powder; ${ }^{1} \mathrm{H}-\mathrm{NMR}$ and ${ }^{13}$ C-NMR shifts: see Supplementary Material; +ESIMS $m / z 828.4$ $\left[\mathrm{M}+\mathrm{NH}_{4}\right]^{+} ;+\mathrm{ESIMS}^{2}(828.4 \rightarrow) \mathrm{m} / z 449.1(100) ;+\mathrm{ESIMS}^{3}$ $(828.4 \rightarrow 449.1 \rightarrow) m / z 389.0$ (11), 301.0 (47), 241.0 (25), and $131.0(100)$; and -ESIMS $m / z \quad 845.3[\mathrm{M}+\mathrm{Cl}]^{-}, 855.3$ $\left[\mathrm{M}+\mathrm{HCOO}^{-}\right.$.

Scrovalentinoside (14): whitish amorphous powder; ${ }^{1} \mathrm{H}-\mathrm{NMR}$ and ${ }^{13} \mathrm{C}-\mathrm{NMR}$ shifts: see Supplementary Material; +ESIMS $\mathrm{m} / \mathrm{z}$ $770.3\left[\mathrm{M}+\mathrm{NH}_{4}\right]^{+}$; + ESIMS $^{2}(770.3 \rightarrow) \mathrm{m} / z 770.3(14), 591.2$ $(5), 573.1(4)$, and $391.1(100) ;+\operatorname{ESIMS}^{3}(770.3 \rightarrow 391.1 \rightarrow)$ $\mathrm{m} / z 391.1$ (28), 271.1 (6), 213.0 (21), 161.0 (100), 153.0 (71), $133.0(7)$, and $111.1(19)$; and -ESIMS $m / z 787.3[\mathrm{M}+\mathrm{Cl}]^{-}, 797.3$ $\left[\mathrm{M}+\mathrm{HCOO}^{-}\right.$.

\section{Cell Culture}

Cultivation of human MCF-7 breast cancer cells was carried out in MEM medium supplemented with 10\% FCS, $1 \%$ penicillin/streptomycin, and 1\% NEAA (all from Invitrogen Life Technologies, Karlsruhe, Germany) and of telomerase immortalized human LECs in EGM2 MV medium (Clonetics CC-4147, Allendale, NJ, United States) at $37^{\circ} \mathrm{C}$ in a humidified atmosphere containing $5 \% \mathrm{CO}_{2}$.

\section{3-D Co-cultivation of MCF-7 Cancer Cells With LECs}

MCF-7 cells were transferred to $30 \mathrm{~mL}$ MEM medium containing $6 \mathrm{~mL}$ of a $1.6 \%$ methylcellulose solution $(0.3 \%$ final 
<smiles>[R]Oc1cc2oc(-c3ccc(O)c([R])c3)c([R3])c(=O)c2c(O)c1[R2]</smiles>

\begin{tabular}{|c|c|c|c|c|}
\hline & $\mathrm{R}_{1}$ & $\mathrm{R}_{2}$ & $\mathrm{R}_{3}$ & $\mathrm{R}_{4}$ \\
\hline 1 & $\mathrm{H}$ & $\mathrm{H}$ & $\beta-G \mid c p-O$ & $\mathrm{H}$ \\
\hline 2 & $\beta-G \mid c \rho$ & $\mathrm{CH}_{3} \mathrm{O}$ & H & H \\
\hline 3 & $2-A c-\beta-G \mid c p$ & $\mathrm{CH}_{3} \mathrm{O}$ & H & $\mathrm{H}$ \\
\hline 4 & $\beta$-GIcp & $\mathrm{CH}_{3} \mathrm{O}$ & $\mathrm{H}$ & $\mathrm{OH}$ \\
\hline 6 & $\mathrm{H}$ & $\mathrm{H}$ & $\alpha$-Rhap-1-6- $\beta-G l c p-O$ & H \\
\hline 7 & $\mathrm{H}$ & $\mathrm{H}$ & $\alpha$-Rhap-1-6- $\beta$-Glcp-O & $\mathrm{OH}$ \\
\hline 8 & $\beta-G \mid c p$ & $\mathrm{H}$ & $\mathrm{H}$ & $\mathrm{OH}$ \\
\hline 9 & $\mathrm{H}$ & $\mathrm{CH}_{3} \mathrm{O}$ & $\mathrm{H}$ & $\mathrm{H}$ \\
\hline
\end{tabular}

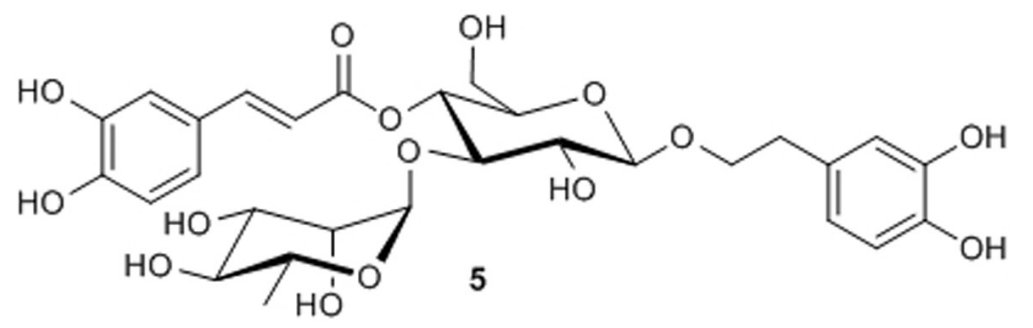

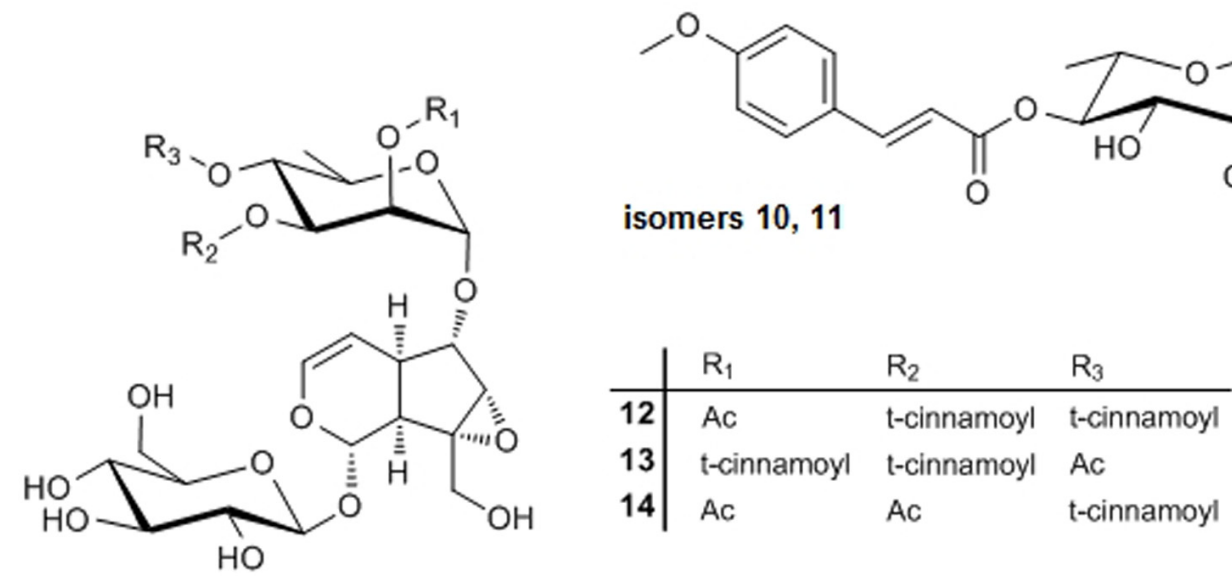

FIGURE 3 | Structures of compounds 1-14. Glcp, glucopyranosyl; Rhap, rhamnopyranosyl; Ac, acetyl.

concentration; cat. no. M-512, 4000 centipoises; Sigma-Aldrich, Munich, Germany). To each well of a 96-well plate (Greiner Bioone, Cellstar 650185, Kremsmünster, Austria), $150 \mu \mathrm{L}$ of the cell suspension was transferred for spheroid formation within $48 \mathrm{~h}$. Then, MCF-7 spheroids were washed in PBS and transferred to CellTracker $^{\mathrm{TM}}$ green (Invitrogen Life Technologies, Karlsruhe, Germany) stained LEC monolayers that had been seeded into 24-well plates (Costar 3524, Sigma) in 2 mL EGM2 MV medium (Vonach et al., 2011).

\section{Circular Chemorepellent-Induced Defect (CCID) Assay}

After $4 \mathrm{~h}$ of incubation of the co-cultures of MCF-7 spheroids (3,000 cells/spheroid) and the LEC monolayer, the size of CCIDs in the LEC monolayer underneath the MCF-7 spheroids was photographed with an Axiovert (Zeiss, Jena, Germany) fluorescence microscope to visualize CellTracker ${ }^{\mathrm{TM}}$ (green) stained LECs underneath the spheroids (Madlener et al., 2010).
CCID areas were calculated with the Axiovision Re. 4.5 software (Zeiss, Jena, Germany). MCF-7 spheroids were treated with solvent (ethanol) as negative control. The CCID areas of at least 15 spheroids per experiment were measured.

\section{RESULTS AND DISCUSSION}

From the methanolic extract of the aerial parts from S. lucida, apolar compounds were depleted by extraction with petroleum ether. After subsequent partition with chloroform and ethyl acetate, the two organic fractions and the residue of the aqueous phase were tested in the CCID assay. The crude methanol extract significantly and linearly inhibited CCID formation between concentrations of $0.25-1 \mathrm{mg} / \mathrm{mL}$ (Figure 1). At a concentration of $0.25 \mathrm{mg} / \mathrm{mL}$, the CF seemed to be even more effective than $0.25 \mathrm{mg} / \mathrm{mL}$ of the methanol extract; however, this difference was not significant. The EF was only active at $1 \mathrm{mg} / \mathrm{mL}$ and the $\mathrm{AF}$ was inactive at the tested concentrations. Therefore, the $\mathrm{CF}$ 
was chosen for further separation by $\mathrm{CC}$ on silica and five of the resulting sub-fractions (A-E) were active in the CCID assay with sub-fraction A exhibiting the strongest inhibition (Figure 2). The EF was separated by size exclusion chromatography followed by solid phase extraction or another subsequent size exclusion chromatography. Due to the lower activity of the EF in the CCID assay, these sub-fractions were submitted to chemical investigations only.

The processes resulted in the elucidation of 14 compounds, one of which is a new natural product (Figure 3). The 13 known compounds were identified by chromatographic, mass spectrometric and/or spectroscopic dereplication with authentic samples or data from literature. This led to the elucidation of seven flavonoids. The widespread natural compounds astragalin (1), luteolin-7-O-glucoside (8), kaempferol-3-O-rutinoside (6), and rutin (7) were confirmed by TLC- and LC-DAD comparison with the authentic substances. To the best of our knowledge, only the two latter have been reported in the genus Scrophularia until now, namely, in Scrophularia ilwensis C. Koch (Calis et al., 1993). Two further, less prevalent flavonoids, nepitrin (4) and homoplantaginin (2), had been isolated from S. ningpoensis ( $\mathrm{Li}$ et al., 2009) and nepitrin also from S. striata (Monsef-Esfahani et al., 2010) before. ${ }^{1} \mathrm{H}-\mathrm{NMR}$ - and ${ }^{13} \mathrm{C}-\mathrm{NMR}$ data of $\mathbf{2}$ correlated excellently with the data published earlier for homoplantaginin (Wang et al., 1998; Dawa et al., 2009). Hispidulin (9) was detected for the first time in a Scrophularia species and elucidated by LC-DAD comparison and LC-MS data.

A new flavonoid (3) was identified by thorough MS and NMR experiments: a molecular formula of $\mathrm{C}_{24} \mathrm{H}_{24} \mathrm{O}_{12}$ was determined from the HRESIMS data. Fragmentation of the $[\mathrm{M}+\mathrm{H}]^{+}$ion at $\mathrm{m} / \mathrm{z} 505.0$ showed the loss of a $204 \mathrm{Da}$ to yield a $\mathrm{Y}_{0}$ ion at $m / z$ 301.3, which again lost a $\mathrm{CH}_{3}^{\bullet}$ in $\mathrm{MS}^{3}$, typical for a methoxy group bound to an aromatic system. The $\mathrm{MS}^{2}, \mathrm{MS}^{3}$, and $\mathrm{MS}^{4}$ spectra were nearly identical to those of homoplantaginin. Fragmentation of the $[\mathrm{M}-\mathrm{H}]^{-}$ion at $m / z 503.0$ confirmed this information. In addition, the loss of $60 \mathrm{Da}\left(\mathrm{CH}_{3} \mathrm{COOH}\right)$ in $\mathrm{MS}^{2}$ indicated acetylation of the glucose of homoplantaginin. The signals of 3 in the ${ }^{1} \mathrm{H}-\mathrm{NMR}$ spectrum excellently correlated with those of compound 2 (Table 1), despite a large downfield shift of the proton at $\mathrm{C}-2^{\prime \prime}$ in the sugar for $1.55 \mathrm{ppm}$. The signals of the protons at C- $1^{\prime \prime}$ and C- $3^{\prime \prime}$ were shifted downfield for 0.24 and $0.22 \mathrm{ppm}$, respectively, as well. Additionally, from the singlet at $\delta=2.038 \mathrm{ppm}$, linkage of an acetyl group was deduced. The connection of an additional acetyl group in position $2^{\prime \prime}$ of homoplantaginin was confirmed by the ${ }^{13} \mathrm{C}-\mathrm{NMR}$ data. The signals of C-1" and C- $3^{\prime \prime}$ were shifted upfield for 2.28 and

TABLE $1 \mid{ }^{1} \mathrm{H}$ NMR and ${ }^{13} \mathrm{C}$ NMR data of compounds 2 and $\mathbf{3}$ in DMSO-d 6 .

\begin{tabular}{|c|c|c|c|c|c|c|c|}
\hline & & \multicolumn{3}{|c|}{ Compound 2} & \multicolumn{3}{|c|}{ Compound 3} \\
\hline \multicolumn{2}{|c|}{ Position } & ${ }^{1} \mathrm{H}(\mathrm{ppm})$ & $\mathrm{J}_{H . H}(\mathrm{~Hz})$ & ${ }^{13} \mathrm{C}(\mathrm{ppm})$ & ${ }^{1} \mathrm{H}$ (ppm) & $\mathrm{J}_{H . H}(\mathrm{~Hz})$ & ${ }^{13} \mathrm{C}$ (ppm) \\
\hline 3 & $\mathrm{CH}$ & 6.86 & s & 102.7 & 6.87 & s & 102.7 \\
\hline 4 & C & - & - & 182.3 & - & - & 182.3 \\
\hline $4 a$ & C & - & - & 105.7 & - & - & 106.1 \\
\hline \multirow[t]{2}{*}{6} & C & - & - & 132.5 & - & - & 132.5 \\
\hline & $\mathrm{CH}_{3}$ & 3.77 & s & 60.3 & 3.65 & s & 60.2 \\
\hline 7 & C & - & - & 156.5 & - & - & 155.9 \\
\hline 8 & $\mathrm{CH}$ & 7.02 & & 94.3 & 7.03 & s & 94.7 \\
\hline $8 a$ & C & - & - & 152.1 & - & - & 152.0 \\
\hline $4^{\prime}$ & C & - & - & 161.4 & - & - & 161.5 \\
\hline $1^{\prime \prime}$ & $\mathrm{CH}$ & 5.11 & d 7.1 & 100.2 & 5.35 & d 8.1 & 97.9 \\
\hline \multirow[t]{3}{*}{$2^{\prime \prime}$} & $\mathrm{CH}$ & 3.34 & dd 7.1/9.6 & 73.2 & 4.89 & dd 8.1/9.6 & 73.3 \\
\hline & C & - & - & & - & - & 169.3 \\
\hline & $\mathrm{CH}_{3}$ & - & - & & 2.04 & s & 20.8 \\
\hline $3^{\prime \prime}$ & $\mathrm{CH}$ & 3.32 & dd 9.6/9.2 & 76.7 & 3.54 & dd 9.6/9.1 & 73.8 \\
\hline $4^{\prime \prime}$ & $\mathrm{CH}$ & 3.21 & dd 9.2/9.6 & 69.5 & 3.31 & dd 9.1/9.7 & 69.6 \\
\hline $5^{\prime \prime}$ & $\mathrm{CH}$ & 3.47 & ddd 9.6/ 6.0/2.1 & 77.3 & 3.58 & ddd 9.7/6.0/2.1 & 77.5 \\
\hline $6^{\prime \prime} a$ & $\mathrm{CH}_{2}$ & 3.73 & dd 2.1/11.6 & 60.6 & 3.77 & dd 2.1/11.8 & 60.4 \\
\hline $\mathrm{b}$ & & 3.49 & dd 6.0/11.6 & & 3.53 & dd 6.0/11.8 & \\
\hline \multicolumn{2}{|c|}{ Further $\mathrm{OH}$ signals } & $\begin{array}{c}10.40 \\
5.43 \\
5.12 \\
4.63\end{array}$ & & & $\begin{array}{r}10.43 \\
5.39 \\
4.77\end{array}$ & & \\
\hline
\end{tabular}


$2.95 \mathrm{ppm}$, respectively. Signals of an acetyl group occurred at $\delta=169.26 \mathrm{ppm}$ (quarternary C) and $\delta=20.82 \mathrm{ppm}$ (methyl C). An HMBC crosspeak from the $\mathrm{CH}_{3}$ protons to the $\mathrm{C}-2^{\prime \prime}$ finally proofed the connectivity. Thus, compound $\mathbf{3}$ was unambiguously identified as $2^{\prime \prime}$-O-acetyl-homoplantaginin.

The phenylpropanoid verbascoside, which had been detected in many species of the Scrophulariaceae family (see, e.g., Zhang et al., 2013; Pasdaran et al., 2016, 2017; Venditti et al., 2016), was isolated from S. lucida as well and identified by HPLC-DAD and MS.

The genus is well-known for synthesizing a broad range of iridoids (De Santos Galíndez et al., 2002) and two such compounds were isolated from and two further ones tentatively identified in the CF.

The major iridoid was scrovalentinoside (14). The compound was elucidated by MS and NMR experiments (see Supplementary Material). The spectroscopic data were in excellent accordance with those published after isolation of the compound from S. auriculata L. ssp. pseudoauriculata (Senn.) Bolós et Vigo (Giner et al., 1998) and from S. nodosa L. (Miyase and Mimatsu, 1999). A second iridoid was unambiguously elucidated as koelzioside (13) based on MS experiments and the analogy of the ${ }^{1} \mathrm{H}-\mathrm{NMR}$ and ${ }^{13} \mathrm{C}-\mathrm{NMR}$ data as reported for the compound, until now only known from Scrophularia koelzii L. (Bhandari et al., 1992) and Scrophularia deserti Del. (Ahmed et al., 2003). Tentatively identified by thorough LC-MS experiments were two isomers of buergeriside $C_{1}$ (10 and 11), known from Scrophularia buergeriana (Miquel) (Kim and Kim, 2000), and 6-O-(2" -acetyl- $3^{\prime \prime}, 4^{\prime \prime}$-di-O-trans-cinnamoyl)$\alpha$-L-rhamnopyranosyl-catalpol (scropolioside B, 12), reported in Scrophularia scopolii [Hoppe ex] Pers. var. scopolii (Calis et al., 1988). Compounds $\mathbf{1 0}$ and $\mathbf{1 1}$ both showed a UV spectrum typical for a para-substituted cinnamic acid derivative and yielded identical fragment ions, although the relative intensities were different in the $\mathrm{MS}^{2}$ spectra of the $[\mathrm{M}+\mathrm{COOH}]^{-}$ ion of $\mathbf{1 0}$ and 11. Fragmentation of the $\left[\mathrm{M}-\mathrm{H}_{2} \mathrm{O}+\mathrm{H}\right]^{+}$ ion yielded the acylium ion of methoxycinnamic acid $\left(\mathrm{Z}_{0}\right)$, while $\mathrm{CID}$ of the $[\mathrm{M}+\mathrm{COOH}]^{-}$ion produced deprotonated methoxycinnamic acid $\left(\mathrm{Y}_{0}\right)$, dehydrated deoxyhexose $\left(\mathrm{B}_{1}\right)$, and typical cross-ring cleavage of the deoxyhexose. From the compounds described for the genus Scrophularia, this pattern only fits to buergeriside $C_{1}$, why we have tentatively identified $\mathbf{1 0}$ and $\mathbf{1 1}$ as buergeriside $\mathrm{C}_{1}$ isomers. The UV spectrum of 12 points toward cinnamic acid. Fragmentation of the $[\mathrm{M}-\mathrm{H}]^{-}$ion (resulting from CID of the $[\mathrm{M}+\mathrm{COOH}]^{-}$ ion) yielded the neutral loss of 60, 148, and $162 \mathrm{Da}$, fitting to an acetylated, cinnamoylated, and hexosylated compound. Scropolioside B is the only previously described compound in the genus Scrophularia being in accordance with these data. All iridoids detected in S. lucida are very rare natural compounds.

The quantitative determination by HPLC of the major compounds in the methanolic extract revealed $3 \%$ homoplantaginin, $1.4 \%$ scrovalentinoside, and $0.7 \%$ koelzioside.

In an earlier study, $20 \mu \mathrm{g} / \mathrm{mL}$ of the methanolic extract of S. lucida was reported to induce $70 \%$ apoptosis and to almost completely inhibit proliferation of HL-60 cells in S-phase within
48 and 72 h, respectively (Giessrigl et al., 2012). Here, we demonstrate that $250 \mu \mathrm{g} / \mathrm{mL}$ of the methanolic extract inhibits $60 \%$ CCID formation in the MCF-7/LEC model within $4 \mathrm{~h}$ and the CF of the methanolic extract inhibited CCIDs almost similarly (with insignificant difference; Figure 1). Sub-fraction A of the CF was the most active one and other four fractions (B-E) also exhibited significant CCID-inhibitory properties (Figure 2).

Hispidulin turned out to be the major component in subfraction $\mathrm{A}$, whereas koelzioside and scrovalentinoside, the main components in sub-fractions $\mathrm{C}$ and $\mathrm{D}$, respectively, showed significantly less activity. To evaluate the potential of hispidulin in suppressing CCID formation, the pure compound was tested as well-demonstrating an $\mathrm{IC}_{50}$ of $88.7 \mu \mathrm{M}$ confirming a contribution of hispidulin to the effects of the methanolic extract. This further implicated that other components present in sub-fraction A must have synergized strongly with hispidulin as only $250 \mu \mathrm{g} / \mathrm{mL}$ of sub-fraction A inhibited $\sim 90 \%$ CCID formation (compared to $26.6 \mathrm{mg} / \mathrm{mL}$ of purified hispidulin, which inhibited just 50\%). Conceivably, residual amounts of sub-fraction B still been present in sub-fraction A caused this synergy. Hispidulin, in vitro and in vivo, had been shown to inhibit proliferation and to induce apoptosis of various cancer lines (Yu et al., 2013; Gao et al., 2015; Wang et al., 2015). The compound exhibited cytotoxicity toward HepG2 liver cancer cells but not normal liver cells (Gao et al., 2014). It also inhibited pancreas cancer cell mobility and invasion in vitro as well as neovascularization in C57/BL6 mice (He et al., 2011). Additionally, colon cancer cell intravasation through blood endothelial barriers was hindered in vitro (Holzner et al., 2017).

\section{CONCLUSION}

In the activity-guided search for components of S. lucida inhibitory in the CCID assay, we showed that intravasation of breast cancer cells through the lymph endothelial wall, which is the major route of breast cancer dissemination, is significantly inhibited by hispidulin and other compounds present in the CF of $S$. lucida methanolic extract.

In addition, this is the first study characterizing the chemical composition of S. lucida. It resulted in the proof or tentative identification of several rare iridoids and flavonoids for the first time in the genus Scrophularia and a new compound, $2^{\prime \prime}-O$ acetyl-homoplantaginin, was unambiguously elucidated.

For a more profound assessment of the potential of S. lucida to inhibit metastasis in breast cancer, testing in an appropriate animal model is necessary as a next step.

\section{AUTHOR CONTRIBUTIONS}

VL, LS, and TL performed the phytochemical fractionation of the extract and isolation of secondary metabolites. JE performed the CCID assay of hispidulin. HK performed all NMR measurements and structure elucidation. MZ performed all MS and LC-MS measurements and structure elucidation. 
$\mathrm{CN}$ performed the CCID assays of the extracts and fractions. GK supervised all pharmacological experiments. AÖ collected the plant material, was responsible for correct identification, and prepared the extract. LK supervised all phytochemical work. LK, HK, MZ, CN, and GK compiled the manuscript.

\section{FUNDING}

The project was funded by the global budget of the University of Vienna and the Medical University of Vienna.

\section{REFERENCES}

Ahmed, B., Al-Rehaily, A. J., Al-Howiriny, T. A., El-Sayed, K. A., and Ahmad, M. S. (2003). Scropolioside-D2 and harpagoside-B: two new iridoid glycosides from Scrophularia deserti and their antidiabetic and antiinflammatory activity. Biol. Pharm. Bull. 26, 462-467. doi: 10.1248/bpb.26.462

Azadmehr, A., Hajiaghaee, R., Baradaran, B., and Haghdoost-Yazdi, H. (2015). Apoptosis cell death effect of Scrophularia variegata on breast cancer cells via mitochondrial intrinsic pathway. Adv. Pharm. Bull. 5, 443-446. doi: 10.15171/ apb. 2015.060

Azadmehr, A., Hajiaghaee, R., and Mazandarani, M. (2013). Induction of apoptosis and G2 /M cell cycle arrest by Scrophularia striata in a human leukaemia cell line. Cell Prolif. 46, 637-643. doi: 10.1111/cpr.12074

Baradaran, P. C., Mohammadi, A., Mansoori, B., Baradaran, S. C., and Baradaran, B. (2017). Growth inhibitory effect of Scrophularia oxysepala extract on mouse mammary carcinoma $4 \mathrm{~T} 1$ cells in vitro and in vivo systems. Biomed. Pharmacother. 85, 718-724. doi: 10.1016/j.biopha.2016. 11.086

Baytop, T. (1999). Türkiyede Bitkiler ile Tedavi. Istanbul: Istanbul University Press.

Bhandari, S. P. S., Mishra, A., Roy, R., and Garg, H. S. (1992). Koelzioside, an iridoid diglycoside from Scrophularia koelzii. Phytochemistry 31, 689-691. doi: 10.1016/0031-9422(92)90061-T

Calis, I., Gross, G. A., Winkler, T., and Sticher, O. (1988). Isolation and structure elucidation of two highly acylated iridoid diglycosides from Scrophularia scopolii. Planta Med. 54, 168-170. doi: 10.1055/s-2006-962382

Calis, I., Zor, M., Basaran, A. A., Wright, A. D., and Sticher, O. (1993). Karsoside and scropolioside D, two new iridoid glycosides from Scrophularia ilwensis. J. Nat. Prod. 56, 606-609. doi: 10.1021/np50094a022

Crisan, G., Kiss, B., Vlase, L., Balica, G., and Tamas, M. (2009). HPLC determination of some phenolic compounds of Scrophularia nodosa and S. scopolii. Chem. Nat. Compd. 45, 885-888. doi: 10.1007/s10600-0109478-8

Davis, P. (1968-1985). Flora of Turkey. Edinburgh: Edinburgh University Press, $1-10$.

Dawa, Z., Bai, Y., Zhou, Y., Gesang, S., Ping, A., and Ding, L. (2009). Chemical constituents of the whole plants of Saussurea medusa. J. Nat. Med. 63, 327-330. doi: 10.1007/s11418-009-0320-1

De Santos Galíndez, J., Díaz Lanza, A. M., and Matellano, L. F. (2002). Biologically active substances from the genus Scrophularia. Pharm. Biol. 40, 45-59. doi: 10.1076/phbi.40.1.45.5864

Gao, H., Jiang, Q., Han, Y., Peng, J., and Wang, C. (2015). Hispidulin potentiates the antitumor effect of sunitinib against human renal cell carcinoma in laboratory models. Cell Biochem. Biophys. 71, 757-764. doi: 10.1007/s12013014-0260-6

Gao, H., Wang, H., and Peng, J. (2014). Hispidulin induces apoptosis through mitochondrial dysfunction and inhibition of P13k/Akt signalling pathway in HepG2 cancer cells. Cell Biochem. Biophys. 69, 27-34. doi: 10.1007/s12013-0139762-x

Giessrigl, B., Yazici, G., Teichmann, M., Kopf, S., Ghassemi, S., Atanasov, A. G., et al. (2012). Effects of Scrophularia extracts on tumor cell proliferation, death and intravasation through lymphoendothelial cell barriers. Int. J. Oncol. 40, 2063-2074. doi: 10.3892/ijo.2012.1388

\section{ACKNOWLEDGMENTS}

The authors are grateful to Mag. A. M. Spitznagl and Mag. E. Sumetsberger for technical assistance in the identification of some of the compounds.

\section{SUPPLEMENTARY MATERIAL}

The Supplementary Material for this article can be found online at: https://www.frontiersin.org/articles/10.3389/fphar. 2018.00304/full\#supplementary-material

Giner, R. M., Villalba, M. L., Del Carmen Recio, M., Mainez, S., Gray, A. I., and Rios, J. L. (1998). A New Iridoid from Scrophularia auriculata ssp. pseudoauriculata. J. Nat. Prod. 61, 1162-1163. doi: 10.1021/np980067o

Giner, R. M., Villalba, M. L., Recio, M. C., Máñez, S., Cerdá-Nicolás, M., and Ríos, J. L. (2000). Anti-inflammatory glycoterpenoids from Scrophularia auriculata. Eur. J. Pharmacol. 389, 243-252. doi: 10.1016/S0014-2999(99)00846-8

Guarrera, P. M., and Lucia, L. M. (2007). Ethnobotanical remarks on central and southern Italy. J. Ethnobiol. Ethnomed. 3:23. doi: 10.1186/1746-426 9-3-23

Hajiaghaee, R., Monsef-Esfahani, H. R., Khorramizadeh, M. R., Saadat, F., Shahverdi, A. R., and Attar, F. (2007). Inhibitory effect of aerial parts of Scrophularia striata on matrix metalloproteinases expression. Phytother. Res. 21, 1127-1129. doi: 10.1002/ptr.2221

He, L., Wu, Y., Lin, L., Wang, J., Wu, Y., Chen, Y., et al. (2011). Hispidulin, a small flavonoid molecule, suppresses the angiogenesis and growth of human pancreatic cancer by targeting vascular endothelial growth factor receptor 2-mediated PI3K/Akt/mTOR signaling pathway. Cancer Sci. 102, 219-225. doi: 10.1111/j.1349-7006.2010.01778.x

Holzner, S., Brenner, S., Atanasov, A. G., Senfter, D., Stadler, S., Nguyen, C. H., et al. (2017). Intravasation of SW620 colon cancer cell spheroids through the blood endothelial barrier is inhibited by clinical drugs and flavonoids in vitro. Food Chem. Toxicol. 111, 114-124. doi: 10.1016/j.fct.2017.11.015

Kim, S. R., and Kim, Y. C. (2000). Neuroprotective phenylpropanoid esters of rhamnose isolated from roots of Scrophularia buergeriana. Phytochemistry 54, 503-509. doi: 10.1016/S0031-9422(00)00110-2

Lange, I., Moschny, J., Tamanyan, K., Khutsishvili, M., Atha, D., Borris, R. P., et al. (2016). Scrophularia orientalis extract induces calcium signaling and apoptosis in neuroblastoma cells. Int. J. Oncol. 48, 1608-1616. doi: 10.3892/ijo.2016. 3373

Li, J., Huang, X., Du, X., Sun, W., and Zhang, Y. (2009). Study of chemical composition and antimicrobial activity of leaves and roots of Scrophularia ningpoensis. Nat. Prod. Res. 23, 775-780. doi: 10.1080/1478641080269 6247

Madlener, S., Saiko, P., Vonach, C., Viola, K., Huttary, N., Stark, N., et al. (2010). Multifactorial anticancer effects of digalloyl-resveratrol encompass apoptosis, cell-cycle arrest, and inhibition of lymphendothelial gap formation in vitro. $\mathrm{Br}$. J. Cancer 102, 1361-1370. doi: 10.1038/sj.bjc.6605656

Miyase, T., and Mimatsu, A. (1999). Acylated iridoid and phenylethanoid glycosides from the aerial parts of Scrophularia nodosa. J. Nat. Prod. 62, 1079-1084. doi: 10.1021/np9805746

Monsef-Esfahani, H. R., Hajiaghaee, R., Shahverdi, A. R., Khorramizadeh, M. R., and Amini, M. (2010). Flavonoids, cinnamic acid and phenyl propanoid from aerial parts of Scrophularia striata. Pharm. Biol. 48, 333-336. doi: 10.3109/ 13880200903133829

Ozbilgin, A., Durmuskahya, C., Kayalar, H., and Ostan, I. (2014). Assessment of in vivo antimalarial activities of some selected medicinal plants from Turkey. Parasitol. Res. 113, 165-173. doi: 10.1007/s00436-013-3639-1

Pasdaran, A., Delazar, A., Ayatollahi, S. A., Nahar, L., and Sarker, S. D. (2016). Phytochemical and bioactivity evaluation of Scrophularia amplexicaulis Benth. Rec. Nat. Prod. 10, 519-525.

Pasdaran, A., Pasdaran, A., Delazar, A., Ayatollahi, S. A., Ayatollahi, S. A., and Pasdaran, A. (2017). Chemical composition and biological activities of 
methanolic extract of Scrophularia oxysepala Boiss. Iran. J. Pharm. Res. 16, 338-346.

Passalacqua, N. G., Guarrera, P. M., and De Fine, G. (2007). Contribution to the knowledge of the folk plant medicine in Calabria region (Southern Italy). Fitoterapia 78, 52-68. doi: 10.1016/j.fitote.2006.07.005

Stevenson, P. C., Simmonds, M. S. J., Sampson, J., Houghton, P. J., and Grice, P. (2002). Wound healing activity of acylated iridoid glycosides from Scrophularia nodosa. Phytother. Res. 16, 33-35. doi: 10.1002/ptr.798

Stöger, E. A. (2017). Arzneibuch der Chinesischen Medizin. Stuttgart: Deutscher Apotheker Verlag.

Tong, S., Yan, J., and Lou, J. (2006). Preparative isolation and purification of harpagoside from Scrophularia ningpoensis Hemsley by high-speed countercurrent chromatography. Phytochem. Anal. 17, 406-408. doi: 10.1002/pca.938

Valiyari, S., Jahanban-Esfahlan, R., Shahneh, F. Z., Yaripour, S., Baradaran, B., and Delazar, A. (2013). Cytotoxic and apoptotic activity of Scrophularia oxysepala in MCF-7 human breast cancer cells. Toxicol. Environ. Chem. 95, 1208-1220. doi: 10.1080/02772248.2013.854362

Venditti, A., Frezza, C., Riccardelli, M., Foddai, S., Nicoletti, M., Serafini, M., et al. (2016). Secondary metabolites from Scrophularia canina L. Nat. Prod. Res. 30, 1665-1669. doi: 10.1080/14786419.2015.1122598

Viola, K., Kopf, S., Huttary, N., Vonach, C., Kretschy, N., Teichmann, M., et al. (2013). Bay11-7082 inhibits the disintegration of the lymphendothelial barrier triggered by MCF-7 breast cancer spheroids; the role of ICAM-1 and adhesion. Br. J. Cancer 108, 564-569. doi: 10.1038/bjc.2012.485

Vonach, C., Viola, K., Giessrigl, B., Huttary, N., Raab, I., Kalt, R., et al. (2011). NF-kappaB mediates the 12(S)-HETE-induced endothelial to mesenchymal transition of lymphendothelial cells during the intravasation of breast carcinoma cells. Br. J. Cancer 105, 263-271. doi: 10.1038/bjc.2011.194
Wang, M., Li, J., Rangarajan, M., Shao, Y., Lavoie, E. J., Huang, T.-C., et al. (1998). Antioxidative phenolic compounds from sage (Salvia officinalis). J. Agric. Food Chem. 46, 4869-4873. doi: 10.1021/jf98 0614b

Wang, Y., Liu, W., He, X., and Fei, Z. (2015). Hispidulin enhances the anti-tumor effects of temozolomide in glioblastoma by activating AMPK. Cell Biochem. Biophys. 71, 701-706. doi: 10.1007/s12013-0140252-6

Yu, C. Y., Su, K. Y., Lee, P. L., Jhan, J. Y., Tsao, P. H., Chan, D. C., et al. (2013). Potential therapeutic role of hispidulin in gastric cancer through induction of apoptosis via NAG-1 signaling. Evid. Based Complement. Alternat. Med. 2013:518301. doi: 10.1155/2013/518301

Zhang, L., Yang, Z., Jia, Q., Dorje, G., Zhao, Z., Guo, F., et al. (2013). Two new phenylpropanoid glycosides with interesterification from Scrophularia dentata Royle ex Benth. J. Mol. Struct. 1049, 299-302. doi: 10.1016/j.molstruc.2013. 05.039

Conflict of Interest Statement: The authors declare that the research was conducted in the absence of any commercial or financial relationships that could be construed as a potential conflict of interest.

Copyright (c) 2018 Lewenhofer, Schweighofer, Ledermüller, Eichsteininger, Kählig, Zehl, Nguyen, Krupitza, Özmen and Krenn. This is an open-access article distributed under the terms of the Creative Commons Attribution License (CC BY). The use, distribution or reproduction in other forums is permitted, provided the original author(s) and the copyright owner are credited and that the original publication in this journal is cited, in accordance with accepted academic practice. No use, distribution or reproduction is permitted which does not comply with these terms. 


\section{OPEN ACCESS}

Edited by:

Judit Hohmann,

University of Szeged, Hungary

Reviewed by:

Linlin Lu,

International Institute for Translational

Chinese Medicine, China

Codruta Soica

Victor Babeș University of Medicine and Pharmacy, Timisoara, Romania

*Correspondence:

Georg Krupitza

georg.krupitza@meduniwien.ac.at

Liselotte Krenn

liselotte.krenn@univie.ac.at

Specialty section:

This article was submitted to

Ethnopharmacology,

a section of the journal

Frontiers in Pharmacology

Received: 05 October 2017

Accepted: 27 February 2018

Published: 14 March 2018

Citation:

Hong J, Fristiohady A, Nguyen $\mathrm{CH}$, Milovanovic D, Huttary N, Krieger S, Hong J, Geleff S, Birner P, Jäger W,

Özmen A, Krenn L and Krupitza $G$ (2018) Apigenin and Luteolin

Attenuate the Breaching of MDA-MB231 Breast Cancer Spheroids Through the Lymph Endothelial Barrier in Vitro. Front. Pharmacol. 9:220. doi: 10.3389/fphar.2018.00220

\section{Apigenin and Luteolin Attenuate the Breaching of MDA-MB231 Breast Cancer Spheroids Through the Lymph Endothelial Barrier in Vitro}

\author{
Junli Hong 1,2,3, Adryan Fristiohady $3,4,5$, Chi H. Nguyen ${ }^{3,4}$, Daniela Milovanovic ${ }^{3}$, \\ Nicole Huttary ${ }^{3}$, Sigurd Krieger ${ }^{3}$, Junqiang Hong ${ }^{6}$, Silvana Geleff ${ }^{3}$, Peter Birner ${ }^{3}$, \\ Walter Jäger ${ }^{4}$, Ali Özmen ${ }^{7}$, Liselotte Krenn ${ }^{1 *}$ and Georg Krupitza ${ }^{3 *}$
}

\begin{abstract}
1 Department of Pharmacognosy, Faculty of Life Sciences, University of Vienna, Vienna, Austria, ${ }^{2}$ School of Pharmacy, Nanjing Medical University, Nanjing, China, ${ }^{3}$ Clinical Institute of Pathology, Medical University of Vienna, Vienna, Austria, ${ }^{4}$ Department for Clinical Pharmacy and Diagnostics, Faculty of Life Sciences, University of Vienna, Vienna, Austria,

${ }^{5}$ Faculty of Pharmacy, Halu Oleo University, Kendari, Indonesia, ${ }^{6}$ Department of Medical Oncology, The 188th Hospital of People's Liberation Army of China, Chaozhou, China, ${ }^{7}$ Department of Biology, Faculty of Science and Art, Adnan Menderes University, Aydin, Turkey
\end{abstract}

Flavonoids, present in fruits, vegetables and traditional medicinal plants, show anticancer effects in experimental systems and are reportedly non-toxic. This is a favorable property for long term strategies for the attenuation of lymph node metastasis, which may effectively improve the prognostic states in breast cancer. Hence, we studied two flavonoids, apigenin and luteolin exhibiting strong bio-activity in various test systems in cancer research and are readily available on the market. This study has further advanced the mechanistic understanding of breast cancer intravasation through the lymphatic barrier. Apigenin and luteolin were tested in a three-dimensional (3-D) assay consisting of MDA-MB231 breast cancer spheroids and immortalized lymph endothelial cell (LEC) monolayers. The 3-D model faithfully resembles the intravasation of breast cancer emboli through the lymphatic vasculature. Western blot analysis, intracellular $\mathrm{Ca}^{2+}$ determination, EROD assay and siRNA transfection revealed insights into mechanisms of intravasation as well as the anti-intravasative outcome of flavonoid action. Both flavonoids suppressed pro-intravasative trigger factors in MDA-MB231 breast cancer cells, specifically MMP1 expression and CYP1A1 activity. A pro-intravasative contribution of FAK expression in LECs was established as FAK supported the retraction of the LEC monolayer upon contact with cancer cells thereby enabling them to cross the endothelial barrier. As mechanistic basis, MMP1 caused the phosphorylation (activation) of FAK at Tyr397 in LECs. Apigenin and luteolin prevented MMP1-induced FAK activation, but not constitutive FAK phosphorylation. Luteolin, unlike apigenin, inhibited MMP1-induced $\mathrm{Ca}^{2+}$ release. Free intracellular $\mathrm{Ca}^{2+}$ is a central signal amplifier triggering LEC retraction through activation of the mobility protein MLC2, thereby enhancing intravasation. FAK activity and $\mathrm{Ca}^{2+}$ levels did not correlate. This implicates that the pro-intravasative contribution of FAK and of $\mathrm{Ca}^{2+}$ release in LECs was independent of each other and explains the better anti-intravasative effects 
of luteolin in vitro. In specific formulations, flavonoid concentrations causing significant anti-intravasative effects, can certainly be achieved in vivo. As the therapeutic strategy has to be based on permanent flavonoid treatment both the beneficial and adverse effects have to be investigated in future studies.

Keywords: intravasation, 3D model, flavonoids, FAK, MMP1, CYP1A1, $\mathrm{Ca}^{2+}$ release

\section{INTRODUCTION}

Species of the Scrophularia family are used in ethno-medicine since ancient times (Galindez et al., 2002). In the search for anti-metastatic natural compounds, extracts of six Scrophularia species were tested. For a methanolic extract from S. lucida a significant anti-intravasative activity was discovered (Giessrigl et al., 2012). The chloroform fraction of the methanolic extract inhibited in vitro intravasation of breast cancer spheroids most effectively. The major compound of the most active sub-fraction was hispidulin which, as pure compound, attenuated this early stage of the metastatic process significantly (Lewenhofer et al., 2018, same issue). Therefore, we decided to test two structurally related flavonoids, luteolin and apigenin, in in-depth mechanistic studies. These flavonoids are present in many medicinal plants used in the traditional medicine of Central and Eastern Europe. One of the oldest references to a healing plant in occidental literature can be found in Homer's Iliad (book XI, line 822 et seq.; Murray, 1924). There is said that 'A $A\llcorner\lambda \lambda \varepsilon \dot{s} \varsigma$ used a bitter, astringent root to treat wounds. This background in mythology led to the name of a very important medicinal plant: Achillea. In fact, this plant is used to treat, among other ailments, bleeding disorders and bruises (Akram, 2013). Later, Achillea sp. ("Achilleios") was also listed in De Materia Medica; Dioscurides, 1st century A.D. and used by Classical Greeks, in the Roman Empire, the Byzantium, and the Middle Ages up until our present day. A. millefolium is very rich in glycosides of luteolin and apigenin, which are easily metabolized into the respective genins (Benedek et al., 2007; Benetis et al., 2008). Many other apigenin- and luteolin-rich plant genera are also listed in $D e$ Materia Medica: Cynara, Apium, Allium, Petroselinum, Punica, Thymus, Solidago, Chamaemelum, Viola, and Vitis (the entire $5^{\text {th }}$ book is dedicated to Vitis).

Triple negative breast cancer (TNBC) comprises about 15\% of all breast cancer types. In general, TNBC responds well to chemotherapy as long it has not disseminated to other organs. However, as soon as TNBC metastasizes the success of drug treatment is very limited. This implicates that TNBC, in addition to conventional therapy, needs a metastasis-preventive approach to maintain a tumor state associated with a better prognosis. At an early stage of metastasis breast cancer cells intravasate the lymphatic vasculature and colonize lymph nodes. Importantly, the "lymph node status" is a prognostic breast tumor marker (Sobin et al., 2009). Hence, we hypothesized that compounds which prevent intravasation may interfere with tumor progression such as lymph node- and distant organ metastasis. As such a preventive concept for TNCB has to be based on long term drug administration, the therapeutic regimen has to be mild and rather free of side effects to allow continuous treatment. Luteolin inhibited proliferation and induced apoptosis of the highly metastatic TNBC cell line MDA-MB231 (Attoub et al., 2011). Anti-invasive/anti-metastatic activities of luteolin (Tsai et al., 2016; Lin et al., 2017) and the structurally related analog apigenin (Lindenmeyer et al., 2001; Qin et al., 2016; Zhao et al., 2017) have also been demonstrated in various cancer cell lines. As these flavonoids are also wide-spread constituents in fruits and vegetables they are considered free of adverse effects. This tempted us to investigate luteolin and apigenin in terms of their anti-intravasative properties. In the daily diet, the concentration of flavonoids is too low to show tumor preventive effects (Vogiatzoglou et al., 2015). However, apigenin and luteolin are also commercially available as food supplements with rather high concentrations (40-200 mg apigenin/capsule; $50 \mathrm{mg}$ luteolin/capsule) and may provide a preventive option.

The TNCB cell line MDA-MB231 over-expresses the pro-metastatic matrix metalloprotease 1 (MMP1), and inhibition of MMP1 attenuates tumor intravasation (Nguyen et al., 2015). Furthermore, MDA-MB231 cells secrete the pro-intravasative arachidonic acid metabolite 12(S)-HETE (Nguyen et al., 2016a), which is synthesized by CYP1A1. Therefore, we investigated whether luteolin and apigenin interfered with MMP1 expression and CYP1A1 activity in MDA-MB231 cells.

MMP1 and 12(S)-HETE activate the PAR1 and the 12(S)-HETE receptor, respectively, in LECs, which both trigger the release of $\mathrm{Ca}^{2+}$, causing the rapid retraction of LECs. This opens gaps in the monolayer [circular chemorepellent-induced defects (CCIDs)] through which tumor cells cross the endothelial barrier (Nguyen et al., 2015, 2016b). Hence, the influence of apigenin and luteolin on levels of intracellular $\mathrm{Ca}^{2+}$ in LECs was investigated. The expression of FAK in LECs seems to be crucial for rapid cell movement (Desai et al., 2009) as observed during CCID formation. Thus, FAK expression and activity were studied as well.

\section{MATERIALS AND METHODS}

\section{Antibodies and Reagents}

Polyclonal rabbit anti-focal adhesion kinase (FAK) and polyclonal rabbit anti-phospho-Tyr397-FAK were purchased from Cell Signaling (Danvers, MA, United States), polyclonal rabbit anti-MMP1 from Abcam (Cambridge, United Kingdom) and monoclonal anti- $\beta$-actin from Sigma (Munich, Germany). Polyclonal rabbit anti-mouse, anti-goat and anti-rabbit IgGs were acquired from Dako (Glostrup, Denmark). Apigenin and luteolin were purchased from Sigma (Munich, Germany) and human recombinant MMP1 from Sigma-Aldrich (SRP3117, 
<smiles>O=c1cc(-c2ccc(O)cc2)oc2cc(O)cc(O)c12</smiles><smiles>O=c1cc(-c2ccc(O)c(O)c2)oc2cc(O)cc(O)c12</smiles>

\section{c Treatment of the MDA/LEC model with apigenin and luteolin}

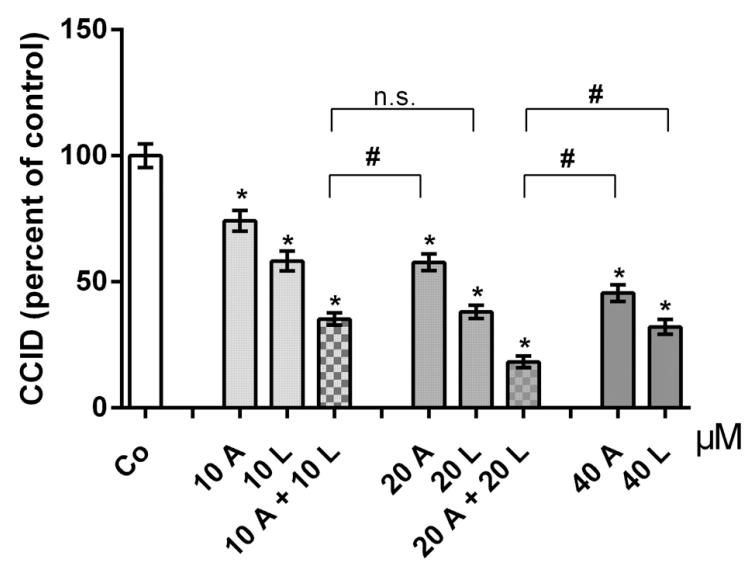

FIGURE 1 | Effects of flavonoid combinations on CCID formation. Chemical structures of (A) apigenin and (B) luteolin. (C) MDA-MB231 spheroids were placed on LEC monolayers and treated with solvent (DMSO, Co), or with 10, 20, and $40 \mu \mathrm{M}$ apigenin (A) or luteolin (L), or combinations of 10 and $20 \mu \mathrm{M} \mathrm{A}$ and L (solvent was kept constant) for $4 \mathrm{~h}$, after which CCID areas were measured using an Axiovert microscope and Zen little 2012 software. Three independent experiments with at least five replicates were analyzed. Error bars indicate means \pm SEM and asterisks and hash significances $(p<0.05 ; t$-test).

St. Louis, MO, United States). U73122 was obtained from Calbiochem (Darmstadt, Germany), BAPTA-AM was from Santa Cruz Biotechnology (Heidelberg, Germany).

siRNAs targeting human MLC2 (MYL2; SMART pool, ON-TARGET PLUS, Cat. No.: L-011087000005, siRNAs targeting human FAK (SMART pool, ON-TARGET PLUS, Cat. No.: L-003164000005) were ordered from Dharmacon (Gene Expression and Gene Editing, GE Healthcare, Lafayette, CO, United States). Non-targeting (n.t.) control siRNA (Silencer ${ }^{\circledR}$ Select Negative Control No. 1 siRNA, Cat. No.: 4390843) was purchased from Ambion (Life Technologies, Carlsbad, CA, United States). All siRNAs were re-suspended in RNAse-free water to yield a stock concentration of $20 \mu \mathrm{M}$.

\section{Cell Culture}

Human MDA-MB231 breast cancer cells from the American Type Culture Collection (ATCC, Rockville, MD, United States) were grown in MEM medium supplemented with $10 \%$ fetal calf serum (FCS), 1\% penicillin/streptomycin (PS) and 1\% non-essential amino acids (Gibco, Invitrogen, Karlsruhe, Germany). Telomerase immortalized human lymph endothelial cells (LECs) were grown in EGM2 MV (Clonetics CC-4147, Allendale, NJ, United States). The cells were kept at $37^{\circ} \mathrm{C}$ in a humidified atmosphere containing $5 \% \mathrm{CO}_{2}$. For CCID formation assays, LECs were stained with CellTracker ${ }^{\mathrm{TM}}$ green purchased from Invitrogen (Karlsruhe, Germany).

\section{Spheroid Formation}

MDA-MB231 cells (input of 6.000 cells per spheroid) were transferred to $30 \mathrm{~mL}$ serum free MEM medium containing $6 \mathrm{~mL}$ of a $1.6 \%$ methylcellulose solution $(0.3 \%$ final concentration; Cat. No.: M-512, 4000 centipoises; Sigma-Aldrich, Munich, Germany). $150 \mu \mathrm{L}$ of cell suspension were transferred to each well of a 96-well plate (Greiner Bio-one, Cellstar 650185, Kremsmünster, Austria) to allow spheroid formation within $48 \mathrm{~h}$. 


\section{CCID (Circular Chemorepellent Induced Defect) Assay}

In this assay, the sizes of the cell free areas (CCIDs) formed in the endothelial monolayer underneath the tumor spheroids, were measured. MDA-MB231 spheroids were washed in PBS and transferred to CellTracker ${ }^{\mathrm{TM}}$ (green)-stained LEC monolayers seeded into 24-well plates (Costar 3524, Sigma-Aldrich, Munich, Germany) in $1 \mathrm{~mL}$ EGM2 MV medium. After $4 \mathrm{~h}$ of incubation, the CCID areas in the LEC monolayers underneath the MDA-MB231 spheroids were photographed using an Axiovert (Zeiss, Jena, Germany) fluorescence microscope. CCID areas were calculated using the Zen Little 2012 (Zeiss, Jena, Germany). For each treatment and control the CCID size of at least 25 spheroids (unless otherwise specified) was measured.

\section{SDS Gel Electrophoresis and Western Blotting}

MDA-MB231 cells and LECs were grown in T-25 tissue culture flasks (Nunc, Roskilde, Denmark) to $80 \%$ confluence and then pre-treated with the flavonoids for $0.5,1,2$, and $4 \mathrm{~h}$. Then, cells were processed for SDS gel electrophoresis and Western blotting as described before (Nguyen et al., 2015). Chemo-luminescence was developed by Amersham ECL prime Kit (GE Healthcare, Freiburg, Germany) and detected using a Lumi-Imager F1 Workstation (Roche, Basel, Switzerland). Densitometry of the Western blots was analyzed with the Image-J software (National Institutes of Health, Bethesda, MD, United States).

\section{Ethoxyresorufin-O-Deethylase (EROD) Assay Selective for CYP1A1/A2 Activity}

MDA-MB231 cells were grown in phenol red-free DMEM/F12 medium (Gibco, Karlsruhe, Germany) containing 10\% FCS and $1 \%$ PS (Invitrogen, Karlsruhe, Germany). Before treatment, the cells were transferred to DMEM/F12 medium supplemented with $10 \%$ charcoal-stripped FCS (PAN Biotech, Aldenbach, Germany) and $1 \%$ PS. After $4 \mathrm{~h}$ of treatment with apigenin and luteolin CYP1A1 activity was measured with minor modifications as previously described (Teichmann et al., 2014). Briefly, ethoxyresorufin (final concentration $5.0 \mu \mathrm{M}$, Sigma-Aldrich, Munich, Germany) was added, $0.4 \mathrm{~mL}$ aliquots of the medium were collected after $180 \mathrm{~min}$ and the formation of resorufin was analyzed by spectro-fluorometry (PerkinElmer LS50B, Waltham, $\mathrm{MA}$, United States) at an excitation wavelength of $530 \mathrm{~nm}$ and an emission wavelength of $585 \mathrm{~nm}$.

\section{Intracellular $\mathrm{Ca}^{2+}$ Assay}

Free intracellular $\mathrm{Ca}^{2+}$ levels were measured using the FluoForte ${ }^{\mathrm{TM}}$ Calcium Assay Kit (Enzo Life Sciences, Ann Arbor, MI, United States). $8 \times 10^{3}$ LECs/well/100 $\mu$ L EGM2 medium were seeded into 96-well black-wall clear-bottom plates (Nunc, Thermo Fisher Scientific, Rochester, NY, United States) and after 24 h LECs were pre-treated with apigenin, luteolin or DMSO for $1 \mathrm{~h}$. Then, medium was removed and $100 \mu \mathrm{L}$ FluoForte ${ }^{\mathrm{TM}}$ Dye-loading (containing apigenin, luteolin, or DMSO) were added to each well. Cells were further incubated for $45 \mathrm{~min}$ at
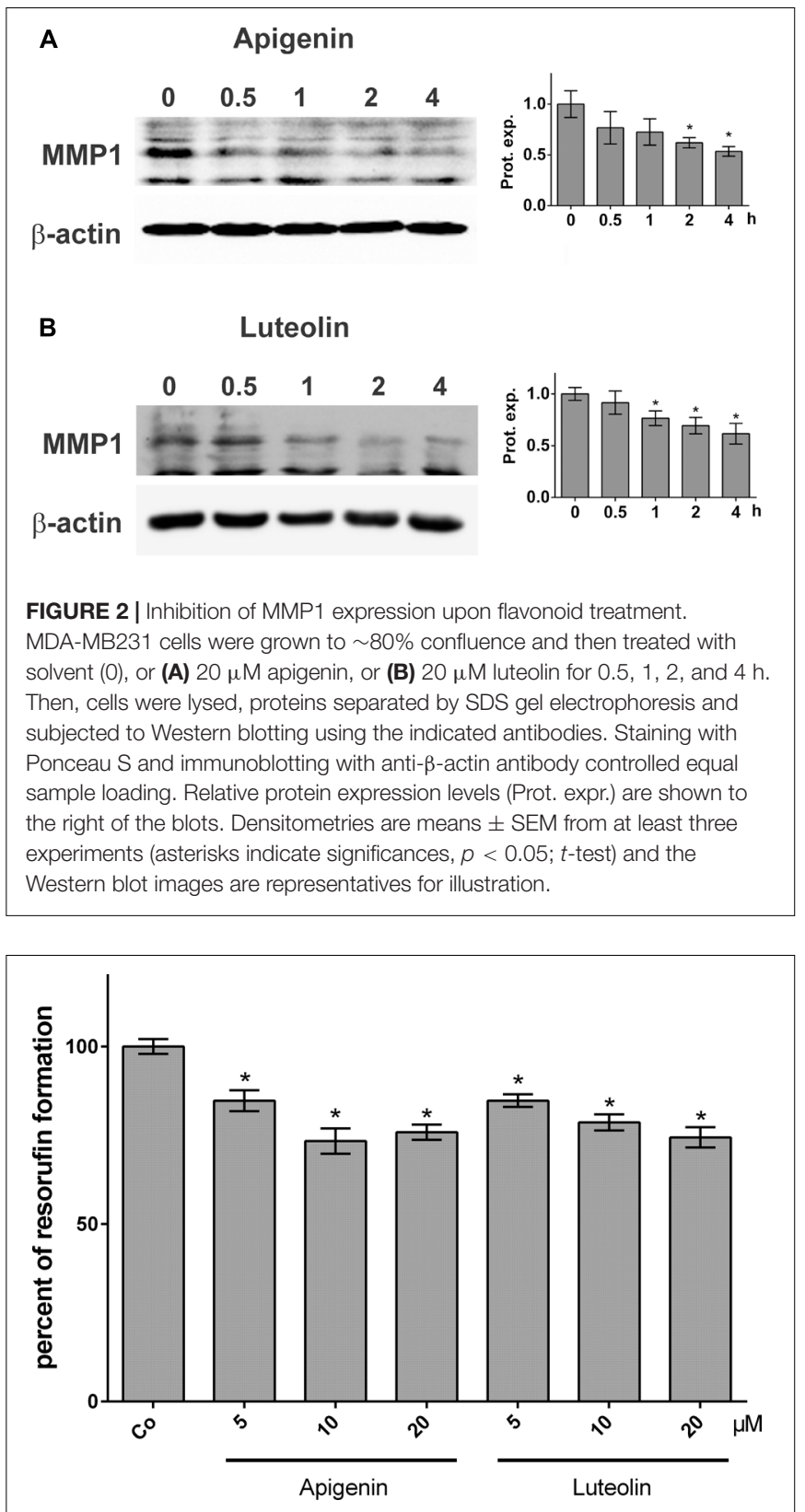

FIGURE 3 | Inhibition of CYP1A1 activity in MDA-MB231 cells by flavonoids. MDA-MB231 cells were treated with solvent (DMSO, Co), or the indicated concentrations of apigenin and luteolin. Ethoxyresorufin $(5 \mu \mathrm{M})$ was added after $4 \mathrm{~h}$ and the formation of resorufin was measured. Three independent experiments with at least three replicates were analyzed. Error bars indicate means \pm SEM and asterisks significances $\left({ }^{*} p<0.05\right.$; ANOVA together with Tukey's post-test).

$37^{\circ} \mathrm{C}$ followed by $15 \mathrm{~min}$ at room temperature. Then, cells were stimulated with $1 \mu \mathrm{M}$ 12(S)-HETE for $5 \mathrm{~min}$, and fluorescence was measured with a fluorescence plate reader at $490 / 525 \mathrm{~nm}$.

\section{Transfection of LEC Monolayer}

Lymph endothelial cells were seeded in 24-well plates $(1 \mathrm{~mL} /$ well) and grown in EGM2 medium. Transfections were performed 

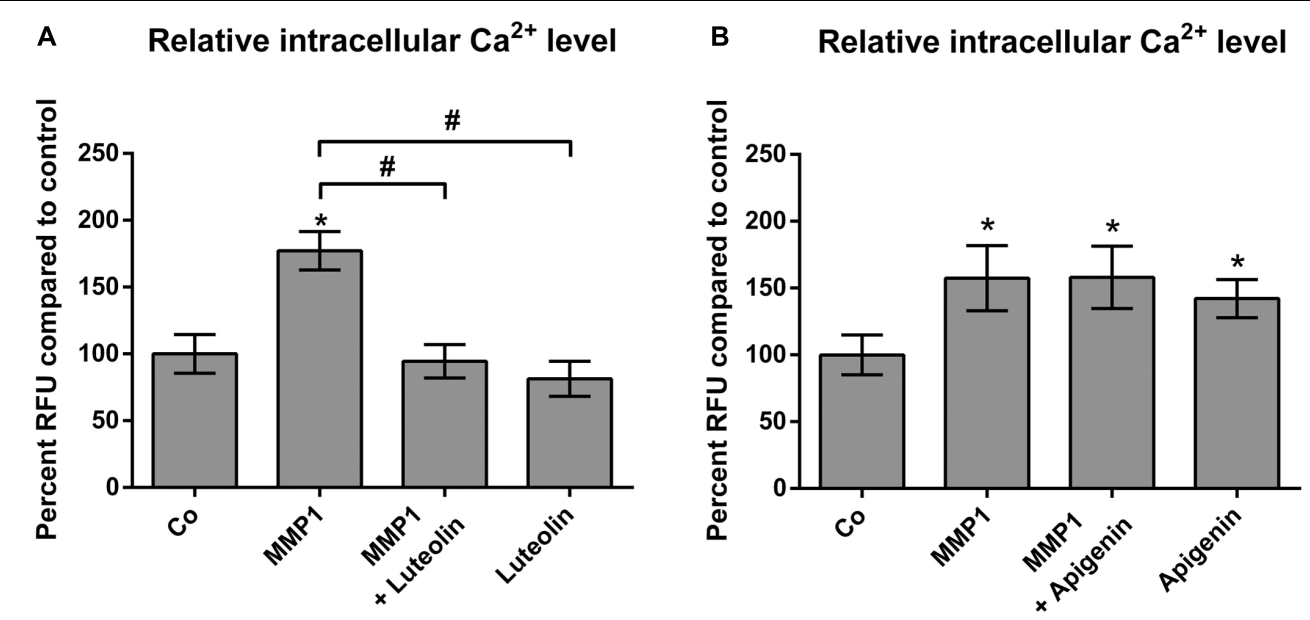

FIGURE 4 | Modulation of MMP1-induced $\mathrm{Ca}^{2}+$ release by flavonoids in LECs. LECs $\left(8 \times 10^{3}\right.$ cells/well) were pre-treated with solvent (DMSO, Co), or (A) $20 \mu \mathrm{M}$ luteolin, or (B) $20 \mu \mathrm{M}$ apigenin and then charged with FluoForte Dye-loading for $45 \mathrm{~min}$ at $37^{\circ} \mathrm{C}$ and 15 min at room temperature when cells were stimulated with $100 \mathrm{ng} / \mathrm{mL}$ activated recombinant MMP1 for $5 \mathrm{~min}$. Intracellular free calcium was measured with a fluorescence plate reader at $490 / 525 \mathrm{~nm}$. Three independent experiments with at least two replicates were analyzed. Error bars indicate means \pm SEM, and asterisks and hash significances ( $p<0.05$; $t$-test).

at a confluence of $70-80 \%$. $0.75 \mu \mathrm{g}$ siRNA and $6 \mu \mathrm{L}$ HiPerFect Transfection Reagent (Cat. No.: 301705; Qiagen, Hilden, Germany) were mixed in $100 \mu \mathrm{L}$ serum-free medium and incubated for $30 \mathrm{~min}$ at room temperature to allow the formation of transfection complexes. Cell culture medium was discarded and $500 \mu \mathrm{L}$ of fresh EGM2 medium were added into each well. Then, the transfection complexes were added drop-wise to the cells (to a final siRNA concentration of $100 \mathrm{nM}$ ) and incubated for $24 \mathrm{~h}$ at $37^{\circ} \mathrm{C}$. After $24 \mathrm{~h}$, the medium was replaced and cells were incubated for another $24 \mathrm{~h}$ to recover. The LEC monolayers were used for CCID assays or isolated RNA for qPCR.

\section{Statistical Analysis}

For statistical analyses Excel 2013 software and Prism 6 software packages (GraphPad, San Diego, CA, United States) were used. The values were expressed as mean \pm SEM, and the Student's $t$-test and ANOVA with Tukey's post-test was used to compare differences between control samples and treatment groups as well as differences among treatment groups. The statistical significance level was set to $p<0.05$.

\section{RESULTS}

\section{Apigenin/Luteolin Inhibit CCID Formation Induced by MDA-MB231 Spheroids}

Breast cancer cells have to intravasate the lymphatic barrier to colonize lymph nodes, and the CCID in vitro assay realistically resembles the intravasation of tumor spheroids through the lymphatic vasculature (Kerjaschki et al., 2011). Treatment of the three-dimensional MDA-MB231/LEC model with apigenin (Figure 1A) and luteolin (Figure 1B) dose dependently inhibited CCID formation in the LEC monolayer, with luteolin showing a stronger effect than apigenin. Simultaneous treatment with
$20 \mu \mathrm{M}$ luteolin and $20 \mu \mathrm{M}$ apigenin synergized and inhibited CCID formation significantly stronger than $40 \mu \mathrm{M}$ luteolin or $40 \mu \mathrm{M}$ apigenin, respectively, thus suggesting that luteolin and apigenin interfered with distinct pro-intravasative mechanisms (Figure 1C).

\section{Apigenin/Luteolin Inhibit MMP1 Expression and CYP1A1 Activity in MDA-MB231 Cells}

MDA-MB231 cell spheroids express MMP1, which is one mechanism causing CCIDs in the adjacent LEC monolayer (Nguyen et al., 2015). Thus, the expression of MMP1 was investigated after treatment of MDA-MB231 cells with apigenin and luteolin. $20 \mu \mathrm{M}$ apigenin and $20 \mu \mathrm{M}$ luteolin caused a significant and time-dependent down-regulation of MMP1 protein (Figures 2A,B).

Another mechanism of breast cancer emboli to cross the endothelial barrier is the secretion of 12 (S)-HETE which, in MDA-MB231 cells, is produced by CYP1A1 (Nguyen et al., 2016a). Hence, the activity of CYP1A1 in MDA-MB231 cells was analyzed by EROD assay after treatment with increasing concentrations of apigenin and luteolin. 5-20 $\mu \mathrm{M}$ apigenin and luteolin inhibited CYP1A1 activity significantly within $4 \mathrm{~h}$ (Figure 3).

The results proved that apigenin and luteolin inhibited MMP1 expression and CYP1A1 activity, which trigger the retraction of the adjacent LEC barrier thereby enabling MDA-MB231 tumor spheroid intravasation.

\section{Influence of Apigenin and Luteolin on Intracellular $\mathrm{Ca}^{2+}$ Release in LEC}

The intravasation of breast cancer cells relies on an interplay between cancer emboli and the LEC barrier (Vonach et al., 2011; Viola et al., 2013). Therefore, it was investigated whether apigenin 
A

Treatment of the MDA-MB231/LEC model with
BAPTA-AM and apigenin

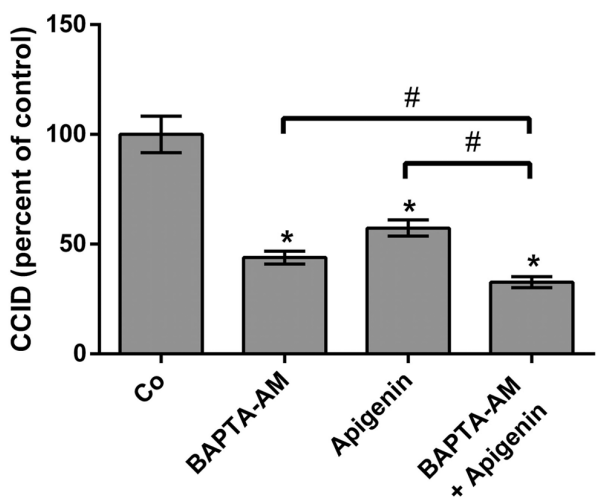

C

Treatment of the MDA-MB231/LEC model with BAPTA-AM and luteolin

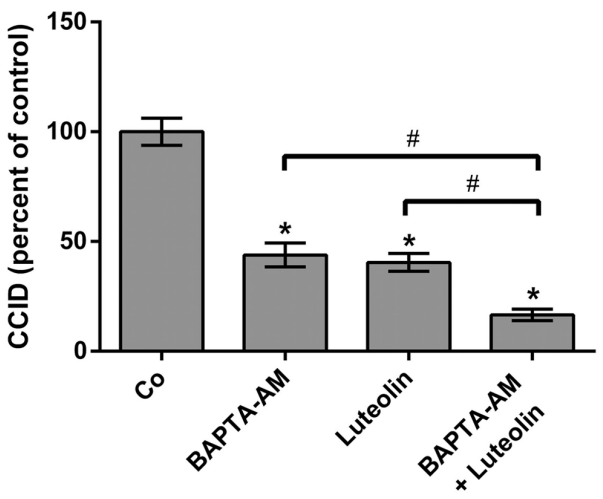

B

Treatment of the MDA-MB231/LEC model with
U73122 and apigenin

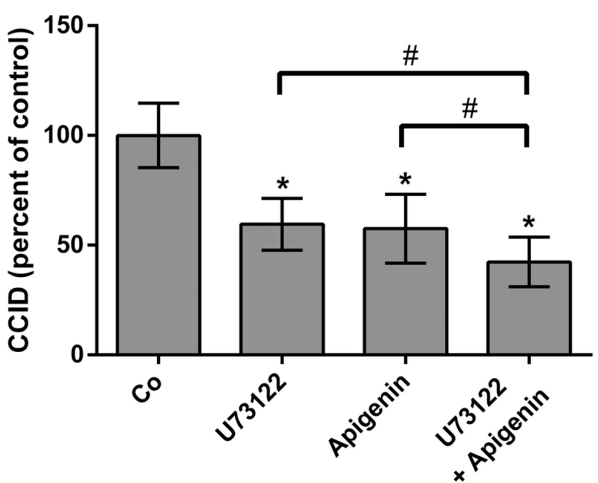

D Treatment of the MDA-MB231/LEC model with U73122 and luteolin

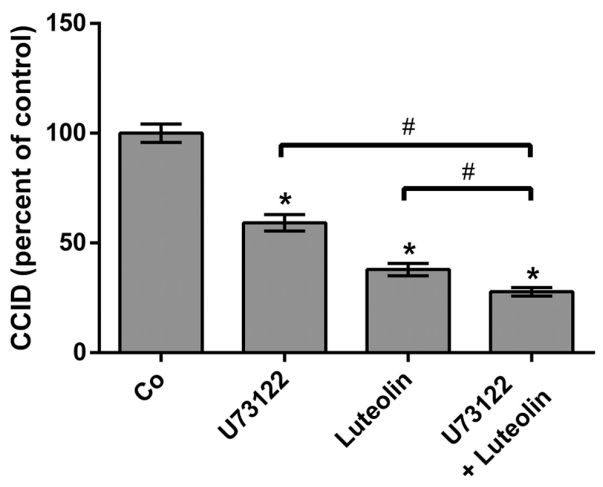

FIGURE 5 | Analysis of drug combinations on CCID formation. MDA-MB231 spheroids were placed on LEC monolayers and treated with solvent (DMSO, Co), or (A,B) $20 \mu \mathrm{M}$ apigenin, or (C,D) $20 \mu \mathrm{M}$ luteolin, alone, or together with (A,C) $5 \mu \mathrm{M}$ BAPTA-AM, or together with (B,D) $2.5 \mu \mathrm{M}$ U73122 for $4 \mathrm{~h}$, after which CCID areas were measured using an Axiovert microscope and Zen little 2012 software. Three independent experiments with at least five replicates were analyzed. Error bars indicate means \pm SEM and asterisks and hash significances $\left({ }^{*} p<0.05 ; t\right.$-test).

or luteolin would also inhibit the pro-intravasative response of LECs.

The release of $\mathrm{Ca}^{2+}$ from intracellular stores plays a central role in MMP1- and 12(S)-HETE-induced CCID formation (Nguyen et al., 2016b; Stadler et al., 2017). Hence, it was tested whether apigenin and luteolin affected intracellular $\mathrm{Ca}^{2+}$ release. Luteolin prevented MMP1-induced $\mathrm{Ca}^{2+}$ increase (Figure 4A), which is consistent with previous reports (Jiang et al., 2005). In contrast, apigenin did not reduce MMP1-induced $\mathrm{Ca}^{2+}$ levels in LECs, but in contrast increased $\mathrm{Ca}^{2+}$ release (Figure 4B). This is in agreement with data that apigenin activates potassium channels leading to their hyper-polarization and causing the release of $\mathrm{Ca}^{2+}$ (Erdogan et al., 2007). Accordingly, treating the MDA-MB231/LEC model with apigenin and BAPTA-AM (intracellular $\mathrm{Ca}^{2+}$ inhibitor) caused a significantly stronger inhibition of CCID formation than apigenin alone, or BAPTA-AM alone (Figure $\mathbf{5 A}$ ). $\mathrm{Ca}^{2+}$ release is dependent on PLC $\beta$ activity and, therefore, also the co-treatment with apigenin and U73122 (PLC inhibitor) attenuated CCID formation significantly stronger than the treatment with just apigenin or U73122 (Figure 5B). Luteolin ameliorated the CCID-inhibitory effect of BAPTA-AM (Figure 5C) or U73122 (Figure 5D) even more efficiently than apigenin. Overall, these data support the notion that an additional intravasative mechanism, which was independent of $\mathrm{Ca}^{2+}$ signaling, must have been inhibited by apigenin and luteolin as well.

\section{Luteolin/Apigenin Inhibit}

\section{Phosphorylation of Tyr397-FAK in LEC}

The FAK controls cell-matrix interactions and mobility (Schwock et al., 2010) as observed in CCID formation (Nguyen et al., 2015). Recombinant MMP1 induced the phosphorylation of FAK at Tyr397 indicative for its activation. Pre-treatment 


\section{A}

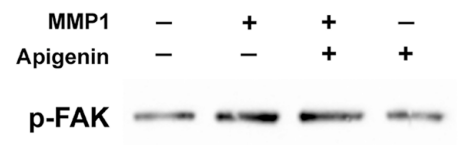

FAK

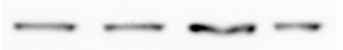

$\beta$-actin

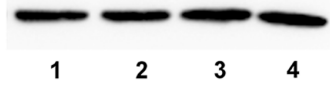

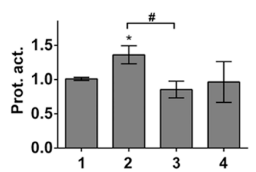

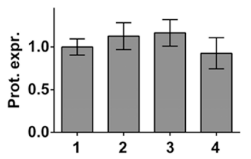

B
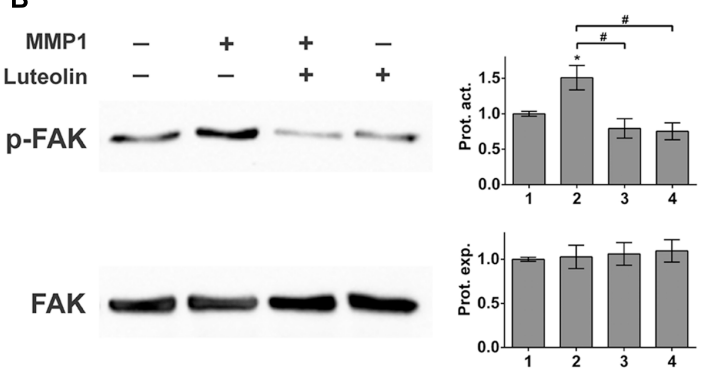

$\beta$-actin

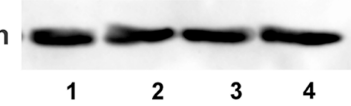

FIGURE 6 | MMP1 activates focal adhesion kinase (FAK) in LECs. LECs were grown to 70-80\% confluence and then pre-treated with solvent (DMSO), or (A) $20 \mu \mathrm{M}$ apigenin, or (B) $20 \mu \mathrm{M}$ luteolin and then stimulated with $100 \mathrm{ng} / \mathrm{mL}$ activated recombinant MMP1 for $4 \mathrm{~h}$. Cells were lysed, proteins were separated by SDS gel electrophoresis and analyzed by Western blotting using the indicated antibodies. Equal sample loading was controlled by Ponceau $S$ staining and $\beta$-actin immunoblotting. The relative protein expression (Prot. expr.) was quantified by densitometry facilitating the comparison with $\beta$-actin control, which was set to a value of "1." The calculated FAK protein expression levels are shown in the bar graphs next to the FAK Western blots. The relative phosphorylation of FAK ( $p$-FAK; corresponding to FAK activity) was quantified by densitometry facilitating the comparison with FAK protein expression, which was set to a value of "1." The calculated FAK protein activity levels (Prot. Act.) are shown in the bar graphs next to the p-FAK Western blots. The numbers below the bar graphs refer to the respective treatment conditions indicated in the blot lanes. Densitometries are means \pm SEM from at least three experiments (asterisks indicate significances, $p<0.05$; $t$-test) and the Western blot images are representatives for illustration. Hashes (\#; rhomboids) indicate significance.
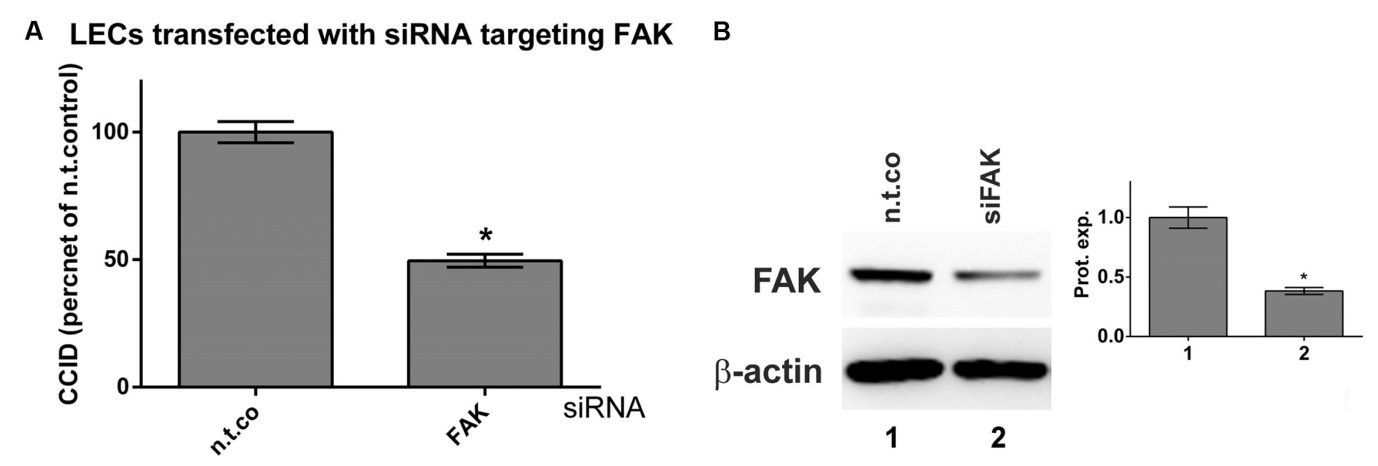

FIGURE 7 | Inhibition of focal adhesion kinase (FAK) expression in LECs by specific siRNA. LECs were transiently transfected with either non-targeting siRNA (n.t.co) or siRNA inhibiting the expression of FAK. (A) MDA-MB231 spheroids were placed on confluent LEC monolayers and co-incubated for $4 \mathrm{~h}$ when the areas of CCIDs were analyzed using an Axiovert microscope and Zen Little 2012 software. Three independent experiments with at least five replicates were analyzed. (B) After $24 \mathrm{~h}$ cells were lysed, total protein was isolated, separated by SDS-PAGE and transferred to PVDF membranes for Western blotting using FAK antibody. $\beta$-Actin was used to control equal sample loading. Relative protein expression levels (Prot. expr.) are shown to the right of the blots and the numbers below the bar graphs refer to the respective treatment conditions shown below the lanes. Densitometries are means $\pm \mathrm{SEM}$ from at least three experiments (asterisks indicate significances, $p<0.05$; $t$-test) and the Western blot images are representatives for illustration.

with apigenin or luteolin prevented MMP1-induced, but not constitutive FAK phosphorylation (Figures 6A,B). The inhibition of Tyr397-FAK phosphorylation by apigenin did not correlate with the inhibition of $\mathrm{Ca}^{2+}$ release. Therefore, the relevance of FAK for CCID formation was studied by siRNA-mediated knock-down of FAK (siFAK). In fact, siFAK transfection of LECs suppressed MDA-MB231-induced CCID formation by $50 \%$ (Figure 7A). The knock-down of FAK was confirmed by Western blotting (Figure 7B). Hence, FAK significantly contributed to intravasation in vitro and apigenin and luteolin inhibited this mechanism.

Earlier work demonstrated that CCID formation through MLC2 depends on intracellular $\mathrm{Ca}^{2+}$ release (Nguyen et al.,
2015). Our results show that FAK-dependent CCID formation did not correlate with intracellular $\mathrm{Ca}^{2+}$ levels. Although both pathways were induced by MMP1, they, obviously, are independent of each other. Thus, we hypothesized that simultaneous inhibition of MLC2 $\left(\mathrm{Ca}^{2+}\right.$-dependent $)$ and FAK $\left(\mathrm{Ca}^{2+}\right.$-independent $)$ by respective siRNAs, should bring about a greater reduction in CCID formation than inhibition of either FAK or MLC2 alone, which was actually confirmed (Figure 8). This supports former data (Figure 1C) showing that luteolin (inhibiting FAK and $\mathrm{Ca}^{2+}$ release) and apigenin (inhibiting FAK but not $\mathrm{Ca}^{2+}$ release) synergize in CCID attenuation via distinct signaling pathways. 


\section{LECs transfected with siRNA targeting FAK and MLC2}

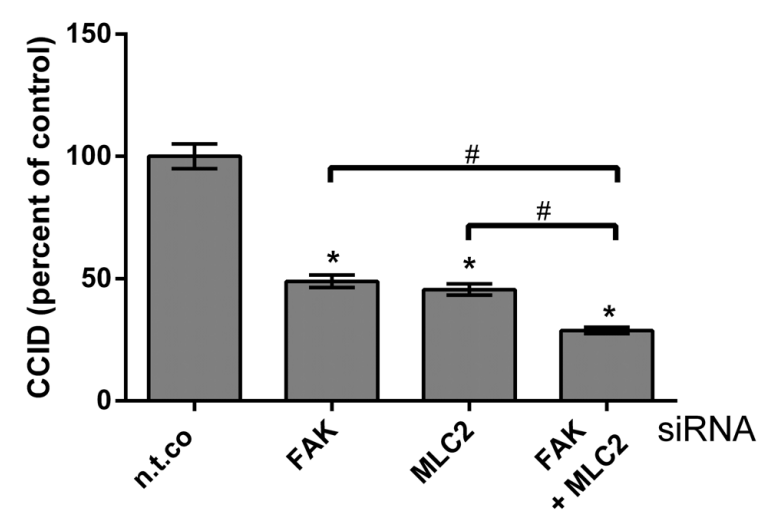

FIGURE 8 | Inhibition of focal adhesion kinase (FAK) and myosin light chain 2 (MLC2) protein expression in LECs by specific siRNAs. LECs were transiently transfected with either non-targeting siRNA (n.t.co), or siRNAs inhibiting the expression of FAK and MLC2. Then, MDA-MB231 spheroids were placed on confluent LEC monolayers and co-incubated for $4 \mathrm{~h}$, after which the areas of CCIDs were analyzed using an Axiovert microscope and Zen Little 2012 software. Three independent experiments with at least five replicates were analyzed. Error bars indicate means \pm SEM, asterisks and hash significances $(p<0.05 ;$ t-test).

\section{DISCUSSION}

We show for the first time that luteolin and apigenin attenuate the intravasation of breast cancer cells in vitro. At the tested concentrations, luteolin and apigenin inhibited CYP1A1 activity in MDA-MB231 cells. CYP1A1 synthesizes the pro-metastatic arachidonic acid metabolite 12(S)-HETE and is induced, e.g., by the activated aryl hydrocarbon receptor, the expression of which is high in many cancer entities (Murray et al., 2014) and also in MDA-MB231 cells (Nguyen et al., 2016a). In HepG2 cells with a luciferase reporter fused to the CYP1A1-regulator region, $5 \mu \mathrm{M}$ apigenin increased reporter gene expression by threefold. In contrast, the higher concentration of $20 \mu \mathrm{M}$ apigenin inhibited the expression by 50\% (Allen et al., 2001). In cell-free assays luteolin inhibited CYP1A1 more potently than apigenin, whereas CYP1A2 was inhibited more efficiently by apigenin (Kim et al., 2005). CYP1A2 mediates the formation of flavonoid metabolites with different properties to the genuin compounds. The comparability of flavonoid effects is rather difficult among different cell lines, which depend on the expression and activity profiles of the various CYP isoforms converting these flavonoids to other (more or less active) products (Androutsopoulos et al., 2009). Based on the very different individual expression levels of CYP1A2 in humans, Breinholt et al. (2002) conclude that this may result in different individual sensitivity.

Apigenin and luteolin inhibited also MMP1 expression in MDA-MB231 cells. MMP1 expression is induced by NF-кB (Lee et al., 2011), which is activated not only in inflamed- but also in cancerous tissue (Karin and Greten, 2005; Karin, 2006). Recently, we provided evidence that specific inhibition of
NF-кB (by siRELA, siNFKB1, siNEMO) down-regulated MMP1 expression and attenuated CCID formation (Nguyen et al., 2015). Flavones such as apigenin and luteolin inhibited NF- $\kappa \mathrm{B}$ activity and consequently the expression of its target MMP9 (Amrutha et al., 2014). Hence, the inhibition of NF- $\mathrm{B}$ by apigenin and luteolin may have been the reason for suppressed MMP1 levels in MDA-MB231 cells.

As shown here, recombinant MMP1 significantly induced the phosphorylation of FAK at Tyr397 in LECs. FAK activity contributes to cancer cell migration by establishing loose cell-matrix interactions necessary for rapid cell movement (Desai et al., 2009; Schwock et al., 2010), as described in CCID formation (Kerjaschki et al., 2011). In our experiments suppression of FAK significantly inhibited CCID formation. The activation of FAK (through phosphorylation of Tyr397) is mediated through Kv2.1 channels (Wei et al., 2008). Since FAK as well as potassium channel activity contribute to LEC retraction and CCID formation (Nguyen et al., 2017), the reduction of Tyr397-FAK phosphorylation by apigenin may have been due to the inhibition of potassium channels. This was supported by reports demonstrating that trimethyl-apigenin interfered with cardiac potassium currents (Liu et al., 2012) and the inhibition of potassium channel activity by the flavonoids acacetin, scutellarin, genistein, and naringenin (Teisseyre and Michalak, 2005; Zhu et al., 2008; Wu et al., 2011; Gasiorowska et al., 2012). However, quite to the contrary, apigenin and luteolin exhibited vasodilatory effects, which are discussed to be due to activation (rather than inactivation) of potassium channels (Calderone et al., 2004; Jiang et al., 2005). A more recent research concluded that the vasodilatory properties of apigenin and luteolin were independent of potassium channel inhibition, but due to extracellular calcium influx (Roberts et al., 2013). Therefore, the mechanism by which apigenin and luteolin inhibit FAK phosphorylation remains to be established.

MMP1 also activates the mobility protein MLC2 in LECs, which contributes to CCID formation as well (Nguyen et al., 2015, 2016c). MMP1 binds to PAR1, activates PLC $\beta$, triggers $\mathrm{Ca}^{2+}$ release and activates the $\mathrm{Ca}^{2+}$-calmodulin kinase MYLK, which phosphorylates MLC2. MMP1 was also shown to cause migration by binding to PAR1 in breast cancer cells (Boire et al., 2005) and blood endothelial cells (Juncker-Jensen et al., 2013). Whereas MMP1-induced MLC2 activation depends on $\mathrm{Ca}^{2+}$ release, FAK activation was not found to correlate with intracellular $\mathrm{Ca}^{2+}$ levels. This implicates that the pathway activating FAK represents a pro-intravasative mechanism independent of the one activating MLC2. Therefore, the inhibition both of FAK (either by siFAK or apigenin) and of MLC2 (by siMLC2, or BAPTA-AM, or U73122) reduced CCID formation in LEC monolayers to a greater extent than the inhibition of just one pathway.

Summing up, luteolin and apigenin inhibited pro-intravasative mechanisms in MDA-MB231 breast cancer cells at the levels of CYP1A1 activity and MMP1 expression. In LECs, apigenin and luteolin inhibited MMP1-induced, aberrant FAK activity but not constitutive, normal FAK phosphorylation. Only luteolin inhibited $\mathrm{Ca}^{2+}$ signaling in LEC, which contributed 
to CCID formation (Nguyen et al., 2016b). This may explain why luteolin affected CCID formation more potently than apigenin as well as the synergism between apigenin and luteolin.

The results implicate that luteolin and apigenin maintained the resilience of the endothelial barrier whilst attacking cancer cells and additionally, inhibited the malignant armamentarium of the tumor. Luteolin and apigenin exerted their effects at low $\mu \mathrm{M}$ concentrations which usually cannot be reached by daily dietary intake (Vogiatzoglou et al., 2015). Nevertheless, the daily intake of particular flavonoid subclasses (flavonols, flavan-3-ols, anthocyanidins) significantly correlates inversely with the risk of colorectal cancer (Woo and Kim, 2013). However, no other flavonoid subclasses or total flavonoids are associated with a lower risk of breast cancer in post-menopausal women (Hui et al., 2013). Intake of highly enriched apigenin and luteolin formulations achieved flavonoid concentrations in blood plasma which are comparable to those described herein in vitro. In detail, a single oral dose of apigenin delivered via a carbon nanopowder solid dispersion carrier $(60 \mathrm{mg} / \mathrm{kg})$ to rats resulted in peak plasma concentrations of up to $3.2 \mu \mathrm{g} / \mathrm{mL}$ which corresponds to $11.8 \mu \mathrm{M}$ (Ding et al., 2014). Similarly, a single oral dose luteolin in a peanut hull extract $(14.3 \mathrm{mg} / \mathrm{kg})$ to rats resulted in peak plasma concentrations of up to $8.3 \mu \mathrm{g} / \mathrm{mL}(29.1 \mu \mathrm{M})$ (Zhou et al., 2008). The evaluation as to whether these concentrations are free of negative side effects throughout long term treatment requires controlled phase I studies.

\section{REFERENCES}

Akram, M. (2013). Minireview on Achillea millefolium Linn. J. Membr. Biol. 246, 661-663. doi: 10.1007/s00232-013-9588-x

Allen, S. W., Mueller, L., Williams, S. N., Quattrochi, L. C., and Raucy, J. (2001). The use of a high-volume screening procedure to assess the effects of dietary flavonoids on human CYP1A1 expression. Drug Metab. Dispos. 29, 1074-1079.

Amrutha, K., Nanjan, P., Shaji, S. K., Sunilkumar, D., Subhalakshmi, K., Rajakrishna, L., et al. (2014). Discovery of lesser known flavones as inhibitors of NF- $\kappa \mathrm{B}$ signaling in MDA-MB-231 breast cancer cells-A SAR study. Bioorg. Med. Chem. Lett. 24, 4735-4742. doi: 10.1016/j.bmcl.2014.07.093

Androutsopoulos, V. P., Ruparelia, K., Arroo, R. R., Tsatsakis, A. M., and Spandidos, D. A. (2009). CYP1-mediated antiproliferative activity of dietary flavonoids in MDA-MB-468 breast cancer cells. Toxicology 264, 162-170. doi: 10.1016/j.tox.2009.07.023

Attoub, S., Hassan, A. H., Vanhoecke, B., Iratni, R., Takahashi, T., Gaben, A. M., et al. (2011). Inhibition of cell survival, invasion, tumor growth and histone deacetylase activity by the dietary flavonoid luteolin in human epithelioid cancer cells. Eur. J. Pharmacol. 651, 18-25. doi: 10.1016/j.ejphar.2010. 10.063

Benedek, B., Gjoncaj, N., Saukel, J., and Kopp, B. (2007). Distribution of phenolic compounds in Middleeuropean taxa of the Achillea millefolium L. aggregate. Chem. Biodivers. 4, 849-857. doi: 10.1002/cbdv.200790072

Benetis, R., Radusiene, J., and Janulis, V. (2008). Variability of phenolic compounds in flowers of Achillea millefolium wild populations in Lithuania. Medicina 44, 775-781.

Boire, A., Covic, L., Agarwal, A., Jacques, S., Sherifi, S., and Kuliopulos, A. (2005). PAR 1 is a matrix metalloprotease- 1 receptor that promotes invasion and tumorigenesis of breast cancer cells. Cell 120, 303-313. doi: 10.1016/j.cell.2004. 12.018

Breinholt, V. M., Offord, E. A., Brouwer, C., Nielsen, S. E., Brøsen, K., and Friedberg, T. (2002). In vitro investigation of cytochrome P450-mediated metabolism of dietary flavonoids. Food Chem. Toxicol. 40, 609-616. doi: 10.1016/S0278-6915(01)00125-9

\section{AUTHOR CONTRIBUTIONS}

$\mathrm{JlH}, \mathrm{AF}$, and $\mathrm{DM}$ performed the experiments. $\mathrm{CN}, \mathrm{NH}$, and SK analyzed the data. JqH, SG, PB, and WJ were involved in experiment planning and supervision. AÖ, LK, and GK supervised experimental work, and compiled and interpreted the data. LK and GK wrote the manuscript.

\section{FUNDING}

$\mathrm{JlH}$ was supported by a State Scholarship Fund of China Scholarship Council, the National Natural Science Foundation of China (No. 81202853), the Natural Science Foundation of Jiangsu Province (No. BK2012444), and Jiangsu Planned Projects for Postdoctoral Research Funds, AF by a DIKTI-OeAD fellowship, and $\mathrm{CN}$ by a technology grant (TSA Doktorat) financed by the Austrian Federal Ministry of Science and Research (BMFW) within the framework of ASEAUNINET.

\section{ACKNOWLEDGMENTS}

We wish to thank Toni Jäger for preparing the figures and Gertrud Krainz for improvements in grammar and style.

Calderone, V., Chericoni, S., Martinelli, C., Testai, L., Nardi, A., Morelli, I., et al. (2004). Vasorelaxing effects of flavonoids: investigation on the possible involvement of potassium channels. Naunyn Schmiedebergs Arch. Pharmacol. 370, 290-298.

Desai, L. P., White, S. R., and Waters, C. M. (2009). Mechanical stretch decreases FAK phosphorylation and reduces cell migration through loss of JIP3-induced JNK phosphorylation in airway epithelial cells. Am. J. Physiol. Lung Cell. Mol. Physiol. 297, 520-529. doi: 10.1152/ajplung.00076.2009

Ding, S. M., Zhang, Z. H., Song, J., Cheng, X. D., Jiang, J., and Jia, X. B. (2014). Enhanced bioavailability of apigenin via preparation of a carbon nanopowder solid dispersion. Int. J. Nanomed. 9, 2327-2333. doi: 10.2147/IJN. S60938

Dioscurides, P. (1st century A.D.). De Materia Medica, Copy from the 6th Century, Konstantinopel; Since 16th Century (by Emperor Ferdinand I) at the Department of Manuscripts and Rare Books Collection of the Austrian National Library. Vienna: Codex Vindobonensis med. Gr. 1.

Erdogan, A., Most, A. K., Wienecke, B., Fehsecke, A., Leckband, C., Voss, R., et al. (2007). Apigenin-induced nitric oxide production involves calcium-activated potassium channels and is responsible for antiangiogenic effects. J. Thromb. Haemost. 5, 1774-1781. doi: 10.1111/j.1538-7836.2007.02615.x

Galindez, J. D., Lanza, A. M. D., and Matellano, L. F. (2002). Biologically active substances from the genus Scrophularia. Pharm. Biol. 40, 45-99. doi: 10.1076/ phbi.40.1.45.5864

Gasiorowska, J., Teisseyre, A., Uryga, A., and Michalak, K. (2012). The influence of 8-prenylnaringenin on the activity of voltage-gated Kv1.3 potassium channels in human Jurkat T cells. Cell. Mol. Biol. Lett. 17, 559-570. doi: 10.2478/s11658012-0029-0

Giessrigl, B., Yazici, G., Teichmann, M., Kopf, S., Ghassemi, S., Atanasov, A. G., et al. (2012). Effects of Scrophularia extracts on tumor cell proliferation, death and intravasation through lymphoendothelial cell barriers. Int. J. Oncol. 40, 2063-2074. doi: 10.3892/ijo.2012.1388

Hui, C., Qi, X., Qianyong, Z., Xiaoli, P., Jundong, Z., and Mantian, M. (2013). Flavonoids, flavonoid subclasses and breast cancer risk: a meta-analysis of epidemiologic studies. PLoS One 8:e54318. doi: 10.1371/journal.pone. 0054318 
Jiang, H., Xia, Q., Wang, X., Song, J., and Bruce, I. C. (2005). Luteolin induces vasorelaxion in rat thoracic aorta via calcium and potassium channels. Pharmazie 60, 444-447.

Juncker-Jensen, A., Deryugina, E. I., Rimann, I., Zajac, E., Kupriyanova, T. A., Engelholm, L. H., et al. (2013). Tumor MMP-1 activates endothelial PAR1 to facilitate vascular intravasation and metastatic dissemination. Cancer Res. 73, 4196-4211. doi: 10.1158/0008-5472.CAN-12-4495

Karin, M. (2006). Nuclear factor-kappaB in cancer development and progression. Nature 441, 431-436. doi: 10.1038/nature04870

Karin, M., and Greten, F. R. (2005). NF-kappaB: linking inflammation and immunity to cancer development and progression. Nat. Rev. Immunol. 5, 749-759. doi: 10.1038/nri1703

Kerjaschki, D., Bago-Horvath, Z., Rudas, M., Sexl, V., Schneckenleithner, C., Wolbank, S., et al. (2011). Lipoxygenase mediates invasion of intrametastatic lymphatic vessels and propagates lymph node metastasis of human mammary carcinoma xenografts in mouse. J. Clin. Invest. 121, 2000-2012. doi: 10.1172/ JCI44751

Kim, H. J., Lee, S. B., Park, S. K., Kim, H. M., Park, Y. I., and Dong, M. S. (2005). Effects of hydroxyl group numbers on the B-ring of 5,7-dihydroxyflavones on the differential inhibition of human CYP 1A and CYP1B1 enzymes. Arch. Pharm. Res. 28, 1114-1121. doi: 10.1007/BF02972971

Lee, S. T., Li, Z., Wu, Z., Aau, M., Guan, P., Karuturi, R. K., et al. (2011). Contextspecific regulation of NF- $\kappa \mathrm{B}$ target gene expression by EZH2 in breast cancers. Mol. Cell 43, 798-810. doi: 10.1016/j.molcel.2011.08.011

Lewenhofer, V., Schweighofer, L., Ledermüller, T., Eichsteininger, J., Kählig, H., Zehl, M., et al. (2018). Chemical composition of Scrophularia lucida and the effects on tumor invasiveness in vitro. Front. Pharmacol.

Lin, D., Kuang, G., Wan, J., Zhang, X., Li, H., Gong, X., et al. (2017). Luteolin suppresses the metastasis of triple-negative breast cancer by reversing epithelial-to-mesenchymal transition via downregulation of $\beta$-catenin expression. Oncol. Rep. 37, 895-902. doi: 10.3892/or.2016.5311

Lindenmeyer, F., Li, H., Menashi, S., Soria, C., and Lu, H. (2001). Apigenin acts on the tumor cell invasion process and regulates protease production. Nutr. Cancer 39, 139-147. doi: 10.1207/S15327914nc391_19

Liu, Y., Xu, X. H., Liu, Z., Du, X. L., Chen, K. H., Xin, X., et al. (2012). Effects of the natural flavone trimethylapigenin on cardiac potassium currents. Biochem. Pharmacol. 84, 498-506. doi: 10.1016/j.bcp.2012.05.002

Murray, A. T. (1924). The Iliad, English trans. Homer Ph.D. in two volumes. Cambridge, MA: Harvard University Press.

Murray, I. A., Patterson, A. D., and Perdew, G. H. (2014). Aryl hydrocarbon receptor ligands in cancer: friend and foe. Nat. Rev. Cancer 14, 801-814. doi: $10.1038 / \mathrm{nrc} 3846$

Nguyen, C. H., Brenner, S., Huttary, N., Atanasov, A. G., Dirsch, V. M., Chatuphonprasert, W., et al. (2016a). AHR/CYP1A1 interplay triggers lymphatic barrier breaching in breast cancer spheroids by inducing 12(S)-HETE synthesis. Hum. Mol. Genet. 25, 5006-5016. doi: $10.1093 / \mathrm{hmg} / \mathrm{ddw} 329$

Nguyen, C. H., Brenner, S., Huttary, N., Li, Y., Atanasov, A. G., Dirsch, V. M., et al. (2016b). 12(S)-HETE increases intracellular $\mathrm{Ca}^{2+}$ in lymph-endothelial cells disrupting their barrier function in vitro; stabilization by clinical drugs impairing calcium supply. Cancer Lett. 380, 174-183. doi: 10.1016/j.canlet.2016. 06.022

Nguyen, C. H., Huttary, N., Atanasov, A. G., Chatuphonprasert, W., Brenner, S., Fristiohady, A., et al. (2017). Fenofibrate inhibits tumor intravasation by several independent mechanisms in a 3-dimensional co-culture model. Int. J. Oncol. 50, 1879-1888. doi: 10.3892/ijo.2017.3956

Nguyen, C. H., Senfter, D., Basilio, J., Holzner, S., Stadler, S., Krieger, S., et al. (2015). NF-кB contributes to MMP1 expression in breast cancer spheroids causing paracrine PAR1 activation and disintegrations in the lymph endothelial barrier in vitro. Oncotarget 6, 39262-39275. doi: 10.18632/oncotarget. 5741

Nguyen, C. H., Stadler, S., Brenner, S., Huttary, N., Krieger, S., Jäger, W., et al. (2016c). Cancer cell-derived 12(S)-HETE signals via 12-HETE receptor, RHO, ROCK and MLC2 to induce lymph endothelial barrier breaching. Br. J. Cancer 115, 364-370. doi: 10.1038/bjc.2016.201

Qin, Y., Zhao, D., Zhou, H. G., Wang, X. H., Zhong, W. L., Chen, S., et al. (2016). Apigenin inhibits NF- $\mathrm{B}$ and snail signaling, EMT and metastasis in human hepatocellular carcinoma. Oncotarget 7, 41421-41431. doi: 10.18632/ oncotarget.9404
Roberts, R. E., Allen, S., Chang, A. P., Henderson, H., Hobson, G. C., Karania, B., et al. (2013). Distinct mechanisms of relaxation to bioactive components from chamomile species in porcine isolated blood vessels. Toxicol. Appl. Pharmacol. 272, 797-805. doi: 10.1016/j.taap.2013.06.021

Schwock, J., Dhani, N., and Hedley, D. W. (2010). Targeting focal adhesion kinase signaling in tumor growth and metastasis. Expert Opin. Ther. Targets 14, 77-94. doi: $10.1517 / 14728220903460340$

Sobin, L. H., Gospodarowicz, M. K., and Wittekind, C. (eds). (2009). TNM Classification of Malignant Tumours (UICC International Union against Cancer), 7th Edn. New York, NY: Wiley-Blackwell.

Stadler, S., Nguyen, C. H., Schachner, H., Milovanovic, D., Holzner, S., Brenner, S., et al. (2017). Colon cancer cell-derived 12(S)-HETE induces the retraction of cancer-associated fibroblast via MLC2, $\mathrm{RHO} / \mathrm{ROCK}$ and $\mathrm{Ca}^{2+}$ signaling. Cell. Mol. Life Sci. 74, 1907-1921. doi: 10.1007/s00018-016-2441-5

Teichmann, M., Kretschy, N., Kopf, S., Jarukamjorn, K., Atanasov, A. G., Viola, K., et al. (2014). Inhibition of tumor spheroid induced prometastatic intravasation gates in the lymph endothelial cell barrier by carbamazepine: drug testing in a 3D model. Arch. Toxicol. 88, 691-699.

Teisseyre, A., and Michalak, K. (2005). Genistein inhibits the activity of kv1.3 potassium channels in human T lymphocytes. J. Membr. Biol. 205, 71-79. doi: 10.1007/s00232-005-0764-5

Tsai, P. H., Cheng, C. H., Lin, C. Y., Huang, Y. T., Lee, L. T., Kandaswami, C. C., et al. (2016). Dietary flavonoids luteolin and quercetin suppressed cancer stem cell properties and metastatic potential of isolated prostate cancer cells. Anticancer Res. 36, 6367-6380. doi: 10.21873/anticanres.11234

Viola, K., Kopf, S., Huttary, N., Vonach, C., Kretschy, N., Teichmann, M., et al. (2013). Bay11-7082 inhibits the disintegration of the lymphendothelial barrier triggered by MCF-7 breast cancer spheroids; the role of ICAM-1 and adhesion. Br. J. Cancer 108, 564-569. doi: 10.1038/bjc.2012.485

Vogiatzoglou, A., Mulligan, A. A., Lentjes, M. A., Luben, R. N., Spencer, J. P., Schroeter, H., et al. (2015). Flavonoid intake in European adults (18 to 64 years). PLoS One 10:e0128132. doi: 10.1371/journal.pone.0128132

Vonach, C., Viola, K., Giessrigl, B., Huttary, N., Raab, I., Kalt, R., et al. (2011). NF-кB mediates the 12(S)-HETE-induced endothelial to mesenchymal transition of lymphendothelial cells during the intravasation of breast carcinoma cells. Br. J. Cancer 105, 263-271. doi: 10.1038/bjc.2011.194

Wei, J. F., Wei, L., Zhou, X., Lu, Z. Y., Francis, K., Hu, X. Y., et al. (2008). Formation of Kv2.1-FAK complex as a mechanism of FAK activation, cell polarization and enhanced motility. J. Cell Physiol. 217, 544-557. doi: 10.1002/jcp.21530

Woo, H. D., and Kim, J. (2013). Dietary flavonoid intake and risk of stomach and colorectal cancer. World J. Gastroenterol. 19, 1011-1019. doi: 10.3748/wjg.v19. i7. 1011

Wu, H. J., Wu, W., Sun, H. Y., Qin, G. W., Wang, H. B., Wang, P., et al. (2011). Acacetin causes a frequency- and use-dependent blockade of hKv1.5 channels by binding to the S6 domain. J. Mol. Cell. Cardiol. 51, 966-973. doi: 10.1016/j. yjmcc.2011.08.022

Zhao, G., Han, X., Cheng, W., Ni, J., Zhang, Y., Lin, J., et al. (2017). Apigenin inhibits proliferation and invasion, and induces apoptosis and cell cycle arrest in human melanoma cells. Oncol. Rep. 37, 2277-2285. doi: 10.3892/or.2017.5450

Zhou, P., Li, L. P., Luo, S. Q., Jiang, H. D., and Zeng, S. (2008). Intestinal absorption of luteolin from peanut hull extract is more efficient than that from individual pure luteolin. J. Agric. Food Chem. 56, 296-300. doi: 10.1021/jf072612+

Zhu, B. H., Ma, L., Pan, X. D., Huang, Y. L., and Liu, J. (2008). Scutellarin induced $\mathrm{Ca}^{2+}$ release and blocked KCl-induced $\mathrm{Ca}^{2+}$ influx in smooth muscle cells isolated from rat thoracic artery. J. Asian Nat. Prod. Res. 10, 583-589. doi: $10.1080 / 10286020801966633$

Conflict of Interest Statement: The authors declare that the research was conducted in the absence of any commercial or financial relationships that could be construed as a potential conflict of interest.

Copyright (C) 2018 Hong, Fristiohady, Nguyen, Milovanovic, Huttary, Krieger, Hong, Geleff, Birner, Jäger, Özmen, Krenn and Krupitza. This is an open-access article distributed under the terms of the Creative Commons Attribution License (CC BY). The use, distribution or reproduction in other forums is permitted, provided the original author(s) and the copyright owner are credited and that the original publication in this journal is cited, in accordance with accepted academic practice. No use, distribution or reproduction is permitted which does not comply with these terms. 


\section{OPEN ACCESS}

Edited by:

Atanas G. Atanasov,

Institute of Genetics and Animal

Breeding (PAS), Poland

Reviewed by:

Zhaojun Wei,

Hefei University of Technology, China

Claudio Ferrante,

Università degli Studi "G. d'Annunzio"

Chieti-Pescara, Italy

*Correspondence:

Laurian Vlase

laurian.vlase@umfluj.ro

†Shared first authorship

Specialty section:

This article was submitted to

Ethnopharmacology,

a section of the journal

Frontiers in Pharmacology

Received: 15 December 2017

Accepted: 04 January 2018

Published: 02 March 2018

Citation:

Toiu A, Mocan A, Vlase L, Pârvu AE, Vodnar DC, Gheldiu A-M,

Moldovan C and Oniga I (2018)

Phytochemical Composition,

Antioxidant, Antimicrobial and in Vivo

Anti-inflammatory Activity

of Traditionally Used Romanian Ajuga laxmannii (Murray) Benth.

("Nobleman's Beard" - Barba

Împăratului). Front. Pharmacol. 9:7.

doi: 10.3389/fphar.2018.00007

\section{Phytochemical Composition,} Antioxidant, Antimicrobial and in Vivo Anti-inflammatory Activity of Traditionally Used Romanian Ajuga laxmannii (Murray) Benth. ("Nobleman's Beard" - Barba Împăratului)

Anca Toiu ${ }^{1 \dagger}$, Andrei Mocann', Laurian Vlase ${ }^{3 *}$, Alina E. Pârvu ${ }^{4}$, Dan C. Vodnar ${ }^{5}$, Ana-Maria Gheldiu ${ }^{3}$, Cadmiel Moldovan ${ }^{2}$ and llioara Oniga ${ }^{1}$

\footnotetext{
'Department of Pharmacognosy, "Uliu Haţieganu" University of Medicine and Pharmacy, Cluj-Napoca, Romania, 2 Department of Pharmaceutical Botany, "Iuliu Haţieganu" University of Medicine and Pharmacy, Cluj-Napoca, Romania, ${ }^{3}$ Department of Pharmaceutical Technology and Biopharmacy, "Illiu Haţieganu" University of Medicine and Pharmacy, Cluj-Napoca, Romania, " Department of Pathophysiology, "Iuliu Hațieganu" University of Medicine and Pharmacy, Cluj-Napoca, Romania, ${ }^{5}$ Department of Food Sciences, Faculty of Food Science and Technology, University of Agricultural Sciences and Veterinary Medicine, Cluj-Napoca, Romania
}

In the Romanian folk medicine, aerial parts of Ajuga laxmannii ("nobleman's beard," Romanian - "barba boierului" or "avrămească" or "creştinească") are traditionally used as galactagogue and anti-inflammatory agents. The present study aimed to evaluate the chemical composition (polyphenols, iridoids, and phytosterols), antioxidant, antimicrobial and in vivo anti-inflammatory activity of different extracts of $A$. laxmannii aerial parts. The major identified bioactive compounds were rutin, 8-O-acetylharpagide and $\beta$-sitosterol. The antioxidant activity of $A$. laxmannii extracts was evaluated using several methods, and the results showed good antiradical effects. Moreover, the antimicrobial evaluation showed a potent antifungal activity against C. albicans and P. funiculosum. Furthermore, the anti-inflammatory effect was determined by monitoring some parameters involved in the inflammatory process. The results obtained showed differences between the analyzed extracts; and therefore the importance of choosing the best solvent in order to extract the appropriate amount of bioactive compounds. A. laxmannii ethanol extract showed an anti-inflammatory effect by reducing total leukocytes, PMN, phagocytosis, and oxidative stress. Compared to diclofenac, only the $50 \mathrm{mg} / \mathrm{mL}$ A. laxmannii extract had better anti-inflammatory and anti-oxidative stress effects, and this could justify the importance of a correlation between the activity and the used concentration. These findings strongly suggest that $A$. laxmannii could be considered as a valuable source of bioactive compounds, which could be further valued as anti-inflammatory agents in the composition of several herbal drugs.

Keywords: Ajuga laxmannii, polyphenols, iridoids, sterols, anti-inflammatory, antimicrobial 


\section{INTRODUCTION}

Medicinal plants have proven their value as sources of molecules with therapeutic potential, and still represent an important pool for the identification of novel drug leads (Atanasov et al., 2015). Moreover, nowadays herbal medicines have received great scientific interest because they provide both important biomolecules which are used in the treatment of several diseases, as well as a broad spectrum of long-term use and safety. Nevertheless, plant secondary metabolites are excellent candidates for developing new phytopharmaceuticals with various biological activities, including antioxidant, antimicrobial, and anti-inflammatory (Shahidi and Ambigaipalan, 2015; Liao et al., 2017; Zhang et al., 2017).

In many parts of the world such as Europe, Asia, Africa, South America, plants and/or plant compounds are used to cure or prevent different diseases, being in the same time part of the cultural inheritage of different nations or ethnicities. Still, herbal medicines were used and applied only based on traditional knowledge and empirical observations of "indigenous healers," without elucidating the chemical composition of the used extracts or mechanistic knowledge of their pharmacological activities and main bioactive compounds (Atanasov et al., 2015). Often, the empirical value of medicinal plants is seen as a proof of effectiveness and safety, and still many plant preparations are traditionally used without having a certain scientific proof of their pharmacological effects or long-term safety and efficacy.

Phytochemicals are biomolecules that occur in herbal drugs or phytopharmaceuticals, and possess the ability to modulate one or more metabolic processes or pathways in the human organism resulting in health benefits and promotion of well-being (Abuajah et al., 2014; Ferrante et al., 2017; Locatelli et al., 2017). Within this frame, new studies on uninvestigated traditionally used medicinal plants or plant products provide remarkable interest for the development of novel herbal drugs or herbal formulations.

The genus Ajuga part of the Lamiaceae family comprises 50 species, and about 300 taxa (including subspecies and varieties), distributed in Asia, Africa, Australia, North America, and Europe (Riaz et al., 2007; Atay et al., 2016). Some Ajuga species are used in folk medicine for the treatment of diabetes, inflammation, malaria, high blood pressure, pain, fever, and as antihelmintic (Cocquyt et al., 2011). Moreover, several studies have been carried out on Ajuga species indicating pharmacological activities such as antimalarial (Njoroge and Bussmann, 2006; Cocquyt et al., 2011), hypoglycemic (El-Hilaly et al., 2006), anti-inflammatory (Gautam et al., 2011), anti-arthritis, antipyretic, anabolic, antibacterial, hepatoprotective, antifungal, antioxidant, cardiotonic (Israili and Lyoussi, 2009), and analgesic properties (Ono et al., 2008).

Additionally, several phytochemical studies on Ajuga species have shown the presence of iridoids, diterpenes, phytoecdysteroids, flavonoids, sterol glycosides and phenylethanoid glycosides as the main secondary metabolites of the genus (Manguro et al., 2006, 2011; Ono et al., 2011; Atay et al., 2016).

Ajuga laxmannii (Murray) Benth. is an erect rhizomatous pubescent herb that belongs to the genus Ajuga, growing in the grasslands of Romania and other parts of Europe. In Romania, the local name for this species is "nobleman's beard" (Romanian "barba boierului" or "avrămească" or "creştinească"), and it is used as a galactogogue and anti-inflammatory agent. To the best of our knowledge, there is only one study on A. laxmannii (Murray) Benth. regarding its antiprotozoal activity (Atay et al., 2016).

Within this frame, the aim of this study was to perform a phytochemical analysis of $A$. laxmannii aerial parts extracts, particularly polyphenolic compounds, iridoids and phytosterols, and to investigate the antioxidant, antimicrobial and antiinflammatory activities.

\section{MATERIALS AND METHODS}

\section{Chemicals}

The standards used were chlorogenic acid, p-coumaric acid, caffeic acid, rutin, apigenin, quercetin, isoquercitrin, hyperoside, kaempferol, quercetol, myricetol, fisetin, gallic acid, aucubin, catalpol, harpagoside, $\beta$-sitosterol, brassicasterol, stigmasterol, campesterol and ergosterol from Sigma-Aldrich (Germany), ferulic acid, sinapic acid, gentisic acid, patuletin, luteolin from Roth (Germany), caftaric acid from Dalton (United States), harpagide, 8-O-acetyl-harpagide from PhytoLab GmbH \& Co. (Germany). Methanol of HPLC analytical-grade, acetonitrile of HPLC analytical-grade, ammonium acetate of HPLC analytical-grade, silver nitrate of HPLC analytical-grade, chloroform, petroleum ether, $n$-hexane, potassium hydroxide of analytical-grade and hydrochloric acid of analytical-grade, acetic acid of analytical-grade, Folin-Ciocâlteu reagent were purchased from Merck (Germany). Sodium carbonate, Copper (II) sulfate pentahydrate, sodium acetate trihydrate and anhydrous aluminum chloride were acquired from SigmaAldrich (Germany). The DPPH (2,2-diphenyl-1-picrylhydrazyl), Trolox (6-hydroxy-2,5,7,8-tetramethylchroman-2-carboxylic acid) were obtained from Alfa-Aesar (Karlsruhe, Germany), HRP (horseradish peroxidase), Fremy's salt were purchased from Sigma-Aldrich (Germany).

Methanolic stock solutions $(100 \mathrm{mg} / \mathrm{mL})$ of the flavonoid standards were prepared and stored at $4{ }^{\circ} \mathrm{C}$, protected from daylight. They were appropriately diluted with double distilled water before being used as working solutions. Methanolic stock solutions of iridoids $(1 \mathrm{mg} / \mathrm{mL})$ were prepared and stored at $4^{\circ} \mathrm{C}$, protected from daylight. They were appropriately diluted with double distilled water before being used as working solutions. Chloroform stock solutions $(1 \mathrm{mg} / \mathrm{mL})$ of the phytosterol standards were prepared and stored at $4{ }^{\circ} \mathrm{C}$, protected from daylight. Before being used as working solutions, they were appropriately diluted with acetonitrile. Distilled, deionised water was produced by a Direct Q-5 Millipore (Millipore SA, Molsheim, France) water system.

\section{Plant Sample and Extraction Procedure}

Ajuga laxmannii aerial parts were collected from Cluj County (Romania) spontaneous flora during flowering stage in June 2015. The air-dried powder of herbal material was extracted with different solvents. The methanol extract was obtained from $5 \mathrm{~g}$ 
herbal material and $50 \mathrm{~mL} \mathrm{70 \%} \mathrm{methanol} \mathrm{for} 30 \mathrm{~min}$ on a water bath at $60^{\circ} \mathrm{C}$ (Methanol Extract, ME). The $10 \%$ tincture was prepared at room temperature from $100 \mathrm{~g}$ of herbal material and $1000 \mathrm{~mL} \mathrm{70 \%} \mathrm{ethanol} \mathrm{by} \mathrm{maceration} \mathrm{at} \mathrm{room} \mathrm{temperature}$ for 7 days (Ethanol Extract, EE) as previously described by Toiu et al. (2017). In order to evaluate the anti-inflammatory activity of $A$. laxmannii aerial parts, three extracts were used: A. laxmannii ethanol extract $100 \%$ (100 mg dry weight herbal material $/ \mathrm{mL}$ ), A. laxmannii ethanol extract $50 \%$ (50 mg dw herbal material $/ \mathrm{mL}$ ), and A. laxmannii ethanol extract 25\% (25 mg dw herbal material $/ \mathrm{mL}$ ).

For identification and quantification of phytosterols, and for determination of antifungal properties, chloroform (CE) and petroleum ether extracts (PEE) were also prepared. Thus, $2.5 \mathrm{~g}$ of herbal material were extracted with $25 \mathrm{~mL}$ chloroform and $25 \mathrm{~mL}$ petroleum ether, respectively, for $30 \mathrm{~min}$ in a sonication bath at $60^{\circ} \mathrm{C}$. The extracts were cooled down, and then centrifuged at $4500 \mathrm{rpm}$ for $15 \mathrm{~min}$, and the supernatant was recovered (Mocan et al., 2014).

\section{Quantitative Determinations of Total Bioactive Compounds Total Phenolic Content (TPC)}

The total phenolic content (TPC) of the A. laxmannii extracts was determined photometrically, by Folin-Ciocâlteu method with slight modifications (Tămaş et al., 2009). Briefly, $2 \mathrm{~mL}$ of each ethanolic and methanolic extracts were diluted 25 times, then mixed with Folin-Ciocâlteu reagent $(1 \mathrm{~mL})$ and distilled water $(10.0 \mathrm{~mL})$, and further diluted to $25 \mathrm{~mL}$ with a $290 \mathrm{~g} / \mathrm{L}$ solution of sodium carbonate. The samples were incubated in the dark for $30 \mathrm{~min}$. The absorbance was measured at $760 \mathrm{~nm}$, using a JASCO V-530 (Jasco International Co., Ltd., Tokyo, Japan) UV-VIS spectrophotometer. TPC values were expressed as gallic acid equivalents $\left(R^{2}=0.999\right)$, mg gallic acid/g dry weight herbal material (mg GAE/g dw herbal material). Assay was performed in triplicate.

\section{Total Flavonoid Content (TFC)}

The total flavonoid content (TFC) of the A. laxmannii extracts was calculated and expressed as rutin equivalents after the method previously described (Mocan et al., 2014). Briefly, each extract (EE and ME) (5 mL) was mixed with sodium acetate $(5.0 \mathrm{~mL}, 100 \mathrm{~g} / \mathrm{L})$, aluminum chloride $(3.0 \mathrm{~mL}, 25 \mathrm{~g} / \mathrm{L})$, and mixed up to $25 \mathrm{~mL}$ in a calibrated flask with methanol. The absorbance was measured at $430 \mathrm{~nm}$. The TFC was expressed as rutin equivalents $\left(R^{2}=0.999\right), \mathrm{mg}$ rutin equivalents/g dry weight herbal material (mg RE/g dw herbal material). Assay was performed in triplicate.

\section{Total Iridoid Content (TIC)}

The total iridoid content (TIC) of the A. laxmannii extracts was determined by a photometric method based on a Trim-Hill reaction and the results were expressed as aucubin equivalents (mg AE/g dw herbal material). Each extract $(0.4 \mathrm{~mL})$ was mixed with $4 \mathrm{~mL}$ of Trim-Hill reagent (acetic acid $-0.2 \%$ $\mathrm{CuSO}_{4}$ - conc. $\left.\mathrm{HCl}, 10: 1: 0.5\right)$, afterwards the absorbance was measured at $609 \mathrm{~nm}$, and the blue color indicated the presence of iridoids. The amount of iridoids was calculated using an aucubin $(0.1-1 \mathrm{mg} / \mathrm{mL})$ calibration curve $\left(R^{2}=0.999\right)$ (Erdenechimeg et al., 2017). Assay was performed in triplicate.

\section{Antioxidant Capacity Assays}

For testing the antioxidant capacity, the A. laxmannii extracts were further tested using three different assays.

\section{DPPH Radical Scavenging Activity}

The effect of the A. laxmannii extracts against the 2,2diphenyl-1-picrylhydrazyl (DPPH) radical was tested according to Savran et al. (2016). Two milliliters of sample solution (2 mL, $3.75-30 \mu \mathrm{g} / \mathrm{mL}$ ) was added to $2 \mathrm{~mL}$ of a $0.1 \mathrm{mg} / \mathrm{mL}$ $\mathrm{DPPH}$ methanol solution. After $30 \mathrm{~min}$ of incubation at room temperature in the dark, the absorbance was read at $517 \mathrm{~nm}$. The DPPH radical scavenging activity was expressed as $\mathrm{IC}_{50}$ $(\mu \mathrm{g} / \mathrm{mL})$. The percentage of DPPH consumption was converted to trolox equivalents (TE) using a calibration curve $\left(R^{2}=0.985\right)$ of Trolox standard solutions $(0.5-5 \mu \mathrm{g} / \mathrm{mL})$. Considering the results, an $\mathrm{IC}_{50}<50 \mu \mathrm{g} \mathrm{TE} / \mathrm{mL}$ shows a very good antioxidant potential; an $\mathrm{IC}_{50}$ of $50-100 \mu \mathrm{g} \mathrm{TE} / \mathrm{mL}$ displays a good antioxidant potential; an $\mathrm{IC}_{50}$ of $100-200 \mu \mathrm{g} \mathrm{TE} / \mathrm{mL}$ reveals a weak antioxidant potential; an $\mathrm{IC}_{50}>200 \mu \mathrm{g} \mathrm{TE} / \mathrm{mL}$ means no antioxidant potential (Araniciu et al., 2014). Assay was performed in triplicate.

\section{ABTS Radical Cation Scavenging Activity}

The scavenging activity of the A. laxmannii ethanol extract against the ABTS radical cation (2,2'-azino-bis(3-ethylbenzothiazoline)-6-sulphonic acid) was measured according to Zengin et al. (2014). ABTS $^{\bullet}+$ was produced directly by reacting $7 \mathrm{mM}$ ABTS solution with $2.45 \mathrm{mM}$ potassium persulfate and allowing the mixture to stand for $12-16 \mathrm{~h}$ in the dark at room temperature. Prior to beginning the assay, ABTS solution was diluted with methanol to an absorbance of $0.700 \pm 0.02$ at $734 \mathrm{~nm}$. Sample solution was added to ABTS solution $(2 \mathrm{~mL})$ and mixed. After $30 \mathrm{~min}$ incubation at room temperature, the sample absorbance was read at $734 \mathrm{~nm}$. The ABTS radical cation scavenging activity was expressed as milligrams of TE per gram of extract (mg TE/g). Assay was performed in triplicate.

\section{Electron Paramagnetic Resonance (EPR) Spectroscopy}

In order to compare with the results of the spectrophotometric measurements, the radical scavenging activity of the A. laxmannii ethanol extract was additionally measured with EPR spectrometry using the stable synthetic radical Fremy's salt as reported previously (Barakat and Rohn, 2014; MoussaAyoub et al., 2014). A mixture of $25 \mathrm{~mL}$ of the diluted extract and a $25 \mathrm{~mL}$ of a $1 \mathrm{mM}$ Fremy's salt solution [potassium nitrosodisulfonate, $\mathrm{K}_{2} \mathrm{NO}\left(\mathrm{SO}_{3}\right)_{2}$ ] in phosphate buffer ( $\mathrm{pH} 7.4$ ) were filled into a $50 \mathrm{~mL}$ capillary. After a reaction time of $30 \mathrm{~min}$, the EPR spectrum of Fremy's salt radical was recorded and intensity was obtained by integration of the signal. The antioxidant activity of the extract, expressed as milligrams of Fremy's salt equivalents per gram dry weight (mg FSE/g 
$\mathrm{dw}$ ), was calculated in comparison to a control reaction with the solvent. Spectra were recorded at $21^{\circ} \mathrm{C}$ on a Miniscope MS 200 X-band spectrometer (Magnettech $\mathrm{GmbH}$, Berlin, Germany) with the microwave power set to $10 \mathrm{~dB}$ and using modulation amplitude of $1500 \mathrm{mG}$. Assay was performed in triplicate.

\section{Identification and Quantification of Polyphenolic Compounds}

\section{General Apparatus and Chromatographic Conditions}

An Agilent 1100 HPLC Series system was used (Agilent Technologies, Darmstadt, Germany), coupled with an Agilent Ion Trap SL mass spectrometer equipped with an electrospray or APCI ion source.

\section{Chromatographic Conditions for the Analysis of Polyphenolic Compounds}

The experiment was carried out using an Agilent 1100 HPLC Series system (Agilent, United States) equipped with degasser, binary gradient pump, column thermostat, autosampler and UV detector. The HPLC system was coupled with an Agilent 1100 mass spectrometer (LC/MSD Ion Trap VL). For the separation, a reverse-phase analytical column was employed (Zorbax SB-C18 $100 \mathrm{~mm} \times 3.0 \mathrm{~mm}$ i.d., $3.5 \mu \mathrm{m}$ particle); the work temperature was $48^{\circ} \mathrm{C}$. The detection of the compounds was performed on both UV and MS mode. The UV detector was set at $330 \mathrm{~nm}$ until $17.5 \mathrm{~min}$, then at $370 \mathrm{~nm}$. The MS system operated using an electrospray ion source in negative mode. The chromatographic data were processed using ChemStation and Data Analysis software from Agilent, United States.

The mobile phase was a binary gradient prepared from methanol and solution of $0.1 \%$ acetic acid $(v / v)$. The elution started with a linear gradient, beginning with $5 \%$ methanol and ending at $42 \%$ methanol, for $35 \mathrm{~min}$; isocratic elution followed for the next 3 min with $42 \%$ methanol. The flow rate was $1 \mathrm{~mL} / \mathrm{min}$ and the injection volume was $5 \mu \mathrm{L}$.

The MS signal was used only for qualitative analysis based on specific mass spectra of each compound. The MS spectra obtained from a standard solution of polyphenols were integrated in a mass spectra library. Later, the MS traces/spectra of the compounds from analyzed samples were compared to spectra from library, which allows positive identification of each substance, based on spectral match. The UV trace was used for quantification of identified compounds from MS detection. Using the chromatographic conditions described above, the polyphenols eluted in less than $35 \mathrm{~min}$. Four polyphenols cannot be quantified in current chromatographic conditions due overlapping (caftaric acid with gentisic acid and caffeic acid with chlorogenic acid). However, all four compounds can be selectively identified in MS detection (qualitative analysis) based on differences between their molecular mass and MS spectra. The detection limits were calculated as minimal concentration producing a reproductive peak with a signal-to-noise ratio greater than three. Quantitative determinations were performed using an external standard method. Calibration curves in the range of $0.5-50 \mu \mathrm{g} / \mathrm{mL}$ range with good linearity $\left(R^{2}>0.999\right)$ for a five point plot were used to determine the concentration of polyphenols in plant samples.

\section{Mass Spectrometry Analyses}

The detection and quantification of polyphenols was made in UV assisted by mass spectrometry. Due peak overlapping, four polyphenol-carboxylic acids (caftaric, gentisic, caffeic, and chlorogenic) were determined only based on MS spectra, whereas for the rest of compounds the linearity of calibration curves was very good $\left(R^{2}>0.998\right)$, with detection limits in the range of 18 to $92 \mathrm{ng} / \mathrm{mL}$. The detection limits were calculated as minimal concentration producing a reproductive peak with a signalto-noise ratio greater than three. Quantitative determinations were performed using an external standard method; retention times were determined with a standard deviation ranging from 0.04 to $0.19 \mathrm{~min}$. Accuracy was checked by spiking samples with a solution containing each polyphenol in a concentration of $10 \mu \mathrm{g} / \mathrm{mL}$. In all analyzed samples, the compounds were identified by comparison of their retention times and the recorded electrospray mass spectra with those of standards in the same chromatographic conditions (Vlase et al., 2013; Andriamadio et al., 2015).

\section{Identification and Quantification of Phytosterols \\ Chromatographic Conditions for the Analysis of Phytosterols}

Compounds were separated using a Zorbax SB-C18 reversedphase analytical column $(100 \mathrm{~mm} \times 3.0 \mathrm{~mm}$ i.d., $5 \mu \mathrm{m}$ particle $)$ fitted with a guard column Zorbax SB-C18, both operated at $40^{\circ} \mathrm{C}$. Sterols were separated under isocratic conditions using a mobile phase consisting of 10:90 $(v / v)$ methanol and acetonitrile. The flow rate was $1 \mathrm{~mL} / \mathrm{min}$ and the injection volume was $5 \mu \mathrm{L}$. Mass spectrometry analysis was performed on an Agilent Ion Trap 1100 VL mass spectrometer with atmospheric pressure chemical ionization (APCI) interface. The instrument was operated in positive ion mode. Operating conditions were optimized in order to achieve maximum sensitivity values: gas temperature (nitrogen) $325^{\circ} \mathrm{C}$ at a flow rate of $7 \mathrm{~L} / \mathrm{min}$, nebulizer pressure $60 \mathrm{psi}$ and capillary voltage $-4000 \mathrm{~V}$.

The identification of sterols was performed by comparing the retention times and mass spectra with those of standards in the same chromatographic conditions. To avoid or limit the interference from background, the multiple reactions monitoring analysis mode was used instead of single ion monitoring (e.g., MS/MS instead of MS). Linearity of calibration curves was very good $\left(R^{2}>0.998\right)$, with detection limits in the range of 69 to $3312 \mathrm{ng} / \mathrm{mL}$ for ergosterol, 62 to $2952 \mathrm{ng} / \mathrm{mL}$ for brassicasterol, 59 to $2808 \mathrm{ng} / \mathrm{mL}$ for campesterol, 136 to $6528 \mathrm{ng} / \mathrm{mL}$ for stigmasterol, and 132 to $6336 \mathrm{ng} / \mathrm{mL}$ for $\beta$-sitosterol. The results are expressed as $\mu \mathrm{g}$ per $\mathrm{mL}$ of extract $(\mu \mathrm{g} / \mathrm{mL})$.

The software ChemStation (vA09.03) and DataAnalysis (v5.3) from Agilent, United States were used for the acquisition and analysis of chromatographic data (Vlase et al., 2013). 


\section{Identification and Quantification of Iridoids}

Targeted A. laxmannii iridoids (aucubin, catalpol, harpagide, harpagoside, and 8-O-acetylharpagide) were analyzed by HPLCMS on a Agilent 1100 liquid chromatography system equipped with a binary pump, autosampler, thermostat and detector (all 1100 Series from Agilent Inc., United States). The system was controlled with Data Analysis software (version B01.03, Agilent Inc., United States). The separation was carried out on an Atlantis HILIC $3.5 \mu \mathrm{m}(100 \mathrm{~mm} \times 3.0 \mathrm{~mm}$ i.d.) (Waters Inc., United States) column equipped with an online $0.2 \mu \mathrm{m}$ filter (Agilent Inc.), at a working temperature of $40^{\circ} \mathrm{C}$, a flow rate of $0.75 \mathrm{~mL} / \mathrm{min}$ and an injection volume of $8 \mu \mathrm{L}$. A binary gradient system with eluent (A) $0.1 \%$ acetic acid $(v / v)$ and $20 \mu \mathrm{M}$ sodium acetate in water, and eluent (B) $0.1 \%$ acetic acid $(v / v)$ and $20 \mu \mathrm{M}$ sodium acetate in acetonitrile was used for the analyzed samples with the following gradient: $95-80 \%$ B (0-5 min). The HPLC system was coupled with an Agilent Ion Trap 1100 SL mass spectrometer equipped with an electrospray ionisation (ESI) source and operated in the positive mode with a scan range between 360 and $680 \mathrm{~m} / z$. The newly developed LC-ESI-MS/MS method was used to identify the targeted compounds based on their sodium adducts $(M+23 \mathrm{~m} / z)$ : aucubin $(369 \mathrm{~m} / \mathrm{z})$, catalpol $(385 \mathrm{~m} / \mathrm{z})$, harpagide $(387.2 \mathrm{~m} / \mathrm{z})$, harpagoside $(517.4 \mathrm{~m} / \mathrm{z})$ and 8-O-acetylharpagide $(429.3 \mathrm{~m} / \mathrm{z})$, and by comparison with standards in the same chromatographic conditions. The capillary voltage was set to $4 \mathrm{kV}$ and the capillary temperature to $300^{\circ} \mathrm{C}$. Nitrogen $\left(\mathrm{N}_{2}\right)$ was used as dry gas with a dry flow of $12 \mathrm{~L} / \mathrm{min}$ and a pressure of $60 \mathrm{psi}$ for the nebulizer. For quantification of the iridoids, stock solutions of the five commercially available standards substances were prepared in acetonitrile, and different concentrations of each standard were used. All calibration curves yielded a coefficient of determination of $R^{2} \geq 0.990$. The results are expressed as $\mu \mathrm{g}$ per $\mathrm{mL}$ of extract $(\mu \mathrm{g} / \mathrm{mL})$. All phytochemical assays were performed in triplicate.

\section{Antibacterial Activity}

\section{Microorganisms and Culture Conditions}

For the bioassay, five aerobic bacterial strains were used, two Gram positive: Staphylococcus aureus (ATCC 49444), Listeria monocytogenes (ATCC 19114) and three gram negative: Pseudomonas aeruginosa (ATCC 27853), Salmonella typhimurium (ATCC 14028) and Escherichia coli (ATCC 25922). All of the tested microorganisms were obtained from Food Biotechnology Laboratory, Life Sciences Institute, University of Agricultural Sciences and Veterinary Medicine Cluj Napoca, Romania. The bacteria were cultured on Muller-Hinton Agar and cultures were stored at $4^{\circ} \mathrm{C}$ and subcultured once a month.

\section{Microdilution Method}

The modified microdilution technique was used to evaluate antimicrobial activity. Bacterial species were cultured overnight at $37^{\circ} \mathrm{C}$ in Tryptic Soy Broth (TSB) medium at $37^{\circ} \mathrm{C}$. The bacterial cell suspensions were adjusted with sterile saline to a concentration of approximately $2.5 \times 10^{5} \mathrm{CFU} / \mathrm{mL}$ in a final volume of $100 \mu \mathrm{L}$ per well. The inoculum was stored at $+4^{\circ} \mathrm{C}$ for further use. Dilutions of the inoculum were cultured on solid Muller-Hinton (MH) for bacteria in order to verify the absence of contamination and to check the validity of the inoculum. Determinations of minimum inhibitory concentrations (MICs) were performed by a serial dilution technique using 96-well microtitre plates. Different $A$. laxmannii ethanol extract dilutions were carried out over the wells containing $100 \mu \mathrm{L}$ of TSB and afterwards, $10 \mu \mathrm{L}$ of inoculum was added to all the wells. The microplates were incubated for $24-48 \mathrm{~h}$ at $37^{\circ} \mathrm{C}$. The MIC of the samples was detected following the addition of $20 \mu \mathrm{L}$ $(0.2 \mathrm{mg} / \mathrm{mL})$ of resazurin solution to each well, and the plates were incubated $2 \mathrm{~h}$ at $37^{\circ} \mathrm{C}$. A change from blue to pink indicates reduction of resazurin, therefore, the bacterial growth. The MIC was defined as the lowest drug concentration that prevented this color change. The minimum bactericidal concentrations (MBCs) were determined by serial subcultivation of a $2 \mu \mathrm{L}$ into microtitre plates containing $100 \mu \mathrm{L}$ of broth per well and further incubation for $48 \mathrm{~h}$ at $37^{\circ} \mathrm{C}$. The lowest concentration, with no visible growth, was defined as MBC, indicating $99.5 \%$ killing of the original inoculum. Gentamycin $(25 \mu \mathrm{L} /$ well at concentration of $4 \mu \mathrm{g} / \mathrm{mL}$ ) was used as positive control for bacterial growth. A $50 \%$ solution of ethanol in water was used as negative control. All determinations were performed in triplicate, and values are the averages of three replicates (Mocan et al., 2014).

\section{Antifungal Activity}

To investigate the antifungal activities of $A$. laxmannii extracts, the following fungi were used: Aspergillus flavus (ATCC 9643), Aspergillus niger (ATCC 6275), Candida albicans (ATCC 10231), Candida parapsilosis (ATCC 22019) and Penicillium funiculosum (ATCC 56755). These fungi were obtained from the Food Biotechnology Laboratory, Life Sciences Institute, University of Agricultural Sciences and Veterinary Medicine Cluj-Napoca, Romania. Cultures were maintained on malt agar at $4^{\circ} \mathrm{C}$ and subcultured every month. Spore suspension $\left(1.0 \times 10^{5}\right.$ $\mathrm{CFU} / \mathrm{mL}$ ) was obtained by washing agar plates with sterile solution containing $[0.85 \%$ saline, $0.10 \%$ Tween $80(v / v)]$, then added to each well to a final volume of $100 \mu \mathrm{L}$. Inocula were screened for contamination by culturing on a solid medium. The minimum inhibitory (MIC) and minimum fungicidal (MFC) concentrations assays were determined using the microdilution method by preparing a serial of dilutions in 96-well microtiter plates. The extracts (EE, PEE, CE) were diluted in $0.85 \%$ saline $(10 \mathrm{mg} / \mathrm{mL})$, then added to microplates containing Broth Malt medium with inoculum and incubated for $72 \mathrm{~h}$ at $28^{\circ} \mathrm{C}$ on a rotary shaker. The lowest concentrations without visible growth (at the binocular microscope) were defined as MICs. The MFCs were determined by serial sub-cultivation of $2 \mu \mathrm{L}$ of tested extracts dissolved in medium and inoculated for $72 \mathrm{~h}$, into microtiter plates containing $100 \mu \mathrm{L}$ of broth per well and further incubation $72 \mathrm{~h}$ at $28^{\circ} \mathrm{C}$. The lowest concentration with no visible growth was defined as MFC indicating 99.5\% killing of the original inoculum. The fungicide fluconazole was used as positive control $(1-3500 \mu \mathrm{g} / \mathrm{mL})$. All the experiments were performed in duplicate and repeated three times (Stana et al., 2017). 


\section{In Vivo Anti-inflammatory Effects Experimental Design}

All procedures that involved the use of life animals followed the European guidelines and rules as established by the EU Directive 2010/63/EU. The study protocol was approved by the Research Ethics Committee of the Iuliu Haţieganu University of Medicine and Pharmacy Cluj-Napoca (No. 382/2017).

The experiments were performed on adult male Wistar (strain Crl:WI) albino rats (Charles River Laboratories, United States), weighing 200-250 g, that were bred in the Animal Facility of Iuliu Haţieganu University of Medicine and Pharmacy. The animals were randomly assigned to six groups $(n=8)$. Rats from group I were injected intramuscularly (i.m.) with $0.9 \%$ saline solution as a negative control. Inflammation was induced by i.m. injection of turpentine oil $(6 \mathrm{~mL} / \mathrm{kg} \mathrm{BW})$ in groups II-VI. Animals were housed under controlled conditions (12 h light/dark cycle, at an average temperature of $21-22^{\circ} \mathrm{C}$ and humidity of $50-55 \%$ ), and had free access to standard pellet (Cantacuzino Institute, Bucharest, Romania) basal diet and water ad libitum. After the i.m. injection, the animals received the following intraperitoneal (i.p.) injections: groups I and II received $1 \mathrm{~mL}$ of a $0.9 \%$ saline solution; groups III-V received $5 \mathrm{~mL} / \mathrm{kg}$ BW of $A$. laxmannii ethanol extract diluted in distilled water to concentrations of $25 \%$ (25 mg dw/mL), 50\% (50 mg dw/mL) and $100 \%$ (100 mg dw/mL), respectively; group VI received $20 \mathrm{mg} / \mathrm{kg} \mathrm{BW}$ diclofenac (Araniciu et al., 2014).

In previous studies on anti-inflammatory activity of A. bracteosa, the evaluated dose was $200 \mathrm{mg} / \mathrm{kg}$ BW (Kayani et al., 2016), therefore in this research the animals received $400 \mathrm{mg} / \mathrm{kg} \mathrm{BW}$ (100\%), $200 \mathrm{mg} / \mathrm{kg} \mathrm{BW} \mathrm{(50 \% ),} \mathrm{and} 100 \mathrm{mg} / \mathrm{kg}$ BW (25\%) of A. laxmannii ethanol extract.

Twenty-four hours after the incubation of inflammation, the rats were anesthetized using a combination of $50 \mathrm{mg} / \mathrm{kg} \mathrm{BW}$ ketamine and $20 \mathrm{mg} / \mathrm{kg}$ BW xylazine (Parvu et al., 2014), and blood was withdrawn by retro-orbital puncture. Blood collected for use in the phagocytosis test and for white blood cells count (WBC) was collected on ethylenediaminetetraacetic acid (EDTA), while blood collected for use in the nitro-oxidative stress tests was collected without anticoagulant. Coagulated blood was centrifuged and the separated serum was stored in $-80^{\circ} \mathrm{C}$ until use. The total nitrites and nitrates (NOx), total oxidative status (TOS), total antioxidant response (TAR) and oxidative stress index (OSI) calculation was measured in the serum.

The experiments were performed in triplicate. At the end of the experiments under anesthesia using a combination of ketamine (60 mg/kg BW) and xylazine (15 mg/kg BW) (Francischi et al., 2017) animals were killed by cervical dislocation.

\section{In Vitro Phagocytosis Test}

Phagocytic activity (PA) was determined as previously described with minor modifications (Moldovan et al., 2011). The blood samples that were harvested on EDTA were incubated with an E. coli suspension $\left(4 \times 10^{6}\right.$ bacteria/mL, in $0.9 \%$ saline solution in the ratio of $0.2 \mathrm{~mL}$ of blood $/ 20 \mu \mathrm{L}$ E. coli suspension) in a silicon tube at $37^{\circ} \mathrm{C}$ for $30 \mathrm{~min}$. May-Grünwald-Giemsa stained smears were then prepared and counted by light microscopy using an Olympus microscope. We used two parameters to assess phagocytic capacity: the PA which was the number of the E. coli bacteria that were phagocytized by 100 leukocytes, and the phagocytic index (PI) which was the percentage of leukocytes that phagocytized at least one bacterium.

\section{WBC Count}

For the WBC count, a blood sample dilution 1:10 in Türk solution was prepared in a Potain leukocyte-dropper. The count was performed with an optical microscope (Olympus), using a Bürcker-Türk counting chamber. The differential leukocyte count was expressed as a percentage and carried out on MayGrünwald-Giemsa stained smears.

\section{Oxidative Stress Evaluation}

First serum samples were passed through $10-\mathrm{kDd}$ filters (Sartorius AG, Goettingen, Germany) and contaminant proteins were removed by extraction with a 3:1 ( $v: v)$ solution of methanol/diethyl ether. The sample methanol/diethyl ether ratio was 1:9 (v:v) (Harma et al., 2003). The Griess reaction was used to indirectly determine NO synthesis (NOx). In brief, $100 \mu \mathrm{L}$ of $8 \mathrm{mg} / \mathrm{mL} \mathrm{VCl}_{3}$ was added to $100 \mu \mathrm{L}$ of filtered and extracted serum supernatant in order to reduce nitrate to nitrite, followed by the addition of the Griess reagents, $50 \mu \mathrm{L}$ of SULF (2\%) and $50 \mu \mathrm{L}$ of $\operatorname{NEDD}(0.1 \%)$. After $30 \mathrm{~min}$ incubation at $37^{\circ} \mathrm{C}$, the sample absorbance was read at $540 \mathrm{~nm}$. The concentration of serum NOx was determined using a sodium nitrite-based curve, and expressed as nitrite $\mu \mathrm{mol} / \mathrm{L}$ (Miranda et al., 2001). The TOS of the serum was measured using a colorimetric assay (Erel, 2005). This assay measures the oxidation of ferrous ions to ferric ions in the presence of various reactive oxygen species in an acidic medium. The ferric ions are detected by reaction with xylenol orange. Assay measurements were standardized using hydrogen peroxide $\left(\mathrm{H}_{2} \mathrm{O}_{2}\right)$ as the oxidative species, and the obtained results were expressed in $\mu \mathrm{mol} \mathrm{H}_{2} \mathrm{O}_{2}$ equivalents/ $\mathrm{L}$. The TAR was measured in serum using a colorimetric assay (Erel, 2004). In this assay, the rate of hydroxyl radical producion by the Fenton reaction was monitored by following the changes in the absorbance of colored dianisidyl radicals. Upon addition of a serum sample, the hydroxyl radical initiated oxidative reactions are suppressed by antioxidant present in the serum. Inhibition of dianisidyl oxidation prevents the subsequent color change, thereby effectively measuring the total antioxidant capacity of the serum. This assay is calibrated using trolox and results are expressed as micromol TE/L. The ratio of the TOS to the TAR represents the OSI, an indicator of the degree of oxidative stress (Harma et al., 2003): OSI (Arbitrary Unit) $=$ TOS (micromol $\mathrm{H}_{2} \mathrm{O}_{2}$ equivalents/L)/TAR (micromol $\mathrm{TE} / \mathrm{L})$.

All of the spectroscopic measurements were performed using a Jasco V-530 UV-Vis spectrophotometer (Jasco International Co., Ltd., Tokyo, Japan).

\section{Statistical Analysis}

All results were expressed as the mean \pm SD. Otherwise, the median and first quartile (Q1) and third quartile (Q3) were reported. Statistical comparisons between two independent 
groups were performed using the Student's $t$-test (with equal and unequal variances, depending upon to the results of the F-test) for normally distributed data. Mann-Whitney's test was used for non-parametric data. Pearson and Spearman's correlation analyses were used to calculate statistical relationships between parameters. A $p$-value $<0.05$ was considered as statistically significant. Analyses were performed using SPSS 16.0 for Windows (SPSS Inc., United States).

\section{RESULTS AND DISCUSSION}

\section{Quantitative Determinations of Total Bioactive Compounds}

Various studies showed that phenolic compounds are widely distributed in the Ajuga species and these compounds could contribute to their antioxidant activity. In this part, a preliminary comparative overview of the total phenolic, flavonoid and iridoid contents of the different extracts of the A. laxmannii is presented. The TPC is presented in Table 1 and was $67.68 \pm 1.57 \mathrm{mg}$ GAE/g dw for ethanol extract, and $56.76 \pm 0.92 \mathrm{mg}$ GAE/g dw for methanol extract. The TPC values of analyzed A. laxmannii aerial parts extracts were higher than those obtained previously for A. reptans flower extracts $(20.86 \pm 0.53$ and $24.11 \pm 0.57 \mathrm{mg}$ GAE/g dw, for methanol and ethanol extracts, respectively) by Toiu et al. (2017). Another study performed by Movahhedin et al. (2016) revealed that ethanol extract of Ajuga chamaecistus subsp. scoparia (Boiss.) Rech.f. aerial parts (6.5 g extract obtained from $50 \mathrm{~g}$ plant material) had a TPC value of $20.32 \pm 0.39 \mathrm{mg}$ GAE/g extract (representing $2.64 \mathrm{mg}$ GAE/g dw), and the water extract had TPC values of $18.94 \pm 0.13 \mathrm{mg}$ GAEs/g extract, which is lower than both previously considered Ajuga species.

The TFC for ethanol extract of A. laxmannii $36.14 \pm 0.53 \mathrm{mg}$ $\mathrm{RE} / \mathrm{g} \mathrm{dw}$ ), was lower than the one reported for the ethyl acetate, methanol and acetone extract of A. chamaepitys (L.) Schreb $(91.76 \pm 0.81,63.87 \pm 0.66$, and $61.77 \pm 0.51 \mathrm{mg} \mathrm{RE} / \mathrm{g}$, respectively), and considerably higher than the water extract from same species (9.32 $\pm 0.33 \mathrm{mg} \mathrm{RE} / \mathrm{g}$ ) (Jakovljević, 2015). However, a clear comparison between the results of the present study is rather impossible, due to different extraction protocols and ways of expressing results.

In previous studies, Toiu et al. (2017) found a TFC value of $12.38 \pm 0.22 \mathrm{mg} \mathrm{RE} / \mathrm{g} \mathrm{dw}$ for a methanol extract of $A$. reptans flowers.

Concerning the TIC of different species of Ajuga, the available data is limited. In a former research on $A$. reptans flowers, Toiu

TABLE 1 | TPC, TFC and TIC in A. laxmannii extracts ( \pm SD).

\begin{tabular}{lccc}
\hline Extract & $\begin{array}{c}\text { TPC } \\
\text { (mg GAE/g dw) }\end{array}$ & $\begin{array}{c}\text { TFC } \\
\text { (mg RE/g dw) }\end{array}$ & $\begin{array}{c}\text { TIC } \\
\text { (mg AE/g dw) }\end{array}$ \\
\hline Methanol extract (ME) & $56.76 \pm 0.92$ & $31.22 \pm 0.39$ & $15.37 \pm 0.77$ \\
Ethanol extract (EE) & $67.68 \pm 1.57$ & $36.14 \pm 0.53$ & $16.28 \pm 0.85$ \\
\hline
\end{tabular}

Values are the mean $\pm S D(n=3)$. et al. (2017) revealed that the methanol extract content in iridoids is lower than ethanol extract from the same species $(22.17 \pm 0.89$ vs. $27.49 \pm 0.94 \mathrm{mg} \mathrm{AE} / \mathrm{g} \mathrm{dw}$ ). The same trend was observed in this study for the A. laxmannii, the TIC being $15.37 \pm 0.77$ and $16.28 \pm 0.85 \mathrm{mg} \mathrm{AE} / \mathrm{g} \mathrm{dw}$ for methanol and ethanol extract, respectively.

\section{Identification and Quantification of Polyphenolic Compounds}

In order to determine the polyphenolic compounds from A. laxmannii extracts, an optimized HPLC/MS method for the identification and quantification of 18 polyphenols was employed. The A. laxmannii extracts contain one caffeic acid derivative (chlorogenic acid), corresponding to peak 1, with $\mathrm{m} / z$ 353, three flavonoid glycosides (isoquercitrin, rutin, and quercitrin), corresponding to peaks 2 , 3 , and 4 , with $m / z 463,609$, and 447, respectively. Additionally, two free aglycones (luteolin and apigenin), with $m / z 285$ and 279 , corresponding to peaks 5 and 6 were identified.

The HPLC chromatogram of A. laxmannii ethanol extract (Figure 1), and the amounts of polyphenols identified in the analyzed extracts expressed as $\mu \mathrm{g} / \mathrm{g} d \mathrm{w}$ are presented (Table 2).

Rutin, an important flavonoid glycoside, was the major compound found in a significant quantity, both in ethanol and methanol extract $(6883.48 \pm 9.12$ and $6721.49 \pm 8.92 \mu \mathrm{g} / \mathrm{g}$ $\mathrm{dw}$, respectively). Various studies showed the effectiveness of rutin in various diseases such as inflammatory bowel disease and Alzheimer's disease (Kim et al., 2005; Xu et al., 2014). Another flavonoid glycoside compound which was found and quantified in ethanol and methanol A. laxmannii extracts was isoquercitrin, which is known to have good anti-inflammatory effects (Rogerio et al., 2007). The quantities obtained are significant lower than those obtained for rutin, but still important (685.35 \pm 5.72 and $636.1 \pm 5.44 \mu \mathrm{g} / \mathrm{g} \mathrm{dw}$ ), for ethanol and methanol extracts, respectively.

Considering the correlation between the type of the extract and the quantity of a particular compound, with one exception, all extracts showed similar values. Only in case of luteolin, the methanol extract showed a lower value than the ethanol extract $(88.24 \pm 1.09$ vs. $122.27 \pm 1.14 \mu \mathrm{g} / \mathrm{g} \mathrm{dw})$.

\section{Identification and Quantification of Phytosterols}

Under the proposed chromatographic conditions, retention times of the five analyzed sterols were: $3.2 \mathrm{~min}$ for ergosterol, $3.9 \mathrm{~min}$ for brassicasterol, $4.9 \mathrm{~min}$ for stigmasterol and campesterol (co-elution) and $5.7 \mathrm{~min}$ for $\beta$-sitosterol. The ions monitorized in the MS method are presented in Table 3. In the ionization conditions all sterols have lost a water molecule, therefore the ions detected by the mass spectrometer are always in the form $\left[\mathrm{M}-\mathrm{H}_{2} \mathrm{O}+\mathrm{H}\right]^{+}$.

The pseudo-molecular ions of sterols (379 for ergosterol, 381 for brassicasterol, 395 for stigmasterol, 383 for campesterol, and 397 for $\beta$-sitosterol) have been fragmented, and based on their daughter ions from the MS spectrum the extracted 


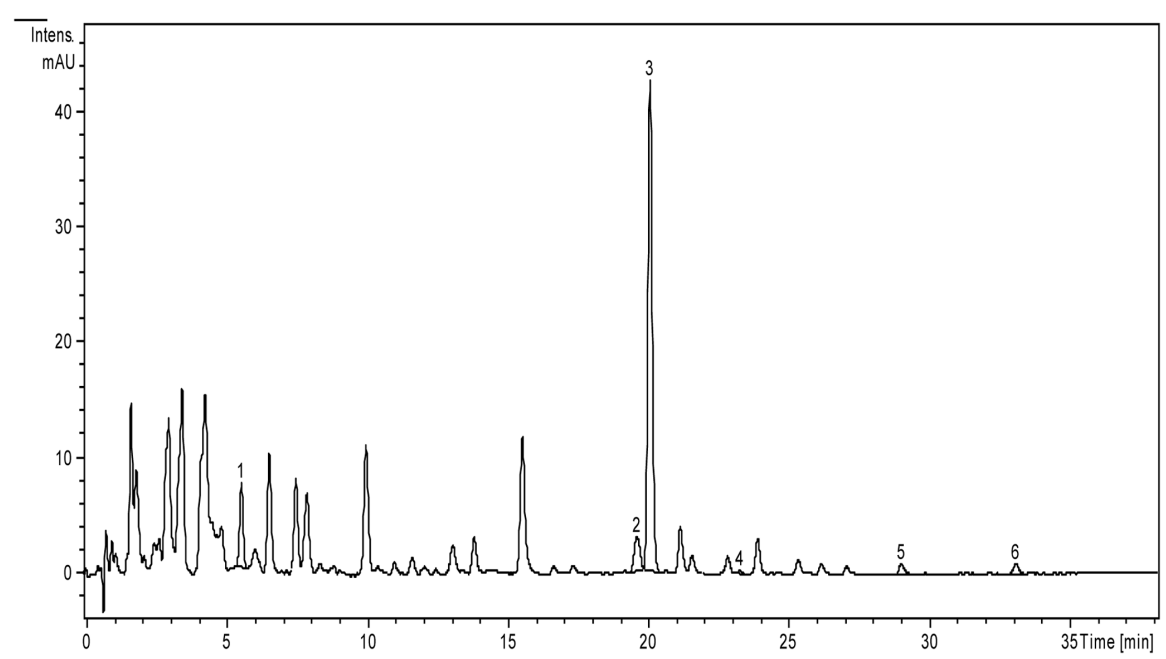

FIGURE 1 | HPLC chromatogram of polyphenols from A. laxmannii aerial parts extract. The identified compounds: chlorogenic acid (1), isoquercitrin (2), rutin (3), quercitrin (4), luteolin (5), apigenin (6).

TABLE 2 | The content of polyphenolic compounds in A. laxmannii extracts by HPLC ( $\mu \mathrm{g} / \mathrm{g}$ dw).

\begin{tabular}{|c|c|c|c|c|}
\hline Polyphenolic compounds & $m / z$ value & $R_{\mathrm{T}} \pm \mathrm{SD}(\mathrm{min})$ & A. laxmannii EE ( $\mu \mathrm{g} / \mathrm{g} \mathrm{dw})$ & A. Iaxmannii ME ( $\mu \mathrm{g} / \mathrm{g} \mathrm{dw})$ \\
\hline Chlorogenic acid (1) & 353 & $5.6 \pm 0.05$ & $23.27 \pm 1.72$ & $19.33 \pm 1.57$ \\
\hline Isoquercitrin (2) & 463 & $19.60 \pm 0.10$ & $685.35 \pm 5.72$ & $636.1 \pm 5.44$ \\
\hline Rutin (3) & 609 & $20.20 \pm 0.15$ & $6883.48 \pm 9.12$ & $6721.49 \pm 8.92$ \\
\hline Quercitrin (4) & 447 & $23.64 \pm 0.13$ & $41.13 \pm 1.87$ & $36.5 \pm 1.68$ \\
\hline Luteolin (5) & 285 & $29.64 \pm 0.15$ & $122.27 \pm 1.14$ & $88.24 \pm 1.09$ \\
\hline Apigenin (6) & 279 & $33.10 \pm 0.17$ & $129.32 \pm 2.49$ & $126.53 \pm 2.31$ \\
\hline
\end{tabular}

Values are the mean $\pm S D(n=3)$.

TABLE 3 | Characteristic ions of standard sterols in full scan mode by LC-MS/MS.

\begin{tabular}{|c|c|c|c|c|}
\hline Compound & $R_{\mathrm{T}}(\min )$ & $M$ & $\mathrm{M}-\mathrm{H}_{2} \mathrm{O}+\mathrm{H}^{+}$ & Specific ions for identification lon $\left[\mathrm{M}-\mathrm{H}_{2} \mathrm{O}+\mathrm{H}^{+}\right]>$Ions from spectrum \\
\hline Ergosterol & 3.2 & 396 & 379 & 379 > 158.9; 184.9; 199; 213; 225; 239; 253; 295; 309; 323 \\
\hline Brassicasterol & 3.9 & 398 & 381 & 381 > 201.3; 203.3; 215.2; 217.3; 241.2; 255.3; 257.4; 271.1; 297.3; 299.3 \\
\hline Stigmasterol & 4.9 & 412 & 395 & $395>255 ; 297 ; 283 ; 311 ; 241 ; 201$ \\
\hline Campesterol & 4.9 & 400 & 383 & $383>147 ; 149 ; 161 ; 175 ; 189 ; 203 ; 215 ; 229 ; 243 ; 257$ \\
\hline$\beta$-Sitosterol & 5.7 & 414 & 397 & $397>160.9 ; 174.9 ; 188.9 ; 202.9 ; 214.9 ; 243 ; 257 ; 287.1 ; 315.2$ \\
\hline
\end{tabular}

TABLE 4 | The content in sterols in A. laxmannii extracts ( $\mu \mathrm{g} / \mathrm{mL}$ extract).

\begin{tabular}{lccc}
\hline Phytosterol & A. laxmannii EE & A. laxmannii PEE & A. laxmannii CE \\
\hline$\beta$-Sitosterol & $367.24 \pm 2.97$ & - & $11589.96 \pm 8.66$ \\
Campesterol & - & $598.04 \pm 4.22$ & $1717.28 \pm 5.25$ \\
Stigmasterol & $55.49 \pm 2.01$ & $55.49 \pm 1.99$ & $55.5 \pm 2.09$ \\
Ergosterol & $1.88 \pm 0.09$ & $1.88 \pm 0.11$ & $1.88 \pm 0.13$ \\
Brassicasterol & $47.65 \pm 2.79$ & $47.65 \pm 2.71$ & $47.66 \pm 2.39$
\end{tabular}

Not found, below the limit of detection. Values are the mean $\pm S D(n=3)$.

chromatograms of each compound were constructed. The method can also be applied for quantitative determination because the intensity of ions in the mass spectrum is proportional to the concentration of the substance in the sample.
In order to quantify the five sterols from $A$. laxmannii extracts (EE, PEE, CE), we have constructed the extracted chromatograms for each compound, taking into account the intensity of major ions in the mass spectrum (Table 3 ).

Calibration curves were obtained from standard solutions at different concentration levels, selected as representative of the range of concentration in the sample. Regression analysis of various concentrations of standard solutions $(0.08-8 \mu \mathrm{g} / \mathrm{mL})$ gave good correlation coefficients for the calibration curves of sterols. Concentrations of phytosterols in A. laxmannii extracts are presented in Table 4. Significant differences between the three A. laxmannii extracts were observed: all five sterols were identified in $\mathrm{CE}$, whereas the $\mathrm{EE}$ contains $\beta$-sitosterol, stigmasterol, ergosterol and brassicasterol and PEE contains campesterol, stigmasterol, ergosterol and brassicasterol. 
The A. laxmannii CE was the richest in phytosterols, with $\beta$-sitosterol as major compound in very high concentration $(11589.96 \pm 8.66 \mu \mathrm{g} / \mathrm{mL})$, while in EE it was found in smaller quantities $(367.24 \pm 2.97 \mu \mathrm{g} / \mathrm{mL})$, and in PEE campesterol was the main sterol $(598.04 \pm 4.22 \mu \mathrm{g} / \mathrm{mL})$. The concentrations of stigmasterol, ergosterol, and brassicasterol were comparable in all three extracts. To the best of our knowledge, this is the first report on phytosterols from A. laxmannii aerial parts extracts. Previous studies showed the presence of stigmasterol and $\beta$-sitosterol in other Ajuga species, such as A. bracteosa, A. relicta, A. taiwanensis (Israili and Lyoussi, 2009).

\section{Identification and Quantification of Iridoids}

Iridoids are important compounds for the genus Ajuga, and many Ajuga species contain the iridoid glycoside harpagide (Manguro et al., 2011; Mamadalieva et al., 2013). The main known ethnopharmacological indications for Ajuga species are oedema, hypertension, fever, intestinal and biliary disorders, ulcer, they are used as antipyretic, diuretic, and astringent (Toiu et al., 2017), and several studies have shown that iridoid glycosides in Ajuga species are linked to this therapeutic effects (Makni et al., 2013; Hailu and Engidawork, 2014). The HPLC-MS results from the present study show the characterization of Ajuga laxmannii aerial parts in five commercially available iridoid glycosides, namely aucubin, catalpol, harpagide, harpagoside, and 8-O-acetylharpagide. From a pharmaceutical point of view, the concentrations of the compounds determined in plant extract cannot be neglected as they are directly linked to their pharmaceutical efficacy and effectiveness. 8-Oacetylharpagide was the major compound found in ethanol extract $(266.3 \pm 3.92 \mu \mathrm{g} / \mathrm{mL}$ extract), followed by harpagide (87.4 $\pm 2.39 \mu \mathrm{g} / \mathrm{mL}$ extract). The concentrations of aucubin and catalpol $(7.2 \pm 0.41$ and $3.1 \pm 0.23 \mu \mathrm{g} / \mathrm{mL}$ ethanol extract, respectively) were significantly lower than the other iridoids. We observed that A. laxmannii aerial parts ethanol extract contains higher amounts of iridoids than methanol extract (Table 5). As far as one can tell, this is the first report of a rapid, simple and highly accurate HPLC-MS/MS method for the identification and quantification of iridoids from A. laxmannii extracts.

\section{Antioxidant Activity}

\section{DPPH and ABTS Radical Scavenging Activity}

Several studies showed that the number and position of the substituents on the aromatic ring affects the antioxidant properties of phenolics; different substituents affect the reactivity and thus, the antioxidant capacity of the phenolic compounds (Shahidi and Ambigaipalan, 2015).

The DPPH and ABTS radical scavenging assays are reliable and commonly used methods for evaluation of the radical scavenging activity. These measurements are based on the reduction of radical species by electron-transferring or hydrogendonating radical scavengers. $\mathrm{DPPH}^{\bullet}$ method is used for anion radicals and $\mathrm{ABTS}^{\bullet}+$ is used for cation radicals. $\mathrm{DPPH}^{\bullet}$ is a stable free radical, the largest absorbance occurs at $517 \mathrm{~nm}$, and when it reacts with antioxidants free radicals its absorbance decreases.

TABLE 5 | The quantification of iridoids in A. laxmannii extracts ( $\mu \mathrm{g} / \mathrm{mL}$ extract).

\begin{tabular}{|c|c|c|c|c|c|}
\hline Extract & Harpagide & Aucubin & Catalpol & Harpagoside & 8-O-acetyl-harpagide \\
\hline A. laxmannii EE & $87.4 \pm 2.39$ & $7.2 \pm 0.41$ & $3.1 \pm 0.23$ & $37.2 \pm 2.35$ & $266.3 \pm 3.92$ \\
\hline A. laxmannii ME & $76.5 \pm 2.01$ & $6.9 \pm 0.37$ & $2.7 \pm 0.19$ & $29.1 \pm 1.98$ & $241.4 \pm 3.65$ \\
\hline
\end{tabular}

Values are the mean $\pm S D(n=3)$.

TABLE 6 | DPPH, TEAC, EPR activity of $A$. laxmannii (mean \pm SD).

\begin{tabular}{|c|c|c|c|c|c|}
\hline Sample & DPPH IC ${ }_{50}(\mu \mathrm{g} / \mathrm{mL}) \mathrm{EE}$ & DPPH IC ${ }_{50}(\mu \mathrm{g} / \mathrm{mL}) \mathrm{ME}$ & TEAC mg TE/g dw & EPR mg FS/25 $\mu \mathrm{L}$ & EPR $\mathrm{mg} \mathrm{FS} / \mathrm{g} \mathrm{dw}$ \\
\hline A. laxmannii & $22.64 \pm 0.88$ & $24.89 \pm 0.83$ & $71.07 \pm 2.40$ & 0.266 & $98.073 \pm 1.23$ \\
\hline Trolox & $11.2 \pm 0.21$ & & & & \\
\hline
\end{tabular}

Values are the mean $\pm S D(n=3)$.

TABLE 7 | Antibacterial activity of $A$. laxmannii extracts (MIC, MBC).

\begin{tabular}{|c|c|c|c|c|c|}
\hline \multirow[t]{2}{*}{ Bacterial strains } & \multicolumn{2}{|c|}{ MIC (mg/mL) } & \multicolumn{2}{|c|}{ MBC (mg/mL) } & \multirow[t]{2}{*}{ Gentamycin $(\mu \mathrm{g} / \mathrm{mL})$} \\
\hline & ME & EE & ME & EE & \\
\hline S. aureus & 1.56 & 0.78 & 3.12 & 1.56 & 0.038 \\
\hline P. aeruginosa & 3.12 & 3.12 & 6.25 & 6.25 & 1.2 \\
\hline L. monocytogenes & 6.25 & 6.25 & 12.5 & 12.5 & 0.076 \\
\hline E. coli & 6.25 & 6.25 & 12.5 & 12.5 & 1.2 \\
\hline S. typhimurium & 6.25 & 6.25 & 12.5 & 12.5 & 2.4 \\
\hline
\end{tabular}


TABLE 8 | Antifungal activity of $A$. laxmannii extracts (MIC, MFC).

\begin{tabular}{|c|c|c|c|c|c|c|c|c|}
\hline \multirow[t]{2}{*}{ Bacterial Strains } & \multicolumn{3}{|c|}{ MIC (mg/mL) } & \multicolumn{3}{|c|}{ MFC (mg/mL) } & \multicolumn{2}{|c|}{ Fluconazole $(\mu \mathrm{g} / \mathrm{mL})$} \\
\hline & EE & PEE & CE & EE & PEE & CE & MIC $(\mu \mathrm{g} / \mathrm{mL})$ & MFC $(\mu \mathrm{g} / \mathrm{mL})$ \\
\hline Aspergillus flavus & 0.05 & 0.025 & 0.12 & 0.1 & 0.05 & 0.025 & 0.15 & 0.3 \\
\hline Aspergillus niger & 0.05 & 0.05 & 0.025 & 0.1 & 0.1 & 0.05 & 0.15 & 0.3 \\
\hline Candida albicans & 0.025 & 0.1 & 0.012 & 0.05 & 0.2 & 0.025 & 0.1 & 0.2 \\
\hline Candida parapsilosis & 0.012 & 0.025 & 0.025 & 0.025 & 0.5 & 0.05 & 0.1 & 0.2 \\
\hline Penicillium funiculosum & 0.1 & 0.025 & 0.012 & 0.2 & 0.05 & 0.025 & 0.15 & 0.3 \\
\hline
\end{tabular}

TABLE 9 | Anti-inflammatory activity of $A$. laxmannii extracts (WBC, PMN, Monocytes, PA, PI, TAR, TOS, NO, OSI).

\begin{tabular}{|c|c|c|c|c|c|}
\hline Parameter & A.I. $100 \%$ (100 mg dw/mL) & A.I. $50 \%(50 \mathrm{mg} \mathrm{dw} / \mathrm{mL})$ & A.I. $25 \%$ (25 mg dw/mL) & Inflam & Diclo \\
\hline WBC & 10444 & 5103.6 & 5634 & 11602 & 4866.8 \\
\hline PMN & 68.2 & 62 & 68.6 & 79.6 & 54.4 \\
\hline Monocytes & 3 & 2.4 & 2.4 & 2.8 & 2.4 \\
\hline PA & 42.4 & 23.6 & 35.2 & 50.4 & 23.8 \\
\hline $\mathrm{PI}$ & 54.2 & 31.2 & 41.2 & 86.8 & 37.2 \\
\hline TAR & 1.088668 & 1.086654 & 1.08674 & 1.087136 & 1.089406 \\
\hline TOS & 22.36506 & 16.70665 & 18.16688 & 22.46936 & 16.20834 \\
\hline NO & 42.56259 & 44.77172 & 57.1134 & 59.29308 & 33.23183 \\
\hline OSI & 20.539 & 15.37416 & 16.71447 & 20.66744 & 14.87844 \\
\hline
\end{tabular}

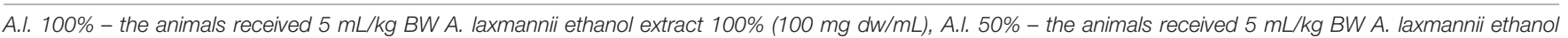

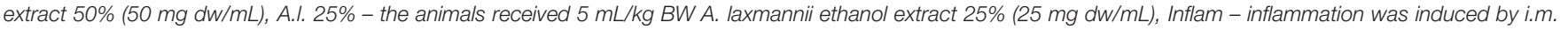
injection of turpentine oil ( $6 \mathrm{~mL} / \mathrm{kg} \mathrm{BW}$ ), Diclo - the animals received $20 \mathrm{mg} / \mathrm{kg}$ BW diclofenac.

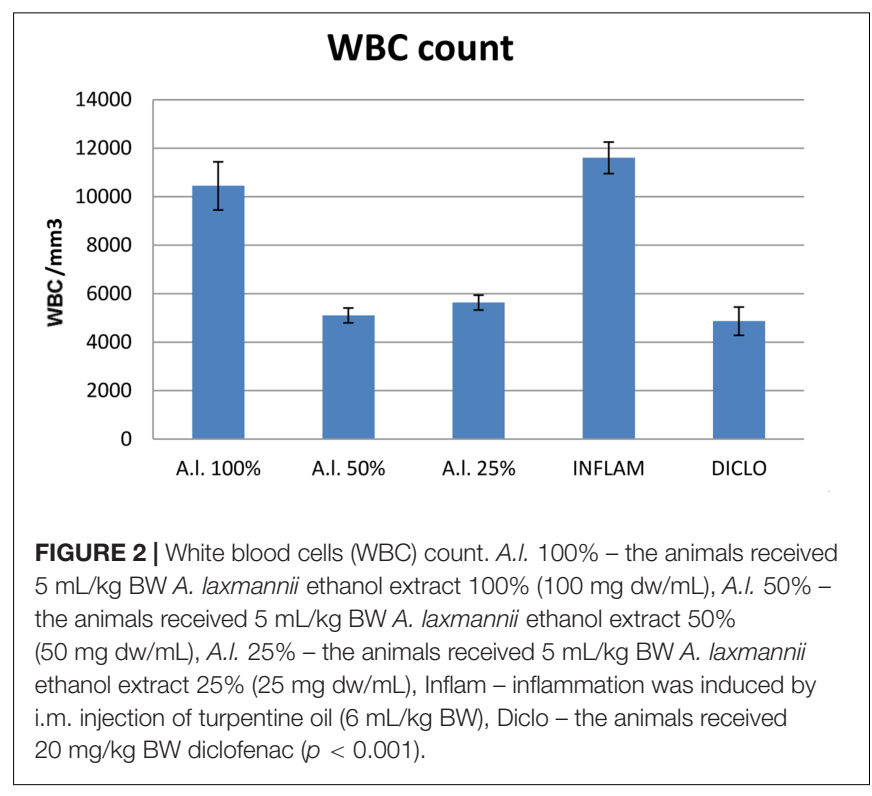

The antioxidant activity of A. laxmannii was investigated for the first time and the $\mathrm{IC}_{50}$ values were determined: $22.64 \pm 0.88$ and $24.89 \pm 0.83 \mu \mathrm{g} / \mathrm{mL}$ for ethanol and methanol extract, respectively, while for Trolox $11.2 \pm 0.21 \mu \mathrm{g} / \mathrm{mL}$ (Table 6). In comparison with other research, $A$. laxmannii showed a higher antiradical capacity. For example, a study concerning antioxidant capacity of A. turkestanica reported a value of $57.84 \pm 4.19 \mu \mathrm{g} / \mathrm{mL}$ (Mamadalieva et al., 2013), whereas a similar study concerning A. reptans reported a slightly higher value $(65.7 \pm 3.82 \mu \mathrm{g} / \mathrm{mL})$ (Ono et al., 2011). A similar antiradical activity was reported by Movahhedin et al. (2016) for A. chamaecistus subsp. scoparia (Boiss.) Rech.f. $(22.69 \pm 1.30 \mu \mathrm{g} / \mathrm{mL})$.

2,2'-Azino-bis-(3-ethylbenzothiazoline-6-sulfonic acid) (ABTS) is another free radical used for evaluation of antioxidant capacity. In the $\mathrm{ABTS}^{\bullet}+$ (TEAC) scavenging assay, the value of antiradical capacity of $A$. laxmannii ethanol extract was $71.07 \pm 2.40 \mathrm{mg} \mathrm{TE} / \mathrm{g}$ extract (Table 6), which is higher than the one reported by Movahhedin et al. (2016) for A. chamaecistus subsp. scoparia (Boiss.) Rech.f. (53.87 $\pm 2.11 \mathrm{mg}$ TE/g extract).

\section{Electron Paramagnetic Resonance (EPR) Spectroscopy}

To support the results from the TEAC assay, the Ajuga laxmannii ethanol extract was additionally analyzed using EPR with Fremy's salt as a stable radical. EPR is a very common method to assess the antioxidant activity of different samples. The main advantage of this assay is the matrix-independent measurement of the reaction between potential antioxidants and radicals in an electro-magnetic field instead of the absorbance of light (Yu and Cheng, 2008). The degraded amount of Fremy's salt after $30 \mathrm{~min}$ incubation time was $98.07 \pm 1.23 \mathrm{mg} \mathrm{FS} / \mathrm{g}$ extract (Table 6). The EPR is a common, well-characterized, established methodology for detecting radicals and their kinetics. Unfortunately, it is not so frequently used in combination with other assays to detect the overall antioxidant activity. Therefore, in this case, a comparison with the results of 


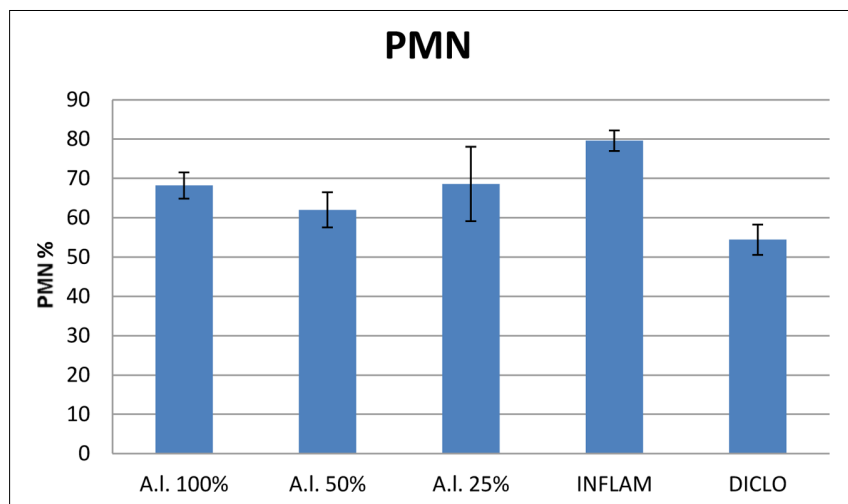

FIGURE 3 | Polymorphonuclear leukocytes (PMN). A.I. 100\% - the animals received $5 \mathrm{~mL} / \mathrm{kg}$ BW A. laxmannii ethanol extract 100\% (100 mg dw/mL), A.l. $50 \%$ - the animals received $5 \mathrm{~mL} / \mathrm{kg}$ BW A. laxmannii ethanol extract $50 \%$ (50 mg dw/mL), A.l. $25 \%$ - the animals received $5 \mathrm{~mL} / \mathrm{kg}$ BW A. laxmannii ethanol extract $25 \%$ ( $25 \mathrm{mg} \mathrm{dw} / \mathrm{mL}$ ), Inflam - Inflammation was induced by i.m. injection of turpentine oil $(6 \mathrm{~mL} / \mathrm{kg} \mathrm{BW})$, Diclo - the animals received $20 \mathrm{mg} / \mathrm{kg}$ BW diclofenac $(p<0.001)$.

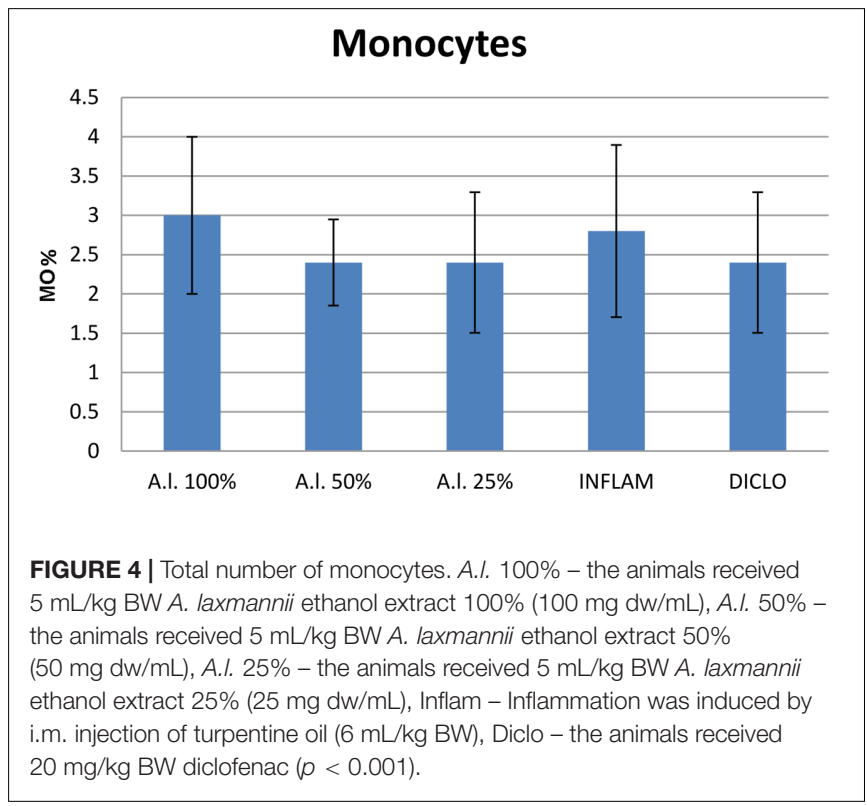

other researchers with regard to other Ajuga species is lacking.

\section{Antibacterial Activity}

The obtained results for the antibacterial activity of $A$. laxmannii extracts and gentamicin against Gram + and Gram- bacteria are presented in Table 7. The antimicrobial effect was measured by microdilution assay, and the determination of MIC $(\mathrm{mg} / \mathrm{mL})$ and MBC $(\mathrm{mg} / \mathrm{mL})$ was assessed.

The MIC values obtained for the ethanol extract ranged from 0.78 to $6.25 \mathrm{mg} / \mathrm{mL}$, and from 1.56 to $6.25 \mathrm{mg} / \mathrm{mL}$ for the methanol extract of $A$. laxmannii. Against some bacterial strains, such as Pseudomonas aeruginosa, Listeria monocytogenes, Escherichia coli and Salmonella typhimurium, both extracts
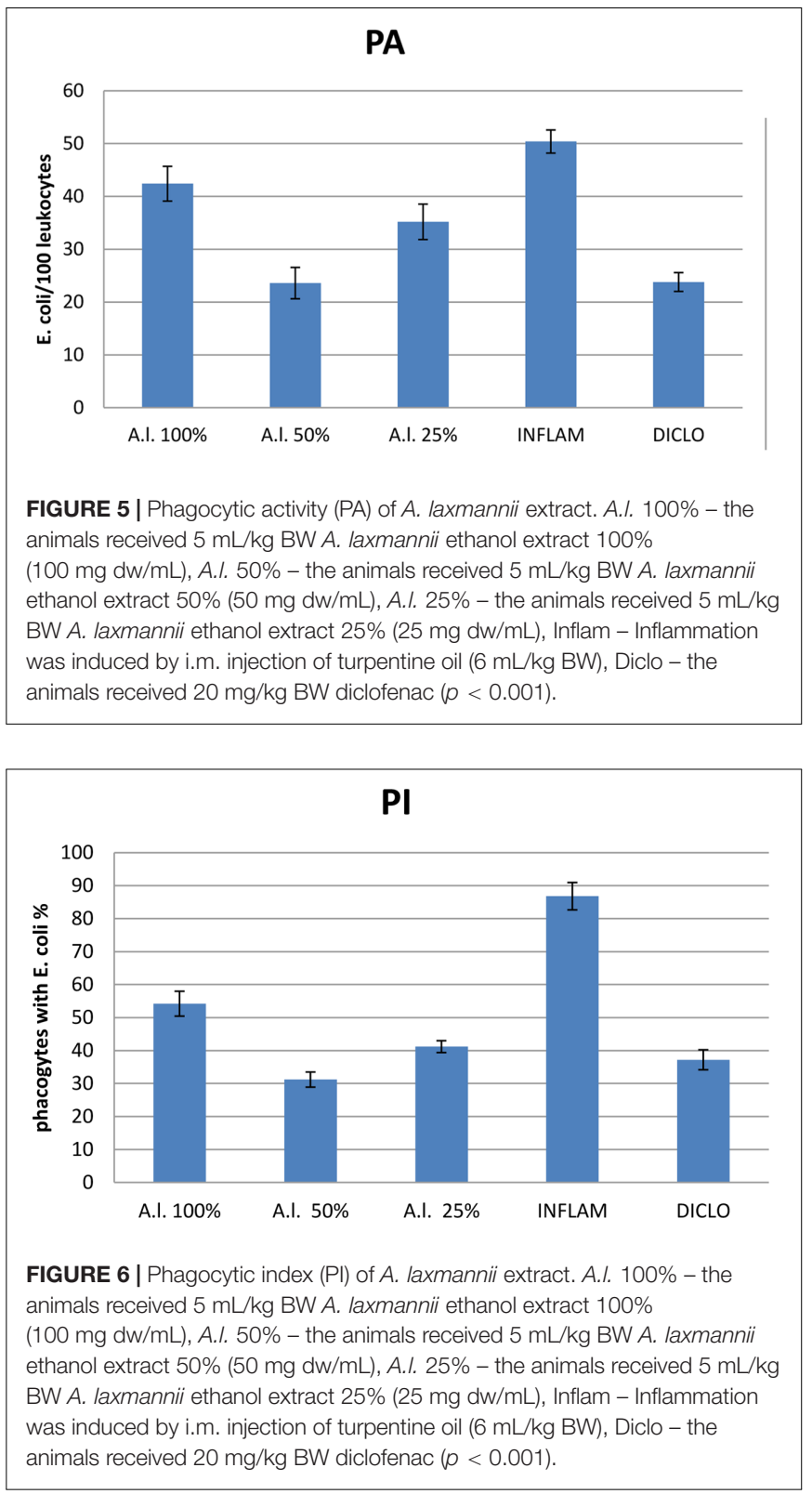

showed comparable activities. The best antimicrobial activity against $S$. aureus is associated with A. laxmannii ethanol extract $(\mathrm{MIC}$ value $=0.78 \mathrm{mg} / \mathrm{mL}$ and $\mathrm{MBC}$ value $=1.56 \mathrm{mg} / \mathrm{mL})$. The less susceptible strains were: Listeria monocytogenes, Escherichia coli and Salmonella typhimurium, for both methanol and ethanol extracts. Previous studies showed a similar trend in terms of MIC and MBC for A. reptans (Toiu et al., 2017). According to Salvat et al. (2004), herbal extracts with MIC values less than/around $0.50 \mathrm{mg} / \mathrm{mL}$ indicate good antimicrobial effect. Consequently, the results presented herein showed moderate antibacterial activity.

Generally, the bacterial strains were more sensitive to ethanol extract of $A$. laxmannii. Considering HPLC-MS results presented in this study, we can derive some assumptions regarding antibacterial activity of $A$. laxmannii aerial parts. The content 


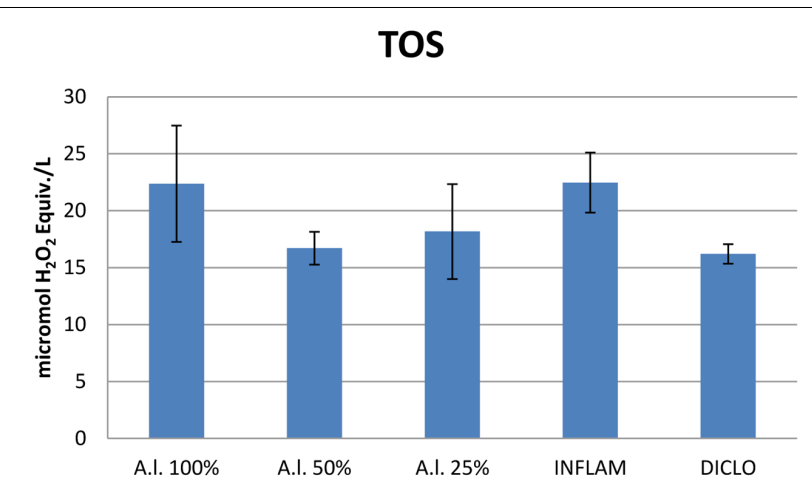

FIGURE 7 | Total oxidative status (TOS). A.l. 100\% - the animals received $5 \mathrm{~mL} / \mathrm{kg}$ BW A. laxmannii ethanol extract 100\% (100 mg dw/mL), A.l. 50\% the animals received $5 \mathrm{~mL} / \mathrm{kg}$ BW A. laxmannii ethanol extract $50 \%$ (50 mg dw/mL) $(p<0.001), A . l .25 \%$ - the animals received $5 \mathrm{~mL} / \mathrm{kg} \mathrm{BW}$ A. laxmannii ethanol extract $25 \%(25 \mathrm{mg} \mathrm{dw} / \mathrm{mL})(p<0.01)$, Inflam Inflammation was induced by i.m. injection of turpentine oil ( $6 \mathrm{~mL} / \mathrm{kg} \mathrm{BW})$, Diclo - the animals received $20 \mathrm{mg} / \mathrm{kg}$ BW diclofenac $(p<0.001)$.

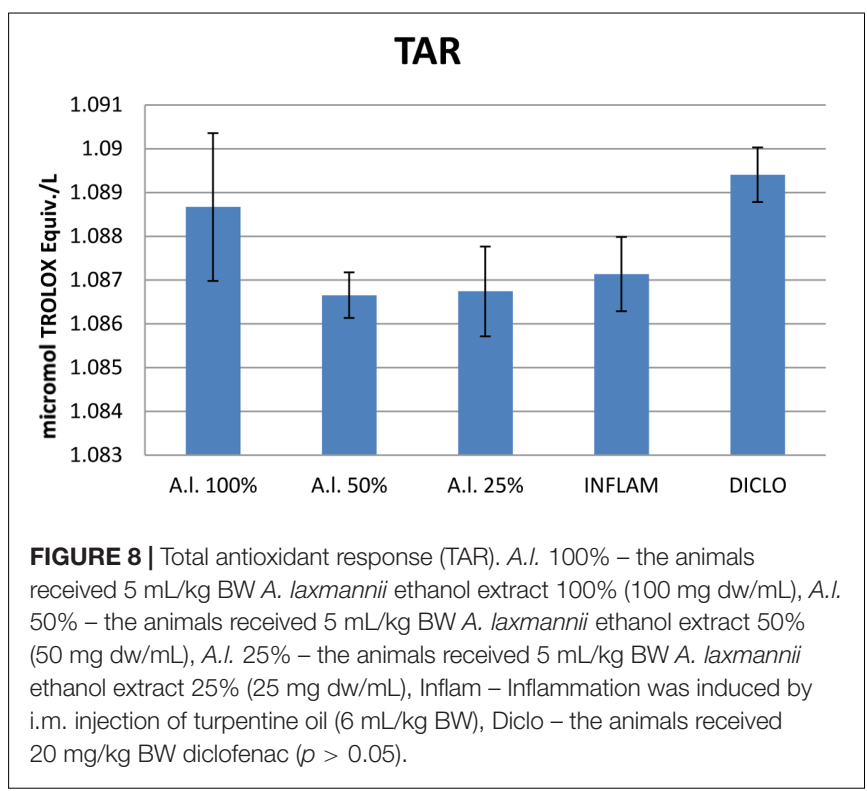

of polyphenolic compounds in ethanol extract of A. laxmannii was higher than in methanol extract. Many recent investigations confirmed antibacterial and antifungal properties of phenolic compounds from plant origin, among which are the main constituents of examined extracts (rutin and isoquercitrin) (Alves et al., 2013; Stojković et al., 2013). The exerted antibacterial potential of A. laxmannii could be associated with higher level of mentioned phenolic compounds in ethanol extract.

\section{Antifungal Activity}

The antifungal activity of the A. laxmannii extracts (EE, PEE, and $\mathrm{CE}$ ) was tested against a panel of five fungi, selected on the basis of their relevance for public health. The results obtained for the antifungal efficacy of $A$. laxmannii extracts and fluconazole against tested strains are presented in Table 8.
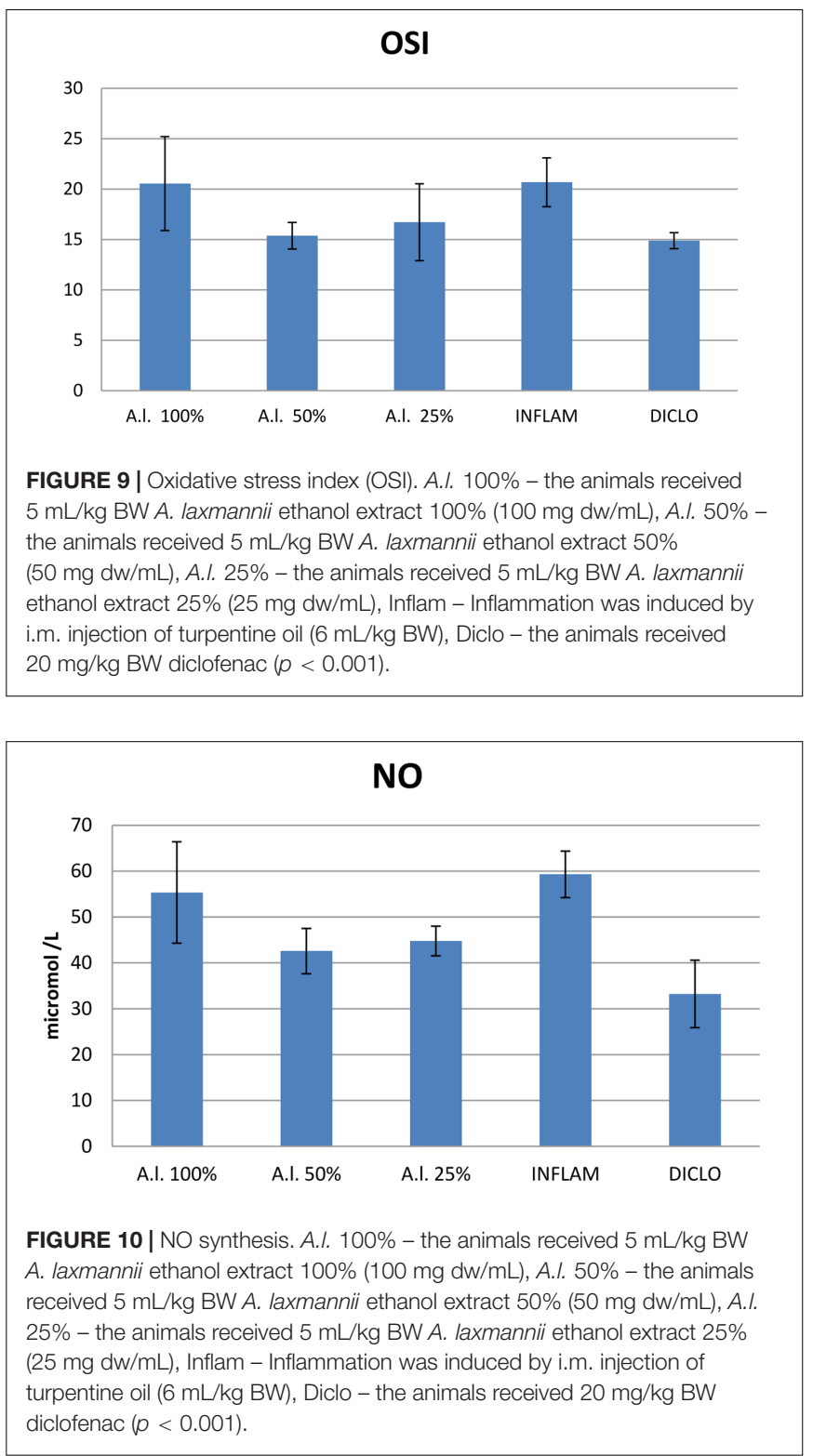

Candida parapsilosis possessed the highest sensitivity to the ethanolic extract of A. laxmannii, with MIC $=0.012 \mathrm{mg} / \mathrm{mL}$ and MFC $=0.025 \mathrm{mg} / \mathrm{mL}$. Moreover, Candida albicans and Penicillium funiculosum were similarly susceptible to the inhibitory (MICs $=0.012 \mathrm{mg} / \mathrm{mL}$ ) and fungicidal effects $($ MFCs $=0.025 \mathrm{mg} / \mathrm{mL})$ of the chloroform extract. Nonetheless, the most resistant fungal strains were Candida parapsilosis and Candida albicans, toward petroleum ether extract with MFCs 0.5 and $0.2 \mathrm{mg} / \mathrm{mL}$, respectively. According to Kawamura and Ohara (2005), the antifungal activity of plant extracts might be ascribed to the high level of iridoids. Both methanol and ethanol extracts from A. laxmannii aerial parts showed high values for iridoid glycosides, especially for 8 -O-acetylharpagide. These results are in accordance to previous studies performed by Makni et al. (2013), who similarly demonstrated the antifungal effect of $A$. iva extracts. 


\section{Anti-inflammatory Activity}

The anti-inflammatory effects of three A. laxmannii ethanol extract concentrations $(25 \mathrm{mg} \mathrm{dw} / \mathrm{mL}, 50 \mathrm{mg} \mathrm{dw} / \mathrm{mL}$, and $100 \mathrm{mg} \mathrm{dw} / \mathrm{mL}$ ) were evaluated in vivo on a model turpentine oil-induced inflammation in rats by determining WBC count, differential WBC count, serum total nitrites and nitrates, TOS, TAR and OSI. These three extract dilutions were also evaluated in vitro for the ability to inhibit phagocytosis. The $50 \mathrm{mg} \mathrm{dw} / \mathrm{mL}$ diluted extract had the best inhibitory activity on phagocytosis and oxidative stress. In conclusion, these results support the hypothesis that extracts from $A$. laxmannii aerial parts exert antiinflammatory activities by inhibiting phagocytosis through the reduction of oxidative stress (Table 9).

Compared to the inflammation group, A. laxmannii ethanol extracts significantly reduced $(p<0.001)$ total WBC count (Figure 2) by lowering PMN\% (Figures 3, 4). That was associated with an important decrease of the phagocytosis indices PA (Figure 5) and PI (Figure 6) $(p<0.001)$. Extracts $50 \mathrm{mg} \mathrm{dw} / \mathrm{mL}$ and $25 \mathrm{mg} \mathrm{dw} / \mathrm{mL}$ had the best inhibitory effects $(p<0.001)$ and this was comparable to diclofenac activity $(p>0.05)$.

Turpentine-induced inflammation significantly increased $(p<0.001)$ and treatment with diclofenac significantly reduced $(p<0.001)$ the TOS (Figure 7). Importantly, the TOS was also reduced by the treatment with all dilutions of the A. laxmannii extract. The inhibitory effects were strongest for the $50 \mathrm{mg} \mathrm{dw} / \mathrm{mL}(p<0.001)$ and $25 \mathrm{mg} \mathrm{dw} / \mathrm{mL}(p<0.01)$ plant extracts. Diclofenac treatment had a similar effect on TOS inhibition $(p<0.01)$ compared to treatment with either the $25 \mathrm{mg} \mathrm{dw} / \mathrm{mL}(p<0.01)$ or the $50 \mathrm{mg} \mathrm{dw} / \mathrm{mL}(p<0.01)$ extract. The A. laxmannii extract had no important effect on TAR (Figure 8) $(p>0.05)$.

In the inflammation group, the OSI was significantly elevated $(p<0.001)$, and diclofenac treatment decreased the OSI $(p<0.001)$ (Figure 9). The $25 \mathrm{mg} \mathrm{dw} / \mathrm{mL}$ and $50 \mathrm{mg} \mathrm{dw} / \mathrm{mL}$ extracts of A. laxmannii aerial parts induced a significant decline in OSI $(p<0.001)$. The $50 \mathrm{mg} \mathrm{dw} / \mathrm{mL}$ extract was the best OSI inhibitor $(p<0.001)$. The $50 \mathrm{mg} \mathrm{dw} / \mathrm{mL}(p<0.001)$ and $25 \mathrm{mg} \mathrm{dw} / \mathrm{mL}(p<0.01)$ A. laxmannii extract dilutions significantly reduced NOx (Figure 10). The OSI correlated with the TOS $(r=0.92)$ and NOx $(r=0.81)$.

Compared to diclofenac treatment, A. laxmannii extract had a lower anti-inflammatory and anti-nitro-oxidative stress effect upon all measured parameters $(p<0.001)$. Among the three extracts, the $50 \mathrm{mg} \mathrm{dw} / \mathrm{mL}$ A. laxmannii one had the best effect in comparison with diclofenac.

\section{REFERENCES}

Abuajah, C. I., Ogbonna, A. C., and Osuji, C. M. (2014). Functional components and medicinal properties of food: a review. J. Food Sci. Technol. 52, 2522-2529. doi: 10.1007/s13197-014-1396-5

Alves, M. J., Ferreira, I. C., Froufe, H. J., Abreu, R. M., Martins, A., and Pintado, M. (2013). Antimicrobial activity of phenolic compounds identified in wild mushrooms, SAR analysis and docking studies. J. Appl. Microbiol. 115, 346-357. doi: 10.1111/jam.12196

Andriamadio, J. H., Rasoanaivo, L. H., Benedec, D., Vlase, L., Gheldiu, A. M., Duma, M., et al. (2015). HPLC/MS analysis of polyphenols, antioxidant and

\section{CONCLUSION}

In the present study, the chemical composition, antioxidant, antimicrobial, and anti-inflammatory properties of different A. laxmannii (from Romania) extracts were evaluated for the first time. The major identified compounds were rutin, 8-Oacetylharpagide and $\beta$-sitosterol. The content in polyphenolic compounds, iridoids and phytosterols could be correlated with the evaluated pharmacological effects. The antioxidant activity of A. laxmannii extracts was assessed using several methods, and results showed good antiradical effects. The results of the antimicrobial evaluation showed a potent antifungal activity against $C$. albicans and $P$. funiculosum. The antiinflammatory effect was determined by monitoring some parameters involved in the inflammatory process, and these findings could indicate a possible mechanism of action. The obtained results showed differences between the analyzed extracts; and therefore the importance of choosing the best solvent in order to extract the appropriate amounts of bioactive compounds. A. laxmannii ethanol extract possessed anti-inflammatory effect by reducing total leukocytes, PMN, phagocytosis, and oxidative stress. Compared to diclofenac, only the $50 \mathrm{mg} / \mathrm{mL}$ A. laxmannii extract showed better antiinflammatory and anti-oxidative stress effects, and this could justify the importance of the correlation between the activity and the dose. The results confirm the use of A. laxmannii in traditional medicine as an anti-inflammatory agent. Further research is needed in order to deeply comprehend the bioavailability and how processes are involved in the metabolic pathways.

\section{AUTHOR CONTRIBUTIONS}

AT, AM, LV, AP, DV, A-MG, and IO conceived and designed the structure of the manuscript and data collection. AT, AM, CM, and AP drafted and revised the manuscript. AT, AM, and IO critically reviewed the manuscript. All authors have seen and agreed on the final version of the manuscript.

\section{ACKNOWLEDGMENTS}

AT acknowledges the support by UEFISCDI, Romania, project no. PNII-RU-TE-2014-4-1247.

antimicrobial activities of Artabotrys hildebrandtii O. Hffm. extracts. Nat. Prod. Res. 29, 2188-2196. doi: 10.1080/14786419.2015.1007458

Araniciu, C., Parvu, A. E., Palage, M. D., Oniga, S. D., Benedec, D., Oniga, I., et al. (2014). The effect of some 4,2 and 5,2 bisthiazole derivatives on nitro-oxidative stress and phagocytosis in acute experimental inflammation. Molecules 19, 9240-9256. doi: 10.3390/molecules 190 79240

Atanasov, A. G., Waltenberger, B., Pferschy-wenzig, E., Linder, T., Wawrosch, C., Uhrin, P., et al. (2015). Discovery and resupply of pharmacologically active plant-derived natural products?: a review. Biotechnol. Adv. 33, 1582-1614. doi: 10.1016/j.biotechadv.2015.08.001 
Atay, I., Kirmizibekmez, H., Kaiser, M., Akaydin, G., Yesilada, E., and Tasdemir, D. (2016). Evaluation of in vitro antiprotozoal activity of Ajuga laxmannii and its secondary metabolites. Pharm. Biol. 54, 1808-1814. doi: 10.3109/13880209. 2015.1129542

Barakat, H., and Rohn, S. (2014). Effect of different cooking methods on bioactive compounds in vegetarian, broccoli-based bars. J. Funct. Foods 11, 407-416. doi: 10.1016/j.jff.2014.10.009

Cocquyt, K., Cos, P., Herdewijn, P., Maes, L., Van Den Steen, P. E., and Laekeman, G. (2011). Ajuga remota Benth.: from ethnopharmacology to phytomedical perspective in the treatment of malaria. Phytomedicine 18, 1229-1237. doi: 10.1016/j.phymed.2011.08.063

El-Hilaly, J., Tahraoui, A., Israili, Z. H., and Lyoussi, B. (2006). Hypolipidemic effects of acute and sub-chronic administration of an aqueous extract of Ajuga iva L. whole plant in normal and diabetic rats. J. Ethnopharmacol. 105, 441-448. doi: 10.1016/j.jep.2005.11.023

Erdenechimeg, C., Guiqide, A., Dejidmaa, B., Chimedragchaa, C., and Purevsuren, S. (2017). Total phenolic, flavonoid, alkaloid and iridoid content and preventive effect of Lider-7-tang on lipopolysaccharide-induced acute lung injury in rats. Braz. J. Med. Biol. Res. 50, 6-11. doi: 10.1590/1414-431x201 75916

Erel, O. (2004). A novel automated method to measure total antioxidant response against potent free radical reactions. Clin. Biochem. 37, 112-119. doi: 10.1016/j. clinbiochem.2003.10.014

Erel, O. (2005). A new automated colorimetric method for measuring total oxidant status. Clin. Biochem. 38, 1103-1111. doi: 10.1016/j.clinbiochem.2005.08.008

Ferrante, C., Recinella, L., Locatelli, M., Guglielmi, P., Secci, D., Leporini, L., et al. (2017). Protective effects induced by microwave-assisted aqueous harpagophytum extract on rat cortex synaptosomes challenged with amyloid $\beta$-peptide. Phyther. Res. 31, 1257-1264. doi: 10.1002/ptr.5850

Francischi, J. N., Frade, T. I. C., Almeida, M. P. A., Queiroz, B. F. G., and Bakhle, Y. S. (2017). Ketamine-xylazine anaesthesia and orofacial administration of substance P: a lethal combination in rats. Neuropeptides 62, 21-26. doi: 10.1016/ j.npep.2017.01.003

Gautam, R., Jachak, S. M., and Saklani, A. (2011). Anti-inflammatory effect of Ajuga bracteosa Wall Ex Benth. mediated through cyclooxygenase (COX) inhibition. J. Ethnopharmacol. 133, 928-930. doi: 10.1016/j.jep.2010. 11.003

Hailu, W., and Engidawork, E. (2014). Evaluation of the diuretic activity of the aqueous and $80 \%$ methanol extracts of Ajuga remota Benth (Lamiaceae) leaves in mice. BMC Complement. Altern. Med. 14:135. doi: 10.1186/1472-6882-14135

Harma, M., Harma, M., and Erel, O. (2003). Increased oxidative stress in patients with hydatidiform mole. Swiss Med. Wkly. 133, 563-566.

Israili, Z. H., and Lyoussi, B. (2009). Ethnopharmacology of the plants of genus Ajuga. Pak. J. Pharm. Sci. 22, 425-462.

Jakovljević, D. Z., Vasić, S. M., Stanković, M. S., Čomić, L. R., and Topuzović, M. D. (2015). Secondary metabolite content and in vitro biological effects of Ajuga chamaepitys (L.) Schreb. subsp. chamaepitys. Arch. Biol. Sci. 67, 1195-1202. doi: 10.2298/ABS150225095J

Kawamura, F., and Ohara, S. (2005). Antifungal activity of iridoid glycosides from the heartwood of Gmelina arborea. Holzforschung 59, 153-155. doi: 10.1515/ HF.2005.023

Kayani, W. K., Dilshad, E., Ahmed, T., Ismail, H., and Mirza, B. (2016). Evaluation of Ajuga bracteosa for antioxidant, anti-inflammatory, analgesic, antidepressant and anticoagulant activities. BMC Complement. Altern. Med. 16:375. doi: 10.1186/s12906-016-1363-y

Kim, H., Kong, H., Choi, B., Yang, Y., Kim, Y., Mi, J. L., et al. (2005). Metabolic and pharmacological properties of rutin, a dietary quercetin glycoside, for treatment of inflammatory bowel disease. Pharm. Res. 22, 1499-1509. doi: 10.1007/s11095-005-6250-Z

Liao, B.-Y., Zhu, D.-Y., Thakur, K., Li, L., Zhang, J.-G., and Wei, Z.-J. (2017). Thermal and antioxidant properties of polysaccharides sequentially extracted from mulberry leaves (Morus alba L.). Molecules 22:E2271. doi: 10.3390/ molecules22122271

Locatelli, M., Ferrante, C., Carradori, S., Secci, D., Leporini, L., Chiavaroli, A., et al. (2017). Optimization of aqueous extraction and biological activity of Harpagophytum procumbens root on ex vivo rat colon inflammatory model. Phyther. Res. 31, 937-944. doi: 10.1002/ptr.5821
Makni, M., Haddar, A., Kriaa, W., and Zeghal, N. (2013). Antioxidant, free radical scavenging, and antimicrobial activities of Ajuga iva leaf extracts. Int. J. Food Prop. 16, 756-765. doi: 10.1080/10942912.2011.561465

Mamadalieva, N. Z., El-readi, M. Z., Ovidi, E., Ashour, M. L., Hamoud, R., Sagdullaev, S. S., et al. (2013). Antiproliferative, antimicrobial and antioxidant activities of the chemical constituents of Ajuga turkestanica. Phytopharmacology $4,1-18$.

Manguro, L. O. A., Lemmen, P., and Hao, P. (2011). Iridoid glycosides from underground parts of Ajuga remota. Rec. Nat. Prod. 5, 147-157.

Manguro, L. O. A., Otieno, S., and Lemmen, P. (2006). Flavonol and iridoid glycosides of Ajuga remota aerial parts. Phytochemistry 67, 830-837. doi: 10.1016/j.phytochem.2006.01.005

Miranda, K. M., Espey, M. G., and Wink, D. A. (2001). A rapid, simple spectrophotometric method for simultaneous detection of nitrate and nitrite. Nitric Oxide 5, 62-71. doi: 10.1006/niox.2000.0319

Mocan, A., Crişan, G., Vlase, L., Crişan, O., Vodnar, D. C., Raita, O., et al. (2014). Comparative studies on polyphenolic composition, antioxidant and antimicrobial activities of Schisandra chinensis leaves and fruits. Molecules 19, 15162-15179. doi: 10.3390/molecules190915162

Moldovan, C. M., Oniga, O., Pârvu, A., Tiperciuc, B., Verite, P., Pîrnău, A., et al. (2011). Synthesis and anti-inflammatory evaluation of some new acyl-hydrazones bearing 2-aryl-thiazole. Eur. J. Med. Chem. 46, 526-534. doi: 10.1016/j.ejmech.2010.11.032

Moussa-Ayoub, T. E., Abd El-Hady, E. S. A., Omran, H. T., El-Samahy, S. K., Kroh, L. W., and Rohn, S. (2014). Influence of cultivar and origin on the flavonol profile of fruits and cladodes from cactus Opuntia ficus-indica. Food Res. Int. 64, 864-872. doi: 10.1016/j.foodres.2014.08.021

Movahhedin, N., Zengin, G., Bahadori, M. B., Sarikurkcu, C., Bahadori, S., and Dinparast, L. (2016). Ajuga chamaecistus subsp. scoparia (Boiss.) Rech.f.: a new source of phytochemicals for antidiabetic, skin-care, and neuroprotective uses. Ind. Crops Prod. 94, 89-96. doi: 10.1016/j.indcrop.2016.08.028

Njoroge, G. N., and Bussmann, R. W. (2006). Diversity and utilization of antimalarial ethnophytotherapeutic remedies among the Kikuyus (Central Kenya). J. Ethnobiol. Ethnomed. 2:8. doi: 10.1186/1746-4269-2-8

Ono, M., Furusawa, C., Ozono, T., Oda, K., Yasuda, S., Okawa, M., et al. (2011). Four new iridoid glucosides from Ajuga reptans. Chem. Pharm. Bull. 59, 1065-1068. doi: 10.1248/cpb.59.1065

Ono, Y., Fukaya, Y., Imai, S., and Yamakuni, T. (2008). Beneficial effects of Ajuga decumbens on osteoporosis and arthritis. Biol. Pharm. Bull. 31, 1199-1204. doi: 10.1248/bpb.31.1199

Parvu, A. E., Parvu, M., Vlase, L., Miclea, P., Mot, A. C., and Silaghi-Dumitrescu, R. (2014). Anti-inflammatory effects of Allium schoenoprasum L. leaves. J. Physiol. Pharmacol. 65, 309-315.

Riaz, N., Nawaz, S. A., Mukhtar, N., Malik, A., Afza, N., Ali, S., et al. (2007). Isolation and enzyme-inhibition studies of the chemical constituents from Ajuga bracteosa. Chem. Biodivers. 4, 72-83. doi: 10.1002/cbdv.200790008

Rogerio, A. P., Kanashiro, A., Fontanari, C., Da Silva, E. V. G., Lucisano-Valim, Y. M., Soares, E. G., et al. (2007). Anti-inflammatory activity of quercetin and isoquercitrin in experimental murine allergic asthma. Inflamm. Res. 56, 402-408. doi: 10.1007/s00011-007-7005-6

Salvat, A., Antonacci, L., Fortunato, R. H., Suarez, E. Y., and Godoy, H. M. (2004). Antimicrobial activity in methanolic extracts of several plant species from northern Argentina. Phytomedicine 11, 230-234. doi: 10.1078/0944-711300327

Savran, A., Zengin, G., Aktumsek, A., Mocan, A., Glamočlija, J., Ćirić, A., et al. (2016). Phenolic compounds and biological effects of edible Rumex scutatus and Pseudosempervivum sempervivum: potential sources of natural agents with health benefits. Food Funct. 7, 3252-3262. doi: 10.1039/C6FO00 $695 \mathrm{G}$

Shahidi, F., and Ambigaipalan, P. (2015). Phenolics and polyphenolics in foods, beverages and spices: antioxidant activity and health effects - a review. J. Funct. Foods 18, 820-897. doi: 10.1016/j.jff.2015.06.018

Stana, A., Vodnar, D. C., Tamaian, R., Pîrnău, A., Vlase, L., Ionuţ, I., et al. (2017). Design, synthesis and antifungal activity evaluation of new thiazolin-4-ones as potential lanosterol $14 \alpha$-demethylase inhibitors. Int. J. Mol. Sci. 18:E177. doi: 10.3390/ijms18010177

Stojković, D., Petrović, J., Soković, M., Glamočlija, J., Kukić-Marković, J., and Petrović, S. (2013). In situ antioxidant and antimicrobial activities of naturally 
occurring caffeic acid, $p$-coumaric acid and rutin, using food systems. J. Sci. Food Agric. 93, 3205-3208. doi: 10.1002/jsfa.6156

Tămaș, M., Toiu, A., Oniga, I., Deliu, C., Oltean, B., and Coldea, G. (2009). Quantitative determination of total polyphenols and flavonoids from indigenous species of Epilobium of wild origin and "in vitro" regenerated plantlets. Contrib. Bot. 44, 119-123.

Toiu, A., Vlase, L., Gheldiu, A. M., and Vodnar, D. (2017). Evaluation of the antioxidant and antibacterial potential of bioactive compounds from Ajuga reptans extracts. Farmacia 65, 351-355.

Vlase, L., Parvu, M., Parvu, E. A., and Toiu, A. (2013). Chemical constituents of three Allium species from Romania. Molecules 18, 114-127. doi: 10.3390/ molecules18010114

Xu, P. X., Wang, S. W., Yu, X. L., Su, Y. J., Wang, T., Zhou, W. W., et al. (2014). Rutin improves spatial memory in Alzheimer's disease transgenic mice by reducing $A \beta$ oligomer level and attenuating oxidative stress and neuroinflammation. Behav. Brain Res. 264, 173-180. doi: 10.1016/j.bbr.2014. 02.002

Yu, L., and Cheng, Z. (2008). Application of electron spin resonance (ESR) spectrometry in nutraceutical and food research. Mol. Nutr. Food Res. 52, 62-78. doi: 10.1002/mnfr.200700395
Zengin, G., Uysal, A., Gunes, E., and Aktumsek, A. (2014). Survey of phytochemical composition and biological effects of three extracts from a wild plant (Cotoneaster nummularia Fisch. et Mey.): a potential source for functional food ingredients and drug formulations. PLOS ONE 9:e113527. doi: 10.1371/journal. pone. 0113527

Zhang, Y. Y., Zhang, F., Thakur, K., Ci, A. T., Wang, H., Zhang, J. G., et al. (2017). Effect of natural polyphenol on the oxidative stability of pecan oil. Food Chem. Toxicol. doi: 10.1016/j.fct.2017.10.001 [Epub ahead of print].

Conflict of Interest Statement: The authors declare that the research was conducted in the absence of any commercial or financial relationships that could be construed as a potential conflict of interest.

Copyright (c) 2018 Toiu, Mocan, Vlase, Pârvu, Vodnar, Gheldiu, Moldovan and Oniga. This is an open-access article distributed under the terms of the Creative Commons Attribution License (CC BY). The use, distribution or reproduction in other forums is permitted, provided the original author(s) and the copyright owner are credited and that the original publication in this journal is cited, in accordance with accepted academic practice. No use, distribution or reproduction is permitted which does not comply with these terms. 


\section{OPEN ACCESS}

Edited by:

Atanas G. Atanasov,

Institute of Genetics and Animal

Breeding (PAN), Poland

Reviewed by:

Fawzi Mohamad Mahomoodally,

University of Mauritius, Mauritius

Stefan Gafner,

American Botanical Council,

United States

*Correspondence:

Alexander N. Shikov alexs79@mail.ru

Specialty section:

This article was submitted to

Ethnopharmacology,

a section of the journal

Frontiers in Pharmacology

Received: 28 August 2017 Accepted: 03 November 2017 Published: 21 November 2017

Citation:

Shikov AN, Tsitsilin AN,

Pozharitskaya ON, Makarov VG and Heinrich M (2017) Traditional and

Current Food Use of Wild Plants Listed in the Russian Pharmacopoeia.

Front. Pharmacol. 8:841.

doi: 10.3389/fphar.2017.00841

\section{Traditional and Current Food Use of Wild Plants Listed in the Russian Pharmacopoeia}

\author{
Alexander N. Shikov ${ }^{1 *}$, Andrey N. Tsitsilin ${ }^{2}$, Olga N. Pozharitskaya ${ }^{1}$, Valery G. Makarov ${ }^{1}$ \\ and Michael Heinrich ${ }^{3}$ \\ ${ }^{1}$ Saint-Petersburg Institute of Pharmacy, Kuzmolovsky, Russia, ${ }^{2}$ All Russian Research Institute Medicinal and Aromatic Plants \\ (VILAR), Moscow, Russia, ${ }^{3}$ Research Cluster Biodiversity and Medicines, Centre for Pharmacognosy and Phytotherapy, UCL \\ School of Pharmacy, University of London, London, United Kingdom
}

Historically Russia can be regarded as a "herbophilious" society. For centuries the multinational population of Russia has used plants in daily diet and for self-medication. The specificity of dietary uptake of medicinal plants (especially those in the unique and highly developed Russian herbal medical tradition) has remained mostly unknown in other regions. Based on 11th edition of the State Pharmacopoeia of the USSR, we selected 70 wild plant species which have been used in food by local Russian populations. Empirical searches were conducted via the Russian-wide applied online database E-library.ru, library catalogs of public libraries in St-Petersburg, the databases Scopus, Web of Science, PubMed, and search engine Google Scholar. The large majority of species included in Russian Pharmacopoeia are used as food by local population, however, aerial parts are more widely used for food. In this review, we summarize data on medicinal species published in Russia and other countries that are included in the Russian Pharmacopoeia and have being used in food for a long time. Consequently, the Russian Pharmacopoeia is an important source of information on plant species used traditionally at the interface of food and medicine. At the same time, there are the so-called "functional foods", which denotes foods that not only serves to provide nutrition but also can be a source for prevention and cure of various diseases. This review highlights the potential of wild species of Russia monographed in its pharmacopeia for further developing new functional foods and-through the lens of their incorporation into the pharmacopeia-showcases the species' importance in Russia.

Keywords: medicinal plants, traditional use, functional food, health benefits, Russian Federation

\section{INTRODUCTION}

Until today over 7000 species have been recorded to be edible (Lim, 2012) and for many of these specific health benefits have been claimed, with, however, generally only limited pharmacological evidence for such claims. Only a small share of these is used on a large, extensive scale. Wild and cultivated food plants may have medicinal, nutraceutical, pharmaceutical, and health benefits. Plants are excellent sources of active phytochemicals with importance in the prevention of different diseases. Herbal medicinal products are going recognition for the treatment and prophylaxis of different diseases. Direct nutritional benefits of such plants are often known 
only to a limited extend. Herbal infusions will add small quantities of essential micronutrients and vitamins to the daily intake. For sick or malnourished individuals this may contribute to restoring the balance between nutrients and thereby improve or sustain health (Lim, 2012).

Blurring of the food and medicine interface is a common theme across multiple contexts and cultures (Heinrich, 2016). The association between food and disease is widely recognized as the bedrock of preventive nutrition. Throughout the ages, and at least since the proclamation "let food be your medicine, let medicine be your food" which has been attributed to Hippocrates' (ca. 480-377 BC), physicians recognized the impact of food on human health. The question of the continuum between food and medicine is of great interest to (ethno-)pharmacologists (Etkin and Ross, 1982; Leonti, 2012; Valussi and Scire, 2012; Jennings et al., 2014; Alarcón et al., 2015).

In many countries medicinal plants are widely used as dietary supplements, in daily foods and as functional foods, with the aim of promoting health. In Eastern cultures food and medicine are seen to come from the same source. Their use is based on the same fundamental theories, and they are equally important in maintaining and improving health and for preventing and curing diseases, as well as facilitating rehabilitation. In China, Japan, Korea, and Southeast Asia, medicinal plants are widely used both daily foods (cereals, vegetables, and fruits) and functional foods, for replacement and medical purposes (Shi et al., 2011). The last few decades has seen the introduction to Europe of a wide range of species used in other continents, including from South Africa e.g. rooibos (Aspalathus linearis (Burm.f.) R.Dahlgren) and to a lesser extent honey bush tea (Cyclopia sp.) or Hoodia gordonii (Masson) Sweet ex Decne. to regulate the appetite. Also some exotic fruits, such as acerola (Malpighia glabra L.) from South America, rich in vitamin C are now marketed as food supplements (Franz et al., 2011).

In the early history of Western biomedicine, diet figured prominently in the prevention of diseases and therapeutics. The history of biomedicine customarily is traced to Greece and the Hyppocratic Corpus, some 60 medical texts dating to the fifth to fourth centuries BCE. Diet regulation was the most prominent element in all the Hippocratic texts (Etkin, 2006). Due to its location between East and West Russian tradition of medicine and diet has accumulated and adopted approaches that originated in Europe and Asia. The Greek influence on Russian medicine is of particular relevance in this context (Shikov et al., 2014a).

Russia in the past and present can be regarded as a "herbophilious" society. The term "herbophilia" was used by Łuczaj (2008) for such cultures, in which medicinal and food plant species are often used and highly prized. Approximately $58 \%$ of the Russian population was reported to use phytopharmaceuticals as a form of treatment (Shikov et al., 2011) including wild plants. Plants are an integral part of Russian everyday life. A large number of species of green vegetables are used in agricultural communities, particularly those in which food shortages are frequent. Due to the harsh climatic conditions coupled with poor crop harvests Russian peasants were educated on how to survive by relying on wild plants (Kovaleva, 1972).
Wild food plants became highest priority in the USSR during the Second World War most notably during the siege of Leningrad (1941-1944). In 1942 a special manual about most important wild food plants of the Leningrad region was published and distributed to the army and civilians. The scientists from V.L. Komarov Botanical Institute described thirty seven common herbs and two edible lichens, their nutritional and functional properties, specificity of harvesting, and food processing (Tikhomirov, 1942).

As a result of socio-economic changes after the Second World War, the active role of wild plants gradually diminished in regular diet. However, some species are still gathered and made into food products by small companies. Wild berry harvesting is very popular especial among of rural populations. The practice of preparing jam, compotes, and home made liqueurs from wild berries has been passed down by housewives from generation to generation (Grigorieva, 2008).

A wide choice of herbal medicines or botanicals has provided increasing opportunities for both the development and marketing of herbal food products, dietary supplements, and functional foods.

Wild species were selected for food application by humans not only because of their pleasant taste and aroma, but some pharmacological effects were considered as well (Gammerman and Grom, 1976). Herbal medicine products have become popular over the past decade and they are widely used for the treatment and prevention of various diseases. However, safety and efficacy of herbal medicine should be confirmed. Utilization of knowledge about positive pharmacological effects of edible plants included in in a pharmacopeia is one of probably safe and effective way for development of functional products with new beneficial effects (Kunakova et al., 2011).

Searching for new food supplements is essential. Systematic explorations of pharmacopoeial plants are one particularly relevant opportunity in Eastern Europe, especially in those areas which, for historical reasons, remain relatively isolated. The use and importance of Russian medicinal plants remains largely unknown in the West. The aim of this review is to summarize data about species that are referenced in the Russian Pharmacopoeia and about their health food value with a primary focus on literature published in Russian.

\section{OVERVIEW OF SPECIES FROM THE RUSSIAN PHARMACOPOEIA USED AS FOODS}

For centuries the multinational population of Russia has used plants in daily diet and for self-medication. The beneficial effects of plants were carefully collected by "knowledgeable experts" (znahar = "знахарь" in Russian) and recorded in chronicles and manuscripts. In 1778, Russia was among the first countries to implement a national pharmacopeia: The Pharmacopoeia Rossica in St. Petersburg. This work contains 770 monographs, of which 316 texts are on herbal medicinal preparations (Shikov et al., 2014a). 
Since 1990, the Russian Federation has followed the State Pharmacopoeia of the USSR (1990) 11th edition. This Pharmacopeia includes 83 individual monographs for plants describing 119 species. There are 26 Rosaceae, 12 Compositae (syn. Asteraceae), eight Lamiaceae, six Polygonaceae, four each of the Apiaceae, Betulaceae, Ericaceae, and Malvaceae species, three representatives each of the Solanaceae, Leguminosae (Fabaceae), and Plantaginaceae, two species each of the Adoxaceae (Caprifoliaceae), Asparagaceae (Liliaceae, s.l), Araliaceae Fagaceae, Gentianaceae, Hypericaceae, Laminariaceae, Pinaceae, Rhamnaceae, and Violaceae, and one member each of the Acoraceae (Araceae), Brassicaceae, Caprifoliaceae (Valerianaceae), Crassulaceae, Cucurbitaceae, Cupressaceae, Equisetaceae, Hymenochaetaceae, Linaceae, Menyanthaceae, Myrtaceae, Papaveraceae, poaceae, Polemoniaceae, Ranunculaceae, Rubiaceae, Saxifragaceae, Schisandraceae, and Urticaceae, respectively.

In the most of monographs, just one species is described as a source of plant material. However, 13 species are approved for Fructus rosae, 11 species are approved for Fructus crataegi, two species are approved for Herba hyperici, Fructus alni, Herba violae etc. Different parts are described for Viburnum opulus L. (Cortex viburni as diuretic and Fructus viburni as diaphoretic and anti-inflammatory) and Crataegus (Flos crataegi-13 species and Fructus crataegi-11 species for cardiovascular problems).

The large majority of species included in the Russian Pharmacopoeia (98 out of 119) are known to have been used as food by local population in Russia. For a majority of species, the part of plant used in food and as a medicine is the same. For example, the aerial parts of Origanum vulgare L., Thymus serpyllum L., Leonurus cardiaca L., are used as a medicine and as a food. Acorns and leaves of Quercus robur L. and Q. petraea (Matt.) Liebl. are used in food, while oak bark is used as a medicine. Fruits and bark of Viburnum opulus L. are used as medicine, with only the fruits being edible. Roots and rhizomes of Acorus calamus L., Taraxacum campylodes G.E.Haglund (syn. Taraxacum officinale L.), and Aralia elata (Miq.) Seem. are used as a medicine while young sprouts, leaves, flowers and fruits are used in food. The aerial parts of Equisetum arvense L. are used in medicine, while its cone-bearing shoots and black nodules attached to the roots are eaten raw in spring.

In general, considering all 98 edible plant species in the Russian Pharmacopoeia 11th edition, a higher number of plant parts are used in nutrition as compared to medicine. In the context of this article, the term "wild medicinal plants" referrings mainly to non-cultivated species. Twenty one plants including Calendula officinalis L., Eucalyptus viminalis Labill., Pimpinella anisum L., Zea mays L., and some others do not occur in Russia outside of cultivation and are excluded from this review. All poisonous and toxic species are not used in food as such as the species belonging to the Solanaceae (Atropa belladonna L., Datura stramonium L., Hyoscyamus niger L.), Asparagaceae (Convallaria majalis L., Convallaria keiskei Miq.), Rhamnaceae (Frangula alnus Mill., Rhamnus cathartica L.), Ranunculaceae (Adonis vernalis L.), Papaveraceae (Chelidonium majus L.) and some species from the newly circumscribed Plantaginaceae (Digitalis purpurea L., Digitalis grandiflora Mill.).
Consumer and marketing studies invariably showed that taste, as opposed to perceived nutrition or health value is the key influence on food selection (Drewnowski and Gomez-Carneros, 2000). Sensitized to the astringent taste of tannins or some bitter poisons, humans reject foods that are perceived to be excessively astringent or bitter. Polemonium caeruleum L. (Polemoniaceae), Rubia tinctorum L. (Rubiaceae), Alnus incana (L.) Moench and Alnus glutinosa (L.) Gaertn. (Betulaceae) are not considered to be edible due to their taste, as well as Gnaphalium uliginosum L. and Bidens tripartita L. (Asteraceae) which, however, are without any strong taste.

Edible wild species included in the Russian Pharmacopoeia have a wide range of uses: expectorant, diuretic, astringent, haemostatic, bitterness and choleretic, anti-inflammatory, diaphoretic, tonic, sedative, spasmolytic, polyvitamin, cardiovascular, and antihelminthic (Table 1).

Plants with expectorant properties alleviate the symptoms of patients in certain stages of bronchitis or tracheitis. Beneficial expectorant effects are mentioned for nine edible wild species. Certain edible species (nine species) are natural diuretics. Astringent species (eight species) provide not only specific taste for wine, tea, and other beverages due to high tannins content, but are effective in stopping the flow of blood or other secretions, and resolve diarrhea. Bitterness and choleretic herbs (seven species) are useful for development of functional foods with digestion-enhancing properties. The haemostatic effects of herbs (six species) are often due to mechanisms such as tannin astringency rather than enhancement of coagulation, although there are a few haemostatic herbs have been shown to reduce clotting times and have inhibitory effects on the Platelet Aggregation Factor. Four species included in the Russian Pharmacopoeia are used for their anti-inflammatory action. In the last decades, so-called natural anti-inflammatory diets have gained popularity (Sears, 2015). Diaphoretic herbs (four species) induce involuntary perspiration and thus reduce fever, promote circulation, relieve muscle tension, aching joints, and inflammatory skin conditions. These herbs are used as well to relieve diarrhea, dysentery, kidney nephritis, liver, urinary, and gall bladder. Tonic herbs (four species) as supplement to food are consumed throughout the world on a daily basis to promote radiant health and are included in many beverages. Motherwort and valerian provide mild sedative effects. The species with spasmolytic effects (two species) are noticeable as components of diets for treatment of gastro-intestinal ailments.

Hawthorn berries and flowers are useful for the creation of functional products with cardiovascular properties. Antihelmintic properties of tansy are complementary to its choleretic effects. The well documented anxiolytic effect of $L$. cardiaca L. may lead to the development of antistress functional food products. Increased access to information through systematic evaluation of medicinal plants and its nutritional properties will support the generation of ideas for functional food with new value added properties.

Underground parts like roots, shoots and leafy greens, berries and other fleshy fruits, seeds, and other species commonly yield wild-harvested foods, a practice which is based on specialized 


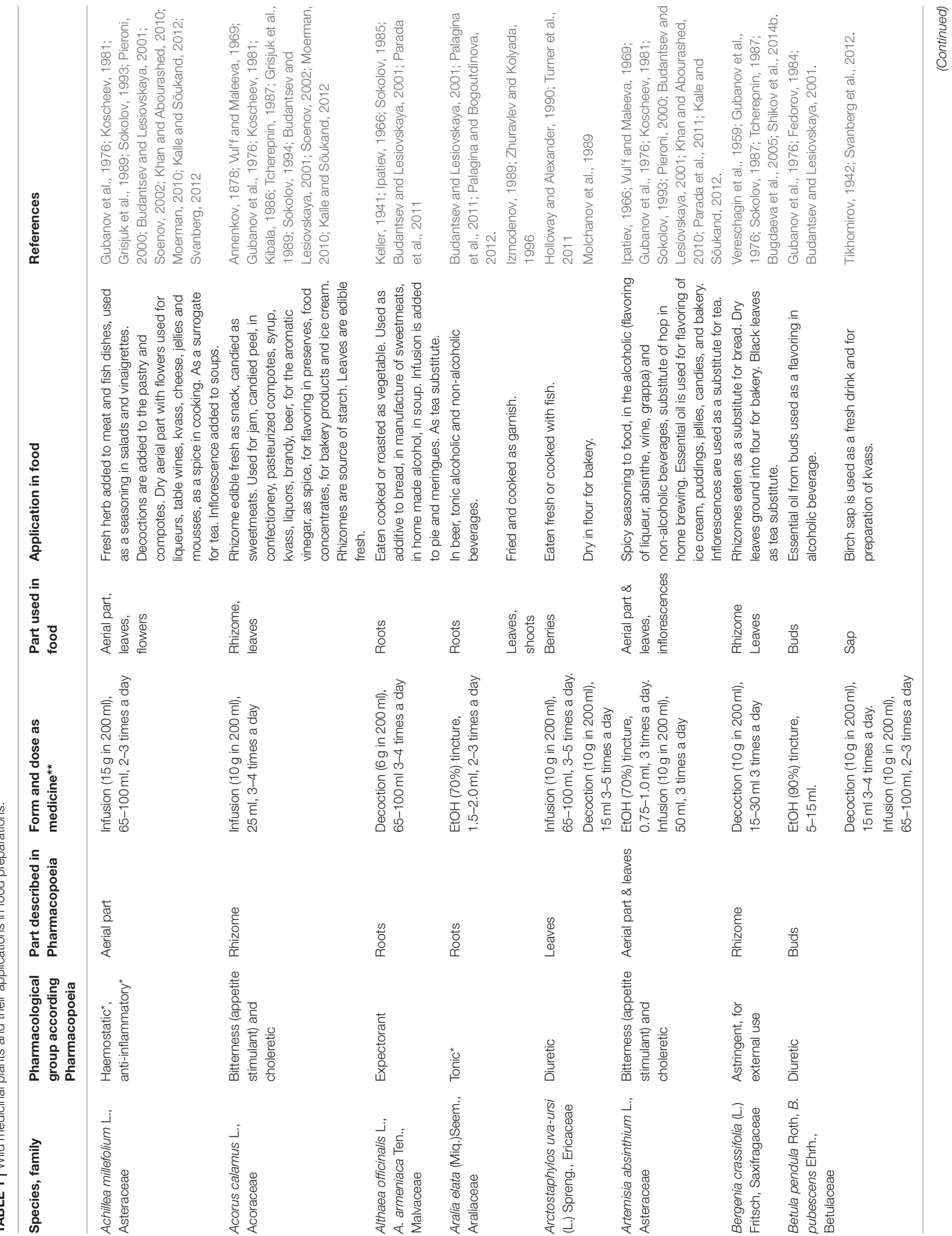




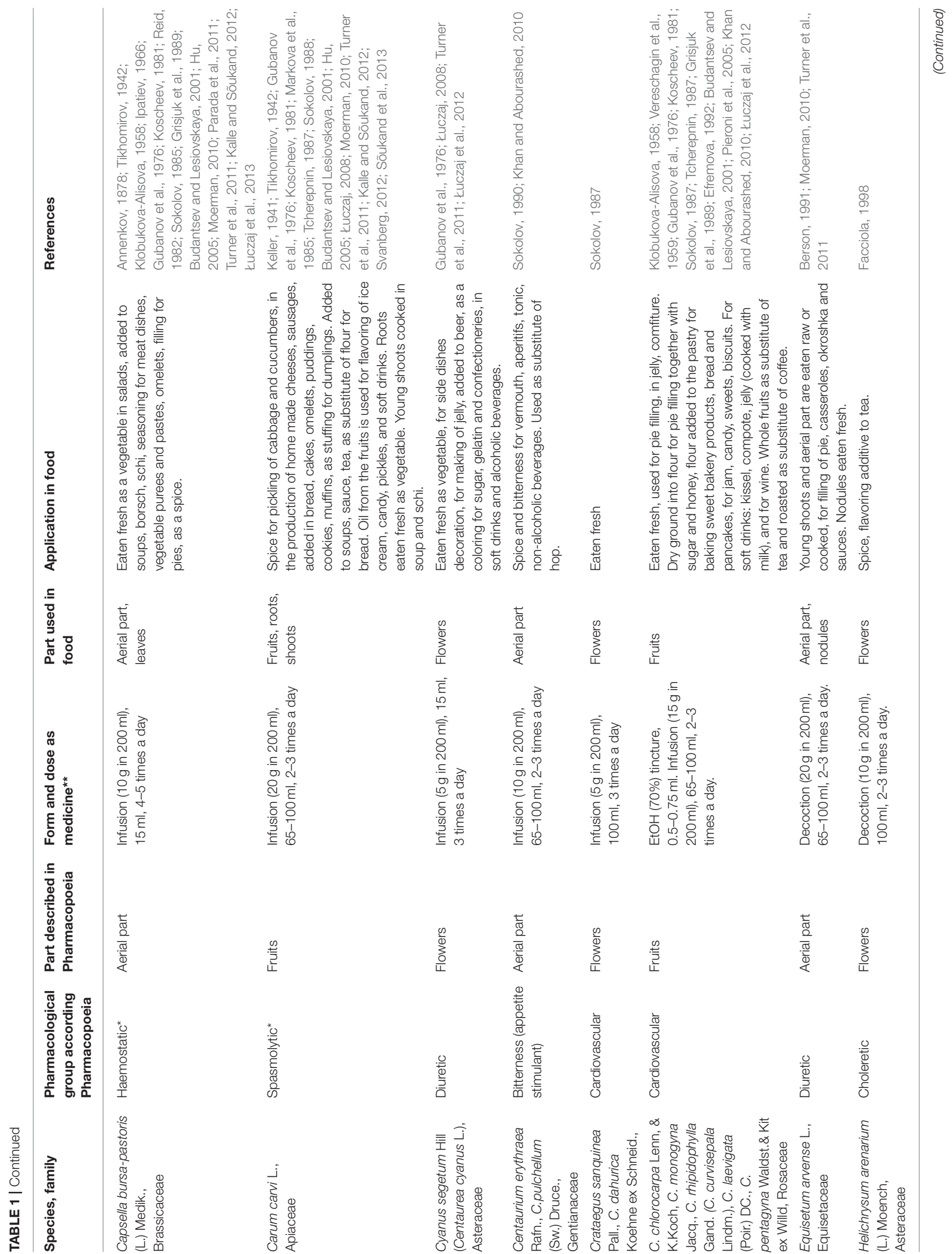




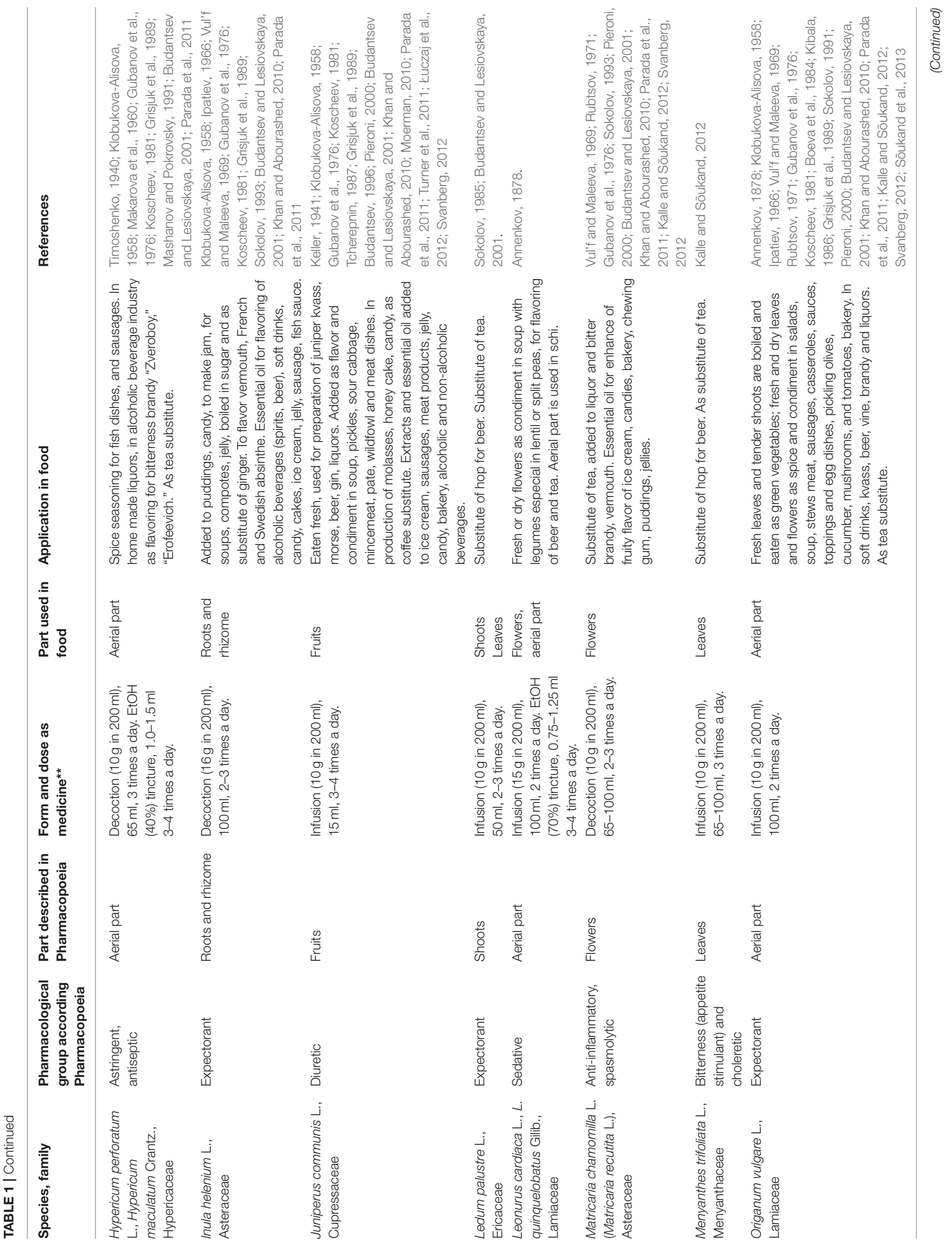




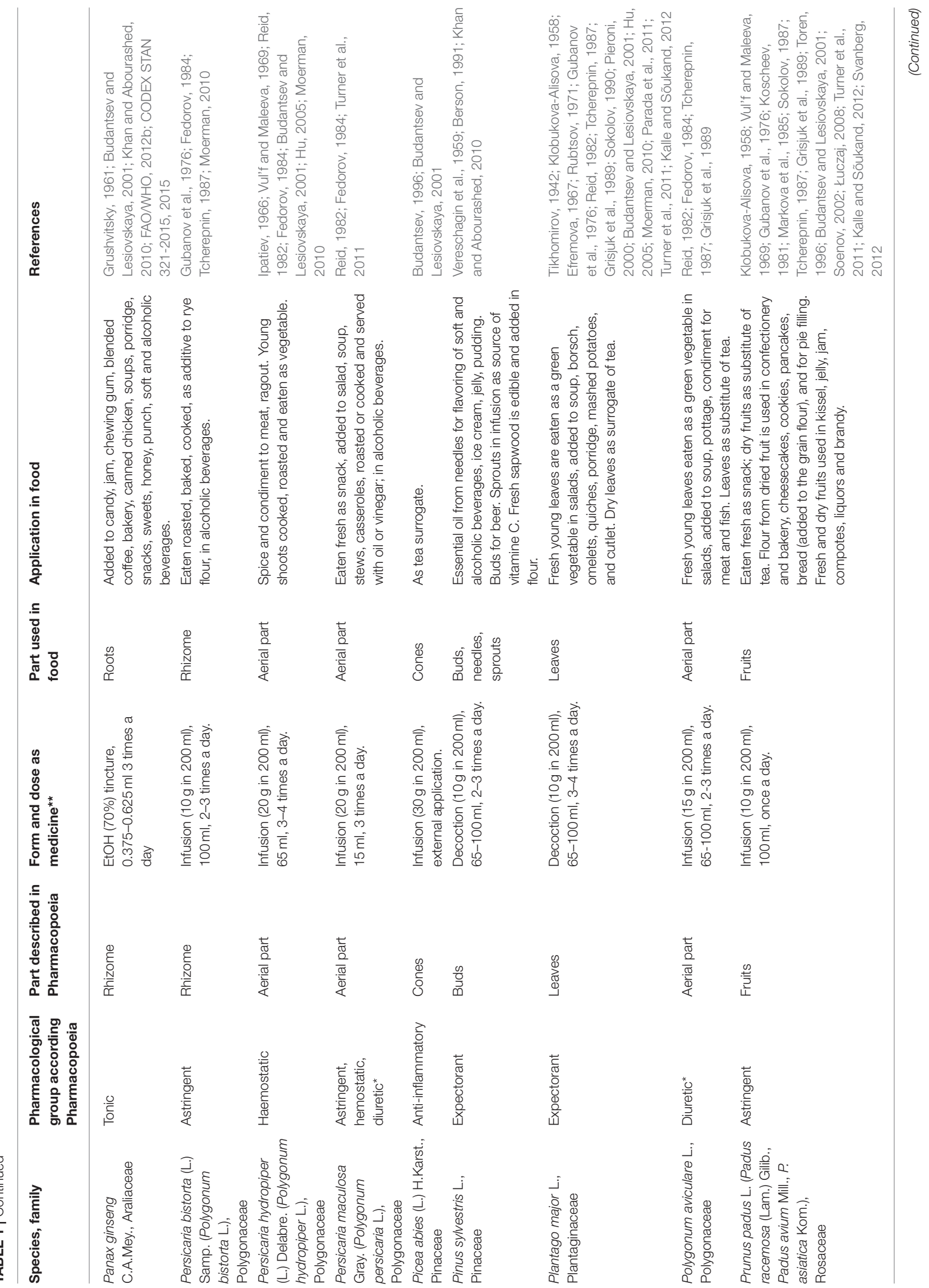




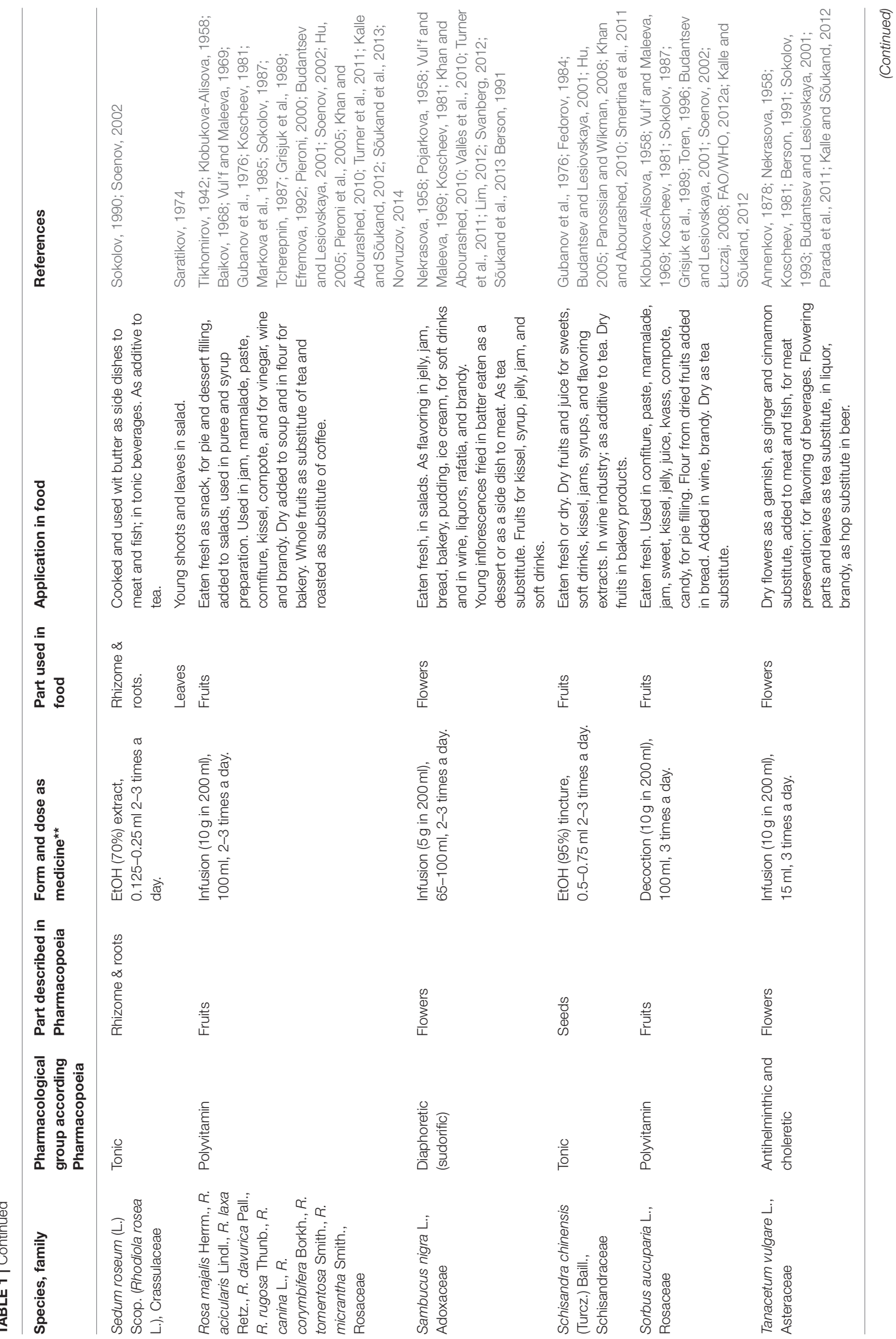




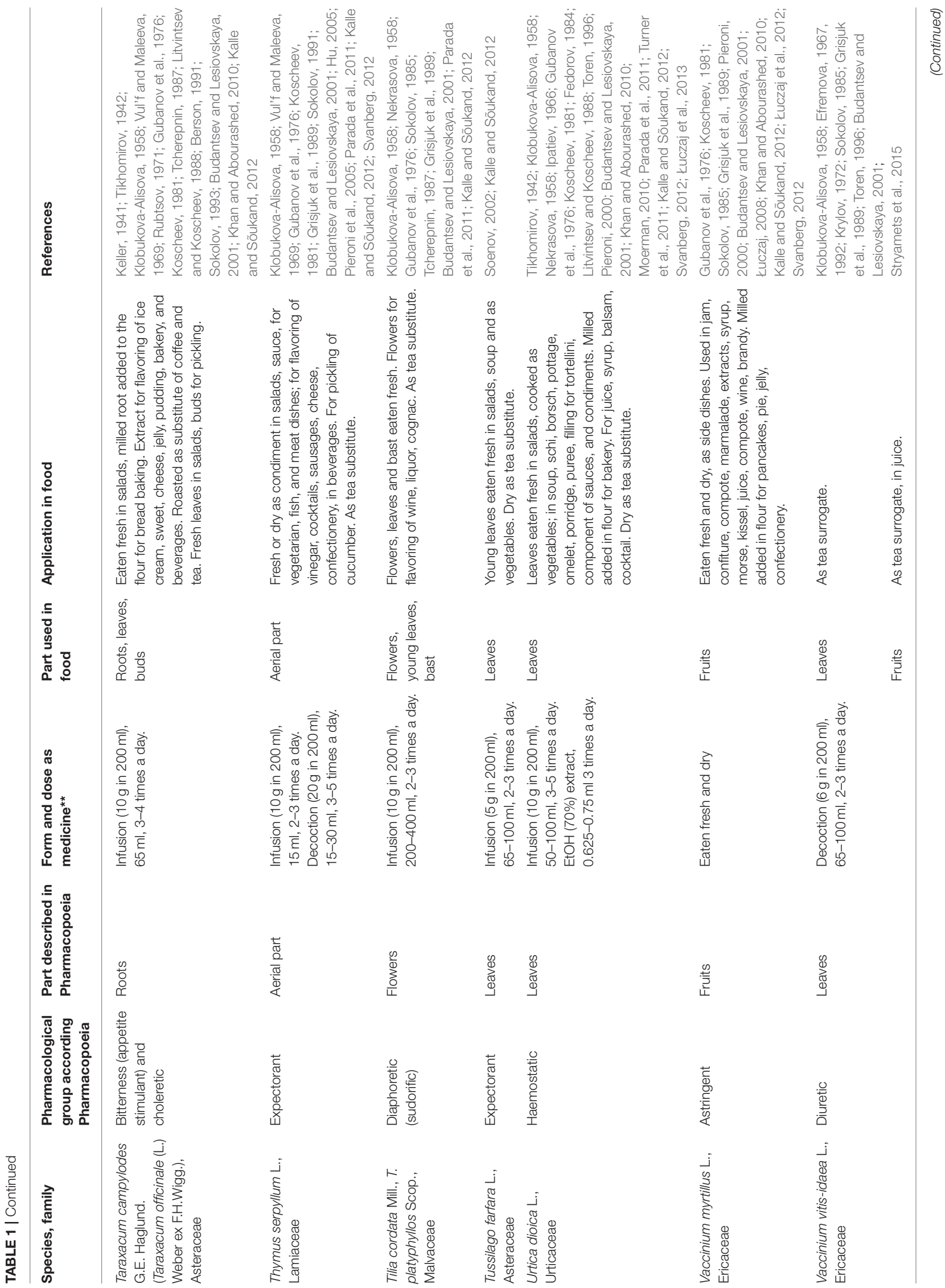




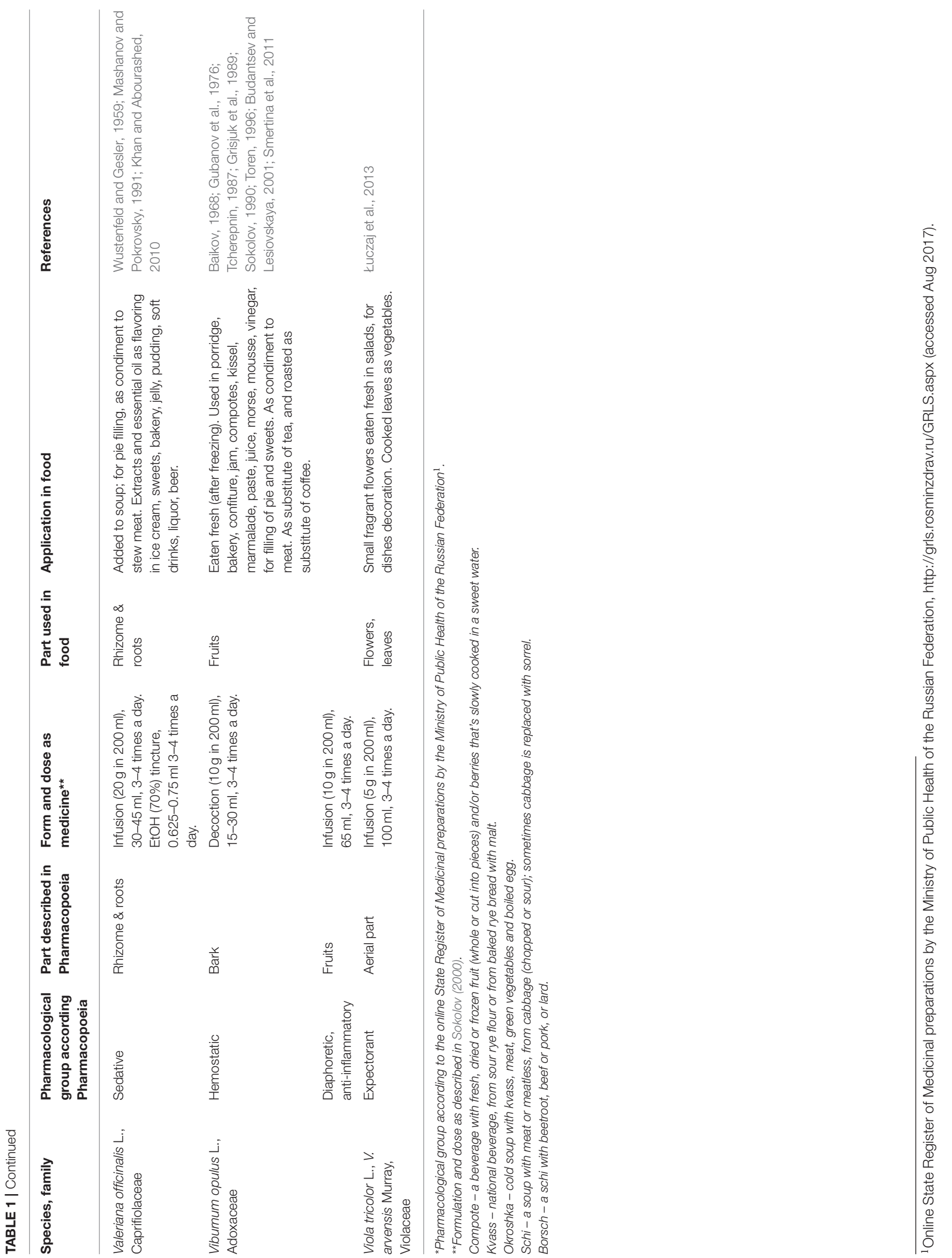


cultural knowledge of their harvesting, preparation, cooking and other forms of processing.

\section{EDIBLE WILD PLANTS-APPROACHES AND METHODS FOR ASSESSING USES AND EVIDENCE}

Based on the State Pharmacopoeia of the USSR, we selected seventy wild species that are used as food in Russia and systematically searched the scientific literature (published between 1878-2016) for data using the Russia-wide applied online database E-library.ru, library catalogs of public libraries in St-Petersburg, the databases Scopus, Web of Science, PubMed, and search engine Google Scholar. The primary search criterion was a food application of medicinal plants.

Edible wild plants include food categories familiar to everyone: green vegetables and potherbs, wild berries and fruits, beverages, tea and coffee substitutes, seasonings and spices, sweets, bread surrogates, plants used for preserves. Family names of the species are based on www.theplantlist.org database with the names in the Russian Pharmacopoeia given in brackets (Table 1). Also included are the applications in food.

\section{Green Vegetables and Potherbs}

This category includes the majority of examined plants. Especially in the spring or at the beginning of their growing season, many wild species produce tender, edible leaves and shoots, and flowers at the beginning of flowering. Some, like shepherd's-purse (Capsella bursa-pastoris (L.) Medik.), cornflower (Cyanus segetum Hill., syn. Centaurea cyanus L.), and broadleaf plantain (Plantago major L.) can be eaten raw, after being peeled from soil (Sokolov, 1985, 1990; Tcherepnin, 1987; Grisjuk et al., 1989; Budantsev and Lesiovskaya, 2001; Turner et al., 2011), whereas others, like stinging nettles (Urtica dioica L.) must be steamed or cooked in some way (Litvintsev and Koscheev, 1988; Fedorov, 1984; Toren, 1996). These plants are used for salads, added to soups, borsch, omelets, cooked and used as a garnish.

Many edible greens emerging right after the melting of snow are particularly important for their vitamin C content in the spring and traditionally have been used to prevent and alleviate scurvy. In particularly, soup and borsch with nettles are popular not only among rural but also many urban people (Gubanov et al., 1976; Koscheev, 1981; Tcherepnin, 1987; Litvintsev and Koscheev, 1988).

Tonic properties of shoots of aralia (Aralia elata (Miq.)Seem.) were recognized by Ussuri aborigines (Far East). Young shots no more than $20 \mathrm{~cm}$ are cooked and served as garnish (Izmodenov, 1989).

\section{Wild Berries and Fruits}

Presumably, wild berries and fruits are the most favored group of edible medicinal plants in Russia, and today probably used most frequently. The wild fruits most commonly collected in Russia include bilberry (Vaccinium myrtillus L.), hawthorn (Crataegus spp.), and rose hips (Rosa spp.) (Gubanov et al., 1976; Koscheev,
1981; Sokolov, 1987; Tcherepnin, 1987; Grisjuk et al., 1989; Efremova, 1992; Budantsev and Lesiovskaya, 2001; Soenov, 2002; Łuczaj et al., 2012; Sõukand et al., 2013; Novruzov, 2014). All of them are consumed fresh, but some are also preserved for the winter by making jams, marmalade or pasteurized compotes (Turova and Sapozhnikova, 1989). Furthermore, they are used to make beverages. Some berries are popular among all groups of the population (like bilberry), other species that once were used as a valuable food source in some regions of Russia have seen that food use diminished because of the bitter or astringent taste as such as guelder rose (Viburnum opulus L.), bird cherry (Prunus padus L.), and rowan (Sorbus aucuparia L.) (Baikov, 1968; Rubtsov, 1971; Gubanov et al., 1976; Sokolov, 1985, 1990; Tcherepnin, 1987; Grisjuk et al., 1989; Toren, 1996; Smertina et al., 2011). The berries and seeds of Schisandra chinensis (Turcz.) Bail. were eaten by Nanai (Goldes or Samagir) hunters from Far Eastern Russia since "it gives forces to follow a sable all the day without food" and were acclaimed as a tonic, to improve night vision and to reduce hunger, thirst and exhaustion (Panossian and Wikman, 2008).

\section{Beverage}

Aerial parts, leaves or flowers are used mainly for making beverages, while the underground parts are used rarely. Aromatic plants belonging to the Asteraceae and Lamiaceae are used to sweeten or to flavor alcoholic and non-alcoholic beverages: yarrow (Achillea millefolium L.), tansy (Tanacetum vulgare L.), and oregano (Origanum vulgare L.) (Timoshenko, 1940; Wustenfeld and Gesler, 1959; Koscheev, 1981; Berson, 1991; Sokolov, 1991; Budantsev and Lesiovskaya, 2001; Soenov, 2002; Kalle and Sõukand, 2012; Sõukand et al., 2013). Juicy fruits of species belonging to the Rosaceae (rowan and bird cherry) are used often for coloring of alcoholic beverages and giving a more pronounced flavor. The fruits of these plants are used both in home made alcoholic beverages as well as in wine- and brewing industry for the production of liqueurs, bitters, wines, and beers (Wustenfeld and Gesler, 1959; Gubanov et al., 1976; Koscheev, 1981; Tcherepnin, 1987; Toren, 1996; Budantsev and Lesiovskaya, 2001). Making liqueurs out of rowan berries seems to be an old habit that has been in vogue in Russia (Timoshenko, 1940). The aroma is an important attribute of refreshing soft drinks (kvass, lemonade, and fruit morse), which are popular in the summer season.

Tonic properties of ginseng and aralia were recorded in the Far Eastern Russia by hunters and their roots were regularly added to alcoholic and nonalcoholic beverages, and balsams (Grushvitsky, 1961; Zhuravlev and Kolyada, 1996; Budantsev and Lesiovskaya, 2001; Palagina et al., 2011; Palagina and Bogoutdinova, 2012).

Birch (Betula pendula Roth., and B. pubescens Ehrh.) sap has been gathered all over Russia and was usually seen as a refreshing drink (Fedorov, 1984; Tcherepnin, 1987; Budantsev and Lesiovskaya, 2001). Today, Russia, Estonia, Latvia, Lithuania, Ukraine and Belarus are the only countries where the gathering and use of birch sap has remained an important. Large birch forests, low population density and the incorporation of sap into the former Soviet economic system facilitated this (Svanberg et al., 2012). Birch sap was utilized the diverse ways as described 
by the Russian ethnographer Zelenin (1927). It was drunk fresh, but also fermented by adding malt, wax, beans or rye bread.

\section{Tea and Coffee Substitutes}

After water, tea is the second most-consumed beverage worldwide (Keating et al., 2015). Although the English term "tea" generally denotes an infusion made of the leaves of Camellia sinensis (L.) Kuntze, many herbal teas, which usually are monoor polyherbal formulations made from (medicinal) plant(s), are available worldwide. Historically in Russia such herbal teas often made from local species were widespread. Samovar and teadrinking are an indispensable element of Russian culture. With C. sinensis not being available or affordable to the vast majority of population, local surrogates/substitutes of tea have been used for centuries. The best known tea surrogates were prepared from fireweed (Epilobium angustifolium L.) and, in Siberia Bergenia spp. (Tcherepnin, 1987; Pohlebkin, 1998; Shikov et al., 2006, 2014b; Sõukand et al., 2013). Prior to the introduction of oriental (black or green) tea in Europe, E. angustifolium was esteemed as the "original Russian tea" and used widely throughout Russia and beyond (Litvintsev and Koscheev, 1988; Sõukand et al., 2013). However, this botanical drug is not in the Russian Pharmacopoeia. Extracts of black and fermented leaves of $B$. crassifolia are reported as appetite and energy intake suppressants (Shikov et al., 2012). Other species that are used as substitutes for tea in Russia belong to different families. First of all they are expected to bring delicious flavor, taste and nice color to the tea. Marsh Labrador tea (Ledum palustre L.), thyme (Thymus serpyllum L.), wormwood (Artemisia absinthium L.), are highly aromatic. Aerial parts of Saint John's wort (Hypericum perforatum L.), oregano, thyme, leaves of lingonberry (Vaccinium vitis-idaea L.), flowers of linden (Tilia cordata Mill. and T. platyphyllos Scop.) are among the most popular components of herbal teas in Russia (Krylov, 1972; Koscheev, 1981; Sokolov, 1985; Grisjuk et al., 1989; Efremova, 1992; Kalle and Sõukand, 2012). Dry fruits of rose hips, hawthorn, and rowan are used as substitutes of tea (Gubanov et al., 1976; Tcherepnin, 1987; Budantsev and Lesiovskaya, 2001). Ethnobotanical data also lists dried and roasted rhizomes of dandelion (Taraxacum campylodes G.E. Haglund), dry seeds of guelder rose, roasted fruits of hawthorn, and fruits of $R$. canina L.as coffee substitutes (Klobukova-Alisova, 1958; Vul'f and Maleeva, 1969; Koscheev, 1981; Sokolov, 1990, 1993).

\section{Seasoning, Spices}

Aromatic herbs are very important part of local gastronomy, especial for seasoning and as spices in southern Russia. In particular, sweet flag (A. calamus L.), valerian (Valeriana officinalis L.), elecampane (Inula helenium L.) roots and rhizomes, juniper (Juniperus communis L.), and caraway (Carum carvi L.) fruits, dwarf everlast (Helichrysum arenarium (L.) Moench) and tansy flowers, yarrow, oregano, thyme, and artemisia aerial parts are normally used as aroma and flavor enhancer or digestives (Sokolov, 1988, 1994; Berson, 1991; Mashanov and Pokrovsky, 1991). These species are used both in culinary preparations at home: they are added to the soups and main dishes, salads, meats, bakery, as well as in the food industry in the production of sausages, confectionery and bakery products.

\section{Sweets}

The Russian make a kind of sweet flag and elecampane candied peel by boiling the transverse root slices in syrup, and drying (Gubanov et al., 1976; Koscheev, 1981; Berson, 1991). Candied fruits of hawthorn, bird cherry, guelder rose are used as sweetness (Gubanov et al., 1976; Koscheev, 1981; Tcherepnin, 1987; Budantsev and Lesiovskaya, 2001).

\section{Bread Surrogates}

In the eighteenth and the first half of the nineteenth century flour and products like bread derived from it were the main foods for Russian peasants. Consequently, every an extended period of bad weather resulting in crop failure resulted in largescale famines. This explains why a vast variety of plant parts was used for filling bread, including roots, fruits, leaves, and barks. Roots and rhizomes of some plants are known to be good sources of starch and carbohydrates. Powdered roots of Persicaria bistorta (L.) Samp. were added to the rye flour for baking bread (Rubtsov, 1971; Fedorov, 1984; Tcherepnin, 1987). Bergenia rhizomes were eaten as a substitute for bread (Vereschagin et al., 1959; Tcherepnin, 1987). Flour from black leaves of bergenia is used for cookies (Bugdaeva et al., 2005). Roots of sweet flag were used as source of starch (Berson, 1991). Whole or crushed caraway seeds, dried and ground into flour fruits of hawthorn, bird cherry, rowan, guelder rose were added to the pastry when baking bread and sweet bakery (cakes and pancakes) (Gubanov et al., 1976; Koscheev, 1981; Tcherepnin, 1987; Budantsev and Lesiovskaya, 2001).

In the middle of nineteenth century, fruits of guelder rose (Viburnum opulus) were popular in Tver province of Russia as ingredients for making "kulaga," a porridge based on malt flour. Guelder rose fruits tenderized with flour and honey were "a tasty cuisine and refined taste for urban residents, not excluding the nobility" (Toren, 1996). Residents of Pskov province received the nickname "kalinniki" because they cooked a delicious porridge with guelder rose ("kalina" in Russian) berries and licorice flour, which was sold at the bazaar (Toren, 1996).

Despite the fact that today wild bread additives have almost completely disappeared from the European diet (Luczaj et al., 2012), in Russia some local bakeries produce bread and buns with nettle, hawthorn, and dog rose.

\section{Species Used in Preserves}

Home made preserves have a long tradition in Russia because of the long and severe cold season. There are many canning recipes in every household for foods which have been handed down from generation to generation. Plants used for preserves can be divided into two large groups. Fresh juicy berries of rose hips, guelder rose, rowan, bird cherry, and bilberry are widely used in jam, comfiture, and marmalade. All these berries are useful for filling pies (Klobukova-Alisova, 1958; Baikov, 1968; Gubanov et al., 1976; Sokolov, 1985, 1990; Budantsev and Lesiovskaya, 2001; Soenov, 2002). The second group includes species used as spices and condiments for pickling and salting of cabbage, cucumbers, 
tomatoes and other vegetables, as well as mushrooms: caraway, juniper, and thyme (Rubtsov, 1971; Gubanov et al., 1976; Koscheev, 1981; Tcherepnin, 1987; Litvintsev and Koscheev, 1988; Grisjuk et al., 1989; Sõukand et al., 2013).

\section{CONCLUSIONS}

This review focuses on botanical drugs with a monograph in the Russian Pharmacopoeia 11th edition and their potential as untapped resources beyond their strictly medical uses. In general, the Soviet period was characterized by the country being closed not only from a political point of view, but also scientifically. Most commonly, research output was only available in Russian and not translated into English dramatically restricting its availability to the international community. Here such information published in Russian was evaluated focusing on medicinal species from the Russian (Soviet) Pharmacopoeia and used as a food. This highlights the importance of the Russian Pharmacopoeia as a source of information on plant species used traditionally at the interface of food and medicine.

Clearly our approach has the limitation of only focusing on species included in the pharmacopeia and, consequently, local

\section{REFERENCES}

Alarcón, R., Pardo-de-Santayana, M., Priestley, C., Morales, R., and Heinrich, M. (2015). Medicinal and local food plants in the south of Alava (Basque Country, Spain). J. Ethnopharmacol. 176, 207-224. doi: 10.1016/j.jep.2015.10.022

Annenkov, N. I. (1878). Botanical Dictionary. Reference Book for Botanist, Villagers, Horticulturists, Foresters, Chemists, Doctors, Droggists, Travelers about Russia and All the Countryfolk. St-Petersburg: Typography of the Imperial Academy of Sciences.

Baikov, G. K. (1968). "Wild fruit and berries plants from mountain forest areas of the South Urals," in Wild and Introduced Useful Plants in Bashkiria, Vol. 2. Kazan: Kazan University publishing.

Berson, G. Z. (1991). Wild Edible Plants. Leningrad: Gidrometeoizdat.

Boeva, A., Noninska, L., and Tzanowa, M. (1984). Podprawkite kato Chrana I Lekarstwo (Species as Food and Medicine). Sofia: Medizina i fiskultura.

Budantsev, A. L. (1996). Plant Resources of Russia and Neighboring Countries: Part I - Families Lycopodiaceae-Ephedraceae, Part II - Supplement, Vol. 1-7. St-Petersburg: Mir i semja-95. Nauka.

Budantsev, A. L., and Lesiovskaya, E. E. (2001). Wild Useful Plants of Russia. St-Petersburg: Izdatelstvo SPCPA.

Bugdaeva, N. P., Dambaev, B. D., and Esheeva, V. (2005). Methods of use of Bergenia crassifolia leaves in food industry. Mod. High Technol. 4:62.

CODEX STAN 321-2015 (2015). Standard for Ginseng Products. Available online at: http://www.fao.org/fao-who- codexalimentarius/sh-proxy/en/?lnk= 1\&url=https\%253A\%252F\%252Fworkspace.fao.org\%252Fsites\%252Fcodex \%252FStandards\%252FCODEX\%2BSTAN\%2B321-2015\%252FCXS_321e_ 2015.pdf

Drewnowski, A., and Gomez-Carneros, C. (2000). Bitter taste, phytonutrients, and the consumer: a review. Am. J. Clin. Nutr. 72, 1424-1435.

Efremova, N. A. (1967). Medicinal Plants of Kamchatka and the Commander Islands. Petropavlovsk-Kamchatsky: Far East book publishing.

Efremova, N. A. (1992). Cherished Grass. Wild and Cultivated Plants of the North-Eastern Part of Russia and Their Therapeutic Properties. PetropavlovskKamchatsky: Kamshat.

Etkin, N. (2006). Edible Medicines. An Ethnopharmacology of Food. Tucson: The University of Arizona Press.

Etkin, N. L., and Ross, P. J. (1982). Food as medicine and medicine as food: an adaptive framework for the interpretation of plant utilization or endemic species are mostly excluded. However, in the context of developing high value products with potential health benefits priority needs to be given to species which are widely available and not at risk of being overexploited if the demand increases.

The evidence for the individual species varies, and while we do not claim that their use could be, based on the current data, evidence-based, the review provides a basis for further research and development.

"Functional food" are foods that not only serve to provide nutrition but also can be a source for prevention and cure of various diseases. This review highlights the potential of wild Russian species monographed in its pharmacopeia for further developing new functional foods and-through the lens of their incorporation into the pharmacopeia-showcases the species' importance in Russia.

\section{AUTHOR CONTRIBUTIONS}

AS, AT, OP, and VM have written the first draft of the manuscript. AS, OP, and MH revised and improved the various drafts and revisions. All authors have seen and agreed on the final version of the manuscript.

among the Hausa of northern Nigeria. Soc. Sci. Med. 16, 1559-1573. doi: 10.1016/0277-9536(82)90167-8

Facciola, S. (1998). Cornucopia II: A Source Book of Edible Plants. Vista, CA: Kampong Publications.

FAO/WHO (2012a). JOINT FAO/WHO Food Standards Programme Codex Alimentarius Commission, 35th Session. Geneva.

FAO/WHO (2012b). JOINT FAO/WHO Food Standards Programme Codex Committee on Processed Fruits and Vegetables, 26th Session. Montego Bay.

Fedorov, A. A. (1984). Plant Resources of USSR. Flowering Plants, its Chemical Composition, Application. Families Magnoliaceae-Limoniaceae. Leningrad: Nauka.

Franz, C., Chizzola, R., Novak, J., and Sponza, S. (2011). Botanical species being used for manufacturing plant food supplements (PFS) and related products in the EU member states and selected third countries. Food Funct. 2, 720-730. doi: 10.1039/c1fol $0130 \mathrm{~g}$

Gammerman, A. F., and Grom, I. I. (1976). Wild Medicinal Plants of USSR. Moscow: Medizina.

Grigorieva, A. (2008). Secrets of Home Made Preserves. Russian Traditions. Moscow: White city.

Grisjuk, N. M., Grinchak, I. L., and Elin, E. Y. (1989). Wild Food, Industrial and Honey Bee Plants of Ukraine. Kiev: Urozhaj.

Grushvitsky, I. V. (1961). Ginseng. Aspects of Biology. Leningrad: Nauka.

Gubanov, I. A., Krylova, I. L., and Tikhonova, V. L. (1976). Wild Valuable Plants of USSR. Moscow: Mysl.

Heinrich, M. (2016). “Food-herbal medicine interface," in Encyclopedia of Food and Health, eds L. Trugo, P. Finglas, and B. Caballero (Oxford: Elsevier), 94-98.

Holloway, P. S., and Alexander, G. (1990). Ethnobotany of the Fort Yukon Region, Alaska. Econ. Bot. 44, 214-225. doi: 10.1007/BF028 60487

Hu, S. Y. (2005). Food Plants of China. Hong Kong: Chinese University Press.

Ipatiev, A. N. (1966). Vegetable Plants of the Earth. Minsk: Vysheishaya shkola.

Izmodenov, A. G. (1989). Forest Samobranka. Honey, Vegetables and Juices Ussuri Forests. Khabarovsk: Khabarovsk Book Publishers.

Jennings, H. M., Merrell, J., Thompson, J. L., and Heinrich, M. (2014). Food or medicine? The food-medicine interface in households in Sylhet. J. Ethnopharmacol. 167, 97-104. doi: 10.1016/j.jep.2014. 09.011 
Kalle, R., and Sõukand, R. (2012). Historical ethnobotanical review of wild edible plants of Estonia (1770s-1960s). Acta Soc. Bot. Polon. 81, 271-281. doi: 10.5586/asbp. 2012.033

Keating, B., Lindstrom, A., Lynch, M. E., and Blumenthal, M. (2015). Sales of tea \& herbal tea increase 3.6\% in United States in 2014. HerbalGram 105, 59-67.

Keller, B. A. (1941). Wild Edible Plants. Moscow; Leningrad: Academy of Science of USSR.

Khan, I. A., and Abourashed, E. A. (2010). Leung's Encyclopedia of Common Natural Ingredients: Used in Food, Drugs and Cosmetics, 3rd Edn. Hoboken, NJ: John Wiley \& Sons.

Kibala, J. A. (1986). Spices and Condiments. Prague: Artia.

Klobukova-Alisova, E. N. (1958). Wild Useful and Harmful Plants of Bashkiria. Moscow; Leningrad: Academy of Science of USSR.

Koscheev, A. K. (1981). Wild Edible Plants in Our Nutrition. Moscow: Pischevaja promyshlennost.

Kovaleva, N. G. (1972). Healing with Plants. Moscow: Medizina.

Krylov, G. V. (1972). Herbs of Life and Their Seekers. Novosibirsk: West-Siberian book publishing.

Kunakova, R. V., Zainullin, R. A., Abramova, L. M., and Anischenko, O. E. (2011). Food and Medicinal Plants in Functional Food Products. Ufa: Gilem.

Leonti, M. (2012). The co-evolutionary perspective of the food-medicine continuum and wild gathered and cultivated vegetables. Genet. Resour. Crop Evol. 59, 1295-1302. doi: 10.1007/s10722-012-9894-7

Lim, T. K. (2012). Edible Medicinal and Non-Medicinal Plants, Vol. 1, Fruits. Dordrecht; Heidelberg; London, New York, NY: Springer.

Litvintsev, A. N., and Koscheev, A. K. (1988). Greens on the Table. Irkutsk: East Siberian Book Publishing House.

Łuczaj, Ł. (2008). Archival data on wild food plants used in Poland in 1948. J. Ethnobiol. Ethnomed. 4:4. doi: 10.1186/1746-4269-4-4

Łuczaj, Ł., Fressel, N., and Perković, S. (2013). Wild food plants used in the villages of the Lake Vrana Nature Park (northern Dalmatia, Croatia). Acta Soc. Bot. Pol. 82, 275-281. doi: 10.5586/asbp.2013.036

Łuczaj, Ł., Pieroni, A., Tardío, J., Pardo-de-Santayana, M., Sõukand, R., Svanberg, I., and Kalle, R. (2012). Wild food plant use in 21st century Europe: the disappearance of old traditions and the search for new cuisines involving wild edibles. Acta Soc. Bot. Pol. 81, 359-370. doi: 10.5586/asbp. 2012.031

Makarova, T. I., Surzhin, S. N., Pavlova, U. G., and Sergeeva, T. V. (1960). The Use of Domestic Spices in Fishing Industry, Vol. 5. Leningrad: Trudy BIN RAN USSR, Raw Plant Material.

Markova, L. P., Belenovskaya, L. M., and Nadezhdina, T. P. (1985). Wild Useful Plants of Flora of Mongolian People's Republic. Leningrad: Nauka.

Mashanov, V. I., and Pokrovsky, A. A. (1991). Spicy and Aromatic Plants. Moscow: Agropromizdat.

Moerman, D. E. (2010). Native American Food Plants: An Ethnobotanical Dictionary. Portland; London: Timber Press.

Molchanov, G. I., Molchanova, L. P., Gulko, N. M., Molchanov, A. G., and Suchkov, I. F. (1989). Edible Curative Plants of Caucasus. Rostov on Don: Rostov University Publisher.

Nekrasova, V. L. (1958). The History of the Study of Wild Plant Resources in the USSR, Vol. 1. Moscow: Academy of science of USSR.

Novruzov, A. R. (2014). Contents and dynamics of accumulation of the ascorbic acid in fruits of Rosa canina L. Khimija Rastitel'nogo Syrja 3, 221-226. doi: $10.14258 /$ jcprm. 1403221

Palagina, M. V., and Bogoutdinova, A. A. (2012). Application of spikenard extracts in production of new kinds of non-alcoholic beer. News Far East. Fed. Univ. Econ. Manag. 2, 122-126.

Palagina, M. V., Teltevskaya, O. P., and Boyarova, M. D. (2011). Characterization of plant extracts of the family Araliaceae and their possible applications in technology spirits. News Far East. Fed. Univ. Econ. Manag. 1, 88-92.

Panossian, A., and Wikman, G. (2008). Pharmacology of Schisandra chinensis Bail.: an overview of Russian research and uses in medicine. J. Ethnopharmacol. 118, 183-212. doi: 10.1016/j.jep.2008.04.020

Parada, M., Carrió, E., and Vallès, J. (2011). Ethnobotany of food plants in the Alt Emporda region (Catalonia, Iberian Peninsula). J. Appl. Bot. Food Qual. 84, $11-25$.
Pieroni, A. (2000). Medicinal plants and food medicines in the folk traditions of the upper Lucca Province, Italy. J. Ethnopharmacol. 70, 235-273. doi: 10.1016/S0378-8741(99)00207-X

Pieroni, A., Nebel, S., Santoro, R. F., and Heinrich, M. (2005). Food for two seasons: culinary uses of non-cultivated local vegetables and mushrooms in a south Italian village. Int. J. Food Sci. Nutr. 56, 245-272. doi: 10.1080/09637480500146564

Pohlebkin, V. V. (1998). Tea, its History, Properties and Consumption. Moscow: Molodaja gvardia.

Pojarkova, A. I. (1958). "Elderberry - Sambucus L", in Flora of USSR, ed B. K. Shishkin (Moscow-Leningrad: Academy of science of USSR). 422-442.

Reid, B. E. (1982). Famine Foods of the Chiu-huang Pen-ts' ao. Southern Materials Centre. Taipei: INC.

Rubtsov, N. I. (1971). "Wild useful plants of Crimea," in Proceedings of Nikitsky Botanical Garden, Vol. XLIX. Yalta.

Saratikov, A. S. (1974). Golden Root (Rhodiola rosea). Tomsk: Tomsk University Publishing.

Sears, B. (2015). Anti-inflammatory diets. J. Am. Coll. Nutr. 34, 14-21. doi: $10.1080 / 07315724.2015 .1080105$

Shi, J., Ho, C. T., and Shahidi, F. (eds.). (2011). Functional Foods of the East. Boca Raton, FL; London; New York, NY: CRC Press.

Shikov, A. N., Poltanov, E. A., Dorman, H. D., Makarov, V. G., Tikhonov, V. P., and Hiltunen, R. (2006). Chemical composition and in vitro antioxidant evaluation of commercial water-soluble willow herb (Epilobium angustifolium L.) extracts. J. Agric. Food Chem. 54, 3617-3624. doi: 10.1021/jf052606i

Shikov, A. N., Pozharitskaya, O. N., Kamenev, I., Yu., and Makarov, V. G. (2011). Arznei und Gewurzpflanzen in Russland. Z. Arzn. Gewurzpflanzen 16, 135-137.

Shikov, A. N., Pozharitskaya, O. N., Makarov, V. G., Wagner, H., Verpoorte, R., and Heinrich, M. (2014a). Medicinal plants of the Russian pharmacopoeia, history and applications. J. Ethnopharmacol. 154, 481-536. doi: $10.1016 /$ j.jep.2014.04.007

Shikov, A. N., Pozharitskaya, O. N., Makarova, M. N., Kovaleva, M. A., Laakso, I., Dorman, H. J. D., et al. (2012). Effect of Bergenia crassifolia L. extracts on weight gain and feeding behavior of rats with high-caloric diet- induced obesity. Phytomedicine 19, 1250-1255. doi: 10.1016/j.phymed.2012.09.019

Shikov, A. N., Pozharitskaya, O. N., Makarova, M. N., Makarov, V. G., and Wagner H. (2014b). Bergenia crassifolia (L.) Fritsch-pharmacology and phytochemistry. Phytomedicine 21, 1534-1542. doi: 10.1016/j.phymed.2014.06.009

Smertina, E. S., Fedyanina, L. N., Kalenik, T. K., Kushnerova, N. F., and Vigerina, N. S. (2011). Application of wild plants in bakery products of functional purpose. News Far East. Fed. Univ. Econ. Manag. 3, 129-133.

Soenov, V. I. (2002). Gathering of Plant Food in Altai. Antiques of Altai, 9. Available online at: http://e-lib.gasu.ru/da/archive/2002/09/02.html (Accessed Aug 2017).

Sokolov, P. D. (1985). Plant Resources of USSR. Flowering Plants, Its Chemical Composition, Application. Families Paeoniaceae-Thymelaeaceae. Leningrad: Nauka.

Sokolov, P. D. (1987). Plant Resources of USSR. Flowering Plants, Its Chemical Composition, Application. Families Hydrangeaceae-Haloragaceae. Leningrad: Nauka.

Sokolov, P. D. (1988). Plant Resources of USSR. Flowering Plants, Its Chemical Composition, Application. Families Rutaceae-Elaegnaceae. Leningrad: Nauka.

Sokolov, P. D. (1990). Plant Resources of USSR. Flowering Plants, its Chemical Composition, Application. Families Caprifoliaceae-Plantaginaceae. Leningrad: Nauka.

Sokolov, P. D. (1991). Plant Resources of USSR. Flowering Plants, Its Chemical Composition, Application. Families Hippuridaceae-Lobeliaceae. St-Petersburg: Nauka.

Sokolov, P. D. (1993). Plant Resources of USSR. Flowering Plants, Its Chemical Composition, Application. Family Asteraceae. St-Petersburg: Nauka.

Sokolov, P. D. (1994). Plant Resources of Russia and Neighboring Countries: Flowering Plants, Its Chemical Composition, Application. Families ButomaceaeTyphaceae. St-Petersburg: Nauka

Sokolov, S. Y. (2000). Phytotherapy and Phytopharmacology: The Manual for Doctors. Moscow: MIA.

Sõukand, R., Quave, C. L., Pieroni, A., Pardo-de-Santayana, M., Tardío, J., Kalle, R., et al. (2013). Plants used for making recreational tea in Europe: 
a review based on specific research sites. J. Ethnobiol. Ethnomed. 9:58. doi: 10.1186/1746-4269-9-58

State Pharmacopoeia of the USSR (1990). State Pharmacopoeia of the USSR, 11th Edn., Part 2. Moscow: Medicina.

Stryamets, N., Elbakidze, M., Ceuterick, M., Angelstam, P., and Axelsson, R. (2015). From economic survival to recreation: contemporary uses of wild food and medicine in rural Sweden, Ukraine and NW Russia. J. Ethnobiol. Ethnomed. 11:53. doi: 10.1186/s13002-015-0036-0

Svanberg, I. (2012). The use of wild plants as food in pre-industrial Sweden. Acta Soc. Bot. Pol. 81, 317-327. doi: 10.5586/asbp.2012.039

Svanberg, I., Sõukand, R., Luczaj, L., Kalle, R., Zyryanova, O., Dénes, A., et al. (2012). Uses of tree saps in northern and eastern parts of Europe. Acta Soc. Bot. Pol. 81, 343-357. doi: 10.5586/asbp.2012.036

Tcherepnin, V. L. (1987). Food Plants of Siberia. Novosibirsk: Nauka.

Tikhomirov, B. A. (1942). The Cardinal Wild Food Plants of the Leningrad Region. Leningrad: Leningrad Newspaper-Magazine and Book Publisher.

Timoshenko, B. N. (1940). Herbs for Alcoholic Liquor Industry. Moscow; Leningrad: Pischepromizdat.

Toren, M. D. (1996). Russian Folk Medicine and Psychotherapy. St-Petersburg: Litera.

Turner, N. J., Łuczaj, Ł. J., Migliorini, P., Pieroni, A., Dreon, A. L., Sacchetti, L. E., et al. (2011). Edible and tended wild plants, traditional ecological knowledge and agroecology. Crit. Rev. Plant Sci. 30, 198-225. doi: 10.1080/07352689.2011.554492

Turova, A. D., and Sapozhnikova, E. N. (1989). Medicinal Plants of USSR and Their Applications. Moscow: Medizina.
Vallès, J., Bonet, M. A., Garnatje, T., Muntanè, J. O. A. N., Parada, M., and Rigat, M. (2010). Sambucus nigra L. in Catalonia (Iberian Peninsula). Under. Underexpl. Hortic. Crops 5, 393-424.

Valussi, M., and Scire, A. S. (2012). Quantitative ethnobotany and traditional functional foods. Nutrafoods 11, 85-93. doi: 10.1007/s13749-012-0032-0

Vereschagin, V. I., Sobolevskaya, K. A., and Yakubova, A. I. (1959). Useful Plants of West Siberia. Moscow; Leningrad: Publishing of Academy of Science of USSR.

Vul'f, E. V., and Maleeva, O. F. (1969). World Resources of Useful Plants. Leningrad: Nauka.

Wustenfeld, G., and Gesler, G. (1959). Production of Cordial and Brandy. Moscow: Pischepromizdat.

Zelenin, D. K. (1927). Russische (Ostslavische) Volkskunde. Berlin: W. de Gruyter. Zhuravlev, Y.u,.., N., and Kolyada, A.S. (1996). Araliaceae: Ginseng and Others. Vladivostok: Dalnauka.

Conflict of Interest Statement: The authors declare that the research was conducted in the absence of any commercial or financial relationships that could be construed as a potential conflict of interest.

Copyright (c) 2017 Shikov, Tsitsilin, Pozharitskaya, Makarov and Heinrich. This is an open-access article distributed under the terms of the Creative Commons Attribution License (CC BY). The use, distribution or reproduction in other forums is permitted, provided the original author(s) or licensor are credited and that the original publication in this journal is cited, in accordance with accepted academic practice. No use, distribution or reproduction is permitted which does not comply with these terms. 


\section{OPEN ACCESS}

Edited by:

Judith Maria Rollinger, University of Vienna, Austria

Reviewed by:

Anastasia Karioti, Aristotle University of Thessaloniki,

Greece

Michał Tomczyk,

Medical University of Bialystok, Poland

*Correspondence:

Monika A. Olszewska monika.olszewska@umed.lodz.pl

Specialty section:

This article was submitted to

Ethnopharmacology,

a section of the journal

Frontiers in Pharmacology

Received: 11 July 2017 Accepted: 11 September 2017 Published: 11 October 2017

Citation:

Marchelak A, Owczarek A, Matczak M, Pawlak A, Kolodziejczyk-Czepas J, Nowak P and Olszewska MA (2017) Bioactivity Potential of Prunus spinosa L. Flower Extracts: Phytochemical Profiling, Cellular Safety, Pro-inflammatory Enzymes Inhibition and Protective Effects Against Oxidative Stress In Vitro. Front. Pharmacol. 8:680. doi: 10.3389/fphar.2017.00680

\section{Bioactivity Potential of Prunus spinosa L. Flower Extracts: Phytochemical Profiling, Cellular Safety, Pro-inflammatory Enzymes Inhibition and Protective Effects Against Oxidative Stress In Vitro}

\author{
Anna Marchelak ${ }^{1}$, Aleksandra Owczarek ${ }^{1}$, Magdalena Matczak ${ }^{1}$, Adam Pawlak ${ }^{1}$, \\ Joanna Kolodziejczyk-Czepas ${ }^{2}$, Pawel Nowak ${ }^{2}$ and Monika A. Olszewska ${ }^{1 *}$ \\ ${ }^{1}$ Department of Pharmacognosy, Faculty of Pharmacy, Medical University of Lodz, Lodz, Poland, ${ }^{2}$ Department of General \\ Biochemistry, Faculty of Biology and Environmental Protection, University of Lodz, Lodz, Poland
}

Flower extracts of Prunus spinosa L. (blackthorn) - a traditional medicinal plant of Central and Eastern Europe indicated for the treatment of urinary tract disorders, inflammation, and adjunctive therapy of cardiovascular diseases - were evaluated in terms of chemical composition, antioxidant activity, potential anti-inflammatory effects, and cellular safety in function of fractionated extraction. The UHPLC-PDA-ESI-MS ${ }^{3}$ fingerprinting led to full or partial identification of 57 marker constituents (36 new for the flowers), mostly flavonoids, A-type proanthocyanidins, and phenolic acids, and provided the basis for authentication and standardization of the flower extracts. With the contents up to 584.07 $\mathrm{mg} / \mathrm{g}$ dry weight (dw), 490.63, 109.43, and $66.77 \mathrm{mg} / \mathrm{g}$ dw of total phenolics (TPC), flavonoids, proanthocyanidins, and phenolic acids, respectively, the extracts were proven to be rich sources of polyphenols. In chemical in vitro tests of antioxidant (DPPH, FRAP, TBARS) and enzyme (lipoxygenase and hyaluronidase) inhibitory activity, the extracts effects were profound, dose-, phenolic-, and extraction solvent-dependent. Moreover, at in vivo-relevant levels $(1-5 \mu \mathrm{g} / \mathrm{mL})$ the extracts effectively protected the human plasma components against peroxynitrite-induced damage (reduced the levels of oxidative stress biomarkers: 3-nitrotyrosine, lipid hydroperoxides, and thiobarbituric acid-reactive substances) and enhanced the total antioxidant status of plasma. The effects observed in biological models were in general dose- and TPC-dependent; only for protein nitration the relationships were not significant. Furthermore, in cytotoxicity tests, the extracts did not affect the viability of human peripheral blood mononuclear cells (PBMC), and might be regarded as safe. Among extracts, the defatted methanol-water (7:3, v/v) extract and its diethyl ether and ethyl acetate fractions appear to be the most advantageous for biological applications. As compared to the positive controls, activity of the extracts was favorable, which might be attributed to some synergic effects of their constituents. In conclusion, this research proves that the antioxidant and enzyme inhibitory capacity of phenolic fractions should be counted as one of the mechanisms behind the activity 
of the flowers reported by traditional medicine and demonstrates the potential of the extracts as alternative ingredients for functional products supporting the treatment of oxidative stress-related pathologies cross-linked with inflammatory changes, especially in cardiovascular protection.

Keywords: Prunus spinosa, oxidative stress, antioxidants, human plasma, lipoxygenase, hyaluronidase, polyphenols, LC-MS

\section{INTRODUCTION}

Medicinal plants as primary sources of natural bioactive compounds are attracting growing interest as constituents of functional products active in prevention and adjunctive therapy of numerous chronic diseases, including cardiovascular disorders (CVD), the leading cause of mortality in the world today. In the search of new candidates for closer investigation, ethnobotanical knowledge constitutes an important guideline indicating species with the relevant activity (Franz et al., 2011).

One of such plants might be Prunus spinosa L. (blackthorn or sloe) - a wild plume tree native to Europe, western Asia, north-western Africa, and naturalized in New Zealand and North America (Tutin et al., 1968). In European tradition it has been known for over 7,000 years, at first as a source of edible fruit and then also as a medicinal plant (Poonam et al., 2011; Zohary et al., 2012), used i.a. in the treatment of various circulatory system disorders. For medicinal applications the plant has been used throughout Europe with the flowers being the most popular in central and eastern parts of the continent (Hoppe, 1981). Ethnopharmacological sources indicate vasoprotective, anti-inflammatory, diuretic, detoxifying (blood purifying), and spasmolytic activities for the flowers, and document their use as ingredients of compound herbal prescriptions traditionally applied, e.g., to treat intestinal and respiratory tract disorders, but also various cardiac complaints, such as myocarditis, cardiac neurosis and atherosclerosis (Berger, 1949; Hoppe, 1981; Wawrzyniak, 1992; Blumenthal and Busse, 1998). The fruits, according to German Commission $\mathrm{E}$, have been indicated mainly in mild inflammation of the oral and pharyngeal mucosa, as well as an astringent (Blumenthal and Busse, 1998); however, local European sources report their use also as a heart-strengthening remedy (Kültür, 2007; Jarić et al., 2015). Branches, on the other hand, have been more popular in the south of Europe and suggested to possess anti-hypertensive properties (Calvo and Cavero, 2014).

Active components of the plant are believed to be polyphenols, including flavonoids, A-type proanthocyanidins, anthocyanins, coumarins, and phenolic acids, forming unique and diversified profiles in particular organs, among which the flowers are the least characterized (Kolodziej et al., 1991; Sakar and Kolodziej, 1993; Olszewska and Wolbiś, 2001, 2002a,b; Guimarães et al., 2013; Pinacho et al., 2015; Owczarek et al., 2017). Some blackthorn constituents, such as flavonoid pentosides (arabinosides, xylosides, rhamnosides) and A-type procyanidin dimers with twice-bonded structures are quite rare in nature and their distribution is generally limited to selected species and plant families (Pinacho et al., 2015). This unique composition may correspond to the distinctive activity profile of $P$. spinosa reported by traditional medicine. Indeed, the earliest studies suggested that the flavonoid fraction of blackthorn flowers significantly reduces capillary permeability and shows antiinflammatory effects in animal internal organs, normalizes the blood cholesterol and cholesterol/phospholipid ratio in atherogenic rabbits, exhibits spasmolytic effects on isolated intestinal segments from different animals, and increases the amplitude of heart contractions in perfusion of isolated frog hearts (Lisevitskaya et al., 1968; Makarov, 1968, 1972; Makarov and Khadzhaí, 1969). However, despite these promising in vivo and ex vivo results, the potential of blackthorn, especially the blackthorn flowers, as a source of biologically active extracts (that means standardized dry extracts-more effective than unprocessed plant materials and recommended in modern phytotherapy) remains unexplored, and the plant materials are still used mostly in the form of traditional herbal teas, partly due to the lacking molecular background for their activity and safety. Recently, special attention has been given to the antioxidant activity of the extracts from the branches, leaves, and fruits, as one of the possible mechanism of action of the blackthorn polyphenols (Barros et al., 2010; Guimarães et al., 2014; Pinacho et al., 2015). Nevertheless, as these studies were based only on simple, mostly chemical tests, and did not cover the flower extracts, the subject requires more detailed investigations.

Polyphenols, including flavonoids, are specialized plant metabolites, the beneficial effects of which in CVD is commonly linked with their ability to influence two interdependent pathological processes of oxidative/nitrative stress and inflammation (Biswas, 2016). As free radical scavengers, metal chelators, inhibitors of pro-inflammatory enzymes, and modifiers of cell signaling pathways, polyphenols may protect cellular and functional elements of the circulatory system against lipid peroxidation, protein nitration, chronic inflammation, and oxidative damage to DNA, which results i.a. in vasodilatory, vasoprotective, anti-atherogenic, antithrombotic, and antiapoptotic effects (Alissa and Ferns, 2012; Quiñones et al., 2013). Moreover, the reducing polyphenols and their metabolites can increase the total antioxidant capacity of blood plasma and thus the tolerance of body tissues against ischemic/reperfusion injuries (Pandey and Rizvi, 2009).

Therefore, the aim of this project was a comprehensive characteristic of the flowers of $P$. spinosa in a function of fractionated extraction with respect to the chemical composition and biological activity of the dry extracts obtained with solvents of different polarity. Phenolic profiles of the extracts were investigated by UHPLC-PDA-ESI-MS ${ }^{3}$, HPLC-PDA, and UVspectrophotometric methods, while their biological effects were studied in vitro by nine complementary tests (both chemical and 
human plasma models) covering some of the main mechanisms of the beneficial action of polyphenols in CVD, including direct scavenging of free radicals, inhibition of pro-inflammatory enzymes, enhancement of the total antioxidant capacity of blood plasma and protection against oxidative and nitrative damage of its lipid and protein components. Moreover, the relationship between activity of the extracts and the presence of different groups of polyphenolic constituents was explored statistically. Additionally, cellular safety of the extracts was evaluated in vitro in cytotoxicity tests against human peripheral blood mononuclear cells (PBMCs).

\section{MATERIALS AND METHODS}

\section{General}

HPLC grade reagents and standards, such as 2,2-diphenyl1-picrylhydrazyl (DPPH); 2,2'-azobis-(2-amidinopropane) dihydrochloride (AAPH); 2,4,6-tris-(2-pyridyl)-s-triazine (TPTZ); $\quad( \pm)$-6-hydroxy-2,2,7,8-tetramethylchroman-2carboxylic acid (Trolox $\left.{ }^{\circledR}\right)$; gallic acid monohydrate; chlorogenic acid hemihydrate; caffeic acid; kaempferol; quercetin trihydrate; isorhamnetin; rutin trihydrate; cyanidin chloride; indomethacin; bovine testis hyaluronidase; lipoxygenase from soybean; linoleic acid; 2-thiobarbituric acid; were purchased from Sigma-Aldrich (Seelze, Germany/St. Louis, MO, USA), as were analytical grade butylated hydroxyanisole (BHA); 2,6-di-tert-butyl-4methylphenol (BHT); Tween ${ }^{\circledR}$ 40; xylenol orange disodium salt; and Histopaque ${ }^{\circledR}-1077$ medium. HPLC grade solvents (acetonitrile and methanol) used for UHPLC and HPLC analyses were obtained from Avantor Performance Materials (Gliwice, Poland). A $\left(\mathrm{Ca}^{2+}\right)$-free phosphate buffered saline (PBS) was purchased from Biomed (Lublin, Poland). Peroxynitrite was synthesized according to Pryor et al. (1995). All immunoreagents for 3-nitrotyrosine detection were purchased from Abcam (Cambridge, UK). Pierce BCA Protein Assay Kit was obtained from Thermo Scientific (Waltham, MA, USA). All other chemicals and solvents were of analytical grade and obtained from Avantor (Poland). In all analyses redistilled water was used. For chemical tests samples were incubated in a constant temperature using a BD 23 incubator (Binder, Tuttlingen, Germany) and measured using a UV-1601 Rayleigh spectrophotometer (Beijing, China), in $10 \mathrm{~mm}$ quartz cuvettes. Activity tests in blood plasma models and enzyme inhibitory assays were done using 96-well plates and a microplate reader, SPECTROStar Nano (BMG LabTech, Ortenberg, Germany).

\section{Plant Material and Extracts Preparation}

Commercial samples of Prunus spinosa L. flowers were purchased in 2015 (harvest in April 2015) from Dary Natury (Koryciny, Poland). According to the manufacturer, the raw material was collected in the district Rudka, Malopolska province $\left(50^{\circ} 02^{\prime} \mathrm{N}\right.$, $20^{\circ} 52^{\prime} \mathrm{E}$ ). The raw material was powdered with an electric grinder, and sieved through a $0.315-\mathrm{mm}$ sieve. A portion (100 g) was extracted with chloroform $(3 \mathrm{~L}, 30 \mathrm{~h})$ in a Soxhlet apparatus and the pellet was next four times refluxed with methanolwater $(7: 3, \mathrm{v} / \mathrm{v} ; 4 \times 1 \mathrm{~L})$ to give the defatted methanol extract (MED, $27.3 \mathrm{~g} \mathrm{dw}$ ). The extraction solvent was selected from methanol-water mixtures of different alcohol concentration after optimization performed in terms of extracts yield and total phenolic content (results not shown). The MED (25.0 g) was suspended in water $(1 \mathrm{~L})$ and subjected to sequential liquidliquid extraction with organic solvents $(8 \times 100 \mathrm{~mL}$ each $)$ to yield diethyl ether fraction (DEF, $1.23 \mathrm{~g} \mathrm{dw}$ ), ethyl acetate fraction (EAF, $4.00 \mathrm{~g} \mathrm{dw}$ ), $n$-butanol fraction (BF, $4.86 \mathrm{~g} \mathrm{dw}$ ) and water residue (WR, $13.08 \mathrm{~g} \mathrm{dw}$ ). The organic solvent extracts were evaporated in vacuo, and the water-containing fractions were lyophilized using an Alpha 1-2/LD Plus freeze dryer (Christ, Osterode am Harz, Germany) before weighing. In further analyses freshly prepared solutions of the extracts in methanolwater $(7: 3, \mathrm{v} / \mathrm{v})$ were used. All quantitative results were calculated per dry weight $(\mathrm{dw})$ of the extracts.

\section{Phytochemical Profiling Qualitative LC-MS Study}

The UHPLC-PDA-ESI-MS 3 analysis was performed on UHPLC3000 RS system (Dionex, Dreieich, Germany) equipped with a dual low-pressure gradient pump, an autosampler, a column compartment, a diode array detector, and an AmaZon SL ion trap mass spectrometer with an ESI interface (Bruker Daltonik, Bremen, Germany). Separations were carried out on a Kinetex XB-C18 column $(1.7 \mu \mathrm{m}, 150 \mathrm{~mm} \times 2.1 \mathrm{~mm}$ i.d.; Phenomenex, Torrance, CA, USA) at $25^{\circ} \mathrm{C}$. The mobile phase consisted of solvent A (water/formic acid, 100:0.1, v/v), and solvent B (acetonitrile/formic acid, 100:0.1, v/v) with the elution profile as follows: 0-10 $\min , 6-13 \% \mathrm{~B}(\mathrm{v} / \mathrm{v}) ; 10-15 \min , 13 \% \mathrm{~B}(\mathrm{v} / \mathrm{v}) ; 15-19$ min, 13-15\% B (v/v); 19-24 min, 15\% B (v/v); 24-40 min, 15$23 \%$ B (v/v); 40-55 min, 23-40\% B (v/v); 55-60 min, 40\% B (v/v); 60-63 min, 40-6\% B (v/v); 63-70 min, 6\% B (equilibration). The flow rate was $0.3 \mathrm{~mL} / \mathrm{min}$. Before injection, sample solutions of the extracts $(3.0 \mathrm{mg} / \mathrm{mL})$ were filtered through a PTFE syringe filter $(13 \mathrm{~mm}, 0.2 \mu \mathrm{m}$, Whatman, Pittsburgh, PA, USA). UVVis spectra were recorded over a range of $200-600 \mathrm{~nm}$, and chromatograms were acquired at 254, 280, and $350 \mathrm{~nm}$. The LC eluate was introduced directly into the ESI interface without splitting and analyzed in both negative and positive ion modes. ESI parameters: the nebulizer pressure was $40 \mathrm{psi}$; dry gas flow 9 $\mathrm{L} / \mathrm{min}$; dry temperature $300^{\circ} \mathrm{C}$; and capillary voltage $4.5 \mathrm{kV}$. $\mathrm{MS}^{2}$ and $\mathrm{MS}^{3}$ fragmentations were obtained in Auto MS/MS mode for the most abundant ions at the time. Analysis was carried out using scan from $\mathrm{m} / \mathrm{z} 200-2,200$.

\section{Quantitative Standardization}

The total phenolic contents (TPC) and total proanthocyanidin contents (TPA) were quantified by the Folin-Ciocalteu and $n$-butanol-HCl methods, respectively, as described previously (Olszewska et al., 2012). Results were expressed as equivalents of gallic acid (GAE) and cyanidin chloride (CYE), respectively. The total flavonoid contents (TFA) were determined by HPLCPDA as the total content of flavonoid aglycones after acid hydrolysis. Samples of the extracts (1-5 mg) were heated under reflux for $30 \mathrm{~min}$ with methanol-water $(9: 1, \mathrm{v} / \mathrm{v} ; 30 \mathrm{~mL})$ and $25 \%(\mathrm{w} / \mathrm{v})$ hydrochloric acid $(9 \mathrm{~mL})$. The hydrolysates were diluted with methanol-water $(7: 3, \mathrm{v} / \mathrm{v})$ to $50 \mathrm{~mL}$, filtered through a PTFE syringe filter (as above) and injected $(5 \mu \mathrm{L})$ into 
the HPLC system. The HPLC-PDA assays were carried out according to Olszewska (2012) with quercetin, kaempferol and isorhamnetin used for external calibration. Results were recalculated for total contents of glycosides with the molecular masses of avicularin, juglanin, and isorhamnetin diglucoside, correspondingly. The total contents of phenolic acids (TAC) were assayed by HPLC-PDA according to Olszewska et al. (2012) with caffeic acid and chlorogenic acid used as calibration standards for quantification of simple hydroxycinnamic acids and quinic acid pseudodepsides, respectively.

\section{Antioxidant Activity in Chemical Models}

The DPPH free-radical scavenging activity was determined according to the method optimized previously (Olszewska et al., 2012) and expressed as normalized $\mathrm{EC}_{50}$ values calculated from concentration-inhibition curves. The FRAP (Ferric Reducing Antioxidant Power) was determined according to Olszewska et al. (2012) and expressed in $\mu \mathrm{mol}$ of ferrous ions $\left(\mathrm{Fe}^{2+}\right)$ produced by $1 \mathrm{~g}$ of the dry extract or standard, which was calculated from the calibration curve of ferrous sulfate. The ability of the extracts to inhibit AAPH-induced peroxidation of linoleic acid (LA) was assayed as described previously (Olszewska et al., 2012) with peroxidation monitored by quantification of thiobarbituric acid-reactive substances (TBARS) according to the method of Kljak and Grbeša (2015) with some changes. Briefly, before and after the samples were incubated for $3 \mathrm{~h}$ at $50.0 \pm 0.1^{\circ} \mathrm{C}$ in the dark, the reaction solution $(0.5 \mathrm{~mL})$ was mixed with $0.67 \%(\mathrm{w} / \mathrm{w})$ aqueous thiobarbituric acid $(1 \mathrm{~mL}), 0.05 \mathrm{M} \mathrm{HCl}(0.5 \mathrm{~mL})$, Tween ${ }^{\circledR} 40(1 \mathrm{~mL})$, and then heated for $30 \mathrm{~min}$ in the water bath at $95^{\circ} \mathrm{C}$. The absorbance was measured at 535 $\mathrm{nm}$ vs. the control with methanol-water $(7: 3, \mathrm{v} / \mathrm{v})$ instead of the reaction mixture. The inhibition ratio (I\%) of the LA-peroxidation was calculated as follows: $\mathrm{I} \%=\left(\Delta \mathrm{A}_{\text {control }}\right.$ $\left.-\Delta \mathrm{A}_{\text {sample }}\right) / \Delta \mathrm{A}_{\text {control}}$, where $\Delta \mathrm{A}$ is the difference between the absorbance measured before and after incubation. Finally, antioxidant activity was expressed as $\mathrm{IC}_{50}$ values calculated from concentration-inhibition curves.

\section{Antioxidant Activity in Human Plasma Models Isolation of Blood Plasma and Preparation of Samples}

Blood from five healthy, non-smoking volunteers was obtained from the Regional Centre of Blood Donation and Blood Treatment in Lodz (Poland), collected on CPD (citrate/phosphate/dextrose) solution in the Fresenius-Kabi Compoflex bags, and next plasma was isolated by differential centrifugation of blood (Kolodziejczyk-Czepas et al., 2013). All experiments were approved by the committee on the Ethics of Research at the University of Lodz 8/KBBN-UŁ/II/2015. For the FRAP assay and measurements of 3-nitrotyrosine, plasma samples were diluted with a $\left(\mathrm{Ca}^{2+}\right)$-free $\operatorname{PBS}$ buffer $(1: 4, \mathrm{v} / \mathrm{v})$, whereas for hydroperoxide and TBARS assays plasma was diluted with $\left(\mathrm{Ca}^{2+}\right)$-free PBS in a volume ratio 1:1. For all tests, samples were pre-incubated for $5 \mathrm{~min}$ at $37^{\circ} \mathrm{C}$ with the examined extracts added to the final concentration range of $1-50 \mu \mathrm{g} / \mathrm{mL}$, and then exposed to $100 \mu \mathrm{M}$ peroxynitrite $\left(\mathrm{ONOO}^{-}\right)$. Control samples were prepared with plasma untreated with the extracts and/or peroxynitrite. In the experiments with blood plasma and the extracts only (without adding $\mathrm{ONOO}^{-}$) no pro-oxidative effect was found. Protein concentration in blood plasma was estimated using bicinchoninic acid (BCA) assay (according to protocol provided by the manufacturer).

\section{Determination of 3-Nitrotyrosine in Human Plasma Proteins}

Detection of nitrotyrosine-containing proteins by the competitive ELISA (C-ELISA) method in plasma samples (control or antioxidants and $\mathrm{ONOO}^{-}$-treated plasma) was performed according to Kolodziejczyk-Czepas et al. (2013). The concentrations of nitrated proteins were estimated from the standard curve and are expressed as the $3 \mathrm{NT}-\mathrm{Fg}$ equivalents (in $\mathrm{nmol} / \mathrm{mg}$ of plasma protein).

\section{Ferric-Xylenol Orange Hydroperoxide Assay}

Concentration of hydroperoxides in plasma samples (control or antioxidants and $\mathrm{ONOO}^{-}$-treated plasma) was determined by a ferric-xylenol orange (FOX-1) protocol (KolodziejczykCzepas et al., 2013). The FOX-1 reagent contained $125 \mu \mathrm{M}$ xylenol orange and $100 \mathrm{mM}$ sorbitol in $25 \mathrm{mM}$ sulfuric acid, and was freshly prepared each time before use by the addition of ammonium ferrous sulfate to the final concentration of $250 \mu \mathrm{M}$. To perform FOX-1 assay, blood plasma samples were mixed with the reagent in a volume ratio 1:9 and incubated for $30 \mathrm{~min}$ in the dark $\left(25^{\circ} \mathrm{C}\right)$. Absorbance of the sample was measured at $560 \mathrm{~nm}$ against blank (water instead of plasma). The amount of lipid hydroperoxides was calculated from the standard curve of hydrogen peroxide and expressed in $\mathrm{nmol} / \mathrm{mg}$ of plasma proteins.

\section{TBARS Test}

Determination of thiobarbituric acid reactive substances (TBARS) in plasma samples (control or antioxidants and $\mathrm{ONOO}^{-}$-treated plasma) was performed according to Kolodziejczyk et al. (2011). Results were expressed in $\mu \mathrm{mol}$ TBARS/mL of plasma.

\section{Ferric Reducing Ability of Plasma (FRAP) Assay}

The influence of the extracts on the total antioxidant activity of plasma (dependent on non-enzymatic antioxidants) was determined according to Kolodziejczyk-Czepas et al. (2014) with some modifications. The freshly collected plasma samples were diluted with a $\left(\mathrm{Ca}^{2+}\right)$-free PBS buffer in a volume ratio of 1:4, prepared as described above, and then added to the reagent mixture in a volume ratio of 1:10:1:1 for plasma, acetate buffer (300 mM, pH 3.6), TPTZ (10 mM, in 0.04 M hydrochloric acid), and ferric chloride $(20 \mathrm{mM})$, respectively. After incubation for 15 min at $37^{\circ} \mathrm{C}$, the measured FRAPs of plasma samples (control or antioxidants and $\mathrm{ONOO}^{-}$-treated plasma) were expressed in $\mathrm{mM} \mathrm{Fe}^{2+}$ in plasma.

\section{Inhibition of Pro-Inflammatory Enzymes}

The ability of the extracts to inhibit lipoxygenase (LOX) and hyaluronidase (HYAL) was examined as described by Michel et al. (2017) with some changes. Briefly, in the LOX tests the reagents were used in a volume ratio of 1:2:1 for working solutions of the tested analyte, linoleic acid, and enzyme. Results 
of the both assays were expressed as $\mathrm{IC}_{50}$ values calculated from concentration-inhibition curves.

\section{Cell Viability Assay}

PBMCs were isolated from human blood (obtained and collected as described above) using the Histopaque ${ }^{\circledR}-1077$ medium (a sterile solution of polysucrose, $57 \mathrm{~g} / \mathrm{L}$, and sodium diatrizoate, $90 \mathrm{~g} / \mathrm{L}$, with a density of $1.077 \mathrm{~g} / \mathrm{mL}$ ). From each of eight donors, two independent PBMCs isolations and incubations with the extracts were performed. Blood was carefully layered (in a volume ratio of 1:1) onto the medium, and centrifuged for $30 \mathrm{~min}(400 \times \mathrm{g}$, at room temperature). Then, the pellet was washed two times with $0.02 \mathrm{M}$ PBS buffer. The obtained fraction of PBMCs was suspended in PBS. Cell suspensions (1 $\times 10^{6} \mathrm{PBMCs} / \mathrm{mL}$ ) were incubated with plant extracts, added to the final concentration of $5 \mu \mathrm{g} / \mathrm{mL}$. Cell viability (\%) was determined during a spectrofluorimetric analysis, involving the use of propidium iodide as a fluorescent dye. Measurements were conducted using a microchip-type automatic cell counter AdamMC DigitalBio (NanoEnTek Inc., Seoul, Korea), after 60 and 120 min of incubation of PBMCs with the examined substances (at $37^{\circ} \mathrm{C}$ ). The procedure was carried out according to the manufacturer's protocol.

\section{Statistical Analysis}

Normality of the distribution of the results was verified using the Shapiro-Wilk test, and the homogeneity of variances using the
Levene's test. The results are reported as means \pm SD (standard deviation) or $\pm \mathrm{SE}$ (standard error) for the indicated number of experiments. The significance of differences between samples and controls was determined with one-way ANOVA (for chemical tests) or one-way ANOVA for repeated measures (for human plasma model), followed by the post-hoc Tukey's test for multiple comparisons. The correlations were evaluated using F-test. All calculations were performed using the Satistica12Pl software for Windows (StatSoft Inc., Krakow, Poland) with $p$-values less than 0.05 regarded as significant.

\section{RESULTS}

\section{LC-MS Metabolite Profiling}

Phytochemical profiling of the dry extracts revealed significant differences in their chemical composition depending on the extraction solvent (Figure 1, Tables 1, 2). The qualitative UHPLC-PDA-ESI-MS ${ }^{3}$ analysis resulted in full or partial identification of over fifty phenolic constituents (Figure 1, Table 1, peaks 1-59) belonging to three main classes of phytochemicals-flavonols (thirty eight analytes), flavan-3-ol derivatives (catechins and proanthocyanidins, seven peaks) and phenolic acids (twelve compounds). The analytes were structurally characterized based on the comparison of their chromatographic behavior and ESI-MS 3 fragmentation patterns (in positive and negative ionization modes) with the literature data or reference standards, both commercial and isolated

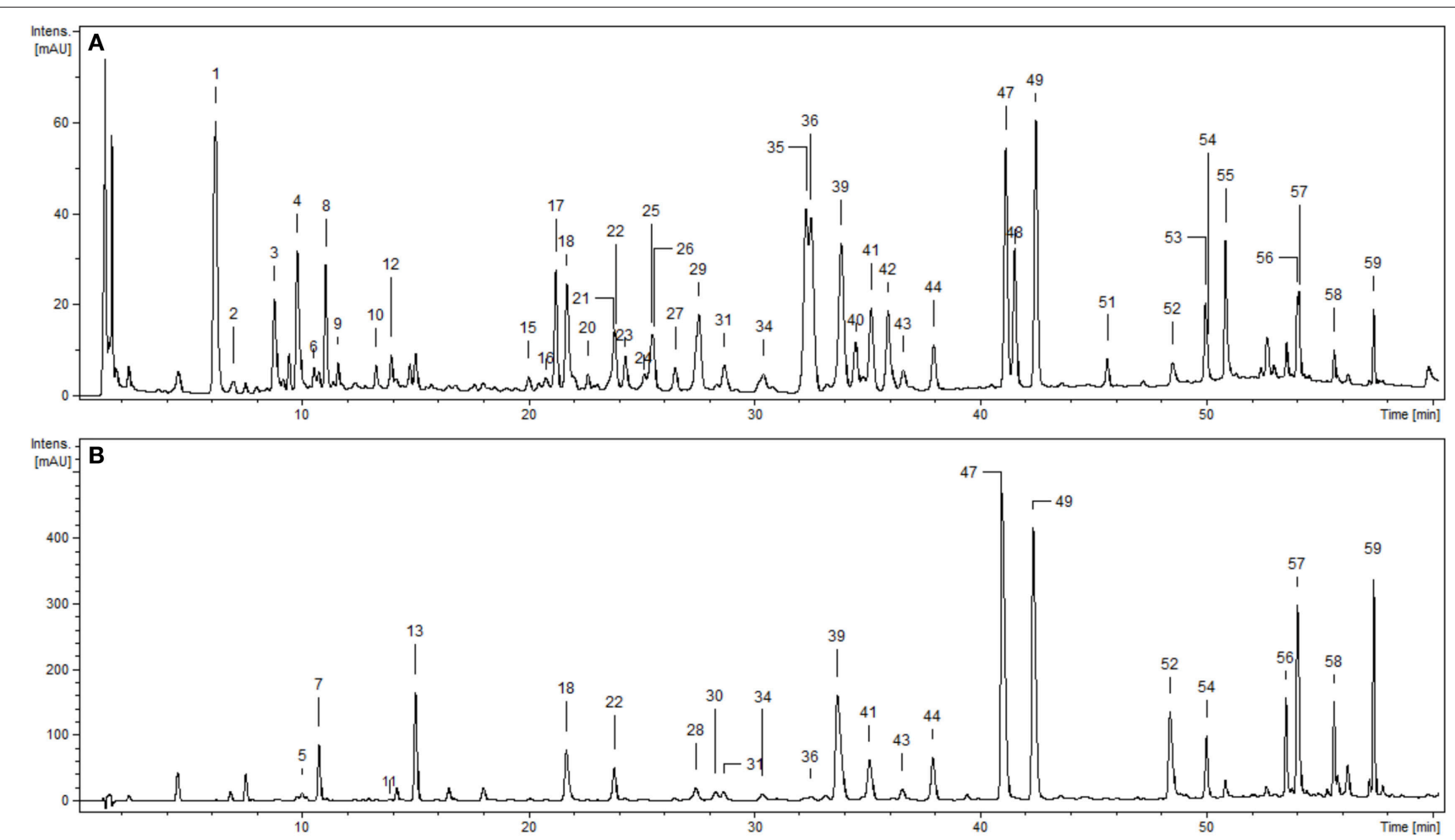

FIGURE 1 | Representative UHPLC chromatograms of the $P$. spinosa flower dry extracts at $280 \mathrm{nm:}$ (A) MED, defatted methanol-water (7:3, v/v) extract; (B) DEF, diethyl ether fraction. Peak numbers refer to those implemented in Table 1. 


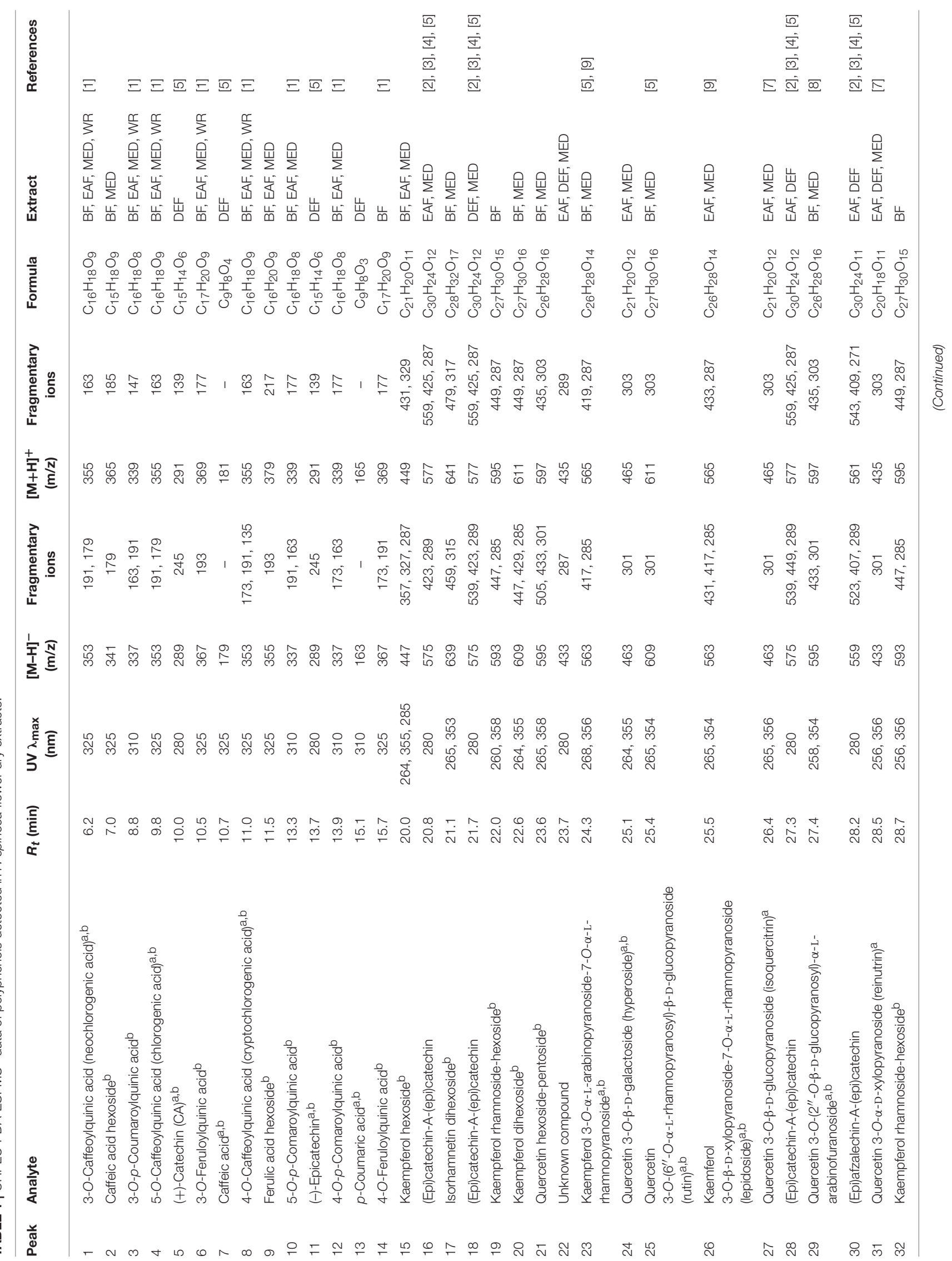




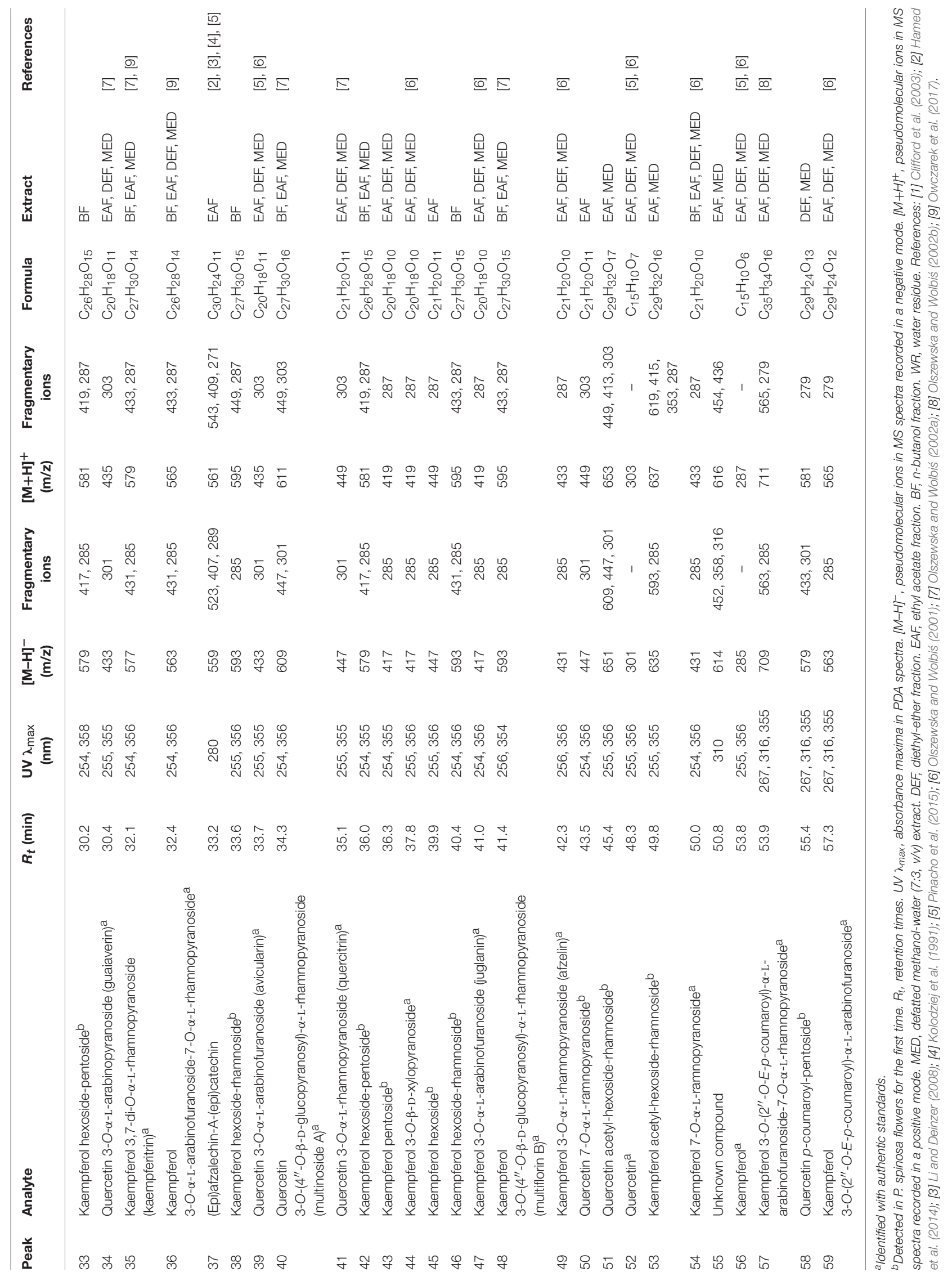


TABLE 2 | Quantitative standardization data for P. spinosa flower dry extracts.

\begin{tabular}{|c|c|c|c|c|c|c|c|}
\hline \multirow[t]{2}{*}{ Extract } & \multirow{2}{*}{$\begin{array}{l}\text { Total phenolics }{ }^{a} \\
\text { TPC (mg GAE/g dw) }\end{array}$} & \multicolumn{4}{|c|}{ Total flavonoids $(\mathrm{mg} / \mathrm{g} \mathrm{dw})^{b}$} & \multirow{2}{*}{$\begin{array}{c}\text { Total } \\
\text { proanthocyanidins } \\
\text { TPA (mg CYE/g } \\
\text { dw) }\end{array}$} & \multirow{2}{*}{$\begin{array}{l}\text { Total phenolic acids } \\
\text { TAC (mg/g dw) }\end{array}$} \\
\hline & & Kaempferol & Quercetin & Isorhamnetin & TFC & & \\
\hline DEF & $464.57 \pm 20.57^{D}$ & $259.68 \pm 3.30^{\mathrm{E}}$ & $61.14 \pm 2.22^{\mathrm{E}}$ & $5.16 \pm 0.01^{D}$ & $490.63 \pm 8.16^{\mathrm{E}}$ & $49.5 \pm 2.23^{\mathrm{B}}$ & $8.76 \pm 0.27^{A}$ \\
\hline EAF & $584.07 \pm 12.98^{\mathrm{E}}$ & $158.69 \pm 1.32^{\mathrm{D}}$ & $53.21 \pm 1.15^{\mathrm{D}}$ & $4.57 \pm 0.29^{C}$ & $325.53 \pm 4.23^{\mathrm{D}}$ & $109.43 \pm 3.71^{C}$ & $17.20 \pm 0.47^{\mathrm{B}}$ \\
\hline
\end{tabular}

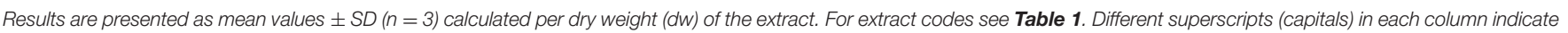
significant differences in the means at $p<0.05$.

a Values expressed in gallic acid equivalents.

${ }^{b}$ Values expressed as the levels of individual aglycones released after acid hydrolysis, and TFC, total glycosides.

${ }^{c}$ Values expressed in cyanidine chloride equivalents.

${ }^{d}$ Values calculated as a sum of caffeic acid and chlorogenic acid equivalents.

previously in our laboratory from flowers and leaves of $P$. spinosa (Olszewska and Wolbiś, 2001, 2002a,b; Owczarek et al., 2017). The greatest chemical diversity was observed for the defatted methanol-water (7:3, v/v) extract (MED), while its fractions of diethyl ether (DEF), ethyl acetate (EAF), n-butanol (BF), and water residue (WR) obtained after sequential liquid-liquid partitioning were enriched in selected analytes, depending on the fractionation solvent.

Compounds 1-4, 6-10, and 12-14 displayed absorption maxima at 325 or $310 \mathrm{~nm}$ and UV-Vis spectra characteristic of caffeic acid or p-coumaric acid derivatives, respectively. According to the spectral profiles and hierarchical discrimination key proposed by Clifford et al. (2003), the peaks showing parent $[\mathrm{M}-\mathrm{H}]^{-}$ions at $\mathrm{m} / \mathrm{z} 353,337$, and 367 were identified as isomeric caffeoylquinic acids (chlorogenic acids; 1, 4, 8), $p$-coumaroylquinic acids $(3,10,12)$, and feruloylquinic acids $(6,14)$, correspondingly. The identity of chlorogenic acids was additionally confirmed by experiments with standards, as was the presence of simple caffeic (7) and $p$-coumaric acids (13) giving the typical $[\mathrm{M}-\mathrm{H}]^{-}$ions at $\mathrm{m} / \mathrm{z} 179$ and 163 , respectively. The same ions were found in $\mathrm{MS}^{2}$ spectra of compounds 2 and $\mathbf{9}$ after neutral losses of sugar moieties (-162 Da each), which led to the tentative identification of hexosides of caffeic acid and ferulic acid, respectively.

Peaks 5, 11, 16, 18, 28, 30, and 37 with UV maxima at $280 \mathrm{~nm}$ were classified as flavan-3-ols and proanthocyanidins. Based on standard spiking and literature data, compounds 5 and 11 were characterized as (+)-catechin and (-)epicatechin, respectively. Compounds 16, 18, and 28 with parent ions $[\mathrm{M}+\mathrm{H}]^{+}$at $\mathrm{m} / \mathrm{z} 577$ were classified as dimeric A-type procyanidins. These compounds gave secondary ions in $\mathrm{MS}^{2}$ spectra at $\mathrm{m} / \mathrm{z} 559,425$, and 287 . The ions at $\mathrm{m} / \mathrm{z} 425$, arising from retro-Diels-Alder (RDA) fission of the $[\mathrm{M}+\mathrm{H}]^{+}(577-152 \mathrm{Da})$, and the ions at $\mathrm{m} / \mathrm{z} 287(577-290$ $\mathrm{Da})$ resulting from quinone methide $(\mathrm{QM})$ fission, confirmed the presence of two (epi)catechin units in the structures ( $\mathrm{Li}$ and Deinzer, 2008; Hamed et al., 2014). Compounds 16, 18, and 28 were thus tentatively identified as (epi)catechin-A(epi)catechins. Two A-type proanthocyanidins of that kind have already been isolated from the flowers of $P$. spinosa and identified as ent-epicatechin- $(4 \alpha \rightarrow 8 ; 2 \alpha \rightarrow \mathrm{O} \rightarrow 7)$-catechin and ent-epicatechin- $(4 \alpha \rightarrow 8 ; 2 \alpha \rightarrow \mathrm{O} \rightarrow 7)$-epicatechin (Kolodziej et al., 1991). Compounds 30 and 37 with pseudomolecular $[\mathrm{M}+\mathrm{H}]^{+}$ions at $\mathrm{m} / \mathrm{z} 561$ and ions in $\mathrm{MS}^{2}$ spectra at $\mathrm{m} / \mathrm{z} 543$, 409 and 271 were classified as dimeric A-type proanthocyanidins. The mass difference between compounds 16, 18, 28, and 30, 37 was $16 \mathrm{Da}$, which indicated that in the latter molecules one (epi)catechin unit is probably replaced by (epi)afzelechin, the flavan-3-ol reported to occur in blackthorn flowers (Kolodziej et al., 1991) and branches (Pinacho et al., 2015). m/z 409 (561-152 Da) arising from RDA fission of the $[\mathrm{M}+\mathrm{H}]^{+}$ion, confirmed the presence of (epi)catechin in both compounds. On the other hand, the product ions at $\mathrm{m} / \mathrm{z} 271$ (561-290 $\mathrm{Da})$ resulting from $\mathrm{QM}$ fission of the $[\mathrm{M}+\mathrm{H}]^{+}$ions, showed that the upper unit in both cases was (epi)afzelechin, and hence the terminal unit must be (epi)catechin, $\mathrm{m} / \mathrm{z} 287$ (Li and Deinzer, 2008; Hamed et al., 2014). The structures of compounds $\mathbf{3 0}$ and $\mathbf{3 7}$ were thus tentatively identified as (epi)afzelechin-A-(epi)catechin. Two proanthocyanidins of that type, i.e., ent-epiafzelechin- $(4 \alpha \rightarrow 8 ; 2 \alpha \rightarrow \mathrm{O} \rightarrow 7)$-epicatechin and ent-epiafzelechin- $(4 \alpha \rightarrow 8 ; 2 \alpha \rightarrow \mathrm{O} \rightarrow 7)$-catechin have previously been isolated from blackthorn flowers (Kolodziej et al., 1991).

Peaks 15, 17, 19-21, 23-27, 29, 31-36, 38-54, and 56-59 with two UV-Vis maxima at 250-268 and 355-365 nm were classified as flavonoids. Compounds $\mathbf{5 2}$ and $\mathbf{5 6}$ showed parent $[\mathrm{M}-\mathrm{H}]^{-}$ ions at $\mathrm{m} / \mathrm{z} 301$ and 285, respectively, and were identified with standards as free aglycones quercetin and kaempferol. Peaks $\mathbf{2 3}$, $\mathbf{2 6}, 35,36,44,47-49,54,57$, and 59 with fragment ions in $\mathrm{MS}^{2}$ $\mathrm{m} / \mathrm{z} 285$ (typical for kaempferol) were identified with authentic standards of kaempferol glycosides (Table 1). Compounds 15, $32,33,38,42,43,45$, and 46 were tentatively characterized based on their fragmentation pattern as kaempferol mono- and diglycosides. Peak 53 with parent $[\mathrm{M}-\mathrm{H}]^{-}$ion at $\mathrm{m} / \mathrm{z} 635$ and fragment ions at $\mathrm{m} / \mathrm{z} 593,285$ in MS spectra was tentatively identified as kaempferol acetyl hexoside-rhamnoside due to the neutral loss of an acetyl moiety $(-42 \mathrm{Da})$, hexose $(-162 \mathrm{Da})$ and rhamnose (-146 Da). Peaks 24, 25, 27, 31, 34, 39-41, and 50 with fragment ions in $\mathrm{MS}^{3}$ spectra at $\mathrm{m} / \mathrm{z} 301$ (typical for 
quercetin) were identified with standards of quercetin glycosides (Table 1). Compound 21 with pseudomolecular $[\mathrm{M}-\mathrm{H}]^{-}$ion at $\mathrm{m} / \mathrm{z} 595$ and fragment ion at $\mathrm{m} / \mathrm{z} 301$ was tentatively assigned to quercetin hexoside-pentoside. Peak 51 with parent ion at $\mathrm{m} / \mathrm{z}$ 651 and fragment ions at $\mathrm{m} / \mathrm{z} 609,447$, and 301 was determined as a quercetin analog of $\mathbf{5 3}$, i.e., quercetin acetyl hexosiderhamnoside. Similarly, compound $\mathbf{5 8}$ was tentatively identified as an analog of 59, i.e., quercetin $p$-coumaroyl-pentoside, probably quercetin 3-O-(2" $-E$ - $p$-coumaroyl $)-\alpha$-L-arabinofuranoside, due to the additional UV-Vis absorption maximum at $316 \mathrm{~nm}$ (typical for an aromatic acyl unit), and the neutral loss of $p$-coumaroyl moiety $(-146 \mathrm{Da})$ and a pentose $(-132 \mathrm{Da})$. Peak 17 gave the parent ion at $\mathrm{m} / \mathrm{z} 639$ and its MS spectra revealed the cleavage of two hexoses ( -162 amu twice) and the aglycone signal consistent with isorhamnetin, which enabled tentative identification of isorhamnetin dihexoside.

\section{Quantitative Standardization}

According to the LC-MS findings, total contents of polyphenols (TPC), flavonoids (TFC), proanthocyanidins (TPA), and phenolic acids (TAC) were selected as standardization targets (Table 2). The TPC levels assayed by the standard FolinCiocalteu method and expressed in gallic acid equivalents (GAE) varied in the range of 64.6-584.07 $\mathrm{mg} \mathrm{GAE} / \mathrm{g} \mathrm{dw}$ of the extracts with the highest values found for EAF (584.07 $\mathrm{mg} \mathrm{GAE} / \mathrm{g} \mathrm{dw}$ ) and DEF (464.57 mg GAE/g dw). The total flavonoid content (TFC) was determined by HPLC-PDA as a sum of flavonoid aglycones released after acid hydrolysis of native extracts, and recalculated on the dominant glycosides. With the levels constituting $55.7-105.5 \%$ of the TPC values, flavonoids were the dominant phenolic components of all extracts except WR, in which phenolic acids prevailed. The highest TFC contents were observed for DEF (490.63 mg/g dw) and EAF (325.53 mg/g dw). As regards individual aglycones, kaempferol was the dominant one and constituted $64.4-79.7 \%$ of the total aglycones. The contents of quercetin and isorhamnetin were much lower and constituted $18.8-30.9 \%$ and $1.6-4.7 \%$ of the sum of aglycones, respectively. The total TPA, determined by the $n$-butanol- $\mathrm{HCl}$ assay and expressed as cyanidin chloride equivalents (CYE), ranged from 12.43 to $109.43 \mathrm{mg} \mathrm{CYE/g} \mathrm{dw}$, with the peak value found again for EAF. Contrastingly, the highest level of phenolic acids (TAC), assayed by HPLC-PDA, was observed for BF (66.77 $\mathrm{mg} / \mathrm{g} \mathrm{dw}$ ), while in other extracts the TAC contents varied in the range of $8.76-29.24 \mathrm{mg} / \mathrm{g} \mathrm{dw}$.

\section{Antioxidative Effects in Chemical Models}

All of the extracts showed significant and dose-dependent antioxidant activity and ability to scavenge free radicals (DPPH, a stable synthetic radical), directly reduce transition metal ions $\left(\mathrm{Fe}^{3+}, \mathrm{FRAP}\right)$, and inhibit AAPH-induced linoleic acid peroxidation (chemical model of lipid peroxidation) significantly diminishing levels of thiobarbituric acid-reactive substances (TBARS), with the final parameters strongly influenced by the extraction solvent (Table 3). Regardless of the test, activity of the extracts decreased in similar order, i.e., EAF $\geq \mathrm{DEF}$ $>\mathrm{BF}>\mathrm{MED}>\mathrm{WR}$, and correlated with the amounts of polyphenols (TPC) and flavonoids (TFC) in the samples
(Table 4). The strongest relationships were observed for the FRAP test, whereas for the DPPH and TBARS assays, the correlation coefficients were negatively influenced by WR, a virtually flavonoid-free extract. If WR was excluded from the data matrix, the impact of TPC $(|r|>0.91, p<0.05)$ on the antioxidant parameters of the other extracts was evident for all tests (results not shown). In a comparison to the positive standards, the antioxidant capacity of the most active extracts EAF and DEF was higher $(p<0.05)$ or not statistically different $(p>0.05)$ than that of an industrial antioxidant BHT (DPPH and TBARS tests), and Trolox, a synthetic analog of vitamin E (FRAP and TBARS tests). Moreover, if activity parameters of the native extracts were recalculated to GAE (Table 3) using the TPC values, the obtained antioxidant capacities of the phenolic fractions constituting the dry extracts were comparable to all of the positive standards except quercetin.

\section{Protective Effects on Human Plasma Components}

After demonstrating the promising antioxidant capacity in chemical assays, the extracts were examined in terms of their effects on human plasma exposed to oxidative stress induced by peroxynitrite $\left(\mathrm{ONOO}^{-} ; 100 \mu \mathrm{M}\right)$. In comparison to the control (untreated) samples, the peroxynitrite-stimulated plasma exhibited a considerably enhanced level (Figure 2A; $p<0.001$ ) of 3-nitrotyrosine in plasma proteins (3-NT-Fg, a marker of protein nitration), a strong increase of lipid hydroperoxides (ferric-xylenol orange assay, FOX-1) and thiobarbituric acidreactive substances (TBARS) - markers of lipid peroxidation (Figures 2B,C; $p<0.001$ ), as well as a noticeable decrease (Figure 2D; $p<0.001$ ) in ferric reducing ability (FRAP, a marker of total antioxidant status of plasma). In plasma samples incubated with $\mathrm{ONOO}^{-}$in the presence of the extracts (at $1-50 \mu \mathrm{g} / \mathrm{mL}$ ), the rate of oxidative/nitrative damage was significantly reduced (Figures 2A-C; $p<0.05$ ). All tested extracts effectively diminished the nitration of tyrosine residues (Figure 2A; $p<0.001$ ) - by about $15-50 \%$ and $36-54 \%$ at 1 and $50 \mu \mathrm{g} / \mathrm{mL}$, respectively. The impact of extraction solvent on the anti-nitrative activity was less pronounced than that observed in chemical tests; still, WR was again the least effective at each concentration level $(p<0.05)$. Among the other extracts, MED displayed the strongest activity (50-58\% decrease in tyrosine nitration); however, only the effects of EAF, BF, and WR were dose-dependent (Figure 2A; $p<$ 0.05). In consequence, the correlation between the percentage inhibition of tyrosine nitration and the phenolic contents (TPC, TFC, TPA, TAC) was not significant (Table 4). All of the tested extracts exhibited also protective properties against peroxynitrite-induced lipid peroxidation (Figures 2B,C; $p<$ 0.001 ), regardless of the concentration level. In samples preincubated with the medium extracts concentration $(5 \mu \mathrm{g} / \mathrm{mL})$, the levels of hydroperoxides and TBARS decreased by about $47-53 \%$ and $21-34 \%$, respectively. The solvent effects were negligible, although EAF at $50 \mu \mathrm{g} / \mathrm{mL}$ was the most effective antioxidant, able to reduce the peroxidation parameters by 
TABLE 3 | Antioxidant activity of $P$. spinosa flower dry extracts and standard antioxidants in DPPH, FRAP, and TBARS tests.

\begin{tabular}{|c|c|c|c|c|c|c|}
\hline \multirow[t]{2}{*}{ Analyte } & \multicolumn{2}{|c|}{ DPPH-Radical scavenging activity } & \multicolumn{2}{|c|}{ Ferric reducing antioxidant power } & \multicolumn{2}{|c|}{ LA-Peroxidation TBARS } \\
\hline & $E_{50}(\mu \mathrm{g} / \mathrm{mL})^{a}$ & $\mathrm{EC}_{50}(\mu \mathrm{g} \mathrm{GAE} / \mathrm{mL})^{\mathrm{b}}$ & $\operatorname{FRAP}\left(\mathrm{mmol} \mathrm{Fe} e^{2+/ g}\right)^{\mathrm{C}}$ & FRAP $\left(\mathrm{mmol} \mathrm{Fe}^{2+} / \mathrm{g} \text { GAE }\right)^{\mathrm{d}}$ & $\mathrm{IC}_{50}(\mu \mathrm{g} / \mathrm{mL})^{\mathrm{e}}$ & $\mathrm{IC}_{50}(\mu \mathrm{g} \text { GAE } / \mathrm{mL})^{\mathrm{d}, \mathrm{f}}$ \\
\hline MED & $15.46 \pm 0.38^{F}$ & 3.19 & $4.40 \pm 0.10^{\mathrm{B}}$ & 21.35 & $20.02 \pm 1.95^{\mathrm{F}}$ & 4.13 \\
\hline DEF & $6.91 \pm 0.22^{\mathrm{D}}$ & 3.42 & $9.46 \pm 0.04^{\mathrm{D}, \mathrm{E}}$ & 19.12 & $6.87 \pm 0.59^{C, D}$ & 3.40 \\
\hline EAF & $6.04 \pm 0.07^{\mathrm{D}}$ & 3.53 & $9.02 \pm 0.11^{\mathrm{D}}$ & 15.44 & $5.81 \pm 0.40^{\mathrm{B}, \mathrm{C}}$ & 3.40 \\
\hline $\mathrm{BF}$ & $11.79 \pm 0.50^{\mathrm{E}}$ & 3.50 & $6.15 \pm 0.23^{C}$ & 20.75 & $11.34 \pm 0.76^{\mathrm{E}}$ & 3.36 \\
\hline WR & $51.32 \pm 0.93^{G}$ & 3.32 & $1.31 \pm 0.04^{\mathrm{A}}$ & 20.33 & $49.73 \pm 2.05^{\mathrm{G}}$ & 3.21 \\
\hline QU & $1.63 \pm 0.07^{\mathrm{A}}$ & - & $36.02 \pm 1.1^{\mathrm{H}}$ & - & $1.85 \pm 0.12^{\mathrm{A}}$ & - \\
\hline TX & $4.34 \pm 0.22^{C}$ & - & $10.83 \pm 0.32^{\mathrm{E}}$ & - & $8.47 \pm 0.45^{\mathrm{C}, \mathrm{D}, \mathrm{E}}$ & - \\
\hline $\mathrm{BHA}$ & $2.90 \pm 0.14^{\mathrm{B}}$ & - & $16.13 \pm 0.83^{\mathrm{F}}$ & - & $3.16 \pm 0.22^{A, B}$ & - \\
\hline $\mathrm{BHT}$ & $6.54 \pm 0.28^{\mathrm{D}}$ & - & $18.89 \pm 0.42^{\mathrm{G}}$ & - & $9.31 \pm 0.16^{\mathrm{D}, \mathrm{E}}$ & - \\
\hline
\end{tabular}

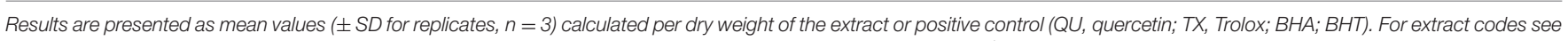

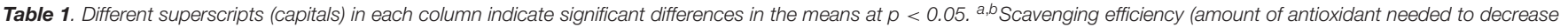

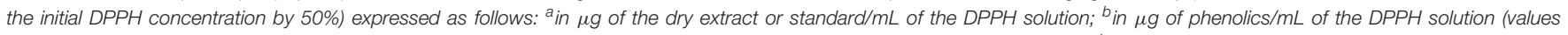

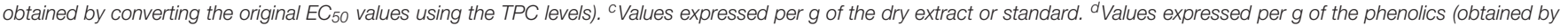

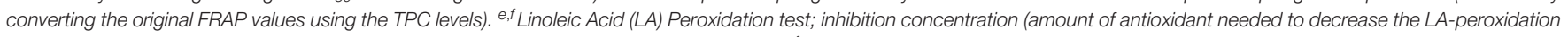

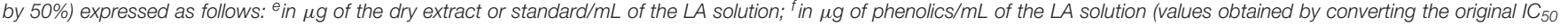
values using the TPC levels).

TABLE 4 | Correlation coefficients $(r)$ and probability ( $p$ ) values of linear relationships between antioxidant activity parameters and phenolic contents.

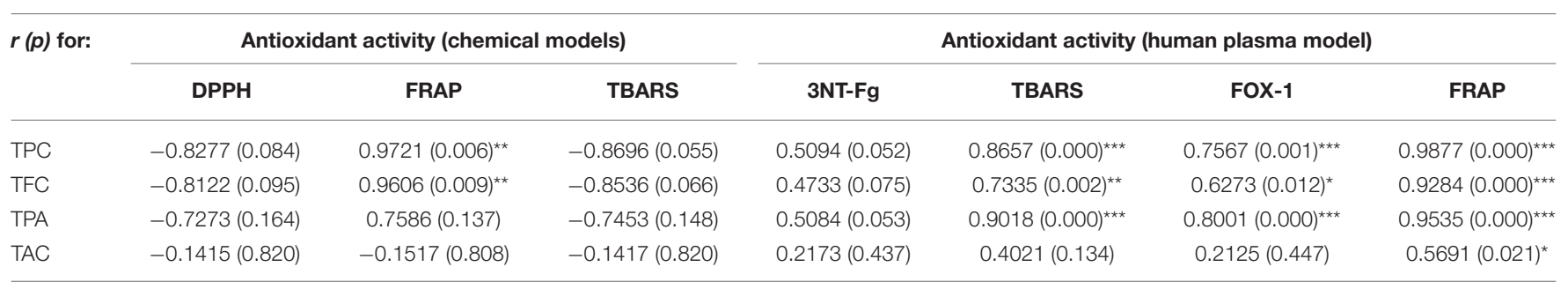

Activity and concentration parameters according to Tables 2, 3, and Figure 2. Asterisks mean statistical significance of the estimated linear relationships ${ }^{*} p<0.05,{ }^{* *} p<0.01$, $\left.{ }^{\star * *} p<0.001\right)$.

about $70 \%$ in FOX-1 and $45 \%$ in TBARS tests. The inhibitory activity on lipid peroxidation was dose-dependent for all extracts if measured by TBARS (Figure 2C; $p<0.05$ ); however, in the FOX-1 assay only some dose-effects for MED, DEF and EAF were significant, i.e., differences observed between the two lowest concentration levels $(1-5 \mu \mathrm{g} / \mathrm{mL})$ and the highest dose of $50 \mu \mathrm{g} / \mathrm{mL}$ (Figure 2B; $p<0.05$ ). Consequently, relatively strong correlations $(|r|>0.73, p<0.01)$ were observed between the percentage of lipid peroxidation assayed by TBARS and the phenolic contents (TPC, TFC, TPA), whereas the analog relationships for FOX-1 test were weaker $(|r|>0.62, p<$ 0.01) (Table 4). All of the extracts were also able to normalize and/or enhance the total antioxidant status of $\mathrm{ONOO}^{-}$treated plasma (FRAP), depending on the concentration used, and only MED at $1 \mu \mathrm{g} / \mathrm{mL}$ did not change significantly the FRAP value of the oxidized plasma (Figure 2D; $p>0.05$ ). For all other extracts, a significant improvement in the plasma reducing ability was observed with the increase up to about $190 \%$ vs. the $\mathrm{ONOO}^{-}$-stimulated plasma and $140 \%$ vs. the control sample for EAF at $50 \mu \mathrm{g} / \mathrm{mL}$ (Figure 2D; $p$ $<0.001)$. Strong dose-dependency of the analytical response was found, and thus strong relationships $(|r|>0.92, p<$ 0.001 ) between the percentage increase in the FRAP values of the oxidized plasma and the TPC, TFC, and TPA levels (Table 4).

Simultaneously, the $\mathrm{ONOO}^{-}$-treated plasma was incubated with $5 \mu \mathrm{g} / \mathrm{mL}$ of standard phenolics (Figures $2 \mathrm{~A}-\mathbf{D}$ ). In contrast to results of the chemical tests, in the plasma model the pure compounds were merely comparable or inferior to the blackthorn extracts in terms of the antioxidant activity. For instance, the range of percentage inhibition of tyrosine nitration achieved for the standards (ca. 11-34\%) was lower than for the extracts (ca. 19-58\%) applied at the same concentration of $5 \mu \mathrm{g} / \mathrm{mL}$ (Figure 2A). Moreover, the activity of MED and $\mathrm{EAF}$ at $5 \mu \mathrm{g} / \mathrm{mL}$ was significantly higher $(p<0.001)$ than that of all standards. Furthermore, quercetin-the strongest antioxidant in chemical models-counteracted the plasma nitration significantly less effectively than all extracts (Figure 2A; $p<0.001)$. Similar trend was observed for $\mathrm{ONOO}^{-}$-induced lipid peroxidation, both with FOX-1 detection-only rutin exhibited protective activity comparable to that of the extracts at $5 \mu \mathrm{g} / \mathrm{mL}$ (Figure 2B; $p>0.05$ ), and with TBARS testoverlapped ranges of percentage inhibition of peroxidation were found for the standards (ca. 26-32\%) and the extracts (21-34\%) at $5 \mu \mathrm{g} / \mathrm{mL}$ (Figure 2C; $p>0.05$ ). Only in the FRAP assay, two of the standards-quercetin and chlorogenic acid-enhanced the 

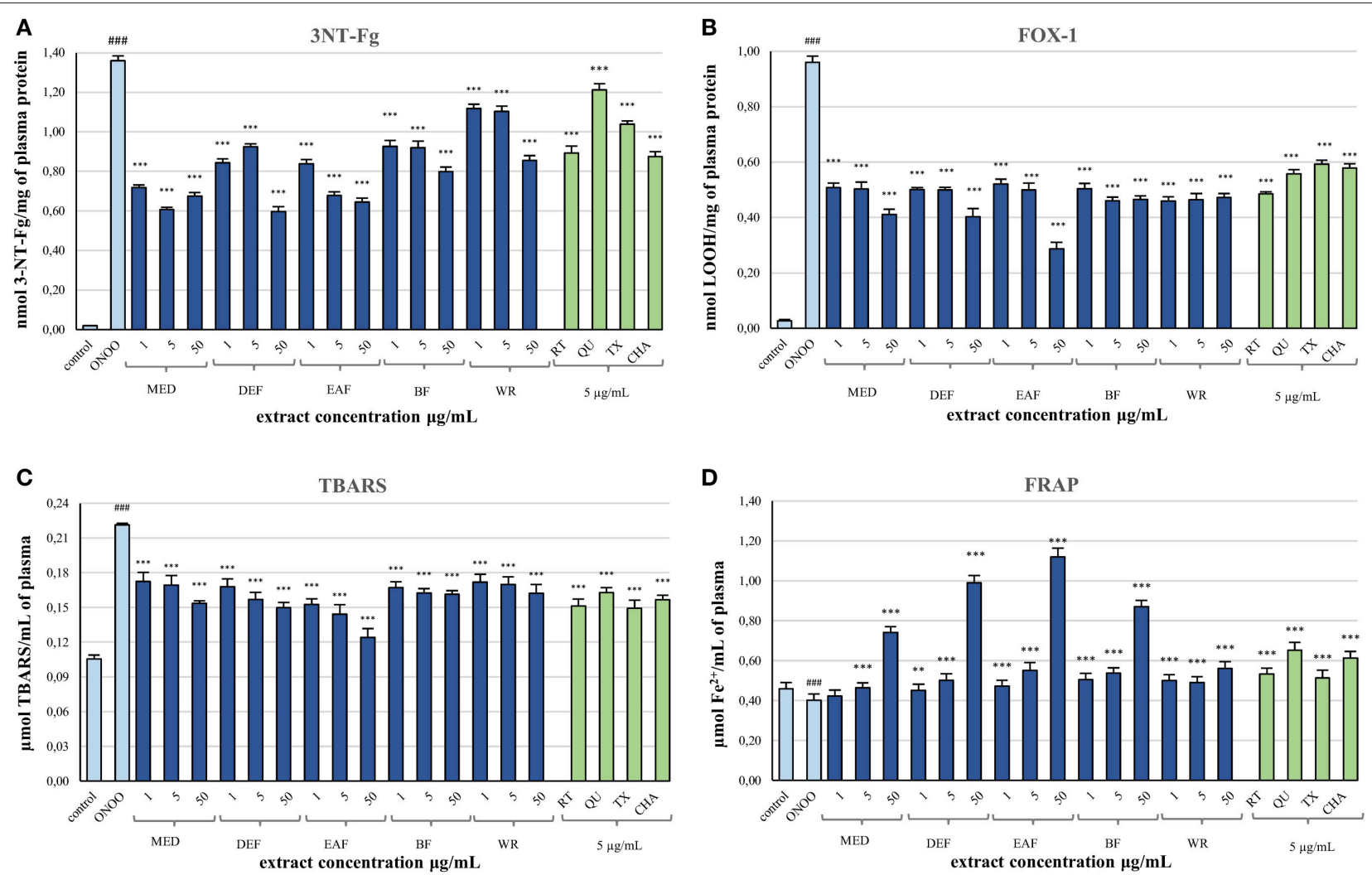

FIGURE 2 | Effects of $P$. spinosa flower dry extracts on human plasma exposed to oxidative stress: (A) effects on the nitration of tyrosine residues in plasma proteins and formation of 3-nirotyrosine, 3-NT-Fg; effects on the peroxidation of plasma lipids including formation of lipid hydroperoxides, LOOH (B), and thiobarbituric acid-reactive substances, TBARS (C); (D) effects on ferric reducing ability of plasma, FRAP. Results are presented as means \pm SE $(n=10)$ for repeated measures: $\# \# \# p<0.001$ for control plasma vs. $\mathrm{ONOO}^{-}$-treated plasma (without the extracts); ${ }^{* *} p<0.01,{ }^{* \star} p<0.001$ for $\mathrm{ONOO}^{-}$-treated plasma in the presence of the extracts (1, 5, or $50 \mu \mathrm{g} / \mathrm{mL})$ or standards $(5 \mu \mathrm{g} / \mathrm{mL})$ vs. ONOO-- treated plasma in the absence of the extracts. Standards: RT, rutin; QU, quercetin; TX, Trolox; CHA, chlorogenic acid.

reducing ability of plasma more strongly than the extracts at the corresponding concentration (Figure 2D; $p<0.001$ ).

\section{Inhibitory Effects on Pro-Inflammatory Enzymes}

The extracts inhibited the activity of lipoxygenase (LOX) and hyaluronidase (HYAL) in a dose-dependent manner, but with different responses toward particular enzyme (Table 5). Considering the $\mathrm{IC}_{50}$ values expressed in $\mu \mathrm{g} / \mathrm{U}$, the extracts were stronger inhibitors of LOX than HYAL. In both tests the strongest effects observed for EAF and DEF were intermediate between those of phenolic standards and indomethacin, a strong nonsteroidal anti-inflammatory drug. The inhibitory activity toward both enzymes was strongly correlated with the TPC levels $(|r|>0.88, p<0.05)$. The responses in the LOX test were also strongly TFC-dependent $(r=-0.8868, p<0.05)$, while in the HYAL assay this relationship was noticeable $(r=-0.8691)$ but not significant $(p=0.056)$.

\section{Influence on Cells Viability}

The potential cytotoxicity of the extracts was evaluated in a model of PBMCs after 60 and 120 min of incubation with the extracts at $5 \mu \mathrm{g} / \mathrm{mL}$. Cellular safety of the extracts was evidenced by the lack of significant differences $(p>0.05)$ in cell viability observed between PBMCs incubated with the extracts (85.6$90.5 \%$ viability) and the control (untreated) samples (87.85-90.85 viability), regardless of the incubation time and extraction solvent (Figure 3).

\section{DISCUSSION}

As beneficial for human health, including prevention of CVD, polyphenol-rich extracts are widely used in the preparation of dietary supplements and pharmaceuticals (Franz et al., 2011). Extraction from natural matrices is thus the crucial step in their utilization as it affects extract composition, activity, and yield. Generally, alcohol and alcohol-water mixtures are the best extractants for low-molecular-weight polyphenols of high antioxidant potential and good bioavailability (Manach et al., 2005). The crude extracts are often fractionated to remove fatsoluble ballast substances (chlorophylls and waxes) and thereby purify and concentrate the phenolic fractions (Rana et al., 2016). The results from the present study confirmed these observations: the defatted methanol-water $(7: 3, \mathrm{v} / \mathrm{v})$ extract from the blackthorn flowers (MED), demonstrated to be a rich source 
TABLE 5 | Inhibitory activity of $P$. spinosa flower dry extracts on lipoxygenase (LOX) and hyaluronidase (HYAL).

\begin{tabular}{|c|c|c|c|c|}
\hline \multirow[t]{2}{*}{ Analyte } & \multicolumn{2}{|c|}{ LOX } & \multicolumn{2}{|c|}{ HYAL } \\
\hline & $I C_{50}(\mu \mathrm{g} / \mathrm{mL})^{a}$ & $I C_{50}(\mu \mathrm{g} / \mathrm{U})^{\mathrm{b}}$ & $\mathrm{IC}_{50}(\mu \mathrm{g} / \mathrm{mL})^{\mathrm{a}}$ & $I C_{50}(\mu \mathrm{g} / \mathrm{U})^{\mathrm{b}}$ \\
\hline MED & $327.36 \pm 5.93^{F}$ & 7.85 & $51.74 \pm 2.16^{\mathrm{E}}$ & 23.00 \\
\hline DEF & $150.36 \pm 4.47^{C}$ & 3.60 & $21.40 \pm 0.76^{\mathrm{B}}$ & 9.51 \\
\hline EAF & $135.36 \pm 5.55^{\mathrm{B}}$ & 3.25 & $21.27 \pm 0.16^{\mathrm{B}}$ & 9.45 \\
\hline BF & $171.10 \pm 1.36^{\mathrm{E}}$ & 4.11 & $42.23 \pm 0.99^{D}$ & 18.77 \\
\hline WR & $479.50 \pm 3.38^{G}$ & 11.50 & $121.72 \pm 5.73^{F}$ & 54.10 \\
\hline QU & $89.23 \pm 2.13^{A}$ & 2.14 & $30.78 \pm 1.84^{C}$ & 13.65 \\
\hline RT & $162.70 \pm 3.70^{\mathrm{D}}$ & 3.90 & $54.63 \pm 2.61^{E}$ & 24.23 \\
\hline $\mathrm{CHA}$ & $166.83 \pm 7.15^{\mathrm{D}, \mathrm{E}}$ & 4.00 & $28.59 \pm 1.21^{C}$ & 12.68 \\
\hline IND & $92.60 \pm 3.71^{A}$ & 2.22 & $12.77 \pm 0.91^{\mathrm{A}}$ & 5.66 \\
\hline
\end{tabular}

Results are presented as mean values $\pm S D(n=3)$ calculated per dry weight of the extract or positive control (QU, quercetin; RT, rutin; CHA, chlorogenic acid; IND, indomethacin). For extract codes see Table 1. Different superscripts (capitals) in each column indicate significant differences in the means at $p<0.05{ }^{a, b}$ Inhibition concentration (amount of analyte needed for $50 \%$ inhibition of enzyme activity) expressed as follows: ${ }^{a}$ in $\mu \mathrm{g}$ of the dry extract or standard/ $m L$ of the enzyme solution; ${ }^{b}$ in $\mu g$ of the extracts/enzyme unit (U).

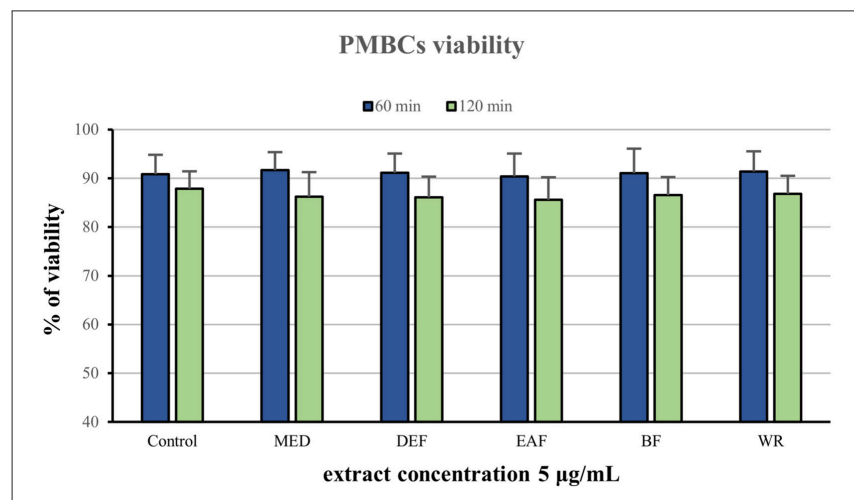

FIGURE 3 | Viability of peripheral blood mononuclear cells (PMBCs) after 60 and $120 \mathrm{~min}$ of incubation with $P$. spinosa flower dry extracts at $5 \mu \mathrm{g} / \mathrm{mL}$.

of phenolic compounds, could be further enriched by liquidliquid partitioning with organic solvents (Table 2). The TPC in the richest fractions EAF and DEF, as determined by the FolinCiocalteu (FC) assay, were comparable to those found in the extracts considered important in CVD prevention, for instance in commercial ethanol extracts of grape seed (ca. 630-670 mg GAE/g dw) (Baydara et al., 2004) or in ethyl acetate fractions of green tea and green mate (ca. 480-580 mg GAE/g dw) (Erol et al., 2009). The previous literature data referring to P. spinosa are limited to studies on fruits, branches, and leaves. According to Pinacho et al. (2015), the highest TPC level quantified by the Prussian Blue method (PB) was found for ethanol fraction from branches (732.34 mg GAE/g dw), followed by those from fruits (359.11 mg GAE/g dw) and leaves (228.56 mg GAE/g $\mathrm{dw})$. Despite some differences in chemistry of the PB assay in comparison to the standardized FC test, in the preliminary studies focused on selection of the plant material for the present work, we have observed the magnitude of phenolic levels to some extent similar, which enables ordering of the blackthorn tissues in respect to the TPC values as follows: branches $>$ flowers $>$ fruits $\geq$ leaves. Therefore, the flowers were found promising for further studies.

The present paper is the first presenting comprehensive LCMS profile of $P$. spinosa flowers (Figure 1, Table 1). The previous studies aimed at isolation and resulted in structure elucidation of 16 flavonoids (Sakar and Kolodziej, 1993; Olszewska and Wolbiś, 2001, 2002a) and five proanthocyanidins (Kolodziej et al., 1991), the presence of which was now confirmed in the flower dry extracts. In addition, 36 constituents, mainly flavonoids, and phenolic acids, were reported here for the first time for the blackthorn flowers (Table 1). With nearly 60 components detected, in contrast to only 25 observed in the leaves (Owczarek et al., 2017), 26 in branches (Pinacho et al., 2015), and 29 in fruits (Guimarães et al., 2013), the phenolic matrix of the flowers is obviously the most complex. A distinctive qualitative feature of the flower extracts is a vast diversity of the flavonoid fraction (thirty seven peaks). While according to Owczarek et al. (2017) flavonoids prevail also in the leaf samples, only 14 structures have been detected in this organ. The branches and fruits of $P$. spinosa may be in turn distinguished by the presence of numerous proanthocyanidins (twelve peaks in branches) and anthocyanins (eight peaks in fruits), respectively (Guimarães et al., 2013; Pinacho et al., 2015). In phytotherapy, the blackthorn flowers are indeed recognized as a flavonoid herbal product (Hoppe, 1981; Poonam et al., 2011). High content of flavonoids found in the present study and their prevalence in flower dry extracts (Table 2), were in agreement with these findings. In addition to flavonoids, the analyzed extracts contained moderate levels of proanthocyanidins and phenolic acids (Table 2). The TPA level of MED is in accordance with the results reported recently by Ropiak et al. (2016) for aqueous acetone extracts of blackthorn flowers $(40 \mathrm{mg} / \mathrm{g} \mathrm{dw})$. As indicated in the latter paper, the average degree of polymerization of flower procyanidins is 2.9 , which means that the majority of TPA fraction is within the bioavailable range of molecular masses (Manach et al., 2005). Thus, the composition of the flower extracts of blackthorn appears promising in the context of CVD and potential industrial application. Regular intake of flavonoids with the diet or supplements is associated with reduced risk of cardiovascular episodes and mortality (Alissa and Ferns, 2012). In in vivo studies, they have been shown to improve antioxidant status, exert anti-atherosclerotic and anti-atherothrombotic effects in early stages of atherosclerosis development (e.g., decrease LDL oxidation), modulate lipid metabolism (e.g., normalize LDL/HDL profile), improve capillary permeability (vasoprotective effects) and endothelial function, and increase nitric oxide release (vasodilatory effects) (Middleton et al., 2000; Alissa and Ferns, 2012). As the most common group of polyphenols in the human diet, flavonoids are also regarded as safe in internal applications. Like flavonoids, both low-molecular-mass proanthocyanidins and phenolic acids have been shown to modulate lipid metabolism, increase plasma antioxidant capacity, improve vascular functions, and reduce platelet activity in humans (Manach et al., 2005).

As antioxidant activity of polyphenols is one of the most important aspects of their beneficial effects in CVD (Middleton et al., 2000; Alissa and Ferns, 2012; Quiñones et al., 2013; 
Santilli et al., 2015) we decided to verify it in complementary chemical and biological models that reflect various direct and complex mechanisms. Although simple chemical in vitro assays are hardly relevant to in vivo conditions, they enable preliminary screening for potential mechanisms and comparison with the literature data. In the present study, we employed three the most frequently used tests of both single electron transfer (DPPH, FRAP) and hydrogen atom transfer basic mechanisms (TBARS) to explore direct interactions of the extracts with free-radicals and transition metal ions as well as to study some of these effects in a model of lipid peroxidation. The results demonstrated that the flower extracts are potent and universal antioxidants, and that their dose-dependent activity is determined by the contents of phenolics, primarily flavonoids, and strongly influenced by the fractionation solvent (Tables 3, 4). Also the extracts produced from other organs of $P$. spinosa have recently been reported effective DPPH scavengers, as well as inhibitors of lipid peroxidation (Guimarães et al., 2014; Pinacho et al., 2015). Comparing our results to those of Pinacho et al. (2015) and in terms of capacity of BHA after 60 min of incubation, it seems that the activity of flower extracts (EAF, DEF) is comparable to that of branches, and superior to those of leaves and fruits. Moreover, the comparison of our results from TBARS assay and the results obtained by Guimarães et al. (2014) in terms of activity of Trolox, shows that the lipid peroxidation inhibitory activity of flower extracts (EAF, DEF) is higher than that of phenolic-enriched extracts from wild fruits. On the other hand, the outcomes from DPPH test suggest similar scavenging activity for flower and fruit extracts.

To give a more accurate approximation of the possible in vivo effects of the flower extracts, we extended the in vitro study to the model of human plasma exposed to oxidative stress. The stress conditions were induced by peroxynitrite $\left(\mathrm{ONOO}^{-}\right)$, a powerful oxidative and nitrative species, generated in vivo by the reaction of nitric oxide (NO) and superoxide anion $\left(\mathrm{O}_{2}^{\bullet-}\right)$, and involved in the pathophysiology of various inflammatory, neurodegenerative, and especially cardiovascular disorders including atherosclerosis, myocardial infarction and chronic heart failure (Ronson et al., 1999). The concentration of $\mathrm{ONOO}^{-}$used in our in vitro study $(100 \mu \mathrm{M})$ enables quantitative measurements of the changes induced in plasma components and corresponds to its levels in vivo, that can be reached in local compartments in the conditions of accelerated production of $\mathrm{NO}$ and $\mathrm{O}_{2}^{\bullet-}$, e.g., during a serious inflammation of blood vessels, when its synthesis can increase up to $50-100 \mu \mathrm{M} / \mathrm{min}$ (Szabo et al., 2007). During the study, the plasma samples were pre-incubated with the extracts at the levels of $1-50 \mu \mathrm{g} / \mathrm{mL}$, equivalent to $0.06-29.2 \mu \mathrm{g} \mathrm{GAE} / \mathrm{mL}$, depending on the extract. From the physiological point of view, the concentrations of phenolic substances that are likely to occur in blood plasma in vivo after oral supplementation can reach up to 5-7 $\mu \mathrm{M}$, depending on the food matrix (Manach et al., 2005). For example, it has been reported that $100-\mathrm{mg}$ dose of quercetin from onions and 150-mg dose of pure isoquercitrin (quercetin-3-glucoside) resulted in the concentrations up to 7.6 and $5 \mu \mathrm{M}$ in plasma, respectively, which is an equivalent of $1.5-2.3 \mu \mathrm{g} / \mathrm{mL}$ of quercetin (Hollmann, 2004; Manach et al., 2005). It has also been suggested that the bioavailability of kaempferol and its glycosides, the dominant flavonoids of blackthorn flowers according to our results, is higher than that of quercetin (DuPont et al., 2004). Therefore, the lower levels of the extracts $(1-5 \mu \mathrm{g} / \mathrm{mL})$ used in the study appear to closely correspond to the range of physiological level of plant-derived phenolic compounds available after oral administration. In accordance with the common practice of in vitro studies (Kolodziejczyk et al., 2011; Kolodziejczyk-Czepas et al., 2013, 2014), the extracts were studied also at $50 \mu \mathrm{g} / \mathrm{mL}$ to enable that of observation of all possible effects of their interaction with peroxynitrite.

The results from biological model confirmed our hypothesis that antioxidant activity of the $P$. spinosa flower extracts may be crucial in understanding their beneficial effects in CVD in vivo. The analyzed extracts not only enhanced the total antioxidant status of the $\mathrm{ONOO}^{-}$-treated plasma but also effectively reduced the levels of well-known oxidative stress biomarkers-products of protein nitration (3-nitrotyrosine) and lipid peroxidation (hydroperoxides and TBARS) (Figure 3). In CVD patients, the increased levels of these biomarkers are good predictors of cardiovascular events and are correlated with prothrombotic, proatherogenic, and pro-inflammatory intravascular effects, plaque instability, and endothelial dysfunctions (Lee et al., 2012; Thomson, 2015). The mechanism of action of the blackthorn extracts in the protection of plasma proteins and lipids probably involves a direct scavenging of peroxynitrite or/and secondary radicals formed in the induced chain reactions. It is consistent with the noticeable scavenging potential of the extracts toward the model radical (DPPH), and with the accumulating evidence of anti-radical effects of polyphenols against $\mathrm{ONOO}^{-}$and $\mathrm{ONOO}^{-}$-related radicals operating in plasma, such as $\mathrm{O}_{2}^{--}$, NO, $\bullet \mathrm{NO}_{2},{ }^{\bullet} \mathrm{OH}$, etc. (Heijnen et al., 2001). Despite some arguments being presented for the impact of blackthorn polyphenols on the target activity in plasma, the differences between the examined extracts were not as evident as in the case of chemical tests. The discrepancies may be an effect of complexity and ontogenetic diversity of blood plasma, as well as differences in the sensitivity to $\mathrm{ONOO}^{-}$between the plasma samples. Some interactions between endogenous plasma constituents and blackthorn phenolics could also be the reason. On the other hand, the complexity of the extracts and synergic effects of their individual components are probably behind the superior activity profile revealed by the extracts comparing to the pure standards. Finally, it is of note that all beneficial effects of the extracts on plasma were measurable at physiological concentrations (1$5 \mu \mathrm{g} / \mathrm{mL}$ ), which do not deteriorate the viability of PBMCs and may be regarded as safe.

Pathogenesis of CVD covers, apart from oxidative stress, also a closely related process of inflammation (Biswas, 2016). To evaluate the potential anti-inflammatory effects of the blackthorn extracts, we decided to study their impact on two model pro-inflammatory enzymes. The first belongs to the family of lipoxygenases, enzymes catalyzing dioxygenation of polyenoic fatty acids in biological membranes and lipoproteins, and producing key chemokines and ROS, such as leukotrienes and $\mathrm{O}_{2}^{\bullet-}$, associated with the development of oxidative stress-related inflammatory pathologies of CVD, e.g., myocardial infarction/reperfusion injury (Schneider and Bucar, 2005). The second one-hyaluronidase-is a spreading factor increasing 
tissues (i.a. vascular) permeability during the inflammatory processes by degrading hyaluronan, an anti-inflammatory extracellular matrix component (Girish et al., 2009). Both enzymes are targets of many synthetic drugs used in antiinflammatory therapies, but it has been shown that plant extracts, especially those containing phenolics, can also significantly inhibit their activity (Schneider and Bucar, 2005; Piwowarski et al., 2011). Indeed, the investigated extracts, rich in polyphenols, were found to be inhibitors of both enzymes (Table 5). The activity of the most active fractions was between those of the commercial agents-indomethacin (nonsteroidal antiinflammatory drug) and rutin (flavonoid vasoprotective agent), while the basic methanol-water extract was slightly less active. However, with the results of antioxidant activity tests, these findings suggest that the extracts might influence both interdependent processes of oxidative stress and inflammation.

\section{CONCLUSIONS}

This work is the first comprehensive phytochemical and activity study of the flower extracts of P. spinosa. The detailed LC-MS patterns presented here can be recommended as reference fingerprints useful in authentication and standardization studies of blackthorn flowers and flower extracts. Distinct phenolic profiles, significant antioxidant effects comparable to or surpassing the activity of pure phenolics in both chemical and biological models, noticeable inhibitory effects on proinflammatory enzymes, and cellular safety suggest that the investigated extracts may be promising candidates for the production of pharma- and nutraceuticals effective in cardiovascular protection. Considering both the yield and the activity, the defatted methanol-water (7:3, v/v) extract and

\section{REFERENCES}

Alissa, E. M., and Ferns, G. A. (2012). Functional foods and nutraceuticals in the primary prevention of cardiovascular diseases. J. Nutr. Metab. 2012:569486. doi: $10.1155 / 2012 / 569486$

Barros, L., Carvalho, A. M., Morais, J. S., and Ferreira, I. C. F. R. (2010). Strawberry-tree, blackthorn and rose fruits: detailed characterisation in nutrients and phytochemicals with antioxidant properties. Food Chem. 120, 247-254. doi: 10.1016/j.foodchem.2009.10.016

Baydara, N. G., Özkanb, G., and Sağdiçc, O. (2004). Total phenolic contents and antibacterial activities of grape (Vitis vinifera L.) extracts. Food Control 15, 335-339. doi: 10.1016/S0956-7135(03)00083-5

Berger, F. (1949). Handbuch der Drogenkunde, 1st Edn. Wien: Maudrich.

Biswas, S. K. (2016). Does the interdependence between oxidative stress and inflammation explain the antioxidant paradox? Oxid. Med. Cell. Longev. 2016:5698931. doi: 10.1155/2016/5698931

Blumenthal, M., and Busse, W. R. (1998). The Complete German Commission E monographs: Therapeutic Guide to Herbal Medicines. Austin, TX: The American Botanical Council.

Calvo, M. I., and Cavero, R. Y. (2014). Medicinal plants used for cardiovascular diseases in Navarra and their validation from official sources. J. Ethnopharmacol. 157, 268-273. doi: 10.1016/j.jep.2014.09.047

Clifford, M. N., Johnston, K. L., Knight, S., and Kuhnert, N. (2003). Hierarchical scheme for LC-MS ${ }^{n}$ identification of chlorogenic acids. J. Agric. Food Chem. 51, 2900-2911. doi: 10.1021/jf026187q

DuPont, M. S., Day, A. J., Bennett, R. N., Mellon, F. A., and Kroon, P. A. (2004). Absorption of kaempferol from endive, a source of kaempferol-3-glucuronide, in humans. Eur. J. Clin. Nutr. 58, 947-954. doi: 10.1038/sj.ejen.1601916 its diethyl ether and ethyl acetate fractions appear to be the most advantageous for biological applications. As the observed capacities might be considered as some of the mechanisms behind the activity of blackthorn phenolics within the circulatory system, the present study forms a basis for their further studies in the context of CVD. For instance, the demonstrated protective effects of the extracts against $\mathrm{ONOO}^{-}$-induced changes in plasma components and ability to inhibit pro-inflammatory enzymes might be cross-linked with their possible antiatherogenic, anticoagulant, and antiplatelet functions or ability to influence endothelium, which should be addressed in future research.

\section{AUTHOR CONTRIBUTIONS}

$\mathrm{AM}, \mathrm{AO}$, and $\mathrm{MO}$ planned the experiments and wrote the manuscript. AM, AO, MM, AP, and JK-C performed the experiments. AM and $\mathrm{AO}$ carried out data analysis. $\mathrm{PN}$ acted as the consultant on biological methods. MO supervised all work. All authors critically revised and approved the final version of the manuscript.

\section{ACKNOWLEDGMENTS}

The authors would like to express their gratitude to the Department of Pharmacognosy and Molecular Basis of Phytotherapy, Faculty of Pharmacy, Medical University of Warsaw, for enabling access to the UHPLC-MS equipment. This work was supported by Medical University of Lodz (grant Nos. 503/3-022-01/503-31-001, 503/3-022-01/503-36-001, and 502-03/3-022-01/502-34-082) and University of Lodz (grant No. $506 / 1136)$.

Erol, N. T., Sari, F., Calikoglu, E., and Velioglu, Y. S. (2009). Green and roasted mate: phenolic profile and antioxidant activity. Turk. J. Agric. For. 33, 353-362. doi: 10.3906/tar-0901-4

Franz, C., Chizzola, R., Novak, J., and Sponza, S. (2011). Botanical species being used for manufacturing plant food supplements (PFS) and related products in the EU member states and selected third countries. Food Funct. 2, 720-730. doi: $10.1039 / \mathrm{clfo} 10130 \mathrm{~g}$

Girish, K. S., Kemparaju, K., Nagaraju, S., and Vishwanath, B. S. (2009). Hyaluronidase inhibitors: a biological and therapeutic perspective. Curr. Med. Chem. 16, 2261-2288. doi: 10.2174/092986709788453078

Guimarães, R., Barros, L., Calhelha, R. C., Carvalho, A. M., Queiroz, M. J. R. P., and Ferreira, I. C. (2014). Bioactivity of different enriched phenolic extracts of wild fruits from northeastern Portugal: a comparative study. Plant Foods Hum. Nutr. 69, 37-42. doi: 10.1007/s11130-013-0394-5

Guimarães, R., Barros, L., Dueñas, M., Carvalho, A. M., Queiroz, M. J. R. P., Santos-Buelga, C., et al. (2013). Characterisation of phenolic compounds in wild fruits from Northeastern Portugal. Food Chem. 141, 3721-3730. doi: 10.1016/j.foodchem.2013.06.071

Hamed, A. I., Al-Ayed, A. S., Moldoch, J., Piacente, S., Oleszek, W., and Stochmal, A. (2014). Profiles analysis of proanthocyanidins in the argun nut (Medemia argun-an ancient Egyptian palm) by LC-ESI-MS/MS. J. Mass Spectrom. 49, 306-315. doi: 10.1002/jms.3344

Heijnen, C. G., Haenen, G. R., Vekemans, J. A., and Bast, A. (2001). Peroxynitrite scavenging of flavonoids: structure activity relationship. Environ. Toxicol. Pharmacol. 10, 199-206. doi: 10.1016/S1382-6689(01)00083-7

Hollmann, P. C. H. (2004). Absorption, bioavailability, and metabolism of flavonoids. Pharm. Biol. 42 , 74-83. doi: 10.3109/138802004908 93492 
Hoppe, H. A. (1981). Taschenbuch der Drogenkunde. Berlin; New York, NY: De Gruyter.

Jarić, S., Mačukanović-Jocić, M., Djurdjević, L., Mitrović, M., Kostić, O., KaradŽić, B., et al. (2015). An ethnobotanical survey of traditionally used plants on Suvaplanina mountain (south-eastern Serbia). J. Ethnopharmacol. 175, 93-108. doi: 10.1016/j.jep.2015.09.002

Kljak, K., and Grbeša, D. (2015). Carotenoid content and antioxidant activity of hexane extracts from selected Croatian corn hybrids. Food Chem. 167, 402-408. doi: 10.1016/j.foodchem.2014.07.002

Kolodziej, H., Sakar, M. K., Burger, J. F. W., Engelshowe, R., and Ferreira, D. (1991). A-type proanthocyanidins from Prunus spinosa. Phytochemistry 30, 2041-2047. doi: 10.1016/0031-9422(91)85064-7

Kolodziejczyk-Czepas, J., Nowak, P., Kowalska, I., and Stochmal, A. (2014). Biological activity of clovers-Free radical scavenging ability and antioxidant action of six Trifolium species. Pharm. Biol. 52, 1308-1314. doi: 10.3109/13880209.2014.891042

Kolodziejczyk-Czepas, J., Olas, B., Malinowska, J., Wachowicz, B., MoniuszkoSzajwaj, B., Kowalska, I., et al. (2013). Trifolium pallidum and Trifolium scabrum ectracts in the protection of human plasma components. J. Thromb. Thrombolysis 35, 193-199. doi: 10.1007/s11239-012-0792-9

Kolodziejczyk, J., Olas, B., Wachowicz, B., Szajwaj, B., Stochmal, A., and Oleszek, W., (2011). Clovamide-rich extract from Trifolium pallidum reduces oxidative stress-induced damage to blood platelets and plasma. J. Physiol. Biochem. 67, 391-399. doi: 10.1007/s13105-011-0089-z

Kültür, S. (2007). Medicinal plants used in Kirklareli Province (Turkey). J. Ethnopharmacol. 111, 341-364. doi: 10.1016/j.jep.2006.11.035

Lee, R., Margaritis, M., Channon, K. M., and Antoniades, C. (2012). Evaluating oxidative stress in human cardiovascular disease: methodological aspects and considerations. Curr. Med. Chem. 19, 2504-2520. doi: $10.2174 / 092986712800493057$

Li, H. J., and Deinzer, M. L. (2008). The mass spectral analysis of isolated hops atype proanthocyanidins by electrospray ionization tandem mass spectrometry. J. Mass Spectrom. 43, 1353-1363. doi: 10.1002/jms.1411

Lisevitskaya, L. J., Shinkarenko, A. L., Bandyukova, V. A., and Makarov, V. A. (1968). Flavonol substances during experimental atherosclerosis. Aktual. Vopr. Farm. 1968, 176-177.

Makarov, V. A. (1968). Spasmolytic and antitonic action of Prunus spinosa flavonoids. Aktual. Vopr. Farm. 1968, 1988-1990.

Makarov, V. A. (1972). Chemical and pharmacological study of Prunus spinosa flavonoids. Rast. Res. 8, 42-49.

Makarov, V. A., and Khadzhai, I. A. (1969). Anti-inflammatory and P-vitamin activity of blackthorn flavonols. Farmakol. Toksikol. 39, 438-441.

Manach, C., Williamson, G., Morand, C., Scalbert, A., and Rémésy, C. (2005). Bioavailability and bioefficacy of polyphenols in humans. I. review of 97 bioavailability studies. Am. J. Clin. Nutr. 81 (1 Suppl.), 230S-242S.

Michel, P., Owczarek, A., Kosno, M., Szymański, P., Mikiciuk-Olasik, E., Kilanowicz, A., et al. (2017). Metabolite profiling of Eastern Teaberry (Gaultheria procumbens L.) lipophilic leaf extracts with hyaluronidase and lipoxygenase inhibitory activity. Molecules 22:412. doi: 10.3390/molecules220 30412

Middleton, E., Kandaswami, C., and Theoharides, T. C. (2000). The effects of plant flavonoids on mammalian cells: implications for inflammation, heart disease, and cancer. Pharmacol. Rev. 52, 673-751.

Olszewska, M. (2012). New validated high-performance liquid chromatographic method for simultaneous analysis of ten flavonoid aglycones in plant extracts using a $\mathrm{C} 18$ fused-core column and acetonitrile-tetrahydrofuran gradient. J. Sep. Sci. 35, 2174-2183. doi: 10.1002/jssc.201200287

Olszewska, M. A., Presler, A., and Michel, P. (2012). Profiling of phenolic metabolites and antioxidant activity of dry extracts from the selected Sorbus species. Molecules 17, 3093-3113. doi: 10.3390/molecules 17033093

Olszewska, M., and Wolbiś, M. (2001). Flavonoids from the flowers of Prunus spinosa L. Acta Pol. Pharm. 58, 367-372.

Olszewska, M., and Wolbiś, M. (2002a). Further flavonoids from the flowers of Prunus spinosa L. Acta Pol. Pharm. 59, 133-137.

Olszewska, M., and Wolbiś, M. (2002b). Flavonoids from the leaves of Prunus spinosa L. Pol. J. Chem. 76, 967-974.

Owczarek, A., Magiera, A., Matczak, M., Piotrowska, D. G., Olszewska, M. A., and Marchelak, A. (2017). Optimisation of preparative HPLC separation of four isomeric kaempferol diglycosides from Prunus spinosa L. by application of the response surface methodology. Phytochem. Lett. 20, 415-424. doi: 10.1016/j.phytol.2017.01.010

Pandey, K. B., and Rizvi, S. I. (2009). Plant polyphenols as dietary antioxidants in human health and disease. Oxid. Med. Cell. Longev. 2, 270-278. doi: 10.4161/oxim.2.5.9498

Pinacho, R., Cavero, R. Y., Astiasarán, I., Ansorena, D., and Calvo, M. I. (2015). Phenolic compounds of blackthorn (Prunus spinosa L.) and influence of in vitro digestion on their antioxidant capacity. J. Funct. Foods 19, 49-62. doi: 10.1016/j.jff.2015.09.015

Piwowarski, J. P., Kiss, A. K., and Kozlowska-Wojciechowska, M. (2011). Anti-hyaluronidase and anti-elastase activity screening of tannin-rich plant materials used in traditional Polish medicine for external treatment of diseases with inflammatory background. J. Ethnopharmacol. 137, 937-941. doi: 10.1016/j.jep.2011.05.039

Poonam, V., Raunak Kumar, G., Reddy, L. C. S., Jain, R., Sharma, S. K., and Parmar, V. S. (2011). Chemical constituents of the genus Prunus and their medicinal properties. Curr. Med. Chem. 18, 3758-3824. doi: 10.2174/092986711803414386

Pryor, W. A., Cueto, R., Jin, X., Koppenol, W. H., Ngu-Schwemlein, M., Squadrito, G. L., et al. (1995). A practical method for preparing peroxynitrite solutions of low ionic strength and free of hydrogen peroxide. Free Radic. Biol. Med. 18, 75-83. doi: 10.1016/0891-5849(94)00105-S

Quiñones, M., Miguel, M., and Aleixandre, A. (2013). Beneficial effects of polyphenols on cardiovascular disease. Pharmacol. Res. 68, 125-131. doi: 10.1016/j.phrs.2012.10.018

Rana, S., Kumar, S., Rana, A., Sharma, V., Katoch, P., Padwad, Y., et al. (2016). Phenolic constituents from apple tree leaves and their in vitro biological activity. Ind. Crops Prod. 90, 118-125. doi: 10.1016/j.indcrop.2016.06.027

Ronson, R. S., , Nakamura, M., and Vinten-Johansen, J. (1999). The cardiovascular effects and implications of peroxynitrite. Cardiovasc. Res. 44, 47-59. doi: 10.1016/S0008-6363(99)00184-4

Ropiak, H. M., Ramsay, A., and Mueller-Harvey, I. (2016). Condensed tannins in extracts from European medicinal plants and herbal products. J. Pharm. Biomed. Anal. 121, 225-231. doi: 10.1016/j.jpba.2015.12.034

Sakar, M. K., and Kolodziej, H. (1993). Flavonoid glycosides from the flowers of Prunus spinosa. Fitoterapia 64, 180-181.

Santilli, F., D’Ardes, D., and Davì, G. (2015). Oxidative stress in chronic vascular disease: from prediction to prevention. Vasc. Pharm. 74, 23-37. doi: 10.1016/j.vph.2015.09.003

Schneider, I., and Bucar, F. (2005). Lipoxygenase inhibitors from natural plant sources. Part 2: medicinal plants with inhibitory activity on arachidonate 12lipoxygenase, 15-lipoxygenase and leukotriene receptor antagonists. Phytother. Res. 19, 263-272. doi: 10.1002/ptr.1604

Szabo, C., Ischiropoulos, H., and Radi, R. (2007). Peroxynitrite: biochemistry, pathophysiology and development of therapeutics. Nat. Rev. Drug Discov. 6, 662-680. doi: $10.1038 / \mathrm{nrd} 2222$

Thomson, L. (2015). 3-Nitrotyrosine modified proteins in atherosclerosis. Dis. Markers 2015:708282. doi: 10.1155/2015/708282

Tutin, T. G., Heywood, V. H., Burges, N. A., Moore, D. H., Walters, S. M., and Webb, D. A. (1968). Flora Europea. Vol. 2. Cambridge: Cambridge University Press.

Wawrzyniak, E. (1992). Leczenie ziołami: Kompendium fitoterapii. Warszawa: Contrast.

Zohary, D., Hopf, M., and Weiss, E. (2012). Domestication of Plants in the Old World. New YorK, NY: Oxford University Press.

Conflict of Interest Statement: The authors declare that the research was conducted in the absence of any commercial or financial relationships that could be construed as a potential conflict of interest.

Copyright (๐ 2017 Marchelak, Owczarek, Matczak, Pawlak, Kolodziejczyk-Czepas, Nowak and Olszewska. This is an open-access article distributed under the terms of the Creative Commons Attribution License (CC BY). The use, distribution or reproduction in other forums is permitted, provided the original author(s) or licensor are credited and that the original publication in this journal is cited, in accordance with accepted academic practice. No use, distribution or reproduction is permitted which does not comply with these terms. 
OPEN ACCESS

Edited by:

Atanas G. Atanasov, Institute of Genetics and Animal Breeding (PAN), Poland

Reviewed by:

Parimal C. Sen,

Bose Institute, India

SubbaRao V. Madhunapantula,

Jagadguru Sri Shivarathreeswara

University, India

*Correspondence:

Chandi C. Mandal

chandicmanda@gmail.com

Specialty section:

This article was submitted to

Ethnopharmacology,

a section of the journal

Frontiers in Pharmacology

Received: 23 May 2017

Accepted: 16 August 2017

Published: 05 September 2017

Citation:

Chowdhury K, Sharma A, Kumar S,

Gunjan GK, Nag A and Mandal CC

(2017) Colocynth Extracts Prevent

Epithelial to Mesenchymal Transition and Stemness of Breast Cancer Cells.

Front. Pharmacol. 8:593.

doi: 10.3389/fphar.2017.00593

\section{Colocynth Extracts Prevent Epithelial to Mesenchymal Transition and Stemness of Breast Cancer Cells}

\author{
Kaushik Chowdhury ${ }^{1}$, Ankit Sharma ${ }^{1}$, Suresh Kumar ${ }^{1}$, Gyanesh K. Gunjan ${ }^{1}$, Alo Nag ${ }^{2}$ \\ and Chandi C. Mandal ${ }^{\text {* }}$ \\ ${ }^{1}$ Department of Biochemistry, School of Life Sciences, Central University of Rajasthan, Ajmer, India, ${ }^{2}$ Department of \\ Biochemistry, University of Delhi, New Delhi, India
}

Modern treatment strategies provide better overall survival in cancer patients, primarily by controlling tumor growth. However, off-target and systemic toxicity, tumor recurrence, and resistance to therapy are still inadvertent hurdles in current treatment regimens. Similarly, metastasis is another deadly threat to patients suffering from cancer. This has created an urgent demand to come up with new drugs having anti-metastatic potential and minimum side effects. Thus, this study was aimed at exploring the anti-proliferative and anti-metastatic potential of colocynth medicinal plant. Results from MTT assay, morphological visualization of cells and scratch assay indicated a role of ethanol and acetone extracts of fruit pulp of the colocynth plant in inhibiting cell viability, enhancing cell cytotoxicity and preventing cell migration in various cancer cell types, including breast cancer cell lines MCF-7 and MDA-MB-231, and cervical cancer cell line SiHa, subsequently having a low cytotoxic effect on mononuclear PBMC and macrophage J774A cells. Our study in metastatic MDA-MB-231 cells showed that both ethanol and acetone pulp extracts decreased transcript levels of the anti-apoptotic genes BCL2 and $B C L X L$, and a reverse effect was observed for the pro-apoptotic genes BAX and caspase 3. Additionally, enhanced caspase 3 activity and downregulated BCL2 protein were seen, indicating a role of these extracts in inducing apoptotic activity. Moreover, MDA-MB-231 cells treated with both these extracts demonstrated up-regulation of the epithelial gene keratin 19 and down-regulation of the mesenchymal genes, vimentin, $\mathrm{N}$-cadherin, Zeb1 and Zeb2 compared to control, suggesting a suppressive impact of these extracts in epithelial to mesenchymal transition (EMT). In addition, these extracts inhibited colony and sphere formation with simultaneous reduction in the transcript level of the stemness associated genes, BMI-1 and CD44. It was also found that both the plant extracts exhibited synergistic potential with the chemotherapeutic drug doxorubicin to inhibit cancer viability. Furthermore, GC-MS/MS analysis revealed the presence of certain novel compounds in both the extracts that are responsible for the anti-cancer role of the extracts. Overall, the results of this report suggest, for the first time, that colocynth fruit pulp extracts may block the proliferative as well as metastatic activity of breast cancer cells.

Keywords: cancer, colocynth, cell death, apoptosis, cell viability, epithelial to mesenchymal transition, metastasis 


\section{INTRODUCTION}

Cancer constitutes a group of deadly diseases that is not only the second leading cause of death worldwide, but also largely contributes to the global health economic burden. The World Health Organization (WHO) has estimated over 14.1 million cancer cases and 8.2 million cancer-induced deaths worldwide in 2012, and this number is expected to rise catastrophically in the coming decades (Torre et al., 2015). Breast and cervical cancers are the most frequently diagnosed cancers in females nationwide, accounting for about 1.7 million new cases and 521,900 breast cancer deaths in 2008 (Torre et al., 2015). India infamously boasts of a surmounting 27 and 23\% breast and cervical cancer incidence respectively ${ }^{1}$. Cervical cancer is the second most diagnosed cancer and third leading cause of deaths in females, accounting for about 527600 new cases and 265700 deaths (Torre et al., 2015).

Cancer is predominantly caused due to abnormalities in the genome (Balmain et al., 2003) and epigenome (Feinberg and Tycko, 2004) due to exposure to various damaging agents. This set of accumulated cells that has escaped the normal regulatory control mechanisms undergoes unchecked proliferation to form tumors. Despite several advancements in recent years, contemporary anticancer therapy suffers from several limitations owing to their associated toxicity and off-target effects. This provokes an urgent need to design novel drugs with high efficacy specific for cancer cells and less toxicity to off-target cells. Phytochemicals have shown promise in this regard as they fit the above criteria, and their usage in anticancer therapy is an emerging trend.

Citrullus colocynth (L.) is a valuable cucurbit plant, widely distributed in the desert areas of the world, including India, known to possess nutritional values and diverse medicinal activities, including antibacterial, antifungal, larvicidal and anti-inflammatory properties (Sawaya et al., 1986; Marzouk et al., 2010; Chawech et al., 2017). Literature documents the presence of many bioactive compounds, such as cucurbitacin, phenolic acids, flavonoids, pyridine and quinolone type alkaloids and fatty acids in fruits of these herbal plants (Hussain et al., 2013, 2014; Jeon and Lee, 2014). This plant is traditionally used to control diabetes (Shi et al., 2014). Recent clinical trial studies have witnessed a fall in fasting blood glucose and $\mathrm{Hb} 1 \mathrm{Ac}$, triglyceride and cholesterol in case of colocynth users (Rahbar and Nabipour, 2010; Barghamdi et al., 2016). Intriguingly, a study by TanninSpitz et al. (2007) documented cancer specific apoptotic activity of the isolate cucurbitacin, extracted from this plant. However, no study has yet been conducted to explore the effect of colocynth extract in cancer metastasis. Thus, this study was primarily aimed at investigating the unexplored anti-metastatic potential of this plant extract.

This study testified that ethanol and acetone fruit pulp extracts exhibited impressive inhibition of cell viability and cell migration of various cancer cell types, including breast and cervical cancer cells with considerably less effect on mononuclear cells and macrophage cells. Moreover, these pulp

${ }^{1}$ breastcancerindia.net extracts noticeably hindered colony and sphere formation and epithelial to mesenchymal transition (EMT) of metastatic breast cancer MDA-MB-231 cells. Our GC-MS analysis also reveals some unique compounds, which may account for the anticancer activity of the extracts. The current study is the first report advocating that fruit pulp extracts containing the novel compounds may have anti-metastatic potential along with apoptotic activity.

\section{MATERIALS AND METHODS}

\section{Materials}

Verso cDNA synthesis kit (AB1453A, Thermo Scientific), TRIzol Reagent (T9424, Sigma Aldrich), Taq Polymerase (MBT060A, Himedia), ready Mix dNTP (MBT078, Himedia), caspase-3 antibody (\#9661, Cell signaling), BCL-2 antibody (SC-7382, Santa Cruz Technology), actin antibody (A02066, Sigma Aldrich), WesternSure-Premium Chemiluminescent substrate (WesternSure-Li-COR-Part No: 926-95000).

\section{Cell Lines}

The human breast cancer MDA-MB-231 (metastatic) and MCF-7 (non-metastatic) cell lines, and cervical cancer SiHa cell line were procured from NCCS cell repository, Pune, India. J774A cell (Macrophage cell line) was obtained from Dr. Vijay Kumar Prajapati, Department of Biochemistry, Central University of Rajasthan, India. All cells were cultured in Dulbecco's Modified Eagles Medium (DMEM), supplemented with 10\% fetal bovine serum (FBS) (RM1112, Himedia) and maintained at $37^{\circ} \mathrm{C}$ in a humidified incubator with $5 \% \mathrm{CO}_{2}$.

\section{Isolation of Human Mononuclear Cells (PBMC)}

Mononuclear cells were isolated from human peripheral blood by using a simple and rapid density gradient centrifugation technique using Ficoll-Paque (Sigma-F5414-50ML) methodology established by Boyum (1968) and Boyum (1976). The isolation was done according to the manufacturer's protocol. Cells $\left(0.5 \times 10^{5}\right.$ cells $)$ were seeded in 96 well culture plate in DMEM supplemented with $10 \%$ FBS and incubated for overnight at $5 \% \mathrm{CO}_{2}$, and treated with increasing concentration (mentioned in other cell lines ) by ethanolic and acetone plant extracts respectively for $24 \mathrm{~h}$. Blood samples from two healthy volunteers were taken and mixed before isolation of PBMC. Written consent was obtained from the participants, and they were informed about the use of blood in this study. Moreover, the work related to blood samples had been conducted by following the regulation of Institutional Ethical Committee at Central University of Rajasthan and, this study was approved by Institutional Ethical Committee.

\section{Plant Extracts}

The plant Citrullus colocynth was obtained from a rural area of India [Jaisalmer $\left(26.9157^{\circ} \mathrm{N}, 70.9083^{\circ} \mathrm{E}\right)$, Rajasthan, India]. The taxonomic name of this plant had been confirmed by Dr. Amit 
Kotia, Department of Botany, University of Rajasthan, India. The pulp was isolated from fruits, dried and crushed, using a mixer grinder. One gram of dried pulp powder was suspended in $10 \mathrm{ml}$ of ethanol and acetone, followed by solvent extraction at $50^{\circ} \mathrm{C}$ for $72 \mathrm{~h}$. The solvent fractions were evaporated using a rotaryevaporator at $42^{\circ} \mathrm{C}$ to get the crude products. The crude extracts obtained were then dissolved in ethanol and acetone and kept at $-20^{\circ} \mathrm{C}$ for further use.

\section{MTT Cell Viability Assay}

Cell viability was measured by MTT assay (MB186, Himedia) described earlier (Ghosh-Choudhury et al., 2010; Mehta et al., 2015). In brief, cancer cells (5000 cells/well) were seeded in 96 well tissue culture plate in DMEM supplemented with 10\% FBS at an atmosphere of $5 \% \mathrm{CO}_{2}$ at $37^{\circ} \mathrm{C}$. After $24 \mathrm{~h}$ of seeding, cells were separately incubated with different concentrations [25, 100, and $250 \mu \mathrm{g} / \mathrm{ml}$; equivalent to 1,4 , and $10 \mu \mathrm{l}$ of solvents (ethanol/acetone)] of fruit pulp ethanol extract (PEE) and pulp acetone extract (PAE) for $24 \mathrm{~h}$. Control cells were similarly incubated with equal volume of the solvents as in the experimental wells. At the end of the incubation period, $10 \mu \mathrm{l}$ of MTT solution $(5 \mathrm{mg} / \mathrm{ml})$ was added to each well and the plate was incubated at $37^{\circ} \mathrm{C}$ for $1 \mathrm{~h}$. The formazan crystals formed were solubilized by adding DMSO, and subsequently, absorbance was measured at a wavelength of $530 \mathrm{~nm}$ (Studzinski, 1995; Moongkarndi et al., 2004; Ghosh-Choudhury et al., 2010; Mehta et al., 2015). The effective absorbance was calculated for the experimental wells with respect to corresponding control wells. $\mathrm{LC}_{50}$ (Lethal concentration at which $50 \%$ cells are killed) at $24 \mathrm{~h}$ duration was calculated using MTT data set for different cancer cell lines (Zhang et al., 2007).

\section{Scratch Assay}

Cell migration was determined by scratch assay as described earlier (Mandal et al., 2010b, 2011b). In brief, cells $\left(2 \times 10^{5}\right.$ cells/plate) were seeded in $35 \mathrm{~mm}$ tissue culture plates and allowed to grow till they reach $95 \%$ confluency. A cell scratchwound was generated using a micropipette tip, and washed once with PBS buffer to remove the floating cells. Cells were then separately treated with PEE and PAE $(25 \mu \mathrm{g} / \mathrm{ml})$ for $24 \mathrm{~h}$ in experimental plates, and equal volume of respective solvents was added in control plates. Cells were visualized under an inverted microscope (Carl-Zeiss), and cell migration was assessed by measuring gap sizes in multiple fields and the relative area calculated using ImageJ, and statistically analyzed (Hirsch et al., 2009).

\section{RT-PCR Analysis}

Transcript levels of several genes were measured by semi-quantitative RT-PCR analysis as described previously (Mandal et al., 2010a; Chowdhury et al., 2017). In brief, breast cancer cells $\left(2 \times 10^{5}\right.$ cells/plate $)$ were plated in $35 \mathrm{~mm}$ tissue culture plates in DMEM supplemented with 10\% FBS at an atmosphere of $5 \% \mathrm{CO}_{2}$ at $37^{\circ} \mathrm{C}$. After $90-100 \%$ cell confluency is reached, the cells were treated with different test factors (such as PEE and PAE $(50 \mu \mathrm{g} / \mathrm{ml})$ ) for $24 \mathrm{~h}$. Total RNA was extracted using TRIzol reagent as described earlier (Mandal et al., 2010a, 2011a, 2016a; Ghosh-Choudhury et al., 2013). First stand cDNA synthesis was performed using $1 \mu \mathrm{g}$ of total RNAs using the Verso cDNA synthesis kit, according to manufacturer's instruction. Semi-quantitative PCR was performed using this cDNA as template and gene specific primers in a ProFlex PCR system (Applied Biosystem by Life Technologies). The list of primers used in this study is given in Supplementary Table S1. Densitometric analysis of the PCR bands was done using ScnImage (Chowdhury et al., 2017).

\section{Western Blot Analysis}

Methodology of western blotting has been described previously (Chowdhury et al., 2017). In brief, cells were seeded at a density of $2 \times 10^{5}$ cells/plate in $35 \mathrm{~mm}$ tissue culture plates. Cells were treated with different test factors and harvested after $24 \mathrm{~h}$ of treatment. Protein extraction was carried out in RIPA buffer and subsequently, cell lysates were centrifuged at $12,000 \times g$ at $4^{\circ} \mathrm{C}$ for $20 \mathrm{~min}$. The protein concentration was measured by Bradford reagent (BIORAD), according to manufacturer's instruction. Equal amounts of whole cell lysates were resolved by $12 \%$ SDS-PAGE, and transferred to PVDF membrane. This PVDF membrane was incubated with primary antibody (Anti-caspase-3 antibody (\#9661, Cell signaling), Anti-BCL-2 antibody (SC-7382, Santa Cruz Technology), Anti-actin antibody (A02066, Sigma Aldrich) used at 1:1000 dilution) overnight at $4^{\circ} \mathrm{C}$. After washing, the PVDF membrane was further incubated with HRP-conjugated secondary antibody (used at 1:10000 dilution) for $1 \mathrm{~h}$ at room temperature. The washed membrane was further incubated with chemiluminescent substrate and subsequently, the blot was scanned by C-DIGIT Blot Scanner (Li-COR-Model: $3600)$.

\section{Colony Formation Assay}

Cells were seeded at a density of 500 cells/well in a 12 well tissue culture plate in DMEM with $10 \%$ FBS at $37^{\circ} \mathrm{C}$ incubator with $5 \% \mathrm{CO}_{2}$ for $24 \mathrm{~h}$. Cells were then treated with PEE and PAE extracts $(25 \mu \mathrm{g} / \mathrm{ml})$, and incubated under same conditions for 5 days. Next, colonies were fixed with methanol and were stained with crystal violet solution (Hirsch et al., 2009). Photographs of stained plate were taken by a camera. Photographs of colonies were also taken by a bright field inverted microscope (Carl-Zeiss).

\section{Soft Agar Sphere Formation Assay}

For performing soft agar assay (Sebolt-Leopold et al., 1999), MDA-MB-231 cells (10,000 cells/plate) were mixed with $2 \mathrm{X}$ DMEM containing $0.6 \%(\mathrm{w} / \mathrm{v})$ agar and layered with $2 \mathrm{X}$ DMEM containing $1.2 \%$ base agar medium in $35 \mathrm{~mm}$ culture plates. $25 \mu \mathrm{g} / \mathrm{ml}$ of PEE and PAE was added in $300 \mu \mathrm{l}$ of DMEM to the top agar and cells were allowed to grow for 8 days. The top $300 \mu \mathrm{l}$ medium was replaced with fresh medium with or without test factors at intervals of every 3 days. The morphology and size of the spheres were visualized using bright field inverted microscope (Carl-Zeiss) and photographed. Subsequently, spheres were manually counted under the microscope. 

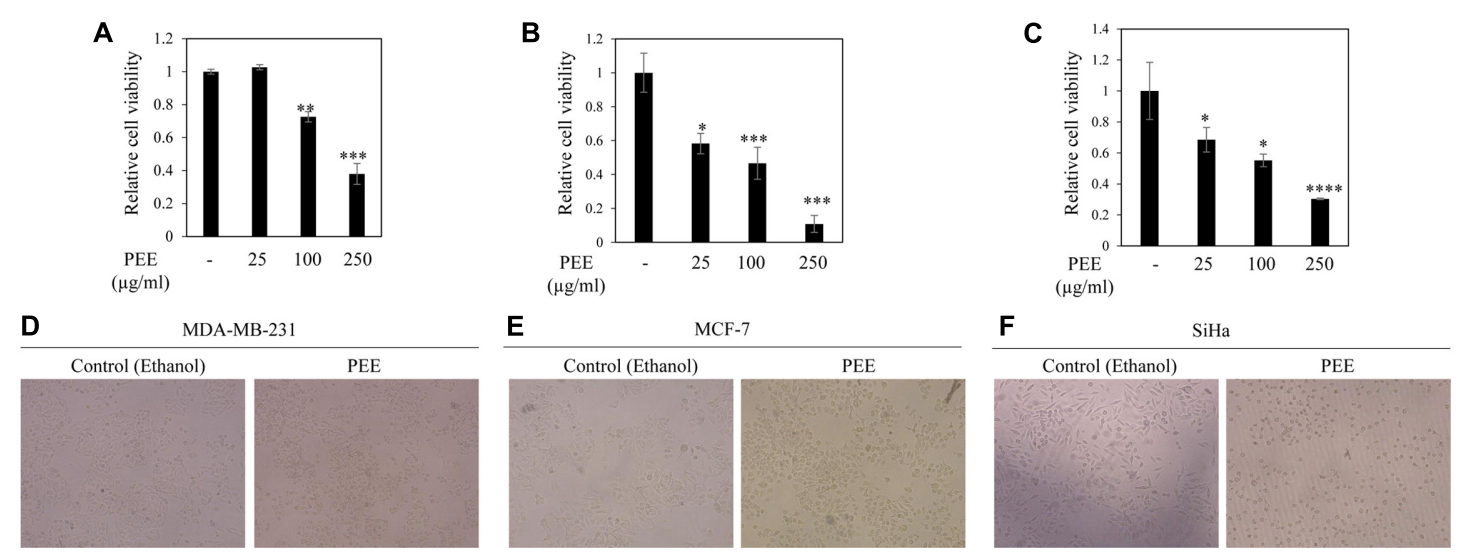

FIGURE 1 | Effect of fruit pulp ethanol extract of colocynth on cell viability and cytotoxicity of breast and cervical cancer cell lines. Cell viability was measured by MTT assay after $24 \mathrm{~h}$ of treatment of breast cancer MDA-MB-231 (A) and MCF-7 (B), and cervical SiHa (C) cell lines with pulp ethanol extract (PEE) with different concentrations $(25-250 \mu \mathrm{g} / \mathrm{ml})$. Values represent mean \pm SE of triplicate measurements, ${ }^{*} p<0.05,{ }^{* *} p<0.01$, and $* * * p<0.001$ vs. control. (D-F) Morphologic visualization (cytotoxicity) was recorded after $24 \mathrm{hr}$ of treatment of breast cancer MDA-MB-231 (D) and MCF-7 (E), and cervical SiHa (F) cell lines with PEE (100 $\mu \mathrm{g} / \mathrm{ml})$. Photographs were taken at 10X magnification.

\section{GC-MS/MS Analysis}

Solvent Extraction of Citrullus colocynthis fruit pulp was done by ethanol and acetone. Dried extracts were dissolved in methanol followed by GC-MS/MS analysis using Thermo GC 1300 and “TSQ 8000" Triple quadrupole GC-MS/MS SYSTEM with auto sampler Al 1310. The Gas Chromatography 1300 was used with a fused GC column TG-5MS AMINE. The column length was of $30 \mathrm{~m}$ with an internal diameter coated film of $0.25 \mu \mathrm{m}$, with the flow rate of $10 \mathrm{ml} / \mathrm{min}$ and the condition kept were as follows: PTV Temp. Program: $70^{\circ} \mathrm{C}$, hold $2.00 \mathrm{~min}, 10^{\circ} \mathrm{C} / \mathrm{min}$ to $270^{\circ} \mathrm{C}$, hold $8 \mathrm{~min}$. Carrier gas helium flow rate was $1 \mathrm{ml} / \mathrm{min}$ and split ratio of 1:50. GC was equipped with an auto sampler AI 1300 and sample volume loaded was $1 \mu$ l. Eluates were automatically passed into the mass spectrometer. Mass Spectrum analysis was conducted using TSQ-8000 with a transfer line temperature of $280^{\circ} \mathrm{C}$ and ion source temperature of $230^{\circ} \mathrm{C}$ in EI mode. Mass scan time was $4 \mathrm{~min}$ with feature of full Scan MS. The mass spectrum was also equipped with a computer fed NIST mass Spectra data library.

\section{Component Identification}

Chemical constituents of the extracts were identified by matching the peaks with Computer NIST MS libraries and confirmed by comparing mass spectra of the peaks and those from literature. List of 50 compounds isolated from the extracts are listed in Supplementary Tables S2, S3. Scifinder was used to analyze the compounds and thus interpret the literature available for them.

\section{Statistical Analysis}

Statistical analysis was done by GraphPad Prism (GhoshChoudhury et al., 2010; Mandal et al., 2011a,b, 2016b). A p-value of $<0.05$ was considered to be statistically significant. All values were represented as mean \pm standard error mean of three measurements.

\section{RESULTS}

\section{Effect of Colocynth Fruit Pulp Extracts on Viability of Breast and Cervical Cancer Cells}

To test the anticancer potential of colocynth fruit pulp extracts (prepared separately by solvent ethanol and acetone), cell viability was measured by MTT assay. For this, different cancer cells, including breast cancer cell lines, MCF-7 and MDA-MB-231, and cervical cancer cell line, $\mathrm{SiHa}$, were separately treated with increasing concentrations (range 25-250 $\mu \mathrm{g} / \mathrm{ml}$ ) of PEE and PAE for $24 \mathrm{~h}$ (Figures 1A-C, 2A-C). MTT assay result showed noteworthy reduction in the number of viable cells in a dose dependent manner for both PEE and PAE treatment when compared to their respective solvent controls. To determine the effectiveness of the pulp extracts in these cancer cells, $\mathrm{LC}_{50}$ values (using MTT data) were also calculated. In case of ethanol extract, $\mathrm{LC}_{50}(\mu \mathrm{g} / \mathrm{ml})$ values were $142 \pm 16,105 \pm 8.3$ and $157 \pm 4.3$ for MDA-MB-231, MCF-7 and SiHa cell lines respectively (Table 1). For acetone extract, these values were $140 \pm 0.8,94 \pm 6.4$ and $121 \pm 4.6$ respectively. These findings indicated relatively higher efficacy of PAE in cancer cell lines compared to PEE. Moreover, MCF-7 cells showed higher sensitivity to both PAE and PEE as compared to MDA-MB-231 cells. LC $_{50}$ values of both the extracts in PBMC and J774A cell line were $>300 \mu \mathrm{g} / \mathrm{ml}$ (Table 1). Furthermore, microscopic visualization indicated a cytotoxic effect of the extracts upon these cancer cell lines (Figures 1D-F, 2D-F). All these findings suggested that the fruit pulp extracts induced reduced viability of cells that is presumably caused by inhibiting cell proliferation and inducing cytotoxicity.

To examine the influence of these pulp extracts on cell apoptosis, RT-PCR analysis was performed to compare the transcript levels of anti-apoptotic BCL2 and BCLXL and apoptotic BAX genes, using total RNA of MDA-MB-231 


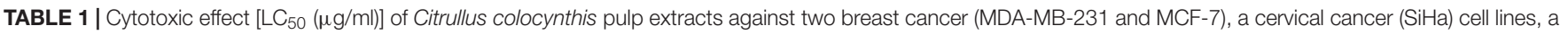
human mononuclear cell PBMC and a macrophage cell J774A.

$\mathrm{LC50}(\mu \mathrm{g} / \mathrm{ml}) \pm \mathrm{SEM}$

\begin{tabular}{|c|c|c|c|c|c|c|}
\hline & \multirow[b]{3}{*}{ Solvents (extraction) } & \multirow{2}{*}{\multicolumn{3}{|c|}{ Cancer cell lines }} & & \\
\hline & & & & & \multicolumn{2}{|c|}{ Normal cell lines } \\
\hline & & MDA-MB-231 & MCF-7 & $\mathrm{SiHa}$ & PBMC & J774A \\
\hline \multirow[t]{2}{*}{ Colocynth fruit pulp } & Ethanol & $142 \pm 16$ & $105 \pm 8.3$ & $157 \pm 4.3$ & $>300$ & $>300$ \\
\hline & Acetone & $140 \pm 0.8$ & $94 \pm 6.4$ & $121 \pm 4.6$ & $>300$ & $>300$ \\
\hline
\end{tabular}

Values represent mean \pm SEM of three independent experiments.

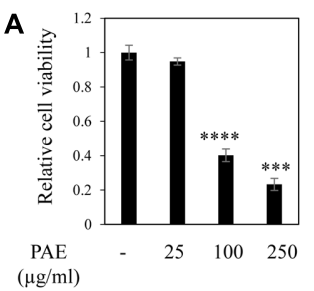

D

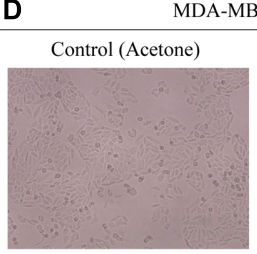

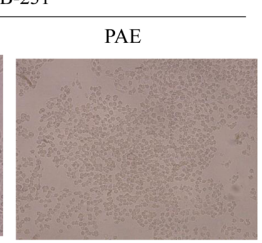

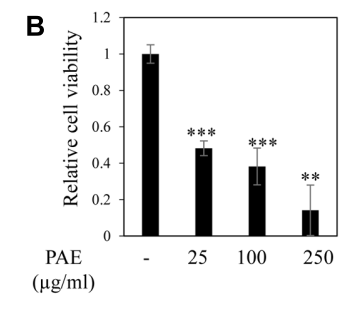

E

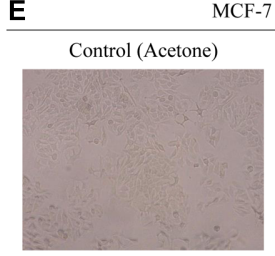

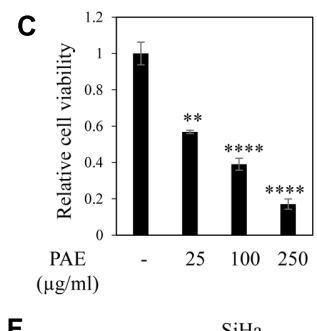

F

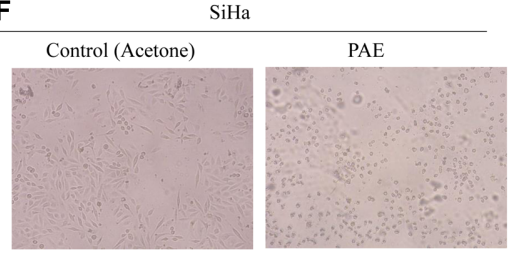

FIGURE 2 | Effect of fruit pulp acetone extract of colocynth on cell viability and cytotoxicity of breast and cervical cancer cell lines. MTT assay was performed to measure cell viability by after $24 \mathrm{~h}$ of treatment of breast cancer MDA-MB-231 (A) and MCF-7 (B), and cervical SiHa (C) cell lines with pulp acetone extract (PAE) with different concentrations $(25-250 \mu \mathrm{g} / \mathrm{ml})$. Values represent mean \pm SE of triplicate measurements, ${ }^{* *} p<0.01,{ }^{* * *} p<0.001$, and ${ }^{* * * *} p<0.0001$, vs. control. (D-F) Morphologic visualization (cytotoxicity) was recorded after $24 \mathrm{~h}$ of treatment of breast cancer MDA-MB-231 (D) and MCF-7 (E), and cervical SiHa (F) cell lines with PAE (100 $\mu \mathrm{g} / \mathrm{ml})$. Photographs were taken at 10X magnification.

cells with or without treatment with ethanol and acetone extracts $(50 \mu \mathrm{g} / \mathrm{ml})$. Both PEE and PAE considerably impeded BCL2 and BCLXL expression with simultaneous upsurge in BAX expression (Figures 3A-D and Supplementary Figures S1A-C,E-G). Moreover, expression of apoptotic caspase 3 gene was augmented upon treatment with both PAE and PEE (Figures 3E,F and Supplementary Figures S1D,H). Furthermore, western blot results demonstrated increased level of cleaved caspase 3 (Figures $\mathbf{3 G}, \mathbf{H}$ ) with a concomitant decrease in the protein level of anti-apoptotic gene BCL2 (Figures 3I,J) for both ethanol and acetone extract treated cells compared to control. These observations led us to propose an apoptotic activity of fruit pulp extracts in breast cancer cells.

\section{Inhibitory Effect of Colocynth Pulp Extracts on Cancer Cell Migration}

A common problem faced by a large percentage of cancer patients is development of metastasis if cancers are not detected in early and/or not treated with a suitable therapy. Migration of cancer cells is a critical step in the metastatic cascade (Chaffer and Weinberg, 2011). Thus, we investigated the effect of pulp ethanol and acetone extracts on the migratory property of cancer cells by performing scratch assay. Scratches were made on cell monolayer by a small pipet tip, and subsequently, cells were incubated with or without pulp extracts. Microscopic photos indicated migration of cells to close the induced scratch after $24 \mathrm{~h}$ in control MDA-MB-231, MCF-7 and SiHa cells (Figures 4A-C, 5A-C). Cells treated with both ethanol and acetone extracts $(25 \mu \mathrm{g} / \mathrm{ml})$ showed substantial prevention of cell migration as evident from the unfilled gap areas between the two ends of the scratches that were larger in cases of pulp extracts as compared to control. The data displayed in Figures 4D-F, 5D-F denoted a significant inhibition of cell migration in response to treatment with both ethanol and acetone extracts.

\section{Effect of Colocynth Pulp Extracts on EMT}

Transition from epithelial to mesenchymal phenotype is a critical step for metastasis (Chaffer and Weinberg, 2011). To examine the effect of fruit pulp extracts on prevention of EMT of metastatic MDA-MB-231 cells, we analyzed mRNA levels of several EMT markers by RT-PCR. Interestingly, we observed a decline in expression of mesenchymal markers, Vimentin and $N$-cadherin, with a concurrent increase in the expression of epithelial marker 


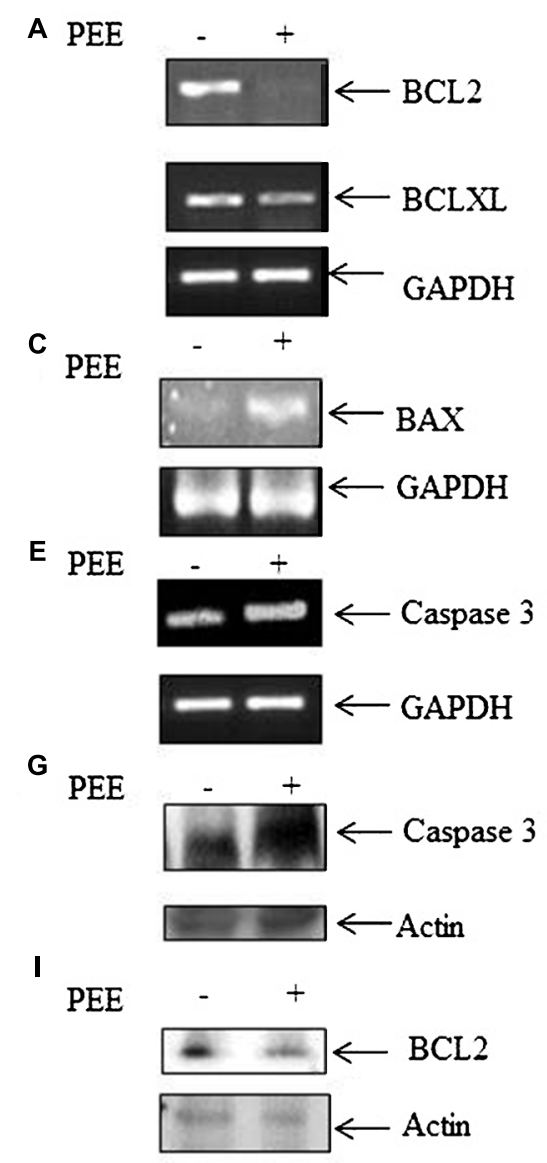

B PAE
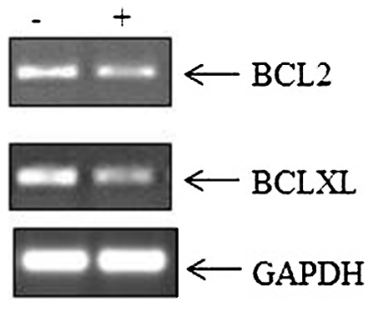

D PAE

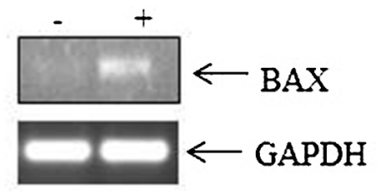

F PAE

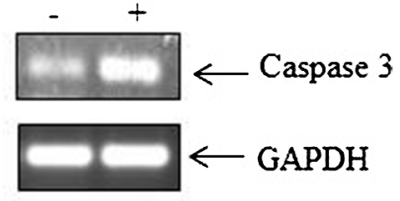

H PAE

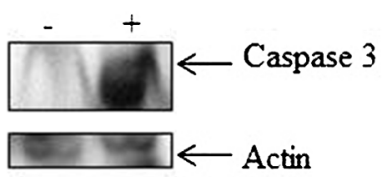

J PAE

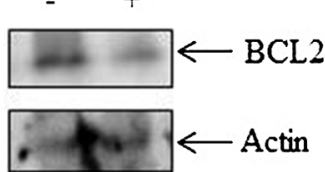

FIGURE 3 | Effect of fruit pulp extracts on anti-apoptotic and apoptotic genes in MDA-MB-231 cells. (A) Cells were separately treated with pulp ethanol extract (PEE) (A,C,E,G) and pulp acetone extract (PAE) (B,D,F,H) for $24 \mathrm{~h}$, and subsequently expression of anti-apoptotic genes (A,B) BCL2 and BCLXL, and pro-apoptotic genes BAX (C,D) and Caspase $3 \mathbf{( E , F ) ~ w e r e ~ m e a s u r e d ~ b y ~ R T - P C R ~ a n a l y s i s , ~ u s i n g ~ t o t a l ~ R N A ~ i s o l a t e d ~ f r o m ~ c e l l s ~ w i t h ~ o r ~ w i t h o u t ~ P E E ~ a n d ~ P A E ~ t r e a t m e n t , ~ a n d ~ g e n e ~}$ specific primers. Here, GAPDH was used as an internal loading control. Densitometric analysis data are given in Supplementary Figure S1. (G,H) Level of cleaved caspase 3 and $(\mathbf{I}, \mathbf{J})$ BCL-2 was measured by western blotting using equal amount of cellular protein isolated from cells with or without treatment with PEE or PAE. B-actin serves as an internal loading control.

keratin-19 in treated samples (Figures 6A,B and Supplementary Figures S2A-C,F-H). Moreover, both PEE and PAE treatment strongly inhibited expression of the mesenchymal phenotype inducing transcription factors, Zeb1 and Zeb2 (Figures 6A,B and Supplementary Figures S2D,E,I,J). These observations clearly indicate a restraining impact of these pulp extracts on EMT of breast cancer cells.

\section{Colocynth Pulp Extracts Inhibited Stemness Property of Breast Cancer Cells}

Accumulating evidences suggest that cancers develop from cancer initiating cells or cancer stem cells (CSCs) (Reya et al., 2001; Singh and Settleman, 2010). Although CSCs comprise of a small percentage of the tumor population, their resistance to contemporary chemo- and radiation- therapy is a leading cause for tumor recurrence and metastasis ( $\mathrm{Li}$ et al., 2007; Singh and Settleman, 2010). Thus, we investigated the effect of colocynth pulp extracts on the stemness property of metastatic MDA-MB-231 cells by colony formation assay. In agreement with previous observations, the number of colony formed was markedly reduced when cells were treated with pulp extracts (25 $\mu \mathrm{g} / \mathrm{ml}$ ) as compared to control, indicating an inhibitory role of both PEE and PAE on cancer stemness (Figures 7A-D). To further support these results, we carried out soft agar sphere formation assay. Microscopic visualization documented formation of a great number of spheres with large size after 8 days of cell culture in the control plates. The number as well as the size of such spheres was, however, greatly diminished when cells were incubated with both PAE and PEE (25 $\mu \mathrm{g} / \mathrm{ml})$ as compared to control (Figures 8A-D). These results show that fruit pulp extracts might attenuate stemness property of cancer cells. To verify these findings, we further examined the effect of these pulp extracts on the expression of stemness associated genes CD44 and BMI-1. RT-PCR analysis documented that transcript levels of both CD44 and BMI1 genes (Al-Hajj et al., 2003; Liu et al., 2006) were significantly 


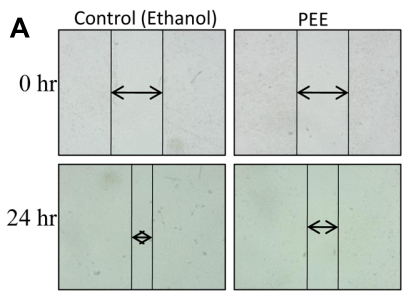

D

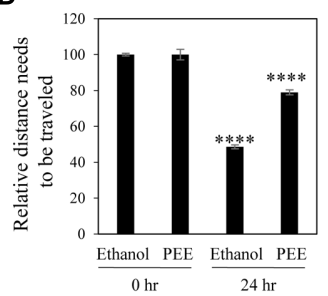

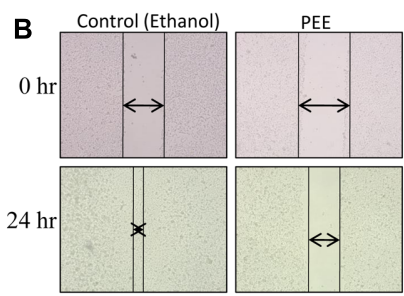

E

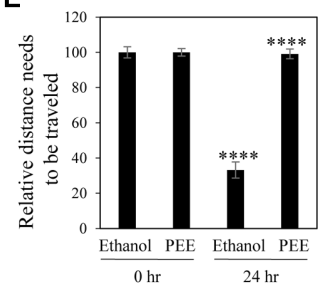

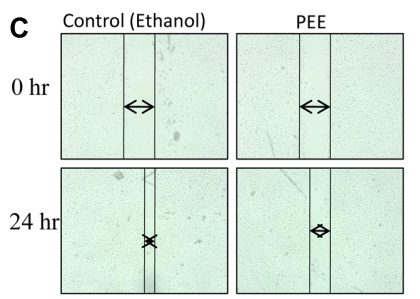

F

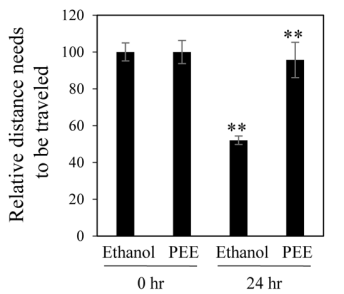

FIGURE 4 | Pulp ethanol extract inhibited migration of breast and cervical cancer cells. Breast cancer MDA-MB-231 (A), MCF-7 (B) and cervical cancer (SiHa) (C) cells were treated with pulp ethanol extract (PEE) for $24 \mathrm{~h}$. Photos of cells at the starting time point and after $24 \mathrm{~h}$ of treatment were captured by inverted bright field microscope. The unfilled gap areas between two ends of the scratch were measured and subsequently plotted. Significant inhibition of cell migration was observed in case of PEE treated plates wells as compared to control in MDA-MB-231 (D), MCF-7 (E) and SiHa (F) cancer cell lines. Percent inhibition by PEE after 24 h was calculated with the $0 \mathrm{~h}$ being the control. Values represent mean $\pm \mathrm{SE}$ of triplicate measurements. In (D) ****p<0.0001; control (ethanol) $24 \mathrm{~h}$ vs. control (ethanol) 0 h and ${ }^{* * * *} p<0.0001$; PEE 24 h vs. control 24 h. In (E) $* * * * p<0.0001$; control (ethanol) 24 h vs. control (ethanol) 0 h and $* * * * p<0.0001$; PEE 24 h vs. control (ethanol) 24 h. In (F) ${ }^{* *} p<0.01$; control (ethanol) 24 h vs. control (ethanol) 0 h and ${ }^{* *} p<0.01$; PEE 24 h vs. control (ethanol) 24 h.
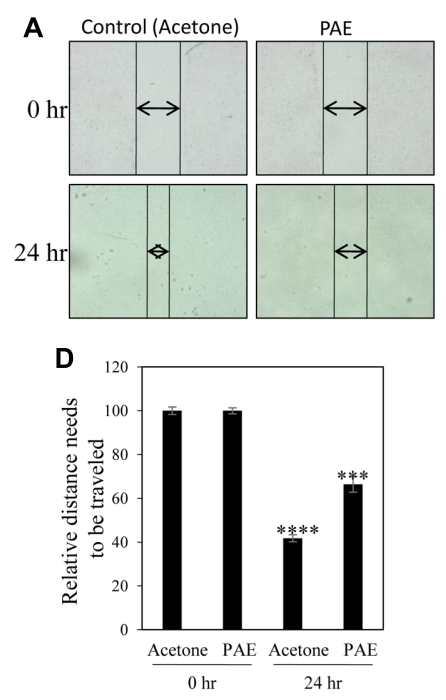

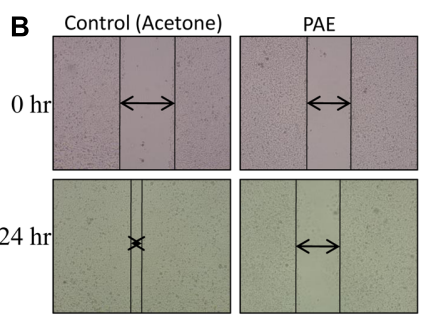

E

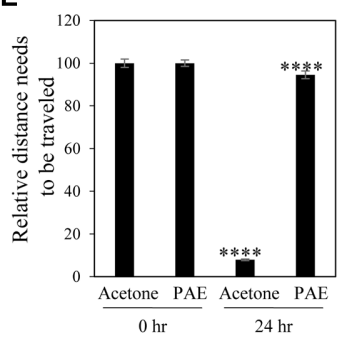

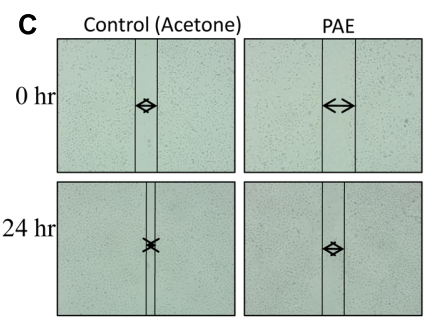

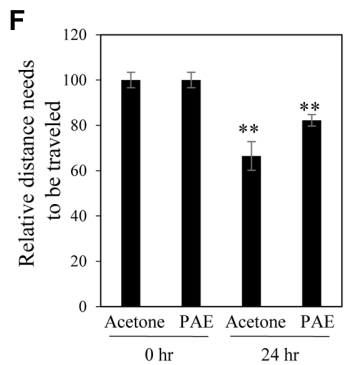

FIGURE 5 | Pulp acetone extract inhibited migration of breast and cervical cancer cells. Breast cancer MDA-MB-231 (A), MCF-7 (B) and cervical cancer (SiHa) (C) cells were treated with pulp acetone extract (PAE) for $24 \mathrm{~h}$. Photos of cells at the starting timepoint and after $24 \mathrm{~h}$ of treatment were captured by inverted bright field microscope. The unfilled gap areas between two ends of the scratch were measured and subsequently plotted. Significant inhibition of cell migration was observed in case of PAE treated plates wells as compared to control in MDA-MB-231 (D), MCF-7 (E) and SiHa (F) cancer cell lines. Percent inhibition by PAE after 24 h was calculated with the $0 \mathrm{~h}$ being the control. Values represent mean \pm SE of triplicate measurements. In (D) ${ }^{* * * *} p<0.0001$; control (acetone) $24 \mathrm{~h}$ vs. control (acetone) $0 \mathrm{~h}$ and ${ }^{* * *} p<0.001$; PAE 24 h vs. control (acetone) $24 \mathrm{~h}$. In (E) **** $p<0.0001$; control (acetone) 24 h vs. control (acetone) 0 h and **** $p$ <.0001; PAE 24 h vs. control (acetone) $24 \mathrm{~h}$. In (F) ** $p<0.01$; control (acetone) $24 \mathrm{~h}$ vs. control (acetone) $0 \mathrm{~h}$. and ** $p<0.01$; PAE 24 h vs. control (acetone) 24 h.

lowered in MDA-MB-231 cells when treated with both PAE and PEE as compared to control (Figures $\mathbf{8 E}, \mathbf{F}$ and Supplementary Figures S3A-D). Together, these data clearly suggested that fruit pulp extracts (PEE and PAE) obliterate the stemness property of breast cancer cells.

\section{DISCUSSION}

Considering the current status of diagnostic and treatment options available, cancer associated deaths are on the rise. In India, carcinoma of the breast and cervix contributes to 
A
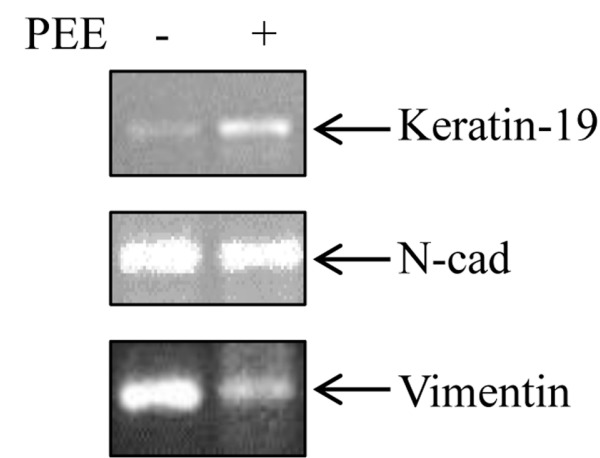

$\leftarrow$ Vimentin
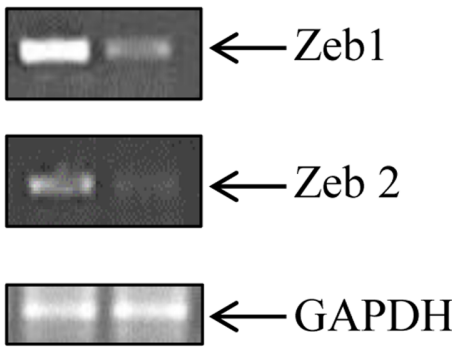

B

\section{PAE}
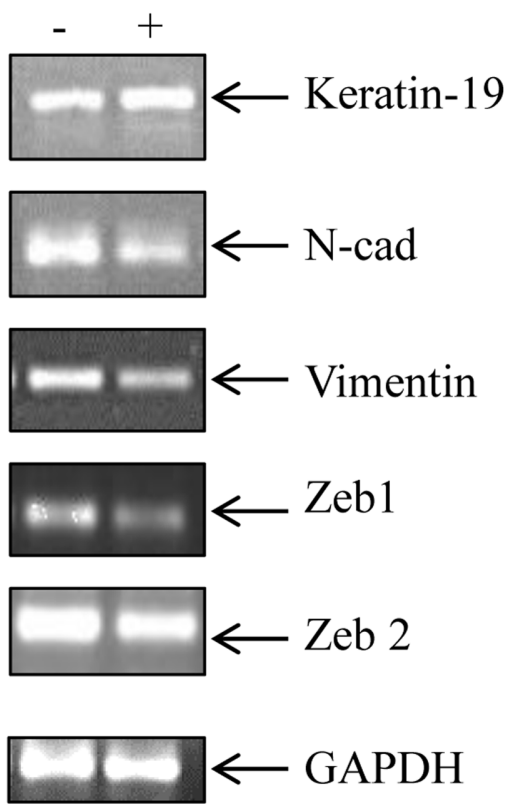

FIGURE 6 | Effect of colocynth pulp extracts on expression of EMT associated genes in breast cancer cells. Breast cancer MDA-MB-231 cells were either treated with pulp ethanol extract (PEE) (A) or pulp acetone extract (PAE) (B) for $24 \mathrm{~h}$, and subsequently the expression of EMT associated genes was measured by RT-PCR analysis using total RNA isolated from cells. GAPDH was used an internal loading control. Densitometric data is given in Supplementary Figure S2.

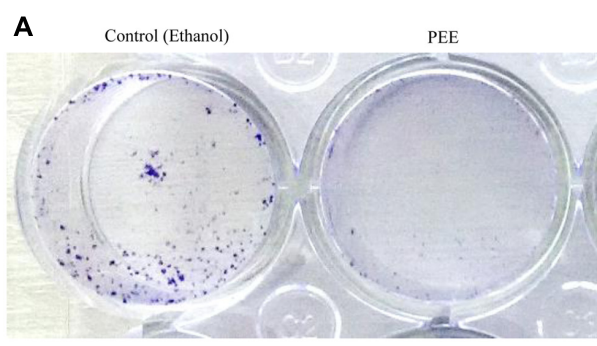

C

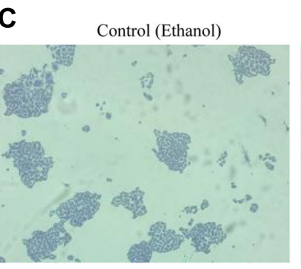

PEE

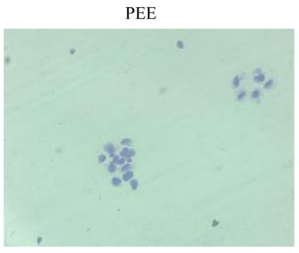

B

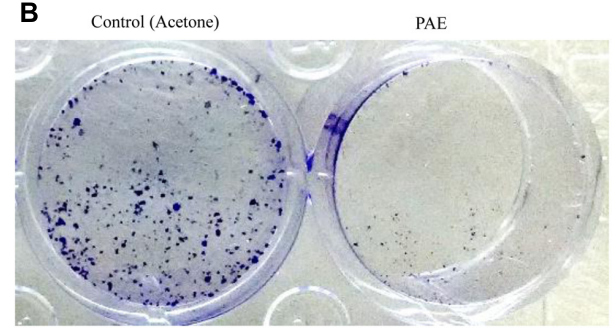

D Control (Acetone)

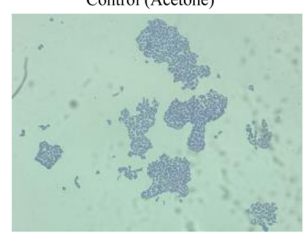

PAE

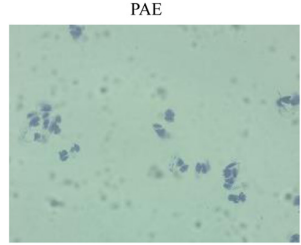

FIGURE 7 | Effect of colocynth pulp extracts on colony formation of breast cancer cell. MDA-MB-231 cells were treated with pulp ethanol extract (PEE) (A,C) and pulp acetone extract (B,D). After 5 days of cell seeding, photos of the wells were taken by a camera $(\mathbf{A}, \mathbf{B})$, and colonies were also visualized by inverted bright field microscope (C,D).

$50 \%$ of total cancer incidences ${ }^{2}$. However, surgical removal, radiation-, chemo-, endocrine-, and other targeted- therapies are continuously being improvised to improve the overall survival and quality of life of cancer patients. Although modern treatment modalities are capable of taking care of the primary tumors, tumor recurrence and metastasis are the major limitations in the limelight at present. Furthermore,

\footnotetext{
${ }^{2}$ breastcancerindia.net
}

chemotherapeutic drugs often show significant off-target effects along with systemic toxicity, such as cardiovascular, liver and nephrotoxicity (King and Perry, 2001; Schimmel et al., 2004). Thus, finding novel anticancer drugs, which prevent both tumor growth and metastasis while circumventing the known side effects, is an active area of research. In this area, application of natural products like secondary metabolites with effective tumor suppressive ability has shown promise in recent years as they are found to be effective even at sufficiently lower 


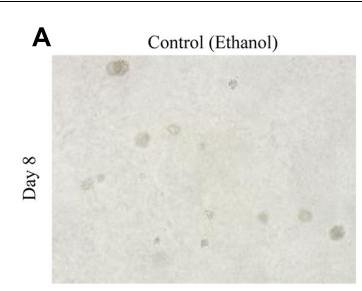

C

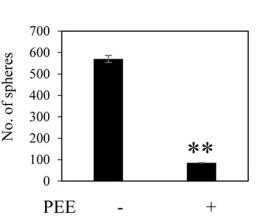

PEE

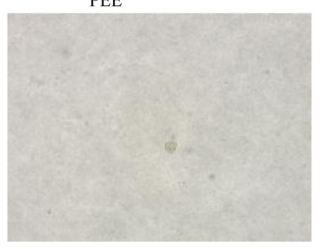

D

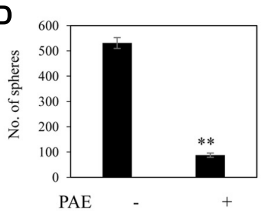

B

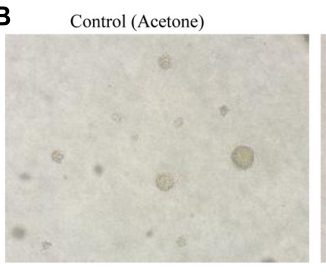

E

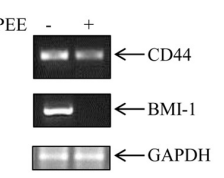

F

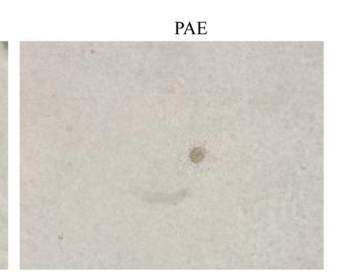

PAE

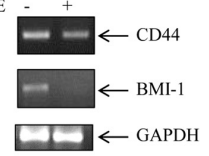

FIGURE 8 | Inhibitory activity of colocynth pulp extract on spheroid formation and stemness of breast cancer cell. (A) Soft agar assay was performed on MDA-MB-231 cells by treating cells with pulp ethanol extract (PEE; A,C) and pulp acetone extract (PAE; B,D) and allowing cells to grow for 8 days. Spheroid colonies were visualized by inverted bright field microscope (A,B). Number of spheroids of all plates was counted and analyzed with respect to control plates (C,D), in (C) $* * p<0.01$; PEE vs. control (ethanol) after 8 days, and in (D) ** ${ }^{*} p$ 0.01; PAE vs. control (acetone) after 8 days. (E,F) MDA-MB-231 cells were treated with PEE (E) and PAE (F) for $24 \mathrm{~h}$ and subsequently, expression of CD44 and BMl-1 was compared by RT-PCR analysis using total RNA isolated from cells and CD44 and BMl-1 gene specific primers. GAPDH was used as an internal loading control. Densitometric data is given in Supplementary Figure S3.

doses and due to their safety profile (Koehn and Carter, 2005).

Thus, this study was aimed at exploring the anti-cancer properties of extracts from Citrullus colocynth plant of the cucurbitaceae family that has been used as a traditional medicine to control diabetes (Zaini et al., 2011; Shi et al., 2014). Reports about the nutritional and medicinal values of this plant and presence of many bioactive molecules had initially attracted our attention (Sawaya et al., 1983, 1986; Asyaz et al., 2010; Marzouk et al., 2010; Hussain et al., 2013, 2014; Jeon and Lee, 2014; Chawech et al., 2017). Moreover, Tannin-Spitz et al. (2007) found that an isolate (cucurbitacin glucosides) extracted from the leaves of this plant exhibits apoptotic activity in cancer cells. Nevertheless, no detailed study was done regarding the anticancer effects of this plant extract. Our study found that both ethanol and acetone extracts of fruit pulp of the colocynth plant showed a significant inhibition of cell viability of various cancer cell lines, including breast cancer MCF-7 and MDA-MB-231, and cervical cancer SiHa cell lines (Figures 1, 2 and Table 1). Correspondingly, both extracts had reverse cytotoxic effect on mononuclear cells PBMC and macrophage cell line J774A. Breast cancer MCF-7 cells showed more sensitivity to both ethanol and acetone pulp extracts as compared to other cancer cells. This was in agreement with previous reports that too claim higher sensitivity of MCF-7 cells to colocynth fruit extract at relatively lower concentrations (Mukherjee and Patil, 2012). The probable explanation may be either use of alkaloid rich extracts or longer exposure in MCF-7 cells (Mukherjee and Patil, 2012). Our study also found that both ethanol and acetone extracts exhibited synergistic potential with the chemotherapeutic drug doxorubicin to inhibit viability in the breast cancer cells (Supplementary Figure S4).

Moreover, on molecular analysis, we found suppression of transcripts of anti-apoptotic genes BCL2 and BCLXL, and an induction of apoptotic gene BAX and caspase 3 mRNA in metastatic breast cancer MDA-MB-231 cells following treatment with ethanol and acetone pulp extracts (Figure 3). In addition, these pulp extracts displayed a higher level of cleaved caspase 3 protein and a lower level of BCL- 2 protein as compared to control, implying an enhancement of active caspase 3 upon treatment with these extracts (Figure 3). Thus, this study indicated that colocynth fruit pulp extracts exhibit an anticancer potential presumably by promoting apoptosis.

As stated earlier, metastasis remains one of the major threats to cancer patients. However, no study has yet been conducted to investigate the anti-metastatic potential of this plant extract. Our study indicated, for the first time, a preventive role of these pulp extracts in cancer cell migration, a key step in the metastatic process (Figures 4, 5) (Chaffer and Weinberg, 2011). These pulp extracts also block EMT (Chaffer and Weinberg, 2011), another vital step in the metastatic cascade, since both PEE and PAE treatment significantly enhanced epithelial gene expression with simultaneous inhibition of mesenchymal marker expression in breast cancer MDA-MB-231 cells (Figure 6). Additionally, treatment of cancer cells with these ethanol and acetone pulp extracts showed a noticeable reduction in colony and sphere formation with concomitant inhibition of cancer stemness associated genes CD44 and BMI-1 at their transcript levels, suggesting a preventive role of these pulp extracts in cancer stemness (Monteiro and Fodde, 2010), a pivotal property responsible for metastasis, therapy resistance and tumor recurrence (Figures 7,8 ).

All these findings summarized a role of the ethanol and acetone fruit pulp extracts of colocynth in prevention of cell proliferation, induction of cell apoptosis, prevention of cell migration, reduction of EMT, and inhibition of cancer stemness property in breast cancer cells.

However, further study is required to identify the bioactive molecule(s) present in fruit pulp, liable for the observed anticancer potential. Subsequent GC-MS/MS analysis of both the extracts revealed about fifty compounds (Supplementary Figures S5A,B and Tables S2, S3) out of which, we analyzed the top 20 
TABLE 2 | Top 20 compounds isolated from GC-MS/MS analysis of ethanol and acetone crude extract.

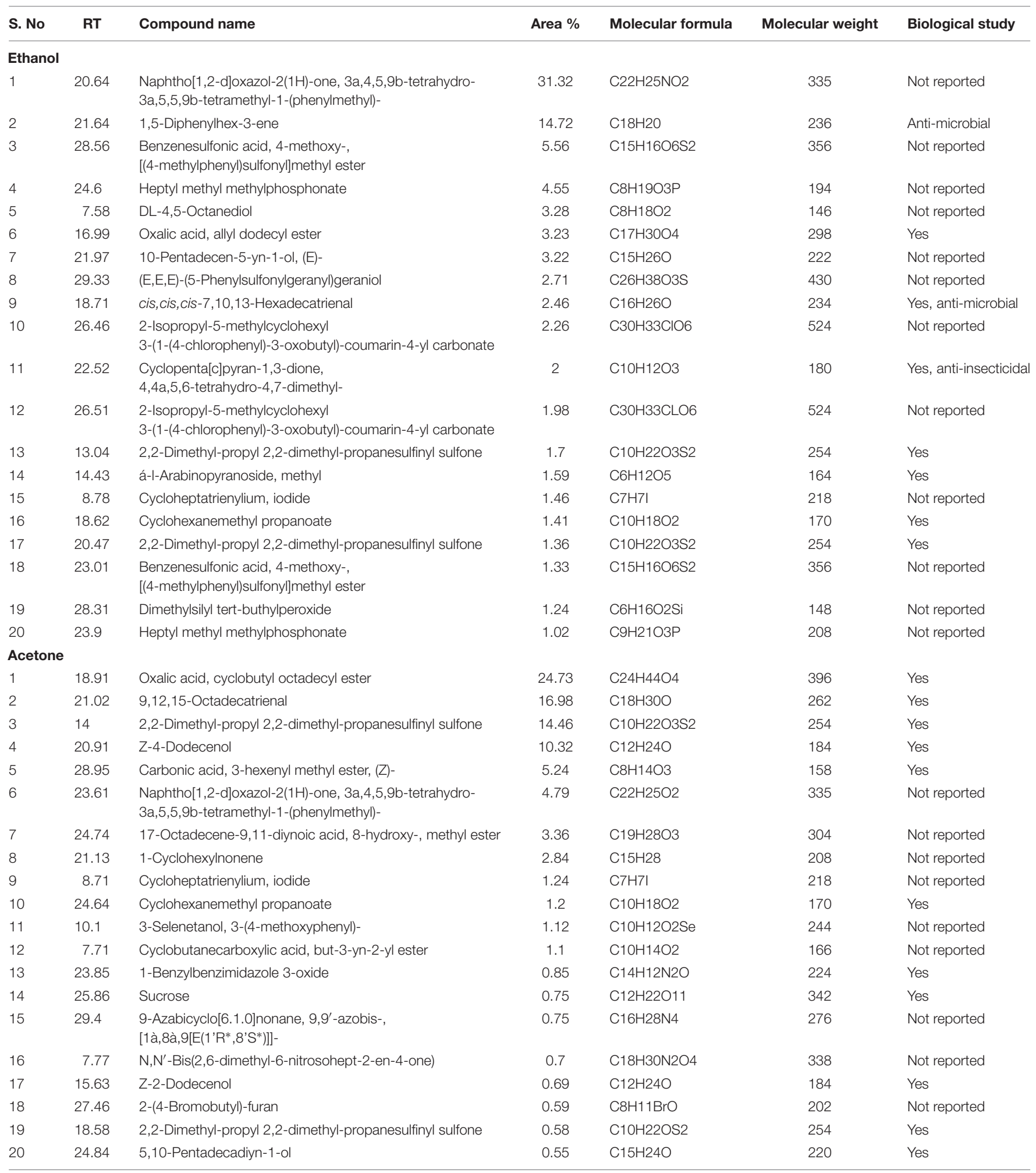

Compounds were selected on the basis of top twenty peak area extracted from the ethanol crude extract of fruit from the plant Citrullus colocynthis. Interpretation of the compound activity was done, and thus a comparative analysis is given with their reported molecular formula.

compounds on the basis of abundance in the extracts (Table 2). More in-depth study narrowed the list to those compounds that are novel to the extracts, with no previous reports of biological study (Table 3). We have also noticed that some compounds are common both in ethanolic and acetone extracts. Thus, purification and more detailed investigation of these compounds 
TABLE 3 | List of extracted novel compounds.

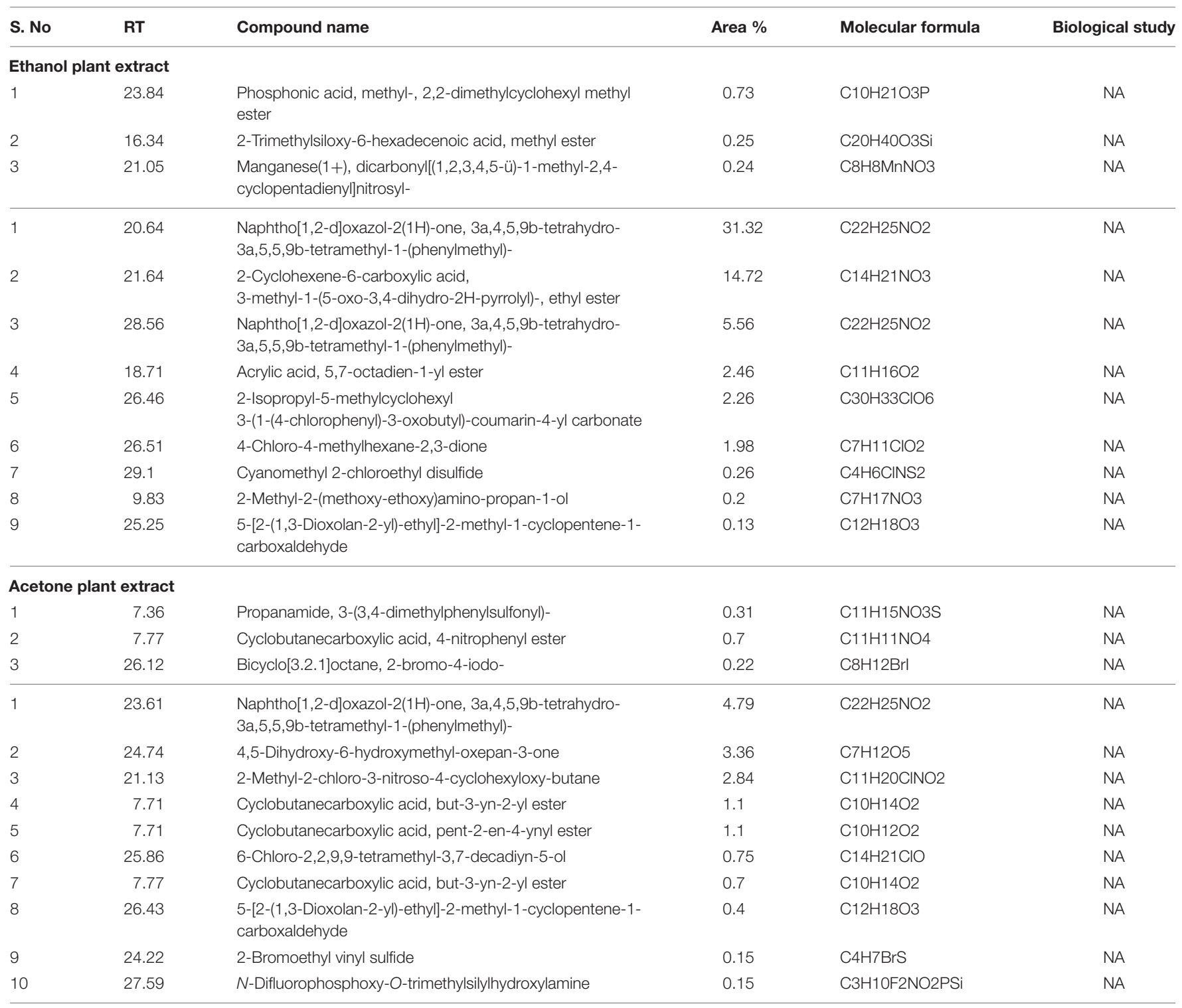

Compounds were isolated by GC-MS/MS analysis from ethanol and acetone pulp extract and tabulated on the basis of non-reported data.

will enable identification of the potent constituents in the extracts that are accountable for retarded cell growth and metastasis of cancer cells. Further mechanistic studies will prove extremely useful for their utility in future therapeutic interventions.

In brief, using cell culture based in vitro study and GC-MS/MS analysis, we report, for the first time, some novel compounds in the fruit pulp extracts of colocynth plant, that show promising potential as anti-cancer therapeutics for their tumor suppressive properties.

\section{AUTHOR CONTRIBUTIONS}

KC, AS, SK, and GG have substantial contribution in performing experiments, analyzing data, and interpreting results. KC has substantial involvement in drafting manuscript. AN has significant contribution in analyzing data and editing the manuscript. $\mathrm{CM}$ has formulated and supervised the work, and written the manuscript. All authors approved the final version of the manuscript and agree to be accountable for the content of this work.

\section{ACKNOWLEDGMENTS}

CM is supported by UGC [30-49/2014 (BSR)], DBT [6242P9/RGCB/PMD/DBT/CCML/2015], and Central University of Rajasthan, India. GC-MS analysis was performed by Mr. Arun Devana at USIC, University of Rajasthan, Jaipur. The authors are thankful to Dr. Amit Kotia for identification of the plant and 
taxonomically classifying it. Authors thank Dr. Vijay K Prajapati, Department of Biochemistry, Central University of Rajasthan, India for providing us J774A cells. The authors also acknowledge Dr. Umesh Yadav, Department of Pharmacy, Central University of Rajasthan, India for providing the reagent doxorubicin.

\section{REFERENCES}

Al-Hajj, M., Wicha, M. S., Benito-Hernandez, A., Morrison, S. J., and Clarke, M. F. (2003). Prospective identification of tumorigenic breast cancer cells. Proc. Natl. Acad. Sci. U.S.A. 100, 3983-3988. doi: 10.1073/pnas.0530291100

Asyaz, S., Khan, F., Hussain, I., Khan, M., and Khan, I. (2010). Evaluation of chemical analysis profile of Citrullus colocynthis growing in southern areas of Khyber Pukhtunkhwa Pakistan. World Appl. Sci. J. 10, 402-405.

Balmain, A., Gray, J., and Ponder, B. (2003). The genetics and genomics of cancer. Nat. Genet. 33, 238-244. doi: 10.1038/ng1107

Barghamdi, B., Ghorat, F., Asadollahi, K., Sayehmiri, K., Peyghambari, R., and Abangah, G. (2016). Therapeutic effects of Citrullus colocynthis fruit in patients with type II diabetes: a clinical trial study. J. Pharm. Bioallied Sci. 8, 130-134. doi: 10.4103/0975-7406.171702

Boyum, A. (1968). Isolation of mononuclear cells and granulocytes from human blood. Isolation of monuclear cells by one centrifugation, and of granulocytes by combining centrifugation and sedimentation at $1 \mathrm{~g}$. Scand. J. Clin. Lab. Invest. Suppl. 97, 77-89.

Boyum, A. (1976). Isolation of lymphocytes, granulocytes and macrophages. Scand. J. Immunol. Suppl. 5, 9-15. Epub 1976/06/01. PubMed PMID: 1052391 doi: 10.1111/j.1365-3083.1976.tb03851.x

Chaffer, C. L., and Weinberg, R. A. (2011). A perspective on cancer cell metastasis. Science 331, 1559-1564. doi: 10.1126/science.1203543

Chawech, R., Njeh, F., Hamed, N., Damak, M., Ayadi, A., Hammami, H., et al. (2017). A study of the molluscicidal and larvicidal activities of Citrullus colocynthis (L.) leaf extract and its main cucurbitacins against the mollusc Galba truncatula, intermediate host of Fasciola hepatica. Pest Manag. Sci. 73, 1473-1477. doi: 10.1002/ps.4479

Chowdhury, K., Sharma, A., Sharma, T., Kumar, S., and Mandal, C. C. (2017). Simvastatin and MBCD inhibit breast cancer-induced osteoclast activity by targeting osteoclastogenic factors. Cancer Invest. 35, 403-413. doi: 10.1080/ 07357907.2017.1309548

Feinberg, A. P., and Tycko, B. (2004). The history of cancer epigenetics. Nat. Rev. Cancer 4, 143-153. doi: 10.1038/nrc1279

Ghosh-Choudhury, N., Mandal, C. C., Das, F., Ganapathy, S., Ahuja, S., and Choudhury, G. G. (2013). c-Abl-dependent molecular circuitry involving Smad5 and phosphatidylinositol 3-kinase regulates bone morphogenetic protein-2-induced osteogenesis. J. Biol. Chem. 288, 24503-24517. doi: 10.1074/ jbc.M113.455733

Ghosh-Choudhury, N., Mandal, C. C., Ghosh-Choudhury, N., and Choudhury, G. G. (2010). Simvastatin induces derepression of PTEN expression via NFKB to inhibit breast cancer cell growth. Cell. Signal. 22, 749-758. doi: 10.1016/j.cellsig. 2009.12.010

Hirsch, H. A., Iliopoulos, D., Tsichlis, P. N., and Struhl, K. (2009). Metformin selectively targets cancer stem cells, and acts together with chemotherapy to block tumor growth and prolong remission. Cancer Res. 69, 7507-7511. doi: 10.1158/0008-5472.CAN-09-2994

Hussain, A. I., Rathore, H. A., Sattar, M. Z., Chatha, S. A., Sarker, S. D., and Gilani, A. H. (2014). Citrullus colocynthis (L.) Schrad (bitter apple fruit): a review of its phytochemistry, pharmacology, traditional uses and nutritional potential. J. Ethnopharmacol. 155, 54-66. doi: 10.1016/j.jep.2014.06.011

Hussain, A. I., Rathore, H. A., Sattar, M. Z., Chatha, S. A., ud din Ahmad, F., Ahmad, A., et al. (2013). Phenolic profile and antioxidant activity of various extracts from Citrullus colocynthis (L.) from the Pakistani flora. Ind. Crops Prod. 45, 416-422. doi: 10.1016/j.indcrop.2013.01.002

Jeon, J.-H., and Lee, H.-S. (2014). Biofunctional constituent isolated from Citrullus colocynthis fruits and structure-activity relationships of its analogues show acaricidal and insecticidal efficacy. J. Agric. Food Chem. 62, 8663-8667. doi: $10.1021 /$ jf502536e

\section{SUPPLEMENTARY MATERIAL}

The Supplementary Material for this article can be found online at: http://journal.frontiersin.org/article/10.3389/fphar. 2017.00593/full\#supplementary-material

King, P. D., and Perry, M. C. (2001). Hepatotoxicity of chemotherapy. Oncologist 6, 162-176. doi: 10.1634/theoncologist.6-2-162

Koehn, F. E., and Carter, G. T. (2005). The evolving role of natural products in drug discovery. Nat. Rev. Drug Discov. 4, 206-220. doi: 10.1038/nrd1657

Li, F., Tiede, B., Massagué, J., and Kang, Y. (2007). Beyond tumorigenesis: cancer stem cells in metastasis. Cell Res. 17,3-14. doi: 10.1038/sj.cr.7310118

Liu, S., Dontu, G., Mantle, I. D., Patel, S., Ahn, N.-S., Jackson, K. W., et al. (2006). Hedgehog signaling and Bmi-1 regulate self-renewal of normal and malignant human mammary stem cells. Cancer Res. 66, 6063-6071. doi: 10.1158/00085472.CAN-06-0054

Mandal, C. C., Das, F., Ganapathy, S., Harris, S. E., Choudhury, G. G., and Ghosh-Choudhury, N. (2016a). Bone morphogenetic protein-2 (BMP-2) activates NFATc1 transcription factor via an autoregulatory loop involving Smad/Akt/Ca ${ }^{2+}$ signaling. J. Biol. Chem. 291, 1148-1161. doi: 10.1074/jbc. M115.668939

Mandal, C. C., Drissi, H., Choudhury, G. G., and Ghosh-Choudhury, N. (2010a). Integration of phosphatidylinositol 3-kinase, Akt kinase, and Smad signaling pathway in BMP-2-induced osterix expression. Calcif. Tissue Int. 87, 533-540. doi: 10.1007/s00223-010-9419-3

Mandal, C. C., Ganapathy, S., Gorin, Y., Mahadev, K., Block, K., Abboud, H. E., et al. (2011a). Reactive oxygen species derived from Nox4 mediate BMP2 gene transcription and osteoblast differentiation. Biochem. J. 433, 393-402. doi: 10.1042/BJ20100357

Mandal, C. C., Ghosh-Choudhury, T., Yoneda, T., Choudhury, G. G., and GhoshChoudhury, N. (2010b). Fish oil prevents breast cancer cell metastasis to bone. Biochem. Biophys. Res. Commun. 402, 602-607. doi: 10.1016/j.bbrc.2010. 10.063

Mandal, C. C., Ghosh-Choudhury, N., Yoneda, T., Choudhury, G. G., and GhoshChoudhury, N. (2011b). Simvastatin prevents skeletal metastasis of breast cancer by an antagonistic interplay between p53 and CD44. J. Biol. Chem. 286, 11314-11327. doi: 10.1074/jbc.M110.193714

Mandal, C. C., Sharma, A., Panwar, M. S., and Radosevich, J. A. (2016b). Is cholesterol a mediator of cold-induced cancer? Tumor Biol. 37, 9635-9648. doi: 10.1007/s13277-016-4799-2 doi: 10.1007/s13277-016-4799-2

Marzouk, B., Marzouk, Z., Haloui, E., Fenina, N., Bouraoui, A., and Aouni, M. (2010). Screening of analgesic and anti-inflammatory activities of Citrullus colocynthis from southern Tunisia. J. Ethnopharmacol. 128, 15-19. doi: 10.1016/ j.jep.2009.11.027

Mehta, J., Asthana, S., Mandal, C. C., and Saxena, S. (2015). A molecular analysis provides novel insights into androgen receptor signalling in breast cancer. PLoS ONE 10:e0120622. doi: 10.1371/journal.pone.0120622

Monteiro, J., and Fodde, R. (2010). Cancer stemness and metastasis: therapeutic consequences and perspectives. Eur. J. Cancer 46, 1198-1203. doi: 10.1016/j. ejca.2010.02.030

Moongkarndi, P., Kosem, N., Kaslungka, S., Luanratana, O., Pongpan, N., and Neungton, N. (2004). Antiproliferation, antioxidation and induction of apoptosis by Garcinia mangostana (mangosteen) on SKBR3 human breast cancer cell line. J. Ethnopharmacol. 90, 161-166. doi: 10.1016/j.jep.2003.09.048

Mukherjee, A., and Patil, S. D. (2012). Effects of alkaloid rich extract of Citrullus colocynthis fruit on Artemia salina and human cancerous (MCF-7 and HEPG-2) cells. J. PharmaSciTech 1, 15-19.

Rahbar, A., and Nabipour, I. (2010). The hypolipidemic effect of Citrullus colocynthis on patients with hyperlipidemia. Pak. J. Biol. Sci. 13, 1202-1207. doi: 10.3923/pjbs.2010.1202.1207

Reya, T., Morrison, S. J., Clarke, M. F., and Weissman, I. L. (2001). Stem cells, cancer, and cancer stem cells. Nature 414, 105-111. doi: 10.1038/35102167

Sawaya, W., Daghir, N., and Khan, P. (1983). Chemical characterization and edibility of the oil extracted from Citrullus colocynthis seeds. J. Food Sci. 48, 104-106. doi: 10.1111/j.1365-2621.1983.tb14799.x 
Sawaya, W. N., Daghir, N. J., and Khalil, J. K. (1986). Citrullus colocynthis seeds as a potential source of protein for food and feed. J. Agric. Food Chem. 34, 285-288. doi: 10.1021/jf00068a035

Schimmel, K. J., Richel, D. J., van den Brink, R. B., and Guchelaar, H.-J. (2004). Cardiotoxicity of cytotoxic drugs. Cancer Treat. Rev. 30, 181-191. doi: 10.1016/ j.ctrv.2003.07.003

Sebolt-Leopold, J. S., Dudley, D. T., Herrera, R., Van Becelaere, K., Wiland, A., Gowan, R. C., et al. (1999). Blockade of the MAP kinase pathway suppresses growth of colon tumors in vivo. Nat. Med. 5, 810-816. doi: 10.1038/ 10533

Shi, C., Karim, S., Wang, C., Zhao, M., and Murtaza, G. (2014). A review on antidiabetic activity of Citrullus colocynthis Schrad. Acta Pol. Pharm. 71, 363-367.

Singh, A., and Settleman, J. (2010). EMT, cancer stem cells and drug resistance: an emerging axis of evil in the war on cancer. Oncogene 29, 4741-4751. doi: 10.1038/onc.2010.215

Studzinski, G. P. (ed.). (1995). Cell Growth and Apoptosis: A Practical Approach. New York, NY: Oxford University.

Tannin-Spitz, T., Grossman, S., Dovrat, S., Gottlieb, H. E., and Bergman, M. (2007). Growth inhibitory activity of cucurbitacin glucosides isolated from Citrullus colocynthis on human breast cancer cells. Biochem. Pharmacol. 73, 56-67. doi: 10.1016/j.bcp.2006.09.012
Torre, L. A., Bray, F., Siegel, R. L., Ferlay, J., Lortet-Tieulent, J., and Jemal, A. (2015). Global cancer statistics, 2012. CA Cancer J. Clin. 65, 87-108. doi: 10.3322/caac. 21262

Zaini, N. A. M., Anwar, F., Hamid, A. A., and Saari, N. (2011). Kundur [Benincasa hispida (Thunb.) Cogn.]: a potential source for valuable nutrients and functional foods. Food Res. Int. 44, 2368-2376. doi: 10.1016/j.foodres.2010. 10.024

Zhang, M., Aguilera, D., Das, C., Vasquez, H., Zage, P., Gopalakrishnan, V., et al. (2007). Measuring cytotoxicity: a new perspective on LC50. Anticancer Res. 27, $35-38$.

Conflict of Interest Statement: The authors declare that the research was conducted in the absence of any commercial or financial relationships that could be construed as a potential conflict of interest.

Copyright $\odot 2017$ Chowdhury, Sharma, Kumar, Gunjan, Nag and Mandal. This is an open-access article distributed under the terms of the Creative Commons Attribution License (CC BY). The use, distribution or reproduction in other forums is permitted, provided the original author(s) or licensor are credited and that the original publication in this journal is cited, in accordance with accepted academic practice. No use, distribution or reproduction is permitted which does not comply with these terms. 


\section{OPEN ACCESS}

Edited by:

Ruiwen Zhang

University of Houston, United States

Reviewed by:

Jian Li,

Beijing University of Chinese Medicine,

China

Ouyang Chen

Second Military Medical University,

China

*Correspondence:

Atanas G. Atanasov

atanas.atanasov@univie.ac.at

Specialty section:

This article was submitted to

Ethnopharmacology,

a section of the journal

Frontiers in Pharmacology

Received: 10 June 2017

Accepted: 17 August 2017

Published: 01 September 2017

Citation:

Wang L, Palme V, Schilcher N,

Ladurner A, Heiss EH, Stangl $H$,

Bauer R, Dirsch VM and Atanasov AG

(2017) The Dietary Constituent

Falcarindiol Promotes Cholesterol

Efflux from THP-1 Macrophages by

Increasing ABCA1 Gene Transcription

and Protein Stability.

Front. Pharmacol. 8:596.

doi: 10.3389/fphar.2017.00596
The Dietary Constituent Falcarindiol Promotes Cholesterol Efflux from THP-1 Macrophages by Increasing ABCA1 Gene Transcription and Protein Stability

\author{
Limei Wang ${ }^{1,2}$, Veronika Palme ${ }^{1}$, Nicole Schilcher ${ }^{1}$, Angela Ladurner ${ }^{1}$, Elke H. Heiss ${ }^{1}$, \\ Herbert Stangl ${ }^{3}$, Rudolf Bauer ${ }^{4}$, Verena M. Dirsch ${ }^{1}$ and Atanas G. Atanasov ${ }^{1,5 *}$ \\ 'Department of Pharmacognosy, University of Vienna, Vienna, Austria, ${ }^{2}$ Department of Pharmacology, School of Pharmacy, \\ Qingdao University, Qingdao, China, ${ }^{3}$ Center for Pathobiochemistry and Genetics, Institute of Medical Chemistry, Medical \\ University of Vienna, Vienna, Austria, ${ }^{4}$ Department of Pharmacognosy, Institute of Pharmaceutical Sciences, \\ Karl-Franzens-University Graz, Graz, Austria, ${ }^{5}$ Department of Molecular Biology, Institute of Genetics and Animal Breeding of \\ the Polish Academy of Sciences, Lesznowola, Poland
}

We report increased cholesterol efflux from macrophages in the presence of falcarindiol, an important dietary constituent present in commonly used vegetables and medicinal plants. Falcarindiol (3-20 $\mu \mathrm{M}$ ) increased cholesterol efflux from THP-1-derived macrophages. Western blot analysis showed an increased protein level of ABCA1 upon falcarindiol exposure. Quantitative real-time PCR revealed that also ABCA1 mRNA level rise with falcarindiol $(10 \mu \mathrm{M})$ treatment. The effect of falcarindiol on ABCA1 protein as well as mRNA level were counteracted by co-treatment with BADGE, an antagonist of PPAR $\gamma$. Furthermore, falcarindiol significantly inhibited ABCA1 protein degradation in the presence of cycloheximide. This post-translational regulation of ABCA1 by falcarindiol occurs most likely by inhibition of lysosomal cathepsins, resulting in decreased proteolysis and extended protein half-life of ABCA1. Taken together, falcarindiol increases $A B C A 1$ protein level by two complementary mechanisms, i.e., promoting $\mathrm{ABCA} 1$ gene expression and inhibiting $\mathrm{ABCA} 1$ protein degradation, which lead to enhanced cholesterol efflux.

Keywords: falcarindiol, cholesterol efflux, ABCA1, protein degradation, PPAR $\gamma$

\section{INTRODUCTION}

Cardiovascular disease (CVD) associated with atherosclerosis is a leading cause of mortality in the world. Cholesterol metabolism has been considered as a major player in the pathogenesis of CVD. Excessive cholesterol deposition resulting in plaques is a critical cause of atherosclerosis. Reverse cholesterol transport (RTC) is a complex multi-step process overall resulting in the relocation of cholesterol from peripheral tissues to the liver for excretion into the bile and ultimately the feces. Cholesterol efflux, the initial step of RTC, is known to enhance the export of excessive cholesterol from peripheral tissues, in particular macrophages, and thereby to inhibit the accumulation of cholesterol in the wall of arteries (Fielding and Fielding, 1995). There is strong evidence that the cholesterol efflux capacity is inversely associated with the 

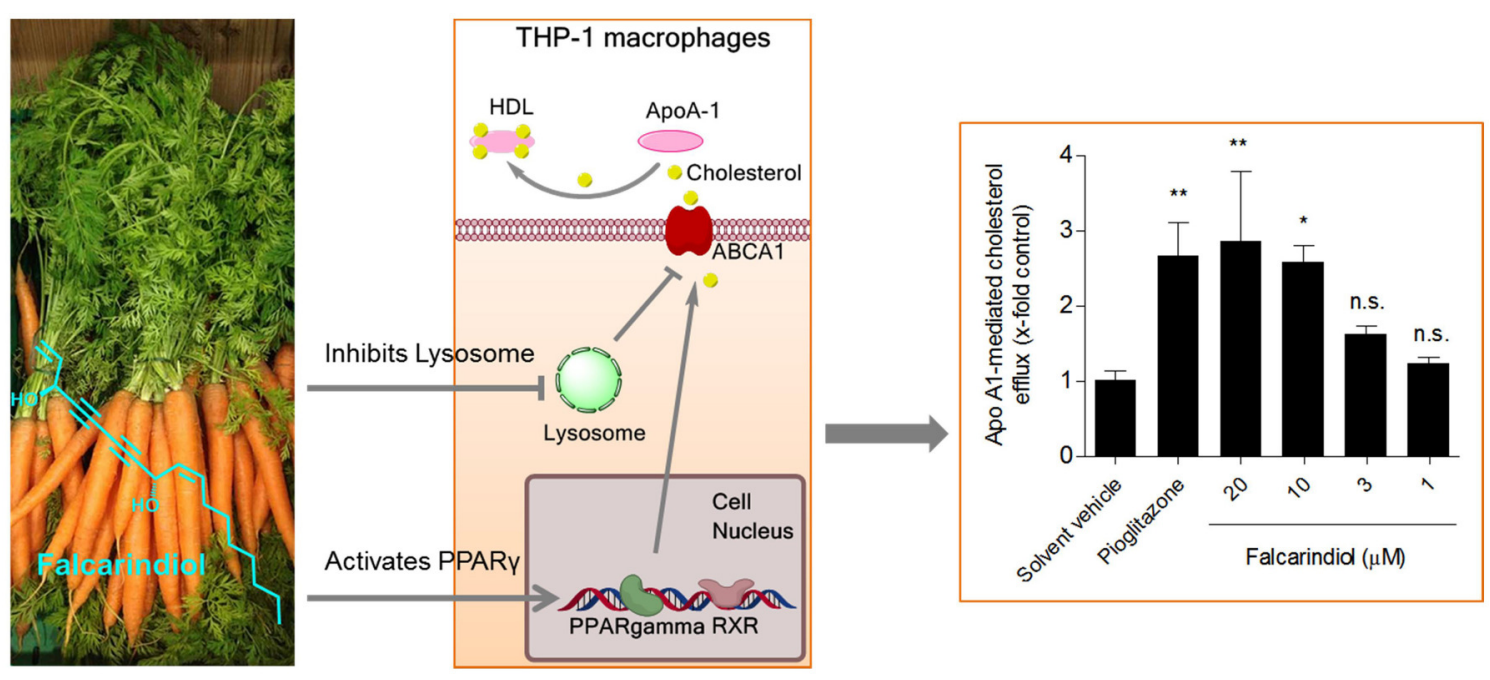

Graphical Abstract: The dietary constituent falcarindiol promotes macrophage cholesterol efflux by increasing ABCA1 transporter gene transcription (via PPAR $\gamma$

activation) and protein stability (via inhibition of lysosomal proteolysis).

incidence of CVD (Rohatgi et al., 2014). Therefore, the enhancement of cholesterol efflux from macrophages might prevent CVD, making it a potential strategy for pharmacological interventions. Key determinant of cholesterol efflux is the expression level and activity of transmembrane transporter proteins such as the ATP-binding cassette transporter A1 (ABCA1) and G1 (ABCG1) and the scavenger receptor class B member 1 (SR-B1) (Duffy and Rader, 2006). Among them, $A B C A 1$ is the predominant one and it contributes to most of the cholesterol transport in macrophages (Rosenson et al., 2012; Du et al., 2015). ABCA1 mediates mostly transfer of cholesterol to lipid-poor apolipoprotein A1 (apo A1), but it can also mediate cholesterol transport using high density lipoproteins (HDL) as an acceptor (Du et al., 2015). Modulation of the ABCA1 protein level is considered an important regulator for cholesterol efflux. In general, the level of $\mathrm{ABCA} 1$ protein is determined by de novo synthesis and protein degradation. Several nuclear receptors are implicated in the regulation of ABCA1 gene transcription. Thus, the activation of the ligand-dependent nuclear receptors peroxisome proliferator-activated receptor gamma (PPAR $\gamma)$ and subsequently the liver $\mathrm{X}$ receptor (LXR), can lead to increased ABCA1 gene transcription (Chawla et al., 2001; Chinetti et al., 2001; Ozasa et al., 2011). The ABCA1 protein half-life is largely regulated via the proteolytic activity of calpain-, lysosome-, and ubiquitin-degradation pathways (Wang et al., 2003; Mizuno et al., 2011; Liu and Tang, 2012; Yokoyama et al., 2012). Cathepsins represent key lysosomal proteases (Turk et al., 2012) and diverse cathepsin isoforms (e.g., cathepsin B, D, K, L, S) are known to be expressed in macrophages (Punturieri et al., 2000; Beers et al., 2003; Bracke et al., 2005; Vasiljeva et al., 2006).

Natural products have proven to be an excellent source of molecules with promising pharmacological activities and novel mechanisms of action (Atanasov et al., 2015). Here, we report the identification and characterization of the natural product falcarindiol (Figure 1A) as a novel enhancer of macrophage cholesterol efflux. Falcarindiol is a C(17)-polyacetylene and a typical constituent of roots and rhizomes of Apiaceae plants used worldwide, including in Europe, such as the commonly used vegetables and herbs carrot (Daucus carota L.) (Garrod et al., 1978), celery (Apium graveolens L.) (Zidorn et al., 2005), Notopterygium incisum K.C.Ting ex H.T.Chang (Zschocke et al., 1997), and Aegopodium podagraria L. (Kemp, 1978), among others. Taking the important role of PPAR $\gamma$ in the regulation of macrophage cholesterol efflux into consideration, we have chosen this natural product as a study subject in the context of cholesterol efflux because falcarindiol, extracted from the rhizomes and roots of Notopterygium incisum K.C.Ting ex H.T.Chang, was recently identified as a partial PPAR $\gamma$ agonist in HEK-293 cells transfected with a PPAR $\gamma$-responsive luciferase reporter (Atanasov et al., 2013).

\section{MATERIALS AND METHODS}

\section{Chemical Reagents}

Roswell Park Memorial Institute 1640 (RPMI-1640) medium was obtained from Lonza (Basel, Switzerland). Fetal bovine serum (FBS) was purchased from Gibco via Invitrogen (Lofer, Austria). $\left[{ }^{3} \mathrm{H}\right]$-Cholesterol was bought from Perkin Elmer Life Sciences (Vienna, Austria) and pioglitazone was from Molekula (Munich, Germany). Albumin faction V, fatty acid free BSA was obtained from Carl Roth (Graz, Austria). Bisphenol A diglycidyl ether (BADGE), resazurin sodium salt, water soluble unesterified cholesterol, apolipoprotein (apo) A1, phorbol 12myristate 13-acetate (PMA), lactacystin, calpeptin, chloroquine, and cycloheximide were provided by Sigma-Aldrich (Vienna, Austria). Falcarindiol was isolated from the roots and rhizomes of the traditional Chinese herbal drug Radix Notopterygii, with a purity over $95 \%$ as determined by High Performance Liquid Chromatography (HPLC) (Zschocke et al., 1997; Liu et al., 2014). Cathepsin B, L, D, and K Activity Fluorometric kits were 
A<smiles>C=CC(O)C#CC#CC(O)/C=C\CCCCCCC</smiles>

C

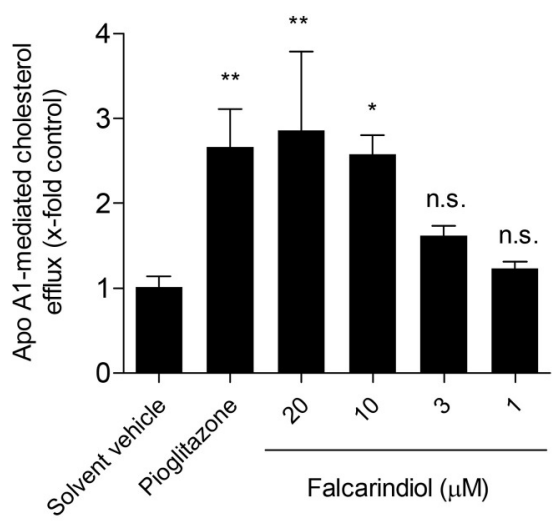

B

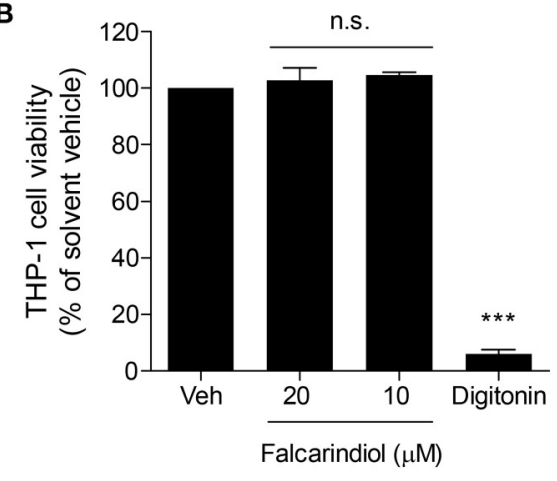

D

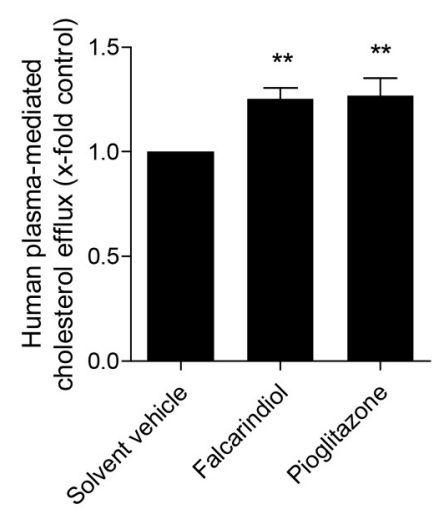

FIGURE 1 | Chemical structure of falcarindiol (A), its effect on cell viability (B), and cholesterol efflux in THP-1-derived macrophages (C,D). (B) Differentiated THP-1 macrophages were incubated with solvent vehicle control (DMSO), $50 \mu \mathrm{g} / \mathrm{mL}$ digitonin, or the indicated concentrations (10-20 $\mu \mathrm{M}$ ) of falcarindiol. After $24 \mathrm{~h}$, the increased fluorescent signal from resazurin conversion was detected and calculated as the percentage of cell viability. (C,D) THP-1-derived macrophages were loaded with $\left[{ }^{3} \mathrm{H}\right]$-cholesterol together with the indicated treatments for $24 \mathrm{~h}$. On the next day, macrophages were washed twice with pre-warmed PBS and incubated with the same compounds [solvent vehicle (DMSO), falcarindiol (1-20 $\mu \mathrm{M}$ for apo A1-mediated cholesterol efflux, or $10 \mu \mathrm{M}$ for human plasma-mediated cholesterol efflux), or the PPAR $\gamma$ agonist pioglitazone (10 $\mu \mathrm{M})$ as positive control] with or without $10 \mu \mathrm{g} / \mathrm{mL}$ apo A1 (C) or $1 \%$ human plasma (D) dissolved in serum-free medium for $6 \mathrm{~h}$. Extracellular as well as intracellular radioactivity were quantified with a scintillation counter. All values are means $\pm S D(n=3)$ and vs. solvent vehicle control (DMSO): ${ }^{\star} p<0.05,{ }^{\star \star} p<0.01,{ }^{\star \star *} p<0.001$, n.s, not significant (ANOVAVBonferroni).

purchased from BioVision via THP Medical Products GmbH (Vienna, Austria). Cathepsin S activity assay kit (fluorometric) was bought from Abcam (Cambridge, UK). Antibodies were obtained from the following companies: ABCA1, ABCG1, SRB1 from Novus Biologicals via THP Medical Products GmbH (Vienna, Austria), anti-actin antibody from MP biologicals (Illkirch, France), horseradish peroxidase-conjugated goat antirabbit secondary antibody from New England Biolabs (Frankfurt, Germany), horseradish peroxidase conjugated goat anti-mouse secondary antibody from Upstate (Millipore, Vienna, Austria).

\section{Cell Culture}

Human monocytic THP-1 cells were acquired from ATCC ${ }^{\circledR}$ and grown in suspension in RPMI-1640 medium supplemented with $2 \mathrm{mM}$ glutamine, $100 \mathrm{U} / \mathrm{mL}$ benzylpenicillin, $100 \mathrm{mg} / \mathrm{mL}$ streptomycin, and 10\% FBS in T175 flasks. For experiments, differentiation was induced by seeding THP-1 monocytes in 96-, 24-, or 6- well plates under treatment of $200 \mathrm{nM}$ PMA for $72 \mathrm{~h}$ (seeding cell density of $0.2 \times 10^{6}$ cells per $\mathrm{mL}$ ). All of the tested compounds were dissolved in dimethyl sulfoxide (DMSO) and stored at $-20^{\circ} \mathrm{C}$. Control cells were always treated with an equal volume of solvent (DMSO) and its final concentrations did not exceed $0.1 \%$.

\section{Cholesterol Efflux Assay}

The cholesterol efflux assay (Wang et al., 2015) was adapted from previous studies (Chawla et al., 2001; Chinetti et al., 2001). Briefly, THP-1-derived macrophages were incubated for $24 \mathrm{~h}$ with the compounds at the indicated concentrations [solvent vehicle (DMSO), falcarindiol (1-20 $\mu \mathrm{M})$, the PPAR $\gamma$ agonist pioglitazone $(10 \mu \mathrm{M})$ as positive control] together with $\left[{ }^{3} \mathrm{H}\right]$ cholesterol in serum free medium supplemented with $0.1 \%$ BSA and $10 \mu \mathrm{g} / \mathrm{mL}$ unesterified cholesterol. On the next day, cells were washed twice with pre-warmed PBS and incubated with the same compounds with or without $10 \mu \mathrm{g} / \mathrm{mL}$ apo $\mathrm{A} 1$ or $1 \%$ human plasma dissolved in serum-free medium for $6 \mathrm{~h}$. Extracellular as well as intracellular radioactivity were quantified 
with scintillation counting. The apo A1- as well as human plasma-mediated cholesterol efflux was calculated as follows:
QuantiTect primer assay, Cat.no.: \#QT00064869, QIAGEN) and 18S (Hs_RRN18S_1_SG QuantiTect Primer assay,

$$
\begin{aligned}
\text { Apo A1 mediated cholesterol efflux } \% & =\left(\frac{(\text { extracellular cpm }) \text { apo A1 }}{(\text { total } c p m) \text { apo A1 }}-\frac{(\text { extracellular cpm }) \text { no apo A1 }}{(\text { total cpm }) \text { no apo A1 }}\right) \times 100 \\
\text { Human plasma mediated cholesterol efflux } \% & =\left(\frac{(\text { extracellular } c p m) \text { plasma }}{(\text { total } c p m) \text { plasma }}-\frac{(\text { extracellular cpm }) \text { no plasma }}{(\text { total } c p m) \text { no plasma }}\right) \times 100
\end{aligned}
$$

\section{Resazurin Conversion Assay}

The viability of THP-1-derived macrophages was assessed by resazurin conversion. The assay was performed as previously described (Wang et al., 2015) by quantification of the conversion of the low fluorescent resazurin to high fluorescent resorufin.

\section{Gel Electrophoresis and Immunoblot Analysis}

THP-1 cells were seeded in 6-well plates and differentiated into macrophages with $200 \mathrm{nM}$ PMA for $72 \mathrm{~h}$. The macrophages were treated with the indicated compounds at the corresponding time points, as described in detail in each figure legend. Cellular proteins were extracted using NP40 buffer (150 $\mathrm{mM} \mathrm{NaCl} ; 50 \mathrm{mM}$ HEPES (pH 7.4); $1 \%$ NP40; $1 \%$ protease inhibitor Complete ${ }^{\mathrm{TM}} \quad$ (Roche); $1 \%$ phenylmethylsulfonyl fluoride (PMSF); $0.5 \% \quad \mathrm{Na}_{3} \mathrm{VO}_{4} ; 0.5 \% \mathrm{NaF}$ ) and quantified with the Bradford method. For western blot analysis, an equal amount of protein $(20 \mu \mathrm{g})$ was separated via SDS-PAGE and transferred onto a PVDF membrane. The membrane was blocked for $1 \mathrm{~h}$ at room temperature with $5 \%$ non-fat milk, and then incubated at $4{ }^{\circ} \mathrm{C}$ overnight with primary polyclonal antibody against ABCA1, ABCG1 or SR-B1 (1:500) or actin $(1: 10,000)$. After being washed for three times (15 min each time), the membranes were incubated with a secondary antibody [horseradish peroxidase-conjugated goat anti-rabbit (1:500) or anti-mouse $(1: 10,000)]$ for $1 \mathrm{~h}$ at room temperature. The signals were detected and visualized using a LAS-3000 luminescent image analyzer (Fujifilm, Düsseldorf, Germany) using AIDA image analyzer 4.06 software (Raytest, Sprockhövel, Germany). The indicated protein band intensity was normalized to that of actin.

\section{RNA Extraction and Analysis}

THP-1 cells were seeded in 6-well plates and differentiated into macrophages with a $200 \mathrm{nM}$ PMA treatment for $72 \mathrm{~h}$. The macrophages were treated with the indicated compounds at the indicated time points, which are described in detail in the figure legends. Total cellular RNA was extracted from the treated THP-1 macrophages using peqGOLD Total RNA kits (PeqLab, Linz, Austria) according to the manufacturer's instructions. One microgram of total RNA was used for CDNA synthesis according to the protocol of the High Capacity cDNA Reverse Transcription Kit together with RNase Inhibitor (Applied Biosystems). LightCycler ${ }^{\circledR} 480$ SYBR Green I Master kit (Roche) was used for quantitative real-time PCR (qRTPCR) with $40 \mathrm{ng}$ cDNA from each sample for triplicate measurements. Amplification cycles were detected using the LightCycler 480 system (Roche). ABCA1 (HS_ABCA1_1_SG
Cat.no.: \#QT00199367, QIAGEN) primers were used, and the quantification was performed with the $\Delta C_{T}$ method.

\section{Determination of Cathepsin Activity}

The cathepsin activity assays were performed according to the manufacturer's instructions. In general, THP-1-derived macrophages were cultured for $24 \mathrm{~h}$ with solvent control (DMSO) or falcarindiol $(10 \mu \mathrm{M})$. After incubation, cells were washed once with cold PBS and lysed with chilled cell lysis buffer. Protein concentration was determined using the Bradford method. Hundred micrograms of protein per reaction in $50 \mu \mathrm{L}$ of cell lysis buffer was used for the activity measurement. Fifty microliters of reaction buffer and $2 \mu \mathrm{L}$ of the respective cathepsin substrate were added to $50 \mu \mathrm{L}$ cell lysates. The assay plate was incubated at $37^{\circ} \mathrm{C}$ for $1 \mathrm{~h}$ protected from light. The fluorescence was quantified with Tecan Infinite $200^{\circledR}$ Pro plate reader (Vienna, Austria).

\section{Statistical Methods}

Statistical analysis was performed with data acquired from at least three independent experiments using GraphPad Prism software version 4.03 (GraphPad Software Inc., La Jolla, CA, USA). Figures with bar graphs represent mean $\pm S D$. To determine statistical significance, one- or two- way analysis of variance (ANOVA) were performed and $\mathrm{p}<0.05$ was considered significant.

\section{RESULTS}

\section{Falcarindiol Promotes Cholesterol Efflux in Thp-1-Derived Macrophages}

In a previous study, we showed that falcarindiol activates PPAR $\gamma$ in HEK-293 cells transfected with a PPAR $\gamma$-responsive luciferase reporter (Atanasov et al., 2013). Considering that PPAR $\gamma$ is a known regulator of cholesterol efflux (Chawla et al., 2001; Chinetti et al., 2001), we aimed to investigate whether falcarindiol can promote cholesterol efflux in THP-1derived macrophages. Since apo A1, the nascent and lipid free form of HDL, is the most significant acceptor for cholesterol effluxed from macrophages (Du et al., 2015), we first studied cholesterol efflux mediated by apo A1. Falcarindiol enhanced apo A1-mediated cholesterol efflux in a concentration-dependent manner when applied at $1-20 \mu \mathrm{M}$ (without effect on cell viability; Figure 1B) with a half maximal effective concentration $\left(\mathrm{EC}_{50}\right)$ of $5.8 \mu \mathrm{M}$ (Figure 1C). Moreover, $10 \mu \mathrm{M}$ falcarindiol also significantly enhanced $1 \%$ human plasma-mediated cholesterol efflux (Figure 1D) as plasma containing HDL particles served as cholesterol acceptors in the process of cholesterol efflux. Pioglitazone, a PPAR $\gamma$ agonist known to upregulate cholesterol 
efflux (Ozasa et al., 2011), was used as positive control both in presence of apo A1 and human plasma (Figures 1C,D).

\section{Falcarindiol Enhances ABCA1 Protein Expression}

The transmembrane proteins ABCA1, ABCG1, and SR-B1 are transporters mediating cholesterol efflux (Duffy and Rader, 2006). Therefore, we investigated protein expression of these three transporters upon treatment with falcarindiol in THP-1derived macrophages. As evident in Figure 2A, ABCA1 protein level significantly increased in response to falcarindiol $(10 \mu \mathrm{M})$. At the same time, there was no significant upregulation of ABCG1 (Figure 2B) or the bi-directional transporter SR-B1 (Figure 2C; Stangl et al., 1999). Next, we investigated whether increased ABCA1 protein level is associated with an increase in ABCA1 mRNA level. ABCA1 mRNA level indeed significantly increased upon falcarindiol $(10 \mu \mathrm{M})$ treatment (Figure 3B, left).

In macrophages, enhanced ABCA1 mRNA level might result from PPAR $\gamma$ and subsequent LXR activation (Chawla et al., 2001; Chinetti et al., 2001). We, therefore, asked whether the increased ABCA1 mRNA level induced by falcarindiol might be due to $\operatorname{PPAR} \gamma$-activation. To check this possibility, we used the PPAR $\gamma$ antagonist, BADGE $(50 \mu \mathrm{M})$, to block the activity of PPAR $\gamma$ in THP-1-derived macrophages (Hinz et al., 2003). Indeed, BADGE blocked the falcarindiol-mediated increase of ABCA1 protein as well as mRNA level (Figures 3A,B).

\section{Falcarindiol Inhibits ABCA1 Protein Degradation}

Interestingly, the ABCA1 mRNA up-regulation by falcarindiol was significantly lower than the mRNA induction caused by the PPAR $\gamma$ agonist pioglitazone (Figure 3B, on the left), while the induction of ABCA1 protein level by falcarindiol and pioglitazone was equally potent (Figure $\mathbf{3 A}$, on the left). This inconsistency indicated that an additional effect of falcarindiol might exist, which could enhance ABCA1 protein level besides the increase of ABCA1 mRNA level. To explore this possibility, we measured the rate of ABCA1 protein degradation in the presence and absence of falcarindiol. THP-1derived macrophages were incubated with falcarindiol or solvent vehicle (DMSO) for $24 \mathrm{~h}$ and were then further treated with cycloheximide $(140 \mu \mathrm{M})$ to block de novo protein synthesis (Arakawa et al., 2009). As evident in Figure 4, ABCA1 protein degradation was significantly inhibited in the presence of falcarindiol showing that the compound interferes with the ABCA1 protein turnover rate.

\section{Falcarindiol Inhibits Lysosomal Cathepsin-Dependent ABCA1 Degradation}

To further explore how falcarindiol inhibits ABCA1 protein degradation, we first investigated, which protein degradation pathway could be affected by falcarindiol in THP-1-derived macrophages. As presented in Figure 5A, the application of lactacystin, an ubiquitin proteasome inhibitor, failed to affect ABCA1 protein level in our experimental setting. However, the application of calpeptin (a calpain inhibitor) and chloroquine (a lysosome inhibitor) (Figures 5B,C) increased ABCA1 protein level, similarly to falcarindiol. Falcarindiol had an additive effect on ABCA1 protein level upon co-treatment with calpeptin, indicating that its mechanism of action is likely different from the calpain inhibitor calpeptin. However, there was no difference observed with or without chloroquine in the presence of falcarindiol, suggesting that falcarindiol might interfere with protein degradation in the lysosome, mimicking the effect of chloroquine (Figure 5C).

The lysosomal degradation pathway is a well-known regulator of ABCA1 protein turnover (Neufeld et al., 2001; SantamarinaFojo et al., 2001; Mizuno et al., 2011) and cathepsins are well established proteases mediating the lysosomal proteolysis in general (Turk et al., 2012; Appelqvist et al., 2013). Therefore,
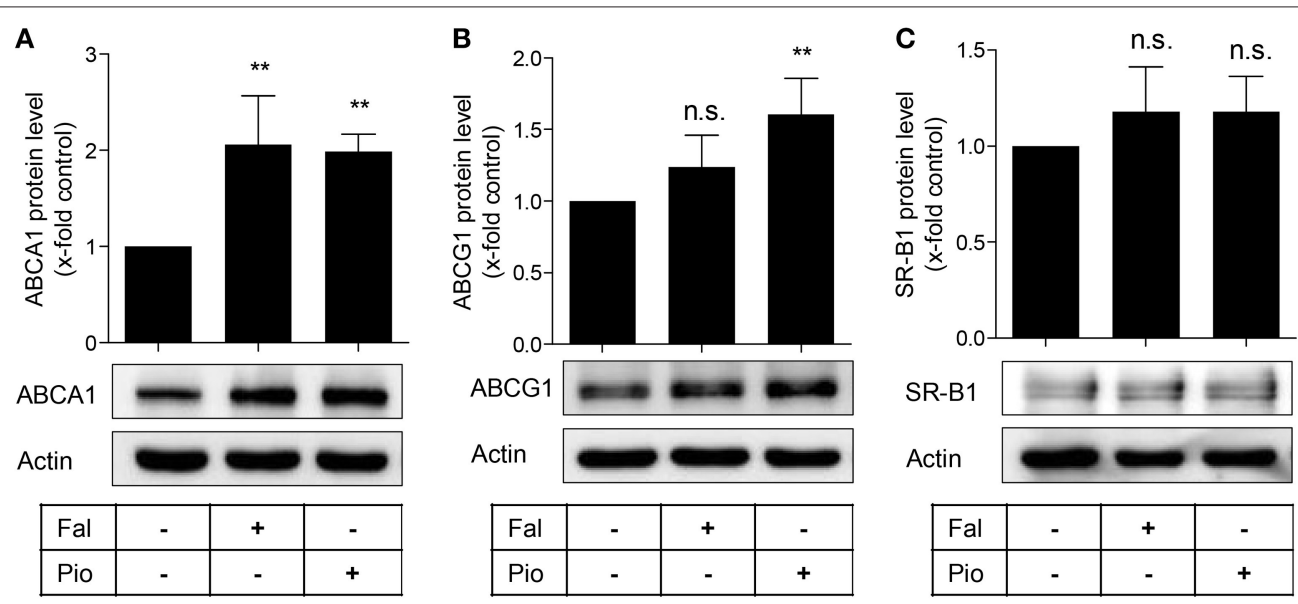

FIGURE 2 | Falcarindiol increases ABCA1 protein level. Differentiated THP-1 macrophages were treated with solvent vehicle control (DMSO), falcarindiol (Fal, $10 \mu \mathrm{M})$, or the PPAR $\gamma$ agonist pioglitazone (Pio, $10 \mu \mathrm{M}$ ). After $24 \mathrm{~h}$, cells were lysed and $20 \mu \mathrm{g}$ protein was resolved via SDS-PAGE. Immunoblotting was performed with antibodies against the indicated proteins, ABCA1 (A), ABCG1 (B), and SR-B1 (C) as well as actin as loading control. All experiments were performed four times and values are presented as means $\pm S D$ and vs. solvent vehicle control (DMSO): ${ }^{* *} p<0.01$, n.s. not significant (ANOVA/Bonferroni). 


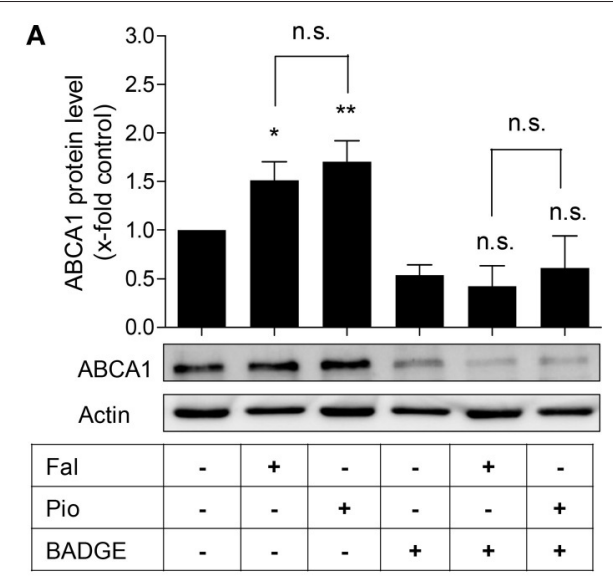

B

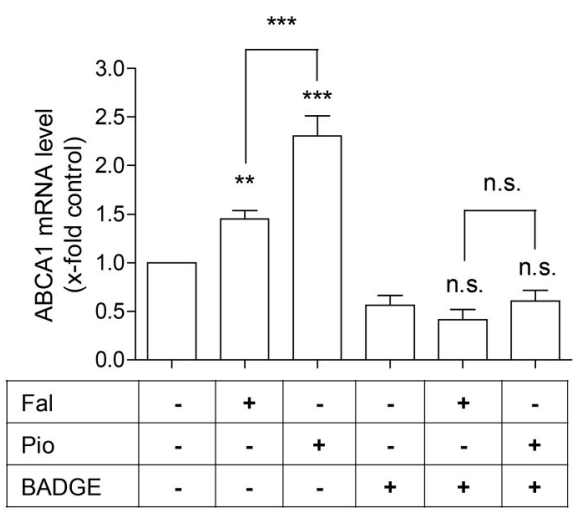

FIGURE 3 | The PPAR $\gamma$ antagonist, BADGE, blocks falcarindiol-mediated ABCA1 protein and mRNA induction. Differentiated THP-1 macrophages were incubated for $24 \mathrm{~h}$ with falcarindiol (Fal, $10 \mu \mathrm{M}$ ) or pioglitazone (Pio, $10 \mu \mathrm{M}$ ) with or without $50 \mu \mathrm{M}$ BADGE. ABCA1 protein (A) and mRNA (B) level were analyzed by western blotting and qPCR, respectively. All data are mean $\pm S D(n=3),{ }^{\star} p<0.05,{ }^{\star \star} p<0.01$, ${ }^{\star \star *} p<0.001$, n.s. not significant vs. solvent vehicle control (DMSO) (ANOVA/Bonferroni).

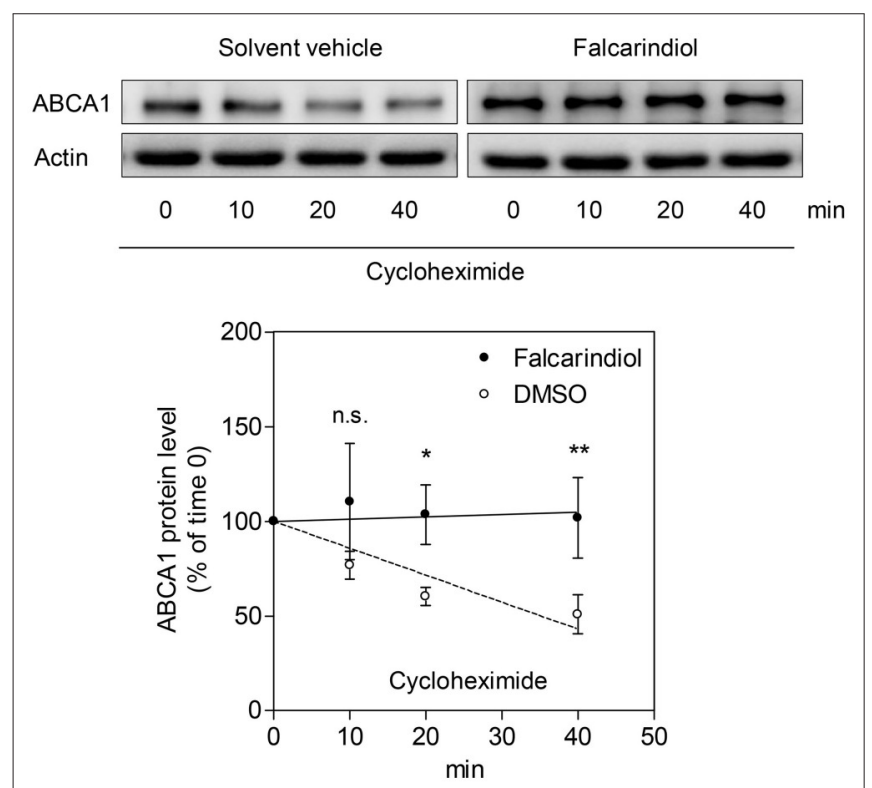

FIGURE 4 | Falcarindiol inhibits ABCA1 protein degradation. Differentiated THP-1 macrophages were stimulated with $10 \mu \mathrm{M}$ falcarindiol (black circles) or an equal amount of the solvent vehicle control (DMSO; white circles). After 24 $\mathrm{h}$ incubation, cells were treated with cycloheximide $(140 \mu \mathrm{M})$ and lysed at different time points $(0,10,20,40 \mathrm{~min})$. Western blotting was used to monitor the decline of ABCA1 protein level upon cycloheximide treatment in the presence or absence of falcarindiol. All data are mean $\pm S D(n=3)$ and vs. solvent vehicle control (DMSO): ${ }^{\star} p<0.05,{ }^{\star *} p<0.01$, n.s. not significant (two-way ANOVA/Bonferroni).

we investigated whether the proteolytic activity of lysosomal cathepsins is affected by falcarindiol. Figures 6A-E shows that among the tested cathepsins (B, D, K, L, S) the activity of cathepsin B, S, and $\mathrm{K}$ were significantly suppressed by 35,14 , and $8 \%$, respectively. Overall, these data indicate that falcarindiol leads to the suppression of ABCA1 degradation and inhibits lysosomal cathepsins.

\section{DISCUSSION}

In this study, we describe for the first time that falcarindiol, a typical constituent of Apiaceae vegetables, stimulates cholesterol efflux by promoting ABCA1 gene expression in THP-1-derived macrophages. In addition, we show that falcarindiol increases ABCA1 protein stability and inhibits the proteolytic activity of lysosomal cathepsins.

Macrophage cholesterol efflux is a process counteracting the transformation of macrophages into pro-atherogenic foam cells. Thus, the study of this process and molecules that can regulate it is of significance for potential approaches to combat CVD (Rosenson et al., 2012; Du et al., 2015). ABCA1 is the most important membrane transporter for cholesterol efflux from macrophages (Du et al., 2015). Treatment of THP-1derived macrophages with falcarindiol increased ABCA1 protein expression (Figure 2). In contrast, the expression level of the other two cholesterol transmembrane transporters, SR-B1 and ABCG1, remained unchanged. In THP-1 macrophages, SR-B1 was shown to be co-expressed with caveolin-1 and to enhance selectively cholesterol ester uptake rather than cholesterol efflux (Matveev et al., 1999). Interestingly, both ABCA1 and ABCG1 proteins seem to be tightly regulated by PPAR $\gamma$ as shown in studies using full agonists of PPAR $\gamma$ (Chawla et al., 2001; Ozasa et al., 2011). Falcarindiol, in contrast, works as a partial PPAR $\gamma$ agonist in HEK-293 cells (Atanasov et al., 2013). Thus, the fact that the abundance of ABCG1 protein did not significantly increase in response to falcarindiol might be explained by the partial agonism of the compound. The contribution of PPAR $\gamma$ to falcarindiol-induced ABCA1 protein expression was demonstrated by co-treatment experiment with BADGE, a PPAR $\gamma$ antagonist (Figure 3). Numerous reports 
A

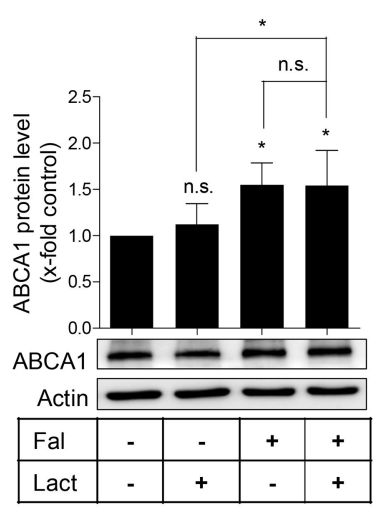

B

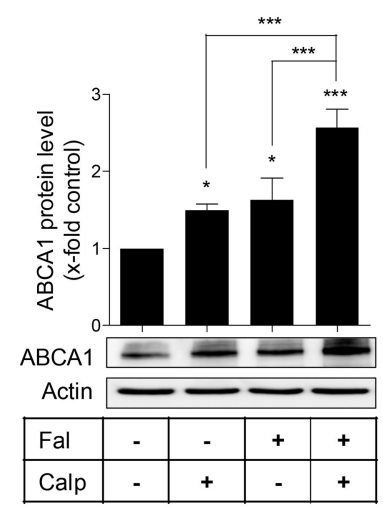

C

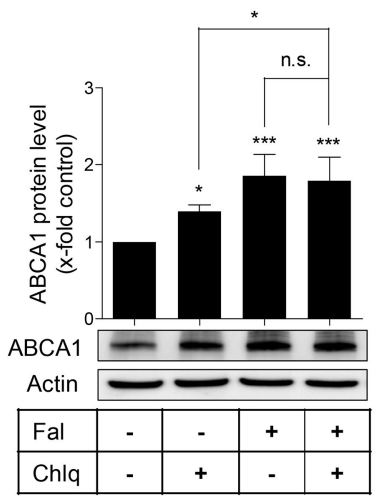

FIGURE 5 | Effect of specific inhibitors of the proteasome (A) calpain (B), and lysosomal (C) protein degradation pathways on ABCA1 protein level in the presence or absence of falcarindiol. Differentiated THP-1 macrophages were pre-treated with or without falcarindiol (Fal) at $10 \mu \mathrm{M}$ for $24 \mathrm{~h}$ and incubated for another $3 \mathrm{~h}$ with the proteasome inhibitor lactacystin (Lact) at $10 \mu \mathrm{M}$ (A), the calpain inhibitor calpeptin (Calp) at $30 \mu \mathrm{g} / \mathrm{mL}$ (B), or the lysosomal inhibitor chloroquine (Chlq) at $100 \mu \mathrm{M}$ (C). Cells were lysed and protein was resolved via SDS-PAGE. Western blot analysis was used to monitor ABCA1 protein level under the indicated treatment conditions. All data are mean $\pm S D(n=4)$ and vs. solvent vehicle control (DMSO): ${ }^{*} p<0.05,{ }^{* \star \star} p<0.001$, n.s. not significant (one-way ANOVA/Bonferroni).
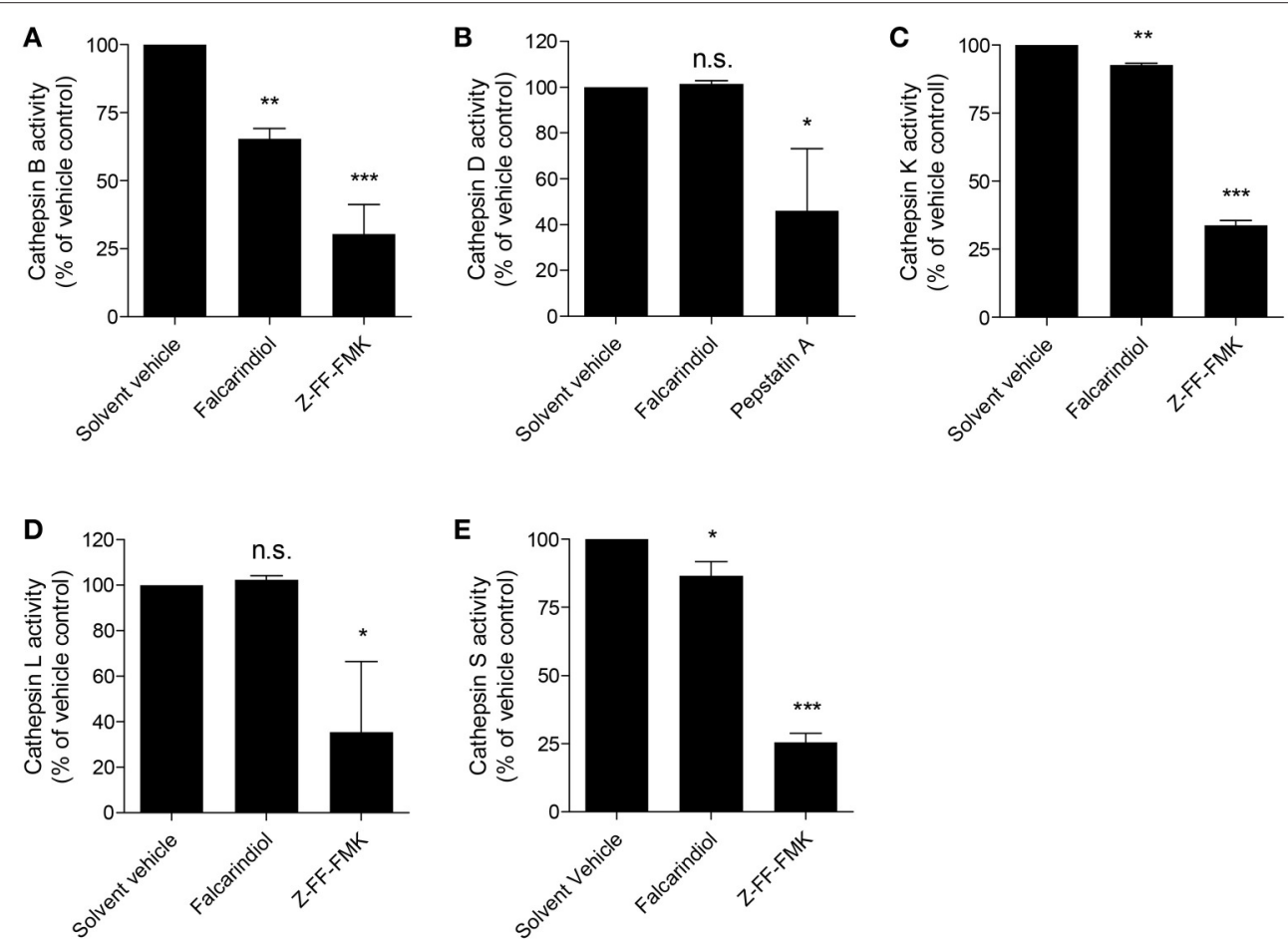

FIGURE 6 | Falcarindiol inhibits several lysosomal cathepsins. (A-E) Differentiated THP-1 macrophages were incubated for $24 \mathrm{~h}$ with or without falcarindiol (10 $\mu$ M). Cells were lysed and cathepsin activity ( $, B, L, K$, and D) was determined by fluorescence measurements. Irreversible inhibitors of cathepsin B, S, L, and K (Z-FF-FMK; $1 \mu \mathrm{M}$ ) and cathepsin D (pepstatin A; $10 \mu \mathrm{M}$ ) were used as positive controls and directly added to the control lysates. All data are mean $\pm S D(n=3)$ and vs. solvent vehicle control (DMSO): ${ }^{\star} p<0.05,{ }^{\star \star} p<0.01,{ }^{\star \star \star} p<0.001$, n.s. not significant (one way-ANOVA/Bonferroni).

suggest a synergistic effect between ABCA1 and ABCG1 in the context of cholesterol efflux (Gelissen et al., 2006; Hsieh et al., 2014). Although they exhibited a similar degradation rate in CHO cells, ABCG1 degradation seems to work by different degradation mechanisms than that of ABCA1. In addition, there are differences in the protein stability of two ABCG1 isoforms (Gelissen et al., 2010).

We also demonstrate that falcarindiol inhibits protein degradation of ABCA1 in the presence of cycloheximide, a de novo protein synthesis inhibitor (Figure 4). Several proteolytic 
pathways have been described to mediate ABCA1 degradation in different cell types, particularly the calpain-, lysosome-, and proteasome-mediated pathways (Wang et al., 2003; Mizuno et al., 2011; Liu and Tang, 2012; Yokoyama et al., 2012). Applying specific inhibitors of these three proteolytic pathways, we found that falcarindiol mimics the effect of lysosomal proteolysis inhibitor chloroquine on ABCA1 levels (Figure 5).

Cathepsins are well-established lysosomal proteases (Turk et al., 2012). A variety of cathepsins (e.g., cathepsin B, D, $\mathrm{K}, \mathrm{L}, \mathrm{S}$ ) are known to be expressed and to serve important regulatory functions in macrophages (Punturieri et al., 2000; Beers et al., 2003; Bracke et al., 2005; Vasiljeva et al., 2006). Measuring the activity of lysosomal cathepsins known to be expressed in macrophages revealed that falcarindiol simultaneously suppresses the activity of cathepsin B, S, and K. Among these three cathepsins, cathepsin $S$ and $K$ were proposed to reduce cholesterol efflux from macrophages (Lindstedt et al., 2003; Lutgens et al., 2007) and knockout of cathepsin S or K genes was shown to lead to decreased atherosclerosis in mice (Sukhova et al., 2003; Lutgens et al., 2006). Cathepsin B was also found to be highly expressed and active in murine atherosclerotic lesions (Chen et al., 2002; Lutgens et al., 2007). The potential of cathepsin $\mathrm{B}, \mathrm{S}$, and $\mathrm{K}$ to influence $\mathrm{ABCA} 1$ protein level regulation further supports the concept for their implication in atherosclerosis. To the best of our knowledge, interference of falcarindiol with cathepsins or with lysosomal function was never reported before.

In summary, our results demonstrate for the first time that falcarindiol is able to increase macrophage cholesterol efflux. Falcarindiol upregulates ABCA1 protein level by promoting its $\operatorname{PPAR} \gamma$-driven gene expression and by extending its protein halflife. Moreover, our findings reveal the inhibition of lysosomal proteases as a new possible mechanism of action of this important dietary constituent. These data advance our knowledge for the molecular mechanisms, by which small molecules regulate

\section{REFERENCES}

Appelqvist, H., Waster, P., Kagedal, K., and Ollinger, K. (2013). The lysosome: from waste bag to potential therapeutic target. J. Mol. Cell Biol. 5, 214-226. doi: $10.1093 / \mathrm{jmcb} / \mathrm{mjt} 022$

Arakawa, R., Tsujita, M., Iwamoto, N., Ito-Ohsumi, C., Lu, R., Wu, C. A., et al. (2009). Pharmacological inhibition of ABCA1 degradation increases HDL biogenesis and exhibits antiatherogenesis. J. Lipid Res. 50, 2299-2305. doi: 10.1194/jlr.M900122-JLR200

Atanasov, A. G., Blunder, M., Fakhrudin, N., Liu, X., Noha, S. M., Malainer, C., et al. (2013). Polyacetylenes from Notopterygium incisum-new selective partial agonists of peroxisome proliferator-activated receptor-gamma. Plos ONE 8:e61755. doi: 10.1371/journal.pone.0061755

Atanasov, A. G., Waltenberger, B., Pferschy-Wenzig, E. M., Linder, T., Wawrosch, C., Uhrin, P., et al. (2015). Discovery and resupply of pharmacologically active plant-derived natural products: a review. Biotechnol. Adv. 33, 1582-1614. doi: 10.1016/j.biotechadv.2015.08.001

Beers, C., Honey, K., Fink, S., Forbush, K., and Rudensky, A. (2003). Differential regulation of cathepsin $\mathrm{S}$ and cathepsin $\mathrm{L}$ in interferon gamma-treated macrophages. J. Exp. Med. 197, 169-179. doi: 10.1084/jem.20020978

Bracke, K., Cataldo, D., Maes, T., Gueders, M., Noel, A., Foidart, J. M. et al. (2005). Matrix metalloproteinase-12 and cathepsin D expression in pulmonary macrophages and dendritic cells of cigarette smoke-exposed mice. Int. Arch. Allergy Immunol. 138, 169-179. doi: 10.1159/000088439 macrophage cholesterol efflux, and might have implication for the development of novel therapeutics or dietary supplements for the prevention or treatment of CVD.

\section{CONCLUSION}

In the present study, we demonstrated that falcarindiol enhances cholesterol efflux and increases ABCA1 protein level by two complementary mechanisms, i.e., promoting ABCA1 gene expression and inhibiting ABCA1 protein degradation.

\section{AUTHOR CONTRIBUTIONS}

LW: Performed most of the experiments, analyzed the results, and drafted the manuscript. VP and NS: Conducted the experiments concerning the expression of transporters. AA and VD: Supervised the study. All authors revised the manuscript.

\section{ACKNOWLEDGMENTS}

The authors acknowledge the financial support by the Austrian Science Fund (FWF) project (P25971-B23), the Vienna Anniversary Foundation for Higher Education (Hochschuljubiläumsstiftung der Stadt Wien) project (H297332/2014), the Polish KNOW (Leading National Research Centre) Scientific Consortium "Healthy Animal-Safe Food," decision of Ministry of Science and Higher Education No. 05-1/KNOW2/2015, the Qingdao Applied Basic Research on Innovation Program (17-1-1-53-jch), and the Qingdao University Research Start-up Fee for Zhuoyue Talents (41117010020). The authors also would like to thank Dr. Xin Liu (Institute of Pharmaceutical Sciences, Department of Pharmacognosy, Karl-Franzens-University Graz) for the assistance in the execution of this project.
Chawla, A., Boisvert, W. A., Lee, C. H., Laffitte, B. A., Barak, Y., Joseph, S. B., et al. (2001). A PPAR gamma-LXR-ABCA1 pathway in macrophages is involved in cholesterol efflux and atherogenesis. Mol. Cell. 7, 161-171. doi: 10.1016/S1097-2765(01)00164-2

Chen, J., Tung, C. H., Mahmood, U., Ntziachristos, V., Gyurko, R., Fishman, M. C., et al. (2002). In vivo imaging of proteolytic activity in atherosclerosis. Circulation 105, 2766-2771. doi: 10.1161/01.CIR.0000017860.20619.23

Chinetti, G., Lestavel, S., Bocher, V., Remaley, A. T., Neve, B., Torra, I. P., et al. (2001). PPAR- $\alpha$ and PPAR- $\gamma$ activators induce cholesterol removal from human macrophage foam cells through stimulation of the ABCA1 pathway. Nat. Med. 7, 53-58. doi: 10.1038/83348

Du, X. M., Kim, M. J., Hou, L. M., Le Goff, W., Chapman, M. J., Van Eck, M., et al. (2015). HDL particle size is a critical determinant of ABCA1mediated macrophage cellular cholesterol export. Circ. Res. 116, 1133-1142. doi: 10.1161/CIRCRESAHA.116.305485

Duffy, D., and Rader, D. J. (2006). Emerging therapies targeting high-density lipoprotein metabolism and reverse cholesterol transport. Circulation 113, 1140-1150. doi: 10.1161/CIRCULATIONAHA.105.593855

Fielding, C. J., and Fielding, P. E. (1995). Molecular physiology of reverse cholesterol transport. J. Lipid Res. 36, 211-228.

Garrod, B., Lewis, B. G., and Coxon, D. T. (1978). Cis-Heptadeca-1,9Diene-4,6-Diyne-3,8-Diol, an antifungal polyacetylene from carrot roottissue. Physiol. Plant. Pathol. 13, 241-246. doi: 10.1016/0048-4059(78)90 $039-5$ 
Gelissen, I. C., Cartland, S., Brown, A. J., Sandoval, C., Kim, M., Dinnes, D. L., et al. (2010). Expression and stability of two isoforms of ABCG1 in human vascular cells. Atherosclerosis 208, 75-82. doi: 10.1016/j.atherosclerosis.2009.06.028

Gelissen, I. C., Harris, M., Rye, K. A., Quinn, C., Brown, A. J., Kockx, M., et al. (2006). ABCA1 and ABCG1 synergize to mediate cholesterol export to apoA-I. Arterioscler. Thromb. Vasc. Biol. 26, 534-540. doi: 10.1161/01.ATV.0000200082.58536.e1

Hinz, B., Brune, K., and Pahl, A. (2003). 15-Deoxy- $\Delta^{(12,14)}$-prostaglandin J2 inhibits the expression of proinflammatory genes in human blood monocytes via a PPAR- $\gamma$-independent mechanism. Biochem. Biophys. Res. Commun. 302, 415-420. doi: 10.1016/S0006-291X(03)00195-5

Hsieh, V., Kim, M. J., Gelissen, I. C., Brown, A. J., Sandoval, C., Hallab, J. C., et al. (2014). Cellular cholesterol regulates ubiquitination and degradation of the cholesterol export proteins ABCA1 and ABCG1. J. Biol. Chem. 289, 7524-7536. doi: 10.1074/jbc.M113.515890

Kemp, M. S. (1978). Falcarindiol - antifungal polyacetylene from Aegopodiumpodagraria. Phytochemistry 17, 1002-1002. doi: 10.1016/S00319422(00)88669-0

Lindstedt, L., Lee, M., Oorni, K., Bromme, D., and Kovanen, P. T. (2003). Cathepsins $\mathrm{F}$ and S block HDL3-induced cholesterol efflux from macrophage foam cells. Biochem. Biophys. Res. Commun. 312, 1019-1024. doi: 10.1016/j.bbrc.2003.11.020

Liu, X., Kunert, O., Blunder, M., Fakhrudin, N., Noha, S. M., Malainer, C., et al. (2014). Polyyne hybrid compounds from Notopterygium incisum with peroxisome proliferator-activated receptor gamma agonistic effects. J. Nat. Prod. 77, 2513-2521. doi: 10.1021/np500605v

Liu, Y. H., and Tang, C. R. (2012). Regulation of ABCA1 functions by signaling pathways. Biochim. Biophys. Acta 1821, 522-529. doi: 10.1016/j.bbalip.2011.08.015

Lutgens, E., Lutgens, S. P., Faber, B. C., Heeneman, S., Gijbels, M. M., de Winther, M. P., et al. (2006). Disruption of the cathepsin K gene reduces atherosclerosis progression and induces plaque fibrosis but accelerates macrophage foam cell formation. Circulation 113, 98-107. doi: 10.1161/CIRCULATIONAHA.105.561449

Lutgens, S. P., Cleutjens, K. B., Daemen, M. J., and Heeneman, S. (2007). Cathepsin cysteine proteases in cardiovascular disease. FASEB J. 21, 3029-3041. doi: 10.1096/fj.06-7924com

Matveev, S., van der Westhuyzen, D. R., and Smart, E. J. (1999). Co-expression of scavenger receptor-BI and caveolin-1 is associated with enhanced selective cholesteryl ester uptake in THP-1 macrophages. J. Lipid Res. 40, 1647-1654.

Mizuno, T., Hayashi, H., Naoi, S., and Sugiyama, Y. (2011). Ubiquitination is associated with lysosomal degradation of cell surface-resident atpbinding cassette transporter al (abcal) through the endosomal sorting complex required for transport (escrt) pathway. Hepatology 54, 631-643. doi: 10.1002/hep. 24387

Neufeld, E. B., Remaley, A. T., Demosky, S. J., Stonik, J. A., Cooney, A. M., Comly, M., et al. (2001). Cellular localization and trafficking of the human ABCA1 transporter. J. Biol. Chem. 276, 27584-27590. doi: 10.1074/jbc.M103264200

Ozasa, H., Ayaori, M., Iizuka, M., Terao, Y., Uto-Kondo, H., Yakushiji, E., et al. (2011). Pioglitazone enhances cholesterol efflux from macrophages by increasing ABCA1/ABCG1 expressions via PPAR $\gamma / \mathrm{LXR} \alpha$ pathway: findings from in vitro and ex vivo studies. Atherosclerosis 219, 141-150. doi: 10.1016/j.atherosclerosis.2011.07.113

Punturieri, A., Filippov, S., Allen, E., Caras, I., Murray, R., Reddy, V., et al. (2000). Regulation of elastinolytic cysteine proteinase activity in normal and cathepsin K-deficient human macrophages. J. Exp. Med. 192, 789-799. doi: 10.1084/jem.192.6.789

Rohatgi, A., Khera, A., Berry, J. D., Givens, E. G., Ayers, C. R., Wedin, K. E., et al. (2014). HDL cholesterol efflux capacity and incident cardiovascular events. New Engl. J. Med. 371, 2383-2393. doi: 10.1056/NEJMoa1409065

Rosenson, R. S., Brewer, H. B., Davidson, W. S., Fayad, Z. A., Fuster, V., Goldstein, J., et al. (2012). Cholesterol efflux and atheroprotection advancing the concept of reverse cholesterol transport. Circulation 125, 1905-1919. doi: 10.1161/CIRCULATIONAHA.111.066589

Santamarina-Fojo, S., Remaley, A. T., Neufeld, E. B., and Brewer, H. B. (2001). Regulation and intracellular trafficking of the ABCA1 transporter. J. Lipid Res. 42, 1339-1345.

Stangl, H., Hyatt, M., and Hobbs, H. H. (1999). Transport of lipids from high and low density lipoproteins via scavenger receptor-BI. J. Biol. Chem. 274, 32692-32698. doi: 10.1074/jbc.274.46.32692

Sukhova, G. K., Zhang, Y., Pan, J. H., Wada, Y., Yamamoto, T., Naito, M., et al. (2003). Deficiency of cathepsin S reduces atherosclerosis in LDL receptordeficient mice. J. Clin. Invest. 111, 897-906. doi: 10.1172/JCI200314915

Turk, V., Stoka, V., Vasiljeva, O., Renko, M., Sun, T., Turk, B., et al. (2012). Cysteine cathepsins: from structure, function and regulation to new frontiers. Biochim. Biophys. Acta 1824, 68-88. doi: 10.1016/j.bbapap.2011.10.002

Vasiljeva, O., Papazoglou, A., Krueger, A., Brodoefel, H., Korovin, M., Deussing, J., et al. (2006). Tumor cell-derived and macrophage-derived cathepsin B promotes progression and lung metastasis of mammary cancer. Cancer Res. 66, 5242-5250. doi: 10.1158/0008-5472.CAN-05-4463

Wang, L., Rotter, S., Ladurner, A., Heiss, E. H., Oberlies, N. H., Dirsch, V. M., et al. (2015). Silymarin constituents enhance ABCA1 expression in THP-1 macrophages. Molecules 21:e55. doi: 10.3390/molecules21010055

Wang, N., Chen, W. G., Linsel-Nitschke, P., Martinez, L. O., Agerholm-Larsen, B., Silver, D. L., et al. (2003). A PEST sequence in ABCA1 regulates degradation by calpain protease and stabilization of ABCA1 by apoA-I. J. Clin. Invest. 111, 99-107. doi: 10.1172/JCI200316808

Yokoyama, S., Arakawa, R., Wu, C., Iwamoto, N., Lu, R., Tsujita, M., et al. (2012). Calpain-mediated ABCA1 degradation: post-translational regulation of ABCA1 for HDL biogenesis. Biochim. Biophys. Acta 1821, 547-551. doi: 10.1016/j.bbalip.2011.07.017

Zidorn, C., Johrer, K., Ganzera, M., Schubert, B., Sigmund, E. M., Mader, J., et al. (2005). Polyacetylenes from the Apiaceae vegetables carrot, celery, fennel, parsley, and parsnip and their cytotoxic activities. J. Agric. Food Chem. 53, 2518-2523. doi: 10.1021/jf048041s

Zschocke, S., Lehner, M., and Bauer, R. (1997). 5-lipoxygenase and cyclooxygenase inhibitory active constituents from Qianghuo (Notopterygium incisum). Planta Med. 63, 203-206. doi: 10.1055/s-2006-957653

Conflict of Interest Statement: The authors declare that the research was conducted in the absence of any commercial or financial relationships that could be construed as a potential conflict of interest.

Copyright (c) 2017 Wang, Palme, Schilcher, Ladurner, Heiss, Stangl, Bauer, Dirsch and Atanasov. This is an open-access article distributed under the terms of the Creative Commons Attribution License (CC BY). The use, distribution or reproduction in other forums is permitted, provided the original author(s) or licensor are credited and that the original publication in this journal is cited, in accordance with accepted academic practice. No use, distribution or reproduction is permitted which does not comply with these terms. 


\title{
A Multidirectional Perspective for Novel Functional Products: In vitro Pharmacological Activities and In silico Studies on Ononis natrix subsp. hispanica
}

\author{
Serife Yerlikaya ${ }^{1 *}$, Gokhan Zengin ${ }^{2 *}$, Adriano Mollica ${ }^{3}$, Mehmet C. Baloglu ${ }^{1}$, \\ Yasemin Celik Altunoglu ${ }^{1}$ and Abdurrahman Aktumsek ${ }^{2}$ \\ ${ }^{1}$ Department of Genetics and Bioengineering, Faculty of Engineering and Architecture, Kastamonu University, Kastamonu, \\ Turkey, ${ }^{2}$ Department of Biology, Science Faculty, Selcuk University, Konya, Turkey, ${ }^{3}$ Department of Pharmacy University \\ "G. d'Annunzio" of Chieti-Pescara, Chieti, Italy
}

OPEN ACCESS

Edited by:

Atanas G. Atanasov Institute of Genetics and Animal

Breeding (PAN), Poland

Reviewed by:

M. Babak Bahadori,

Tabriz University of Medical Sciences,

Kannan R. R. Rengasamy, China Agricultural University, China

*Correspondence: Serife Yerlikaya serifeyerlikaya@gmail.com Gokhan Zengin

gokhanzengin@selcuk.edu.tr

Specialty section:

This article was submitted to

Ethnopharmacology,

a section of the journal

Frontiers in Pharmacology

Received: 08 July 2017

Accepted: 21 August 2017 Published: 01 September 2017

Citation:

Yerlikaya S, Zengin G, Mollica A, Baloglu MC, Celik Altunoglu Y and Aktumsek A (2017) A Multidirectional

Perspective for Novel Functional

Products: In vitro Pharmacological Activities and In silico Studies on

Ononis natrix subsp. hispanica.

Front. Pharmacol. 8:600

doi: 10.3389/fphar.2017.00600
The genus Ononis has important value as traditional drugs and foods. In the present work, we aimed to assess the chemical profiles and biological effects of Ononis natrix subsp. hispanica extracts (ethyl acetate, methanol, and water). For chemical profile, total and individual phenolic components were detected. For biological effects, antioxidant (DPPH, ABTS, CUPRAC, FRAP, phosphomolybdenum, and metal chelating assays), enzyme inhibitory (against cholinesterase, tyrosinase, $\alpha$-amylase and $\alpha$-glucosidase), antimicrobial, DNA protection and cytotoxic abilities were tested. The predominant phenolics were apigenin, luteolin, and quercetin in the tested extracts. Generally, the ethyl acetate and methanol extracts were noted as the most active in the antioxidant and enzyme inhibitory assays. Water extract with different concentrations indicated high level of DNA protection activity. Methanol and ethyl acetate extracts showed antibacterial effect against to Staphylococcus aureus and Staphylococcus epidermidis strains. The cytotoxic effects of $O$. natrix subsp. hispanica extracts on the survival of HeLa and PC3 cells were determined by MTT cell viability assay. Water and methanol extracts caused initiation of apoptosis for PC3 cell line. Furthermore, molecular docking was performed to better understand interactions between dominant phenolic compounds and selected enzymes. Our results clearly indicate that $O$. natrix subsp. hispanica could be considered a potential candidate for designing novel pharmaceuticals, cosmeceuticals and nutraceuticals.

Keywords: Ononis, bioactive compounds, natural agents, pharmaceuticals, molecular docking

\section{INTRODUCTION}

In the twenty first century, the usage of plant or plant products are undergoing a revolution as sources of prominent (functional and bioactive) compounds. For example, artemisinin was awarded as an anti-malarial compound in Nobel Prize for Medicine 2015 (Omura and Campbell, 2015). From this point, to better combat the lifestyle, communicable or infectious diseases, many phytochemicals have been suggested as antioxidant, antimicrobial, anticancer 
and anti-mutagenic agents (Fernandes et al., 2017; LosadaBarreiro and Bravo-Díaz, 2017; Meeran et al., 2017; Yin et al., 2017). These facts provide encouragement for designing further studies on the plants. Within these perspectives, the term of functional products has emerged and it is defined as any products which provide health benefits including treatment of afore-mentioned diseases. In this framework, uninvestigated wild plants could be considered as a springboard for designing novel functional products with low toxicity.

The genus Ononis (Fabaceae) is represented by 75 species in the world especially Europe and Central Asia (Wollenweber et al., 2003). The genus comprises of 23 taxa in Tukey and known as "semisk" and "kayışkıran" in Turkish name (Baytop, 1999; Sohretoglu, 2007; Mükemre et al., 2015). In the literature, several studies were reported that member of the genus Ononis exhibited significant biological activities, such as antimicrobial effect (Mhamdi et al., 2015; Sayari et al., 2016), antioxidant (Mhamdi et al., 2015; Mezrag et al., 2017), wound healing (Süntar et al., 2011), cytotoxic (Kuete et al., 2013; Ghribi et al., 2016), and analgesic (Yõlmaz et al., 2006). In addition, the decoctions from Ononis species is used for treating urinary problems, skin disorders as well as gout (Baytop, 1999; Erdemgil et al., 2002; Liebezeit, 2008). With this in mind, Ononis species have been sold as herbal tea on the market as single or combined with other diuretic plants (Gampe et al., 2016). Several publications have appeared in recent years documenting on biological activities of some Ononis species along with their phytochemicals profiles (Daruházi et al., 2008; Al-Rehaily et al., 2014; Ghribi et al., 2015; Yousaf et al., 2015; Gampe et al., 2016; Casiglia et al., 2017; Mezrag et al., 2017). However, to the best of our knowledge, there is no report on the chemical profile and biological ability of Ononis natrix subsp. hispanica. Thus, the present study aims to provide more details about chemical and biological properties of $O$. natrix subsp. hispanica. The observed findings could be provide new insights for $O$. natrix subsp. hispanica.

\section{MATERIALS AND METHODS}

\section{Plant Material and Preparation of the Extracts}

Ononis natrix subsp. hispanica was collected from AntalyaTurkey during of flowering season (June 2015). Taxonomic identification of the plant material was confirmed by the senior taxonomist Dr. Murad Aydin Sanda, from the Department of Biology, Selcuk University. The voucher specimen has been deposited at the Herbarium of the Department of Biology, Selcuk University, Konya-Turkey.

To obtain ethyl acetate and methanol extracts, the air-dried aerial parts $(10 \mathrm{~g})$ were macerated with $200 \mathrm{~mL}$ of these solvents at room temperature $\left(25^{\circ} \pm 1^{\circ} \mathrm{C}\right)$ for $24 \mathrm{~h}$. The extracts were concentrated under vacuum at $40^{\circ} \mathrm{C}$ by using a rotary evaporator. To obtain water extract, the powdered samples were boiled with $250 \mathrm{~mL}$ of distilled water for $30 \mathrm{~min}$. The aqueous extract was filtered, lyophilized $\left(-80^{\circ} \mathrm{C}, 48 \mathrm{~h}\right)$, and all extracts stored at + $4^{\circ} \mathrm{C}$ in the dark until use.

\section{Total Phenolics, Flavonoids, and Phenolic Composition}

The total phenolics content was determined by Folin-Ciocalteu method (Slinkard and Singleton, 1977) with slight modification and expressed as gallic acid equivalents (GAE/g extract), while total flavonoids content was determined using $\mathrm{AlCl}_{3}$ method (Zengin et al., 2014) with slight modification and expressed as rutin equivalents ( $\mathrm{RE} / \mathrm{g}$ extract).

Phenolic compounds were evaluated by RP-HPLC (Shimadzu Scientific Instruments, Tokyo, Japan). Detection and quantification were carried out with a LC-10ADvp pump, a Diode Array Detector, a CTO-10Avp column heater, SCL-10Avp system controller, DGU-14A degasser and SIL-10ADvp auto sampler (Shimadzu Scientific Instruments, Columbia, MD). Separations were conducted at $30^{\circ} \mathrm{C}$ on Agilent ${ }^{\circledR}$ Eclipse XDB C-18 reversed-phase column $(250 \times 4.6 \mathrm{~mm}$ length, 5 $\mu \mathrm{m}$ particle size). Phenolic compositions of the extracts were determined by a modified method of Zengin et al. (2014). Gallic acid, protocatechuic acid, (+)-catechin, p-hydroxybenzoic acid, chlorogenic acid, caffeic acid, (-)-epicatechin, syringic acid, vanillin, $p$-coumaric acid, ferulic acid, sinapic acid, benzoic acid, $o$-coumaric acid, rutin, hesperidin, rosmarinic acid, eriodictyol, trans-cinnamic acid, quercetin, luteolin, kaempferol, and apigenin were used as standards. Identification and quantitative analysis were done by comparison with standards. The amount of each phenolic compound was expressed as microgram per gram of extract using external calibration curves, which were obtained for each phenolic standard.

\section{Antioxidant and Enzyme Inhibitory Assays}

Antioxidant [DPPH, ABTS radical scavenging, reducing power (CUPRAC and FRAP), phosphomolybdenum and metal chelating (ferrozine method) and enzyme inhibitory assays (cholinesterase (Elmann's method), tyrosinase (dopachrome method), $\alpha$-amylase (iodine/potassium iodide method) and $\alpha$-glucosidase (chromogenic PNPG method)] were performed according to our previous researches (Zengin et al., 2014; Zengin, 2016). Antioxidant capacities were expressed as equivalents of trolox and EDTA (for metal chelating). In addition, the enzyme inhibitory activities of the extracts were evaluated as equivalents of standard inhibitors per gram of the plant extract (galantamine for AChE and BChE, kojic acid for tyrosinase, and acarbose for $\alpha$-amylase and $\alpha$-glucosidase inhibition assays).

\section{Molecular Modelling}

\section{Receptors Preparation}

The crystalline structure of the selected enzymes together with their inhibitors have been downloaded from the Protein Databank RCSB PDB (Berman et al., 2000): acetylcholinesterase (pdb:4X3C) (Pesaresi and Lamba, in press) in complex with tacrine-nicotinamide hybrid inhibitor, butyrilcholinesterase (pdb:4BDS) (Nachon et al., 2013) in complex with tacrine, amylase (pdb:1VAH) (Zhuo et al., 2004) in complex with rnitrophenyl- $\alpha$-D-maltoside, glucosidase (pdb:3AXI) (Yamamoto et al., 2011) in complex with maltose and tyrosinase (pdb:2Y9X) (Ismaya et al., 2011) in complex with tropolone. The raw crystal structures were prepared for the docking experiments as 
previously reported (Mocan et al., 2016; Uysal et al., 2016). Noncatalytic water molecules, inhibitors and all the other molecules present in the pdb files were removed by using UCSF Chimera (DeLano, 2002) and the proteins alone were neutralized at $\mathrm{pH}$ 7.4 by PropKa implemented in Maestro 10.2 suite (Maestro, 2015). Seleno-cysteines and seleno-methionines, if present, were converted to cysteines and methionines, respectively. All the missing fragments and other errors present in the crystal structures were automatically solved by the Wizard Protein Preparation implemented in Maestro 10.2 suite (Maestro, 2015).

\section{Ligands Preparation}

$(+)$-Epicatechin, apigenin, luteolin, quercetin and rosmarinic acid were selected as representative compounds to carry out molecular docking study, as these compounds were present in abundance in the herbs extracts. The chemical structure the selected compounds are reported in Figure 1. The three dimensional structures have been downloaded from Zinc databases (Irwin et al., 2012) and used for molecular modeling experiments after preparation. The ligands were prepared by the LigPrep tool embedded in Maestro 10.2, neutralized at $\mathrm{pH} 7.4$ by Ionizer and minimized by OPLS3 force field (Shelley et al., 2007).

\section{Molecular Docking}

Dockings of the representative substances have been performed for each selected enzyme employed for the in vitro enzymatic inhibition tests in this work. Glide embedded in the maestro suite 10.2 has been employed for the docking calculations by using the "eXtra Precision" scoring function for all the enzymes and the mm-GBSA binding energy has been calculated by the use of Prime embedded in maestro 10.2 (Table 1; Jones et al., 1997). In all cases, the binding pocket was determined automatically by centering the grid on the crystallographic inhibitor, extended in a radius of 10 Angstroms from the center. The best pose for each compound docked to the selected enzymes was the best ranked among the 10,000 generated.

\section{DNA Protection}

DNA protection activity of the studied extracts was analyzed using pUC19 plasmid DNA (pDNA). Plasmid isolation was performed by Thermo Scientific Genejet Plasmid Miniprep Kit. The reaction mixture contained $5 \mu \mathrm{L}$ of Fenton's reagent $(30 \mathrm{mM}$ $\mathrm{H}_{2} \mathrm{O}_{2}, 50 \mathrm{mM}$ ascorbic acid, and $\left.80 \mathrm{mM} \mathrm{FeCI}_{3}\right), 5 \mu \mathrm{L}$ of these extracts at two different concentrations (5 and $10 \mathrm{mg} / \mathrm{mL}$ ) and $3 \mu \mathrm{L}$ of pDNA $(300 \mu \mathrm{g} / \mu \mathrm{L})$. Final volume of reaction mixture was brought up to $20 \mu \mathrm{L}$ using double-distilled water. Positive control was composed of $12 \mu \mathrm{L}$ of distilled water, $5 \mu \mathrm{L}$ of Fenton's reagent and $3 \mu \mathrm{L}$ of pDNA. Negative control involved only $17 \mu \mathrm{L}$ of distilled water and $3 \mu \mathrm{L}$ of pDNA. Samples were incubated for $30 \mathrm{~min}$ at $37^{\circ} \mathrm{C}$ and $4 \mu \mathrm{L}$ loading dye (Thermo Scientific, USA) was added to the all mixtures. The DNA mixtures were run on $0.8 \%$ agarose gel and then visualized under ultraviolet light cabin. Biological replication of test was carried out at three times and band density was determined by the gel image analysis software (Quantum, Vision-Capt., Vilber Lourmat SAS, France) (Ozkan et al., 2015).

\section{Cytotoxic Evaluation \\ Cell Culture Materials}

HeLa and PC3 cancer cell lines were obtained from Selcuk University, Faculty of Science, and Department of Biochemistry. Penicillin/Streptomycin, EMEM cell culture media, Ham's F12 cell culture media, Fetal bovine serum (FBS), trypsin, MTT, ethanol, 2-prophanol, $60 \times 15 \mathrm{~mm}$ corning plates and $75 \mathrm{~cm}^{2}$<smiles>O=c1cc(-c2ccc(O)cc2)oc2cc(O)cc(O)c12</smiles><smiles>O=c1cc(-c2cc(O)cc(O)c2)oc2cc(O)cc(O)c12</smiles><smiles>O=c1c(O)c(-c2ccc(O)c(O)c2)oc2cc(O)cc(O)c12</smiles>

FIGURE 1 | Chemical structure of apigenin, luteolin and quercetin.

TABLE 1 | Binding energy and glide XP docking scores.

\begin{tabular}{|c|c|c|c|c|c|c|c|c|c|c|}
\hline \multirow[t]{2}{*}{ Compounds } & \multicolumn{2}{|c|}{$\mathrm{AChE}^{\star}$} & \multicolumn{2}{|c|}{ BChE* } & \multicolumn{2}{|c|}{$\alpha$-amylase* } & \multicolumn{2}{|c|}{$\alpha$-glucosidase* } & \multicolumn{2}{|c|}{ Tyrosinase* } \\
\hline & $\mathbf{X P}$ & $\Delta \mathbf{G}$ & XP & $\Delta \mathbf{G}$ & XP & $\Delta \mathbf{G}$ & $\mathbf{X P}$ & $\Delta \mathbf{G}$ & $\mathbf{X P}$ & $\Delta \mathbf{G}$ \\
\hline Apigenin & -9.6 & -60.6 & -9.4 & -52.5 & -5.98 & -35.2 & -5.7 & -17.2 & -5.7 & -26.1 \\
\hline Benzoic A. & -4.0 & -14.2 & -4.9 & -22.3 & -3.88 & -0.03 & -2.3 & +71.8 & -5.1 & +41.7 \\
\hline (-)-Epicat. & -9.3 & -41.5 & -7.3 & -41.0 & -7.03 & -38.1 & -6.9 & -21.8 & -5.5 & -20.9 \\
\hline Luteolin & -9.6 & -52.9 & -8.0 & -53.3 & -6.54 & -54.0 & -6.7 & -30.3 & -5.1 & -29.3 \\
\hline Quercetin & -10.3 & -62.9 & -7.9 & -43.5 & -8.79 & -52.9 & -6.9 & -31.2 & -5.4 & -28.3 \\
\hline Rosmarinic A. & -11.3 & -64.5 & -10.9 & -42.8 & -8.26 & -46.8 & -6.5 & -22.6 & -6.3 & -12.2 \\
\hline
\end{tabular}

${ }^{*} \Delta G$ values (Binding energy) are expressed in $\mathrm{Kcal} / \mathrm{mol}$. 
corning flasks were purchased from Sigma-Aldrich (SigmaAldrich, USA).

\section{Cell Culture Maintenance}

Culture media were prepared with 10\% fetal bovine serum (FBS) and $1 \%$ penicillin-streptomycin (Pen-Strep) solution. Prepared medium was kept at $+4^{\circ} \mathrm{C}$ and warmed at $37^{\circ} \mathrm{C}$ before using. Cells (HeLa, PC3) were proliferated in incubator $\left(37^{\circ} \mathrm{C}\right.$ including $5 \% \mathrm{CO}_{2}$ ). Stock cells were proliferated in $75 \mathrm{~cm}^{2}$ sterile corning flasks and experiment cell cultures were proliferated in $60 \times 15$ $\mathrm{mm}$ sterile petri dishes. In the logarithmic phase of the growth (when reached to $\sim 80 \%$ cell confluency), cells were sub-cultured (Karakurt and Adali, 2016).

\section{Preparation of Plant Extraction}

Dilutions were made to obtain extract concentration of $10 \mathrm{mg} / \mathrm{ml}$. The water extract was suspended in PBS and other extracts were suspended in DMSO (Dimethylsulfoxide)/water (1:5) and the diluted extracts were centrifuged at 10,000 $\times$ g during $2 \mathrm{~min}$, and supernatants were filtrated (having the diameter of $0.22 \mu \mathrm{m}$ pore size) and they were kept in $-20^{\circ} \mathrm{C}$.

\section{MTT Cell Viability Test}

A total of 10.000 PC3 and HeLa cells were seeded in 96-well plates and allowed to fasten on the wells for $24 \mathrm{~h}$. After $24 \mathrm{~h}$, cells were treated with different doses of the tested extracts $(0.1$, $1,2.5,5$, and $10 \mathrm{mg}$ for water) $(0.1,1,10,100$, and $1,000 \mu \mathrm{g}$ for ethyl acetate and methanol) for $24 \mathrm{~h}$. After exposure time completed, the medium was changed with EMEM and Ham's F12 medium supplemented with $0.5 \% \mathrm{FBS}+0.5 \mathrm{mg} / \mathrm{ml}$ MTT for HeLa and PC3 cancer cells, respectively. Then, they were incubated at $37^{\circ} \mathrm{C}$ with $5 \% \mathrm{CO}_{2}$, for $4 \mathrm{~h}$. After that time, the cells were treated with $3 \% \mathrm{SDS}+40 \mathrm{mM} \mathrm{HCl} /$ isopropanol for $15 \mathrm{~min}$ in order to dissolve the MTT-formazan crystals (Yerlikaya et al., 2016). The absorbance of each sample was recorded at $570 \mathrm{~nm}$. Cell survival rate was calculated by using GraphPad Prism 3.03 software (GraphPad Software, Inc., La Jolla, CA, USA).

\section{Antimicrobial Activity}

Antimicrobial activity test was performed by disc diffusion method. Nutrient agar medium was used for test microorganisms and microorganisms were refreshed in $100 \mathrm{~mm}$ sterile petri dishes. Single colony of bacterial strain was inoculated in $20 \mathrm{ml}$ nutrient broth medium for $24 \mathrm{~h}$ at $37^{\circ} \mathrm{C}$ incubator. After bacteria concentration was made visible in \%9 sterile $\mathrm{NaCI}$ and its turbidity was standardized to $0.5 \mathrm{McF}$ arland by adding bacterial suspension. Bacterial suspension seeded to $100 \mathrm{~mm}$ petri dishes. After the tested extracts were dissolved in DMSO and saturated on discs, they were incubated at room temperature for drying. They were placed on the inoculated petri plates with microorganisms for $24 \mathrm{~h}$ at $37^{\circ} \mathrm{C}$ incubator. Test was replicated at least three times and results were analyzed by measuring inhibition zone. Microorganisms were illustrated on Table 2.

\section{Statistical Analysis}

All the assays were carried out in triplicate. The results were expressed as mean value and standard deviation (mean \pm
TABLE 2 | Microorganisms used for antimicrobial activity test.

\begin{tabular}{lcc}
\hline Microorganisms & Gram (+) & Gram (-) \\
\hline Klebsiella pneumonia & & $x$ \\
Staphylococcus aureus ATCC 25923 & $x$ & \\
Staphylococcus hominis & $x$ & $x$ \\
Proteus vulgaris & & $X$ \\
Escherichia coli & & $x$ \\
Serratia marcescens & $x$ & \\
Staphylococcus epidermidis & $x$ & \\
Alfa streptococcus haemolyticua & $x$ & \\
Enterococcus faecium & & \\
Pseudomonas aeruginosa & $x$ & \\
Listeria monocytogenes ATCC 7644 & $x$ & $x$ \\
Enterococcus durans & & \\
Salmonella kentucky & & \\
Enterobacter aerogenes ATCC 13048 & & \\
\hline
\end{tabular}

SD). Statistical differences between the extracts were analyzed by using one-way analysis of variance (ANOVA) followed by Tukey's honestly significant difference post-hoc test $(\alpha=$ 0.05). All the analysis was carried out using SPSS v22.0 software.

\section{RESULTS AND DISCUSSION \\ Phytochemical Composition}

In the last decades, many studies highlighted biological activities of phenolic compounds, such as antioxidant, anticancer, antimicrobial, and anti-inflammatory. Flavonoids are the most abundant group of phenolics and considered as natural bioactive agents for designing novel functional products. In this sense, the amounts of total phenolics and flavonoids contents in $O$. natrix extracts were determined by Folin-Ciocalteu and $\mathrm{AlCl}_{3}$ methods. The greatest content of total phenolics was noticed in the ethyl acetate extract (60.19 mgGAE/g extract), followed by methanol (59.22 mgGAE/g extract) and water (35.12 mgGAE/g extract) extracts. Similar results were observed for flavonoid as well (Table 3). These findings were confirmed by several researchers who reported the higher concentration of total phenolics in ethyl acetate and methanol extracts (Do et al., 2014; Murugan and Parimelazhagan, 2014). In a previous study (Mhamdi et al., 2015), the total phenolic content of O. natrix in Tunisia was reported as $51 \mathrm{mgGAE} / \mathrm{g}$, which was lower than that found in the present study. Moreover, some researchers were found to be different levels of total phenolics in several Ononis species, such as O. spinosa (3.09 mgGAE/g extract) (Orhan et al., 2012), O. pubescens (17.23 mgGAE/g extract), and O. ornithopodioides (20.96 mgGAE/g extract) (Sarikürkcü et al., 2016).

Individual phenolic constituents in the tested extracts were analyzed by HPLC-DAD and the results are given in Table 4. A total of 23 standard phenolics were used and 21 of them were identified in these extracts. Two compounds (rutin and hesperidin) were not detected in the extracts. Apigenin was 
TABLE 3 | Total phenolic and flavonoid contents, and free radical scavenging (DPPH and ABTS) activities of the extracts.

\begin{tabular}{|c|c|c|c|c|}
\hline Extracts & Total phenolic content (mgGAE/g extract) & Total flavonoid content (mgRE/g extract) & DPPH (mgTE/g extract) & ABTS (mgTE/g extract) \\
\hline Ethyl acetate & $60.19 \pm 1.67^{\star}$ & $30.07 \pm 0.39$ & $56.60 \pm 1.46$ & $530.37 \pm 0.40$ \\
\hline Methanol & $59.22 \pm 0.09$ & $28.40 \pm 0.27$ & $63.95 \pm 1.45$ & $521.80 \pm 2.90$ \\
\hline Water & $35.12 \pm 0.07$ & $10.25 \pm 0.23$ & $63.38 \pm 1.10$ & $457.92 \pm 2.29$ \\
\hline
\end{tabular}

GAE, gallic acid equivalents; RE, rutin equivalents; TE, trolox equivalents.

*Values expressed are means \pm S.D. of three parallel measurements.

TABLE 4 | Quantitative analysis for determination of phenolic components in the extracts ( $\mu \mathrm{g} / \mathrm{g}$ extract).

\begin{tabular}{|c|c|c|c|c|c|c|c|c|}
\hline \multirow[t]{2}{*}{ No. } & \multirow[t]{2}{*}{ Phenolic compounds } & \multicolumn{3}{|c|}{ Extracts } & \multicolumn{4}{|c|}{ Analytical characteristics } \\
\hline & & Ethyl acetate & Methanol & Water & Linear range (ppm) & $r^{2}$ & LOD (ppm) & LOQ (ppm) \\
\hline 1 & Gallic acid & $8 \pm 0.4$ & $100 \pm 0.8$ & $58 \pm 0.8$ & $0.20-25.0$ & 0.9993 & 0.075 & 0.227 \\
\hline 2 & Protocatechuic acid & $10 \pm 0.4$ & $80 \pm 0.6$ & $234 \pm 8$ & $0.20-25.0$ & 0.9991 & 0.086 & 0.260 \\
\hline 3 & $(+)$-Catechin & nd & $152 \pm 2$ & $492 \pm 20$ & $0.90-113$ & 0.9988 & 0.172 & 0.522 \\
\hline 4 & p-Hydroxybenzoic acid & $44 \pm 4$ & $106 \pm 4$ & $200 \pm 4$ & $0.20-25.0$ & 0.9994 & 0.007 & 0.020 \\
\hline 5 & Chlorogenic acid & $210 \pm 2$ & nd & $166 \pm 2$ & $0.35-45.0$ & 0.9988 & 0.080 & 0.241 \\
\hline 6 & Caffeic acid & $42 \pm 4$ & $34 \pm 4$ & $90 \pm 4$ & $0.16-21.0$ & 0.9993 & 0.054 & 0.162 \\
\hline 7 & (-)-Epicatechin & $310 \pm 16$ & $950 \pm 24$ & nd & $0.50-66.0$ & 0.9990 & 0.170 & 0.514 \\
\hline 8 & Syringic acid & $22 \pm 0.2$ & $66 \pm 4$ & $86 \pm 4$ & $0.05-12.0$ & 0.9995 & 0.030 & 0.090 \\
\hline 9 & Vanillin & $2 \pm 0.1$ & $10 \pm 0.2$ & nd & $0.08-10.0$ & 0.9995 & 0.020 & 0.060 \\
\hline 10 & $p$-coumaric acid & $8 \pm 0.6$ & $34 \pm 2$ & $64 \pm 2$ & $0.04-6.0$ & 0.9996 & 0.066 & 0.199 \\
\hline 11 & Ferulic acid & $8 \pm 0.4$ & $90 \pm 0.4$ & $130 \pm 6$ & $0.12-17.0$ & 0.9993 & 0.004 & 0.011 \\
\hline 12 & Sinapic acid & nd & nd & $54 \pm 2$ & $0.12-17.0$ & 0.9993 & 0.017 & 0.053 \\
\hline 13 & Benzoic acid & $50 \pm 0.6$ & $50 \pm 0.6$ & $1386 \pm 26$ & $0.85-55.0$ & 0.9998 & 0.111 & 0.335 \\
\hline 14 & o-Coumaric acid & $82 \pm 2$ & $82 \pm 2$ & nd & $0.24-32.0$ & 0.9988 & 0.023 & 0.069 \\
\hline 15 & Rutin & nd & nd & nd & $0.40-56.0$ & 0.9989 & 1.113 & 3.373 \\
\hline 16 & Hesperidin & nd & nd & nd & $0.43-55.0$ & 0.9992 & 1.080 & 3.280 \\
\hline 17 & Rosmarinic acid & $76 \pm 2$ & $1960 \pm 22$ & nd & $0.02-7.0$ & 0.9998 & 0.148 & 0.447 \\
\hline 18 & Eriodictyol & nd & nd & $116 \pm 4$ & $0.33-21.0$ & 0.9998 & 0.140 & 0.410 \\
\hline 19 & trans-Cinnamic acid & $18 \pm 1$ & $66 \pm 1.2$ & $10 \pm 1$ & $0.02-7.0$ & 0.9998 & 0.148 & 0.447 \\
\hline 20 & Quercetin & nd & nd & $1356 \pm 42$ & $0.40-55.0$ & 0.9999 & 0.013 & 0.040 \\
\hline 21 & Luteolin & $756 \pm 56$ & $908 \pm 56$ & $1006 \pm 56$ & $0.13-17.0$ & 0.9999 & 0.020 & 0.060 \\
\hline 22 & Kaempferol & nd & $288 \pm 6$ & $222 \pm 4$ & $0.05-15.0$ & 0.9996 & 0.021 & 0.062 \\
\hline 23 & Apigenin & $4136 \pm 94$ & $3314 \pm 86$ & $850 \pm 24$ & $0.17-11.0$ & 0.9997 & 0.034 & 0.104 \\
\hline
\end{tabular}

nd, not detected.

quantified as the dominant phenolic in the ethyl acetate and methanol extracts. Other dominant phenolics were luteolin, epicatechin, and rosmarinic acid in these extracts. However, the most abundant phenolics in the water extract were benzoic acid and quercetin. In recent studies, the detected phenolics in $O$. natrix extracts possess a broad range of biological activities including antioxidant, anticancer, antimicrobial and enzyme inhibitory (López-Lázaro, 2009; Amoah et al., 2016; David et al., 2016). In this framework, the observed biological activities for $O$. natrix extracts might be explained with the presence of these phenolic components. To the best of our knowledge, this study is the first report which indicates comprehensive analysis of phenolic constituents of $O$. natrix. In this sense, the present findings could be opened new horizons for the genus Ononis and it' usages.

\section{Antioxidant Capacity}

In order to evaluate antioxidant ability of $O$. natrix extracts, several methods were performed: free radical scavenging (DPPH and ABTS), reducing power (CUPRAC and FRAP), phosphomolybdenum and metal chelating assays. DPPH and ABTS assays are widely utilized to examine radical scavenging activities of plant extracts or synthetics. These assays are based on the reduction of $\mathrm{ABTS}^{+}$and DPPH in the presence of antioxidants and the changes (from blue to white in ABTS; from purple to yellow in DPPH) can spectrophotometrically be recorded. In addition, the assays reflect to hydrogen donating abilities of antioxidants. As shown in Table 3, the methanol and water extracts had similar DPPH scavenging abilities whereas the lowest ability was observed in the ethyl acetate extract. However, ABTS radical scavenging abilities can be ranked as ethyl acetate> 
methanol>water. The apparent differences can be attributed to nature of these radicals (ABTS assay is considered as both hydrophilic and lipophilic antioxidant systems, while DPPH is only hydrophobic systems). Our findings are consistent with previous results, which were reported different results for DPPH and ABTS (Kim et al., 2002; Zengin et al., 2015, 2017; Bouhlali et al., 2017).

Reducing power is an important way in the antioxidant mechanisms and it reflects electron-donating abilities of antioxidants. From this point, CUPRAC and FRAP assays were performed to detect reducing abilities of $O$. natrix extracts (Table 5). By using CUPRAC assay (from $\mathrm{Cu}^{2+}$ to $\mathrm{Cu}$ ), the ethyl acetate extract showed the strongest reducing ability, followed by methanol and water extracts. These results are in line with the total phenolic and flavonoid contents. Nevertheless, the FRAP (from $\mathrm{Fe}^{3+}$ to $\mathrm{Fe}^{2+}$ ) abilities decreased in that order water (106.12 mgTE/g extract) > ethyl acetate $(90.71 \mathrm{mgTE} / \mathrm{g}$ extract) $>$ methanol (79.73 $\mathrm{mgTE} / \mathrm{g}$ extract). This finding for water extract in FRAP assay might be related to complex interactions of phytochemical (antagonistic etc.) described as Peña-Cerda et al. (2017) and (Hmid et al., 2017).

Phosphomolybdenum assays is proposed by Prieto et al. (1999), which is based on the reduction of Mo (VI) to Mo (V) by the antioxidants and then a green phosphate/Mo (V) complex occurs at the acidic $\mathrm{pH}$. The assay is also considered as one of total antioxidant capacity assays. Within $O$. natrix extracts, the methanol extract ( $1.53 \mathrm{mmolTE} / \mathrm{g}$ extract) exerted the strongest ability in phosphomolybdenum assay, followed by ethyl acetate (1.27 mmolTE/g extract) and water (1.13 mmolTE/g extract) extracts (Table 4). In the literature, several researchers reported different approaches for a correlation between total phenolic and the reduction of Mo (VI) to Mo (V) (Albayrak et al., 2010; Kocak et al., 2016; Amessis-Ouchemoukh et al., 2017). At this point, this activity for the methanol extract may be related to the presence of other antioxidants as well as phenolics.

Transition metal ions, such as iron and copper are involved in the production of hydroxyl radical via Fenton and Haber-Weiss reactions. In this sense, the metal chelating ability is considered as an important way in the antioxidant mechanisms. Contrary to expectations, the metal chelating ability significantly differs from other antioxidant assays and the strongest activity was observed in water extract with $14.49 \mathrm{mgEDTAE} / \mathrm{g}$, while the ethyl acetate extract had the lowest ability (Table 5). These findings are consistent with previous studies, which reported no correlation between total phenolic and metal chelating ability (Silva et al., 2011; Hatami et al., 2014; Khorasani Esmaeili et al., 2015). In fact, the metal chelating abilities of phenolics were described as a minor way in theirs antioxidant properties by Rice-Evans et al. (1996).

\section{Enzyme Inhibitory Effect}

Alzheimer's diseases (AD) and diabetes mellitus (DM) are considered as major public health problems and their incidence tends to increase at an alarming rate (more than 35.6 million $\mathrm{AD}$ patients in worldwide and is expected to be more than 100 million $\mathrm{AD}$ patients by 2050). The development of new therapeutic approaches (also in terms of non-pharmacological therapy supplement) for these diseases has become a pressing issue. Among the therapeutic approaches, the inhibition of key enzymes is considered as one of most effective strategy (Wimo and Prince, 2010). Cholinesterase inhibitors are currently used as a drug for the treatment of $\mathrm{AD}$. The inhibitors increase brain acetylcholine (ACh) levels by preventing the breakdown of $\mathrm{ACh}$ and this case is very important to cognitive function (Menichini et al., 2014). Again, $\alpha$-amylase and $\alpha$-glucosidase release glucose from larger carbohydrates (especially starch) and are key enzymes in DM glucose level control (Kubinova et al., 2014). In this direction, both $\alpha$ amylase and $\alpha$-glucosidase inhibitors are promising drugs in the treatment of DM. In addition, tyrosinase is a main enzyme in the synthesis of melanin and thus tyrosinase inhibitors are useful for controlling hyperpigmentation problems. Within the framework of these information's, many enzyme inhibitors are synthetically produced. However, most of them show limited effectiveness especially related to side effects, such as gastrointestinal disturbances, and toxic properties (Kumar et al., 2011). Therefore, many scientists have focused on naturally occurring compounds from plants as potential sources of either new or safe effective inhibitors.

Thus, we tested enzyme inhibitory effects of Ononis extracts against to cholinesterases, tyrosinase, amylase and glucosidase. The results are represented in Table 6. The ethyl acetate extract exhibited the strongest cholinesterase inhibitory effect on both AChE (1.46 mgGALAE/g extract) and BChE (0.93 mgGALAE/g extract). However, the water extract was not active on BChE. The observed activity for ethyl acetate extract may be linked to higher level of phenolics in the extracts. Our findings were confirmed by several researchers (Kennedy and Wightman, 2011; Roseiro et al., 2012; Mazlan et al., 2013; Hlila et al., 2015), who reported a linear correlation between phenolic content and anti-cholinesterase abilities. Interestingly, the ethyl acetate extract did not have any inhibitory effect on tyrosinase and the

TABLE 5 | Total antioxidant capacity (phosphomolybdenum assay), reducing power (CUPRAC and FRAP assays) and metal chelating activities of the extracts.

\begin{tabular}{|c|c|c|c|c|}
\hline Extracts & Phosphomolybdenum (mmolTE/g extract) & CUPRAC (mgTE/g extract) & FRAP (mgTE/g extract) & Metal chelating (mgEDTAE/g extract) \\
\hline Ethyl acetate & $1.27 \pm 0.07^{*}$ & $183.37 \pm 0.02$ & $90.71 \pm 1.79$ & $9.16 \pm 0.17$ \\
\hline Methanol & $1.53 \pm 0.08$ & $170.51 \pm 0.57$ & $79.73 \pm 1.17$ & $12.02 \pm 1.40$ \\
\hline Water & $1.13 \pm 0.03$ & $94.16 \pm 0.99$ & $106.12 \pm 0.44$ & $14.49 \pm 0.64$ \\
\hline
\end{tabular}

TE, trolox equivalents; EDTAE, EDTA equivalents.

*Values expressed are means \pm S.D. of three parallel measurements. 
water extract had remarkable anti-tyrosinase effect with 52.81 mgKAE/g extract. As for amylase and glucosidase inhibition, the ethyl acetate $(0.74 \mathrm{mmolACAE} / \mathrm{g}$ and $17.52 \mathrm{mmolACAE} / \mathrm{g})$ and methanol extracts (0.59 mmolACAE/g and $19.94 \mathrm{mmolACAE} / \mathrm{g})$ had the greatest abilities compared to water extract. The higher concentrations of phenolics in these extracts may be responsible for their anti-diabetic effects. In accordance with our findings, some phenolic components were reported as anti-diabetic agents (Tundis et al., 2010; Etxeberria et al., 2012; Ríos et al., 2015). From this point, molecular approaches could be valuable to understand interactions of enzymes and phenolics. To the best of our knowledge, this study is the first report on Ononis natrix

TABLE 6 | Enzyme inhibitory effects of the extracts.

\begin{tabular}{|c|c|c|c|c|c|}
\hline Extracts & $\begin{array}{l}\text { AChE inhibition } \\
\text { (mgGALAE/g } \\
\text { extract) }\end{array}$ & $\begin{array}{l}\text { BChE ınhibition } \\
\text { (mgGALAE/g } \\
\text { extract) }\end{array}$ & $\begin{array}{l}\text { Tyrosinase inhibition } \\
\text { (mgKAE/g extract) }\end{array}$ & $\begin{array}{c}\alpha \text {-amylase Inhibition } \\
\text { (mmolACAE/g } \\
\text { extract) }\end{array}$ & $\begin{array}{c}\alpha \text {-glucosidase } \\
\text { Inhibition (mmolACAE/g } \\
\text { extract) }\end{array}$ \\
\hline Ethyl acetate & $1.46 \pm 0.07^{*}$ & $0.93 \pm 0.01$ & na & $0.74 \pm 0.02$ & $17.52 \pm 0.28$ \\
\hline Methanol & $1.29 \pm 0.05$ & $0.64 \pm 0.06$ & $11.20 \pm 2.30$ & $0.59 \pm 0.01$ & $19.94 \pm 0.11$ \\
\hline Water & $0.02 \pm 0.01$ & na & $52.81 \pm 5.15$ & $0.17 \pm 0.01$ & $3.69 \pm 0.17$ \\
\hline
\end{tabular}

GALAE, galanthamine equivalents; ACAE, acarbose equivalents; KAE, kojic acid equivalents; na, not active.

*Values expressed are means \pm S.D. of three parallel measurements.

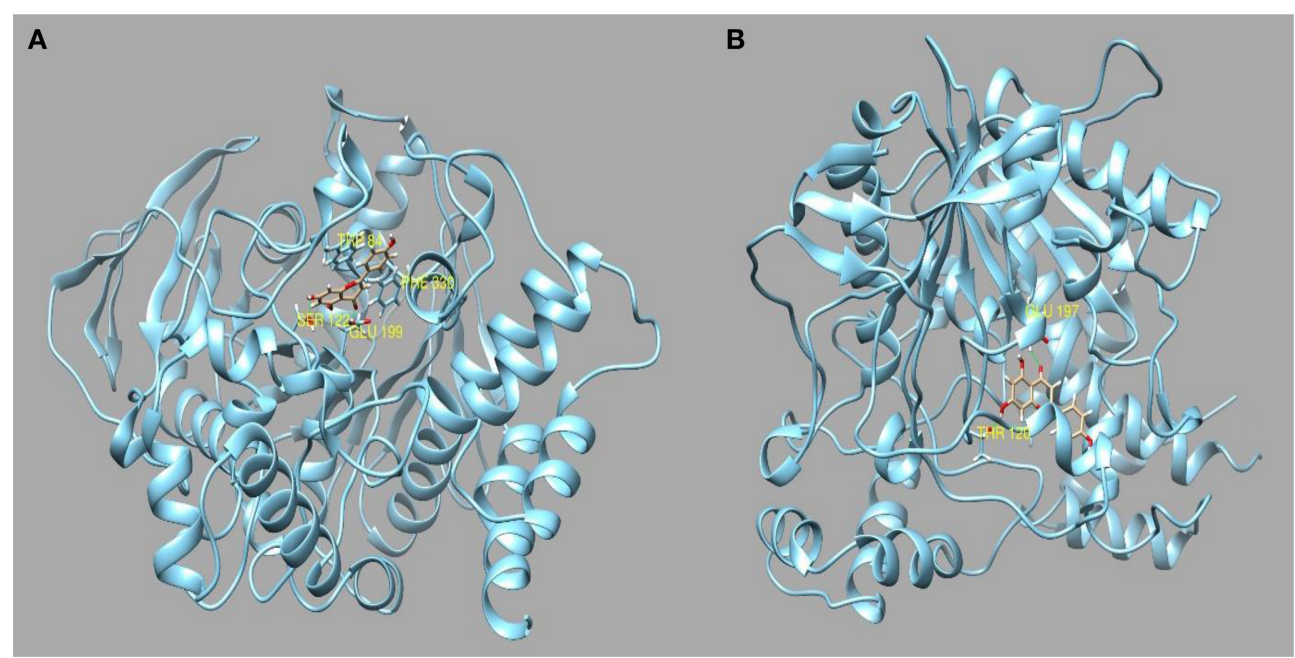

FIGURE 2 | (A) Best pose of apigenin docked to AChE and (B) to BChE.

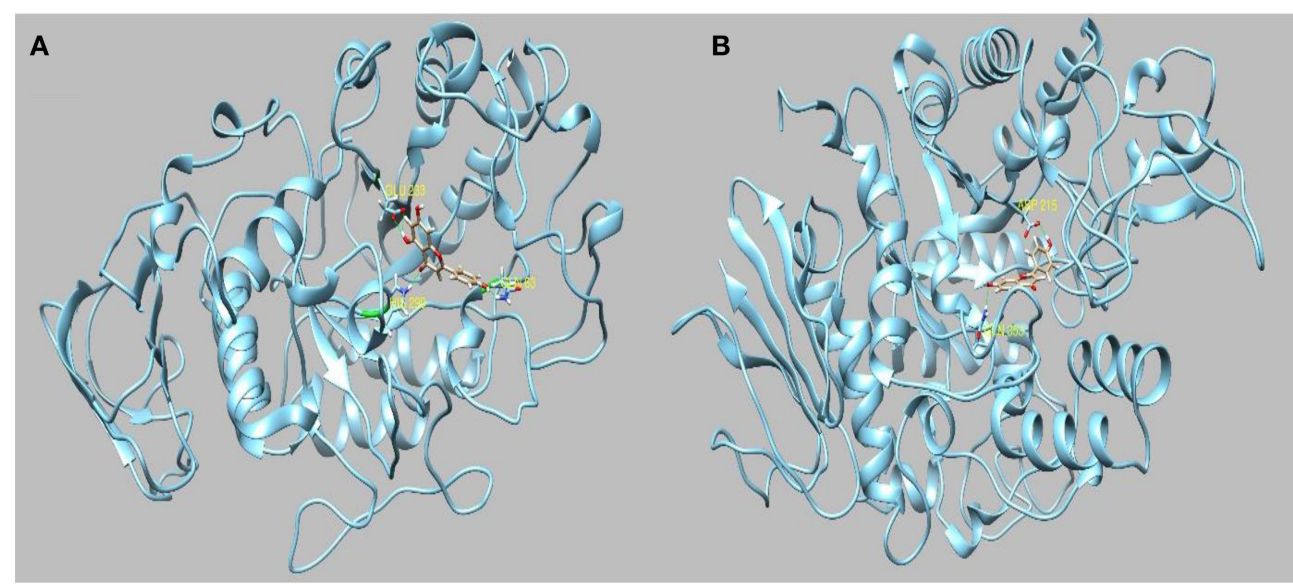

FIGURE 3 | (A) Best pose of apigenin docked to $\alpha$-amylase and (B) to $\alpha$-glucosidase. 
subsp. hispanica. Taken together, this study could provide a starting point on this species and could open new perspectives for designing novel functional products.

\section{Molecular Docking}

Evidence from the literature supports the inhibitory action of apigenin on AChE and BChE (Katalinić et al., 2010; Xie et al., 2014). Apigenin was also reported to induce moderate inhibitory effect on $\alpha$-amylase (Li et al., 2014; Wulan et al., 2014) and on $\alpha$-glucosidase. On the other hand, we have found that the water extract possesses good inhibition activity toward tyrosinase. This may be attributed to the presence of luteolin and quercetin. In light of the observed inhibitory activity, in silico molecular docking simulation was used to investigate the interactions between apigenin to $\mathrm{AChE}, \mathrm{BChE}$, amylase and glucosidase, whereas luteolin and quercetin have been selected as principal

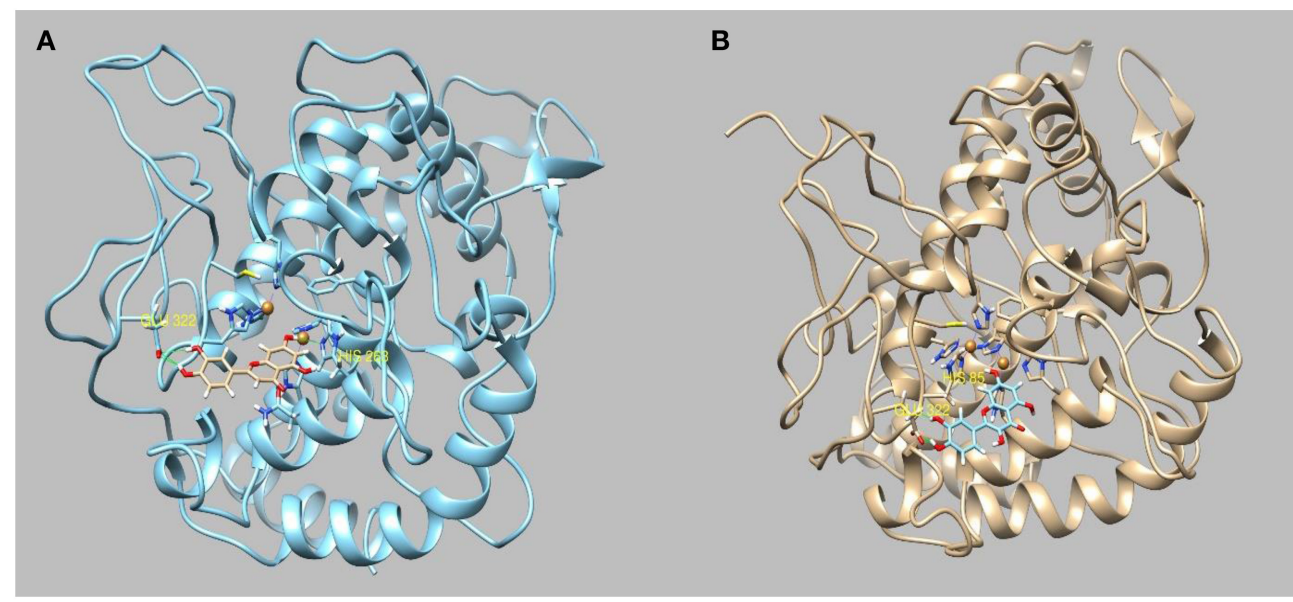

FIGURE 4 | Best pose of (A) luteolin and (B) quercetin docked to tyrosinase.
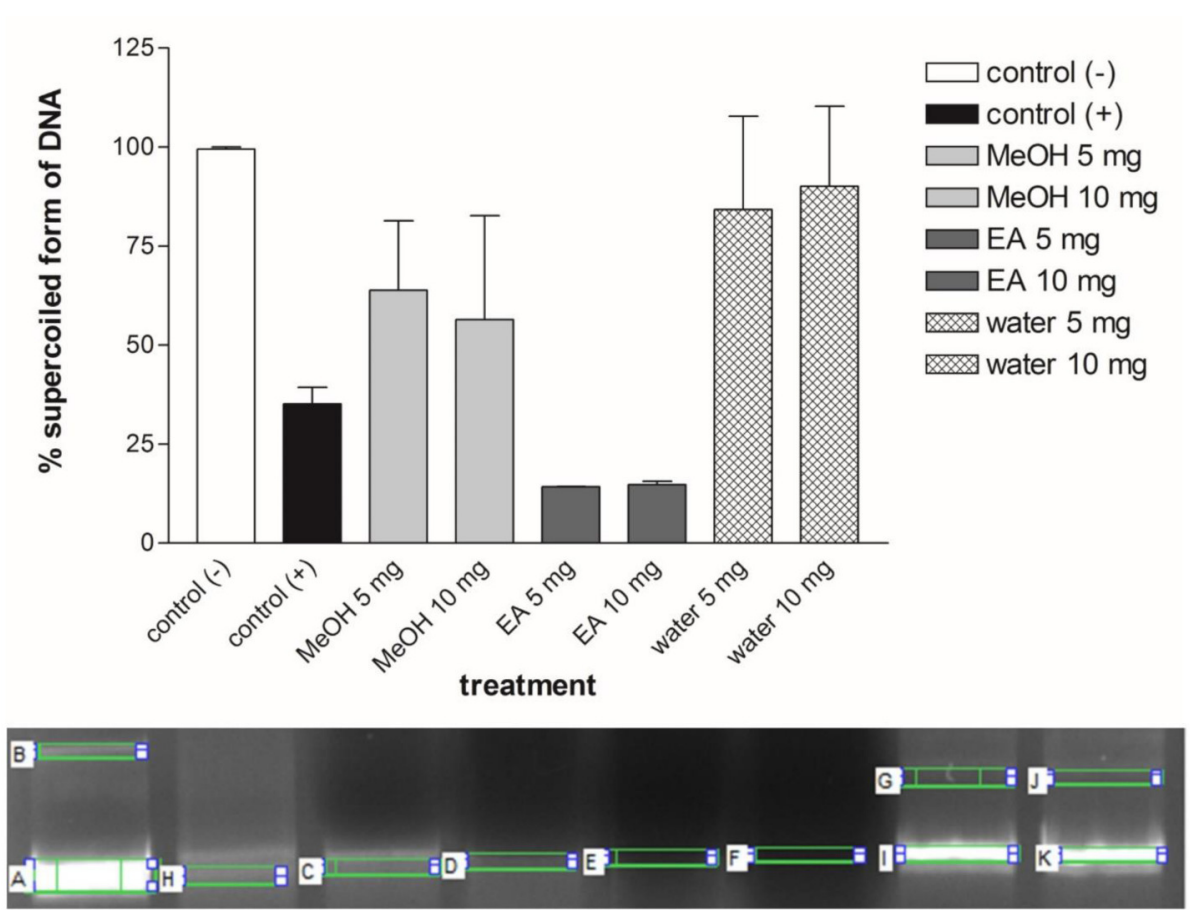

FIGURE 5 | percentage of DNA protection analysis and gel view of plasmid DNA protection test for each Ononis natrix extracts. A,B: (+) control, H: (-) control, C: $5 \mathrm{mg}$ methanol extract, D: $10 \mathrm{mg}$ methanol extract, E: $5 \mathrm{mg}$ ethyl acetate extract, F: $10 \mathrm{mg}$ ethyl acetate extract, I-G: 5 mg water extract, K-J: 10 mg water extract. Striped bars indicate significantly different applications. 
inhibitors of tyrosinase. The most representative enzyme-ligand complexes were reported in Figures 2-4.

Apigenin has been previously reported to inhibit both AChE and BChE by IC50 of $7.72 \mathrm{micro} / \mathrm{Mol}$ (Balkis et al., 2015) and $37.4 \mathrm{micro} / \mathrm{Mol}$ (Katalinić et al., 2010), respectively. The best docked pose interacts with AChE in the binding pocked by establishing two hydrogen bonds to Glu199 and Ser122 and two pi-pi stacks to Phe330 and Trp 84 with a docking score of -9.607 and a binding energy of $-60.64 \mathrm{Kcal} / \mathrm{Mol}$. Apigenin also interacts with several residues present in the binding pocket of BChE forming two hydrogen bonds to Thr120 and Glu197 with a docking score of -9.459 and a binding energy of -52.54 $\mathrm{kcal} / \mathrm{Mol}$. Also, considering the abundance in the ethyl acetate and methanol extracts, apigenin could be also responsible for the amylase and glucosidase inhibiting activity. Indeed, it has been previously reported that apigenin was an efficacious inhibitor of $\alpha$-glucosidase with a IC50 of and a moderate $\alpha$-amylase inhibitor with a IC50 of $10.5 \mu / \mathrm{Mol}$ (Zeng et al., 2016).

Apigenin interacts with $\alpha$-glucosidase by forming two hydrogen bonds to Asp215 and Gln353 and with the enzymatic cavity of $\alpha$-amylase by forming three hydrogen bonds to Gln63, His299, and Glu233. The aqueous extract has shown a strong activity toward tyrosinase. The most abundant substances present in the extracts are benzoic acid, luteolin and quercetin. All of these substances have been demonstrated to have an tyrosinase inhibitory activity in in vitro experiments (IC50 = quercetin 0.13 $\mathrm{mM}$ ) (Chen and Kubo, 2002). However, benzoic acid reported a scarce docking score $(-5.1)$ and a positive binding energy $(+41.7 \mathrm{Kcal} / \mathrm{Mol})$ which indicate none or very little affinity for the binding pocket of the enzyme, whereas the luteolin and quercetin have obtained the best binding energy. ( $-28 \mathrm{Kcal} / \mathrm{Mol}$ and $-29.31 \mathrm{Kcal} / \mathrm{Mol}$, respectively).

However, it is noteworthy to highlight that the plant extracts contain several phenolic compounds which in less extent are able to inhibit the tested enzymes all with non-competitive mechanism as demonstrated in our previous paper (Picot et al., 2017). Inhibitory action of the plant extracts on AChE, BChE, tyrosinase, $\alpha$-amylase, and $\alpha$-glucosidase might be due to the concerted action of several phenolic compounds rather than a single molecule.

\section{DNA Protective Effects}

Various stress factors, such as oxidative stress, acid, alkaline, UV, and heavy metals can damage DNA. ROS formed by hydroxyl radicals cause DNA strand breakage that is brought about carcinogenesis, mutagenesis and cytotoxicity (Golla and Bhimathati, 2014). Here, we targeted to measure the DNA damage by Fenton reaction-mediated oxidative stress. Fenton's reagent $\left(\mathrm{Fe}^{2+}+\mathrm{H}_{2} \mathrm{O}_{2} \rightarrow \mathrm{Fe}^{3+}+\mathrm{OH}^{-}+\cdot \mathrm{OH}\right)$ produces the highly deleterious hydroxyl radicals that damage the cellular components, such as DNA, lipid and proteins. The scavenging effect of extract was tested in plasmid nicking assay. Figure 5 illustrates the DNA protection activity of the Ononis extracts with two different concentrations ( 5 and $10 \mathrm{mg} / \mathrm{ml}$ ). Ononis extracted in water $(10 \mathrm{mg} / \mathrm{ml})$ had the most effective on protection of DNA with $78 \%$, followed by water $(5 \mathrm{mg} / \mathrm{ml})(70 \%)$ and methanol extract with $5 \mathrm{mg} / \mathrm{ml}$ (53\%). Except for the ethyl acetate extract ( 5 and $10 \mathrm{mg} / \mathrm{ml}$ ), water and methanol extracts protected supercoiled form of pDNA and indicated DNA protection activity.
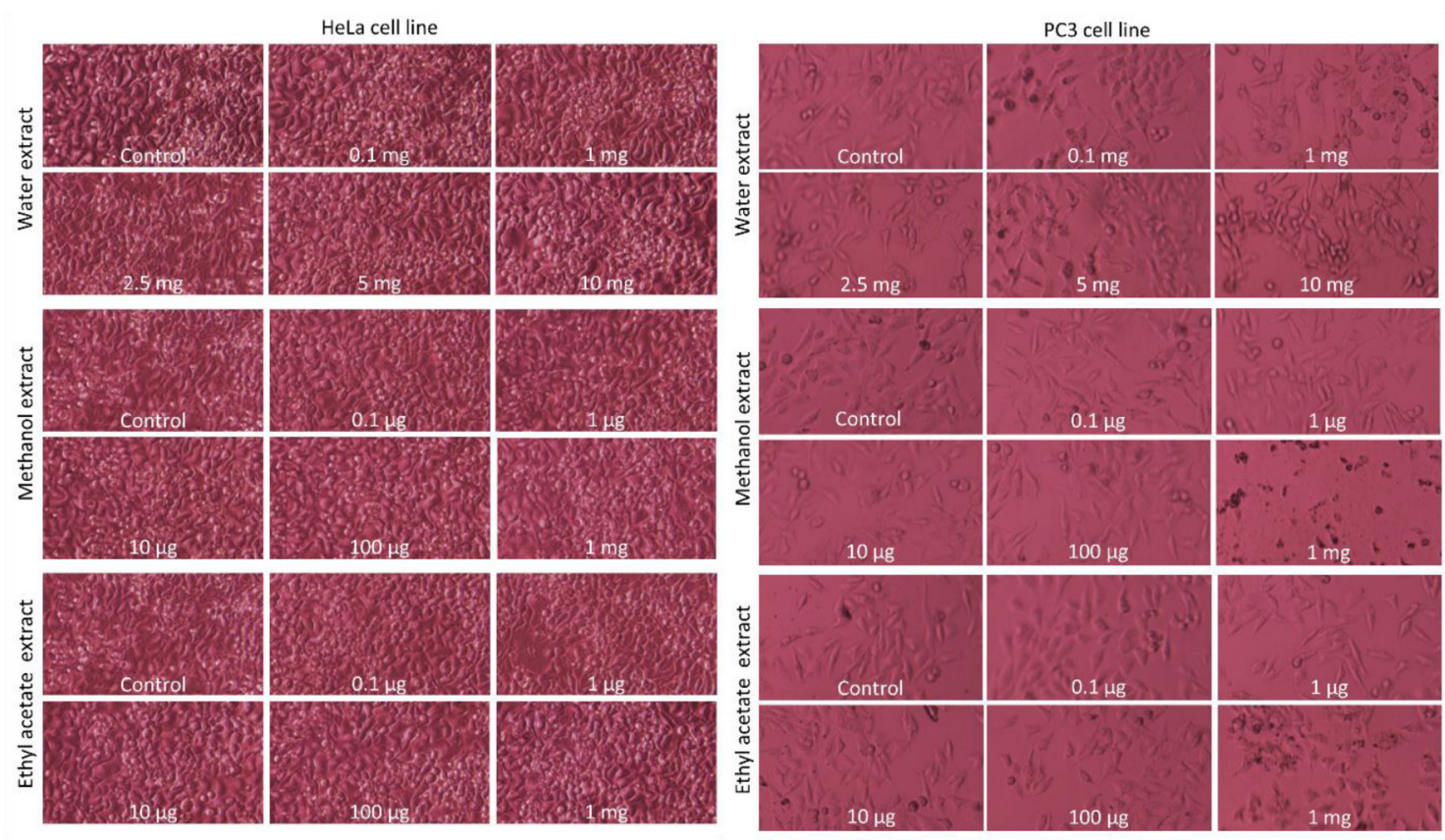

FIGURE 6 | Cells morphology of HeLA and PC3 cancer cell lines after application of different extracts of Ononis natrix. Control and different concentrations of extracts were tested in HeLA or PC3 cancer cell lines and indicated for each figures. 
In the present study, the water extract had the most protection activity on DNA. This can be arisen from availability of different metabolites in water extract. Quercetin and sinapic acid are only metabolites which found in this extract. So, it can be concluded that these chemicals may be responsible for protection of DNA from Fenton reagent. Like water extract, the methanol extract also contained specific metabolite, such as rosmarinic acid. In this sense, the observed DNA protection ability for the methanol extract can be arisen from presence of higher amount of rosmarinic acid. Therefore, it seems that different ingredients of the extracts may cause formation of DNA protection property for the tested extracts. According to DNA protection assay results, water extract of Ononis natrix showed the most protective activity for DNA. This result may be explained by determination of phenolic components in the extracts using HPLC analysis. As indicated in Table 4, rosmarinic acid and quercetin were the most dissolved components in methanol and water extracts of Ononis natrix, respectively. So, it can be concluded that rosmarinic acid and quercetin can be considered as potential chemicals for protection of DNA from free radicals, hydroxyl radicals and mutagens. Quercetin is a well-investigated antioxidant known as promising molecule for pharmacological studies. In a comprehensive research for DNA protection ability of quercetin, its supplementation indicated protection of nuclear DNA from oxidative damage and significant genoprotective activity on mitochondrial DNA (Potenza et al., 2008). In a different study, three polyphenolic compounds including luteolin, quercetin and rosmarinic acid, were investigated for their protective effects against oxidative DNA damage (Silva et al., 2008). They found that all of these phenolics were protection agents against oxidative stress-induced DNA damage. In another search, protective activity of pure rosmarinic acid and elagic acid were evaluated. It has been found that they have equal effect on declining AAPH-induced oxidative DNA damage (Vattem et al., 2006). These results are in accord with our study indicating that methanol and water extracts of Ononis natrix can be considered
HeLa cell line
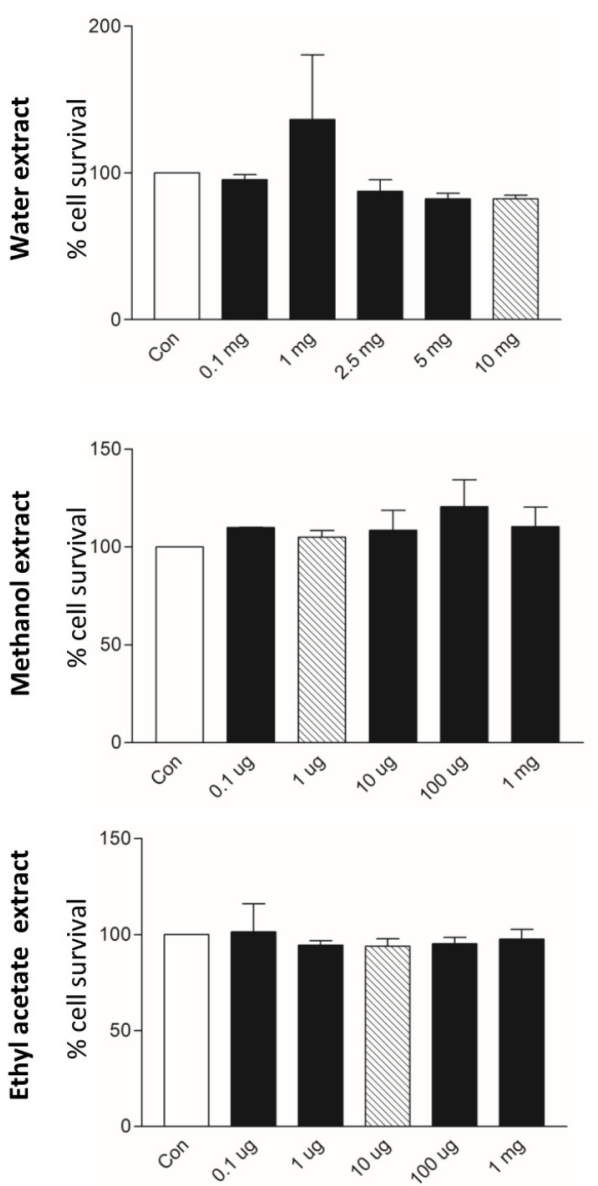

PC3 cell line
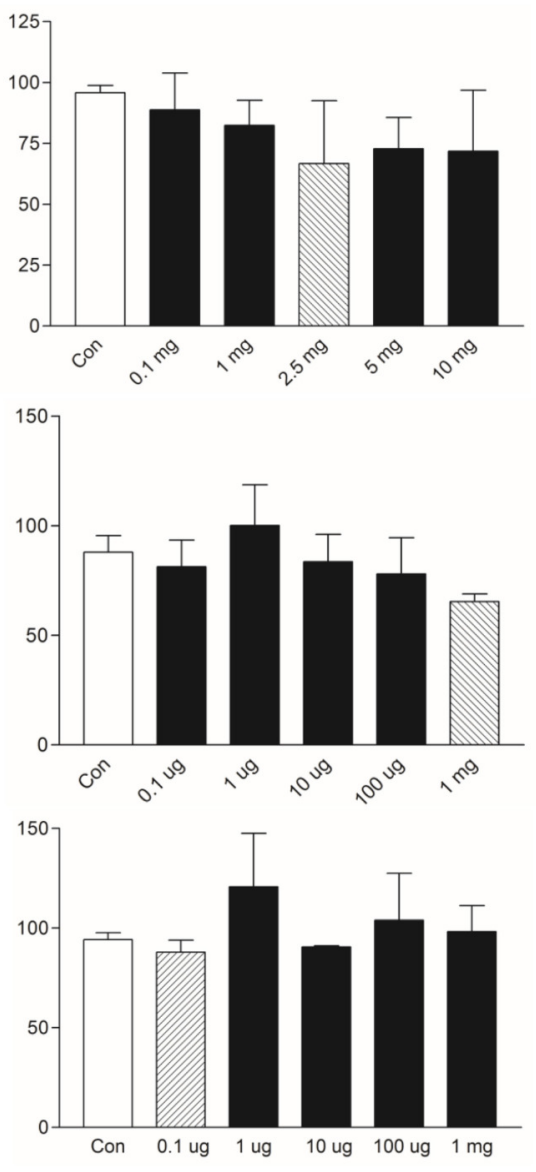

FIGURE 7 | Cell viability assay (MTT) analysis of HeLA and PC3 cancer cell lines after application of different extracts of Ononis natrix. White bars and striped bars indicate controls and significantly different applications, respectively. 
as valuable DNA protectors because of presence of rosmarinic acid and quercetin.

\section{Cytotoxic Effects}

The cytotoxic effects of Ononis natrix on the survival of HeLa and PC3 cells were determined by MTT cell viability assay. HeLa cervical cancer cell line and PC3 prostate cancer cell line were treated with these extracts with different concentrations for $24 \mathrm{~h}$. Cells morphology was observed under inverted microscope and illustrated in Figure 6. According to morphological appearance, there was no significant apoptotic effect on Ononis natrix extracts for HeLa cell line. However, the water and methanol extracts caused initiation of apoptosis for PC3 cell line. To indicate effect of three extracts on cancer cell lines, cell viability assay (MTT) was also conducted. Although there was a cytotoxic activity for HeLa cells after application of the water extract, no significant decline was observed in cancer cell numbers compared to control (Figure 7). On the other hand, $2.5 \mathrm{mg}$ water extract, $1 \mathrm{mg}$ methanol extract and $0.1 \mu \mathrm{g}$ ethyl acetate extract caused a significant cell number reduction on PC3 cancer line (Figure 7). Ethyl acetate extract indicated cytotoxic activity with the smallest concertation $(0.1 \mu \mathrm{g})$ among all extracts. $2.5 \mathrm{mg}$ of the water extract showed dose-independent cytotoxic effect on PC3 cell line since there was no effect for $5 \mathrm{mg}$ and $10 \mathrm{mg}$ water extracts. It has been considered that concentration of water extract more than $2.5 \mathrm{mg}$ may be toxic against healthy or normal viable cells. Thus, it can be concluded that $2.5 \mathrm{mg}$ is a moderate concentration for cytotoxicity on PC3 cancer cell line. It was also monitored that other treatments had cytotoxic effect whereas, they were not significantly different from control sample anymore.

According to reports, about $60 \%$ of anticancer drugs are isolated from plant-derived natural compounds. These medicinal plants highly contain polyphenolic compounds which are actually inhibitors for cancer development (Sukumaran et al., 2016). Cytotoxic and apoptotic effects of the studied extracts on HeLa and PC3 cell lines were investigated. Although there was no significant variation on HeLa cells, $2.5 \mathrm{mg}$ of water and $1 \mathrm{mg}$ methanol extracts leaded to cytotoxic activity compared to control in PC3. Some of phenolic compounds show antiproliferative effect which depends on polarity of compounds. Cytotoxic effect of methanol extract can be explained with the presence of rosmarinic acid, apigenin, and epicatechin. Effect of anti-carcinogenic and scavenging of reactive oxygen radicals of rosmaniric acid were particularly searched in Perilla frutescens (Osakabe et al., 2004). The body's response to cancer showed parallel effects with the inflammatory response. Since, inflammatory cytokine networks may affect survival, growth, mutation, proliferation, differentiation and relocation of tumor and stromal cells (David, 1988; Balkwill and Mantovani, 2001; Osakabe et al., 2004). Rosmaniric acid is a water-soluble polyphenolic compound and has function on inhibition of angiogenesis (Huang and Zheng, 2006). There was a potential evidence that ROS acts as initiating angiogenesis and onset of cancer mechanism. It was proved that hydrogen peroxide stimulated angiogenesis in vitro condition (Shono et al., 1996; Yasuda et al., 1999; Huang and Zheng, 2006). Rosmaniric acid reduced ROS expression, hydrogen peroxide level and VEGF
(Vascular Endothelial Growth Factor) level that are important on cancer development (Huang and Zheng, 2006). Furthermore, the previous report which indicated effect of methanolic extract of plant species on antioxidant activity and examination of level of rosmaniric acid (Tepe, 2008). In addition, in our previous study, three extracts (ethyl acetate, methanol and water) of two Potentilla species (Potentilla reptans and P. speciosa) showed cytotoxic activity against A549 andMCF-7 due to some phenolic compounds (Uysal et al., 2017). The findings of this analysis suggest that one or more than one phenolic compounds and their interaction may cause initiation of apoptosis in cancer cell lines. However, further investigation should be performed in in vivo test to arrive at a definite judgment for inhibition of cancer development.

\section{Antimicrobial Effects}

Antimicrobial activity of the extracts from O. natrix subsp. hispanica were searched against to 13 bacteria and 1 fungus by the disk diffusion method. The extracts were loaded the disks at the concentration of 10,50, and $100 \mathrm{mg}$. According to results, 50 and $100 \mathrm{mg}$ of methanol and ethyl acetate extracts showed the best antibacterial effect against to Staphylococcus aureus ATCC 25923 and Staphylococcus epidermidis strains (Figure 8). In addition, $100 \mathrm{mg}$ water extracts had a small effect on Salmonella kentucky. It was realized that extracts possessed more protective effect against to gram positive bacteria strains. In addition, concentration-dependent effect was also observed. For example, S. epidermis stain was more sensitive against to $100 \mathrm{mg}$ of methanol and ethyl acetate extracts than $50 \mathrm{mg}$ ones. Methanol and ethyl acetate extracts of $O$. natrix subsp. hispanica were more effective than water extract for antimicrobial analysis. The results of this analysis support the idea that because of higher total phenolic and flavonoid contents of methanol and ethyl acetate

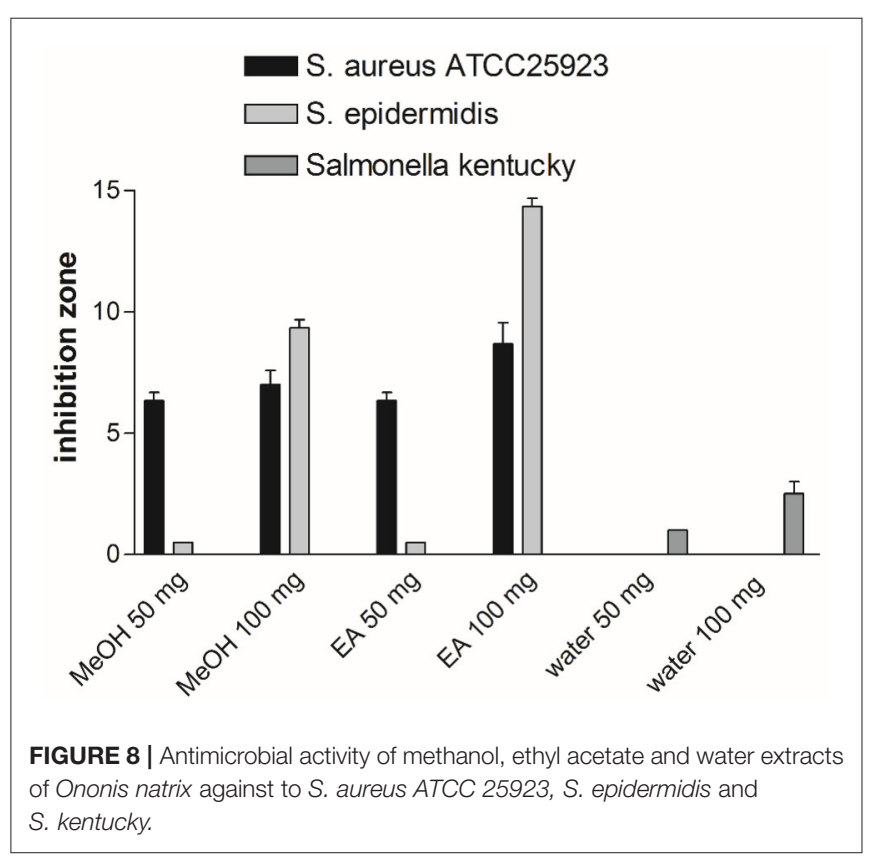


extracts, they indicated more antimicrobial activity than water one.

Staphylococcus aureus (ATCC 25923) is a gram positive bacterium and causes several human infections and leads to sanitary problems. S. aureus also shows resistance to some antibiotics and drugs particularly to methicillin (Hannan et al., 2008). Resistance problems induced to researchers to tend for seeking novel therapy. S. epidermidis together with $S$. aureus lead to severe infectious diseases (Otto, 2009). S. epidermidis is more being tend to cause infectious diseases due to developing persistent infection on human skin (Otto, 2009). Researchers are seeking the mechanisms by which $S$. epidermidis promotes diseases (Otto, 2009). In a study of Sayari et al. (2016) Ononis natrix leaves extracts were tested against to 9 bacterial strains (5 gram-negative and 4 gram-positive). Consistently with our study, $S$. aureus were the most susceptible against to Ononis natrix leaves extracts. In another study, S. aureus (ATCC 25923), and E. coli (ATCC 25922) were found to have more sensitivity against the Ononis natrix essential oils with inhibition zone as 27 and 25, respectively (Elamrani and Benaissa, 2010).

\section{REFERENCES}

Albayrak, S., Aksoy, A., Sagdic, O., and Hamzaoglu, E. (2010). Compositions, antioxidant and antimicrobial activities of Helichrysum (Asteraceae) species collected from Turkey. Food Chem. 119, 114-122. doi: 10.1016/j.foodchem.2009.06.003

Al-Rehaily, A. J., Shamim Ahmad, M., Yousaf, M., Iqrar Khan, S., Mustafa, J., Tekwani, B. L., et al. (2014). Bioactive chemical constituents of Ononis natrix. J. Chem. Soc. Pak. 36, 1114-1121.

Amessis-Ouchemoukh, N., Ouchemoukh, S., Meziant, N., Idiri, Y., Hernanz, D., Stinco, C. M., et al. (2017). Bioactive metabolites involved in the antioxidant, anticancer and anticalpain activities of Ficus carica L., Ceratonia siliqua L. and Quercus ilex L. extracts. Ind. Crops Prod. 95, 6-17. doi: 10.1016/j.indcrop.2016.10.007

Amoah, S. K., Sandjo, L. P., Kratz, J. M., and Biavatti, M. W. (2016). Rosmarinic acid-pharmaceutical and clinical aspects. Planta Med. 82, 388-406. doi: 10.1055/s-0035-1568274

Balkis, A., Tran, K., Lee, Y. Z., and Ng, K. (2015). Screening flavonoids for inhibition of acetylcholinesterase identified baicalein as the most potent inhibitor. J. Agric. Sci. 7, 26-35. doi: 10.5539/jas.v7n9p26

Balkwill, F., and Mantovani, A. (2001). Inflammation and cancer: back to Virchow? Lancet 357, 539-545. doi: 10.1016/S0140-6736(00)04046-0

Baytop, T. (1999). Therapy with Medicinal Plants in Turkey (Past and Present). Istanbul: Nobel Tip Basimevi.

Berman, H. M., Westbrook, J., Feng, Z., Gilliland, G., Bhat, T. N., Weissig, H., et al. (2000). The protein data bank. Nucleic Acids Res. 28, 235-242. doi: $10.1093 / \mathrm{nar} / 28.1 .235$

Bouhlali, E. D. T., Ramchoun, M., Alem, C., Ghafoor, K., Ennassir, J., and Zegzouti, Y. F. (2017). Functional composition and antioxidant activities of eight Moroccan date fruit varieties (Phoenix dactylifera L.). J. Saudi Soc. Agric. Sci. 16, 257-264. doi: 10.1016/j.jssas.2015.08.005

Casiglia, S., Bruno, M., and Senatore, F. (2017). Chemical composition of the essential oil from the aerial parts of Ononis reclinata L. (Fabaceae) grown wild in Sicily. Nat. Prod. Res. 31, 7-15. doi: 10.1080/14786419.2016.1205054

Chen, Q.-X., and Kubo, I. (2002). Kinetics of mushroom tyrosinase inhibition by quercetin. J. Agric. Food Chem. 50, 4108-4112. doi: 10.1021/jf011378z

Daruházi, Á. E., Szarka, S., Héthelyi, É., Simándi, B., Gyurján, I., László, M., et al. (2008). GC-MS identification and GC-FID quantitation of terpenoids in Ononidis spinosae radix. Chromatographia 68, 71-76. doi: 10.1365/s10337-008-0679-2

\section{CONCLUSION}

In recent years, pharmaceuticals and functional ingredients from plant sources have been of paramount interest. In this sense, chemical profile and biological abilities of $O$. natrix subsp. hispanica were investigated in the current work. As far as we aware, this work is the first report on this species. The tested extracts, especially ethyl acetate and methanol, exhibited notable biological effects correlated with higher levels of bioactive compounds. The results obtained from the present work provide a new framework for utilization of the genus Ononis and as a result, $O$. natrix subp. hispanica could be suggested as a natural source of bioactive agents, such as antioxidant, antimicrobial and anticancer. We hope that our research will serve a base for future studies for in vivo and bioavailability studies on O. natrix subsp. hispanica.

\section{AUTHOR CONTRIBUTIONS}

SY, GZ, MB, and YC set up and carried out experiments. AM and AA executed data analysis.

David, A. V. A., Arulmoli, R., and Parasuraman, S. (2016). Overviews of biological importance of quercetin: a bioactive flavonoid. Pharmacogn. Rev. 10, 84-89. doi: 10.4103/0973-7847.194044

David, H. (1988). Rudolf Virchow and modern aspects of tumor pathology. Pathol. Res. Pract. 183, 356-364. doi: 10.1016/S0344-0338(88)80138-9

DeLano, W. L. (2002). The PyMOL Molecular Graphics System. Palo Alto, CA.

Do, Q. D., Angkawijaya, A. E., Tran-Nguyen, P. L., Huynh, L. H., Soetaredjo, F. E., Ismadji, S., et al. (2014). Effect of extraction solvent on total phenol content, total flavonoid content, and antioxidant activity of Limnophila aromatica. J. Food Drug Anal. 22, 296-302. doi: 10.1016/j.jfda.2013.11.001

Elamrani, A., and Benaissa, M. (2010). Chemical composition and antibacterial activity of the essential oil of Ononis natrix from morocco. J. Essent. Oil Bear. Plants 13, 477-488. doi: 10.1080/0972060X.2010.10643852

Erdemgil, F., Kurkcuoglu, M., and Baser, K. (2002). Composition of the essential oil of Ononis viscosa subsp. breviflora. Chem. Nat. Comp. 38, 565-567. doi: 10.1023/A:1022686721070

Etxeberria, U., De La Garza, A. L., Campión, J., Martinez, J. A., and Milagro, F. I. (2012). Antidiabetic effects of natural plant extracts via inhibition of carbohydrate hydrolysis enzymes with emphasis on pancreatic alpha amylase. Expert Opin. Ther. Targets 16, 269-297. doi: 10.1517/14728222.2012.664134

Fernandes, L., Casal, S., Pereira, J. A., Saraiva, J. A., and Ramalhosa, E. (2017). Edible flowers: a review of the nutritional, antioxidant, antimicrobial properties and effects on human health. J. Food Composit. Anal. 60, 38-50. doi: 10.1016/j.jfca.2017.03.017

Gampe, N., Darcsi, A., Lohner, S., Béni, S., and Kursinszki, L. (2016). Characterization and identification of isoflavonoid glycosides in the root of Spiny restharrow (Ononis spinosa L.) by HPLC-QTOF-MS, HPLC-MS/MS and NMR. J. Pharm. Biomed. Anal. 123, 74-81. doi: 10.1016/j.jpba.2016. 01.058

Ghribi, L., Nejma, A. B., Besbes, M., Harzalla-Skhiri, F., Flamini, G., and Jannet, H. B. (2016). Chemical composition, cytotoxic and antibacterial activities of the essential oil from the Tunisian Ononis angustissima L. (Fabaceae). J. Oleo Sci. 65, 339-345. doi: 10.5650/jos.ess 15242

Ghribi, L., Waffo-Téguo, P., Cluzet, S., Marchal, A., Marques, J., Mérillon, J.-M., et al. (2015). Isolation and structure elucidation of bioactive compounds from the roots of the Tunisian Ononis angustissima L. Bioorg. Med. Chem. Lett. 25, 3825-3830. doi: 10.1016/j.bmcl.2015.07.076

Golla, U., and Bhimathati, S. S. (2014). Evaluation of antioxidant and DNA damage protection activity of the hydroalcoholic extract of Desmostachya bipinnata L. Stapf. Sci. World J. 2014:215084. doi: 10.1155/2014/215084 
Hannan, A., Saleem, S., Chaudhary, S., Barkaat, M., and Arshad, M. U. (2008). Anti bacterial activity of Nigella sativa against clinical isolates of methicillin resistant Staphylococcus aureus. J. Ayub Med. Coll. Abbottabad 20, 72-74.

Hatami, T., Emami, S. A., Miraghaee, S. S., and Mojarrab, M. (2014). Total phenolic contents and antioxidant activities of different extracts and fractions from the aerial parts of Artemisia biennis Willd. Iran. J. Pharm. Res. 13:551.

Hlila, M. B., Mosbah, H., Mssada, K., Jannet, H. B., Aouni, M., and Selmi, B. (2015). Acetylcholinesterase inhibitory and antioxidant properties of roots extracts from the Tunisian Scabiosa arenaria Forssk. Ind. Crops Prod. 67, 62-69. doi: 10.1016/j.indcrop.2015.01.009

Hmid, I., Elothmani, D., Hanine, H., Oukabli, A., and Mehinagic, E. (2017). Comparative study of phenolic compounds and their antioxidant attributes of eighteen pomegranate (Punica granatum L.) cultivars grown in Morocco. Arab. J. Chem. 10, 2675-2684. doi: 10.1016/j.arabjc.2013.10.011

Huang, S. S., and Zheng, R. L. (2006). Rosmarinic acid inhibits angiogenesis and its mechanism of action in vitro. Cancer Lett 239, 271-280. doi: 10.1016/j.canlet.2005.08.025

Irwin, J. J., Sterling, T., Mysinger, M. M., Bolstad, E. S., and Coleman, R. G. (2012). ZINC: a free tool to discover chemistry for biology. J. Chem. Inf. Model. 52, 1757-1768. doi: 10.1021/ci3001277

Ismaya, W. T., Rozeboom, H. J., Weijn, A., Mes, J. J., Fusetti, F., Wichers, H. J., et al. (2011). Crystal structure of Agaricus bisporus mushroom tyrosinase: identity of the tetramer subunits and interaction with tropolone. Biochemistry 50, 5477-5486. doi: 10.1021/bi200395t

Jones, G., Willett, P., Glen, R. C., Leach, A. R., and Taylor, R. (1997). Development and validation of a genetic algorithm for flexible docking. J. Mol. Biol. 267, 727-748. doi: 10.1006/jmbi.1996.0897

Karakurt, S., and Adali, O. (2016). Tannic acid inhibits proliferation, migration, invasion of prostate cancer and modulates drug metabolizing and antioxidant enzymes. Anticancer. Agents Med. Chem. 16, 781-789. doi: 10.2174/1871520616666151111115809

Katalinić, M., Rusak, G., Barović, J. D., Šinko, G., Jelić, D., Antolović, R., and Kovarik, Z. (2010). Structural aspects of flavonoids as inhibitors of human butyrylcholinesterase. Eur. J. Med. Chem. 45, 186-192. doi: 10.1016/j.ejmech.2009.09.041

Kennedy, D. O., and Wightman, E. L. (2011). Herbal extracts and phytochemicals: plant secondary metabolites and the enhancement of human brain function. Adv. Nutr. 2, 32-50. doi: 10.3945/an.110.000117

Khorasani Esmaeili, A., Mat Taha, R., Mohajer, S., and Banisalam, B. (2015). Antioxidant activity and total phenolic and flavonoid content of various solvent extracts from in vivo and in vitro grown Trifolium pratense L. (red clover). Biomed Res. Int. 2015:643285. doi: 10.1155/2015/643285

Kim, D.-O., Lee, K. W., Lee, H. J., and Lee, C. Y. (2002). Vitamin C equivalent antioxidant capacity (VCEAC) of phenolic phytochemicals. J. Agric. Food Chem. 50, 3713-3717. doi: 10.1021/jf020071c

Kocak, M. S., Sarikurkcu, C., Cengiz, M., Kocak, S., Uren, M. C., and Tepe, B. (2016). Salvia cadmica: phenolic composition and biological activity. Ind. Crops Prod. 85, 204-212. doi: 10.1016/j.indcrop.2016.03.015

Kubinova, R., Porizkova, R., Navratilova, A., Farsa, O., Hanakova, Z., Bacinska, A., et al. (2014). Antimicrobial and enzyme inhibitory activities of the constituents of Plectranthus madagascariensis (Pers.) Benth. J. Enzym. Inhib. Med. Chem. 29, 749-752. doi: 10.3109/14756366.2013.848204

Kuete, V., Wiench, B., Alsaid, M. S., Alyahya, M. A., Fankam, A. G., Shahat, A. A., et al. (2013). Cytotoxicity, mode of action and antibacterial activities of selected Saudi Arabian medicinal plants. BMC Complement. Altern. Med. 13:354. doi: 10.1186/1472-6882-13-354

Kumar, S., Narwal, S., Kumar, V., and Prakash, O. (2011). $\alpha$-glucosidase inhibitors from plants: a natural approach to treat diabetes. Pharmacogn. Rev. 5, 19-29. doi: 10.4103/0973-7847.79096

Li, H.-R., Habasi, M., Xie, L.-Z., and Aisa, H. A. (2014). Effect of chlorogenic acid on melanogenesis of B16 melanoma cells. Molecules 19, 12940-12948. doi: 10.3390/molecules190912940

Liebezeit, G. (2008). Ethnobotany and phytochemistry of plants dominant in salt marshes of the Lower Saxonian Wadden Sea, southern North Sea. Mar. Biodiversity 38, 1-30. doi: 10.1007/BF03043865

López-Lázaro, M. (2009). Distribution and biological activities of the flavonoid luteolin. Mini Rev. Med. Chem. 9, 31-59. doi: 10.2174/138955709787001712

Losada-Barreiro, S., and Bravo-Díaz, C. (2017). Free radicals and polyphenols: the redox chemistry of neurodegenerative diseases. Eur. J. Med. Chem. 133, 379-402. doi: 10.1016/j.ejmech.2017.03.061
Maestro, S. (2015). Version 9.2. LLC. New York, NY.

Mazlan, N. A., Mediani, A., Abas, F., Ahmad, S., Shaari, K., Khamis, S., et al. (2013). Antioxidant, antityrosinase, anticholinesterase, and nitric oxide inhibition activities of three Malaysian Macaranga species. Sci. World J. 2013:312741. doi: 10.1155/2013/312741

Meeran, M. F. N., Javed, H., Al Taee, H., Sheikh, A., and Ojha, S. K. (2017). Pharmacological properties and molecular mechanisms of thymol: prospects for its therapeutic potential and pharmaceutical development. Front. Pharmacol. 8:380. doi: 10.3389/fphar.2017.00380

Menichini, F., Losi, L., Bonesi, M., Pugliese, A., Loizzo, M. R., and Tundis, R. (2014). Chemical profiling and in vitro biological effects of Cardiospermum halicacabum L. (Sapindaceae) aerial parts and seeds for applications in neurodegenerative disorders. J. Enzym. Inhib. Med. Chem. 29, 677-685. doi: 10.3109/14756366.2013.840614

Mezrag, A., Malafronte, N., Bouheroum, M., Travaglino, C., Russo, D., Milella, L., et al. (2017). Phytochemical and antioxidant activity studies on Ononis angustissima L. aerial parts: isolation of two new flavonoids. Nat. Prod. Res. 31, 507-514. doi: 10.1080/14786419.2016.1195381

Mhamdi, B., Abbassi, F., and Abdelly, C. (2015). Chemical composition, antioxidant and antimicrobial activities of the edible medicinal Ononis natrix growing wild in Tunisia. Nat. Prod. Res. 29, 1157-1160. doi: 10.1080/14786419.2014.981188

Mocan, A., Zengin, G., Uysal, A., Gunes, E., Mollica, A., Degirmenci, N. S., et al. (2016). Biological and chemical insights of Morina persica L.: a source of bioactive compounds with multifunctional properties. J. Funct. Foods 25, 94-109. doi: 10.1016/j.jff.2016.05.010

Mükemre, M., Behçet, L., and Çakılcıŏlu, U. (2015). Ethnobotanical study on medicinal plants in villages of Çatak (Van-Turkey). J. Ethnopharmacol. 166, 361-374. doi: 10.1016/j.jep.2015.03.040

Murugan, R., and Parimelazhagan, T. (2014). Comparative evaluation of different extraction methods for antioxidant and anti-inflammatory properties from Osbeckia parvifolia Arn.-An in vitro approach. J. King Saud Univer. Sci. 26, 267-275. doi: 10.1016/j.jksus.2013.09.006

Nachon, F., Carletti, E., Ronco, C., Trovaslet, M., Nicolet, Y., Jean, L., et al. (2013). Crystal structures of human cholinesterases in complex with huprine $\mathrm{W}$ and tacrine: elements of specificity for anti-Alzheimer's drugs targeting acetyl-and butyryl-cholinesterase. Biochem. J. 453, 393-399. doi: 10.1042/BJ201 30013

Omura, S., and Campbell, W. C. (2015). Nobel Prize for artemisinin brings phytotherapy into the spotlight. Phytomedicine 22, A1-A3. doi: 10.1016/j.phymed.2015.10.003

Orhan, D. D., Özçeli̇k, B., Hoşbaş, S., and Vural, M. (2012). Assessment of antioxidant, antibacterial, antimycobacterial, and antifungal activities of some plants used as folk remedies in Turkey against dermatophytes and yeast-like fungi. Turkish J. Biol. 36, 672-686. doi: 10.3906/biy1203-33

Osakabe, N., Yasuda, A., Natsume, M., and Yoshikawa, T. (2004). Rosmarinic acid inhibits epidermal inflammatory responses: anticarcinogenic effect of Perilla frutescens extract in the murine two-stage skin model. Carcinogenesis 25, 549-557. doi: 10.1093/carcin/bgh034

Otto, M. (2009). Staphylococcus epidermidis-the 'accidental' pathogen. Nat. Rev. Microbiol. 7, 555-567. doi: 10.1038/nrmicro2182

Ozkan, O. E., Zengin, G., Akca, M., Baloglu, M. C., Olgun, C., Altuner, E. M., et al. (2015). DNA protection, antioxidant, antibacterial and enzyme inhibition activities of heartwood and sapwood extracts from juniper and olive woods. RSC Adv. 5, 72950-72958. doi: 10.1039/C5RA12302J

Peña-Cerda, M., Arancibia-Radich, J., Valenzuela-Bustamante, P., PérezArancibia, R., Barriga, A., Seguel, I., et al. (2017). Phenolic composition and antioxidant capacity of Ugni molinae Turcz. leaves of different genotypes. Food Chem. 215, 219-227. doi: 10.1016/j.foodchem.2016.07.159

Pesaresi, A., and Lamba, D. (in press). Torpedo Californica acetylcholinesterase in complex with a tacrine-nicotinamide hybrid inhibitor. Protein Data Bank. doi: $10.2210 / \mathrm{pdb} 4 \mathrm{x} 3 \mathrm{c} / \mathrm{pdb}$

Picot, M., Zengin, G., Mollica, A., Stefanucci, A., Carradori, S., and Mahomoodally, M. (2017). In vitro and in silico studies of mangiferin from Aphloia theiformis on key enzymes linked to diabetes type 2 and associated complications. Med. Chem. 13, 1-8. doi: 10.2174/1573406413666170307163929

Potenza, L., Calcabrini, C., De Bellis, R., Mancini, U., Cucchiarini, L., and Dachà, M. (2008). Effect of quercetin on oxidative nuclear and mitochondrial DNA damage. Biofactors 33, 33-48. doi: 10.1002/biof.5520330104 
Prieto, P., Pineda, M., and Aguilar, M. (1999). Spectrophotometric quantitation of antioxidant capacity through the formation of a phosphomolybdenum complex: specific application to the determination of vitamin E. Anal. Biochem. 269, 337-341. doi: 10.1006/abio.1999.4019

Rice-Evans, C. A., Miller, N. J., and Paganga, G. (1996). Structure-antioxidant activity relationships of flavonoids and phenolic acids. Free Radic. Biol. Med. 20, 933-956. doi: 10.1016/0891-5849(95)02227-9

Ríos, J. L., Francini, F., and Schinella, G. R. (2015). Natural products for the treatment of type 2 diabetes mellitus. Planta Med. 81, 975-994. doi: $10.1055 / \mathrm{s}-0035-1546131$

Roseiro, L. B., Rauter, A. P., and Serralheiro, M. L. M. (2012). Polyphenols as acetylcholinesterase inhibitors: structural specificity and impact on human disease. Nutr. Aging 1, 99-111

Sarikürkcü, C., Cengiz, M., Çomak, Z., Zengin, G., and Aktumsek, A. (2016). İki Ononis Türünün Antioksidan Aktivitelerinin Karşılaştırılması. Süleyman Demirel Üniversitesi Fen Bilimleri Enstitüsü Dergisi 20, 298-302. doi: 10.19113/sdufbed.51573

Sayari, N., Saidi, M. N., Sila, A., Ellouz-Chaabouni, S., and Bougatef, A. (2016). Chemical composition, angiotensin I-converting enzyme (ACE) inhibitory, antioxydant and antimicrobial activities of Ononis natrix leaves extracts. Free Radic. Antioxid. 6:23. doi: 10.5530/fra.2016.1.3

Shelley, J. C., Cholleti, A., Frye, L. L., Greenwood, J. R., Timlin, M. R., and Uchimaya, M. (2007). Epik: a software program for $\mathrm{pK}$ a prediction and protonation state generation for drug-like molecules. J. Comput. Aided Mol. Des. 21, 681-691. doi: 10.1007/s10822-007-9133-z

Shono, T., Ono, M., Izumi, H., Jimi, S. I., Matsushima, K., Okamoto, T., et al. (1996). Involvement of the transcription factor NF- $\mathrm{KB}$ in tubular morphogenesis of human microvascular endothelial cells by oxidative stress. Mol. Cell. Biol. 16, 4231-4239. doi: 10.1128/MCB.16.8.4231

Silva, C. H. T. P. D., Sobrinho, T. J. D. S.P., Lima, D. D. C. A., and Amorim, E. L. C. D. (2011). Antioxidant capacity and phenolic content of Caesalpinia pyramidalis Tul. and Sapium glandulosum (L.) Morong from Northeastern Brazil. Molecules 16, 4728-4739. doi: 10.3390/molecules16064728

Silva, J. P., Gomes, A. C., and Coutinho, O. P. (2008). Oxidative DNA damage protection and repair by polyphenolic compounds in PC12 cells. Eur. J. Pharmacol. 601, 50-60. doi: 10.1016/j.ejphar.2008.10.046

Slinkard, K., and Singleton, V. L. (1977). Total phenol analysis: automation and comparison with manual methods. Am. J. Enol. Vitic. 28, 49-55.

Sohretoglu, D. (2007). Tedavide Kullanilan Bitkiler. Ononis Spinosa FFD Monograflarıtedavide Kullanılan Bitkiler. Ononis Spinosa FFD Monografları, ed O. Demirezer. Istanbul: Nobel Tip Kitapevi.

Sukumaran, S. T., Girija, S., and Sheela, N. G. (2016). In vitro cytotoxic activity of root methanol extract of Pseudarthria viscida (L.) wight and arn. against hela, hep g2 and 1929 cell lines. Eur. J. Pharm. Med. Res. 3, 498-501.

Süntar, İ., Baldemir, A., Coşkun, M., Keleş, H., and Akkol, E. K. (2011). Wound healing acceleration effect of endemic Ononis species growing in Turkey. J. Ethnopharmacol. 135, 63-70. doi: 10.1016/j.jep.2011.02.023

Tepe, B. (2008). Antioxidant potentials and rosmarinic acid levels of the methanolic extracts of Salvia virgata (Jacq), Salvia staminea (Montbret and Aucher ex Bentham) and Salvia verbenaca (L.) from Turkey. Bioresour. Technol. 99, 1584-1588. doi: 10.1016/j.biortech.2007.04.008

Tundis, R., Loizzo, M., and Menichini, F. (2010). Natural products as $\alpha$ amylase and $\alpha$-glucosidase inhibitors and their hypoglycaemic potential in the treatment of diabetes: an update. Mini Rev. Med. Chem. 10, 315-331. doi: $10.2174 / 138955710791331007$

Uysal, A., Zengin, G., Mollica, A., Gunes, E., Locatelli, M., Yilmaz, T., et al. (2016). Chemical and biological insights on Cotoneaster integerrimus: a new (-)-epicatechin source for food and medicinal applications. Phytomedicine 23, 979-988. doi: 10.1016/j.phymed.2016.06.011

Uysal, S., Zengin, G., Locatelli, M., Bahadori, M. B., Mocan, A., Bellagamba, G., et al. (2017). Cytotoxic and enzyme inhibitory potential of two Potentilla species (P. speciosa L. and $P$. reptans Willd.) and their chemical composition. Front. Pharmacol. 8:290. doi: 10.3389/fphar.2017. 00290

Vattem, D., Jang, H. D., Levin, R., and Shetty, K. (2006). Synergism of cranberry phenolics with ellagic acid and rosmarinic acid for antimutagenic and DNA protection functions. J. Food Biochem. 30, 98-116. doi: $10.1111 / j .1745-4514.2005 .00063 . x$
Wimo, A., and Prince, M. (2010). Alzheimer's Disease International. 2010. World Alzheimer Report.

Wollenweber, E., Dörr, M., Rivera, D., and Roitman, J. N. (2003). Externally accumulated flavonoids in three Mediterranean Ononis species. Zeitschrift fuer Naturforschung C 58, 771-775. doi: 10.1515/znc-2003-11-1202

Wulan, D. R., Utomo, E. P., and Mahdi, C. (2014). Molecular modeling of Ruellia tuberosa L compounds as a-amylase inhibitor: an in silico comparation between human and rat enzyme model. Bioinformation 10, 209-215. doi: 10.6026/97320630010209

Xie, Y., Yang, W., Chen, X., and Xiao, J. (2014). Inhibition of flavonoids on acetylcholine esterase: binding and structure-activity relationship. Food Funct. 5, 2582-2589. doi: 10.1039/C4FO00287C

Yamamoto, K., Miyake, H., Kusunoki, M., and Osaki, S. (2011). Steric hindrance by 2 amino acid residues determines the substrate specificity of isomaltase from Saccharomyces cerevisiae. J. Biosci. Bioeng. 112, 545-550. doi: 10.1016/j.jbiosc.2011.08.016

Yasuda, M., Ohzeki, Y., Shimizu, S., Naito, S., Ohtsuru, A., Yamamoto, T., et al. (1999). Stimulation of in vitro angiogenesis by hydrogen peroxide and the relation with ETS-1 in endothelial cells. Life Sci. 64, 249-258. doi: 10.1016/S0024-3205(98)00560-8

Yerlikaya, A., Erdoğan, E., Okur, E., Yerlikaya, S., and Savran, B. (2016). A novel combination treatment for breast cancer cells involving BAPTA$\mathrm{AM}$ and proteasome inhibitor bortezomib. Oncol. Lett. 12, 323-330. doi: 10.3892/OL.2016.4597

Yin, S.-Y., Yang, N.-S., and Lin, T.-J. (2017). Phytochemicals approach for developing cancer immunotherapeutics. Front. Pharmacol. 8:386. doi: $10.3389 /$ fphar.2017.00386

Yõlmaz, B. S., Özbek, H., Çitoğlu, G. S., Uğraş, S., Bayram, İ., and Erdoğan, E. (2006). Analgesic and hepatotoxic effects of Ononis spinosa L. Phytother. Res. 20, 500-503. doi: 10.1002/ptr.1891

Yousaf, M., Al-Rehaily, A. J., Ahmad, M. S., Mustafa, J., Al-Yahya, M. A., Al-Said, M. S., et al. (2015). A 5-alkylresorcinol and three3, 4dihydroisocoumarins derived from Ononis natrix. Phytochem. Lett. 13, 1-5. doi: 10.1016/j.phytol.2015.05.002

Zeng, L., Zhang, G., Lin, S., and Gong, D. (2016). Inhibitory mechanism of apigenin on $\alpha$-glucosidase and synergy analysis of flavonoids. J. Agric. Food Chem. 64, 6939-6949. doi: 10.1021/acs.jafc.6b02314

Zengin, G. (2016). A study on in vitro enzyme inhibitory properties of Asphodeline anatolica: new sources of natural inhibitors for public health problems. Ind. Crops Prod. 83, 39-43. doi: 10.1016/j.indcrop.2015.12.033

Zengin, G., Ceylan, R., Katanić, J., Mollica, A., Aktumsek, A., Boroja, T., et al. (2017). Combining in vitro, in vivo and in silico approaches to evaluate nutraceutical potentials and chemical fingerprints of Moltkia aurea and Moltkia coerulea. Food Chem. Toxicol. 107(Pt B), 540-553. doi: 10.1016/j.fct.2017.04.004

Zengin, G., Sarikurkcu, C., Aktumsek, A., Ceylan, R., and Ceylan, O. (2014). A comprehensive study on phytochemical characterization of Haplophyllum myrtifolium Boiss. endemic to Turkey and its inhibitory potential against key enzymes involved in Alzheimer, skin diseases and type II diabetes. Ind. Crops Prod. 53, 244-251. doi: 10.1016/j.indcrop.2013.12.043

Zengin, G., Uysal, S., Ceylan, R., and Aktumsek, A. (2015). Phenolic constituent, antioxidative and tyrosinase inhibitory activity of Ornithogalum narbonense L. from Turkey: a phytochemical study. Ind. Crops Prod. 70, 1-6. doi: 10.1016/j.indcrop.2015.03.012

Zhuo, H., Payan, F., and Qian, M. (2004). Crystal structure of the pig pancreatic $\alpha-$ amylase complexed with $\rho$-nitrophenyl- $\alpha$-d-maltoside-flexibility in the active site. Protein J. 23, 379-387. doi: 10.1023/B:JOPC.0000039552.94529.95

Conflict of Interest Statement: The authors declare that the research was conducted in the absence of any commercial or financial relationships that could be construed as a potential conflict of interest.

Copyright (c) 2017 Yerlikaya, Zengin, Mollica, Baloglu, Celik Altunoglu and Aktumsek. This is an open-access article distributed under the terms of the Creative Commons Attribution License (CC BY). The use, distribution or reproduction in other forums is permitted, provided the original author(s) or licensor are credited and that the original publication in this journal is cited, in accordance with accepted academic practice. No use, distribution or reproduction is permitted which does not comply with these terms. 


\title{
Veronica officinalis Product Authentication Using DNA Metabarcoding and HPLC-MS Reveals Widespread Adulteration with Veronica chamaedrys
}

\begin{abstract}
Ancuta C. Raclariu1,2, Andrei Mocan 3,4, Madalina O. Popa', Laurian Vlase, Mihael C. Ichim ${ }^{2}$, Gianina Crisan ${ }^{3}$, Anne K. Brysting ${ }^{6}$ and Hugo de Boer ${ }^{1,7 *}$
\end{abstract}

\begin{abstract}
'Plant Evolution and Metabarcoding Group, Natural History Museum, University of Oslo, Oslo, Norway, ${ }^{2}$ Stejarul Research Centre for Biological Sciences, National Institute of Research and Development for Biological Sciences (NIRDBS), Piatra Neamt, Romania, ${ }^{3}$ Department of Pharmaceutical Botany, Faculty of Pharmacy, Iuliu Hatieganu University of Medicine and Pharmacy, Cluj-Napoca, Romania, ${ }^{4}$ ICHAT and Institute for Life Sciences, University of Agricultural Sciences and Veterinary Medicine of Cluj-Napoca, Cluj-Napoca, Romania, ${ }^{5}$ Department of Pharmaceutical Technology and Biopharmaceutics, Iuliu Hatieganu University of Medicine and Pharmacy, Cluj-Napoca, Romania, ${ }^{6}$ Department of Biosciences, Centre for Ecological and Evolutionary Synthesis (CEES), University of Oslo, Oslo, Norway, ${ }^{7}$ Department of Organismal Biology, Evolutionary Biology Centre, Uppsala University, Uppsala, Sweden
\end{abstract}

OPEN ACCESS

Edited by:

Atanas G. Atanasov,

Institute of Genetics and Animal Breeding (PAN), Poland

Reviewed by:

Marcello Locatelli,

Università degli Studi "G. d'Annunzio"

Chieti - Pescara, Italy

C. Haris Saslis-Lagoudakis, Natural History Museum of Denmark,

Denmark

*Correspondence: Hugo de Boer hugo.deboer@nhm.uio.no

Specialty section: This article was submitted to

Ethnopharmacology,

a section of the journal

Frontiers in Pharmacology

Received: 23 April 2017

Accepted: 31 May 2017

Published: 19 June 2017

Citation

Raclariu AC, Mocan A, Popa MO,

Vlase L, Ichim MC, Crisan G, Brysting AK and de Boer H (2017) Veronica officinalis Product Authentication Using DNA Metabarcoding and HPLC-MS Reveals Widespread Adulteration with Veronica chamaedrys.

Front. Pharmacol. 8:378. doi: 10.3389/fphar.2017.00378
Studying herbal products derived from local and traditional knowledge and their value chains is one of the main challenges in ethnopharmacology. The majority of these products have a long history of use, but non-harmonized trade and differences in regulatory policies between countries impact their value chains and lead to concerns over product efficacy, safety and quality. Veronica officinalis L. (common speedwell), a member of Plantaginaceae family, has a long history of use in European traditional medicine, mainly in central eastern Europe and the Balkans. However, no specified control tests are available either to establish the quality of derived herbal products or for the discrimination of its most common substitute, $V$. chamaedrys L. (germander speedwell). In this study, we use DNA metabarcoding and high performance liquid chromatography coupled with mass spectrometry (HPLC-MS) to authenticate sixteen $V$. officinalis herbal products and compare the potential of the two approaches to detect substitution, adulteration and the use of unreported constituents. HPLC-MS showed high resolution in detecting phytochemical target compounds, but did not enable detection of specific plant species in the products. DNA metabarcoding detected $V$. officinalis in only $15 \%$ of the products, whereas it detected $V$. chamaedrys in $62 \%$ of the products. The results confirm that DNA metabarcoding can be used to test for the presence of Veronica species, and detect substitution and/or admixture of other Veronica species, as well as simultaneously detect all other species present. Our results confirm that none of the herbal products contained exactly the species listed on the label, and all included substitutes, contaminants or fillers. This study highlights the need for authentication of raw herbals along the value chain of these products. An integrative methodology can assess both the quality of herbal products in terms of target compound concentrations and species composition, as well as admixture and substitution with other chemical compounds and plants.

Keywords: adulteration, DNA metabarcoding, herbal products, HPLC-MS, Veronica chamaedrys, Veronica officinalis 


\section{INTRODUCTION}

Traditional herbal medicines play an important role in meeting healthcare needs around the world, and complementary and alternative medicines based on these are gaining in importance in many industrialized countries as a perceived healthy alternative to synthetic pharmaceuticals (World Health Organization, 2013). The commercialization of such products is undergoing a transition process from unregulated locally traded products to internationally traded mass produced herbal pharmaceuticals and food supplements (Heinrich, 2010; Leonti and Casu, 2013). A challenge in ethnopharmacology is to study such novel herbal products and their value chains (Heinrich, 2010; Atanasov et al., 2015), as these are impacted by nonharmonized regulatory and trade policies (Booker et al., 2012, 2016).

The regulation of herbal products varies globally and between EU member countries, these falling under specific legislation depending on their intended use, for instance, as food, food supplements, herbal medicines, or homeopathic products. Similar to the United States and Canada, the EU currently does not have a centralized marketing authorization procedure for herbal products, but instead provides relevant methodological specifications to guarantee manufacturing quality. The EU also lacks a central public institution for pharmacovigilance of the herbal products, and the primary legal responsibility for safety of the marketed herbal products is delegated to the pharmaceutical industry, whereas national pharmacovigilance agencies monitor drug safety.

A lack of standards for studies of medicinal plants has been previously identified (cf. Ríos and Recio, 2005; Gertsch, 2009) and several authors have advocated a stronger guidance of value chains to mitigate this shortcoming (Rios et al., 1988; Cos et al., 2006; Verpoorte, 2008). Current quality assessment methods rely mainly on morphology and analytical chemistry based methods detailed in national or EU pharmacopoeias (EDQM - Council of Europe, 2014). However, the resolution of these methods is highly influenced by factors such as the phenotypic plasticity, morphological cryptic taxa, species-specific chemical markers, growing conditions, harvesting process, storage condition, or the extraction procedure (Barnes, 2003; Bickford et al., 2007; Jiang et al., 2010; Shaw et al., 2012).

In addition to ensuring that herbal products contain the intended ingredients in the right quantities, an important problem is the use of substitutes and undeclared fillers (Coghlan et al., 2012, 2015; Newmaster et al., 2013). Misidentification (Saslis-Lagoudakis et al., 2015) or incongruences in the vernacular names of the raw material (Farah et al., 2006; Ouarghidi et al., 2012; Walker and Applequist, 2012; De Boer et al., 2015) can lead to accidental substitution, but rising prices of raw materials and the high demand for such products also provide an incentive for deliberate adulteration. The use of unlabelled ingredients presents a serious safety challenge as adverse drug reactions cannot be associated to the product label and ingredients (Heubl, 2010; Gilbert, 2011). Recent studies of herbal medicine and herbal food supplement value chains using a combination of phytochemical and metabolomics analyses have found that adulteration of these products often occurs in the first stages of their value chains (Booker et al., 2012, 2014, 2016).

Veronica officinalis L. (common speedwell) is a popular medicinal plant in several European countries, especially in central eastern Europe and the Balkans, and used in herbal medicine both traditionally and increasingly in commercial herbal products. Veronica L. (Plantaginaceae) is a genus of about 450 species (Albach and Meudt, 2010) occurring over most of the Northern Hemisphere and in many parts of the Southern Hemisphere (Mabberley, 2008). Modern systematic studies of the genus have focused on molecular phylogenies of several nuclear and plastid markers: the nuclear ribosomal internal transcribed spacer, nrITS (Wagstaff and Garnock-Jones, 1998; Albach and Chase, 2001; Albach et al., 2004; Buhk et al., 2015), the plastid regions trnL-F (Albach et al., 2004, 2006; Buhk et al., 2015), ndhF-rpl32 (Sonibare et al., 2014; Albach and Al-Gharaibeh, 2015; Buhk et al., 2015), trnH-psbA (Sonibare et al., 2014; Albach and AlGharaibeh, 2015), rps16 intron (Albach and Chase, 2004), rps16-trnK (Sonibare et al., 2014; Albach and Al-Gharaibeh, 2015).

\section{Ethnopharmacological Importance of Veronica Species}

Several Veronica species are used in European traditional medicine to treat respiratory affections, as an expectorant, an antiscorbutic, a diuretic, and for wound healing (Baytop, 1984; Harput et al., 2002a). In Turkey, the aerial parts of Veronica anagallis-aquatica L. are boiled in milk to obtain a poultice used to alleviate abdominal pain (Küpeli et al., 2005), or used as a bath cure to relieve rheumatic pain (Fujita et al., 1995). In the Valdesi valley of Italy, in the poorer villages Veronica species are traditionally used as an inexpensive substitute for imported teas and this has been posed as an explanation of the modern use of Veronica species in recreational teas (Bellia and Pieroni, 2015). In traditional Chinese medicine $V$. anagallis-aquatica is used for the treatment of influenza, hemoptysis, laryngopharyngitis, and hernia (Su et al., 1999). In Europe, V. officinalis is the best-known and most commonly used species (Table 1). The main ethnopharmacological indications for $V$. officinalis are inflammatory conditions, rheumatism, stomach ulcers, respiratory ailments (Scarlat et al., 1985; Mocan et al., 2015b), and nervous, cardiovascular and metabolism system disorders (Vogl et al., 2013).

\section{Phytochemical and Pharmacological Studies}

Veronica species are rich in iridoid glucosides, mainly aucubin, catalpol and benzoic and cinnamic acid esters of catalpol, all compounds that have previously been investigated for their bioactivities (Harput et al., 2002a,b, 2014; Saracoglu et al., 2002). Saracoglu and Harput (2012) showed that some iridoid glucosides of $V$. anagallis-aquatica, $V$. persica Poir, and $V$. thymoides P.H.Davis subsp. pseudocinerea M.A.Fisch. exhibited cytostatic 
TABLE 1 | Traditional uses of Veronica officinalis in different European countries.

\begin{tabular}{|c|c|c|}
\hline Country & Use & Reference \\
\hline Austria & $\begin{array}{l}\text { Cardiovascular system } \\
\text { Metabolism } \\
\text { Nervous system } \\
\text { Respiratory tract }\end{array}$ & Vogl et al., 2013 \\
\hline Bulgaria & $\begin{array}{l}\text { Appetizer } \\
\text { Anti-inflammatory } \\
\text { Antitussive } \\
\text { Asthma } \\
\text { Coughs } \\
\text { Expectorant } \\
\text { Pharyngitis } \\
\text { Tonsillitis }\end{array}$ & $\begin{array}{l}\text { Ivancheva and Stantcheva, } \\
\text { 2000; Leporatti and Ivancheva, } \\
2003\end{array}$ \\
\hline Italy & Recreational tea & Bellia and Pieroni, 2015 \\
\hline Montenegro & $\begin{array}{l}\text { Bronchitis } \\
\text { Rheumatic complaints } \\
\text { Skin diseases } \\
\text { Wounds }\end{array}$ & Menković et al., 2011 \\
\hline Romania & $\begin{array}{l}\text { Antiulcerous activity } \\
\text { Catarrh } \\
\text { Cough } \\
\text { Hepatoprotective activity } \\
\text { Hypocholesterolemic effect } \\
\text { Kidney diseases } \\
\text { Lung diseases } \\
\text { Wound healing properties }\end{array}$ & $\begin{array}{l}\text { Tamas et al., 1984; Scarlat } \\
\text { et al., 1985; Crişan et al., 2007; } \\
\text { Gründemann et al., } 2013\end{array}$ \\
\hline Serbia & $\begin{array}{l}\text { Against anemia } \\
\text { Hypolipemic } \\
\text { Treatment of skin diseases }\end{array}$ & Zlatković et al., 2014 \\
\hline Sweden & Recreational tea & Sõukand et al., 2013 \\
\hline
\end{tabular}

and cytotoxic activities on human and murine cancer cell lines, with verminoside being the most cytotoxic compound and Harput et al. (2002a) showed that V. cymbalaria Bodard, $V$. hederifolia L., V. pectinata L. var. glandulosa, V. persica and $V$. polita Fr. possess anti-inflammatory, cytotoxic and radical scavenging activities. Vogl et al. (2013) showed for the first time in vitro anti-inflammatory activity of the extracts prepared from $V$. officinalis. Nikolova (2011) found that $V$. bellidioides L., V. kellereri Degen \& Urum., V. vindobonensis (M.A.Fisch.) M.A.Fisch., V. beccabunga L., $V$. rhodopea Degen ex Stoj. $\&$ Stef. and $V$. austriaca L. showed significant antioxidant properties.

\section{Quality Issues of Veronica officinalis Herbal Products}

As a folk remedy, $V$. officinalis is used mostly in traditional medicine, and no current national pharmacopeia monographs include standards for quality control or authentication of Veronica herbal products. Identification tests currently available to detect substitutes and adulterants rely on chromatographic methods, usually as a combination of separate approaches (e.g., HPLC with TLC-densitometry) or a combination of different approaches into a single procedure (e.g., HPLC-UV, HPLC-MS or GC-MC) (European Medicine Agency [EMA], 2006; EDQM Council of Europe, 2014).

All raw materials of $V$. officinalis used in the marketed herbal products are harvested from the spontaneous flora, and sourced from several countries in central eastern Europe and the Balkans. Kathe et al. (2003) and Ichim et al. (2011) cite that the exploitation pressure of wild-harvesting could potentially threaten the species. The limited availability of $V$. officinalis is a likely driver behind the widespread substitution with $V$. chamaedrys L. (germander speedwell), an abundant herb of grassland and forest edges, with no proven therapeutic uses Crişan et al. (2007, 2011). Furthermore, even though their vegetative morphology is clearly distinct, the flowers of $V$. officinalis and $V$. chamaedrys can easily be confused (Leyel, 1937). Crişan et al. (2001, 2011) report histological, anatomical and phytochemical characters that distinguish $V$. officinalis and $V$. chamaedrys and propose a rapid differentiation method using thin-layer chromatography (TLC) of species-specific phenyl-propanoic compounds. These analytical methods enable chemical identification of some target compounds, but cannot rule out that other Veronica species, or mixtures thereof, might be present in the investigated products. Marketed herbal products are complex formulations that are usually highly processed and consist of numerous ingredients.

\section{DNA Barcoding and Metabarcoding}

Rapid advances in DNA sequencing have enabled cost-effective use of DNA sequences for species identification. Hebert et al. (2003) coined the concept of DNA barcoding as a rapid and accurate way of species-level identifications using short standard DNA regions. Different combinations of plastid markers (e.g., rbcL, matK, and trnH-psbA) and the nuclear ribosomal internal transcribed spacer (nrITS) have been proposed as the primary barcodes for plants, and the current core barcodes are rbcL and matK, supplemented by nrITS (Kress and Erickson, 2007; CBOL Plant Working Group, 2009; China Plant BOL Group, 2011; Hollingsworth et al., 2011). Several studies have shown that about $75-85 \%$, and in some floras over $90 \%$, of plant species can be identified to species level using DNA barcoding (Lahaye et al., 2008; Kress et al., 2009, 2010; Chen et al., 2010; Burgess et al., 2011). In taxonomically difficult plant groups, where hybridization is frequent, or in lineages of relatively young age the use of traditional barcodes may not be reliable (Kress and Erickson, 2007; Fazekas et al., 2009). In such groups the use of low-coverage shotgun sequencing of genomic DNA could resolve relationships and augment current plant barcodes (Coissac et al., 2016; Hollingsworth et al., 2016).

Techen et al. (2014) reviewed the progress made in DNA barcoding of medicinal plants. DNA barcoding of herbal products has revealed alarming levels of substitution within marketed herbal products, for instance, $6 \%$ in saw palmetto herbal dietary supplements (Little and Jeanson, 2013), 16\% in ginkgo products (Little, 2014), 25\% in black cohosh (Baker et al., 2012), $33 \%$ in herbal teas (Stoeckle et al., 2011), 50\% in ginseng (Wallace et al., 2012), 37\% in Senna and 50\% in Cassia market products (Seethapathy et al., 2014). Newmaster et al. (2013) 
tested 44 herbal products sold in North America using DNA barcoding and found that 59\% contained species not listed on the label, and only 2 out of 12 screened companies had products free of substitution, contamination or unreported fillers. DNA metabarcoding, a combination of high-throughput sequencing (HTS) and polymerase chain reaction based DNA amplification, provides simultaneous taxonomic identification of taxa from samples containing DNA from different origins (Taberlet et al., 2012). DNA metabarcoding studies are providing insights into species composition of complex mixtures of DNA such as processed herbal products. For example, Coghlan et al. (2012) evaluated the species composition of fifteen highly processed traditional Chinese medicines (TCM) and found that these contained species and genera included on CITES appendices I and II. Similarly, Cheng et al. (2014) found a high level of contamination within 27 investigated herbal preparations, Ivanova et al. (2016) found that all 15 tested herbal supplements contained species not listed on the product label, and Raclariu et al. (2017) authenticated 78 Hypericum perforatum herbal products and found the target species in only $68 \%$ products while detecting incongruence between constituent species and those listed on the label in all products.

In this study we test the hypothesis that dwindling availability of wild $V$. officinalis has resulted in admixture of $V$. chamaedrys and other Veronica species in Veronica herbal products. Substitution of $V$. officinalis with $V$. chamaedrys would not only be fraudulent from a commercial perspective, but would also leave anticipating consumers with a herbal product without proven therapeutic activity. We approach this hypothesis using DNA metabarcoding and HPLC-MS to authenticate and detect species diversity in European Veronica herbal products, and aim to answer the following research questions: (1) Can HPLC-MS be used to distinguish $V$. officinalis from $V$. chamaedrys and to identify exclusive presence of $V$. officinalis in herbal products?; (2) Can DNA metabarcoding be used to test for the presence of $V$. officinalis in herbal products, to detect substitution and adulteration for other Veronica species and/or presence of other off label plant species?

\section{MATERIALS AND METHODS}

\section{Sample Collection}

Sixteen single and multi-ingredient herbal products including $V$. officinalis on the label were purchased from retail stores and pharmacies (14) and via e-commerce (2). The products included herbal teas (12), extracts (2), capsules (1), and candies (1). An overview of the samples is included in the Supplementary Table S1, but identifying information on the producer/importer, product name, lot number, expiration date is withheld for anonymity. The herbal products were imported to Norway under Norwegian Medicines Agency license no. 16/04551-2.

For DNA barcoding, nrITS reference sequences of 118 accessions belonging to 56 Veronica species were compiled into a barcode reference database, consisting partly of sequences mined from NCBI/GenBank and partly of sequences generated within the project from specimens collected in 2014 and 2015 (Supplementary Table S2). For the phytochemical analyses, aerial parts of $V$. officinalis and $V$. chamaedrys (samples marked with an asterisk in Supplementary Table S2) were used as references for the identification and quantification of the main compounds. Voucher specimens of the plant material used in this study are deposited in the Herbarium of the Alexandru Borza Botanical Garden (CL) of Babes-Bolyai University, Cluj-Napoca, Romania (Supplementary Table S2).

\section{Phytochemical Analysis High-Performance Liquid Chromatography-Mass Spectrometry (HPLC-MS)}

Extracts were prepared from plant material and herbal products following established procedures (Crişan et al., 2010; Mocan et al., 2015a,b), with slight modifications. One gram of each sample was extracted with $10 \mathrm{ml}$ of $70 \%$ ethanol (HPLC grade) at room temperature for $1 \mathrm{~h}$ in a sonication bath (Polsonic 3, Polsonic Inc., Poland). The extracts were filtered through MN 615 filter paper (Macherey-Nagel $\mathrm{GmbH}$, Germany) and stored at $4^{\circ} \mathrm{C}$ until further analysis. Prior to HPLC-MS analysis the extracts were syringe filtered with a $0.45 \mu \mathrm{m}$ nylon membrane (Whatman Inc., United States).

Target Veronica iridoids (aucubin, catalpol, catalposide, and veronicoside) from all sixteen herbal products as well as aerial plant parts were analyzed by HPLC-MS on a Agilent 1100 liquid chromatography system equipped with a binary pump, autosampler, thermostat and detector (all 1100 Series from Agilent Inc., United States). The system was controlled with Data Analysis software (version B01.03, Agilent Inc., United States). The separation was carried out on an Atlantis HILIC $3.5 \mu \mathrm{m}(100 \mathrm{~mm} \times 3.0 \mathrm{~mm}$ i.d.) (Waters Inc., United States) column equipped with an online $0.2 \mu \mathrm{m}$ filter (Agilent Inc.), at a working temperature of $40^{\circ} \mathrm{C}$, a flow rate of $0.75 \mathrm{ml} / \mathrm{min}$ and an injection volume of $6 \mu \mathrm{l}$. A binary gradient system with eluent (A) $0.1 \%$ acetic acid and $20 \mu \mathrm{M}$ sodium acetate in water, and eluent (B) $0.1 \%$ acetic acid and $20 \mu \mathrm{M}$ sodium acetate in acetonitrile was used for the analyzed samples with the following gradient: $95-80 \%$ B (1$5 \mathrm{~min})$. The HPLC system was coupled with an Agilent Ion Trap 1100 SL mass spectrometer equipped with an electrospray ionisation (ESI) source and operated in the positive mode with a scan range between 360 and $680 \mathrm{~m} / \mathrm{z}$, to identify the target compounds based on their sodium adducts $(M+23 \mathrm{~m} / \mathrm{z})$ : aucubin $(369 \mathrm{~m} / \mathrm{z})$, catalpol $(385 \mathrm{~m} / \mathrm{z})$, catalposide $(505 \mathrm{~m} / \mathrm{z})$ and veronicoside $(489 \mathrm{~m} / \mathrm{z})$, and by comparison with analytical standards in the same chromatographic conditions. The capillary voltage was set to $4 \mathrm{kV}$ and the capillary temperature to $325^{\circ} \mathrm{C}$. Nitrogen $\left(\mathrm{N}_{2}\right)$ was used as dry gas with a dry flow of $12 \mathrm{l} / \mathrm{min}$ and a pressure of $60 \mathrm{psi}$ for the nebulizer. For quantitation of the iridoids, stock solutions of the four commercially available standards substances (Sigma-Aldrich, St. Louis, Missouri, United States) were prepared in acetonitrile, and different concentrations of each standard were used. All calibration curves yielded a coefficient of determination of 
$R^{2} \geq 0.99$. The results are expressed as $\mu \mathrm{g}$ per $\mathrm{g}$ of dry weight material $(\mu \mathrm{g} / \mathrm{g} \mathrm{dw})$.

\section{Genetic Analysis DNA Extraction, PCR Amplification and DNA Sequencing}

The total DNA was extracted from $300 \mathrm{mg}$ of each homogenized herbal product or silica gel dried leaves, using a modified CTAB extraction protocol (Doyle and Doyle, 1987; Kool et al., 2012). Two extraction negative controls were included to screen for contamination and cross-contamination. Total DNA extracts of the herbal products were quantified and assessed for fragmentation with a Fragment Analyzer (Advanced Analytical Technologies, Inc., Ankeny, IA, United States) using the DNF488-33 HS (High Sensitivity) genomic DNA Reagent Kit (50 $40000 \mathrm{bp}$ ). Total DNA extracts of silica gel dried leaves were quantified with a Qubit 2.0 fluorometer (Life Technologies, Carlsbad, CA, United States) using the dsDNA BR (BroadRange) kit. PCR amplification using nrITS primers, ITS-4 and ITS-5 (White et al., 1990), was done on purified total DNA from the dried leaves, using a final reaction volume of $25 \mu \mathrm{l}$, including $2.5 \mu \mathrm{l} 10 \mathrm{X}$ reaction buffer II (supplied with the polymerase), $2.5 \mu \mathrm{l} 25 \mathrm{mM} \mathrm{MgCl} 2$ (supplied with the polymerase), $0.2 \mu 15 \mathrm{U} / \mathrm{ml}$ AmpliTaq DNA Polymerase (Applied Biosystems, Foster City, CA, United States), $2.5 \mu 110 \mu \mathrm{M}$ dNTPs (Applied Biosystems, Foster City, CA, United States), $2.5 \mu 1$ $10 \mu \mathrm{M}$ of each primer (Sigma-Aldrich, St. Louis, Mo, United States), $2.5 \mu l 1 \mathrm{mg} / \mathrm{ml}$ bovine serum albumen (BSA) (Roche Diagnostic GmbH, Mannheim, Germany), $9.3 \mu \mathrm{l}$ of Milli-Q ultrapure water and $0.5 \mu \mathrm{l}$ of template DNA solution $(1 \mathrm{ng} / \mu \mathrm{l})$. The PCR cycling protocol consisted of initial denaturation at $94^{\circ} \mathrm{C}$ for $2.5 \mathrm{~min}$, followed by 32 cycles of denaturation at $94^{\circ} \mathrm{C}$ for $30 \mathrm{~s}$, annealing at $53^{\circ} \mathrm{C}$ for $30 \mathrm{~s}$, and elongation at $72^{\circ} \mathrm{C}$ for $50 \mathrm{~s}$, followed by a final elongation step at $72^{\circ} \mathrm{C}$ for $4 \mathrm{~min}$. Three PCR negative controls were included per amplification to control for external and cross-sample contamination. $6 \mu \mathrm{l}$ of each PCR products was purified using $2 \mu 110$ times diluted ExoSAP-IT (USB Corporation, Cleveland, OH, United States) by incubation at $37^{\circ} \mathrm{C}$ for $45 \mathrm{~min}$ followed by $15 \mathrm{~min}$ at $80^{\circ} \mathrm{C}$. Sequencing was performed using the ABI BigDye Terminator sequencing buffer and the v3.1 Cycle Sequencing kit (Applied Biosystems, Foster City, CA, United States) on an ABI3130XL automated sequencer (Applied Biosystems, Foster City, CA, United States). The sequences were edited, and the forward and reverse sequences assembled using SeqTrace (Stucky, 2012). The sequences were submitted to NCBI/GenBank and their accession numbers are listed in the Supplementary Table S2.

\section{Barcode Gap Analysis}

The reference sequences of 118 accessions belonging to 56 Veronica species were aligned using AliView (Larsson, 2014), and pairwise genetic distances between the accessions were calculated using MEGA 6.0 (Tamura et al., 2013) for each of the following regions: nrITS1, nrITS2, and the entire nrITS. Automatic Barcode Gap Discovery (ABGD) (Puillandre et al., 2012) was used to determine the genetic distance threshold and automatically define species delimitation hypotheses. ABGD determines the number of groups (hypothetical species) within a dataset using pairwise sequence distances and two input variables, prior intraspecific diversity $(P)$ and minimum gap width $(X)$ (Puillandre et al., 2012). Based on this information ABGD makes an initial partition of the supplied sequences into candidate species groups based on a statistically inferred barcode gap and then applies recursive partitions to each group to generate subsequent partitions into candidate species until no further splitting occurs (Puillandre et al., 2012). The minimum gap width $(X)$ was set to $X=1.0$, the prior intraspecific diversity $(P)$ was set to range from 0.001 to 0.1 , and the $p$-distance, JukesCantor (JC) and Kimura 2 Parameter (K2P) distance metrics were used in the ABGD analyses (Jukes and Cantor, 1969; Kimura, 1980), resulting in 18 analyses (6 per dataset). Default values were used for all other parameters (steps: 10 and Nb bins: 20). The total numbers of groups corresponding to the prior intraspecific divergence $(P)$ values, ranging from 0.001 to 0.1 , were recorded (Supplementary Table S3).

To determine whether $V$. officinalis can be unambiguously differentiated from its main adulterant, $V$. chamaedrys, using molecular operational taxonomic units (MOTUs), the intra- and interspecific variation of all reference sequences of these species, respectively, 16 accessions belonging to $V$. officinalis and 9 to $V$. chamaedrys, were analyzed based on pairwise comparison of nrITS genetic distances using Taxon DNA/SpeciesIdentifier v 1.7.8 (Meier et al., 2006).

\section{DNA Metabarcoding}

Fusion primers of the internal transcribed spacers nrITS1 and nrITS2, based on primers 17SE and 5.8I1, and 5.8I2 and 26SE (Sun et al., 1994) (Biolegio B.V., the Netherlands), were used to generate PCR based amplicons. Forward primers were fused with a unique $10 \mathrm{bp}$ multiplex identifier (MID) tags and the reverse primers with a truncated version of adapter P1 (trP1) tags. Expected amplicon sizes were 300-350 bp. PCR reactions on DNA extracted from the herbal products were carried out in final reaction volumes of $25 \mu \mathrm{l}$ including $0.5 \mu \mathrm{l}$ of template DNA solution (ranging from 0.5 to $2 \mathrm{ng} / \mu \mathrm{l}$ ), $5 \mu \mathrm{l} 5 \mathrm{X}$ Q5 reaction buffer (New England Biolabs Inc., United Kingdom), $1.5 \mu 110 \mu \mathrm{M}$ of each primer (Biolegio B.V., the Netherlands), $0.5 \mu \mathrm{l} 10 \mathrm{mM}$ dNTPs, $0.25 \mu \mathrm{l} 20 \mathrm{U} / \mu \mathrm{l}$ Q5 High-Fidelity DNA Polymerase (New England Biolabs Inc., United Kingdom), $5 \mu \mathrm{l}$ 5X Q5 High GC enhancer and $10.75 \mu$ l of Milli-Q ultrapure water. The PCR cycling protocol consisted of initial denaturation at $98^{\circ} \mathrm{C}$ for 30 s, followed by 35 cycles of denaturation at $98^{\circ} \mathrm{C}$ for 10 s, annealing at $56^{\circ} \mathrm{C}$ for nrITS1 or $71^{\circ} \mathrm{C}$ for nrITS2 for $30 \mathrm{~s}$, and elongation at $72^{\circ} \mathrm{C}$ for $30 \mathrm{~s}$, followed by a final elongation step at $72^{\circ} \mathrm{C}$ for $2 \mathrm{~min}$. Eight PCR negative controls were included per amplification to control for external and crosssample contamination.

Agencourt AMPure XP PCR purification (Beckman Coulter Inc., United States) was used for high-throughput purification of PCR amplicons. The size, purity and the molar concentration $(\mathrm{nmol} / \mathrm{l})$ of each amplicon library was measured using a Fragment Analyzer ${ }^{\mathrm{TM}}$ (Advanced Analytical Technologies Inc., United States) with a DNF-910 dsDNA Reagent Kit (35$1,500 \mathrm{bp}$ ), equimolar pools ( $2 \mathrm{ng} / \mu \mathrm{l} / \mathrm{library})$ were prepared 
from the amplicon libraries using a Biomek 4000 Laboratory Automation Workstation (Beckman Coulter Inc., United States). To identify the optimum bead-to-template ratio for template preparation, the concentration of the purified pooled amplicon libraries was analyzed with a Fragment Analyzer ${ }^{\mathrm{TM}}$ (Advanced Analytical Technologies, Inc., United States) using a DNF-488 High Sensitivity Genomic DNA Analysis Kit. The pooled Ion AmpliSeq libraries (Life Technologies, Thermo Fisher Scientific Inc., United States) for emulsion PCR and sequencing chip loading were prepared using an Ion Chef (LT). The DNA template concentration was adjusted to the number of Ion Sphere Particles (ISPs) and added to the emulsion PCR master mix. The templatepositive ISPs were enriched and sequenced on an Ion Torrent Personal Genome Machine (LT) using Ion 318 v2 chips (LT) and the Ion PGM Sequencing 400 kit (LT). Sequencing read data was initially processed and demultiplexed based on MIDs into FASTQ files using Ion Torrent Suite Software version 5.0.4 (LT).

\section{Bioinformatics Analysis}

Sequencing reads were processed using the HTS barcode-checker pipeline freely available at https:/github.com/naturalis/HTSbarcode-checker (Lammers et al., 2014). The nrITS1 and nrITS2 primer sequences were used to split the reads based on the primer sequence. Read lengths and Phred quality scores were assessed using PRINSEQ (Schmieder and Edwards, 2011), and based on these values reads with a mean Phred quality score of less than 26 and a length of less than 300 bp were filtered out. The remaining reads were trimmed to a maximum length of $350 \mathrm{bp}$. Reads were clustered into MOTUs using three different sequence similarity thresholds during clustering, respectively, 97, 99 and $100 \%$, with a minimum of 10 reads per cluster using CDHIT-EST (Li and Godzik, 2006). A representative sequence from each MOTU was taxonomically assigned using local Basic Local Alignment Search Tool (local BLAST) (Altschul et al., 1990), with a maximum e-value of 0.05 and a minimum hit length of $100 \mathrm{bp}$, against a reference database consisting of a local copy of the NCBI nucleotide database (Benson et al., 2013). Species level identifications were assigned when similarity to the reference barcode matched at $>99 \%$.

\section{RESULTS AND DISCUSSION}

\section{Phyotochemical Analysis High-Performance Liquid Chromatography-Mass Spectrometry (HPLC-MS)}

Iridoids and iridoid glycosides are specific chemotaxonomic markers for the genus Veronica, and most Veronica species contain the iridoid glycosides aucubin and/or catalpol as well as one or more 6-O-esters of catalpol (i.e., veronicoside, catalposide) (Afifi-Yazar and Sticher, 1980; Taskova et al., 2002, 2004; Jensen et al., 2005). The main known ethnopharmacological indication for Veronica species are inflammatory diseases (rheumatism, stomach ulcer) or respiratory ailments (Scarlat et al., 1985; Mocan et al., 2015a), and several studies have shown that iridoid glycosides in Veronica are linked to this therapeutic effects (Scarlat et al., 1985; Gründemann et al., 2013; Ignjatović,
2015). Although regulatory guidelines to establish quality control or authentication of Veronica herbal products are lacking, the presence and concentration of specific iridoid glycosides has been shown to enable authentication of Veronica herbal products (Crişan et al., 2010; Gründemann et al., 2013).

The HPLC-MS results from the present study show the characterization of Veronica herbal products against four commercially available iridoid glycosides, namely aucubin, catalpol, veronicoside and catalposide (Table 2). The two most commonly identified compounds in the examined herbal products and reference plant material were aucubin and catalpol. From a pharmaceutical point of view, the concentrations of the compounds present in different herbal remedies or plant materials cannot be neglected as they are directly linked to their pharmaceutical efficacy and effectiveness. Aucubin was found in fifteen herbal products samples as well as in the reference plant material of both species ( $V$. officinalis and $V$. chamaedrys), with the highest concentration in sample 9. Aucubin concentrations in the herbal products and reference plant material show a large variation with concentrations ranging from 0.33 to $39.73 \mu \mathrm{g} / \mathrm{g}$. Catalpol was found in 13 herbal products, with the highest concentration in sample 11, but was missing in two of the reference plant samples ( $V$. off 4 and $V$. cha 1). No iridoid glycosides were identified in sample 14, a lozenge with nineteen ingredients listed on the label. Jensen et al. (2005) concludes that aucubin and/or catalpol is/are present in all Veronica species, implying that if sample 14 contains any material of Veronica this would be below the level of detection of the HPLC-MS platform we used in this study, and consequently unlikely to exert any pharmacological activity. Concerning the other two 6-O-esters of catalpol, veronicoside was present in five herbal products and in all $V$. officinalis reference material, with highest concentration in sample $V$. off 1, while catalposide was present in nine herbal products, with the highest concentration in sample 8 , but none of the investigated reference plant materials contained catalposide. Jensen et al. (2005) states that although aucubin and/or catalpol are present in all Veronica species, $V$. micrantha is the only species in Veronica subgenus Chamaedrys, to which $V$. chamaedrys belongs, that contains aucubin. However, this assumption was later rejected by Crişan et al. (2010) that identified both aucubin and catalpol in $V$. chamaedrys, and as well as by the results of this study.

\section{Genetic Analyses Barcode Gap Analysis}

Species-level identification using DNA barcoding and metabarcoding requires that the level of intraspecific genetic divergence is lower than the interspecific genetic divergence (Meyer and Paulay, 2005), and several identification approaches are based on setting a cut-off threshold above which a query sequence is considered to be distinct from a reference sequence. Defining an optimal threshold is a controversial debate (Hebert et al., 2004; Meyer and Paulay, 2005; Smith et al., 2005; Wiemers and Fiedler, 2007; Chen et al., 2013). Previous studies proposed different thresholds of genetic distances, for instance, $1 \%$ (Ratnasingham and Hebert, 2013), 3\% (Smith et al., 2005) or the 


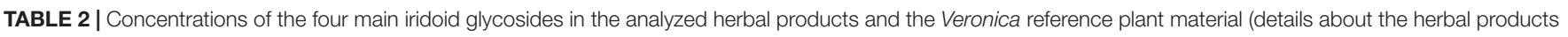
can be found in Supplementary Table S1).

\begin{tabular}{|c|c|c|c|c|}
\hline \multirow[t]{2}{*}{ Product no. } & \multicolumn{4}{|c|}{ Concentrations (in $\mu \mathrm{g} / \mathrm{g}$ ) } \\
\hline & Veronicoside & Catalposide & Aucubin & Catalpol \\
\hline 1 & $\mathrm{n} / \mathrm{c}$ & $0.48 \pm 0.02$ & $1.19 \pm 0.03$ & $0.28 \pm 0.01$ \\
\hline 2 & $n / c$ & $\mathrm{n} / \mathrm{c}$ & $1.58 \pm 0.02$ & $1.05 \pm 0.04$ \\
\hline 3 & $n / c$ & $3.38 \pm 0.11$ & $13.77 \pm 0.52$ & $1.52 \pm 0.04$ \\
\hline 4 & $0.24 \pm 0.01$ & $2.10 \pm 0.08$ & $29.17 \pm 1.03$ & $5.42 \pm 0.18$ \\
\hline 5 & $\mathrm{n} / \mathrm{c}$ & $\mathrm{n} / \mathrm{c}$ & $0.33 \pm 0.01$ & $0.56 \pm 0.02$ \\
\hline 6 & $\mathrm{n} / \mathrm{c}$ & $\mathrm{n} / \mathrm{c}$ & $20.79 \pm 0.73$ & $\mathrm{n} / \mathrm{c}$ \\
\hline 7 & $\mathrm{n} / \mathrm{c}$ & $\mathrm{n} / \mathrm{c}$ & $6.01 \pm 0.13$ & $\mathrm{n} / \mathrm{c}$ \\
\hline 8 & $\mathrm{n} / \mathrm{c}$ & $4.56 \pm 0.16$ & $18.24 \pm 0.71$ & $5.82 \pm 0.12$ \\
\hline 9 & $\mathrm{n} / \mathrm{c}$ & $0.22 \pm 0.01$ & $39.73 \pm 1.17$ & $2.33 \pm 0.09$ \\
\hline 10 & $n / c$ & $\mathrm{n} / \mathrm{c}$ & $26.88 \pm 0.95$ & $3.97 \pm 0.13$ \\
\hline 11 & $8.81 \pm 0.39$ & $0.77 \pm 0.02$ & $11.78 \pm 0.43$ & $11.46 \pm 0.37$ \\
\hline 12 & $9.06 \pm 0.41$ & $2.01 \pm 0.08$ & $7.31 \pm 0.29$ & $9.63 \pm 0.38$ \\
\hline 13 & $2.74 \pm 0.11$ & $2.08 \pm 0.07$ & $4.16 \pm 0.17$ & $1.92 \pm 0.08$ \\
\hline 14 & $\mathrm{n} / \mathrm{c}$ & $\mathrm{n} / \mathrm{c}$ & $\mathrm{n} / \mathrm{c}$ & $\mathrm{n} / \mathrm{c}$ \\
\hline 15 & $\mathrm{n} / \mathrm{c}$ & $\mathrm{n} / \mathrm{c}$ & $0.64 \pm 0.02$ & $0.43 \pm 0.01$ \\
\hline 16 & $1.01 \pm 0.03$ & $0.37 \pm 0.01$ & $1.11 \pm 0.04$ & $1.12 \pm 0.02$ \\
\hline V. off 1 & $36.07 \pm 1.8$ & $\mathrm{n} / \mathrm{c}$ & $11.32 \pm 0.55$ & $10.04 \pm 0.45$ \\
\hline V. off 2 & $0.29 \pm 0.01$ & $\mathrm{n} / \mathrm{c}$ & $1.94 \pm 0.07$ & $3.32 \pm 0.15$ \\
\hline V. off 3 & $1.72 \pm 0.03$ & $\mathrm{n} / \mathrm{c}$ & $1.53 \pm 0.06$ & $0.48 \pm 0.01$ \\
\hline V. off 4 & $0.27 \pm 0.01$ & $\mathrm{n} / \mathrm{c}$ & $0.41 \pm 0.02$ & $\mathrm{n} / \mathrm{c}$ \\
\hline V. cha 1 & $n / c$ & $\mathrm{n} / \mathrm{c}$ & $3.39 \pm 0.11$ & $\mathrm{n} / \mathrm{c}$ \\
\hline V. cha 2 & $n / c$ & $\mathrm{n} / \mathrm{c}$ & $6.12 \pm 0.29$ & $0.87 \pm 0.03$ \\
\hline V. cha 3 & $n / c$ & $\mathrm{n} / \mathrm{c}$ & $7.44 \pm 0.32$ & $0.93 \pm 0.02$ \\
\hline V. cha 4 & $n / c$ & $\mathrm{n} / \mathrm{c}$ & $7.52 \pm 0.27$ & $2.38 \pm 0.10$ \\
\hline
\end{tabular}

$10 \mathrm{X}$ rule where the interspecific distances are considered to be 10 times greater than intraspecific distances (Hebert et al., 2004), but each has shortcomings for general application (Meier et al., 2008; Rach et al., 2008; Bertolazzi et al., 2009).

In this study we used ABGD to assess the 'barcode gap' for the Veronica genus, with the specific aim of evaluating if nrITS has sufficient discriminatory power to differentiate $V$. officinalis from its main adulterant, $V$. chamaedrys. The three distance metrics used for the analyses produced identical initial partitions within each dataset and for all the values of prior intraspecific diversity $(P)$, but with a slight difference between the datasets, respectively, 14 groups for nrITS and nrITS2 and 13 for nrITS1. The recursive partitions resulted in a variable number of groups within each dataset depending on $P$, respectively, with 18,22, 37, and 53 groups for nrITS dataset, 14, 19, and 37 groups for nrITS1, and $16,23,31$, and 54 groups for nrITS2 data set.

Infra- and interspecific divergence based on pairwise comparison of nrITS genetic distances of $V$. officinalis and $V$. chamaedrys (Supplementary Table S4) helped to identify an optimal clustering threshold for analysis of high-throughput sequence data and accurate molecular identification and discrimination of the two species. Pairwise intraspecific genetic distances did not exceed 1.5\%, and furthermore $93.8 \%$ of the pairwise intraspecific distances were below 1\%. Pairwise interspecific genetic distances, on the other hand, ranged from 18 to $22 \%$. The pairwise comparisons showed that applying
97\% or more similarity as a clustering threshold will confidently discriminate $V$. officinalis and $V$. chamaedrys. These results are consistent with the ABGD analysis in which the sequences belonging to $V$. officinalis and $V$. chamaedrys were assigned to well defined independent groups using an a priori defined genetic threshold of $1 \%$.

\section{DNA Metabarcoding}

The quantity of the total DNA extracted from the sixteen herbal products varied between samples. Total DNA concentration measurement gave detectable results for 15 of the samples (93.7\%), with DNA concentrations ranging from 0.06 to $87.32 \mathrm{ng} / \mu \mathrm{l}$, and nrITS1 and nrITS2 amplicons were obtained for all these samples. One sample, marketed as a lozenge, did not yield a measurable DNA concentration and no amplicons of nrITS1 and nrITS2 were obtained from this sample (Supplementary Table S5).

The raw data before demultiplexing consisted of 2,638,101 sequences, with an average of 82,440 sequences per sample for each marker. Sequencing was successful for thirteen herbal products $(81.3 \%)$ for both nrITS1 and nrITS2 and they were used for further analysis (Table 3). Three products, two herbal teas $(5,16)$ and the lozenge $(14)$, did not yield reads or MOTUs after applying the quality filtering criteria and were excluded from further analyses. A total of 63,929 sequences passed our trimming and filtering quality criteria (2.4\% of reads), including 19,788 
TABLE 3 | Overview of the results of three clustering thresholds (97, 99, and 100\%).

\begin{tabular}{|c|c|c|c|c|c|c|c|c|c|}
\hline \multirow[t]{2}{*}{ Sample \# } & \multirow{2}{*}{$\begin{array}{l}\text { nrITS1+nrITS2 \# reads } \\
\text { before demultiplexing }\end{array}$} & \multirow{2}{*}{$\begin{array}{l}\text { nrlTS21 \# } \\
\text { reads }\end{array}$} & \multirow{2}{*}{$\begin{array}{l}\text { nrlTS2 \# } \\
\text { reads }\end{array}$} & \multicolumn{2}{|c|}{$97 \%$} & \multicolumn{2}{|c|}{$99 \%$} & \multicolumn{2}{|c|}{$100 \%$} \\
\hline & & & & \# MOTUs & \# Species & \# MOTUs & \# Species & \# MOTUs & \# Species \\
\hline 1 & 163443 & 80255 & 78683 & 15 & 6 & 75 & 11 & 108 & 12 \\
\hline 2 & 180106 & 104096 & 67500 & 17 & 11 & 71 & 17 & 79 & 16 \\
\hline 3 & 158877 & 85618 & 69168 & 19 & 7 & 53 & 13 & 188 & 12 \\
\hline 4 & 440956 & 299997 & 114335 & 47 & 12 & 142 & 23 & 191 & 16 \\
\hline $5^{*}$ & 34 & 8 & 18 & 0 & 0 & 0 & 0 & 0 & 0 \\
\hline 6 & 217323 & 131223 & 74700 & 16 & 7 & 27 & 7 & 47 & 7 \\
\hline 7 & 400607 & 174347 & 192110 & 49 & 25 & 99 & 32 & 191 & 26 \\
\hline 8 & 309978 & 73352 & 213401 & 30 & 16 & 107 & 23 & 303 & 19 \\
\hline 9 & 80179 & 58175 & 16627 & 25 & 14 & 36 & 13 & 49 & 12 \\
\hline 10 & 62198 & 45321 & 15903 & 12 & 5 & 47 & 7 & 49 & 4 \\
\hline 11 & 254965 & 186423 & 47991 & 33 & 14 & 64 & 16 & 77 & 10 \\
\hline 12 & 122393 & 63939 & 55331 & 14 & 7 & 33 & 5 & 108 & 4 \\
\hline 13 & 26001 & 16464 & 7830 & 0 & 0 & 4 & 2 & 12 & 2 \\
\hline $14^{*}$ & 19 & 10 & 6 & 0 & 0 & 0 & 0 & 0 & 0 \\
\hline 15 & 220984 & 70947 & 131918 & 10 & 4 & 66 & 4 & 170 & 4 \\
\hline $16^{*}$ & 38 & 12 & 18 & 0 & 0 & 0 & 0 & 0 & 0 \\
\hline Total & 2638101 & 1390187 & 1085539 & 287 & 91 & 824 & 109 & 1572 & 80 \\
\hline
\end{tabular}

* Samples excluded from subsequent data analysis.

nrITS1 reads and 44,141 nrITS2 reads (Supplementary Table S6). The metabarcoding data is used for qualitative evaluation only, to determine presence of identified taxa, and not for quantitative assessment of relative species abundance based on read numbers, as too many variables impact the values, such as the availability of DNA that can be removed or degraded during the harvesting, drying, storage, transportation and processing (Bombardelli, 1991; Novak et al., 2007), as well as variation in nrITS copy numbers, primer annealing and PCR amplification biases (Pawluczyk et al., 2015). Furthermore, incomplete reference databases and incorrectly identified sequences in GenBank can impact taxonomic assignments.

The choice of similarity clustering thresholds $(>97,>99$, and $100 \%)$ impacted the number and size of assigned MOTUs (Table 3). Setting the clustering threshold to $100 \%$ yielded 1572 MOTUs, $99 \%$ yielded 824 MOTUs, and $97 \%$ yielded only 287 MOTUs. The low number of MOTUs obtained using the $97 \%$ clustering threshold is likely the result of multiple species being grouped together into the same MOTU as 3\% divergence far exceeds interspecific variation in many genera (Yang and Rannala, 2017). Setting the clustering threshold to $100 \%$, on the other hand, likely resulted in more than one MOTU being formed for a single taxonomic species as a result of infraspecific variation and/or sequencing errors between reads. Here we followed previous studies in using a 99\% clustering threshold (Ghorbani et al., 2016; Raclariu et al., 2017; Veldman et al., 2017). Furthermore, to limit the impact of sequencing errors, which are known to affect the Ion Torrent sequencing platform (Loman et al., 2012; Salipante et al., 2014) and which could lead to the formation of false MOTUs, we used only the clusters that contained a minimum 10 reads.
The 824 MOTUs that were retained using a 99\% similarity clustering threshold were further identified using BLAST as 109 different species (Table 3 and Supplementary Table S6). Based on only nrITS1 we detected a total of 64 different species and on only nrITS2 61 species (Supplementary Table S6). Based on both markers, the number of species detected per sample ranged from 2 to 32 , with an average of 8 species per sample. Only two of the seven (28.6\%) single ingredient samples (those listing $V$. officinalis as the only ingredient) contained $V$. officinalis, three others contained $V$. chamaedrys and one contained other Veronica species, and all products contained additional species, ranging from 1 to 21 species per product. None of the nine retained multiple ingredient samples (i.e., those listing $V$. officinalis and other species on the label) contained only the listed ingredients, and none contained $V$. officinalis (Figure 1). The overall ingredient fidelity (detected species from product label/total number of species on label) was $9.5 \%$ for the multiple ingredient products, and $17 \%$ for all products. The target species $V$. officinalis was detected in only 2 products (15\%), whereas $V$. chamaedrys was detected in eight $(62 \%)$ of the retained samples. These results are supported by the pairwise comparison of the genetic distances that showed that $V$. officinalis could be unambiguously differentiated from $V$. chamaedrys using standard arbitrary clustering thresholds (Supplementary Table S4). In sample 10 a mixture of several Veronica species was detected, including $V$. bombycina Boiss. \& Kotschy, V. filiformis Sm., V. pectinata, V. tenuifolia Asso, $V$. krumovii (Peev) Peev and V. chamaedrys. The presence of these species can be explained as substitutes, admixtures or contaminants. It should be noted that the general monograph number 1433 on 'Herbal drugs' of the European Pharmacopoeia 

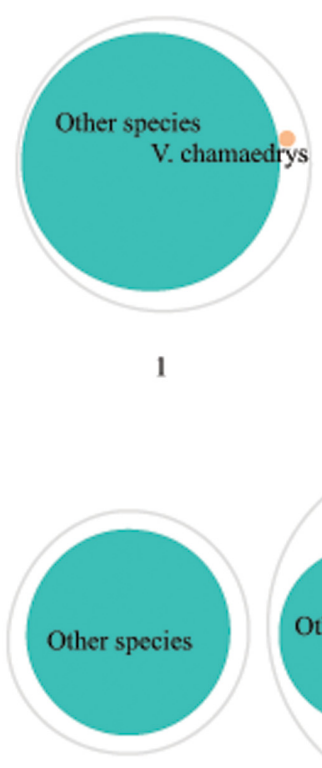

6

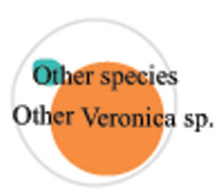

10

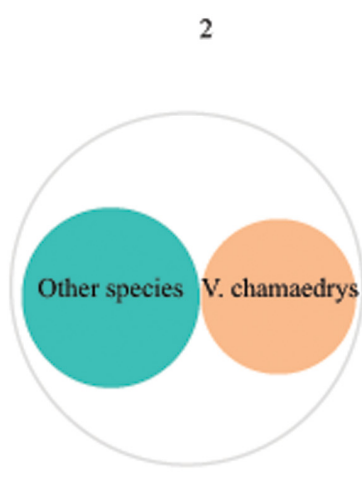

7

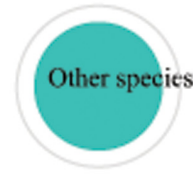

11
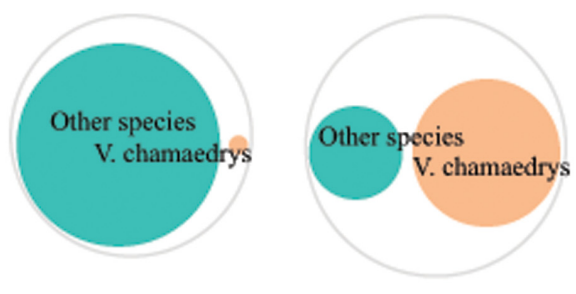

Other species V. chamaedrys

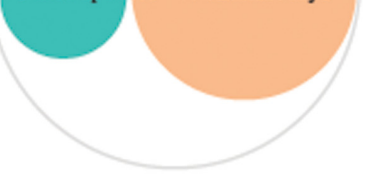

4

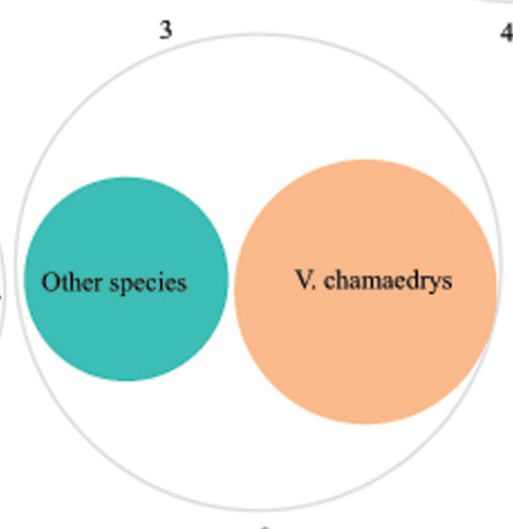

8

9

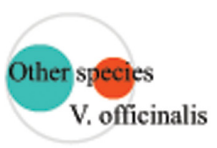

12

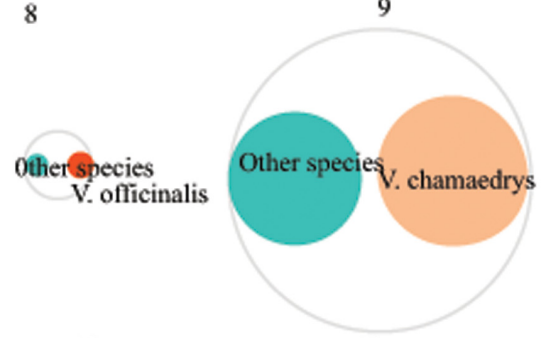

15

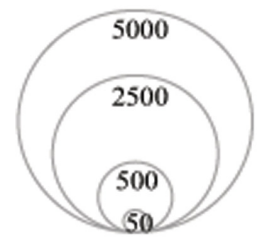

FIGURE 1 | Veronica and other species detected in the herbal products using DNA metabarcoding. Each numbered circle represents a product (more information about these products can be found in Supplementary Table S1). The size of circles corresponds to the relative abundance of the reads.

allows up to $2 \%$ foreign matter, unless this is differently indicated in a specific herb monograph (EDQM - Council of Europe, 2014). Presence of these other Veronica species might not be in breach of the European Pharmacopoeia but it does indicate a lapse in the value chain of this product. The same applies to the $62 \%$ products that contained $V$. chamaedrys instead of V. officinalis.

\section{CONCLUSION}

This study used HPLC-MS and DNA metabarcoding to authenticate and detect adulteration or admixture of $V$. officinalis with $V$. chamaedrys in Veronica herbal products. HPLC-MS is useful for qualitative and quantitative analysis of constituents in medicinal plants and herbal products and its use has increased in recent years (Steinmann and Ganzera, 2011; Bansal et al., 2014), but constraints are the expensive chemical reference standards (some of which are still unavailable for biologically active target components), sensitivity to the type of raw material and manufacturing process, and lack of resolution in distinguishing plant species (Bansal et al., 2014). Our HPLC-MS results show that distinction of $V$. officinalis from other Veronica species based on the targeted iridoid glycosides, aucubin, catalpol, veronicoside, and catalposide is difficult in pure products, and impossible in multiple ingredient products. General application 
of HPLC-MS to detect substitution and/or adulteration of highly processed and multi-ingredient herbal products is challenging, and this is corroborated by our findings.

Several authors have advocated the use of DNA barcoding and metabarcoding in herbal product authentication and pharmacovigilance (Coghlan et al., 2012; Newmaster et al., 2013; Cheng et al., 2014; De Boer et al., 2015; Palhares et al., 2015; Ivanova et al., 2016), due to its cost effectiveness and ability to disclose plant species diversity within products. Our DNA metabarcoding results corroborate previous research that used metabarcoding to authenticate herbal products (Coghlan et al., 2012, 2015; Cheng et al., 2014; Ivanova et al., 2016; Veldman et al., 2017). In this study we confirm its applicability to test for presence of $V$. officinalis and simultaneously to detect substitution, adulteration and/or admixture of other Veronica species. Using DNA metabarcoding we found that only $15 \%$ of the products contained $V$. officinalis, whereas $62 \%$ of the products contained $V$. chamaedrys. Furthermore, we found that all the investigated herbal products contained other species not listed on the label. Limitations of DNA metabarcoding are its susceptibility to various factors, such as quality, processing state and product type of the extracted material, as well as several variables related to the methodological framework, such as DNA extraction procedures, primers, markers, amplification protocols for the library preparation sequencing platform, filtering, quality thresholds and chimera removal, and clustering thresholds.

Despite limitations of the methodology, our DNA metabarcoding results show that there is a need to study herbal products derived from traditional medicine, and to increase consumer confidence by advocating and promoting a higher standard of quality in herbal products. In recent years, the scientific community has been showing an increasing interest in finding adequate and comprehensive methodologies to investigate such complex products, focusing on their entire value chain, from the raw material to the product on the shelf.

\section{REFERENCES}

Afifi-Yazar, F. Ü., and Sticher, O. (1980). Verproside, a new iridoid glucoside from Veronica officinalis L. (Scrophulariaceae). Helv. Chim. Acta 63, 1905-1907. doi: 10.1002/hlca.19800630716

Albach, D. C., and Al-Gharaibeh, M. (2015). Systematics and biogeography of Veronica subg. Pentasepalae from the Levant. Willdenowia 45, 5-14. doi: 10.3372/wi.45.45101

Albach, D. C., and Chase, M. W. (2001). Paraphyly of Veronica (Veroniceae; Scrophulariaceae): evidence from the internal transcribed spacer (ITS) sequences of nuclear ribosomal DNA. J. Plant Res. 114, 9-18. doi: 10.1007/ PL00013971

Albach, D. C., and Chase, M. W. (2004). Incongruence in Veroniceae (Plantaginaceae): evidence from two plastid and a nuclear ribosomal DNA region. Mol. Phylogenet. Evol. 32, 183-197. doi: 10.1016/j.ympev.2003.12.001

Albach, D. C., Martínez-Ortega, M. M., and Chase, M. W. (2004). Veronica: parallel morphological evolution and phylogeography in the Mediterranean. Plant Syst. Evol. 246, 177-194. doi: 10.1007/s00606-004-0148-9

Albach, D. C., and Meudt, H. M. (2010). Phylogeny of Veronica in the Southern and Northern Hemispheres based on plastid, nuclear ribosomal and nuclear

\section{SUPPORTING INFORMATION}

Ion-Torrent amplicon read data is deposited in DRYAD doi: $10.5061 /$ dryad.606ks.

\section{AUTHOR CONTRIBUTIONS}

MI, HB, GC, and AR conceived the experiment. AR collected the material and carried out the molecular lab work and analysis. MP assisted with the molecular lab work. AM, LV, and GC carried out the phytochemical lab work and analyses. AR wrote the manuscript together with $\mathrm{HB}$ and $\mathrm{AB}$. All authors contributed to and approved the final version of the manuscript.

\section{FUNDING}

The authors acknowledge funding from the Romanian - EEA Research Programme operated by the MECS-ANCSI PO under the EEA Financial Mechanism 2009-2014 and project contract number 2SEE/2014.

\section{ACKNOWLEDGMENT}

B. L. Thorbek, Audun Schrøder-Nielsen, and Jarl Andreas Anmarkrud are acknowledged for help and assistance with the molecular laboratory analyses.

\section{SUPPLEMENTARY MATERIAL}

The Supplementary Material for this article can be found online at: http://journal.frontiersin.org/article/10.3389/fphar. 2017.00378/full\#supplementary-material

TABLE S6 | Veronica HTS reads and identified MOTUs per product at 99\% threshold.

low-copy DNA. Mol. Phylogenet. Evol. 54, 457-471. doi: 10.1016/j.ympev.2009. 09.030

Albach, D. C., Schönswetter, P., and Tribsch, A. (2006). Comparative phylogeography of the Veronica alpina complex in Europe and North America. Mol. Ecol. 15, 3269-3286. doi: 10.1111/j.1365-294X.2006.02980.x

Altschul, S. F., Gish, W., Miller, W., Myers, E. W., and Lipman, D. J. (1990). Basic local alignment search tool. J. Mol. Biol. 215, 403-410. doi: 10.1016/S00222836(05)80360-2

Atanasov, A. G., Waltenberger, B., Pferschy-Wenzig, E.-M., Linder, T., Wawrosch, C., Uhrin, P., et al. (2015). Discovery and resupply of pharmacologically active plant-derived natural products: a review. Biotechnol. Adv. 33, 1582-1614. doi: 10.1016/j.biotechadv.2015.08.001

Baker, D. A., Stevenson, D. W., and Little, D. P. (2012). DNA barcode identification of black cohosh herbal dietary supplements. J. AOAC Int. 95, 1023-1034. doi: 10.5740/jaoacint.11-261

Bansal, A., Chhabra, V., Rawal, R. K., and Sharma, S. (2014). Chemometrics: a new scenario in herbal drug standardization. J. Pharm. Anal. 4, 223-233. doi: 10.1016/j.jpha.2013.12.001

Barnes, J. (2003). Pharmacovigilance of herbal medicines. Drug Saf. 26, 829-851. doi: 10.2165/00002018-200326120-00001 
Baytop, T. (1984). Treatment with plants in Turkey. Istanbul. Univ. Publ. 3255:420. Bellia, G., and Pieroni, A. (2015). Isolated, but transnational: the glocal nature of Waldensian ethnobotany, Western Alps, NW Italy. J. Ethnobiol. Ethnomed. 11:37. doi: 10.1186/s13002-015-0027-1

Benson, D. A., Cavanaugh, M., Clark, K., Karsch-Mizrachi, I., Lipman, D. J., Ostell, J., et al. (2013). GenBank. Nucleic Acids Res. 41, D36-D42. doi: 10.1093/ nar/gks1195

Bertolazzi, P., Felici, G., and Weitschek, E. (2009). Learning to classify species with barcodes. BMC Bioinformatics 10:S7. doi: 10.1186/1471-2105-10S14-S7

Bickford, D., Lohman, D. J., Sodhi, N. S., Ng, P. K. L., Meier, R., Winker, K., et al. (2007). Cryptic species as a window on diversity and conservation. Trends Ecol. Evol. 22, 148-155. doi: 10.1016/j.tree.2006.11.004

Bombardelli, E. (1991). "Technologies for the processing of medicinal plants," in The Medicinal Plant Industry, ed. R. O. B. Wijesekera (Boca Raton, FL: CRC Press), 85-98.

Booker, A., Frommenwiler, D., Johnston, D., Umealajekwu, C., Reich, E., and Heinrich, M. (2014). Chemical variability along the value chains of turmeric (Curcuma longa): a comparison of nuclear magnetic resonance spectroscopy and high performance thin layer chromatography. J. Ethnopharmacol. 152, 292-301. doi: 10.1016/j.jep.2013.12.042

Booker, A., Johnston, D., and Heinrich, M. (2012). Value chains of herbal medicines-Research needs and key challenges in the context of ethnopharmacology. J. Ethnopharmacol. 140, 624-633. doi: 10.1016/j.jep.2012.01.039

Booker, A., Zhai, L., Gkouva, C., Li, S., and Heinrich, M. (2016). From traditional resource to global commodities:-a comparison of Rhodiola species using NMR spectroscopy-metabolomics and HPTLC. Front. Pharmacol. 7:254. doi: 10.3389/fphar.2016.00254

Buhk, N., Zhao, L., Li, H., and Albach, D. C. (2015). Molecular systematics and morphometrics in Veronica subsect. Canae (Plantaginaceae). Plant Syst. Evol. 301, 1967-1979. doi: 10.1007/s00606-015-1214-1

Burgess, K. S., Fazekas, A. J., Kesanakurti, P. R., Graham, S. W., Husband, B. C., Newmaster, S. G., et al. (2011). Discriminating plant species in a local temperate flora using the rbcL+matK DNA barcode. Methods Ecol. Evol. 2, 333-340. doi: 10.1111/j.2041-210X.2011.00092.x

CBOL Plant Working Group (2009). A DNA barcode for land plants. Proc. Natl. Acad. Sci. U.S.A. 106, 12794-12797. doi: 10.1073/pnas.09058 45106

Chen, S., Yao, H., Han, J., Liu, C., Song, J., Shi, L., et al. (2010). Validation of the ITS2 region as a novel DNA barcode for identifying medicinal plant species. PLoS ONE 5:e8613. doi: 10.1371/journal.pone.0008613

Chen, W., Zhang, C. K., Cheng, Y., Zhang, S., and Zhao, H. (2013). A comparison of methods for clustering 16S rRNA sequences into OTUs. PLoS ONE 8:e70837. doi: 10.1371/journal.pone.0070837

Cheng, X., Su, X., Chen, X., Zhao, H., Bo, C., Xu, J., et al. (2014). Biological ingredient analysis of traditional Chinese medicine preparation based on highthroughput sequencing: the story for Liuwei Dihuang Wan. Sci. Rep. 4:5147. doi: $10.1038 /$ srep05147

China Plant BOL Group (2011). Comparative analysis of a large dataset indicates that internal transcribed spacer (ITS) should be incorporated into the core barcode for seed plants. Proc. Natl. Acad. Sci. U.S.A. 108, 19641-19646. doi: 10.1073/pnas.1104551108

Coghlan, M. L., Haile, J., Houston, J., Murray, D. C., White, N. E., Moolhuijzen, P., et al. (2012). Deep sequencing of plant and animal DNA contained within traditional Chinese medicines reveals legality issues and health safety concerns. PLoS Genet. 8:e1002657. doi: 10.1371/journal.pgen.1002657

Coghlan, M. L., Maker, G., Crighton, E., Haile, J., Murray, D. C., White, N. E., et al. (2015). Combined DNA, toxicological and heavy metal analyses provides an auditing toolkit to improve pharmacovigilance of traditional Chinese medicine (TCM). Sci. Rep. 5:17457. doi: 10.1038/srep17475

Coissac, E., Hollingsworth, P. M., Lavergne, S., and Taberlet, P. (2016). From barcodes to genomes: extending the concept of DNA barcoding. Mol. Ecol. 25, 1423-1428. doi: $10.1111 / \mathrm{mec} .13549$

Cos, P., Vlietinck, A. J., Berghe, D. V., and Maes, L. (2006). Anti-infective potential of natural products: how to develop a stronger in vitro 'proof-of-concept'. J. Ethnopharmacol. 106, 290-302. doi: 10.1016/j.jep.2006.04.003

Crişan, G., Tamas, M., Garbacki, N., and Angenot, L. (2001). Criterii de differentiere a unor specii de Veronica. Farmacia 49, 67-73.
Crişan, G., Tămaş, M., Miclăuş, V., Krausz, T., and Sandor, V. (2007). A comparative study of some Veronica species. Rev. Med. Chir. Soc. Med. Nat. Iasi 111, 280-284.

Crişan, G., Vlase, L., Balica, G., Muntean, D., Ştefănescu, C., Păltinean, R., et al. (2010). LC/MS analysis of aucubin and catalpol of some Veronica species. Farmacia 58, 237-242.

Crişan, G., Vlase, L., Crişan, O., and Ichim, M. (2011). Comparative phytochemical study on Veronica officinalis L. and Veronica chamaedrys L. Planta Med. 77:PL14. doi: 10.1055/s-0031-1282663

De Boer, H. J., Ichim, M. C., and Newmaster, S. G. (2015). DNA barcoding and pharmacovigilance of herbal medicines. Drug Saf. 38, 611-620. doi: 10.1007/ s40264-015-0306-8

Doyle, J. J., and Doyle, J. L. (1987). A rapid DNA isolation procedure for small quantities of fresh leaf tissue. Phytochem. Bull. 19, 11-15.

EDQM - Council of Europe. (2014). European Pharmacopoeia, 8th Edn. Strasbourg: Council of Europe.

European Medicine Agency [EMA] (2006). EMEA/CVMP/815/00 - Guideline on Specifications: Test Procedures and Acceptance Criteria for Herbal Substances, Herbal Preparations and Herbal Medicinal Products. London: EMA.

Farah, M. H., Olsson, S., Bate, J., Lindquist, M., Edwards, R., Simmonds, M. S., et al. (2006). Botanical nomenclature in pharmacovigilance and a recommendation for standardisation. Drug Saf. 29, 1023-1029. doi: 10.2165/ 00002018-200629110-00002

Fazekas, A. J., Kesanakurti, P. R., Burgess, K. S., Percy, D. M., Graham, S. W., Barrett, S. C. H., et al. (2009). Are plant species inherently harder to discriminate than animal species using DNA barcoding markers? Mol. Ecol. Resour. 9, 130-139. doi: 10.1111/j.1755-0998.2009.02652.x

Fujita, T., Sezik, E., Tabata, M., Yesilada, E., Honda, G., Takeda, Y., et al. (1995). Traditional medicine in Turkey VII. Folk medicine in middle and west Black Sea regions. Econ. Bot. 49, 406-422. doi: 10.1007/BF02863092

Gertsch, J. (2009). How scientific is the science in ethnopharmacology? Historical perspectives and epistemological problems. J. Ethnopharmacol. 122, 177-183. doi: 10.1016/j.jep.2009.01.010

Ghorbani, A., Gravendeel, B., Selliah, S., Zarre, S., and de Boer, H. J. (2016). DNA barcoding of tuberous Orchidoideae: a resource for identification of orchids used in Salep. Mol. Ecol. Resour. 17, 342-352. doi: 10.1111/1755-0998.12615

Gilbert, N. (2011). Regulations: herbal medicine rule book. Nature 480, S98-S99. doi: $10.1038 / 480598$ a

Gründemann, C., Garcia-Käufer, M., Sauer, B., Stangenberg, E., Könczöl, M., Merfort, I., et al. (2013). Traditionally used Veronica officinalis inhibits proinflammatory mediators via the NF- $\mathrm{KB}$ signalling pathway in a human lung cell line. J. Ethnopharmacol. 145, 118-126. doi: 10.1016/j.jep.2012.10.039

Harput, U. S., Nagatsu, A., Ogihara, Y., and Saracoglu, I. (2014). Iridoid glucosides from Veronica pectinata var. glandulosa. Z. Naturforsch. C 58, 481-484.

Harput, U. S., Saracoglu, I., Inoue, M., and Ogihara, Y. (2002a). Anti-inflammatory and cytotoxic activities of five Veronica species. Biol. Pharm. Bull. 25, 483-486. doi: 10.1248/bpb.25.483

Harput, U. S., Saracoglu, I., Inoue, M., and Ogihara, Y. (2002b). Phenylethanoid and iridoid glycosides from Veronica persica. Chem. Pharm. Bull. 50, 869-871. doi: $10.1248 / \mathrm{cpb} .50 .869$

Hebert, P. D. N., Cywinska, A., Ball, S. L., and deWaard, J. R. (2003). Biological identifications through DNA barcodes. Proc. R. Soc. Lond. B Biol. Sci. 270, 313-321. doi: 10.1098/rspb.2002.2218

Hebert, P. D. N., Stoeckle, M. Y., Zemlak, T. S., and Francis, C. M. (2004). Identification of birds through DNA barcodes. PLoS Biol. 2:e312. doi: 10.1371/ journal.pbio.0020312

Heinrich, M. (2010). Ethnopharmacology in the 21st century - grand challenges. Front. Pharmacol. 1:8. doi: 10.3389/fphar.2010.00008

Heubl, G. (2010). New aspects of DNA-based authentication of Chinese medicinal plants by molecular biological techniques. Planta Med. 76, 1963-1974. doi: 10.1055/s-0030- 1250519

Hollingsworth, P. M., Graham, S. W., and Little, D. P. (2011). Choosing and using a plant DNA barcode. PLoS ONE 6:e19254. doi: 10.1371/journal.pone.0019254

Hollingsworth, P. M., Li, D.-Z., Bank, M., van der, and Twyford, A. D. (2016). Telling plant species apart with DNA: from barcodes to genomes. Philos. Trans. R. Soc. B Biol. Sci. 371:20150338. doi: 10.1098/rstb.2015.0338

Ichim, M. C., Raclariu, A. C., Paramon, P. P., and Toth, E. T. (2011). Biodiversity assessment of Veronica sp. in Romania for their characterization, preservation 
and sustainable use in pharmacognosy. Planta Med. 77, L26. doi: 10.1055/s0031-1282675

Ignjatović, Đ., Živković, J., Tovilović, G., Šavikin, K., Tomić, M., Maksimović, Z., et al. (2015). Evaluation of angiogenic and neuroprotective potential of different extracts from three Veronica species. Front. Life Sci. 8:107-116. doi: 10.1080/21553769.2014.998297

Ivancheva, S., and Stantcheva, B. (2000). Ethnobotanical inventory of medicinal plants in Bulgaria. J. Ethnopharmacol. 69, 165-172. doi: 10.1016/S03788741(99)00129-4

Ivanova, N. V., Kuzmina, M. L., Braukmann, T. W. A., Borisenko, A. V., and Zakharov, E. V. (2016). Authentication of herbal supplements using nextgeneration sequencing. PLOS ONE 11:e0156426. doi: 10.1371/journal.pone. 0156426

Jensen, S. R., Albach, D. C., Ohno, T., and Grayer, R. J. (2005). Veronica: iridoids and cornoside as chemosystematic markers. Biochem. Syst. Ecol. 33, 1031-1047. doi: 10.1016/j.bse.2005.03.001

Jiang, Y., David, B., Tu, P., and Barbin, Y. (2010). Recent analytical approaches in quality control of traditional Chinese medicines-a review. Anal. Chim. Acta 657, 9-18. doi: 10.1016/j.aca.2009.10.024

Jukes, T. H., and Cantor, C. R. (1969). "Evolution of protein molecules," in Mammalian Protein Metabolism, ed. H. N. Munro (New York, NY: Academic Press), 21-132. doi: 10.1016/b978-1-4832-3211-9.50009-7

Kathe, W., Honnef, S., and Heym, A. (2003). Medicinal and Aromatic Plants in Albania, Bosnia-Herzegovina, Bulgaria, Croatia and Romania: A Study of the Collection of and Trade in Medicinal and Aromatic Plants (MAPs), Relevant Legislation and the Potential of MAP Use for Financing Nature Conservation and Protected Areas. Bonn: Federal Agency for Nature Conservation.

Kimura, M. (1980). A simple method for estimating evolutionary rates of base substitutions through comparative studies of nucleotide sequences. J. Mol. Evol. 16, 111-120. doi: 10.1007/BF01731581

Kool, A., de Boer, H. J., Krüger, Å., Rydberg, A., Abbad, A., Björk, L., et al. (2012). Molecular identification of commercialized medicinal plants in southern Morocco. PLoS ONE 7:e39459. doi: 10.1371/journal.pone.0039459

Kress, W. J., and Erickson, D. L. (2007). A two-locus global DNA barcode for land plants: the coding rbcL gene complements the non-coding trnH-psbA spacer region. PLoS ONE 2:e508. doi: 10.1371/journal.pone.0000508

Kress, W. J., Erickson, D. L., Jones, F. A., Swenson, N. G., Perez, R., Sanjur, O., et al. (2009). Plant DNA barcodes and a community phylogeny of a tropical forest dynamics plot in Panama. Proc. Natl. Acad. Sci. U.S.A. 106, 18621-18626. doi: 10.1073/pnas.0909820106

Kress, W. J., Erickson, D. L., Swenson, N. G., Thompson, J., Uriarte, M., and Zimmerman, J. K. (2010). Advances in the use of DNA barcodes to build a community phylogeny for tropical trees in a puerto rican forest dynamics plot. PLoS ONE 5:e15409. doi: 10.1371/journal.pone.0015409

Küpeli, E., Harput, U. S., Varel, M., Yesilada, E., and Saracoglu, I. (2005). Bioassay-guided isolation of iridoid glucosides with antinociceptive and antiinflammatory activities from Veronica anagallis-aquatica L. J. Ethnopharmacol. 102, 170-176. doi: 10.1016/j.jep.2005.05.042

Lahaye, R., Bank, M., van der, Bogarin, D., Warner, J., Pupulin, F., et al. (2008). DNA barcoding the floras of biodiversity hotspots. Proc. Natl. Acad. Sci. U.S.A. 105, 2923-2928. doi: 10.1073/pnas.0709936105

Lammers, Y., Peelen, T., Vos, R. A., and Gravendeel, B. (2014). The HTS barcode checkerpipeline, a tool for automated detection of illegally traded species from high-throughput sequencing data. BMC Bioinformatics 15:44. doi: 10.1186/ 1471-2105-15-44

Larsson, A. (2014). AliView: a fast and lightweight alignment viewer and editor for large datasets. Bioinformatics 30, 3276-3278. doi: 10.1093/bioinformatics/ btu531

Leonti, M., and Casu, L. (2013). Traditional medicines and globalization: current and future perspectives in ethnopharmacology. Front. Pharmacol. 4:92. doi: 10.3389/fphar.2013.00092

Leporatti, M. L., and Ivancheva, S. (2003). Preliminary comparative analysis of medicinal plants used in the traditional medicine of Bulgaria and Italy. J. Ethnopharmacol. 87, 123-142. doi: 10.1016/S0378-8741(03) 00047-3

Leyel, C. (1937). Herbal Delights: Tisanes, Syrups, Confections, Electuaries, Robs, Juleps, Vinegars, and Conserve. London: Faber \& Faber.
Li, W., and Godzik, A. (2006). CD-HIT: a fast program for clustering and comparing large sets of protein or nucleotide sequences. Bioinformatics 22, 1658-1659. doi: 10.1093/bioinformatics/btl158

Little, D. P. (2014). Authentication of Ginkgo biloba herbal dietary supplements using DNA barcoding. Genome 57, 513-516. doi: 10.1139/gen-2014-0130

Little, D. P., and Jeanson, M. L. (2013). DNA barcode authentication of Saw Palmetto herbal dietary supplements. Sci. Rep. 3:3518. doi: 10.1038/srep03518

Loman, N. J., Misra, R. V., Dallman, T. J., Constantinidou, C., Gharbia, S. E., Wain, J., et al. (2012). Performance comparison of benchtop high-throughput sequencing platforms. Nat. Biotechnol. 30, 434-439. doi: 10.1038/nbt.2198

Mabberley, D. J. (2008). Mabberley's Plant-Book: A Portable Dictionary of Plants, Their Classifications and Uses. Cambridge: Cambridge University Press.

Meier, R., Shiyang, K., Vaidya, G., Ng, P. K. L., and Hedin, M. (2006). DNA barcoding and taxonomy in Diptera: a tale of high intraspecific variability and low identification success. Syst. Biol. 55, 715-728. doi: 10.1080/ 10635150600969864

Meier, R., Zhang, G., Ali, F., and Zamudio, K. (2008). The use of mean instead of smallest interspecific distances exaggerates the size of the "barcoding gap" and leads to misidentification. Syst. Biol. 57, 809-813. doi: 10.1080/ 10635150802406343

Menković, N., Šavikin, K., Tasić, S., Zdunić, G., Stešević, D., Milosavljević, S., et al. (2011). Ethnobotanical study on traditional uses of wild medicinal plants in Prokletije Mountains (Montenegro). J. Ethnopharmacol. 133, 97-107. doi: 10.1016/j.jep.2010.09.008

Meyer, C. P., and Paulay, G. (2005). DNA barcoding: error rates based on comprehensive sampling. PLoS Biol. 3:e422. doi: 10.1371/journal.pbio.0030422

Mocan, A., Vlase, L., Arsene, A. L., Vodnar, D., Bischin, C., Silaghi-Dumitrescu, R., et al. (2015a). HPLC/MS analysis of caffeic and chlorogenic acids from three Romanian Veronica species and their antioxidant and antimicrobial proprieties. Farmacia 63, 890-896.

Mocan, A., Vodnar, D. C., Vlase, L., Crişan, O., Gheldiu, A. M., and Crişan, G. (2015b). Phytochemical characterization of Veronica officinalis L., V. teucrium L. and $V$. orchidea Crantz from Romania and their antioxidant and antimicrobial properties. Int. J. Mol. Sci. 16, 21109-21127. doi: 10.3390/ ijms160921109

Newmaster, S. G., Grguric, M., Shanmughanandhan, D., Ramalingam, S., and Ragupathy, S. (2013). DNA barcoding detects contamination and substitution in North American herbal products. BMC Med. 11:222. doi: 10.1186/17417015-11-222

Nikolova, M. (2011). Screening of radical scavenging activity and polyphenol content of Bulgarian plant species. Pharmacogn. Res. 3, 256-259. doi: 10.4103/ 0974-8490.89746

Novak, J., Grausgruber-Gröger, S., and Lukas, B. (2007). DNA-based authentication of plant extracts. Food Res. Int. 40, 388-392. doi: 10.1016/j.foodres.2006.10.015

Ouarghidi, A., Powell, B., Martin, G. J., de Boer, and Abbad, A. (2012). Species substitution in medicinal roots and possible implications for toxicity of herbal remedies in Morocco. Econ. Bot. 66, 370-382. doi: 10.1007/s12231-0129215-2

Palhares, R. M., Drummond, M. G., Brasil, B., dos, S. A. F., Cosenza, G. P., Brandão, M., et al. (2015). Medicinal plants recommended by the World Health Organization: DNA barcode identification associated with chemical analyses guarantees their quality. PLoS ONE 10:e0127866. doi: 10.1371/journal.pone. 0127866

Pawluczyk, M., Weiss, J., Links, M. G., Egaña Aranguren, M., Wilkinson, M. D., and Egea-Cortines, M. (2015). Quantitative evaluation of bias in PCR amplification and next-generation sequencing derived from metabarcoding samples. Anal. Bioanal. Chem. 407, 1841-1848. doi: 10.1007/s00216-014-8435-y

Puillandre, N., Lambert, A., Brouillet, S., and Achaz, G. (2012). ABGD, Automatic Barcode Gap Discovery for primary species delimitation. Mol. Ecol. 21, 1864-1877. doi: 10.1111/j.1365-294X.2011.05239.x

Rach, J., DeSalle, R., Sarkar, I. N., Schierwater, B., and Hadrys, H. (2008). Characterbased DNA barcoding allows discrimination of genera, species and populations in Odonata. Proc. R. Soc. Lond. B Biol. Sci. 275, 237-247. doi: 10.1098/rspb.2007. 1290

Raclariu, A. C., Paltinean, R., Vlase, L., Labarre, A., Manzanilla, V., Ichim, M. C., et al. (2017). Comparative authentication of Hypericum perforatum herbal 
products using DNA metabarcoding, TLC and HPLC-MS. Sci. Rep. 7:1291. doi: 10.1038/s41598-017-01389-w

Ratnasingham, S., and Hebert, P. D. N. (2013). A DNA-Based registry for all animal species: the barcode index number (BIN) system. PLoS ONE 8:e66213. doi: 10.1371/journal.pone. 0066213

Ríos, J. L., and Recio, M. C. (2005). Medicinal plants and antimicrobial activity. J. Ethnopharmacol. 100, 80-84. doi: 10.1016/j.jep.2005.04.025

Rios, J. L., Recio, M. C., and Villar, A. (1988). Screening methods for natural products with antimicrobial activity: a review of the literature. J. Ethnopharmacol. 23, 127-149. doi: 10.1016/0378-8741(88)90001-3

Salipante, S. J., Kawashima, T., Rosenthal, C., Hoogestraat, D. R., Cummings, L. A., Sengupta, D. J., et al. (2014). Performance comparison of illumina and ion torrent next-generation sequencing platforms for $16 \mathrm{~S}$ rRNA-based bacterial community profiling. Appl. Environ. Microbiol. 80, 7583-7591. doi: 10.1128/ AEM.02206-14

Saracoglu, I., and Harput, U. S. (2012). In vitro cytotoxic activity and structure activity relationships of iridoid glucosides derived from Veronica species. Phytother. Res. 26, 148-152. doi: 10.1002/ptr.3546

Saracoglu, I., Harput, U. S., Inoue, M., and Ogihara, Y. (2002). New phenylethanoid glycosides from Veronica pectinata var. glandulosa and their free radical scavenging activities. Chem. Pharm. Bull. 50, 665-668. doi: 10.1248/cpb.50.665

Saslis-Lagoudakis, C. H., Bruun-Lund, S., Iwanycki, N. E., Seberg, O., Petersen, G., Jäger, A. K., et al. (2015). Identification of common horsetail (Equisetum arvense L.; Equisetaceae) using thin layer chromatography versus DNA barcoding. Sci. Rep. 5:11942. doi: 10.1038/srep11942

Scarlat, M., Şandor, V., Tămaş, M., and Cuparencu, B. (1985). Experimental antiulcer activity of Veronica officinalis L. extracts. J. Ethnopharmacol. 13, 157-163. doi: 10.1016/0378-8741(85)90003-0

Schmieder, R., and Edwards, R. (2011). Quality control and preprocessing of metagenomic datasets. Bioinformatics 27, 863-864. doi: 10.1093/ bioinformatics/btr026

Seethapathy, G. S., Ganesh, D., Kumar, J. U. S., Senthilkumar, U., Newmaster, S. G., Ragupathy, S., et al. (2014). Assessing product adulteration in natural health products for laxative yielding plants, Cassia, Senna, and Chamaecrista, in Southern India using DNA barcoding. Int. J. Legal Med. 129, 693-700. doi: $10.1007 / \mathrm{s} 00414-014-1120-\mathrm{z}$

Shaw, D., Graeme, L., Pierre, D., Elizabeth, W., and Kelvin, C. (2012). Pharmacovigilance of herbal medicine. J. Ethnopharmacol. 140, 513-518. doi: $10.1016 /$ j.jep.2012.01.051

Smith, M. A., Fisher, B. L., and Hebert, P. D. N. (2005). DNA barcoding for effective biodiversity assessment of a hyperdiverse arthropod group: the ants of Madagascar. Philos. Trans. R. Soc. Lond. B Biol. Sci. 360, 1825-1834. doi: 10.1098/rstb.2005.1714

Sonibare, M. A., Armagan, M., Özgökce, F., Yaprak, A. E., Mayland-Quellhorst, E., and Albach, D. C. (2014). Analysis of taxonomic and geographic patterns of Turkish Veronica orientalis using nuclear and plastid DNA and morphological data. Plant Syst. Evol. 300, 645-664. doi: 10.1007/s00606-013-0909-4

Sõukand, R., Quave, C. L., Pieroni, A., Pardo-de-Santayana, M., Tardío, J., Kalle, R., et al. (2013). Plants used for making recreational tea in Europe: a review based on specific research sites. J. Ethnobiol. Ethnomed. 9:58. doi: 10.1186/1746-42699-58

Steinmann, D., and Ganzera, M. (2011). Recent advances on HPLC/MS in medicinal plant analysis. J. Pharm. Biomed. Anal. 55, 744-757. doi: 10.1016/j. jpba.2010.11.015

Stoeckle, M. Y., Gamble, C. C., Kirpekar, R., Young, G., Ahmed, S., and Little, D. P. (2011). Commercial teas highlight plant DNA barcode identification successes and obstacles. Sci. Rep. 1:42. doi: 10.1038/srep00042

Stucky, B. J. (2012). SeqTrace: a graphical tool for rapidly processing DNA sequencing chromatograms. J. Biomol. Tech. 23, 90-93. doi: 10.7171/jbt.122303-004

Su, B.-N., Zhu, Q.-X., and Jia, Z.-J. (1999). Aquaticol, a novel bis-sesquiterpene from Veronica anagallis-aquatica. Tetrahedron Lett. 40, 357-358. doi: 10.1016/ S0040-4039(98)02303-X

Sun, Y., Skinner, D. Z., Liang, G. H., and Hulbert, S. H. (1994). Phylogenetic analysis of Sorghum and related taxa using internal transcribed spacers of nuclear ribosomal DNA. Theor. Appl. Genet. 89, 26-32. doi: 10.1007/ BF00226978
Taberlet, P., Coissac, E., Pompanon, F., Brochmann, C., and Willerslev, E. (2012). Towards next-generation biodiversity assessment using DNA metabarcoding. Mol. Ecol. 21, 2045-2050. doi: 10.1111/j.1365-294X.2012. 05470.x

Tamas, M., Rosca, M., and Scarlat, M. A. (1984). Studiul fitochimic al plantei Veronica officinalis L. Clujul Med. 47, 169-172.

Tamura, K., Stecher, G., Peterson, D., Filipski, A., and Kumar, S. (2013). MEGA6: molecular evolutionary genetics analysis version 6.0. Mol. Biol. Evol. 30, 2725-2729. doi: 10.1093/molbev/mst197

Taskova, R., Peev, D., and Handjieva, N. (2002). Iridoid glucosides of the genus Veronica s.l. and their systematic significance. Plant Syst. Evol. 231, 1-17. doi: $10.1007 / \mathrm{s} 006060200008$

Taskova, R. M., Albach, D. C., and Grayer, R. J. (2004). Phylogeny of Veronica - a combination of molecular and chemical evidence. Plant Biol. 6, 673-682. doi: 10.1055/s-2004-830330

Techen, N., Parveen, I., Pan, Z., and Khan, I. A. (2014). DNA barcoding of medicinal plant material for identification. Curr. Opin. Biotechnol. 25, 103-110. doi: 10.1016/j.copbio.2013.09.010

Veldman, S., Gravendeel, B., Otieno, J. N., Lammers, Y., Duijm, E., Nieman, A., et al. (2017). High-throughput sequencing of African chikanda cake highlights conservation challenges in orchids. Biodivers. Conserv. 215, 1-18.

Verpoorte, R. (2008). Setting standards: diabetes. J. Ethnopharmacol. 115, 161-162. doi: $10.1016 /$ j.jep.2007.10.035

Vogl, S., Picker, P., Mihaly-Bison, J., Fakhrudin, N., Atanasov, A. G., Heiss, E. H., et al. (2013). Ethnopharmacological in vitro studies on Austria's folk medicine-an unexplored lore in vitro anti-inflammatory activities of 71 Austrian traditional herbal drugs. J. Ethnopharmacol. 149, 750-771. doi: 10. 1016/j.jep.2013.06.007

Wagstaff, S. J., and Garnock-Jones, P. J. (1998). Evolution and biogeography of the Hebe complex (Scrophulariaceae) inferred from ITS sequences. N. Z. J. Bot. 36, 425-437. doi: 10.1080/0028825X.1998.9512581

Walker, K. M., and Applequist, W. L. (2012). Adulteration of selected unprocessed botanicals in the U.S. retail herbal trade. Econ. Bot. 66, 321-327. doi: 10.1007/ s12231-012-9211-6

Wallace, L. J., Boilard, S. M. A. L., Eagle, S. H. C., Spall, J. L., Shokralla, S., and Hajibabaei, M. (2012). DNA barcodes for everyday life: routine authentication of natural health products. Food Res. Int. 49, 446-452. doi: 10.1016/j.foodres. 2012.07.048

White, T. J., Bruns, T., Lee, S., and Taylor, J. W. (1990). "Amplification and direct sequencing of fungal ribosomal RNA genes for phylogenetics," in $P C R$ Protocols: A Guide to Methods and Applications, eds M. A. Innis, D. H. Gelfand, J. J. Sninsky, and T. J. White (New York, NY: Academic Press), $315-322$.

Wiemers, M., and Fiedler, K. (2007). Does the DNA barcoding gap exist? - a case study in blue butterflies (Lepidoptera: Lycaenidae). Front. Zool. 4:8. doi: 10.1186/1742-9994-4-8

World Health Organization (2013). WHO Traditional Medicine Strategy: 20142023. Geneva: World Health Organization.

Yang, Z., and Rannala, B. (2017). Bayesian species identification under the multispecies coalescent provides significant improvements to DNA barcoding analyses. Mol. Ecol. 26, 3028-3036. doi: 10.1111/mec.14093

Zlatković, B. K., Bogosavljević, S. S., Radivojević, A. R., and Pavlović, M. A. (2014). Traditional use of the native medicinal plant resource of Mt. Rtanj (Eastern Serbia): ethnobotanical evaluation and comparison. J. Ethnopharmacol. 151, 704-713. doi: 10.1016/j.jep.2013.11.037

Conflict of Interest Statement: The authors declare that the research was conducted in the absence of any commercial or financial relationships that could be construed as a potential conflict of interest.

Copyright (c) 2017 Raclariu, Mocan, Popa, Vlase, Ichim, Crisan, Brysting and de Boer. This is an open-access article distributed under the terms of the Creative Commons Attribution License (CC BY). The use, distribution or reproduction in other forums is permitted, provided the original author(s) or licensor are credited and that the original publication in this journal is cited, in accordance with accepted academic practice. No use, distribution or reproduction is permitted which does not comply with these terms. 


\section{OPEN ACCESS}

Approved by:

Pharmacology Editorial Office,

Frontiers, Switzerland

*Correspondence:

Frontiers Production Office production.office@frontiersin.org

Specialty section:

This article was submitted to

Ethnopharmacology,

a section of the journal

Frontiers in Pharmacology

Received: 15 December 2017 Accepted: 15 December 2017

Published: 19 December 2017

Citation:

Frontiers Production Office (2017) Erratum: Veronica officinalis Product

Authentication Using DNA

Metabarcoding and HPLC-MS

Reveals Widespread Adulteration with

Veronica chamaedrys.

Front. Pharmacol. 8:957.

doi: 10.3389/fphar.2017.00957

\section{Erratum: Veronica officinalis Product} Authentication Using DNA Metabarcoding and HPLC-MS Reveals Widespread Adulteration with Veronica chamaedrys

\author{
Frontiers Production Office* \\ Frontiers Production Office, Frontiers, Lausanne, Switzerland
}

Keywords: adulteration, DNA metabarcoding, herbal products, HPLC-MS, Veronica chamaedrys, Veronica officinalis

\section{An erratum on}

Veronica officinalis Product Authentication Using DNA Metabarcoding and HPLC-MS Reveals Widespread Adulteration with Veronica chamaedrys

by Raclariu, A. C., Mocan, A., Popa, M. O., Vlase, L., Ichim, M. C., Crisan, G., Brysting, A. K., et al. (2017). Front. Pharmacol. 8:378. doi: 10.3389/fphar.2017.00378

Due to a typesetting error, affiliation 5 was incorrectly identified as "Centre for Ecological and Evolutionary Synthesis (CEES), Department of Biosciences, University of Oslo, Oslo, Norway," the correct affiliation is: "Department of Pharmaceutical Technology and Biopharmaceutics, Iuliu Hatieganu University of Medicine and Pharmacy, Cluj-Napoca, Romania.” Also, affiliation 6 was missing "University of Oslo," the correct affiliation 6 is: "Department of Biosciences, Centre for Ecological and Evolutionary Synthesis (CEES), University of Oslo, Oslo, Norway."

The publisher apologizes for this error and the original article has been updated to reflect this. This error does not change the scientific conclusions of the article in any way.

Copyright $\odot 2017$ Frontiers Production Office. This is an open-access article distributed under the terms of the Creative Commons Attribution License (CC BY). The use, distribution or reproduction in other forums is permitted, provided the original author(s) or licensor are credited and that the original publication in this journal is cited, in accordance with accepted academic practice. No use, distribution or reproduction is permitted which does not comply with these terms. 


\title{
Exploring Pharmacological Mechanisms of Lavender (Lavandula angustifolia) Essential Oil on Central Nervous System Targets
}

\author{
Víctor López ${ }^{1,2 *}$, Birgitte Nielsen ${ }^{2}$, Maite Solas ${ }^{3}$, Maria J. Ramírez ${ }^{3}$ and Anna K. Jäger ${ }^{2}$ \\ ${ }^{1}$ Department of Pharmacy, Faculty of Health Sciences, Universidad San Jorge, Zaragoza, Spain, ${ }^{2}$ Department of Drug \\ Design and Pharmacology, Faculty of Health and Medical Sciences, University of Copenhagen, Copenhagen, Denmark, \\ ${ }^{3}$ Department of Pharmacology and Toxicology, School of Pharmacy, University of Navarra, Pamplona, Spain
}

\section{OPEN ACCESS}

Edited by:

Atanas G. Atanasov,

Institute of Genetics and Animal

Breeding (PAN), Poland

Reviewed by:

Pinarosa Avato

Università Degli Studi di Bari Aldo

Moro, Italy

Roser Vila,

University of Barcelona, Spain

*Correspondence:

Víctor López

ilopez@usj.es

Specialty section:

This article was submitted to

Ethnopharmacology,

a section of the journal

Frontiers in Pharmacology

Received: 28 March 2017 Accepted: 03 May 2017

Published: 19 May 2017

Citation:

López V, Nielsen B, Solas M, Ramírez MJ and Jäger AK (2017)

Exploring Pharmacological Mechanisms of Lavender (Lavandula angustifolia) Essential Oil on Centra

Nervous System Targets.

Front. Pharmacol. 8:280.

doi: 10.3389/fphar.2017.00280
Lavender essential oil is traditionally used and approved by the European Medicines Agency (EMA) as herbal medicine to relieve stress and anxiety. Some animal and clinical studies reveal positive results in models of anxiety and depression although very little research has been done on molecular mechanisms. Our work consisted of evaluating the effects of lavender (Lavandula angustifolia) essential oil on central nervous system well-established targets, such as MAO-A, SERT, GABA $A_{A}$ and NMDA receptors as well as in vitro models of neurotoxicity. The results showed that lavender essential oil and its main components exert affinity for the glutamate NMDA-receptor in a dose-dependent manner with an $\mathrm{IC}_{50}$ value of $0.04 \mu \mathrm{l} / \mathrm{mL}$ for lavender oil. In addition, lavender and linalool were also able to bind the serotonin transporter (SERT) whereas they did not show affinity for $\mathrm{GABA}_{\mathrm{A}}$-benzodiazepine receptor. In three different models of neurotoxicity, lavender did not enhance the neurotoxic insult and improved viability of SH-SY5Y cells treated with hydrogen peroxide. According to our data, the anxiolytic and antidepressant-like effects attributed to lavender may be due to an antagonism on the NMDA-receptor and inhibition of SERT. This study suggests that lavender essential oil may exert pharmacological properties via modulating the NMDA receptor, the SERT as well as neurotoxicity induced by hydrogen peroxide.

Keywords: essential oils, central nervous system, lavender, Lavandula angustifolia, Lavandula officinalis, SH-SY5Y cells, glutamate receptor

\section{INTRODUCTION}

Essential oils have a long tradition in pharmaceutical sciences as natural products with pharmacological, cosmetic, agrochemical and nutritional applications (Bakkali et al., 2008). The use of EO in form of aromatherapy or phytotherapy is widely extended, some of them being used as agents to relieve anxiety and stress (Setzer, 2009). Phytotherapy consists of the use of medicinal plants in order to prevent, cure or threat illnesses. Aromatherapy can be understood as a subdivision of phytotherapy and defined as the use of essential oils regarding therapeutic effects.

Abbreviations: CNS, central nervous system; LEO, lavender essential oil; GABA, $\gamma$-aminobutyric acid; iGLURs, ionotropic glutamate receptors; MAO-A, monoamine oxidase A; NMDA, n-methyl-D-aspartate; SERT, serotonin transporter; SSRI, selective serotonin reuptake inhibitor. 
These products have been used for centuries and are accepted in traditional or modern healthcare systems of medicine. Medicinal plants are widely used for the treatment of central nervous system disorders (Wheatley, 2005) but in some cases there is still lack of preclinical and clinical studies.

Central nervous system disorders have a great impact in society due to a general aging process of the population as well as lifestyle. Stress is one of the most prevalent psychological disorders in developed countries leading to other clinical features, such as anxiety, insomnia or depression.

Benzodiazepines (BZD) and selective serotonin reuptake inhibitors (SSRIs) are highly prescribed as anxiolytic and antidepressant drugs, respectively. BZD, such as diazepam, lorazepam or alprazolam produce calming effects via binding to $\mathrm{GABA}_{\mathrm{A}}$ receptors, but they may also produce somnolence and cognitive impairment as adverse drug reactions. SSRIs (e.g., fluoxetin, paroxetin, citalopram) are prescribed as antidepressants because they are able to selectively block the serotonin transporter (SERT), but side effects include sexual dysfunction and neuropsychiatric disorders, such as suicide tendencies and sleep disorders. Both groups of medicines are also involved in withdrawal and "rebound effects" as a result of discontinuing their administration.

Certain EO are being used as anxiolytic remedies and the administration mode can be orally but also by inhalation or combined with massage. One of the most popular essential oils for mental disorders and anxiety is lavender (Lavandula angustifolia Miller or Lavandula officinalis Chaix). Lavender essential oil can be considered as one of the best-seller over the counter herbal remedies for anxiety, stress and depression. Studies reveal high content of linalool and linalyl acetate (Da Porto et al., 2009) and international organizations, such as the World Health Organization (WHO), the European Scientific Cooperative on Phytotherapy (ESCOP) or the European Medicines Agency (EMA) approve this medicinal plant to relieve stress, restlessness and anxiety (Community) ${ }^{1}$.

The growing awareness of the adverse effects of central nervous system drugs has led to develop new strategies and safer pharmacological agents in mental health. Enzymes, such as monoamine oxidase (MAO), proteins, such as the SERT and ligand-gated ion channels, such as $\mathrm{GABA}_{\mathrm{A}}$ and NMDA receptors are therapeutic targets in neuropharmacology. With the aim to contribute to evidence-based herbal medicine we have studied the effects of lavender essential oil on pharmacological targets involved in anxiolytic and antidepressive properties as well as in vitro models of neurotoxicity.

\section{METHODS}

\section{Lavender Essential Oil (LEO) and Chemicals}

Pure lavender (L. angustifolia) essential oil was kindly supplied by Pranarom International (Belgium). Isolated monoterpenes (linalool, linalyl acetate) were purchased from Fluka. Enzymes,

${ }^{1}$ Community herbal monograph on Lavandula angustifolia Mill., aetheroleum., EMA/HMPC/143181/2010 (http://www.ema.europa.eu/ema/). proteins and reagents were acquired from Sigma. Linalool and linalyl acetate were also tested when lavender essential oil had a clear and significant activity in the assays.

\section{Chemical Profile by GC-MS}

Although the essential oils are chemically characterized by Pranarom International, LEO was analyzed in the laboratory by GC-MS on an Agilent 6890N Network GC system coupled to a 5973 Network Mass Selective Detector, accelerating voltage $69.9 \mathrm{eV}$, recoding masses of 35.00-400.00. GC conditions: injector temperature: $150^{\circ} \mathrm{C}$; temperature programme: start $50^{\circ} \mathrm{C}$, $20^{\circ} \mathrm{C} / \mathrm{min}$ to $300^{\circ} \mathrm{C}$; column: HP5MS (5\% phenylmethylsiloxane) capillary, $30.0 \mathrm{~m} \times 250 \mu \mathrm{l} \times 0.25 \mu \mathrm{m}$ nominal. Carrier gas: helium at $1.0 \mathrm{ml} / \mathrm{min}$. A NIST library was used for comparison of MS data.

\section{Animals and Brain Membrane Homogenates}

Adult male Sprague Dawley rats were obtained from Taconic (Denmark). Ethical permission for the studies was granted by the Animal Welfare Committee, appointed by the Danish Ministry of Justice, and all animal procedures were carried out in compliance with the EC Directive 86/609/EEC and the Danish laws regulating experiments on animals. Rats were put down by competent staff, the heads were separated from the body and the brains were removed whereas cerebellum was discarded. The cortex were weighed and homogenized with an Ultra-Turrax using different buffers at $4^{\circ} \mathrm{C}$ and the tissue preparation in each case was carried out as earlier described (Ransom and Stec, 1988). The tissue homogenates were resuspended and stored in aliquots at $-80^{\circ} \mathrm{C}$ until use.

\section{Bioassays Regarding Serotonergic Targets Monoamine Oxidase-A Inhibition (MAO-A Assay)}

The bioassay was performed in a 96-well microplate (Saaby et al., 2009). Each well contained $50 \mu \mathrm{l}$ of essential oil dilution or DMSO as blank (making a final concentration in the wells of $0.002,0.01,0.02$, and $0.1 \%), 50 \mu \mathrm{l}$ of chromogenic solution $(0.8 \mathrm{mM}$ vanillic acid, $417 \mathrm{mM} 4$-aminoantipyrine and $4 \mathrm{U} / \mathrm{ml}$ horseradish peroxidase in potassium phosphate buffer $\mathrm{pH} 7.6), 100 \mu \mathrm{l}$ of $3 \mathrm{mM}$ tyramine and $50 \mu \mathrm{l}$ of $8 \mathrm{U} / \mathrm{ml} \mathrm{MAO-}$ A. Absorbance was read at $490 \mathrm{~nm}$ every $5 \mathrm{~min}$ for $30 \mathrm{~min}$. Background interferences were deducted in the same way described above but without MAO enzyme. Data was analyzed using GraphPad Prism. IC 50 values could not be obtained because the higest tested concentration did not reach $50 \%$ of MAO inhibition. Clorgyline was used as positive control.

\section{Serotonin Transporter Assay $\left(\left[^{3} \mathrm{H}\right]\right.$-Citalopram Binding Assay)}

The assay was performed on the basis of the method from Nielsen et al. (2004). $25 \mu \mathrm{l}$ of three different essential oil concentrations were mixed with $50 \mu \mathrm{l}$ of $4 \mathrm{nM}\left[{ }^{3} \mathrm{H}\right]$-Citalopram and $225 \mu \mathrm{l}$ of rat cortex suspension making a final concentration of the essential oils in the assay of $0.08,0.4$, and $0.8 \%$. All components were previously dissolved in buffer (50 mM Tris-base; $120 \mathrm{mM}$ $\mathrm{NaCl}: 5 \mathrm{mM} \mathrm{KCl}$ at $\mathrm{pH} \mathrm{7.5).} \mathrm{Plastic} \mathrm{tubes} \mathrm{were} \mathrm{placed} \mathrm{on} \mathrm{an}$ 
ice bath, the reagents were added and the tubes were mixed and then left at room temperature (approximately $22^{\circ} \mathrm{C}$ ) for $2 \mathrm{~h}$. After incubation, $5 \mathrm{ml}$ of ice cold buffer were added to the samples and they were filtered through GC-50 Advantec glass filters under vacuum and immediately washed once with additional $5 \mathrm{ml}$ of ice cold buffer. Control test tubes (with buffer instead of EO) and blind tubes (with $25 \mu \mathrm{l}$ of $120 \mu \mathrm{M}$ paroxetine instead of EO) were done in every run in order to determine total and unspecific binding. The amount of radioactivity was determined transferring the glass filters into scintillation tubes and adding $4 \mathrm{ml}$ of Ultimo Gold XR. The scintillation tubes were placed in the dark for $30 \mathrm{~min}$ before they were measured on a Tri-CARB 2100 TR analyzer. Three independent experiments were performed in triplicates. Nonradioactive citalopram was used as reference. Linalool and linalyl acetate were tested. The specific binding of $\left[{ }^{3} \mathrm{H}\right]$-citalopram was determined using the formula: $\%$ binding $=\left[\mathrm{dpm}_{(\text {essential oil })^{-}}\right.$ $\left.\mathrm{dpm}_{\text {(unspecific binding) }} / \mathrm{dpm}_{\text {(total binding) }}-\mathrm{dpm}_{\text {(unspeficic binding) }}\right] \times$ 100.

\section{Bioassays on lonotropic Receptors Affinity for $\mathrm{GABA}_{A}$ Receptor $\left(\left[{ }^{3} \mathrm{H}\right]-\right.$ Ro 15-1788 Binding Assay)}

The membrane preparation was washed with ice-cold buffer (50 mM Tris-citrate $\mathrm{pH} 7.1$ ). The suspension was centrifuged at $0-4^{\circ} \mathrm{C}$ for $10 \mathrm{~min}$ at $27,000 \times g$. The pellet was resuspended in Tris-citrate buffer ( $2 \mathrm{mg}$ original tissue per $\mathrm{ml}$ ) and used for the assay. $25 \mu \mathrm{l}$ of ${ }^{3} \mathrm{H}$-Ro $15-1788$ (flumazenil) was added to $25 \mu \mathrm{l}$ of test solutions $(10,1$, and $0.1 \mathrm{mg} / \mathrm{ml})$ and $500 \mu \mathrm{l}$ of membrane preparation. Total and unspecific binding was measured using buffer or diazepam ( $1 \mu \mathrm{M}$ assay final concentration). After incubation for $40 \mathrm{~min}$ in an ice bath, $5 \mathrm{ml}$ of ice-cold buffer was added to the samples and poured onto Adventic glass fiber filters (GC-50) under vacuum, and immediately washed with another $5 \mathrm{ml}$ of ice-cold buffer. The amount of radioactivity of the filters was measured by conventional liquid scintillation counting using Ultimo Gold XR as scintillation fluid. Clonazepam was used as positive control.

\section{Affinity for NMDA Receptor $\left(\left[{ }^{3} \mathrm{H}\right]-\mathrm{CGP} 39653\right.$ Binding Assay)}

Affinity for native NMDA receptors was determined using $2 \mathrm{nM}$ $\left[{ }^{3} \mathrm{H}\right]$-CGP 39653 (Sills et al., 1991) with some modification. On the day of the assay, frozen membranes were quickly thawed and homogenized in 30 volumes of ice-cold Tris- $\mathrm{HCl}$ buffer, $\mathrm{pH} 7.4(50 \mathrm{mM}$ containing $2.5 \mathrm{mM} \mathrm{CaCl} 2)$, and centrifuged $(48,000 \times g$ for $10 \mathrm{~min})$. This step was repeated three times. The final pellet was re-suspended in ice-cold buffer, corresponding to approximately $0.4-0.5 \mathrm{mg}$ protein $/ \mathrm{ml}$. Binding was carried out in aliquots consisting of $25 \mu \mathrm{L}\left[{ }^{3} \mathrm{H}\right]$-CGP 39653, $25 \mu \mathrm{L}$ test solution, and $200 \mu \mathrm{L}$ membrane suspension and incubated at $0^{\circ}$ for $60 \mathrm{~min}$. Non-specific binding was determined using $1 \mathrm{mM}$ (S)-Glu. Binding was terminated by filtration through Whatman GF/C filters using a 96-well Packard Filter-Mate Cell Harvester and filters were washed with $3 \times 250 \mu \mathrm{L}$ of ice-cold buffer. After drying, $30 \mu \mathrm{L}$ Microscint 0 (Perkin-Elmer) per well was added and the filter was counted on a Topcounter (Perkin-Elmer). Linalool and linalyl acetate were also tested in this assay.

\section{Neuroprotection on SH-SY5Y Cells SH-SY5Y Neuroblastoma Cell Culture}

Cells were cultured in DMEM (Gibco, ref. 41966-029) containing phenol red $(\mathrm{PR})$, L-Glutamine $(1 \mathrm{mM})$ and sodium pyruvate $(1 \mathrm{mM})$ and was supplemented with $10 \% \mathrm{FBS}$ (Gibco), penicillin (100 U/mL, Gibco) and streptomycin (100 U/mL, Gibco). Cells were maintained at $37^{\circ} \mathrm{C}$ in saturated humidity $\left(5 \% \mathrm{CO}_{2}\right)$. Nondifferentiated cells were plated on 48 -well plates (Corning), at a density of $5 \times 10^{4}$ cells per well and were used $48 \mathrm{~h}$ after seeding.

\section{$A \beta_{25-35}$ SolubleSpecies Preparation}

$\mathrm{A} \beta_{25-35}$ fragment was purchased from Sigma-Aldrich laboratories (ref. A4559-1MG). $1 \mathrm{mg}$ of $\mathrm{A} \beta_{25-35}$ fragment was dissolved in $1 \mathrm{~mL}$ of type II water and was frozen at $-20^{\circ} \mathrm{C}$ until further use. Different concentrations of $A \beta_{25-35}(5,10$ and $15 \mu \mathrm{M}$ ) were incubated in DMEM phenol red free (Gibco, 31053-028) at $37^{\circ} \mathrm{C}$ for 3 days to obtain the oligomeric forms.

\section{Cell Treatment and MTT Cell Viability Assay}

SH-SY5Y cells were treated with either different LEO concentration $(0.05,0.1,0.5$, and $1 \mu \mathrm{L} / \mathrm{mL})$ or incubation time $(0,2$, and $24 \mathrm{~h})$, followed by hydrogen peroxide $(100,200$, $400,800$, and $1,600 \mu \mathrm{M})$, malonate $(0$ or $50 \mathrm{mM})$ or $\mathrm{A} \beta_{25-35}(5$, 10 , and $15 \mu \mathrm{M})$.

Cell viability was examined by the 3,4,5-dimethylthiazol-2yl-2,5-diphenyltetrazolium bromide (MTT) assay. MTT assay is a colorimetric assay for measuring the activity of cellular enzymes in living cells in response to potential toxic. After the cell treatment, culture medium was replaced by a solution of $5 \mathrm{mg} / \mathrm{mL}$ MTT (Sigma-Aldrich) in DMEM (Gibco, 31053-028). Cells were incubated with MTT solution for $2 \mathrm{~h}$ in the cell incubator $\left(5 \% \mathrm{CO}_{2}\right.$ and $\left.37^{\circ} \mathrm{C}\right)$. Then, the MTT solution was discarded and DMSO was added to the wells. Aliquots were transferred to a 96-well plate, and absorbance was measured at $595 \mathrm{~nm}$ in a plate reader. Results were expressed as percentages of non-treated control cells.

\section{Statistical Analyses}

Data are expressed as mean $\pm \mathrm{SE}$ (figures) or as mean $\pm \mathrm{SD}$ (tables) of at least three independent experiments performed in different days and in triplicates. GraphPad Prism was used to calculate $\mathrm{IC}_{50}$ values and to detect significant differences. Student $t$-test or ANOVA was performed for data analysis.

\section{RESULTS}

\section{Chemical Profile by GC-MS}

According to the GC-MS analyses, lavender essential oil mainly contained the following monoterpenes: linalyl acetate $(52.1 \%)$, linalool (37.4\%), geranyl acetate (5.4\%), and $\beta$-caryophyllene (5.1\%).

\section{Monoamine Oxidase-A Inhibition (MAO-A Assay)}

Inhibition of MAO A was not detected (data not shown). 
TABLE 1 | Activity of lavender essential oil (LEO) on the serotonin transporter (SERT) and $\mathrm{GABA}_{\mathrm{A}}$ ionotropic receptor.

\begin{tabular}{|c|c|c|c|}
\hline \multirow[t]{2}{*}{ Samples } & \multicolumn{3}{|c|}{ SERT ( $\%{ }^{3} \mathrm{H}$-Citalopram binding) } \\
\hline & $0.8 \mu \mathrm{l} / \mathrm{ml}$ & $4 \mu \mathrm{l} / \mathrm{ml}$ & $8 \mu \mathrm{l} / \mathrm{ml}$ \\
\hline \multirow[t]{3}{*}{ LEO } & $105.4 \pm 0.9$ & $77.6 \pm 2.7^{\star}$ & $37.8 \pm 16.0^{\star \star \star}$ \\
\hline & \multicolumn{3}{|c|}{ GABA $_{A}\left(\%{ }^{3} \mathrm{H}\right.$-Flumazenil binding) } \\
\hline & $0.045 \mu \mathrm{l} / \mathrm{ml}$ & $0.45 \mu \mathrm{l} / \mathrm{ml}$ & $4.5 \mu \mathrm{l} / \mathrm{ml}$ \\
\hline LEO & $100.3 \pm 9.5$ & $100.1 \pm 0.8$ & $105.2 \pm 9.3$ \\
\hline
\end{tabular}

${ }^{*} p<0.05 ;{ }^{* \star *} p<0.001$ vs. the lowest concentration tested $(0.8 \mu \mathrm{l} / \mathrm{ml}$ in the SERT assay or $0.045 \mathrm{ll} / \mathrm{ml}$ in the GABA assay). Data are mean $\pm S D$ of three independent experiments performed in triplicates.

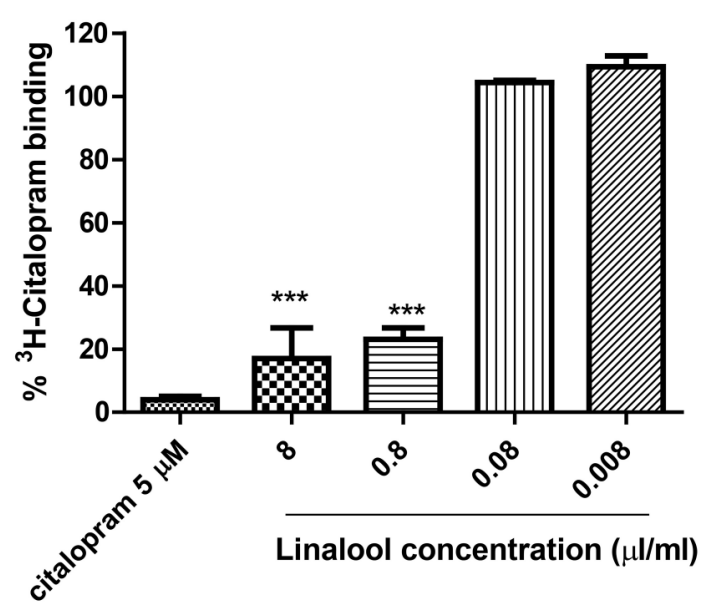

FIGURE 1 | Percentage of ${ }^{3} \mathbf{H}$-Citalopram binding induced by different concentrations of linalool on the SERT assay. Linalyl acetate did not show affinity to the serotonin transporter (data not shown). ${ }^{\star \star \star} p<0.0001$ vs. the lowest concentration tested.

\section{Serotonin Transporter Assay $\left(\left[{ }^{3} \mathrm{H}\right]\right.$-Citalopram Binding Assay)}

The effects of LEO on the SERT are displayed in Table 1, showing moderate activity in this assay (citalopram $\mathrm{IC}_{50}$ value was $1.3 \mathrm{nM}$ ). LEO significantly displaced ${ }^{3} \mathrm{H}$-citalopram from binding to the SERT in a dose-dependent manner, which means that LEO might have an antidepressantlike effect via this specific transporter. This effect was also detected for linalool, one of the main constituents of lavender essential oil (Figure 1), but not for linalyl acetate.

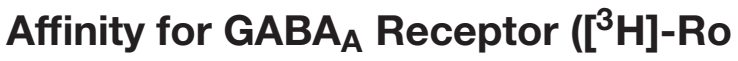 15-1788 Binding Assay)}

The affinity for the $\mathrm{GABA}_{A}$-benzodiazepine receptor, which may lead to a nerve calming effect, was not detected (Table $\mathbf{1}$ ). LEO had no effect in binding this ionotropic receptor. Clonazepam $\left(\mathrm{IC}_{50} 0.002 \mathrm{nM}\right)$ was used as positive control drug.
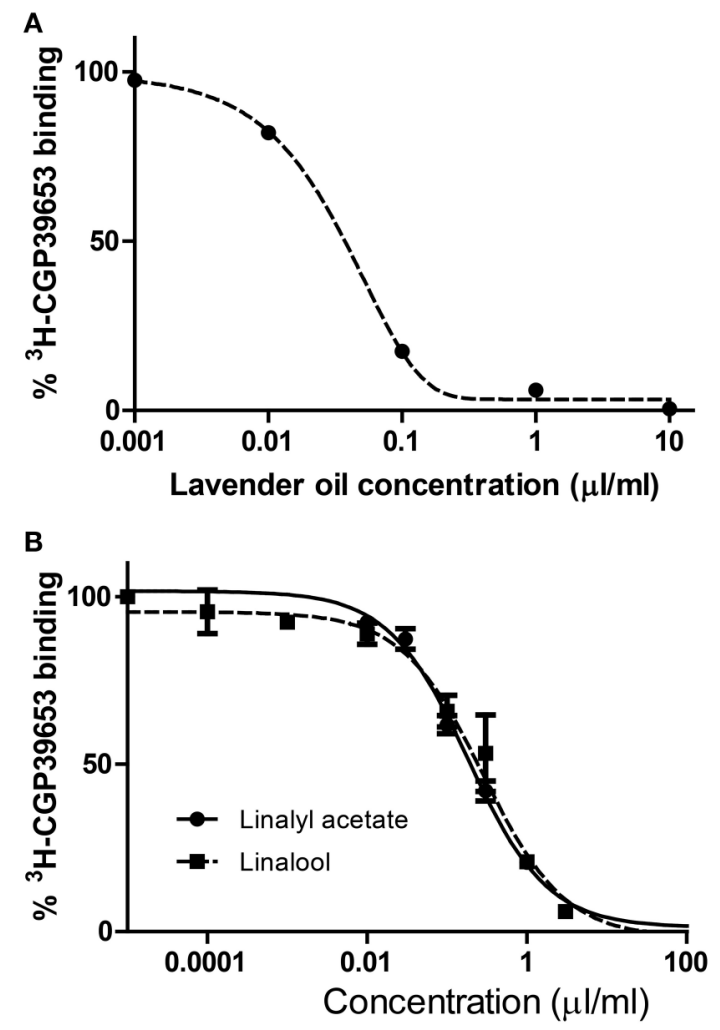

FIGURE 2 | Affinity of lavender essential oil (A) and linalool and linalyl acetate (B) for the glutamate NMDA receptor. The displacement studies of $\left[{ }^{3} \mathrm{H}\right] \mathrm{CGP} 39653$ were performed on membranes obtained from rat brain homogenates.

TABLE 2 | IC 50 and $K_{i}$ values of lavender essential oil (LEO) and monoterpenes in the $\left[{ }^{3} \mathrm{H}\right] \mathrm{CGP} 39653$ binding assay.

\begin{tabular}{lcc}
\hline Essential oil & $\mathbf{I C}_{\mathbf{5 0}}(\boldsymbol{\mu l} \mathbf{m} \mathbf{m l})$ & $\mathbf{K}_{\boldsymbol{i}}(\boldsymbol{\mu l} \mathbf{l} \mathbf{m l})$ \\
\hline LEO & $0.04 \pm 0.09$ & $0.026[0.022 ; 0.030]$ \\
Monoterpenes & $\mathrm{IC}_{50}(\mathrm{mM})$ & $\mathrm{K}_{\mathrm{i}}(\mathrm{mM})$ \\
Linalyl acetate & $0.74 \pm 0.18^{\star \star}$ & $0.54[0.47 ; 0.62]$ \\
Linalool & $2.97 \pm 0.63$ & $2.3[2.1 ; 2.6]$
\end{tabular}

${ }^{* *} p<0.01$ vs. linalool; each $I C_{50}$ value, determined from the "dose-response" curve, has been converted into the $K_{i}$ value using the Cheng Prusoff equation: $\left(K_{i}=I C_{50} \times\left[1 /\left([L] / K_{D}\right)\right.\right.$ + 1)]. (S)-Glu has a Ki value of $0.02 \mu \mathrm{M}$.

\section{Affinity for NMDA Receptor (CGP39653 Binding Assay)}

Lavender Essential Oil (LEO) was found to be active in the CGP39653 binding assay. Figure 2 shows the profile of the affinities for the NMDA receptor and Table 2 presents results in terms of $\mathrm{IC}_{50}$ and $\mathrm{K}_{\mathrm{i}}$ values. It can be observed that lavender was significantly active showing a clear dose-response activity (Figure 2A). In this case, linalool and linalyl acetate were also tested, showing binding properties to the NMDA receptor (Figure 2B). 


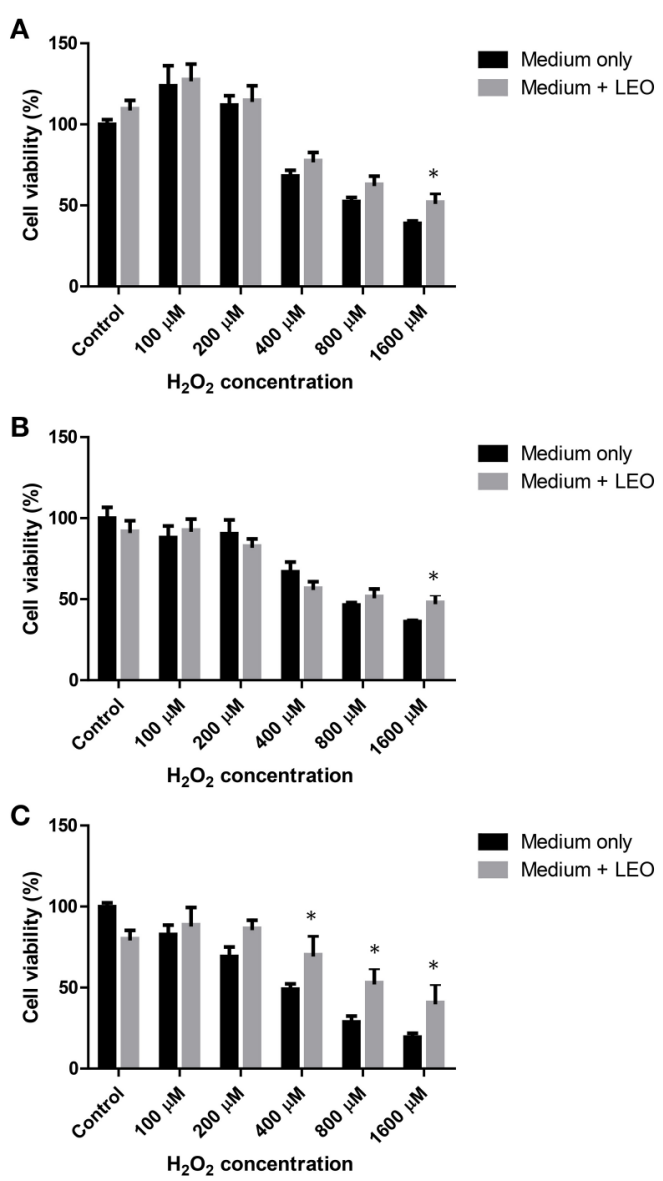

FIGURE 3 | Effect of $1 \mu \mathrm{l} / \mathrm{ml}$ lavender essential oil (LEO) on hydrogen peroxide induced toxicity on SH-SY5Y cells at 0 (A), 2 (B), and $24 \mathrm{~h}$ (C) ${ }^{*} p<0.05$ vs. medium only.

\section{Effects of Lavender Oil against Hydrogen Peroxide in SH-SY5Y Cells}

As depicted in Figure 3A, when $1 \mu \mathrm{l} / \mathrm{ml}$ lavender oil was incubated $0 \mathrm{~h}$ before the addition of hydrogen peroxide $\left(\mathrm{H}_{2} \mathrm{O}_{2}\right)$, lavender oil only produced a significance difference $\left({ }^{*} p<0.05\right.$, Two-way ANOVA) at $1,600 \mu \mathrm{M}$ of $\mathrm{H}_{2} \mathrm{O}_{2}$. Same result was obtained when lavender oil was incubated $2 \mathrm{~h}$ prior the addition of $\mathrm{H}_{2} \mathrm{O}_{2}\left(^{*} p<0.05\right.$, Two-way ANOVA) (Figure 3B). As shown in Figure 3C, when lavender oil was incubated $24 \mathrm{~h}$ prior the addition of $\mathrm{H}_{2} \mathrm{O}_{2}$ a significant reversion of cell death was obtained at 400,800 , and $1,600 \mu \mathrm{M}$ of $\mathrm{H}_{2} \mathrm{O}_{2}\left(^{*} p<0.05\right.$, Two-way ANOVA).

\section{Effects of Lavender Oil against Malonate in SH-SY5Y Cells}

Lavender oil did not protect from malonate induced toxicity in any of the concentrations $(0.05,0.1,0.5$, and $1 \mu \mathrm{L} / \mathrm{mL})$ or incubation times tested $(0,2$, or $24 \mathrm{~h}$; Figure 4).

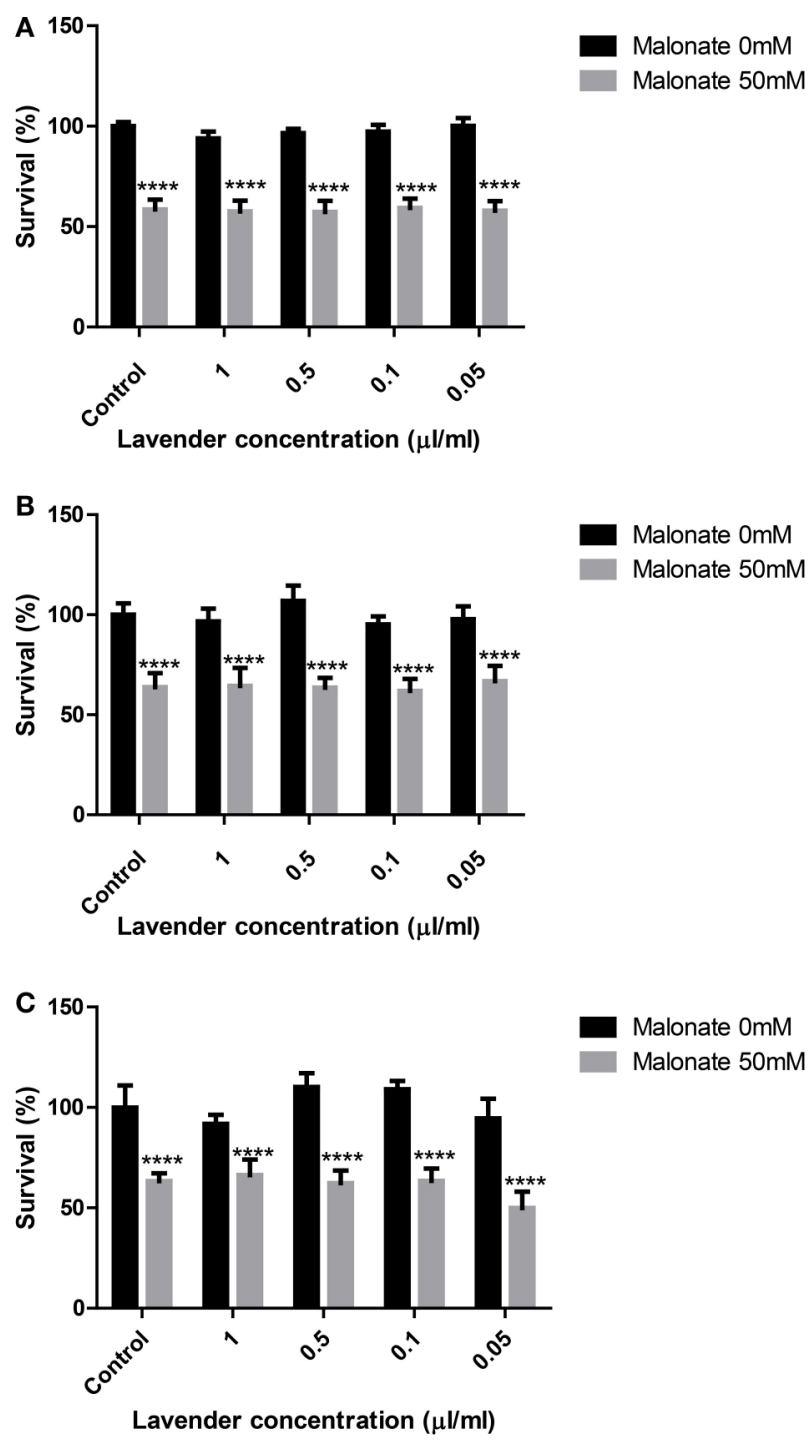

FIGURE 4 | Effect of lavender essential oil on malonate induced toxicity on SH-SY5Y cells at 0 (A), 2 (B), and $24 \mathrm{~h}$ (C). ${ }^{\star \star \star \star} p<0.0001$ vs. $0 \mathrm{mM}$ malonate in the respective lavender concentration.

\section{Effects of Lavender Oil against $\mathbf{A} \boldsymbol{\beta}_{25-35}$ in SH-SY5Y Cells}

As observed in Figure 5, lavender oil only produced a significant effect against $A \beta_{25-35} 15 \mu \mathrm{M}$ induced toxicity when it was incubated $2 \mathrm{~h}$ before toxin addition $\left(^{* *} \mathrm{p}<0.01\right.$, Two-way ANOVA; Figure 5B).

\section{DISCUSSION}

Lavender is used in pharmacy, phytotherapy and aromatherapy to treat central nervous system disorders, such as anxiety, stress, and sleep disorders. This essential oil is one of the bestselling natural remedies and a common therapeutic tool for physiotherapists and chiropractors. 

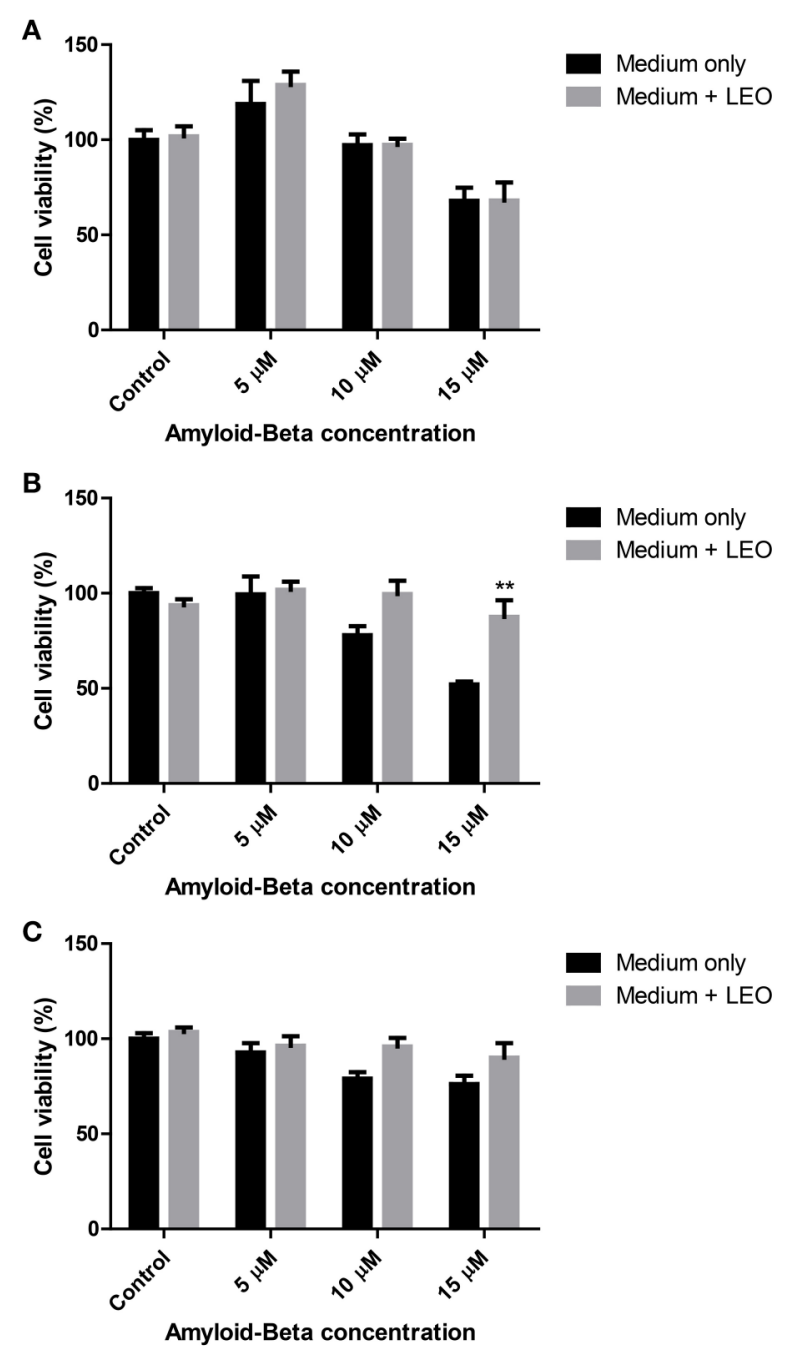

FIGURE 5 | Effect of $1 \mu \mathrm{l} / \mathrm{ml}$ lavender essential oil (LEO) on beta-amyloid induced toxicity on SH-SY5Y cells at $\mathbf{0}$ (A), 2 (B), and $24 \mathrm{~h}$ (C). ${ }^{\star \star} p<0.01$ vs. $15 \mu \mathrm{M}$ beta-amyloid (medium only).

There has recently been an increase in the use of natural therapies due to well-known side effects of common CNS drugs, such as (BZD) and selective serotonin re-uptake inhibitors (SSRI). Although BZD and SSRI have been widely used in neuropharmacology, there is a need of developing new therapeutic tools that play a role both in prevention and treatment of mild CNS disorders.

In this sense, aromatherapy and some essentials oils have been used for decades to induce nerve-calming effects and moodenhancing properties (Dobetsberger and Buchbauer, 2011). Aromatherapy is a form of complementary and alternative medicine (CAM) that uses plant essentials oils to affect the mood or health of the patient (Wheatley, 2005) and some clinical studies suggest that inhaled or topically applied essential oils like lavender exert psychological and central nervous system effects (Perry and Perry, 2006). It has also recently been stated that essential oils may behave as antidepressants due to playing an important role on neurotransmitter pathways, mainly in the serotonin system (Lv et al., 2013).

Certain studies have reported pharmacological properties of essential oils but there is little research on the molecular mechanisms underlying the CNS effects. The ability of lavender essential oil to interact with neuropharmacological targets, such as MAO-A, the SERT and ionotropic receptors $\left(\mathrm{GABA}_{\mathrm{A}}\right.$ and NMDA) has been tested, as well as the protective potential against neurotoxic agents, such as hydrogen peroxide, malonate and amyloid peptide.

It was surprising that LEO had the capacity to bind to the NMDA receptor, which was measured through the CGP39653 binding assay. CGP39653 is a competitive antagonist, being currently the ligand of choice for labeling NMDA receptors. NMDA receptors are neurochemically classified as ionotropic glutamate receptors (iGLURs) and are involved in certain neurological and psychiatric disorders, such as epilepsy, sustained-seizure damage, Parkinsonism, etc. For this reason iGLURs are considered pharmacological targets in drug research and development. LEO was able to displace CGP39653 binding in a dose-dependent mode, which means that this oil may exert nerve-calming effects via modulating NMDA receptors. This is the first time that this affinity is reported and this fact could explain the anti-agitation properties that have been found for these products in animal and some clinical studies (Bradley et al., 2009; Faturi et al., 2010; Kasper et al., 2010; Tsang and Ho, 2010; Woelk and Schlaefke, 2010; Chioca et al., 2011; Goes et al., 2012; Hritcu et al., 2012; Schuwald et al., 2013). NMDA-receptor activation by glutamate is also involved in neurotoxicity so our essential oil might exert neuroprotection through the blockade of this ionotropic receptor.

According to our data, these results are in the range of other natural products that have been reported to show activity in this target (Cho et al., 2001; Pedersen et al., 2008; Marchetti et al., 2011).

Lavender essential oil has shown activity in animal and human studies but according to Koulivand et al. (2013), due to methodological inadequacies, small sample sizes, variations on administration methods or the absence of placebos or control groups in the studies, more standard experiments are needed to confirm the benefits of lavender in CNS disorders. The molecular mechanisms involved in lavender effects have been suggested in other studies. For example, Huang et al. (2009) suggest that lavender essential oil reversibly inhibited GABA-induced currents in a concentration-dependent manner $(0.01-1 \mathrm{mg} / \mathrm{mL})$, whereas no inhibition of NMDA- or AMPA-induced currents was noted. In a recent study, Schuwald et al. (2013) identified a standarized lavender essential oil (Silexan) as a potent anxiolytic inhibiting voltage dependent calcium channels in synaptosomes, primary hippocampal neurons and stably overexpressing cell lines, in the same range as pregabaline.

Lavender essential oil is known to contain monoterpenes like linalool and linalyl acetate, which seem to be responsible for the activity according to our data and previous works (Cline et al., 2008; Linck et al., 2010; Souto-Maior et al., 2011). The capacity of linalool to interact with the glutamatergic system and the NMDA receptor is not new as it has been previously described by other 
authors (Elisabetsky et al., 1995, 1999; Silva Brum et al., 2001; Aprotosoaie et al., 2014); however, the ability of linalyl acetate to bind the NMDA receptor has not been found to be reported in previous works. It seems that lavender essential oil anxiolytic effects is due to the fact that its main monoterpenes, linalool and linalyl acetate, interact with the NMDA receptor.

It is very difficult to establish structure activity relationships because linalyl acetate was inactive in the SERT assay, whereas the activity of this compound was slightly higher than linalool in the NMDA binding receptor assay. Linalool and linalyl acetate are secondary metabolites classified as monoterpenes with a similar structure consisting of a linear hydrocarbon chain of 10 carbons (linalool) or 11 (linalyl acetate), being linalyl acetate the ester of linalool. The free hydroxyl group in linalool seems to be determinant for the activity in the SERT. On the contrary, the activity on the NMDA receptor is increased when the acetate group exists in the compound named linalyl acetate; limonene, another monoterpenic compound found at high concentration in Citrus essential oils, has also shown anxiolytic-like activity with other targets involved (de Almeida et al., 2012; Lima et al., 2013).

Other essential oils have been demonstrated to produce changes in brain neurotransmitters which could explain its antiagitation properties. For example, ylang-ylang (Cananga odorata) essential oil has shown anxiolytic effects in male mice decreasing the dopamine concentration in the striatum and increasing the serotonin concentration in the hippocampus (Zhang et al., 2016). Surprisingly, linalool is one of the main components of this essential oil. The essential oils obtained from Eugenia uniflora has also demonstrated antidepressant-like activities in animal models with the serotonergic and adrenergic systems being involved (Victoria et al., 2013). Melissa officinalis essential oil, which is also used as a natural sedative, has demonstrated a depressant effect on neurotransmission but no inhibition of NMDA- or AMPA-induced currents was detected (Abuhamdah et al., 2008). Citrus aurantifolia (bitter orange) essential oil has also exhibited anxiolytic-like effects mediated by $5-\mathrm{HT}\left({ }_{1 \mathrm{~A}}\right)$-receptors (Costa et al., 2013). All these works put forward the idea that the serotonergic system is involved in the nerve calming effects of essential oils.

We have also observed that lavender and linalool inhibits serotonergic targets, such as the SERT, which might explain why lavender has shown antidepressant-like effects in animal and

\section{REFERENCES}

Abuhamdah, S., Huang, L., Elliott, M. S., Howes, M. J., Ballard, C., Holmes, C., et al. (2008). Pharmacological profile of an essential oil derived from Melissa officinalis with anti-agitation properties: focus on ligand-gated channels. J. Pharm. Pharmacol. 60, 377-384. doi: 10.1211/jpp.60.3.0014

Akhondzadesh, S., Kashani, L., Fotouhi, A., Jarvandi, S., Mobaseri, M., Moin, M., et al. (2003). Comparison of Lavandula angustifolia Mill. tincture and imipramine in the treatment of mild to moderate depression: a double-blind, randomized trial. Prog. Neuro Psychopharmacol. Biol. Psychiatry 27, 123-127. doi: 10.1016/S0278-5846(02)00342-1

Aprotosoaie, A. C., Hãncianu, M., Costache, I., and Miron, A. (2014). Linalool: a review on a key odorant molecule with valuable biological properties. Flavour Fragr. J. 29, 193-219. doi: 10.1002/ffj.3197 human models (Cavanagh and Wilkinson, 2002; Akhondzadesh et al., 2003; Hritcu et al., 2012). However, we did not observe effects on the $\mathrm{GABA}_{\mathrm{A}}$-receptor, which is in accordance with results from a recent study (Chioca et al., 2013).

This study has been completed using neuroblasts from neural human tissue (SH-SY5Y) exposed to different neurotoxic agents. Lavender was able to protect the cells from the toxic insult generated by hydrogen peroxide although it was not capable to reduce malonate toxicity. Results from amyloid- $\beta$ peptide induced toxicity are not conclusive. However, this activity seems to be in accordance with a previous study where lavender essential oil showed neuroprotective properties against hydrogen peroxide induced toxicity in PC12 cells (Xu et al., 2016). The authors conclude that lavender protected the cells reducing $\mathrm{LDH}$, NO release, intracellular ROS accumulation and MMP loss.

\section{CONCLUSION}

Our study reveals for the first time that lavender exerts receptor binding affinities with a relevant activity on the NMDA receptor. According to our data, we can state that the anti-agitation and antidepressant activities of lavender may be attributed at least in part to the NMDA receptor modulation as well as an inhibition of the SERT. Lavender essential oil also protected SH-SY5Y cells from hydrogen peroxide induced neurotoxicity.

\section{AUTHOR CONTRIBUTIONS}

VL conceived the study, performed in vitro pharmacological activities (enzyme inhibition tests, affinities to receptors and transporters), carried out data analysis and wrote the manuscript. $\mathrm{BN}$ run the NMDA activity assay. MS and MR perfomed the cell assays neuroblasts. AJ supervised all work.

\section{ACKNOWLEDGMENTS}

Pranarom International is acknowledged for providing lavender essential oil and funding the open access publishing fee. The funders had no role in study design, data collection, analysis, decision to publish, or preparation of the manuscript. The Department of Drug Design and Pharmacology of the University of Copenhagen is thanked for allowing VL to perform a stay as Guest Researcher.

Bakkali, F., Averbeck, S., Averbeck, D., and Waomar, M. (2008). Biological effects of essential oils-A review. Food Chem. Toxicol. 46, 446-475. doi: 10.1016/j.fct.2007.09.106

Bradley, B. F., Brown, S. L., Chu, S., and Lea, R. W. (2009). Effects of orally administered lavender essential oil on responses to anxietyprovoking film clips. Hum. Psychopharmacol. 24, 319-330. doi: 10.1002/h up. 1016

Cavanagh, H. M. A., and Wilkinson, J. N. (2002). Biological activities of lavender essential oil. Phytother. Res. 16, 301-308. doi: 10.1002/ptr.1103

Chioca, L. R., Ferro, M. M., Baretta, I. P., Oliveira, S. M., Silva, C. R., Ferreira, J., et al. (2013). Anxiolytic-like effect of lavender essential oil inhalation in mice: participation of serotonergic but not GABAA/benzodiazepine neurotransmission. J. Ethnopharmacol. 147, 412-418. doi: 10.1016/j.jep.2013.03.028 
Chioca, L. R., Pereira, M., Baretta, I. P., Antunes, V. D. C., Menezes, J. V. B. N., Ferreira, J., et al. (2011). Anxiolytic-like effect of lavender and orange essential oil: participation of nitric oxide but not GABA-A benzodiazepine complex. Eur Neuropsychopharmacol. 21, S538-S538. doi: 10.1016/s0924-977x(11)70875-6

Cho, J., Kong, J. Y., Jeong, D. Y., Lee, K. D., Lee, D. U., and Kang, B. S. (2001). NMDA recepter-mediated neuroprotection by essential oils from the rhizomes of Acorus gramineus. Life Sci. 68, 1567-1573. doi: 10.1016/S0024-3205(01)00944-4

Cline, M., Taylor, J. E., Flores, J., Bracken, S., McCall, S., and Ceremuga, T. E. (2008). Investigation of the anxiolytic effects of linalool, a lavender extract, in the male Sprague-Dawley rat. AANA J. 76, 47-52. Available online at: http:// www.aana.com/newsandjournal/Documents/anxiolytic_effects_0208_47-52. pdf

Costa, C. A., Cury, T. C., Cassettari, B. O., Takahira, R. K., Flório, J. C., and Costa, M. (2013). Citrus aurantium L. essential oil exhibits anxiolytic-like activity mediated by $5-\mathrm{HT}_{1 \mathrm{~A}}$-receptors and reduces cholesterol after repeated oral treatment. BMC Complement Altern Med. 13:42. doi: 10.1186/1472-6882-13-42

Da Porto, C., Decorti, D., and Kikic, I. (2009). Flavour compounds of Lavandula angustifolia $\mathrm{L}$. to use in food manufacturing: comparison of three different extraction methods. Food Chem. 112, 1072-1078. doi: 10.1016/j.foodchem.2008.07.015

de Almeida, A. A. C., Costa, J. P., de Carvalho, R. B., de Sousa, D. P., and de Freitas, R. M. (2012). Evaluation of acute toxicity of a natural compound (+)-limonene epoxide and its anxiolytic-like action. Brain Res. 1448, 56-62. doi: 10.1016/j.brainres.2012.01.070

Dobetsberger, C., and Buchbauer, G. (2011). Actions of essential oils on the central nervous system: an updated review. Flavour Frag. J. 26, 300-316. doi: 10.1002/ffj. 2045

Elisabetsky, E., Brum, L. F., and Souza, D. O. (1999). Anticonvulsant properties of linalool in glutamate-related seizure models. Phytomedicine 6, 107-113. doi: 10.1016/S0944-7113(99)80044-0

Elisabetsky, E., Marschner, J., and Souza, D. O. (1995). Effects of Linalool on glutamatergic system in the rat cerebral cortex. Neurochem. Res. 20, 461-465. doi: 10.1007/BF00973103

Faturi, C. B., Leite, J. R., Alves, P. B., Canton, A. C., and Teixeira-Silva, F. (2010). Anxiolytic-like effect of sweet orange aroma in Wistar rats. Prog. Neuro Psychopharmacol. Biol. Psychiatry 34, 605-609. doi: 10.1016/j.pnpbp.2010.02.020

Goes, T. C., Antunes, F. D., Alves, P. B., and Teixeira-Silva, F. (2012). Effect of sweet orange aroma on experimental anxiety in humans. J. Altern. Complement. Med. 18, 798-804. doi: 10.1089/acm.2011.0551

Hritcu, L., Cioanca, O., and Hancianu, M. (2012). Effects of lavender oil inhalation on improving scopolamine-induced spatial memory impairment in laboratory rats. Phytomedicine 19, 529-534. doi: 10.1016/j.phymed.2012.02.002

Huang, L., Abuhamdah, S., Howes, M. J., Dixon, C. L., Elliot, M. S., Ballard, C., et al. (2009). Pharmacological profile of essential oils derived from Lavandula angustifolia and Melissa officinalis with anti-agitation properties: focus on ligand-gated channels. J. Pharm. Pharmacol. 61, 267-267. doi: 10.1111/j.2042-7158.2009.tb00311.x

Kasper, S., Gastpar, M., Mueller, W. E., Volz, H.-P., Moeller, H.-J., Dienel, A., et al. (2010). Silexan, an orally administered Lavandula oil preparation, is effective in the treatment of 'subsyndromal' anxiety disorder: a randomized, double-blind, placebo controlled trial. Int. Clin. Psychopharmacol. 25, 277-287. doi: 10.1097/YIC.0b013e32833b3242

Koulivand, P. H., Ghadiri, M. K., and Gorji, A. (2013). Lavender and the Nervous System. Evid. Complement. Alternat. Med. 2013:681304. doi: 10.1155/2013/681304

Lima, N. G., De Sousa, D. P., Pimenta, F. C., Alves, M. F., De Souza, F. S., Macedo, R. O., et al. (2013). Anxiolytic-like activity and GC-MS analysis of (R)-(+)limonene fragrance, a natural compound found in foods and plants. Pharmacol. Biochem. Behav. 103, 450-454. doi: 10.1016/j.pbb.2012.09.005

Linck, V. M., da Silva, A. L., Figueiro, M., Caramao, E. B., Moreno, P. R. H., and Elisabetsky, E. (2010). Effects of inhaled Linalool in anxiety, social interaction and aggressive behavior in mice. Phytomedicine 17, 679-683. doi: 10.1016/j.phymed.2009.10.002

Lv, X. N., Zhang, H. J., and Tzeng, C. M. (2013). Aromatherapy on Central Nerve System (Cns): therapeutic mechanism and its associated genes. Curr. Drug Targets 14, 872-879. doi: 10.2174/1389450111314080007

Marchetti, C., Gavazzo, P., Stafford, G. I., and Van Staden, J. (2011). South African plants used in traditional medicine to treat epilepsy have an antagonistic effect on NMDA receptor currents. J. Ethnopharmacol. 137, 382-388. doi: 10.1016/j.jep.2011.05.038

Nielsen, N. D., Sandager, M., Stafford, G. I., van Staden, J., and Jäger, A. K. (2004). Screening of indigenous plants from South Africa for affinity to the serotonin reuptake transport protein. J. Ethnopharmacol. 94, 159-163. doi: 10.1016/j.jep.2004.05.013

Pedersen, M. E., Vestergaard, H. T., Stafford, G. I., van Staden, J., and Jäger, A. K. (2008). The effect of extracts of Searsia species on epileptiform activity in slices of the mouse cerebral cortex. J. Ethnopharmacol. 119, 538-541. doi: 10.1016/j.jep.2008.08.009

Perry, N., and Perry, E. (2006). Aromatherapy in the management of psychiatric disorders-Clinical and neuropharmacological perspectives. CNS Drugs 20, 257-280. doi: 10.2165/00023210-200620040-00001

Ransom, R. W., and Stec, N. L. (1988). Cooperative modulation of $\left[{ }^{3} \mathrm{H}\right] \mathrm{MK}$ 801 binding to the $\mathrm{N}$-methyl-D-aspartate receptor-ion channel complex by L-glutamate, glycine, and polyamines. J. Neurochem. 51, 830-836. doi: 10.1111/j.1471-4159.1988.tb01818.x

Saaby, L., Rasmussen, H. B., and Jäger, A. K. (2009). MAO-A inhibitory activity of quercetin from Calluna vulgaris (L.) Hull. J. Ethnopharmacol. 121, 178-181. doi: 10.1016/j.jep.2008.10.012

Schuwald, A. M., Noldner, M., Wilmes, T., Klugbauer, N., Leuner, K., and Muller, W. E. (2013). Lavender oil-potent anxiolytic properties via modulating voltage dependent calcium channels. PLoS ONE 8:e59998-e59998. doi: 10.1371/journal.pone.0059998

Setzer, W. N. (2009). Essential oils and anxiolytic aromatherapy. Nat. Prod. Commun. 4, 1305-1316.

Sills, M. A., Fagg, G., Pozza, M., Angst, C., Brundish, D. E., Hurt, S. D., et al. (1991). [3H] 39653: a new N-methyl-D-aspartate antagonist radioligand with low nanomolar affinity in rat brain. Eur. J. Pharmacol. 192, 19-24. doi: 10.1016/0014-2999(91)90063-V

Silva Brum, L. F., Emanuelli, T., Souza, D. O., and Elisabetsky, E. (2001). Effects of linalool on glutamate release and uptake in mouse cortical synaptosomes. Neurochem. Res. 26, 191-194. doi: 10.1023/A:1010904214482

Souto-Maior, F. N., de Carvalho, F. L., Soares Lima de Morais, L. C., Netto, S. M., de Sousa, D. P., and de Almeida, R. N. (2011). Anxiolytic-like effects of inhaled linalool oxide in experimental mouse anxiety models. Pharmacol. Biochem. Behav. 100, 259-263. doi: 10.1016/j.pbb.2011.08.029

Tsang, H. W. H., and Ho, T. Y. C. (2010). A systematic review on the anxiolytic effects of aromatherapy on rodents under experimentally induced anxiety models. Rev. Neurosci. 21, 141-152. doi: 10.1515/REVNEURO.2010.21.2.141

Victoria, F. N., de Siqueira Brahm, A., Savegnago, L., and Lenardão, E. J. (2013). Involvement of serotoninergic and adrenergic systems on the antidepressant-like effect of E. uniflora $L$. leaves essential oil and further analysis of its antioxidant activity. Neurosci. Lett. 544, 105-109. doi: 10.1016/j.neulet.2013.03.054

Wheatley, D. (2005). Medicinal plants for insomnia: a review of their pharmacology, efficacy and tolerability. J. Psychopharmacol. 19, 414-421. doi: $10.1177 / 0269881105053309$

Woelk, H., and Schlaefke, S. (2010). A multi-center, double-blind, randomised study of the Lavender oil preparation Silexan in comparison to Lorazepam for generalized anxiety disorder. Phytomedicine 17, 94-99. doi: 10.1016/j.phymed.2009.10.006

Xu, P., Wang, K., Lu, C., Dong, L., Gao, L., Yan, M., et al. (2016). Protective effect of lavender oil on scopolamine induced cognitive deficits in mice and $\mathrm{H}_{2} \mathrm{O}_{2}$ induced cytotoxicity in PC12 cells. J. Ethnopharmacol. 193, 408-415 doi: 10.1016/j.jep.2016.08.030

Zhang, N., Zhang, L., Feng, L., and Yao, L. (2016). The anxiolytic effect of essential oil of Cananga odorata exposure on mice and determination of its major active constituents. Phytomedicine 23, 1727-1734. doi: 10.1016/j.phymed.2016.10.017

Conflict of Interest Statement: The authors declare that the research was conducted in the absence of any commercial or financial relationships that could be construed as a potential conflict of interest.

Copyright (c) 2017 López, Nielsen, Solas, Ramírez and Jäger. This is an open-access article distributed under the terms of the Creative Commons Attribution License (CC $B Y)$. The use, distribution or reproduction in other forums is permitted, provided the original author(s) or licensor are credited and that the original publication in this journal is cited, in accordance with accepted academic practice. No use, distribution or reproduction is permitted which does not comply with these terms. 


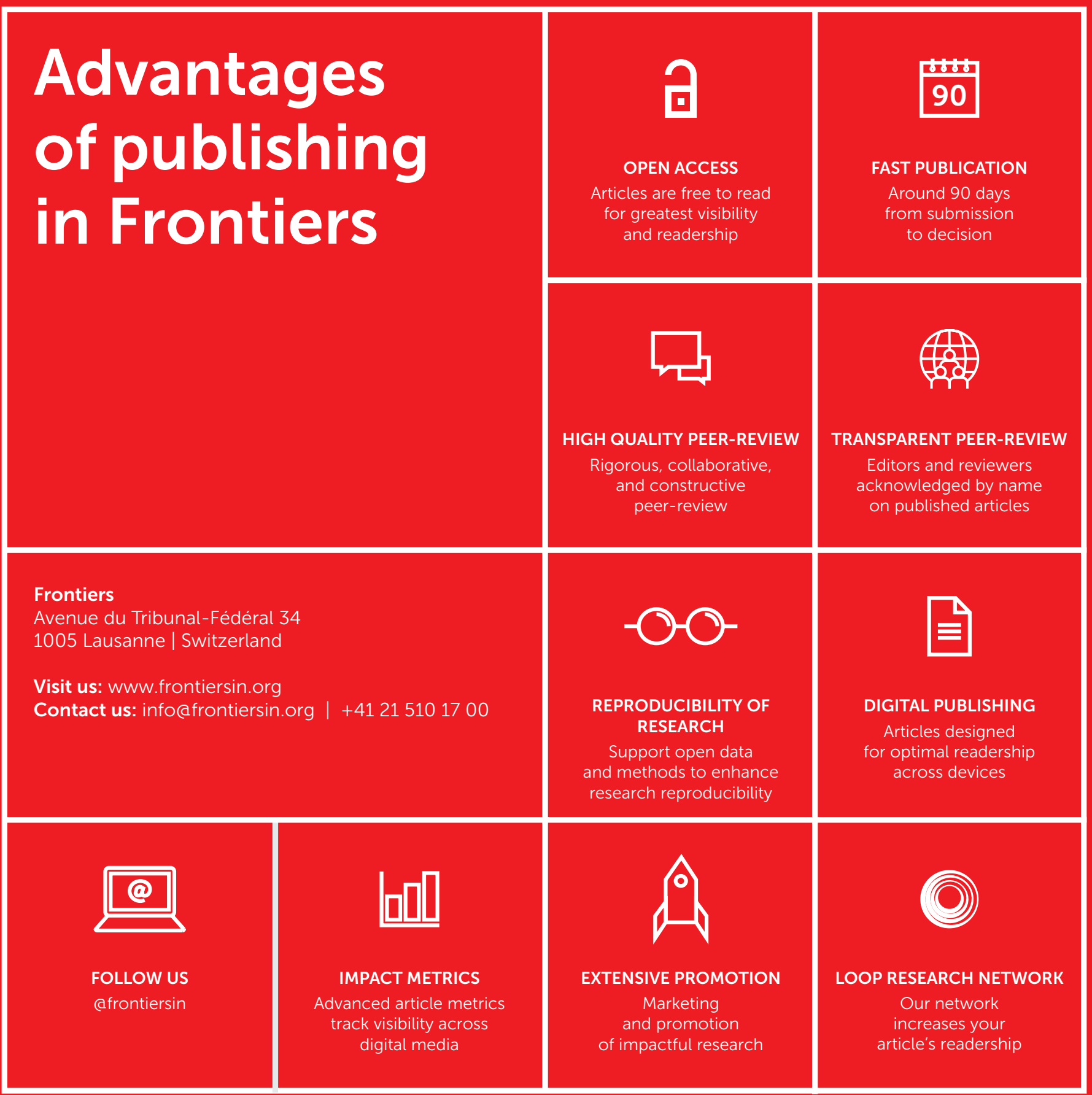

